\title{
Enantioselective Folding of Enynes by Gold(I) Catalysts with a Remote $C_{2}$-Chiral Element
}

\author{
Giuseppe Zuccarello, ${ }^{1,2}$ Joan G. Mayans, ${ }^{1,2}$ Imma Escofet, ${ }^{1,2}$ Dagmar Scharnagel, ${ }^{1}$ \\ Mariia S. Kirillova, ${ }^{1}$ Alba H. Pérez-Jimeno, ${ }^{1,2}$ Pilar Calleja, ${ }^{1}$ Jordan R. Boothe ${ }^{1,2}$ and \\ Antonio M. Echavarren ${ }^{1,2}$ \\ ${ }^{1}$ Institute of Chemical Research of Catalonia (ICIQ), Barcelona Institute of Science \\ and Technology, Av. Països Catalans 16, 43007 Tarragona (Spain). \\ ${ }^{2}$ Departament de Química Orgànica i Analítica, Universitat Rovira i Virgili, \\ C/Marcel·lí Domingo s/n, 43007 Tarragona (Spain). \\ SUPPLEMENTARY INFORMATION
}




\section{Table of contents}

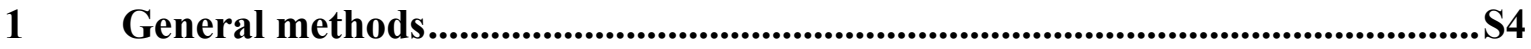

2 Synthesis of chiral complexes ...................................................................................56

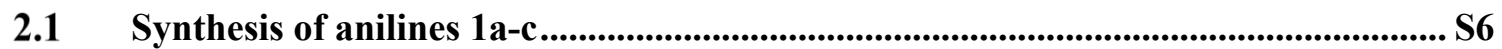

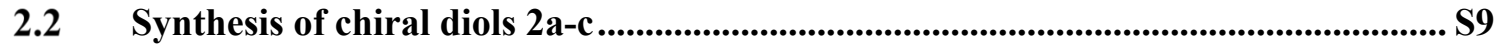

2.3 Synthesis of chiral complexes A-G .................................................................................... S16

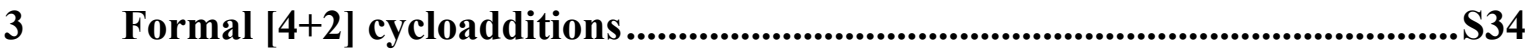

3.1 Preparation of 1,6-enynes 5b-q .......................................................................................... S34

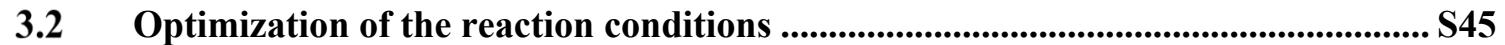

$3.3 \quad$ Scope of the formal [4+2] cyclization ............................................................................... S48

4 Synthesis of azabycyclo[4.1.0]hept-4-enes..........................................................S59

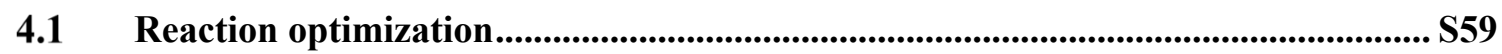

4.2 Scope of the cyclopropanation reaction ............................................................................. S59

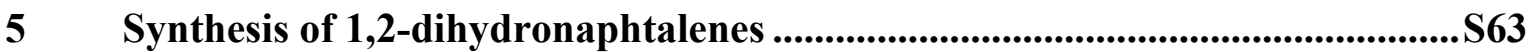

5.1 Synthesis of 1,6-enynes 9a-d, 9i-l and 17....................................................................... S63

5.2 Optimization of the reaction conditions ........................................................................... S72

$5.3 \quad$ Reaction scope ......................................................................................................................... S86

$6 \quad$ Enantioselective total syntheses of carexane I, $O$ and $P$...............................S94

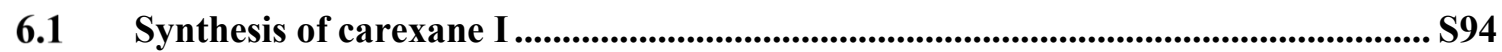

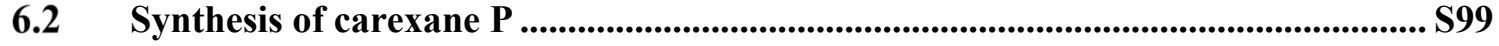

6.3 Synthesis of carexane O.................................................................................................. S102

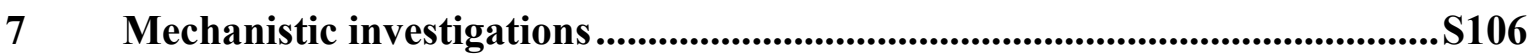

7.1 Hammett study ….................................................................................................................. S106

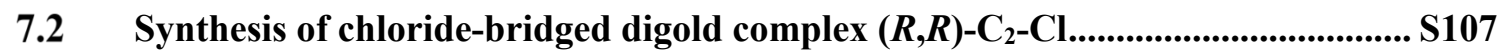

7.3 Evidence for the arene-gold(I) complex........................................................................... S108

7.4 Kinetic studies and reaction monitoring......................................................................... S113

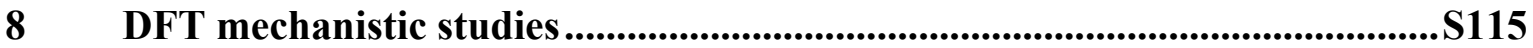

8.1 Computational methods ............................................................................................... S115

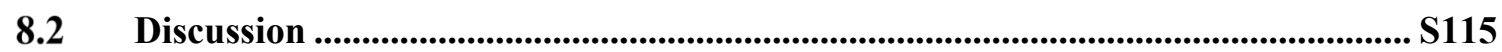

8.3 Coordinates and Energies of Intermediates and Transition States (BP86-D3) ... S120

9 X-ray crystallographic data. 


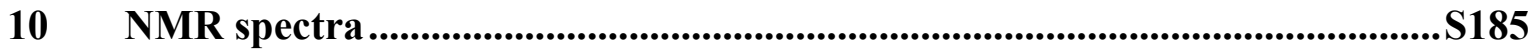

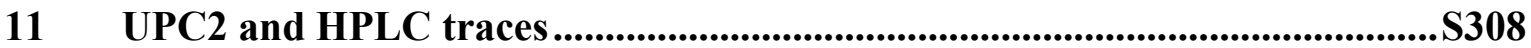

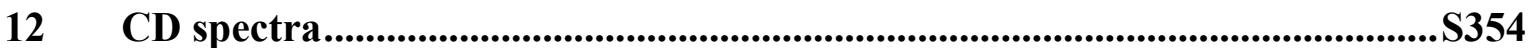




\section{General methods}

Unless otherwise stated, all the reactions were performed under an inert atmosphere of argon. All solvents and other chemicals were used as received. All the reactions were run in dry solvents (passed through an activated alumina column on a PureSolv ${ }^{\mathrm{TM}}$ solvent purification system). Reactions were followed using a GC-MS apparatus, by TLC (thin layer chromatography) or by NMR analysis. Analytical thin layer chromatography was carried out using TLC aluminum sheets coated with $0.2 \mathrm{~mm}$ of silica gel (Merck $60 \mathrm{~F}_{254}$ ) using UV light as the visualizing agent and an acidic solution of vanillin in ethanol or basic solution of $\mathrm{KMnO}_{4}$ in water as stain. Chromatographic purifications were carried out using flash grade silica gel (PanReac Silica Gel 60, 40-63 $\mu \mathrm{m}$ ) or automated flash column chromatographer CombiFlash Companion. Preparative thin layer chromatography was performed on TLC plates (Analtec Silica Gel GF UV254, 20×20 cm, $1000 \mu \mathrm{m})$. Melting points were determined using a Mettler Toledo MP70 melting point apparatus. NMR spectra were recorded at $298 \mathrm{~K}$ on BrukerAvance Ultrashield NMR spectrometers $(300 \mathrm{MHz}, 400 \mathrm{MHz}, 500 \mathrm{MHz}$ and $500 \mathrm{MHz}$ with CryoProbe). Chemical shifts $(\delta)$ are reported in parts per million (ppm) and referenced to residual solvent (For ${ }^{1} \mathrm{H}$ NMR: $\mathrm{CDCl}_{3}$ at $7.26 \mathrm{ppm}, \mathrm{CD}_{2} \mathrm{Cl}_{2}$ at $5.31 \mathrm{ppm}, \mathrm{C}_{6} \mathrm{D}_{6}$ at $7.16 \mathrm{ppm}$, $\mathrm{CD}_{3} \mathrm{OD}$ at $3.31 \mathrm{ppm}$, for ${ }^{13} \mathrm{C} \mathrm{NMR}$ : $\mathrm{CDCl}_{3}$ at $77.16 \mathrm{ppm}, \mathrm{CD}_{2} \mathrm{Cl}_{2}$ at $54.00 \mathrm{ppm}, \mathrm{C}_{6} \mathrm{D}_{6}$ at 128.06 ppm, $\mathrm{CD}_{3} \mathrm{OD}$ at $\left.49.00 \mathrm{ppm}\right)$. The following abbreviations were used to explain multiplicities: $\mathrm{s}=$ singlet, $\mathrm{d}=$ doublet, $\mathrm{t}=$ triplet, $\mathrm{q}=$ quartet, $\mathrm{p}=$ pentet, $\mathrm{m}=$ multiplet, $\mathrm{br} \mathrm{s}=$ broad singlet. Coupling constants $(J)$ are reported in Hertz $(\mathrm{Hz})$. Infrared spectra were recorded neat on a Bruker ALPHA FTIR-ATR TR0 spectrometer. The peaks are reported as absorption maxima $\left(\mathrm{n}, \mathrm{cm}^{-1}\right)$. Mass spectra were recorded on MicroTOF Focus or Maxis Impact spectrometers (both from Bruker Daltonics). Elemental analyses were performed on a LECO CHNS 932 microanalyzer at the Universidad Complutense de Madrid. Specific optical rotation measurements were carried out on a Jasco P-1030 model polarimeter equipped with a PMT detector using the sodium line at $589 \mathrm{~nm}$, and $1 \mathrm{~mL}$ (10 mm pathlength) or $2 \mathrm{~mL}$ (100 mm pathlength) cells. Chiral HPLC analyses were performed on an Agilent Technologies 1200 series, a Waters ACQUITY UPC2 System with diode array detector and by Chiral Technologies Europe analytical service. X-ray diffraction data were collected at $100 \mathrm{~K}$ on a Rigaku MicroMax-007HF, Mo Ka rotating anode, equipped with a Pilatus $200 \mathrm{~K}$ detector or on a Bruker APEX DUO, Mo $K \alpha$ Microfocus source E025 IuS anode, equipped with an APEX DUO detector using omega scans. Circular dichroism spectra measurements were carried out on an Applied Photophysics Chirascan 
Circular Dichroism spectrometer equipped with a photomultiplier detector, dual polarizing prism design monochromator, photo-elastic modulator (PEM) and 150W Xenon light source. 


\section{Synthesis of chiral complexes}

\subsection{Synthesis of anilines 1a-c}

\subsubsection{Synthesis of aniline 1a}

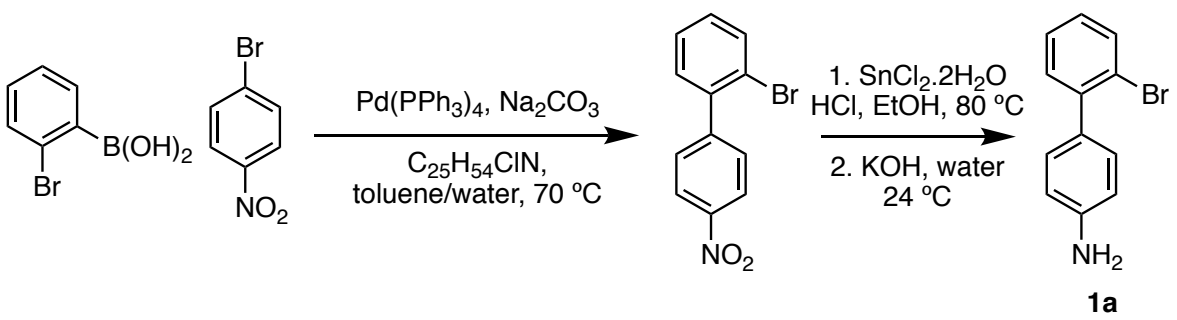

\section{2'-Bromo-[1,1'-biphenyl]-4-amine (1a)}

A two-necked round bottom flask equipped with condenser and stirring bar was charged with 1-bromo-4-nitrobenzene (12.5 g, $61.9 \mathrm{mmol}, 1.0$ equiv), (2-bromophenyl)boronic acid (13.1 g, 65.0 mmol, 1.05 equiv), $\mathrm{Na}_{2} \mathrm{CO}_{3}$ (26.2 g, $248 \mathrm{mmol}, 4.0$ equiv), methyltrioctylammonium chloride (2.5 g, $6.1 \mathrm{mmol}, 0.1$ equiv), $\mathrm{Pd}\left(\mathrm{PPh}_{3}\right)_{4}(358 \mathrm{mg}, 0.31 \mathrm{mmol}, 0.5 \mathrm{~mol} \%)$ and toluene $(248 \mathrm{~mL})$ and water $(124 \mathrm{~mL})$ were added. The reaction mixture was stirred at 70 ${ }^{\circ} \mathrm{C}$ for $2 \mathrm{~h}$. The reaction was monitored by TLC (cyclohexane/Et ${ }_{2} \mathrm{O} 10: 1$ ) and GC-MS analysis. After full conversion of the starting material the reaction was diluted with EtOAc. The phases were separated, the organic phase was washed with water $(2 \times 200 \mathrm{~mL})$, dried over $\mathrm{Na}_{2} \mathrm{SO}_{4}$, filtered and concentrated. The crude product was taken into the next step without further purification.

A solution of tin(II) chloride dihydrate $(73.0 \mathrm{~g}, 324 \mathrm{mmol}, 4.5$ equiv) in conc. $\mathrm{HCl}(81 \mathrm{~mL}$, 4M) was added to a solution of 2-bromo-4'-nitro-1,1'-biphenyl (20 g, $71.9 \mathrm{mmol}, 1.0$ equiv) in $\mathrm{EtOH}(160 \mathrm{~mL})$. The reaction mixture was stirred at $80^{\circ} \mathrm{C}$ for $2.5 \mathrm{~h}$. Then, the reaction mixture was cooled to $24{ }^{\circ} \mathrm{C}$ and diluted with water $(20 \mathrm{~mL})$. The precipitate was filtered off and a solution of $\mathrm{KOH}(66 \mathrm{~g})$ in water $(100 \mathrm{~mL})$ was added. The reaction mixture was stirred overnight at $24^{\circ} \mathrm{C}$. $\mathrm{Et}_{2} \mathrm{O}$ was added to the reaction, the aqueous phase was extracted with $\mathrm{Et}_{2} \mathrm{O}$ (2x), dried over $\mathrm{Na}_{2} \mathrm{SO}_{4}$, filtered and concentrated. The crude was purified by flash column chromatography (cyclohexane $/ \mathrm{CH}_{2} \mathrm{Cl}_{2}$ 1:1) to afford aniline $\mathbf{1 a}(10.3 \mathrm{~g}, 41.5 \mathrm{mmol}, 58 \%)$ as a pale brown viscous oil over two steps.

${ }^{1}$ H NMR $\left(500 \mathrm{MHz}, \mathrm{CDCl}_{3}\right) \delta 7.67-7.63(\mathrm{~m}, 1 \mathrm{H}), 7.34-7.30(\mathrm{~m}, 2 \mathrm{H}), 7.25-7.22(\mathrm{~m}$, 2H), 7.15 (ddd, $J=8.0,5.5,3.6 \mathrm{~Hz}, 1 \mathrm{H}), 6.77-6.71(\mathrm{~m}, 2 \mathrm{H}), 3.75$ (br s, 2H). ${ }^{13} \mathrm{C}$ NMR (101 $\left.\mathrm{MHz}, \mathrm{CDCl}_{3}\right) \delta 146.1,142.7,133.2,131.51,131.47,130.6,128.2,127.5,123.1,114.6$. HRMS (ESI+) calculated for $\left[\mathrm{C}_{12} \mathrm{H}_{11} \mathrm{BrN}\right]^{+} 248.0069 \mathrm{~m} / \mathrm{z}$; found $[\mathrm{M}+\mathrm{H}]^{+} 248.0069 \mathrm{~m} / z$. 


\subsubsection{Synthesis of aniline $1 \mathrm{~b}$}

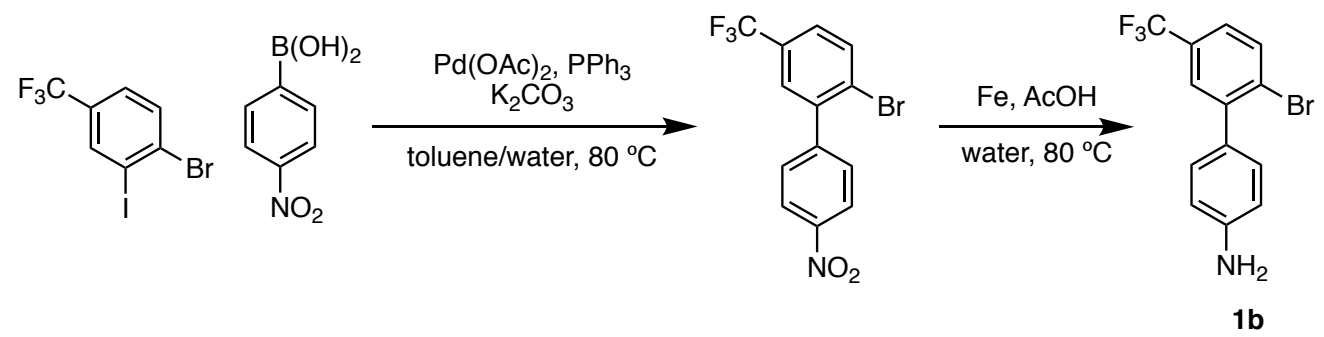

\section{2-Bromo-4'-nitro-5-(trifluoromethyl)-1,1'-biphenyl}

A Schlenk flask equipped with a stirring bar was charged with (4-nitrophenyl)boronic acid (1.00 g, $6.00 \mathrm{mmol}, 1.2$ equiv), 1-bromo-2-iodo-4-(trifluoromethyl)benzene (1.76 g, 5.00 mmol, 1.0 equiv), $\mathrm{K}_{2} \mathrm{CO}_{3}$ (1.38 g, $10.0 \mathrm{mmol}, 2.0$ equiv), $\mathrm{Pd}(\mathrm{OAc})_{2}$ (112 mg, $0.500 \mathrm{mmol}, 0.1$ equiv), $\mathrm{PPh}_{3}$ (262 mg, $1.00 \mathrm{mmol}, 0.2$ equiv) and toluene $(6 \mathrm{~mL})$ and water $(6 \mathrm{~mL})$ were added. The reaction mixture was stirred at $80{ }^{\circ} \mathrm{C}$ for $4 \mathrm{~d}$ and monitored by TLC (cyclohexane/ $\mathrm{CH}_{2} \mathrm{Cl}_{2}$ 9:1). The reaction mixture was diluted with EtOAc $(20 \mathrm{~mL})$ and the phases were separated. The organic phase was washed with water $(2 \times 10 \mathrm{~mL})$, dried over $\mathrm{Na}_{2} \mathrm{SO}_{4}$, filtered and concentrated. The crude product was purified by column chromatography (cyclohexane/ $\mathrm{CH}_{2} \mathrm{Cl}_{2}$ 9:1) to give the coupling product $(1.14 \mathrm{~g}, 3.29 \mathrm{mmol}, 66 \%)$ as an off-white solid.

M.p. $=98-101{ }^{\circ} \mathrm{C}\left(\right.$ cyclohexane $\left./ \mathrm{CH}_{2} \mathrm{Cl}_{2}\right) .{ }^{1} \mathbf{H}$ NMR $\left(400 \mathrm{MHz}, \mathrm{CDCl}_{3}\right) \delta 8.33(\mathrm{~d}, J=8.8$ $\mathrm{Hz}, 2 \mathrm{H}), 7.86(\mathrm{~d}, J=8.3 \mathrm{~Hz}, 1 \mathrm{H}), 7.64-7.51(\mathrm{~m}, 4 \mathrm{H}) .{ }^{13} \mathbf{C ~ N M R}\left(101 \mathrm{MHz}, \mathrm{CDCl}_{3}\right) \delta 147.9$, 146.1, 141.4, 134.3, 130.6, 130.3 (q, $J=52.2 \mathrm{~Hz}$ ), 127.7 (q, $J=3.6 \mathrm{~Hz}$ ), 126.7 (q, $J=3.5 \mathrm{~Hz}$ ), 126.3, 123.71, 123.67 (q, $J=125.0 \mathrm{~Hz}) .{ }^{19} \mathbf{F}\left\{{ }^{1} \mathbf{H}\right\}$ NMR $\left(376 \mathrm{MHz}, \mathrm{CDCl}_{3}\right) \delta-62.8 . \mathbf{H R M S}$ $(\mathrm{APCI}+)$ calculated for $\left[\mathrm{C}_{13} \mathrm{H}_{7} \mathrm{BrF}_{3} \mathrm{NO}_{2}\right]^{+} 344.9607 \mathrm{~m} / \mathrm{z}$; found $[\mathrm{M}]^{+}: 344.9601$.

\section{2'-Bromo-5'-(trifluoromethyl)-[1,1'-biphenyl]-4-amine (1b)}

2-Bromo-4'-nitro-5-(trifluoromethyl)-1,1'-biphenyl (1.13 g, $3.26 \mathrm{mmol}, 1.0$ equiv) was dissolved in acetic acid $(7 \mathrm{~mL})$ and water $(2.3 \mathrm{~mL})$ at $80{ }^{\circ} \mathrm{C}$ and iron $(1.82 \mathrm{~g}, 32.6 \mathrm{mmol}, 10$ equiv) was added in portions. The mixture was stirred for $1 \mathrm{~h}$ at $80^{\circ} \mathrm{C}$ and the solvent was removed under vacuum. The residue was suspended in $\mathrm{CH}_{2} \mathrm{Cl}_{2}(30 \mathrm{~mL})$ and $\mathrm{H}_{2} \mathrm{O}(20 \mathrm{~mL})$ and basified with $\mathrm{NaOH}(3 \mathrm{M}, 30 \mathrm{~mL})$. The aqueous phase was extracted with $\mathrm{CH}_{2} \mathrm{Cl}_{2}(2 \times 50 \mathrm{~mL})$ and the combined organic phases were washed with $\mathrm{H}_{2} \mathrm{O}(30 \mathrm{~mL})$, dried over $\mathrm{Na}_{2} \mathrm{SO}_{4}$ and concentrated to give aniline $\mathbf{1 b}(914 \mathrm{mg}, 2.89 \mathrm{mmol}, 89 \%)$ as a yellowish oil which solidified upon standing.

M.p. $=57-59{ }^{\circ} \mathrm{C}\left(\mathrm{CH}_{2} \mathrm{Cl}_{2}\right) .{ }^{1} \mathbf{H}$ NMR $\left(400 \mathrm{MHz}, \mathrm{CDCl}_{3}\right) \delta 7.77(\mathrm{~d}, J=8.3 \mathrm{~Hz}, 1 \mathrm{H}), 7.58$ $(\mathrm{d}, J=1.9 \mathrm{~Hz}, 1 \mathrm{H}), 7.40(\mathrm{dd}, J=8.3,1.9 \mathrm{~Hz}, 1 \mathrm{H}), 7.24(\mathrm{~d}, J=8.5 \mathrm{~Hz}, 2 \mathrm{H}), 6.76(\mathrm{~d}, J=8.5 \mathrm{~Hz}$, 
2H), 3.81 (br s, 2H). ${ }^{13} \mathbf{C}$ NMR (101 MHz, $\left.\mathrm{CDCl}_{3}\right) \delta 146.6,143.5,133.8,130.5,129.99,129.98$ (q, $J=33.0 \mathrm{~Hz}), 128.1$ (q, $J=3.5 \mathrm{~Hz}), 126.9,124.7$ (q, $J=3.5 \mathrm{~Hz}), 124.0(\mathrm{q}, J=273.1 \mathrm{~Hz})$, 114.6. ${ }^{19} \mathbf{F}\left\{{ }^{1} \mathbf{H}\right\}$ NMR $\left(376 \mathrm{MHz}, \mathrm{CDCl}_{3}\right) \delta-62.7$. HRMS (ESI+) calculated for $\left[\mathrm{C}_{13} \mathrm{H}_{10} \mathrm{BrF}_{3} \mathrm{~N}\right]^{+}$ $315.9943 \mathrm{~m} / \mathrm{z}$; found $[\mathrm{M}+\mathrm{H}]^{+} 315.9937 \mathrm{~m} / \mathrm{z}$.

\subsubsection{Synthesis of aniline 1c}

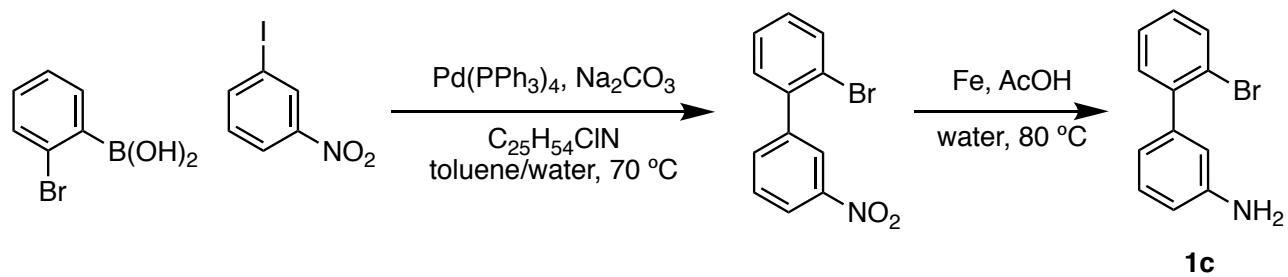

\section{2'-Bromo-[1,1'-biphenyl]-3-amine (1c)}

A two-necked round bottom flask equipped with condenser and stirring bar was charged with 1-iodo-3-nitrobenzene (10 g, $40.2 \mathrm{mmol}, 1.0$ equiv), 2-bromophenyl boronic acid ( $8.5 \mathrm{~g}$, $42.2 \mathrm{mmol}, 1.05$ equiv), $\mathrm{Na}_{2} \mathrm{CO}_{3}(17.03 \mathrm{~g}, 161 \mathrm{mmol}, 4.0$ equiv), methyltrioctylammonium chloride (1.6 g, $4.02 \mathrm{mmol}, 0.1$ equiv), $\mathrm{Pd}\left(\mathrm{PPh}_{3}\right)_{4}(0.5 \mathrm{~g}, 0.40 \mathrm{mmol}, 1 \mathrm{~mol} \%)$ and toluene (50 $\mathrm{mL})$ and water $(25 \mathrm{~mL})$ were added. The reaction mixture was stirred at $70{ }^{\circ} \mathrm{C}$ for $60 \mathrm{~h}$. The reaction was monitored by TLC (cyclohexane/ $\mathrm{Et}_{2} \mathrm{O}$ 10:1) and GC-MS analysis. After full conversion of the starting material the reaction was diluted with EtOAc. The phases were separated, the organic phase was washed with water $(2 \times 250 \mathrm{~mL})$, dried over $\mathrm{Na}_{2} \mathrm{SO}_{4}$, filtered and concentrated. The crude product was taken into the next step without further purification.

2-Bromo-3'-nitro-1,1'-biphenyl (1.0 g, $3.6 \mathrm{mmol}, 1.0$ equiv) was dissolved in acetic acid (6 $\mathrm{mL})$ and water $(2 \mathrm{~mL})$. Iron powder $\left(2.0 \mathrm{~g}, 36.0 \mathrm{mmol}, 10\right.$ equiv) was added at $80^{\circ} \mathrm{C}$ (attention: foaming). The mixture was stirred for $1 \mathrm{~h}$ at the same temperature and the solvent was removed under reduced pressure. The residue was partially dissolved in $\mathrm{CH}_{2} \mathrm{Cl}_{2}(35 \mathrm{~mL})$ and sat. $\mathrm{NaHCO}_{3}(20 \mathrm{~mL})$, and $\mathrm{NaOH}(200 \mathrm{~mL}, 10 \%)$ were added until basic $\mathrm{pH}$ was obtained. The phases were separated, the aqueous phase was extracted with $\mathrm{CH}_{2} \mathrm{Cl}_{2}(2 \mathrm{x} 100 \mathrm{~mL})$ and the organic phase was washed with water $(2 \times 100 \mathrm{~mL})$. The organic phase was dried over $\mathrm{Na}_{2} \mathrm{SO}_{4}$ and concentrated. The crude product was purified by flash column chromatography (cyclohexane $\left./ \mathrm{CH}_{2} \mathrm{Cl}_{2} 1: 1\right)$ to afford aniline $1 \mathrm{c}(0.90 \mathrm{~g}, 3.5 \mathrm{mmol}, 95 \%$, two steps) as a yellow solid.

M.p. $=52-54{ }^{\circ} \mathrm{C}\left(\right.$ cyclohexane $\left./ \mathrm{CH}_{2} \mathrm{Cl}_{2}\right) .{ }^{1} \mathbf{H}$ NMR $\left(400 \mathrm{MHz} \mathrm{CDCl}_{3}\right) \delta 7.65(\mathrm{~d}, J=8.0$ $\mathrm{Hz}, 1 \mathrm{H}), 7.36-7.29(\mathrm{~m}, 2 \mathrm{H}), 7.24-7.15(\mathrm{~m}, 2 \mathrm{H}), 6.82-6.76(\mathrm{~m}, 1 \mathrm{H}), 6.74-6.69(\mathrm{~m}, 2 \mathrm{H})$, 
3.72 (br s, 2H). ${ }^{13} \mathbf{C}$ NMR (126 MHz, $\left.\mathrm{CDCl}_{3}\right) \delta 146.3,143.2,142.6,133.4,131.5,129.3,129.0$, 127.6, 122.9, 120.2, 116.5, 114.7. HRMS (ESI+) calculated for $\left[\mathrm{C}_{12} \mathrm{H}_{11} \mathrm{BrN}\right]^{+} 248.0069$, found $[\mathrm{M}+\mathrm{H}]^{+} 248.0070$.

\subsection{Synthesis of chiral diols 2a-c}

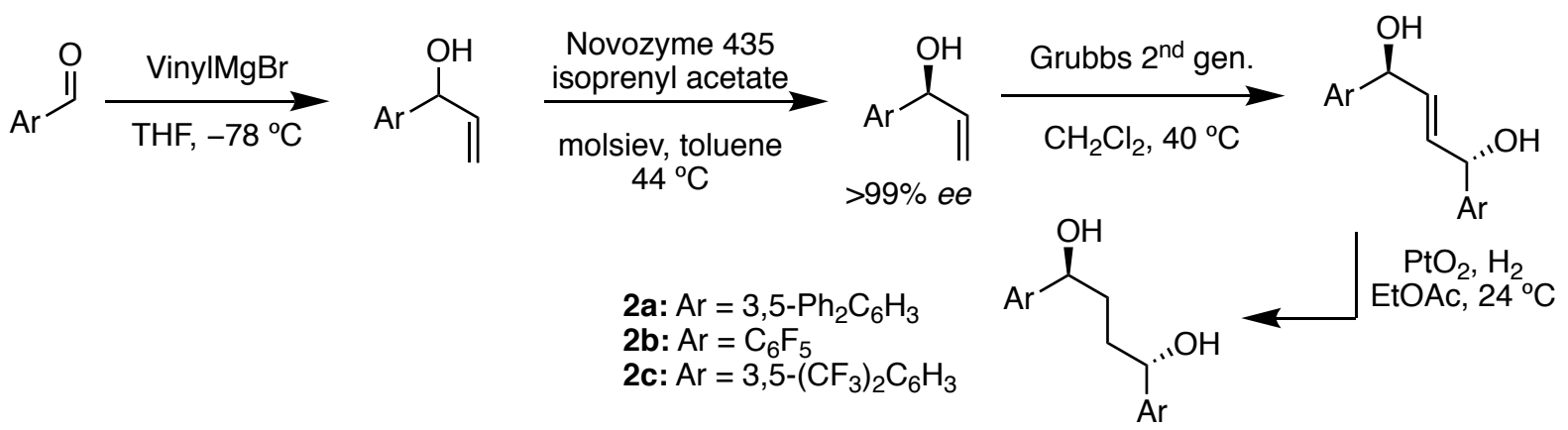

\subsubsection{Synthesis of diol $2 a$}

\section{1-([1,1':3',1''-Terphenyl]-5'-yl)prop-2-en-1-ol}<smiles>C=CC(O)c1cc(-c2ccccc2)cc(-c2ccccc2)c1</smiles>

[1,1':3',1"-Terphenyl]-5'-carbaldehyde ${ }^{1}$ (1.9 g, $7.4 \mathrm{mmol}, 1.0$ equiv) was dissolved in THF $(52 \mathrm{~mL})$ in a flame-dried two-necked $250 \mathrm{~mL}$ round bottom flask and the solution was cooled to $-78{ }^{\circ} \mathrm{C}$. Vinylmagnesium bromide $(8.1 \mathrm{~mL}, 8.1 \mathrm{mmol}, 1.1$ equiv, $1 \mathrm{M}$ in THF) was added dropwise to the solution and the reaction mixture was stirred at the same temperature for $2 \mathrm{~h}$. The reaction was monitored by TLC (cyclohexane/EtOAc 4:1) and after full conversion of the starting material the reaction was allowed to warm up to $24^{\circ} \mathrm{C}$, diluted with $\mathrm{Et}_{2} \mathrm{O}$ and quenched with sat. $\mathrm{NH}_{4} \mathrm{Cl}$ solution. The aqueous phase was extracted with $\mathrm{Et}_{2} \mathrm{O}(3 \mathrm{x})$ and the combined organic phases were dried over $\mathrm{Na}_{2} \mathrm{SO}_{4}$, filtered and concentrated. The crude was purified by flash column chromatography (cyclohexane/EtOAc 7:1) to yield the title compound (1.75 g, $6.1 \mathrm{mmol}, 83 \%)$ as a white solid.

M.p. $=100-102{ }^{\circ} \mathrm{C}\left(\right.$ cyclohexane/EtOAc). ${ }^{1} \mathbf{H}$ NMR $\left(500 \mathrm{MHz}, \mathrm{CDCl}_{3}\right) \delta 7.74(\mathrm{t}, J=1.8$ $\mathrm{Hz}, 1 \mathrm{H}), 7.68-7.63(\mathrm{~m}, 4 \mathrm{H}), 7.60(\mathrm{~d}, J=1.7 \mathrm{~Hz}, 2 \mathrm{H}), 7.49-7.44(\mathrm{~m}, 4 \mathrm{H}), 7.41-7.36$ (m, 2H), $6.15(\mathrm{ddd}, J=17.1,10.3,6.0 \mathrm{~Hz}, 1 \mathrm{H}), 5.45(\mathrm{dt}, J=17.1,1.4 \mathrm{~Hz}, 1 \mathrm{H}), 5.38-5.34(\mathrm{~m}, 1 \mathrm{H})$, $5.26(\mathrm{dt}, J=10.3,1.3 \mathrm{~Hz}, 1 \mathrm{H}), 2.06(\mathrm{~d}, J=3.4 \mathrm{~Hz}, 1 \mathrm{H}) .{ }^{13} \mathbf{C ~ N M R}\left(126 \mathrm{MHz}, \mathrm{CDCl}_{3}\right) \delta 143.8$, 142.3, 141.0, 133.5, 128.9, 127.6, 127.4, 125.7, 124.2, 74.6. HRMS (ESI+) calculated for $\left[\mathrm{C}_{21} \mathrm{H}_{18} \mathrm{NaO}\right]^{+} 309.1250 \mathrm{~m} / z$; found $[\mathrm{M}+\mathrm{Na}]^{+} 309.1245 \mathrm{~m} / z$. 
(S)-1-([1,1':3',1'-Terphenyl]-5'-yl)prop-2-en-1-ol<smiles>C=CC(O)c1cc(-c2ccccc2)cc(-c2ccccc2)c1</smiles>

A $250 \mathrm{~mL}$ two-necked round bottom flask equipped with a stirring bar was charged with powdered $4 \AA$ molecular sieve $\left(1.6 \mathrm{~g}\right.$ ) and heated to $200{ }^{\circ} \mathrm{C}$ under high vacuum (ca $0.1 \mathrm{mbar}$ ) during $40 \mathrm{~min}^{2}$ The flask was allowed to cool to $24{ }^{\circ} \mathrm{C}$ under argon and novozyme 435 resin (357 mg, >5000 U/g) was added. The solids were suspended in anhydrous toluene $(120 \mathrm{~mL})$ and isoprenyl acetate $(8.5 \mathrm{~mL}, 76 \mathrm{mmol}, 4.55$ equiv) was added followed by addition of 1([1,1':3',1"-terphenyl]-5'-yl)prop-2-en-1-ol (4.81 g, $16.8 \mathrm{mmol}, 1.0$ equiv) in toluene (18 mL). The resulting mixture was stirred at $44{ }^{\circ} \mathrm{C}$ for $37 \mathrm{~h}$. The solvent was removed under vacuum and the crude product was purified by flash column chromatography (cyclohexane/EtOAc 20:1, then $10: 1)$ to yield the title compound (3.33 g, $8.14 \mathrm{mmol}, 49 \%$ ) as a white solid in $>99: 1 \mathrm{er}$.

M.p. $=101-103{ }^{\circ} \mathrm{C}($ cyclohexane/EtOAc) $) \boldsymbol{\alpha}_{\mathbf{D}}{ }^{\mathbf{5 8 9}}=+6.2 \mathrm{deg} \cdot \mathrm{cm}^{2} \cdot \mathrm{g}^{-1}\left(\mathrm{CHCl}_{3}, \mathrm{c} 0.98,299\right.$ K). HPLC Chiralpak IA $(250 \times 4.6 \mathrm{~mm}, 5 \mu \mathrm{m})$ at $25^{\circ} \mathrm{C}$, flow $1.0 \mathrm{~mL} / \mathrm{min}$, isocratic hexane/EtOH 98:2, $220 \mathrm{~nm}, \mathrm{t}_{\mathrm{R}}$ (major) 22.6; $\mathrm{t}_{\mathrm{R}}$ (minor) 23.7.

\section{$(1 S, 4 S, E)-1,4-D i\left(\left[1,1^{\prime}: 3^{\prime}, 1^{\prime \prime}-\right.\right.$-terphenyl]-5'-yl)but-2-ene-1,4-diol}<smiles>OC(/C=C/C(O)c1cc(-c2ccccc2)cc(-c2ccccc2)c1)c1cc(-c2ccccc2)cc(-c2ccccc2)c1</smiles>

(S)-1-([1,1':3',1"-Terphenyl]-5'-yl)prop-2-en-1-ol (2.31 g. $8.1 \mathrm{mmol}, 1.0$ equiv) was dissolved in $\mathrm{CH}_{2} \mathrm{Cl}_{2}(8.1 \mathrm{~mL})$ and Grubbs $2^{\text {nd }}$ gen. catalyst (68 $\mathrm{mg}, 81 \mu \mathrm{mol}, 1 \mathrm{~mol} \%$ ) was added. The reaction mixture was stirred for $3 \mathrm{~h}$ at $40{ }^{\circ} \mathrm{C}$. The crude was purified by flash column chromatography (cyclohexane/EtOAc 2:1) and then further purified by precipitation from cold $n$ hexane, filtered and dried to afford the title compound $(1.31 \mathrm{~g}, 2.4 \mathrm{mmol}, 60 \%)$ as a white solid.

M.p. $=145-147^{\circ} \mathrm{C}($ hexane/Et $2 \mathrm{O}) .{ }^{1} \mathbf{H}$ NMR $\left(500 \mathrm{MHz}, \mathrm{CDCl}_{3}\right) \delta 7.73(\mathrm{t}, J=1.7 \mathrm{~Hz}, 2 \mathrm{H})$, $7.62-7.54(\mathrm{~m}, 12 \mathrm{H}), 7.43-7.37(\mathrm{~m}, 8 \mathrm{H}), 7.37-7.32(\mathrm{~m}, 4 \mathrm{H}), 6.16(\mathrm{dd}, J=3.4,1.6 \mathrm{~Hz}, 2 \mathrm{H})$, $5.36(\mathrm{~s}, 2 \mathrm{H}), 2.56(\mathrm{~s}, 2 \mathrm{H}) .{ }^{13} \mathbf{C} \mathbf{N M R}\left(126 \mathrm{MHz}, \mathrm{CDCl}_{3}\right) \delta 143.8,142.3,141.0,133.5,128.9$, 127.6, 127.4, 125.8, 124.2, 74.6. HRMS (ESI+) calculated for $\left[\mathrm{C}_{40} \mathrm{H}_{32} \mathrm{NaO}_{2}\right]^{+} 567.2295 \mathrm{~m} / \mathrm{z}$; found $[\mathrm{M}+\mathrm{Na}]^{+} 567.2283 \mathrm{~m} / z \cdot \boldsymbol{\alpha}_{\mathbf{D}}^{\mathbf{5 8 9}}=+22.8 \mathrm{deg} \cdot \mathrm{cm}^{2} \cdot \mathrm{g}^{-1}\left(\mathrm{CHCl}_{3}, \mathrm{c} 1.0,298 \mathrm{~K}\right)$. 


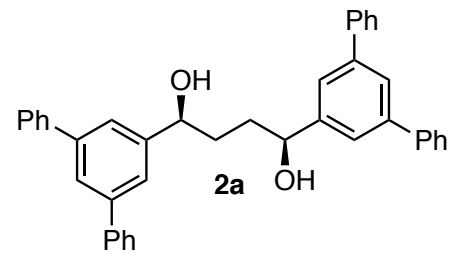

A $20 \mathrm{~mL}$ microwave vial was charged with $\mathrm{PtO}_{2}(12 \mathrm{mg}, 0.06 \mathrm{mmol}, 5 \mathrm{~mol} \%)$ and evacuated and back-filled with argon (3x). A solution of $(1 S, 4 S, E)-1,4-\operatorname{di}\left(\left[1,1^{\prime}: 3^{\prime}, 1^{\prime \prime}-\right.\right.$ terphenyl]-5'-yl)but2-ene-1,4-diol (0.59 g, $1.1 \mathrm{mmol}, 1.0$ equiv) in EtOAc $(10.9 \mathrm{~mL})$ was added and the reaction mixture was evacuated and back-filled with hydrogen. The reaction was stirred at $24^{\circ} \mathrm{C}$ for $1 \mathrm{~h}$ under hydrogen atmosphere ( $1 \mathrm{~atm})$. The reaction was monitored by ${ }^{1} \mathrm{H}$ NMR analysis and after full conversion of the starting material, the reaction mixture was filtered over Celite ${ }^{\circledR}$ and concentrated. The crude was purified by flash column chromatography (cyclohexane/EtOAc $3: 1)$ to yield the title compound $\mathbf{2 a}(0.56 \mathrm{~g}, 1.0 \mathrm{mmol}, 94 \%)$ as a white solid.

M.p. $=83-85^{\circ} \mathrm{C}$ (cyclohexane/EtOAc). ${ }^{1} \mathbf{H}$ NMR $\left(400 \mathrm{MHz}, \mathrm{CDCl}_{3}\right) \delta 7.71(\mathrm{t}, J=1.7 \mathrm{~Hz}$, 2H), $7.66-7.60(\mathrm{~m}, 8 \mathrm{H}), 7.57(\mathrm{~d}, J=1.7 \mathrm{~Hz}, 4 \mathrm{H}), 7.48-7.40(\mathrm{~m}, 8 \mathrm{H}), 7.40-7.33(\mathrm{~m}, 4 \mathrm{H})$, $4.92-4.86$ (m, 2H), 2.41 (br s, 2H), $2.16-1.96$ (m, 4H). $\left.{ }^{13} \mathbf{C ~ N M R ~ ( 1 0 1 ~ M H z , ~} \mathrm{CDCl}_{3}\right) \delta 145.9$, 142.2, 141.1, 128.9, 127.6, 127.4, 125.6, 123.7, 74.9, 36.2. HRMS (ESI+) calculated for $\left[\mathrm{C}_{40} \mathrm{H}_{34} \mathrm{NaO}_{2}\right]^{+} 569.2451 \mathrm{~m} / z$; found $[\mathrm{M}+\mathrm{Na}]^{+} 569.2459 \mathrm{~m} / z . \boldsymbol{\alpha}_{\mathbf{D}}{ }^{\mathbf{5 8 9}}=-7.6 \mathrm{deg} \cdot \mathrm{cm}^{2} \cdot \mathrm{g}^{-1}\left(\mathrm{CHCl}_{3}\right.$, c $1.0,297 \mathrm{~K})$.

\subsubsection{Synthesis of diol $2 \mathrm{~b}$}

\section{1-(Perfluorophenyl)prop-2-en-1-ol}

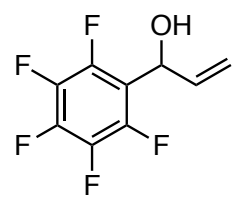

2,3,4,5,6-Pentafluorobenzaldehyde ( $3.2 \mathrm{~mL}, 25.5 \mathrm{mmol}, 1.0$ equiv) was dissolved in THF $(128 \mathrm{~mL})$ in a flame-dried $500 \mathrm{~mL}$ two-necked round bottom flask. The solution was cooled to $-78^{\circ} \mathrm{C}$ and vinylmagnesium bromide $(28.1 \mathrm{~mL}, 28.1 \mathrm{mmol}, 1.1$ equiv, $1 \mathrm{M}$ in THF) was added dropwise. The reaction mixture was stirred at the same temperature for $1 \mathrm{~h}$. The reaction was monitored by TLC (cyclohexane/Et ${ }_{2} \mathrm{O} 10: 1$ ). The reaction was allowed to warm to $24{ }^{\circ} \mathrm{C}$, diluted with $\mathrm{Et}_{2} \mathrm{O}$ and quenched with sat. $\mathrm{NH}_{4} \mathrm{Cl}$ solution. The phases were separated and the aqueous phase was extracted with $\mathrm{Et}_{2} \mathrm{O}(3 \mathrm{x})$. The combined organic phases were dried over $\mathrm{Na}_{2} \mathrm{SO}_{4}$, filtered and concentrated. The crude was purified by flash column chromatography (pentane/Et $\left.{ }_{2} \mathrm{O} 10: 1\right)$ to yield the title compound (4.7 g, $20.8 \mathrm{mmol}, 79 \%$ ) as a colorless oil. 
${ }^{1} \mathbf{H}$ NMR $\left(500 \mathrm{MHz}, \mathrm{CD}_{3} \mathrm{OD}\right) \delta 6.27-6.18(\mathrm{~m}, 1 \mathrm{H}), 5.56(\mathrm{~d}, J=6.0 \mathrm{~Hz}, 1 \mathrm{H}), 5.36(\mathrm{~d}, J=$ $17.1,1 \mathrm{H}), 5.25(\mathrm{dt}, J=10.4,1.3 \mathrm{~Hz}, 1 \mathrm{H}) .{ }^{13} \mathbf{C}$ NMR $\left(101 \mathrm{MHz}, \mathrm{CD}_{3} \mathrm{OD}\right) \delta 144.8(\mathrm{dm}, J=$ 248.4 Hz), $140.6(\mathrm{dm}, J=251.5 \mathrm{~Hz}), 137.5(\mathrm{dm}, J=250.4 \mathrm{~Hz}), 137.2,117.3$ - 116.7 (m), 115.4, 65.8. ${ }^{19} \mathbf{F}\left\{{ }^{1} \mathbf{H}\right\}$ NMR $\left(471 \mathrm{MHz}, \mathrm{CD}_{3} \mathrm{OD}\right) \delta-145.0--145.2(\mathrm{~m}),-158.9(\mathrm{t}, J=20.0 \mathrm{~Hz}),-165.4$ $--165.6(\mathrm{~m})$. HRMS (APCI+) calculated for $\left[\mathrm{C}_{9} \mathrm{H}_{4} \mathrm{~F}_{5}\right]^{+} 207.0228 \mathrm{~m} / \mathrm{z}$; found $[\mathrm{M}-\mathrm{OH}]^{+}$ $207.0222 \mathrm{~m} / \mathrm{z}$.

\section{(S)-1-(Perfluorophenyl)prop-2-en-1-ol}<smiles>C=CC(O)c1c(F)c(F)c(F)c(F)c1F</smiles>

A $250 \mathrm{~mL}$ two-necked round bottom flask equipped with a stirring bar was charged with powdered $4 \AA$ molecular sieve $(1.6 \mathrm{~g})$ and heated to $200^{\circ} \mathrm{C}$ under high vacuum $(0.1 \mathrm{mbar})$ during $40 \mathrm{~min} .{ }^{2}$ The flask was allowed to cool to $24{ }^{\circ} \mathrm{C}$ under argon and novozyme $435 \mathrm{resin}(351 \mathrm{mg}$, $>5000 \mathrm{U} / \mathrm{g})$ was added. The solids were suspended in anhydrous toluene $(137 \mathrm{~mL})$ and isoprenyl acetate ( $8.3 \mathrm{~mL}, 74.9 \mathrm{mmol}, 4.55$ equiv) was added followed by addition of 1(perfluorophenyl)prop-2-en-1-ol (3.7 g, $16.5 \mathrm{mmol}, 1.0$ equiv) in toluene $(5 \mathrm{~mL})$. The resulting mixture was stirred at $45{ }^{\circ} \mathrm{C}$ for $48 \mathrm{~h}$. The solvent was removed under vacuum and the crude product was purified by flash column chromatography (cyclohexane/EtOAc 30:1) to yield the title compound as a colorless oil (1.6 g, $7.1 \mathrm{mmol}, 43 \%)$ in 99:1 er.

$\boldsymbol{\alpha}_{\mathbf{D}}{ }^{\mathbf{5 8 9}}=+17.9 \mathrm{deg} \cdot \mathrm{cm}^{2} \cdot \mathrm{g}^{-1}\left(\mathrm{CHCl}_{3}, \mathrm{c} 0.9,298 \mathrm{~K}\right)$. HPLC Chiralpak IA $(250 \times 4.6 \mathrm{~mm}, 5 \mu \mathrm{m})$ at $25{ }^{\circ} \mathrm{C}$, flow $1.0 \mathrm{~mL} / \mathrm{min}$, isocratic hexane $/ \mathrm{PrOH} 98: 2,254 \mathrm{~nm}, \mathrm{t}_{\mathrm{R}}$ (major) $9.9 ; \mathrm{t}_{\mathrm{R}}$ (minor) 10.9 .

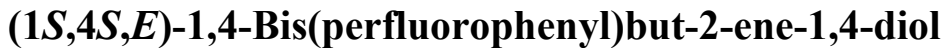<smiles>OC(/C=C/C(O)c1c(F)c(F)c(F)c(F)c1F)c1c(F)c(F)c(F)c(F)c1F</smiles>

(S)-1-(Perfluorophenyl)prop-2-en-1-ol (3.0 g, $13.4 \mathrm{mmol}, 1.0$ equiv) was dissolved in $\mathrm{CH}_{2} \mathrm{Cl}_{2}(13.4 \mathrm{~mL}, 1 \mathrm{M})$ and Grubbs $2^{\text {nd }}$ gen. catalyst $(57 \mathrm{mg}, 0.067 \mathrm{mmol}, 0.5 \mathrm{~mol} \%$ ) was added. The reaction mixture was stirred for $2 \mathrm{~h}$ at $40^{\circ} \mathrm{C}$. The crude was directly loaded on silica and purified by flash column chromatography (cyclohexane/EtOAc 5:1). The compound was 
further purified by precipitation from $\mathrm{CHCl}_{3}$ to afford the title compound $(1.54 \mathrm{~g}, 3.7 \mathrm{mmol}$, $55 \%)$ as a white solid.

M.p. $=125-127^{\circ} \mathrm{C}$ (cyclohexane/EtOAc). ${ }^{1} \mathbf{H}$ NMR $\left(500 \mathrm{MHz}, \mathrm{CD}_{2} \mathrm{Cl}_{2}\right) \delta 6.19-6.10(\mathrm{~m}$, $2 \mathrm{H}), 5.68-5.59(\mathrm{~m}, 2 \mathrm{H}), 2.55(\mathrm{~d}, J=6.7 \mathrm{~Hz}, 2 \mathrm{H}) .{ }^{13} \mathbf{C} \mathbf{N M R}\left(126 \mathrm{MHz}, \mathrm{CD}_{2} \mathrm{Cl}_{2}\right) \delta 144.8(\mathrm{dm}$, $J=248.9 \mathrm{~Hz}), 141.0(\mathrm{dm}, J=253.8 \mathrm{~Hz}), 137.7(\mathrm{dm}, J=252.7 \mathrm{~Hz}), 131.1,115.8-115.2(\mathrm{~m})$, 65.4. ${ }^{19} \mathbf{F}\left\{{ }^{1} \mathbf{H}\right\}$ NMR $\left(471 \mathrm{MHz}, \mathrm{CD}_{2} \mathrm{Cl}_{2}\right) \delta-143.7$ (dd, $\left.J=21.6,7.6 \mathrm{~Hz}\right),-155.3(\mathrm{t}, J=20.7$ $\mathrm{Hz}),-162.3--162.6(\mathrm{~m})$. HRMS (APCI+) calculated for $\left[\mathrm{C}_{16} \mathrm{H}_{5} \mathrm{~F}_{10} \mathrm{O}\right]^{+} 403.0175 \mathrm{~m} / \mathrm{z}$; found $[\mathrm{M}-\mathrm{OH}]^{+} 403.0168 \mathrm{~m} / z . \boldsymbol{\alpha}_{\mathbf{D}}{ }^{\mathbf{5 8 9}}=-6.7 \mathrm{deg} \cdot \mathrm{cm}^{2} \cdot \mathrm{g}^{-1}\left(\mathrm{CH}_{2} \mathrm{Cl}_{2}, \mathrm{c} 1.1,298 \mathrm{~K}\right)$.

\section{$(1 S, 4 S)-1,4-B i s(p e r f l u o r o p h e n y l) b u t a n e-1,4-d i o l ~(2 b)$}<smiles>OC(CCC(O)c1c(F)c(F)c(F)c(F)c1F)c1c(F)c(F)c(F)c(F)c1F</smiles>

A $100 \mathrm{~mL}$ round bottom flask equipped with a stirring bar was charged with $\mathrm{PtO}_{2}(46 \mathrm{mg}$, $0.21 \mathrm{mmol}, 5 \mathrm{~mol} \%)$ and the flask was evacuated and back filled with argon (3x). Then, a solution of (1S,4S,E)-1,4-bis(perfluorophenyl)but-2-ene-1,4-diol (1.72 g, $4.1 \mathrm{mmol}, 1.0$ equiv) in EtOAc $(41 \mathrm{~mL})$ was added. The reaction mixture was evacuated and back filled with hydrogen $(3 \mathrm{x})$ and stirred at $24{ }^{\circ} \mathrm{C}$ for $1 \mathrm{~h}$ under hydrogen atmosphere $(1 \mathrm{~atm})$. The reaction was monitored by ${ }^{1} \mathrm{H}$ NMR and after full conversion of the starting material the reaction was degassed with argon and filtered over Celite ${ }^{\circledR}$. The solvent was removed under vacuum and the crude was purified by flash column chromatography (cyclohexane/EtOAc 3:1) to afford the title compound $\mathbf{2 b}$ (1.67 g, $3.96 \mathrm{mmol}, 97 \%)$ as a white solid.

M.p. $=104-106{ }^{\circ} \mathrm{C}($ EtOAc $) .{ }^{1} \mathbf{H}$ NMR $\left(500 \mathrm{MHz}, \mathrm{CDCl}_{3}\right) \delta 5.16-5.06(\mathrm{~m}, 1 \mathrm{H}), 2.68(\mathrm{~d}$, $J=6.7 \mathrm{~Hz}, 2 \mathrm{H}), 2.35-2.20(\mathrm{~m}, 2 \mathrm{H}), 1.92-1.80(\mathrm{~m}, 2 \mathrm{H}) .{ }^{13} \mathbf{C} \mathbf{N M R}\left(126 \mathrm{MHz}, \mathrm{CDCl}_{3}\right) \delta$ $144.7(\mathrm{dm}, J=248.3 \mathrm{~Hz}), 140.7(\mathrm{dm}, J=254.7 \mathrm{~Hz}), 137.7(\mathrm{dm}, J=253.7 \mathrm{~Hz}), 116.8-116.3$ (m), 66.0, 33.5. ${ }^{19} \mathbf{F}\left\{{ }^{1} \mathbf{H}\right\}$ NMR $\left(376 \mathrm{MHz}, \mathrm{CD}_{3} \mathrm{OD}\right) \delta-147.0(\mathrm{dd}, J=21.5,7.9 \mathrm{~Hz}),-160.7$ (dt, $J=29.4,20.0 \mathrm{~Hz}),-166.9--167.1(\mathrm{~m})$. HRMS (ESI+) calculated for $\left[\mathrm{C}_{16} \mathrm{H}_{8} \mathrm{~F}_{10} \mathrm{NaO}_{2}\right]^{+}$ $445.0257 \mathrm{~m} / z$; found $[\mathrm{M}+\mathrm{Na}]^{+} 445.0248 \mathrm{~m} / z . \boldsymbol{\alpha}_{\mathbf{D}}{ }^{\mathbf{5 8 9}}=+6.5 \mathrm{deg} \cdot \mathrm{cm}^{2} \cdot \mathrm{g}^{-1}\left(\mathrm{CHCl}_{3}, \mathrm{c} 1.22,299\right.$ $\mathrm{K})$. 


\subsubsection{Synthesis of diol $2 \mathrm{c}$}

\section{1-(3,5-Bis(trifluoromethyl)phenyl)prop-2-en-1-ol}<smiles>C=CC(O)c1cc(C(F)(F)F)cc(C(F)(F)F)c1</smiles>

A $250 \mathrm{~mL}$ two-necked round bottom flask equipped with a stirring bar was flame-dried, charged with 3,5-bis(trifluoromethyl)benzaldehyde (3.4 mL, $20.7 \mathrm{mmol}, 1.0$ equiv) and THF $(103 \mathrm{~mL})$ was added under an argon atmosphere. Vinylmagnesium bromide $(23.0 \mathrm{~mL}, 23$ mmol, 1.1 equiv, $1 \mathrm{M}$ in THF) was added at $-78{ }^{\circ} \mathrm{C}$ dropwise and the resulting mixture was stirred for $2 \mathrm{~h}$ at the same temperature. The reaction was monitored by TLC (cyclohexane/Et ${ }_{2} \mathrm{O}$ 5:1) and GC-MS analysis. The reaction was allowed to warm up to $24{ }^{\circ} \mathrm{C}$, diluted with $\mathrm{Et}_{2} \mathrm{O}$ and quenched with $\mathrm{NH}_{4} \mathrm{Cl}$. The aqueous phase was extracted with $\mathrm{Et}_{2} \mathrm{O}(3 \mathrm{x} 150 \mathrm{~mL})$, dried over $\mathrm{Na}_{2} \mathrm{SO}_{4}$ and concentrated. The crude was purified by flash column chromatography (cyclohexane/EtOAc 10:1) to afford the title compound (4.5 g, $20.3 \mathrm{mmol}, 79 \%$ ) as a white solid.

M.p. $36-38{ }^{\circ} \mathrm{C}$ (cyclohexane/EtOAc). ${ }^{1} \mathbf{H}$ NMR $\left(400 \mathrm{MHz}, \mathrm{CDCl}_{3}\right) \delta 7.85(\mathrm{~s}, 2 \mathrm{H}), 7.80(\mathrm{~s}$, 1H), $6.05-5.95(\mathrm{~m}, 1 \mathrm{H}), 5.44(\mathrm{dt}, J=17.2,1.1 \mathrm{~Hz}, 1 \mathrm{H}), 5.35-5.30(\mathrm{~m}, 2 \mathrm{H}), 2.07$ (br s, 1H).${ }^{13} \mathbf{C}$ NMR $\left(101 \mathrm{MHz}, \mathrm{CDCl}_{3}\right) \delta 145.0,139.1,131.9$ (q, $\left.J=33.3 \mathrm{~Hz}\right), 126.6(\mathrm{~d}, J=4.1 \mathrm{~Hz})$, $123.5(\mathrm{q}, J=272.6), 121.7(\mathrm{p}, J=3.9 \mathrm{~Hz}), 117.6,74.5 .{ }^{19} \mathbf{F}\left\{{ }^{1} \mathbf{H}\right\}$ NMR $\left(376 \mathrm{MHz}, \mathrm{CDCl}_{3}\right) \delta-$ 63.0. HRMS (EI+) calculated for $\left[\mathrm{C}_{11} \mathrm{H}_{7} \mathrm{~F}_{6} \mathrm{O}\right]^{+} 269.0401 \mathrm{~m} / \mathrm{z}$; found $[\mathrm{M}-\mathrm{H}]^{+} 269.0460 \mathrm{~m} / z$.

\section{(S)-1-(3,5-Bis(trifluoromethyl)phenyl)prop-2-en-1-ol}<smiles>C=CC(O)c1cc(C(F)(F)F)cc(C(F)(F)F)c1</smiles>

A $250 \mathrm{~mL}$ two-necked round bottom flask equipped with a stirring bar was charged with powdered $4 \AA$ molecular sieve $(2.5 \mathrm{~g})$ and heated to $200{ }^{\circ} \mathrm{C}$ under high vacuum (ca $0.1 \mathrm{mbar}$ ) during 40 minutes. ${ }^{2}$ The flask was allowed to cool to $24{ }^{\circ} \mathrm{C}$ under argon and novozyme 435 resin $(548 \mathrm{mg},>5000 \mathrm{U} / \mathrm{g}$ ) was added. The solids were suspended in anhydrous toluene (222 $\mathrm{mL}$ ) and isoprenyl acetate $(13.0 \mathrm{~mL}, 117 \mathrm{mmol}, 4.55$ equiv) was added followed by addition of 1-(3,5-bis(trifluoromethyl)phenyl)prop-2-en-1-ol (6.95 g, $25.7 \mathrm{mmol}, 1.0$ equiv) in toluene (7 $\mathrm{mL}$ ). The resulting mixture was stirred at $45^{\circ} \mathrm{C}$ for $16 \mathrm{~h}$. The solvent was removed under vacuum and the crude product was purified by flash column chromatography 
(cyclohexane/EtOAc 20:1) to yield the title compound (3.43 g, $12.7 \mathrm{mmol}, 49 \%$ ) as a white solid in >99:1 er.

M.p. $=51-53{ }^{\circ} \mathrm{C}$ (cyclohexane/EtOAc). $\boldsymbol{\alpha}_{\mathbf{D}}{ }^{\mathbf{5 8 9}}=+26.0$ deg. $\mathrm{cm}^{2} \cdot \mathrm{g}^{-1}\left(\mathrm{CHCl}_{3}, \mathrm{c} 1.1,298 \mathrm{~K}\right)$. HPLC Chiralpak OD-H $(250 \times 4.6 \mathrm{~mm}, 5 \mu \mathrm{m})$ at $25{ }^{\circ} \mathrm{C}$, flow $1.0 \mathrm{~mL} / \mathrm{min}$, isocratic hexane/iPrOH 99:1, $220 \mathrm{~nm}, \mathrm{t}_{\mathrm{R}}$ (major) 12.4 ; $\mathrm{t}_{\mathrm{R}}$ (minor) 13.8.

\section{(1S,4S,E)-1,4-Bis(3,5-bis(trifluoromethyl)phenyl)but-2-ene-1,4-diol}<smiles>OC(/C=C/C(O)c1cc(C(F)(F)F)cc(C(F)(F)F)c1)c1cc(C(F)(F)F)cc(C(F)(F)F)c1</smiles>

$(S)$-1-(3,5-Bis(trifluoromethyl)phenyl)prop-2-en-1-ol (3.4 g, $12.4 \mathrm{mmol}, 1.0$ equiv) was dissolved in $\mathrm{CH}_{2} \mathrm{Cl}_{2}(12.5 \mathrm{~mL}, 1 \mathrm{M})$ and Grubbs $2^{\text {nd }}$ gen. catalyst $(106 \mathrm{mg}, 0.12 \mathrm{mmol}, 1 \mathrm{~mol}$ $\%$ ) was added. The reaction mixture was stirred for $3 \mathrm{~h}$ at $40{ }^{\circ} \mathrm{C}$. The product was precipitated from cold $n$ hexane and filtered to afford the title compound $(2.4 \mathrm{~g}, 4.7 \mathrm{mmol}, 75 \%)$ as a white solid.

M.p. $=168-170{ }^{\circ} \mathrm{C}$ (nhexane). ${ }^{1} \mathbf{H}$ NMR $\left(400 \mathrm{MHz}, \mathrm{CD}_{3} \mathrm{OD}\right) \delta 7.95(\mathrm{~s}, 4 \mathrm{H}), 7.86(\mathrm{~s}, 2 \mathrm{H})$, $6.08-5.99(\mathrm{~m}, 2 \mathrm{H}), 5.45-5.35(\mathrm{~m}, 2 \mathrm{H}) .{ }^{13} \mathrm{C}$ NMR (101 MHz, CD $\left.3 \mathrm{OD}\right) \delta 148.5,134.5,132.8$ $(\mathrm{q}, J=33.2 \mathrm{~Hz}), 127.9-127.5(\mathrm{~m}), 124.9(\mathrm{q}, J=271.7 \mathrm{~Hz}) 122.4-121.6(\mathrm{~m}), 73.5 .{ }^{19} \mathbf{F}\left\{{ }^{1} \mathbf{H}\right\}$ NMR (376 MHz, CD $\left.{ }_{3} \mathrm{OD}\right) \delta-64.5$. HRMS (ESI-) calculated for $\left[\mathrm{C}_{20} \mathrm{H}_{11} \mathrm{~F}_{12} \mathrm{O}_{2}\right]^{-}$511. 0573 $m / z$; found $[\mathrm{M}-\mathrm{H}]^{-} 511.0586 \mathrm{~m} / z . \boldsymbol{\alpha}_{\mathbf{D}}{ }^{589}=+12.1 \mathrm{deg} \cdot \mathrm{cm}^{2} \cdot \mathrm{g}^{-1}(\mathrm{MeOH}, \mathrm{c} 0.95,298 \mathrm{~K})$.

\section{(1S,4S)-1,4-Bis(3,5-bis(trifluoromethyl)phenyl)butane-1,4-diol (2c)}<smiles>OC(CCC(O)c1cc(C(F)(F)F)cc(C(F)(F)F)c1)c1cc(C(F)(F)F)cc(C(F)(F)F)c1</smiles>

A $100 \mathrm{~mL}$ round bottom flask equipped with a stirring bar was charged with $\mathrm{PtO}_{2}(53 \mathrm{mg}$, $0.233 \mathrm{mmol}, 5 \mathrm{~mol} \%$ ) and the flask was evacuated and back filled with argon (3x). Then, a solution of $(1 S, 4 S, E)$-1,4-bis(3,5-bis(trifluoromethyl)phenyl)but-2-ene-1,4-diol (2.4 g, 4.67 mmol, 1.0 equiv) in EtOAc $(47 \mathrm{~mL})$ was added. The reaction mixture was evacuated and back filled with hydrogen $(3 \mathrm{x})$ and stirred at $24^{\circ} \mathrm{C}$ for $1 \mathrm{~h}$ under hydrogen atmosphere ( $\left.1 \mathrm{~atm}\right)$. The reaction was monitored by TLC (cyclohexane/EtOAc 2:1) and ${ }^{1} \mathrm{H}$ NMR. After full conversion of the starting material the reaction was degassed with argon and filtered over Celite ${ }^{\circledR}$. The 
solvent was removed under vacuum and the crude was purified by flash column chromatography (cyclohexane/EtOAc 3:1) to afford the title compound $2 \mathrm{c}$ (2.3 g, $4.54 \mathrm{mmol}$, 97\%) as a white solid.

M.p. $=116-118{ }^{\circ} \mathrm{C}$ (cyclohexane/EtOAc). ${ }^{1} \mathbf{H}$ NMR $\left(400 \mathrm{MHz}, \mathrm{CDCl}_{3}\right) \delta 7.82(\mathrm{~s}, 4 \mathrm{H})$, 7.80 (s, 2H), $4.98-4.86$ (m, 2H), 3.03 (br s, 2H), $2.05-1.82(\mathrm{~m}, 4 \mathrm{H}) .{ }^{13} \mathrm{C}$ NMR (101 MHz, $\left.\mathrm{CDCl}_{3}\right) \delta 147.1,132.0(\mathrm{q}, J=33.4 \mathrm{~Hz}), 126.2-125.9(\mathrm{~m}), 123.4(\mathrm{q}, \mathrm{J}=271.5 \mathrm{~Hz}) 121.8(\mathrm{p}, J$ $=3.8 \mathrm{~Hz}), 73.5,36.1 .{ }^{19} \mathbf{F}\left\{{ }^{1} \mathbf{H}\right\}$ NMR $\left(376 \mathrm{MHz}, \mathrm{CD}_{2} \mathrm{Cl}_{2}\right) \delta-63.3$. HRMS (ESI-) calculated for $\left[\mathrm{C}_{20} \mathrm{H}_{13} \mathrm{~F}_{12} \mathrm{O}_{2}\right]^{-} 513.0729 \mathrm{~m} / z$; found $[\mathrm{M}-\mathrm{H}]^{-} 513.0738 \mathrm{~m} / z . \boldsymbol{\alpha}_{\mathbf{D}}{ }^{\mathbf{5 8 9}}=-36.2 \mathrm{deg} \cdot \mathrm{cm}^{2} . \mathrm{g}^{-1}\left(\mathrm{CHCl}_{3}\right.$, $0.64,298 \mathrm{~K})$.

\subsection{Synthesis of chiral complexes A-G}
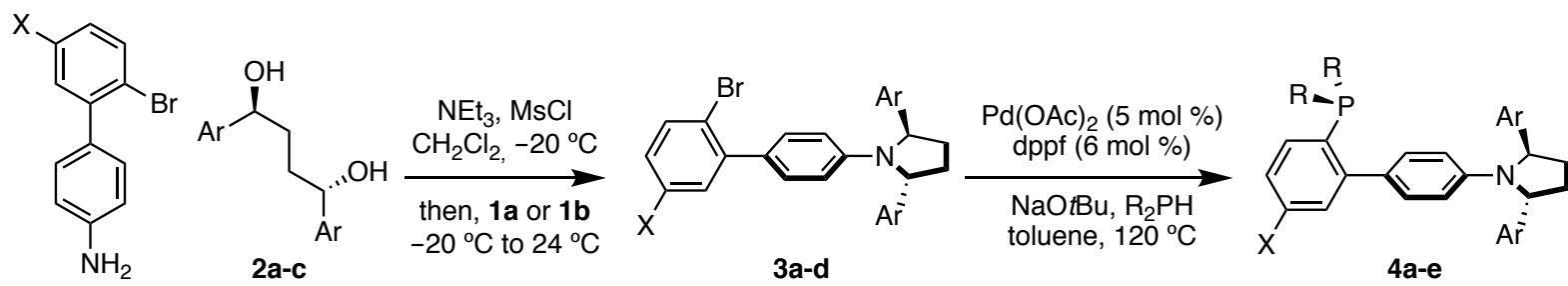

1a: $X=\mathrm{H}$

1a: $\mathrm{X}=\mathrm{H}$
1b: $\mathrm{X}=\mathrm{CF}_{3}$
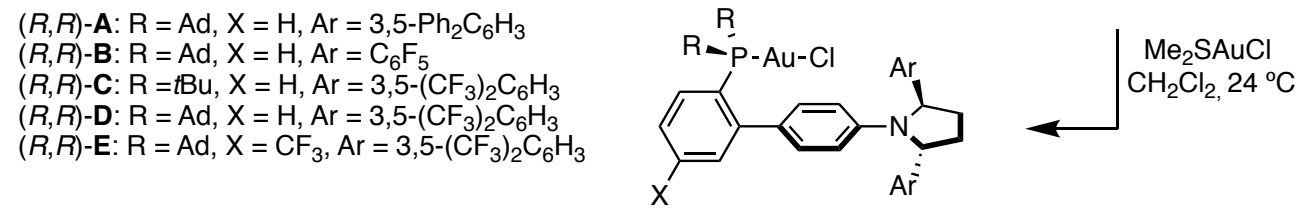

\subsubsection{Synthesis of chiral complex $(R, R)-A$}

\section{Chiral precursor 3a}

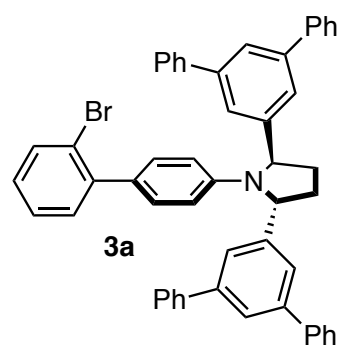

To a solution of $(1 S, 4 S)$-1,4-di([1,1':3',1"-terphenyl]-5'-yl)butane-1,4-diol (2a) (1.21 g, 2.21 mmol, 1.0 equiv) in $\mathrm{CH}_{2} \mathrm{Cl}_{2}(22 \mathrm{~mL})$ at $-20{ }^{\circ} \mathrm{C}$ were added $\mathrm{NEt}_{3}(0.93 \mathrm{~mL}, 6.64 \mathrm{mmol}, 3.0$ equiv) and methanesulfonyl chloride $\left(0.45 \mathrm{~mL}, 5.75 \mathrm{mmol}, 2.6\right.$ equiv) under anhydrous $\mathrm{N}_{2}$ atmosphere. The mixture was stirred for $2.5 \mathrm{~h}$ at $-20^{\circ} \mathrm{C}$. Then, a solution of aniline $1 \mathrm{a}(2.7 \mathrm{~g}$, $10.84 \mathrm{mmol}, 4.9$ equiv) in $\mathrm{CH}_{2} \mathrm{Cl}_{2}(4 \mathrm{~mL})$ was added and the resulting solution was stirred at $24{ }^{\circ} \mathrm{C}$ overnight. The volatile products were removed under reduced pressure and the organic 
materials were dissolved in EtOAc. The organic phase was washed with brine and sat. $\mathrm{NaHCO}_{3}$, dried over $\mathrm{Na}_{2} \mathrm{SO}_{4}$ and concentrated. The excess aniline 1a was removed by flash column chromatography (cyclohexane/ $\mathrm{CH}_{2} \mathrm{Cl}_{2} 3: 1$, then 1:1). The title compound was further purified by washing with $\mathrm{CH}_{2} \mathrm{Cl}_{2} / \mathrm{Et} 2 \mathrm{O}$ mixtures to yield $\mathbf{3 a}(973 \mathrm{mg}, 1.3 \mathrm{mmol}, 58 \%$ ) as a white solid.

M.p. $=228-230{ }^{\circ} \mathrm{C}\left(\mathrm{CH}_{2} \mathrm{Cl}_{2} /\right.$ pentane $) .{ }^{1} \mathbf{H}$ NMR $\left(500 \mathrm{MHz}, \mathrm{CDCl}_{3}\right) \delta 7.72-7.68(\mathrm{~m}, 2 \mathrm{H})$, $7.64-7.59(\mathrm{~m}, 9 \mathrm{H}), 7.50-7.44(\mathrm{~m}, 12 \mathrm{H}), 7.41-7.35(\mathrm{~m}, 4 \mathrm{H}), 7.29-7.24(\mathrm{~m}, 2 \mathrm{H}), 7.18-$ $7.14(\mathrm{~m}, 2 \mathrm{H}), 7.11-7.06(\mathrm{~m}, 1 \mathrm{H}), 6.59-6.54(\mathrm{~m}, 2 \mathrm{H}), 5.44(\mathrm{~d}, J=6.8 \mathrm{~Hz}, 2 \mathrm{H}), 2.83-2.63$ (m, 2H), $2.11-1.97(\mathrm{~m}, 2 \mathrm{H}) .{ }^{13} \mathbf{C}$ NMR $\left(126 \mathrm{MHz}, \mathrm{CDCl}_{3}\right) \delta 144.9,144.7,143.1,142.2,141.4$, $133.1,131.4,130.1,129.0,127.8,127.6,127.5,127.3,124.9,124.3,123.3,113.7,63.6,32.8$. HRMS (ESI+) calculated for $\left[\mathrm{C}_{52} \mathrm{H}_{41} \mathrm{BrN}\right]^{+} 758.2417 \mathrm{~m} / \mathrm{z}$; found $[\mathrm{M}+\mathrm{H}]^{+} 758.2431 \mathrm{~m} / \mathrm{z} \boldsymbol{\alpha}_{\mathbf{D}}{ }^{\mathbf{5 8 9}}$ $=+75.0$ deg. $\mathrm{cm}^{2} \cdot \mathrm{g}^{-1}\left(\mathrm{CHCl}_{3}, \mathrm{c} 0.95,299 \mathrm{~K}\right)$.

\section{Ligand 4a}

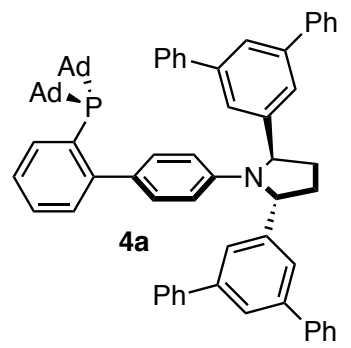

A flame-dried $10 \mathrm{~mL}$ Schlenk flask was charged with $3 \mathbf{a}$ (170 mg, $0.22 \mathrm{mmol}, 1.0$ equiv), $\mathrm{Pd}(\mathrm{OAc})_{2}(2.5 \mathrm{mg}, 11 \mu \mathrm{mol}, 5 \mathrm{~mol} \%)$, dppf(7.5 mg, $\left.13 \mu \mathrm{mol}, 6 \mathrm{~mol} \%\right)$, NaOt $\mathrm{Bu}(26 \mathrm{mg}, 0.27$ mmol, 1.2 equiv) and toluene $(1.2 \mathrm{~mL})$ and the resulting suspension was stirred for $15 \mathrm{~min}$ at $24{ }^{\circ} \mathrm{C}$. Di(1-adamantyl)phosphine (75 mg, $0.25 \mathrm{mmol}, 1.1$ equiv) was added and the reaction mixture was stirred at $120^{\circ} \mathrm{C}$ for $16 \mathrm{~h}$. Then, the reaction was allowed to cool to $24{ }^{\circ} \mathrm{C}$ and the solids were removed by filtration. The crude was dissolved in EtOAc and activated charcoal was added. The mixture was stirred for 15 min at $24{ }^{\circ} \mathrm{C}$, filtered over Celite ${ }^{\circledR}$ and concentrated. The residue was dissolved in $\mathrm{Et}_{2} \mathrm{O}$ and precipitated with $\mathrm{MeOH}$ to yield ligand $4 \mathbf{a}$ (140 mg, $0.14 \mathrm{mmol}, 64 \%$ ) as a white solid.

M.p. $=>110^{\circ} \mathrm{C}$ (decomposition; methanol). ${ }^{1} \mathbf{H}$ NMR $\left(400 \mathrm{MHz}, \mathrm{CD}_{2} \mathrm{Cl}_{2}\right) \delta 7.81(\mathrm{dd}, J=$ 7.5, 1.6 Hz, 1H), $7.75-7.71(\mathrm{~m}, 2 \mathrm{H}), 7.69-7.62(\mathrm{~m}, 8 \mathrm{H}), 7.55-7.51(\mathrm{~m}, 4 \mathrm{H}), 7.50-7.43$ (m, 8H), $7.40-7.34(\mathrm{~m}, 4 \mathrm{H}), 7.29-7.18(\mathrm{~m}, 2 \mathrm{H}), 7.17-7.11(\mathrm{~m}, 1 \mathrm{H}), 7.02-6.95(\mathrm{~m}, 2 \mathrm{H})$, $6.53(\mathrm{dd}, J=8.7,1.8 \mathrm{~Hz}, 2 \mathrm{H}), 5.49(\mathrm{~d}, J=6.6 \mathrm{~Hz}, 2 \mathrm{H}), 2.86-2.71(\mathrm{~m}, 2 \mathrm{H}), 2.07-1.95$ (m, $2 \mathrm{H}), 1.94-1.87$ (m, 3H), 1.84 (br s, 6H), $1.78-1.68$ (m, 8H), 1.64 (br s, 6H), $1.57-1.48$ (m, 7H). ${ }^{13} \mathrm{C}$ NMR $\left(126 \mathrm{MHz}, \mathrm{CDCl}_{3}\right) \delta 152.3(\mathrm{~d}, J=32.0 \mathrm{~Hz}), 145.5,143.5,142.1,141.4,136.7$ 
$(\mathrm{d}, J=2.6 \mathrm{~Hz}), 133.6,133.4,131.8(\mathrm{~d}, J=7.0 \mathrm{~Hz}), 131.4(\mathrm{~d}, J=4.3 \mathrm{~Hz}), 131.2(\mathrm{~d}, J=5.9 \mathrm{~Hz})$, $128.9,128.1,127.5,124.7,124.5,124.3,113.0,63.6,42.4$ (d, $J=13.5 \mathrm{~Hz}), 41.7$ (d, $J=13.2$ $\mathrm{Hz}), 37.5(\mathrm{~d}, J=29.0 \mathrm{~Hz}), 37.3(\mathrm{~d}, \mathrm{~J}=29.0 \mathrm{~Hz}), 37.1,37.0,32.9,29.1$ (d, $J=8.6 \mathrm{~Hz}), 28.9$ (d, $J=8.5 \mathrm{~Hz}) .{ }^{31} \mathbf{P}\left\{{ }^{1} \mathbf{H}\right\}$ NMR $\left(162 \mathrm{MHz}, \mathrm{CDCl}_{3}\right) \delta$ 24.8. HRMS (ESI+) calculated for $\left[\mathrm{C}_{72} \mathrm{H}_{71} \mathrm{NP}\right]^{+} 980.5319 \mathrm{~m} / z$; found $[\mathrm{M}+\mathrm{H}]^{+} 980.5300 \mathrm{~m} / z . \boldsymbol{\alpha}_{\mathbf{D}}{ }^{\mathbf{5 8 9}}=+33.1 \mathrm{deg} \cdot \mathrm{cm}^{2} \cdot \mathrm{g}^{-1}\left(\mathrm{CHCl}_{3}\right.$, c $0.96,302 \mathrm{~K})$.

\section{$(\boldsymbol{R}, \boldsymbol{R})-\mathrm{A}$}

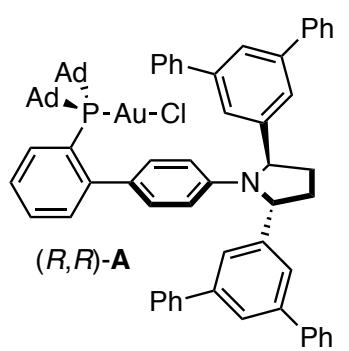

$\mathrm{Me}_{2} \mathrm{SAuCl}$ (14 mg, $46 \mu \mathrm{mol}, 1.0$ equiv) and ligand $4 \mathbf{a}$ (45 mg, $46 \mu \mathrm{mol}, 1.0$ equiv) were dissolved in $\mathrm{CH}_{2} \mathrm{Cl}_{2}(1.0 \mathrm{~mL})$ under an atmosphere of argon. The reaction mixture was stirred at $24{ }^{\circ} \mathrm{C}$ for $15 \mathrm{~min}$. The mixture was filtered through a syringe filter and concentrated. The residue was dissolved in $\mathrm{CH}_{2} \mathrm{Cl}_{2}$, filtered through a path of neutral $\mathrm{Al}_{2} \mathrm{O}_{3}$ and concentrated to yield complex $(R, R)-\mathbf{A}(38 \mathrm{mg}, 31 \mu \mathrm{mol}, 62 \%)$ as a white solid.

M.p. $=>160{ }^{\circ} \mathrm{C}\left(\right.$ decomposition; pentane/hexane). ${ }^{1} \mathbf{H} \mathbf{N M R}\left(500 \mathrm{MHz}, \mathrm{CD}_{2} \mathrm{Cl}_{2}\right) \delta 7.81$ (td, $J=7.3,1.6 \mathrm{~Hz}, 1 \mathrm{H}), 7.76-7.70(\mathrm{~m}, 10 \mathrm{H}), 7.62(\mathrm{~s}, 4 \mathrm{H}), 7.50-7.44(\mathrm{~m}, 8 \mathrm{H}), 7.44-7.39(\mathrm{~m}$, 2H), $7.39-7.35$ (m, 4H), 7.22 (ddd, $J=7.5,4.3,1.7 \mathrm{~Hz}, 1 \mathrm{H}), 6.89$ (dd, $J=8.5,2.2 \mathrm{~Hz}, 1 \mathrm{H})$, $6.82(\mathrm{dd}, J=8.4,2.2 \mathrm{~Hz}, 1 \mathrm{H}), 6.57(\mathrm{dd}, J=8.4,2.6 \mathrm{~Hz}, 1 \mathrm{H}), 6.53(\mathrm{dd}, J=8.5,2.6 \mathrm{~Hz}, 1 \mathrm{H})$, $5.48(\mathrm{~d}, J=6.5 \mathrm{~Hz}, 2 \mathrm{H}), 2.84-2.73(\mathrm{~m}, 2 \mathrm{H}), 2.32-2.24(\mathrm{~m}, 3 \mathrm{H}), 2.24-2.17(\mathrm{~m}, 3 \mathrm{H}), 2.07-$ $1.94(\mathrm{~m}, 9 \mathrm{H}), 1.94-1.90(\mathrm{~m}, 2 \mathrm{H}), 1.89-1.83(\mathrm{~m}, 3 \mathrm{H}), 1.74-1.64(\mathrm{~m}, 6 \mathrm{H}), 1.58-1.50(\mathrm{~m}$, $6 \mathrm{H}) .{ }^{13} \mathrm{C}$ NMR $\left(126 \mathrm{MHz}, \mathrm{CD}_{2} \mathrm{Cl}_{2}\right) \delta 151.8(\mathrm{~d}, J=13.0 \mathrm{~Hz}), 146.3,145.7,142.5,141.7,135.1$ (d, $J=2.1 \mathrm{~Hz}), 134.6$ (d, $J=7.1 \mathrm{~Hz}), 130.6$ (d, $J=2.0 \mathrm{~Hz}), 130.3(\mathrm{~d}, J=6.6 \mathrm{~Hz}), 130.2,130.0$, $129.3,128.0,127.9,126.1$ (d, $J=6.6 \mathrm{~Hz}), 125.0,124.7,124.5,124.2,116.1,113.9,64.7,43.3$ (d, $J=23.7 \mathrm{~Hz}), 43.1$ (d, $J=2.5 \mathrm{~Hz}), 42.7$ (d, $J=23.4 \mathrm{~Hz}), 42.3$ (d, $J=2.4 \mathrm{~Hz}), 36.8$ (d, $J=$ 21.4 Hz), 33.3, 29.4 (d, $J=9.8 \mathrm{~Hz}), 29.2$ (d, $J=9.8 \mathrm{~Hz}) .{ }^{31} \mathbf{P}\left\{{ }^{1} \mathbf{H}\right\} \mathbf{N M R}\left(162 \mathrm{MHz}, \mathrm{CD}_{2} \mathrm{Cl}_{2}\right) \delta$ 65.3. HRMS (ESI+) calculated for $\left[\mathrm{C}_{72} \mathrm{H}_{70} \mathrm{AuNP}\right]^{+} 1176.4906 \mathrm{~m} / \mathrm{z}$; found $[\mathrm{M}-\mathrm{Cl}]^{+} 1176.4909$ $m / z \cdot \boldsymbol{\alpha}_{\mathbf{D}}{ }^{589}=+66.6$ deg. $\mathrm{cm}^{2} \mathrm{~g}^{-1}\left(\mathrm{CHCl}_{3}, \mathrm{c} 0.87,302 \mathrm{~K}\right)$. 


\subsubsection{Synthesis of chiral complex $(R, R)-\mathrm{B}$}

\section{Chiral precursor $3 \mathbf{b}$}

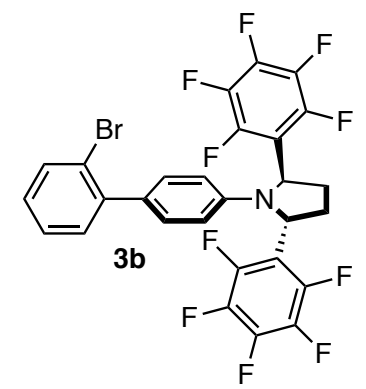

To a solution of (1S,4S)-1,4-bis(perfluorophenyl)butane-1,4-diol (2b) (1.5 g, 3.6 mmol, 1.0 equiv) in $\mathrm{CH}_{2} \mathrm{Cl}_{2}(36 \mathrm{~mL})$ at $-20{ }^{\circ} \mathrm{C}$ were added $\mathrm{NEt}_{3}(1.5 \mathrm{~mL}, 10.8 \mathrm{mmol}, 3.0$ equiv) and methanesulfonyl chloride $(0.72 \mathrm{~mL}, 9.4 \mathrm{mmol}, 2.6 \mathrm{mmol})$ under anhydrous $\mathrm{N}_{2}$ atmosphere. The mixture was stirred for $2.5 \mathrm{~h}$ at $-20{ }^{\circ} \mathrm{C}$. Then, a solution of aniline $1 \mathrm{a}(4.4 \mathrm{~g}, 17.6 \mathrm{mmol}, 4.9$ equiv) in $\mathrm{CH}_{2} \mathrm{Cl}_{2}(3 \mathrm{~mL})$ was added and the resulting solution was stirred at $24{ }^{\circ} \mathrm{C}$ overnight. The volatiles were removed under reduced pressure and the organic materials were dissolved in EtOAc. The organic phase was washed with sat. $\mathrm{NaHCO}_{3}$ and brine, dried over $\mathrm{Na}_{2} \mathrm{SO}_{4}$ and concentrated. The excess aniline 1a was removed by flash column chromatography (cyclohexane/ $\mathrm{CH}_{2} \mathrm{Cl}_{2} 2: 1$, then 1:1) and the obtained compound was further purified by flash column chromatography (cyclohexane/toluene $20: 1$ ) to yield $\mathbf{3 b}(815 \mathrm{mg}, 1.28 \mathrm{mmol}, 36 \%$ ) as a white solid.

M.p. $=182-184{ }^{\circ} \mathrm{C}$ (cyclohexane/toluene). ${ }^{1} \mathbf{H}$ NMR $\left(400 \mathrm{MHz}, \mathrm{CD}_{2} \mathrm{Cl}_{2}\right) \delta 7.63(\mathrm{dd}, J=$ 8.0, 1.3 Hz, 1H), 7.33 (td, $J=7.5,1.3 \mathrm{~Hz}, 1 \mathrm{H}), 7.26$ (dd, $J=7.7,1.9 \mathrm{~Hz}, 1 \mathrm{H}), 7.22-7.18$ (m, 2H), $7.16(\mathrm{ddd}, J=8.0,7.2,1.9 \mathrm{~Hz}, 1 \mathrm{H}), 6.52-6.46(\mathrm{~m}, 2 \mathrm{H}), 5.64-5.54(\mathrm{~m}, 2 \mathrm{H}), 2.94-2.77$ (m, 2H), $2.28-2.12(\mathrm{~m}, 2 \mathrm{H}) .{ }^{13} \mathbf{C}$ NMR $\left(101 \mathrm{MHz}, \mathrm{CD}_{2} \mathrm{Cl}_{2}\right) \delta 145.3(\mathrm{dm}, J=247.1 \mathrm{~Hz}), 143.3$, 142.6, 140.9 (dm, $J=251.7 \mathrm{~Hz}), 138.4$ (dm, $J 250.6 \mathrm{~Hz}), 133.7,131.9$, 131.0, 128.7, 128.0, 123.0, 117.5 - $116.9(\mathrm{~m}), 113.0,54.8,33.3 .{ }^{19} \mathbf{F}\left\{{ }^{1} \mathbf{H}\right\}$ NMR $\left(376 \mathrm{MHz}, \mathrm{CD}_{2} \mathrm{Cl}_{2}\right) \delta-145.7$, $156.6(\mathrm{t}, J=20.9 \mathrm{~Hz}),-162.5(\mathrm{td}, J=21.4,6.7 \mathrm{~Hz})$. HRMS (ESI+) calculated for $\left[\mathrm{C}_{28} \mathrm{H}_{15} \mathrm{BrF}_{10} \mathrm{~N}\right]^{+} 634.0223 \mathrm{~m} / \mathrm{z}$; found $[\mathrm{M}+\mathrm{H}]^{+} 634.0220 \mathrm{~m} / \mathrm{z} \cdot \boldsymbol{\alpha}_{\mathbf{D}}{ }^{\mathbf{5 8 9}}=+80.9 \mathrm{deg} \cdot \mathrm{cm}^{2} \cdot \mathrm{g}^{-1}$ $\left(\mathrm{CHCl}_{3}, \mathrm{c} 1.07,300 \mathrm{~K}\right)$. 


\section{Ligand 4b}

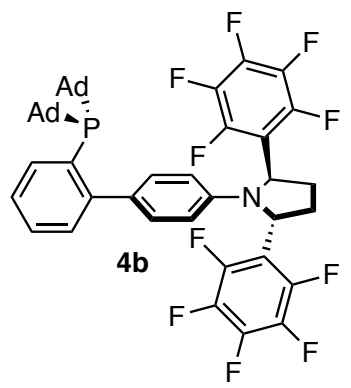

A flame-dried $10 \mathrm{~mL}$ Schlenk flask was charged with $\mathbf{3 b}$ (150 mg, $0.24 \mathrm{mmol}, 1.0$ equiv), $\mathrm{Pd}(\mathrm{OAc})_{2}(2.7 \mathrm{mg}, 12 \mu \mathrm{mol}, 5 \mathrm{~mol} \%)$, dppf (7.9 mg, $\left.14 \mu \mathrm{mol}, 6 \mathrm{~mol} \%\right), \mathrm{NaO} t \mathrm{Bu}(27 \mathrm{mg}, 0.28$ mmol, 1.2 equiv) and toluene $(1.2 \mathrm{~mL})$ and the resulting suspension was stirred for $15 \mathrm{~min}$ at $24{ }^{\circ} \mathrm{C}$. Di(1-adamantyl)phosphine (79 $\mathrm{mg}, 0.26 \mathrm{mmol}, 1.1$ equiv) was added and the reaction mixture was stirred at $120^{\circ} \mathrm{C}$ for $16 \mathrm{~h}$. Then, the reaction was allowed to cool to $24^{\circ} \mathrm{C}$ and the crude was purified by flash column chromatography (cyclohexane $/ \mathrm{CH}_{2} \mathrm{Cl}_{2} \quad 3: 1$, then cyclohexane/Et $2 \mathrm{O} 40: 1)$ to yield ligand $\mathbf{4 b}(90 \mathrm{mg}, 0.105 \mathrm{mmol}, 45 \%)$ as a white solid.

M.p. $=138-140{ }^{\circ} \mathrm{C}\left(\right.$ cyclohexane/ $\left.\mathrm{Et}_{2} \mathrm{O}\right) .{ }^{1} \mathbf{H}$ NMR $\left(500 \mathrm{MHz}, \mathrm{CD}_{2} \mathrm{Cl}_{2}\right) \delta 7.83(\mathrm{~d}, J=7.5$ $\mathrm{Hz}, 1 \mathrm{H}), 7.32-7.22(\mathrm{~m}, 2 \mathrm{H}), 7.12(\mathrm{ddd}, J=7.1,4.0,1.2 \mathrm{~Hz}, 1 \mathrm{H}), 6.93$ (d, $J=8.4 \mathrm{~Hz}, 2 \mathrm{H})$, $6.39(\mathrm{~d}, J=8.5 \mathrm{~Hz}, 2 \mathrm{H}), 5.58(\mathrm{~d}, J=6.5 \mathrm{~Hz}, 2 \mathrm{H}), 2.90-2.74(\mathrm{~m}, 2 \mathrm{H}), 2.23-2.14(\mathrm{~m}, 2 \mathrm{H})$, $1.91-1.84(\mathrm{~m}, 7 \mathrm{H}), 1.84-1.79(\mathrm{~m}, 7 \mathrm{H}), 1.79-1.70(\mathrm{~m}, 6 \mathrm{H}), 1.66(\mathrm{~s}, 7 \mathrm{H}), 1.63-1.60(\mathrm{~m}$, $3 \mathrm{H}) .{ }^{13} \mathrm{C}$ NMR $\left(126 \mathrm{MHz}, \mathrm{CD}_{2} \mathrm{Cl}_{2}\right) \delta 151.9(\mathrm{~d}, J=32.1 \mathrm{~Hz}), 145.5(\mathrm{dm}, J=246.9 \mathrm{~Hz}), 142.4$, $140.7(\mathrm{dm}, J=252.7 \mathrm{~Hz}), 138.3(\mathrm{dm}, J=250.8 \mathrm{~Hz}), 137.3$ (d, $J=2.6 \mathrm{~Hz}), 134.5$ (d, $J=7.0$ $\mathrm{Hz}), 134.0,133.8,132.1$ (d, $J=4.1 \mathrm{~Hz}), 131.2(\mathrm{~d}, J=6.0 \mathrm{~Hz}), 128.7,125.5,117.5$ (t, $J=14.1$ Hz), 112.7, 54.8, 42.5 (d, $J=13.1 \mathrm{~Hz}), 42.3(\mathrm{~d}, J=13.1 \mathrm{~Hz}), 37.8(\mathrm{~d}, J=9.9 \mathrm{~Hz}), 37.6(\mathrm{~d}, J=$ $10.1 \mathrm{~Hz}), 37.5(\mathrm{~d}, J=7.4 \mathrm{~Hz}), 33.2,29.62(\mathrm{~d}, J=8.6 \mathrm{~Hz}), 29.55(\mathrm{~d}, J=8.5 \mathrm{~Hz}) .{ }^{31} \mathbf{P}\left\{{ }^{1} \mathbf{H}\right\} \mathbf{~ N M R}$ $\left(162 \mathrm{MHz}, \mathrm{CD}_{2} \mathrm{Cl}_{2}\right) \delta 24.7 .{ }^{19} \mathbf{F}\left\{{ }^{1} \mathbf{H}\right\} \mathbf{N M R}\left(376 \mathrm{MHz}, \mathrm{CD}_{2} \mathrm{Cl}_{2}\right) \delta-145.3(\mathrm{dd}, J=22.6,7.8 \mathrm{~Hz})$, $-156.7(\mathrm{t}, J=21.0 \mathrm{~Hz}),-162.7(\mathrm{t}, J=21.2 \mathrm{~Hz})$. HRMS (ESI+) calculated for $\left[\mathrm{C}_{48} \mathrm{H}_{45} \mathrm{~F}_{10} \mathrm{NP}\right]^{+}$ $856.3124 \mathrm{~m} / z$; found $[\mathrm{M}+\mathrm{H}]^{+} 856.3099 \mathrm{~m} / z . \boldsymbol{\alpha}_{\mathbf{D}}{ }^{\mathbf{5 8 9}}=+60.7 \mathrm{deg} \cdot \mathrm{cm}^{2} \cdot \mathrm{g}^{-1}\left(\mathrm{CHCl}_{3}, \mathrm{c} 1.0,299 \mathrm{~K}\right)$. 
$(R, R)-\mathrm{B}$

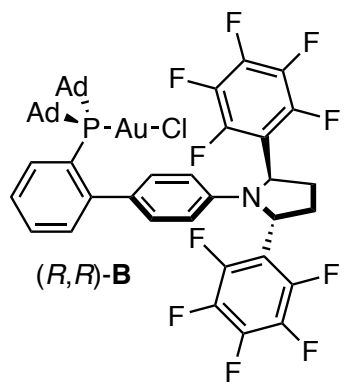

$\mathrm{Me}_{2} \mathrm{SAuCl}(13 \mathrm{mg}, 43 \mu \mathrm{mol}, 1.0$ equiv) and ligand $4 \mathbf{b}$ (37.2 mg, $43 \mu \mathrm{mol}, 1.0$ equiv) were dissolved in $\mathrm{CH}_{2} \mathrm{Cl}_{2}(0.44 \mathrm{~mL})$ under an atmosphere of argon. The reaction mixture was stirred at $24{ }^{\circ} \mathrm{C}$ for $15 \mathrm{~min}$. The mixture was filtered through a syringe filter and concentrated. The residue was dissolved in $\mathrm{CH}_{2} \mathrm{Cl}_{2}$, filtered through a path of neutral $\mathrm{Al}_{2} \mathrm{O}_{3}$ and concentrated to yield complex $(R, R)$-B (36 mg, $33 \mu \mathrm{mol}, 76 \%)$ as a white solid.

M.p. $=202-204{ }^{\circ} \mathrm{C}\left(\mathrm{CH}_{2} \mathrm{Cl}_{2} / \mathrm{Et}_{2} \mathrm{O}\right) .{ }^{1} \mathbf{H}$ NMR $\left(500 \mathrm{MHz}, \mathrm{CD}_{2} \mathrm{Cl}_{2}\right) \delta 7.89-7.83(\mathrm{~m}, 1 \mathrm{H})$, $7.49-7.42(\mathrm{~m}, 2 \mathrm{H}), 7.17-7.12(\mathrm{~m}, 1 \mathrm{H}), 6.95(\mathrm{dd}, J=8.4,2.2 \mathrm{~Hz}, 1 \mathrm{H}), 6.79$ (dd, $J=8.4,2.2$ $\mathrm{Hz}, 1 \mathrm{H}), 6.55$ (dd, $J=8.4,2.8 \mathrm{~Hz}, 1 \mathrm{H}), 6.42(\mathrm{dd}, J=8.4,2.8 \mathrm{~Hz}, 1 \mathrm{H}), 5.63$ (d, $J=7.5 \mathrm{~Hz}, 2 \mathrm{H})$, $2.98-2.85$ (m, 2H), $2.21-2.14(\mathrm{~m}, 8 \mathrm{H}), 2.14-2.06(\mathrm{~m}, 7 \mathrm{H}), 2.00$ (br s, $7 \mathrm{H}), 1.70$ (br s, 10H). ${ }^{13} \mathrm{C}$ NMR $\left(126 \mathrm{MHz}, \mathrm{CD}_{2} \mathrm{Cl}_{2}\right) \delta 151.2(\mathrm{~d}, J=13.5 \mathrm{~Hz}), 145.4(\mathrm{dm}, J=248.0 \mathrm{~Hz}), 143.9,140.7$ (dm, $J=251.2 \mathrm{~Hz}), 138.3(\mathrm{dm}, J=250.3 \mathrm{~Hz}), 135.2(\mathrm{~d}, J=2.3 \mathrm{~Hz}), 134.8(\mathrm{~d}, J=7.3 \mathrm{~Hz})$, $132.5(\mathrm{~d}, J=6.6 \mathrm{~Hz}), 130.9$ (d, $J=6.6 \mathrm{~Hz}), 130.6(\mathrm{~d}, J=2.1 \mathrm{~Hz}), 126.4(\mathrm{~d}, J=6.6 \mathrm{~Hz}), 124.6$, 124.2, 117.4 (t, $J=14.2 \mathrm{~Hz}), 113.3$ (d, $J=13.5 \mathrm{~Hz}), 54.5,43.1$ (d, $J=23.8 \mathrm{~Hz}), 42.74$ (d, $J=$ $23.8 \mathrm{~Hz}), 42.74(\mathrm{~d}, J=2.5 \mathrm{~Hz}), 42.5(\mathrm{~d}, J=2.4 \mathrm{~Hz}), 36.9,33.3,29.4(\mathrm{~d}, J=12.3 \mathrm{~Hz}), 29.3(\mathrm{~d}$, $J=11.9 \mathrm{~Hz}) .{ }^{31} \mathbf{P}\left\{{ }^{1} \mathbf{H}\right\}$ NMR $\left(203 \mathrm{MHz}, \mathrm{CD}_{2} \mathrm{Cl}_{2}\right) \delta$ 64.8. ${ }^{19} \mathbf{F}\left\{{ }^{1} \mathbf{H}\right\} \mathbf{N M R}\left(471 \mathrm{MHz}, \mathrm{CD}_{2} \mathrm{Cl}_{2}\right) \delta$ $-142.9--146.3(\mathrm{~m}),-157.4(\mathrm{t}, J=20.9 \mathrm{~Hz}),-160.8--164.5(\mathrm{~m})$. HRMS (ESI+) calculated for $\left[\mathrm{C}_{48} \mathrm{H}_{44} \mathrm{AuClF}_{10} \mathrm{NNaP}\right]^{+} 1110.2298 \mathrm{~m} / \mathrm{z}$; found $[\mathrm{M}+\mathrm{H}]^{+} 1110.2275 \mathrm{~m} / \mathrm{z}$. Elemental analysis calculated for $\mathrm{C}_{48} \mathrm{H}_{45} \mathrm{AuClF}_{10} \mathrm{NP}$ : C 52.98; $\mathrm{H} 4.08$; $\mathrm{N} 1.29$; found: $\mathrm{C} 53.04 ; \mathrm{H} 4.25 ; \mathrm{N}$ 1.49. $\boldsymbol{\alpha}_{\mathbf{D}}{ }^{\mathbf{5 8 9}}=+49.9$ deg. $\mathrm{cm}^{2} \cdot \mathrm{g}^{-1}\left(\mathrm{CHCl}_{3}, \mathrm{c} 1.1,297 \mathrm{~K}\right)$. 


\subsubsection{Synthesis of chiral complex $(R, R)-C$}

\section{Chiral precursor $3 \mathrm{c}$}

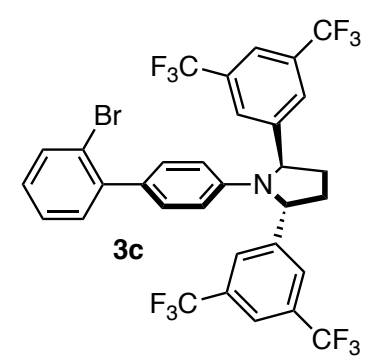

To a solution of (1S,4S)-1,4-bis(3,5-bis(trifluoromethyl)phenyl)butane-1,4-diol (2c) (2.4 g, $4.61 \mathrm{mmol}, 1.0$ equiv) in $\mathrm{CH}_{2} \mathrm{Cl}_{2}(46 \mathrm{~mL})$ at $-20{ }^{\circ} \mathrm{C}$ were added $\mathrm{NEt}_{3}(1.93 \mathrm{~mL}, 13.8 \mathrm{mmol}$, 3.0 equiv) and methanesulfonyl chloride $(0.93 \mathrm{~mL}, 12.0 \mathrm{mmol}, 2.6$ equiv) under an atmosphere of argon. The mixture was stirred for $2.5 \mathrm{~h}$ at $-20^{\circ} \mathrm{C}$. Then, a solution of aniline $1 \mathrm{a}(5.6 \mathrm{~g}, 22.6$ mmol, 4.9 equiv) in $\mathrm{CH}_{2} \mathrm{Cl}_{2}(22.5 \mathrm{~mL})$ was added and the resulting mixture was stirred at 24 ${ }^{\circ} \mathrm{C}$ overnight. The volatile products were removed under reduced pressure and the organic materials were extracted with EtOAc. The combined organic phases were washed with brine and sat. $\mathrm{NaHCO}_{3}$, dried over $\mathrm{Na}_{2} \mathrm{SO}_{4}$ and concentrated. The excess aniline $1 \mathrm{a}$ was removed by flash column chromatography (cyclohexane $/ \mathrm{CH}_{2} \mathrm{Cl}_{2}$ 1:1) and the obtained compound was purified by flash column chromatography (cyclohexane/EtOAc 20:1) to afford 3c (1.31 g, 1.80 mmol, 39\%) as a white solid.

M.p. $=68-70{ }^{\circ} \mathrm{C}$ (cyclohexane/EtOAc). ${ }^{1} \mathbf{H}$ NMR $\left(500 \mathrm{MHz}, \mathrm{CDCl}_{3}\right) \delta 7.80(\mathrm{~s}, 2 \mathrm{H}), 7.67$ (s, 4H), $7.58(\mathrm{dd}, J=8.0,1.2 \mathrm{~Hz}, 1 \mathrm{H}), 7.30-7.21(\mathrm{~m}, 2 \mathrm{H}), 7.15-7.06(\mathrm{~m}, 3 \mathrm{H}), 6.32(\mathrm{~d}, J=$ $8.8 \mathrm{~Hz}, 2 \mathrm{H}), 5.45(\mathrm{~d}, J=7.1 \mathrm{~Hz}, 2 \mathrm{H}), 2.66-2.54(\mathrm{~m}, 2 \mathrm{H}), 2.00-1.89(\mathrm{~m}, 2 \mathrm{H}) .{ }^{13} \mathbf{C}$ NMR $(126$ $\left.\mathrm{MHz}, \mathrm{CDCl}_{3}\right) \delta 146.2,142.8,142.3,133.2,132.3(\mathrm{q}, J=33.3 \mathrm{~Hz}), 131.3,130.7,130.5,128.2$, 127.4, 126.4, 123.4 (q, J = 273.2 Hz), 123.1, $121.7-121.4(\mathrm{~m}), 113.6,62.7,32.3 .{ }^{19} \mathbf{F}\left\{{ }^{1} \mathbf{H}\right\}$ NMR $\left(376 \mathrm{MHz}, \mathrm{CDCl}_{3}\right) \delta-62.9$. HRMS $(\mathrm{ESI}+)$ calculated for $\left[\mathrm{C}_{32} \mathrm{H}_{21} \mathrm{BrF}_{12} \mathrm{~N}\right]^{+} 726.0660$ $m / z$; found $[\mathrm{M}+\mathrm{H}]^{+} 726.0647 \mathrm{~m} / z . \boldsymbol{\alpha}_{\mathbf{D}}^{589}=+26.0 \mathrm{deg} \cdot \mathrm{cm}^{2} \cdot \mathrm{g}^{-1}\left(\mathrm{CHCl}_{3}, \mathrm{c} 0.95,297 \mathrm{~K}\right)$. 


\section{Ligand 4c}

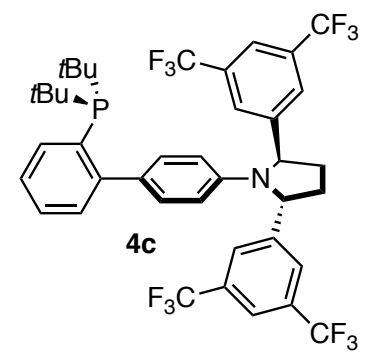

A flame-dried $10 \mathrm{~mL}$ Schlenk flask was charged with 3c (150 mg, $0.21 \mathrm{mmol}, 1.0$ equiv), $\mathrm{Pd}(\mathrm{OAc})_{2}(2.32 \mathrm{mg}, 10.3 \mu \mathrm{mol}, 5 \mathrm{~mol} \%)$, dppf (6.9, $\left.0.012 \mathrm{mmol}, 6 \mathrm{~mol} \%\right), \mathrm{NaO} t \mathrm{Bu}(24 \mathrm{mg}$, $0.25 \mathrm{mmol}, 1.2$ equiv) and toluene $(1 \mathrm{~mL})$ and the resulting suspension was stirred for $15 \mathrm{~min}$ at $24{ }^{\circ} \mathrm{C}$. Di-tert-butylphosphane $(42 \mu \mathrm{L}, 0.227 \mathrm{mmol}, 1.1$ equiv) was added and the reaction mixture was stirred at $120^{\circ} \mathrm{C}$ for $16 \mathrm{~h}$. Then, the reaction was allowed to cool to $24^{\circ} \mathrm{C}$ and the crude was purified by flash column chromatography (cyclohexane $/ \mathrm{CH}_{2} \mathrm{Cl}_{2} \quad 3: 1$, then cyclohexane/Et $\left.\mathrm{E}_{2} \mathrm{O} 40: 1\right)$ to yield ligand $4 \mathrm{c}(157 \mathrm{mg}, 0.198 \mathrm{mmol}, 96 \%)$ as a white solid.

M.p. $=172-174{ }^{\circ} \mathrm{C}$ (pentane). ${ }^{1} \mathbf{H}$ NMR $\left(400 \mathrm{MHz}, \mathrm{CD}_{2} \mathrm{Cl}_{2}\right) \delta 7.85-7.79(\mathrm{~m}, 3 \mathrm{H}), 7.73$ (s, 4H), $7.32-7.27(\mathrm{~m}, 1 \mathrm{H}), 7.26-7.21(\mathrm{~m}, 1 \mathrm{H}), 7.15(\mathrm{ddd}, J=7.6,4.2,1.7 \mathrm{~Hz}, 1 \mathrm{H}), 7.02-$ $6.97(\mathrm{~m}, 2 \mathrm{H}), 6.31-6.25(\mathrm{~m}, 2 \mathrm{H}), 5.47$ (d, $J=7.2 \mathrm{~Hz}, 2 \mathrm{H}), 2.68-2.50(\mathrm{~m}, 2 \mathrm{H}), 1.98-1.84$ $(\mathrm{m}, 2 \mathrm{H}), 1.13(\mathrm{~d}, J=11.5 \mathrm{~Hz}, 9 \mathrm{H}), 0.96$ (d, $J=11.5 \mathrm{~Hz}, 9 \mathrm{H}) .{ }^{13} \mathbf{C} \mathbf{N M R}\left(126 \mathrm{MHz}, \mathrm{CD}_{2} \mathrm{Cl}_{2}\right) \delta$ $151.3(\mathrm{~d}, J=31.3 \mathrm{~Hz}), 147.2,142.5,136.5,136.3,136.1$ (d, $J=3.0 \mathrm{~Hz}), 133.4$ (d, $J=6.7 \mathrm{~Hz})$, $132.5(\mathrm{~d}, J=4.5 \mathrm{~Hz}), 132.3(\mathrm{q}, J=33.5 \mathrm{~Hz}), 131.2(\mathrm{~d}, J=5.8 \mathrm{~Hz}), 128.9,127.3-127.0(\mathrm{~m})$, 125.8, 124.1 (q, $J=273.0 \mathrm{~Hz}), 122.0$ - $121.5(\mathrm{~m}), 113.3,63.2,33.2,33.0,32.9,32.7,31.2(\mathrm{~d}$, $J=15.8 \mathrm{~Hz}), 30.6(\mathrm{~d}, J=15.5 \mathrm{~Hz}) \cdot{ }^{31} \mathbf{P}\left\{{ }^{1} \mathbf{H}\right\} \mathbf{N M R}\left(203 \mathrm{MHz}, \mathrm{CD}_{2} \mathrm{Cl}_{2}\right) \delta 21.2 .{ }^{19} \mathbf{F}\left\{{ }^{1} \mathbf{H}\right\} \mathbf{N M R}$ $\left(376 \mathrm{MHz}, \mathrm{CD}_{2} \mathrm{Cl}_{2}\right) \delta-63.2$. HRMS (ESI+) calculated for $\left[\mathrm{C}_{40} \mathrm{H}_{39} \mathrm{~F}_{12} \mathrm{NP}\right]^{+} 792.2623 \mathrm{~m} / z$; found $[\mathrm{M}+\mathrm{H}]^{+} 792.2629 \mathrm{~m} / z \cdot \boldsymbol{\alpha}_{\mathbf{D}}{ }^{589}=+25.8 \mathrm{deg} \cdot \mathrm{cm}^{2} \cdot \mathrm{g}^{-1}\left(\mathrm{CHCl}_{3}, \mathrm{c} 1.03,298 \mathrm{~K}\right)$.

\section{$(R, R)-C$}

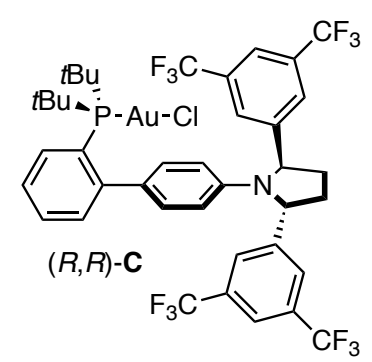

$\mathrm{Me}_{2} \mathrm{SAuCl}(15 \mathrm{mg}, 51 \mu \mathrm{mol}, 1.0$ equiv) and ligand $4 \mathbf{c}(40 \mathrm{mg}, 51 \mu \mathrm{mol}, 1.0$ equiv) were dissolved in $\mathrm{CH}_{2} \mathrm{Cl}_{2}(0.51 \mathrm{~mL})$ under an atmosphere of argon. The reaction mixture was stirred at $24{ }^{\circ} \mathrm{C}$ for $15 \mathrm{~min}$. The mixture was filtered through a syringe filter and concentrated. The 
residue was dissolved in $\mathrm{CH}_{2} \mathrm{Cl}_{2}$, filtered through a path of neutral $\mathrm{Al}_{2} \mathrm{O}_{3}$ and concentrated to yield complex $(R, R)-\mathbf{C}(49 \mathrm{mg}, 48 \mu \mathrm{mol}, 94 \%)$ as a white solid.

M.p. $=259-262{ }^{\circ} \mathrm{C}\left(\mathrm{CH}_{2} \mathrm{Cl}_{2} /\right.$ Pentane). ${ }^{1} \mathbf{H}$ NMR $\left(400 \mathrm{MHz}, \mathrm{CD}_{2} \mathrm{Cl}_{2}\right) \delta 7.90(\mathrm{~s}, 4 \mathrm{H}), 7.85$ - $7.77(\mathrm{~m}, 3 \mathrm{H}), 7.49-7.38(\mathrm{dtt}, J=20.2,7.6,1.4 \mathrm{~Hz}, 2 \mathrm{H}), 7.27-7.22(\mathrm{~m}, 1 \mathrm{H}), 6.92(\mathrm{dd}, J=$ 8.4, $2.2 \mathrm{~Hz}, 1 \mathrm{H}), 6.74(\mathrm{dd}, J=8.4,2.2 \mathrm{~Hz}, 1 \mathrm{H}), 6.28(\mathrm{dd}, J=8.4,2.7 \mathrm{~Hz}, 1 \mathrm{H}), 6.21(\mathrm{dd}, J=$ 8.4, 2.7 Hz, 1H), 5.50 (d, $J=6.8 \mathrm{~Hz}, 2 \mathrm{H}), 2.67-2.53(\mathrm{~m}, 2 \mathrm{H}), 1.91-1.79$ (m, 2H), $1.45(\mathrm{~d}, J$ $=15.5 \mathrm{~Hz}, 9 \mathrm{H}), 1.19(\mathrm{~d}, J=15.6 \mathrm{~Hz}, 9 \mathrm{H}) .{ }^{13} \mathbf{C}$ NMR $\left(126 \mathrm{MHz}, \mathrm{CD}_{2} \mathrm{Cl}_{2}\right) \delta 150.6(\mathrm{~d}, J=12.9$ $\mathrm{Hz}), 147.2,144.0,134.3$ (d, $J=2.9 \mathrm{~Hz}), 133.9$ (d, $J=7.2 \mathrm{~Hz}), 132.2$ (q, $J=33.2 \mathrm{~Hz}), 130.98$ $(\mathrm{d}, J=6.8 \mathrm{~Hz}), 130.96(\mathrm{~d}, J=2.3 \mathrm{~Hz}), 130.4,130.2,127.5-127.4(\mathrm{~m}), 127.0$ (d, $J=22.5 \mathrm{~Hz})$, $126.8(\mathrm{~d}, J=16.5 \mathrm{~Hz}), 124.1$ (q, $J=273.3 \mathrm{~Hz}), 121.8-121.4(\mathrm{~m}), 116.3,114.0,63.7,38.3(\mathrm{~d}$, $J=25.8 \mathrm{~Hz}), 37.8(\mathrm{~d}, J=25.4 \mathrm{~Hz}), 32.7,31.9(\mathrm{~d}, J=6.8 \mathrm{~Hz}), 30.4(\mathrm{~d}, J=6.7 \mathrm{~Hz}) .{ }^{31} \mathbf{P}\left\{{ }^{1} \mathbf{H}\right\}$ NMR $\left(162 \mathrm{MHz}, \mathrm{CD}_{2} \mathrm{Cl}_{2}\right) \delta 63.3 .{ }^{19} \mathbf{F}\left\{{ }^{1} \mathbf{H}\right\}$ NMR $\left(376 \mathrm{MHz}, \mathrm{CD}_{2} \mathrm{Cl}_{2}\right) \delta-62.8$. HRMS (ESI+) calculated for $\left[\mathrm{C}_{40} \mathrm{H}_{38} \mathrm{AuClF}_{12} \mathrm{NNaP}\right]^{+} 1046.1797 \mathrm{~m} / \mathrm{z}$; found $[\mathrm{M}+\mathrm{Na}]^{+} 1046.1797 \mathrm{~m} / \mathrm{z}$. Elemental analysis calculated for $\mathrm{C}_{40} \mathrm{H}_{38} \mathrm{AuClF}_{12} \mathrm{NP}$ : C 46.91; H 3.74; $\mathrm{N} 1.37$; found: $\mathrm{C} 46.87$; H 3.81; N 1.47. $\boldsymbol{\alpha}_{\mathbf{D}}{ }^{\mathbf{8 8 9}}=+34.0$ deg.cm ${ }^{2} \cdot \mathrm{g}^{-1}\left(\mathrm{CHCl}_{3}\right.$, c 1.1, $\left.297 \mathrm{~K}\right)$.

\subsubsection{Synthesis of chiral complex $(R, R)-D$}

\section{Ligand 4d}

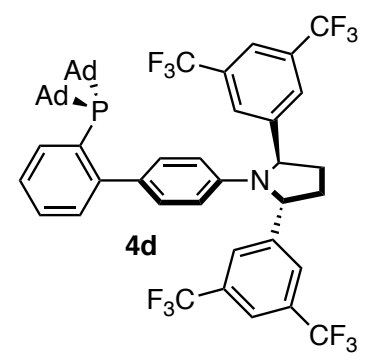

A flame-dried $10 \mathrm{~mL}$ Schlenk flask was charged with $3 \mathbf{c}$ (400 mg, $0.55 \mathrm{mmol}, 1.0$ equiv), $\mathrm{Pd}(\mathrm{OAc})_{2}(6.18 \mathrm{mg}, 0.028 \mathrm{mmol}, 5 \mathrm{~mol} \%)$, dppf (18 mg, $\left.0.033 \mathrm{mmol}, 6 \mathrm{~mol} \%\right), \mathrm{NaO} t \mathrm{Bu}(64$ $\mathrm{mg}, 0,661 \mathrm{mmol}, 1.2$ equiv) and toluene $(2.75 \mathrm{~mL})$, the resulting suspension was stirred for 15 min at $24{ }^{\circ} \mathrm{C}$. Di(1-adamantyl)phosphine (183 mg, $0.606 \mathrm{mmol}, 1.1$ equiv) was added and the reaction mixture was stirred at $120^{\circ} \mathrm{C}$ for $16 \mathrm{~h}$. Then, the reaction was allowed to cool to $24{ }^{\circ} \mathrm{C}$ and the crude was purified by flash column chromatography (cyclohexane $/ \mathrm{CH}_{2} \mathrm{Cl}_{2} 3: 1$, then cyclohexane/Et $\left.\mathrm{E}_{2} \mathrm{O} 40: 1\right)$ to yield ligand $\mathbf{4 d}(493 \mathrm{mg}, 0.520 \mathrm{mmol}, 94 \%)$ as a white solid.

M.p. $=174-176{ }^{\circ} \mathrm{C}($ cyclohexane/Et $2 \mathrm{O}) .{ }^{1} \mathbf{H}$ NMR $\left(500 \mathrm{MHz}, \mathrm{CD}_{2} \mathrm{Cl}_{2}\right) \delta 7.88-7.79(\mathrm{~m}$, 3H), $7.75(\mathrm{~s}, 4 \mathrm{H}), 7.34-7.27(\mathrm{~m}, 1 \mathrm{H}), 7.24(\mathrm{td}, J=7.4,1.6 \mathrm{~Hz}, 1 \mathrm{H}), 7.14(\mathrm{ddd}, J=7.6,4.1$, $1.6 \mathrm{~Hz}, 1 \mathrm{H}), 7.02-6.94(\mathrm{~m}, 2 \mathrm{H}), 6.33-6.25(\mathrm{~m}, 2 \mathrm{H}), 5.48(\mathrm{~d}, J=7.2 \mathrm{~Hz}, 2 \mathrm{H}), 2.68-2.50(\mathrm{~m}$, 
2H), $1.96-1.90(\mathrm{~m}, 5 \mathrm{H}), 1.89-1.83(\mathrm{~m}, 6 \mathrm{H}), 1.80-1.73(\mathrm{~m}, 4 \mathrm{H}), 1.73-1.71(\mathrm{~m}, 3 \mathrm{H}), 1.70$ - $1.63(\mathrm{~m}, 10 \mathrm{H}), 1.63-1.56(\mathrm{~m}, 5 \mathrm{H}), 1.55-1.51(\mathrm{~m}, 1 \mathrm{H}) .{ }^{13} \mathbf{C ~ N M R}\left(126 \mathrm{MHz}, \mathrm{CD}_{2} \mathrm{Cl}_{2}\right) \delta$ $151.9(\mathrm{~d}, J=31.4 \mathrm{~Hz}), 147.2,142.4,137.4$ (d, $J=2.5 \mathrm{~Hz}), 133.9,133.64$ (d, $J=2.1 \mathrm{~Hz}), 133.59$, $132.4(\mathrm{~d}, J=4.8 \mathrm{~Hz}), 132.3(\mathrm{q}, J=33.2 \mathrm{~Hz}), 131.4(\mathrm{~d}, J=5.9 \mathrm{~Hz}), 128.7,127.2-127.0$ (m), 125.4, 124.0 (q, $J=273.0 \mathrm{~Hz}), 121.8$ (p, $J=3.7 \mathrm{~Hz}), 113.2,63.2,42.7$ (d, $J=13.3 \mathrm{~Hz}), 42.1$ (d, $J=13.0 \mathrm{~Hz}), 38.0,37.8(\mathrm{~d}, J=2.7 \mathrm{~Hz}), 37.5,37.4,32.6,29.6$ (d, $J=8.6 \mathrm{~Hz}), 29.5$ (d, $J=$ $8.5 \mathrm{~Hz}) .{ }^{31} \mathbf{P}\left\{{ }^{1} \mathbf{H}\right\}$ NMR $\left(203 \mathrm{MHz}, \mathrm{CD}_{2} \mathrm{Cl}_{2}\right) \delta 24.0 .{ }^{19} \mathbf{F}\left\{{ }^{1} \mathbf{H}\right\} \mathbf{N M R}\left(471 \mathrm{MHz}, \mathrm{CD}_{2} \mathrm{Cl}_{2}\right) \delta-63.1$. HRMS (ESI+) calculated for $\left[\mathrm{C}_{52} \mathrm{H}_{51} \mathrm{~F}_{12} \mathrm{NP}\right]^{+} 948.3562 \mathrm{~m} / \mathrm{z}$; found $[\mathrm{M}+\mathrm{H}]^{+} 948.3562 \mathrm{~m} / \mathrm{z}$. $\boldsymbol{\alpha}_{\mathbf{D}}{ }^{\mathbf{5 8 9}}=+26.7 \mathrm{deg} \cdot \mathrm{cm}^{2} \cdot \mathrm{g}^{-1}\left(\mathrm{CHCl}_{3}, \mathrm{c} 0.97,300 \mathrm{~K}\right)$.

\section{$(R, R)-\mathrm{D}$}

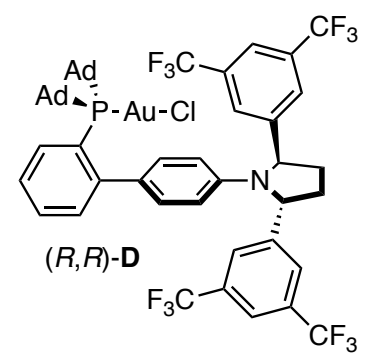

$\mathrm{Me}_{2} \mathrm{SAuCl}$ (31 mg, $0.11 \mathrm{mmol}, 1.0$ equiv) and ligand 4d (101 mg, $0.11 \mathrm{mmol}, 1.0$ equiv) were dissolved in $\mathrm{CH}_{2} \mathrm{Cl}_{2}(1.1 \mathrm{~mL})$ under an atmosphere of argon. The reaction mixture was stirred at $24{ }^{\circ} \mathrm{C}$ for $15 \mathrm{~min}$. The mixture was filtered through a syringe filter and concentrated. The residue was dissolved in $\mathrm{CH}_{2} \mathrm{Cl}_{2}$, filtered through a path of neutral $\mathrm{Al}_{2} \mathrm{O}_{3}$ and concentrated to yield complex $(R, R)$-D (114 mg, $0.097 \mathrm{mmol}, 90 \%)$ as a white solid.

M.p. $=>245^{\circ} \mathrm{C}\left(\right.$ decomposition; $\left.\mathrm{CH}_{2} \mathrm{Cl}_{2} / \mathrm{Et}_{2} \mathrm{O}\right) .{ }^{1} \mathbf{H} \mathbf{N M R}\left(500 \mathrm{MHz}, \mathrm{CDCl}_{3}\right) \delta 7.89(\mathrm{~s}, 4 \mathrm{H})$, $7.82-7.75(\mathrm{~m}, 3 \mathrm{H}), 7.48-7.37(\mathrm{~m}, 2 \mathrm{H}), 7.31-7.26(\mathrm{~m}, 1 \mathrm{H}), 6.91(\mathrm{dd}, J=8.4,2.1 \mathrm{~Hz}, 1 \mathrm{H})$, $6.70(\mathrm{dd}, J=8.4,2.2 \mathrm{~Hz}, 1 \mathrm{H}), 6.28(\mathrm{dd}, J=8.4,2.7 \mathrm{~Hz}, 1 \mathrm{H}), 6.17(\mathrm{dd}, J=8.4,2.7 \mathrm{~Hz}, 1 \mathrm{H})$, $5.49(\mathrm{~d}, J=6.6 \mathrm{~Hz}, 2 \mathrm{H}), 2.67-2.51(\mathrm{~m}, 2 \mathrm{H}), 2.28-2.17(\mathrm{~m}, 6 \mathrm{H}), 2.00-1.88(\mathrm{~m}, 12 \mathrm{H}), 1.87$ - $1.83(\mathrm{~m}, 2 \mathrm{H}), 1.73-1.63(\mathrm{~m}, 6 \mathrm{H}), 1.63-1.55(\mathrm{~m}, 6 \mathrm{H}) .{ }^{13} \mathbf{C ~ N M R}\left(126 \mathrm{MHz}, \mathrm{CDCl}_{3}\right) \delta 150.8$ $(\mathrm{d}, J=12.5 \mathrm{~Hz}), 146.5,143.5,134.5(\mathrm{~d}, J=2.2 \mathrm{~Hz}), 133.8$ (d, $J=7.0 \mathrm{~Hz}), 132.0(\mathrm{q}, J=33.2$ Hz), 130.7 (d, $J=6.5 \mathrm{~Hz}), 130.4$ (d, $J=1.9 \mathrm{~Hz}), 130.0,129.7,126.9,125.9$ (d, $J=6.6 \mathrm{~Hz})$, $124.3,124.0,123.5$ (q, $J=272.83 \mathrm{~Hz}), 121.5-120.8(\mathrm{~m}), 115.9,113.5,63.3,43.0$ (d, $J=23.5$ Hz), 42.8 (d, $J=2.5 \mathrm{~Hz}), 42.1$ (d, $J=23.3 \mathrm{~Hz}), 41.6$ (d, $J=2.4 \mathrm{~Hz}), 36.5,36.3,32.5,28.9$ (d, $J=9.8 \mathrm{~Hz}), 28.6(\mathrm{~d}, J=9.9 \mathrm{~Hz}) .{ }^{31} \mathbf{P}\left\{{ }^{1} \mathbf{H}\right\} \mathbf{N M R}\left(162 \mathrm{MHz}, \mathrm{CDCl}_{3}\right) \delta$ 64.9. ${ }^{19} \mathbf{F}\left\{{ }^{1} \mathbf{H}\right\} \mathbf{N M R}(471$ $\left.\mathrm{MHz}, \mathrm{CDCl}_{3}\right) \delta-62.3$. HRMS (ESI+) calculated for $\left[\mathrm{C}_{52} \mathrm{H}_{50} \mathrm{AuClF}_{12} \mathrm{NNaP}\right]^{+} 1202.2736 \mathrm{~m} / \mathrm{z}$; found $[\mathrm{M}+\mathrm{Na}]^{+} 1202.2764 \mathrm{~m} / z$. Elemental analysis calculated for $\mathrm{C}_{52} \mathrm{H}_{50} \mathrm{AuClF}_{12} \mathrm{NP}$ : C 
52.91; H 4.27; N 1.19; found: C 52.89; H 4.51; N 1.20. $\boldsymbol{\alpha}_{\mathbf{D}}{ }^{\mathbf{5 8 9}}=+42.4 \mathrm{deg} . \mathrm{cm}^{2} . \mathrm{g}^{-1}\left(\mathrm{CHCl}_{3}, \mathrm{c}\right.$ $1.24,299 \mathrm{~K})$.

\subsubsection{Synthesis of chiral complex $(R, R)-\mathrm{E}$}

\section{Chiral precursor 3d}

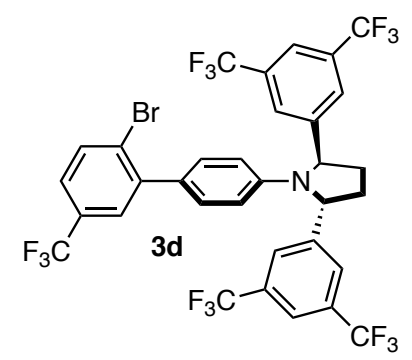

To a solution of (1S,4S)-1,4-bis(3,5-bis(trifluoromethyl)phenyl)butane-1,4-diol (2c) (200 $\mathrm{mg}, 0.389 \mathrm{mmol}, 1.0$ equiv) in $\mathrm{CH}_{2} \mathrm{Cl}_{2}(3.9 \mathrm{~mL})$ at $-20{ }^{\circ} \mathrm{C}$ were added $\mathrm{NEt}_{3}(0.163 \mathrm{~mL}, 1.17$ mmol, 3.0 equiv) and methanesulfonyl chloride $(0.078 \mathrm{~mL}, 1.01 \mathrm{mmol}, 2.6$ equiv) under an atmosphere of argon. The mixture was stirred for $2.5 \mathrm{~h}$ at $-20{ }^{\circ} \mathrm{C}$. Then, a solution of aniline $1 \mathbf{b}$ (602 mg, $1.91 \mathrm{mmol}, 4.9$ equiv) in $\mathrm{CH}_{2} \mathrm{Cl}_{2}(2 \mathrm{~mL})$ was added and the resulting mixture was stirred at $24{ }^{\circ} \mathrm{C}$ overnight. The volatile products were removed under reduced pressure and the organic materials were dissolved in EtOAc $(15 \mathrm{~mL})$. The organic phase was washed with sat. $\mathrm{NaHCO}_{3}$ and brine, dried over $\mathrm{Na}_{2} \mathrm{SO}_{4}$ and concentrated. The excess aniline $\mathbf{1 b}$ was removed by flash column chromatography (cyclohexane $/ \mathrm{CH}_{2} \mathrm{Cl}_{2} 1: 1$, then $2: 1$ ) and the obtained compound was purified by flash column chromatography (cyclohexane/EtOAc 20:1) to give 3d (114 mg, $0.136 \mathrm{mmol}, 35 \%)$ as a white foam.

M.p. $=73-76^{\circ} \mathrm{C}\left(\mathrm{CH}_{2} \mathrm{Cl}_{2}\right) .{ }^{1} \mathbf{H}$ NMR $\left(500 \mathrm{MHz}, \mathrm{CDCl}_{3}\right) \delta 7.82(\mathrm{~s}, 2 \mathrm{H}), 7.71(\mathrm{~d}, J=8.3$ $\mathrm{Hz}, 1 \mathrm{H}), 7.68$ (s, 4H), 7.48 (d, $J=1.8 \mathrm{~Hz}, 1 \mathrm{H}), 7.34$ (dd, $J=8.3,2.0 \mathrm{~Hz}, 1 \mathrm{H}), 7.13$ (d, $J=8.7$ $\mathrm{Hz}, 2 \mathrm{H}), 6.35$ (d, $J=8.7 \mathrm{~Hz}, 2 \mathrm{H}), 5.46$ (d, $J=6.9 \mathrm{~Hz}, 2 \mathrm{H}), 2.68-2.56$ (m, 2H), $2.02-1.90$ $(\mathrm{m}, 2 \mathrm{H}) .{ }^{13} \mathrm{C}$ NMR $\left(126 \mathrm{MHz}, \mathrm{CDCl}_{3}\right) \delta$ 146.0, 143.3, 143.2, 133.8, 132.4 (q, $\left.J=33.4 \mathrm{~Hz}\right)$, $130.5,130.0$ (q, $J=32.8 \mathrm{~Hz}), 129.3,127.9$ (q, $J=3.6 \mathrm{~Hz}), 127.0,126.5-126.2(\mathrm{~m}), 124.7$ (q, $J=3.6 \mathrm{~Hz}), 124.0(\mathrm{q}, J=272.1 \mathrm{~Hz}), 123.4$ (q, $J=272.1 \mathrm{~Hz}), 121.8-121.5(\mathrm{~m}), 113.7,62.8$, 32.3. ${ }^{19} \mathbf{F}\left\{{ }^{1} \mathbf{H}\right\}$ NMR (471 MHz, $\left.\mathrm{CDCl}_{3}\right) \delta-62.7,-62.8$. HRMS (ESI+) calculated for $\left[\mathrm{C}_{33} \mathrm{H}_{19} \mathrm{BrF}_{15} \mathrm{NNa}\right]^{+} 816.0354 \mathrm{~m} / z$; found $[\mathrm{M}+\mathrm{Na}]^{+} 816.0363 \mathrm{~m} / \mathrm{z} \cdot \boldsymbol{\alpha}_{\mathbf{D}}{ }^{\mathbf{5 8 9}}=+26.1 \mathrm{deg} \cdot \mathrm{cm}^{2} \cdot \mathrm{g}^{-1}$ $\left(\mathrm{CHCl}_{3}, \mathrm{c} 1.0,300 \mathrm{~K}\right)$. 


\section{Ligand 4e}

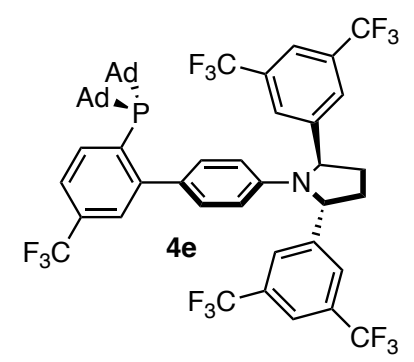

A flame-dried $10 \mathrm{~mL}$ Schlenk flask was charged with 3d (109 mg, $0.137 \mathrm{mmol}, 1.0$ equiv), $\mathrm{Pd}(\mathrm{OAc})_{2}(1.54 \mathrm{mg}, 6.86 \mu \mathrm{mol}, 5 \mathrm{~mol} \%)$, dppf (4.56 mg, $\left.8.23 \mu \mathrm{mol}, 6 \mathrm{~mol} \%\right), \mathrm{NaO} t \mathrm{Bu}(15.8$ $\mathrm{mg}, 0.165 \mathrm{mmol}, 1.2$ equiv) and toluene $(1 \mathrm{~mL})$ and the resulting suspension was stirred for 15 min at $24^{\circ} \mathrm{C}$. Di-(1-adamantyl)phosphine ( $45.6 \mathrm{mg}, 0.151 \mathrm{mmol}, 1.1$ equiv) was added and the reaction mixture was stirred at $120^{\circ} \mathrm{C}$ for $14 \mathrm{~h}$. Then, the reaction was allowed to cool to $24{ }^{\circ} \mathrm{C}$ and the crude was purified by flash column chromatography (cyclohexane $/ \mathrm{CH}_{2} \mathrm{Cl}_{2} 4: 1$, then cyclohexane/Et $\left.\mathrm{E}_{2} \mathrm{O} 40: 1\right)$ to yield ligand $4 \mathrm{e}(134 \mathrm{mg}, 0.121 \mathrm{mmol}, 88 \%)$ as an off-white foam.

M.p. $=173-178{ }^{\circ} \mathrm{C}($ cyclohexane/Et $2 \mathrm{O}) .{ }^{1} \mathbf{H}$ NMR $\left(500 \mathrm{MHz}, \mathrm{CDCl}_{3}\right) \delta 7.93(\mathrm{~d}, J=7.9$ $\mathrm{Hz}, 1 \mathrm{H}), 7.80$ (s, 2H), 7.70 (s, 4H), $7.49-7.43$ (m, 2H), 6.99 (d, $J=8.0 \mathrm{~Hz}, 2 \mathrm{H}), 6.26$ (d, $J=$ $8.6 \mathrm{~Hz}, 2 \mathrm{H}), 5.47(\mathrm{~d}, J=7.0 \mathrm{~Hz}, 2 \mathrm{H}), 2.65-2.53(\mathrm{~m}, 2 \mathrm{H}), 1.97-1.82(\mathrm{~m}, 11 \mathrm{H}), 1.81-1.76$ $(\mathrm{m}, 3 \mathrm{H}), 1.73-1.63(\mathrm{~s}, 12 \mathrm{H}), 1.63-1.51(\mathrm{~m}, 6 \mathrm{H}) .{ }^{13} \mathbf{C} \mathbf{N M R}\left(126 \mathrm{MHz}, \mathrm{CDCl}_{3}\right) \delta 151.9(\mathrm{~d}, J$ $=31.5 \mathrm{~Hz}), 146.3,142.1,138.7(\mathrm{~d}, J=33.5 \mathrm{~Hz}), 137.1$ (d, $J=2.6 \mathrm{~Hz}), 132.2$ (q, $J=33.3 \mathrm{~Hz})$, $132.1(\mathrm{~d}, J=5.0 \mathrm{~Hz}), 131.8(\mathrm{~d}, J=3.0 \mathrm{~Hz}), 130.2(\mathrm{q}, J=32.4 \mathrm{~Hz}), 127.4-127.2(\mathrm{~m}), 126.6-$ $126.4(\mathrm{~m}), 124.4(\mathrm{q}, J=273.0 \mathrm{~Hz}), 123.4$ (q, $J=273.0 \mathrm{~Hz}), 121.5-121.3(\mathrm{~m}), 121.3-121.1$ (m), 113.0, 62.6, $42.2(\mathrm{~d}, J=13.4 \mathrm{~Hz}), 41.6(\mathrm{~d}, J=13.0 \mathrm{~Hz}), 37.6(\mathrm{~d}, J=27.0 \mathrm{~Hz}), 37.3(\mathrm{~d}, J$ $=27.6 \mathrm{~Hz}), 37.0(\mathrm{~d}, J=16.9 \mathrm{~Hz}), 32.4,29.0(\mathrm{~d}, J=8.6 \mathrm{~Hz}), 28.8(\mathrm{~d}, J=8.5 \mathrm{~Hz}) .{ }^{31} \mathbf{P}\left\{{ }^{1} \mathbf{H}\right\} \mathbf{N M R}$ $\left(202 \mathrm{MHz}, \mathrm{CDCl}_{3}\right) \delta 20.3 .{ }^{19} \mathbf{F}\left\{{ }^{1} \mathbf{H}\right\}$ NMR $\left(376 \mathrm{MHz}, \mathrm{CDCl}_{3}\right) \delta-62.7,-62.8$. HRMS (ESI+) calculated for $\left[\mathrm{C}_{53} \mathrm{H}_{50} \mathrm{~F}_{15} \mathrm{NP}\right]^{+} 1016.3436$; found $[\mathrm{M}+\mathrm{H}]^{+} 1016.3457 . \boldsymbol{\alpha}_{\mathbf{D}}^{\mathbf{5 8 9}}=+23.8 \mathrm{deg} . \mathrm{cm}^{2} \cdot \mathrm{g}^{-}$ ${ }^{1}\left(\mathrm{CHCl}_{3}, \mathrm{c} 1.0,300 \mathrm{~K}\right)$. 
$(R, R)-\mathrm{E}$

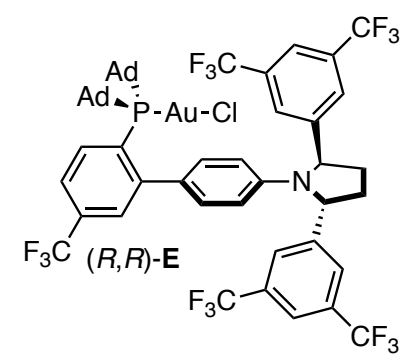

$\mathrm{Me}_{2} \mathrm{SAuCl}$ (35.4 mg, $0.120 \mathrm{mmol}, 1.0$ equiv) and ligand 4e (122 mg, $0.120 \mathrm{mmol}, 1.0$ equiv) were dissolved in $\mathrm{CH}_{2} \mathrm{Cl}_{2}(1.2 \mathrm{~mL})$ under an atmosphere of argon. The reaction mixture was stirred at $24{ }^{\circ} \mathrm{C}$ for $15 \mathrm{~min}$. The mixture was filtered through a syringe filter and concentrated. The residue was dissolved in $\mathrm{CH}_{2} \mathrm{Cl}_{2}$, filtered through a path of neutral $\mathrm{Al}_{2} \mathrm{O}_{3}$ and concentrated to yield complex $(R, R)$-E (146 $\mathrm{mg}, 0.115 \mathrm{mmol}, 95 \%)$ as a white solid.

M.p. $=>306{ }^{\circ} \mathrm{C}\left(\right.$ decomposition; pentane $\left./ \mathrm{Et}_{2} \mathrm{O}\right) .{ }^{1} \mathbf{H}$ NMR $\left(500 \mathrm{MHz}, \mathrm{CDCl}_{3}\right) \delta 7.96-7.84$ (m, 5H), $7.79(\mathrm{~s}, 2 \mathrm{H}), 7.65(\mathrm{dd}, J=8.3,2.1 \mathrm{~Hz}, 1 \mathrm{H}), 7.55(\mathrm{dd}, J=3.7,2.1 \mathrm{~Hz}, 1 \mathrm{H}), 6.92(\mathrm{dd}, J$ $=8.4,2.2 \mathrm{~Hz}, 1 \mathrm{H}), 6.70(\mathrm{dd}, J=8.5,2.2 \mathrm{~Hz}, 1 \mathrm{H}), 6.30(\mathrm{dd}, J=8.4,2.7 \mathrm{~Hz}, 1 \mathrm{H}), 6.19(\mathrm{dd}, J=$ 8.5, $2.7 \mathrm{~Hz}, 1 \mathrm{H}), 5.49$ (d, $J=6.9 \mathrm{~Hz}, 2 \mathrm{H}), 2.67-2.55(\mathrm{~m}, 2 \mathrm{H}), 2.27-2.16(\mathrm{~m}, 6 \mathrm{H}), 2.03-1.82$ $(\mathrm{m}, 14 \mathrm{H}), 1.74-1.53(\mathrm{~m}, 12 \mathrm{H}) .{ }^{13} \mathbf{C} \mathbf{N M R}\left(126 \mathrm{MHz}, \mathrm{CDCl}_{3}\right) \delta 151.6(\mathrm{~d}, J=12.8 \mathrm{~Hz}), 146.3$, 143.9, 134.9 (d, $J=2.4 \mathrm{~Hz}), 132.1$ (qd, $J=33.2,2.1 \mathrm{~Hz}), 132.0$ (q, $J=32.5 \mathrm{~Hz}), 130.4-130.1$ (m), 129.9, 129.6, 129.2 (d, $J=6.2 \mathrm{~Hz}), 128.8(\mathrm{~d}, J=40.9 \mathrm{~Hz}), 127.0-126.8(\mathrm{~m}), 123.6$ (q, $J$ $=274.4 \mathrm{~Hz}), 123.5(\mathrm{q}, J=272.1 \mathrm{~Hz}), 122.4-122.3(\mathrm{~m}), 121.4-121.1(\mathrm{~m}), 116.1,113.7,63.3$, $43.3(\mathrm{~d}, J=22.3 \mathrm{~Hz}), 42.8$ (d, $J=2.5 \mathrm{~Hz}), 42.4$ (d, $J=22.2 \mathrm{~Hz}), 41.6$ (d, $J=2.3 \mathrm{~Hz}), 36.3$ (d, $J=19.2 \mathrm{~Hz}), 32.5,28.8(\mathrm{~d}, J=9.8 \mathrm{~Hz}), 28.5(\mathrm{~d}, J=9.9 \mathrm{~Hz}) .{ }^{31} \mathbf{P}\left\{{ }^{1} \mathbf{H}\right\} \mathbf{N M R}\left(203 \mathrm{MHz}, \mathrm{CDCl}_{3}\right)$ $\delta$ 64.9. ${ }^{19} \mathbf{F}\left\{{ }^{1} \mathbf{H}\right\}$ NMR $\left(471 \mathrm{MHz}, \mathrm{CDCl}_{3}\right) \delta-62.4,-63.1$. HRMS (ESI+) calculated for $\left[\mathrm{C}_{53} \mathrm{H}_{49} \mathrm{AuClF}_{15} \mathrm{NNaP}^{+} 1270.2610 \mathrm{~m} / \mathrm{z}\right.$; found $[\mathrm{M}+\mathrm{Na}]^{+} 1270.2583 \mathrm{~m} / \mathrm{z}$. Elemental analysis calculated for $\mathrm{C}_{53} \mathrm{H}_{49} \mathrm{AuClF}_{15} \mathrm{NP}$ : C 50.99; H 3.96; N 1.12; found: C 50.99; H 3.96; N 1.19. $\boldsymbol{\alpha}_{\mathbf{D}}{ }^{\mathbf{5 8 9}}=+40.2 \mathrm{deg} \cdot \mathrm{cm}^{2} \cdot \mathrm{g}^{-1}\left(\mathrm{CHCl}_{3}, \mathrm{c} 1.0,301 \mathrm{~K}\right)$. 


\subsubsection{Synthesis of chiral complex $(S, S)-\mathrm{F}$}

\section{Chiral precursor $3 \mathrm{e}$}

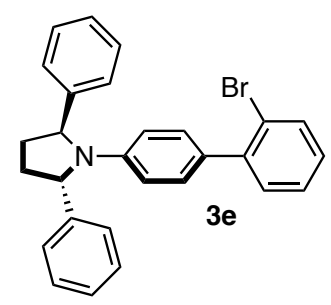

To a solution of $(1 R, 4 R)-1,4-$ diphenylbutane-1,4-diol ${ }^{3}(0.20 \mathrm{~g}, 0.83 \mathrm{mmol}, 1.0$ equiv) in $\mathrm{CH}_{2} \mathrm{Cl}_{2}(8.3 \mathrm{~mL})$ at $-20{ }^{\circ} \mathrm{C}$ were added $\mathrm{NEt}_{3}(0.35 \mathrm{~mL}, 2.48 \mathrm{mmol}, 3.0$ equiv) and methanesulfonyl chloride $(0.17 \mathrm{~mL}, 2.15 \mathrm{mmol}, 2.6$ equiv) under an anhydrous argon atmosphere. The mixture was stirred for $2.5 \mathrm{~h}$ at $-20^{\circ} \mathrm{C}$. Then, a solution of aniline $1 \mathrm{a}(1.02 \mathrm{~g}$, 4.1 mmol, 5.0 equiv) in $\mathrm{CH}_{2} \mathrm{Cl}_{2}(1.5 \mathrm{~mL})$ was added and the resulting solution was stirred at 24 ${ }^{\circ} \mathrm{C}$ overnight. The volatiles were removed under reduced pressure and the organic materials were dissolved in EtOAc. The organic phase was washed with brine and sat. $\mathrm{NaHCO}_{3}$, dried over $\mathrm{Na}_{2} \mathrm{SO}_{4}$ and concentrated. The excess of aniline 1a was removed by flash column chromatography (cyclohexane/ $\mathrm{CH}_{2} \mathrm{Cl}_{2}$ 1:1). The title compound was recrystallized from $\mathrm{CH}_{2} \mathrm{Cl}_{2} / \mathrm{Et}_{2} \mathrm{O}$ to yield $3 \mathrm{e}$ (94 mg, $0.21 \mathrm{mmol}, 52 \%$ ) as a white crystalline solid.

M.p. $=130-132{ }^{\circ} \mathrm{C}\left(\mathrm{CH}_{2} \mathrm{Cl}_{2} / \mathrm{Et}_{2} \mathrm{O}\right) .{ }^{1} \mathbf{H} \mathbf{N M R}\left(400 \mathrm{MHz}, \mathrm{CD}_{2} \mathrm{Cl}_{2}\right) \delta 7.58(\mathrm{dd}, J=8.0,1.2$ $\mathrm{Hz}, 1 \mathrm{H}), 7.38-7.33(\mathrm{~m}, 4 \mathrm{H}), 7.30-7.20(\mathrm{~m}, 8 \mathrm{H}), 7.11-7.04(\mathrm{~m}, 3 \mathrm{H}), 6.43-6.37(\mathrm{~m}, 2 \mathrm{H})$, $5.27(\mathrm{~d}, J=7.3 \mathrm{~Hz}, 2 \mathrm{H}), 2.66-2.54(\mathrm{~m}, 2 \mathrm{H}), 1.88-1.75(\mathrm{~m}, 2 \mathrm{H}) .{ }^{13} \mathbf{C}$ NMR $(101 \mathrm{MHz}$, $\left.\mathrm{CD}_{2} \mathrm{Cl}_{2}\right) \delta 145.1,144.6,143.0,133.6,132.0,130.3,129.1,128.7,128.3,127.9,127.3,126.7$, 123.1, 113.7, 63.9, 32.8. HRMS (ESI+) calculated for $\left[\mathrm{C}_{28} \mathrm{H}_{25} \mathrm{BrN}\right]^{+} m / z$ 454.1165; found [M $+\mathrm{H}]^{+} m / z$ 454.1175. $\boldsymbol{\alpha}_{\mathbf{D}}{ }^{589}=-93.2$ deg. $\mathrm{cm}^{2} \cdot \mathrm{g}^{-1}\left(\mathrm{CHCl}_{3}, \mathrm{c} 0.9,300 \mathrm{~K}\right)$.

\section{Ligand $4 f$}

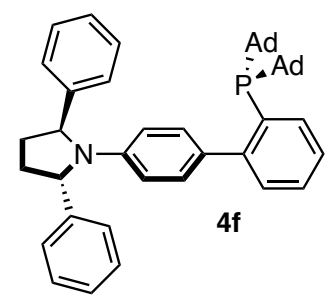

A flame-dried $20 \mathrm{~mL}$ Schlenk flask was charged with $3 \mathbf{e}(0.40 \mathrm{~g}, 0.88 \mathrm{mmol}, 1.0$ equiv), $\mathrm{Pd}(\mathrm{OAc})_{2}(9.9 \mathrm{mg}, 0.04 \mathrm{mmol}, 5 \mathrm{~mol} \%)$, dppf (29 mg, $\left.0.05 \mathrm{mmol}, 6 \mathrm{~mol} \%\right), \mathrm{NaO} t \mathrm{Bu}(102$ $\mathrm{mg}, 1.06 \mathrm{mmol}, 1.2$ equiv) and toluene $(4.4 \mathrm{~mL})$, the resulting suspension was stirred for 15 min at $24{ }^{\circ} \mathrm{C}$. Di(1-adamantyl)phosphine (293 mg, $0.97 \mathrm{mmol}, 1.1$ equiv) was added and the reaction mixture was stirred at $120^{\circ} \mathrm{C}$ for $16 \mathrm{~h}$. Then, the reaction was allowed to cool to $24{ }^{\circ} \mathrm{C}$ 
and the crude was purified by flash column chromatography (cyclohexane/ $\mathrm{CH}_{2} \mathrm{Cl}_{2} 3: 1$, then cyclohexane/Et $2 \mathrm{O} 40: 1)$ to yield ligand $\mathbf{4 f}(157 \mathrm{mg}, 0.23 \mathrm{mmol}, 72 \%)$ as a white solid.

M.p. $=173-175{ }^{\circ} \mathrm{C}($ cyclohexane/Et $2 \mathrm{O}) .{ }^{1} \mathbf{H}$ NMR $\left(400 \mathrm{MHz}, \mathrm{CDCl}_{3}\right) 7.82-7.76(\mathrm{~m}, 1 \mathrm{H})$, $7.35-728(\mathrm{~m}, 4 \mathrm{H}), 7.28-7.26(\mathrm{~m}, 3 \mathrm{H}), 7.26-7.24(\mathrm{~m}, 2 \mathrm{H}), 7.24-7.16(\mathrm{~m}, 4 \mathrm{H}), 6.94-6.88$ $(\mathrm{m}, 2 \mathrm{H}), 6.32-6.27(\mathrm{~m}, 2 \mathrm{H}), 5.25(\mathrm{~d}, J=7.0 \mathrm{~Hz}, 2 \mathrm{H}), 2.65-2.46(\mathrm{~m}, 2 \mathrm{H}), 2.05-1.91(\mathrm{~m}$, $5 \mathrm{H}), 1.91-1.86(\mathrm{~m}, 3 \mathrm{H}), 1.84-1.79(\mathrm{~m}, 2 \mathrm{H}), 1.76$ (br s, 3H), $1.70-1.64(\mathrm{~m}, 10 \mathrm{H}), 1.58-$ $1.53(\mathrm{~m}, 8 \mathrm{H}) .{ }^{13} \mathrm{C}$ NMR $\left(101 \mathrm{MHz}, \mathrm{CDCl}_{3}\right) \delta 152.1(\mathrm{~d}, J=31.1 \mathrm{~Hz}), 144.2,143.6,136.8(\mathrm{~d}, J$ $=2.9 \mathrm{~Hz}), 133.1(\mathrm{~d}, J=27.4 \mathrm{~Hz}), 131.5(\mathrm{~d}, J=4.8 \mathrm{~Hz}), 131.2(\mathrm{~d}, J=6.0 \mathrm{~Hz}), 131.1(\mathrm{~d}, J=6.9$ Hz). 128.5, 128.2, 126.6, 126.5, 124.4, 112.7, 63.4, 42.5 (d, $J=13.5 \mathrm{~Hz}), 41.5$ (d, $J=12.9 \mathrm{~Hz})$, $37.6(\mathrm{~d}, J=26.1 \mathrm{~Hz}) 37.2(\mathrm{~d}, J=26.9 \mathrm{~Hz}), 37.0(\mathrm{~d}, J=10.0 \mathrm{~Hz}), 32.6,29.1(\mathrm{~d}, J=8.6 \mathrm{~Hz})$, $28.9(\mathrm{~d}, J=8.5 \mathrm{~Hz}) .{ }^{31} \mathbf{P}\left\{{ }^{1} \mathbf{H}\right\}$ NMR $\left(162 \mathrm{MHz}, \mathrm{CDCl}_{3}\right) \delta 24.5$. HRMS (ESI+) calculated for $\left[\mathrm{C}_{48} \mathrm{H}_{55} \mathrm{NP}\right]^{+} 676.4067 \mathrm{~m} / z$; found $[\mathrm{M}+\mathrm{H}]^{+} 676.4042 \mathrm{~m} / z . \boldsymbol{\alpha}_{\mathbf{D}}{ }^{589}=-47.5 \mathrm{deg} \cdot \mathrm{cm}^{2} \cdot \mathrm{g}^{-1}\left(\mathrm{CHCl}_{3}\right.$, c $1.0,298 \mathrm{~K})$.

\section{$(S, S)-\mathbf{F}$}

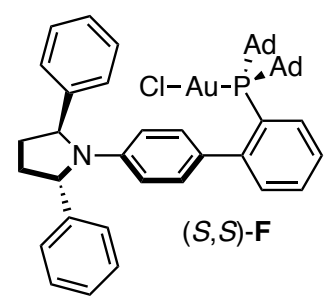

$\mathrm{Me}_{2} \mathrm{SAuCl}$ (100 mg, $0.15 \mathrm{mmol}, 1.0$ equiv) and ligand $4 \mathbf{f}$ (44 mg, $0.15 \mathrm{mmol}, 1.0$ equiv) were dissolved in $\mathrm{CH}_{2} \mathrm{Cl}_{2}(1.5 \mathrm{~mL})$ under an atmosphere of argon. The reaction mixture was stirred at $24{ }^{\circ} \mathrm{C}$ for $30 \mathrm{~min}$. The mixture was filtered through a syringe filter and concentrated. The residue was dissolved in $\mathrm{CH}_{2} \mathrm{Cl}_{2}$, filtered through a path of neutral $\mathrm{Al}_{2} \mathrm{O}_{3}$ and concentrated. The residue was dissolved in a minimal amount of $\mathrm{CH}_{2} \mathrm{Cl}_{2}$ and precipitated from $\mathrm{Et}_{2} \mathrm{O}$ to afford complex $(S, S)-\mathbf{F}(121 \mathrm{mg}, 0.13 \mathrm{mmol}, 90 \%)$ as a white solid.

M.p. $=>260{ }^{\circ} \mathrm{C}$ (decomposition; $\left.\mathrm{Et}_{2} \mathrm{O} / \mathrm{MeOH}\right) .{ }^{1} \mathbf{H}$ NMR $\left(400 \mathrm{MHz}, \mathrm{CD}_{2} \mathrm{Cl}_{2}\right) \delta 7.81(\mathrm{td}, J$ $=7.2,2.1 \mathrm{~Hz}, 1 \mathrm{H}), 7.45-7.38(\mathrm{~m}, 6 \mathrm{H}), 7.37-7.32(\mathrm{~m}, 4 \mathrm{H}), 7.26-7.21(\mathrm{~m}, 2 \mathrm{H}), 7.19-7.15$ (m, 1H), $6.81-6.74(\mathrm{~m}, 2 \mathrm{H}), 6.35-6.28(\mathrm{~m}, 2 \mathrm{H}), 5.24(\mathrm{~d}, J=6.9 \mathrm{~Hz}, 2 \mathrm{H}), 2.65-2.49(\mathrm{~m}$, 2H), $2.31-2.22(\mathrm{~m}, 3 \mathrm{H}), 2.22-2.15(\mathrm{~m}, 3 \mathrm{H}), 2.09-1.93(\mathrm{~m}, 9 \mathrm{H}), 1.93-1.87(\mathrm{~m}, 3 \mathrm{H}), 1.78$ $-1.72(\mathrm{~m}, 2 \mathrm{H}), 1.70(\mathrm{~s}, 6 \mathrm{H}), 1.61(\mathrm{~s}, 6 \mathrm{H}) .{ }^{13} \mathbf{C}$ NMR $\left(101 \mathrm{MHz}, \mathrm{CD}_{2} \mathrm{Cl}_{2}\right) \delta 151.2(\mathrm{~d}, J=13.2$ $\mathrm{Hz}), 144.5$ (d, $J=107.6 \mathrm{~Hz}), 134.6(\mathrm{~d}, J=2.5 \mathrm{~Hz}), 134.2$ (d, $J=7.2 \mathrm{~Hz}), 130.0$ (d, $J=2.3 \mathrm{~Hz})$, 129.9, $129.4(\mathrm{~d}, J=6.6 \mathrm{~Hz}), 129.3,128.4,126.6,126.5,125.5$ (d, $J=6.6 \mathrm{~Hz}), 123.4$ (d, $J=$ $44.7 \mathrm{~Hz}), 113.7(\mathrm{~d}, J=74.3 \mathrm{~Hz}), 63.5,42.6-42.3(\mathrm{~m}), 42.5(\mathrm{~d}, J=2.6 \mathrm{~Hz}), 41.8(\mathrm{~d}, J=2.6$ 
$\mathrm{Hz}), 36.3(\mathrm{~d}, J=9.5 \mathrm{~Hz}), 36.2(\mathrm{~d}, J=9.5 \mathrm{~Hz}), 32.4,28.8(\mathrm{~d}, J=13.8), 28.7(\mathrm{~d}, J=13.9) .{ }^{31} \mathbf{P}\left\{{ }^{1} \mathbf{H}\right\}$

NMR $\left(202 \mathrm{MHz}, \mathrm{CDCl}_{3}\right) \delta$ 65.1. HRMS (ESI+) calculated for $\left[\mathrm{C}_{48} \mathrm{H}_{55} \mathrm{AuClNP}\right]^{+} 908.3421$ $m / z$; found $[\mathrm{M}+\mathrm{H}]^{+} 908.3393 \mathrm{~m} / z \cdot \boldsymbol{\alpha}_{\mathbf{D}}{ }^{589}=-57.1 \mathrm{deg} \cdot \mathrm{cm}^{2} \cdot \mathrm{g}^{-1}\left(\mathrm{CHCl}_{3}, \mathrm{c} 0.8,299 \mathrm{~K}\right)$.

\subsubsection{Synthesis of chiral complex $(R, R)-G$}<smiles>Nc1cccc(-c2ccccc2Br)c1</smiles>

$1 c$

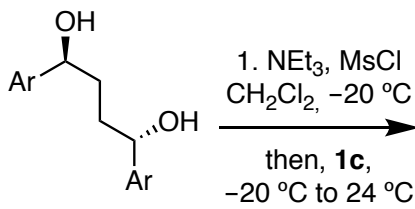

$2 c$

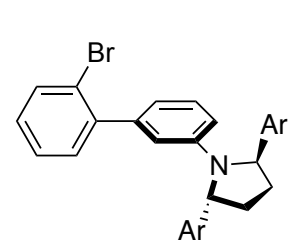

$3 f$

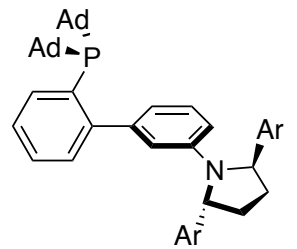

$4 g$

$\mathrm{Ar}=3,5-\left(\mathrm{CF}_{3}\right)_{2} \mathrm{C}_{6} \mathrm{H}_{3}$

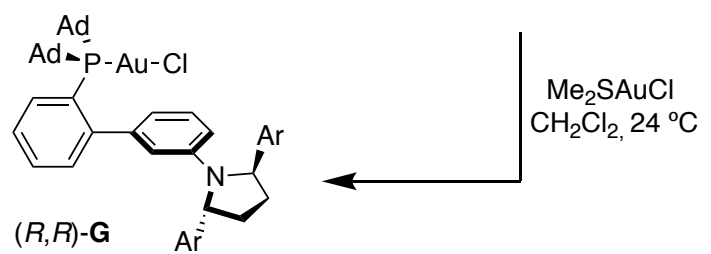

\section{Chiral precursor $3 f$}

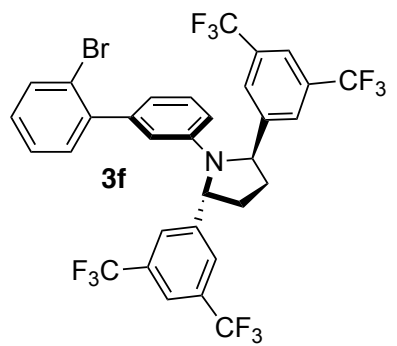

A $25 \mathrm{~mL}$ flame-dried two necked round bottom flask was charged with solution of 1,4bis(3,5-bis(trifluoromethyl)phenyl)butane-1,4-diol (2c) (360 mg, $0.7 \mathrm{mmol}, 1.0$ equiv) in $\mathrm{CH}_{2} \mathrm{Cl}_{2}(6 \mathrm{~mL})$ at $-20{ }^{\circ} \mathrm{C}$. $\mathrm{NEt}_{3}(0.40 \mathrm{~mL}, 2.80 \mathrm{mmol}, 4.0$ equiv $)$ and methanesulfonyl chloride ( $0.11 \mathrm{~mL}, 1.40 \mathrm{mmol}, 2.0$ equiv) were added under argon atmosphere and the mixture was stirred for $2.5 \mathrm{~h}$ at $-20^{\circ} \mathrm{C}$. Aniline $1 \mathrm{c}(870 \mathrm{mg}, 3.50 \mathrm{mmol}, 5.0$ equiv) was then added and the solution was stirred for $20 \mathrm{~h}$ at $24{ }^{\circ} \mathrm{C}$. The reaction was quenched with sat. $\mathrm{NH}_{4} \mathrm{Cl}$ and the aqueous phase was extracted with EtOAc. After evaporation of the solvent, the crude was purified by flash column chromatography (cyclohexane $/ \mathrm{CH}_{2} \mathrm{Cl}_{2}$ 1:1) to afford the title compound $3 \mathbf{f}$ (163 $\mathrm{mg}, 0.224 \mathrm{mmol}, 32 \%$ ) as a white solid.

M.p. $=55-57{ }^{\circ} \mathrm{C}\left(\right.$ cyclohexane $\left./ \mathrm{CH}_{2} \mathrm{Cl}_{2}\right) .{ }^{1} \mathbf{H}$ NMR $\left(500 \mathrm{MHz} \mathrm{CDCl}_{3}\right) \delta 7.78(\mathrm{~s}, 2 \mathrm{H}), 7.66$ (s, 4H), $7.51(\mathrm{dd}, J=7.9,1.01 \mathrm{H}), 7.26-7.23(\mathrm{~m}, 1 \mathrm{H}), 7.15-7.09(\mathrm{~m}, 3 \mathrm{H}), 6.63(\mathrm{~d}, J=8.0$ $\mathrm{Hz}, 1 \mathrm{H}), 6.38-6.34(\mathrm{~m}, 1 \mathrm{H}), 6.32(\mathrm{dd}, J=8.3,2.5 \mathrm{~Hz}, 1 \mathrm{H}), 5.43(\mathrm{~d}, J=7.2 \mathrm{~Hz}, 2 \mathrm{H}), 2.64-$ $2.54(\mathrm{~m}, 2 \mathrm{H}), 1.97-1.88(\mathrm{~m}, 2 \mathrm{H}) .{ }^{13} \mathbf{C} \mathbf{N M R}\left(126 \mathrm{MHz}, \mathrm{CDCl}_{3}\right) \delta 146.3,142.8(\mathrm{~d}, J=2.8 \mathrm{~Hz})$, 
$142.2,133.2,132.2(\mathrm{q}, J=33.3 \mathrm{~Hz}), 131.1,129.4,128.7,127.3,126.6-126.4$ (m), 123.4 (q, $J$ $=273.2 \mathrm{~Hz}), 122.5,121.5(\mathrm{p}, J=3.7 \mathrm{~Hz}), 118.9,115.8,113.5,62.6,32.3 .{ }^{19} \mathbf{F}\left\{{ }^{1} \mathbf{H}\right\} \mathbf{N M R}(376$ $\left.\mathrm{MHz}, \mathrm{CDCl}_{3}\right) \delta-62.5$. HRMS (ESI+) calculated for $\left[\mathrm{C}_{32} \mathrm{H}_{21} \mathrm{BrF}_{12} \mathrm{~N}\right]^{+} 726.0660 \mathrm{~m} / \mathrm{z}$; found [M $+\mathrm{H}]^{+} 726.0683 \mathrm{~m} / z \cdot \boldsymbol{\alpha}_{\mathbf{D}}{ }^{\mathbf{5 8 9}}=-54.92 \mathrm{deg} \cdot \mathrm{cm}^{2} \cdot \mathrm{g}^{-1}\left(\mathrm{CHCl}_{3}, \mathrm{c} 1.00,300 \mathrm{~K}\right)$.

\section{Ligand 4g}

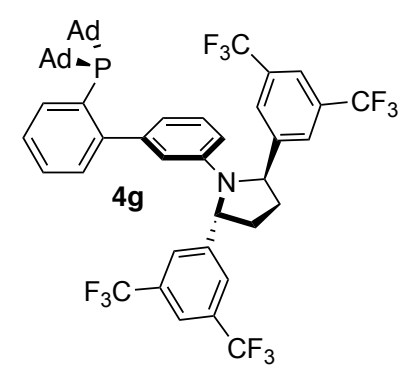

A $10 \mathrm{~mL}$ Schlenk flask was charged with $\mathbf{3 f}(100 \mathrm{mg}, 0.14 \mathrm{mmol}), \operatorname{Pd}(\mathrm{OAc})_{2}(1.55 \mathrm{mg}, 6.88$ $\mu \mathrm{mol}, 5 \mathrm{~mol} \%$ ), $\mathrm{NaO} t \mathrm{Bu}(15.88 \mathrm{mg}, 0.17 \mathrm{mmol}, 1.2$ equiv), dppf (4.60 mg, $8.26 \mu \mathrm{mol}, 5 \mathrm{~mol}$ $\%$ ) and toluene $(0.69 \mathrm{~mL})$. The resulting suspension was stirred for $15 \mathrm{~min}$ at $24{ }^{\circ} \mathrm{C}$. Di(adamantan-1-yl)phosphane (46 mg, $0.151 \mathrm{mmol}, 1.1$ equiv) was added and the resulting mixture was stirred at $120^{\circ} \mathrm{C}$ for $24 \mathrm{~h}$. Then, the reaction was allowed to cool to $24{ }^{\circ} \mathrm{C}$, filtered through syringe filter and washed with EtOAc. The crude product was purified by flash column chromatography (cyclohexane/ $\mathrm{CH}_{2} \mathrm{Cl}_{2} 3: 1$, then cyclohexane/Et ${ }_{2} \mathrm{O} 40: 1$ ) to afford ligand $\mathbf{4 g}$ (78 $\mathrm{mg}, 0.14 \mathrm{mmol}, 60 \%$ ) as a white solid.

M.p. $=114-116^{\circ} \mathrm{C}\left(\right.$ cyclohexane $\left./ \mathrm{CH}_{2} \mathrm{Cl}_{2} / \mathrm{Et}_{2} \mathrm{O}\right) .{ }^{1} \mathbf{H} \mathbf{N M R}\left(500 \mathrm{MHz} \mathrm{CDCl}_{3}\right) \delta 7.84-7.80$ $(\mathrm{m}, 1 \mathrm{H}), 7.76(\mathrm{~s}, 2 \mathrm{H}), 7.65(\mathrm{~s}, 4 \mathrm{H}), 7.27-7.24(\mathrm{~m}, 2 \mathrm{H}), 7.04-6.98(\mathrm{~m}, 2 \mathrm{H}), 6.57(\mathrm{~d}, J=7.4 \mathrm{~Hz}$, $1 \mathrm{H}), 6.32(\mathrm{~s}, 1 \mathrm{H}), 6.23(\mathrm{dd}, J=8.2,2.1 \mathrm{~Hz}, 1 \mathrm{H}), 5.46(\mathrm{~d}, J=7.0 \mathrm{~Hz}, 2 \mathrm{H}), 2.60-2.50(\mathrm{~m}, 2 \mathrm{H})$, $1.98-1.86(\mathrm{~m}, 12 \mathrm{H}), 1.77-1.61(\mathrm{~m}, 15 \mathrm{H}), 1.61-1.48(\mathrm{~m}, 5 \mathrm{H}) .{ }^{13} \mathbf{C} \mathbf{N M R}\left(126 \mathrm{MHz}, \mathrm{CDCl}_{3}\right)$ $\delta 151.9(\mathrm{~d}, J=31.8 \mathrm{~Hz}), 146.5,144.9(\mathrm{~d}, J=6.8 \mathrm{~Hz}), 142.1,136.8(\mathrm{~d}, J=2.7 \mathrm{~Hz}), 133.2(\mathrm{~d}, J$ $=29.5 \mathrm{~Hz}), 132.1(\mathrm{q}, J=33.2 \mathrm{~Hz}), 130.5(\mathrm{~d}, J=6.0 \mathrm{~Hz}), 128.1(\mathrm{~d}, J=16.5 \mathrm{~Hz}), 126.6-126.4$ (m), 125.3, 123.4 (q, $J=274.8 \mathrm{~Hz}), 121.5-121.2(\mathrm{~m}), 121.0(\mathrm{~d}, J=3.5 \mathrm{~Hz}), 117.3$ (d, $J=5.2$ Hz), 112.6, 62.4, 42.4 (d, $J=13.6 \mathrm{~Hz}), 41.5$ (d, $J=13.1 \mathrm{~Hz}), 37.4,37.2,36.99,36.95$ (d, $J=$ $4.4 \mathrm{~Hz}), 32.3,29.0$ (d, $J=8.5 \mathrm{~Hz}), 28.9(\mathrm{~d}, J=8.6 \mathrm{~Hz}) .{ }^{31} \mathbf{P}\left\{{ }^{1} \mathbf{H}\right\} \mathbf{N M R}\left(162 \mathrm{MHz}, \mathrm{CDCl}_{3}\right) \delta$ 24.6. ${ }^{19} \mathbf{F}\left\{{ }^{1} \mathbf{H}\right\}$ NMR $\left(376 \mathrm{MHz}, \mathrm{CDCl}_{3}\right) \delta$-62.8. HRMS (ESI+) calculated for $\left[\mathrm{C}_{52} \mathrm{H}_{51} \mathrm{~F}_{12} \mathrm{NP}\right]^{+}$ $948.3562 \mathrm{~m} / z$; found $[\mathrm{M}+\mathrm{H}]^{+} 948.3565 \mathrm{~m} / z . \boldsymbol{\alpha}_{\mathbf{D}}{ }^{\mathbf{5 8 9}}=+20.7 \mathrm{deg} \cdot \mathrm{cm}^{2} \cdot \mathrm{g}^{-1}\left(\mathrm{CHCl}_{3}, \mathrm{c} 0.65,302\right.$ $\mathrm{K})$. 


\section{$(R, R)-G$}

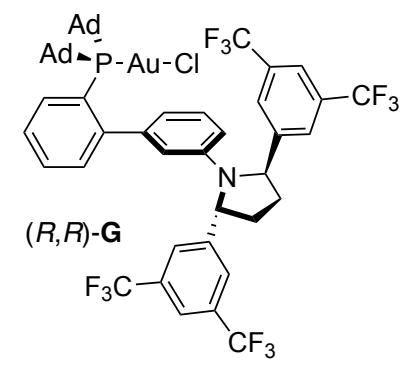

$\mathrm{Me}_{2} \mathrm{SAuCl}$ (6 mg, $0.021 \mathrm{mmol}, 1.0$ equiv) and ligand $4 \mathrm{~g}$ (20 mg, $0.021 \mathrm{mmol}, 1.0$ equiv) were dissolved in $\mathrm{CH}_{2} \mathrm{Cl}_{2}(0.4 \mathrm{~mL})$ under an atmosphere of argon. The reaction mixture was stirred at $24{ }^{\circ} \mathrm{C}$ for $15 \mathrm{~min}$. The mixture was dissolved in $\mathrm{CH}_{2} \mathrm{Cl}_{2}$, filtered through a path of neutral $\mathrm{Al}_{2} \mathrm{O}_{3}$ and concentrated to yield complex $(R, R)-\mathbf{G}(24 \mathrm{mg}, 0.021 \mathrm{mmol}, 96 \%)$ as a white solid in a 5:1 mixture of rotamers.

M.p. $=>95{ }^{\circ} \mathrm{C}$ (decomposition; $\left.\mathrm{CH}_{2} \mathrm{Cl}_{2}\right)$. Major rotamer: ${ }^{1} \mathbf{H}$ NMR $\left(400 \mathrm{MHz}, \mathrm{CDCl}_{3}\right) \delta$ $7.76(\mathrm{~d}, J=12.7 \mathrm{~Hz}, 7 \mathrm{H}), 7.50$ (t, $J=7.4 \mathrm{~Hz}, 1 \mathrm{H}), 7.42(\mathrm{t}, J=7.5 \mathrm{~Hz}, 1 \mathrm{H}), 7.34-7.29(\mathrm{~m}, 1 \mathrm{H})$, 6.98 (t, $J=7.9 \mathrm{~Hz}, 1 \mathrm{H}), 6.42$ (dd, $J=8.4,2.2 \mathrm{~Hz}, 1 \mathrm{H}), 6.32$ (d, $J=7.4 \mathrm{~Hz}, 1 \mathrm{H}), 6.08$ (s, 1H), $5.47(\mathrm{~d}, J=5.2 \mathrm{~Hz}, 2 \mathrm{H}), 2.57-2.45(\mathrm{~m}, 2 \mathrm{H}), 2.20-1.99(\mathrm{~m}, 6 \mathrm{H}), 1.96$ (br s, 3H), $1.79-1.61$ $(\mathrm{m}, 14 \mathrm{H}), 1.60-1.50(\mathrm{~s}, 6 \mathrm{H}), 1.46-1.38(\mathrm{~s}, 3 \mathrm{H}) .{ }^{13} \mathbf{C}$ NMR $\left(126 \mathrm{MHz}, \mathrm{CDCl}_{3}\right) \delta 150.9(\mathrm{~d}, J$ $=13.6 \mathrm{~Hz}), 146.3,143.7-143.6(\mathrm{~m}), 143.5,134.2(\mathrm{~d}, J=2.5 \mathrm{~Hz}), 133.3(\mathrm{~d}, J=7.3 \mathrm{~Hz}), 131.8$ (q, $J=32.7 \mathrm{~Hz}), 130.1(\mathrm{~d}, J=2.5 \mathrm{~Hz}), 128.7,126.6-126.3(\mathrm{~m}), 126.1$ (d, $J=6.4 \mathrm{~Hz}), 124.2$, $123.9,123.3(\mathrm{q}, J=274.1 \mathrm{~Hz}), 121.4-121.1(\mathrm{~m}), 119.6,115.1,112.8,62.5,42.2-42.0(\mathrm{~m})$, $41.5(\mathrm{~d}, J=2.7 \mathrm{~Hz}), 36.1$ (d, $J=40.3 \mathrm{~Hz}), 32.0,28.5$ (d, $J=9.9 \mathrm{~Hz}), 28.3$ (d, $J=9.7 \mathrm{~Hz})$. ${ }^{31} \mathbf{P}\left\{{ }^{1} \mathbf{H}\right\}$ NMR $\left(162 \mathrm{MHz}, \mathrm{CDCl}_{3}\right) \delta$ 64.4. ${ }^{19} \mathbf{F}\left\{{ }^{1} \mathbf{H}\right\}$ NMR $\left(471 \mathrm{MHz}, \mathrm{CDCl}_{3}\right) \delta-62.4$. Minor rotamer (only significant signals): ${ }^{1} \mathbf{H}$ NMR $\left(400 \mathrm{MHz}, \mathrm{CDCl}_{3}\right) 7.36(\mathrm{~d}, J=7.7 \mathrm{~Hz}, 0.2 \mathrm{H})$, $7.08(\mathrm{t}, J=7.9 \mathrm{~Hz}, 0.2 \mathrm{H}), 6.71(\mathrm{dd}, J=8.3,3.3 \mathrm{~Hz}, 0.2 \mathrm{H}), 6.51(\mathrm{dd}, J=8.2,2.2 \mathrm{~Hz}, 0.2 \mathrm{H})$, $5.87(\mathrm{~s}, 0.2 \mathrm{H}), 5.32(\mathrm{~d}, J=7.0 \mathrm{~Hz}, 0.4 \mathrm{H}), 2.65-2.58(\mathrm{~m}, 0.2 \mathrm{H}), 1.85(\mathrm{~d}, J=6.4 \mathrm{~Hz}, 1 \mathrm{H}) .{ }^{13} \mathrm{C}$ NMR $\left(126 \mathrm{MHz}, \mathrm{CDCl}_{3}\right) \delta 150.8(\mathrm{~d}, J=13.2 \mathrm{~Hz}), 143.0(\mathrm{~d}, J=6.4 \mathrm{~Hz}), 134.2(\mathrm{~d}, J=2.1 \mathrm{~Hz})$, $133.1(\mathrm{~d}, J=7.5 \mathrm{~Hz}), 132.0$ (q, $J=33.3 \mathrm{~Hz}), 129.7,126.0$ (d, $J=6.7 \mathrm{~Hz}), 123.3$ (q, $J=272.6$ $\mathrm{Hz}), 119.4,116.2,113.7,63.3,42.3,41.9,32.4,28.7$ (d, $J=9.7 \mathrm{~Hz}) .{ }^{31} \mathbf{P}\left\{{ }^{1} \mathbf{H}\right\} \mathbf{N M R}(162 \mathrm{MHz}$, $\left.\mathrm{CDCl}_{3}\right) \delta$ 64.3. ${ }^{19} \mathbf{F}\left\{{ }^{1} \mathbf{H}\right\}$ NMR $\left(471 \mathrm{MHz}, \mathrm{CDCl}_{3}\right) \delta$, -62.5. HRMS (ESI+) calculated for $\left[\mathrm{C}_{52} \mathrm{H}_{50} \mathrm{AuClF}_{12} \mathrm{NNaP}^{+} 1202.2736 \mathrm{~m} / \mathrm{z}\right.$; found $[\mathrm{M}+\mathrm{Na}]^{+} 1202.2719 \mathrm{~m} / \mathrm{z} . \boldsymbol{\alpha}_{\mathbf{D}}{ }^{\mathbf{5 8 9}}=-27.2$ deg. $\mathrm{cm}^{2} \cdot \mathrm{g}^{-1}\left(\mathrm{CHCl}_{3}\right.$, c $\left.0.51,302 \mathrm{~K}\right)$. 


\section{Formal [4+2] cycloadditions}

\subsection{Preparation of 1,6-enynes 5b-q}

\subsubsection{Synthesis of 1,6-enynes 5b-5d}

(4,4-Bis(methoxymethyl)-7-methyloct-6-en-1-yn-1-yl)benzene (5b)

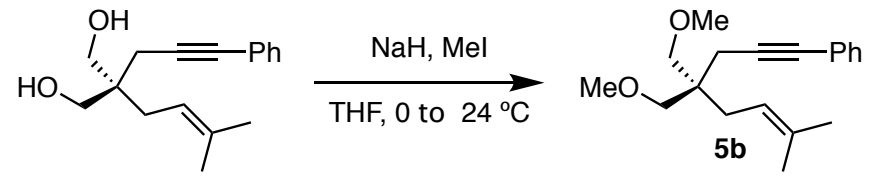

A solution of 2-(3-methylbut-2-en-1-yl)-2-(3-phenylprop-2-yn-1-yl)propane-1,3-diol ${ }^{4}$ (0.56 $\mathrm{g}, 2.18 \mathrm{mmol}, 1.0$ equiv) in THF $(7 \mathrm{~mL})$ was added to a suspension of $\mathrm{NaH}(0.21 \mathrm{~g}, 5.2 \mathrm{mmol}$, 2.4 equiv, $60 \%$ in mineral oil) in THF $(7 \mathrm{~mL})$ at $0{ }^{\circ} \mathrm{C}$. After stirring for $10 \mathrm{~min} \mathrm{MeI}(0.41 \mathrm{~mL}$ 6.54 mmol, 3.0 equiv) was added slowly. The reaction was stirred at $24{ }^{\circ} \mathrm{C}$ for $1 \mathrm{~h}$, then diluted with $\mathrm{Et}_{2} \mathrm{O}$ and quenched with sat. $\mathrm{NH}_{4} \mathrm{Cl}$ solution. The aqueous phase was extracted with $\mathrm{Et}_{2} \mathrm{O}$ (3x) and the combined organic phases were dried over $\mathrm{Na}_{2} \mathrm{SO}_{4}$, filtered and concentrated. The crude was purified by flash column chromatography (cyclohexane/EtOAc 30:1) to afford 1,6enyne $\mathbf{5 b}(0.56 \mathrm{~g}, 1.95 \mathrm{mmol}, 90 \%)$ as a colorless oil.

${ }^{1} \mathbf{H}$ NMR $\left(400 \mathrm{MHz}, \mathrm{CD}_{2} \mathrm{Cl}_{2}\right) \delta 7.42-7.35(\mathrm{~m}, 2 \mathrm{H}), 7.33-7.25(\mathrm{~m}, 3 \mathrm{H}), 5.22-5.15(\mathrm{~m}$, 1H), $3.32(\mathrm{~s}, 6 \mathrm{H}), 3.30-3.23(\mathrm{~m}, 4 \mathrm{H}), 2.37(\mathrm{~s}, 2 \mathrm{H}), 2.13(\mathrm{~d}, J=7.9 \mathrm{~Hz}, 2 \mathrm{H}), 1.73(\mathrm{~s}, 3 \mathrm{H}), 1.66$ (s, 3H). ${ }^{13} \mathrm{C}$ NMR $\left(101 \mathrm{MHz}, \mathrm{CD}_{2} \mathrm{Cl}_{2}\right) \delta 135.0,132.0,128.8,128.1,124.7,119.8,88.2,82.7$, 74.9, 59.6, 43.6, 30.8, 26.4, 23.5, 18.1. HRMS (APCI+) calculated for $\left[\mathrm{C}_{19} \mathrm{H}_{27} \mathrm{O}_{2}\right]^{+} 287.2006$ $m / z$; found $[\mathrm{M}+\mathrm{H}]^{+} 287.2010 \mathrm{~m} / z$.

\section{6-(3-Methylbut-2-en-1-yl)-6-(3-phenylprop-2-yn-1-yl)-2,4,8,10-tetraoxaundecane (5c)}

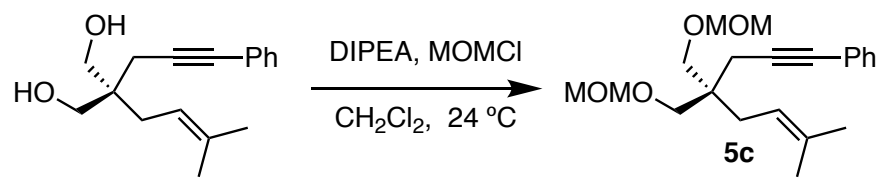

To a stirred mixture 2-(3-methylbut-2-en-1-yl)-2-(3-phenylprop-2-yn-1-yl)propane-1,3$\operatorname{diol}^{4}$ (60 mg, $0.23 \mathrm{mmol}, 1.0$ equiv) and DIPEA (0.16 mL, $0.92 \mathrm{mmol}, 4.0$ equiv) in $\mathrm{CH}_{2} \mathrm{Cl}_{2}$ $(2.3 \mathrm{~mL})$ was added $\mathrm{MOMCl}\left(0.053 \mathrm{~mL}, 0.69 \mathrm{mmol}, 3.0\right.$ equiv) at $24{ }^{\circ} \mathrm{C}$ and the reaction was stirred for $24 \mathrm{~h}$. $\mathrm{HCl}(5 \%)$ was added to the mixture and the aqueous phase was extracted with $\mathrm{CH}_{2} \mathrm{Cl}_{2}(3 \mathrm{x})$. The combined organic phases were washed with sat. $\mathrm{NaHCO}_{3}(2 \mathrm{x})$ and brine, dried over $\mathrm{Na}_{2} \mathrm{SO}_{4}$, filtered and evaporated. The crude was purified by flash column chromatography (cyclohexane/EtOAc 20:1) to yield 1,6-enyne 5c (68 mg, $0.19 \mathrm{mmol}, 85 \%)$ as a colorless oil. 
${ }^{1} \mathbf{H}$ NMR $\left(500 \mathrm{MHz}, \mathrm{CD}_{2} \mathrm{Cl}_{2}\right) \delta 7.40-7.37(\mathrm{~m}, 2 \mathrm{H}), 7.32-7.26(\mathrm{~m}, 3 \mathrm{H}), 5.25-5.20(\mathrm{~m}$, 1H), $4.61(\mathrm{~s}, 4 \mathrm{H}), 3.46$ (q, $J=9.3 \mathrm{~Hz}, 4 \mathrm{H}), 3.35$ (s, 6H), $2.44(\mathrm{~s}, 2 \mathrm{H}), 2.20$ (d, $J=7.8 \mathrm{~Hz}, 2 \mathrm{H})$, $1.74(\mathrm{~s}, 3 \mathrm{H}), 1.67(\mathrm{~s}, 3 \mathrm{H}) .{ }^{13} \mathrm{C}$ NMR $\left(126 \mathrm{MHz}, \mathrm{CD}_{2} \mathrm{Cl}_{2}\right) \delta 135.1,132.0,128.8,128.1,124.6$, 119.6, 97.4, 87.9, 82.8, 69.9, 55.5, 43.0, 30.8, 26.4, 23.5, 18.2. HRMS (APCI+) calculated for $\left[\mathrm{C}_{21} \mathrm{H}_{31} \mathrm{O}_{4}\right]^{+} 347.2217 \mathrm{~m} / z$; found $[\mathrm{M}+\mathrm{H}]^{+} 347.2218 \mathrm{~m} / \mathrm{z}$.

\section{2-(3-Methylbut-2-en-1-yl)-2-(3-phenylprop-2-yn-1-yl)propane-1,3-diyl diacetate (5d)}

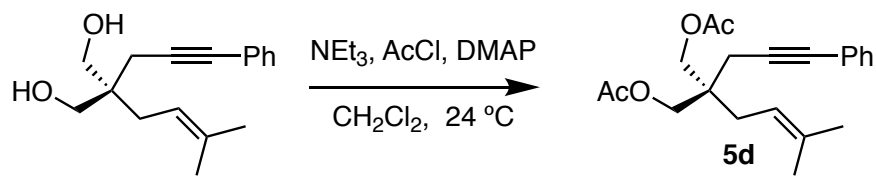

2-(3-methylbut-2-en-1-yl)-2-(3-phenylprop-2-yn-1-yl)propane-1,3-diol ${ }^{4} \quad(100 \quad \mathrm{mg}, \quad 0.39$ mmol, 1.0 equiv), acetyl chloride $(58 \mu \mathrm{L}, 0.81 \mathrm{mmol}, 2.1$ equiv) and DMAP (71 mg, $0.58 \mathrm{mmol}$, 1.5 equiv) were dissolved in $\mathrm{CH}_{2} \mathrm{Cl}_{2}(4 \mathrm{~mL})$ and $\mathrm{NEt}_{3}(0.16 \mathrm{~mL}, 1.16 \mathrm{mmol}, 3.0$ equiv) was added. The mixture was stirred at $24^{\circ} \mathrm{C}$ for $3 \mathrm{~h}$. Water was added to the mixture and the aqueous phase was extracted with $\mathrm{CH}_{2} \mathrm{Cl}_{2}(3 \mathrm{x})$. The combined organic phases were washed with brine, dried over $\mathrm{Na}_{2} \mathrm{SO}_{4}$, filtered and concentrated. The crude was purified by flash column chromatography (cyclohexane/EtOAc 20:1) to afford 1,6-enyne 5d (102 mg, 0.3 mmol, 77\%) as a colorless oil.

${ }^{1} \mathbf{H}$ NMR $\left(300 \mathrm{MHz}, \mathrm{CDCl}_{3}\right) \delta 7.43-7.33(\mathrm{~m}, 2 \mathrm{H}), 7.30-7.23(\mathrm{~m}, 3 \mathrm{H}), 5.19-5.08(\mathrm{~m}$, 1H), $4.15-3.99(\mathrm{~m}, 4 \mathrm{H}), 2.46$ (s, 2H), 2.23 (d, J=7.9 Hz, 2H), 2.07 (s, 6H), 1.73 (s, 3H), 1.64 (s, 3H). ${ }^{13} \mathbf{C}$ NMR $\left(101 \mathrm{MHz}, \mathrm{CDCl}_{3}\right) \delta 170.9,136.1,131.7,128.4,128.0,123.6,117.7,85.4$, 83.4, 65.9, 41.2, 30.3, 26.2, 23.2, 21.0, 18.0. HRMS (ESI+) calculated for $\left[\mathrm{C}_{21} \mathrm{H}_{26} \mathrm{NaO}_{4}\right]^{+}$ $365.1723 \mathrm{~m} / \mathrm{z}$; found $[\mathrm{M}+\mathrm{Na}]^{+} 365.1721 \mathrm{~m} / \mathrm{z}$. 


\subsubsection{Synthesis of 1,6-enynes 5e-o}
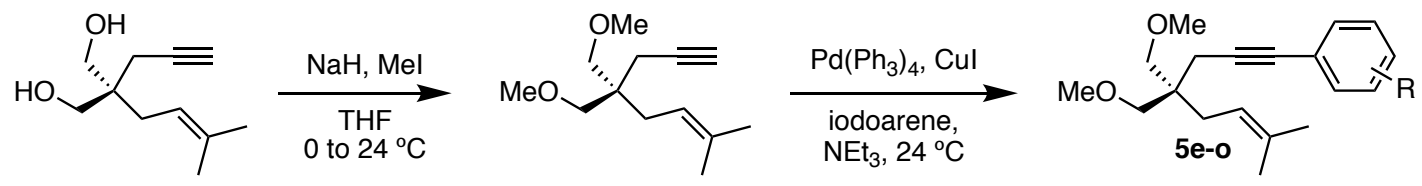

\section{4,4-Bis(methoxymethyl)-7-methyloct-6-en-1-yne}

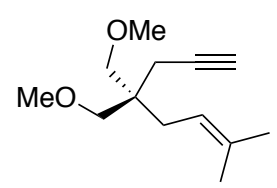

A solution of 2-(3-methylbut-2-en-1-yl)-2-(prop-2-yn-1-yl)propane-1,3-diol ${ }^{5}$ (1.0 g, 5.5 mmol, 1.0 equiv) in THF $(18 \mathrm{~mL})$ was added to a suspension of $\mathrm{NaH}(0.53 \mathrm{~g}, 13,2 \mathrm{mmol}, 2.4$ equiv, $60 \%$ in mineral oil) in THF $(18 \mathrm{~mL})$ at $0{ }^{\circ} \mathrm{C}$. After stirring the mixture for $10 \mathrm{~min}$ MeI (1.0 mL, $16.5 \mathrm{mmol}, 3.0$ equiv) was added slowly. The reaction was stirred at $24^{\circ} \mathrm{C}$ for $1 \mathrm{~h}$, then diluted with $\mathrm{Et}_{2} \mathrm{O}$ and quenched with sat. $\mathrm{NH}_{4} \mathrm{Cl}$ solution. The aqueous phase was extracted with $\mathrm{Et}_{2} \mathrm{O}(3 \mathrm{x})$ and the combined organic phases were dried over $\mathrm{Na}_{2} \mathrm{SO}_{4}$, filtered and concentrated. The crude was purified by flash column chromatography (cyclohexane/EtOAc $50: 1)$ to afford the title compound $(1.1 \mathrm{~g}, 5.2 \mathrm{mmol}, 94 \%)$ as a colorless oil.

${ }^{1} \mathbf{H}$ NMR $\left(500 \mathrm{MHz}, \mathrm{CDCl}_{3}\right) \delta 5.14-5.07(\mathrm{~m}, 1 \mathrm{H}), 3.31(\mathrm{~s}, 6 \mathrm{H}), 3.25-3.18(\mathrm{~m}, 4 \mathrm{H}), 2.19$ $-2.16(\mathrm{~m}, 2 \mathrm{H}), 2.08(\mathrm{~d}, J=7.9 \mathrm{~Hz}, 2 \mathrm{H}), 1.94(\mathrm{t}, J=2.7 \mathrm{~Hz}, 1 \mathrm{H}), 1.71(\mathrm{~s}, 3 \mathrm{H}), 1.62(\mathrm{~s}, 3 \mathrm{H}) .{ }^{13} \mathrm{C}$ NMR $\left(126 \mathrm{MHz}, \mathrm{CDCl}_{3}\right) \delta 134.6,119.2,81.8,74.4,70.0,59.3,42.6,30.1,26.2,22.1,17.8$. HRMS (ESI+) calculated for $\left[\mathrm{C}_{13} \mathrm{H}_{22} \mathrm{NaO}_{2}\right]^{+} 233.1512 \mathrm{~m} / z$; found $[\mathrm{M}+\mathrm{Na}]^{+} 233.1503 \mathrm{~m} / \mathrm{z}$.

\section{General procedure A: Sonogashira coupling}

$\operatorname{Pd}\left(\mathrm{PPh}_{3}\right)_{2} \mathrm{Cl}_{2}(1 \mathrm{~mol} \%)$ and copper(I) iodide $(2 \mathrm{~mol} \%)$ were sequentially added to a stirred solution of the terminal alkyne (1.0 equiv) in $\mathrm{NEt}_{3}(0.25 \mathrm{M})$ under argon at $24{ }^{\circ} \mathrm{C}$ and the mixture was stirred for $10 \mathrm{~min}$. Then, iodoarene (1.2 equiv) was added and the mixture was stirred for the corresponding time. The reaction was quenched with sat. $\mathrm{NH}_{4} \mathrm{Cl}$ and extracted with EtOAc (3x). The combined organic phases were washed with brine, dried over $\mathrm{Na}_{2} \mathrm{SO}_{4}$, and concentrated. The residue was purified by flash column chromatography to yield the desired Sonogashira coupling products. 
1-(4,4-Bis(methoxymethyl)-7-methyloct-6-en-1-yn-1-yl)-4-methoxybenzene (5e)

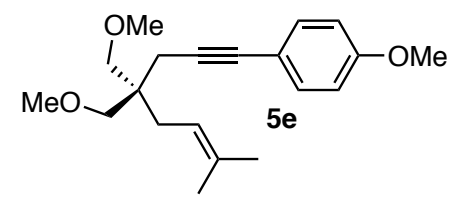

1,6-Enyne 5e was synthesized following general procedure A using 4,4-bis(methoxymethyl)-7-methyloct-6-en-1-yne (200 mg, $1 \mathrm{mmol}$ ) whereby the reaction was stirred for $2 \mathrm{~h}$. Purification by flash column chromatography (cyclohexane/EtOAc $60: 1$, then toluene/ $\mathrm{CH}_{2} \mathrm{Cl}_{2}$ 4:1) afforded the title compound $\mathbf{5 e}(241 \mathrm{mg}, 0.76 \mathrm{mmol}, 80 \%)$ as a colorless oil.

${ }^{1} \mathbf{H}$ NMR $\left(500 \mathrm{MHz}, \mathrm{CDCl}_{3}\right) \delta 7.36-7.31(\mathrm{~m}, 2 \mathrm{H}), 6.84-6.79(\mathrm{~m}, 2 \mathrm{H}), 5.21-5.15(\mathrm{~m}$, 1H), $3.80(\mathrm{~s}, 3 \mathrm{H}), 3.34(\mathrm{~s}, 6 \mathrm{H}), 3.32-3.26(\mathrm{~m}, 4 \mathrm{H}), 2.37$ (s, 2H), $2.15(\mathrm{~d}, J=7.9 \mathrm{~Hz}, 2 \mathrm{H}), 1.75$ $-1.72(\mathrm{~m}, 3 \mathrm{H}), 1.68-1.64(\mathrm{~m}, 3 \mathrm{H}) .{ }^{13} \mathbf{C} \mathbf{N M R}\left(126 \mathrm{MHz}, \mathrm{CDCl}_{3}\right) \delta 159.2,134.6,133.0,119.5$, 116.5, 114.0, 85.9, 82.1, 74.7, 59.5, 55.4, 43.2, 30.4, 26.3, 23.1, 17.9. HRMS (ESI+) calculated for $\left[\mathrm{C}_{20} \mathrm{H}_{28} \mathrm{NaO}_{3}\right]^{+} 339.1931 \mathrm{~m} / z$; found $[\mathrm{M}+\mathrm{Na}]^{+} 339.1926 \mathrm{~m} / \mathrm{z}$.

\section{1-(4,4-Bis(methoxymethyl)-7-methyloct-6-en-1-yn-1-yl)-4-nitrobenzene (5f)}

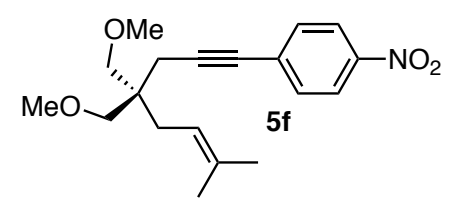

1,6-Enyne 5f was synthesized following general procedure A using 4,4-bis(methoxymethyl)-7-methyloct-6-en-1-yne (99 $\mathrm{mg}, 0.47 \mathrm{mmol}$ ) whereby the reaction was stirred for 18 h. Purification by flash column chromatography (cyclohexane/EtOAc 60:1) afforded the title compound $\mathbf{5 f}$ (142 $\mathrm{mg}, 0.43 \mathrm{mmol}, 91 \%)$ as a colorless oil.

${ }^{1} \mathbf{H}$ NMR $\left(400 \mathrm{MHz}, \mathrm{CDCl}_{3}\right) \delta 8.21-8.10(\mathrm{~m}, 2 \mathrm{H}), 7.57-7.47(\mathrm{~m}, 2 \mathrm{H}), 5.20-5.12(\mathrm{~m}$, $1 \mathrm{H}), 3.35(\mathrm{~s}, 6 \mathrm{H}), 3.31-3.25(\mathrm{~m}, 4 \mathrm{H}), 2.45(\mathrm{~s}, 2 \mathrm{H}), 2.15(\mathrm{~d}, J=7.9 \mathrm{~Hz}, 2 \mathrm{H}), 1.74(\mathrm{~s}, 3 \mathrm{H}), 1.65$ (s, 3H). ${ }^{13} \mathbf{C}$ NMR $\left(101 \mathrm{MHz}, \mathrm{CDCl}_{3}\right) \delta$ 146.8, 135.0, 132.4, 131.3, 123.7, 119.1, 94.3, 81.1, 74.6, 59.5, 43.2, 30.5, 26.3, 23.4, 17.9. HRMS (ESI+) calculated for $\left[\mathrm{C}_{19} \mathrm{H}_{25} \mathrm{NNaO}_{4}\right]^{+} 354.1676$ $\mathrm{m} / z$; found $[\mathrm{M}+\mathrm{Na}]^{+} 354.1690 \mathrm{~m} / z$. 
1-(4,4-Bis(methoxymethyl)-7-methyloct-6-en-1-yn-1-yl)-4-(tert-butyl)benzene (5g)

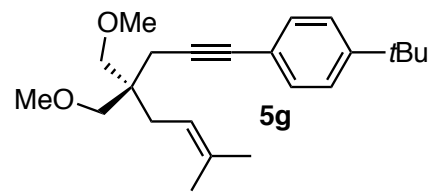

1,6-Enyne 5g was synthesized following general procedure A using 4,4-bis(methoxymethyl)-7-methyloct-6-en-1-yne (105 mg, $0.5 \mathrm{mmol})$ whereby the reaction was stirred for $3 \mathrm{~h}$. Purification by flash column chromatography (hexane/EtOAc 70:1) afforded the title compound 5g (160 mg, $0.47 \mathrm{mmol}, 94 \%$ ) as a colorless oil.

${ }^{1}$ H NMR (400 MHz, $\left.\mathrm{CDCl}_{3}\right) \delta 7.37-7.28(\mathrm{~m}, 4 \mathrm{H}), 5.22$ - $5.14(\mathrm{~m}, 1 \mathrm{H}), 3.37$ - $3.33(\mathrm{~m}$, $6 \mathrm{H}), 3.32-3.25(\mathrm{~m}, 4 \mathrm{H}), 2.40-2.37(\mathrm{~m}, 2 \mathrm{H}), 2.15(\mathrm{~d}, J=7.8 \mathrm{~Hz}, 2 \mathrm{H}), 1.74(\mathrm{~s}, 3 \mathrm{H}), 1.67$ (s, $3 \mathrm{H}), 1.33-1.28(\mathrm{~m}, 9 \mathrm{H}) .{ }^{13} \mathbf{C}$ NMR $\left(101 \mathrm{MHz}, \mathrm{CDCl}_{3}\right) \delta 150.8,134.6,131.4,125.3,121.3$, 119.5, 86.8, 82.4, 74.7, 59.5, 43.2, 34.8, 31.4, 30.4, 26.3, 23.1, 17.9. HRMS (ESI+) calculated for $\left[\mathrm{C}_{23} \mathrm{H}_{34} \mathrm{NaO}_{2}\right]^{+} 365.2451 \mathrm{~m} / z$; found $[\mathrm{M}+\mathrm{Na}]^{+} 365.2451 \mathrm{~m} / \mathrm{z}$.

1-(4,4-Bis(methoxymethyl)-7-methyloct-6-en-1-yn-1-yl)-4-chlorobenzene (5h)

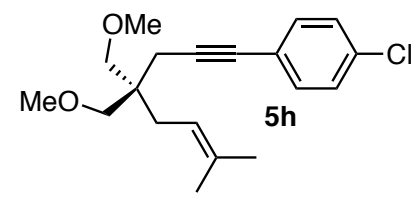

1,6-Enyne 5h was synthesized following general procedure A using 4,4-bis(methoxymethyl)-7-methyloct-6-en-1-yne $(110 \mathrm{mg}, 0.523 \mathrm{mmol})$ whereby the reaction was stirred for 15 h. Purification by flash column chromatography (cyclohexane/EtOAc 40:1) afforded the title compound $\mathbf{5 h}$ (146 $\mathrm{mg}, 0.455 \mathrm{mmol}, 87 \%)$ as a yellowish oil.

${ }^{1} \mathbf{H}$ NMR $\left(400 \mathrm{MHz}, \mathrm{CDCl}_{3}\right) \delta 7.34-7.29(\mathrm{~m}, 2 \mathrm{H}), 7.27-7.23(\mathrm{~m}, 2 \mathrm{H}), 5.20-5.13(\mathrm{~m}$, 1H), $3.34(\mathrm{~s}, 6 \mathrm{H}), 3.31-3.24(\mathrm{~m}, 4 \mathrm{H}), 2.38(\mathrm{~s}, 2 \mathrm{H}), 2.14(\mathrm{~d}, J=7.9 \mathrm{~Hz}, 1 \mathrm{H}), 1.73(\mathrm{~s}, 3 \mathrm{H}), 1.65$ (s, 3H). ${ }^{13} \mathrm{C}$ NMR $\left(101 \mathrm{MHz}, \mathrm{CDCl}_{3}\right) \delta 134.7,133.6,132.9,128.6,122.8,119.3,88.8,81.3$, 74.6, 59.5, 43.2, 30.4, 26.3, 23.1, 17.9. HRMS (ESI+) calculated for $\left[\mathrm{C}_{19} \mathrm{H}_{25} \mathrm{ClNaO}_{2}\right]^{+}$ $343.1435 \mathrm{~m} / \mathrm{z}$; found [M $+\mathrm{Na}]^{+} 343.1445 \mathrm{~m} / \mathrm{z}$. 
1-(4,4-Bis(methoxymethyl)-7-methyloct-6-en-1-yn-1-yl)-4-(trifluoromethyl)benzene (5i)

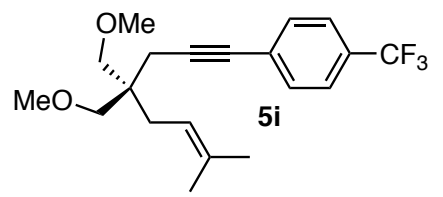

1,6-Enyne 5i was synthesized following general procedure A using 4,4-bis(methoxymethyl)7-methyloct-6-en-1-yne $(110 \mathrm{mg}, 0.523 \mathrm{mmol})$ whereby the reaction was stirred for $15 \mathrm{~h}$. Purification by flash column chromatography (cyclohexane/EtOAc 40:1) afforded the title compound $5 \mathbf{i}$ (159 mg, $0.449 \mathrm{mmol}, 86 \%$ ) as a colorless oil.

${ }^{1}$ H NMR $\left(400 \mathrm{MHz}, \mathrm{CDCl}_{3}\right) \delta 7.54(\mathrm{~d}, J=8.3 \mathrm{~Hz}, 1 \mathrm{H}), 7.49(\mathrm{~d}, J=8.2 \mathrm{~Hz}, 1 \mathrm{H}), 5.20-5.14$ (m, 1H), $3.35(\mathrm{~s}, 6 \mathrm{H}), 3.32-3.25(\mathrm{~m}, 4 \mathrm{H}), 2.42(\mathrm{~s}, 2 \mathrm{H}), 2.16(\mathrm{~d}, J=7.9 \mathrm{~Hz}, 1 \mathrm{H}), 1.74(\mathrm{~s}, 3 \mathrm{H})$, $1.66(\mathrm{~s}, 3 \mathrm{H}) .{ }^{13} \mathrm{C} \mathbf{N M R}\left(101 \mathrm{MHz}, \mathrm{CDCl}_{3}\right) \delta 134.8,131.9,129.4(\mathrm{q}, J=32.7 \mathrm{~Hz}), 128.2-128.1$ (m), 125.3 (q, $J=3.8 \mathrm{~Hz}), 124.2(\mathrm{q}, J=272.0 \mathrm{~Hz}), 119.2,90.7,81.3,74.6,59.5,43.2,30.4$, 26.3, 23.2, 17.9. ${ }^{19} \mathbf{F}\left\{{ }^{1} \mathbf{H}\right\}$ NMR (376 MHz, $\left.\mathrm{CDCl}_{3}\right) \delta-62.8$. HRMS (ESI+) calculated for $\left[\mathrm{C}_{20} \mathrm{H}_{25} \mathrm{~F}_{3} \mathrm{NaO}_{2}\right]^{+} 377.1699 \mathrm{~m} / z$; found $[\mathrm{M}+\mathrm{Na}]^{+} 377.1717 \mathrm{~m} / z$.

\section{1-(4,4-Bis(methoxymethyl)-7-methyloct-6-en-1-yn-1-yl)-2-fluorobenzene (5j)}

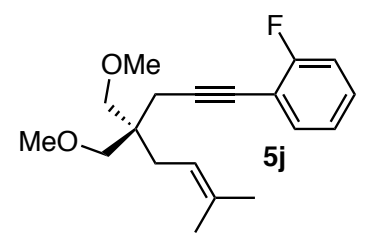

1,6-Enyne 5j was synthesized following general procedure A using 4,4-bis(methoxymethyl)-7-methyloct-6-en-1-yne (200 mg, $1 \mathrm{mmol}$ ) whereby the reaction was stirred for $6 \mathrm{~h}$. Purification by flash column chromatography (cyclohexane/EtOAc 100:1) afforded the title compound 5j (269 mg, $0.88 \mathrm{mmol}, 93 \%$ ) as a colorless oil.

${ }^{1}$ H NMR (400 MHz, $\left.{ }_{6} \mathrm{D}_{6}\right) \delta 7.34-7.28(\mathrm{~m}, 1 \mathrm{H}), 6.75-6.68(\mathrm{~m}, 2 \mathrm{H}), 6.67-6.59(\mathrm{~m}, 1 \mathrm{H})$, $5.44-5.33(\mathrm{~m}, 1 \mathrm{H}), 3.40(\mathrm{q}, J=8.7 \mathrm{~Hz}, 4 \mathrm{H}), 3.16(\mathrm{~s}, 6 \mathrm{H}), 2.65(\mathrm{~s}, 2 \mathrm{H}), 2.48(\mathrm{~d}, J=7.9 \mathrm{~Hz}$, $2 \mathrm{H}), 1.72-1.70(\mathrm{~m}, 3 \mathrm{H}), 1.70-1.68(\mathrm{~m}, 3 \mathrm{H}) .{ }^{13} \mathbf{C}$ NMR $\left(101 \mathrm{MHz}, \mathrm{C}_{6} \mathrm{D}_{6}\right) \delta 163.6(\mathrm{~d}, J=$ $250.0 \mathrm{~Hz}), 134.4,133.7$ (d, $J=1.5 \mathrm{~Hz}), 129.3$ (d, $J=7.8 \mathrm{~Hz}), 124.0$ (d, $J=3.7 \mathrm{~Hz}), 120.2$, $115.6(\mathrm{~d}, J=21.1 \mathrm{~Hz}), 113.3(\mathrm{~d}, J=15.9 \mathrm{~Hz}), 94.2$ (d, $J=3.3 \mathrm{~Hz}), 76.3,74.6,59.0,43.6,30.8$, 26.3, 23.8, 17.9. ${ }^{19} \mathbf{F}\left\{{ }^{1} \mathbf{H}\right\}$ NMR $\left(376 \mathrm{MHz}, \mathrm{C}_{6} \mathrm{D}_{6}\right) \delta$-110.6. HRMS (ESI+) calculated for $\left[\mathrm{C}_{19} \mathrm{H}_{25} \mathrm{FNaO}_{2}\right]^{+} 327.1731 \mathrm{~m} / \mathrm{z}$; found $[\mathrm{M}+\mathrm{Na}]^{+} 327.1733 \mathrm{~m} / z$. 
1-(4,4-Bis(methoxymethyl)-7-methyloct-6-en-1-yn-1-yl)-2-methylbenzene (5k)

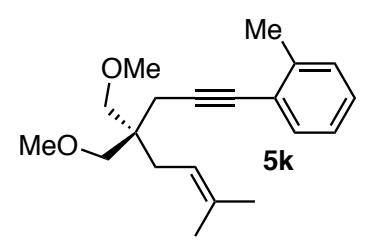

1,6-Enyne 5k was synthesized following general procedure A using 4,4-bis(methoxymethyl)-7-methyloct-6-en-1-yne (200 mg, $1 \mathrm{mmol}$ ) whereby the reaction was stirred for $2 \mathrm{~h}$. Purification by flash column chromatography (cyclohexane/EtOAc $80: 1$, then toluene/ $\mathrm{CH}_{2} \mathrm{Cl}_{2}$ 1:1) afforded the title compound $5 \mathbf{k}(242 \mathrm{mg}, 0.81 \mathrm{mmol}, 85 \%)$ as a colorless oil.

${ }^{1} \mathbf{H}$ NMR $\left(400 \mathrm{MHz}, \mathrm{CDCl}_{3}\right) \delta 7.38(\mathrm{~d}, J=7.3 \mathrm{~Hz}, 1 \mathrm{H}), 7.20-7.14(\mathrm{~m}, 2 \mathrm{H}), 7.14-7.07$ (m, 1H), $5.22-5.15(\mathrm{~m}, 1 \mathrm{H}), 3.35(\mathrm{~s}, 6 \mathrm{H}), 3.33-3.27(\mathrm{~m}, 4 \mathrm{H}), 2.45(\mathrm{~s}, 2 \mathrm{H}), 2.44(\mathrm{~s}, 3 \mathrm{H}), 2.17$ (d, $J=7.9 \mathrm{~Hz}, 2 \mathrm{H}), 1.74(\mathrm{~s}, 3 \mathrm{H}), 1.66(\mathrm{~s}, 3 \mathrm{H}) .{ }^{13} \mathbf{C} \mathbf{~ N M R}\left(101 \mathrm{MHz}, \mathrm{CDCl}_{3}\right) \delta 140.0,134.7$, 132.1, 129.4, 127.6, 125.6, 124.1, 119.4, 91.5, 81.3, 74.7, 59.4, 43.2, 30.4, 26.3, 23.3, 21.0, 17.9. HRMS (ESI+) calculated for $\left[\mathrm{C}_{20} \mathrm{H}_{28} \mathrm{NaO}_{2}\right]^{+} 323.1982 \mathrm{~m} / z$; found $[\mathrm{M}+\mathrm{Na}]^{+} 323.1978$ $m / z$.

\section{1-(4,4-Bis(methoxymethyl)-7-methyloct-6-en-1-yn-1-yl)-2,4-dimethoxybenzene (5l)}

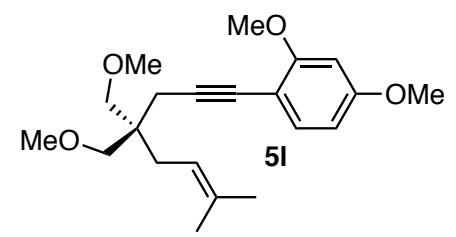

1,6-Enyne $5 \mathbf{l}$ was synthesized following general procedure A using 4,4-bis(methoxymethyl)7-methyloct-6-en-1-yne $(150 \mathrm{mg}, 0.7 \mathrm{mmol})$ whereby the reaction was stirred for $7 \mathrm{~h}$. Purification by flash column chromatography (cyclohexane/EtOAc 60:1, then 20:1) afforded the title compound $\mathbf{5 l}$ (193 $\mathrm{mg}, 0.56 \mathrm{mmol}, 78 \%$ ) as a pale yellow oil.

${ }^{1}$ H NMR $\left(400 \mathrm{MHz}, \mathrm{CD}_{2} \mathrm{Cl}_{2}\right) \delta 7.28-7.23(\mathrm{~m}, 1 \mathrm{H}), 6.45-6.40(\mathrm{~m}, 2 \mathrm{H}), 5.22-5.15(\mathrm{~m}$, 1H), $3.82(\mathrm{~s}, 3 \mathrm{H}), 3.79$ (s, 3H), $3.32(\mathrm{~s}, 6 \mathrm{H}), 3.30-3.24(\mathrm{~m}, 4 \mathrm{H}), 2.36(\mathrm{~s}, 2 \mathrm{H}), 2.13$ (d, J= 7.9 $\mathrm{Hz}, 2 \mathrm{H}), 1.73$ (s, 3H), 1.65 (s, 3H). ${ }^{13} \mathbf{C}$ NMR (101 MHz, $\left.\mathrm{CD}_{2} \mathrm{Cl}_{2}\right) \delta 161.9,161.2,134.9,134.4$, 120.0, 106.3, 105.2, 98.9, 90.2, 78.8, 74.9, 59.5, 56.2, 56.0, 43.6, 30.7, 26.4, 23.7, 18.0. HRMS $\left(\mathrm{ESI}+\right.$ ) calculated for $\left[\mathrm{C}_{21} \mathrm{H}_{30} \mathrm{NaO}_{4}\right]^{+} 369.2036 \mathrm{~m} / z$; found $[\mathrm{M}+\mathrm{Na}]^{+} 369.2035 \mathrm{~m} / z$. 
1-(4,4-Bis(methoxymethyl)-7-methyloct-6-en-1-yn-1-yl)-3,5-dibromobenzene (5m)

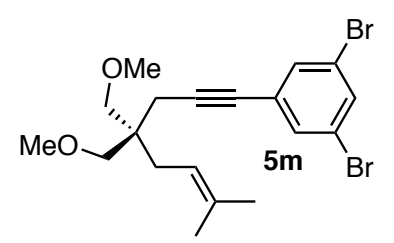

1,6-Enyne 5m was synthesized following general procedure A using 4,4-bis(methoxymethyl)-7-methyloct-6-en-1-yne $(150 \mathrm{mg}, 0.71 \mathrm{mmol})$ whereby the reaction was stirred for 18 h. Purification by flash column chromatography (cyclohexane/EtOAc 70:1) afforded the title compound 5m (294 mg, $0.66 \mathrm{mmol}, 93 \%$ ) as a brown oil.

${ }^{1} \mathbf{H}$ NMR $\left(500 \mathrm{MHz}, \mathrm{C}_{6} \mathrm{D}_{6}\right) \delta 7.39(\mathrm{~d}, J=1.8 \mathrm{~Hz}, 2 \mathrm{H}), 7.28(\mathrm{t}, J=1.7 \mathrm{~Hz}, 1 \mathrm{H}), 5.35-5.29$ (m, 1H), $3.32-3.27(\mathrm{~m}, 4 \mathrm{H}), 3.12(\mathrm{~s}, 6 \mathrm{H}), 2.55(\mathrm{~s}, 2 \mathrm{H}), 2.37$ (d, J=7.9 Hz, 2H), $1.71(\mathrm{~s}, 3 \mathrm{H})$, $1.64(\mathrm{~s}, 3 \mathrm{H}) .{ }^{13} \mathrm{C}$ NMR $\left(126 \mathrm{MHz}, \mathrm{C}_{6} \mathrm{D}_{6}\right) \delta 134.6,133.6,133.3,128.4,123.0,119.9,91.5,80.2$, $74.5,59.0,43.5,30.9,26.3,23.4,17.9$. HRMS (ESI+) calculated for $\left[\mathrm{C}_{19} \mathrm{H}_{24} \mathrm{Br}_{2} \mathrm{NaO}_{2}\right]^{+}$ $465.0035 \mathrm{~m} / \mathrm{z}$; found $[\mathrm{M}+\mathrm{Na}]^{+} 465.0057 \mathrm{~m} / \mathrm{z}$.

5-(4,4-Bis(methoxymethyl)-7-methyloct-6-en-1-yn-1-yl)-1,2,3-trimethoxybenzene (5n)

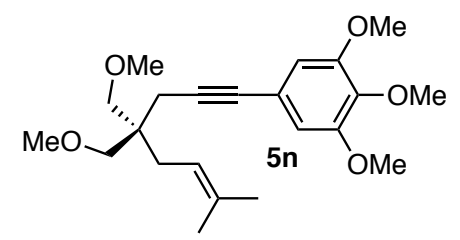

1,6-Enyne 5n was synthesized following general procedure A using 4,4-bis(methoxymethyl)-7-methyloct-6-en-1-yne (105 mg, $0.5 \mathrm{mmol}$ ) whereby the reaction was stirred for $3 \mathrm{~h}$. Purification by flash column chromatography (cyclohexane/EtOAc 30:1) afforded the title compound 5n (154 mg, $0.41 \mathrm{mmol}, 83 \%)$ as a pale yellow oil.

${ }^{1} \mathbf{H}$ NMR $\left(400 \mathrm{MHz}, \mathrm{CDCl}_{3}\right) \delta 6.63(\mathrm{~s}, 2 \mathrm{H}), 5.18(\mathrm{tm}, J=6.5 \mathrm{~Hz}, 1 \mathrm{H}), 3.84(\mathrm{~s}, 6 \mathrm{H}), 3.83(\mathrm{~s}$, $3 \mathrm{H}), 3.35(\mathrm{~s}, 6 \mathrm{H}), 3.32-3.25(\mathrm{~m}, 4 \mathrm{H}), 2.39(\mathrm{~s}, 2 \mathrm{H}), 2.16(\mathrm{~d}, J=7.9 \mathrm{~Hz}, 2 \mathrm{H}), 1.74(\mathrm{~s}, 3 \mathrm{H}), 1.67$ (s, 3H). ${ }^{13} \mathbf{C}$ NMR $\left(101 \mathrm{MHz}, \mathrm{CDCl}_{3}\right) \delta 153.2,138.5,134.6,119.4,109.0,86.7,82.4,74.7$, $61.1,59.5,56.3,43.2,30.4,26.3,23.1,17.9$. HRMS (ESI+) calculated for $\left[\mathrm{C}_{22} \mathrm{H}_{33} \mathrm{O}_{5}\right]^{+}$ $377.2323 \mathrm{~m} / \mathrm{z}$; found $[\mathrm{M}+\mathrm{H}]^{+} 377.2331 \mathrm{~m} / \mathrm{z}$. 


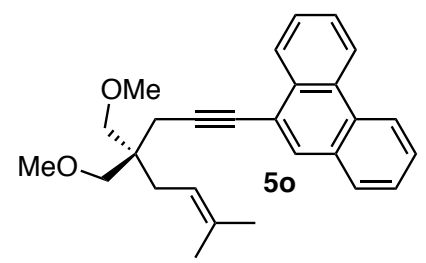

1,6-Enyne 5o was synthesized following general procedure A using 4,4-bis(methoxymethyl)-7-methyloct-6-en-1-yne (100 $\mathrm{mg}, 0.5 \mathrm{mmol})$ whereby the reaction was stirred for 20 h. Purification by flash column chromatography (pentane/EtOAc 100:1, then $\mathrm{CH}_{2} \mathrm{Cl}_{2}$ /pentane 3:1) afforded the title compound $\mathbf{5 0}(154 \mathrm{mg}, 0.4 \mathrm{mmol}, 80 \%)$ as a colorless gum.

${ }^{1} \mathbf{H}$ NMR $\left(400 \mathrm{MHz}, \mathrm{C}_{6} \mathrm{D}_{6}\right) \delta 8.84(\mathrm{dd}, J=8.1,1.1 \mathrm{~Hz}, 1 \mathrm{H}), 8.44(\mathrm{~d}, J=8.2 \mathrm{~Hz}, 1 \mathrm{H}), 8.37$ $(\mathrm{d}, J=8.2 \mathrm{~Hz}, 1 \mathrm{H}), 8.00(\mathrm{~s}, 1 \mathrm{H}), 7.55-7.49(\mathrm{~m}, 2 \mathrm{H}), 7.47-7.39(\mathrm{~m}, 1 \mathrm{H}), 7.37-7.32(\mathrm{~m}, 1 \mathrm{H})$, $7.31-7.26(\mathrm{~m}, 1 \mathrm{H}), 5.51-5.43(\mathrm{~m}, 1 \mathrm{H}), 3.48(\mathrm{q}, J=8.7 \mathrm{~Hz}, 4 \mathrm{H}), 3.20(\mathrm{~s}, 6 \mathrm{H}), 2.82(\mathrm{~s}, 2 \mathrm{H})$, $2.57(\mathrm{~d}, J=7.9 \mathrm{~Hz}, 2 \mathrm{H}), 1.76-1.73(\mathrm{~m}, 3 \mathrm{H}), 1.73-1.70(\mathrm{~m}, 3 \mathrm{H}) .{ }^{13} \mathbf{C}$ NMR $\left(101 \mathrm{MHz}, \mathrm{C}_{6} \mathrm{D}_{6}\right)$ $\delta 134.5,132.4,132.0,131.9,130.8,130.5,128.7,127.6,127.3,127.23,127.19,127.1,123.2$, 123.0, 121.3, 120.2, 93.0, 81.3, 74.9, 59.1, 43.7, 31.1, 26.3, 24.0, 18.0. HRMS (ESI+) calculated for $\left[\mathrm{C}_{27} \mathrm{H}_{30} \mathrm{NaO}_{2}\right]^{+} 409.2138 \mathrm{~m} / z$; found $[\mathrm{M}+\mathrm{Na}]^{+} 409.2129 \mathrm{~m} / \mathrm{z}$.

\subsubsection{Synthesis of 1,6-enyne $5 p$}

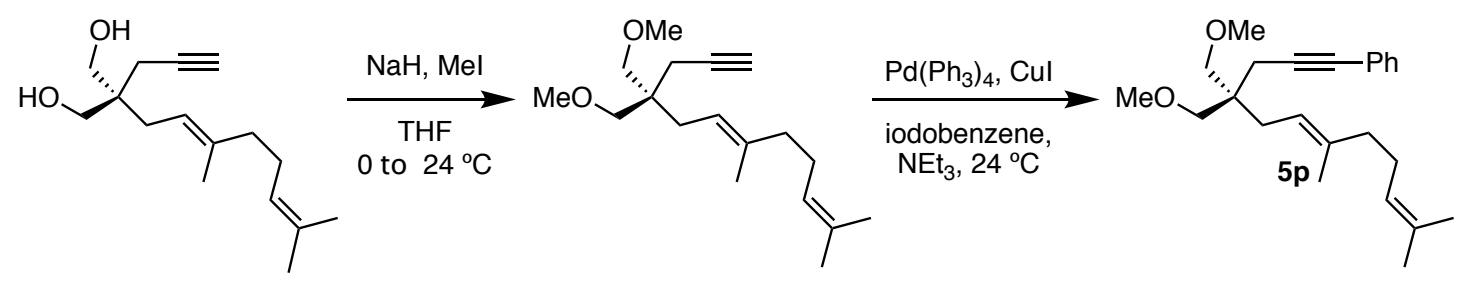

\section{(E)-4,4-Bis(methoxymethyl)-7,11-dimethyldodeca-6,10-dien-1-yne}

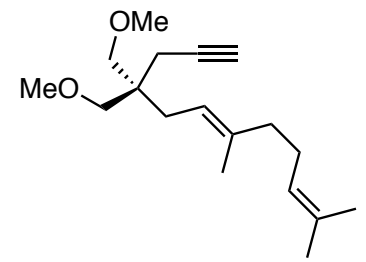

A solution of (E)-2-(3,7-dimethylocta-2,6-dien-1-yl)-2-(prop-2-yn-1-yl)propane-1,3-diol ${ }^{6}$ ( $2.0 \mathrm{~g}, 8.0 \mathrm{mmol}, 1.0$ equiv) in THF $(26 \mathrm{~mL})$ was added to a suspension of $\mathrm{NaH}(0.67 \mathrm{~g}, 16.8$ mmol, 2.1 equiv, $60 \%$ in mineral oil) in THF $(26 \mathrm{~mL})$ at $0{ }^{\circ} \mathrm{C}$. After stirring for $10 \mathrm{~min}$ MeI (1.5 mL, $23.9 \mathrm{mmol}, 3.0$ equiv) was added slowly. The reaction was stirred at $24{ }^{\circ} \mathrm{C}$ for $1 \mathrm{~h}$, then diluted with $\mathrm{Et}_{2} \mathrm{O}$ and quenched with sat. $\mathrm{NH}_{4} \mathrm{Cl}$ solution. The aqueous phase was extracted with $\mathrm{Et}_{2} \mathrm{O}(3 \mathrm{x})$ and the combined organic phases were dried over $\mathrm{Na}_{2} \mathrm{SO}_{4}$, filtered and 
concentrated. The crude was purified by flash column chromatography (cyclohexane/EtOAc $40: 1)$ to afford the title compound $(1.8 \mathrm{~g}, 6.5 \mathrm{mmol}, 81 \%)$ as a colorless oil.

NMR $\left(400 \mathrm{MHz}, \mathrm{CDCl}_{3}\right) \delta 5.16-5.04(\mathrm{~m}, 2 \mathrm{H}), 3.32(\mathrm{~s}, 6 \mathrm{H}), 3.26-3.19(\mathrm{~m}, 4 \mathrm{H}), 2.18(\mathrm{~d}$, $J=2.7 \mathrm{~Hz}, 2 \mathrm{H}), 2.14-2.06(\mathrm{~m}, 4 \mathrm{H}), 2.05-1.99(\mathrm{~m}, 2 \mathrm{H}), 1.95(\mathrm{t}, J=2.7 \mathrm{~Hz}, 1 \mathrm{H}), 1.70-1.66$ (m, 3H), $1.64-1.59$ (m, 6H). ${ }^{13} \mathbf{C}$ NMR (101 MHz, $\left.\mathrm{CDCl}_{3}\right) \delta 138.2,131.4,124.6,119.4,81.8$, 74.4, 70.0, 59.4, 42.6, 40.2, 30.0, 26.7, 25.9, 22.1, 17.9, 16.0. HRMS (ESI+) calculated for $\left[\mathrm{C}_{18} \mathrm{H}_{30} \mathrm{NaO}_{2}\right]^{+} 301.2138 \mathrm{~m} / z$; found $[\mathrm{M}+\mathrm{Na}]^{+} 301.2130 \mathrm{~m} / z$.

\section{(E)-(4,4-Bis(methoxymethyl)-7,11-dimethyldodeca-6,10-dien-1-yn-1-yl)benzene (5p)}

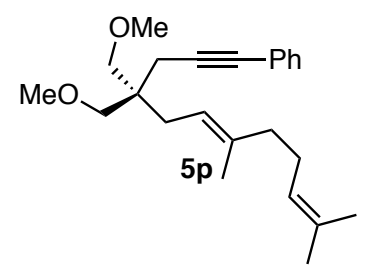

Dienyne 5p was synthesized following general procedure A using (E)-4,4-bis(methoxymethyl)-7,11-dimethyldodeca-6,10-dien-1-yne (258 $\mathrm{mg}, 0.9 \mathrm{mmol})$ whereby the reaction was stirred overnight. Purification by flash column chromatography (cyclohexane/EtOAc 50:1) afforded the title compound $\mathbf{5 p}(291 \mathrm{mg}, 0.8 \mathrm{mmol}, 89 \%)$ as a colorless oil.

${ }^{1} \mathbf{H}$ NMR $\left(400 \mathrm{MHz}, \mathrm{CD}_{2} \mathrm{Cl}_{2}\right) \delta 7.42-7.36(\mathrm{~m}, 2 \mathrm{H}), 7.33-7.25(\mathrm{~m}, 3 \mathrm{H}), 5.22-5.14(\mathrm{~m}$, $1 \mathrm{H}), 5.14-5.06(\mathrm{~m}, 1 \mathrm{H}), 3.32(\mathrm{~s}, 6 \mathrm{H}), 3.30-3.23(\mathrm{~m}, 4 \mathrm{H}), 2.37(\mathrm{~s}, 2 \mathrm{H}), 2.17-2.08(\mathrm{~m}, 4 \mathrm{H})$, $2.07-2.02(\mathrm{~m}, 2 \mathrm{H}), 1.68(\mathrm{~s}, 3 \mathrm{H}), 1.65(\mathrm{~s}, 3 \mathrm{H}), 1.61(\mathrm{~s}, 3 \mathrm{H}) .{ }^{13} \mathbf{C ~ N M R}\left(101 \mathrm{MHz}, \mathrm{CD}_{2} \mathrm{Cl}_{2}\right) \delta$ 138.6, 132.0, 131.8, 128.8, 128.1, 125.0, 124.8, 120.0, 88.2, 82.7, 74.9, 59.6, 43.7, 40.7, 30.6, 27.2, 26.0, 23.4, 18.0, 16.3. HRMS (ESI+) calculated for $\left[\mathrm{C}_{24} \mathrm{H}_{34} \mathrm{NaO}_{2}\right]^{+} 377.2451 \mathrm{~m} / \mathrm{z}$; found $[\mathrm{M}+\mathrm{Na}]^{+} 377.2458 \mathrm{~m} / \mathrm{z}$. 


\subsubsection{Synthesis of 1,6-enyne 5q}
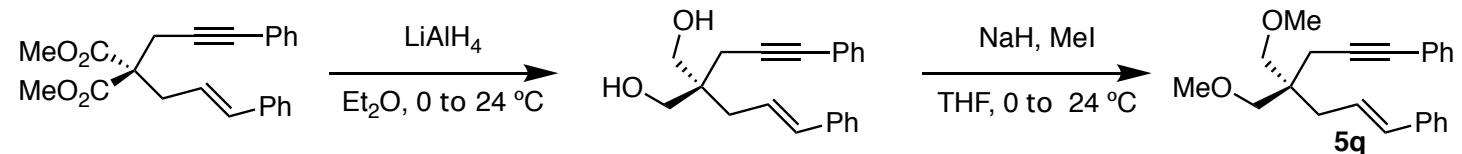

\section{2-Cinnamyl-2-(3-phenylprop-2-yn-1-yl)propane-1,3-diol}

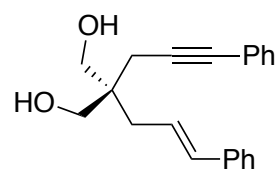

A solution of dimethyl 2-cinnamyl-2-(3-phenylprop-2-yn-1-yl)malonate ${ }^{7}$ (250 mg, 0.69 mmol, 1.0 equiv) in $\mathrm{Et}_{2} \mathrm{O}(5 \mathrm{~mL})$ was added dropwise to a suspension of $\mathrm{LiAlH}_{4}(105 \mathrm{mg}, 2.8$ mmol, 4.0 equiv) in $\mathrm{Et}_{2} \mathrm{O}(2 \mathrm{~mL})$ at $0{ }^{\circ} \mathrm{C}$. The reaction was stirred for $1 \mathrm{~h}$ and after consumption of the starting material the reaction was quenched with $\mathrm{HCl}(0.5 \mathrm{M})$. After stirring for $30 \mathrm{~min}$ the mixture was filtered over Celite ${ }^{\circledR}$ and the organic phase was separated. The aqueous phase was extracted with $\mathrm{Et}_{2} \mathrm{O}(3 \mathrm{x})$ and the combined organic phases were washed with brine, dried over $\mathrm{Na}_{2} \mathrm{SO}_{4}$, filtered and concentrated. The crude was purified by flash column chromatography (cyclohexane/EtOAc 2:1, then 1:1) to yield the title compound (201 mg, $0.66 \mathrm{mmol}$, $95 \%)$ as a white solid.

M.p. $=75-77{ }^{\circ} \mathrm{C}$ (cyclohexane/EtOAc). ${ }^{1} \mathbf{H}$ NMR $\left(400 \mathrm{MHz}, \mathrm{CDCl}_{3}\right) \delta 7.45-7.39(\mathrm{~m}$, 2H), $7.39-7.34(\mathrm{~m}, 2 \mathrm{H}), 7.33-7.27(\mathrm{~m}, 5 \mathrm{H}), 7.25-7.19(\mathrm{~m}, 1 \mathrm{H}), 6.52(\mathrm{~d}, J=15.7 \mathrm{~Hz}, 1 \mathrm{H})$, $6.29(\mathrm{dt}, J=15.6,7.7 \mathrm{~Hz}, 1 \mathrm{H}), 3.84-3.72(\mathrm{~m}, 4 \mathrm{H}), 2.52(\mathrm{~s}, 2 \mathrm{H}), 2.39(\mathrm{~d}, J=7.7 \mathrm{~Hz}, 2 \mathrm{H}), 2.36$ - 2.17 (br s, 2H). ${ }^{13} \mathrm{C}$ NMR $\left(101 \mathrm{MHz}, \mathrm{CDCl}_{3}\right) \delta 137.4,133.8,131.8,128.7,128.4,128.0$, 127.4, 126.3, 125.2, 123.7, 86.5, 83.5, 68.0, 43.5, 35.7, 22.9. HRMS (ESI+) calculated for $\left[\mathrm{C}_{21} \mathrm{H}_{22} \mathrm{NaO}_{2}\right]^{+} 329.1512 \mathrm{~m} / \mathrm{z}$; found $[\mathrm{M}+\mathrm{Na}]^{+} 329.1528 \mathrm{~m} / \mathrm{z}$.

\section{(E)-(4,4-Bis(methoxymethyl)hept-1-en-6-yne-1,7-diyl)dibenzene (5q)}

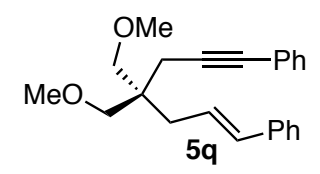

A solution of 2-cinnamyl-2-(3-phenylprop-2-yn-1-yl)propane-1,3-diol (100 mg, $0.33 \mathrm{mmol}$, 1.0 equiv) in THF ( $2 \mathrm{~mL})$ was added to a suspension of $\mathrm{NaH}(31 \mathrm{mg}, 0.78 \mathrm{mmol}, 2.4$ equiv, $60 \%$ in mineral oil $)$ in $\mathrm{THF}(1 \mathrm{~mL})$ at $0{ }^{\circ} \mathrm{C}$. After stirring the mixture for $10 \mathrm{~min} \mathrm{MeI}(61 \mu \mathrm{L}$, $0.98 \mathrm{mmol}, 3.0$ equiv) was added slowly. The reaction was stirred at $24{ }^{\circ} \mathrm{C}$ for $30 \mathrm{~min}$ and then quenched with sat. $\mathrm{NH}_{4} \mathrm{Cl}$. The aqueous phase was extracted with EtOAc (3x) and the combined organic phases were dried over $\mathrm{Na}_{2} \mathrm{SO}_{4}$, filtered and concentrated. The crude was purified by 
flash column chromatography (cyclohexane/EtOAc 60:1) to afford the title compound 5q (105 $\mathrm{mg}, 0.31 \mathrm{mmol}, 96 \%)$ as a pale yellow oil.

${ }^{1} \mathbf{H}$ NMR (400 MHz, $\left.\mathrm{CD}_{2} \mathrm{Cl}_{2}\right) \delta 7.45-7.39(\mathrm{~m}, 2 \mathrm{H}), 7.39-7.35(\mathrm{~m}, 2 \mathrm{H}), 7.34-7.26(\mathrm{~m}$, $5 \mathrm{H}), 7.23-7.17(\mathrm{~m}, 1 \mathrm{H}), 6.48(\mathrm{~d}, J=15.8 \mathrm{~Hz}, 1 \mathrm{H}), 6.30(\mathrm{dt}, J=15.6,7.6 \mathrm{~Hz}, 1 \mathrm{H}), 3.37-3.32$ $(\mathrm{m}, 10 \mathrm{H}), 2.45(\mathrm{~s}, 2 \mathrm{H}), 2.40-2.35(\mathrm{~m}, 2 \mathrm{H}) .{ }^{13} \mathbf{C} \mathbf{N M R}\left(101 \mathrm{MHz}, \mathrm{CD}_{2} \mathrm{Cl}_{2}\right) \delta 138.4,133.5$, 132.1, 129.0, 128.8, 128.2, 127.5, 126.7, 126.6, 124.6, 87.7, 83.0, 75.1, 59.7, 43.5, 36.4, 23.7. HRMS (ESI+) calculated for $\left[\mathrm{C}_{23} \mathrm{H}_{26} \mathrm{NaO}_{2}\right]^{+} 357.1825 \mathrm{~m} / \mathrm{z}$; found $[\mathrm{M}+\mathrm{Na}]^{+} 357.1833 \mathrm{~m} / \mathrm{z}$.

\subsection{Optimization of the reaction conditions}

\section{General procedure B: enantioselective formal $[4+2]$ reaction}

1,6-Enyne 5a (1.0 equiv) and catalyst (mol \% see tables) were dissolved in the respective solvent, a solution of the silver salt (mol \% see tables) in the same solvent (total concentration as indicated) was added dropwise and the reaction was stirred for the given time at $24^{\circ} \mathrm{C}$ in the dark. The reaction was quenched by filtration over a plug of silica and concentrated.

\section{Structure-selectivity investigations with the novel chiral complexes}

Table 1: Screening of complexes ${ }^{a}$

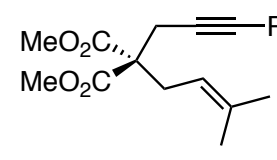

$5 \mathbf{a}$

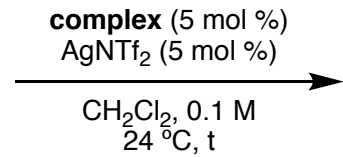
$24{ }^{\circ} \mathrm{C}, \mathrm{t}$

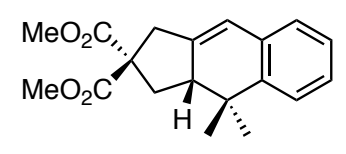

$6 a$

\begin{tabular}{ccccc}
\hline entry & complex & T $(\boldsymbol{h})$ & ${\text { yield }(\%)^{\mathbf{b}}}$ & $\boldsymbol{e r}$ \\
\hline $\mathbf{1}$ & $(R, R)-\mathbf{A}$ & 40 & 61 & $84: 16$ \\
$\mathbf{2}$ & $(R, R)-\mathbf{B}$ & 40 & 93 & $85: 15$ \\
$\mathbf{3}$ & $(R, R)-\mathbf{C}$ & 43 & $39^{\mathrm{c}}$ & $86: 14$ \\
$\mathbf{4}$ & $(R, R)-\mathbf{D}$ & 43 & $56^{\mathrm{d}}$ & $90: 10$ \\
$\mathbf{5}$ & $(R, R)-\mathbf{E}$ & 40 & $57^{\mathrm{e}}$ & $92: 8$ \\
$\mathbf{6}$ & $(\mathrm{S}, \mathrm{S})-\mathbf{F}$ & 40 & 75 & $20: 80$ \\
$\mathbf{7}$ & $(R, R)-\mathbf{G}$ & 46 & 74 & $24: 76$ \\
\hline
\end{tabular}

${ }^{\text {a }} \mathbf{5 a}=0.1 \mathrm{mmol}$ scale. ${ }^{\mathrm{b}}$ Yields determined by ${ }^{1} \mathrm{H}$ NMR using 1,3,5-tribromobenzene as internal standard. ${ }^{\mathrm{c}} 58 \%$ conversion. ${ }^{\mathrm{d}} 68 \%$ conversion. ${ }^{\mathrm{e}} 6 \%$ of an unexpected side product; $82 \%$ conversion. ${ }^{\mathrm{f}} \mathrm{er}$ determined by chiral HPLC.

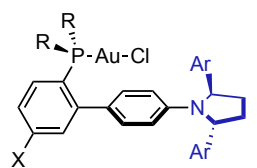

$(R, R)-\mathbf{A}: \mathrm{R}=\mathrm{Ad}, \mathrm{X}=\mathrm{H}, \mathrm{Ar}=3,5-\mathrm{Ph}_{2} \mathrm{C}_{6} \mathrm{H}_{3}$ $(R, R)-\mathrm{B}: \mathrm{R}=\mathrm{Ad}, \mathrm{X}=\mathrm{H}, \mathrm{Ar}=\mathrm{C}_{6} \mathrm{~F}_{5}$

R, R)-C: $\mathrm{R}=\mathrm{Ad}, \mathrm{X}=\mathrm{H}, \mathrm{Ar}=3,5-\left(\mathrm{CF}_{3}\right)_{2} \mathrm{C}_{6} \mathrm{H}_{3}$ $(R, R)$-D: $\mathrm{R}=\mathrm{Ad}, \mathrm{X}=\mathrm{H}, \mathrm{Ar}=3,5-\left(\mathrm{CF}_{3}\right)_{2} \mathrm{C}_{6} \mathrm{H}_{3}$ $(R, R)-\mathrm{E}: \mathrm{R}=\mathrm{Ad}, \mathrm{X}=\mathrm{CF}_{3}, \mathrm{Ar}=3,5-\left(\mathrm{CF}_{3}\right)_{2} \mathrm{C}_{6} \mathrm{H}_{3}$
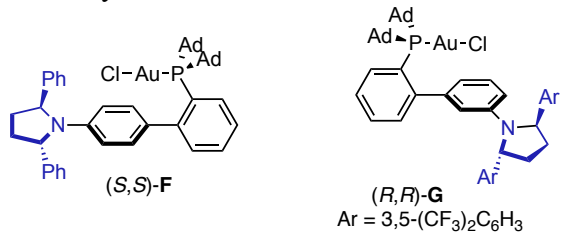
Diene $6 \mathbf{a}^{\prime}$ was obtained as a side product in the [4+2] cycloadditions of 1,6-enyne 5a catalyzed by complex $(R, R)$-E. However, isolation of $\mathbf{6 a}^{\prime}$ by preparative TLC or preparative HPLC could not be achieved. Its structure is proposed based on characteristic proton shifts and is in accordance with minor compounds previously observed in analogous reactions by our group. ${ }^{8}$ The achiral diene can be formed via 6-endo-dig cyclization followed by 1,2-hydrogen shift.

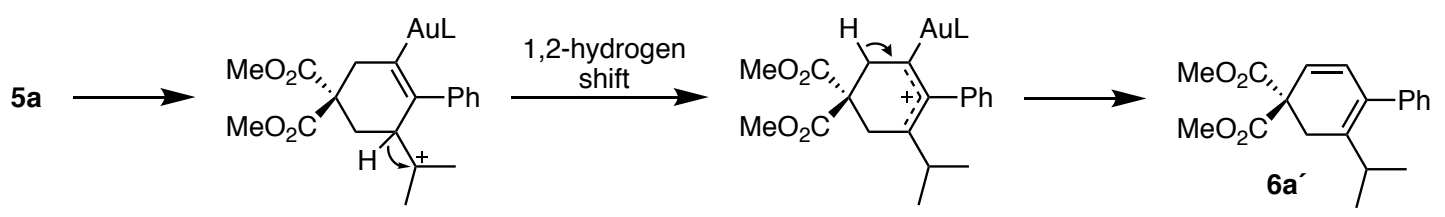

Characteristic signals for $\mathbf{6 a}^{\prime}:{ }^{1} \mathbf{H}$ NMR $\left(500 \mathrm{MHz}, \mathrm{CDCl}_{3}\right) \delta 6.16(\mathrm{~d}, J=9.5 \mathrm{~Hz}, 1 \mathrm{H}), 5.85$ $(\mathrm{d}, J=9.5 \mathrm{~Hz}, 1 \mathrm{H}), 2.86(\mathrm{~s}, 2 \mathrm{H}), 2.76$ (hept, $J=6.9 \mathrm{~Hz}, 1 \mathrm{H}), 1.00(\mathrm{~d}, J=6.9 \mathrm{~Hz}, 6 \mathrm{H})$.

In the [4+2] cycloadditions catalyzed by the other gold complexes only minuscule traces of side product 6a' were observed in the crude products.

\section{Optimization of the reaction conditions}

Table 2: Screening of chloride scavenger

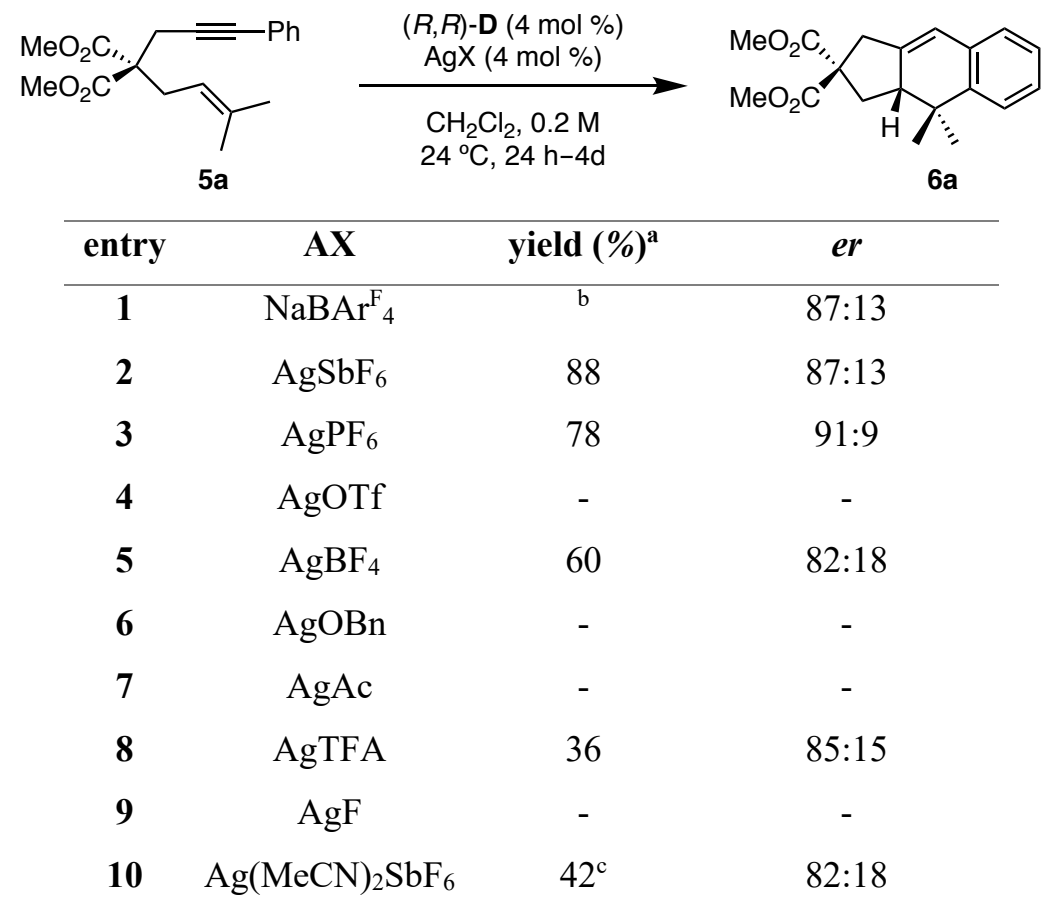

a Yields determined by ${ }^{1} \mathrm{H}$ NMR using 1,3,5-tribromobenzene as internal standard. ${ }^{\mathrm{b}}$ After $60 \mathrm{~h}$ reaction time the $\mathrm{SM} /$ product ratio, was estimated by ${ }^{1} \mathrm{H}$ NMR to be $5: 1 .{ }^{\text {c }}$ After $60 \mathrm{~h}$ reaction time $42 \%$ conversion. 
Table 3: Screening of solvents
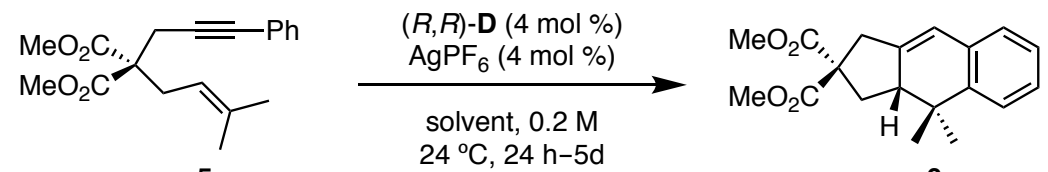

$5 a$ $24{ }^{\circ} \mathrm{C}, 24 \mathrm{~h}-5 \mathrm{~d}$

$6 a$

\begin{tabular}{cccc}
\hline entry & solvent & yield $(\%)^{\mathbf{a}}$ & $\boldsymbol{e r}$ \\
\hline $\mathbf{1}$ & 1,2 -dichloroethane & 83 & $92: 8$ \\
$\mathbf{2}$ & PhCl & - & - \\
$\mathbf{3}$ & benzene & - & - \\
$\mathbf{4}$ & EtOAc & 19 & $87: 13$ \\
$\mathbf{5}$ & THF & - & - \\
$\mathbf{6}$ & 1,2-dichloroethane/HFIP 3:1 & 78 & $90: 10$ \\
$\mathbf{7}$ & 1,2-dichloroethane & $21^{\mathrm{b}}$ & $93: 7$ \\
$\mathbf{8}$ & $\alpha, \alpha, \alpha$-trifluorotoluene & 65 & $91: 9$ \\
$\mathbf{9}$ & $1,3-\alpha, \alpha, \alpha$-bis(trifluoromethyl)benzene & 21 & $85: 15$ \\
$\mathbf{1 0}$ & anisole & - & - \\
$\mathbf{1 1}$ & CHCl & - & - \\
$\mathbf{1 2}$ & $1,1,2$-trichloroethane & - & - \\
$\mathbf{1 3}$ & $1,1,2,2$-tetrachloroethane & - & - \\
\hline
\end{tabular}

${ }^{\mathrm{a}}$ Yields determined by ${ }^{1} \mathrm{H}$ NMR using 1,3,5-tribromo-benzene as internal standard. ${ }^{\mathrm{b}}$ Run at $-5^{\circ} \mathrm{C}, \mathrm{t}=4 \mathrm{~d}, 90 \%$ conversion.

Table 4: Screening of catalyst loading and molarity

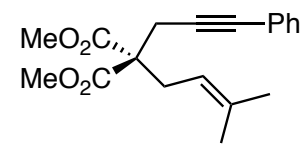

$5 a$

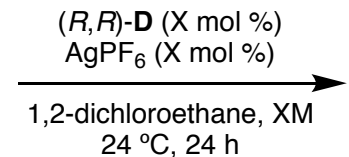

$24{ }^{\circ} \mathrm{C}, 24 \mathrm{~h}$

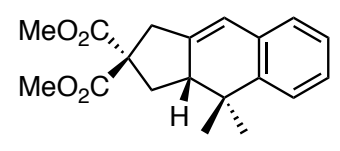

$6 a$

\begin{tabular}{|c|c|c|c|c|}
\hline entry & $(R, R)-\mathrm{D}(\mathrm{mol} \%)$ & concentration $(\mathbf{M})$ & yield $(\%)^{a}$ & $e r$ \\
\hline 1 & 4 & 0.5 & 73 & $92: 8$ \\
\hline 2 & 4 & 0.1 & 76 & $92: 8$ \\
\hline 3 & 4 & 0.05 & 83 & $93: 7$ \\
\hline 4 & 4 & 0.01 & - & - \\
\hline 5 & 2 & 0.05 & $\mathrm{~b}$ & 91:9 \\
\hline 6 & 1 & 0.05 & $-^{c}$ & - \\
\hline
\end{tabular}

${ }^{\mathrm{a}}$ Yields determined by ${ }^{1} \mathrm{H}$ NMR using 1,3,5-tribromobenzene as internal standard. ${ }^{\mathrm{b}}$ After $16 \mathrm{~h}$ reaction time the $\mathrm{SM} /$ product ratio, was estimated by ${ }^{1} \mathrm{H}$ NMR to be $3: 1 .{ }^{\mathrm{c}}$ No reaction after $16 \mathrm{~h}$. 


\subsection{Scope of the formal $[4+2]$ cyclization}

General procedure $C$ : enantioselective formal $[4+2]$ reaction under optimized conditions

1,6-Enyne 5 (1.0 equiv) and ( $R, R)$-D (4 mol \%) were dissolved in 1,2-dichloroethane and a solution of $\mathrm{AgPF}_{6}(4 \mathrm{~mol} \%$ ) in 1,2-dichloroethane (total concentration $0.05 \mathrm{M}$ ) was added dropwise. The reaction was stirred for the given time at $24{ }^{\circ} \mathrm{C}$ in the dark, quenched by addition of 3 drops of $\mathrm{NEt}_{3}$ and concentrated. The crude was purified by flash column chromatography or preparative TLC.

\section{Dimethyl $(R)-4,4-d i m e t h y l-1,3,3 a, 4-t e t r a h y d r o-2 H$-cyclopenta[b]naphthalene-2,2-} dicarboxylate (6a)

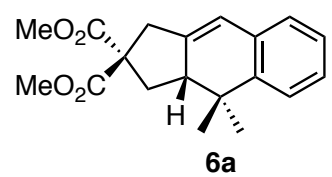

Compound 6a was synthesized following general procedure $\mathbf{C}$ using 1,6-enyne 5a (27 mg, $0.088 \mathrm{mmol}$ ) whereby the reaction was stirred for $25 \mathrm{~h}$. Purification by preparative TLC (cyclohexane/ $\mathrm{CH}_{2} \mathrm{Cl}_{2}$ 1:1) afforded the title compound 6a (17.6 mg, $0.056 \mathrm{mmol}, 64 \%$ ) as a colorless oil in 93:7 er.

$\boldsymbol{\alpha}_{\mathbf{D}}{ }^{589}=-14.1$ deg. $\mathrm{cm}^{2} \cdot \mathrm{g}^{-1}\left(\mathrm{CHCl}_{3}\right.$, c $\left.0.79,300 \mathrm{~K}\right) . \mathbf{U P C}{ }^{2}$ Chiralpak IC $(150 \times 4.6 \mathrm{~mm}, 3 \mu \mathrm{m})$ at $35^{\circ} \mathrm{C}$, flow $3 \mathrm{~mL} / \mathrm{min}$, isocratic $\mathrm{CO}_{2} / \mathrm{MeCN}$ 90:10, ABRP pressure $1500 \mathrm{psi}, 266 \mathrm{~nm}, \mathrm{t}_{\mathrm{R}}$ (major) $0.9 ; \mathrm{t}_{\mathrm{R}}$ (minor) 1.1 . The spectral data of $\mathbf{6 a}$ were fully consistent with those previously reported. ${ }^{7}$

\section{(R)-2,2-Bis(methoxymethyl)-4,4-dimethyl-2,3,3a,4-tetrahydro-1H-cyclopenta $[b]-$} naphthalene (6b)

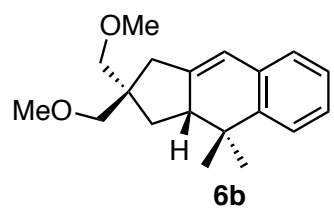

Compound $\mathbf{6 b}$ was synthesized following general procedure $\mathbf{C}$ using 1,6-enyne $\mathbf{5 b}$ ( $25 \mathrm{mg}$, $0.087 \mathrm{mmol}$ ) whereby the reaction was stirred for $16 \mathrm{~h}$. Purification by flash column chromatography (cyclohexane/EtOAc 30:1) afforded the title compound $\mathbf{6 b}$ (23 mg, $0.08 \mathrm{mmol}$, $92 \%$ ) as a colorless oil in 94:6 er.

${ }^{1} \mathbf{H}$ NMR $\left(400 \mathrm{MHz}, \mathrm{CD}_{2} \mathrm{Cl}_{2}\right) \delta 7.31-7.25(\mathrm{~m}, 1 \mathrm{H}), 7.14-7.07(\mathrm{~m}, 2 \mathrm{H}), 7.00-6.95(\mathrm{~m}$, $1 \mathrm{H}), 6.29-6.25(\mathrm{~m}, 1 \mathrm{H}), 3.36-3.31(\mathrm{~m}, 5 \mathrm{H}), 3.29(\mathrm{~s}, 3 \mathrm{H}), 3.24-3.19(\mathrm{~m}, 2 \mathrm{H}), 2.74-2.67$ 
(m, 1H), $2.50-2.42(\mathrm{~m}, 1 \mathrm{H}), 2.33(\mathrm{dt}, J=18.1,2.8 \mathrm{~Hz}, 1 \mathrm{H}), 1.83(\mathrm{dd}, J=12.6,8.4 \mathrm{~Hz}, 1 \mathrm{H})$, $1.55(\mathrm{t}, J=12.2 \mathrm{~Hz}, 1 \mathrm{H}), 1.38(\mathrm{~s}, 3 \mathrm{H}), 0.89$ (s, 3H). ${ }^{13} \mathbf{C}$ NMR $\left(126 \mathrm{MHz}, \mathrm{CD}_{2} \mathrm{Cl}_{2}\right) \delta$ 147.7, $145.2,135.2$, 127.0, 126.6, 126.4, 124.0, 119.2, 77.7, 75.7, 59.6, 48.9, 47.2, 38.3, 37.2, 32.1, 26.0, 22.2. HRMS (ESI+) calculated for $\left[\mathrm{C}_{19} \mathrm{H}_{26} \mathrm{NaO}_{2}\right]^{+} 309.1825 \mathrm{~m} / \mathrm{z}$; found $[\mathrm{M}+\mathrm{Na}]^{+}$ $309.1821 \mathrm{~m} / z \cdot \boldsymbol{\alpha}_{\mathbf{D}}{ }^{\mathbf{5 8 9}}=+1.7 \mathrm{deg} \cdot \mathrm{cm}^{2} \cdot \mathrm{g}^{-1}\left(\mathrm{CHCl}_{3}\right.$, c 1.0, $\left.300 \mathrm{~K}\right) \cdot \mathbf{U P C}{ }^{2}$ Chiralpak OJ-3 $(150 \times$ $4.6 \mathrm{~mm}, 3 \mu \mathrm{m}$ ) at $35^{\circ} \mathrm{C}$, flow $2 \mathrm{~mL} / \mathrm{min}$, isocratic $\mathrm{CO}_{2} / \mathrm{PrOH} 98: 2$, ABRP pressure 2000 psi, $267 \mathrm{~nm}, \mathrm{t}_{\mathrm{R}}$ (major) 2.3; $\mathrm{t}_{\mathrm{R}}$ (minor) 2.6.

\section{(R)-2,2-Bis((methoxymethoxy)methyl)-4,4-dimethyl-2,3,3a,4-tetrahydro-1H-} cyclopenta $[b]$ naphthalene $(6 c)$

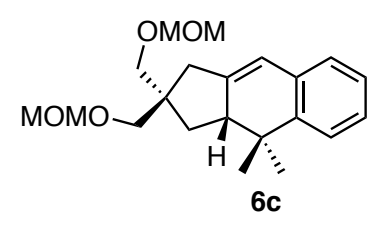

Compound $\mathbf{6 c}$ was synthesized following general procedure $\mathbf{C}$ using 1,6-enyne $\mathbf{5 c}$ (32 $\mathrm{mg}$, $0.09 \mathrm{mmol}$ ) whereby the reaction was stirred for $16 \mathrm{~h}$. Purification by flash column chromatography (cyclohexane/EtOAc 20:1) afforded the title compound $\mathbf{6 c}$ (29 mg, $0.08 \mathrm{mmol}$, 91\%) as a colorless oil in 93:7 er.

${ }^{1} \mathbf{H}$ NMR $\left(500 \mathrm{MHz}, \mathrm{CD}_{2} \mathrm{Cl}_{2}\right) \delta 7.31-7.26(\mathrm{~m}, 1 \mathrm{H}), 7.15-7.08(\mathrm{~m}, 2 \mathrm{H}), 7.01-6.96(\mathrm{~m}$, 1H), $6.31-6.28(\mathrm{~m}, 1 \mathrm{H}), 4.63$ (s, 2H), 4.58 (s, 2H), 3.53 (q, J=9.1 Hz, 2H), 3.41 (s, 2H), 3.35 $(\mathrm{s}, 3 \mathrm{H}), 3.31(\mathrm{~s}, 3 \mathrm{H}), 2.80-2.70(\mathrm{~m}, 1 \mathrm{H}), 2.55-2.47(\mathrm{~m}, 1 \mathrm{H}), 2.40(\mathrm{dt}, J=18.1,2.9 \mathrm{~Hz}, 1 \mathrm{H})$, $1.91(\mathrm{dd}, J=12.6,8.3 \mathrm{~Hz}, 1 \mathrm{H}), 1.59(\mathrm{t}, J=12.2 \mathrm{~Hz}, 1 \mathrm{H}), 1.39(\mathrm{~s}, 3 \mathrm{H}), 0.91(\mathrm{~s}, 3 \mathrm{H}) .{ }^{13} \mathbf{C}$ NMR $\left(126 \mathrm{MHz}, \mathrm{CD}_{2} \mathrm{Cl}_{2}\right) \delta 147.3,145.1,135.1,127.0,126.6,126.4,124.0,119.4,97.3,97.2,72.6$, 70.3, 55.51, 55.47, 48.8, 46.6, 38.4, 37.2, 32.0, 26.0, 22.3. HRMS (ESI+) calculated for $\left[\mathrm{C}_{21} \mathrm{H}_{30} \mathrm{NaO}_{4}\right]^{+} 369.2036 \mathrm{~m} / z$; found $[\mathrm{M}+\mathrm{Na}]^{+} 369.2036 \mathrm{~m} / z . \boldsymbol{\alpha}_{\mathbf{D}}{ }^{\mathbf{5 8 9}}=-3.3 \mathrm{deg} \cdot \mathrm{cm}^{2} \cdot \mathrm{g}^{-1}\left(\mathrm{CHCl}_{3}\right.$, c $1.0,300 \mathrm{~K})$. $\mathbf{U P C}^{2}$ Chiralpak IG $(150 \times 4.6 \mathrm{~mm}, 3 \mu \mathrm{m})$ at $35^{\circ} \mathrm{C}$, flow $2 \mathrm{~mL} / \mathrm{min}$, isocratic $\mathrm{CO}_{2} / \mathrm{iPrOH}$ 85:15, ABRP pressure $2000 \mathrm{psi}, 267 \mathrm{~nm}, \mathrm{t}_{\mathrm{R}}$ (major) 2.0; $\mathrm{t}_{\mathrm{R}}$ (minor) 2.4. 
(R)-(4,4-Dimethyl-2,3,3a,4-tetrahydro-1 $H$-cyclopenta $[b]$ naphthalene-2,2-diyl)bis(methylene) diacetate (6d)

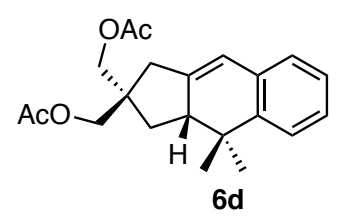

Compound 6d was synthesized following general procedure $\mathbf{C}$ using 1,6-enyne $\mathbf{5 d}$ (32 mg, $0.87 \mathrm{mmol}$ ) whereby the reaction was stirred for $48 \mathrm{~h}$. Purification by flash column chromatography (cyclohexane/EtOAc 20:1) afforded title compound 6d (28 mg, $0.086 \mathrm{mmol}$, 94\%) as a colorless oil in 79:21 er.

${ }^{1} \mathbf{H}$ NMR $\left(500 \mathrm{MHz}, \mathrm{CDCl}_{3}\right) \delta 7.31-7.27(\mathrm{~m}, 1 \mathrm{H}), 7.18-7.10(\mathrm{~m}, 2 \mathrm{H}), 7.03-6.97(\mathrm{~m}$, $1 \mathrm{H}), 6.34-6.29(\mathrm{~m}, 1 \mathrm{H}), 4.15-4.07(\mathrm{~m}, 2 \mathrm{H}), 4.04-3.94(\mathrm{~m}, 2 \mathrm{H}), 2.80-2.73(\mathrm{~m}, 1 \mathrm{H}), 2.58$ $-2.51(\mathrm{~m}, 1 \mathrm{H}), 2.40(\mathrm{dt}, J=18.2,2.8 \mathrm{~Hz}, 1 \mathrm{H}), 2.09$ (s, 3H), 2.07 (s, 3H), 1.87 (dd, $J=12.8$, $8.3 \mathrm{~Hz}, 1 \mathrm{H}), 1.60(\mathrm{t}, J=12.4 \mathrm{~Hz}, 1 \mathrm{H}), 1.39$ (s, 3H), $0.91(\mathrm{~s}, 3 \mathrm{H}) .{ }^{13} \mathbf{C ~ N M R}\left(126 \mathrm{MHz}, \mathrm{CDCl}_{3}\right)$ $\delta 171.2,171.1,144.4,144.3,134.2,127.0,126.4,126.3,123.6,120.0,68.1,65.7,48.1,45.0$, 37.7, 36.8, 31.9, 25.7, 22.0, 21.0. HRMS (ESI+) calculated for $\left[\mathrm{C}_{21} \mathrm{H}_{26} \mathrm{NaO}_{4}\right]^{+} 365.1723 \mathrm{~m} / \mathrm{z}$; found $[\mathrm{M}+\mathrm{Na}]^{+} 365.1735 \mathrm{~m} / z \cdot \boldsymbol{\alpha}_{\mathbf{D}}{ }^{589}=+3.1 \mathrm{deg} \cdot \mathrm{cm}^{2} \cdot \mathrm{g}^{-1}\left(\mathrm{CHCl}_{3}\right.$, c 1.0, $\left.297 \mathrm{~K}\right)$. UPC Chiralpak IB $(100 \times 4.6 \mathrm{~mm}, 3 \mu \mathrm{m})$ at $35^{\circ} \mathrm{C}$, flow $3 \mathrm{~mL} / \mathrm{min}$, isocratic $\mathrm{CO}_{2} / \mathrm{MeOH}$ 90:10, ABRP pressure $1500 \mathrm{psi}, 266 \mathrm{~nm}, \mathrm{t}_{\mathrm{R}}$ (minor) $0.8 ; \mathrm{t}_{\mathrm{R}}$ (major) 0.9 .

(R)-6-Methoxy-2,2-bis(methoxymethyl)-4,4-dimethyl-2,3,3a,4-tetrahydro-1 $H$-cyclopenta$[b]$ naphthalene (6e)

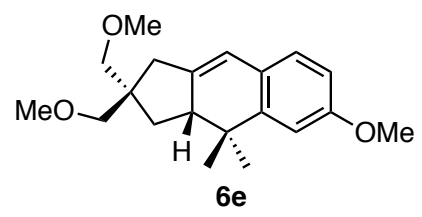

Compound 6e was synthesized following general procedure $\mathbf{C}$ using 1,6-enyne $\mathbf{5 e}(28 \mathrm{mg}$, $0.087 \mathrm{mmol}$ ) whereby the reaction was stirred for $12 \mathrm{~h}$. Purification by flash column chromatography (cyclohexane/EtOAc 40:1) afforded title compound 6e (27.5 mg, $0.087 \mathrm{mmol}$, 97\%) as a colorless oil in 96:4 er.

${ }^{1} \mathbf{H}$ NMR $\left(400 \mathrm{MHz}, \mathrm{CDCl}_{3}\right) \delta 6.93(\mathrm{~d}, J=8.2 \mathrm{~Hz}, 1 \mathrm{H}), 6.88(\mathrm{~d}, J=2.5 \mathrm{~Hz}, 1 \mathrm{H}), 6.66(\mathrm{dd}$, $J=8.2,2.6 \mathrm{~Hz}, 1 \mathrm{H}), 6.27-6.20(\mathrm{~m}, 1 \mathrm{H}), 3.80(\mathrm{~s}, 3 \mathrm{H}), 3.38(\mathrm{~s}, 3 \mathrm{H}), 3.37(\mathrm{~s}, 2 \mathrm{H}), 3.32(\mathrm{~s}, 3 \mathrm{H})$, $3.27-3.21(\mathrm{~m}, 2 \mathrm{H}), 2.72-2.63(\mathrm{~m}, 1 \mathrm{H}), 2.47(\mathrm{~d}, J=18.0 \mathrm{~Hz}, 1 \mathrm{H}), 2.31$ (dt, $J=17.9,2.7 \mathrm{~Hz}$, $1 \mathrm{H}), 1.83(\mathrm{dd}, J=12.6,8.3 \mathrm{~Hz}, 1 \mathrm{H}), 1.53$ (t, $J=12.2 \mathrm{~Hz}, 1 \mathrm{H}), 1.36(\mathrm{~s}, 3 \mathrm{H}), 0.89(\mathrm{~s}, 3 \mathrm{H}) .{ }^{13} \mathrm{C}$ NMR $\left(101 \mathrm{MHz}, \mathrm{CDCl}_{3}\right) \delta 158.6,146.6,144.1,128.0,126.8,118.4,110.9,110.0,75.1,59.5$, 
55.4, 48.1, 46.8, 37.8, 37.1, 31.7, 25.7, 21.9. HRMS (ESI+) calculated for $\left[\mathrm{C}_{20} \mathrm{H}_{28} \mathrm{NaO}_{3}\right]^{+}$ $339.1931 \mathrm{~m} / z$; found $[\mathrm{M}+\mathrm{Na}]^{+} 339.1941 \mathrm{~m} / z . \boldsymbol{\alpha}_{\mathbf{D}}{ }^{589}=-21.7 \mathrm{deg} \cdot \mathrm{cm}^{2} \cdot \mathrm{g}^{-1}\left(\mathrm{CHCl}_{3}, \mathrm{c} 1.0,300\right.$ K). UPC $^{2}$ Chiralpak OJ $(150 \times 4.6 \mathrm{~mm}, 3 \mu \mathrm{m})$ at $35{ }^{\circ} \mathrm{C}$, flow $2 \mathrm{~mL} / \mathrm{min}$, isocratic $\mathrm{CO}_{2} / \mathrm{MeOH}$ 85:15, ABRP pressure $2000 \mathrm{psi}, 275 \mathrm{~nm}, \mathrm{t}_{\mathrm{R}}$ (major) 1.4; $\mathrm{t}_{\mathrm{R}}$ (minor) 1.5 .

(R)-2,2-Bis(methoxymethyl)-4,4-dimethyl-6-nitro-2,3,3a,4-tetrahydro-1H-cyclopenta[b]naphthalene (6f)

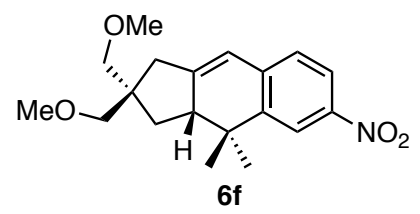

Compound $\mathbf{6 f}$ was synthesized following general procedure $\mathbf{C}$ using 1,6-enyne $\mathbf{5 f}$ (28.8 $\mathrm{mg}$, $0.087 \mathrm{mmol}$ ) whereby the reaction was stirred for $16 \mathrm{~h}$. The crude was purified by preparative TLC (pentane/EtOAc 20:1) to afford title compound $\mathbf{6 f}$ (27.4 mg, $0.082 \mathrm{mmol}, 94 \%$ ) as a yellow oil in 90:10 er.

${ }^{1} \mathbf{H}$ NMR $\left(400 \mathrm{MHz}, \mathrm{CDCl}_{3}\right) \delta 8.13(\mathrm{~d}, J=2.2 \mathrm{~Hz}, 1 \mathrm{H}), 8.00(\mathrm{dd}, J=8.3,2.3 \mathrm{~Hz}, 1 \mathrm{H}), 7.07$ $(\mathrm{d}, J=8.4 \mathrm{~Hz}, 1 \mathrm{H}), 6.39-6.34(\mathrm{~m}, 1 \mathrm{H}), 3.37(\mathrm{~s}, 3 \mathrm{H}), 3.36(\mathrm{~s}, 2 \mathrm{H}), 3.32(\mathrm{~s}, 3 \mathrm{H}), 3.28-3.21$ $(\mathrm{m}, 2 \mathrm{H}), 2.82-2.73(\mathrm{~m}, 1 \mathrm{H}), 2.58-2.50(\mathrm{~m}, 1 \mathrm{H}), 2.40(\mathrm{dt}, J=18.7,2.8 \mathrm{~Hz}, 1 \mathrm{H}), 1.88(\mathrm{dd}, J$ $=12.7,8.5 \mathrm{~Hz}, 1 \mathrm{H}), 1.60(\mathrm{t}, J=12.0 \mathrm{~Hz}, 1 \mathrm{H}), 1.45(\mathrm{~s}, 3 \mathrm{H}), 0.94(\mathrm{~s}, 3 \mathrm{H}) .{ }^{13} \mathbf{C}$ NMR $(101 \mathrm{MHz}$, $\left.\mathrm{CDCl}_{3}\right) \delta 153.7,146.4,145.9,141.1,126.2,122.2,119.4,118.2,75.3,59.5,48.4,46.9,38.3$, 37.3, 31.4, 25.7, 21.9. HRMS (ESI+) calculated for $\left[\mathrm{C}_{19} \mathrm{H}_{25} \mathrm{NNaO}_{4}\right]^{+} 354.1676 \mathrm{~m} / \mathrm{z}$; found [M $+\mathrm{Na}]^{+} 354.1686 \mathrm{~m} / z \cdot \boldsymbol{\alpha}_{\mathbf{D}}{ }^{589}=-68.5 \mathrm{deg} \cdot \mathrm{cm}^{2} \cdot \mathrm{g}^{-1}\left(\mathrm{CHCl}_{3}, \mathrm{c} 1.0,300 \mathrm{~K}\right) . \mathbf{U P C}{ }^{2}$ Chiralpak IG $(150 \times 4.6 \mathrm{~mm}, 3 \mu \mathrm{m})$ at $35^{\circ} \mathrm{C}$, flow $2 \mathrm{~mL} / \mathrm{min}$, isocratic $\mathrm{CO}_{2} / \mathrm{MeOH} 95: 5, \mathrm{ABRP}$ pressure 2000 psi, $338 \mathrm{~nm}, \mathrm{t}_{\mathrm{R}}$ (minor) 4.8; $\mathrm{t}_{\mathrm{R}}$ (major) 5.0.

(R)-6-(tert-Butyl)-2,2-bis(methoxymethyl)-4,4-dimethyl-2,3,3a,4-tetrahydro-1H-cyclopenta[b]naphthalene (6g)

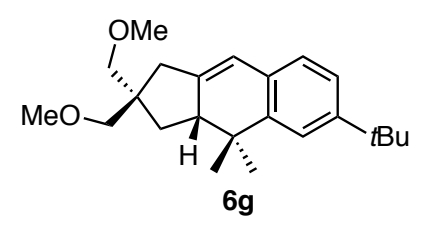

Compound $\mathbf{6 g}$ was synthesized following general procedure $\mathbf{C}$ using 1,6-enyne $\mathbf{5 g}$ (29.8 mg, $0.087 \mathrm{mmol}$ ) whereby the reaction was stirred for $12 \mathrm{~h}$. Purification by flash column chromatography (hexane/EtOAc 70:1) afforded title compound 6g (29.6 mg, 0.087 mmol, 99\%) as a colorless oil in 91:9 er. 
${ }^{1} \mathbf{H}$ NMR $\left(500 \mathrm{MHz}, \mathrm{CD}_{2} \mathrm{Cl}_{2}\right) \delta 7.33(\mathrm{~d}, J=1.5 \mathrm{~Hz}, 1 \mathrm{H}), 7.14(\mathrm{~d}, J=7.9,1.9 \mathrm{~Hz}, 1 \mathrm{H}), 6.91$ $(\mathrm{d}, J=7.9 \mathrm{~Hz}, 1 \mathrm{H}), 6.27-6.23(\mathrm{~m}, 1 \mathrm{H}), 3.37-3.32(\mathrm{~m}, 5 \mathrm{H}), 3.29(\mathrm{~s}, 3 \mathrm{H}), 3.24-3.19(\mathrm{~m}, 2 \mathrm{H})$, $2.74-2.65(\mathrm{~m}, 1 \mathrm{H}), 2.50-2.40(\mathrm{~m}, 1 \mathrm{H}), 2.32(\mathrm{dt}, J=18.0,2.6 \mathrm{~Hz}, 1 \mathrm{H}), 1.83(\mathrm{dd}, J=12.5,8.4$ $\mathrm{Hz}, 1 \mathrm{H}), 1.54(\mathrm{t}, J=12.2 \mathrm{~Hz}, 1 \mathrm{H}), 1.39(\mathrm{~s}, 3 \mathrm{H}), 1.31(\mathrm{~s}, 9 \mathrm{H}), 0.89(\mathrm{~s}, 3 \mathrm{H}) .{ }^{13} \mathbf{C}$ NMR $(126 \mathrm{MHz}$, $\left.\mathrm{CD}_{2} \mathrm{Cl}_{2}\right) \delta 149.9,146.7,144.7,132.5,125.9,123.3,121.0,118.9,77.8,75.7,59.6,49.0,47.2$, 38.3, 37.4 , 35.2, 32.1, 31.8, 26.0, 22.3. HRMS (ESI+) calculated for $\left[\mathrm{C}_{23} \mathrm{H}_{34} \mathrm{NaO}_{2}\right]^{+} 365.2451$ $m / z$; found $[\mathrm{M}+\mathrm{Na}]^{+} 365.2442 \mathrm{~m} / z . \boldsymbol{\alpha}_{\mathbf{D}}{ }^{589}=-17.2 \mathrm{deg} \cdot \mathrm{cm}^{2} \cdot \mathrm{g}^{-1}\left(\mathrm{CHCl}_{3}, \mathrm{c} 1.0,300 \mathrm{~K}\right)$. HPLC Chiralpak OD-H $(250 \times 4.6 \mathrm{~mm}, 5 \mu \mathrm{m})$ at $25^{\circ} \mathrm{C}$, flow $1.0 \mathrm{~mL} / \mathrm{min}$, isocratic hexane, $280 \mathrm{~nm}, \mathrm{t}_{\mathrm{R}}$ (minor) $5.6 ; \mathrm{t}_{\mathrm{R}}$ (major) 6.9 .

\section{(R)-6-Chloro-2,2-bis(methoxymethyl)-4,4-dimethyl-2,3,3a,4-tetrahydro-1H-cyclopenta-} [b]naphthalene (6h)

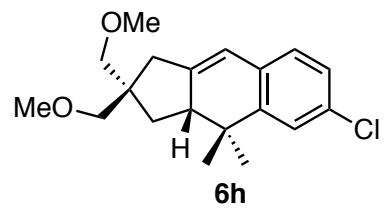

Compound $\mathbf{6 h}$ was synthesized following general procedure $\mathbf{C}$ using 1,6-enyne $\mathbf{5 h}$ (27.9 mg, $0.087 \mathrm{mmol}$ ) whereby the reaction was stirred for $17 \mathrm{~h}$. The crude was purified by preparative TLC (cyclohexane/ $\mathrm{CH}_{2} \mathrm{Cl}_{2}$ 1:1) to afford title compound $\mathbf{6 h}(27 \mathrm{mg}, 0.084 \mathrm{mmol}, 97 \%$ ) as a colorless oil in 94:6 er.

${ }^{1} \mathbf{H}$ NMR $\left(500 \mathrm{MHz}, \mathrm{CDCl}_{3}\right) \delta 7.23(\mathrm{~d}, J=2.1 \mathrm{~Hz}, 1 \mathrm{H}), 7.08(\mathrm{dd}, J=8.0,2.1 \mathrm{~Hz}, 1 \mathrm{H}), 6.90$ $(\mathrm{d}, J=8.1 \mathrm{~Hz}, 1 \mathrm{H}), 6.24(\mathrm{~d}, J=2.4 \mathrm{~Hz}, 1 \mathrm{H}), 3.37$ (s, 3H), $3.36(\mathrm{~s}, 2 \mathrm{H}), 3.32$ (s, 3H), $3.26-$ $3.20(\mathrm{~m}, 2 \mathrm{H}), 2.73-2.64(\mathrm{~m}, 1 \mathrm{H}), 2.52-2.44(\mathrm{~m}, 1 \mathrm{H}), 2.33(\mathrm{dt}, J=18.2,2.8 \mathrm{~Hz}, 1 \mathrm{H}), 1.84$ $(\mathrm{dd}, J=12.5,8.4 \mathrm{~Hz}, 1 \mathrm{H}), 1.54(\mathrm{t}, J=12.1 \mathrm{~Hz}, 1 \mathrm{H}), 1.36(\mathrm{~s}, 3 \mathrm{H}), 0.90(\mathrm{~s}, 3 \mathrm{H}) .{ }^{13} \mathbf{C}$ NMR $(126$ $\left.\mathrm{MHz}, \mathrm{CDCl}_{3}\right) \delta 147.5,146.6,133.2,131.8,127.1,126.1,124.1,118.2,77.3,75.2,59.5,48.1$, 46.8, 37.9, 37.2, 31.6, 25.6, 21.9. HRMS (APCI+) calculated for $\left[\mathrm{C}_{19} \mathrm{H}_{26} \mathrm{ClO}_{2}\right]^{+} 321.1616 \mathrm{~m} / \mathrm{z}$; found $[\mathrm{M}+\mathrm{H}]^{+} 321.1615 \mathrm{~m} / z \cdot \boldsymbol{\alpha}_{\mathbf{D}}{ }^{589}=-3.8 \mathrm{deg} \cdot \mathrm{cm}^{2} \cdot \mathrm{g}^{-1}\left(\mathrm{CHCl}_{3}, \mathrm{c} 1.0,300 \mathrm{~K}\right)$. HPLC Chiralpak IB-N $(150 \times 4.6 \mathrm{~mm}, 5 \mu \mathrm{m})$ at $25^{\circ} \mathrm{C}$, flow $0.5 \mathrm{~mL} / \mathrm{min}$, isocratic $n$ heptane $/ \mathrm{M} t \mathrm{BE} 98: 2,280 \mathrm{~nm}$, $\mathrm{t}_{\mathrm{R}}$ (minor) 14.3; $\mathrm{t}_{\mathrm{R}}$ (major) 16.4 . 
(R)-2,2-Bis(methoxymethyl)-4,4-dimethyl-6-(trifluoromethyl)-2,3,3a,4-tetrahydro-1Hcyclopenta $[b]$ naphthalene (6i)

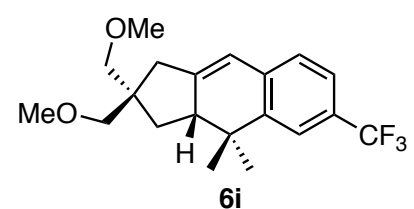

Compound $\mathbf{6} \mathbf{i}$ was synthesized following general procedure $\mathbf{C}$ using 1,6-enyne $\mathbf{5 i}$ (30.8 mg, $0.087 \mathrm{mmol}$ ) whereby the reaction was stirred for $17 \mathrm{~h}$. The crude was purified by preparative TLC (cyclohexane/ $\mathrm{CH}_{2} \mathrm{Cl}_{2}$ 1:1) to afford title compound $6 \mathbf{i}$ (30 mg, $0.084 \mathrm{mmol}, 97 \%$ ) as a colorless oil in 91:9 er.

${ }^{1} \mathbf{H}$ NMR $\left(400 \mathrm{MHz}, \mathrm{CDCl}_{3}\right) \delta 7.50-7.46(\mathrm{~m}, 1 \mathrm{H}), 7.37(\mathrm{ddd}, J=7.9,1.9,0.9 \mathrm{~Hz}, 1 \mathrm{H})$, $7.06(\mathrm{~d}, J=7.9 \mathrm{~Hz}, 1 \mathrm{H}), 6.32$ (d, $J=2.6 \mathrm{~Hz}, 1 \mathrm{H}), 3.38$ (s, 3H), 3.37 (s, 2H), 3.33 (s, 3H), 3.28 $-3.21(\mathrm{~m}, 2 \mathrm{H}), 2.79-2.71(\mathrm{~m}, 1 \mathrm{H}), 2.52(\mathrm{~d}, J=18.4 \mathrm{~Hz}, 1 \mathrm{H}), 2.37(\mathrm{dt}, J=18.5,2.9 \mathrm{~Hz}, 1 \mathrm{H})$, $1.87(\mathrm{dd}, J=12.7,8.4 \mathrm{~Hz}, 1 \mathrm{H}), 1.64-1.52(\mathrm{~m}, 1 \mathrm{H}), 1.42(\mathrm{~s}, 3 \mathrm{H}), 0.93(\mathrm{~s}, 3 \mathrm{H}) .{ }^{13} \mathbf{C}$ NMR $(101$ $\left.\mathrm{MHz}, \mathrm{CDCl}_{3}\right) \delta 150.3,145.2,137.9(\mathrm{~d}, J=1.3 \mathrm{~Hz}), 128.2(\mathrm{q}, J=31.8 \mathrm{~Hz}), 126.0,124.7(\mathrm{q}, J=$ $271.7 \mathrm{~Hz}), 123.3$ (q, $J=3.9,3.5 \mathrm{~Hz}), 120.6$ (q, $J=3.8 \mathrm{~Hz}), 118.3,77.3,75.3,59.5,48.3,46.8$, 38.1, 37.0, 31.5, 25.6, 21.9. ${ }^{19} \mathbf{F}\left\{{ }^{1} \mathbf{H}\right\}$ NMR $\left(376 \mathrm{MHz}, \mathrm{CDCl}_{3}\right) \delta-62.2$. HRMS (APCI+) calculated for $\left[\mathrm{C}_{19} \mathrm{H}_{22} \mathrm{~F}_{3} \mathrm{O}\right]^{+} 323.1617 \mathrm{~m} / \mathrm{z}$; found $\left[\mathrm{M}-\mathrm{CH}_{3} \mathrm{O}\right]^{+} 323.1616 \mathrm{~m} / \mathrm{z} . \boldsymbol{\alpha}_{\mathbf{D}}{ }^{\mathbf{5 8 9}}=-0.9$ deg. $\mathrm{cm}^{2} \cdot \mathrm{g}^{-1}\left(\mathrm{CHCl}_{3}, \mathrm{c} 1.0,300 \mathrm{~K}\right)$. HPLC Chiralpak IB-N $(150 \times 4.6 \mathrm{~mm}, 5 \mu \mathrm{m})$ at $25^{\circ} \mathrm{C}$, flow $0.5 \mathrm{~mL} / \mathrm{min}$, isocratic $n$ heptane/MtBE $98: 2,280 \mathrm{~nm}, \mathrm{t}_{\mathrm{R}}$ (minor) 12.2; $\mathrm{t}_{\mathrm{R}}$ (major) 13.1.

\section{(R)-8-Fluoro-2,2-bis(methoxymethyl)-4,4-dimethyl-2,3,3a,4-tetrahydro-1H-cyclopenta-}

\section{$[b]$ naphthalene $(6 \mathbf{j})$}

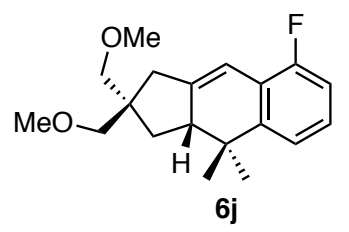

Compound $\mathbf{6 j}$ was synthesized following general procedure $\mathbf{C}$ using 1,6-enyne $\mathbf{5 j}$ (28 mg, $0.087 \mathrm{mmol}$ ) whereby the reaction was stirred for $13 \mathrm{~h}$. The crude was purified by flash column chromatography (cyclohexane/EtOAc 50:1) and further purified by preparative TLC (cyclohexane/EtOAc 30:1) to afford title compound $\mathbf{6 j}(23.3 \mathrm{mg}, 0.076 \mathrm{mmol}, 88 \%$ ) as a colorless oil in 93:7 er.

${ }^{1}$ H NMR $\left(400 \mathrm{MHz}, \mathrm{CDCl}_{3}\right) \delta 7.11-7.02(\mathrm{~m}, 2 \mathrm{H}), 6.89-6.80(\mathrm{~m}, 1 \mathrm{H}), 6.56-6.51(\mathrm{~m}$, $1 \mathrm{H}), 3.38(\mathrm{~s}, 3 \mathrm{H}), 3.37(\mathrm{~s}, 2 \mathrm{H}), 3.33(\mathrm{~s}, 3 \mathrm{H}), 3.28-3.21(\mathrm{~m}, 2 \mathrm{H}), 2.77-2.65(\mathrm{~m}, 1 \mathrm{H}), 2.57$ $2.48(\mathrm{~m}, 1 \mathrm{H}), 2.36(\mathrm{dt}, J=18.2,2.8 \mathrm{~Hz}, 1 \mathrm{H}), 1.86(\mathrm{dd}, J=12.6,8.4 \mathrm{~Hz}, 1 \mathrm{H}), 1.55(\mathrm{t}, J=12.2$ 
$\mathrm{Hz}, 1 \mathrm{H}), 1.38(\mathrm{~s}, 3 \mathrm{H}), 0.90(\mathrm{~s}, 3 \mathrm{H}) .{ }^{13} \mathbf{C} \mathbf{N M R}\left(101 \mathrm{MHz}, \mathrm{CDCl}_{3}\right) \delta 158.5(\mathrm{~d}, J=245.9 \mathrm{~Hz})$, $147.8(\mathrm{~d}, J=1.7 \mathrm{~Hz}), 147.1$ (d, $J=3.5 \mathrm{~Hz}), 127.0,122.4$ (d, $J=14.3 \mathrm{~Hz}), 119.1$ (d, $J=2.9 \mathrm{~Hz})$, $112.9(\mathrm{~d}, J=21.8 \mathrm{~Hz}), 111.0(\mathrm{~d}, J=6.3 \mathrm{~Hz}), 77.3,75.1,59.5$ (d, $J=1.8 \mathrm{~Hz}), 48.1,46.8,38.2$, 37.1, 31.6, 25.8, 21.7. ${ }^{19} \mathbf{F}\left\{{ }^{1} \mathbf{H}\right\}$ NMR $\left(376 \mathrm{MHz}, \mathrm{CDCl}_{3}\right) \delta-123.2$. HRMS (ESI+) calculated for $\left[\mathrm{C}_{19} \mathrm{H}_{25} \mathrm{FNaO}_{2}\right]^{+} 327.1731 \mathrm{~m} / z$; found $[\mathrm{M}+\mathrm{Na}]^{+} 327.1733 \mathrm{~m} / z . \boldsymbol{\alpha}_{\mathbf{D}}{ }^{\mathbf{5 8 9}}=-2.0 \mathrm{deg} \cdot \mathrm{cm}^{2} \cdot \mathrm{g}^{-1}$ $\left(\mathrm{CHCl}_{3}, \mathrm{c} 1.0,300 \mathrm{~K}\right)$. UPC ${ }^{2}$ Chiralpak IG $(150 \times 4.6 \mathrm{~mm}, 3 \mu \mathrm{m})$ at $35^{\circ} \mathrm{C}$, flow $2 \mathrm{~mL} / \mathrm{min}$, isocratic $\mathrm{CO}_{2} / \mathrm{MeOH}$ 95:5, ABRP pressure $2000 \mathrm{psi}, 267 \mathrm{~nm}, \mathrm{t}_{\mathrm{R}}$ (minor) 2.1; $\mathrm{t}_{\mathrm{R}}$ (major) 2.2.

\section{(R)-2,2-Bis(methoxymethyl)-4,4,8-trimethyl-2,3,3a,4-tetrahydro-1H-cyclopenta[b]-} naphthalene (6k)

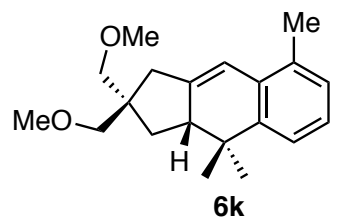

Compound 6k was synthesized following general procedure $\mathbf{C}$ using 1,6-enyne 5k (26 mg, $0.087 \mathrm{mmol}$ ) whereby the reaction was stirred for $20 \mathrm{~h}$. Purification by preparative TLC (cyclohexane/ $\left.\mathrm{CH}_{2} \mathrm{Cl}_{2} 1: 1\right)$ afforded title compound $\mathbf{6 k}(22 \mathrm{mg}, 0.073 \mathrm{mmol}, 85 \%$ ) as a colorless oil in 95:5 er.

${ }^{1} \mathbf{H}$ NMR $\left(500 \mathrm{MHz}, \mathrm{CD}_{2} \mathrm{Cl}_{2}\right) \delta 7.13(\mathrm{~d}, J=7.6 \mathrm{~Hz}, 1 \mathrm{H}), 7.01(\mathrm{t}, J=7.6 \mathrm{~Hz}, 1 \mathrm{H}), 6.96(\mathrm{~d}, J$ $=7.4 \mathrm{~Hz}, 1 \mathrm{H}), 6.51-6.47(\mathrm{~m}, 1 \mathrm{H}), 3.34(\mathrm{~s}, 5 \mathrm{H}), 3.29(\mathrm{~s}, 3 \mathrm{H}), 3.22(\mathrm{~s}, 2 \mathrm{H}), 2.70-2.62(\mathrm{~m}, 1 \mathrm{H})$, $2.55-2.46(\mathrm{~m}, 1 \mathrm{H}), 2.34(\mathrm{dt}, J=18.1,2.8 \mathrm{~Hz}, 1 \mathrm{H}), 2.29(\mathrm{~s}, 3 \mathrm{H}), 1.86-1.79(\mathrm{~m}, 1 \mathrm{H}), 1.57$ $1.51(\mathrm{~m}, 1 \mathrm{H}), 1.35(\mathrm{~s}, 3 \mathrm{H}), 0.87$ (s, 3H). ${ }^{13} \mathbf{C}$ NMR $\left(126 \mathrm{MHz}, \mathrm{CD}_{2} \mathrm{Cl}_{2}\right) \delta 147.6,145.3,133.3$, $133.2,128.4,126.5,121.8,116.0,77.7,75.6,59.6,48.3,47.2,38.7,37.4,32.1,26.2,21.9$, 20.0. HRMS (ESI+) calculated for $\left[\mathrm{C}_{20} \mathrm{H}_{28} \mathrm{NaO}_{2}\right]^{+} 323.1982 \mathrm{~m} / z$; found $[\mathrm{M}+\mathrm{Na}]^{+} 323.1962 \mathrm{~m} / \mathrm{z}$. $\boldsymbol{\alpha}_{\mathbf{D}}{ }^{589}=-34.7 \mathrm{deg} \cdot \mathrm{cm}^{2} \cdot \mathrm{g}^{-1}\left(\mathrm{CHCl}_{3}, \mathrm{c} 1.0,297 \mathrm{~K}\right)$. UPC ${ }^{2}$ Chiralpak IG $(150 \times 4.6 \mathrm{~mm}, 3 \mu \mathrm{m})$ at $35^{\circ} \mathrm{C}$, flow $2 \mathrm{~mL} / \mathrm{min}$, isocratic $\mathrm{CO}_{2} / \mathrm{MeOH}$ 90:10, ABRP pressure $2000 \mathrm{psi}, 272 \mathrm{~nm}, \mathrm{t}_{\mathrm{R}}$ (minor) $1.6 ; \mathrm{t}_{\mathrm{R}}$ (major) 1.7 . 
(R)-6,8-Dimethoxy-2,2-bis(methoxymethyl)-4,4-dimethyl-2,3,3a,4-tetrahydro-1Hcyclopenta $[b]$ naphthalene (6l)

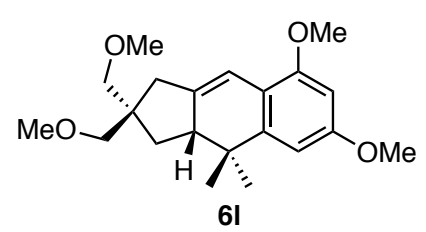

Compound $\mathbf{6 1}$ was synthesized following general procedure $\mathbf{C}$ using 1,6-enyne $\mathbf{5 l}$ (30.1 mg, $0.087 \mathrm{mmol}$ ) whereby the reaction was stirred for $36 \mathrm{~h}$. Purification by flash column chromatography (cyclohexane/EtOAc 20:1) and further purification by preparative TLC (cyclohexane/EtOAc 10:1) afforded title compound $\mathbf{6 1}(27 \mathrm{mg}, 0.078 \mathrm{mmol}, 90 \%)$ as a colorless oil in 93:7 er.

${ }^{1}$ H NMR $\left(400 \mathrm{MHz}, \mathrm{CDCl}_{3}\right) \delta 6.59-6.54(\mathrm{~m}, 1 \mathrm{H}), 6.52(\mathrm{~d}, J=2.2 \mathrm{~Hz}, 1 \mathrm{H}), 6.31(\mathrm{~d}, J=$ $2.3 \mathrm{~Hz}, 1 \mathrm{H}), 3.82(\mathrm{~s}, 3 \mathrm{H}), 3.81(\mathrm{~s}, 3 \mathrm{H}), 3.39-3.35(\mathrm{~m}, 5 \mathrm{H}), 3.31(\mathrm{~s}, 3 \mathrm{H}), 3.26-3.19(\mathrm{~m}, 2 \mathrm{H})$, $2.70-2.60(\mathrm{~m}, 1 \mathrm{H}), 2.54-2.46(\mathrm{~m}, 1 \mathrm{H}), 2.32(\mathrm{dt}, J=17.9,2.9 \mathrm{~Hz}, 1 \mathrm{H}), 1.82(\mathrm{dd}, J=12.5,8.4$ $\mathrm{Hz}, 1 \mathrm{H}), 1.50(\mathrm{t}, J=12.1 \mathrm{~Hz}, 1 \mathrm{H}), 1.34(\mathrm{~s}, 3 \mathrm{H}), 0.88(\mathrm{~s}, 3 \mathrm{H}) .{ }^{13} \mathbf{C ~ N M R}\left(101 \mathrm{MHz}, \mathrm{CDCl}_{3}\right) \delta$ 159.3, 155.6, 147.4, 143.1, 116.9, 112.4, 101.7, 95.5, 75.0, 59.5, 59.4, 55.7, 55.5, 47.8, 46.9, 38.1, 37.4, 31.7, 25.9, 21.4. HRMS (ESI+) calculated for $\left[\mathrm{C}_{21} \mathrm{H}_{30} \mathrm{NaO}_{4}\right]^{+} 369.2036 \mathrm{~m} / z$; found $[\mathrm{M}+\mathrm{Na}]^{+} 369.2027 \mathrm{~m} / z \cdot \boldsymbol{\alpha}_{\mathbf{D}}{ }^{589}=-52.0 \mathrm{deg} \cdot \mathrm{cm}^{2} \cdot \mathrm{g}^{-1}\left(\mathrm{CHCl}_{3}, \mathrm{c} 1.0,297 \mathrm{~K}\right) . \mathbf{U P C}^{2}$ Chiralpak IG $(150 \times 4.6 \mathrm{~mm}, 3 \mu \mathrm{m})$ at $35{ }^{\circ} \mathrm{C}$, flow $2 \mathrm{~mL} / \mathrm{min}$, isocratic $\mathrm{CO}_{2} / \mathrm{MeOH}$ 90:10, ABRP pressure $2000 \mathrm{psi}, 272 \mathrm{~nm}, \mathrm{t}_{\mathrm{R}}$ (minor) 2.8; $\mathrm{t}_{\mathrm{R}}$ (major) 3.0.

\section{(R)-5,7-Dibromo-2,2-bis(methoxymethyl)-4,4-dimethyl-2,3,3a,4-tetrahydro-1 $H$ -} cyclopenta $[b]$ naphthalene $(6 \mathrm{~m})$

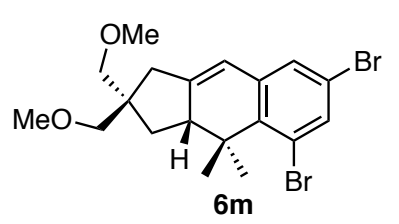

Compound $\mathbf{6 m}$ was synthesized following general procedure $\mathbf{C}$ using 1,6-enyne $\mathbf{5 m}$ (38.6 $\mathrm{mg}, 0.087 \mathrm{mmol}$ ) whereby the reaction was stirred for $16 \mathrm{~h}$. The crude was purified by flash column chromatography (cyclohexane/EtOAc 100:1, then 50:1) to afford title compound $\mathbf{6 m}$ (35 mg, $0.08 \mathrm{mmol}, 91 \%$ ) as a colorless oil in 95:5 er.

${ }^{1} \mathbf{H}$ NMR $\left(400 \mathrm{MHz}, \mathrm{CDCl}_{3}\right) \delta 7.50(\mathrm{~d}, J=2.2 \mathrm{~Hz}, 1 \mathrm{H}), 7.03(\mathrm{~d}, J=2.2 \mathrm{~Hz}, 1 \mathrm{H}), 6.14-6.10$ $(\mathrm{m}, 1 \mathrm{H}), 3.37(\mathrm{~s}, 3 \mathrm{H}), 3.34(\mathrm{~s}, 2 \mathrm{H}), 3.33(\mathrm{~s}, 3 \mathrm{H}), 3.27-3.21(\mathrm{~m}, 2 \mathrm{H}), 2.85-2.75(\mathrm{~m}, 1 \mathrm{H}), 2.55$ $-2.46(\mathrm{~m}, 1 \mathrm{H}), 2.35$ (dt, $J=18.3,2.9 \mathrm{~Hz}, 1 \mathrm{H}), 1.86(\mathrm{dd}, J=12.6,8.3 \mathrm{~Hz}, 1 \mathrm{H}), 1.73(\mathrm{~s}, 3 \mathrm{H})$, $1.64-1.55(\mathrm{~m}, 1 \mathrm{H}), 1.03(\mathrm{~s}, 3 \mathrm{H}) .{ }^{13} \mathbf{C} \mathbf{N M R}\left(101 \mathrm{MHz}, \mathrm{CDCl}_{3}\right) \delta 148.2,141.3,139.6,135.4$, 
$128.7,122.2,120.0,118.5,75.2,59.5,49.8,46.5,40.0,38.0,32.4,27.4,18.6$. HRMS (ESI+) calculated for $\left[\mathrm{C}_{19} \mathrm{H}_{24} \mathrm{Br}_{2} \mathrm{NaO}_{2}\right]^{+} 465.0035 \mathrm{~m} / z$; found $[\mathrm{M}+\mathrm{Na}]^{+} 465.0012 \mathrm{~m} / z$. $\boldsymbol{\alpha}_{\mathbf{D}}{ }^{\mathbf{5 8 9}}=-10.7$ deg. $\mathrm{cm}^{2} \cdot \mathrm{g}^{-1}\left(\mathrm{CHCl}_{3}, \mathrm{c} 1.0,300 \mathrm{~K}\right)$. UPC ${ }^{2}$ Chiralpak IG $(150 \times 4.6 \mathrm{~mm}, 3 \mu \mathrm{m})$ at $35^{\circ} \mathrm{C}$, flow 2 $\mathrm{mL} / \mathrm{min}$, isocratic $\mathrm{CO}_{2} / \mathrm{MeOH}$ 90:10, ABRP pressure $2000 \mathrm{psi}, 278 \mathrm{~nm}, \mathrm{t}_{\mathrm{R}}$ (major) 4.2; $\mathrm{t}_{\mathrm{R}}$ (minor) 4.7.

\section{(R)-5,6,7-Trimethoxy-2,2-bis(methoxymethyl)-4,4-dimethyl-2,3,3a,4-tetrahydro-1 $H$ -} cyclopenta $[b]$ naphthalene (6n)

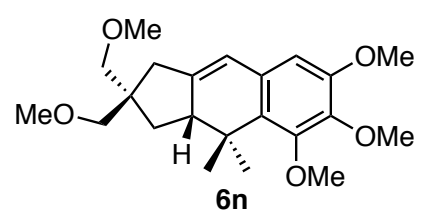

Compound 6n was synthesized following general procedure $\mathbf{C}$ using 1,6-enyne 5n (32.8 mg, $0.087 \mathrm{mmol}$ ) whereby the reaction was stirred for $18 \mathrm{~h}$. Purification by flash column chromatography (cyclohexane/EtOAc 20:1) and further purification by preparative TLC (cyclohexane/EtOAc 10:1) afforded title compound 6n (28 $\mathrm{mg}, 0.074 \mathrm{mmol}, 85 \%$ ) as a colorless oil in 96:4 er.

${ }^{1}$ H NMR $\left(500 \mathrm{MHz}, \mathrm{CDCl}_{3}\right) \delta 6.36(\mathrm{~s}, 1 \mathrm{H}), 6.14(\mathrm{q}, J=2.2 \mathrm{~Hz}, 1 \mathrm{H}), 3.87(\mathrm{~s}, 3 \mathrm{H}), 3.83(\mathrm{~s}$, $3 \mathrm{H}), 3.83(\mathrm{~s}, 3 \mathrm{H}), 3.37(\mathrm{~s}, 3 \mathrm{H}), 3.35(\mathrm{~d}, J=1.6 \mathrm{~Hz}, 2 \mathrm{H}), 3.32(\mathrm{~s}, 3 \mathrm{H}), 3.27-3.21(\mathrm{~m}, 2 \mathrm{H}), 2.76$ $-2.69(\mathrm{~m}, 1 \mathrm{H}), 2.50-2.43(\mathrm{~m}, 1 \mathrm{H}), 2.31$ (dt, $J=18.0,2.8 \mathrm{~Hz}, 1 \mathrm{H}), 1.86(\mathrm{dd}, J=12.5,8.3 \mathrm{~Hz}$, $1 \mathrm{H}), 1.57(\mathrm{~s}, 3 \mathrm{H}), 1.52(\mathrm{t}, J=12.2 \mathrm{~Hz}, 1 \mathrm{H}), 0.95(\mathrm{~s}, 3 \mathrm{H}) .{ }^{13} \mathbf{C ~ N M R}\left(126 \mathrm{MHz}, \mathrm{CDCl}_{3}\right) \delta 153.2$, $151.5,145.7,141.4,131.6,129.5,119.1,106.0,75.2$, 60.9, 60.7, 59.5, 56.0, 49.7, 46.5, 37.90, 37.87, 31.9, 27.4, 20.9. HRMS (ESI+) calculated for $\left[\mathrm{C}_{22} \mathrm{H}_{32} \mathrm{NaO}_{5}\right]^{+} 399.2142 \mathrm{~m} / \mathrm{z}$; found [M $+\mathrm{Na}]^{+} 399.2135 \mathrm{~m} / \mathrm{z} \cdot \boldsymbol{\alpha}_{\mathbf{D}}{ }^{\mathbf{5 8 9}}=-25.6 \mathrm{deg} \cdot \mathrm{cm}^{2} \cdot \mathrm{g}^{-1}\left(\mathrm{CHCl}_{3}, \mathrm{c} 1.0,300 \mathrm{~K}\right)$. UPC ${ }^{2}$ Chiralpak OJ-3 $(150 \times 4.6 \mathrm{~mm}, 3 \mu \mathrm{m})$ at $35^{\circ} \mathrm{C}$, flow $2 \mathrm{~mL} / \mathrm{min}$, isocratic $\mathrm{CO}_{2} / i \mathrm{PrOH} 90: 10$, ABRP pressure $2000 \mathrm{psi}, 225 \mathrm{~nm}, \mathrm{t}_{\mathrm{R}}$ (major) 1.7 ; $\mathrm{t}_{\mathrm{R}}$ (minor) 1.9 .

(R)-11,11-Bis(methoxymethyl)-9,9-dimethyl-9a,10,11,12-tetrahydro-9Hcyclopenta $[b]$ triphenylene $(60)$

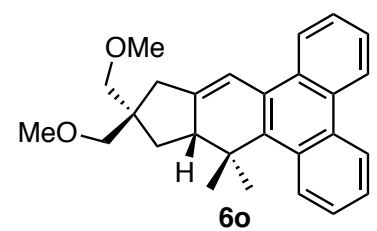

Compound $\mathbf{6 0}$ was synthesized following general procedure $\mathbf{C}$ using 1,6-enyne $\mathbf{5 0}$ (33.6 mg, $0.087 \mathrm{mmol}$ ) whereby the reaction was stirred for $12 \mathrm{~h}$. The crude was purified by flash column 
chromatography (pentane/EtOAc 50:1, then 10:1) to afford title compound 60 (33 mg, 0.087 mmol, 98\%) as a pale-yellow foam in 93:7 er.

M.p. $=112-115^{\circ} \mathrm{C}$ (benzene). ${ }^{1} \mathbf{H}$ NMR $\left(300 \mathrm{MHz}, \mathrm{C}_{6} \mathrm{D}_{6}\right) \delta 8.63-8.55(\mathrm{~m}, 1 \mathrm{H}), 8.55-$ $8.48(\mathrm{~m}, 1 \mathrm{H}), 8.43-8.34(\mathrm{~m}, 1 \mathrm{H}), 8.14-8.05(\mathrm{~m}, 1 \mathrm{H}), 7.48-7.36(\mathrm{~m}, 4 \mathrm{H}), 7.00-6.94(\mathrm{~m}$, $1 \mathrm{H}), 3.49-3.39(\mathrm{~m}, 2 \mathrm{H}), 3.26-3.16(\mathrm{~m}, 5 \mathrm{H}), 3.11(\mathrm{~s}, 3 \mathrm{H}), 2.86-2.74(\mathrm{~m}, 2 \mathrm{H}), 2.61(\mathrm{dt}, J=$ 18.0, 3.0 Hz, 1H), 1.99 (dd, $J=12.3,8.7 \mathrm{~Hz}, 1 \mathrm{H}), 1.89-1.78(\mathrm{~m}, 1 \mathrm{H}), 1.65$ (s, 3H), 1.35 (s, $3 \mathrm{H}) .{ }^{13} \mathrm{C}$ NMR $\left(101 \mathrm{MHz}, \mathrm{C}_{6} \mathrm{D}_{6}\right) \delta 146.2,138.5,131.5,131.3,130.8,130.5,130.3,126.84$, 126.76, 126.2, 125.4, 125.1, 125.0, 124.1, 123.0, 115.8, 77.3, 75.2, 59.1, 59.0, 51.6, 47.2, 39.6, 38.7, 32.3, 30.1, 18.2. HRMS (ESI+) calculated for $\left[\mathrm{C}_{27} \mathrm{H}_{30} \mathrm{NaO}_{2}\right]^{+} 409.2138 \mathrm{~m} / \mathrm{z}$; found $[\mathrm{M}+$ $\mathrm{Na}]^{+} 409.2136 \mathrm{~m} / z \cdot \boldsymbol{\alpha}_{\mathbf{D}}{ }^{\mathbf{5 8 9}}=+139.6 \mathrm{deg} \cdot \mathrm{cm}^{2} \cdot \mathrm{g}^{-1}\left(\mathrm{CHCl}_{3}, \mathrm{c} 1.0,300 \mathrm{~K}\right)$. UPC ${ }^{2}$ Chiralpak IG (150 $\times 4.6 \mathrm{~mm}, 3 \mu \mathrm{m}$ ) at $35^{\circ} \mathrm{C}$, flow $2 \mathrm{~mL} / \mathrm{min}$, isocratic $\mathrm{CO}_{2} / \mathrm{EtOH} 80: 20$, ABRP pressure 2000 psi, $326 \mathrm{~nm}, \mathrm{t}_{\mathrm{R}}$ (major) $4.7 ; \mathrm{t}_{\mathrm{R}}$ (minor) 6.1 .

(3a $R, 4 S)-2,2-B i s(m e t h o x y m e t h y l)-4-m e t h y l-4-(4-m e t h y l p e n t-3-e n-1-y l)-2,3,3 a, 4-$ tetrahydro- $1 H$-cyclopenta $[b]$ naphthalene $(6 p)$

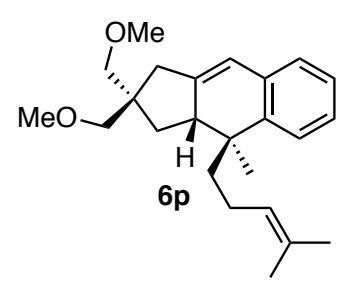

Compound 6p was synthesized following general procedure $\mathbf{C}$ using 1,6-enyne 5p (31 mg, $0.087 \mathrm{mmol}$ ) whereby the reaction was stirred for $7 \mathrm{~d}$. Purification by flash column chromatography (toluene/ $\mathrm{CH}_{2} \mathrm{Cl}_{2} 30: 1$ ) afforded title compound $\mathbf{6 p}$ (23 mg, $0.065 \mathrm{mmol}, 77 \%$ ) as a colorless oil in 93:7 er.

${ }^{1} \mathbf{H}$ NMR $\left(500 \mathrm{MHz}, \mathrm{CD}_{2} \mathrm{Cl}_{2}\right) \delta 7.22-7.19(\mathrm{~m}, 1 \mathrm{H}), 7.15-7.08(\mathrm{~m}, 2 \mathrm{H}), 6.99(\mathrm{dd}, J=7.1$, $1.7 \mathrm{~Hz}, 1 \mathrm{H}), 6.26-6.21(\mathrm{~m}, 1 \mathrm{H}), 5.24-5.18(\mathrm{~m}, 1 \mathrm{H}), 3.34(\mathrm{~s}, 5 \mathrm{H}), 3.30(\mathrm{~s}, 3 \mathrm{H}), 3.23(\mathrm{~s}, 2 \mathrm{H})$, $3.01-2.92(\mathrm{~m}, 1 \mathrm{H}), 2.51-2.45(\mathrm{~m}, 1 \mathrm{H}), 2.33(\mathrm{dt}, J=18.0,2.8 \mathrm{~Hz}, 1 \mathrm{H}), 2.05-1.92(\mathrm{~m}, 3 \mathrm{H})$, $1.79(\mathrm{dd}, J=12.5,8.3 \mathrm{~Hz}, 1 \mathrm{H}), 1.72(\mathrm{~s}, 3 \mathrm{H}), 1.60(\mathrm{~s}, 3 \mathrm{H}), 1.59-1.55(\mathrm{~m}, 1 \mathrm{H}), 1.53(\mathrm{t}, J=12.3$ $\mathrm{Hz}, 1 \mathrm{H}), 0.87(\mathrm{~s}, 3 \mathrm{H}) .{ }^{13} \mathrm{C}$ NMR $\left(126 \mathrm{MHz}, \mathrm{CD}_{2} \mathrm{Cl}_{2}\right) \delta 147.7,142.5,136.1,131.8,126.9,126.8$, 126.5, 125.3, 124.6, 118.7, 77.8, 75.7, 59.61, 59.58, 47.2, 43.9, 40.9, 38.3, 37.2, 31.5, 26.0, 23.9, 23.3, 17.9. HRMS (ESI+) calculated for $\left[\mathrm{C}_{24} \mathrm{H}_{34} \mathrm{NaO}_{2}\right]^{+} 377.2451 \mathrm{~m} / z$; found $[\mathrm{M}+\mathrm{Na}]^{+}$ $377.2451 \mathrm{~m} / z . \boldsymbol{\alpha}_{\mathbf{D}}{ }^{589}=-15.5 \mathrm{deg} \cdot \mathrm{cm}^{2} \cdot \mathrm{g}^{-1}\left(\mathrm{CHCl}_{3}, \mathrm{c} 1.0,297 \mathrm{~K}\right) . \mathbf{U P C}{ }^{2}$ Chiralpak IG $(150 \times$ $4.6 \mathrm{~mm}, 3 \mu \mathrm{m})$ at $35^{\circ} \mathrm{C}$, flow $2 \mathrm{~mL} / \mathrm{min}$, isocratic $\mathrm{CO}_{2} / \mathrm{PrOH}$ 95:5, ABRP pressure 2000 psi, $268 \mathrm{~nm}, \mathrm{t}_{\mathrm{R}}$ (minor) $2.3 ; \mathrm{t}_{\mathrm{R}}$ (major) 2.8 . 
(3aR,4R)-2,2-Bis(methoxymethyl)-4-methyl-4-phenyl-2,3,3a,4-tetrahydro-1Hcyclopenta[b]naphthalene (6q)

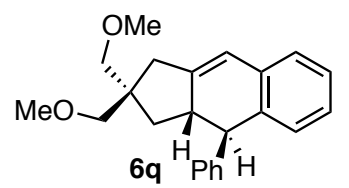

Compound 6q was synthesized following general procedure $\mathbf{C}$ using 1,6-enyne 5q (29.1 mg, $0.087 \mathrm{mmol}$ ) whereby the reaction was stirred for $14 \mathrm{~d}$. Purification by preparative TLC (toluene $\left./ \mathrm{CH}_{2} \mathrm{Cl}_{2} 4: 1\right)$ afforded title compound $\mathbf{6 q}(17.6 \mathrm{mg}, 0.052 \mathrm{mmol}, 61 \%, 91 \% \mathrm{brsm}$ ) as a colorless oil in 81:19 er.

${ }^{1} \mathbf{H}$ NMR $\left(400 \mathrm{MHz}, \mathrm{CD}_{2} \mathrm{Cl}_{2}\right) \delta 7.42-7.35(\mathrm{~m}, 2 \mathrm{H}), 7.34-7.25(\mathrm{~m}, 3 \mathrm{H}), 7.13-7.07(\mathrm{~m}$, 1H), $7.07-7.01(\mathrm{~m}, J=7.4 \mathrm{~Hz}, 1 \mathrm{H}), 6.94$ (t, $J=7.4 \mathrm{~Hz}, 1 \mathrm{H}), 6.52(\mathrm{~d}, J=7.6 \mathrm{~Hz}, 1 \mathrm{H}), 6.38-$ $6.31(\mathrm{~m}, 1 \mathrm{H}), 3.71(\mathrm{~d}, J=15.1 \mathrm{~Hz}, 1 \mathrm{H}), 3.31(\mathrm{~s}, 3 \mathrm{H}), 3.29$ (d, $J=2.3 \mathrm{~Hz}, 2 \mathrm{H}), 3.26(\mathrm{~s}, 3 \mathrm{H})$, $3.19(\mathrm{~s}, 2 \mathrm{H}), 3.17-3.04(\mathrm{~m}, 1 \mathrm{H}), 2.50(\mathrm{~s}, 2 \mathrm{H}), 1.63(\mathrm{dd}, J=13.0,8.2 \mathrm{~Hz}, 1 \mathrm{H}), 1.34-1.24(\mathrm{~m}$, 1H). ${ }^{13} \mathrm{C}$ NMR (101 MHz, $\left.\mathrm{CD}_{2} \mathrm{Cl}_{2}\right) \delta 149.2,143.3,138.9,136.5,130.0,129.1,127.4,127.3$, $127.0,126.5,125.7,119.5,77.6,76.5,59.6,53.6,47.6,45.6,38.4,37.7$. HRMS (ESI+) calculated for $\left[\mathrm{C}_{23} \mathrm{H}_{26} \mathrm{NaO}_{2}\right]^{+} 357.1825 \mathrm{~m} / z$; found $[\mathrm{M}+\mathrm{Na}]^{+} 357.1821 \mathrm{~m} / z$. $\boldsymbol{\alpha}_{\mathbf{D}}{ }^{\mathbf{5 8 9}}=-15.9$ deg. $\mathrm{cm}^{2} \cdot \mathrm{g}^{-1}\left(\mathrm{CHCl}_{3}, \mathrm{c} 1.0,300 \mathrm{~K}\right)$. UPC ${ }^{2}$ Chiralpak IG $(150 \times 4.6 \mathrm{~mm}, 3 \mu \mathrm{m})$ at $35^{\circ} \mathrm{C}$, flow 2 $\mathrm{mL} / \mathrm{min}$, isocratic $\mathrm{CO}_{2} / \mathrm{iPrOH}$ 76:24, ABRP pressure $2000 \mathrm{psi}, 267 \mathrm{~nm}, \mathrm{t}_{\mathrm{R}}$ (major) 3.9; $\mathrm{t}_{\mathrm{R}}$ (minor) 4.2 .

\section{Dimethyl (S)-7,7-dimethyl-1,6,7,7a-tetrahydro-2H-indene-2,2-dicarboxylate (16)}

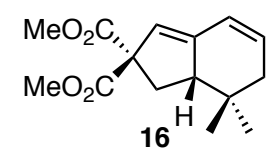

Compound 16 was synthesized following general procedure $\mathbf{C}$ using 1,6-enyne $\mathbf{1 5}^{7}$ (18.1 $\mathrm{mg}, 0.068 \mathrm{mmol}$ ) whereby the reaction was stirred for $48 \mathrm{~h}$. Purification by preparative TLC (cyclohexane/ $\mathrm{CH}_{2} \mathrm{Cl}_{2}$ 1:1) afforded the title compound 16 (10.0 mg, $\left.0.038 \mathrm{mmol}, 55 \%\right)$ as a colorless oil in 67:33 er.

$\boldsymbol{\alpha}_{\mathbf{D}}{ }^{589}=-54.6 \mathrm{deg} \cdot \mathrm{cm}^{2} \cdot \mathrm{g}^{-1}\left(\mathrm{CHCl}_{3}, \mathrm{c} 0.28,300 \mathrm{~K}\right) . \mathbf{U P C}{ }^{2}$ Chiralpak IG $(150 \times 4.6 \mathrm{~mm}, 3 \mu \mathrm{m})$ at $35{ }^{\circ} \mathrm{C}$, flow $2 \mathrm{~mL} / \mathrm{min}$, isocratic $\mathrm{CO}_{2} / \mathrm{MeOH}$ 95:5, ABRP pressure $2000 \mathrm{psi}, 243 \mathrm{~nm}$, $\mathrm{t}_{\mathrm{R}}$ (major) $1.7 ; \mathrm{t}_{\mathrm{R}}$ (minor) 1.8. The spectral data of $\mathbf{1 6}$ were fully consistent with those previously reported. $^{7}$ 


\section{Synthesis of azabycyclo[4.1.0]hept-4-enes}

\subsection{Reaction optimization}

\section{General procedure D: enantioselective cyclopropanation of 1,6-enynes 7}

1,6 enyne 7a (1.0 equiv) and catalyst $(R, R)-\mathbf{D}$ (mol \% see tables) were dissolved in the respective solvent. A solution of the silver salt (mol \% see tables) in the same solvent (total concentration $0.05 \mathrm{M}$ ) was added dropwise and the reaction was stirred for the given time at the given temperature in the dark. The reaction was quenched by addition of 3 drops of $\mathrm{NEt}_{3}$ and concentrated. The crude was purified by flash column chromatography to yield $\mathbf{8 a}$.

Table 5: Screening of reaction conditions

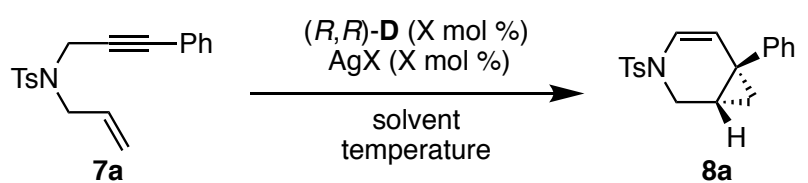

\begin{tabular}{ccccccc}
\hline entry & $\begin{array}{c}(\boldsymbol{R}, \boldsymbol{R})-\mathbf{D} \\
(\mathbf{m o l} \%)\end{array}$ & $\mathbf{A g X}(\mathbf{m o l} \%)$ & solvent & $\begin{array}{c}\text { temperature } \\
\left({ }^{\mathbf{0}} \mathbf{C}\right)\end{array}$ & yield (\%) $^{\mathbf{a}}$ & $\boldsymbol{e r}$ \\
\hline $\mathbf{1}$ & 4 & $\mathrm{AgBF}_{4}(4)$ & $\alpha, \alpha, \alpha$-trifluorotoluene & 24 & - & - \\
$\mathbf{2}$ & 4 & $\mathrm{AgBF}_{4}(4)$ & chlorobenzene & 24 & $21^{\mathrm{b}}$ & $93: 7$ \\
$\mathbf{3}$ & 4 & $\mathrm{AgBF}_{4}(4)$ & toluene & 40 & 35 & $95: 5$ \\
& & & & & $(49 \mathrm{brsm})$ & \\
$\mathbf{4}$ & 8 & $\mathrm{AgBF}_{4}(8)$ & toluene & 24 & $51^{\mathrm{c}}$ & $94: 6$ \\
$\mathbf{5}$ & 4 & $\mathrm{AgBF}_{4}(4)$ & 1,2-dichloroethane & 24 & $-{ }^{\mathrm{d}}$ & $85: 15$ \\
$\mathbf{6}$ & 4 & $\mathrm{AgPF}_{6}(4)$ & 1,2-dichloroethane & 24 & $-{ }^{\mathrm{e}}$ & $87: 13$ \\
\hline
\end{tabular}

a Isolated yields. ${ }^{\mathrm{b}} 34 \%$ conversion. $^{\mathrm{c}} 66 \%$ conversion. ${ }^{\mathrm{d}}$ After $43 \mathrm{~h}$ reaction time the SM/product ratio, was estimated by ${ }^{1} \mathrm{H}$ NMR to be $1: 1.2$. e After $43 \mathrm{~h}$ reaction time the SM/product ratio, was estimated by ${ }^{1} \mathrm{H}$ NMR to be 3.6:1.

\subsection{Scope of the cyclopropanation reaction}

\section{General procedure E: enantioselective cyclopropanation of 1,6-enynes 7}

1,6-Enyne 7 (1.0 equiv) and catalyst $(R, R)$-D ( $4 \mathrm{~mol} \%)$ were dissolved in toluene. A solution of $\mathrm{AgBF}_{4}(4 \mathrm{~mol} \%)$ in toluene (total concentration $0.05 \mathrm{M}$ ) was added dropwise and the reaction was stirred for the given time at $40^{\circ} \mathrm{C}$ in the dark. The reaction was quenched by addition of 3 drops of $\mathrm{NEt}_{3}$ and concentrated. The crude was purified by flash column chromatography to afford the title compounds. 
$(1 S, 6 S)-6-P h e n y l-3-t o s y l-3-a z a b i c y c l o[4.1 .0] h e p t-4-e n e ~(8 a)$

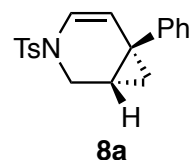

Compound 8a was synthesized following general procedure $\mathbf{E}$ using $N$-allyl-4-methyl- $N$-(3phenylprop-2-yn-1-yl)benzenesulfonamide $(7 \mathbf{a})^{9}(20 \mathrm{mg}, 0.061 \mathrm{mmol}, 1.0$ equiv) whereas the reaction was stirred for $3 \mathrm{~d}$. The crude was purified by preparative TLC (toluene/ $\mathrm{CH}_{2} \mathrm{Cl}_{2}$ 1:1) to afford the product 8a as a white solid ( $7 \mathrm{mg}, 0.022 \mathrm{mmol}, 35 \%, 49 \% \mathrm{brsm}$ ) in 95:5 er.

M.p. $=107-109{ }^{\circ} \mathrm{C}\left(\mathrm{CHCl}_{3}\right) \cdot \boldsymbol{\alpha}_{\mathbf{D}}{ }^{\mathbf{5 8 9}}=-64.3$ deg.cm ${ }^{2} \cdot \mathrm{g}^{-1}\left(\mathrm{CHCl}_{3}\right.$, c $\left.0.32,302 \mathrm{~K}\right)$. HPLC Chiralpak IA $(250 \mathrm{~mm} \times 4.6 \mathrm{~mm}, 5 \mu \mathrm{m})$ at $25^{\circ} \mathrm{C}$, flow $0.9 \mathrm{~mL} / \mathrm{min}$, isocratic hexane $/ \mathrm{EtOH}$ 90:10, $280 \mathrm{~nm}, t_{R}$ (minor) 13.3; $t_{R}$ (major) 16.5. The spectral data of $8 \mathbf{a}$ were fully consistent with those previously reported. ${ }^{10}$

(1S,6S)-1-Methyl-6-phenyl-3-tosyl-3-azabicyclo[4.1.0]hept-4-ene (8b)

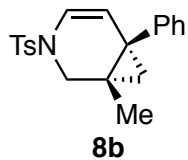

Compound $\mathbf{8 b}$ was synthesized following general procedure $\mathbf{E}$ using 4-methyl- $N$-(2-methylallyl)- $N$-(3-phenylprop-2-yn-1-yl)benzenesulfonamide (7b) ${ }^{11}$ (32 mg, 0.094 mmol, 1.0 equiv) whereas the reaction was stirred for $24 \mathrm{~h}$. The crude was purified by flash column chromatography (cyclohexene/EtOAc 30:1) to afford the product $\mathbf{8 b}$ as a white solid (28.4 $\mathrm{mg}, 0.083$ mmol, 89\%) in 90:10 er.

M.p. $=131-133{ }^{\circ} \mathrm{C}\left(\mathrm{CHCl}_{3}\right) . \boldsymbol{\alpha}_{\mathbf{D}}{ }^{\mathbf{5 8 9}}=-76.7 \mathrm{deg} \cdot \mathrm{cm}^{2} \cdot \mathrm{g}^{-1}\left(\mathrm{CHCl}_{3}\right.$, c $\left.1.0,301 \mathrm{~K}\right)$. HPLC Chiralpak IA $(250 \mathrm{~mm} \times 4.6 \mathrm{~mm}, 5 \mu \mathrm{m})$ at $25^{\circ} \mathrm{C}$, flow $1.0 \mathrm{~mL} / \mathrm{min}$, isocratic hexane $/ \mathrm{PrOH}$ 95:5, $254 \mathrm{~nm}, \mathrm{t}_{\mathrm{R}}$ (minor) 9.8; $\mathrm{t}_{\mathrm{R}}$ (major) 10.8. The spectral data of $\mathbf{8} \mathbf{b}$ were fully consistent with those previously reported. ${ }^{11}$

(1S,6S)-6-(4-Methoxyphenyl)-3-tosyl-3-azabicyclo[4.1.0]hept-4-ene (8c)

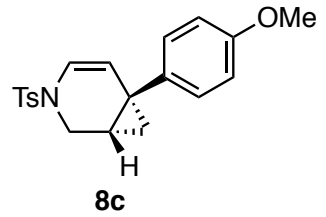

Compound 8c was synthesized following general procedure $\mathbf{E}$ using $\mathrm{N}$-allyl- $\mathrm{N}$-(3-(4methoxyphenyl)prop-2-yn-1-yl)-4-methylbenzenesulfonamide (7c) ${ }^{9}$ (33.4 mg, 0.094 mmol, 1.0 equiv) whereas the reaction was stirred for $16 \mathrm{~h}$ The crude was purified by flash column 
chromatography (cyclohexane/EtOAc 10:1) to afford the product $8 \mathrm{c}$ as a white solid $(24.7 \mathrm{mg}$, $0.069 \mathrm{mmol}, 74 \%$ ) in 94:6 er.

$$
\text { M.p. }=111-113{ }^{\circ} \mathrm{C}\left(\mathrm{CHCl}_{3}\right) \cdot \boldsymbol{\alpha}_{\mathbf{D}}{ }^{\mathbf{5 8 9}}=-76.6 \mathrm{deg} \cdot \mathrm{cm}^{2} \cdot \mathrm{g}^{-1}\left(\mathrm{CHCl}_{3}, \text { c } 1.0,302 \mathrm{~K}\right) . \mathbf{H P L C}
$$

Chiralcel OJ-H $(250 \mathrm{~mm} \times 4.6 \mathrm{~mm}, 5 \mu \mathrm{m})$ at $25^{\circ} \mathrm{C}$, flow $1.0 \mathrm{~mL} / \mathrm{min}$, isocratic hexane $/ \mathrm{PrOH}$ 70:30, $280 \mathrm{~nm}, \mathrm{t}_{\mathrm{R}}$ (major) 16.9; $\mathrm{t}_{\mathrm{R}}$ (minor) 20.8. The spectral data of $\mathbf{8 c}$ were fully consistent with those previously reported. ${ }^{10}$

\section{(1S,6S)-1-Methyl-3-(methylsulfonyl)-6-phenyl-3-azabicyclo[4.1.0]hept-4-ene (8d)}

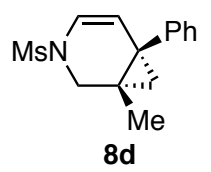

Compound 8d was synthesized following general procedure $\mathbf{E}$ using $N$-(2-methylallyl)- $N$ (3-phenylprop-2-yn-1-yl)methanesulfonamide(7d) ${ }^{11}$ (25 mg, 0.094 mmol, 1.0 equiv) whereas the reaction was stirred for $24 \mathrm{~h}$. The crude was purified by flash column chromatography (cyclohexane/EtOAc 30:1) to afford title compound $\mathbf{8 d}$ as a white solid (21.7 mg, $0.082 \mathrm{mmol}$, $88 \%$ ) in $74: 26$ er.

M.p. $=127-129{ }^{\circ} \mathrm{C}\left(\mathrm{CHCl}_{3}\right) \cdot \boldsymbol{\alpha}_{\mathbf{D}}{ }^{\mathbf{5 8 9}}=-52.32 \mathrm{deg} \cdot \mathrm{cm}^{2} \cdot \mathrm{g}^{-1}\left(\mathrm{CHCl}_{3}\right.$, c 1.0, $\left.301 \mathrm{~K}\right) . \mathbf{H P L C}$ Chiralpak IC $(250 \mathrm{~mm} \times 4.6 \mathrm{~mm}, 5 \mu \mathrm{m})$ at $25^{\circ} \mathrm{C}$, flow $1.0 \mathrm{~mL} / \mathrm{min}$, isocratic hexane $/ i \mathrm{PrOH}$ 80:20, $220 \mathrm{~nm}, \mathrm{t}_{\mathrm{R}}$ (minor) 12.4; $\mathrm{t}_{\mathrm{R}}$ (major) 13.2. The spectral data of $8 \mathbf{d}$ were fully consistent with those previously reported. ${ }^{11}$

\section{Benzyl (1S,6S)-6-phenyl-3-azabicyclo[4.1.0]hept-4-ene-3-carboxylate (8e)}

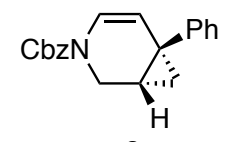

$8 \mathrm{e}$

Compound 8e was synthesized following general procedure $\mathbf{E}$ using benzyl allyl(3-phenylprop-2-yn-1-yl)carbamate $(7 \mathbf{e})^{12}(28.7 \mathrm{mg}, 0.094 \mathrm{mmol}, 1.0$ equiv) whereas the reaction was stirred for $24 \mathrm{~h}$. The crude was purified by flash column chromatography (cyclohexane/EtOAc $20: 1)$ to afford title compound $\mathbf{8 e}(16.1 \mathrm{mg}, 0.052 \mathrm{mmol}, 56 \%)$ as a colorless gum in 91:9 er.

$\boldsymbol{\alpha}_{\mathbf{D}}{ }^{589}=-84.1$ deg. $\mathrm{cm}^{2} \cdot \mathrm{g}^{-1}\left(\mathrm{CHCl}_{3}, \mathrm{c} 0.7,302 \mathrm{~K}\right)$. HPLC Chiralpak IA $(250 \mathrm{~mm} \times 4.6 \mathrm{~mm}$, $5 \mu \mathrm{m}$ ) at $25^{\circ} \mathrm{C}$, flow $1.0 \mathrm{~mL} / \mathrm{min}$, isocratic hexane $/ \mathrm{PrOH} 95: 5,220 \mathrm{~nm}, \mathrm{t}_{\mathrm{R}}$ (minor) 8.5; $\mathrm{t}_{\mathrm{R}}$ (major) 9.5. The spectral data of $\mathbf{8 e}$ were fully consistent with those previously reported. ${ }^{11}$ 


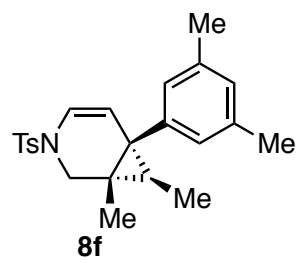

Compound 8f was synthesized following general procedure E using (E)- $N$-(3-(3,5-dimethylphenyl)prop-2-yn-1-yl)-4-methyl- $N$-(2-methylbut-2-en-1-yl)benzenesulfonamide ${ }^{13}$ (32 $\mathrm{mg}, 0.084 \mathrm{mmol}, 1.0$ equiv) whereas the reaction was stirred for $13 \mathrm{~h}$. The crude was purified by preparative TLC (cyclohexane/EtOAc 10:1) to afford the product $\mathbf{8 f}$ as a colorless gum (31 $\mathrm{mg}, 0.081 \mathrm{mmol}, 97 \%$ ) in 97:3 er and 10:1 dr.

$\boldsymbol{\alpha}_{\mathbf{D}}{ }^{\mathbf{5 8 9}}=-85.8 \mathrm{deg} \cdot \mathrm{cm}^{2} \cdot \mathrm{g}^{-1}\left(\mathrm{CHCl}_{3}, \mathrm{c} 1.0,299 \mathrm{~K}\right) . \mathbf{U P C}^{2}$ Chiralpak IC $(100 \times 4.6 \mathrm{~mm}, 3 \mu \mathrm{m})$ at $35{ }^{\circ} \mathrm{C}$, flow $3 \mathrm{~mL} / \mathrm{min}$, isocratic $\mathrm{CO}_{2} / i \mathrm{PrOH} 85: 15$, ABRP pressure $1500 \mathrm{psi}, 220 \mathrm{~nm}$, $\mathrm{t}_{\mathrm{R}}$ (major) 3.9; $\mathrm{t}_{\mathrm{R}}$ (minor) 4.5. The spectral data of $\mathbf{8 f}$ were fully consistent with those previously reported. ${ }^{13}$

(4aS,4bR,7aS)-4a-(3,5-Dimethylphenyl)-2-tosyl-2,4a,4b,5,6,7-hexahydro-1H-cyclopenta$[1,3]$ cyclopropa[1,2-c]pyridine $(8 \mathrm{~g})$

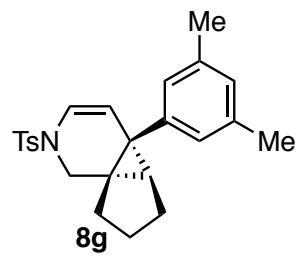

Compound $\mathbf{8 g}$ was synthesized following general procedure $\mathbf{E}$ using $N$-(cyclopent-1-en-1ylmethyl)- $N$-(3-(3,5-dimethylphenyl)prop-2-yn-1-yl)-4-methylbenzenesulfonamide ${ }^{13}$ (33 mg, $0.084 \mathrm{mmol}, 1.0$ equiv) whereas the reaction was stirred for $16 \mathrm{~h}$. The crude was purified by preparative TLC (cyclohexane/ $\mathrm{CH}_{2} \mathrm{Cl}_{2}$ 1:1) to afford the product $8 \mathrm{~g}$ as a colorless gum $(31 \mathrm{mg}$, $0.078 \mathrm{mmol}, 94 \%$ ) in $96: 4 \mathrm{er}$.

${\boldsymbol{\boldsymbol { \alpha } _ { \mathbf { D } }}}^{\mathbf{5 8 9}}=-140.3 \mathrm{deg} \cdot \mathrm{cm}^{2} \cdot \mathrm{g}^{-1}\left(\mathrm{CHCl}_{3}, 1.0 \mathrm{c}, 299 \mathrm{~K}\right)$. HPLC Chiralpak ID $(250 \mathrm{~mm} \times 4.6 \mathrm{~mm}$, $5 \mu \mathrm{m}$ ) at $25^{\circ} \mathrm{C}$, flow $1.0 \mathrm{~mL} / \mathrm{min}$, isocratic hexane/ $\mathrm{PrOH} 95: 5,220 \mathrm{~nm}, \mathrm{t}_{\mathrm{R}}$ (major) $11.5 ; \mathrm{t}_{\mathrm{R}}$ (minor) 12.4. The spectral data of $\mathbf{8 g}$ were fully consistent with those previously reported. ${ }^{13}$ 


\section{Synthesis of 1,2-dihydronaphtalenes}

\subsection{Synthesis of 1,6-enynes 9a-d, 9i-I and 17}

\subsubsection{Synthesis of enynes 9a-d}
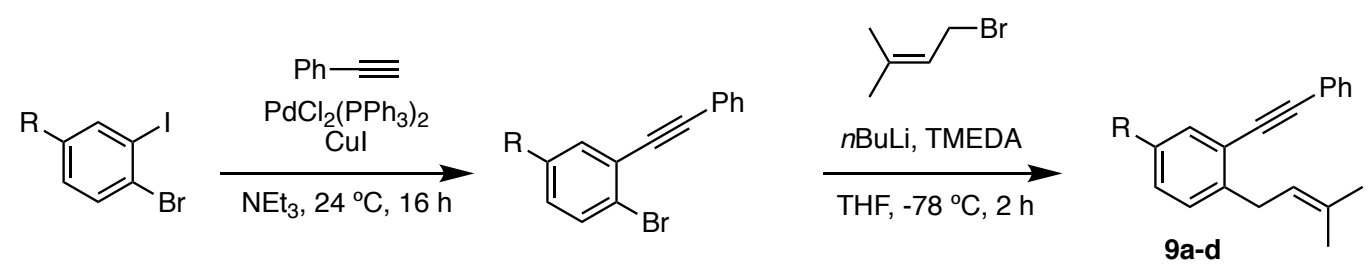

\section{General procedure F: Sonogashira coupling}

To a solution of $\mathrm{Pd}\left(\mathrm{PPh}_{3}\right)_{2} \mathrm{Cl}_{2}$ (2.5 mol \%), copper(I) iodide (5 mol \%) and the corresponding aryl iodide (1.0 equiv) in dry $\mathrm{NEt}_{3}(0.25 \mathrm{M})$ was added ethynylbenzene (1.0 equiv) dropwise at $24{ }^{\circ} \mathrm{C}$ under argon. The reaction was stirred for the given time at the same temperature and quenched with sat. $\mathrm{NH}_{4} \mathrm{Cl}$ solution after full conversion of the starting material. The mixture was extracted with $\mathrm{CH}_{2} \mathrm{Cl}_{2}(3 \mathrm{x})$ and the combined organic phases were washed with brine, dried over $\mathrm{Na}_{2} \mathrm{SO}_{4}$, filtered and concentrated. The crude was purified by flash column chromatography to afford the Sonogashira products.

\section{1-Bromo-2-(phenylethynyl)benzene}

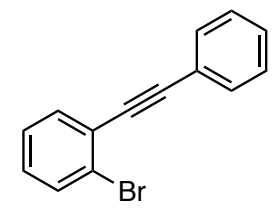

1-Bromo-2-(phenylethynyl)benzene was synthesized following general procedure $\mathbf{F}$ using 1-bromo-2-iodobenzene ( $7 \mathrm{~g}, 24.7 \mathrm{mmol})$ whereby the reaction was stirred for $14 \mathrm{~h}$. Purification by column chromatography (cyclohexane) afforded the title compound (5.91 g, $23.0 \mathrm{mmol}$, 93\%) as a colorless oil.

The spectral data were fully consistent with those previously reported. ${ }^{14}$

\section{1-Bromo-2-(phenylethynyl)-4-(trifluoromethyl)benzene}<smiles>FC(F)(F)c1ccc(Br)c(C#Cc2ccccc2)c1</smiles>

1-Bromo-2-(phenylethynyl)-4-(trifluoromethyl)benzene was synthesized following general procedure $\mathbf{F}$ from 1-bromo-2-iodo-4-(trifluoromethyl)benzene (3.5 g, $10.0 \mathrm{mmol})$ whereby the 
reaction was stirred for $6 \mathrm{~h}$. Purification by column chromatography (cyclohexane) afforded the title compound $(2.70 \mathrm{~g}, 8.4 \mathrm{mmol}, 84 \%)$ as a white solid.

The spectral data were fully consistent with those previously reported..$^{15}$

\section{1-Bromo-4-chloro-2-(phenylethynyl)benzene}

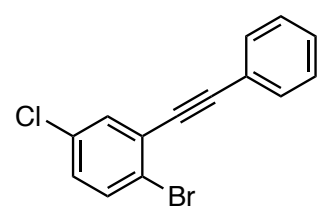

1-Bromo-4-chloro-2-(phenylethynyl)benzene was synthesized following general procedure F from 1-bromo-4-chloro-2-iodobenzene $(1.0 \mathrm{~g}, 3.15 \mathrm{mmol})$ whereby the reaction was stirred for $15 \mathrm{~min}$. Purification by column chromatography (cyclohexane) afforded the title compound (770 mg, $2.64 \mathrm{mmol}, 84 \%$ ) as a white solid.

M.p. $=56-58{ }^{\circ} \mathrm{C}$ (pentane). ${ }^{1} \mathbf{H}$ NMR $\left(500 \mathrm{MHz}, \mathrm{CDCl}_{3}\right) \delta 7.60-7.56(\mathrm{~m}, 2 \mathrm{H}), 7.54(\mathrm{~d}, J$ $=2.5 \mathrm{~Hz}, 1 \mathrm{H}), 7.53(\mathrm{~d}, J=8.6 \mathrm{~Hz}, 1 \mathrm{H}), 7.40-7.36(\mathrm{~m}, 3 \mathrm{H}), 7.16(\mathrm{dd}, J=8.6,2.6 \mathrm{~Hz}, 1 \mathrm{H}) .{ }^{13} \mathbf{C}$ NMR $\left(126 \mathrm{MHz}, \mathrm{CDCl}_{3}\right) \delta 133.6,133.2,132.9,131.9,129.6,129.2,128.6,127.1,123.7,122.6$, 95.3, 87.0. HRMS (APCI+) calculated for $\left[\mathrm{C}_{14} \mathrm{H}_{9} \mathrm{BrCl}\right]^{+} 290.9571 \mathrm{~m} / \mathrm{z}$; found $[\mathrm{M}+\mathrm{H}]^{+}$ $290.9569 \mathrm{~m} / \mathrm{z}$. The compound is known in literature, ${ }^{16}$ but spectral data were not fully reported previously.

\section{1-Bromo-4-methoxy-2-(phenylethynyl)benzene}

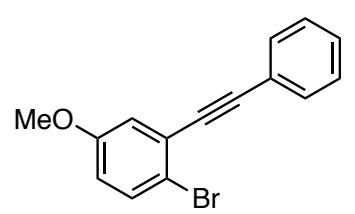

1-Bromo-4-methoxy-2-(phenylethynyl)benzene was synthesized following general procedure $\mathbf{F}$ from 1-bromo-2-iodo-4-methoxybenzene (1.0 g, $3.20 \mathrm{mmol})$ whereby the reaction was stirred for $15 \mathrm{~min}$. Purification by column chromatography (pentane) afforded the title compound (832 mg, $2.90 \mathrm{mmol}, 91 \%$ ) as a colorless oil.

The spectral data were fully consistent with those previously reported. ${ }^{15}$

\section{General procedure G: prenylation reaction}

A solution of the corresponding alkyne (1.0 equiv) in dry THF $(0.2 \mathrm{M})$ was treated with $n \operatorname{BuLi}\left(1.5\right.$ equiv) at $-78^{\circ} \mathrm{C}$ under argon atmosphere and the resulting mixture was stirred for $5 \mathrm{~min}$. TMEDA (1.5 equiv) was added and the mixture was stirred for additional $10 \mathrm{~min}$ before 
adding 1-bromo-3-methylbut-2-ene (1.0 equiv) dropwise. The resulting solution was allowed to reach $24{ }^{\circ} \mathrm{C}$, quenched with sat. $\mathrm{NH}_{4} \mathrm{Cl}$ and extracted with $\mathrm{Et}_{2} \mathrm{O}$. The combined organic phases were washed with brine, dried over $\mathrm{Na}_{2} \mathrm{SO}_{4}$ and concentrated. The crude product was purified by flash column chromatography to afford the corresponding 1,6-enynes.

\section{1-(3-Methylbut-2-en-1-yl)-2-(phenylethynyl)benzene (9a)}

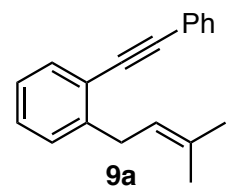

1,6-Enyne 9a was synthesized following general procedure $\mathbf{G}$ from 1-bromo-2-(phenylethynyl)benzene $(2.3 \mathrm{~g}, 8.9 \mathrm{mmol})$ whereby the reaction was stirred for $1 \mathrm{~h}$. Purification by column chromatography (cyclohexane) afforded compound 9a (1.78 g, $7.21 \mathrm{mmol}, 81 \%)$ as a colorless oil.

The spectral data of 9a were fully consistent with those previously reported. ${ }^{17}$

\section{1-(3-Methylbut-2-en-1-yl)-2-(phenylethynyl)-4-(trifluoromethyl)benzene (9b)}

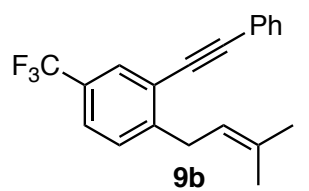

1,6-Enyne 9b was synthesized following general procedure $\mathbf{G}$ from 1-bromo-2-(phenylethynyl)-4-(trifluoromethyl)benzene $(300 \mathrm{mg}, 0.92 \mathrm{mmol})$ whereby the reaction was stirred for 2 h. Purification by column chromatography (cyclohexane) afforded title compound 9b (194 $\mathrm{mg}, 0.62 \mathrm{mmol}, 67 \%$ ) as a colorless oil.

${ }^{1} \mathbf{H}$ NMR $\left(400 \mathrm{MHz}, \mathrm{CDCl}_{3}\right) \delta 7.79(\mathrm{~d}, J=2.0 \mathrm{~Hz}, 1 \mathrm{H}), 7.60-7.54(\mathrm{~m}, 2 \mathrm{H}), 7.51(\mathrm{dd}, J=$ 8.1, 1.4 Hz, 1H), $7.42-7.33(\mathrm{~m}, 4 \mathrm{H}), 5.42-5.36(\mathrm{~m}, 1 \mathrm{H}), 3.66(\mathrm{~d}, J=7.3 \mathrm{~Hz}, 2 \mathrm{H}), 1.81-1.78$ (m, 3H), 1.77 (s, 3H). ${ }^{13} \mathbf{C}$ NMR $\left(101 \mathrm{MHz}, \mathrm{CDCl}_{3}\right) \delta 147.7,134.1,131.7,129.1$ (q, $J=3.9$ Hz), 129.0, 128.8, 128.6, 128.5 (q, $J=32.4 \mathrm{~Hz}), 125.0$ (q, $J=3.7 \mathrm{~Hz}), 124.1$ (q, $J=272.7 \mathrm{~Hz})$, 123.5, 123.1, 121.4, 94.8, 87.0, 33.2, 25.9, 18.1. ${ }^{\mathbf{1 9}} \mathbf{F}\left\{{ }^{\mathbf{1}} \mathbf{H}\right\}$ NMR $\left(376 \mathrm{MHz}, \mathrm{CDCl}_{3}\right) \delta-62.6$. HRMS (APCI + ) calculated for $\left[\mathrm{C}_{20} \mathrm{H}_{18} \mathrm{~F}_{3}\right]^{+} 315.1355 \mathrm{~m} / \mathrm{z}$; found $[\mathrm{M}+\mathrm{H}]^{+} 315.1354 \mathrm{~m} / z$. 
4-Chloro-1-(3-methylbut-2-en-1-yl)-2-(phenylethynyl)benzene (9c)

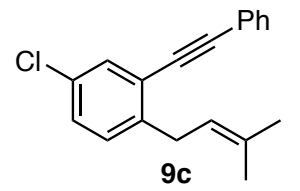

1,6-Enyne 9c was synthesized following general procedure $\mathbf{G}$ from 1-bromo-4-chloro-2(phenylethynyl)benzene $(100 \mathrm{mg}, 0.343 \mathrm{mmol})$ whereby the reaction was stirred for $1 \mathrm{~h}$. Purification by column chromatography (cyclohexane) afforded title compound 9c (62.3 mg, $0.22 \mathrm{mmol}, 65 \%$ ) colorless oil.

${ }^{1}$ H NMR $\left(500 \mathrm{MHz}, \mathrm{CDCl}_{3}\right) \delta 7.56-7.52(\mathrm{~m}, 2 \mathrm{H}), 7.49(\mathrm{~d}, J=2.3 \mathrm{~Hz}, 1 \mathrm{H}), 7.39-7.35$ (m, 3H), $7.23(\mathrm{dd}, J=8.3,2.3 \mathrm{~Hz}, 1 \mathrm{H}), 7.15(\mathrm{dd}, J=8.3,0.4 \mathrm{~Hz}, 1 \mathrm{H}), 5.37-5.31(\mathrm{~m}, 1 \mathrm{H})$, $3.55(\mathrm{~d}, J=7.3 \mathrm{~Hz}, 2 \mathrm{H}), 1.77-1.75(\mathrm{~m}, 3 \mathrm{H}), 1.73(\mathrm{~s}, 3 \mathrm{H}) .{ }^{13} \mathbf{C} \mathbf{N M R}\left(126 \mathrm{MHz}, \mathrm{CDCl}_{3}\right) \delta$ $142.3,133.6,131.8,131.7,131.4,129.8,128.70,128.67,128.6,124.3,123.2$, 122.0, 94.4, 87.2, 32.6, 25.9, 18.1. HRMS (APCI+) calculated for $\left[\mathrm{C}_{19} \mathrm{H}_{18} \mathrm{Cl}\right]^{+} 281.1092 \mathrm{~m} / z$; found $[\mathrm{M}+\mathrm{H}]^{+}$ $281.1092 \mathrm{~m} / \mathrm{z}$.

\section{4-Methoxy-1-(3-methylbut-2-en-1-yl)-2-(phenylethynyl)benzene (9d)}

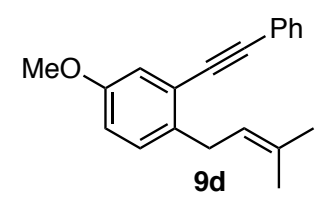

1,6-Enyne 9d was synthesized following general procedure $\mathbf{G}$ from 1-bromo-4-methoxy-2(phenylethynyl)benzene $(140 \mathrm{mg}, 0.49 \mathrm{mmol})$ whereby the reaction was stirred for $1 \mathrm{~h}$. Purification by preparative TLC (pentane $/ \mathrm{CH}_{2} \mathrm{Cl}_{2}$ 95:5) afforded title compound 9d (90 mg, $0.33 \mathrm{mmol}, 68 \%$ ) as a light yellow oil.

${ }^{1} \mathbf{H}$ NMR $\left(500 \mathrm{MHz}, \mathrm{CDCl}_{3} \delta 7.58-7.53(\mathrm{~m}, 2 \mathrm{H}), 7.39-7.34(\mathrm{~m}, 3 \mathrm{H}), 7.14(\mathrm{~d}, J=8.6 \mathrm{~Hz}\right.$, $1 \mathrm{H}), 7.07(\mathrm{~d}, J=2.8 \mathrm{~Hz}, 1 \mathrm{H}), 6.86(\mathrm{dd}, J=8.5,2.8 \mathrm{~Hz}, 1 \mathrm{H}), 5.38$ (tdt, $J=7.3,2.8,1.4 \mathrm{~Hz}, 1 \mathrm{H})$, $3.82(\mathrm{~s}, 3 \mathrm{H}), 3.56(\mathrm{~d}, J=7.3 \mathrm{~Hz}, 2 \mathrm{H}), 1.77(\mathrm{dd}, J=2.8,1.4 \mathrm{~Hz}, 6 \mathrm{H}) .{ }^{13} \mathbf{C}$ NMR $(126 \mathrm{MHz}$, $\left.\mathrm{CDCl}_{3}\right) \delta 157.5,136.3,132.6,131.7,129.6,128.5,128.4,123.6,123.3,123.0,116.6,115.5$, 93.1, 88.5, 55.5, 32.3, 25.9, 18.1. HRMS (ESI+) calculated for $\left[\mathrm{C}_{20} \mathrm{H}_{21} \mathrm{O}\right]^{+} 277.1587 \mathrm{~m} / z$; found $[\mathrm{M}+\mathrm{H}]^{+} 277.1573 \mathrm{~m} / z$. 


\subsubsection{Synthesis of enynes $9 i$ and $9 j$}

\section{General procedure H: Sonogashira coupling}

A mixture of 1-bromo-2-(3-methylbut-2-en-1-yl)benzene ${ }^{18}$ (1.0 equiv), $\mathrm{PdCl}_{2}\left(\mathrm{MeCN}_{2}\right.$ (2 mol \%,), XPhos (3 mol \%) and $\mathrm{Cs}_{2} \mathrm{CO}_{3}$ (3.0 equiv), in anhydrous $\mathrm{MeCN}(1 \mathrm{~mL})$ was stirred under a nitrogen atmosphere at $24{ }^{\circ} \mathrm{C}$ for $25 \mathrm{~min} .{ }^{19}$ Then, alkyne (1.5 equiv) was added and the mixture was stirred at $85^{\circ} \mathrm{C}$ for the indicated time. The reaction was quenched by addition of water and EtOAc and the aqueous phase was extracted with EtOAc (3x). The combined organic phases were dried over anhydrous $\mathrm{Na}_{2} \mathrm{SO}_{4}$, filtered and concentrated. The crude was purified by flash column chromatography to afford the corresponding 1,6-enynes.

\section{1-((4-Chlorophenyl)ethynyl)-2-(3-methylbut-2-en-1-yl)benzene (9i)}

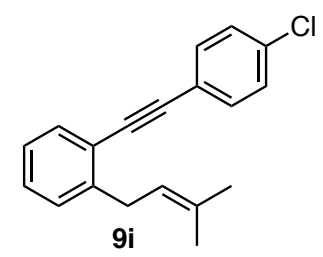

1,6-Enyne 9i was synthesized following general procedure $\mathbf{H}$ from 1-chloro-4-ethynylbenzene $(91 \mathrm{mg}, 0.666 \mathrm{mmol})$ whereby the reaction was stirred for $2 \mathrm{~h}$. The crude was purified by flash column chromatography (cyclohexane/pentane 1:1) to afford the title compound $9 \mathbf{i}$ (73 $\mathrm{mg}, 0.259 \mathrm{mmol}, 59 \%$ ) as a yellow oil.

${ }^{1} \mathbf{H}$ NMR $\left(400 \mathrm{MHz}, \mathrm{CD}_{2} \mathrm{Cl}_{2}\right) \delta 7.52-7.45(\mathrm{~m}, 3 \mathrm{H}), 7.38-7.33(\mathrm{~m}, 2 \mathrm{H}), 7.32-7.23$ (m, 2H), $7.22-7.16(\mathrm{~m}, 1 \mathrm{H}), 5.40-5.34(\mathrm{~m}, 1 \mathrm{H}), 3.59(\mathrm{~d}, J=7.3 \mathrm{~Hz}, 2 \mathrm{H}), 1.75(\mathrm{~s}, 6 \mathrm{H}) .{ }^{13} \mathbf{C}$ NMR $\left(101 \mathrm{MHz}, \mathrm{CD}_{2} \mathrm{Cl}_{2}\right) \delta 144.5,134.7,133.5,133.3,132.7,129.4,129.3,129.2,126.4$, $122.9,122.69,122.68,92.5,89.8,33.6,26.0,18.3$. HRMS (APCI+) calculated for $\left[\mathrm{C}_{19} \mathrm{H}_{18} \mathrm{Cl}\right]^{+}$ $281.1092 \mathrm{~m} / \mathrm{z}$; found $[\mathrm{M}+\mathrm{H}]^{+} 281.1095 \mathrm{~m} / \mathrm{z}$.

\section{1-((4-Methoxyphenyl)ethynyl)-2-(3-methylbut-2-en-1-yl)benzene (9j)}

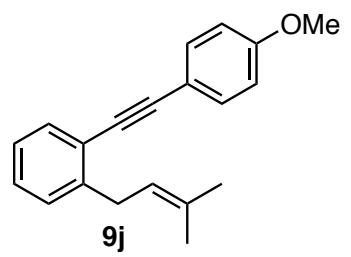

1,6-Enyne 9j was synthesized following general procedure $\mathbf{H}$ from 1-ethynyl-4-methoxybenzene ( $86 \mu \mathrm{L}, 0.666 \mathrm{mmol}, 1.5$ equiv) whereby the reaction was stirred for $2 \mathrm{~h}$. The crude was purified by flash column chromatography (cyclohexane/ $\mathrm{CH}_{2} \mathrm{Cl}_{2} 20: 1$ then 10:1) to afford title compound $9 \mathbf{j}$ (78 $\mathrm{mg}, 0.282 \mathrm{mmol}, 64 \%$ ) as a yellow oil. 
The spectral data of $\mathbf{9 j}$ were fully consistent with those previously reported. ${ }^{17}$

\subsubsection{Preparation of enynes $9 k$ and 91}

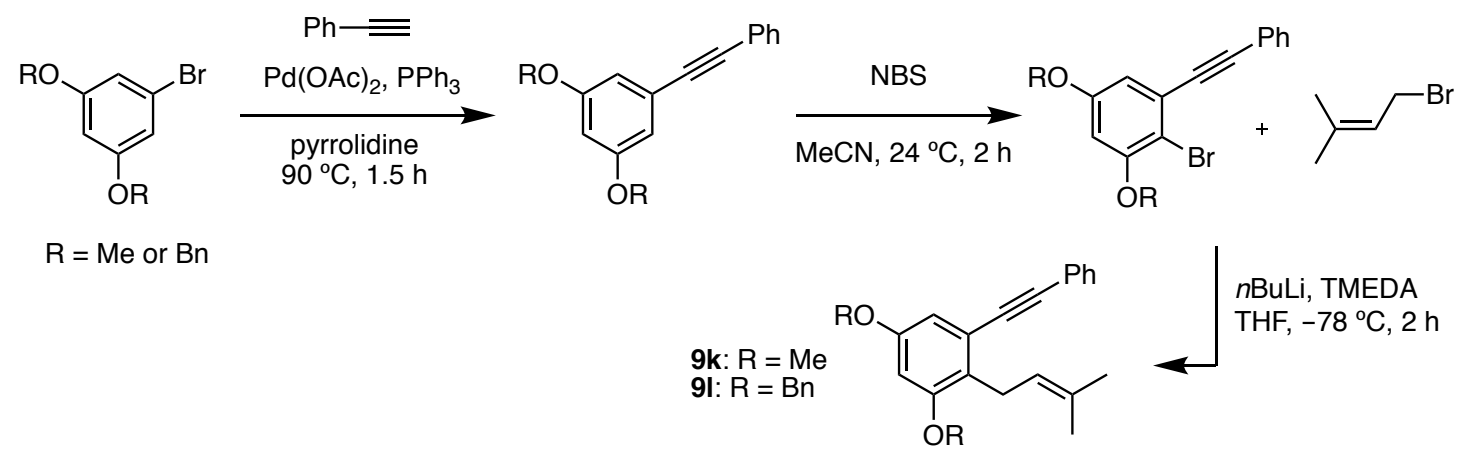

\section{General procedure I: Sonogashira coupling}

To a stirred solution of the corresponding aryl bromide (1.0 equiv) and ethynylbenzene (1.5 equiv) in pyrrolidine $(0.5 \mathrm{M})$ were added $\mathrm{PPh}_{3}(4 \mathrm{~mol} \%)$ and $\mathrm{Pd}(\mathrm{OAc})_{2}(2 \mathrm{~mol} \%)$ under an argon atmosphere. After stirring at $90{ }^{\circ} \mathrm{C}$ for the mentioned time, the mixture was quenched with sat. $\mathrm{NH}_{4} \mathrm{Cl}(30 \mathrm{~mL})$ and extracted with $\mathrm{CH}_{2} \mathrm{Cl}_{2}$. The organic extract was dried over $\mathrm{Na}_{2} \mathrm{SO}_{4}$ and the solvent was removed in vacuo. The crude was purified by flash column chromatography to afford the corresponding alkynes.

\section{1,3-Dimethoxy-5-(phenylethynyl)benzene}

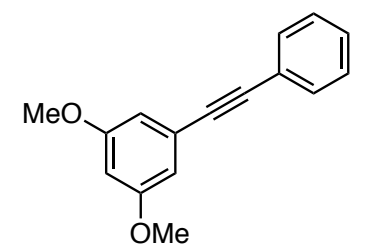

1,3-Dimethoxy-5-(phenylethynyl)benzene was synthesized following general procedure I from 1-bromo-3,5-dimethoxybenzene $(1.0 \mathrm{~g}, 4.61 \mathrm{mmol})$ whereby the reaction was stirred for 90 min. Purification by column chromatography (pentane/ $\mathrm{Et}_{2} \mathrm{O}$ 98:2) afforded the title compound (956 mg, $4.01 \mathrm{mmol}, 87 \%$ ) as a yellow oil.

The spectral data were fully consistent with those previously reported. ${ }^{20}$ 


\section{1,3-Dibenzyloxy-5-(phenylethynyl)benzene}

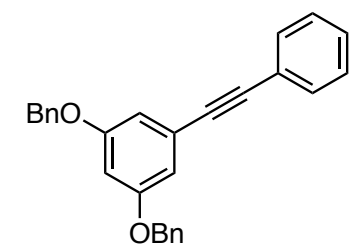

1,3-Dibenzyloxy-5-(phenylethynyl)benzene was synthesized following general procedure I from 3,5-dibenzyloxybromobenzene $(6.3 \mathrm{~g}, 17.1 \mathrm{mmol})$ whereby the reaction was stirred for 90 min. Purification by column chromatography (pentane/Et $2 \mathrm{O} 98: 2$ ) followed by recrystallization from hot $n$ hexane afforded the title compound (5.21 g, $13.3 \mathrm{mmol}, 78 \%$ ) as a white crystalline solid.

M.p. $=80-82{ }^{\circ} \mathrm{C}$ (pentane). ${ }^{1} \mathbf{H}$ NMR $\left(500 \mathrm{MHz}, \mathrm{CDCl}_{3}\right) \delta 7.59-7.52(\mathrm{~m}, 2 \mathrm{H}), 7.48-$ $7.32(\mathrm{~m}, 13 \mathrm{H}), 6.83(\mathrm{~d}, J=2.3 \mathrm{~Hz}, 2 \mathrm{H}), 6.65(\mathrm{t}, J=2.3 \mathrm{~Hz}, 1 \mathrm{H}), 5.07(\mathrm{~s}, 4 \mathrm{H}) .{ }^{13} \mathbf{C}$ NMR (126 $\left.\mathrm{MHz}, \mathrm{CDCl}_{3}\right) \delta 159.9,136.8,131.8,128.8,128.5,128.2,127.7,124.8,123.2,110.8,103.6$, 100.1, 89.5, 89.2, 70.3. HRMS (ESI+) calculated for $\left[\mathrm{C}_{28} \mathrm{H}_{22} \mathrm{NaO}_{2}\right]^{+} 413.1512 \mathrm{~m} / \mathrm{z}$; found [M $+\mathrm{Na}]^{+} 413.1514 \mathrm{~m} / \mathrm{z}$

\section{General procedure $\mathrm{J}$ : bromination reaction}

The aryl alkyne (1.0 equiv) was dissolved in acetonitrile $(0.075 \mathrm{M})$ and the solution was cooled to $0{ }^{\circ} \mathrm{C}$. A solution of NBS (1.05 equiv, $0.5 \mathrm{M}$ in acetonitrile) was added dropwise and the reaction was stirred for the given time at the same temperature. The solvent was evaporated and the crude was purified by flash column chromatography. The yellow solid obtained was recrystallized from hot $n$ hexane to afford the corresponding bromides.

\section{2-Bromo-1,5-dimethoxy-3-(phenylethynyl)benzene}<smiles>COc1cc(C#Cc2ccccc2)c(Br)c(OC)c1</smiles>

2-Bromo-1,5-dimethoxy-3-(phenylethynyl)benzene was synthesized following general procedure $\mathbf{J}$ from 1,3-dimethoxy-5-(phenylethynyl)benzene (1.7 g, $7.13 \mathrm{mmol})$ whereby the reaction was stirred for $2 \mathrm{~h}$. Purification by column chromatography (pentane/Et ${ }_{2} \mathrm{O}$ 98:2) followed by recrystallization from hot $n$ hexane afforded the title compound $(2.06 \mathrm{~g}, 6.49 \mathrm{mmol}$, 91\%) as a white crystalline solid. 
M.p. $=80-82{ }^{\circ} \mathrm{C}$ (pentane). ${ }^{1} \mathbf{H}$ NMR $\left(300 \mathrm{MHz}, \mathrm{CDCl}_{3}\right) \delta 7.63-7.55(\mathrm{~m}, 2 \mathrm{H}), 7.39-$ $7.32(\mathrm{~m}, 3 \mathrm{H}), 6.73(\mathrm{~d}, J=2.7 \mathrm{~Hz}, 1 \mathrm{H}), 6.48(\mathrm{~d}, J=2.7 \mathrm{~Hz}, 1 \mathrm{H}), 3.89(\mathrm{~s}, 3 \mathrm{H}), 3.83(\mathrm{~s}, 3 \mathrm{H}) .{ }^{13} \mathrm{C}$ NMR $\left(75 \mathrm{MHz}, \mathrm{CDCl}_{3}\right) \delta 159.5,157.1,131.9,128.8,128.5,126.7,123.0,108.8,106.7,100.8$, 93.9, 88.5, 56.5, 55.8. HRMS (ESI+) calculated for $\left[\mathrm{C}_{16} \mathrm{H}_{14} \mathrm{BrO}_{2}\right]^{+} 317.0172 \mathrm{~m} / z$; found $[\mathrm{M}+$ $\mathrm{H}]^{+} 317.0172 \mathrm{~m} / \mathrm{z}$.

\section{2-Bromo-1,5-dibenzyloxy-3-(phenylethynyl)benzene}

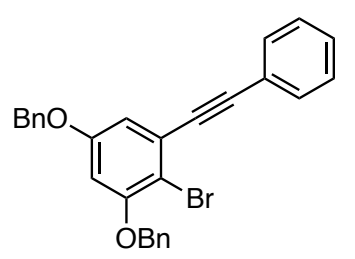

2-Bromo-1,5-dibenzyloxy-3-(phenylethynyl)benzene was synthesized following general procedure $\mathbf{J}$ from 1,3-dibenzyloxy-5-(phenylethynyl)benzene (3.5 g, $8.96 \mathrm{mmol}$ ) whereby the reaction was stirred for $2 \mathrm{~h}$. Purification by column chromatography (pentane/Et $\mathrm{t}_{2} \mathrm{O}$ 98:2) followed by recrystallization from hot $n$ hexane afforded the title compound $(3.49 \mathrm{~g}, 7.44 \mathrm{mmol}$, $83 \%)$ as a white crystalline solid.

M.p. $=101-103{ }^{\circ} \mathrm{C}$ (pentane). ${ }^{1} \mathbf{H}$ NMR $\left(500 \mathrm{MHz}, \mathrm{CDCl}_{3}\right) \delta 7.63-7.59(\mathrm{~m}, 2 \mathrm{H}), 7.49-$ $7.46(\mathrm{~m}, 2 \mathrm{H}), 7.44-7.31(\mathrm{~m}, 11 \mathrm{H}), 6.85(\mathrm{~d}, J=2.7 \mathrm{~Hz}, 1 \mathrm{H}), 6.61(\mathrm{~d}, J=2.7 \mathrm{~Hz}, 1 \mathrm{H}), 5.13(\mathrm{~s}$, 2H), $5.03(\mathrm{~s}, 2 \mathrm{H}) .{ }^{13} \mathrm{C}$ NMR $\left(126 \mathrm{MHz}, \mathrm{CDCl}_{3}\right) \delta 158.5,156.2,136.4,136.3,131.9,128.81$, $128.75,128.5,128.4,128.1,127.7,127.2$, 126.8, 123.0, 110.4, 107.8, 103.1, 94.0, 88.5, 71.1, 70.6. HRMS (ESI+) calculated for $\left[\mathrm{C}_{28} \mathrm{H}_{21} \mathrm{BrNaO}_{2}\right]^{+} 491.0617 \mathrm{~m} / z$; found $[\mathrm{M}+\mathrm{Na}]^{+} 491.0619$ $m / z$.

\section{General procedure K: prenylation reaction}

A flask was charged with the aryl bromide (1.0 equiv). Three cycles vacuum argon were applied and anhydrous THF $(0.05 \mathrm{M})$ was added. The solution was cooled to $-78^{\circ} \mathrm{C}$ and $n \mathrm{BuLi}$ (1.1 equiv) was added dropwise and the reaction mixture was stirred for 3 minutes. TMEDA (1.1 equiv) was then added and stirring was continued for 3 minutes. Finally, 1-bromo-3methylbut-2-ene (1.25 equiv) was added dropwise and the resulting mixture was stirred for the given time while being allowed to slowly warm to $24{ }^{\circ} \mathrm{C}$. The suspension was carefully quenched by addition of sat. $\mathrm{NH}_{4} \mathrm{Cl}$ and extracted with $\mathrm{Et}_{2} \mathrm{O}(2 \times 50 \mathrm{~mL})$. The combined organic phases were washed with brine, dried over $\mathrm{Na}_{2} \mathrm{SO}_{4}$ and concentrated. The crude was purified by flash column chromatography to afford the corresponding 1,6-enynes. 


\section{1,5-Dimethoxy-2-(3-methylbut-2-en-1-yl)-3-(phenylethynyl)benzene (9k)}

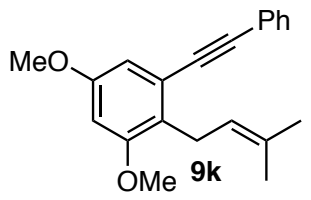

1,6-Enyne 9k was synthesized following general procedure $\mathbf{K}$ from 2-bromo-1,5dimethoxy-3-(phenylethynyl)benzene $(7.6 \mathrm{~g}, 23.9 \mathrm{mmol})$ whereby the reaction was stirred for $2 \mathrm{~h}$. The crude was purified by flash column chromatography (cyclohexane $/ \mathrm{CH}_{2} \mathrm{Cl}_{2}, 9: 1$ ) and further purified by a second flash column chromatography (pentane/ $\mathrm{Et}_{2} \mathrm{O}$ 98:2) to afford compound 9k (4.76 g, $15.5 \mathrm{mmol}, 65 \%)$ as a yellow oil.

${ }^{1} \mathbf{H}$ NMR $\left(400 \mathrm{MHz}, \mathrm{CDCl}_{3}\right) \delta 7.56-7.51(\mathrm{~m}, 2 \mathrm{H}), 7.38-7.32(\mathrm{~m}, 3 \mathrm{H}), 6.65(\mathrm{~d}, J=2.5$ $\mathrm{Hz}, 1 \mathrm{H}), 6.46(\mathrm{~d}, J=2.5 \mathrm{~Hz}, 1 \mathrm{H}), 5.30-5.23(\mathrm{~m}, 1 \mathrm{H}), 3.81(\mathrm{~d}, J=2.0 \mathrm{~Hz}, 6 \mathrm{H}), 3.55(\mathrm{~d}, J=$ $7.1 \mathrm{~Hz}, 2 \mathrm{H}), 1.80(\mathrm{~d}, J=0.7 \mathrm{~Hz}, 3 \mathrm{H}), 1.68(\mathrm{~d}, J=1.1 \mathrm{~Hz}, 3 \mathrm{H}) ;{ }^{13} \mathbf{C} \mathbf{N M R}\left(101 \mathrm{MHz}, \mathrm{CDCl}_{3}\right) \delta$ $158.49,158.48,131.7,131.4,128.5,128.3,125.8,123.7,123.0,107.5,100.2$, 92.4, 88.9, 55.8, 55.6, 27.3, 26.0, 18.2. HRMS (ESI+) calculated for $\left[\mathrm{C}_{21} \mathrm{H}_{23} \mathrm{O}_{2}\right]^{+} 307.1693 \mathrm{~m} / z$; found $[\mathrm{M}+\mathrm{H}]^{+}$ $307.1680 \mathrm{~m} / \mathrm{z}$.

\section{1,5-Dibenzyloxy-2-(3-methylbut-2-en-1-yl)-3-(phenylethynyl)benzene (9l)}<smiles>CC(C)=CCc1c(Br)cc(OCc2ccccc2)cc1C#Cc1ccccc1</smiles>

1,6-Enyne 91 was synthesized following general procedure $\mathbf{K}$ from 2-bromo-1,5-dibenzyloxy-3-(phenylethynyl)benzene $(3.4 \mathrm{~g}, 7.2 \mathrm{mmol})$ whereby the reaction was stirred for $2 \mathrm{~h}$. The crude was purified by flash column chromatography (cyclohexane $/ \mathrm{CH}_{2} \mathrm{Cl}_{2}, 9: 1$ ) and further purified by recrystallization from hot $n$ hexane to afford title compound 91 ( $2.43 \mathrm{~g}, 5.29 \mathrm{mmol}$, $73 \%$ ) as a white crystalline solid.

M.p. $=79-80{ }^{\circ} \mathrm{C}$ (pentane). ${ }^{1} \mathbf{H}$ NMR $\left(400 \mathrm{MHz}, \mathrm{CDCl}_{3}\right) \delta 7.57-7.52(\mathrm{~m}, 2 \mathrm{H}), 7.46-$ $7.31(\mathrm{~m}, 13 \mathrm{H}), 6.79(\mathrm{~d}, J=2.4 \mathrm{~Hz}, 1 \mathrm{H}), 6.61(\mathrm{~d}, J=2.4 \mathrm{~Hz}, 1 \mathrm{H}), 5.36-5.29(\mathrm{~m}, 1 \mathrm{H}), 5.05(\mathrm{~d}$, $J=6.9 \mathrm{~Hz}, 4 \mathrm{H}), 3.62(\mathrm{~d}, J=7.1 \mathrm{~Hz}, 2 \mathrm{H}), 1.71(\mathrm{~d}, J=0.5 \mathrm{~Hz}, 3 \mathrm{H}), 1.68(\mathrm{~d}, J=0.9 \mathrm{~Hz}, 3 \mathrm{H}) .{ }^{13} \mathbf{C}$ NMR $\left(101 \mathrm{MHz}, \mathrm{CDCl}_{3}\right) \delta 157.6,157.5,137.1,137.0,131.7,131.4,128.8,128.6,128.5,128.4$, $128.2,128.0,127.7,127.4,126.3,123.9,123.6,123.0,108.9,102.0,92.6,88.9,70.4,70.3,27.5$, 26.0, 18.2. HRMS $(\mathrm{ESI}+)$ calculated for $\left[\mathrm{C}_{33} \mathrm{H}_{30} \mathrm{NaO}_{2}\right]^{+} 481.2138 \mathrm{~m} / z$; found $[\mathrm{M}+\mathrm{Na}]^{+}$ $481.2152 \mathrm{~m} / \mathrm{z}$. 


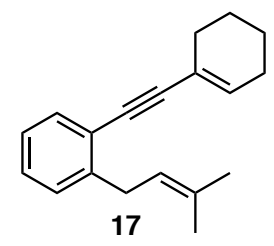

To a solution of cyclohex-1-en-1-yl trifluoromethanesulfonate $(125 \mathrm{mg}, 0.543 \mathrm{mmol}, 1.0$ equiv) and 1-ethynyl-2-(3-methylbut-2-en-1-yl)benzene ${ }^{21}$ (138 mg, $0.81 \mathrm{mmol}, 1.49$ equiv) in $\mathrm{NEt}_{3}(2.17 \mathrm{~mL})$ were added $\left[\mathrm{PdCl}_{2}\left(\mathrm{PPh}_{3}\right)_{2}\right](7.62 \mathrm{mg}, 10.9 \mu \mathrm{mol}, 2.0 \mathrm{~mol} \%)$ and copper(I) iodide $(4.14 \mathrm{mg}, 0.022 \mathrm{mmol}, 4.0 \mathrm{~mol} \%) .{ }^{22}$ The resulting mixture was heated under $\mathrm{N}_{2}$ atmosphere at $55{ }^{\circ} \mathrm{C}$ and the reaction was monitored by TLC. When the reaction was complete, the mixture was allowed to cool to $24{ }^{\circ} \mathrm{C}$, and the ammonium salt was removed by filtration. The solvent was removed under reduced pressure and the residue was purified by column chromatography (cyclohexane) to afford the title compound 17 (125 mg, $0.499 \mathrm{mmol}, 92 \%)$ as a yellow liquid.

The spectral data of $\mathbf{1 7}$ were fully consistent with those previously reported. ${ }^{17}$

\subsection{Optimization of the reaction conditions}

\subsubsection{HTE screening of ligands}

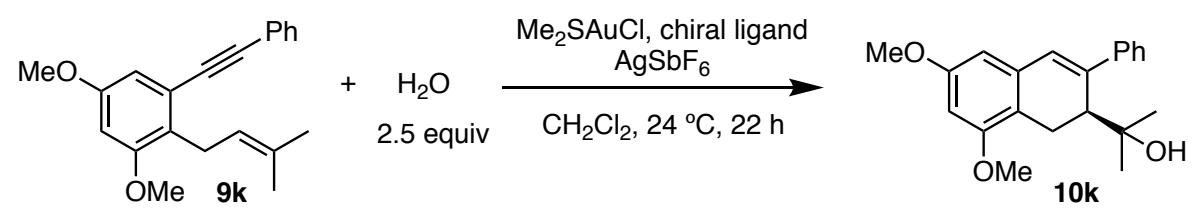

\section{General procedure L: HTE screening (10 $\mu \mathrm{mol}$ scale)}

To a solution of $\mathrm{Me}_{2} \mathrm{SAuCl}\left(0.1\right.$ equiv) in $\mathrm{CH}_{2} \mathrm{Cl}_{2}(0.3 \mathrm{M})$ was added a solution of the ligand ( 0.1 equiv for monodentate ligands or 0.05 equiv for bidentate ligands) in $\mathrm{CH}_{2} \mathrm{Cl}_{2}(0.3 \mathrm{M})$ followed by a solution of $\mathrm{AgSbF}_{6}\left(0.1\right.$ equiv) in $\mathrm{CH}_{2} \mathrm{Cl}_{2}(0.3 \mathrm{M})$. The resulting mixture was stirred for 5 minutes and concentrated under vacuum. To the dried residue were added sequentially a solution of 1,6-enyne 9k (1.0 equiv, $10 \mu \mathrm{mol}$ ) in $\mathrm{CH}_{2} \mathrm{Cl}_{2}, \mathrm{H}_{2} \mathrm{O}$ (2.5 equiv) and $\mathrm{CH}_{2} \mathrm{Cl}_{2}(0.3 \mathrm{M})$. After $22 \mathrm{~h}$, an aliquot was taken and the enantiomeric excess of product 10k was measured. 
Table 6: Screening of chiral ligands at HTE

\begin{tabular}{|c|c|c|c|}
\hline entry & code & ligand structure & $e r^{a}$ \\
\hline 1 & $1 \mathrm{~A}$ & & $<57: 43$ \\
\hline 2 & 1B & & $<57: 43$ \\
\hline 3 & $1 \mathrm{C}$ & & $<57: 43$ \\
\hline 4 & $1 \mathrm{D}$ & & $<57: 43$ \\
\hline 5 & $1 \mathrm{E}$ & & $<57: 43$ \\
\hline 6 & $1 \mathrm{~F}$ & & $<57: 43$ \\
\hline 7 & $1 \mathrm{G}$ & & $61: 39$ \\
\hline 8 & $1 \mathrm{H}$ & & $66: 34$ \\
\hline 9 & $1 \mathrm{I}$ & & $<57: 43$ \\
\hline
\end{tabular}



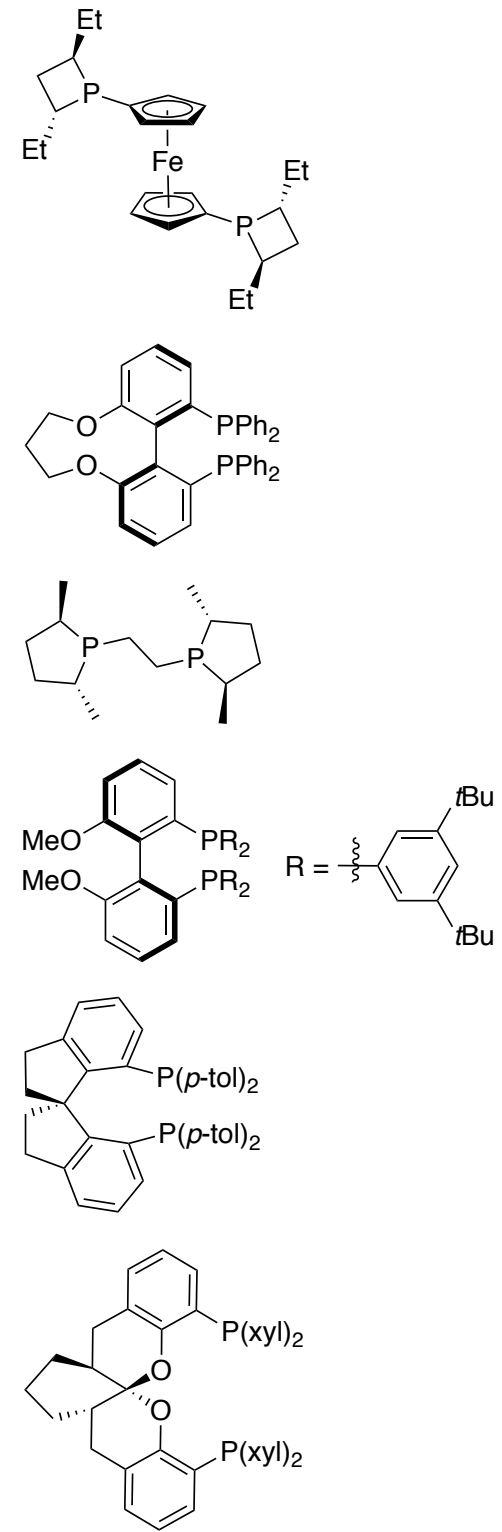

17
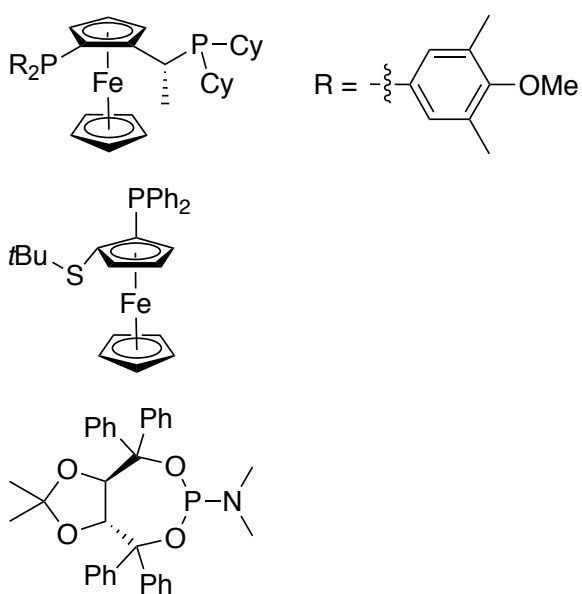


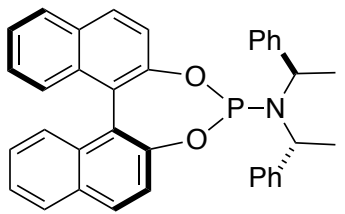

21

$3 \mathrm{C}$<smiles></smiles>

22

3D<smiles>COc1ccc2ccccc2c1-c1c(-c2ccccc2)ccc2ccccc12</smiles>

23

$3 \mathrm{E}$<smiles>Nc1ccc2ccccc2c1-c1c(O)ccc2ccccc12</smiles>

24

$3 \mathrm{~F}$

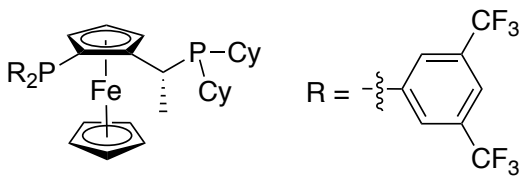
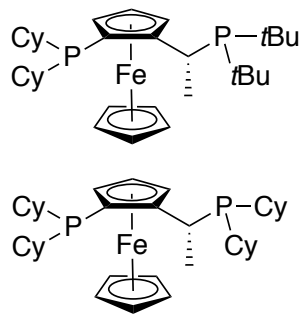<smiles>[R6]c1ccc(P(CC)c2ccco2)cc1C(C)C(C)P</smiles>
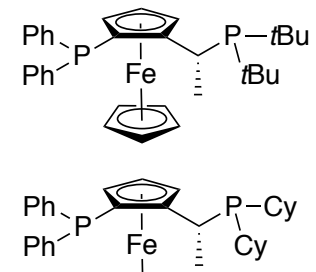
32

$4 \mathrm{E}$

33

34

35

36

37

38

5B

39

5C

40

5D

41

$5 \mathrm{E}$

42
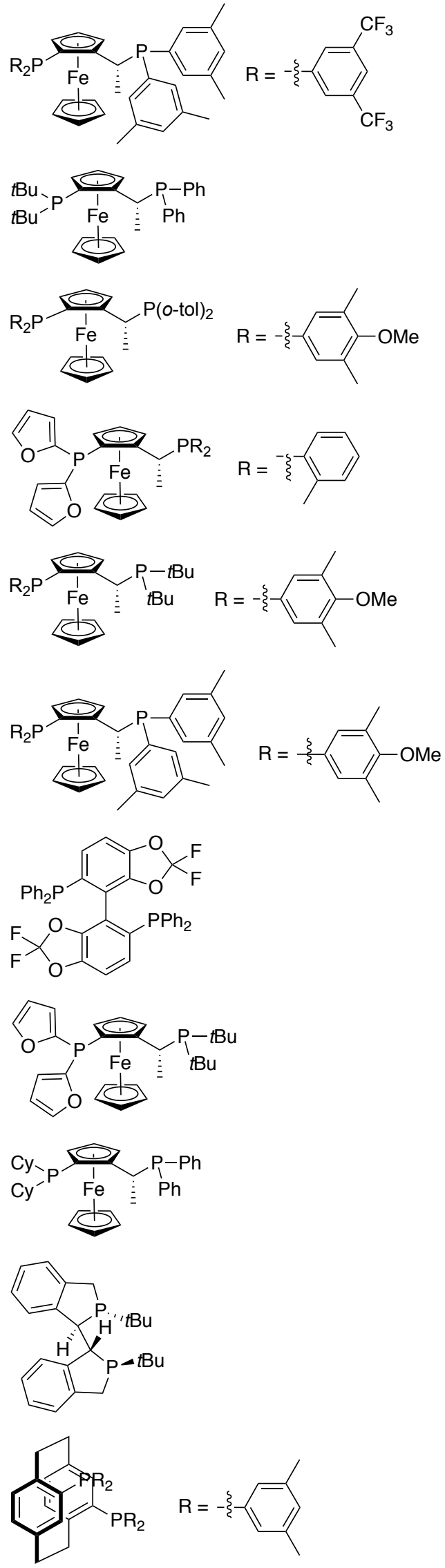

$<57: 43$

$62: 38$

$<57: 43$

$63: 37$

80:20

$<57: 43$

64:36

68:32

$<57: 43$

$<57: 43$

$<57: 43$

S76 


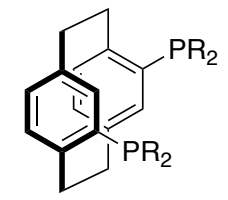<smiles>P[C@@H]1CCCP1CCP1C([PH2+]c2ccccc2)CC[C@H]1P</smiles>
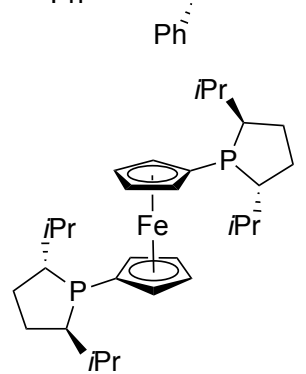

46

$6 \mathrm{~A}$<smiles>CC1CC[C@@H](C)P1C1=CC=C1F</smiles>

47
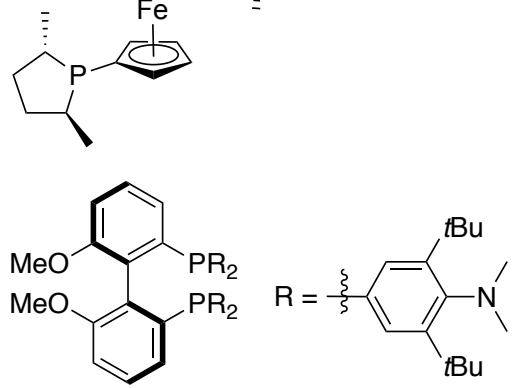

48

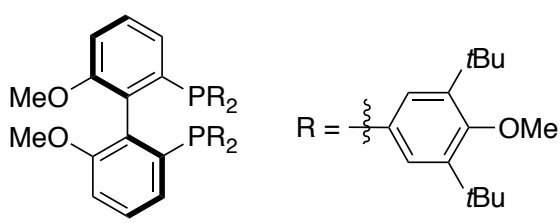

49

$6 \mathrm{D}$

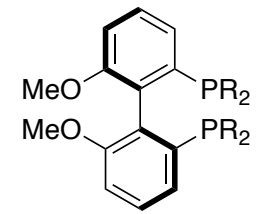<smiles>[2H][14c]1ccco1</smiles>

50

$6 \mathrm{E}$<smiles>COc1cccc(-c2ccccc2)c1-c1c(OC)cccc1-c1ccccc1-c1ccccc1</smiles>

$6 \mathrm{~F}$

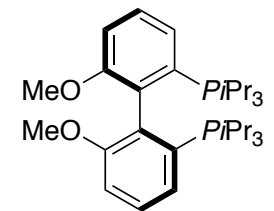



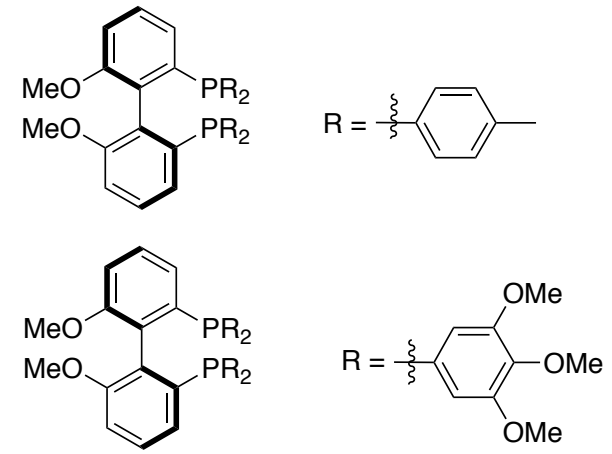

54<smiles>COc1cccc(P)c1-c1c(P)cccc1OC</smiles><smiles>Cc1cc(C)cc(C)c1</smiles>

55

7A

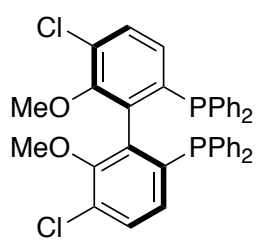

56

7B<smiles>CCC1CCC(CC)P1c1ccccc1P1C(CC)CCC1CC</smiles>

57

$7 \mathrm{C}$

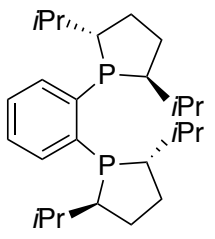

58

7D

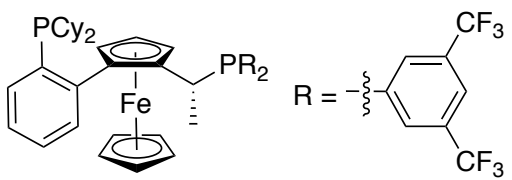

59

7E

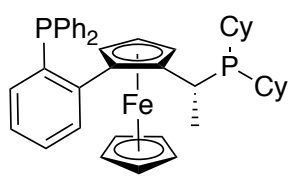

60

7F

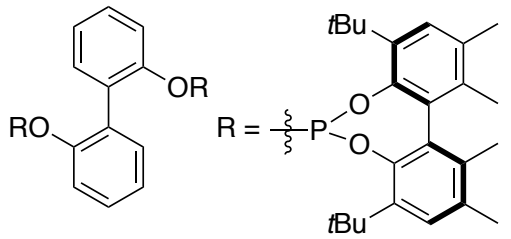


61

$7 \mathrm{G}$

62

7H

63

7I

64

$8 \mathrm{~A}$

65

8B

66

$8 C$

67

8D

68
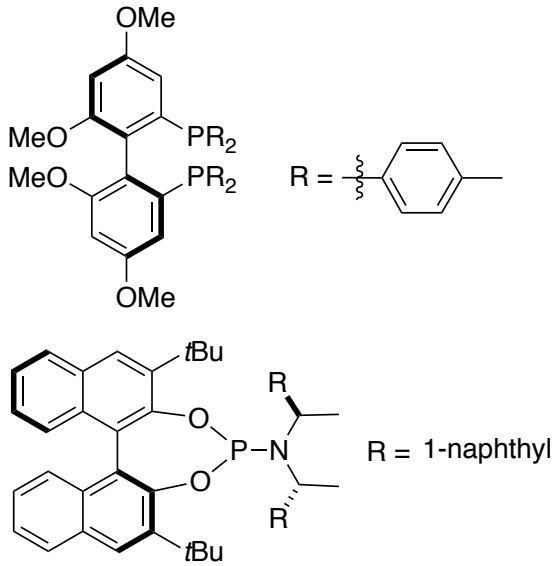

$64: 36$<smiles>COc1cc([PH+])c(C)c(OC)n1</smiles>

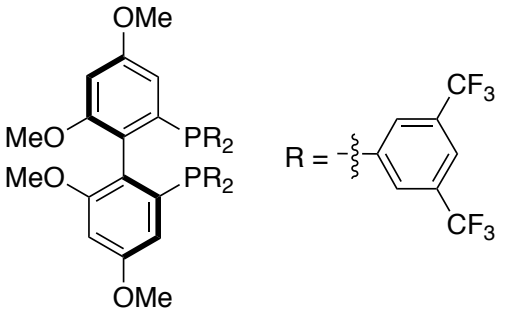

$\mathrm{Fe} \mathrm{PPh}_{2}$

i

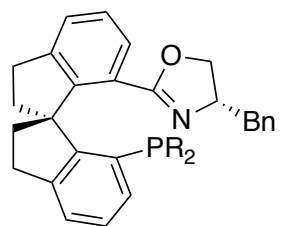<smiles></smiles>

$<57: 43$ 
69

$8 \mathrm{~F}$

70

$8 \mathrm{G}$

71

$8 \mathrm{H}$

72

8I
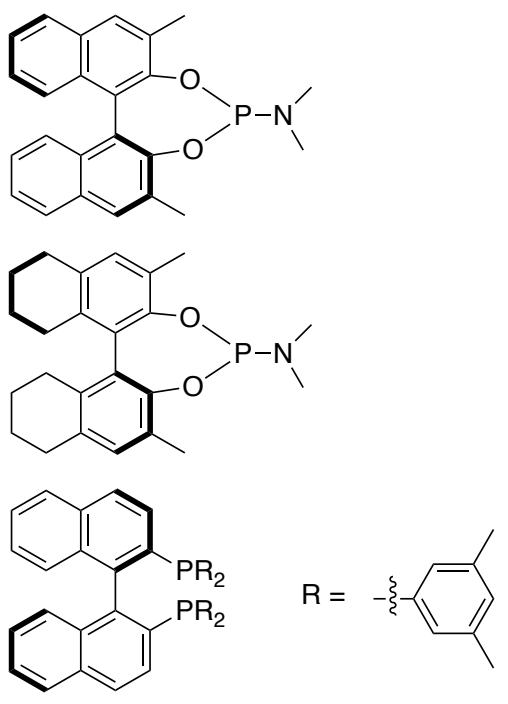

$\sum_{i \operatorname{Pr}}^{\mathrm{Pr}}$

74

9B

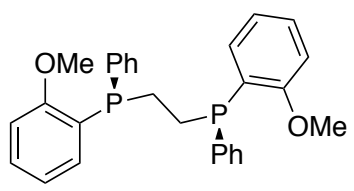

75

9C

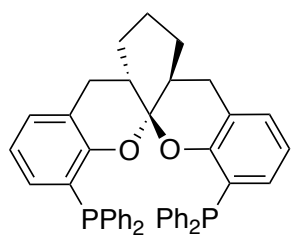

76

9D

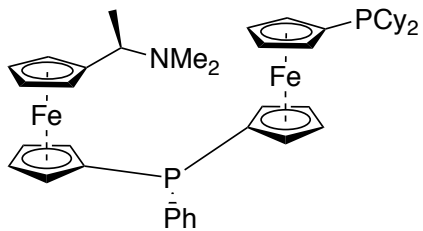

77

9E

78

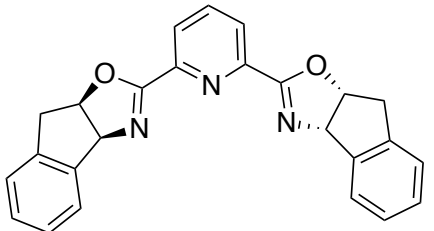

$<57: 43$ 
79 $9 \mathrm{G}$

80 $9 \mathrm{H}$<smiles>Cc1scc(P)c1/C(=C1/Pc2ccccc21)C12CCCC1C2</smiles>

$R=-\{$

$<57: 43$

${ }^{a}$ Enantiomeric ratios determined by SFC analysis using UPC ${ }^{2}$ (Chiralpak ID, PDA detector). Method with gradient: $\mathrm{CO}_{2} / \mathrm{EtOH}$ from $100 \% \mathrm{CO}_{2}(1 \mathrm{~min})$ to $60: 40$ over 4 minutes, flow rate $=3 \mathrm{~mL} / \mathrm{min}, \mathrm{T}=35^{\circ} \mathrm{C}, \mathrm{ABPR}=1500 \mathrm{psi}$, $\lambda=254 \mathrm{~nm}, \mathrm{t}_{\mathrm{R}}($ major $)=4.0 \mathrm{~min}, \mathrm{t}_{\mathrm{R}}($ minor $)=4.9 \mathrm{~min}$.

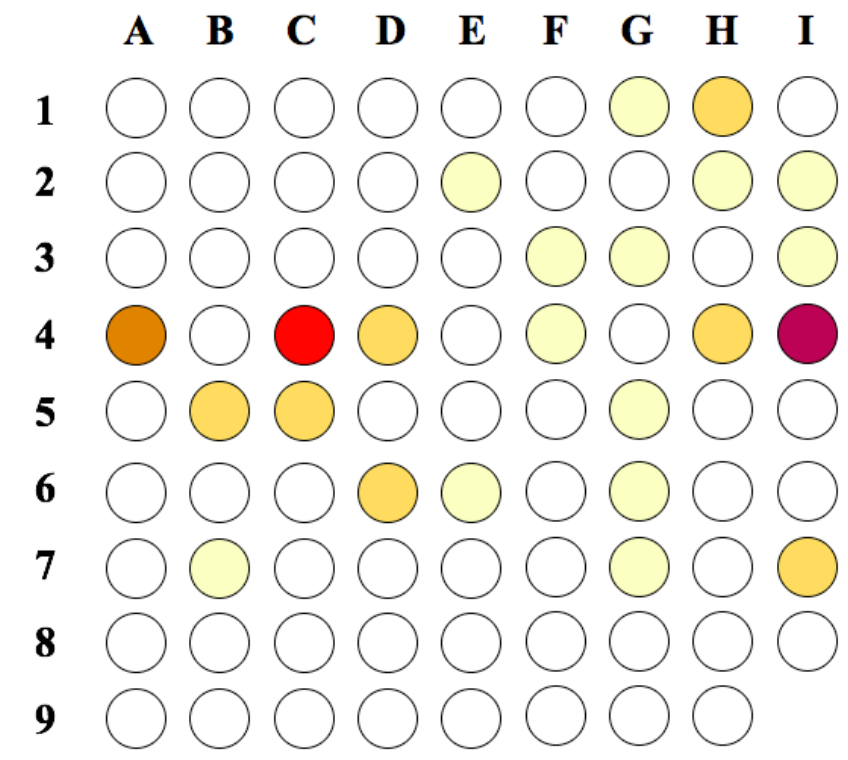

er of $\mathbf{1 0 k}$

color scale

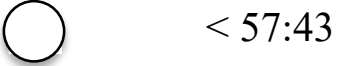

$57: 43-63: 37$

$63: 37-68: 32$

$68: 32-73: 27$

$73: 27-78: 22$

$78: 22-83: 17$

Figure 1: Graphical representation of the enantiomeric ratios of $10 \mathrm{k}$ in the screening of ligands.

\subsubsection{Optimization of the gold(I)-catalyzed 6-endo-dig cyclization}

\section{General procedure M: Screening of the JosiPhos-based ligands (10 $\mu \mathrm{mol}$ scale)}

Under argon atmosphere, 9k (1 equiv) was dissolved in $\mathrm{CH}_{2} \mathrm{Cl}_{2}(0.2 \mathrm{M})$ and water (5.0 equiv) was added. To this solution was added a second solution with the corresponding catalyst (10 mol \%) and $\mathrm{AgSbF}_{6}(20 \mathrm{~mol} \%)$ in $\mathrm{CH}_{2} \mathrm{Cl}_{2}(0.2 \mathrm{M})$. After $22 \mathrm{~h}$, an aliquot was taken and the enantiomeric excess of product 10k was measured. 


\section{Structure-selectivity investigations with JosiPhos-based ligands}

Table 7: Screening of JosiPhos-based chiral ligands

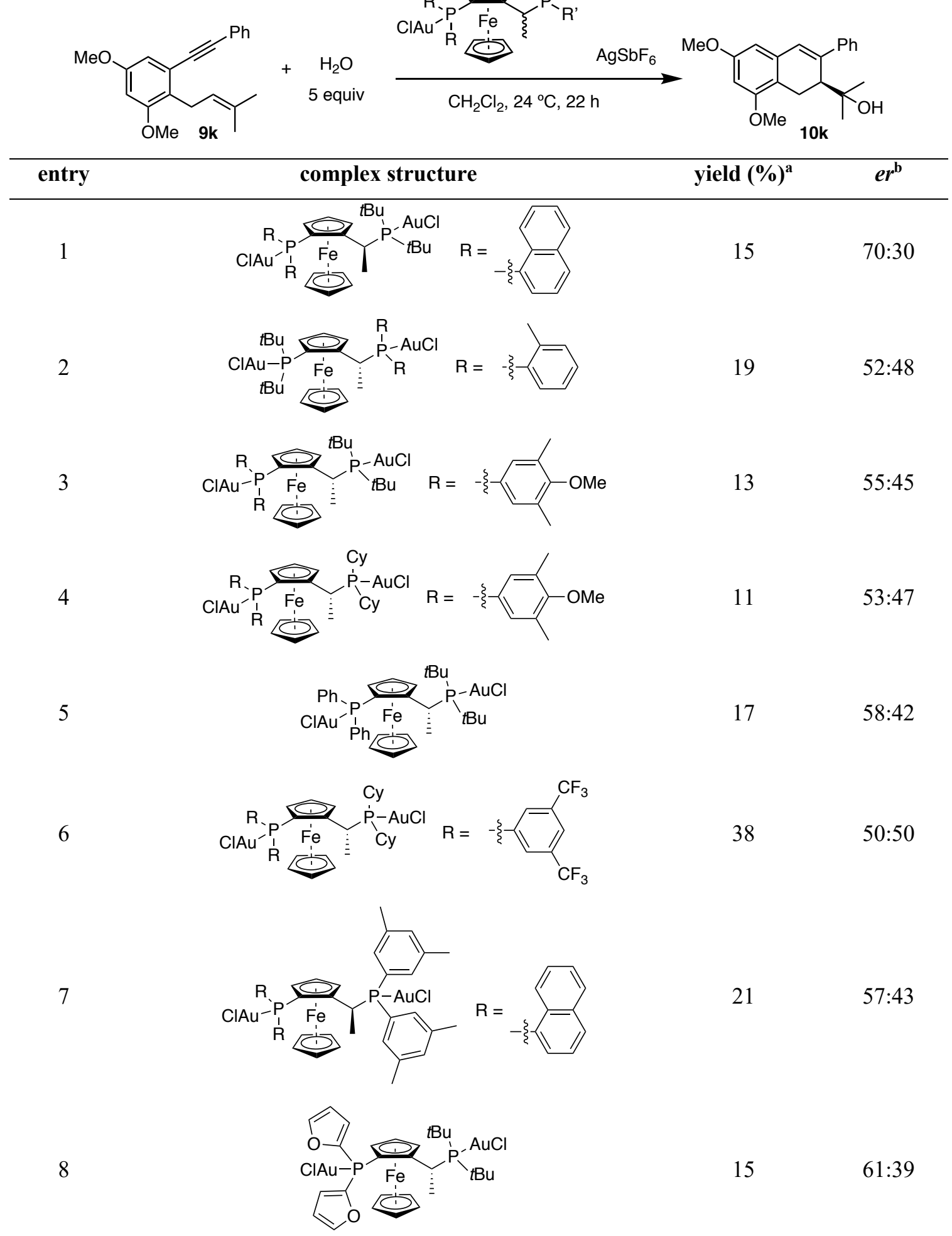




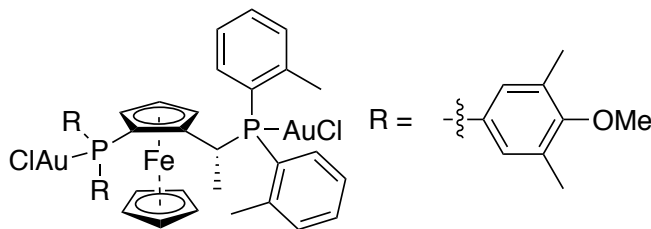

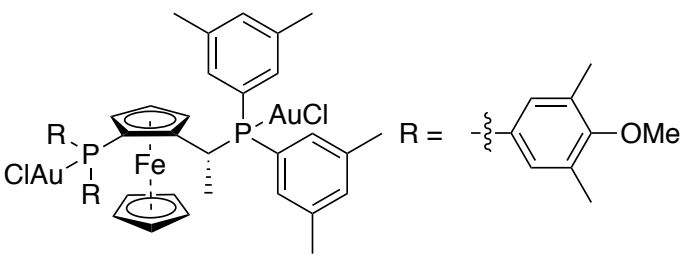

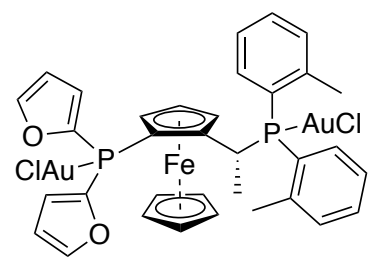

${ }^{\mathrm{a} 1} \mathrm{H}$ NMR yields measured (1,3,5-tribromobenzene as internal standard). ${ }^{\mathrm{b}}$ Enantiomeric excesses determined by SFC analysis using $\mathrm{UPC}^{2}$ (Chiralpak ID, PDA detector). Method with gradient: $\mathrm{CO}_{2} / \mathrm{EtOH}$ from $100 \% \mathrm{CO}_{2}$ (1 min) to 60:40 over 4 minutes, flow rate $=3 \mathrm{~mL} / \mathrm{min}, \mathrm{T}=35^{\circ} \mathrm{C}, \mathrm{ABPR}=1500 \mathrm{psi}, \lambda=254 \mathrm{~nm}, \mathrm{t}_{\mathrm{R}}$ (major) $=4.0 \mathrm{~min}, \mathrm{t}_{\mathrm{R}}($ minor $)=4.9$ $\min$

\section{General procedure N: enantioselective 6-endo-dig cyclization (10 mmol scale)}

Under argon atmosphere, 9k (1 equiv), the corresponding nucleophile (equiv see tables) and the gold catalyst (mol \% see tables) were dissolved in the respective solvent (concentration see tables). The chloride scavenger (mol \% see table) was added and the mixture was stirred for 24 $\mathrm{h}$ at $24{ }^{\circ} \mathrm{C}$. The reaction was quenched by addition of $\mathrm{NEt}_{3}$ and the crude was purified by preparative TLC. 


\section{Structure-selectivity investigations with the novel chiral complexes}

Table 8: Screening of complexes

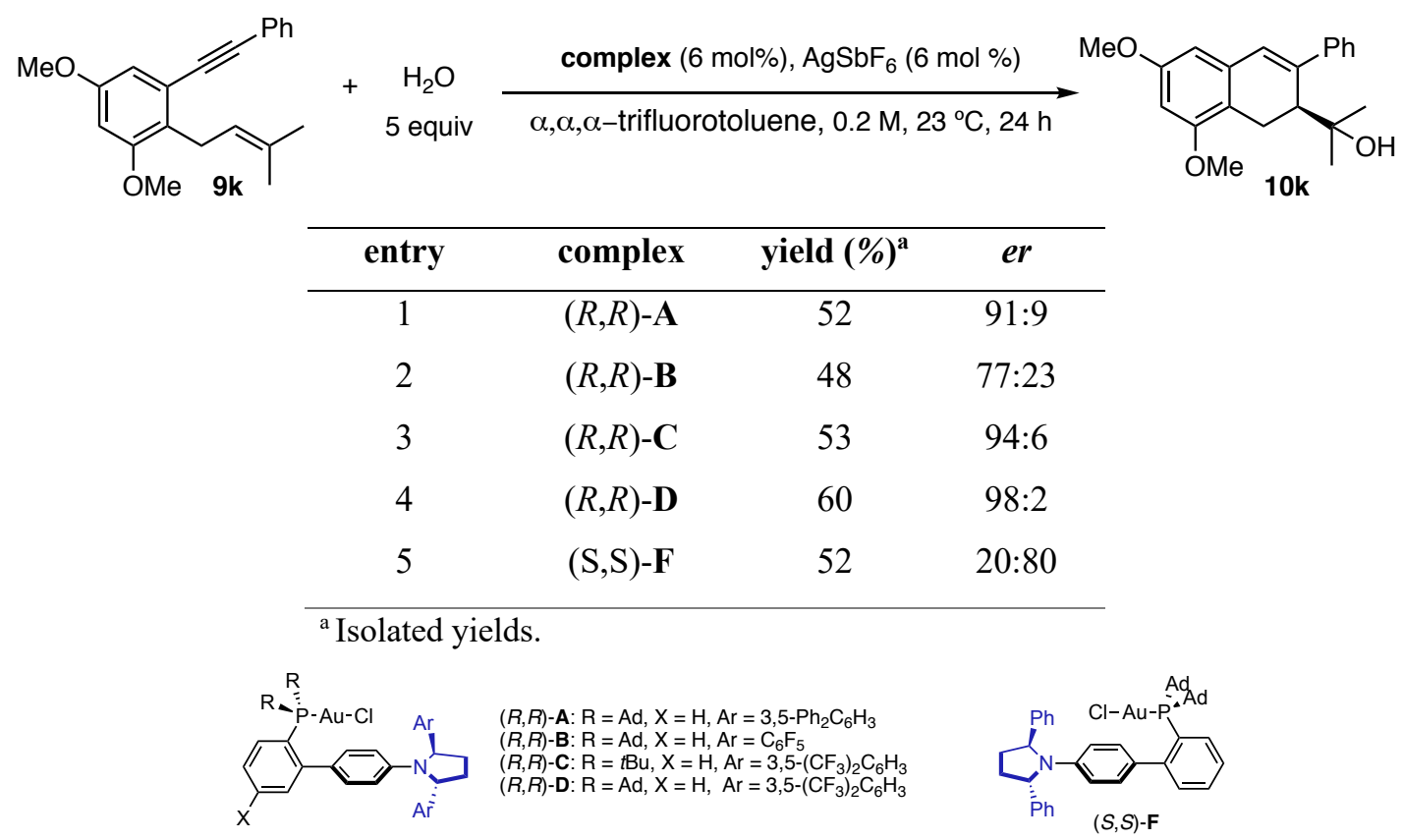

\section{Optimization of the reaction conditions}

Table 9: Screening of chloride scavengers and catalyst loading

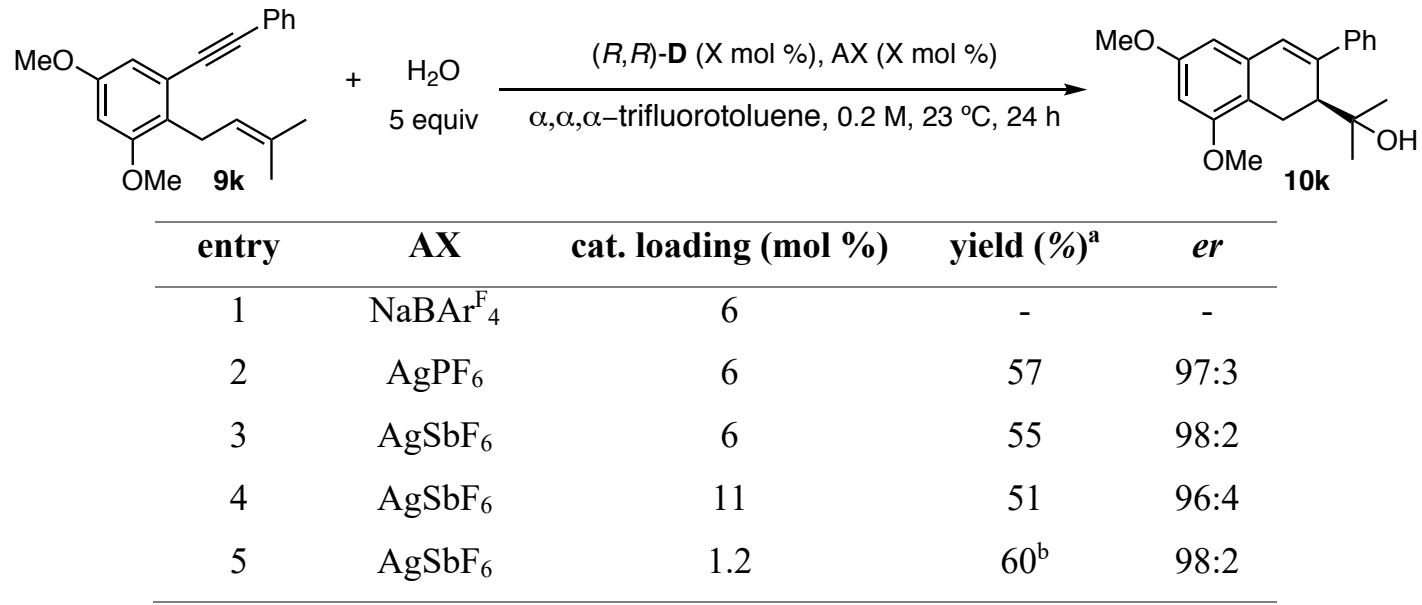

${ }^{\mathrm{a}}$ Isolated yields. ${ }^{\mathrm{b}}$ Reaction conducted at $6.7 \mathrm{mmol}$ scale. 
Table 10: Screening of solvents, concentration and temperature

\begin{tabular}{|c|c|c|c|c|c|}
\hline entry & solvent & concentration $(\mathbf{M})$ & $\mathrm{T}\left({ }^{\circ} \mathrm{C}\right)$ & yield $(\%)^{\mathrm{a}}$ & er \\
\hline 1 & $\alpha, \alpha, \alpha$-trifluorotoluene & 0.2 & 23 & 58 & $98: 2$ \\
\hline 2 & toluene & 0.2 & 23 & 10 & $97: 3$ \\
\hline 3 & 1,2-dichloroethane & 0.2 & 23 & 55 & $97: 3$ \\
\hline 4 & $\mathrm{CH}_{2} \mathrm{Cl}_{2}$ & 0.2 & 23 & 55 & $97: 3$ \\
\hline 5 & THF & 0.2 & 23 & 45 & $96: 4$ \\
\hline 6 & chlorobenzene & 0.2 & 23 & 50 & $96: 4$ \\
\hline 7 & $\alpha, \alpha, \alpha$-trifluorotoluene & 0.1 & 23 & 50 & $97: 3$ \\
\hline 8 & $\alpha, \alpha, \alpha$-trifluorotoluene & 0.4 & 23 & 57 & $98: 2$ \\
\hline 9 & $\alpha, \alpha, \alpha$-trifluorotoluene & 0.2 & 0 & 33 & $98: 2$ \\
\hline 10 & $\alpha, \alpha, \alpha$-trifluorotoluene & 0.2 & -20 & 30 & $98: 2$ \\
\hline 11 & $\alpha, \alpha, \alpha$-trifluorotoluene & 0.2 & 35 & 60 & $96: 4$ \\
\hline 12 & $\alpha, \alpha, \alpha$-trifluorotoluene & 0.2 & 50 & 65 & $95: 5$ \\
\hline
\end{tabular}

${ }^{\mathrm{a}}$ Isolated yields.

Table 11: Screening of chloride scavengers and solvents

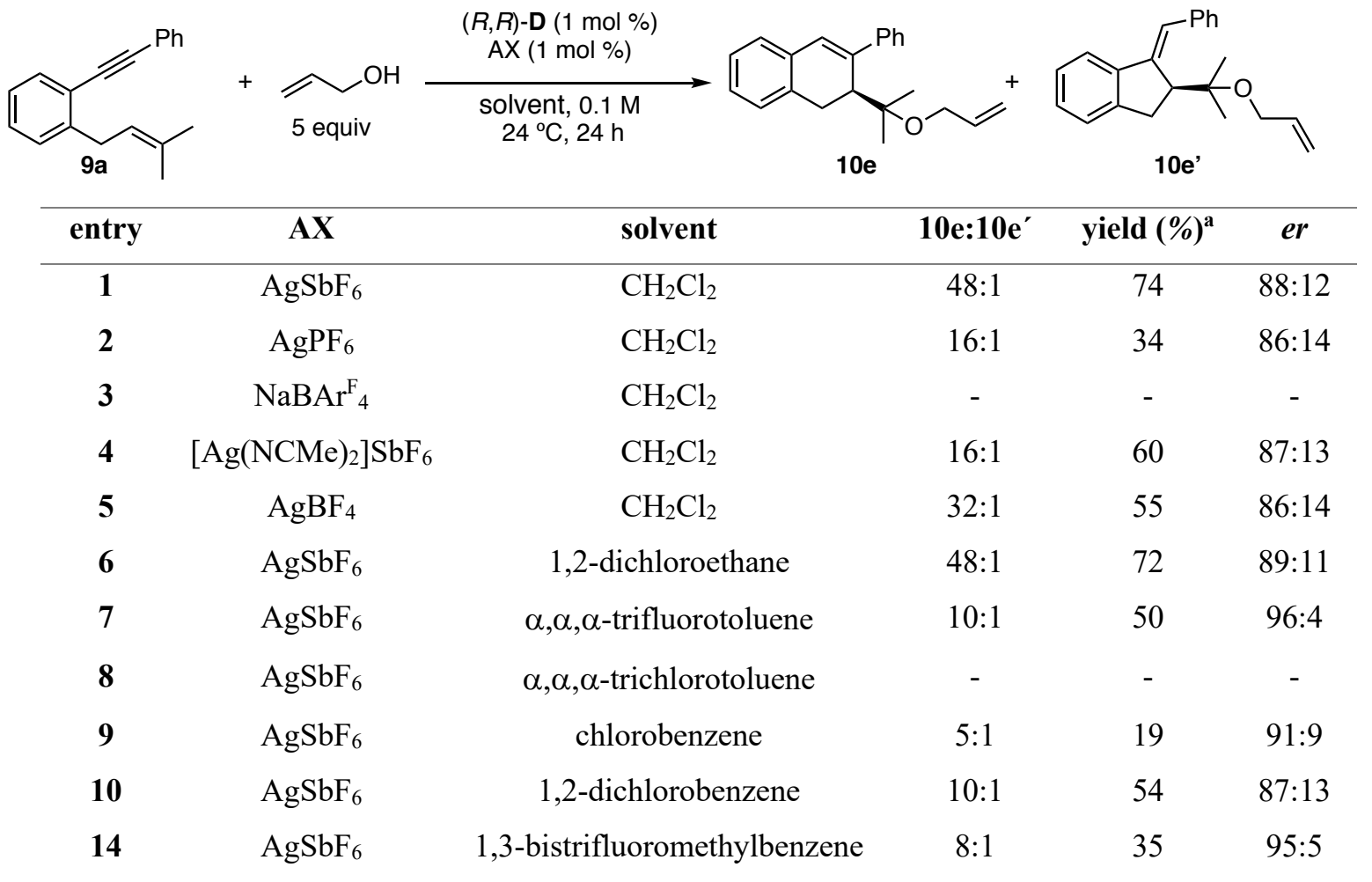

${ }^{\mathrm{a}}$ Isolated yields. 
Table 12: Screening of catalyst loading, equivalents of nucleophile, concentration and temperature

\begin{tabular}{|c|c|c|c|c|c|c|c|}
\hline & $+\overbrace{x \text { equiv }}^{0}$ & $\begin{array}{r}(R, R)-1 \\
\mathrm{AgSbF} \\
\alpha, \alpha, \alpha-\operatorname{tri} \\
\mathrm{XM}\end{array}$ & $\begin{array}{l}\mathrm{X} \mathrm{mol} \mathrm{\% )} \\
\mathrm{X} \mathrm{mol} \mathrm{\% )} \\
\underset{\text { orotoluene }}{2} 24 \mathrm{~h}\end{array}$ & $10 e^{\prime}$ & & 10 & \\
\hline entry & $\begin{array}{l}\text { cat. loading } \\
(\mathrm{mol} \%)\end{array}$ & $\begin{array}{l}\text { alcohol } \\
\text { (equiv) }\end{array}$ & $\begin{array}{c}\text { concentration } \\
\text { (M) }\end{array}$ & $\mathrm{T}\left({ }^{\circ} \mathrm{C}\right)$ & $\begin{array}{l}\text { ratio } \\
8 \mathrm{i}: 8 i^{\prime}\end{array}$ & $\begin{array}{l}\text { yield } \\
(\%)^{\mathrm{a}}\end{array}$ & $e r$ \\
\hline 1 & 2.0 & 5 & 0.1 & 25 & $12: 1$ & 69 & $95: 5$ \\
\hline 2 & 4.0 & 5 & 0.1 & 25 & $12: 1$ & 62 & $97: 3$ \\
\hline 3 & 1.0 & 2.5 & 0.1 & 25 & $33: 1$ & 55 & $97: 3$ \\
\hline 4 & 1.0 & 10 & 0.1 & 25 & $7: 1$ & 29 & $95: 5$ \\
\hline 5 & 1.0 & 5 & 0.05 & 25 & $15: 1$ & 65 & $97: 3$ \\
\hline 6 & 1.0 & 5 & 0.2 & 25 & $6: 1$ & 61 & $96: 4$ \\
\hline $7^{\mathrm{b}}$ & 1.0 & 5 & 0.1 & 0 & traces & - & - \\
\hline
\end{tabular}

${ }^{\mathrm{a}}$ Isolated yields.

\subsection{Reaction scope}

\section{General procedure O: protocol A for the enantioselective 6-endo-dig cyclization}

1,6-Enyne (1.0 equiv) and the corresponding nucleophile (2.5 equiv) were dissolved in $\alpha, \alpha, \alpha$-trifluorotoluene $(0.05 \mathrm{M})$. This solution was added to a vial containing gold catalyst $(R, R)-\mathbf{D}(2 \mathrm{~mol} \%)$ and $\mathrm{AgSbF}_{6}(2 \mathrm{~mol} \%)$ and the reaction was stirred for $24 \mathrm{~h}$ at $25{ }^{\circ} \mathrm{C}$. The reaction was quenched by addition of 1 drop of $\mathrm{NEt}_{3}$ and concentrated. The crude was purified by flash column chromatography.

\section{General procedure P: protocol B for the enantioselective 6-endo-dig cyclization}

1,6-Enyne (1.0 equiv) and $\mathrm{H}_{2} \mathrm{O}$ (5.0 equiv) were dissolved in $\alpha, \alpha, \alpha$-trifluorotoluene ( $\left.0.2 \mathrm{M}\right)$. This solution was added to a vial containing gold catalyst $(R, R)-\mathbf{D}(1 \mathrm{~mol} \%)$ and $\mathrm{AgSbF}_{6}(1$ mol \%) and the reaction was stirred for $24 \mathrm{~h}$ at $25^{\circ} \mathrm{C}$. The reaction was quenched by addition of 1 drop of $\mathrm{NEt}_{3}$ and concentrated. The crude was purified by flash column chromatography. 
(S)-2-(3-Phenyl-1,2-dihydronaphthalen-2-yl)propan-2-ol (10a)

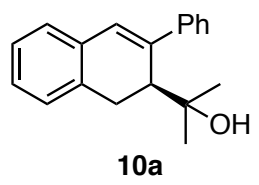

Compound 10a was synthesized following general procedure $\mathbf{O}$ from 9a (30.0 mg, 0.12 mmol). The crude was purified by flash column chromatography (pentane/ $\mathrm{CH}_{2} \mathrm{Cl}_{2} 4: 1$, then pentane $\left./ \mathrm{Et}_{2} \mathrm{O} 1: 1\right)$ to afford $10 \mathrm{a}(21.9 \mathrm{mg}, 0.083 \mathrm{mmol}, 68 \%)$ as a white solid in $97: 3 \mathrm{er}$.

M.p. $=138-140{ }^{\circ} \mathrm{C}$ (pentane). ${ }^{1} \mathbf{H}$ NMR $\left(400 \mathrm{MHz}, \mathrm{CD}_{2} \mathrm{Cl}_{2}\right) \delta 7.58-7.50(\mathrm{~m}, 2 \mathrm{H}), 7.43-$ 7.34 (m, 2H), $7.33-7.25$ (m, 1H), $7.21-7.09$ (m, 4H), $6.86(\mathrm{~s}, 1 \mathrm{H}), 3.32-3.19(\mathrm{~m}, 2 \mathrm{H}), 3.11$ $(\mathrm{dd}, J=7.1,2.3 \mathrm{~Hz}, 1 \mathrm{H}), 1.18-1.12(\mathrm{~m}, 1 \mathrm{H}), 0.96(\mathrm{~s}, 3 \mathrm{H}), 0.88(\mathrm{~s}, 3 \mathrm{H}) .{ }^{13} \mathrm{C}$ NMR $(126 \mathrm{MHz}$, $\left.\mathrm{CD}_{2} \mathrm{Cl}_{2}\right) \delta 143.9,140.9,136.0,135.2,129.1,128.4,128.1,127.8,127.6,127.2,126.9,126.8$, 75.3, 46.0, 31.3, 29.2, 28.1. HRMS (ESI+) calculated for $\left[\mathrm{C}_{19} \mathrm{H}_{20} \mathrm{NaO}\right]^{+} 287.1406 \mathrm{~m} / \mathrm{z}$; found $[\mathrm{M}+\mathrm{Na}]^{+} 287.1394 \mathrm{~m} / z \cdot \boldsymbol{\alpha}_{\mathbf{D}}{ }^{\mathbf{5 8 9}}=-263.5 \mathrm{deg} \cdot \mathrm{cm}^{2} \cdot \mathrm{g}^{-1}\left(\mathrm{CHCl}_{3}\right.$, c $\left.0.83,299 \mathrm{~K}\right) . \mathbf{U P C} \mathbf{C}^{2}$ Chiralpak IC $(150 \times 4.6 \mathrm{~mm}, 3 \mu \mathrm{m})$ at $35^{\circ} \mathrm{C}$, flow $3 \mathrm{~mL} / \mathrm{min}$, isocratic $\mathrm{CO}_{2} / \mathrm{MeOH} 95: 5$, ABRP pressure $1500 \mathrm{psi}, 294 \mathrm{~nm}, \mathrm{t}_{\mathrm{R}}$ (major) 2.8; $\mathrm{t}_{\mathrm{R}}$ (minor) 3.1 .

(S)-2-(3-Phenyl-6-(trifluoromethyl)-1,2-dihydronaphthalen-2-yl)propan-2-ol (10b)

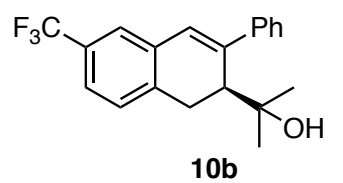

Compound 10b was synthesized following general procedure $\mathbf{O}$ from $9 \mathbf{b}(30.0 \mathrm{mg}, 0.095$ mmol). The crude was purified by flash column chromatography (pentane/ $\mathrm{CH}_{2} \mathrm{Cl}_{2} 4: 1$, then pentane/ $\left./ \mathrm{Et}_{2} \mathrm{O} 1: 1\right)$ to afford $\mathbf{1 0 b}(21.9 \mathrm{mg}, 0.066 \mathrm{mmol}, 69 \%)$ as a colorless oil in 94:6 er.

${ }^{1} \mathbf{H}$ NMR $\left(400 \mathrm{MHz}, \mathrm{CD}_{2} \mathrm{Cl}_{2}\right) \delta 7.56-7.52(\mathrm{~m}, 2 \mathrm{H}), 7.44-7.41(\mathrm{~m}, 4 \mathrm{H}), 7.34-7.27(\mathrm{~m}$, 2H), $6.87(\mathrm{~s}, 1 \mathrm{H}), 3.40(\mathrm{~d}, J=16.7 \mathrm{~Hz}, 1 \mathrm{H}), 3.26(\mathrm{ddt}, J=16.7,8.2,1.7 \mathrm{~Hz}, 1 \mathrm{H}), 3.16$ (dd, $J=$ 8.2, $1.2 \mathrm{~Hz}, 1 \mathrm{H}), 1.16$ (br s, $1 \mathrm{H}), 0.95$ (s, 3H), 0.88 (s, 3H). $\left.{ }^{13} \mathbf{C ~ N M R ~ ( 1 0 1 ~ M H z , ~} \mathrm{CD}_{2} \mathrm{Cl}_{2}\right) \delta$ 143.3, 142.8, 140.4 - $140.3(\mathrm{~m}), 135.8,129.2,129.1$ (q, $J=32.1 \mathrm{~Hz}), 128.2,127.9,127.22$, 127.17, 125.1 (q, $J=272.0 \mathrm{~Hz}), 124.5$ (q, $J=4.0 \mathrm{~Hz}), 123.1$ (q, $J=3.8 \mathrm{~Hz}), 75.2,45.9,31.2$, 29.4, 27.9. ${ }^{19} \mathbf{F}\left\{{ }^{1} \mathbf{H}\right\}$ NMR $\left(376 \mathrm{MHz}, \mathrm{CD}_{2} \mathrm{Cl}_{2}\right) \delta-62.8$. HRMS (APCI+) calculated for $\left[\mathrm{C}_{20} \mathrm{H}_{18} \mathrm{~F}_{3}\right]^{+} 315.1355 \mathrm{~m} / z$; found $[\mathrm{M}-\mathrm{OH}]^{+} 315.1355 \mathrm{~m} / z \cdot \boldsymbol{\alpha}_{\mathbf{D}}{ }^{\mathbf{5 8 9}}=-182.7 \mathrm{deg} \cdot \mathrm{cm}^{2} \cdot \mathrm{g}^{-1}\left(\mathrm{CHCl}_{3}\right.$, c $0.98,299 \mathrm{~K})$. HPLC Chiralpak OJ-H $(250 \mathrm{~mm} \times 4.6 \mathrm{~mm}, 5 \mu \mathrm{m})$ at $25^{\circ} \mathrm{C}$, flow $1.0 \mathrm{~mL} / \mathrm{min}$, isocratic hexane $/ \mathrm{PrOH} 90: 10,280 \mathrm{~nm}, \mathrm{t}_{\mathrm{R}}$ (major) 4.3 ; $\mathrm{t}_{\mathrm{R}}$ (minor) 6.5 . 
(S)-2-(6-Chloro-3-phenyl-1,2-dihydronaphthalen-2-yl)propan-2-ol (10c)

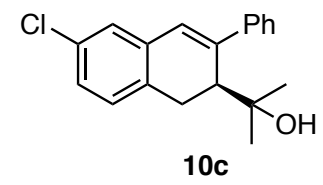

Compound 10c was synthesized following general procedure $\mathbf{O}$ from $9 \mathbf{c}(50 \mathrm{mg}, 0.178$ mmol). The crude was purified by flash column chromatography (pentane/ $\mathrm{CH}_{2} \mathrm{Cl}_{2} 2: 1$, then pentane/Et $2 \mathrm{O} 1: 1)$ to afford $10 \mathrm{c}(23 \mathrm{mg}, 0.077 \mathrm{mmol}, 43 \%)$ as a white solid in 95:5 er.

M.p. $=105-108{ }^{\circ} \mathrm{C}$ (pentane). ${ }^{1} \mathbf{H}$ NMR $\left(400 \mathrm{MHz}, \mathrm{CDCl}_{3}\right) \delta 7.55-7.47$ (m, 2H), $7.43-$ 7.35 (m, 2H), $7.33-7.27(\mathrm{~m}, 1 \mathrm{H}), 7.14-7.05(\mathrm{~m}, 3 \mathrm{H}), 6.77$ (s, 1H), $3.28(\mathrm{~d}, J=15.9 \mathrm{~Hz}, 1 \mathrm{H})$, $3.25-3.09(\mathrm{~m}, 2 \mathrm{H}), 1.00(\mathrm{~s}, 3 \mathrm{H}), 0.90$ (s, 3H). ${ }^{13} \mathbf{C ~ N M R}\left(101 \mathrm{MHz}, \mathrm{CDCl}_{3}\right) \delta 142.8,141.9$, 136.2, 133.7, 132.0, 128.9, 128.3, 127.8, 127.4, 126.9, 126.8, 126.1, 75.1, 45.7, 30.3, 29.2, 27.7. HRMS (ESI+) calculated for $\left[\mathrm{C}_{19} \mathrm{H}_{19} \mathrm{ClNaO}\right]^{+} 321.1017 \mathrm{~m} / z$; found $[\mathrm{M}+\mathrm{Na}]^{+}$found 321.1011 $m / z \cdot \boldsymbol{\alpha}_{\mathbf{D}}{ }^{\mathbf{5 8 9}}=-170.4$ deg. $\mathrm{cm}^{2} \cdot \mathrm{g}^{-1}\left(\mathrm{CHCl}_{3}, \mathrm{c} 0.61,299 \mathrm{~K}\right)$. HPLC Chiralpak IA $(250 \mathrm{~mm} \times 4.6$ $\mathrm{mm}, 5 \mu \mathrm{m}$ ) at $25^{\circ} \mathrm{C}$, flow $1.0 \mathrm{~mL} / \mathrm{min}$, isocratic hexane $/ \mathrm{PrOH} 90: 10,280 \mathrm{~nm}, \mathrm{t}_{\mathrm{R}}$ (major) 6.3; $\mathrm{t}_{\mathrm{R}}$ (minor) 21.1.

(S)-2-(6-Methoxy-3-phenyl-1,2-dihydronaphthalen-2-yl)propan-2-ol (10d)

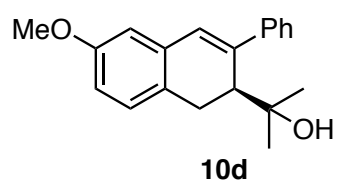

Compound 10d was synthesized following general procedure $\mathbf{O}$ from 9d (50.0 $\mathrm{mg}$ g, 0.181 mmol). The crude was purified by flash column chromatography (pentane $/ \mathrm{CH}_{2} \mathrm{Cl}_{2} 2: 1$, then pentane $\left./ \mathrm{Et}_{2} \mathrm{O} 1: 1\right)$ to afford $\mathbf{1 0 d}(32.1 \mathrm{mg}, 0.109 \mathrm{mmol}, 60 \%)$ as a white solid in 97:3 er.

M.p. $=103-106{ }^{\circ} \mathrm{C}$ (pentane). ${ }^{1} \mathbf{H}$ NMR $\left(500 \mathrm{MHz}, \mathrm{CDCl}_{3}\right) \delta 7.56-7.52(\mathrm{~m}, 2 \mathrm{H}), 7.41-$ $7.36(\mathrm{~m}, 2 \mathrm{H}), 7.31-7.27(\mathrm{~m}, 1 \mathrm{H}), 7.07$ (ddd, $J=8.0,1.3,0.7 \mathrm{~Hz}, 1 \mathrm{H}), 6.82(\mathrm{~s}, 1 \mathrm{H}), 6.73-6.69$ $(\mathrm{m}, 2 \mathrm{H}), 3.81(\mathrm{~s}, 3 \mathrm{H}), 3.26-3.15(\mathrm{~m}, 2 \mathrm{H}), 3.11(\mathrm{dd}, J=7.3,1.8 \mathrm{~Hz}, 1 \mathrm{H}), 1.01(\mathrm{~s}, 3 \mathrm{H}), 0.92$ (s, $3 \mathrm{H}) .{ }^{13} \mathrm{C}$ NMR $\left(126 \mathrm{MHz}, \mathrm{CDCl}_{3}\right) \delta 158.5,143.2,140.9,135.6,128.8,128.1,127.8,127.5$, 127.3, 126.8, 112.7, 112.2, 75.2, 55.4, 45.9, 30.1, 29.1, 27.8. HRMS (ESI+) calculated for $\left[\mathrm{C}_{20} \mathrm{H}_{22} \mathrm{NaO}_{2}\right]^{+} 317.1512 \mathrm{~m} / z$; found $[\mathrm{M}+\mathrm{Na}]^{+}$found $317.1500 \mathrm{~m} / z \cdot \boldsymbol{\alpha}_{\mathbf{D}}{ }^{\mathbf{5 8 9}}=-173.0 \mathrm{deg} \cdot \mathrm{cm}^{2} \cdot \mathrm{g}^{-}$ ${ }^{1}\left(\mathrm{CHCl}_{3}\right.$, c $\left.1.03,299 \mathrm{~K}\right)$. UPC ${ }^{2}$ Chiralpak IA $(150 \times 4.6 \mathrm{~mm}, 3 \mu \mathrm{m})$ at $35^{\circ} \mathrm{C}$, flow $3 \mathrm{~mL} / \mathrm{min}$, isocratic $\mathrm{CO}_{2} / \mathrm{iPrOH}$ 75:25, ABRP pressure $1500 \mathrm{psi}, 242 \mathrm{~nm}, \mathrm{t}_{\mathrm{R}}$ (major) 1.3; $\mathrm{t}_{\mathrm{R}}$ (minor) 2.8 . 
(S)-2-(2-(Allyloxy)propan-2-yl)-3-phenyl-1,2-dihydronaphthalene (10e)

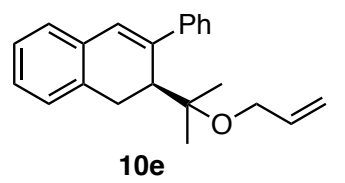

Compound 10e was synthesized following general procedure $\mathbf{O}$ from 9a (30.0 mg, 0.12 mmol). The crude was purified by flash column chromatography (pentane/ $\mathrm{CH}_{2} \mathrm{Cl}_{2} 4: 1$, then pentane/ $\left.\mathrm{Et}_{2} \mathrm{O} 9: 1\right)$ to afford $10 \mathrm{e}(23.4 \mathrm{mg}, 0.077 \mathrm{mmol}, 63 \%)$ as a light-yellow solid in 95:5 er.

M.p. $=78-80{ }^{\circ} \mathrm{C}$ (pentane). ${ }^{1} \mathbf{H}$ NMR $\left(400 \mathrm{MHz}, \mathrm{CD}_{2} \mathrm{Cl}_{2}\right) \delta 7.56-7.50(\mathrm{~m}, 2 \mathrm{H}), 7.42-$ 7.35 (m, 2H), $7.32-7.26$ (m, 1H), $7.19-7.10$ (m, 4H), 6.80 (s, 1H), 5.77 (ddt, $J=17.2$, 10.4, $5.2 \mathrm{~Hz}, 1 \mathrm{H}), 5.16(\mathrm{dq}, J=17.2,1.8 \mathrm{~Hz}, 1 \mathrm{H}), 5.04(\mathrm{dq}, J=10.4,1.6 \mathrm{~Hz}, 1 \mathrm{H}), 3.87$ (ddt, $J=12.5$, 5.3, $1.6 \mathrm{~Hz}, 1 \mathrm{H}), 3.80$ (ddt, $J=12.4,5.1,1.6 \mathrm{~Hz}, 1 \mathrm{H}), 3.40$ (d, $J=16.4 \mathrm{~Hz}, 1 \mathrm{H}), 3.30$ (d, $J=$ $8.4 \mathrm{~Hz}, 1 \mathrm{H}), 3.17(\mathrm{dd}, J=16.3,8.4 \mathrm{~Hz}, 1 \mathrm{H}), 0.89$ (s, 3H), 0.75 (s, 3H). ${ }^{13} \mathbf{C}$ NMR $(126 \mathrm{MHz}$, $\left.\mathrm{CD}_{2} \mathrm{Cl}_{2}\right) \delta 144.4,141.2,136.8,136.4,135.3,129.0,128.91,128.87,128.0,127.6,127.5,127.2$, 126.7, 115.3, 79.6, 62.8, 42.9, 30.4, 25.7, 23.2. HRMS (ESI+) calculated for $\left[\mathrm{C}_{22} \mathrm{H}_{24} \mathrm{NaO}\right]^{+}$ $327.1719 \mathrm{~m} / z$; found $[\mathrm{M}+\mathrm{Na}]^{+} 327.1712 \mathrm{~m} / z \cdot \boldsymbol{\alpha}_{\mathbf{D}}{ }^{\mathbf{5 8 9}}=-181.4 \mathrm{deg} \cdot \mathrm{cm}^{2} \cdot \mathrm{g}^{-1}\left(\mathrm{CHCl}_{3}, \mathrm{c} 0.66,299\right.$ K). $\mathbf{U P C}^{2}$ Chiralpak IC $(150 \times 4.6 \mathrm{~mm}, 3 \mu \mathrm{m})$ at $35^{\circ} \mathrm{C}$, flow $3 \mathrm{~mL} / \mathrm{min}$, isocratic $\mathrm{CO}_{2} / \mathrm{MeOH}$ 95:5, ABRP pressure $1500 \mathrm{psi}, 293 \mathrm{~nm}, \mathrm{t}_{\mathrm{R}}$ (major) 1.1; $\mathrm{t}_{\mathrm{R}}$ (minor) 1.3.

(S)-2-(2-(Benzyloxy)propan-2-yl)-3-phenyl-1,2-dihydronaphthalene (10f)

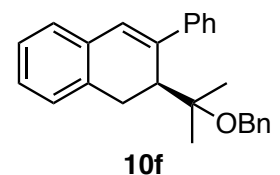

Compound 10f was synthesized following general procedure $\mathbf{O}$ from 9a (30.0 mg, 0.12 mmol). The crude was purified by flash column chromatography (pentane $/ \mathrm{CH}_{2} \mathrm{Cl}_{2} 4: 1$, then pentane/Et $\left.{ }_{2} \mathrm{O} 9: 1\right)$ to afford $\mathbf{1 0 f}(32.4 \mathrm{mg}, 0.091 \mathrm{mmol}, 75 \%)$ as a white solid in 95:5 er.

M.p. $=79-81{ }^{\circ} \mathrm{C}$ (pentane). ${ }^{1} \mathbf{H}$ NMR $\left(400 \mathrm{MHz}, \mathrm{CD}_{2} \mathrm{Cl}_{2}\right) \delta 7.57-7.52(\mathrm{~m}, 2 \mathrm{H}), 7.41-$ $7.34(\mathrm{~m}, 2 \mathrm{H}), 7.31-7.20(\mathrm{~m}, 4 \mathrm{H}), 7.19-7.11(\mathrm{~m}, 6 \mathrm{H}), 6.82(\mathrm{~s}, 1 \mathrm{H}), 4.39(\mathrm{~d}, J=11.2 \mathrm{~Hz}, 1 \mathrm{H})$, $4.31(\mathrm{~d}, J=11.2 \mathrm{~Hz}, 1 \mathrm{H}), 3.45(\mathrm{~d}, J=16.5 \mathrm{~Hz}, 1 \mathrm{H}), 3.36$ (d, $J=8.4 \mathrm{~Hz}, 1 \mathrm{H}), 3.19$ (dd, $J=16.3$, $8.2 \mathrm{~Hz}, 1 \mathrm{H}), 0.95$ (s, 3H), 0.84 (s, 3H). ${ }^{13} \mathrm{C}$ NMR (126 MHz, $\left.\mathrm{CD}_{2} \mathrm{Cl}_{2}\right) \delta 144.4,141.1,140.4$, 136.5, 135.3, 129.0, 128.9, 128.6, 128.0, 127.8, 127.6, 127.50, 127.47, 127.3, 126.7, 79.9, 63.8, 43.3, 30.5, 25.6, 23.2. HRMS (ESI+) calculated for $\left[\mathrm{C}_{26} \mathrm{H}_{26} \mathrm{NaO}\right]^{+} 377.1876 \mathrm{~m} / \mathrm{z}$; found $[\mathrm{M}+$ $\mathrm{Na}]^{+} 377.1869 \mathrm{~m} / z \cdot \boldsymbol{\alpha}_{\mathbf{D}}{ }^{\mathbf{5 8 9}}=-137.1 \mathrm{deg} \cdot \mathrm{cm}^{2} \cdot \mathrm{g}^{-1}\left(\mathrm{CHCl}_{3}, \mathrm{c} 1.19,299 \mathrm{~K}\right)$. UPC ${ }^{2}$ Chiralpak IC $(150 \times 4.6 \mathrm{~mm}, 3 \mu \mathrm{m})$ at $35^{\circ} \mathrm{C}$, flow $3 \mathrm{~mL} / \mathrm{min}$, isocratic $\mathrm{CO}_{2} / \mathrm{MeOH} 90: 10$, ABRP pressure $1500 \mathrm{psi}, 293 \mathrm{~nm}, \mathrm{t}_{\mathrm{R}}$ (major) 1.4; $\mathrm{t}_{\mathrm{R}}$ (minor) 1.7. 
(S)-2-(3-Phenyl-1,2-dihydronaphthalen-2-yl)propan-2-yl acetate (10g)

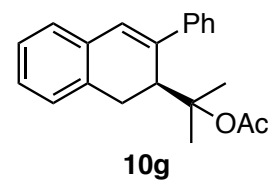

Compound 10g was synthesized following general procedure $\mathbf{O}$ from 9a $(30.0 \mathrm{mg}, 0.12$ mmol). The crude was purified by flash column chromatography (pentane $/ \mathrm{CH}_{2} \mathrm{Cl}_{2} 4: 1$, then pentane/ $\left./ \mathrm{Et}_{2} \mathrm{O} 2: 1\right)$ to afford $\mathbf{1 0 g}(15.3 \mathrm{mg}, 0.050 \mathrm{mmol}, 41 \%)$ as a white solid in 90:10 er.

M.p. $=93-96{ }^{\circ} \mathrm{C}$ (pentane). ${ }^{1} \mathbf{H}$ NMR $\left(400 \mathrm{MHz}, \mathrm{CD}_{2} \mathrm{Cl}_{2}\right) \delta 7.51-7.46(\mathrm{~m}, 2 \mathrm{H}), 7.41-$ $7.35(\mathrm{~m}, 2 \mathrm{H}), 7.30-7.24(\mathrm{~m}, 1 \mathrm{H}), 7.18-7.11(\mathrm{~m}, 4 \mathrm{H}), 6.82(\mathrm{~s}, 1 \mathrm{H}), 3.88(\mathrm{dd}, J=8.4,1.3 \mathrm{~Hz}$, 1H), 3.27 (dd, $J=16.7,8.4 \mathrm{~Hz}, 1 \mathrm{H}), 3.11(\mathrm{dd}, J=16.7,1.2 \mathrm{~Hz}, 1 \mathrm{H}), 1.39$ (s, 3H), 1.34 (s, 3H), $1.00(\mathrm{~s}, 3 \mathrm{H}) .{ }^{13} \mathrm{C}$ NMR $\left(126 \mathrm{MHz}, \mathrm{CD}_{2} \mathrm{Cl}_{2}\right) \delta 170.8,143.9,140.8,135.5,135.2,129.1,128.9$, 128.1, 127.5, 127.4, 127.0, 126.7, 86.0, 41.8, 31.1, 25.2, 25.0, 22.2. HRMS (ESI+) calculated for $\left[\mathrm{C}_{21} \mathrm{H}_{22} \mathrm{NaO}_{2}\right]^{+} 329.1512 \mathrm{~m} / z$; found $[\mathrm{M}+\mathrm{Na}]^{+} 329.1514 \mathrm{~m} / z \cdot \boldsymbol{\alpha}_{\mathbf{D}} \mathbf{5 8 9}=-196.4 \mathrm{deg} \cdot \mathrm{cm}^{2} \cdot \mathrm{g}^{-1}$ $\left(\mathrm{CHCl}_{3}, \mathrm{c} 0.46,299 \mathrm{~K}\right)$. UPC ${ }^{2}$ Chiralpak IC $(150 \times 4.6 \mathrm{~mm}, 3 \mu \mathrm{m})$ at $35{ }^{\circ} \mathrm{C}$, flow $3 \mathrm{~mL} / \mathrm{min}$, isocratic $\mathrm{CO}_{2} / \mathrm{MeOH}$ 95:5, ABRP pressure $1500 \mathrm{psi}, 290 \mathrm{~nm}, \mathrm{t}_{\mathrm{R}}$ (major) 1.7; $\mathrm{t}_{\mathrm{R}}$ (minor) 1.8 .

(S)-2-(2-Fluoropropan-2-yl)-3-phenyl-1,2-dihydronaphthalene (10h)

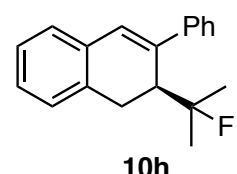

Compound 10h was synthesized following general procedure $\mathbf{O}$ from 9a (20.0 mg, 0.08 mmol) and 5 equiv of HF pyridine complex. The reaction was carried out in an Eppendorf vial using $3 \mathrm{~mol} \%$ of gold catalyst and silver salt each. The crude was purified by flash column chromatography (pentane) to afford $\mathbf{1 0 h}(8.44 \mathrm{mg}, 0.032 \mathrm{mmol}, 40 \%)$ as a white solid in $96: 4$ er.

M.p. $=72-75^{\circ} \mathrm{C}$ (pentane). ${ }^{1} \mathbf{H}$ NMR $\left(400 \mathrm{MHz}, \mathrm{CD}_{2} \mathrm{Cl}_{2}\right) \delta 7.56-7.51(\mathrm{~m}, 2 \mathrm{H}), 7.42-$ 7.35 (m, 2H), $7.32-7.26(\mathrm{~m}, 1 \mathrm{H}), 7.21-7.11(\mathrm{~m}, 4 \mathrm{H}), 6.87(\mathrm{~s}, 1 \mathrm{H}), 3.38-3.29(\mathrm{~m}, 1 \mathrm{H}), 3.28$ - $3.23(\mathrm{~m}, 2 \mathrm{H}), 1.13(\mathrm{~d}, J=21.9 \mathrm{~Hz}, 3 \mathrm{H}), 0.94(\mathrm{~d}, J=22.9 \mathrm{~Hz}, 3 \mathrm{H}) .{ }^{13} \mathbf{C}$ NMR $(126 \mathrm{MHz}$, $\left.\mathrm{CD}_{2} \mathrm{Cl}_{2}\right) \delta 143.3,139.6(\mathrm{~d}, J=6.2 \mathrm{~Hz}), 135.4,135.0,129.0,128.6,128.2,127.8,127.6,127.2$, 127.0, 126.9, 99.0 (d, $J=170.8 \mathrm{~Hz}), 44.4$ (d, $J=22.7 \mathrm{~Hz}), 30.7$ (d, $J=6.6 \mathrm{~Hz}), 27.0$ (d, $J=$ $24.0 \mathrm{~Hz}), 24.9(\mathrm{~d}, J=24.6 \mathrm{~Hz}) .{ }^{19} \mathbf{F}\left\{{ }^{1} \mathbf{H}\right\}$ NMR $\left(376 \mathrm{MHz}, \mathrm{CD}_{2} \mathrm{Cl}_{2}\right) \delta-132.9 . \mathbf{H R M S}(\mathrm{ESI}+)$ calculated for $\left[\mathrm{C}_{19} \mathrm{H}_{19} \mathrm{FNa}\right]^{+} 289.1363 \mathrm{~m} / z$; found $[\mathrm{M}+\mathrm{Na}]^{+} 289.1360 \mathrm{~m} / z \cdot \boldsymbol{\alpha}_{\mathbf{D}}{ }^{\mathbf{5 8 9}}=-164.5$ deg. $\mathrm{cm}^{2} \cdot \mathrm{g}^{-1}\left(\mathrm{CHCl}_{3}\right.$, c $\left.0.165,299 \mathrm{~K}\right)$. UPC ${ }^{2}$ Chiralpak IG $(150 \times 4.6 \mathrm{~mm}, 3 \mu \mathrm{m})$ at $35^{\circ} \mathrm{C}$, flow 
$2 \mathrm{~mL} / \mathrm{min}$, isocratic $\mathrm{CO}_{2} / \mathrm{MeOH}$ 85:15, ABRP pressure $2000 \mathrm{psi}, 292 \mathrm{~nm}, \mathrm{t}_{\mathrm{R}}$ (major) 1.9; $\mathrm{t}_{\mathrm{R}}$ (minor) 4.6.

(S)-3-(4-Chlorophenyl)-2-(2-methoxypropan-2-yl)-1,2-dihydronaphthalene (10i)

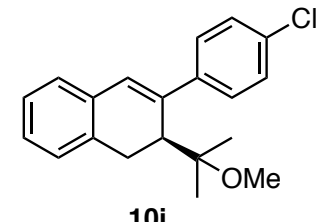

Compound 10i was synthesized following general procedure $\mathbf{O}$ from $9 \mathbf{i}$ (120.0 mg, 0.427 mmol). The crude was purified by preparative TLC (pentane/diethyl ether 95:5) to afford 10i (67.0 $\mathrm{mg}, 0.214 \mathrm{mmol}, 50 \%)$ as a white solid in 96:4 er.

M.p. $=116-118{ }^{\circ} \mathrm{C}$ (pentane). ${ }^{1} \mathbf{H}$ NMR $\left(500 \mathrm{MHz}, \mathrm{CD}_{2} \mathrm{Cl}_{2}\right) \delta 7.48-7.43(\mathrm{~m}, 2 \mathrm{H}), 7.37-$ $7.33(\mathrm{~m}, 2 \mathrm{H}), 7.16-7.09(\mathrm{~m}, 4 \mathrm{H}), 6.77(\mathrm{~s}, 1 \mathrm{H}), 3.32(\mathrm{~d}, J=16.1 \mathrm{~Hz}, 1 \mathrm{H}), 3.19$ (d, $J=8.6 \mathrm{~Hz}$, 1H), $3.12(\mathrm{dd}, J=16.1,8.4 \mathrm{~Hz}, 1 \mathrm{H}), 3.07(\mathrm{~s}, 3 \mathrm{H}), 0.82(\mathrm{~s}, 3 \mathrm{H}), 0.68(\mathrm{~s}, 3 \mathrm{H}) .{ }^{13} \mathrm{C}$ NMR (126 $\left.\mathrm{MHz}, \mathrm{CD}_{2} \mathrm{Cl}_{2}\right) \delta 143.0,139.9,136.4,134.9,133.0,129.2,129.0,128.6,128.2,127.6,126.8$, 126.7, 79.2, 49.1, 42.4, 30.1, 25.02, 22.7. HRMS (APCI+) calculated for $\left[\mathrm{C}_{19} \mathrm{H}_{18} \mathrm{Cl}\right]^{+} 281.1092$ $m / z$; found $\left[\mathrm{M}-\mathrm{CH}_{3} \mathrm{O}\right]^{+} 281.1095 \mathrm{~m} / z . \boldsymbol{\alpha}_{\mathbf{D}}{ }^{589}=-205.0 \mathrm{deg} \cdot \mathrm{cm}^{2} \cdot \mathrm{g}^{-1}\left(\mathrm{CHCl}_{3}\right.$, c $\left.0.50,299 \mathrm{~K}\right)$. HPLC Chiralpak OJ-H $(250 \mathrm{~mm} \times 4.6 \mathrm{~mm}, 5 \mu \mathrm{m})$ at $25{ }^{\circ} \mathrm{C}$, flow $1.0 \mathrm{~mL} / \mathrm{min}$, isocratic hexane/iPrOH 90:10, $280 \mathrm{~nm}, \mathrm{t}_{\mathrm{R}}$ (major) $5.5 ; \mathrm{t}_{\mathrm{R}}$ (minor) 16.6.

(S)-3-(4-Methoxyphenyl)-2-(2-methoxypropan-2-yl)-1,2-dihydronaphthalene (10j)

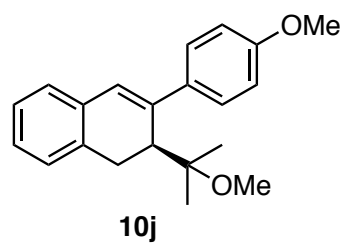

Compound 10j was synthesized following general procedure $\mathbf{O}$ from $\mathbf{9 j}$ (51.0 mg, 0.165 mmol). The crude was purified by preparative TLC (pentane/diethyl ether 95:5) to afford 10j (29.5 mg, $0.096 \mathrm{mmol}, 52 \%)$ as a white solid in $96: 4 \mathrm{er}$.

M.p. $=104-106^{\circ} \mathrm{C}$ (pentane). $\boldsymbol{\alpha}_{\mathbf{D}}{ }^{\mathbf{5 8 9}}=-221.7$ deg.cm ${ }^{2} \cdot \mathrm{g}^{-1}\left(\mathrm{CHCl}_{3}, \mathrm{c} 1.19,299 \mathrm{~K}\right) . \mathbf{H P L C}$ Chiralpak IA $(250 \times 4.6 \mathrm{~mm}, 5 \mu \mathrm{m})$, flow $1.0 \mathrm{~mL} / \mathrm{min}$, isocratic hexane $/ i \operatorname{PrOH} 98: 2,280 \mathrm{~nm}, \mathrm{t}_{\mathrm{R}}$ (major) $5.9 ; t_{R}$ (minor) 7.3. The spectral data of $\mathbf{1 0 j}$ were fully consistent with those previously reported. ${ }^{17}$ 
Interconversion of compound $10 \mathrm{i}$ into compound $10 \mathrm{j}$<smiles>CC(C)(C)C1Cc2ccccc2C=C1c1ccc(Cl)cc1</smiles>

$10 \mathrm{i}$

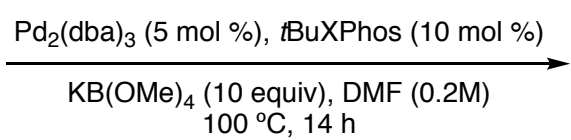

$100^{\circ} \mathrm{C}, 14 \mathrm{~h}$

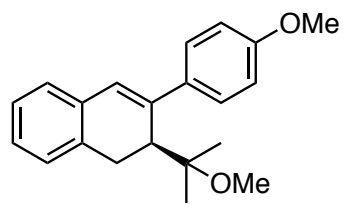

10j

A microwave vial was charged with $\mathrm{Pd}_{2}(\mathrm{dba})_{3}(1.46 \mathrm{mg}, 1.59 \mu \mathrm{mol}, 5 \mathrm{~mol} \%), t$ BuXPhos $(1.36$ $\mathrm{mg}, 3.20 \mu \mathrm{mol}, 10 \mathrm{~mol} \%$ ), compound 10i (10 mg, $32 \mu \mathrm{mol}, 1.0$ equiv) $\mathrm{KB}(\mathrm{OMe})_{4}(55.6 \mathrm{mg}$, $0.32 \mathrm{mmol}, 10.0$ equiv) and DMF $(0.2 \mathrm{~mL}) .{ }^{23}$ The reaction mixture was heated to $100{ }^{\circ} \mathrm{C}$ for 14 h. The reaction was quenched by addition of water and extracted from EtOAc (3x). The combined organic phases were washed with brine, dried over $\mathrm{Na}_{2} \mathrm{SO}_{4}$, filtered and concentrated. The crude was purified by preparative TLC (cyclohexane/Et ${ }_{2} \mathrm{O} 10: 1$ ) to afford compound $\mathbf{1 0 j}$ (7.4 mg, $24 \mu \mathrm{mol}, 75 \%$ ) as a white solid in 96:4 er.

(S)-2-(6,8-Dimethoxy-3-phenyl-1,2-dihydronaphthalen-2-yl)propan-2-ol (10k)<smiles>COc1cc2c(c(OC)c1)CC(C(C)(C)O)C(c1ccccc1)=C2</smiles>

Compound 10k was synthesized following general procedure $\mathbf{P}$ from 9k (205 mg, 0.67 mmol). The crude was purified by flash column chromatography (pentane/ $\mathrm{CH}_{2} \mathrm{Cl}_{2} 2: 1$, then pentane/Et $2 \mathrm{O} \mathrm{1:1)}$ to afford $10 \mathrm{k}(130 \mathrm{mg}, 0.40 \mathrm{mmol}, 60 \%)$ as a white solid in 99:1 $\mathrm{er}(55 \%$, $>99: 1$ er after recrystallization).

M.p. $=102-104{ }^{\circ} \mathrm{C}$ (pentane). ${ }^{1} \mathbf{H}$ NMR $\left(400 \mathrm{MHz}, \mathrm{CDCl}_{3}\right) \delta 7.57-7.52(\mathrm{~m}, 2 \mathrm{H}), 7.41-$ 7.35 (m, 2H), $7.31-7.25(\mathrm{~m}, 1 \mathrm{H}), 6.79(\mathrm{~s}, 1 \mathrm{H}), 6.37$ (q, $J=2.4 \mathrm{~Hz}, 2 \mathrm{H}), 3.84(\mathrm{~s}, 3 \mathrm{H}), 3.82(\mathrm{~s}$, $3 \mathrm{H}), 3.57(\mathrm{~d}, J=16.9 \mathrm{~Hz}, 1 \mathrm{H}), 3.13(\mathrm{dd}, J=8.3,1.2 \mathrm{~Hz}, 1 \mathrm{H}), 2.77(\mathrm{dd}, J=17.0,8.2 \mathrm{~Hz}, 1 \mathrm{H})$, 1.02 (s, 3H), 0.92 (s, 3H). ${ }^{13} \mathbf{C}$ NMR (101 MHz, $\left.\mathrm{CDCl}_{3}\right) \delta 159.1,156.9,143.3,141.0,136.0$, 128.8, 128.0, 127.4, 126.7, 115.1, 103.4, 98.1, 75.3, 55.7, 55.5, 45.3, 28.8, 27.9, 22.8. HRMS $(\mathrm{ESI}+)$ calculated for $\left[\mathrm{C}_{21} \mathrm{H}_{24} \mathrm{NaO}_{3}\right]^{+} 347.1618 \mathrm{~m} / z$; found $[\mathrm{M}+\mathrm{Na}]^{+} 347.1626 \mathrm{~m} / \mathrm{z} \cdot \boldsymbol{\alpha}_{\mathbf{D}} \mathbf{5 8 9}^{\mathbf{2 8 9}}=-$ $253.8 \mathrm{deg} . \mathrm{cm}^{2} \cdot \mathrm{g}^{-1}\left(\mathrm{CHCl}_{3}\right.$, c 1.00, $\left.299 \mathrm{~K}\right)$. HPLC Chiralpak IA $(250 \mathrm{~mm} \times 4.6 \mathrm{~mm}, 5 \mu \mathrm{m})$ at $25^{\circ} \mathrm{C}$, flow $1.0 \mathrm{~mL} / \mathrm{min}$, isocratic hexane $/ \mathrm{PrOH} 90: 10,280 \mathrm{~nm}, \mathrm{t}_{\mathrm{R}}$ (major) 8.5; $\mathrm{t}_{\mathrm{R}}$ (minor) 15.3. 
(S)-2-(6,8-Bis(benzyloxy)-3-phenyl-1,2-dihydronaphthalen-2-yl)propan-2-ol (101)

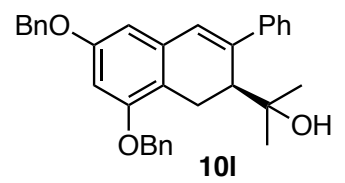

Compound 101 was synthesized following general procedure $\mathbf{P}$ from 91 (1.2 g, $2.62 \mathrm{mmol})$. The crude was purified by flash column chromatography (pentane $/ \mathrm{CH}_{2} \mathrm{Cl}_{2} 2: 1$, then pentane/ $\left.\mathrm{Et}_{2} \mathrm{O} 1: 1\right)$ to afford 101 (484 $\left.\mathrm{mg}, 1.78 \mathrm{mmol}, 68 \%\right)$ as a white solid in 98:2 er (60\%, $>99: 1$ er after recrystallization).

M.p. $=112-113{ }^{\circ} \mathrm{C}$ (pentane). ${ }^{1} \mathbf{H}$ NMR $\left(500 \mathrm{MHz}, \mathrm{CDCl}_{3}\right) \delta 7.55(\mathrm{~d}, J=7.6 \mathrm{~Hz}, 2 \mathrm{H}), 7.48$ $-7.27(\mathrm{~m}, 13 \mathrm{H}), 6.80(\mathrm{~s}, 1 \mathrm{H}), 6.53(\mathrm{~d}, J=2.3 \mathrm{~Hz}, 1 \mathrm{H}), 6.48(\mathrm{~d}, J=2.3 \mathrm{~Hz}, 1 \mathrm{H}), 5.09$ (s, 2H), 5.05 (s, 2H), 3.67 (d, $J=17.0 \mathrm{~Hz}, 1 \mathrm{H}), 3.15(\mathrm{~d}, J=8.1 \mathrm{~Hz}, 1 \mathrm{H}), 2.83(\mathrm{dd}, J=17.0,8.2 \mathrm{~Hz}, 1 \mathrm{H})$, $1.21(\mathrm{~s}, 1 \mathrm{H}), 1.03$ (s, 3H), 0.93 (s, 3H). ${ }^{13} \mathrm{C} \mathrm{NMR}\left(126 \mathrm{MHz}, \mathrm{CDCl}_{3}\right) \delta 158.3,156.0,143.2$, $141.2,137.5,137.2,136.3,128.8,128.73,128.71,128.1,128.0,127.7,127.5,127.4,126.7$, 116.0, 105.1, 100.5, 100.1, 75.3, 70.40, 70.38, 45.3, 28.7, 28.0, 23.1. HRMS (ESI+) calculated for $\left[\mathrm{C}_{33} \mathrm{H}_{32} \mathrm{NaO}_{3}\right]^{+} 499.2244 \mathrm{~m} / \mathrm{z}$; found $[\mathrm{M}+\mathrm{Na}]^{+}$found $499.2265 \mathrm{~m} / \mathrm{z} \cdot \boldsymbol{\alpha}_{\mathbf{D}}{ }^{\mathbf{5 8 9}}=-174.5$ deg. $\mathrm{cm}^{2} \cdot \mathrm{g}^{-1}\left(\mathrm{CHCl}_{3}\right.$, c 1.00, $\left.299 \mathrm{~K}\right)$. HPLC Chiralpak IA $(250 \mathrm{~mm} \times 4.6 \mathrm{~mm}, 5 \mu \mathrm{m})$ at $25^{\circ} \mathrm{C}$, flow $1.0 \mathrm{~mL} / \mathrm{min}$, isocratic hexane $/ \mathrm{iPrOH} 90: 10,280 \mathrm{~nm}, \mathrm{t}_{\mathrm{R}}$ (major) 12.3; $\mathrm{t}_{\mathrm{R}}$ (minor) 33.0.

(S)-2-(3-(Cyclohex-1-en-1-yl)-1,2-dihydronaphthalen-2-yl)propan-2-ol (18)

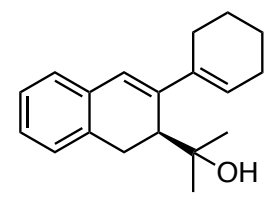

18

Compound 18 was synthesized following general procedure $\mathbf{O}$ from 17 (40.0 mg, 0.160 mmol). After $48 \mathrm{~h}$, the crude was purified by preparative TLC (pentane) to afford 18 (29.0 $\mathrm{mg}$, $0.108 \mathrm{mmol}, 68 \%$ ) as a white solid in 95:5 er.

M.p. $=68-72{ }^{\circ} \mathrm{C}$ (pentane). $\boldsymbol{\alpha}_{\mathbf{D}}{ }^{\mathbf{5 8 9}}=-157.1$ deg.cm ${ }^{2} \cdot \mathrm{g}^{-1}\left(\mathrm{CHCl}_{3}\right.$, c 0.37, $\left.299 \mathrm{~K}\right)$. HPLC Chiralpak IA $(250 \times 4.6 \mathrm{~mm}, 5 \mu \mathrm{m})$, flow $1.0 \mathrm{~mL} / \mathrm{min}$, isocratic hexane $/ \mathrm{PrOH} 90: 10,280 \mathrm{~nm}$, $t_{R}$ (major) 4.6; $t_{R}$ (minor) 7.3. The spectral data of $\mathbf{1 8}$ were fully consistent with those previously reported. ${ }^{17}$ 


\section{Enantioselective total syntheses of carexane I, $O$ and $P$}

\subsection{Synthesis of carexane I}

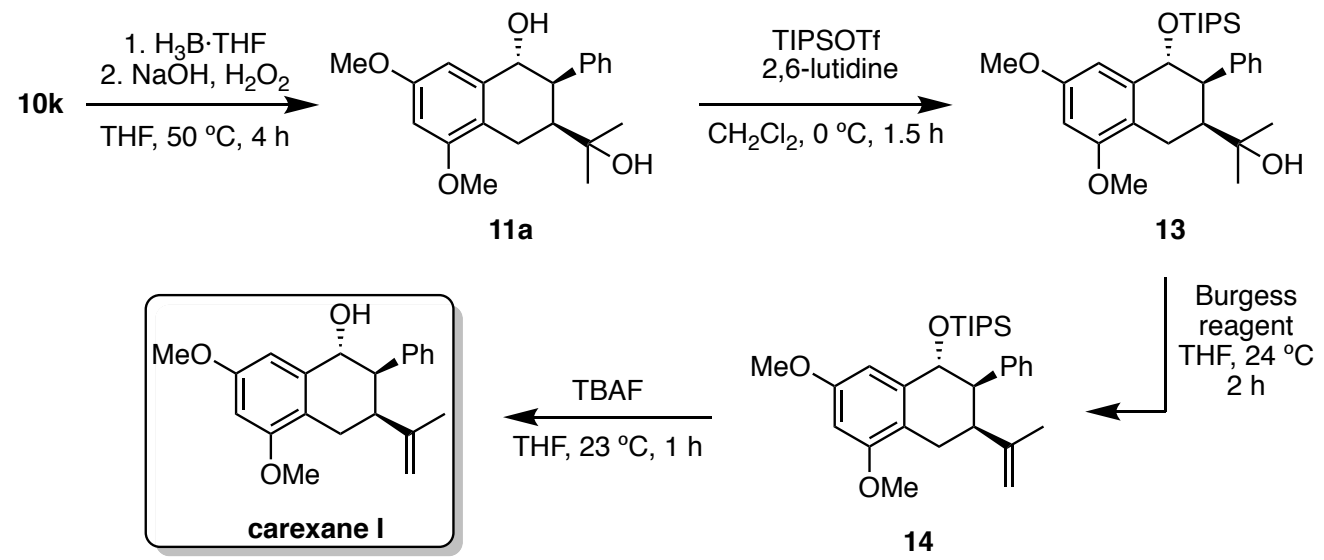

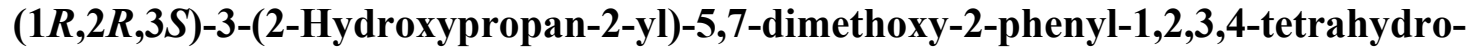

naphthalen-1-ol (11a)

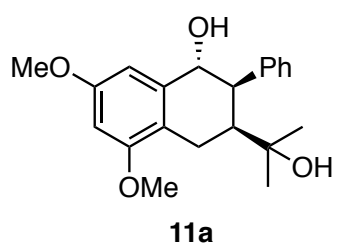

To a solution of $10 \mathrm{k}\left(1.00 \mathrm{~g}, 3.08 \mathrm{mmol}, 1.0\right.$ equiv) in dry THF $(65 \mathrm{~mL}), \mathrm{BH}_{3} \cdot \mathrm{THF}$ complex (12.3 mL, $12.3 \mathrm{mmol}, 4$ equiv, $1 \mathrm{M}$ in THF) was added dropwise at $23{ }^{\circ} \mathrm{C}$. The reaction mixture was warmed to $65{ }^{\circ} \mathrm{C}$ and stirred for $4 \mathrm{~h}$. After consumption of starting material, the reaction was cool down to $0{ }^{\circ} \mathrm{C}$, $\mathrm{NaOH}(5 \mathrm{~mL} ; 10 \%)$ was added dropwise followed by $\mathrm{H}_{2} \mathrm{O}_{2}$ solution ( 5 $\mathrm{mL}, 50 \%)$. The mixture was stirred for $2 \mathrm{~h}$ at $23{ }^{\circ} \mathrm{C}$. The reaction was treated with water and extracted with EtOAc. The combined organic phases were washed with sat. $\mathrm{Na}_{2} \mathrm{~S}_{2} \mathrm{O}_{3}$, water, brine and dried over $\mathrm{MgSO}_{4}$. The crude was purified by flash column chromatography $\left(\mathrm{Et}_{2} \mathrm{O}\right)$ to afford title compound $11 \mathrm{a}(708 \mathrm{mg}, 2.06 \mathrm{mmol}, 67 \%)$ as a white solid.

M.p. $=85-87^{\circ} \mathrm{C}$ (pentane). ${ }^{1} \mathbf{H}$ NMR $\left(500 \mathrm{MHz}, \mathrm{CDCl}_{3}\right) \delta 7.20-7.14(\mathrm{~m}, 3 \mathrm{H}), 7.04-$ $6.99(\mathrm{~m}, 2 \mathrm{H}), 6.46(\mathrm{~d}, J=6.4,2.4 \mathrm{~Hz}, 1 \mathrm{H}), 6.45(\mathrm{~d}, J=6.4,2.4 \mathrm{~Hz}, 1 \mathrm{H}) 4.58(\mathrm{~d}, J=0.8 \mathrm{~Hz}$, 1H), 3.85 (s, 3H), 3.79 (s, 3H), $3.61-3.58(\mathrm{~m}, 1 \mathrm{H}), 3.03$ (dd, $J=17.8,5.3 \mathrm{~Hz}, 1 \mathrm{H}), 2.59$ (dd, $J=17.8,12.8 \mathrm{~Hz}, 1 \mathrm{H}), 2.43(\mathrm{ddd}, J=12.8,5.3,3.7 \mathrm{~Hz}, 1 \mathrm{H}), 2.15($ br s, $1 \mathrm{H}), 1.23(\mathrm{~s}, 3 \mathrm{H}), 1.18$ (s, 3H), 1.15 (br s, 1H). ${ }^{13} \mathbf{C}$ NMR (126 MHz, $\left.\mathrm{CDCl}_{3}\right) \delta 159.2,158.3,140.3,138.2,129.7$, $128.8,127.1,119.4,105.2,98.5,74.3,73.3,55.52,55.48,47.6,42.5,29.1,28.2$, 20.5. HRMS $(\mathrm{ESI}+)$ calculated for $\left[\mathrm{C}_{21} \mathrm{H}_{26} \mathrm{NaO}_{4}\right]^{+} 365.1723 \mathrm{~m} / z$; found $[\mathrm{M}+\mathrm{Na}]^{+}=365.1730 \mathrm{~m} / \mathrm{z} \cdot \boldsymbol{\alpha}_{\mathbf{D}} \mathbf{5 8 9}^{\mathbf{2}}=$ $-49.8 \mathrm{deg} . \mathrm{cm}^{2} \cdot \mathrm{g}^{-1}\left(\mathrm{CHCl}_{3}, \mathrm{c} 1.00,299 \mathrm{~K}\right)$. 


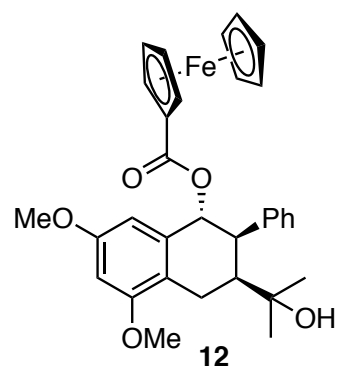

Compound 11a (20 mg, $0.058 \mathrm{mmol}, 1.0$ equiv) and DMAP (8.56 mg, $0.070 \mathrm{mmol}, 1.2$ equiv) were weighted into a small vial and a solution of freshly prepared ferrocenoyl chloride ${ }^{24}$ (21.77 mg, $0.088 \mathrm{mmol}, 1.5$ equiv) in $\mathrm{CH}_{2} \mathrm{Cl}_{2}(1 \mathrm{~mL}$ ) was added dropwise. The resulting deep red solution was stirred at $25^{\circ} \mathrm{C}$ for $3 \mathrm{~h}$. The reaction mixture was quenched by adding a small amount of silica gel and all volatiles were removed under reduced pressure. The crude product was purified by flash column chromatography (pentane/Et $2 \mathrm{O} 4: 1)$ to afford 12 (23.7 mg, 0.042 mmol, 73\%) as a crystalline orange solid in 96:4 er.

M.p. $=182-184{ }^{\circ} \mathrm{C}\left(\mathrm{Et}_{2} \mathrm{O}\right) .{ }^{1} \mathbf{H}$ NMR $\left(500 \mathrm{MHz}, \mathrm{CDCl}_{3}\right) \delta 7.22-7.18(\mathrm{~m}, 3 \mathrm{H}), 7.10-7.04$ (m, 2H), 6.51 (q, $J=2.4 \mathrm{~Hz}, 2 \mathrm{H}), 5.97$ (d, $J=1.6 \mathrm{~Hz}, 1 \mathrm{H}), 4.81$ (dq, $J=13.3,1.9 \mathrm{~Hz}, 2 \mathrm{H}), 4.39$ (t, $J=2.0 \mathrm{~Hz}, 2 \mathrm{H}), 4.18(\mathrm{~s}, 5 \mathrm{H}), 3.89(\mathrm{~s}, 3 \mathrm{H}), 3.76(\mathrm{~s}, 3 \mathrm{H}), 3.67$ (dd, $J=3.8,1.9 \mathrm{~Hz}, 1 \mathrm{H}), 3.13$ $(\mathrm{dd}, J=17.9,5.4 \mathrm{~Hz}, 1 \mathrm{H}), 2.70(\mathrm{dd}, J=17.9,12.8 \mathrm{~Hz}, 1 \mathrm{H}), 2.51(\mathrm{ddd}, J=12.7,5.4,3.8 \mathrm{~Hz}$, 1H), 1.25 (s, 3H), 1.19 (s, 3H), 1.06 (br s, 1H). $\left.{ }^{13} \mathbf{C ~ N M R ~ ( 1 2 6 ~ M H z , ~} \mathrm{CDCl}_{3}\right) \delta 171.1,159.0$, 158.1, 139.4, 134.7, 129.9, 129.0, 127.4, 120.5, 105.7, 99.0, 74.6, 73.1, 71.5, 70.49, 70.48, 69.9, 55.53, 55.51, 45.8, 43.5, 29.3, 28.4, 20.7. HRMS (ESI+) calculated for $\left[\mathrm{C}_{32} \mathrm{H}_{34} \mathrm{NaFeO}_{5}\right]^{+}$ $575.1695 \mathrm{~m} / z$; found $[\mathrm{M}+\mathrm{Na}]^{+}=575.1700 \mathrm{~m} / z \cdot \boldsymbol{\alpha}_{\mathbf{D}}{ }^{\mathbf{5 8 9}}=-33,1 \mathrm{deg} \cdot \mathrm{cm}^{2} \cdot \mathrm{g}^{-1}\left(\mathrm{CHCl}_{3}, \mathrm{c} 1.00,299\right.$ K). HPLC Chiralpak IA $(250 \mathrm{~mm} \times 4.6 \mathrm{~mm}, 5 \mu \mathrm{m})$ at $25{ }^{\circ} \mathrm{C}$, flow $1.0 \mathrm{~mL} / \mathrm{min}$, isocratic hexane/iPrOH 90:10, $220 \mathrm{~nm}, \mathrm{t}_{\mathrm{R}}$ (major) 11.2; $\mathrm{t}_{\mathrm{R}}$ (minor) 14.2.

\section{2-((2S,3R,4R)-6,8-Dimethoxy-3-phenyl-4-((triisopropylsilyl)oxy)-1,2,3,4-tetrahydro-} naphthalen-2-yl)propan-2-ol (13)

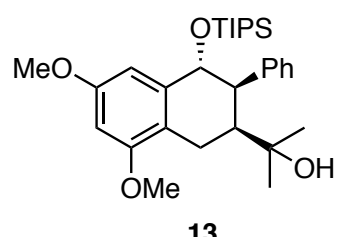

13

To a solution of $11 \mathrm{a}$ (30 mg, $0.088 \mathrm{mmol}, 1.0$ equiv) in anhydrous $\mathrm{CH}_{2} \mathrm{Cl}_{2}(1.2 \mathrm{~mL}), 2,6$ lutidine ( $15 \mu \mathrm{L}, 0.132 \mathrm{mmol}, 1.5$ equiv) and TIPSOTf ( $25 \mu \mathrm{L}, 0.092 \mathrm{mmol}, 1.05$ equiv) were 
added dropwise at $0{ }^{\circ} \mathrm{C}$. The resulting mixture was stirred for $1.5 \mathrm{~h}$ at the same temperature. Upon completion, the reaction was treated with water and extracted with $\mathrm{CH}_{2} \mathrm{Cl}_{2}$. The combined organic phases were washed with brine and dried over $\mathrm{MgSO}_{4}$. The crude was purified by flash column chromatography (cyclohexane/EtOAc 9:1 to 8:2) to afford title compound $\mathbf{1 3}$ (38 $\mathrm{mg}$, $0.077 \mathrm{mmol}, 87 \%$ ) as a white solid.

M.p. $=43-46{ }^{\circ} \mathrm{C}$ (pentane). ${ }^{1} \mathbf{H}$ NMR $\left(500 \mathrm{MHz}, \mathrm{CDCl}_{3}\right) \delta 7.18-7.14(\mathrm{~m}, 3 \mathrm{H}), 6.99-$ $6.94(\mathrm{~m}, 2 \mathrm{H}), 6.44$ (d, $J=2.4 \mathrm{~Hz}, 1 \mathrm{H}), 6.40$ (d, $J=2.4 \mathrm{~Hz}, 1 \mathrm{H}), 4.69$ (d, $J=2.0 \mathrm{~Hz}, 1 \mathrm{H}), 3.86$ (s, 3H), $3.77(\mathrm{~s}, 3 \mathrm{H}), 3.59-3.56(\mathrm{~m}, 1 \mathrm{H}), 3.09-3.02(\mathrm{~m}, 1 \mathrm{H}), 2.68-2.56(\mathrm{~m}, 2 \mathrm{H}), 1.25(\mathrm{~s}$, $3 \mathrm{H}), 1.21$ (s, 3H), $1.19-1.12(\mathrm{~m}, 3 \mathrm{H}), 1.10$ (d, $J=7.0 \mathrm{~Hz}, 9 \mathrm{H}), 1.07$ (d, $J=7.0 \mathrm{~Hz}, 9 \mathrm{H}), 0.91$ (br s, 1H). ${ }^{13} \mathbf{C}$ NMR $\left(126 \mathrm{MHz}, \mathrm{CDCl}_{3}\right) \delta 158.5,158.1,140.4,138.6,129.9,128.9,127.0$, 119.4, 105.9, 98.0, 74.9, 73.4, 55.4, 55.3, 49.1, 42.1, 29.1, 28.5, 20.7, 18.48, 18.45, 13.0. HRMS (ESI+) calculated for $\left[\mathrm{C}_{30} \mathrm{H}_{46} \mathrm{NaO}_{4} \mathrm{Si}\right]^{+} 521.3058 \mathrm{~m} / z$; found $[\mathrm{M}+\mathrm{Na}]^{+} 521.3060$ $m / z \cdot \boldsymbol{\alpha}_{\mathbf{D}}{ }^{589}=-42.0$ deg.cm ${ }^{2} \cdot \mathrm{g}^{-1}\left(\mathrm{CHCl}_{3}, \mathrm{c} 1.00,299 \mathrm{~K}\right)$.

\section{$(((1 R, 2 R, 3 S)-5,7-D i m e t h o x y-2-p h e n y l-3-(p r o p-1-e n-2-y l)-1,2,3,4-t e t r a h y d r o n a p h t h a l e n-$}

\section{1-yl)oxy)triisopropylsilane (14)}

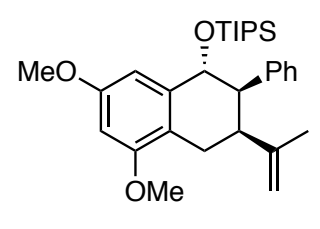

14

The reaction was carried out under argon atmosphere. To a solution of $\mathbf{1 3}$ (618 mg, 1.239 mmol, 1.0 equiv) in anhydrous THF $(18 \mathrm{~mL})$, burgess reagent (400 mg, $1.679 \mathrm{mmol}, 1.36$ equiv) was added at $23{ }^{\circ} \mathrm{C}$. The resulting mixture was stirred for $2 \mathrm{~h}$ (TLC monitoring). Then, the reaction was treated with water and extracted with EtOAc. The combined organic phases were washed with water and brine, dried over $\mathrm{MgSO}_{4}$. The crude product was purified by flash column chromatography (cyclohexane/EtOAc 95:5) to afford 14 (565 mg, $1.177 \mathrm{mmol}, 95 \%$ ) as a white solid.

M.p. $=71-73{ }^{\circ} \mathrm{C}$ (pentane). ${ }^{1} \mathbf{H}$ NMR $\left(400 \mathrm{MHz}, \mathrm{CDCl}_{3}\right) \delta 7.14-7.08$ (m, 3H), $6.82-$ $6.77(\mathrm{~m}, 2 \mathrm{H}), 6.53$ (d, $J=2.4 \mathrm{~Hz}, 1 \mathrm{H}), 6.42(\mathrm{~d}, J=2.4 \mathrm{~Hz}, 1 \mathrm{H}), 4.96(\mathrm{~d}, J=1.7 \mathrm{~Hz}, 1 \mathrm{H}), 4.80$ (s, 1H), $4.49(\mathrm{~s}, 1 \mathrm{H}), 3.82(\mathrm{~s}, 3 \mathrm{H}), 3.81(\mathrm{~s}, 3 \mathrm{H}), 3.42-3.38(\mathrm{~m}, 1 \mathrm{H}), 3.14(\mathrm{dt}, J=13.3,4.3 \mathrm{~Hz}$, 1H), $2.75(\mathrm{dd}, J=17.3,4.6 \mathrm{~Hz}, 1 \mathrm{H}), 2.23(\mathrm{dd}, J=17.3,13.0 \mathrm{~Hz}, 1 \mathrm{H}), 1.82$ (s, 3H), $1.22-1.14$ $(\mathrm{m}, 3 \mathrm{H}), 1.12(\mathrm{~d}, J=6.9 \mathrm{~Hz}, 9 \mathrm{H}), 1.09$ (d, $J=7.0 \mathrm{~Hz}, 9 \mathrm{H}) .{ }^{13} \mathbf{C} \mathbf{N M R}\left(101 \mathrm{MHz}, \mathrm{CDCl}_{3}\right) \delta$ 158.6, 157.9, 146.9, 139.3, 139.2, 129.1, 127.8, 126.4, 119.4, 111.5, 105.5, 98.0, 73.6, 55.44, 
55.38, 51.2, 39.1, 22.9, 22.7, 18.51, 18.47, 13.1. HRMS (ESI+) calculated for $\left[\mathrm{C}_{30} \mathrm{H}_{44} \mathrm{NaO}_{3} \mathrm{Si}^{+}\right.$ $503.2952 \mathrm{~m} / \mathrm{z}$; found $[\mathrm{M}+\mathrm{Na}]^{+} 503.2951 \mathrm{~m} / \mathrm{z} \cdot \boldsymbol{\alpha}_{\mathbf{D}}{ }^{\mathbf{5 8 9}}=-9.1 \mathrm{deg} \cdot \mathrm{cm}^{2} \cdot \mathrm{g}^{-1}\left(\mathrm{CHCl}_{3}, \mathrm{c} 1.00,299 \mathrm{~K}\right)$.

\section{Carexane I}

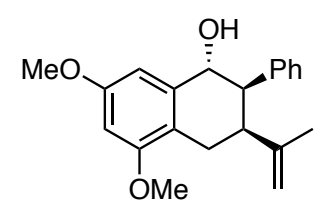

A solution of 14 (17 mg, $0.035 \mathrm{mmol}, 1.0$ equiv) in anhydrous THF (3 mL) was treated with TBAF $\left(70 \mu \mathrm{L}, 0.0699 \mathrm{mmol}, 2.0\right.$ equiv, $1 \mathrm{M}$ in THF) at $0{ }^{\circ} \mathrm{C}$ under argon. After $1.5 \mathrm{~h}$, the mixture was concentrated upon addition of Florisil ${ }^{\circledR}$ and the crude was purified by flash column chromatography (cyclohexane/EtOAc 9:1 to 7:3) to afford enantiopure carexane I (11 mg, $0.034 \mathrm{mmol}, 97 \%$ ) as an off-white solid with $e r>99: 1$.

M.p. $=128-130{ }^{\circ} \mathrm{C}$ (pentane). IR $\left(\mathrm{cm}^{-1}\right) \vee 3297,2932,1604,1491,1457,1273,1194$, 1147, 1021, 888, 834, 669. ${ }^{1} \mathbf{H}$ NMR (400 MHz, CD 3 OD) $\delta 7.12-7.08$ (m, 3H), $6.85-6.80$ $(\mathrm{m}, 2 \mathrm{H}), 6.63(\mathrm{~d}, J=2.4 \mathrm{~Hz}, 1 \mathrm{H}), 6.49(\mathrm{~d}, J=2.4 \mathrm{~Hz}, 1 \mathrm{H}), 4.79-4.77(\mathrm{~m}, 1 \mathrm{H}), 4.76(\mathrm{~d}, J=$ $2.1 \mathrm{~Hz}, 1 \mathrm{H}), 4.42(\mathrm{~s}, 1 \mathrm{H}), 3.82(\mathrm{~s}, 3 \mathrm{H}), 3.81(\mathrm{~s}, 3 \mathrm{H}), 3.37(\mathrm{dd}, J=3.7,1.8 \mathrm{~Hz}, 1 \mathrm{H}), 2.95$ (dt, $J$ $=12.2,3.9 \mathrm{~Hz}, 1 \mathrm{H}), 2.70(\mathrm{dd}, J=17.2,4.5 \mathrm{~Hz}, 1 \mathrm{H}), 2.22(\mathrm{dd}, J=17.3,12.4 \mathrm{~Hz}, 1 \mathrm{H}), 1.80(\mathrm{~s}$, 3H). ${ }^{13} \mathrm{C}$ NMR (101 MHz, CD $\left.3 \mathrm{OD}\right) \delta 160.6,159.2,147.9,140.6,140.0,129.9,128.6,127.4$, 120.2, 111.7, 106.5, 98.8, 73.2, 55.9, 55.8, 51.4, 40.8, 24.1, 22.8. HRMS (ESI+) calculated for $\left[\mathrm{C}_{21} \mathrm{H}_{24} \mathrm{NaO}_{3}\right]^{+} 347.1618 \mathrm{~m} / z$; found $[\mathrm{M}+\mathrm{Na}]^{+} 347.1609 \mathrm{~m} / \mathrm{z} \cdot \boldsymbol{\alpha}_{\mathbf{D}}{ }^{\mathbf{5 8 9}}=+33.3 \mathrm{deg} \cdot \mathrm{cm}^{2} \cdot \mathrm{g}^{-1}$ $(\mathrm{MeOH}$, c 0.15, $299 \mathrm{~K})$; lit. ${ }^{2526} \boldsymbol{\alpha}_{\mathbf{D}}=+15.6 \mathrm{deg} \cdot \mathrm{cm}^{2} \cdot \mathrm{g}^{-1}(\mathrm{MeOH}$, c 0.15, $298 \mathrm{~K})$. HPLC Chiralpak IA $(250 \mathrm{~mm} \times 4.6 \mathrm{~mm}, 5 \mu \mathrm{m})$ at $25^{\circ} \mathrm{C}$, flow $1.0 \mathrm{~mL} / \mathrm{min}$, isocratic hexane $/ \mathrm{PrOH}$ 90:10, $220 \mathrm{~nm}, \mathrm{t}_{\mathrm{R}}$ (minor) 14.3; $\mathrm{t}_{\mathrm{R}}$ (major) 17.3 . 
Table 13: Comparison of NMR shifts of the natural ${ }^{25}$ and the synthetic sample of carexane I

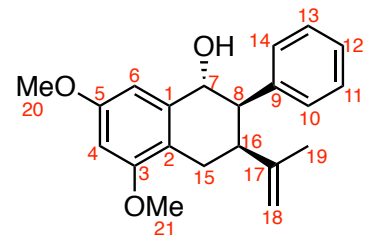

carexane I

\begin{tabular}{|c|c|c|c|c|}
\hline position & $\begin{array}{c}{ }^{1} \mathrm{H} \text { NMR } \\
\text { natural sample } \\
\left(\mathrm{CD}_{3} \mathrm{OD}, 300 \mathrm{MHz}\right)\end{array}$ & $\begin{array}{c}{ }^{1} \mathrm{H} \text { NMR } \\
\text { synthetic sample } \\
\left(\mathrm{CD}_{3} \mathrm{OD}, 300 \mathrm{MHz}\right)\end{array}$ & $\begin{array}{c}{ }^{13} \mathrm{C} \text { NMR } \\
\text { natural sample } \\
\left(\mathrm{CD}_{3} \mathrm{OD}, 75 \mathrm{MHz}\right)\end{array}$ & $\begin{array}{c}{ }^{13} \mathrm{C} \mathrm{NMR} \\
\text { synthetic sample } \\
\left(\mathrm{CD}_{3} \mathrm{OD}, 101 \mathrm{MHz}\right)\end{array}$ \\
\hline 1 & - & - & 140.0 & 140.0 \\
\hline 2 & - & - & 120.2 & 120.2 \\
\hline 3 & - & - & 159.1 & 159.2 \\
\hline 4 & $6.48(\mathrm{~d}, 2.4 \mathrm{~Hz})$ & $6.49(\mathrm{~d}, 2.4 \mathrm{~Hz})$ & 98.0 & 98.8 \\
\hline 5 & - & - & 160.5 & 160.6 \\
\hline 6 & $6.62(\mathrm{~d}, 2.4 \mathrm{~Hz})$ & $6.63(\mathrm{~d}, 2.4 \mathrm{~Hz})$ & 106.5 & 106.5 \\
\hline 7 & $4.76(\mathrm{~d}, 2.7 \mathrm{~Hz})$ & $4.76(\mathrm{~d}, 2.1 \mathrm{~Hz})$ & 73.2 & 73.2 \\
\hline 8 & $\begin{array}{c}3.53 \\
(\mathrm{dd}, 2.7,3.3 \mathrm{~Hz})\end{array}$ & $\begin{array}{c}3.37 \\
(\mathrm{dd}, 3.7,1.8 \mathrm{~Hz})\end{array}$ & 51.4 & 51.4 \\
\hline 9 & - & - & 140.6 & 140.6 \\
\hline 10 & $6.82(\mathrm{~m})$ & $6.82(\mathrm{~m})$ & 129.9 & 129.9 \\
\hline 11 & $7.09(\mathrm{~m})$ & $7.10(\mathrm{~m})$ & 128.6 & 128.6 \\
\hline 12 & $7.09(\mathrm{~m})$ & $7.10(\mathrm{~m})$ & 127.4 & 127.4 \\
\hline 13 & $7.09(\mathrm{~m})$ & $7.10(\mathrm{~m})$ & 128.6 & 128.6 \\
\hline 14 & $6.82(\mathrm{~m})$ & $6.82(\mathrm{~m})$ & 129.9 & 129.9 \\
\hline 15 & $\begin{array}{c}2.69 \\
(\mathrm{dd}, 17.4,4.5 \mathrm{~Hz}) \\
2.21 \\
(\mathrm{dd}, 17.4,12.3 \mathrm{~Hz})\end{array}$ & $\begin{array}{c}2.70 \\
(\mathrm{dd}, 17.2,4.5 \mathrm{~Hz}) \\
2.22 \\
(\mathrm{dd}, 17.3,12.4 \mathrm{~Hz})\end{array}$ & 24.1 & 24.1 \\
\hline 16 & $\begin{array}{c}2.94 \\
(\mathrm{dt}, 12.3,3.3 \mathrm{~Hz})\end{array}$ & $\begin{array}{c}2.95 \\
(\mathrm{dt}, 12.2,3.9 \mathrm{~Hz})\end{array}$ & 40.5 & 40.8 \\
\hline 17 & - & - & 147.9 & 147.9 \\
\hline 18 & $4.75(\mathrm{~s}), 4.41(\mathrm{~s})$ & $4.78(\mathrm{~m}), 4.42(\mathrm{~s})$ & 111.7 & 111.7 \\
\hline 19 & $1.79(\mathrm{~s})$ & $1.80(\mathrm{~s})$ & 22.8 & 22.8 \\
\hline 20 & $3.81(\mathrm{~s})$ & $3.82(\mathrm{~s})$ & 55.8 & 55.9 \\
\hline 21 & $3.80(\mathrm{~s})$ & $3.81(\mathrm{~s})$ & 55.7 & 55.8 \\
\hline
\end{tabular}




\subsection{Synthesis of carexane $P$}

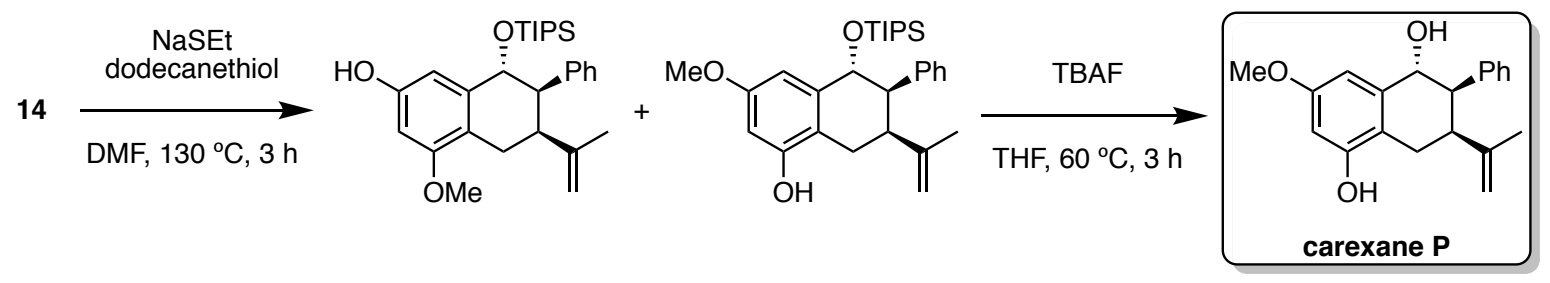

(5R,6R,7S)-3-Methoxy-6-phenyl-7-(prop-1-en-2-yl)-5-((triisopropylsilyl)oxy)-5,6,7,8tetrahydronaphthalen-1-ol and $(6 S, 7 R, 8 R)-4-m e t h o x y-7-p h e n y l-6-(p r o p-1-e n-2-y l)-8$ ((triisopropylsilyl)oxy)-5,6,7,8-tetrahydronaphthalen-2-ol<smiles>C=C(C)C1Cc2c(O)cc(OC)cc2C(O)C1C(=C)C(=O)OCc1ccccc1</smiles>

To a solution of 14 (496 mg, $1.03 \mathrm{mmol}, 1.0$ equiv) in dry DMF (10.3 mL) was added dodecane-1-thiol (0.544 mL, $2.27 \mathrm{mmol}, 2.2$ equiv) and sodium ethanethiolate (278 $\mathrm{mg}, 3.30$ mmol, 3.2 equiv). The solution was heated at $130^{\circ} \mathrm{C}$ and stirred for $3 \mathrm{~h}$. After cooling to $24^{\circ} \mathrm{C}$, water was added and the mixture was extracted with EtOAc. The crude was purified by flash column chromatography (pentane/Et $\mathrm{E}_{2} \mathrm{O}$ 8:2). The regioisomers were obtained as a pale-yellow oil (inseparable mixture, 2.9:1 ratio; $340 \mathrm{mg}, 0.722 \mathrm{mmol}, 70 \%$ ).

Major isomer (3-methoxy compound; 52\% NMR yield): ${ }^{1} \mathbf{H}$ NMR (500 MHz, $\left.\mathrm{CD}_{2} \mathrm{Cl}_{2}\right) \delta$ $7.17-7.09(\mathrm{~m}, 3 \mathrm{H}), 6.83-6.79(\mathrm{~m}, 2 \mathrm{H}), 6.57$ (d, $J=2.4 \mathrm{~Hz}, 1 \mathrm{H}), 6.40(\mathrm{~d}, J=2.5 \mathrm{~Hz}, 1 \mathrm{H})$, 4.97 (br s, 1H), 4.97 (d, $J=1.6 \mathrm{~Hz}, 1 \mathrm{H}), 4.83$ (s, 1H), 4.48 (s, 1H), 3.78 (s, 3H), $3.46-3.44$ (m, 1H), $3.22(\mathrm{dt}, J=12.8,4.0 \mathrm{~Hz}, 1 \mathrm{H}), 2.65(\mathrm{dd}, J=16.5,4.7 \mathrm{~Hz}, 1 \mathrm{H}), 2.29(\mathrm{dd}, J=16.5,12.8$ $\mathrm{Hz}, 1 \mathrm{H}), 1.87(\mathrm{~s}, 3 \mathrm{H}), 1.25-1.17(\mathrm{~m}, 3 \mathrm{H}), 1.15-1.05(\mathrm{~m}, 18 \mathrm{H}) .{ }^{13} \mathbf{C} \mathbf{N M R}\left(126 \mathrm{MHz}, \mathrm{CD}_{2} \mathrm{Cl}_{2}\right)$ $\delta 159.2,154.6,147.3,140.4,139.4,129.5,128.3,127.0,117.0,111.5,107.9,101.8,73.9,55.8$, 51.3, 39.4, 23.2, 23.0, 18.71, 18.67, 13.5. Minor isomer (4-methoxy compound; 18\% NMR yield): ${ }^{1} \mathbf{H}$ NMR (500 MHz, $\left.\mathrm{CD}_{2} \mathrm{Cl}_{2}\right) \delta 7.17-7.09(\mathrm{~m}, 3 \mathrm{H}), 6.83-6.79(\mathrm{~m}, 2 \mathrm{H}), 6.46(\mathrm{~d}, J=$ $2.3 \mathrm{~Hz}, 1 \mathrm{H}), 6.40$ (d, $J=2.5 \mathrm{~Hz}, 1 \mathrm{H}), 4.92$ (d, $J=1.6 \mathrm{~Hz}, 1 \mathrm{H}), 4.88$ (br s, 1H), 4.80 (s, 1H), $4.48(\mathrm{~s}, 1 \mathrm{H}), 3.81(\mathrm{~s}, 3 \mathrm{H}), 3.43-3.41(\mathrm{~m}, 1 \mathrm{H}), 3.15(\mathrm{dt}, J=13.0,4.1 \mathrm{~Hz}, 1 \mathrm{H}), 2.74(\mathrm{dd}, J=$ 17.2, $4.8 \mathrm{~Hz}, 1 \mathrm{H}), 2.23$ (dd, $J=17.4,13.0 \mathrm{~Hz}, 1 \mathrm{H}), 1.84$ (s, 3H), $1.25-1.17$ (m, 3H), 1.15 $1.05(\mathrm{~m}, 18 \mathrm{H}) .{ }^{13} \mathbf{C} \mathbf{N M R}\left(126 \mathrm{MHz}, \mathrm{CD}_{2} \mathrm{Cl}_{2}\right) \delta 158.7,155.1,147.6,140.0,139.6,129.5,128.2$, $126.9,119.4,111.4,108.8,98.4,73.8,56.0,51.3,39.4,23.4,23.0,18.7,18.6,13.5$. HRMS $(\mathrm{ESI}+)$ calculated for $\left[\mathrm{C}_{29} \mathrm{H}_{42} \mathrm{NaO}_{3} \mathrm{Si}\right]^{+} 489.2795 \mathrm{~m} / \mathrm{z}$; found $[\mathrm{M}+\mathrm{Na}]^{+} 489.2793 \mathrm{~m} / \mathrm{z}$. 


\section{Carexane $\mathbf{P}$}

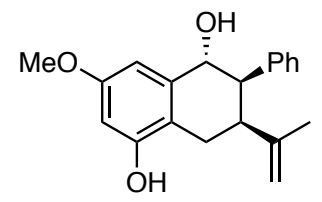

A mixture (2.5:1) of $(5 R, 6 R, 7 S)-3-$-methoxy-6-phenyl-7-(prop-1-en-2-yl)-5-((triisopropylsilyl)oxy)-5,6,7,8-tetrahydronaphthalen-1-ol and (6S,7R,8R)-4-methoxy-7-phenyl-6-(prop-1en-2-yl)-8-((triisopropylsilyl)oxy)-5,6,7,8-tetrahydronaphthalen-2-ol (210 mg, $0.316 \mathrm{mmol}$, 1.0 equiv) was dissolved in dry THF ( $6 \mathrm{~mL})$ and TBAF $(1.9 \mathrm{~mL}, 1.90 \mathrm{mmol}, 6.0$ equiv, $1 \mathrm{M}$ in THF) was added at $0{ }^{\circ} \mathrm{C}$. The solution was heated to $60^{\circ} \mathrm{C}$ and the reaction was stirred at this temperature for $3 \mathrm{~h}$. The reaction was quenched with water, extracted with EtOAc and the crude was purified by flash column chromatography (cyclohexane/EtOAc 3:2) to afford carexane $\mathrm{P}$ (70.2 $\mathrm{mg}, 0.22 \mathrm{mmol}, 70 \%$ ) as white amorphous solid with $\mathrm{er}>99: 1$.

M.p. $=162-164^{\circ} \mathrm{C}$ (pentane). IR $\left(\mathrm{cm}^{-1}\right) \vee 3387,2918,1620,1513,1441,1256,1195,1146$, 1058, 1019, 969, 890, 842, 699. ${ }^{1} \mathbf{H}$ NMR $\left(500 \mathrm{MHz}, \mathrm{CD}_{3} \mathrm{OD}\right) \delta 7.13-7.07$ (m, 3H), 6.87 $6.82(\mathrm{~m}, 2 \mathrm{H}), 6.56(\mathrm{~d}, J=2.5 \mathrm{~Hz}, 1 \mathrm{H}), 6.39(\mathrm{~d}, J=2.5 \mathrm{~Hz}, 1 \mathrm{H}), 4.78(\mathrm{~s}, 1 \mathrm{H}), 4.74(\mathrm{~d}, J=2.1$ $\mathrm{Hz}, 1 \mathrm{H}), 4.45$ (s, 1H), 3.77 (s, 3H), 3.37 (dd, $J=3.7,2.8 \mathrm{~Hz}, 1 \mathrm{H}), 2.98$ (dt, $J=12.4,4.2 \mathrm{~Hz}$, $1 \mathrm{H}), 2.70(\mathrm{dd}, J=17.0,4.6 \mathrm{~Hz}, 1 \mathrm{H}), 2.26(\mathrm{dd}, J=17.0,12.4 \mathrm{~Hz}, 1 \mathrm{H}), 1.80$ (s, 3H). ${ }^{13} \mathbf{C} \mathbf{~ N M R}$ $\left(126 \mathrm{MHz}, \mathrm{CD}_{3} \mathrm{OD}\right) \delta 160.2,156.6,147.9,140.6,140.2,129.9,128.6,127.3,118.7,111.7$, 106.7, 102.0, 73.3, 55.6, 51.4, 40.8, 24.2, 22.9. HRMS (ESI+) calculated for $\left[\mathrm{C}_{20} \mathrm{H}_{22} \mathrm{NaO}_{3}\right]^{+}$ $333.1461 \mathrm{~m} / \mathrm{z}$; found $[\mathrm{M}+\mathrm{Na}]^{+} 333.1464 \mathrm{~m} / \mathrm{z} \cdot \boldsymbol{\alpha}_{\mathbf{D}}{ }^{\mathbf{5 8 9}}=+13.0$ deg. $\mathrm{cm}^{2} \cdot \mathrm{g}^{-1}(\mathrm{MeOH}, \mathrm{c} 0.15,299$ K); lit. ${ }^{26} \boldsymbol{\alpha}_{\mathbf{D}}=-26.7$ deg.cm ${ }^{2} . \mathrm{g}^{-1}(\mathrm{MeOH}, \mathrm{c} 0.02,298 \mathrm{~K})$. HPLC Chiralpak IA (250 mm $\times 4.6$ $\mathrm{mm}, 5 \mu \mathrm{m}$ ) at $25^{\circ} \mathrm{C}$, flow $1.0 \mathrm{~mL} / \mathrm{min}$, isocratic hexane $/ i \mathrm{PrOH} 80: 20,220 \mathrm{~nm}, \mathrm{t}_{\mathrm{R}}$ (major) 13.0; $t_{R}$ (minor) 19.0. 
Table 14: Comparison of NMR shifts of the natural ${ }^{26}$ and the synthetic sample of carexane P

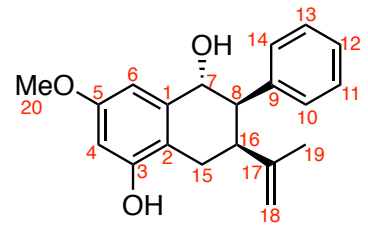

carexane $\mathbf{P}$

\begin{tabular}{|c|c|c|c|c|}
\hline position & $\begin{array}{c}{ }^{1} \mathrm{H} \text { NMR } \\
\text { natural sample } \\
\left(\mathrm{CD}_{3} \mathrm{OD}, 300 \mathrm{MHz}\right)\end{array}$ & $\begin{array}{c}{ }^{1} \mathrm{H} \text { NMR } \\
\text { synthetic sample } \\
\left(\mathrm{CD}_{3} \mathrm{OD}, 500 \mathrm{MHz}\right)\end{array}$ & $\begin{array}{c}{ }^{13} \mathrm{C} \text { NMR } \\
\text { natural sample } \\
\left(\mathrm{CD}_{3} \mathrm{OD}, 75 \mathrm{MHz}\right)\end{array}$ & $\begin{array}{c}{ }^{13} \mathrm{C} \mathrm{NMR} \\
\text { synthetic sample } \\
\left(\mathrm{CD}_{3} \mathrm{OD}, 126 \mathrm{MHz}\right)\end{array}$ \\
\hline 1 & - & - & 140.0 & 140.2 \\
\hline 2 & - & - & 120.2 & 118.7 \\
\hline 3 & - & - & 156.1 & 156.6 \\
\hline 4 & $6.37(\mathrm{~d}, 2.7 \mathrm{~Hz})$ & $6.39(\mathrm{~d}, 2.5 \mathrm{~Hz})$ & 98.0 & 102.0 \\
\hline 5 & - & - & 160.5 & 160.2 \\
\hline 6 & $6.55(\mathrm{~d}, 2.7 \mathrm{~Hz})$ & $6.56(\mathrm{~d}, 2.5 \mathrm{~Hz})$ & 106.5 & 106.7 \\
\hline 7 & $4.73(\mathrm{~d}, 2.7 \mathrm{~Hz})$ & $4.74(\mathrm{~d}, 2.1 \mathrm{~Hz})$ & 73.2 & 73.3 \\
\hline 8 & $3.53(\mathrm{~m})$ & $\begin{array}{c}3.37 \\
(\mathrm{dd}, 3.7,2.8 \mathrm{~Hz})\end{array}$ & 51.4 & 51.4 \\
\hline 9 & - & - & 140.6 & 140.6 \\
\hline 10 & $6.85(\mathrm{~m})$ & $6.85(\mathrm{~m})$ & 129.9 & 129.9 \\
\hline 11 & $7.11(\mathrm{~m})$ & $7.10(\mathrm{~m})$ & 128.6 & 128.6 \\
\hline 12 & $7.11(\mathrm{~m})$ & $7.10(\mathrm{~m})$ & 127.4 & 127.3 \\
\hline 13 & $7.11(\mathrm{~m})$ & $7.10(\mathrm{~m})$ & 128.6 & 128.6 \\
\hline 14 & $6.85(\mathrm{~m})$ & $6.85(\mathrm{~m})$ & 129.9 & 129.9 \\
\hline 15 & $\begin{array}{c}2.68 \\
\text { (dd, 17.4, } 4.5 \mathrm{~Hz}) \\
2.22 \\
(\mathrm{dd}, 17.4,12.2 \mathrm{~Hz})\end{array}$ & $\begin{array}{c}2.70 \\
(\mathrm{dd}, 17.0,4.6 \mathrm{~Hz}) \\
2.26 \\
(\mathrm{dd}, 17.0,12.4 \mathrm{~Hz})\end{array}$ & 24.1 & 24.2 \\
\hline 16 & $\begin{array}{c}2.96 \\
(\mathrm{dt}, 12.2,4.5 \mathrm{~Hz})\end{array}$ & $\begin{array}{c}2.98 \\
(\mathrm{dt}, 12.4,4.2 \mathrm{~Hz})\end{array}$ & 40.5 & 40.8 \\
\hline 17 & - & - & 147.9 & 147.9 \\
\hline 18 & $4.57(\mathrm{~s}), 4.44(\mathrm{~s})$ & $4.78(\mathrm{~s}), 4.45(\mathrm{~s})$ & 111.7 & 111.7 \\
\hline 19 & $1.80(\mathrm{~s})$ & $1.80(\mathrm{~s})$ & 22.8 & 22.9 \\
\hline 20 & $3.77(\mathrm{~s})$ & $3.77(\mathrm{~s})$ & 55.7 & 55.6 \\
\hline
\end{tabular}




\subsection{Synthesis of carexane $O$}

\subsubsection{Synthesis of carexane $O$ from 101}

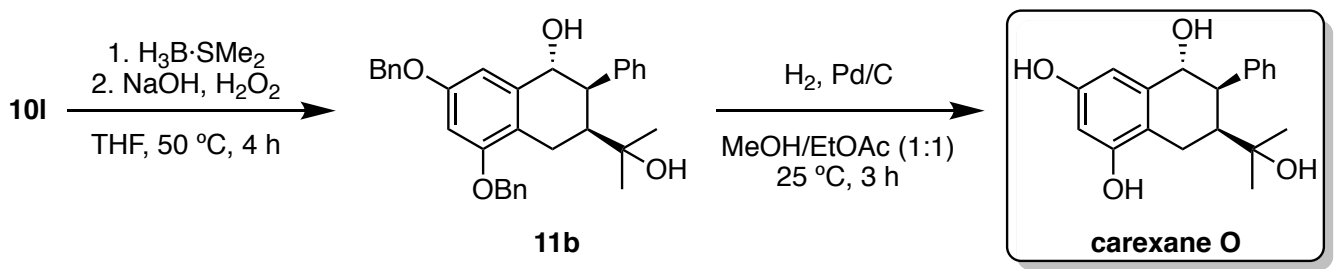

$(1 R, 2 R, 3 S)-5,7-B i s(b e n z y l o x y)-3-(2-h y d r o x y p r o p a n-2-y l)-2-p h e n y l-1,2,3,4-$ tetrahydronaphthalen-1-ol (11b)

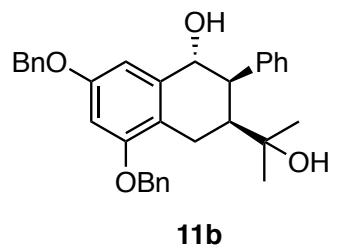

Compound 101 (700 mg, $1.47 \mathrm{mmol}, 1.0$ equiv) was dissolved in anhydrous THF (20 mL). $\mathrm{BH}_{3} \cdot$ THF complex ( $8.2 \mathrm{~mL}, 8.20 \mathrm{mmol}, 5.6$ equiv, $1 \mathrm{M}$ in THF) was added dropwise and the solution was stirred for $4 \mathrm{~h}$ at $50^{\circ} \mathrm{C}$. After cooling to $0{ }^{\circ} \mathrm{C}$, sodium hydroxide $(2.94 \mathrm{~mL}, 7.34$ mmol, 5.0 equiv; $2.5 \mathrm{M})$ was added dropwise followed by hydrogen peroxide $(1.67 \mathrm{~mL}, 14.7$ mmol, 10.0 equiv; 8.8M). The mixture was stirred for $3 \mathrm{~h}$ at $24^{\circ} \mathrm{C}$ and then extracted with $\mathrm{Et}_{2} \mathrm{O}$. The crude was purified by flash column chromatography (pentane/ $\mathrm{Et}_{2} \mathrm{O} 4: 1$ ) to afford $11 \mathbf{b}$ (448 $\mathrm{mg}, 0.91 \mathrm{mmol}, 62 \%$ ) as a white solid.

M.p. $=91-93{ }^{\circ} \mathrm{C}$ (pentane). ${ }^{1} \mathbf{H}$ NMR $\left(500 \mathrm{MHz}, \mathrm{CDCl}_{3}\right) \delta 7.48-7.36(\mathrm{~m}, 8 \mathrm{H}), 7.36-$ $7.31(\mathrm{~m}, 2 \mathrm{H}), 7.22-7.17(\mathrm{~m}, 3 \mathrm{H}), 7.07-7.02(\mathrm{~m}, 2 \mathrm{H}), 6.64(\mathrm{~d}, J=2.3 \mathrm{~Hz}, 1 \mathrm{H}), 6.59$ (d, $J=$ $2.3 \mathrm{~Hz}, 1 \mathrm{H}), 5.15$ (d, $J=12.0 \mathrm{~Hz}, 1 \mathrm{H}), 5.09$ (d, $J=12.0 \mathrm{~Hz}, 1 \mathrm{H}), 5.04-4.98$ (m, 2H), 4.61 (d, $J=1.2 \mathrm{~Hz}, 1 \mathrm{H}), 3.63(\mathrm{dd}, J=3.8,1.9 \mathrm{~Hz}, 1 \mathrm{H}), 3.15(\mathrm{dd}, J=17.9,5.4 \mathrm{~Hz}, 1 \mathrm{H}), 2.70(\mathrm{dd}, J=$ 17.9, $12.8 \mathrm{~Hz}, 1 \mathrm{H}), 2.47$ (ddd, $J=12.8,5.4,3.7 \mathrm{~Hz}, 1 \mathrm{H}), 2.11$ (br s, $1 \mathrm{H}), 1.28$ (s, 3H), 1.20 (s, $3 \mathrm{H}), 1.10$ (br s, $1 \mathrm{H}) .{ }^{13} \mathbf{C}$ NMR $\left(126 \mathrm{MHz}, \mathrm{CDCl}_{3}\right) \delta 158.4,157.4,140.3,138.4,137.1,137.0$, $129.7,128.9,128.73,128.72,128.2,128.0,127.8,127.3,127.1,120.1,106.8,100.7,74.3,73.2$, 70.3, 70.1, 47.5, 42.5, 29.2, 28.3, 20.7. HRMS (ESI+) calculated for $\left[\mathrm{C}_{33} \mathrm{H}_{34} \mathrm{NaO}_{4}\right]^{+} 517.2349$ $m / z$; found $[\mathrm{M}+\mathrm{Na}]^{+} 517.2340 \mathrm{~m} / z \cdot \boldsymbol{\alpha}_{\mathbf{D}}{ }^{\mathbf{5 8 9}}=-39.6 \mathrm{deg} \cdot \mathrm{cm}^{2} \cdot \mathrm{g}^{-1}\left(\mathrm{CDCl}_{3}, \mathrm{c} 1.00,299 \mathrm{~K}\right)$. 


\section{Carexane O}

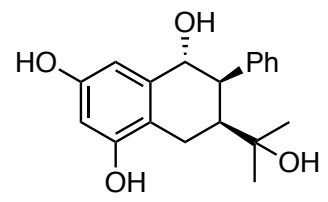

In a microwave vial 11b (40 mg, $0.418 \mathrm{mmol}, 1.0$ equiv) and $\mathrm{Pd} / \mathrm{C}(10 \%$; $4.7 \mathrm{mg}, 4.4 \mu \mathrm{mol}$, $1 \mathrm{~mol} \%$ ) were added. The vial was covered with a septum and evacuated during 5 min through a needle. Then the vial was filled with $\mathrm{H}_{2}$ and a mixture of methanol $(2 \mathrm{~mL})$ and EtOAc $(2 \mathrm{~mL})$ was added. The system was purged with 5 fast cycles of vacuum and hydrogen. The reaction mixture was stirred under hydrogen atmosphere $(1 \mathrm{~atm})$ for $24 \mathrm{~h}$ until full conversion. The reaction was filtered through Celite ${ }^{\circledR}$ and the solvent was evaporated under reduced pressure. Carexane O (130 mg, $0.080 \mathrm{mmol}, 98 \%$ ) was obtained as a white solid with $e r>99: 1$.

M.p. $=189-191^{\circ} \mathrm{C}\left(\mathrm{Et}_{2} \mathrm{O}\right) . \mathbf{I R}\left(\mathrm{cm}^{-1}\right) \vee 3371,2913,1596,1452,1254,1143,840,699 .{ }^{1} \mathbf{H}$ NMR (500 MHz, CD $\left.{ }_{3} \mathrm{OD}\right) \delta 7.18-7.13(\mathrm{~m}, 3 \mathrm{H}), 7.03-6.99(\mathrm{~m}, 2 \mathrm{H}), 6.33(\mathrm{~d}, J=2.4 \mathrm{~Hz}, 1 \mathrm{H})$, $6.32(\mathrm{~d}, J=2.4 \mathrm{~Hz}, 1 \mathrm{H}), 4.39(\mathrm{~d}, J=1.8 \mathrm{~Hz}, 1 \mathrm{H}), 3.48(\mathrm{dd}, J=3.4,1.9 \mathrm{~Hz}, 1 \mathrm{H}), 3.01$ (dd, $J=$ 16.5, 4.4 Hz, 1H), 2.50 (ddd, $J=13.0,4.4,3.4 \mathrm{~Hz}, 1 \mathrm{H}), 2.43$ (dd, $J=16.5,13.0 \mathrm{~Hz}, 1 \mathrm{H}), 1.14$ (s, 3H), 0.93 (s, 3H). ${ }^{13} \mathrm{C}$ NMR (126 MHz, $\left.\mathrm{CD}_{3} \mathrm{OD}\right) \delta 157.2,156.8,142.5,139.6,130.8,129.2$, 127.5, 117.8, 109.5, 102.9, 75.1, 73.9, 49.7, 44.4, 28.2, 27.6, 21.7. HRMS (ESI+) calculated for $\left[\mathrm{C}_{19} \mathrm{H}_{22} \mathrm{NaO}_{4}\right]^{+} 337.1410 \mathrm{~m} / z$; found $[\mathrm{M}+\mathrm{Na}]^{+} 337.1411 \mathrm{~m} / z \cdot \boldsymbol{\alpha}_{\mathbf{D}}{ }^{\mathbf{5 8 9}}=-40,1 \mathrm{deg} \cdot \mathrm{cm}^{2} \cdot \mathrm{g}^{-1}$ $(\mathrm{MeOH}, \mathrm{c} 0.31,299 \mathrm{~K}) ;$ lit. $^{26} \boldsymbol{\alpha}_{\mathbf{D}}=+10.0$ deg.cm ${ }^{2} \cdot \mathrm{g}^{-1}(\mathrm{MeOH}, \mathrm{c} 0.04,298 \mathrm{~K})$. HPLC Chiralpak IA $(250 \mathrm{~mm} \times 4.6 \mathrm{~mm}, 5 \mu \mathrm{m})$ at $25{ }^{\circ} \mathrm{C}$, flow $1.0 \mathrm{~mL} / \mathrm{min}$, isocratic hexane $/ i \mathrm{PrOH} 80: 20,220$ $\mathrm{nm}, \mathrm{t}_{\mathrm{R}}$ (minor) $12.3 ; \mathrm{t}_{\mathrm{R}}$ (major) 15.7 . 
Table 15: Comparison of NMR shifts of the natural ${ }^{26}$ and the synthetic sample of carexane $O$

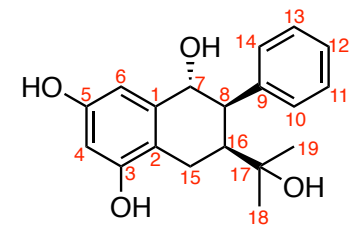

carexane $\mathbf{0}$

\begin{tabular}{|c|c|c|c|c|}
\hline position & $\begin{array}{c}{ }^{1} \mathrm{H} \text { NMR } \\
\text { natural sample } \\
\left(\mathrm{CD}_{3} \mathrm{OD}, 300 \mathrm{MHz}\right)\end{array}$ & $\begin{array}{c}{ }^{1} \mathrm{H} \text { NMR } \\
\text { synthetic sample } \\
\left(\mathrm{CD}_{3} \mathrm{OD}, 500 \mathrm{MHz}\right)\end{array}$ & $\begin{array}{c}{ }^{13} \mathrm{C} \text { NMR } \\
\text { natural sample } \\
\left(\mathrm{CD}_{3} \mathrm{OD}, 75 \mathrm{MHz}\right)\end{array}$ & $\begin{array}{c}{ }^{13} \mathrm{C} \mathrm{NMR} \\
\text { synthetic sample } \\
\left(\mathrm{CD}_{3} \mathrm{OD}, 126 \mathrm{MHz}\right)\end{array}$ \\
\hline 1 & - & - & 138.0 & 139.6 \\
\hline 2 & - & - & 117.5 & 117.8 \\
\hline 3 & - & - & 157.2 & 157.2 \\
\hline 4 & $6.31(\mathrm{~d}, 0.9 \mathrm{~Hz})$ & $6.33(\mathrm{~d}, 2.4 \mathrm{~Hz})$ & 109.7 & 109.5 \\
\hline 5 & - & - & 157.2 & 156.8 \\
\hline 6 & $6.31(\mathrm{~d}, 0.9 \mathrm{~Hz})$ & $6.32(\mathrm{~d}, 2.4 \mathrm{~Hz})$ & 109.7 & 102.9 \\
\hline 7 & $4.38(\mathrm{~d}, 1.8 \mathrm{~Hz})$ & $4.39(\mathrm{~d}, 1.8 \mathrm{~Hz})$ & 74.9 & 75.1 \\
\hline 8 & 3.46 (br s) & $\begin{array}{c}3.48 \\
(\mathrm{dd}, 3.4,1.9 \mathrm{~Hz})\end{array}$ & 49.5 & 44.4 \\
\hline 9 & - & - & 141.9 & 142.5 \\
\hline 10 & $6.99(\mathrm{~m})$ & $7.01(\mathrm{~m})$ & 130.0 & 130.8 \\
\hline 11 & $7.13(\mathrm{~m})$ & $7.15(\mathrm{~m})$ & 128.8 & 129.2 \\
\hline 12 & $7.13(\mathrm{~m})$ & $7.15(\mathrm{~m})$ & 127.5 & 127.5 \\
\hline 13 & $7.13(\mathrm{~m})$ & $7.15(\mathrm{~m})$ & 128.8 & 129.2 \\
\hline 14 & $6.99(\mathrm{~m})$ & $7.01(\mathrm{~m})$ & 130.0 & 130.8 \\
\hline 15 & $\begin{array}{c}2.98 \\
(\mathrm{dd}, 14.5,5.8 \mathrm{~Hz}) \\
2.38(\mathrm{~m})\end{array}$ & $\begin{array}{c}3.01 \\
(\mathrm{dd}, 16.5,4.4 \mathrm{~Hz}) \\
2.43 \\
(\mathrm{dd}, 16.5,13.0 \mathrm{~Hz})\end{array}$ & 23.4 & 21.7 \\
\hline 16 & $2.47(\mathrm{~m})$ & $\begin{array}{c}2.50 \\
(\mathrm{ddd}, 13.0,4.4,3.4 \mathrm{~Hz})\end{array}$ & 48.1 & 49.7 \\
\hline 17 & - & - & 74.0 & 73.9 \\
\hline 18 & $1.14(\mathrm{~s})$ & $1.14(\mathrm{~s})$ & 27.9 & 28.2 \\
\hline 19 & $0.92(\mathrm{~s})$ & $0.93(\mathrm{~s})$ & 27.0 & 27.6 \\
\hline
\end{tabular}




\subsubsection{Synthesis of carexane $O$ from 11a}

\section{Carexane 0}

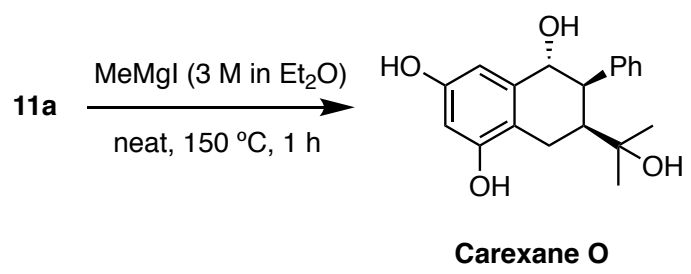

Compound 11a (21.5 mg, 0.063 mmol, 1.0 equiv) was treated with MeMgI (209 $\mu \mathrm{L}, 0.628$ mmol, 10.0 equiv; $3 \mathrm{M}$ in $\mathrm{Et}_{2} \mathrm{O}$ ) at $24{ }^{\circ} \mathrm{C}$. The solvent was evaporated under reduced pressure and the residue was heated to $150^{\circ} \mathrm{C}$ at $2 \mathrm{mbar}$ for $1 \mathrm{~h}$. After cooling to room temperature, $\mathrm{Et}_{2} \mathrm{O}$ $(2 \mathrm{~mL})$ was slowly added. The excess of Grignard reagent was quenched slowly with water and the mixture was extracted with $\mathrm{Et}_{2} \mathrm{O}$. The combined organic phases were combined, washed with brine and the solvent was evaporated under reduced pressure. The crude was purified by preparative TLC (EtOAc/ $\left.\mathrm{Et}_{2} \mathrm{O} 2: 3\right)$ to afford Carexane O (7.7 $\left.\mathrm{mg}, 0.025 \mathrm{mmol}, 39 \%\right)$ as an amorphous white solid.

\begin{tabular}{|c|c|c|}
\hline Natural Product & Literature $\boldsymbol{\alpha}_{\mathbf{D}}\left(\right.$ deg. $\left.\mathrm{cm}^{2} . \mathrm{g}^{-}\right)$ & Measured $\boldsymbol{\alpha}_{\mathbf{D}}\left(\mathrm{deg} . \mathrm{cm}^{2} . \mathrm{g}^{-}\right)$ \\
\hline Carexane I & +15.6 & +33.3 \\
\hline Carexane $\mathbf{P}$ & -26.7 & +13.0 \\
\hline Carexane $\mathbf{O}$ & +10.0 & -40.1 \\
\hline
\end{tabular}

We synthesized the natural products based in the reported absolute configurations. ${ }^{26}$ However, using similar conditions for the measurement of the specific rotations, the values obtained for the three natural compounds are in discrepancy with the ones previously reported. 


\section{Mechanistic investigations}

\subsection{Hammett study}

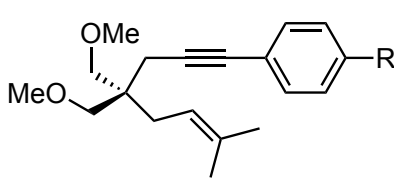

5b: $\mathrm{R}=\mathrm{H}, \mathbf{5 e}: \mathrm{R}=\mathrm{OMe}$ 5f: $\mathrm{R}=\mathrm{NO}_{2}, \mathbf{5 g}: \mathrm{R}=\mathrm{tBu}$ 5h: $\mathrm{R}=\mathrm{Cl}, \mathbf{5 i}: \mathrm{R}=\mathrm{CF}_{3}$

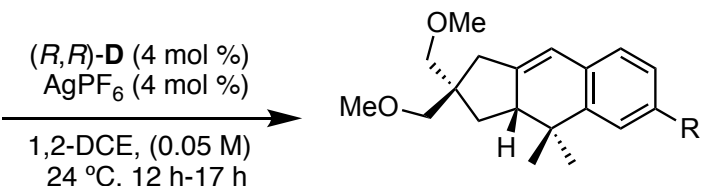

$6 b, 6 e-i$

$\rho=-0.26$

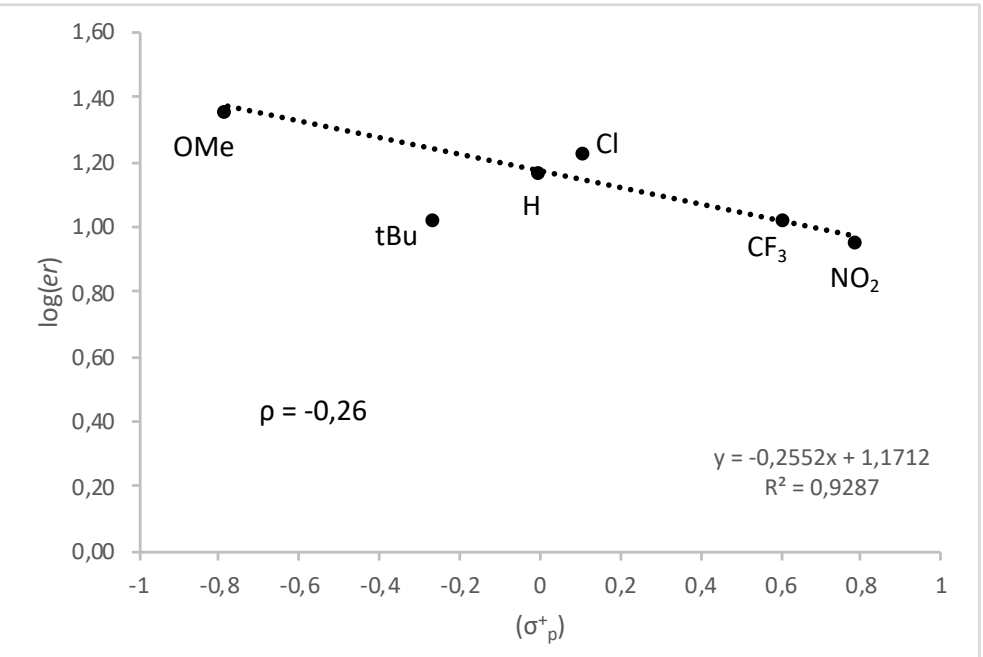

In the Hammett study of the formal [4+2] cycloaddition reaction, the data point corresponding to $\mathbf{5 g}$ was not considered for the linear regression, since the Hammett parameters do not account for steric effects. ${ }^{27}$ 

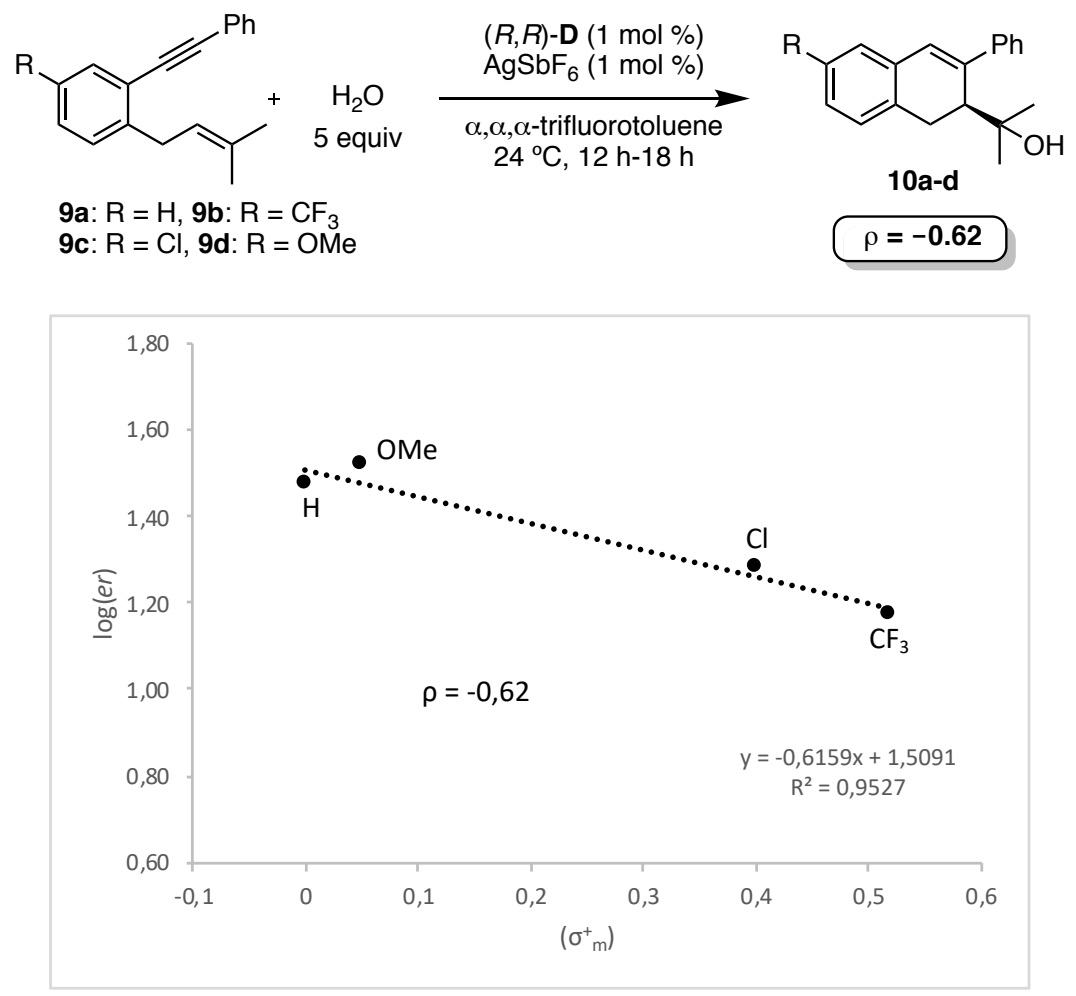

\subsection{Synthesis of chloride-bridged digold complex $(R, R)-C_{2}-C l$}

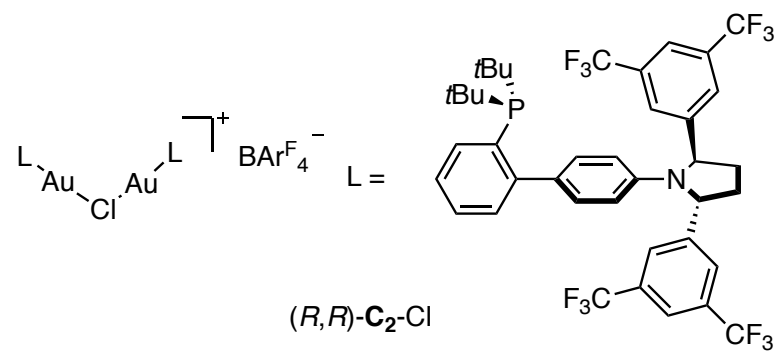

Complex $(R, R)-\mathbf{C}\left(15 \mathrm{mg}, 15 \mu \mathrm{mmol}, 1.0\right.$ equiv) was dissolved in $\mathrm{CD}_{2} \mathrm{Cl}_{2}(0.6 \mathrm{~mL})$ and $\mathrm{NaBAr}_{4}{ }_{4}\left(6.49 \mathrm{mg}, 7.32 \mu \mathrm{mol}, 0.5\right.$ equiv) was added. The reaction mixture was stirred at $24{ }^{\circ} \mathrm{C}$ for $16 \mathrm{~h}$. The yield of the title compound was calculated by ${ }^{1} \mathrm{H}$ NMR with 1,2-dibromoethane as internal standard (65\%).

Characteristic signals for chloride-bridged digold(I) complex $(R, R)-\mathrm{C}_{2}-\mathrm{Cl}$ : ${ }^{1} \mathrm{H}$ NMR $\left(500 \mathrm{MHz}, \mathrm{CD}_{2} \mathrm{Cl}_{2}\right) \delta 7.84-7.76(\mathrm{~m}, 6 \mathrm{H}), 7.75-7.69(\mathrm{~m}, 8 \mathrm{H}), 7.67(\mathrm{~s}, 6 \mathrm{H}), 7.55(\mathrm{~s}, 4 \mathrm{H}), 7.53$ $-7.41(\mathrm{~m}, 4 \mathrm{H}), 7.26-7.12(\mathrm{~m}, 2 \mathrm{H}), 6.86(\mathrm{t}, J=6.3 \mathrm{~Hz}, 4 \mathrm{H}), 6.33(\mathrm{~d}, J=8.8 \mathrm{~Hz}, 2 \mathrm{H}), 6.13(\mathrm{~d}$, $J=8.2 \mathrm{~Hz}, 2 \mathrm{H}), 5.40-5.32(\mathrm{~m}, 4 \mathrm{H}), 2.74-2.48(\mathrm{t}, J=7.5 \mathrm{~Hz}, 4 \mathrm{H}), 2.07-1.85$ (m, 4H), 1.42 (s, 9H), 1.38 (s, 9H), 1.34 (s, 9H), 1.30 (s, 9H). $\left.{ }^{13} \mathbf{C ~ N M R ~ ( 1 2 6 ~ M H z , ~} \mathrm{CD}_{2} \mathrm{Cl}_{2}\right) \delta 162.5(\mathrm{~d}, J=$ $50.0 \mathrm{~Hz}), 161.7$ (d, $J=49.9 \mathrm{~Hz}), 145.8,143.8,135.1,134.4,133.4,132.2,132.0,131.7$ (d, $J=$ 
$3.8 \mathrm{~Hz}), 131.0(\mathrm{~d}, J=6.7 \mathrm{~Hz}), 129.5(\mathrm{~d}, J=5.3 \mathrm{~Hz}), 129.3(\mathrm{dd}, J=5.7,2.8 \mathrm{~Hz}), 129.1(\mathrm{dd}, J=$ 5.7, $2.9 \mathrm{~Hz}), 128.2,127.7-127.5(\mathrm{~m}), 126.8,126.0,123.8,123.7$ (q, $J=273.0 \mathrm{~Hz}), 121.9-$ $121.6(\mathrm{~m}), 117.8(\mathrm{dt}, J=7.9,3.5 \mathrm{~Hz}), 114.9,114.6,63.4,38.3$ (d, $J=25.9 \mathrm{~Hz}), 32.2,31.9$ (d, $J$ $=6.0 \mathrm{~Hz}), 30.2(\mathrm{~d}, J=6.1 \mathrm{~Hz}) \cdot{ }^{31} \mathbf{P}\left\{{ }^{1} \mathbf{H}\right\} \mathbf{N M R}\left(162 \mathrm{MHz}, \mathrm{CD}_{2} \mathrm{Cl}_{2}\right) \delta$ 64.9. ${ }^{19} \mathbf{F}\left\{{ }^{1} \mathbf{H}\right\} \mathbf{N M R}(376$ $\left.\mathrm{MHz}, \mathrm{CD}_{2} \mathrm{Cl}_{2}\right) \delta-62.8,-63.0$. HRMS (ESI+) calculated for $\left[\mathrm{C}_{80} \mathrm{H}_{76} \mathrm{Au}_{2} \mathrm{ClF}_{24} \mathrm{NP}\right]^{+} 2011.4120$ $\mathrm{m} / \mathrm{z}$; found $[\mathrm{M}]^{+} 2011.4094 \mathrm{~m} / \mathrm{z}$.

\subsection{Evidence for the arene-gold(I) complex}

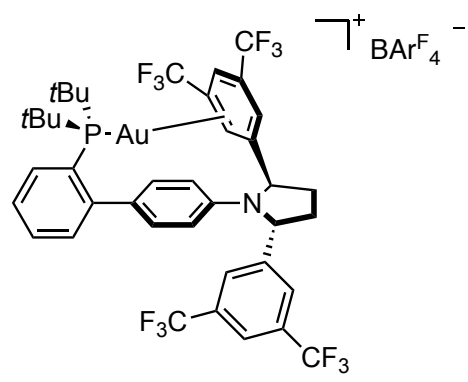

In a capped NMR tube, complex $(R, R)-\mathbf{C}(5.0 \mathrm{mg}, 4.88 \mu \mathrm{mmol}, 1.0$ equiv) was dissolved in dry $\mathrm{CD}_{2} \mathrm{Cl}_{2}(0.5 \mathrm{~mL})$ and $\mathrm{NaBAr}_{4}{ }_{4}(2.2 \mathrm{mg}, 2.44 \mu \mathrm{mol}, 0.5$ equiv) was added. The reaction mixture was stirred at $24{ }^{\circ} \mathrm{C}$ for $16 \mathrm{~h}$ until full conversion to the chloride-bridged digold complex $(R, R)-\mathbf{C}_{2}-\mathrm{Cl}$. The reaction was monitored by ${ }^{31} \mathrm{P}\left\{{ }^{1} \mathrm{H}\right\}$ NMR. Then, a second portion of $\mathrm{NaBAr}_{4}$ (2.2 mg, $2.44 \mu \mathrm{mol}, 0.5$ equiv) was added and the reaction mixture was stirred at $50{ }^{\circ} \mathrm{C}$ for $6 \mathrm{~h}$. The formation of a species with chemical shift of $67.5 \mathrm{ppm}$ was observed, which was attributed to the arene-gold(I) complex based on previous reports. ${ }^{28}$ Extended heating leads to the formation of $(R, R)-\mathbf{C}$. This observation indicates that $(R, R)-\mathbf{C}$, arene-gold(I) complex and chloride-bridged digold complex $(R, R)-\mathbf{C}_{2}-\mathrm{Cl}$ are in equilibrium under these reaction conditions. 


\section{Reaction monitoring by ${ }^{31} \mathbf{P}\left\{{ }^{1} \mathrm{H}\right\}$ NMR}

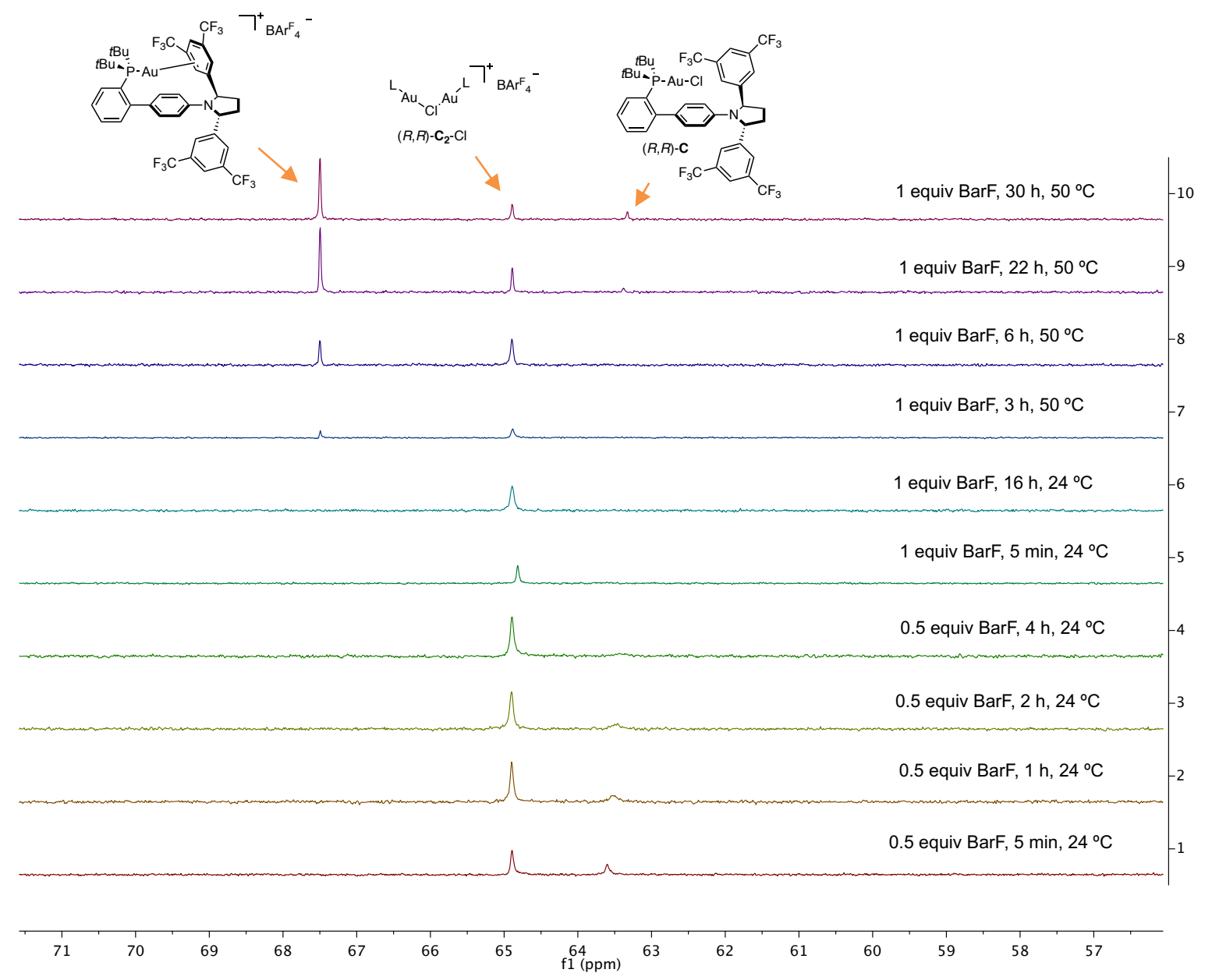

Characteristic signals for arene-gold(I) complex: ${ }^{1} \mathbf{H}$ NMR $\left(400 \mathrm{MHz}, \mathrm{CD}_{2} \mathrm{Cl}_{2}\right) \delta 7.94-$ 7.89 (m, 5H), $7.89-7.85$ (m, 6H), 7.61 (s, 1H), $7.47-7.37$ (m, 2H), $7.32-7.24$ (m, 1H), 7.18 $-7.11(\mathrm{~m}, 1 \mathrm{H}), 6.82-6.77(\mathrm{~m}, 1 \mathrm{H}), 6.15(\mathrm{~d}, J=7.6 \mathrm{~Hz}, 2 \mathrm{H}), 4.94(\mathrm{~d}, J=7.0 \mathrm{~Hz}, 2 \mathrm{H}), 2.47-$ $2.33(\mathrm{~m}, 2 \mathrm{H}), 1.86-1.74(\mathrm{~m}, 2 \mathrm{H}), 1.53(\mathrm{~d}, J=14.4 \mathrm{~Hz}, 9 \mathrm{H}), 1.25(\mathrm{~d}, J=14.7 \mathrm{~Hz}, 9 \mathrm{H}) .{ }^{31} \mathbf{P}\left\{{ }^{1} \mathbf{H}\right\}$ NMR $\left(162 \mathrm{MHz}, \mathrm{CD}_{2} \mathrm{Cl}_{2}\right) \delta 67.5 .{ }^{19} \mathbf{F}\left\{{ }^{1} \mathbf{H}\right\}$ NMR $\left(376 \mathrm{MHz}, \mathrm{CD}_{2} \mathrm{Cl}_{2}\right) \delta-62.4,-63.3,-63.4$. HRMS (ESI+) calculated for $\left[\mathrm{C}_{40} \mathrm{H}_{38} \mathrm{AuClF}_{12} \mathrm{NP}\right]^{+} 1024.1977 \mathrm{~m} / \mathrm{z}$; found $[\mathrm{M}+\mathrm{HCl}]^{+}$ $1024.1962 \mathrm{~m} / \mathrm{z}$. 
${ }^{1} \mathrm{H}$ NMR: $400 \mathrm{MHz}, \mathrm{CD}_{2} \mathrm{Cl}_{2}$, mixture of $(R, R)-\mathbf{C}$, arene-gold(I) complex and $(R, R)-\mathbf{C}_{2}-\mathrm{Cl}$ (only signals belonging to the arene-gold(I) complex are integrated and reported)

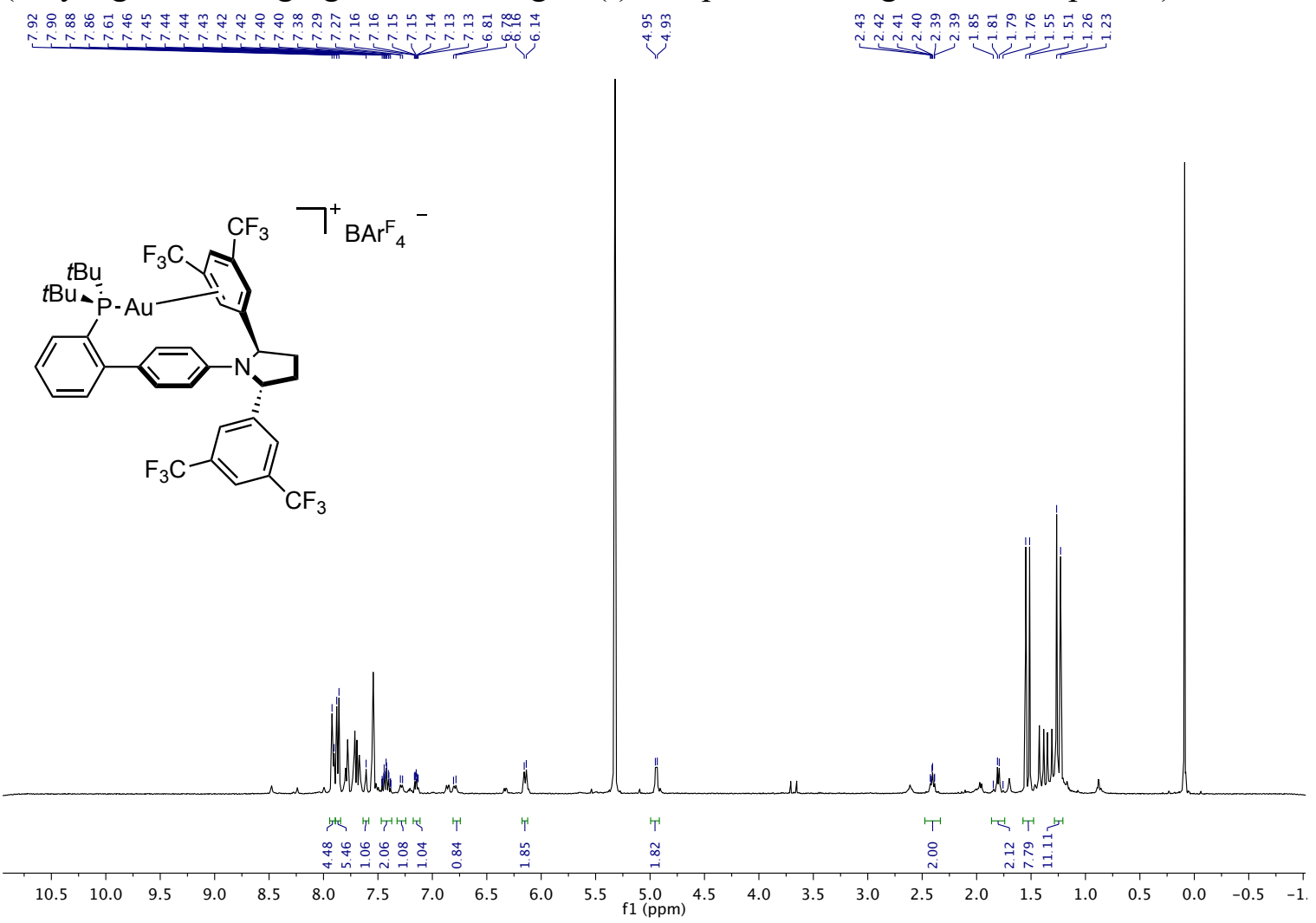

${ }^{1} \mathrm{H}$ NMR: $400 \mathrm{MHz}, \mathrm{CD}_{2} \mathrm{Cl}_{2}$, stacked spectra of $(R, R)-\mathbf{C}$, arene-gold(I) complex and $(R, R)-\mathbf{C}_{2}$ $\mathrm{Cl}$

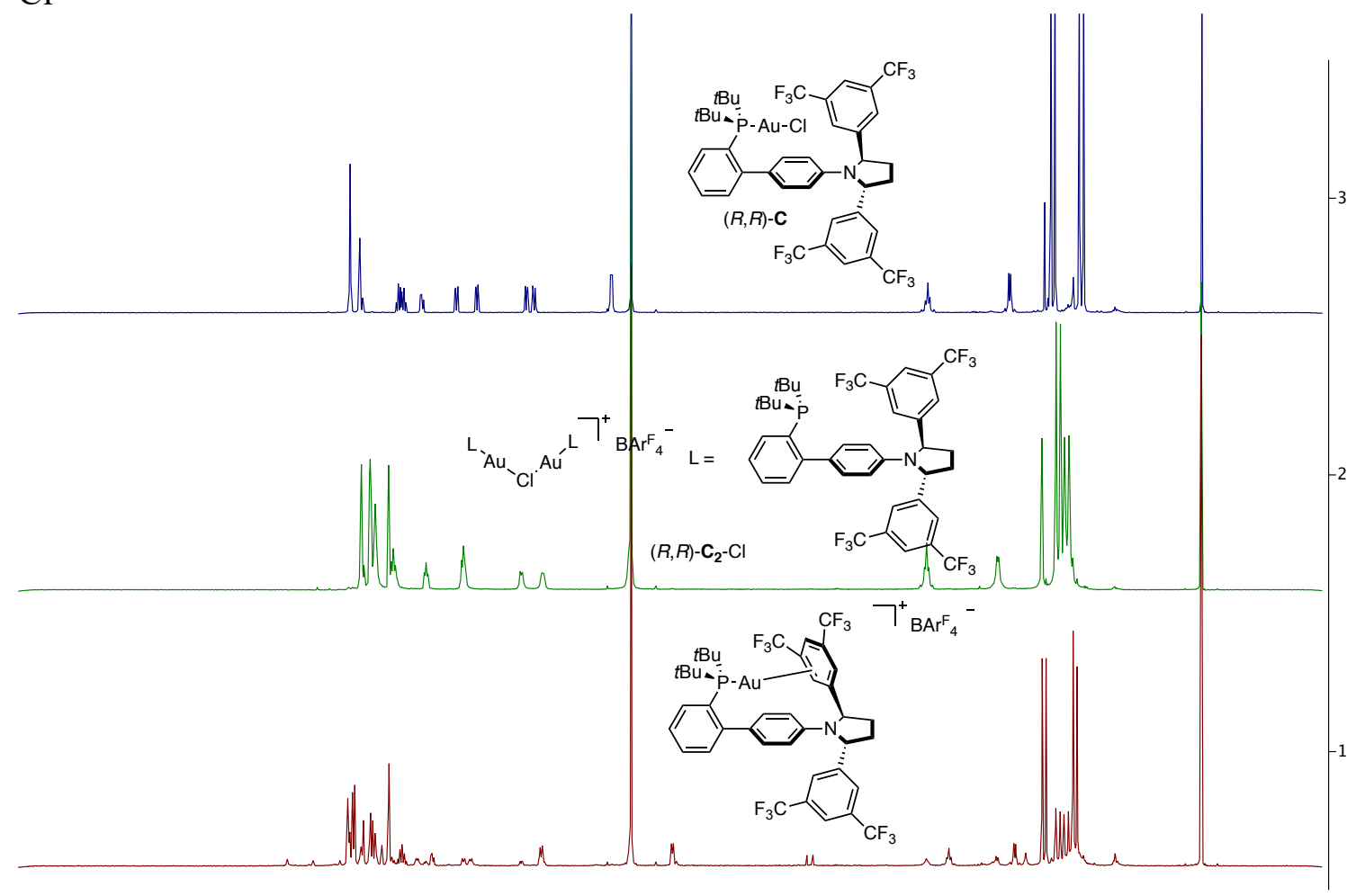

$\begin{array}{lllllllllllllllllllllllll}10.5 & 10.0 & 9.5 & 9.0 & 8.5 & 8.0 & 7.5 & 7.0 & 6.5 & 6.0 & 5.5 & \begin{array}{l}5.0 \\ \mathrm{f} 1\end{array}(\mathrm{ppm}) & 4.5 & 4.0 & 3.5 & 3.0 & 2.5 & 2.0 & 1.5 & 1.0 & 0.5 & 0.0 & -0.5 & -1.0\end{array}$ 
${ }^{31} \mathrm{P}\left\{{ }^{1} \mathrm{H}\right\}$ NMR: $162 \mathrm{MHz}, \mathrm{CD}_{2} \mathrm{Cl}_{2}$, stacked spectra of $(R, R)-\mathbf{C}$, arene-gold(I) complex and $(R, R)-\mathbf{C}_{2}-\mathrm{Cl}$

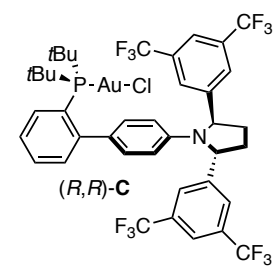

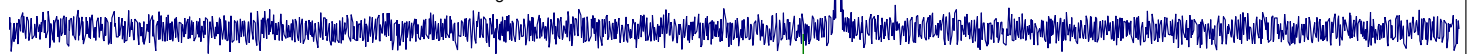

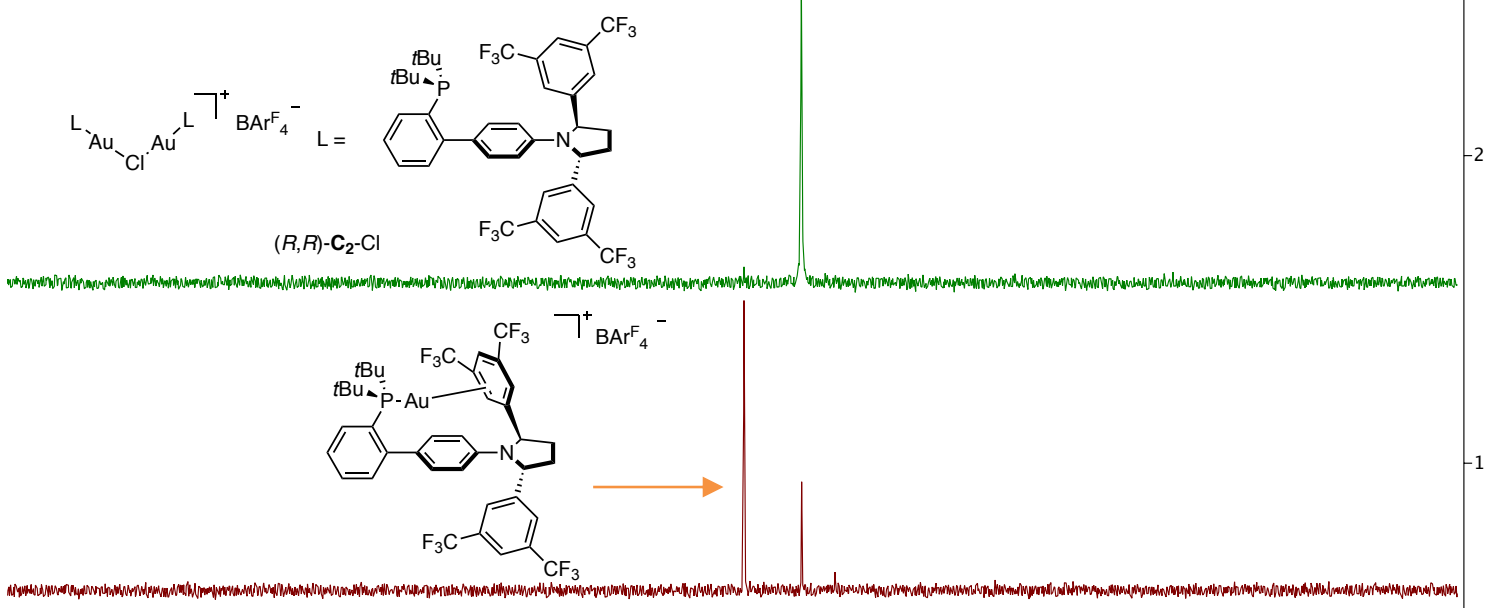

$\begin{array}{lllllllllllllllllllllllllllllllllllllll}100 & 98 & 96 & 94 & 92 & 90 & 88 & 86 & 84 & 82 & 80 & 78 & 76 & 74 & 72 & 70 & 68 & 66 & 64 & 62 & 60 & 58 & 56 & 54 & 52 & 50 & 48 & 46 & 44 & 42 & 40 & 38 & 36\end{array}$

${ }^{19} \mathrm{~F}\left\{{ }^{1} \mathrm{H}\right\}$ NMR: $376 \mathrm{MHz}, \mathrm{CD}_{2} \mathrm{Cl}_{2}$, stacked spectra of $(R, R)-\mathrm{C}$, arene-gold(I) complex and $(R, R)-\mathbf{C}_{2}-\mathrm{Cl}$

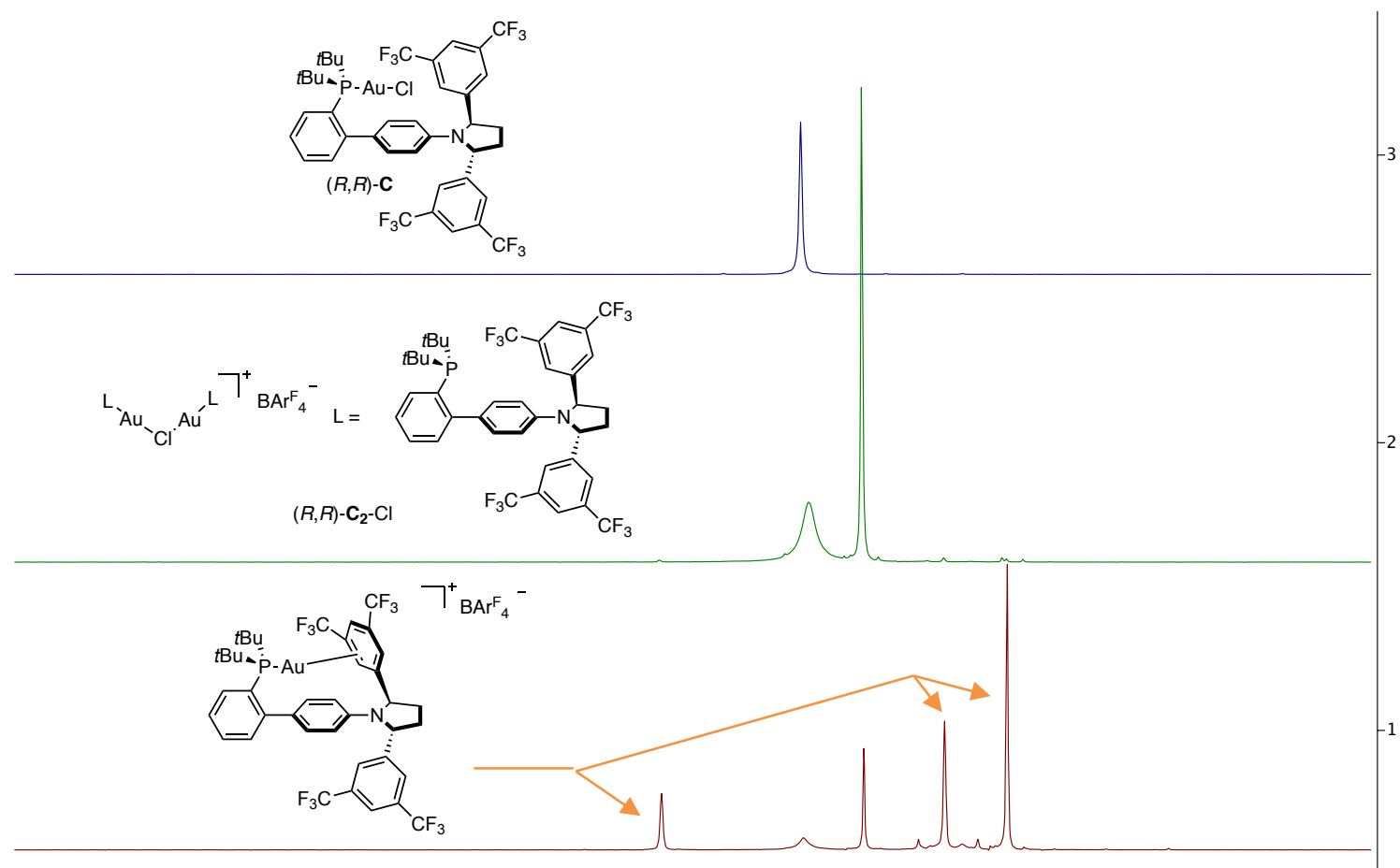

$\begin{array}{lllllllllllllllllllllllllll}-60.6 & -60.8 & -61.0 & -61.2 & -61.4 & -61.6 & -61.8 & -62.0 & -62.2 & -62.4 & -62.6 & -62.8 & -63.0 & -63.2 & -63.4 & -63.6 & -63.8 & -64.0 & -64.2 & -64.4\end{array}$ 


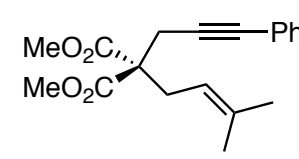

$5 a$

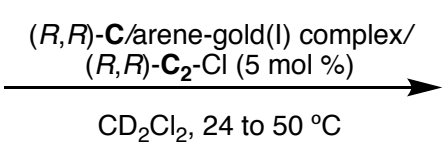

$\mathrm{CD}_{2} \mathrm{Cl}_{2}, 24$ to $50^{\circ} \mathrm{C}$

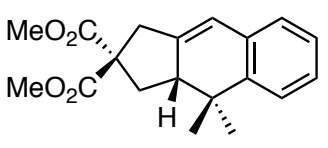

$6 a$

To the obtained mixture of $(R, R)-\mathbf{C} /$ arene-gold(I) complex/(R,R)-C $\mathbf{C}_{\mathbf{2}}-\mathrm{Cl}$, enyne 5a (25 $\mathrm{mg}$, 0.08 mmol, 16.5 equiv) was added and the reaction was monitored by ${ }^{31} \mathrm{P}\left\{{ }^{1} \mathrm{H}\right\}$ NMR. After 18 $\mathrm{h}$ reaction time at $24{ }^{\circ} \mathrm{C}$ no formation of $\mathbf{6 a}$ was observed.

The reaction was heated to $50{ }^{\circ} \mathrm{C}$ for $16 \mathrm{~h}$. Substantial amount of product 6a was formed in a 3:2 ration of starting material 5a to product 6a. According to evidence by ${ }^{31} \mathrm{P}\left\{{ }^{1} \mathrm{H}\right\} \mathrm{NMR}$, the amount of chloride-bridged digold complex $(R, R)-\mathbf{C}_{2}-\mathrm{Cl}$ decreases to form $(R, R-\mathbf{C})$ and arenegold(I) complex. Hence, at $50{ }^{\circ} \mathrm{C}$ arene-gold(I) complex and chloride-bridged digold complex $(R, R)-\mathbf{C}_{2}-\mathrm{Cl}$ can enter the catalytic cycle.

${ }^{31} \mathrm{P}\left\{{ }^{1} \mathrm{H}\right\}$ NMR: $162 \mathrm{MHz}, \mathrm{CD}_{2} \mathrm{Cl}_{2}$, stacked spectra for reaction control

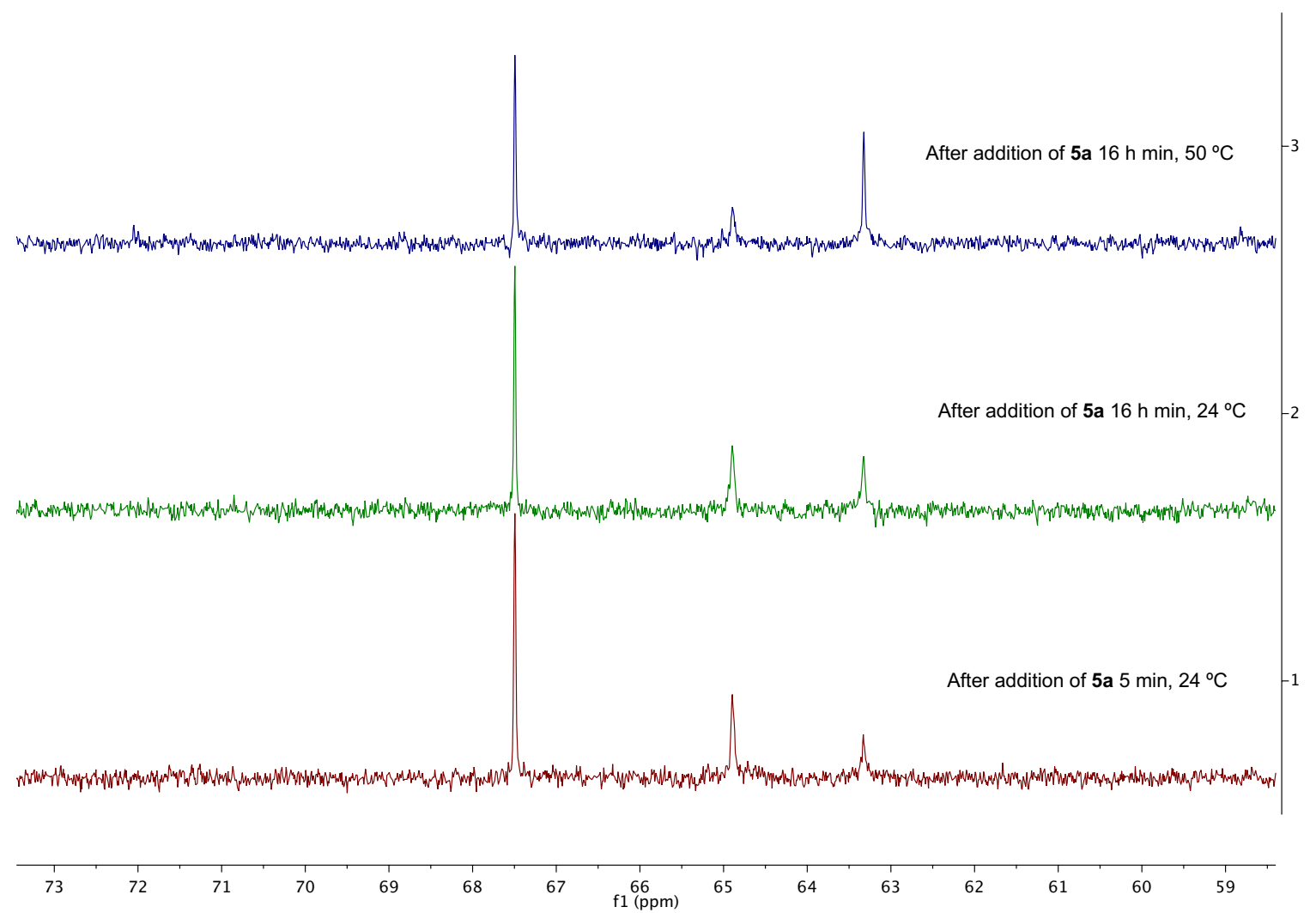




\subsection{Kinetic studies and reaction monitoring}

The following reaction was monitored by ${ }^{1} \mathrm{H}$ NMR with 1,3,5-tribromobenzene as internal standard. The reaction is $1^{\text {st }}$ order, $\mathrm{k}=4 \times 10^{-4} \mathrm{~s}^{-1}$.

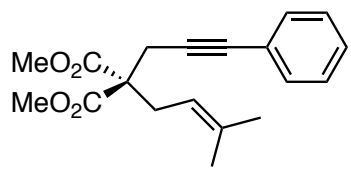

$5 \mathbf{a}$

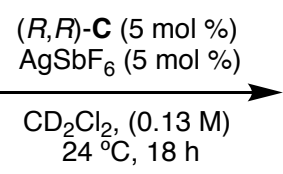

$24{ }^{\circ} \mathrm{C}, 18 \mathrm{~h}$

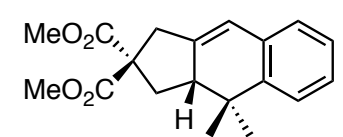

$6 a$

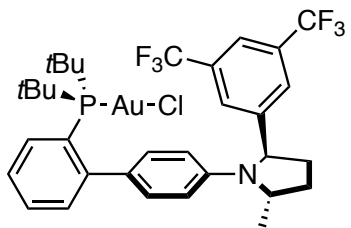

$(R, R)-\mathbf{C}$

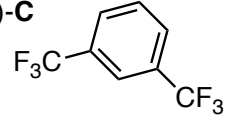

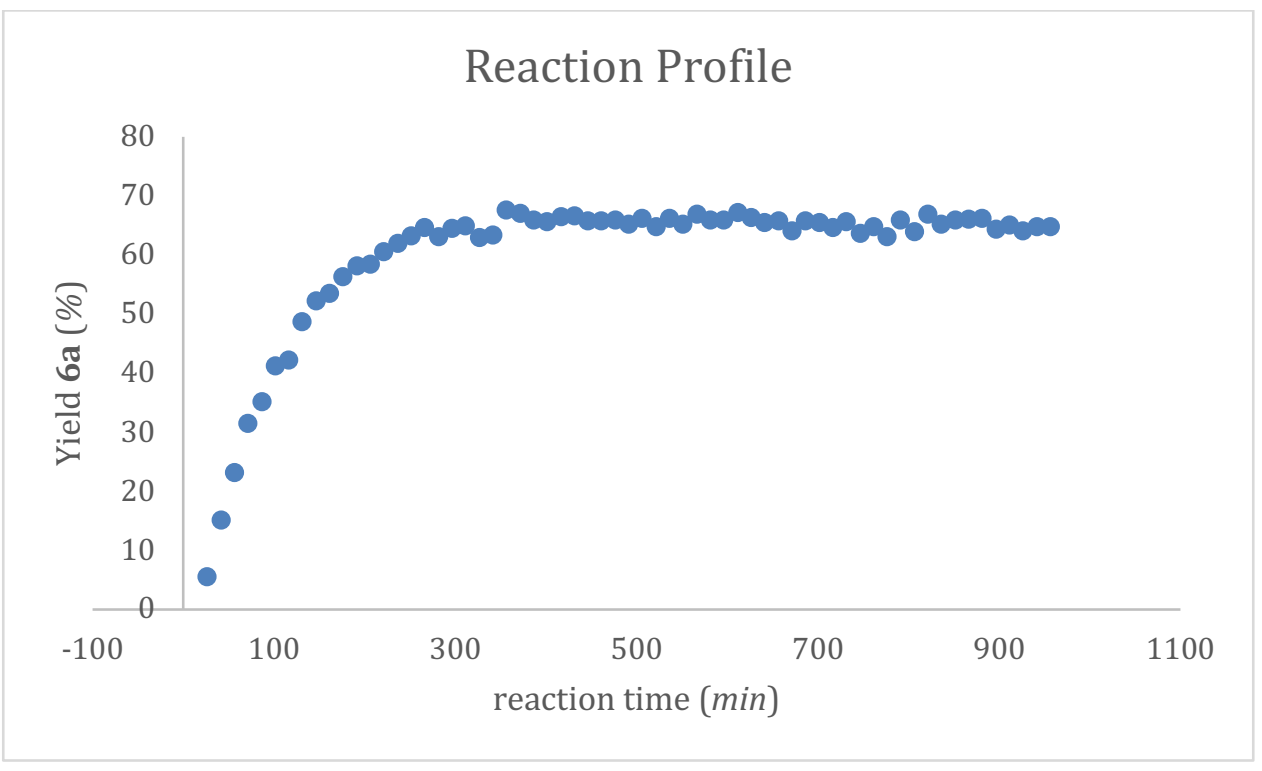

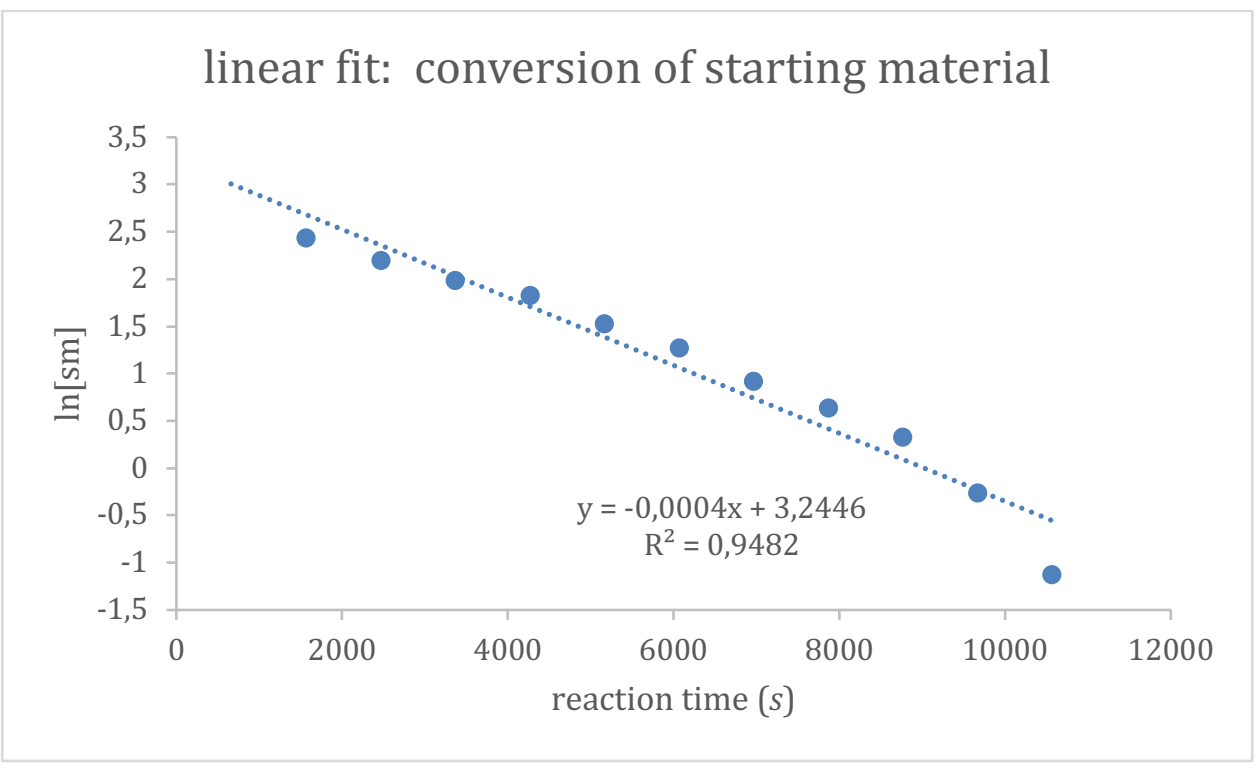


In parallel, the reaction was monitored also by ${ }^{31} \mathrm{P}\left\{{ }^{1} \mathrm{H}\right\}$ NMR. At 11 min reaction time 5a is coordinated to the cationic gold(I) complex generated in situ $(68.5 \mathrm{ppm})$ through the alkene. After full conversion of 5a, the signal is still present in the reaction mixture presumably arising from 6a coordinating to the active catalyst. The signal at $69.2 \mathrm{ppm}$ refers to 1,6 -enyne coordinated to the catalyst through the alkyne. The species at $70.2 \mathrm{ppm}$ which increases with increasing reaction time is attributed to the side product $\mathbf{6 a}$ ' coordinated to the active gold catalyst.

${ }^{31} \mathrm{P}\left\{{ }^{1} \mathrm{H}\right\}$ NMR: $162 \mathrm{MHz}, \mathrm{CD}_{2} \mathrm{Cl}_{2}$, stacked spectra for reaction monitoring of $5 \mathbf{a}$ to $\mathbf{6 a}$

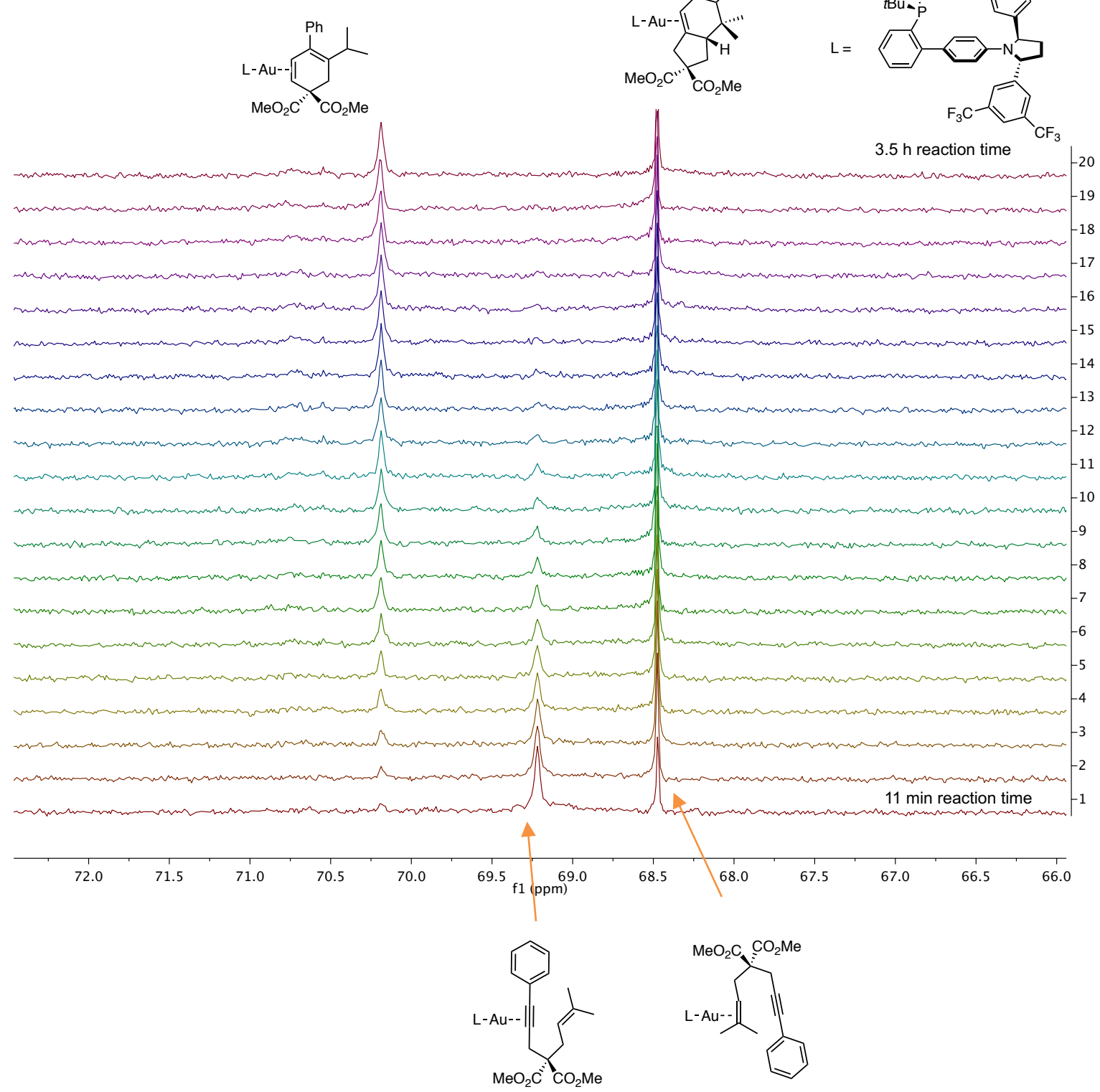




\section{DFT mechanistic studies}

\subsection{Computational methods}

Calculations were performed by means of the Gaussian 09 suite of programs. ${ }^{29}$ DFT was applied using BP86-D3 that has proved its efficiency in other DFT studies of gold-catalyzed transformations. ${ }^{30}$ The SDD basis set and ECP was used to describe Au. ${ }^{31}$ The $6-31 \mathrm{G}(\mathrm{d})$ basis set $^{32}$ was employed for all remaining atoms (C, H, P, F, O and N). Full geometry optimizations were carried out in dichloromethane, through an implicit polarizable continuum model (PCM). ${ }^{33}$ The stationary points were characterized by vibrational analysis. Transition states were identified by the presence of one imaginary frequency while minima by a full set of real frequencies. The connectivity of the transition states was confirmed by the relaxation of each transition state towards both the reactant and the product or, in some cases, by IRC ${ }^{34}$ calculations. NCIPlot was used to obtain the grid data for NCI (non-covalent interactions) analysis $^{35}$ and the corresponding results were visualized with the VMD software ${ }^{36}$. Reported energies are potential energies (E) and free energies (G) in solution, computed at $298 \mathrm{~K}$ and 1 atm.

\subsection{Discussion}

\subsubsection{5-exo-Dig and 6-endo-dig enantioselective cyclization of enynes $5 \mathrm{~b}$ and $9 \mathrm{k}$}
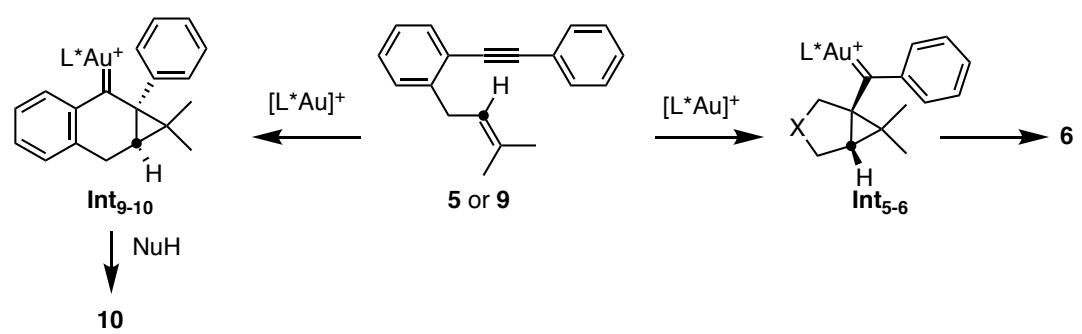

Figure 2: Enantioselective gold(I) catalyzed [4+2] cyclization of enynes 5 or 9 with catalyst $\boldsymbol{C}$ $(L=4 c)$.

The mechanism of this transformation has been previously studied computationally by our group employing simple $\mathrm{PH}_{3}$ as ligand for gold(I). ${ }^{7,8}$ To rationalize the different outcomes $(R$ or $S$ ) observed in the enantioselective version of this reaction depending on the nature of enynes, we performed DFT calculations using $(R, R)-\mathbf{C}$ as gold(I) catalyst, which is similar but simpler to the best catalyst $(R, R)$-D. Our calculations herein center on nucleophilic attack of the 
olefin at on alkyne coordinated to the $\mathrm{Au}(\mathrm{I})$ (Int1a-d and Int3a-d) as the enantio-determining step of the transformations.

Two possible binding orientations $\boldsymbol{A}$ and $\boldsymbol{B}$ (figure 3) of the substrate towards the chiral pocket of the catalyst are possible and also two enantiotopic faces of the olefin (Re and $\mathrm{Si}$ ) can react giving the product $R$ or $S$, correspondingly.

For enyne $\mathbf{5 b}$, upon the coordination of the alkyne to gold, we examined the evolution of the four possible gold(I) complexes Int1a-d by an exocyclic pathway. In this case, the preferred substrate orientation was $\boldsymbol{B}$, being $\boldsymbol{B}$-Int1d the most stable intermediate and the activation energy to reach $\boldsymbol{B}$-TS $\mathbf{S}_{\text {Int1d-2d }}$ lower than other possible pathways by at least $2.5 \mathrm{Kcal} / \mathrm{mol}$. This leads to the formation of the $R$ cyclopropyl gold carbene $\boldsymbol{B}$-Int2d, in agreement with our experimental results (94:6 er with catalyst D). Attractive interactions between the aryl ring of the substrate with the aryl ring of the pyrrolidine (distance $3.62 \AA$ ) and with the lower ring of the biphenyl scaffold of the ligand (distance $3.47 \AA$ ) as well as aryl-OMe interactions (3.01 $\AA$ ) play the major role in the chiral folding of the substrate and in the stabilization of the transition state $(\boldsymbol{B}$-TS Int1d-2d $)$.

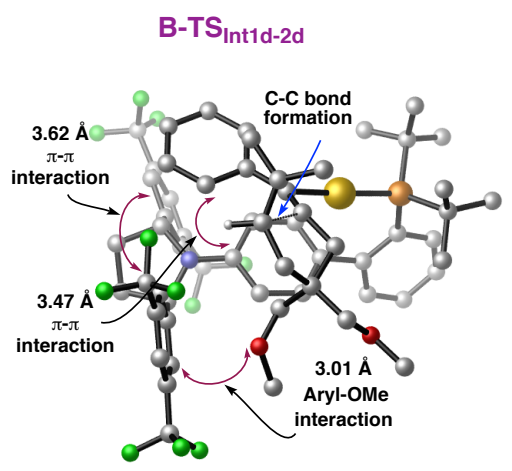




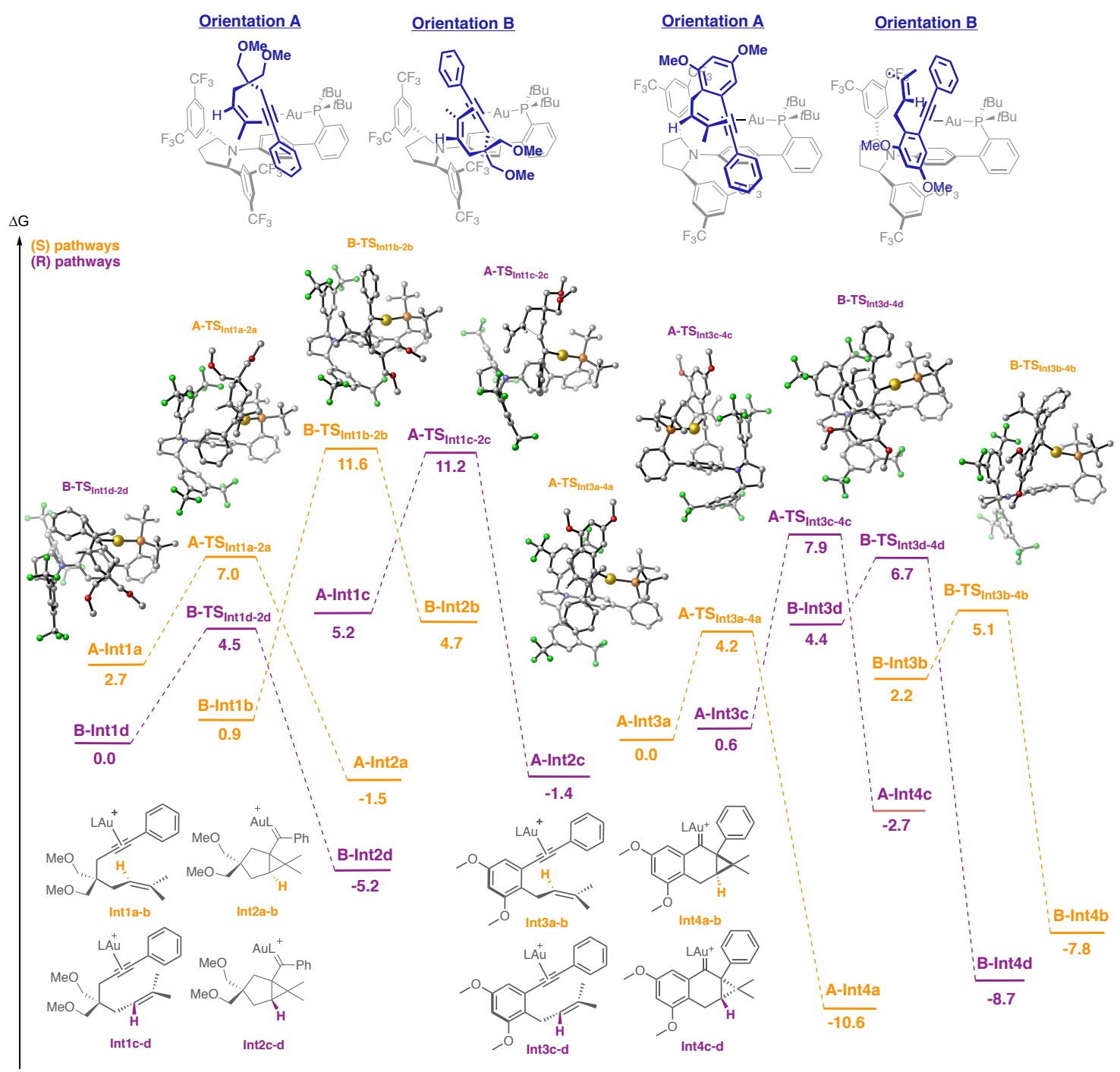

Figure 3: Free energy profiles for the 5-exo-dig (left) and 6-endo-dig (right) enantioselective cyclization reaction of 1,6-enynes $\mathbf{5 b}$ (left) and $\mathbf{9 k}$ (right) coordinated to catalyst $\boldsymbol{C}$.(S) pathways are depicted in orange and $(R)$ pathways in purple. The energy values are given in $\mathrm{Kcal} / \mathrm{mol}$ and represent the relative free energies. $L=4 c$. Hydrogen atoms have been omitted for clarity.

However, orientation $\boldsymbol{A}$ was found to drive the enantioinduction for enyne $9 \mathbf{k}$ by 6 -endo-dig pathway of intermediate $\boldsymbol{A}$-Int3a. The strong aryl-aryl interaction of the most electron-rich aromatic ring of the substrate with the aryl group of the pyrrolidine (distance $3.32 \AA$ ) favors orientation $\mathbf{A}$ in the reactive site leading to the $S$ enantiomer. Hence, cyclopropyl gold carbene $\boldsymbol{A}$-Int4a is preferentially formed via $\boldsymbol{A}$-TS $\mathbf{T S}_{\text {Int3a-4a }}$ that requires 2.5 and $3.7 \mathrm{Kcal} / \mathrm{mol}$ less than the competing $R$ pathways $(\boldsymbol{A}$-TS $\mathbf{I n t 3 c - 4 c}, \boldsymbol{B}$-TS $\mathbf{I n t 3 d - 4 d})$ according with our experimental results (94:6 er). 


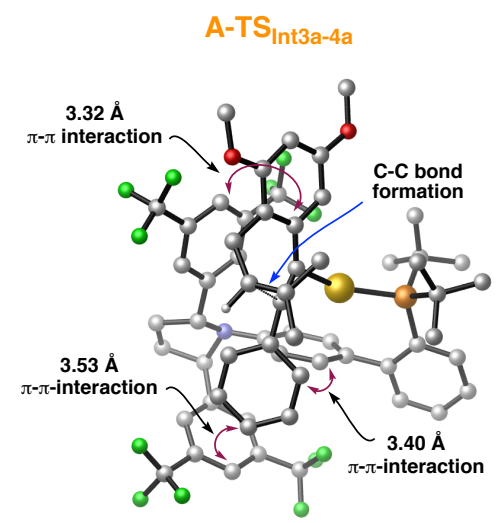

Given the important role that non-covalent interactions could play in the investigated system, we further examined these interactions through the NCI analysis. The NCI plots illustrated the size and shape of the surfaces generated from interactions present in the system.

\subsubsection{NCI plots}

In the lowest-energy transition states, $\boldsymbol{B}$-TS $\mathbf{S}_{\text {Int1d-2d }}$ and $\boldsymbol{A}$-TS Int3a-4a, we could observe larger green surfaces, which mainly correspond to aryl-aryl interactions, than in the other possible transition states coming for the other minima found. Strong attractive interactions are blue, corresponding to the new $\mathrm{C}-\mathrm{C}$ bond formation, and strong repulsive interactions are red.

Thus, we can confirm that non-covalent interactions that occur in the chiral pocket of this new type of catalysts have been found to be the key stereocontrol elements in cycloisomerization of enynes like 5, 7 and 9. 

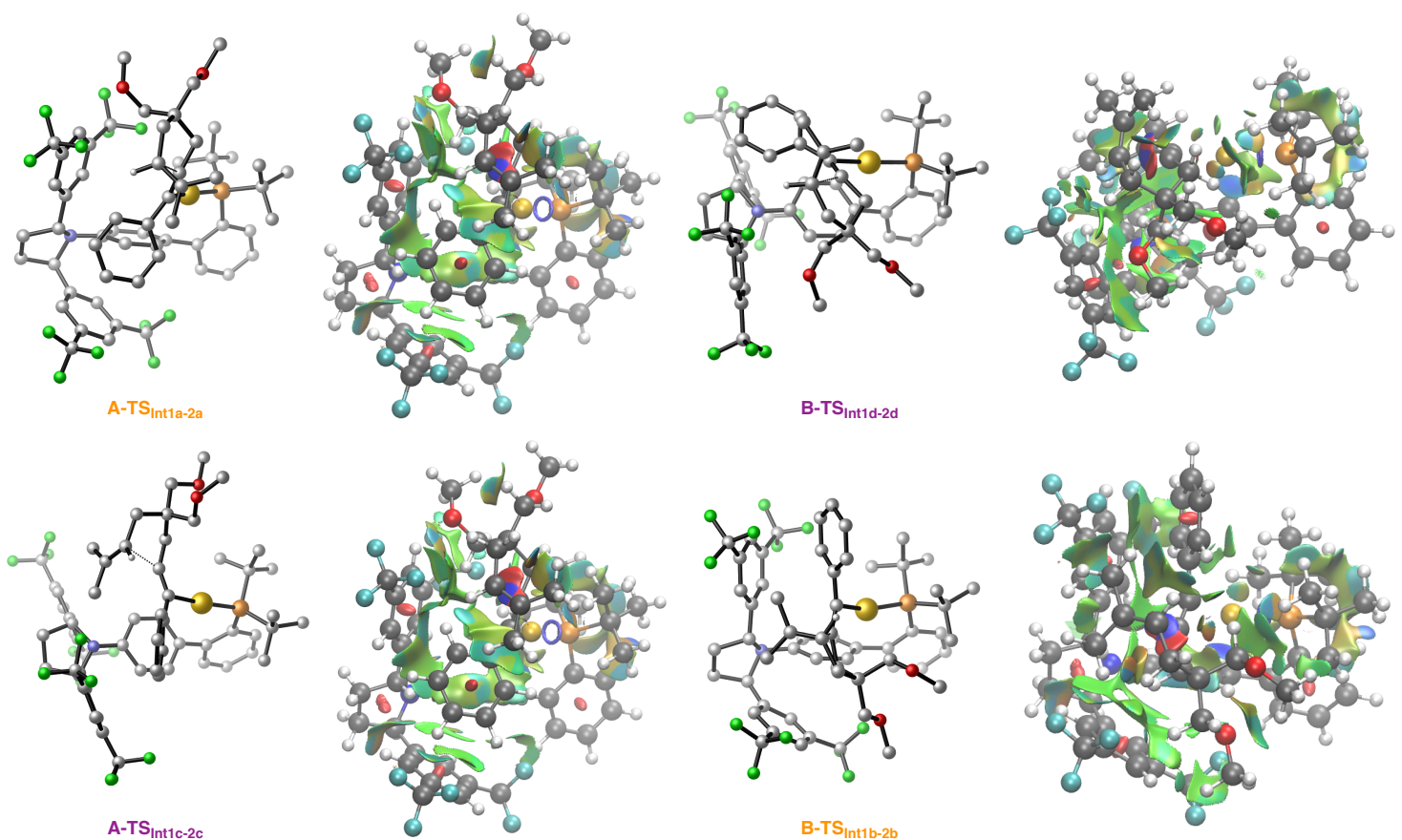

Figure 4: NCI Plots for the four possible transition states in the 5-exo-dig cyclization of enyne 5b. Color code: P: orange, Au: yellow, F: cyan, O: red, C: grey, and H: white.
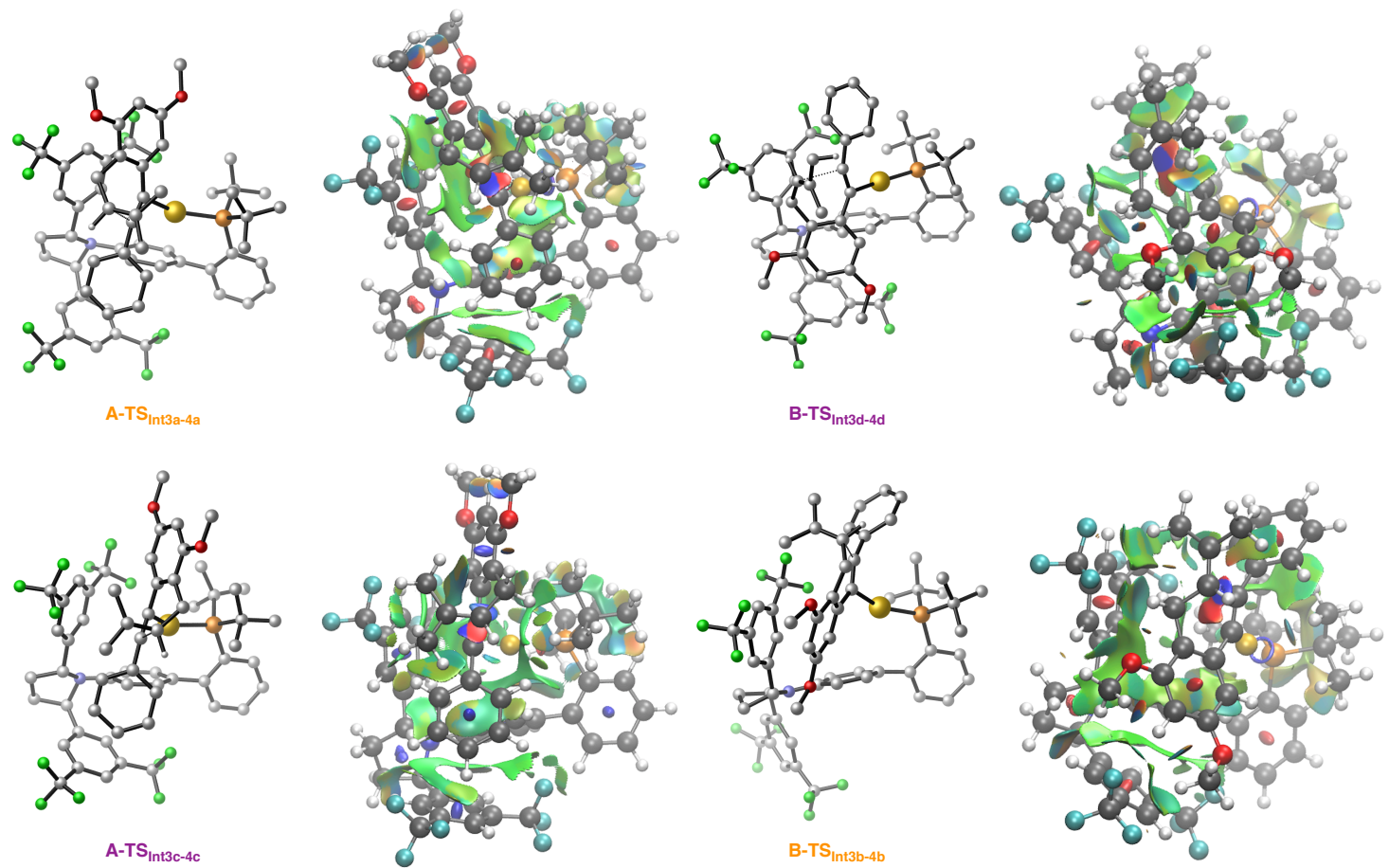

Figure 5: NCI Plots for the four possible transition states in the 6-endo-dig cyclization of enyne 9k. Color code: P: orange, Au: yellow, F: cyan, O: red, C: grey, and H: white. 


\subsection{Coordinates and Energies of Intermediates and Transition States (BP86-D3)}

A-Int1a

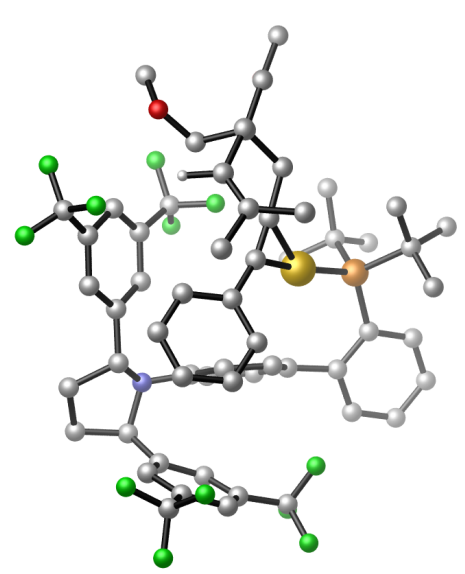

$E=-4167.37188981$ Hartrees

$\mathrm{G}=-4166.394407$ Hartrees
A-Int1c

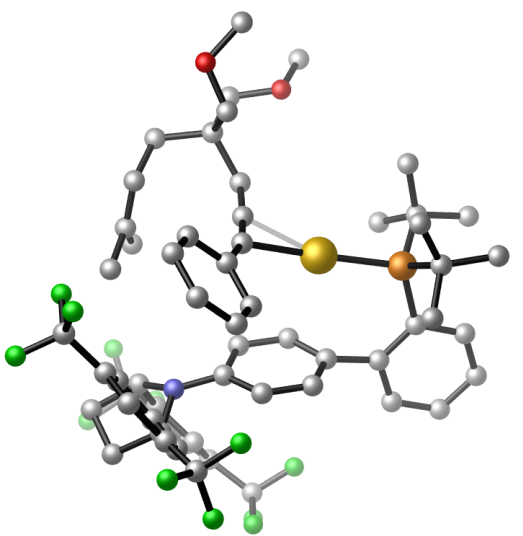

$E=-4167.36318320$ Hartrees

$\mathrm{G}=-4166.390366$ Hartrees

$\begin{array}{rrrrrrrr}79 & -1.113475 & 6.453272 & 7.421234 & 79 & -1.507810 & 7.266157 & 8.037011 \\ 6 & -2.210348 & 3.062947 & 7.559598 & 6 & -4.199927 & 5.021930 & 7.868859 \\ 6 & -2.779664 & 2.014759 & 8.322951 & 6 & -5.335049 & 4.325489 & 8.352529 \\ 6 & -3.528148 & 0.988212 & 7.734604 & 6 & -5.958110 & 3.325392 & 7.595638 \\ 6 & -3.724399 & 0.985514 & 6.346437 & 6 & -5.447293 & 2.993437 & 6.331744 \\ 6 & -3.140647 & 1.991998 & 5.568838 & 6 & -4.340385 & 3.689390 & 5.829156 \\ 6 & -2.372800 & 3.033126 & 6.143460 & 6 & -3.708712 & 4.716399 & 6.568749 \\ 6 & -1.721936 & 3.952283 & 5.163118 & 6 & -2.606080 & 5.434534 & 5.855412 \\ 6 & -0.744095 & 3.410784 & 4.299178 & 6 & -1.266023 & 4.990192 & 5.895295 \\ 6 & -0.175890 & 4.179507 & 3.279431 & 6 & -0.300434 & 5.520050 & 5.032760 \\ 6 & -0.587359 & 5.514096 & 3.077666 & 6 & -0.643705 & 6.500904 & 4.062465 \\ 6 & -1.554752 & 6.066142 & 3.937870 & 6 & -1.965635 & 7.017293 & 4.094330 \\ 6 & -2.119858 & 5.292739 & 4.966930 & 6 & -2.923670 & 6.480066 & 4.963104 \\ 6 & 0.020435 & 7.668692 & 1.940967 & 6 & -0.230998 & 7.572424 & 1.843981 \\ 6 & 0.365296 & 8.012990 & 0.458311 & 6 & 0.768749 & 7.128386 & 0.736768 \\ 6 & -0.034083 & 6.755606 & -0.365397 & 6 & 2.033027 & 6.727398 & 1.512944 \\ 6 & -0.719962 & 5.806819 & 0.636505 & 6 & 1.452403 & 6.056267 & 2.776593 \\ 6 & 1.022994 & 8.212430 & 2.945727 & 6 & -0.316291 & 9.091069 & 1.971334 \\ 6 & 1.303899 & 9.592475 & 2.933690 & 6 & -1.489677 & 9.752551 & 1.571825\end{array}$




\begin{tabular}{|c|c|c|c|c|c|c|c|}
\hline 6 & 2.163763 & 10.158136 & 3.885787 & 6 & -1.546240 & 11.155992 & 1.547920 \\
\hline 6 & 2.776160 & 9.360766 & 4.863815 & 6 & -0.425809 & 11.919216 & 1.896859 \\
\hline 6 & 2.502035 & 7.987890 & 4.873687 & 6 & 0.739096 & 11.260206 & 2.319015 \\
\hline 6 & 1.642944 & 7.413903 & 3.920955 & 6 & 0.791576 & 9.860538 & 2.374157 \\
\hline 6 & 2.295003 & 11.657930 & 3.968759 & 6 & -2.847753 & 11.851482 & 1.234886 \\
\hline 6 & 3.035528 & 7.108953 & 5.975790 & 6 & 1.964779 & 12.078666 & 2.648885 \\
\hline 6 & -2.237398 & 5.945195 & 0.771496 & 6 & 1.152156 & 4.571343 & 2.548212 \\
\hline 6 & -2.946611 & 4.896776 & 1.393096 & 6 & 2.189814 & 3.634389 & 2.697585 \\
\hline 6 & -4.288911 & 5.053130 & 1.765863 & 6 & 1.944186 & 2.263757 & 2.515178 \\
\hline 6 & -4.970732 & 6.246108 & 1.488206 & 6 & 0.654496 & 1.802068 & 2.209528 \\
\hline 6 & -4.282550 & 7.278774 & 0.833230 & 6 & -0.379743 & 2.737305 & 2.064970 \\
\hline 6 & -2.926940 & 7.140438 & 0.489674 & 6 & -0.132684 & 4.110477 & 2.215104 \\
\hline 6 & -4.968627 & 3.975083 & 2.574267 & 6 & 3.091467 & 1.284928 & 2.609561 \\
\hline 6 & -5.048987 & 8.526190 & 0.467320 & 6 & -1.802259 & 2.274329 & 1.861929 \\
\hline 6 & 0.503856 & 3.631127 & 8.691913 & 6 & -2.765077 & 5.305133 & 10.491986 \\
\hline 6 & -2.149867 & 4.731167 & 10.127603 & 6 & -4.673345 & 7.653273 & 9.294603 \\
\hline 15 & -1.241417 & 4.361291 & 8.457950 & 15 & -3.392875 & 6.269406 & 8.959501 \\
\hline 7 & -0.065705 & 6.198195 & 1.936708 & 7 & 0.261215 & 6.883144 & 3.064304 \\
\hline 9 & 3.473394 & 12.051486 & 4.515942 & 9 & -2.653361 & 13.062337 & 0.643576 \\
\hline 9 & 1.291585 & 12.177057 & 4.759795 & 9 & -3.566237 & 12.083996 & 2.382777 \\
\hline 9 & 2.174853 & 12.255945 & 2.751403 & 9 & -3.645028 & 11.110791 & 0.416518 \\
\hline 9 & 2.089638 & 6.950996 & 6.974597 & 9 & 1.637901 & 13.296153 & 3.175767 \\
\hline 9 & 4.142111 & 7.625579 & 6.576240 & 9 & 2.713896 & 12.320041 & 1.532840 \\
\hline 9 & 3.336268 & 5.854584 & 5.543585 & 9 & 2.780525 & 11.451675 & 3.544956 \\
\hline 9 & -6.297432 & 3.879919 & 2.295443 & 9 & 4.007740 & 1.669077 & 3.545266 \\
\hline 9 & -4.863536 & 4.229209 & 3.917596 & 9 & 3.757240 & 1.186575 & 1.420533 \\
\hline 9 & -4.411082 & 2.744853 & 2.364335 & 9 & 2.669115 & 0.031108 & 2.936829 \\
\hline 9 & -4.230446 & 9.548647 & 0.080641 & 9 & -2.466461 & 2.198823 & 3.061365 \\
\hline 9 & -5.923073 & 8.300731 & -0.552970 & 9 & -2.510668 & 3.134479 & 1.076790 \\
\hline 9 & -5.793474 & 8.984071 & 1.524578 & 9 & -1.869746 & 1.039583 & 1.294375 \\
\hline 1 & -0.960649 & 8.142733 & 2.182416 & 1 & -1.244236 & 7.199694 & 1.593234 \\
\hline 1 & 1.442482 & 8.222498 & 0.356379 & 1 & 0.944829 & 7.921547 & -0.006414 \\
\hline 1 & -0.177030 & 8.918403 & 0.139465 & 1 & 0.355755 & 6.251060 & 0.209065 \\
\hline 1 & 0.865553 & 6.260574 & -0.763826 & 1 & 2.625249 & 7.612671 & 1.803035 \\
\hline 1 & -0.686607 & 6.988411 & -1.222258 & 1 & 2.690950 & 6.046386 & 0.950426 \\
\hline 1 & -0.475903 & 4.749419 & 0.436908 & 1 & 2.168982 & 6.135183 & 3.612534 \\
\hline
\end{tabular}




\begin{tabular}{|c|c|c|c|c|c|c|c|}
\hline 1 & -2.633426 & 1.987426 & 9.403246 & 1 & -5.745277 & 4.562440 & 9.335661 \\
\hline 1 & -3.953567 & 0.199569 & 8.363336 & 1 & -6.834314 & 2.807143 & 7.998321 \\
\hline 1 & -4.318763 & 0.199877 & 5.868454 & 1 & -5.914470 & 2.203869 & 5.733800 \\
\hline 1 & -3.282688 & 1.997278 & 4.484430 & 1 & -3.941441 & 3.445824 & 4.838480 \\
\hline 1 & -0.436319 & 2.367652 & 4.428564 & 1 & -0.982681 & 4.197465 & 6.596132 \\
\hline 1 & 0.578133 & 3.750493 & 2.611201 & 1 & 0.711755 & 5.113360 & 5.072473 \\
\hline & -2.973344 & 5.688420 & 5.529189 & 1 & -3.949787 & 6.862215 & 4.929128 \\
\hline & -1.923048 & 7.079323 & 3.767043 & 1 & -2.260208 & 7.830530 & 3.427654 \\
\hline & 0.829907 & 10.237487 & 2.188133 & 1 & -2.365317 & 9.172054 & 1.262147 \\
\hline 1 & 1.460950 & 6.338814 & 3.954732 & 1 & 1.702476 & 9.368518 & 2.720206 \\
\hline 1 & 3.434213 & 9.816615 & 5.604905 & 1 & -0.465757 & 13.010513 & 1.865449 \\
\hline 1 & -6.019117 & 6.368882 & 1.772966 & 1 & 0.458410 & 0.733471 & 2.097315 \\
\hline 1 & -2.419295 & 7.976980 & 0.002475 & 1 & -0.961826 & 4.814422 & 2.109178 \\
\hline 1 & -2.434355 & 3.960072 & 1.629694 & 1 & 3.194783 & 3.972963 & 2.972200 \\
\hline 0 & -1.273352 & 8.602785 & 6.803977 & 6 & 0.734373 & 7.719574 & 7.949887 \\
\hline 6 & -1.944471 & 9.055771 & 5.615028 & 6 & 0.125812 & 8.548867 & 7.226595 \\
\hline 6 & -3.249779 & 8.613189 & 5.293847 & 6 & -0.150301 & 9.739539 & 6.460561 \\
\hline 6 & -1.278811 & 9.965252 & 4.765174 & 6 & -1.357867 & 9.902535 & 5.740545 \\
\hline 6 & -3.861346 & 9.047199 & 4.110941 & 1 & -2.081755 & 9.083348 & 5.722276 \\
\hline 1 & -3.760244 & 7.919252 & 5.969115 & 6 & -0.669958 & 12.140216 & 5.090792 \\
\hline & -1.903550 & 10.400550 & 3.590305 & 1 & -0.866749 & 13.071713 & 4.551917 \\
\hline & -0.293226 & 10.339158 & 5.044843 & 6 & -3.936983 & 8.896826 & 9.849552 \\
\hline 6 & -3.185153 & 9.933665 & 3.251840 & 1 & -3.460838 & 8.708626 & 10.825164 \\
\hline 1 & -4.858310 & 8.686179 & 3.844154 & 1 & -3.161660 & 9.261031 & 9.151816 \\
\hline & -1.386649 & 11.113496 & 2.939940 & 1 & -4.676780 & 9.707227 & 9.988130 \\
\hline & -3.658812 & 10.261284 & 2.323548 & 6 & -5.799925 & 7.259106 & 10.269520 \\
\hline 6 & -0.596296 & 8.603998 & 7.863288 & 1 & -6.375738 & 6.385069 & 9.926613 \\
\hline 6 & 0.182688 & 11.494504 & 9.127369 & 1 & -5.415786 & 7.062161 & 11.284091 \\
\hline & -0.463887 & 11.474677 & 10.024069 & 1 & -6.507883 & 8.105253 & 10.343667 \\
\hline & 0.911884 & 12.312911 & 9.285273 & 6 & -5.258566 & 7.998710 & 7.904222 \\
\hline & -0.615693 & 11.836817 & 7.890611 & 1 & -4.462630 & 8.265405 & 7.184065 \\
\hline 1 & -0.027371 & 12.245475 & 7.056629 & 1 & -5.845472 & 7.165461 & 7.482613 \\
\hline & -1.960482 & 11.770998 & 7.738695 & 1 & -5.926284 & 8.873764 & 8.008921 \\
\hline 0 & -2.908013 & 11.167084 & 8.749794 & 6 & -2.126492 & 6.312160 & 11.474959 \\
\hline & -2.416516 & 10.853387 & 9.685047 & 1 & -1.392393 & 6.973443 & 10.979864 \\
\hline & -3.722948 & 11.870888 & 9.006487 & 1 & -2.886417 & 6.938599 & 11.972765 \\
\hline
\end{tabular}




\begin{tabular}{|c|c|c|c|c|c|c|c|}
\hline 1 & -3.391779 & 10.272219 & 8.309371 & 1 & -1.593403 & 5.748625 & 12.263004 \\
\hline & -1.695889 & 6.126755 & 10.634850 & 6 & -3.838500 & 4.472738 & 11.227558 \\
\hline & -0.603364 & 6.216523 & 10.746318 & 1 & -3.373366 & 4.041056 & 12.133206 \\
\hline 1 & -2.044968 & 6.932270 & 9.966341 & 1 & -4.702607 & 5.071896 & 11.555351 \\
\hline & -2.149364 & 6.297771 & 11.628990 & 1 & -4.199170 & 3.631762 & 10.613759 \\
\hline & -1.884209 & 3.720180 & 11.266096 & 6 & -1.684622 & 4.334003 & 9.952435 \\
\hline & -2.138726 & 2.679446 & 11.015779 & 1 & -1.305872 & 3.725540 & 10.794161 \\
\hline & -0.834136 & 3.746869 & 11.600796 & 1 & -2.095765 & 3.646313 & 9.192301 \\
\hline & -2.511465 & 4.005491 & 12.130703 & 1 & -0.826196 & 4.866768 & 9.507174 \\
\hline 6 & -3.660652 & 4.819734 & 9.804693 & 6 & 1.547575 & 6.861471 & 8.824552 \\
\hline 1 & -3.851198 & 5.510227 & 8.961847 & 1 & 1.962404 & 6.019696 & 8.240190 \\
\hline 1 & -4.104918 & 3.843103 & 9.556939 & 1 & 0.896547 & 6.420101 & 9.600584 \\
\hline & -4.186901 & 5.219073 & 10.691412 & 6 & 2.696822 & 7.601572 & 9.587760 \\
\hline 6 & 1.328967 & 4.596322 & 9.568588 & 6 & 3.964088 & 7.782293 & 8.694377 \\
\hline 1 & 1.331302 & 5.619251 & 9.151441 & 1 & 4.667247 & 8.421561 & 9.259452 \\
\hline & 0.964479 & 4.635829 & 10.608202 & 1 & 4.450436 & 6.796112 & 8.577205 \\
\hline 1 & 2.376583 & 4.243654 & 9.597059 & 6 & 3.662806 & 8.394264 & 7.350861 \\
\hline 6 & 0.477705 & 2.211283 & 9.295511 & 1 & 3.558549 & 9.487885 & 7.338507 \\
\hline 1 & 1.514830 & 1.829121 & 9.331031 & 6 & 3.437953 & 7.733709 & 6.189327 \\
\hline 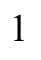 & 0.080758 & 2.187791 & 10.321782 & 6 & 3.551350 & 6.234723 & 6.023480 \\
\hline 1 & -0.113403 & 1.516879 & 8.674308 & 1 & 2.547685 & 5.784970 & 5.891995 \\
\hline 6 & 1.129167 & 3.563404 & 7.278938 & 1 & 4.130407 & 5.983504 & 5.114005 \\
\hline 1 & 2.156362 & 3.163109 & 7.368970 & 1 & 4.030201 & 5.728610 & 6.877107 \\
\hline 1 & 0.563494 & 2.890089 & 6.614494 & 6 & 2.971288 & 8.470780 & 4.956943 \\
\hline 1 & 1.193862 & 4.555630 & 6.802666 & 1 & 3.589825 & 8.220576 & 4.072195 \\
\hline 6 & 0.113336 & 8.898513 & 9.115855 & 1 & 1.928474 & 8.178625 & 4.716030 \\
\hline 1 & 0.764693 & 8.045984 & 9.377932 & 1 & 2.978198 & 9.564200 & 5.086276 \\
\hline 1 & -0.633651 & 8.987879 & 9.928337 & 6 & -1.611740 & 11.097673 & 5.058154 \\
\hline 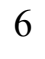 & 1.011522 & 10.179086 & 9.099533 & 1 & -2.533150 & 11.211649 & 4.479915 \\
\hline & -2.638801 & 12.316840 & 6.501035 & 6 & 0.535980 & 11.981503 & 5.795499 \\
\hline & -3.285819 & 13.176179 & 6.768920 & 1 & 1.280008 & 12.782944 & 5.798131 \\
\hline 1 & -1.912139 & 12.653861 & 5.743038 & 6 & 0.799370 & 10.790294 & 6.480585 \\
\hline & -3.296555 & 11.560097 & 6.032344 & 1 & 1.731455 & 10.661428 & 7.036782 \\
\hline & 1.846065 & 10.131823 & 10.396276 & 6 & 2.181729 & 8.972316 & 10.097818 \\
\hline & 2.467742 & 11.050804 & 10.461335 & 1 & 1.961073 & 9.628177 & 9.234564 \\
\hline & 1.170722 & 10.123546 & 11.282035 & 1 & 1.236548 & 8.825498 & 10.656219 \\
\hline
\end{tabular}




$\begin{array}{rrrrrrrr}6 & 1.927169 & 10.115303 & 7.859166 & 6 & 3.083498 & 6.708169 & 10.782200 \\ 1 & 2.487682 & 9.164700 & 7.872116 & 1 & 3.426960 & 5.715097 & 10.414347 \\ 1 & 1.294750 & 10.125744 & 6.953025 & 1 & 3.928159 & 7.180227 & 11.327184 \\ 8 & 2.669543 & 8.967128 & 10.386786 & 8 & 3.165896 & 9.628152 & 10.900221 \\ 8 & 2.815198 & 11.231889 & 7.755155 & 6 & 2.819066 & 9.701217 & 12.287264 \\ 6 & 3.445057 & 8.847050 & 11.579088 & 1 & 3.685539 & 10.143216 & 12.805781 \\ 1 & 2.800343 & 8.774731 & 12.480518 & 1 & 1.933498 & 10.351264 & 12.449920 \\ 1 & 4.039491 & 7.924695 & 11.482774 & 1 & 2.603895 & 8.701589 & 12.712396 \\ 1 & 4.131705 & 9.710721 & 11.706504 & 8 & 1.952543 & 6.552334 & 11.641747 \\ 6 & 4.163597 & 10.933256 & 8.135513 & 6 & 2.228633 & 5.684378 & 12.741247 \\ 1 & 4.244713 & 10.606345 & 9.189773 & 1 & 1.302112 & 5.614271 & 13.332935 \\ 1 & 4.597966 & 10.133949 & 7.499419 & 1 & 2.520423 & 4.669885 & 12.396443 \\ 1 & 4.741712 & 11.860098 & 7.992345 & 1 & 3.041304 & 6.086039 & 13.382277\end{array}$

\section{A-Int2a}

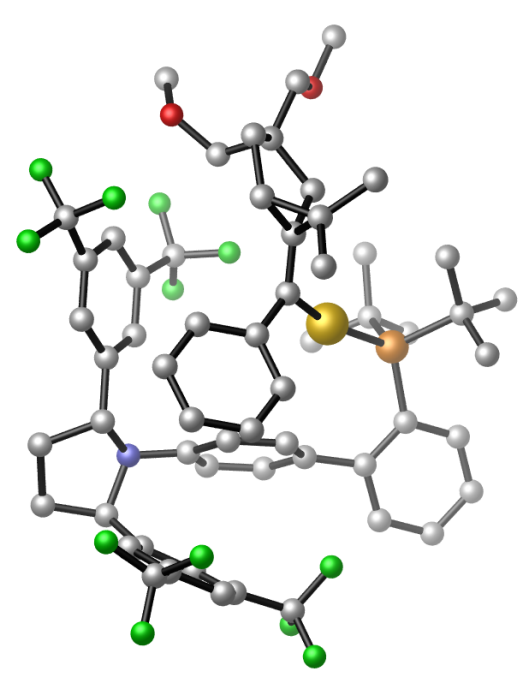

$E=-4167.38032723$ Hartrees

$G=-4166.401105$ Hartrees

\section{A-Int2c}

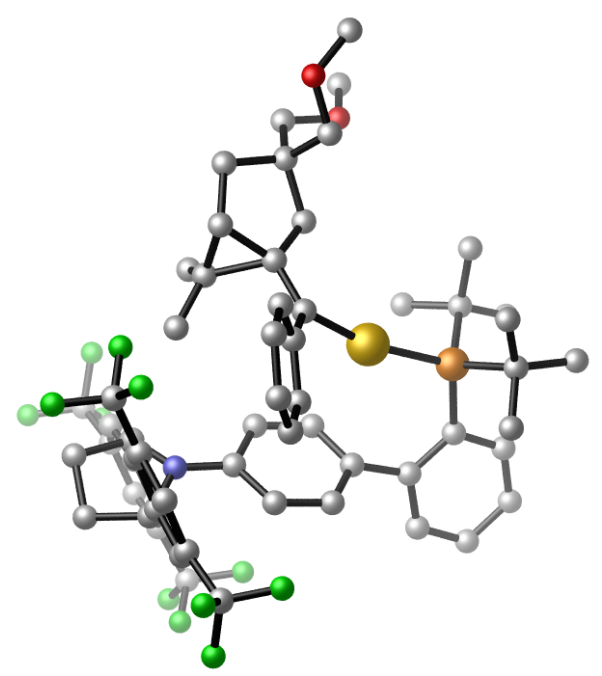

$E=-4167.37405374$ Hartrees

$G=-4166.400970$ Hartrees

$\begin{array}{rrrrrrrr}79 & -1.224581 & 6.694882 & 7.345498 & 79 & -1.497354 & 7.451800 & 7.812738 \\ 6 & -2.318468 & 3.241291 & 7.488698 & 6 & -4.307197 & 5.220581 & 7.763008 \\ 6 & -2.925540 & 2.232497 & 8.275444 & 6 & -5.438387 & 4.568671 & 8.314091 \\ 6 & -3.670392 & 1.189322 & 7.711846 & 6 & -6.357142 & 3.876696 & 7.515636\end{array}$




\begin{tabular}{|c|c|c|c|c|c|c|c|}
\hline 6 & -3.822157 & 1.125104 & 6.319853 & 6 & -6.166127 & 3.831335 & 6.126650 \\
\hline 6 & -3.202494 & 2.090687 & 5.518770 & 6 & -5.048448 & 4.458706 & 5.564892 \\
\hline 6 & -2.441967 & 3.150919 & 6.069057 & 6 & -4.096750 & 5.150336 & 6.354435 \\
\hline 6 & -1.765235 & 4.025844 & 5.064398 & 6 & -2.937935 & 5.713106 & 5.601460 \\
\hline 6 & -0.789152 & 3.444289 & 4.223617 & 6 & -1.620626 & 5.222005 & 5.773876 \\
\hline 6 & -0.183636 & 4.181454 & 3.201750 & 6 & -0.591958 & 5.556394 & 4.884875 \\
\hline 0 & -0.556585 & 5.523496 & 2.975140 & 6 & -0.830436 & 6.415074 & 3.778134 \\
\hline & -1.524854 & 6.112835 & 3.808537 & 6 & -2.132214 & 6.968997 & 3.649470 \\
\hline 6 & -2.129309 & 5.366927 & 4.831974 & 6 & -3.156970 & 6.607297 & 4.531535 \\
\hline 6 & 0.106846 & 7.650328 & 1.827081 & 6 & -0.122267 & 7.359960 & 1.560931 \\
\hline 6 & 0.529550 & 7.963931 & 0.359807 & 6 & 1.031013 & 6.893172 & 0.627997 \\
\hline 6 & 0.085010 & 6.726917 & -0.473113 & 6 & 2.162981 & 6.496180 & 1.590913 \\
\hline 6 & -0.617729 & 5.782833 & 0.524880 & 6 & 1.400528 & 5.865779 & 2.779794 \\
\hline 6 & 1.072684 & 8.191509 & 2.868027 & 6 & -0.215226 & 8.881103 & 1.681097 \\
\hline 6 & 1.279543 & 9.583386 & 2.938729 & 6 & -1.346026 & 9.548032 & 1.181088 \\
\hline & 2.132254 & 10.130681 & 3.907143 & 6 & -1.424289 & 10.950070 & 1.214952 \\
\hline 6 & 2.809854 & 9.306303 & 4.819102 & 6 & -0.366223 & 11.711448 & 1.725615 \\
\hline 6 & 2.587369 & 7.925700 & 4.761881 & 6 & 0.760642 & 11.048040 & 2.233955 \\
\hline 6 & 1.740123 & 7.367479 & 3.788936 & 6 & 0.833343 & 9.647458 & 2.224189 \\
\hline O & 2.202159 & 11.622448 & 4.110805 & 6 & -2.709669 & 11.629677 & 0.813364 \\
\hline 6 & 3.138066 & 7.030698 & 5.841981 & 6 & 1.923533 & 11.861053 & 2.752253 \\
\hline 6 & -2.138710 & 5.912556 & 0.626358 & 6 & 1.153397 & 4.370823 & 2.567904 \\
\hline 6 & -2.852555 & 4.855656 & 1.228721 & 6 & 2.165176 & 3.457893 & 2.913110 \\
\hline 6 & -4.201620 & 5.000760 & 1.578630 & 6 & 1.987288 & 2.082442 & 2.696500 \\
\hline 6 & -4.886786 & 6.189415 & 1.291737 & 6 & 0.792875 & 1.594534 & 2.144741 \\
\hline 6 & -4.194265 & 7.229797 & 0.654583 & 6 & -0.218445 & 2.505761 & 1.809662 \\
\hline 6 & -2.830125 & 7.105043 & 0.338258 & 6 & -0.041764 & 3.883667 & 2.014862 \\
\hline 6 & -4.892254 & 3.919455 & 2.372886 & 6 & 3.119455 & 1.134442 & 3.017424 \\
\hline 6 & -4.968095 & 8.469137 & 0.279441 & 6 & -1.550129 & 2.008611 & 1.298324 \\
\hline 6 & 0.394818 & 3.798656 & 8.603475 & 6 & -2.519971 & 5.163549 & 10.209915 \\
\hline 0 & -2.220148 & 4.945480 & 10.043899 & 6 & -4.478855 & 7.628405 & 9.543830 \\
\hline 10 & -1.339357 & 4.559794 & 8.360171 & 15 & -3.276440 & 6.291387 & 8.860813 \\
\hline 7 & 0.005365 & 6.182581 & 1.837255 & 7 & 0.172721 & 6.679383 & 2.843523 \\
\hline 9 & 3.455902 & 12.052356 & 4.405720 & 9 & -2.512877 & 12.915119 & 0.413672 \\
\hline 9 & 1.400513 & 11.987919 & 5.177108 & 9 & -3.581714 & 11.669324 & 1.874036 \\
\hline 9 & 1.758173 & 12.323203 & 3.032820 & 9 & -3.346345 & 10.970475 & -0.193684 \\
\hline
\end{tabular}




\begin{tabular}{|c|c|c|c|c|c|c|c|}
\hline & 2.216577 & 6.889596 & 6.863948 & 9 & 1.528110 & 13.083112 & 3.209395 \\
\hline & 4.270727 & 7.526413 & 6.413304 & 9 & 2.853339 & 12.079075 & 1.777027 \\
\hline & 3.406791 & 5.773680 & 5.397651 & 9 & 2.571333 & 11.231764 & 3.778318 \\
\hline 9 & -6.204593 & 3.788153 & 2.032765 & 9 & 3.783351 & 1.511531 & 4.149283 \\
\hline & -4.858178 & 4.199620 & 3.713830 & 9 & 4.042314 & 1.105897 & 2.010135 \\
\hline 9 & -4.298062 & 2.699415 & 2.210895 & 9 & 2.684654 & -0.143229 & 3.200873 \\
\hline & -4.154182 & 9.507177 & -0.076234 & 9 & -2.463923 & 1.917303 & 2.311463 \\
\hline & -5.812725 & 8.243024 & -0.765117 & 9 & -2.078883 & 2.854659 & 0.367015 \\
\hline 9 & -5.744925 & 8.906163 & 1.322659 & 9 & -1.455652 & 0.774634 & 0.729915 \\
\hline 1 & -0.879600 & 8.137856 & 2.012909 & 1 & -1.090390 & 6.997060 & 1.162314 \\
\hline 1 & 1.621688 & 8.100791 & 0.304220 & 1 & 1.324751 & 7.674897 & -0.089554 \\
\hline 1 & 0.060817 & 8.900111 & 0.015072 & 1 & 0.696513 & 6.011122 & 0.054487 \\
\hline 1 & 0.964447 & 6.214195 & -0.893499 & 1 & 2.725564 & 7.379962 & 1.937879 \\
\hline 1 & -0.572546 & 6.989302 & -1.317436 & 1 & 2.882586 & 5.792214 & 1.143976 \\
\hline 1 & -0.366391 & 4.725001 & 0.337088 & 1 & 1.982585 & 5.989116 & 3.712309 \\
\hline 1 & -2.808177 & 2.248968 & 9.358978 & 1 & -5.620599 & 4.612196 & 9.388684 \\
\hline 1 & -4.124936 & 0.434838 & 8.362098 & 1 & -7.219165 & 3.386015 & 7.979066 \\
\hline 1 & -4.409974 & 0.324803 & 5.858006 & 1 & -6.876811 & 3.301668 & 5.483462 \\
\hline 1 & -3.310855 & 2.049298 & 4.431251 & 1 & -4.878627 & 4.401519 & 4.484285 \\
\hline 1 & -0.506310 & 2.397295 & 4.378550 & 1 & -1.412811 & 4.504374 & 6.573379 \\
\hline 1 & 0.570269 & 3.722581 & 2.553214 & 1 & 0.388554 & 5.097680 & 5.029198 \\
\hline 1 & -2.959162 & 5.804529 & 5.394443 & 1 & -4.159881 & 7.020469 & 4.377497 \\
\hline 1 & -1.855219 & 7.138203 & 3.628965 & 1 & -2.356976 & 7.682675 & 2.854768 \\
\hline 1 & 0.758463 & 10.247058 & 2.243056 & 1 & -2.176352 & 8.973473 & 0.756105 \\
\hline 1 & 1.597442 & 6.286096 & 3.765176 & 1 & 1.712613 & 9.153132 & 2.641400 \\
\hline 1 & 3.459488 & 9.752703 & 5.576689 & 1 & -0.427664 & 12.801484 & 1.751102 \\
\hline 1 & -5.941401 & 6.303448 & 1.556378 & 1 & 0.650664 & 0.523463 & 1.985980 \\
\hline 1 & -2.316952 & 7.948889 & -0.131375 & 1 & -0.849586 & 4.574035 & 1.757408 \\
\hline 1 & -2.337676 & 3.922346 & 1.471858 & 1 & 3.096895 & 3.820667 & 3.360471 \\
\hline 6 & -1.371478 & 8.698475 & 6.916199 & 6 & 1.356470 & 8.316777 & 7.843440 \\
\hline 6 & -2.038372 & 9.086470 & 5.669769 & 6 & 0.073006 & 8.663555 & 7.321947 \\
\hline 6 & -3.299783 & 8.543082 & 5.306850 & 6 & -0.197384 & 9.844660 & 6.513304 \\
\hline 6 & -1.383913 & 9.954447 & 4.761923 & 6 & -1.192217 & 9.788584 & 5.495617 \\
\hline 6 & -3.896879 & 8.884798 & 4.088428 & 1 & -1.687682 & 8.833294 & 5.293272 \\
\hline 1 & -3.807651 & 7.868869 & 6.005013 & 6 & -0.883402 & 12.149123 & 5.027626 \\
\hline 6 & -1.971431 & 10.261918 & 3.527525 & 1 & -1.147921 & 13.042470 & 4.453286 \\
\hline
\end{tabular}




\begin{tabular}{|c|c|c|c|c|c|c|c|}
\hline 1 & -0.395339 & 10.346686 & 5.008476 & 6 & -3.643813 & 8.803902 & 10.109485 \\
\hline 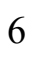 & -3.229681 & 9.739207 & 3.189085 & 1 & -3.021982 & 8.509111 & 10.969143 \\
\hline & -4.870439 & 8.466106 & 3.816388 & 1 & -2.981035 & 9.241210 & 9.341677 \\
\hline & -1.445226 & 10.922882 & 2.831037 & 1 & -4.338293 & 9.594028 & 10.452026 \\
\hline & -3.683318 & 9.989272 & 2.228392 & 6 & -5.452492 & 7.139067 & 10.634150 \\
\hline & -0.884007 & 9.625995 & 7.858776 & 1 & -6.120859 & 6.338064 & 10.280637 \\
\hline & 0.467475 & 11.627980 & 8.530413 & 1 & -4.923260 & 6.790035 & 11.536429 \\
\hline & 0.170274 & 12.248667 & 9.395382 & 1 & -6.095957 & 7.986649 & 10.936267 \\
\hline & 1.147954 & 12.233720 & 7.910705 & 6 & -5.268595 & 8.134740 & 8.313168 \\
\hline & -0.762616 & 11.212705 & 7.719871 & 1 & -4.590849 & 8.465401 & 7.504070 \\
\hline & -0.851234 & 11.611647 & 6.705485 & 1 & -5.943869 & 7.362636 & 7.908034 \\
\hline & -2.026167 & 10.847672 & 8.367605 & 1 & -5.882055 & 9.003167 & 8.617672 \\
\hline 6 & -2.222630 & 10.848561 & 9.870809 & 6 & -1.869423 & 6.057549 & 11.289642 \\
\hline 1 & -1.290565 & 10.905504 & 10.449153 & 1 & -1.208068 & 6.825828 & 10.852249 \\
\hline 1 & -2.823270 & 11.740669 & 10.126364 & 1 & -2.622388 & 6.561239 & 11.919343 \\
\hline 1 & -2.792687 & 9.961258 & 10.196316 & 1 & -1.253146 & 5.422866 & 11.953257 \\
\hline & -1.721802 & 6.331176 & 10.543002 & 6 & -3.497421 & 4.174302 & 10.880331 \\
\hline & -0.628293 & 6.381089 & 10.667442 & 1 & -2.947068 & 3.627381 & 11.668967 \\
\hline & -2.021037 & 7.143463 & 9.857666 & 1 & -4.353449 & 4.672690 & 11.363156 \\
\hline & -2.183020 & 6.529409 & 11.529033 & 1 & -3.877510 & 3.424967 & 10.166811 \\
\hline & -1.976131 & 3.937340 & 11.189588 & 6 & -1.419235 & 4.343828 & 9.491443 \\
\hline & -2.264681 & 2.902044 & 10.953308 & 1 & -0.947385 & 3.660236 & 10.222117 \\
\hline & -0.921945 & 3.933129 & 11.512254 & 1 & -1.840260 & 3.725989 & 8.678070 \\
\hline & -2.583663 & 4.249856 & 12.059339 & 1 & -0.628203 & 4.990665 & 9.070751 \\
\hline & -3.731503 & 5.078552 & 9.735777 & 6 & 1.460785 & 7.433206 & 9.097659 \\
\hline & -3.907902 & 5.774261 & 8.893887 & 1 & 1.549549 & 6.357536 & 8.867330 \\
\hline & -4.207524 & 4.115993 & 9.490563 & 1 & 0.537577 & 7.538139 & 9.690810 \\
\hline & -4.239811 & 5.491912 & 10.626981 & 6 & 2.685685 & 7.964362 & 9.886647 \\
\hline & 1.235354 & 4.743407 & 9.488297 & 6 & 3.646939 & 8.556873 & 8.813253 \\
\hline & 1.251526 & 5.771683 & 9.086252 & 1 & 4.167532 & 9.441390 & 9.214711 \\
\hline & 0.874960 & 4.775137 & 10.529578 & 1 & 4.427980 & 7.829574 & 8.523720 \\
\hline & 2.278073 & 4.375024 & 9.510130 & 6 & 2.780638 & 8.928121 & 7.606923 \\
\hline & 0.349538 & 2.376974 & 9.200190 & 1 & 2.866494 & 9.945818 & 7.215992 \\
\hline & 1.381527 & 1.980522 & 9.240266 & 6 & 2.405154 & 7.897201 & 6.602576 \\
\hline & -0.054569 & 2.354934 & 10.224044 & 6 & 2.879626 & 6.457478 & 6.647454 \\
\hline & -0.248524 & 1.692720 & 8.574295 & 1 & 2.065906 & 5.772714 & 6.351398 \\
\hline
\end{tabular}




$\begin{array}{rrrrrrrr}6 & 1.025229 & 3.730809 & 7.193084 & 1 & 3.702643 & 6.339911 & 5.918550 \\ 1 & 2.055826 & 3.338842 & 7.284346 & 1 & 3.260991 & 6.134957 & 7.626908 \\ 1 & 0.464992 & 3.052480 & 6.529814 & 6 & 2.098128 & 8.384278 & 5.198189 \\ 1 & 1.081821 & 4.722154 & 6.714381 & 1 & 2.998881 & 8.213652 & 4.579403 \\ 6 & 0.092219 & 9.214940 & 8.967169 & 1 & 1.253631 & 7.844738 & 4.740389 \\ 1 & 0.522155 & 8.229277 & 8.722736 & 1 & 1.874437 & 9.457619 & 5.155703 \\ 1 & -0.390454 & 9.096184 & 9.951402 & 6 & -1.512893 & 10.921550 & 4.746381 \\ 6 & 1.183532 & 10.318235 & 8.981806 & 1 & -2.256064 & 10.859753 & 3.945819 \\ 6 & -3.321215 & 11.006811 & 7.597057 & 6 & 0.080282 & 12.233735 & 6.049308 \\ 1 & -3.777968 & 11.957050 & 7.930596 & 1 & 0.556192 & 13.193092 & 6.275347 \\ 1 & -3.177551 & 11.074369 & 6.510447 & 6 & 0.433657 & 11.092637 & 6.777315 \\ 1 & -4.034946 & 10.194913 & 7.816956 & 1 & 1.157380 & 11.168523 & 7.593242 \\ 6 & 1.842702 & 10.493785 & 10.348879 & 6 & 2.183711 & 9.109124 & 10.805098 \\ 1 & 2.568981 & 11.336657 & 10.309552 & 1 & 1.673396 & 9.869858 & 10.178766 \\ 1 & 1.074633 & 10.756322 & 11.113141 & 1 & 1.441721 & 8.702530 & 11.518705 \\ 6 & 2.208247 & 9.906294 & 7.902243 & 6 & 3.375328 & 6.873054 & 10.705867 \\ 1 & 2.671540 & 8.943935 & 8.180774 & 1 & 3.721169 & 6.053285 & 10.034217 \\ 1 & 1.654179 & 9.752122 & 6.956711 & 1 & 4.276588 & 7.290518 & 11.208024 \\ 8 & 2.496814 & 9.276012 & 10.699883 & 8 & 3.256336 & 9.758154 & 11.491165 \\ 8 & 3.207161 & 10.897422 & 7.647218 & 6 & 3.308468 & 9.447503 & 12.886982 \\ 6 & 3.175437 & 9.376640 & 11.951287 & 1 & 4.229424 & 9.908396 & 13.280252 \\ 1 & 2.473866 & 9.610086 & 12.780356 & 1 & 2.435921 & 9.869378 & 13.429251 \\ 1 & 3.647622 & 8.399544 & 12.140972 & 1 & 3.336984 & 8.355501 & 13.071665 \\ 1 & 3.961190 & 10.161752 & 11.925986 & 8 & 2.448100 & 6.367215 & 11.665668 \\ 6 & 4.480848 & 10.572046 & 8.222291 & 6 & 3.026365 & 5.346058 & 12.477415 \\ 1 & 4.410337 & 10.386454 & 9.311301 & 1 & 2.247673 & 5.015444 & 13.183266 \\ 1 & 4.914682 & 9.671561 & 7.742628 & 1 & 3.358559 & 4.477998 & 11.868664 \\ 1 & 5.140396 & 11.434984 & 8.037277 & 1 & 3.899254 & 5.725936 & 13.050408\end{array}$




\section{A-Int3a}

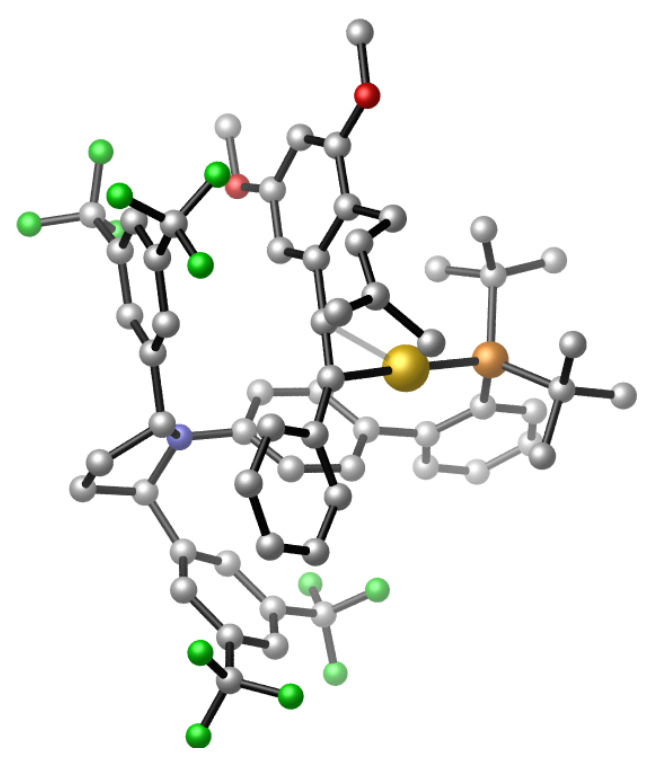

$E=-4241.19803338$ Hartrees

$G=-4240.254451$ Hartrees
A-Int3c

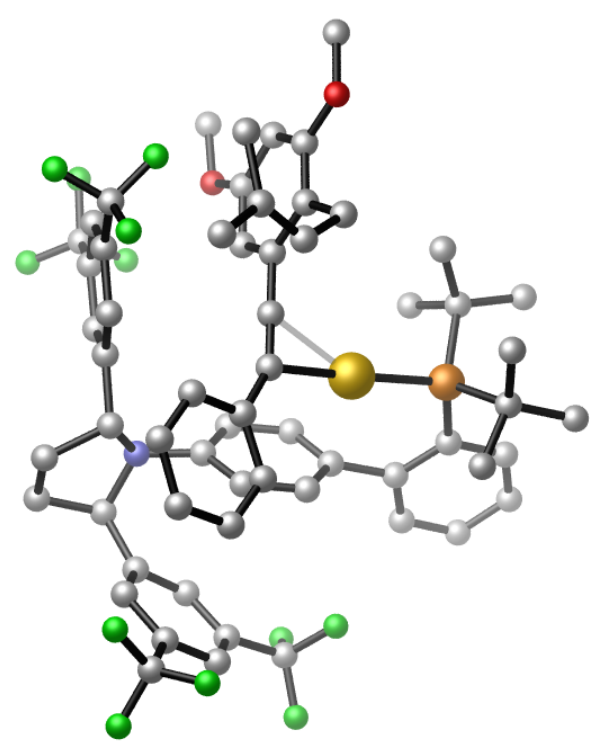

$E=-4241.19411247$ Hartrees

$G=-4240.253519$ Hartrees

$\begin{array}{rrrrrrrr}79 & -1.741504 & 6.975386 & 6.960216 & 79 & -1.561134 & 6.899302 & 6.891637 \\ 6 & -2.613360 & 3.585926 & 7.329941 & 6 & -2.557942 & 3.532589 & 7.288143 \\ 6 & -3.167732 & 2.473795 & 8.010435 & 6 & -3.110821 & 2.431654 & 7.987768 \\ 6 & -3.382571 & 1.248432 & 7.365794 & 6 & -3.447485 & 1.239225 & 7.333648 \\ 6 & -3.040679 & 1.106438 & 6.013531 & 6 & -3.239468 & 1.122226 & 5.951683 \\ 6 & -2.459161 & 2.183548 & 5.333184 & 6 & -2.657487 & 2.185345 & 5.249812 \\ 6 & -2.223845 & 3.423740 & 5.968995 & 6 & -2.292473 & 3.387715 & 5.896478 \\ 6 & -1.527223 & 4.446909 & 5.130457 & 6 & -1.586455 & 4.388158 & 5.039271 \\ 6 & -0.156016 & 4.291321 & 4.840502 & 6 & -0.240270 & 4.162079 & 4.686503 \\ 6 & 0.483785 & 5.140674 & 3.922368 & 6 & 0.405473 & 4.990155 & 3.753288 \\ 6 & -0.236085 & 6.160523 & 3.273996 & 6 & -0.285184 & 6.057910 & 3.152189 \\ 6 & -1.597650 & 6.344827 & 3.597474 & 6 & -1.615981 & 6.316837 & 3.543877 \\ 6 & -2.239826 & 5.497947 & 4.509970 & 6 & -2.264179 & 5.491528 & 4.473311 \\ 6 & 0.433828 & 8.373127 & 2.355028 & 6 & 0.476254 & 8.242696 & 2.245353 \\ 6 & 0.535570 & 8.906067 & 0.890059 & 6 & 0.573986 & 8.798311 & 0.787675 \\ 6 & 0.722682 & 7.645671 & 0.012064 & 6 & 0.625737 & 7.546720 & -0.121945 \\ 6 & 0.090350 & 6.493445 & 0.814333 & 6 & -0.058455 & 6.427469 & 0.684941 \\ 6 & 1.625597 & 8.790624 & 3.212026 & 6 & 1.714276 & 8.558559 & 3.079824 \\ 6 & 1.553857 & 9.950148 & 4.002190 & 6 & 1.758131 & 9.723972 & 3.864948\end{array}$




\begin{tabular}{|c|c|c|c|c|c|c|c|}
\hline 6 & 2.643136 & 10.343017 & 4.790312 & 6 & 2.896965 & 10.026775 & 4.621895 \\
\hline 6 & 3.837598 & 9.603610 & 4.780212 & 6 & 4.023007 & 9.186799 & 4.595826 \\
\hline 6 & 3.918880 & 8.463798 & 3.969409 & 6 & 3.982598 & 8.033103 & 3.805097 \\
\hline 6 & 2.819547 & 8.052401 & 3.196827 & 6 & 2.836778 & 7.716467 & 3.054306 \\
\hline 6 & 2.561193 & 11.559558 & 5.677484 & 6 & 2.932909 & 11.239003 & 5.516111 \\
\hline 6 & 5.210931 & 7.691464 & 3.863455 & 6 & 5.152787 & 7.081636 & 3.754598 \\
\hline 6 & -1.406129 & 6.247893 & 0.619656 & 6 & -1.575589 & 6.298678 & 0.551351 \\
\hline 6 & -1.909422 & 4.985444 & 0.995824 & 6 & -2.158812 & 5.071749 & 0.929576 \\
\hline 6 & -3.287149 & 4.766711 & 1.127395 & 6 & -3.544204 & 4.954105 & 1.106076 \\
\hline 6 & -4.200448 & 5.795436 & 0.852568 & 6 & -4.386528 & 6.051159 & 0.871411 \\
\hline 6 & -3.707837 & 7.041012 & 0.433065 & 6 & -3.816640 & 7.262399 & 0.449328 \\
\hline 6 & -2.325645 & 7.268383 & 0.316219 & 6 & -2.425552 & 7.389359 & 0.292803 \\
\hline 6 & -3.801435 & 3.469319 & 1.702786 & 6 & -4.134587 & 3.690649 & 1.684310 \\
\hline 6 & -4.687058 & 8.133848 & 0.075096 & 6 & -4.722781 & 8.425897 & 0.123249 \\
\hline 6 & -0.855787 & 4.790367 & 9.438570 & 6 & -0.694143 & 4.678408 & 9.357667 \\
\hline 6 & -3.979321 & 5.657184 & 9.105559 & 6 & -3.796635 & 5.640202 & 9.080697 \\
\hline 15 & -2.332017 & 5.153802 & 8.271202 & 15 & -2.179229 & 5.086204 & 8.217194 \\
\hline 7 & 0.394954 & 6.903861 & 2.222673 & 7 & 0.336686 & 6.783744 & 2.084147 \\
\hline 9 & 2.845001 & 11.246449 & 6.979159 & 9 & 3.282172 & 10.899271 & 6.793714 \\
\hline 9 & 1.323976 & 12.140800 & 5.660400 & 9 & 1.723935 & 11.873843 & 5.582153 \\
\hline 9 & 3.460368 & 12.516408 & 5.305524 & 9 & 3.848093 & 12.158609 & 5.093416 \\
\hline 9 & 6.061546 & 7.952673 & 4.899007 & 9 & 6.178798 & 7.464254 & 4.567698 \\
\hline 9 & 5.878993 & 8.011495 & 2.712917 & 9 & 5.656890 & 6.985642 & 2.486807 \\
\hline 9 & 5.002990 & 6.343786 & 3.829787 & 9 & 4.786049 & 5.818766 & 4.122065 \\
\hline 9 & -4.214233 & 3.641549 & 3.000036 & 9 & -4.445746 & 3.859030 & 3.010217 \\
\hline 9 & -2.850393 & 2.490981 & 1.719318 & 9 & -3.276636 & 2.632277 & 1.614437 \\
\hline 9 & -4.875097 & 2.993017 & 1.013069 & 9 & -5.289522 & 3.332613 & 1.057016 \\
\hline 9 & -4.137357 & 9.377111 & 0.189454 & 9 & -4.077710 & 9.624261 & 0.219385 \\
\hline 9 & -5.135020 & 8.009967 & -1.207051 & 9 & -5.220027 & 8.338496 & -1.143486 \\
\hline 9 & -5.792740 & 8.101773 & 0.883589 & 9 & -5.801901 & 8.475407 & 0.966184 \\
\hline 1 & -0.487092 & 8.772971 & 2.829866 & 1 & -0.402744 & 8.695750 & 2.752535 \\
\hline 1 & 1.372143 & 9.613664 & 0.774331 & 1 & 1.462894 & 9.435797 & 0.656168 \\
\hline 1 & -0.385793 & 9.454592 & 0.634444 & 1 & -0.306588 & 9.426142 & 0.574115 \\
\hline 1 & 1.797082 & 7.431674 & -0.116862 & 1 & 1.673766 & 7.254173 & -0.302413 \\
\hline 1 & 0.277756 & 7.750222 & -0.991205 & 1 & 0.148029 & 7.707877 & -1.102397 \\
\hline 1 & 0.615624 & 5.540087 & 0.620977 & 1 & 0.385370 & 5.442080 & 0.451821 \\
\hline
\end{tabular}




\begin{tabular}{|c|c|c|c|c|c|c|c|}
\hline & -3.430084 & 2.551825 & 9.066010 & 1 & -3.279852 & 2.493838 & 9.063556 \\
\hline & -3.816079 & 0.412280 & 7.923783 & 1 & -3.875137 & 0.410112 & 7.906464 \\
\hline & -3.215089 & 0.159845 & 5.491464 & 1 & -3.516522 & 0.204152 & 5.423095 \\
\hline & -2.188972 & 2.084788 & 4.277366 & 1 & -2.485443 & 2.105069 & 4.171901 \\
\hline & 0.404737 & 3.483602 & 5.322167 & 1 & 0.294028 & 3.315593 & 5.130379 \\
\hline & 1.542061 & 5.004122 & 3.676363 & 1 & 1.441861 & 4.796986 & 3.457844 \\
\hline & -3.315970 & 5.594992 & 4.689022 & 1 & -3.323823 & 5.645104 & 4.702523 \\
\hline & -2.170571 & 7.126347 & 3.092129 & 1 & -2.163728 & 7.141992 & 3.082171 \\
\hline & 0.633187 & 10.536890 & 4.009371 & 1 & 0.887481 & 10.383114 & 3.896549 \\
\hline & 2.868896 & 7.140236 & 2.594570 & 1 & 2.793744 & 6.801477 & 2.455931 \\
\hline & 4.680682 & 9.900597 & 5.408625 & 1 & 4.896995 & 9.407467 & 5.212396 \\
\hline & -5.275102 & 5.634064 & 0.972226 & 1 & -5.466188 & 5.966937 & 1.020558 \\
\hline & -1.978857 & 8.260564 & 0.018845 & 1 & -2.015756 & 8.356838 & -0.005403 \\
\hline & -1.212006 & 4.183901 & 1.257280 & 1 & -1.514490 & 4.215666 & 1.151895 \\
\hline & -1.362314 & 8.855806 & 5.835165 & 6 & -1.181857 & 8.818572 & 5.865090 \\
\hline & -2.242601 & 9.555460 & 4.936454 & 6 & -2.029015 & 9.566171 & 4.971464 \\
\hline & -3.413698 & 8.955187 & 4.419744 & 6 & -3.228454 & 9.028690 & 4.451686 \\
\hline & -1.882356 & 10.861219 & 4.518559 & 6 & -1.610577 & 10.861901 & 4.573566 \\
\hline 6 & -4.163125 & 9.612713 & 3.435869 & 6 & -3.949155 & 9.733608 & 3.479459 \\
\hline & -3.711728 & 7.967442 & 4.783771 & 1 & -3.573108 & 8.052581 & 4.805924 \\
\hline & -2.648425 & 11.518143 & 3.547414 & 6 & -2.346707 & 11.564911 & 3.611289 \\
\hline & -1.011780 & 11.342280 & 4.973248 & 1 & -0.714511 & 11.296002 & 5.027636 \\
\hline & -3.773465 & 10.886639 & 2.986141 & 6 & -3.500408 & 10.992978 & 3.043967 \\
\hline & -5.039482 & 9.123083 & 3.002418 & 1 & -4.851339 & 9.293316 & 3.046577 \\
\hline & -2.358990 & 12.522647 & 3.221858 & 1 & -2.012025 & 12.559138 & 3.297737 \\
\hline & -4.350192 & 11.386154 & 2.201800 & 1 & -4.054167 & 11.527389 & 2.266210 \\
\hline & -0.282482 & 8.646460 & 6.449261 & 6 & -0.113623 & 8.626772 & 6.509135 \\
\hline & 0.951421 & 8.413699 & 7.128927 & 6 & 1.083432 & 8.485030 & 7.269138 \\
\hline & 1.876453 & 7.531652 & 6.531505 & 6 & 2.128123 & 7.708258 & 6.726627 \\
\hline & 1.167567 & 9.018646 & 8.394734 & 6 & 1.157248 & 9.099644 & 8.548822 \\
\hline & 3.091067 & 7.282331 & 7.184589 & 6 & 3.319636 & 7.578007 & 7.452766 \\
\hline & 1.657266 & 7.065298 & 5.568936 & 1 & 2.026916 & 7.243754 & 5.742991 \\
\hline & 2.411191 & 8.753842 & 9.017422 & 6 & 2.375173 & 8.942002 & 9.251504 \\
\hline & 3.365349 & 7.898381 & 8.423970 & 6 & 3.448970 & 8.199266 & 8.712623 \\
\hline & 4.313425 & 7.707690 & 8.927248 & 1 & 4.378045 & 8.102153 & 9.275168 \\
\hline & 3.943435 & 6.435830 & 6.535478 & 8 & 4.296763 & 6.841884 & 6.845806 \\
\hline
\end{tabular}




\begin{tabular}{|c|c|c|c|c|c|c|c|}
\hline 8 & 2.608274 & 9.365018 & 10.225372 & 8 & 2.431981 & 9.549298 & 10.478588 \\
\hline 6 & 3.837164 & 9.118314 & 10.924993 & 6 & 3.650109 & 9.457181 & 11.233577 \\
\hline 1 & 3.954573 & 8.045503 & 11.169725 & 1 & 3.888302 & 8.406733 & 11.486270 \\
\hline 1 & 4.710715 & 9.464113 & 10.340552 & 1 & 4.497943 & 9.911014 & 10.686952 \\
\hline 1 & 3.765903 & 9.700391 & 11.854952 & 1 & 3.463998 & 10.023532 & 12.157177 \\
\hline 6 & 5.206986 & 6.141016 & 7.152272 & 6 & 5.571366 & 6.732479 & 7.496691 \\
\hline 1 & 5.734760 & 5.501237 & 6.432092 & 1 & 6.195672 & 6.148492 & 6.807732 \\
\hline 1 & 5.796845 & 7.060421 & 7.317954 & 1 & 6.028353 & 7.728206 & 7.651131 \\
\hline 1 & 5.069797 & 5.602858 & 8.109003 & 1 & 5.485374 & 6.206396 & 8.466192 \\
\hline 6 & 0.084950 & 9.846508 & 9.063828 & 6 & -0.045813 & 9.814311 & 9.144146 \\
\hline 1 & -0.824477 & 9.220494 & 9.152029 & 1 & -0.864108 & 9.070965 & 9.232964 \\
\hline & 0.410247 & 10.065902 & 10.096812 & 1 & 0.205481 & 10.123611 & 10.172109 \\
\hline 6 & -0.222975 & 11.146058 & 8.351569 & 6 & -0.561991 & 10.995944 & 8.331329 \\
\hline 1 & 0.656457 & 11.769302 & 8.148160 & 1 & -1.510825 & 10.835914 & 7.802865 \\
\hline 6 & -1.438735 & 11.610812 & 7.980187 & 6 & 0.046666 & 12.196128 & 8.201819 \\
\hline 6 & -1.579623 & 12.984052 & 7.356045 & 6 & -0.561086 & 13.290637 & 7.352717 \\
\hline 1 & -0.599409 & 13.438952 & 7.133440 & 1 & -1.508325 & 12.974954 & 6.881087 \\
\hline 1 & -2.162056 & 12.937920 & 6.415793 & 1 & -0.757419 & 14.199779 & 7.954617 \\
\hline 1 & -2.133171 & 13.667375 & 8.030726 & 1 & 0.139128 & 13.591169 & 6.549811 \\
\hline 6 & -2.741367 & 10.866303 & 8.171370 & 6 & 1.357196 & 12.546820 & 8.866919 \\
\hline 1 & -3.322779 & 10.865465 & 7.229806 & 1 & 1.255216 & 13.479055 & 9.456167 \\
\hline 1 & -2.602270 & 9.814860 & 8.472872 & 1 & 1.732225 & 11.753201 & 9.532673 \\
\hline 1 & -3.372878 & 11.361555 & 8.935170 & 1 & 2.132996 & 12.736140 & 8.103060 \\
\hline 6 & -3.697013 & 6.878649 & 10.010613 & 6 & -3.472248 & 6.864023 & 9.967661 \\
\hline 1 & -3.161685 & 6.590852 & 10.931258 & 1 & -2.919230 & 6.575553 & 10.877551 \\
\hline 1 & -3.113513 & 7.660634 & 9.491931 & 1 & -2.889924 & 7.632038 & 9.427192 \\
\hline 1 & -4.663016 & 7.323427 & 10.312923 & 1 & -4.423166 & 7.326941 & 10.290034 \\
\hline 6 & -4.701610 & 4.572206 & 9.934827 & 6 & -4.523066 & 4.576452 & 9.932996 \\
\hline 1 & -5.110481 & 3.771070 & 9.298929 & 1 & -4.950904 & 3.773281 & 9.312363 \\
\hline 1 & -4.063678 & 4.125248 & 10.714501 & 1 & -3.880899 & 4.129578 & 10.709129 \\
\hline 1 & -5.558479 & 5.049193 & 10.445747 & 1 & -5.365949 & 5.072030 & 10.449542 \\
\hline 6 & -4.902882 & 6.074181 & 7.932023 & 6 & -4.735967 & 6.064460 & 7.922487 \\
\hline 1 & -4.509146 & 6.953458 & 7.388853 & 1 & -4.331279 & 6.923103 & 7.355176 \\
\hline 1 & -5.041699 & 5.250237 & 7.208911 & 1 & -4.916804 & 5.233499 & 7.217151 \\
\hline 1 & -5.895513 & 6.342691 & 8.338777 & 1 & -5.710222 & 6.368243 & 8.348391 \\
\hline 6 & -0.311083 & 6.108750 & 10.033856 & 6 & -0.106772 & 5.979907 & 9.949129 \\
\hline
\end{tabular}




$\begin{array}{rrrrrrrr}1 & 0.018448 & 6.807875 & 9.246559 & 1 & 0.211809 & 6.684211 & 9.162185 \\ 1 & -1.038951 & 6.626382 & 10.677639 & 1 & -0.807513 & 6.501568 & 10.619165 \\ 1 & 0.572803 & 5.869619 & 10.654071 & 1 & 0.789779 & 5.718219 & 10.541392 \\ 6 & -1.223516 & 3.814170 & 10.572733 & 6 & -1.060002 & 3.707574 & 10.497179 \\ 1 & -0.312144 & 3.597034 & 11.160215 & 1 & -0.143136 & 3.477030 & 11.070803 \\ 1 & -1.966520 & 4.244917 & 11.265452 & 1 & -1.785502 & 4.149706 & 11.200913 \\ 1 & -1.602449 & 2.852697 & 10.190219 & 1 & -1.458548 & 2.751501 & 10.121759 \\ 6 & 0.239053 & 4.176210 & 8.531897 & 6 & 0.363893 & 4.039924 & 8.424983 \\ 1 & 1.149939 & 4.023466 & 9.139819 & 1 & 1.276939 & 3.840634 & 9.015891 \\ 1 & -0.061423 & 3.199438 & 8.116818 & 1 & 0.018954 & 3.083919 & 7.995963 \\ 1 & 0.501647 & 4.849592 & 7.694750 & 1 & 0.639546 & 4.718324 & 7.596476\end{array}$

\section{A-Int4a}

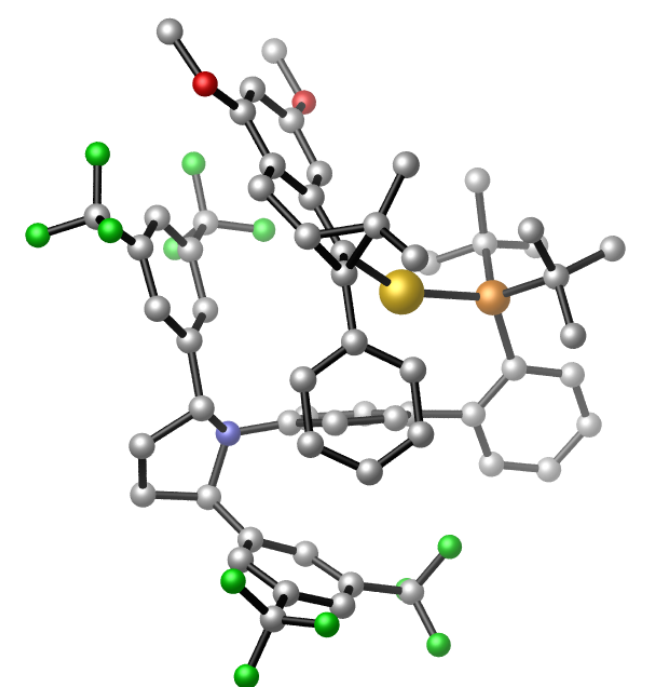

$$
\begin{gathered}
E=-4241.22251705 \text { Hartrees } \\
G=-4240.271284 \text { Hartrees }
\end{gathered}
$$

\section{A-Int4c}

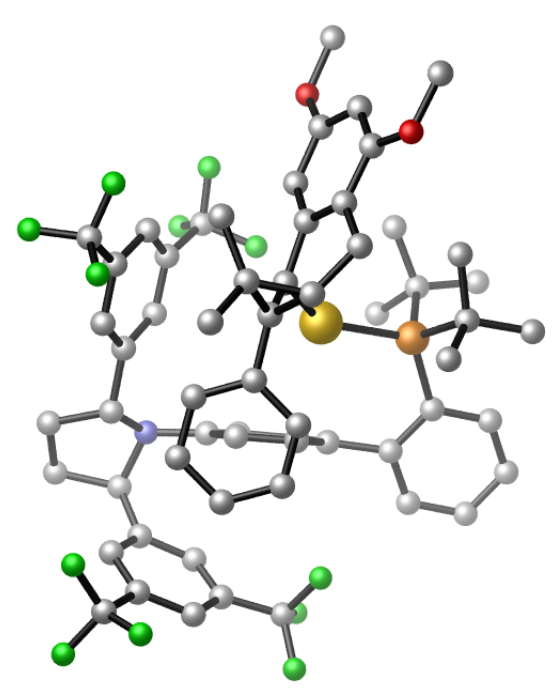

$$
\begin{gathered}
E=-4241.20737493 \text { Hartrees } \\
G=-4240.258695 \text { Hartrees }
\end{gathered}
$$

$\begin{array}{rrrrrrrr}79 & -1.139372 & 6.714809 & 6.957507 & 79 & -0.315231 & 6.924446 & 6.681169 \\ 6 & -2.985485 & 3.529745 & 6.823148 & 6 & -2.473071 & 3.932146 & 7.164766 \\ 6 & -3.839750 & 2.558057 & 7.398447 & 6 & -3.202997 & 3.060613 & 8.010021 \\ 6 & -4.432747 & 1.547892 & 6.630499 & 6 & -4.179624 & 2.187164 & 7.511906 \\ 6 & -4.174862 & 1.480848 & 5.252805 & 6 & -4.445971 & 2.154617 & 6.135999 \\ 6 & -3.325890 & 2.425600 & 4.665004 & 6 & -3.698894 & 2.967441 & 5.275031\end{array}$




\begin{tabular}{|c|c|c|c|c|c|c|c|}
\hline 6 & -2.723848 & 3.454066 & 5.426255 & 6 & -2.703402 & 3.847128 & 5.760182 \\
\hline 6 & -1.855215 & 4.404121 & 4.681131 & 6 & -1.890132 & 4.540838 & 4.723733 \\
\hline 6 & -0.673467 & 3.953420 & 4.057463 & 6 & -1.066001 & 3.760530 & 3.883945 \\
\hline 6 & 0.094463 & 4.825303 & 3.275339 & 6 & -0.335621 & 4.350486 & 2.848469 \\
\hline 6 & -0.310547 & 6.163536 & 3.084442 & 6 & -0.433319 & 5.737784 & 2.607930 \\
\hline 6 & -1.484774 & 6.620173 & 3.712490 & 6 & -1.242208 & 6.525375 & 3.450547 \\
\hline 6 & -2.246224 & 5.749221 & 4.505147 & 6 & -1.962073 & 5.929944 & 4.499314 \\
\hline 6 & 0.581366 & 8.404757 & 2.412313 & 6 & 0.591167 & 7.691229 & 1.444819 \\
\hline 6 & 0.995610 & 9.000581 & 1.023851 & 6 & 1.190465 & 7.869067 & 0.018624 \\
\hline 6 & 0.941463 & 7.815717 & 0.025316 & 6 & 0.570214 & 6.719500 & -0.831457 \\
\hline 6 & 0.082587 & 6.743847 & 0.716260 & 6 & -0.342099 & 5.930501 & 0.134329 \\
\hline 6 & 1.617658 & 8.672636 & 3.500677 & 6 & 1.536483 & 8.074931 & 2.567060 \\
\hline 6 & 2.004818 & 9.998613 & 3.782185 & 6 & 1.744346 & 9.431264 & 2.865468 \\
\hline & 3.050797 & 10.260769 & 4.681583 & 6 & 2.595249 & 9.809677 & 3.916344 \\
\hline 6 & 3.699287 & 9.214425 & 5.358332 & 6 & 3.253334 & 8.844240 & 4.693717 \\
\hline 6 & 3.285207 & 7.901378 & 5.108331 & 6 & 3.059309 & 7.492200 & 4.378171 \\
\hline 6 & 2.269508 & 7.629845 & 4.176845 & 6 & 2.224524 & 7.108089 & 3.317712 \\
\hline & 3.579983 & 11.668900 & 4.798121 & 6 & 2.823955 & 11.279054 & 4.157412 \\
\hline 6 & 3.990403 & 6.723474 & 5.732729 & 6 & 3.706651 & 6.395068 & 5.183084 \\
\hline 6 & -1.430460 & 6.841041 & 0.514940 & 6 & -1.817195 & 6.327445 & 0.150460 \\
\hline 6 & -2.215204 & 5.720117 & 0.860204 & 6 & -2.736248 & 5.446525 & 0.760297 \\
\hline 6 & -3.612516 & 5.803878 & 0.911247 & 6 & -4.041704 & 5.858472 & 1.055152 \\
\hline 6 & -4.267984 & 6.995692 & 0.567473 & 6 & -4.482939 & 7.142608 & 0.700124 \\
\hline 6 & -3.495334 & 8.101707 & 0.185398 & 6 & -3.584721 & 8.010143 & 0.064969 \\
\hline 6 & -2.090783 & 8.033567 & 0.173183 & 6 & -2.260649 & 7.615252 & -0.197818 \\
\hline 6 & -4.409895 & 4.640536 & 1.449189 & 6 & -4.938438 & 4.967963 & 1.879640 \\
\hline 6 & -4.181156 & 9.369284 & -0.260953 & 6 & -4.032709 & 9.383641 & -0.369424 \\
\hline 6 & -0.781357 & 3.798692 & 8.798377 & 6 & 0.287912 & 3.858185 & 8.287982 \\
\hline 6 & -3.509816 & 5.456118 & 9.121318 & 6 & -1.926057 & 5.897061 & 9.450665 \\
\hline & -2.163893 & 4.782526 & 7.917930 & 15 & -1.167443 & 5.037444 & 7.890025 \\
\hline 7 & 0.431059 & 6.960247 & 2.158457 & 7 & 0.252747 & 6.263173 & 1.474225 \\
\hline 9 & 4.308669 & 11.862507 & 5.932141 & 9 & 3.433266 & 11.526825 & 5.348472 \\
\hline 9 & 2.572312 & 12.598933 & 4.790929 & 9 & 1.635425 & 11.974619 & 4.151348 \\
\hline 9 & 4.391919 & 11.977706 & 3.743874 & 9 & 3.594756 & 11.836783 & 3.181270 \\
\hline 9 & 4.878878 & 7.093788 & 6.698487 & 9 & 4.558194 & 6.867598 & 6.133042 \\
\hline 9 & 4.688374 & 6.014207 & 4.796005 & 9 & 4.396300 & 5.524044 & 4.397159 \\
\hline
\end{tabular}




\begin{tabular}{|c|c|c|c|c|c|c|c|}
\hline 9 & 3.106031 & 5.842607 & 6.295868 & 9 & 2.752753 & 5.645811 & 5.841480 \\
\hline 9 & -5.622270 & 4.521666 & 0.839649 & 9 & -4.835113 & 5.274489 & 3.211627 \\
\hline 9 & -4.654862 & 4.789196 & 2.789301 & 9 & -4.613304 & 3.646593 & 1.757510 \\
\hline 9 & -3.752705 & 3.451679 & 1.300535 & 9 & -6.251806 & 5.104627 & 1.545147 \\
\hline 9 & -5.394868 & 9.533730 & 0.347797 & 9 & -3.053115 & 10.318691 & -0.158499 \\
\hline 9 & -3.433237 & 10.481312 & 0.021801 & 9 & -4.334391 & 9.427774 & -1.697307 \\
\hline 9 & -4.407235 & 9.380500 & -1.604841 & 9 & -5.142292 & 9.801620 & 0.310149 \\
\hline 1 & -0.372860 & 8.880637 & 2.724704 & 1 & -0.313482 & 8.338601 & 1.534511 \\
\hline 1 & 2.005301 & 9.439798 & 1.063964 & 1 & 2.287367 & 7.770941 & 0.063557 \\
\hline 1 & 0.303265 & 9.811733 & 0.744677 & 1 & 0.961655 & 8.872297 & -0.376797 \\
\hline 1 & 1.952286 & 7.404372 & -0.128811 & 1 & 1.361870 & 6.052344 & -1.206743 \\
\hline 1 & 0.542680 & 8.101415 & -0.961868 & 1 & 0.013150 & 7.086412 & -1.708577 \\
\hline 1 & 0.405149 & 5.724281 & 0.439377 & 1 & -0.264131 & 4.840846 & -0.020137 \\
\hline 1 & -4.038957 & 2.574663 & 8.470853 & 1 & -2.998283 & 3.037587 & 9.078764 \\
\hline 1 & -5.090568 & 0.817048 & 7.112051 & 1 & -4.722550 & 1.534030 & 8.203004 \\
\hline 1 & -4.636274 & 0.701011 & 4.637806 & 1 & -5.215428 & 1.488368 & 5.731703 \\
\hline 1 & -3.141854 & 2.406525 & 3.586490 & 1 & -3.879943 & 2.940347 & 4.196107 \\
\hline 1 & -0.360126 & 2.911921 & 4.187147 & 1 & -0.996158 & 2.681127 & 4.055786 \\
\hline 1 & 1.006718 & 4.474706 & 2.780155 & 1 & 0.303603 & 3.741225 & 2.200297 \\
\hline 1 & -3.205688 & 6.090150 & 4.904702 & 1 & -2.651179 & 6.540673 & 5.090613 \\
\hline 1 & -1.846739 & 7.634946 & 3.543555 & 1 & -1.350910 & 7.597303 & 3.278384 \\
\hline 1 & 1.521691 & 10.832353 & 3.261238 & 1 & 1.234218 & 10.200509 & 2.275901 \\
\hline 1 & 2.005472 & 6.593610 & 3.961325 & 1 & 2.096946 & 6.048839 & 3.082617 \\
\hline 1 & 4.508522 & 9.421408 & 6.060637 & 1 & 3.898692 & 9.140653 & 5.522871 \\
\hline 1 & -5.358125 & 7.063255 & 0.601867 & 1 & -5.502751 & 7.462950 & 0.925569 \\
\hline 1 & -1.523415 & 8.926930 & -0.099130 & 1 & -1.579857 & 8.332577 & -0.665206 \\
\hline 1 & -1.727545 & 4.780021 & 1.133056 & 1 & -2.413407 & 4.443898 & 1.053477 \\
\hline 6 & -1.243874 & 9.724619 & 6.726565 & 6 & -0.715276 & 9.964865 & 6.600796 \\
\hline 6 & -2.258538 & 9.542462 & 5.625514 & 6 & -1.692681 & 9.744422 & 5.474436 \\
\hline 6 & -3.434287 & 8.786395 & 5.806632 & 6 & -2.968483 & 9.194183 & 5.701718 \\
\hline 6 & -2.025765 & 10.143850 & 4.372272 & 6 & -1.334981 & 10.122864 & 4.164620 \\
\hline 6 & -4.349089 & 8.619298 & 4.755724 & 6 & -3.875731 & 9.035302 & 4.640930 \\
\hline 1 & -3.635673 & 8.334784 & 6.783281 & 1 & -3.255110 & 8.891096 & 6.713886 \\
\hline 6 & -2.940576 & 9.981252 & 3.317609 & 6 & -2.243353 & 9.976713 & 3.104620 \\
\hline 1 & -1.114034 & 10.733681 & 4.222255 & 1 & -0.348420 & 10.560603 & 3.997067 \\
\hline 6 & -4.103483 & 9.215083 & 3.506497 & 6 & -3.519423 & 9.436280 & 3.343346 \\
\hline
\end{tabular}




\begin{tabular}{|c|c|c|c|c|c|c|c|}
\hline 1 & -5.256482 & 8.026242 & 4.914456 & 1 & -4.864138 & 8.603347 & 4.831013 \\
\hline 1 & -2.740898 & 10.437944 & 2.343757 & 1 & -1.964323 & 10.283930 & 2.091600 \\
\hline 1 & -4.815110 & 9.091213 & 2.685650 & 1 & -4.236888 & 9.343625 & 2.524699 \\
\hline 6 & -0.370750 & 8.609632 & 7.033483 & 6 & 0.163772 & 8.892034 & 6.964728 \\
\hline & 0.920188 & 8.857029 & 7.657256 & 6 & 1.042707 & 9.015134 & 8.132426 \\
\hline & 1.669521 & 7.751955 & 8.142749 & 6 & 2.273978 & 8.320038 & 8.164844 \\
\hline & 1.444634 & 10.177405 & 7.797054 & 6 & 0.568312 & 9.690554 & 9.290321 \\
\hline 6 & 2.899394 & 7.937950 & 8.782091 & 6 & 3.048750 & 8.315662 & 9.332100 \\
\hline & 1.283974 & 6.738198 & 8.018558 & 1 & 2.617431 & 7.769744 & 7.290648 \\
\hline 6 & 2.707826 & 10.341126 & 8.410158 & 6 & 1.338011 & 9.624641 & 10.474831 \\
\hline 0 & 3.434705 & 9.239345 & 8.906393 & 6 & 2.577674 & 8.958449 & 10.501591 \\
\hline 1 & 4.404179 & 9.392986 & 9.381114 & 1 & 3.157752 & 8.918867 & 11.423606 \\
\hline$\gamma$ & 3.506411 & 6.807465 & 9.246061 & 8 & 4.229298 & 7.632092 & 9.258794 \\
\hline 9 & 3.139608 & 11.632433 & 8.497612 & 8 & 0.778721 & 10.238661 & 11.561496 \\
\hline 6 & 4.454886 & 11.880752 & 9.018356 & 6 & 1.514199 & 10.235329 & 12.796549 \\
\hline & 4.536647 & 11.566900 & 10.075843 & 1 & 1.671963 & 9.204916 & 13.166744 \\
\hline 1 & 5.224256 & 11.368264 & 8.412419 & 1 & 2.489882 & 10.744527 & 12.684700 \\
\hline 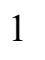 & 4.596276 & 12.968261 & 8.946239 & 1 & 0.889679 & 10.790760 & 13.510644 \\
\hline 6 & 4.806309 & 6.938771 & 9.842971 & 6 & 5.049796 & 7.571833 & 10.436535 \\
\hline 1 & 5.109540 & 5.917003 & 10.112072 & 1 & 5.936390 & 6.990074 & 10.146539 \\
\hline 1 & 5.532847 & 7.361508 & 9.125516 & 1 & 5.362555 & 8.581909 & 10.762586 \\
\hline 1 & 4.770922 & 7.564309 & 10.755058 & 1 & 4.528853 & 7.057990 & 11.266737 \\
\hline 6 & 0.707730 & 11.399704 & 7.320214 & 6 & -0.781834 & 10.347028 & 9.282432 \\
\hline 1 & 0.803096 & 12.198661 & 8.080653 & 1 & -1.533289 & 9.670590 & 9.736796 \\
\hline 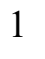 & 1.209434 & 11.797231 & 6.420146 & 1 & -0.764675 & 11.244885 & 9.930663 \\
\hline & -0.757724 & 11.175297 & 7.020945 & 6 & -1.244668 & 10.775725 & 7.911419 \\
\hline & -1.183130 & 11.910039 & 6.325725 & 1 & -2.327392 & 10.933237 & 7.826126 \\
\hline & -1.726321 & 10.644616 & 8.015361 & 6 & -0.405291 & 11.523242 & 6.960905 \\
\hline & -3.166427 & 11.127262 & 7.922570 & 6 & -1.138755 & 12.544062 & 6.092305 \\
\hline 1 & -3.449975 & 11.446644 & 6.908089 & 1 & -2.184390 & 12.267755 & 5.885747 \\
\hline & -3.876675 & 10.349739 & 8.254981 & 1 & -1.138526 & 13.503596 & 6.641925 \\
\hline 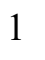 & -3.276981 & 11.994860 & 8.599909 & 1 & -0.619453 & 12.701226 & 5.133267 \\
\hline U & -1.333299 & 10.272487 & 9.434924 & 6 & 1.010947 & 11.982039 & 7.309564 \\
\hline 1 & -2.056583 & 9.557688 & 9.862706 & 1 & 1.372011 & 12.663834 & 6.525971 \\
\hline 1 & -0.325183 & 9.847597 & 9.529932 & 1 & 0.999990 & 12.530483 & 8.268650 \\
\hline & -1.363497 & 11.191520 & 10.049078 & 1 & 1.741998 & 11.167930 & 7.397900 \\
\hline
\end{tabular}




$\begin{array}{cccccccc}6 & -3.011280 & 6.809460 & 9.691567 & 6 & -0.775609 & 6.563826 & 10.241426 \\ 1 & -2.047073 & 6.721604 & 10.217973 & 1 & -0.220819 & 5.827159 & 10.847031 \\ 1 & -2.891663 & 7.566483 & 8.898505 & 1 & -0.059203 & 7.098400 & 9.596841 \\ 1 & -3.761179 & 7.186442 & 10.412306 & 1 & -1.209547 & 7.303660 & 10.940304 \\ 6 & -3.862392 & 4.544596 & 10.317138 & 6 & -2.734201 & 5.022591 & 10.435643 \\ 1 & -4.261605 & 3.562340 & 10.022066 & 1 & -3.704153 & 4.709946 & 10.018450 \\ 1 & -2.999004 & 4.384065 & 10.983250 & 1 & -2.181753 & 4.133427 & 10.780901 \\ 1 & -4.649809 & 5.043886 & 10.912175 & 1 & -2.950284 & 5.637908 & 11.329419 \\ 6 & -4.764269 & 5.722795 & 8.255789 & 6 & -2.875641 & 6.987763 & 8.895163 \\ 1 & -4.516369 & 6.318432 & 7.357850 & 1 & -2.315592 & 7.780582 & 8.370631 \\ 1 & -5.249818 & 4.791282 & 7.921936 & 1 & -3.621816 & 6.569414 & 8.195197 \\ 1 & -5.496314 & 6.298173 & 8.852584 & 1 & -3.419974 & 7.454726 & 9.737306 \\ 6 & -0.177359 & 4.674619 & 9.918092 & 6 & 1.527072 & 4.666456 & 8.737720 \\ 1 & 0.099877 & 5.681122 & 9.558585 & 1 & 1.785777 & 5.462779 & 8.021938 \\ 1 & -0.867289 & 4.790112 & 10.771228 & 1 & 1.402044 & 5.125120 & 9.729974 \\ 1 & 0.740471 & 4.187763 & 10.297118 & 1 & 2.387735 & 3.973920 & 8.797553 \\ 6 & -1.241334 & 2.442782 & 9.372303 & 6 & -0.054688 & 2.796845 & 9.350553 \\ 1 & -0.370734 & 1.950636 & 9.845080 & 1 & 0.813204 & 2.120636 & 9.467855 \\ 1 & -2.022136 & 2.544722 & 10.142917 & 1 & -0.254984 & 3.248095 & 10.337354 \\ 1 & -1.615818 & 1.770649 & 8.582344 & 1 & -0.917546 & 2.175120 & 9.058839 \\ 6 & 0.280459 & 3.539181 & 7.700921 & 6 & 0.605789 & 3.172074 & 6.937009 \\ 1 & 1.117051 & 2.962889 & 8.138259 & 1 & 1.502639 & 2.537065 & 7.062013 \\ 1 & -0.136179 & 2.951416 & 6.863832 & 1 & -0.223278 & 2.527883 & 6.597774 \\ 1 & 0.690542 & 4.477593 & 7.286634 & 1 & 0.817764 & 3.911011 & 6.144009 \\ & & & & & \end{array}$


A-TSInt1a-2a

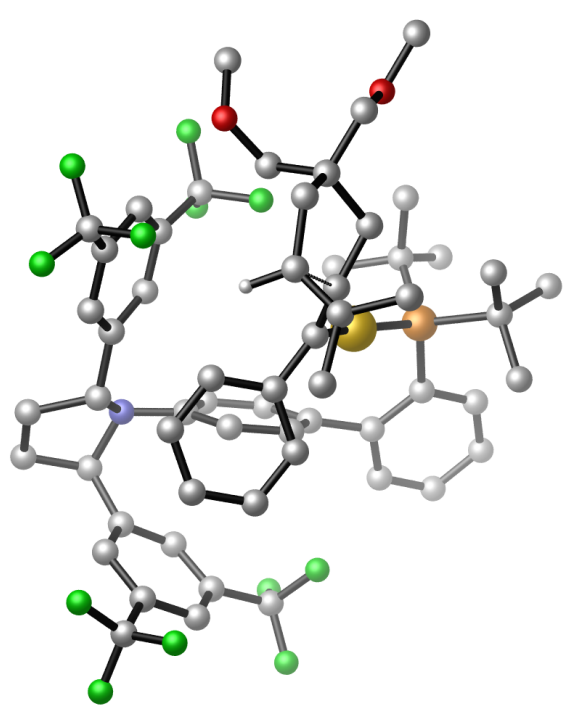

$E=-4167.36450210$ Hartrees

$\mathrm{G}=-4166.387486$ Hartrees
A-TSInt1c-2c

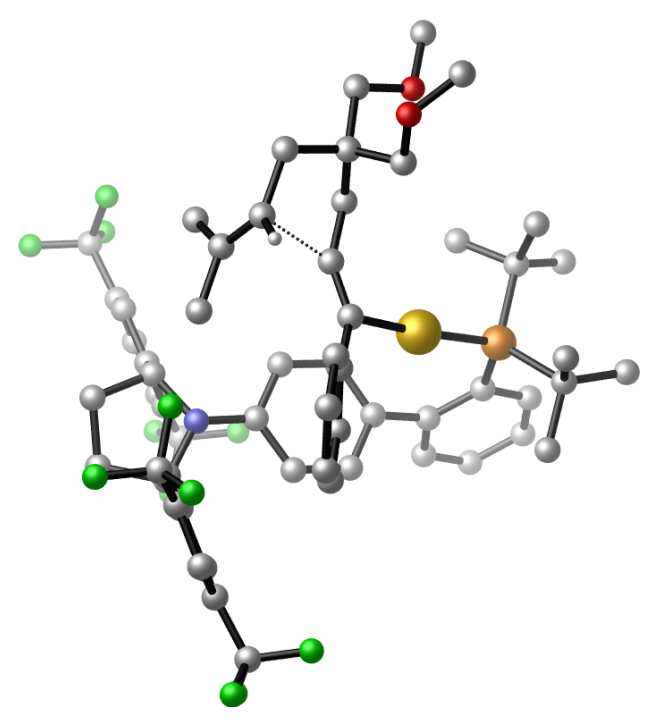

$E=-4167.35316045$ Hartrees

$\mathrm{G}=-4166.380823$ Hartrees

$\begin{array}{rrrrrrrr}79 & -1.134513 & 6.569078 & 7.368798 & 79 & -1.528217 & 7.447738 & 7.959588 \\ 6 & -2.211615 & 3.169504 & 7.504031 & 6 & -4.329498 & 5.291156 & 7.841679 \\ 6 & -2.794271 & 2.145229 & 8.289324 & 6 & -5.485211 & 4.676079 & 8.384652 \\ 6 & -3.579542 & 1.129641 & 7.730313 & 6 & -6.346466 & 3.895271 & 7.604956 \\ 6 & -3.800842 & 1.112254 & 6.346278 & 6 & -6.068572 & 3.718046 & 6.241675 \\ 6 & -3.205782 & 2.093537 & 5.545300 & 6 & -4.933221 & 4.319821 & 5.687023 \\ 6 & -2.401726 & 3.123584 & 6.090247 & 6 & -4.042034 & 5.108073 & 6.456654 \\ 6 & -1.745671 & 4.009424 & 5.081510 & 6 & -2.891617 & 5.681574 & 5.694380 \\ 6 & -0.788553 & 3.431541 & 4.217974 & 6 & -1.553530 & 5.261687 & 5.882658 \\ 6 & -0.198939 & 4.176519 & 3.192862 & 6 & -0.532404 & 5.653833 & 5.007488 \\ 6 & -0.572135 & 5.521092 & 2.984170 & 6 & -0.804452 & 6.488281 & 3.889377 \\ 6 & -1.527781 & 6.106039 & 3.836297 & 6 & -2.136334 & 6.955693 & 3.735151 \\ 6 & -2.112422 & 5.353936 & 4.868036 & 6 & -3.148361 & 6.548673 & 4.611740 \\ 6 & 0.104919 & 7.652865 & 1.855803 & 6 & -0.154791 & 7.421920 & 1.651616 \\ 6 & 0.518333 & 7.980517 & 0.388913 & 6 & 1.004049 & 6.977106 & 0.714642 \\ 6 & 0.058327 & 6.756012 & -0.454683 & 6 & 2.162959 & 6.649575 & 1.670455 \\ 6 & -0.653412 & 5.810174 & 0.535368 & 6 & 1.439503 & 6.023590 & 2.884366 \\ 6 & 1.087156 & 8.159952 & 2.898932 & 6 & -0.323184 & 8.939828 & 1.715421 \\ 6 & 1.301989 & 9.547328 & 3.009452 & 6 & -1.508736 & 9.526858 & 1.242096 \\ 6 & 2.156004 & 10.061879 & 3.995278 & 6 & -1.660431 & 10.922954 & 1.227891\end{array}$




\begin{tabular}{|c|c|c|c|c|c|c|c|}
\hline 6 & 2.826014 & 9.207894 & 4.884523 & 6 & -0.620618 & 11.757972 & 1.654870 \\
\hline 6 & 2.613757 & 7.828732 & 4.773479 & 6 & 0.562201 & 11.175033 & 2.132140 \\
\hline 6 & 1.763558 & 7.304500 & 3.785176 & 6 & 0.705697 & 9.780541 & 2.179445 \\
\hline 6 & 2.237404 & 11.548478 & 4.229199 & 6 & -2.997318 & 11.524923 & 0.873860 \\
\hline 6 & 3.207913 & 6.898969 & 5.800338 & 6 & 1.705178 & 12.064178 & 2.561462 \\
\hline 6 & -2.172516 & 5.956766 & 0.645005 & 6 & 1.235468 & 4.516638 & 2.711797 \\
\hline 6 & -2.892636 & 4.911385 & 1.260401 & 6 & 2.279677 & 3.642709 & 3.061465 \\
\hline 6 & -4.239135 & 5.070593 & 1.613772 & 6 & 2.135668 & 2.256342 & 2.892472 \\
\hline 6 & -4.917021 & 6.260523 & 1.314629 & 6 & 0.943150 & 1.717724 & 2.386189 \\
\hline 6 & -4.219215 & 7.287769 & 0.662505 & 6 & -0.100048 & 2.590058 & 2.044844 \\
\hline 6 & -2.856119 & 7.150641 & 0.345984 & 6 & 0.043373 & 3.978080 & 2.199984 \\
\hline 6 & -4.931334 & 4.002372 & 2.423992 & 6 & 3.300782 & 1.351729 & 3.219914 \\
\hline 6 & -4.984941 & 8.524045 & 0.261269 & 6 & -1.431511 & 2.041377 & 1.588746 \\
\hline 6 & 0.544536 & 3.690663 & 8.549380 & 6 & -2.650539 & 5.298207 & 10.346652 \\
\hline 6 & -2.027059 & 4.820072 & 10.082448 & 6 & -4.553061 & 7.780573 & 9.533874 \\
\hline 1 & -1.191716 & 4.457520 & 8.370941 & 15 & -3.352793 & 6.402154 & 8.946842 \\
\hline 7 & -0.018778 & 6.187236 & 1.847320 & 7 & 0.190238 & 6.804705 & 2.957012 \\
\hline 9 & 3.480981 & 11.953756 & 4.592478 & 9 & -2.877999 & 12.774672 & 0.347559 \\
\hline 9 & 1.388697 & 11.911502 & 5.258579 & 9 & -3.782935 & 11.637338 & 1.994278 \\
\hline 9 & 1.859195 & 12.272854 & 3.141582 & 9 & -3.687873 & 10.760999 & -0.017218 \\
\hline 9 & 2.377122 & 6.789547 & 6.899287 & 9 & 1.277941 & 13.301979 & 2.940856 \\
\hline 9 & 4.409089 & 7.337933 & 6.271812 & 9 & 2.602707 & 12.243446 & 1.547193 \\
\hline 9 & 3.382347 & 5.636008 & 5.326644 & 9 & 2.400894 & 11.533891 & 3.610182 \\
\hline 9 & -6.252424 & 3.891903 & 2.112213 & 9 & 3.966462 & 1.776453 & 4.334147 \\
\hline 9 & -4.863716 & 4.282300 & 3.763883 & 9 & 4.212123 & 1.328172 & 2.202116 \\
\hline 9 & -4.360520 & 2.772217 & 2.251612 & 9 & 2.909036 & 0.065920 & 3.439219 \\
\hline 9 & -4.162693 & 9.568854 & -0.053136 & 9 & -2.312907 & 1.959118 & 2.631548 \\
\hline 9 & -5.778765 & 8.297714 & -0.823241 & 9 & -2.010594 & 2.841889 & 0.647445 \\
\hline 9 & -5.810906 & 8.949784 & 1.269533 & 9 & -1.319779 & 0.792203 & 1.057792 \\
\hline 1 & -0.871374 & 8.152899 & 2.058684 & 1 & -1.108677 & 6.997324 & 1.280400 \\
\hline 1 & 1.610736 & 8.111303 & 0.324772 & 1 & 1.256882 & 7.750694 & -0.027012 \\
\hline 1 & 0.052200 & 8.923469 & 0.059739 & 1 & 0.698101 & 6.067932 & 0.168339 \\
\hline 1 & 0.931636 & 6.236918 & -0.880035 & 1 & 2.694008 & 7.563475 & 1.988119 \\
\hline 1 & -0.596190 & 7.033637 & -1.296460 & 1 & 2.904200 & 5.962162 & 1.233105 \\
\hline 1 & -0.416164 & 4.751377 & 0.334143 & 1 & 2.033741 & 6.185837 & 3.800157 \\
\hline & -2.627942 & 2.128445 & 9.366503 & 1 & -5.731414 & 4.819956 & 9.437644 \\
\hline
\end{tabular}




\begin{tabular}{|c|c|c|c|c|c|c|c|}
\hline & -4.012942 & 0.361027 & 8.378363 & 1 & -7.229252 & 3.436899 & 8.062247 \\
\hline 1 & -4.422765 & 0.335086 & 5.889695 & 1 & -6.729402 & 3.112699 & 5.612287 \\
\hline & -3.364802 & 2.086284 & 4.463169 & 1 & -4.704946 & 4.174796 & 4.625608 \\
\hline & -0.508149 & 2.381751 & 4.357135 & 1 & -1.313633 & 4.570714 & 6.697712 \\
\hline & 0.543264 & 3.722235 & 2.527789 & 1 & 0.475623 & 5.270191 & 5.182223 \\
\hline & -2.935985 & 5.786938 & 5.444601 & 1 & -4.170560 & 6.905436 & 4.444084 \\
\hline & -1.865281 & 7.130871 & 3.665884 & 1 & -2.393831 & 7.647553 & 2.931689 \\
\hline & 0.783700 & 10.235027 & 2.335059 & 1 & -2.325248 & 8.894281 & 0.877225 \\
\hline & 1.625423 & 6.224551 & 3.720048 & 1 & 1.625768 & 9.349527 & 2.578412 \\
\hline & 3.464699 & 9.628966 & 5.664325 & 1 & -0.739887 & 12.843465 & 1.643113 \\
\hline & -5.969830 & 6.385518 & 1.581091 & 1 & 0.826729 & 0.638374 & 2.267432 \\
\hline & -2.338225 & 7.986392 & -0.132968 & 1 & -0.791087 & 4.634865 & 1.940558 \\
\hline & -2.384929 & 3.975706 & 1.509466 & 1 & 3.211121 & 4.042676 & 3.476171 \\
\hline & -1.412314 & 8.631044 & 6.925395 & 6 & 1.181815 & 7.865041 & 7.720490 \\
\hline & -2.088733 & 8.993758 & 5.672512 & 6 & 0.166799 & 8.527829 & 7.256410 \\
\hline & -3.387369 & 8.520927 & 5.374092 & 6 & -0.116492 & 9.736438 & 6.475544 \\
\hline & -1.417800 & 9.808888 & 4.736874 & 6 & -1.132256 & 9.733480 & 5.489048 \\
\hline & -4.004391 & 8.870322 & 4.164295 & 1 & -1.664233 & 8.802225 & 5.273721 \\
\hline 1 & -3.903630 & 7.884233 & 6.101015 & 6 & -0.762155 & 12.099109 & 5.065289 \\
\hline 6 & -2.042506 & 10.158246 & 3.530479 & 1 & -1.002775 & 13.011626 & 4.511547 \\
\hline 1 & -0.408222 & 10.160573 & 4.959371 & 6 & -3.709801 & 8.976893 & 10.041884 \\
\hline & -3.334240 & 9.691745 & 3.239470 & 1 & -3.050564 & 8.706303 & 10.882510 \\
\hline & -5.005322 & 8.494639 & 3.929366 & 1 & -3.082284 & 9.403974 & 9.239418 \\
\hline 1 & -1.517841 & 10.800766 & 2.815340 & 1 & -4.399154 & 9.767809 & 10.392515 \\
\hline & -3.811846 & 9.963994 & 2.297051 & 6 & -5.539098 & 7.363146 & 10.643412 \\
\hline 6 & -0.932940 & 9.307641 & 7.925923 & 1 & -6.190859 & 6.525546 & 10.347936 \\
\hline 6 & 0.406411 & 11.727683 & 8.637206 & 1 & -5.020667 & 7.099055 & 11.580484 \\
\hline 1 & 0.118261 & 12.282391 & 9.547807 & 1 & -6.199760 & 8.222322 & 10.864608 \\
\hline 1 & 1.180917 & 12.322762 & 8.123999 & 6 & -5.323883 & 8.217599 & 8.265179 \\
\hline 6 & -0.789638 & 11.556181 & 7.726474 & 1 & -4.634171 & 8.455854 & 7.433624 \\
\hline 1 & -0.601271 & 11.613751 & 6.649059 & 1 & -6.026721 & 7.440686 & 7.920511 \\
\hline 6 & -2.114117 & 11.564206 & 8.132379 & 1 & -5.903641 & 9.129964 & 8.497914 \\
\hline 6 & -2.554327 & 11.598319 & 9.572771 & 6 & -1.940680 & 6.208839 & 11.374624 \\
\hline 1 & -1.728245 & 11.661171 & 10.296505 & 1 & -1.232745 & 6.908657 & 10.893576 \\
\hline 1 & -3.223932 & 12.463408 & 9.735362 & 1 & -2.660507 & 6.794354 & 11.971971 \\
\hline 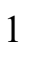 & -3.151923 & 10.695949 & 9.802787 & 1 & -1.365348 & 5.574948 & 12.074559 \\
\hline
\end{tabular}




\begin{tabular}{|c|c|c|c|c|c|c|c|}
\hline & -1.515818 & 6.196190 & 10.591229 & 6 & -3.684559 & 4.407589 & 11.069907 \\
\hline 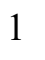 & -0.418628 & 6.244406 & 10.677464 & 1 & -3.167034 & 3.880885 & 11.893445 \\
\hline 1 & -1.847216 & 7.020141 & 9.935788 & 1 & -4.512471 & 4.981230 & 11.515446 \\
\hline & -1.940157 & 6.372951 & 11.597523 & 1 & -4.102613 & 3.637660 & 10.401288 \\
\hline & -1.749654 & 3.791449 & 11.202807 & 6 & -1.612081 & 4.372694 & 9.664691 \\
\hline & -2.038018 & 2.759397 & 10.953503 & 1 & -1.180332 & 3.698189 & 10.427497 \\
\hline & -0.688345 & 3.787526 & 11.501510 & 1 & -2.076032 & 3.745453 & 8.882374 \\
\hline & -2.339083 & 4.084093 & 12.091503 & 1 & -0.783494 & 4.942459 & 9.208489 \\
\hline 6 & -3.545960 & 4.957168 & 9.817725 & 6 & 1.680178 & 6.902324 & 8.734543 \\
\hline 1 & -3.743049 & 5.660092 & 8.986566 & 1 & 2.131377 & 6.011230 & 8.260560 \\
\hline 1 & -4.030405 & 3.997387 & 9.578511 & 1 & 0.831712 & 6.544448 & 9.340990 \\
\hline 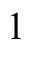 & -4.027705 & 5.364883 & 10.725911 & 6 & 2.717177 & 7.587103 & 9.658892 \\
\hline 6 & 1.412459 & 4.621881 & 9.422562 & 6 & 3.843766 & 8.158645 & 8.742722 \\
\hline 1 & 1.413154 & 5.656716 & 9.036977 & 1 & 4.376387 & 8.953189 & 9.292210 \\
\hline & 1.087089 & 4.634629 & 10.476071 & 1 & 4.577733 & 7.361175 & 8.527523 \\
\hline 1 & 2.455778 & 4.255421 & 9.403409 & 6 & 3.265083 & 8.704060 & 7.459739 \\
\hline 6 & 0.511930 & 2.260260 & 9.127638 & 1 & 3.023980 & 9.772724 & 7.439940 \\
\hline 1 & 1.542106 & 1.857527 & 9.118882 & 6 & 3.157185 & 8.019236 & 6.264192 \\
\hline 1 & 0.151832 & 2.224799 & 10.167333 & 6 & 3.564891 & 6.579554 & 6.077725 \\
\hline 1 & -0.115360 & 1.588711 & 8.516791 & 1 & 2.666673 & 5.954399 & 5.910117 \\
\hline 6 & 1.132691 & 3.638279 & 7.120133 & 1 & 4.193644 & 6.480499 & 5.174239 \\
\hline 1 & 2.165419 & 3.246103 & 7.177232 & 1 & 4.114167 & 6.153490 & 6.930887 \\
\hline 1 & 0.552922 & 2.966254 & 6.467087 & 6 & 2.634590 & 8.704420 & 5.031354 \\
\hline 1 & 1.175005 & 4.634095 & 6.650328 & 1 & 3.367838 & 8.603418 & 4.208664 \\
\hline 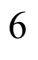 & -0.129690 & 9.335670 & 9.166236 & 1 & 1.693218 & 8.230712 & 4.693967 \\
\hline 1 & 0.276329 & 8.324710 & 9.343566 & 1 & 2.427822 & 9.770792 & 5.192203 \\
\hline & -0.763125 & 9.584894 & 10.037818 & 6 & -1.448353 & 10.903313 & 4.789801 \\
\hline 6 & 1.029627 & 10.355040 & 9.041941 & 1 & -2.225973 & 10.883347 & 4.019469 \\
\hline 0 & -3.237511 & 11.602573 & 7.130469 & 6 & 0.241089 & 12.116331 & 6.048317 \\
\hline 1 & -3.840833 & 12.514413 & 7.304820 & 1 & 0.777569 & 13.044767 & 6.270340 \\
\hline 1 & -2.882984 & 11.603494 & 6.089212 & 6 & 0.556725 & 10.948219 & 6.758688 \\
\hline 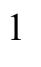 & -3.917954 & 10.742954 & 7.267113 & 1 & 1.305713 & 10.977823 & 7.555798 \\
\hline & 1.755072 & 10.491945 & 10.384432 & 6 & 2.023871 & 8.736270 & 10.437664 \\
\hline & 2.539482 & 11.276283 & 10.302373 & 1 & 1.615519 & 9.474080 & 9.720353 \\
\hline & 1.037731 & 10.819798 & 11.171118 & 1 & 1.172156 & 8.325068 & 11.013412 \\
\hline & 1.987044 & 9.861770 & 7.932645 & 6 & 3.312226 & 6.559614 & 10.628474 \\
\hline
\end{tabular}




$\begin{array}{rrrrrrrr}1 & 2.342472 & 8.843660 & 8.169822 & 1 & 3.797491 & 5.737603 & 10.054527 \\ 1 & 1.424989 & 9.805614 & 6.982237 & 1 & 4.096825 & 7.048269 & 11.246275 \\ 8 & 2.326659 & 9.230784 & 10.727335 & 8 & 2.947744 & 9.417164 & 11.288805 \\ 8 & 3.087001 & 10.751927 & 7.717992 & 6 & 2.733948 & 9.162571 & 12.681293 \\ 6 & 3.035236 & 9.285378 & 11.965988 & 1 & 3.559568 & 9.652682 & 13.222597 \\ 1 & 2.367621 & 9.571215 & 12.806205 & 1 & 1.770977 & 9.593104 & 13.027922 \\ 1 & 3.438487 & 8.276957 & 12.149746 & 1 & 2.734775 & 8.078746 & 12.910340 \\ 1 & 3.875081 & 10.011052 & 11.921721 & 8 & 2.264582 & 6.043907 & 11.448888 \\ 6 & 4.325358 & 10.274082 & 8.265650 & 6 & 2.745665 & 5.079490 & 12.385346 \\ 1 & 4.255157 & 10.079868 & 9.352418 & 1 & 1.876341 & 4.739134 & 12.969988 \\ 1 & 4.646625 & 9.338441 & 7.765500 & 1 & 3.205812 & 4.207875 & 11.873509 \\ 1 & 5.073358 & 11.060699 & 8.077612 & 1 & 3.497456 & 5.520690 & 13.073555\end{array}$

\section{A-TSInt3a-4a}

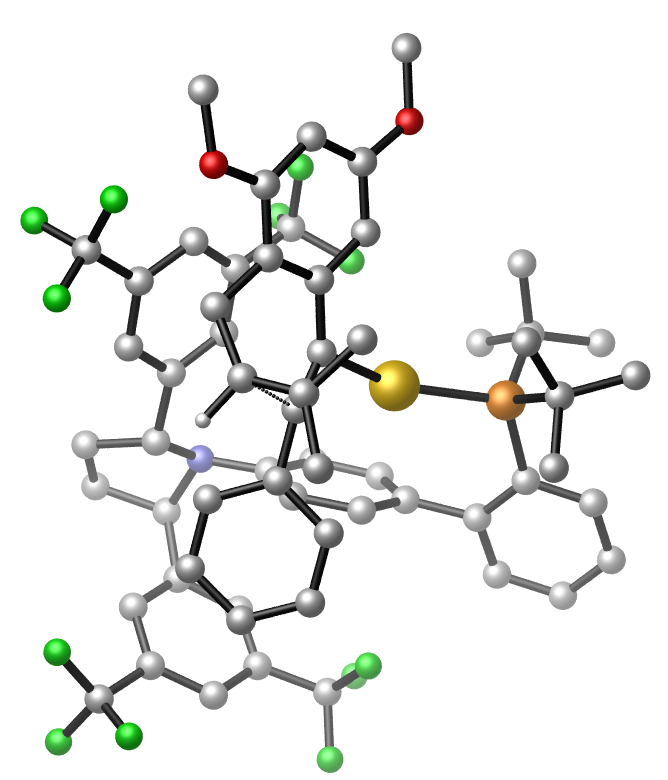

$$
\begin{gathered}
E=-4241.19083258 \text { Hartrees } \\
G=-4240.247829 \text { Hartrees }
\end{gathered}
$$

\section{A-TSInt3c-4c}

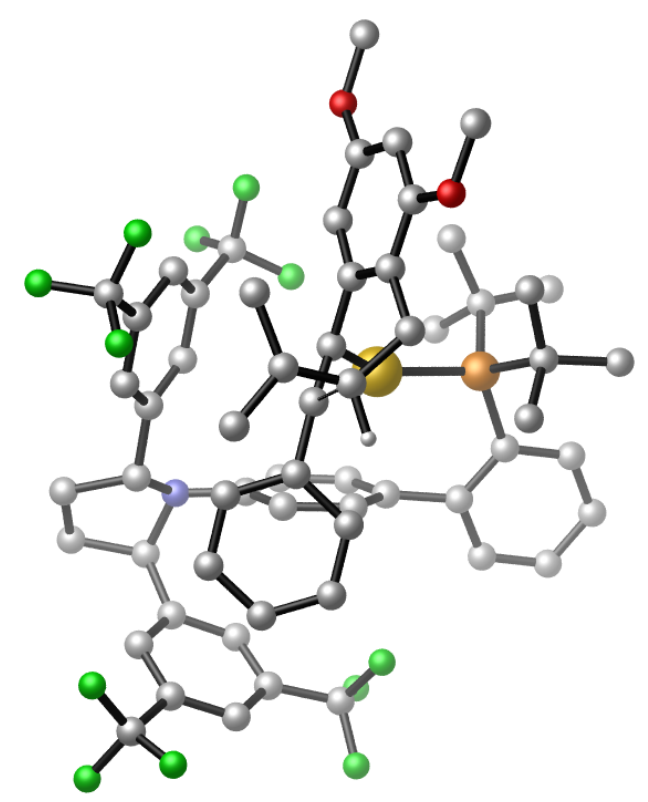

$$
\begin{gathered}
E=-4241.18599520 \text { Hartrees } \\
G=-4240.241784 \text { Hartrees }
\end{gathered}
$$
$\begin{array}{lll}79 & -0.263477 & 6.574671\end{array}$
6.702300
$\begin{array}{lll}6 & -2.404310 & 3.710641\end{array}$
7.191783
$6 \quad-3.131469$
2.817584
8.016918 


\begin{tabular}{|c|c|c|c|c|c|c|c|}
\hline 6 & -4.778168 & 1.961647 & 6.899818 & 6 & -4.199045 & 2.056882 & 7.520560 \\
\hline 6 & -4.815380 & 1.966704 & 5.497606 & 6 & -4.566979 & 2.170813 & 6.172209 \\
\hline 6 & -3.921696 & 2.778980 & 4.789015 & 6 & -3.827680 & 3.008212 & 5.326932 \\
\hline 6 & -2.984814 & 3.603431 & 5.452863 & 6 & -2.736801 & 3.767179 & 5.807585 \\
\hline 6 & -2.079463 & 4.405017 & 4.582438 & 6 & -1.941856 & 4.492183 & 4.773980 \\
\hline 6 & -1.072823 & 3.773679 & 3.823258 & 6 & -1.076412 & 3.746350 & 3.946207 \\
\hline 6 & -0.240720 & 4.519228 & 2.980374 & 6 & -0.361277 & 4.367953 & 2.917584 \\
\hline 6 & -0.424031 & 5.912043 & 2.846572 & 6 & -0.513543 & 5.749333 & 2.677170 \\
\hline 6 & -1.449644 & 6.541543 & 3.576647 & 6 & -1.378018 & 6.499951 & 3.498020 \\
\hline 6 & -2.260362 & 5.795564 & 4.443470 & 6 & -2.080320 & 5.875243 & 4.540816 \\
\hline 6 & 0.653624 & 8.041296 & 2.097352 & 6 & 0.562192 & 7.723219 & 1.591011 \\
\hline 6 & 1.350243 & 8.455576 & 0.760805 & 6 & 1.150233 & 7.947610 & 0.163981 \\
\hline 6 & 0.996718 & 7.336826 & -0.261191 & 6 & 0.532753 & 6.819440 & -0.717235 \\
\hline 6 & -0.004013 & 6.417904 & 0.465325 & 6 & -0.423010 & 6.041977 & 0.213048 \\
\hline 6 & 1.507779 & 8.329689 & 3.324952 & 6 & 1.533919 & 8.021006 & 2.719059 \\
\hline 6 & 1.906300 & 9.657847 & 3.575791 & 6 & 1.825453 & 9.354024 & 3.053681 \\
\hline 6 & 2.747181 & 9.961529 & 4.658098 & 6 & 2.689851 & 9.651692 & 4.118773 \\
\hline 6 & 3.178010 & 8.956663 & 5.539697 & 6 & 3.296909 & 8.628466 & 4.862674 \\
\hline 6 & 2.783911 & 7.638457 & 5.287371 & 6 & 3.027767 & 7.300453 & 4.507036 \\
\hline 6 & 1.970129 & 7.320858 & 4.185729 & 6 & 2.167160 & 6.995977 & 3.440856 \\
\hline 6 & 3.294349 & 11.360863 & 4.801744 & 6 & 2.992962 & 11.096974 & 4.416297 \\
\hline 6 & 3.279203 & 6.503799 & 6.147426 & 6 & 3.658300 & 6.144678 & 5.240385 \\
\hline 6 & -1.485492 & 6.760329 & 0.298994 & 6 & -1.881447 & 6.498989 & 0.225190 \\
\hline 6 & -2.437685 & 5.800114 & 0.704899 & 6 & -2.833729 & 5.662511 & 0.847486 \\
\hline 6 & -3.794423 & 6.130442 & 0.812190 & 6 & -4.130623 & 6.119557 & 1.112877 \\
\hline 6 & -4.247929 & 7.409316 & 0.454432 & 6 & -4.531870 & 7.403223 & 0.710987 \\
\hline 6 & -3.313208 & 8.354860 & 0.010814 & 6 & -3.600861 & 8.225581 & 0.062619 \\
\hline 6 & -1.942458 & 8.043979 & -0.048078 & 6 & -2.282001 & 7.789861 & -0.160243 \\
\hline 6 & -4.756253 & 5.147306 & 1.434275 & 6 & -5.060221 & 5.274820 & 1.949824 \\
\hline 6 & -3.786880 & 9.708304 & -0.458489 & 6 & -4.010537 & 9.585287 & -0.446287 \\
\hline 6 & -0.329864 & 3.304888 & 8.225209 & 6 & 0.383452 & 3.509680 & 8.288878 \\
\hline 6 & -2.563180 & 5.267858 & 9.434259 & 6 & -1.788398 & 5.604117 & 9.485514 \\
\hline 15 & -1.696330 & 4.582795 & 7.845506 & 15 & -1.040844 & 4.728997 & 7.930500 \\
\hline 7 & 0.391624 & 6.606045 & 1.904087 & 7 & 0.179763 & 6.305606 & 1.561381 \\
\hline 9 & 3.623810 & 11.656776 & 6.090664 & 9 & 3.567751 & 11.268746 & 5.639732 \\
\hline 9 & 2.397482 & 12.305760 & 4.380055 & 9 & 1.849568 & 11.859293 & 4.391376 \\
\hline
\end{tabular}




\begin{tabular}{|c|c|c|c|c|c|c|c|}
\hline 9 & 4.423026 & 11.530777 & 4.051636 & 9 & 3.835688 & 11.639806 & 3.492859 \\
\hline 9 & 4.006209 & 6.920424 & 7.215559 & 9 & 4.395084 & 6.538199 & 6.316000 \\
\hline 9 & 4.059776 & 5.643899 & 5.427651 & 9 & 4.470521 & 5.412570 & 4.427977 \\
\hline 9 & 2.237855 & 5.744946 & 6.633029 & 9 & 2.698407 & 5.275686 & 5.708025 \\
\hline 9 & -6.003292 & 5.234820 & 0.893107 & 9 & -4.953315 & 5.600885 & 3.277299 \\
\hline 9 & -4.891273 & 5.379161 & 2.778387 & 9 & -4.776843 & 3.941524 & 1.853378 \\
\hline 9 & -4.335575 & 3.854261 & 1.301781 & 9 & -6.366594 & 5.447390 & 1.606319 \\
\hline 9 & -4.947565 & 10.087714 & 0.158324 & 9 & -3.017283 & 10.511512 & -0.261830 \\
\hline 9 & -2.859386 & 10.686134 & -0.210050 & 9 & -4.283627 & 9.567920 & -1.780576 \\
\hline 9 & -4.025738 & 9.727620 & -1.799358 & 9 & -5.126941 & 10.059787 & 0.185475 \\
\hline 1 & -0.289728 & 8.629028 & 2.193650 & 1 & -0.319130 & 8.396330 & 1.719039 \\
\hline 1 & 2.440545 & 8.520039 & 0.907070 & 1 & 2.248397 & 7.861035 & 0.194163 \\
\hline 1 & 1.002859 & 9.451506 & 0.440151 & 1 & 0.905994 & 8.959597 & -0.198343 \\
\hline 1 & 1.896547 & 6.752584 & -0.510347 & 1 & 1.322232 & 6.136508 & -1.068169 \\
\hline 1 & 0.588414 & 7.727647 & -1.207279 & 1 & 0.015651 & 7.204209 & -1.611002 \\
\hline 1 & 0.146507 & 5.356114 & 0.205096 & 1 & -0.390245 & 4.954745 & 0.026615 \\
\hline 1 & -3.836949 & 2.734443 & 8.663162 & 1 & -2.859619 & 2.697553 & 9.065381 \\
\hline 1 & -5.460895 & 1.327593 & 7.474773 & 1 & -4.737631 & 1.379773 & 8.191639 \\
\hline 1 & -5.535405 & 1.342529 & 4.957975 & 1 & -5.410539 & 1.596660 & 5.774577 \\
\hline 1 & -3.950252 & 2.809804 & 3.695168 & 1 & -4.084799 & 3.089962 & 4.265796 \\
\hline 1 & -0.934184 & 2.690794 & 3.906120 & 1 & -0.963524 & 2.671100 & 4.119532 \\
\hline 1 & 0.551827 & 4.029925 & 2.404017 & 1 & 0.315444 & 3.790099 & 2.278724 \\
\hline 1 & -3.094334 & 6.283589 & 4.955939 & 1 & -2.799135 & 6.454202 & 5.127896 \\
\hline 1 & -1.646265 & 7.605765 & 3.444306 & 1 & -1.534077 & 7.563879 & 3.310366 \\
\hline 1 & 1.582113 & 10.460289 & 2.904596 & 1 & 1.375437 & 10.168904 & 2.476755 \\
\hline 1 & 1.701570 & 6.278192 & 4.004123 & 1 & 1.981314 & 5.952744 & 3.177103 \\
\hline 1 & 3.799715 & 9.200884 & 6.403509 & 1 & 3.954452 & 8.863524 & 5.701477 \\
\hline 1 & -5.308339 & 7.663173 & 0.522952 & 1 & -5.547127 & 7.755960 & 0.906718 \\
\hline 1 & -1.239243 & 8.819670 & -0.363442 & 1 & -1.573059 & 8.475430 & -0.633297 \\
\hline 1 & -2.110792 & 4.794488 & 0.982876 & 1 & -2.546647 & 4.657413 & 1.168597 \\
\hline 6 & -1.152015 & 9.254994 & 6.354263 & 6 & -0.698245 & 9.376679 & 6.270332 \\
\hline 6 & -2.168482 & 9.355638 & 5.344369 & 6 & -1.693045 & 9.512490 & 5.240654 \\
\hline 6 & -3.451370 & 8.790839 & 5.550276 & 6 & -3.012908 & 9.045111 & 5.467631 \\
\hline 6 & -1.881237 & 10.009606 & 4.117620 & 6 & -1.367400 & 10.105942 & 3.996678 \\
\hline 6 & -4.412256 & 8.844866 & 4.531822 & 6 & -3.971681 & 9.133291 & 4.452606 \\
\hline 1 & -3.657012 & 8.281952 & 6.497444 & 1 & -3.257804 & 8.596948 & 6.436190 \\
\hline
\end{tabular}




\begin{tabular}{|c|c|c|c|c|c|c|c|}
\hline 6 & -2.852395 & 10.073060 & 3.113291 & 6 & -2.334112 & 10.193735 & 2.986143 \\
\hline 1 & -0.886036 & 10.439705 & 3.966130 & 1 & -0.356819 & 10.489887 & 3.848905 \\
\hline 6 & -4.115740 & 9.485003 & 3.316368 & 6 & -3.634319 & 9.708207 & 3.213426 \\
\hline 1 & -5.392871 & 8.383389 & 4.685612 & 1 & -4.982171 & 8.748415 & 4.622279 \\
\hline & -2.621264 & 10.557306 & 2.160751 & 1 & -2.079624 & 10.631022 & 2.016770 \\
\hline 1 & -4.864138 & 9.529265 & 2.521265 & 1 & -4.388596 & 9.787579 & 2.427224 \\
\hline 6 & -0.228643 & 8.588486 & 6.982221 & 6 & 0.176123 & 8.651804 & 6.902151 \\
\hline 6 & 0.835478 & 9.008825 & 7.901146 & 6 & 0.999361 & 8.912998 & 8.094637 \\
\hline 6 & 1.477837 & 8.046942 & 8.703690 & 6 & 2.251029 & 8.281844 & 8.224154 \\
\hline 6 & 1.237192 & 10.371530 & 7.945387 & 6 & 0.479180 & 9.727623 & 9.128450 \\
\hline 6 & 2.543058 & 8.409793 & 9.539019 & 6 & 3.020392 & 8.480645 & 9.381310 \\
\hline 1 & 1.168421 & 7.001705 & 8.660513 & 1 & 2.621767 & 7.621833 & 7.440696 \\
\hline 6 & 2.323536 & 10.710871 & 8.790491 & 6 & 1.268228 & 9.901544 & 10.291674 \\
\hline 6 & 2.985210 & 9.746263 & 9.577645 & 6 & 2.532017 & 9.292147 & 10.425770 \\
\hline 1 & 3.822391 & 10.035733 & 10.212405 & 1 & 3.119504 & 9.438160 & 11.332276 \\
\hline 8 & 3.095958 & 7.390748 & 10.268164 & 8 & 4.225729 & 7.830307 & 9.410066 \\
\hline 8 & 2.676761 & 12.035681 & 8.792353 & 8 & 0.714379 & 10.694738 & 11.265376 \\
\hline 6 & 3.836920 & 12.433984 & 9.533671 & 6 & 1.468835 & 10.931593 & 12.462340 \\
\hline 1 & 3.709304 & 12.257283 & 10.618895 & 1 & 1.661925 & 9.990335 & 13.011273 \\
\hline 1 & 4.741613 & 11.906995 & 9.175637 & 1 & 2.429131 & 11.435359 & 12.241956 \\
\hline 1 & 3.945021 & 13.513064 & 9.351325 & 1 & 0.841937 & 11.592209 & 13.078696 \\
\hline 6 & 4.224194 & 7.699905 & 11.099304 & 6 & 5.048262 & 7.995301 & 10.574313 \\
\hline 1 & 4.511859 & 6.750225 & 11.573206 & 1 & 5.953927 & 7.401778 & 10.382570 \\
\hline 1 & 5.072521 & 8.084462 & 10.501293 & 1 & 5.328361 & 9.055614 & 10.724812 \\
\hline 1 & 3.961556 & 8.436608 & 11.882756 & 1 & 4.545207 & 7.614054 & 11.483742 \\
\hline 6 & 0.586375 & 11.447449 & 7.102938 & 6 & -0.900892 & 10.343375 & 9.029181 \\
\hline 1 & 0.906195 & 12.437043 & 7.476295 & 1 & -1.659658 & 9.555088 & 9.203204 \\
\hline 1 & 0.976430 & 11.389880 & 6.071849 & 1 & -1.031951 & 11.072134 & 9.849922 \\
\hline 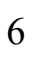 & -0.920898 & 11.429013 & 6.994820 & 6 & -1.232833 & 11.030183 & 7.721488 \\
\hline 1 & -1.309005 & 11.839919 & 6.054599 & 1 & -2.290159 & 10.982584 & 7.431365 \\
\hline 0 & -1.848071 & 11.214676 & 7.992445 & 6 & -0.443872 & 11.939833 & 7.046851 \\
\hline 6 & -3.315281 & 11.374340 & 7.703171 & 6 & -1.009403 & 12.729020 & 5.899528 \\
\hline & -3.518477 & 11.619463 & 6.648416 & 1 & -2.016548 & 12.395216 & 5.602907 \\
\hline 1 & -3.861479 & 10.444838 & 7.958358 & 1 & -1.066004 & 13.797809 & 6.187836 \\
\hline & -3.742790 & 12.171244 & 8.341983 & 1 & -0.339732 & 12.681953 & 5.022445 \\
\hline & -1.494371 & 10.772639 & 9.388058 & 6 & 0.984995 & 12.235052 & 7.431344 \\
\hline
\end{tabular}




$\begin{array}{cccccccc}1 & -1.485223 & 9.665954 & 9.443106 & 1 & 1.341743 & 13.156889 & 6.944171 \\ 1 & -0.496993 & 11.117453 & 9.701785 & 1 & 1.094727 & 12.337513 & 8.525097 \\ 1 & -2.249481 & 11.127998 & 10.110486 & 1 & 1.656007 & 11.416993 & 7.119902 \\ 6 & -1.713816 & 6.443986 & 9.985537 & 6 & -0.654633 & 6.357960 & 10.222280 \\ 1 & -0.675561 & 6.155284 & 10.215027 & 1 & -0.024725 & 5.669550 & 10.810714 \\ 1 & -1.677715 & 7.291095 & 9.278986 & 1 & -0.005313 & 6.937115 & 9.544416 \\ 1 & -2.180329 & 6.800521 & 10.923023 & 1 & -1.114664 & 7.072141 & 10.930775 \\ 6 & -2.748542 & 4.258343 & 10.589863 & 6 & -2.520275 & 4.707223 & 10.509350 \\ 1 & -3.311057 & 3.353598 & 10.313603 & 1 & -3.485543 & 4.341083 & 10.126337 \\ 1 & -1.781797 & 3.946551 & 11.018557 & 1 & -1.916140 & 3.849036 & 10.845302 \\ 1 & -3.317746 & 4.758318 & 11.395680 & 1 & -2.739280 & 5.322228 & 11.402290 \\ 6 & -3.928519 & 5.832491 & 8.975307 & 6 & -2.811781 & 6.623955 & 8.923845 \\ 1 & -3.809863 & 6.520139 & 8.117007 & 1 & -2.320266 & 7.403219 & 8.314337 \\ 1 & -4.639444 & 5.042569 & 8.683747 & 1 & -3.582681 & 6.132697 & 8.303041 \\ 1 & -4.375281 & 6.406482 & 9.808406 & 1 & -3.320364 & 7.121530 & 9.770659 \\ 6 & 0.727967 & 3.955536 & 9.141563 & 6 & 1.637685 & 4.300831 & 8.724623 \\ 1 & 1.150443 & 4.870619 & 8.694400 & 1 & 1.911496 & 5.075806 & 7.990507 \\ 1 & 0.328655 & 4.198885 & 10.140468 & 1 & 1.512841 & 4.782362 & 9.706661 \\ 1 & 1.560238 & 3.241115 & 9.283798 & 1 & 2.485302 & 3.594297 & 8.803707 \\ 6 & -0.864850 & 2.003134 & 8.857304 & 6 & 0.045505 & 2.442241 & 9.347506 \\ 1 & -0.018037 & 1.300962 & 8.971455 & 1 & 0.901191 & 1.745968 & 9.429933 \\ 1 & -1.303112 & 2.159214 & 9.854488 & 1 & -0.115515 & 2.883711 & 10.345297 \\ 1 & -1.617192 & 1.515234 & 8.215313 & 1 & -0.838290 & 1.843034 & 9.072103 \\ 6 & 0.305605 & 2.966786 & 6.856464 & 6 & 0.659120 & 2.831411 & 6.925239 \\ 1 & 1.184409 & 2.316044 & 7.023076 & 1 & 1.547422 & 2.180165 & 7.024857 \\ 1 & -0.402688 & 2.418602 & 6.212958 & 1 & -0.189598 & 2.204581 & 6.601438 \\ 1 & 0.638838 & 3.869134 & 6.317263 & 1 & 0.865153 & 3.574462 & 6.135107\end{array}$




\section{B-Int1b}

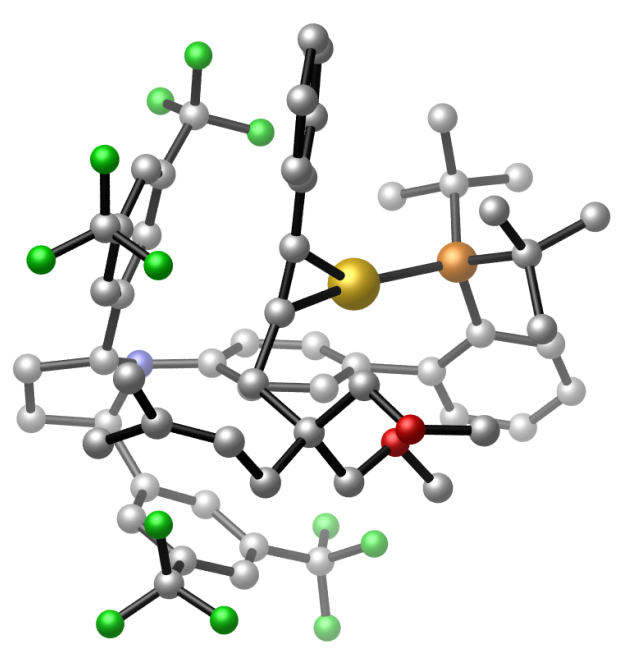

$$
\begin{gathered}
E=-4167.38134937 \text { Hartrees } \\
G=-4166.399435 \text { Hartrees }
\end{gathered}
$$

$\begin{array}{llll}79 & -1.144662 & 6.436469 & 6.726632 \\ 6 & -2.596575 & 3.315419 & 7.440095 \\ 6 & -3.284493 & 2.504415 & 8.373105 \\ 6 & -4.157021 & 1.485010 & 7.970590 \\ 6 & -4.343436 & 1.234792 & 6.603986 \\ 6 & -3.663441 & 2.015822 & 5.661961 \\ 6 & -2.798276 & 3.066058 & 6.046490 \\ 6 & -2.171324 & 3.836040 & 4.933114 \\ 6 & -1.315032 & 3.204939 & 4.006611 \\ 6 & -0.751572 & 3.918150 & 2.938742 \\ 6 & -1.054069 & 5.282489 & 2.750091 \\ 6 & -1.931521 & 5.907168 & 3.652527 \\ 6 & -2.485271 & 5.199225 & 4.728127 \\ 6 & -0.421313 & 7.426120 & 1.660958 \\ 6 & 0.057356 & 7.756678 & 0.225378 \\ 6 & -0.653855 & 6.702443 & -0.672307 \\ 6 & -1.248582 & 5.650164 & 0.299593 \\ 6 & 0.492689 & 7.923287 & 2.762636 \\ 6 & 0.395731 & 9.265165 & 3.174684 \\ 6 & 1.246666 & 9.768248 & 4.171157\end{array}$

B-Int1d

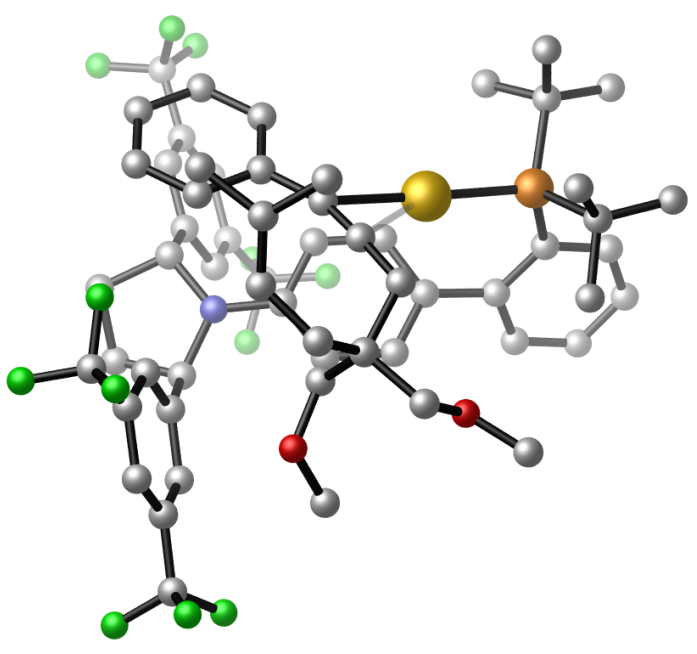

$$
\begin{gathered}
E=-4167.37466010 \text { Hartrees } \\
G=-4166.398710 \text { Hartrees }
\end{gathered}
$$

$\begin{array}{rrrr}79 & -1.465063 & 7.110998 & 7.862150 \\ 6 & -4.597879 & 5.561269 & 7.716915 \\ 6 & -5.794879 & 5.031950 & 8.260616 \\ 6 & -6.930237 & 4.807622 & 7.472840 \\ 6 & -6.894235 & 5.127086 & 6.107220 \\ 6 & -5.712945 & 5.625498 & 5.546791 \\ 6 & -4.542212 & 5.837462 & 6.315850 \\ 6 & -3.340256 & 6.281520 & 5.558786 \\ 6 & -2.154379 & 5.506356 & 5.533217 \\ 6 & -1.093441 & 5.812331 & 4.675293 \\ 6 & -1.183633 & 6.899999 & 3.763613 \\ 6 & -2.338766 & 7.725080 & 3.842399 \\ 6 & -3.387830 & 7.413386 & 4.714975 \\ 6 & -0.486059 & 7.931043 & 1.595241 \\ 6 & 0.500351 & 7.354055 & 0.541056 \\ 6 & 1.668972 & 6.826249 & 1.390012 \\ 6 & 0.955609 & 6.230497 & 2.628326 \\ 6 & -0.273087 & 9.429525 & 1.792835 \\ 6 & -1.146337 & 10.339630 & 1.175707 \\ 6 & -0.901721 & 11.720942 & 1.245270\end{array}$




\begin{tabular}{|c|c|c|c|c|c|c|c|}
\hline 6 & 2.189654 & 8.937387 & 4.794625 & 6 & 0.228457 & 12.210969 & 1.909504 \\
\hline 6 & 2.267893 & 7.595915 & 4.396713 & 6 & 1.107861 & 11.299881 & 2.516618 \\
\hline 6 & 1.442261 & 7.091989 & 3.378311 & 6 & 0.848648 & 9.922671 & 2.484784 \\
\hline 6 & 1.073394 & 11.195165 & 4.629407 & 6 & -1.844505 & 12.682053 & 0.561757 \\
\hline 6 & 3.202198 & 6.636607 & 5.088978 & 6 & 2.388996 & 11.832494 & 3.107342 \\
\hline 6 & -2.746545 & 5.755024 & 0.584523 & 6 & 0.616325 & 4.755037 & 2.420727 \\
\hline 6 & -3.413112 & 4.626352 & 1.113655 & 6 & 1.548414 & 3.787780 & 2.833303 \\
\hline 6 & -4.621424 & 4.766113 & 1.806403 & 6 & 1.272543 & 2.420411 & 2.678636 \\
\hline 6 & -5.200525 & 6.033151 & 1.996403 & 6 & 0.062564 & 1.996215 & 2.109409 \\
\hline 6 & -4.574339 & 7.142912 & 1.422340 & 6 & -0.865909 & 2.963899 & 1.696817 \\
\hline 6 & -3.381190 & 7.005300 & 0.688617 & 6 & -0.595160 & 4.332879 & 1.848989 \\
\hline 6 & -5.290590 & 3.578176 & 2.450913 & 6 & 2.312991 & 1.413687 & 3.109029 \\
\hline 6 & -5.066974 & 8.541408 & 1.683146 & 6 & -2.218286 & 2.538528 & 1.175616 \\
\hline 6 & 0.254368 & 3.479678 & 8.224706 & 6 & -2.661077 & 4.542618 & 9.841557 \\
\hline 6 & -1.932084 & 5.242461 & 9.766535 & 6 & -4.105343 & 7.436339 & 10.002178 \\
\hline & -1.338323 & 4.523956 & 8.076545 & 15 & -3.282536 & 6.083849 & 8.903031 \\
\hline 7 & -0.538224 & 5.959178 & 1.603009 & 7 & -0.210100 & 7.116642 & 2.794980 \\
\hline 9 & 2.116953 & 11.639045 & 5.373483 & 9 & -1.893205 & 13.887898 & 1.203342 \\
\hline 9 & -0.057674 & 11.335113 & 5.405353 & 9 & -3.118368 & 12.194514 & 0.515605 \\
\hline 9 & 0.911025 & 12.052313 & 3.578034 & 9 & -1.464647 & 12.932151 & -0.724952 \\
\hline 9 & 2.488793 & 5.717615 & 5.835845 & 9 & 2.183222 & 12.967546 & 3.834649 \\
\hline 9 & 4.058280 & 7.253759 & 5.944462 & 9 & 3.292581 & 12.143606 & 2.136031 \\
\hline 9 & 3.938554 & 5.911398 & 4.204195 & 9 & 3.002942 & 10.924299 & 3.936745 \\
\hline 9 & -6.521430 & 3.336084 & 1.919462 & 9 & 2.930388 & 1.799807 & 4.266738 \\
\hline 9 & -5.480059 & 3.794014 & 3.792192 & 9 & 3.293847 & 1.276148 & 2.168552 \\
\hline 9 & -4.559595 & 2.432302 & 2.328671 & 9 & 1.780201 & 0.178385 & 3.319865 \\
\hline 9 & -5.356702 & 9.221331 & 0.545057 & 9 & -3.148314 & 2.520047 & 2.178643 \\
\hline 9 & -6.157757 & 8.582737 & 2.493025 & 9 & -2.684616 & 3.395792 & 0.222115 \\
\hline 9 & -4.070484 & 9.262803 & 2.322795 & 9 & -2.191082 & 1.289846 & 0.631855 \\
\hline 1 & -1.410071 & 7.916513 & 1.819028 & 1 & -1.532206 & 7.768896 & 1.269779 \\
\hline 1 & 1.152388 & 7.635786 & 0.176657 & 1 & 0.804460 & 8.109891 & -0.199846 \\
\hline 1 & -0.184198 & 8.796886 & -0.047457 & 1 & 0.012976 & 6.522195 & 0.002783 \\
\hline 1 & 0.062514 & 6.221979 & -1.356481 & 1 & 2.339956 & 7.645245 & 1.701952 \\
\hline 1 & -1.443899 & 7.150426 & -1.296783 & 1 & 2.278412 & 6.073511 & 0.865489 \\
\hline 1 & -0.997873 & 4.617285 & 0.006938 & 1 & 1.598168 & 6.308091 & 3.520203 \\
\hline & -3.127812 & 2.654951 & 9.441724 & 1 & -5.854955 & 4.804099 & 9.325722 \\
\hline
\end{tabular}




\begin{tabular}{|c|c|c|c|c|c|c|c|}
\hline & -4.674971 & 0.885599 & 8.726128 & 1 & -7.836197 & 4.396395 & 7.929384 \\
\hline & -5.016621 & 0.438396 & 6.269984 & 1 & -7.775295 & 4.971177 & 5.475863 \\
\hline & -3.820871 & 1.844233 & 4.593850 & 1 & -5.666675 & 5.833276 & 4.472388 \\
\hline & -1.075502 & 2.143797 & 4.133210 & 1 & -2.088017 & 4.598108 & 6.140139 \\
\hline & -0.082910 & 3.418133 & 2.229893 & 1 & -0.211843 & 5.170919 & 4.691443 \\
\hline & -3.245557 & 5.692784 & 5.340464 & 1 & -4.271906 & 8.060512 & 4.734528 \\
\hline & -2.245305 & 6.937586 & 3.481660 & 1 & -2.420084 & 8.616407 & 3.214442 \\
\hline & -0.352654 & 9.920216 & 2.714915 & 1 & -2.029651 & 9.972057 & 0.642452 \\
\hline & 1.528884 & 6.047046 & 3.068601 & 1 & 1.515319 & 9.225921 & 2.998549 \\
\hline & 2.830618 & 9.322546 & 5.589259 & 1 & 0.417523 & 13.285560 & 1.965971 \\
\hline & -6.108281 & 6.149804 & 2.593419 & 1 & -0.158220 & 0.932099 & 2.000955 \\
\hline & -2.925180 & 7.900550 & 0.255816 & 1 & -1.346777 & 5.069533 & 1.550607 \\
\hline & -2.942327 & 3.641722 & 1.050299 & 1 & 2.485005 & 4.104973 & 3.303502 \\
\hline & -0.385203 & 8.517447 & 6.812595 & 6 & -0.407197 & 9.067688 & 7.386559 \\
\hline & 0.649843 & 8.956563 & 7.708883 & 6 & 0.306872 & 8.121080 & 6.962145 \\
\hline & 1.756490 & 8.140798 & 8.035089 & 6 & 1.425681 & 7.494663 & 6.303112 \\
\hline & 0.553472 & 10.262869 & 8.250430 & 6 & 1.725539 & 6.121519 & 6.455644 \\
\hline & 2.755962 & 8.622576 & 8.887885 & 1 & 1.072857 & 5.495395 & 7.073112 \\
\hline & 1.831932 & 7.147634 & 7.588956 & 6 & 3.675338 & 6.391339 & 5.018999 \\
\hline & 1.558656 & 10.733438 & 9.104828 & 1 & 4.548814 & 5.961067 & 4.518392 \\
\hline & -0.297489 & 10.895805 & 7.985215 & 6 & -3.004629 & 8.244988 & 10.730072 \\
\hline & 2.658691 & 9.917881 & 9.427267 & 1 & -2.436066 & 7.638375 & 11.452331 \\
\hline 1 & 3.615025 & 7.987029 & 9.125392 & 1 & -2.286703 & 8.698003 & 10.026334 \\
\hline & 1.482945 & 11.743984 & 9.519528 & 1 & -3.490745 & 9.064906 & 11.290565 \\
\hline & 3.440964 & 10.293784 & 10.094830 & 6 & -5.089354 & 6.889661 & 11.056963 \\
\hline 6 & -1.363761 & 8.528138 & 6.015605 & 1 & -5.926156 & 6.328588 & 10.612534 \\
\hline & -4.462845 & 10.662238 & 5.400480 & 1 & -4.586838 & 6.254155 & 11.805226 \\
\hline & -4.630953 & 10.476426 & 4.326119 & 1 & -5.528145 & 7.748506 & 11.597976 \\
\hline & -5.457220 & 10.760416 & 5.873594 & 6 & -4.852880 & 8.373709 & 9.020022 \\
\hline & -3.685101 & 11.931591 & 5.643697 & 1 & -4.197936 & 8.754235 & 8.214641 \\
\hline & -3.759248 & 12.321212 & 6.669224 & 1 & -5.718450 & 7.877066 & 8.550434 \\
\hline & -2.899845 & 12.616251 & 4.777256 & 1 & -5.224681 & 9.248429 & 9.584928 \\
\hline & -2.673042 & 12.247880 & 3.327699 & 6 & -1.704706 & 5.016251 & 10.958450 \\
\hline & -3.191375 & 11.330324 & 3.011890 & 1 & -0.933668 & 5.710500 & 10.577578 \\
\hline & -3.004324 & 13.071063 & 2.664672 & 1 & -2.248521 & 5.507540 & 11.782796 \\
\hline & -1.590166 & 12.119559 & 3.138448 & 1 & -1.188698 & 4.134852 & 11.381690 \\
\hline
\end{tabular}




\begin{tabular}{|c|c|c|c|c|c|c|c|}
\hline 6 & -1.136982 & 6.552104 & 10.032061 & 6 & -3.768310 & 3.649912 & 10.442384 \\
\hline 1 & -0.045154 & 6.424616 & 9.962959 & 1 & -3.281139 & 2.827739 & 10.998820 \\
\hline 1 & -1.425322 & 7.354751 & 9.332716 & 1 & -4.417747 & 4.185396 & 11.153107 \\
\hline 1 & -1.374653 & 6.898420 & 11.055113 & 1 & -4.394585 & 3.189878 & 9.660713 \\
\hline & -1.729696 & 4.318634 & 10.989383 & 6 & -1.875152 & 3.708081 & 8.801905 \\
\hline 1 & -2.213412 & 3.333994 & 10.897461 & 1 & -1.485235 & 2.799234 & 9.296666 \\
\hline 1 & -0.661357 & 4.156850 & 11.209180 & 1 & -2.523276 & 3.383566 & 7.968602 \\
\hline 1 & -2.173985 & 4.815537 & 11.871605 & 1 & -1.013738 & 4.263382 & 8.388161 \\
\hline 6 & -3.421576 & 5.625987 & 9.600645 & 6 & -1.188650 & 10.226390 & 7.829308 \\
\hline 1 & -3.575390 & 6.275468 & 8.720055 & 1 & -0.807503 & 10.569491 & 8.808948 \\
\hline 1 & -4.085067 & 4.750871 & 9.507681 & 1 & -2.231839 & 9.900438 & 7.990008 \\
\hline 1 & -3.737534 & 6.195849 & 10.494523 & 6 & -1.268661 & 11.426917 & 6.833020 \\
\hline 6 & 1.354999 & 4.325333 & 8.896764 & 6 & -0.072212 & 12.403328 & 7.028105 \\
\hline 1 & 1.508267 & 5.284126 & 8.375252 & 1 & -0.189765 & 13.208137 & 6.278437 \\
\hline & 1.139397 & 4.532537 & 9.958083 & 1 & -0.171271 & 12.873768 & 8.024495 \\
\hline 1 & 2.306948 & 3.764370 & 8.856240 & 6 & 1.289136 & 11.772868 & 6.863785 \\
\hline 6 & 0.033339 & 2.162597 & 8.996955 & 1 & 1.652680 & 11.711568 & 5.833034 \\
\hline 1 & 0.972931 & 1.580152 & 8.964431 & 6 & 2.109331 & 11.306382 & 7.837298 \\
\hline 1 & -0.219162 & 2.326393 & 10.055395 & 6 & 1.785331 & 11.311786 & 9.314657 \\
\hline 1 & -0.757898 & 1.545768 & 8.538602 & 1 & 1.611504 & 10.277506 & 9.673775 \\
\hline 6 & 0.670256 & 3.146077 & 6.774173 & 1 & 2.636919 & 11.706409 & 9.900551 \\
\hline 1 & 1.663959 & 2.661078 & 6.791893 & 1 & 0.895412 & 11.911619 & 9.567289 \\
\hline 1 & -0.037524 & 2.442399 & 6.307233 & 6 & 3.460585 & 10.717270 & 7.494379 \\
\hline 1 & 0.735777 & 4.046135 & 6.140471 & 1 & 4.265636 & 11.228773 & 8.057222 \\
\hline 0 & -2.453182 & 9.141943 & 5.235747 & 1 & 3.509113 & 9.647020 & 7.776864 \\
\hline 1 & -2.671603 & 8.589541 & 4.308354 & 1 & 3.687998 & 10.795944 & 6.418566 \\
\hline 1 & -2.049147 & 10.126929 & 4.937652 & 6 & 2.849251 & 5.576779 & 5.817074 \\
\hline 6 & -3.789940 & 9.384619 & 6.019388 & 1 & 3.072980 & 4.510973 & 5.929575 \\
\hline 6 & -2.151228 & 13.854167 & 5.221745 & 6 & 3.369500 & 7.756103 & 4.853509 \\
\hline 1 & -2.427954 & 14.732008 & 4.605469 & 1 & 3.999487 & 8.397223 & 4.228580 \\
\hline 1 & -2.341173 & 14.100172 & 6.280777 & 6 & 2.249870 & 8.306938 & 5.488853 \\
\hline 1 & -1.062781 & 13.705088 & 5.089779 & 1 & 2.005059 & 9.364958 & 5.379439 \\
\hline 6 & -4.789921 & 8.227665 & 5.845898 & 6 & -1.332835 & 10.904622 & 5.375759 \\
\hline 1 & -5.764758 & 8.536889 & 6.279065 & 1 & -0.368349 & 10.433172 & 5.105791 \\
\hline 1 & -4.954434 & 8.018654 & 4.766829 & 1 & -2.114870 & 10.126562 & 5.289910 \\
\hline 6 & -3.496809 & 9.607210 & 7.523227 & 6 & -2.569808 & 12.176966 & 7.183899 \\
\hline
\end{tabular}




$\begin{array}{llllllll}1 & -3.084353 & 8.680250 & 7.963184 & 1 & -2.526168 & 12.528865 & 8.238861 \\ 1 & -2.733021 & 10.401399 & 7.633695 & 1 & -2.670749 & 13.067742 & 6.530066 \\ 8 & -4.331986 & 7.043348 & 6.512519 & 8 & -1.560027 & 11.977521 & 4.460188 \\ 8 & -4.676194 & 10.018881 & 8.220282 & 6 & -2.863287 & 11.963180 & 3.871885 \\ 6 & -5.330306 & 6.012265 & 6.501347 & 1 & -2.936942 & 12.871848 & 3.255367 \\ 1 & -5.612243 & 5.731184 & 5.466488 & 1 & -2.998964 & 11.080375 & 3.212539 \\ 1 & -4.889205 & 5.136297 & 7.003652 & 1 & -3.662609 & 11.960751 & 4.637591 \\ 1 & -6.238716 & 6.334136 & 7.049746 & 8 & -3.679037 & 11.290244 & 7.006357 \\ 6 & -5.157747 & 9.038023 & 9.141184 & 6 & -4.902116 & 11.843014 & 7.494603 \\ 1 & -5.402531 & 8.081106 & 8.638501 & 1 & -5.682576 & 11.083771 & 7.328373 \\ 1 & -4.416762 & 8.828516 & 9.941612 & 1 & -4.839902 & 12.069391 & 8.579922 \\ 1 & -6.070991 & 9.452448 & 9.598493 & 1 & -5.173069 & 12.772831 & 6.953069\end{array}$

B-Int2b

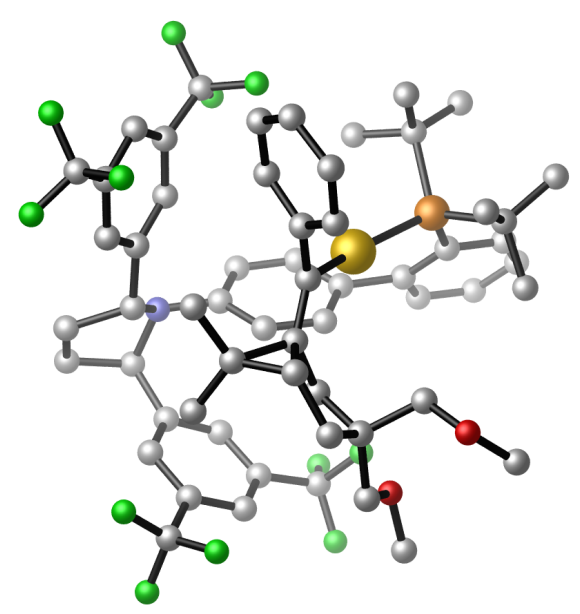

$E=-4167.37236583$ Hartrees

$G=-4166.391239$ Hartrees

\section{B-Int2d}

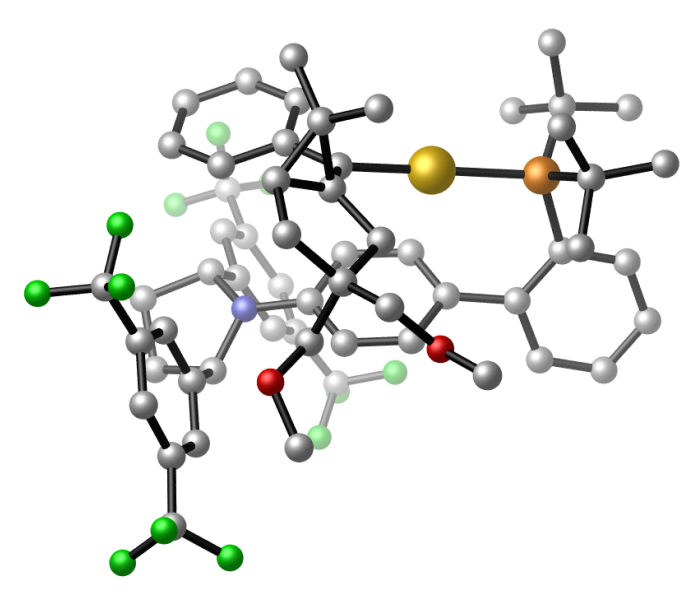

$E=-4167.38367926$ Hartrees

$G=-4166.407002$ Hartrees

$\begin{array}{llll}79 & -1.146116 & 6.626842 & 6.941817 \\ 6 & -2.029101 & 3.131040 & 7.603211 \\ 6 & -2.444867 & 2.156414 & 8.541849 \\ 6 & -3.049012 & 0.953791 & 8.152908 \\ 6 & -3.238915 & 0.684059 & 6.790464 \\ 6 & -2.814840 & 1.622004 & 5.842109 \\ 6 & -2.215240 & 2.847699 & 6.215556\end{array}$

$\begin{array}{llll}79 & -1.194513 & 7.143481 & 7.906199 \\ 6 & -4.321212 & 5.526747 & 7.857777 \\ 6 & -5.510638 & 4.996893 & 8.415736 \\ 6 & -6.649181 & 4.751059 & 7.638309 \\ 6 & -6.623611 & 5.048173 & 6.267514 \\ 6 & -5.449456 & 5.548068 & 5.692555 \\ 6 & -4.277151 & 5.781927 & 6.452572\end{array}$




\begin{tabular}{|c|c|c|c|c|c|c|c|}
\hline 6 & -1.775470 & 3.730870 & 5.098901 & 6 & -3.084002 & 6.228690 & 5.677430 \\
\hline 6 & -0.828836 & 3.255786 & 4.165578 & 6 & -1.907841 & 5.442512 & 5.613392 \\
\hline 6 & -0.409697 & 4.059499 & 3.098887 & 6 & -0.868193 & 5.741898 & 4.727344 \\
\hline 6 & -0.908625 & 5.369850 & 2.952519 & 6 & -0.974216 & 6.835406 & 3.826441 \\
\hline 6 & -1.870459 & 5.841290 & 3.864977 & 6 & -2.111096 & 7.679121 & 3.948720 \\
\hline 6 & -2.315168 & 5.020613 & 4.914004 & 6 & -3.140409 & 7.371126 & 4.847273 \\
\hline 6 & -0.186941 & 7.559707 & 1.956227 & 6 & -0.370581 & 7.811257 & 1.603741 \\
\hline 6 & 0.025713 & 8.017864 & 0.476123 & 6 & 0.615950 & 7.254711 & 0.535332 \\
\hline 6 & -0.653921 & 6.927766 & -0.401468 & 6 & 1.791558 & 6.717806 & 1.368602 \\
\hline 6 & -1.357272 & 5.987272 & 0.596045 & 6 & 1.077119 & 6.096618 & 2.590927 \\
\hline 6 & 1.016122 & 7.875140 & 2.833230 & 6 & -0.236180 & 9.323009 & 1.753934 \\
\hline 6 & 1.441728 & 9.216881 & 2.909242 & 6 & -1.151928 & 10.171724 & 1.110766 \\
\hline 6 & 2.584546 & 9.568767 & 3.642901 & 6 & -0.983223 & 11.565923 & 1.152873 \\
\hline 6 & 3.322731 & 8.591532 & 4.326440 & 6 & 0.085557 & 12.134025 & 1.856146 \\
\hline 6 & 2.880899 & 7.262301 & 4.280978 & 6 & 1.002796 & 11.285487 & 2.497569 \\
\hline 6 & 1.744332 & 6.899875 & 3.537393 & 6 & 0.846969 & 9.892707 & 2.445690 \\
\hline 6 & 2.973805 & 11.020930 & 3.784681 & 6 & -1.931886 & 12.440558 & 0.369440 \\
\hline 6 & 3.567989 & 6.239627 & 5.152590 & 6 & 2.199315 & 11.908273 & 3.171870 \\
\hline 6 & -2.799388 & 6.354238 & 0.947067 & 6 & 0.639763 & 4.657847 & 2.302681 \\
\hline 6 & -3.613902 & 5.380005 & 1.562140 & 6 & 1.560127 & 3.613840 & 2.506386 \\
\hline 6 & -4.847121 & 5.726527 & 2.134504 & 6 & 1.200359 & 2.285007 & 2.233566 \\
\hline 6 & -5.317202 & 7.046499 & 2.072561 & 6 & -0.087046 & 1.973257 & 1.767488 \\
\hline 6 & -4.535815 & 8.010032 & 1.417126 & 6 & -1.004282 & 3.014253 & 1.570565 \\
\hline 6 & -3.285294 & 7.673625 & 0.870539 & 6 & -0.645422 & 4.346787 & 1.830587 \\
\hline 6 & -5.630920 & 4.700749 & 2.917577 & 6 & 2.223198 & 1.184075 & 2.389617 \\
\hline 6 & -5.090361 & 9.401163 & 1.230610 & 6 & -2.426504 & 2.713034 & 1.161254 \\
\hline 6 & 0.670163 & 4.059005 & 8.437966 & 6 & -2.355913 & 4.544368 & 9.962070 \\
\hline 6 & -1.963642 & 5.174681 & 9.894604 & 6 & -3.810350 & 7.422419 & 10.112662 \\
\hline 15 & -1.137238 & 4.637387 & 8.231871 & 15 & -2.984905 & 6.076330 & 9.014146 \\
\hline 7 & -0.476644 & 6.124483 & 1.811328 & 7 & -0.024913 & 7.046093 & 2.824578 \\
\hline 9 & 4.322171 & 11.178520 & 3.891449 & 9 & -1.895537 & 13.740935 & 0.775603 \\
\hline 9 & 2.420874 & 11.564943 & 4.915614 & 9 & -3.225418 & 12.008745 & 0.479965 \\
\hline 9 & 2.545636 & 11.774333 & 2.734047 & 9 & -1.630379 & 12.426012 & -0.962443 \\
\hline 9 & 3.112891 & 6.335285 & 6.448477 & 9 & 1.864054 & 13.031067 & 3.872351 \\
\hline 9 & 4.915216 & 6.424787 & 5.199296 & 9 & 3.147861 & 12.278556 & 2.266661 \\
\hline 9 & 3.333782 & 4.961442 & 4.747034 & 9 & 2.811672 & 11.051518 & 4.053200 \\
\hline
\end{tabular}




\begin{tabular}{|c|c|c|c|c|c|c|c|}
\hline 9 & -6.977252 & 4.866952 & 2.766516 & 9 & 3.138468 & 1.473689 & 3.359356 \\
\hline 9 & -5.375546 & 4.804245 & 4.260806 & 9 & 2.920580 & 0.987945 & 1.230721 \\
\hline 9 & -5.325730 & 3.425626 & 2.553226 & 9 & 1.644320 & -0.007353 & 2.712171 \\
\hline 9 & -4.115432 & 10.317885 & 0.964288 & 9 & -3.258080 & 2.698290 & 2.247155 \\
\hline 9 & -5.983383 & 9.453535 & 0.204146 & 9 & -2.914419 & 3.659122 & 0.306461 \\
\hline 9 & -5.757873 & 9.836692 & 2.349743 & 9 & -2.543620 & 1.502719 & 0.548568 \\
\hline 1 & -1.056960 & 8.118345 & 2.378061 & 1 & -1.416662 & 7.589392 & 1.311025 \\
\hline 1 & 1.103620 & 8.080052 & 0.256152 & 1 & 0.911783 & 8.024001 & -0.194709 \\
\hline 1 & -0.398424 & 9.023046 & 0.317934 & 1 & 0.132041 & 6.428062 & -0.013453 \\
\hline 1 & 0.108128 & 6.354997 & -0.952905 & 1 & 2.453661 & 7.536551 & 1.701987 \\
\hline 1 & -1.354144 & 7.343890 & -1.143898 & 1 & 2.405749 & 5.980465 & 0.827728 \\
\hline 1 & -1.323225 & 4.931789 & 0.275520 & 1 & 1.744204 & 6.087974 & 3.471049 \\
\hline 1 & -2.283125 & 2.324203 & 9.607094 & 1 & -5.561789 & 4.789329 & 9.485653 \\
\hline 1 & -3.360074 & 0.232635 & 8.915616 & 1 & -7.550301 & 4.342244 & 8.106855 \\
\hline 1 & -3.709353 & -0.249973 & 6.466063 & 1 & -7.507338 & 4.876062 & 5.643967 \\
\hline 1 & -2.957122 & 1.422810 & 4.774484 & 1 & -5.411833 & 5.742219 & 4.615134 \\
\hline 1 & -0.411070 & 2.250805 & 4.289360 & 1 & -1.829261 & 4.540174 & 6.226994 \\
\hline 1 & 0.323677 & 3.692685 & 2.372822 & 1 & 0.010675 & 5.094525 & 4.719552 \\
\hline 1 & -3.151968 & 5.339637 & 5.542088 & 1 & -4.016703 & 8.027497 & 4.897642 \\
\hline 1 & -2.301462 & 6.837252 & 3.739724 & 1 & -2.200118 & 8.575466 & 3.328949 \\
\hline 1 & 0.891036 & 9.993043 & 2.368060 & 1 & -2.002416 & 9.745661 & 0.567340 \\
\hline 1 & 1.438525 & 5.853534 & 3.509301 & 1 & 1.554984 & 9.234897 & 2.952737 \\
\hline 1 & 4.214287 & 8.865016 & 4.895926 & 1 & 0.198623 & 13.217961 & 1.913116 \\
\hline 1 & -6.278559 & 7.317045 & 2.515238 & 1 & -0.371292 & 0.936944 & 1.571173 \\
\hline 1 & -2.699062 & 8.456896 & 0.383834 & 1 & -1.385068 & 5.139431 & 1.689344 \\
\hline 1 & -3.262912 & 4.345903 & 1.629494 & 1 & 2.560054 & 3.837258 & 2.893687 \\
\hline 6 & -1.225768 & 8.636026 & 6.548851 & 6 & 0.035571 & 9.682046 & 7.105671 \\
\hline 6 & -0.139226 & 9.333595 & 7.248601 & 6 & 0.286927 & 8.277375 & 7.056617 \\
\hline 6 & 1.197536 & 9.027490 & 6.891276 & 6 & 1.474122 & 7.668239 & 6.478690 \\
\hline 6 & -0.372010 & 10.209295 & 8.339447 & 6 & 1.903153 & 6.375045 & 6.888477 \\
\hline 6 & 2.267535 & 9.652379 & 7.535826 & 1 & 1.343443 & 5.862737 & 7.679238 \\
\hline 1 & 1.371537 & 8.304949 & 6.090841 & 6 & 3.710472 & 6.426878 & 5.262195 \\
\hline 6 & 0.709352 & 10.788315 & 9.019979 & 1 & 4.573668 & 5.945729 & 4.790606 \\
\hline 1 & -1.393607 & 10.385827 & 8.688837 & 6 & -2.696961 & 8.253293 & 10.796378 \\
\hline 6 & 2.029027 & 10.534461 & 8.606668 & 1 & -2.104108 & 7.665848 & 11.515702 \\
\hline 1 & 3.291735 & 9.427155 & 7.222151 & 1 & -2.002660 & 8.685636 & 10.054830 \\
\hline
\end{tabular}




\begin{tabular}{|c|c|c|c|c|c|c|c|}
\hline 1 & 0.517999 & 11.443353 & 9.876555 & 1 & -3.169171 & 9.085896 & 11.350732 \\
\hline & 2.868218 & 11.003076 & 9.131004 & 6 & -4.776267 & 6.901101 & 11.194344 \\
\hline 6 & -2.317425 & 9.259645 & 5.912528 & 1 & -5.613461 & 6.320502 & 10.775503 \\
\hline & -4.403410 & 10.618538 & 6.305605 & 1 & -4.259012 & 6.287470 & 11.951186 \\
\hline & -4.864385 & 11.119724 & 5.434563 & 1 & -5.215667 & 7.769497 & 11.720384 \\
\hline & -4.808680 & 11.095144 & 7.212559 & 6 & -4.566321 & 8.342171 & 9.120462 \\
\hline & -2.882272 & 10.756384 & 6.258352 & 1 & -3.909537 & 8.684821 & 8.299478 \\
\hline & -2.392861 & 11.252790 & 7.099763 & 1 & -5.442826 & 7.842631 & 8.674716 \\
\hline & -2.098240 & 10.676411 & 5.026316 & 1 & -4.920109 & 9.237122 & 9.665403 \\
\hline & -2.736971 & 10.622216 & 3.650615 & 6 & -1.356050 & 5.034401 & 11.033190 \\
\hline & -3.793560 & 10.330139 & 3.653387 & 1 & -0.595020 & 5.711715 & 10.603509 \\
\hline & -2.682671 & 11.634008 & 3.210652 & 1 & -1.864235 & 5.555133 & 11.862486 \\
\hline 1 & -2.184045 & 9.940979 & 2.981257 & 1 & -0.832178 & 4.160120 & 11.462375 \\
\hline & -1.574338 & 6.652246 & 10.175026 & 6 & -3.442524 & 3.667454 & 10.619142 \\
\hline & -0.484773 & 6.809659 & 10.218665 & 1 & -2.941855 & 2.848037 & 11.168409 \\
\hline 1 & -1.984701 & 7.338190 & 9.414552 & 1 & -4.061639 & 4.217772 & 11.345697 \\
\hline & -1.998410 & 6.940224 & 11.155491 & 1 & -4.102580 & 3.201711 & 9.868881 \\
\hline & -1.564677 & 4.356408 & 11.142947 & 6 & -1.607132 & 3.686705 & 8.913224 \\
\hline & -1.768443 & 3.277729 & 11.064475 & 1 & -1.202678 & 2.785728 & 9.411446 \\
\hline & -0.498345 & 4.487150 & 11.390644 & 1 & -2.284427 & 3.348476 & 8.108744 \\
\hline & -2.149639 & 4.732189 & 12.003096 & 1 & -0.761511 & 4.233329 & 8.458759 \\
\hline 6 & -3.493036 & 5.123246 & 9.661522 & 6 & -1.420113 & 10.174122 & 7.112804 \\
\hline 1 & -3.777097 & 5.659891 & 8.737351 & 1 & -1.863501 & 10.214344 & 8.123156 \\
\hline 1 & -3.882334 & 4.094847 & 9.593066 & 1 & -2.038369 & 9.462756 & 6.538359 \\
\hline & -3.999408 & 5.625086 & 10.507161 & 6 & -1.406021 & 11.569164 & 6.434902 \\
\hline 6 & 1.480829 & 5.197704 & 9.094721 & 6 & 0.033071 & 12.133055 & 6.646486 \\
\hline 1 & 1.358003 & 6.154709 & 8.556273 & 1 & 0.369149 & 12.671013 & 5.746853 \\
\hline & 1.202742 & 5.349853 & 10.151471 & 1 & 0.070580 & 12.855607 & 7.482746 \\
\hline 1 & 2.553730 & 4.932357 & 9.066579 & 6 & 0.944789 & 10.932547 & 6.923748 \\
\hline 6 & 0.815095 & 2.750770 & 9.241592 & 1 & 1.864623 & 10.864672 & 6.336822 \\
\hline 1 & 1.880426 & 2.452546 & 9.233238 & 6 & 1.023106 & 10.363197 & 8.301338 \\
\hline & 0.509963 & 2.856935 & 10.294276 & 6 & 0.265867 & 10.923900 & 9.486128 \\
\hline & 0.235083 & 1.927490 & 8.790701 & 1 & -0.041762 & 10.111951 & 10.168393 \\
\hline 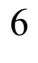 & 1.186372 & 3.818424 & 6.998823 & 1 & 0.948333 & 11.590312 & 10.046170 \\
\hline & 2.263712 & 3.576380 & 7.039444 & 1 & -0.622063 & 11.511745 & 9.216790 \\
\hline & 0.664320 & 2.974336 & 6.518126 & 6 & 2.307524 & 9.656464 & 8.704031 \\
\hline
\end{tabular}




\begin{tabular}{lrllllll}
1 & 1.070925 & 4.707958 & 6.356700 & 1 & 2.945310 & 10.397488 & 9.220068 \\
6 & -3.557021 & 8.450659 & 5.515846 & 1 & 2.115481 & 8.825893 & 9.405623 \\
1 & -3.420296 & 7.401371 & 5.814494 & 1 & 2.877629 & 9.270231 & 7.846667 \\
1 & -3.761595 & 8.448023 & 4.431444 & 6 & 3.018436 & 5.770863 & 6.299918 \\
6 & -4.724600 & 9.092619 & 6.301233 & 1 & 3.344068 & 4.780911 & 6.634732 \\
6 & -0.700829 & 11.273008 & 5.007806 & 6 & 3.279181 & 7.691952 & 4.816432 \\
1 & -0.740698 & 12.226105 & 4.451120 & 1 & 3.801183 & 8.197994 & 3.997342 \\
1 & -0.300957 & 11.486011 & 6.008246 & 6 & 2.178467 & 8.304060 & 5.421248 \\
1 & 0.007724 & 10.608516 & 4.491665 & 1 & 1.826119 & 9.269363 & 5.063006 \\
6 & -6.087811 & 8.814461 & 5.666540 & 6 & -1.646662 & 11.366512 & 4.918878 \\
1 & -6.885457 & 9.300036 & 6.270708 & 1 & -0.850610 & 10.712734 & 4.507498 \\
1 & -6.126850 & 9.247705 & 4.644249 & 1 & -2.616048 & 10.856609 & 4.761476 \\
6 & -4.670570 & 8.532820 & 7.746102 & 6 & -2.461584 & 12.505336 & 7.025105 \\
1 & -4.822529 & 7.437452 & 7.704210 & 1 & -2.270210 & 12.658752 & 8.112634 \\
1 & -3.663943 & 8.713135 & 8.174727 & 1 & -2.400687 & 13.501325 & 6.533936 \\
8 & -6.278643 & 7.400178 & 5.606158 & 8 & -1.602386 & 12.613169 & 4.220258 \\
8 & -5.633168 & 9.153631 & 8.599577 & 6 & -2.878457 & 13.014925 & 3.715376 \\
6 & -7.592471 & 7.057186 & 5.169539 & 1 & -2.743078 & 14.010750 & 3.266157 \\
1 & -7.831978 & 7.516162 & 4.186173 & 1 & -3.236231 & 12.319323 & 2.929879 \\
1 & -7.619479 & 5.962924 & 5.067183 & 1 & -3.640278 & 13.063857 & 4.518156 \\
1 & -8.362879 & 7.386508 & 5.899100 & 8 & -3.750104 & 11.921647 & 6.830285 \\
6 & -6.697935 & 8.273929 & 8.974332 & 6 & -4.789825 & 12.744135 & 7.357909 \\
1 & -7.202444 & 7.833684 & 8.091005 & 1 & -5.741905 & 12.223100 & 7.168445 \\
1 & -6.332176 & 7.445402 & 9.616862 & 1 & -4.671847 & 12.900918 & 8.451565 \\
1 & -7.423287 & 8.876698 & 9.544628 & 1 & -4.814032 & 13.738321 & 6.862418 \\
& & & & & & \\
\hline
\end{tabular}




\section{B-Int3b}

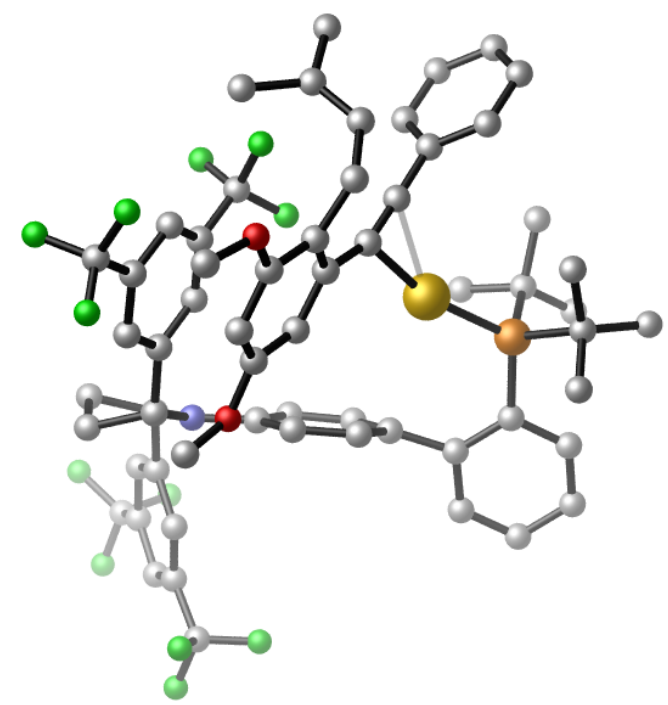

$E=-4241.19177511$ Hartrees

$G=-4240.251004$ Hartrees
B-Int3d

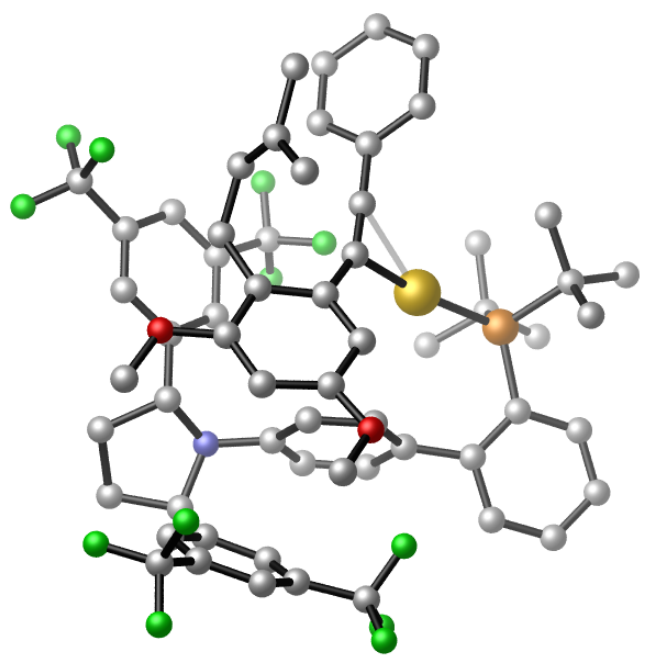

$E=-4241.19076567$ Hartrees

$G=-4240.247438$ Hartrees

$\begin{array}{cccccccc}79 & -1.421859 & 5.944974 & 5.988516 & 79 & -1.687467 & 6.815668 & 6.457709 \\ 6 & -4.587550 & 4.387154 & 6.867345 & 6 & -4.190149 & 4.313580 & 6.444116 \\ 6 & -5.431648 & 3.878531 & 7.886000 & 6 & -5.139560 & 3.567244 & 7.183343 \\ 6 & -6.798150 & 3.658314 & 7.683517 & 6 & -6.344681 & 3.131510 & 6.618816 \\ 6 & -7.359395 & 3.934188 & 6.428736 & 6 & -6.631739 & 3.438523 & 5.281227 \\ 6 & -6.539080 & 4.422974 & 5.406481 & 6 & -5.688165 & 4.138533 & 4.519611 \\ 6 & -5.153685 & 4.663746 & 5.591053 & 6 & -4.458078 & 4.572608 & 5.067567 \\ 6 & -4.456148 & 5.160187 & 4.364695 & 6 & -3.506938 & 5.175084 & 4.084204 \\ 6 & -4.381758 & 4.307113 & 3.242241 & 6 & -2.902427 & 4.321127 & 3.135418 \\ 6 & -4.015752 & 4.776989 & 1.978519 & 6 & -2.085774 & 4.835646 & 2.126029 \\ 6 & -3.735284 & 6.155108 & 1.774214 & 6 & -1.874200 & 6.228254 & 2.011982 \\ 6 & -3.790416 & 7.016044 & 2.903040 & 6 & -2.475879 & 7.088506 & 2.950859 \\ 6 & -4.134314 & 6.522221 & 4.169622 & 6 & -3.286446 & 6.562736 & 3.972795 \\ 6 & -2.935365 & 7.996145 & 0.241670 & 6 & -0.356800 & 7.925685 & 0.921565 \\ 6 & -3.102050 & 8.111764 & -1.292072 & 6 & -0.025727 & 8.214921 & -0.581485 \\ 6 & -2.771231 & 6.688436 & -1.766396 & 6 & -0.853863 & 7.188694 & -1.399292 \\ 6 & -3.449726 & 5.792245 & -0.693893 & 6 & -1.902359 & 6.649251 & -0.415286 \\ 6 & -2.108090 & 2.742839 & 7.055797 & 6 & 0.880600 & 7.806172 & 1.805464 \\ 6 & -2.607446 & 5.214744 & 9.048127 & 6 & 1.039407 & 6.786742 & 2.759139 \\ 15 & -2.784363 & 4.523250 & 7.248749 & 6 & 2.166834 & 6.757839 & 3.599010\end{array}$




\begin{tabular}{|c|c|c|c|c|c|c|c|}
\hline & -3.440342 & 6.642365 & 0.506351 & 6 & 3.173020 & 7.725822 & 3.489626 \\
\hline & -3.555698 & 8.752222 & 0.758683 & 6 & 3.025888 & 8.734144 & 2.522942 \\
\hline & -2.447028 & 8.880758 & -1.731214 & 6 & 1.894237 & 8.779557 & 1.696401 \\
\hline & -4.150391 & 8.368374 & -1.526309 & 6 & 2.241269 & 5.663318 & 4.635289 \\
\hline & -1.679568 & 6.522943 & -1.741602 & 6 & 4.030805 & 9.857890 & 2.469353 \\
\hline & -3.134260 & 6.465301 & -2.781723 & 6 & -3.197354 & 7.457052 & -0.290149 \\
\hline & -2.839504 & 4.881337 & -0.531248 & 6 & -4.278940 & 6.880305 & 0.406383 \\
\hline & -5.014036 & 3.628786 & 8.860897 & 6 & -5.439966 & 7.618213 & 0.678773 \\
\hline & -7.411663 & 3.267300 & 8.501486 & 6 & -5.574717 & 8.931919 & 0.211448 \\
\hline & -8.426044 & 3.768678 & 6.244285 & 6 & -4.508353 & 9.508124 & -0.498451 \\
\hline & -6.967732 & 4.640872 & 4.422513 & 6 & -3.325668 & 8.787102 & -0.733054 \\
\hline & -4.654043 & 3.251570 & 3.354190 & 6 & -6.503363 & 7.005520 & 1.557950 \\
\hline & -3.995490 & 4.074480 & 1.141795 & 6 & -4.664841 & 10.919932 & -1.008076 \\
\hline & -4.213853 & 7.222452 & 5.009695 & 6 & -1.449860 & 3.411360 & 7.278230 \\
\hline & -3.604974 & 8.086598 & 2.785125 & 6 & -3.162193 & 5.414202 & 9.114328 \\
\hline & 0.169594 & 7.425736 & 5.443813 & 15 & -2.660348 & 4.880598 & 7.323965 \\
\hline & -0.054118 & 8.754677 & 4.924852 & 7 & -1.137951 & 6.676244 & 0.875910 \\
\hline & 0.846610 & 9.813187 & 5.224970 & 9 & 3.400100 & 5.686028 & 5.344544 \\
\hline & -1.198200 & 8.949434 & 4.127170 & 9 & 2.106365 & 4.426649 & 4.082461 \\
\hline & 0.563601 & 11.066473 & 4.618635 & 9 & 1.212705 & 5.783630 & 5.549942 \\
\hline 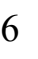 & -1.476610 & 10.215260 & 3.600143 & 9 & 3.669687 & 10.870309 & 3.322986 \\
\hline & -1.849791 & 8.105413 & 3.902747 & 9 & 4.125422 & 10.405897 & 1.226535 \\
\hline 6 & -0.587917 & 11.279615 & 3.832285 & 9 & 5.275735 & 9.452033 & 2.843893 \\
\hline & -0.775671 & 12.259859 & 3.397015 & 9 & -6.192955 & 7.164799 & 2.881665 \\
\hline & 0.845742 & 6.454709 & 5.879525 & 9 & -6.625650 & 5.658992 & 1.348443 \\
\hline & 1.671052 & 5.413475 & 6.406162 & 9 & -7.726887 & 7.567456 & 1.363008 \\
\hline & 2.112249 & 4.348326 & 5.581995 & 9 & -3.606013 & 11.326060 & -1.761442 \\
\hline & 2.041798 & 5.448822 & 7.775963 & 9 & -5.791394 & 11.067694 & -1.754709 \\
\hline & 2.911641 & 3.337195 & 6.127307 & 9 & -4.772807 & 11.819943 & 0.032249 \\
\hline & 1.814728 & 4.326720 & 4.533149 & 1 & -0.952432 & 8.781445 & 1.314330 \\
\hline & 2.844087 & 4.433147 & 8.306344 & 1 & 1.051042 & 8.092503 & -0.780243 \\
\hline & 3.277602 & 3.375446 & 7.484894 & 1 & -0.283914 & 9.257466 & -0.829050 \\
\hline & 3.902085 & 2.580091 & 7.904623 & 1 & -0.211777 & 6.349901 & -1.712831 \\
\hline & -1.166598 & 5.762214 & 9.222918 & 1 & -1.304857 & 7.618410 & -2.308369 \\
\hline & -0.394806 & 4.999851 & 9.034138 & 1 & -2.166618 & 5.599866 & -0.633111 \\
\hline & -0.969317 & 6.624186 & 8.562034 & 1 & -4.936685 & 3.312479 & 8.224327 \\
\hline
\end{tabular}




\begin{tabular}{|c|c|c|c|c|c|c|c|}
\hline 1 & -1.050239 & 6.108504 & 10.266899 & 1 & -7.052714 & 2.560323 & 7.227726 \\
\hline & -2.844482 & 4.202228 & 10.193051 & 1 & -7.577192 & 3.123647 & 4.827340 \\
\hline & -3.846596 & 3.748524 & 10.199208 & 1 & -5.894369 & 4.372902 & 3.470503 \\
\hline & -2.097134 & 3.392020 & 10.188571 & 1 & -3.083566 & 3.242602 & 3.191183 \\
\hline & -2.735924 & 4.742947 & 11.151456 & 1 & -1.615589 & 4.168987 & 1.395394 \\
\hline & -3.593155 & 6.402615 & 9.147324 & 1 & -3.839990 & 7.250590 & 4.618539 \\
\hline & -3.454869 & 7.108261 & 8.306533 & 1 & -2.365391 & 8.171875 & 2.859930 \\
\hline & -4.646992 & 6.080378 & 9.157172 & 1 & 0.286727 & 6.003823 & 2.865784 \\
\hline & -3.393973 & 6.952800 & 10.085571 & 1 & 1.790171 & 9.599369 & 0.979676 \\
\hline & -0.664364 & 2.678925 & 7.595709 & 1 & 4.053052 & 7.698325 & 4.136300 \\
\hline & -0.014923 & 3.443428 & 7.136856 & 1 & -6.500776 & 9.487917 & 0.379681 \\
\hline & -0.620582 & 2.791837 & 8.691346 & 1 & -2.518032 & 9.266861 & -1.290496 \\
\hline & -0.238748 & 1.689202 & 7.347350 & 1 & -4.215577 & 5.845047 & 0.753044 \\
\hline & -2.993174 & 1.692642 & 7.758782 & 6 & -1.083791 & 8.871321 & 6.001104 \\
\hline & -2.544544 & 0.695646 & 7.592055 & 6 & -1.763596 & 9.670825 & 5.010770 \\
\hline & -3.056701 & 1.848741 & 8.847101 & 6 & -0.969989 & 10.339602 & 4.043064 \\
\hline & -4.015658 & 1.673193 & 7.346600 & 6 & -3.169447 & 9.726381 & 5.001099 \\
\hline & -2.101652 & 2.459843 & 5.534937 & 6 & -1.662433 & 10.946655 & 2.964794 \\
\hline & -1.737245 & 1.428860 & 5.368256 & 6 & -3.823790 & 10.401720 & 3.959240 \\
\hline & -3.112362 & 2.536773 & 5.099537 & 1 & -3.752209 & 9.239750 & 5.787622 \\
\hline & -1.435867 & 3.152328 & 4.989038 & 6 & -3.073167 & 10.977236 & 2.910583 \\
\hline & 3.251410 & 2.514401 & 5.490268 & 1 & -3.580480 & 11.433109 & 2.060809 \\
\hline & 3.129532 & 4.463183 & 9.362575 & 6 & -0.135325 & 8.608403 & 6.788424 \\
\hline & -2.625512 & 10.312696 & 2.854147 & 6 & 0.946720 & 8.450586 & 7.699499 \\
\hline$\theta$ & 1.474075 & 12.061464 & 4.863653 & 6 & 2.248342 & 8.201684 & 7.194513 \\
\hline & 1.690428 & 6.272548 & 8.404005 & 6 & 0.744668 & 8.595007 & 9.096092 \\
\hline 6 & 1.976746 & 9.705788 & 6.245161 & 6 & 3.328427 & 8.100058 & 8.075657 \\
\hline & 2.542405 & 10.652226 & 6.214413 & 1 & 2.390280 & 8.111955 & 6.115873 \\
\hline & 1.505579 & 9.672577 & 7.247521 & 6 & 1.833725 & 8.483156 & 9.968177 \\
\hline & 2.932703 & 8.529174 & 6.145376 & 6 & 3.123259 & 8.235136 & 9.461866 \\
\hline & 2.930483 & 7.848452 & 7.004381 & 1 & 3.971394 & 8.150630 & 10.149148 \\
\hline & 3.805255 & 8.263910 & 5.148105 & 6 & -2.054522 & 6.354284 & 9.660207 \\
\hline & 4.691800 & 7.039506 & 5.201431 & 1 & -1.048348 & 5.906003 & 9.642057 \\
\hline & 5.762033 & 7.312859 & 5.116520 & 1 & -2.018527 & 7.296807 & 9.087762 \\
\hline & 4.548651 & 6.464026 & 6.132542 & 1 & -2.296098 & 6.604775 & 10.710050 \\
\hline & 4.467577 & 6.366506 & 4.351788 & 6 & -3.342567 & 4.266379 & 10.132912 \\
\hline
\end{tabular}




\begin{tabular}{|c|c|c|c|c|c|c|c|}
\hline & 3.983275 & 9.148597 & 3.937195 & 1 & -4.087112 & 3.512231 & 9.836325 \\
\hline & 4.958948 & 9.672411 & 3.983457 & 1 & -2.390949 & 3.749798 & 10.340741 \\
\hline & 4.007697 & 8.540465 & 3.013379 & 1 & -3.691661 & 4.706670 & 11.085340 \\
\hline & 3.194190 & 9.911222 & 3.836160 & 6 & -4.458624 & 6.248954 & 8.978621 \\
\hline & 1.290347 & 13.329541 & 4.214939 & 1 & -4.346719 & 7.038517 & 8.211014 \\
\hline & 1.263147 & 13.214361 & 3.116928 & 1 & -5.337966 & 5.638357 & 8.719724 \\
\hline & 0.367610 & 13.831183 & 4.563692 & 1 & -4.660003 & 6.746595 & 9.945299 \\
\hline & 2.163751 & 13.932538 & 4.502540 & 6 & -0.199416 & 3.767596 & 8.110346 \\
\hline & -2.974676 & 11.603348 & 2.326663 & 1 & 0.260347 & 4.714486 & 7.775898 \\
\hline & -3.140749 & 12.335712 & 3.138738 & 1 & -0.422115 & 3.840509 & 9.187763 \\
\hline & -2.193623 & 11.980810 & 1.642049 & 1 & 0.550091 & 2.964802 & 7.981747 \\
\hline & -3.910283 & 11.449900 & 1.770099 & 6 & -2.093167 & 2.101133 & 7.780384 \\
\hline & -4.862125 & 5.361553 & -1.094097 & 1 & -1.365665 & 1.280220 & 7.638988 \\
\hline & -5.034512 & 4.280246 & -1.976783 & 1 & -2.351860 & 2.131890 & 8.849905 \\
\hline & -5.995991 & 6.040653 & -0.621035 & 1 & -3.000729 & 1.844783 & 7.207165 \\
\hline & -6.321073 & 3.893467 & -2.383452 & 6 & -1.052556 & 3.233508 & 5.794786 \\
\hline & -4.160732 & 3.739355 & -2.355818 & 1 & -0.288820 & 2.436269 & 5.728867 \\
\hline & -7.281232 & 5.644179 & -1.025499 & 1 & -1.915501 & 2.923614 & 5.184260 \\
\hline & -5.873453 & 6.877688 & 0.072227 & 1 & -0.625459 & 4.151548 & 5.358259 \\
\hline & -7.455889 & 4.569524 & -1.908264 & 1 & 4.331999 & 7.911360 & 7.681541 \\
\hline & -8.456125 & 4.266935 & -2.225880 & 1 & 1.678637 & 8.596020 & 11.045849 \\
\hline & -1.483147 & 8.178201 & 0.691658 & 8 & -5.188281 & 10.414781 & 4.032304 \\
\hline & -0.863279 & 9.435218 & 0.577615 & 8 & -0.865460 & 11.482998 & 1.985586 \\
\hline & -0.753296 & 7.110737 & 1.231887 & 1 & -0.260147 & 8.798819 & 9.476502 \\
\hline & 0.450114 & 9.621868 & 1.032851 & 6 & 0.537465 & 10.368713 & 4.144626 \\
\hline & -1.416975 & 10.279504 & 0.156170 & 1 & 0.894050 & 9.321797 & 4.090629 \\
\hline & 0.562519 & 7.301880 & 1.679928 & 1 & 0.959337 & 10.861298 & 3.252570 \\
\hline & -1.220207 & 6.128559 & 1.331885 & 6 & 1.176184 & 10.945805 & 5.396206 \\
\hline & 1.175445 & 8.556213 & 1.589287 & 1 & 2.207049 & 10.587109 & 5.522843 \\
\hline & 2.189177 & 8.712750 & 1.958225 & 6 & 0.692449 & 11.752672 & 6.368562 \\
\hline & -8.480866 & 6.346731 & -0.434897 & 6 & 1.534499 & 12.028945 & 7.597118 \\
\hline & -6.482254 & 2.691690 & -3.285011 & 1 & 1.018005 & 11.651402 & 8.503070 \\
\hline & -9.597592 & 6.195979 & -1.200459 & 1 & 2.519274 & 11.533211 & 7.547867 \\
\hline & -8.775714 & 5.857268 & 0.807586 & 1 & 1.690522 & 13.114512 & 7.751873 \\
\hline 9 & -8.258695 & 7.685512 & -0.286617 & 6 & -0.681200 & 12.388529 & 6.394264 \\
\hline & -7.656000 & 2.722712 & -3.976423 & 1 & -0.612853 & 13.427350 & 6.768928 \\
\hline
\end{tabular}




$\begin{array}{rrrrrrrr}9 & -6.471166 & 1.528701 & -2.565948 & 1 & -1.168501 & 12.407363 & 5.408312 \\ 9 & -5.468838 & 2.602258 & -4.194379 & 1 & -1.352311 & 11.843988 & 7.088638 \\ 6 & 1.318165 & 6.116555 & 2.218656 & 6 & -1.498500 & 12.064280 & 0.836373 \\ 6 & 1.124290 & 10.961270 & 0.866252 & 1 & -2.099086 & 11.316788 & 0.289128 \\ 9 & 1.900902 & 5.384418 & 1.227113 & 1 & -2.142983 & 12.919493 & 1.112641 \\ 9 & 0.482013 & 5.255020 & 2.894448 & 1 & -0.676726 & 12.414310 & 0.195403 \\ 9 & 2.306445 & 6.478535 & 3.084443 & 6 & -5.900871 & 11.245596 & 3.102204 \\ 9 & 0.234253 & 12.001994 & 0.920673 & 1 & -5.583906 & 12.302314 & 3.187977 \\ 9 & 1.762697 & 11.057204 & -0.335309 & 1 & -5.756715 & 10.908782 & 2.062145 \\ 9 & 2.064401 & 11.186518 & 1.832478 & 1 & -6.961710 & 11.150627 & 3.374938\end{array}$

\section{B-Int4b}

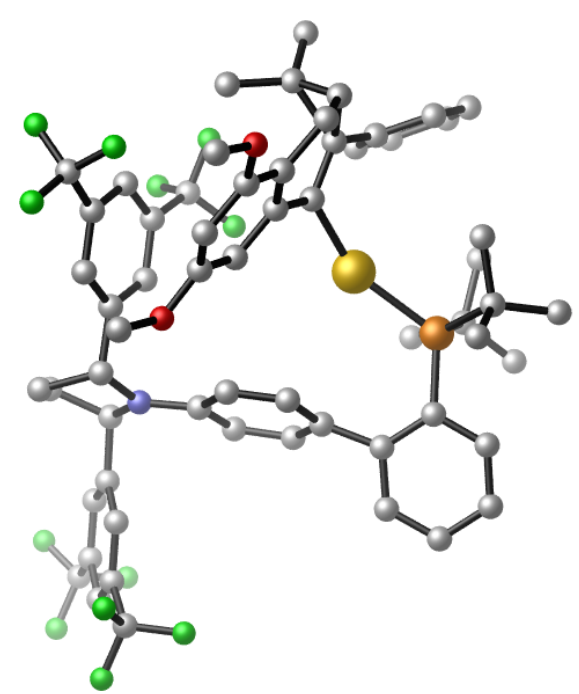

$\mathrm{E}=-4241.21208077$ Hartrees

$G=-4240.266842$ Hartrees

\section{B-Int4d}

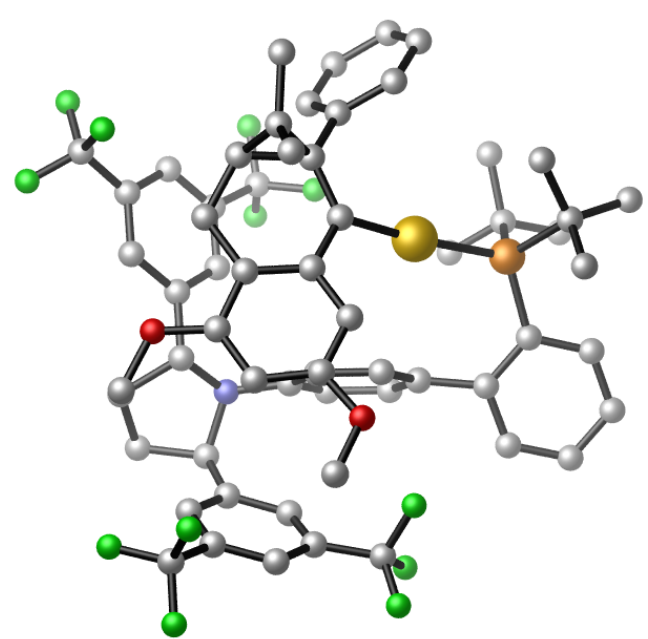

$\mathrm{E}=-4241.21640352$ Hartrees

$G=-4240.268278$ Hartrees

$\begin{array}{llllllll}79 & -1.071591 & 5.956515 & 5.676899 & 79 & -1.768928 & 7.254682 & 6.580083 \\ 6 & -4.372737 & 4.685905 & 6.842357 & 6 & -4.147384 & 4.542593 & 6.537561 \\ 6 & -5.191366 & 4.328399 & 7.942127 & 6 & -5.096323 & 3.788151 & 7.269517 \\ 6 & -6.563353 & 4.088815 & 7.809363 & 6 & -6.219279 & 3.208051 & 6.666307 \\ 6 & -7.157058 & 4.183505 & 6.542499 & 6 & -6.421796 & 3.369098 & 5.288826 \\ 6 & -6.366634 & 4.533927 & 5.443285 & 6 & -5.476300 & 4.075910 & 4.536588 \\ 6 & -4.978104 & 4.802007 & 5.559675 & 6 & -4.328515 & 4.658985 & 5.126053 \\ 6 & -4.317429 & 5.197362 & 4.281718 & 6 & -3.373036 & 5.271777 & 4.151287\end{array}$




\begin{tabular}{|c|c|c|c|c|c|c|c|}
\hline 6 & -4.373299 & 4.314756 & 3.180802 & 6 & -2.694147 & 4.420121 & 3.250479 \\
\hline 6 & -4.044325 & 4.725689 & 1.886732 & 6 & -1.884766 & 4.943166 & 2.238416 \\
\hline 6 & -3.672979 & 6.073392 & 1.634181 & 6 & -1.750513 & 6.340749 & 2.076355 \\
\hline 6 & -3.583329 & 6.958499 & 2.741776 & 6 & -2.417535 & 7.195879 & 2.973696 \\
\hline 6 & -3.890672 & 6.521667 & 4.036088 & 6 & -3.225563 & 6.662543 & 3.991470 \\
\hline 6 & -2.874028 & 7.840580 & 0.033353 & 6 & -0.275184 & 8.057485 & 0.965441 \\
\hline 6 & -3.050481 & 7.922041 & -1.502552 & 6 & -0.042124 & 8.415115 & -0.541410 \\
\hline 6 & -2.791681 & 6.472361 & -1.941580 & 6 & -0.734532 & 7.287068 & -1.345143 \\
\hline 6 & -3.494943 & 5.637379 & -0.835664 & 6 & -1.764773 & 6.689578 & -0.374311 \\
\hline 6 & -2.022972 & 2.924450 & 7.103209 & 6 & 1.021477 & 7.908877 & 1.756054 \\
\hline 6 & -2.249735 & 5.612419 & 8.835648 & 6 & 1.333548 & 6.748846 & 2.481482 \\
\hline & -2.541831 & 4.765083 & 7.117016 & 6 & 2.522246 & 6.670896 & 3.227699 \\
\hline 7 & -3.429025 & 6.517847 & 0.339789 & 6 & 3.429324 & 7.737397 & 3.257480 \\
\hline 1 & -3.459395 & 8.635395 & 0.533995 & 6 & 3.125033 & 8.893194 & 2.520987 \\
\hline 1 & -2.365310 & 8.649359 & -1.966528 & 6 & 1.936654 & 8.980131 & 1.778338 \\
\hline 1 & -4.088736 & 8.220577 & -1.732828 & 6 & 2.773650 & 5.408071 & 4.014706 \\
\hline 1 & -1.708664 & 6.256405 & -1.928074 & 6 & 4.018282 & 10.104304 & 2.616151 \\
\hline 1 & -3.178922 & 6.240330 & -2.946013 & 6 & -3.122081 & 7.395282 & -0.295067 \\
\hline 1 & -2.924386 & 4.704727 & -0.652163 & 6 & -4.167878 & 6.752320 & 0.398390 \\
\hline 1 & -4.745932 & 4.204728 & 8.928352 & 6 & -5.387646 & 7.403183 & 0.634652 \\
\hline 1 & -7.155190 & 3.817587 & 8.689653 & 6 & -5.612622 & 8.696057 & 0.144304 \\
\hline 1 & -8.227316 & 3.992472 & 6.410178 & 6 & -4.580527 & 9.338610 & -0.558892 \\
\hline 1 & -6.824858 & 4.630179 & 4.453092 & 6 & -3.347427 & 8.700227 & -0.771945 \\
\hline 1 & -4.715286 & 3.285505 & 3.338849 & 6 & -6.418008 & 6.730060 & 1.508986 \\
\hline 1 & -4.126980 & 4.007575 & 1.066950 & 6 & -4.815951 & 10.744807 & -1.052287 \\
\hline 1 & -3.854864 & 7.240115 & 4.863207 & 6 & -1.417025 & 3.884719 & 7.553884 \\
\hline 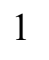 & -3.312908 & 8.006267 & 2.590578 & 6 & -3.353432 & 5.826885 & 9.210854 \\
\hline 6 & 0.456591 & 7.276494 & 5.331412 & 15 & -2.713802 & 5.277415 & 7.469023 \\
\hline 6 & 0.059361 & 8.636396 & 4.989655 & 7 & -1.035308 & 6.797727 & 0.929069 \\
\hline 6 & 0.623421 & 9.748432 & 5.676354 & 9 & 3.952575 & 5.445057 & 4.700502 \\
\hline 6 & -0.934552 & 8.828169 & 4.000119 & 9 & 2.798805 & 4.308209 & 3.210943 \\
\hline 6 & 0.157487 & 11.042680 & 5.353189 & 9 & 1.777487 & 5.196505 & 4.932536 \\
\hline & -1.367035 & 10.120945 & 3.684674 & 9 & 3.554369 & 10.974260 & 3.578461 \\
\hline 1 & -1.336989 & 7.965343 & 3.467078 & 9 & 4.058126 & 10.805332 & 1.450145 \\
\hline & -0.825698 & 11.235367 & 4.360855 & 9 & 5.294400 & 9.784246 & 2.958093 \\
\hline & -1.172879 & 12.241604 & 4.126483 & 9 & -6.156395 & 6.958050 & 2.832922 \\
\hline
\end{tabular}




\begin{tabular}{|c|c|c|c|c|c|c|c|}
\hline & 1.755302 & 7.020108 & 5.879452 & 9 & -6.422478 & 5.372812 & 1.340340 \\
\hline & 2.079117 & 5.628357 & 6.360529 & 9 & -7.678974 & 7.181050 & 1.266907 \\
\hline & 2.142242 & 4.575940 & 5.421776 & 9 & -3.787097 & 11.216148 & -1.808421 \\
\hline & 2.375376 & 5.350372 & 7.709241 & 9 & -5.957184 & 10.847532 & -1.782866 \\
\hline & 2.519324 & 3.284262 & 5.817993 & 9 & -4.957103 & 11.621848 & 0.005757 \\
\hline 1 & 1.907357 & 4.785136 & 4.376309 & 1 & -0.845499 & 8.888873 & 1.436266 \\
\hline 6 & 2.745095 & 4.055043 & 8.108990 & 1 & 1.031900 & 8.473773 & -0.779456 \\
\hline 6 & 2.831477 & 3.020614 & 7.162922 & 1 & -0.473813 & 9.405566 & -0.759194 \\
\hline 1 & 3.128727 & 2.013450 & 7.473606 & 1 & -0.004192 & 6.500895 & -1.596843 \\
\hline 6 & -0.777116 & 6.094819 & 8.902778 & 1 & -1.186563 & 7.638566 & -2.286888 \\
\hline 1 & -0.054313 & 5.277614 & 8.756206 & 1 & -1.942757 & 5.619107 & -0.578533 \\
\hline & -0.560333 & 6.874694 & 8.152285 & 1 & -4.958555 & 3.641553 & 8.341231 \\
\hline & -0.599290 & 6.532004 & 9.903914 & 1 & -6.928272 & 2.638135 & 7.275581 \\
\hline & -2.483125 & 4.728342 & 10.082966 & 1 & -7.301911 & 2.939399 & 4.798842 \\
\hline & -3.507588 & 4.340316 & 10.183159 & 1 & -5.620704 & 4.204014 & 3.459421 \\
\hline & -1.786859 & 3.874716 & 10.118811 & 1 & -2.811745 & 3.335253 & 3.345468 \\
\hline & -2.291098 & 5.346119 & 10.980195 & 1 & -1.366023 & 4.277618 & 1.539834 \\
\hline & -3.165904 & 6.858537 & 8.864668 & 1 & -3.817619 & 7.339600 & 4.611258 \\
\hline 1 & -3.013138 & 7.485455 & 7.966238 & 1 & -2.364296 & 8.279999 & 2.847223 \\
\hline 1 & -4.235507 & 6.600376 & 8.927818 & 1 & 0.651112 & 5.897014 & 2.471839 \\
\hline 1 & -2.907919 & 7.469264 & 9.749937 & 1 & 1.717921 & 9.894981 & 1.217176 \\
\hline U & -0.562801 & 2.825084 & 7.587149 & 1 & 4.349436 & 7.673906 & 3.842258 \\
\hline 1 & 0.099841 & 3.512888 & 7.036635 & 1 & -6.578434 & 9.186084 & 0.292228 \\
\hline 1 & -0.460294 & 3.033300 & 8.665210 & 1 & -2.574849 & 9.227571 & -1.336176 \\
\hline 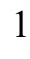 & -0.192401 & 1.798138 & 7.411067 & 1 & -4.029229 & 5.733516 & 0.771272 \\
\hline 6 & -2.937953 & 2.006750 & 7.938909 & 6 & -1.030654 & 9.096417 & 6.054628 \\
\hline & -2.557261 & 0.971147 & 7.858100 & 6 & -1.791772 & 9.874388 & 5.090202 \\
\hline 1 & -2.949262 & 2.271617 & 9.008339 & 6 & -1.087577 & 10.587063 & 4.079134 \\
\hline 1 & -3.976378 & 2.012088 & 7.568107 & 6 & -3.205178 & 9.797463 & 5.060762 \\
\hline 6 & -2.098554 & 2.495294 & 5.618107 & 6 & -1.817399 & 11.038551 & 2.953512 \\
\hline 1 & -1.813570 & 1.429122 & 5.538591 & 6 & -3.917648 & 10.366698 & 3.999055 \\
\hline 1 & -3.117846 & 2.606225 & 5.209714 & 1 & -3.745840 & 9.289592 & 5.864841 \\
\hline 1 & -1.407152 & 3.082798 & 4.987235 & 6 & -3.221466 & 10.937663 & 2.903206 \\
\hline 1 & 2.571152 & 2.482882 & 5.072933 & 1 & -3.767303 & 11.276373 & 2.022162 \\
\hline & 2.966164 & 3.857428 & 9.163421 & 6 & 0.264613 & 9.505972 & 6.484487 \\
\hline & -2.342346 & 10.212775 & 2.725676 & 6 & 1.069197 & 8.531908 & 7.303058 \\
\hline
\end{tabular}




\begin{tabular}{|c|c|c|c|c|c|c|c|}
\hline 8 & 0.725165 & 12.063672 & 6.063494 & 6 & 2.106847 & 7.781981 & 6.723996 \\
\hline 1 & 2.314727 & 6.147185 & 8.457952 & 6 & 0.760616 & 8.339975 & 8.666835 \\
\hline 6 & 1.661705 & 9.541233 & 6.746949 & 6 & 2.830402 & 6.854288 & 7.491916 \\
\hline 1 & 2.364347 & 10.395878 & 6.748529 & 1 & 2.334967 & 7.915123 & 5.662287 \\
\hline 1 & 1.179650 & 9.549376 & 7.744438 & 6 & 1.487832 & 7.422584 & 9.438107 \\
\hline 6 & 2.463209 & 8.264440 & 6.589907 & 6 & 2.527991 & 6.678767 & 8.851647 \\
\hline 1 & 2.980761 & 7.951887 & 7.504675 & 1 & 3.092501 & 5.956976 & 9.451525 \\
\hline 6 & 3.102603 & 7.862028 & 5.331338 & 6 & -2.344358 & 6.859224 & 9.780783 \\
\hline 6 & 4.375692 & 7.028737 & 5.399363 & 1 & -1.313730 & 6.474829 & 9.834013 \\
\hline 1 & 5.233245 & 7.712262 & 5.257231 & 1 & -2.325655 & 7.783470 & 9.178250 \\
\hline 1 & 4.506529 & 6.505681 & 6.358308 & 1 & -2.663108 & 7.125440 & 10.806394 \\
\hline 1 & 4.394834 & 6.282292 & 4.586481 & 6 & -3.506195 & 4.708374 & 10.265804 \\
\hline 6 & 3.056947 & 8.721662 & 4.081368 & 1 & -4.157465 & 3.876725 & 9.957205 \\
\hline 1 & 3.656079 & 9.632288 & 4.268022 & 1 & -2.528554 & 4.288306 & 10.555390 \\
\hline 1 & 3.519479 & 8.175593 & 3.245417 & 1 & -3.955242 & 5.150928 & 11.174753 \\
\hline 1 & 2.056437 & 9.052663 & 3.777156 & 6 & -4.695989 & 6.562528 & 8.979984 \\
\hline 6 & 0.363609 & 13.411990 & 5.720123 & 1 & -4.601055 & 7.328447 & 8.187222 \\
\hline 1 & 0.599812 & 13.632089 & 4.662055 & 1 & -5.517742 & 5.883045 & 8.703948 \\
\hline 1 & -0.709498 & 13.601547 & 5.910362 & 1 & -4.980815 & 7.079329 & 9.915465 \\
\hline 1 & 0.970407 & 14.053720 & 6.374604 & 6 & -0.221203 & 4.376912 & 8.395146 \\
\hline 6 & -2.717488 & 11.522053 & 2.264680 & 1 & 0.177890 & 5.336422 & 8.022672 \\
\hline 1 & -3.180559 & 12.119129 & 3.072063 & 1 & -0.477025 & 4.498320 & 9.461458 \\
\hline 1 & -1.843517 & 12.058178 & 1.850653 & 1 & 0.590939 & 3.628299 & 8.333755 \\
\hline 1 & -3.457147 & 11.350014 & 1.469543 & 6 & -1.973874 & 2.555137 & 8.102332 \\
\hline 6 & -4.928939 & 5.256287 & -1.207988 & 1 & -1.196009 & 1.776860 & 7.987553 \\
\hline 6 & -5.153704 & 4.178433 & -2.083161 & 1 & -2.231614 & 2.605703 & 9.171622 \\
\hline 6 & -6.029619 & 5.975104 & -0.716525 & 1 & -2.864417 & 2.222846 & 7.541126 \\
\hline 6 & -6.459621 & 3.834125 & -2.464903 & 6 & -0.953562 & 3.663979 & 6.094737 \\
\hline 1 & -4.305674 & 3.607181 & -2.475909 & 1 & -0.106193 & 2.953156 & 6.094858 \\
\hline 6 & -7.334819 & 5.621758 & -1.097206 & 1 & -1.757801 & 3.229469 & 5.479220 \\
\hline 1 & -5.863781 & 6.808795 & -0.028102 & 1 & -0.614478 & 4.597398 & 5.612920 \\
\hline 6 & -7.561818 & 4.550701 & -1.972565 & 1 & 3.617423 & 6.261433 & 7.017283 \\
\hline 1 & -8.577297 & 4.281471 & -2.271261 & 1 & 1.239035 & 7.283411 & 10.495729 \\
\hline 6 & -1.410934 & 7.974127 & 0.456440 & 8 & -5.276108 & 10.269577 & 4.076710 \\
\hline 6 & -0.797627 & 9.240463 & 0.448000 & 8 & -1.049664 & 11.557813 & 1.944979 \\
\hline 6 & -0.664441 & 6.858386 & 0.856730 & 1 & -0.058120 & 8.913963 & 9.113382 \\
\hline
\end{tabular}




$\begin{array}{lrrrrrrr}6 & 0.544298 & 9.376009 & 0.820098 & 6 & 0.396352 & 10.790288 & 4.156995 \\ 1 & -1.374626 & 10.122540 & 0.158978 & 1 & 0.899238 & 10.150112 & 3.413289 \\ 6 & 0.687252 & 6.998530 & 1.216451 & 1 & 0.639151 & 11.827185 & 3.852149 \\ 1 & -1.138612 & 5.875122 & 0.908855 & 6 & 1.016857 & 10.532429 & 5.513675 \\ 6 & 1.306879 & 8.250728 & 1.177285 & 1 & 2.088050 & 10.301859 & 5.461899 \\ 1 & 2.361056 & 8.361767 & 1.433112 & 6 & 0.566339 & 11.117625 & 6.783395 \\ 6 & -8.499136 & 6.368336 & -0.490386 & 6 & 1.621391 & 11.323695 & 7.863073 \\ 6 & -6.676351 & 2.635690 & -3.359175 & 1 & 1.194278 & 11.192036 & 8.871846 \\ 9 & -9.635154 & 6.246737 & -1.232625 & 1 & 2.482314 & 10.645945 & 7.757735 \\ 9 & -8.785186 & 5.901097 & 0.762429 & 1 & 1.988449 & 12.363325 & 7.779895 \\ 9 & -8.231087 & 7.700763 & -0.360561 & 6 & -0.587383 & 12.097055 & 6.907575 \\ 9 & -7.862932 & 2.700332 & -4.025921 & 1 & -0.188621 & 13.113913 & 6.735755 \\ 9 & -6.684476 & 1.474474 & -2.637373 & 1 & -1.401271 & 11.939118 & 6.188086 \\ 9 & -5.685393 & 2.514026 & -4.289550 & 1 & -1.005483 & 12.065314 & 7.928345 \\ 6 & 1.458289 & 5.767963 & 1.618168 & 6 & -1.708838 & 11.931791 & 0.723248 \\ 6 & 1.191147 & 10.733963 & 0.927122 & 1 & -2.210768 & 11.059849 & 0.268508 \\ 9 & 1.905690 & 5.061639 & 0.543134 & 1 & -2.448570 & 12.736764 & 0.885372 \\ 9 & 0.676359 & 4.911723 & 2.353077 & 1 & -0.911940 & 12.289292 & 0.056201 \\ 9 & 2.551334 & 6.068013 & 2.384315 & 6 & -6.057550 & 10.936303 & 3.071360 \\ 9 & 0.388213 & 11.736575 & 0.467918 & 1 & -5.842568 & 12.021366 & 3.053977 \\ 9 & 2.365314 & 10.795078 & 0.237850 & 1 & -5.873552 & 10.512440 & 2.070031 \\ 9 & 1.489480 & 11.027926 & 2.233446 & 1 & -7.106224 & 10.767992 & 3.354470\end{array}$


B-TSInt1b-2b

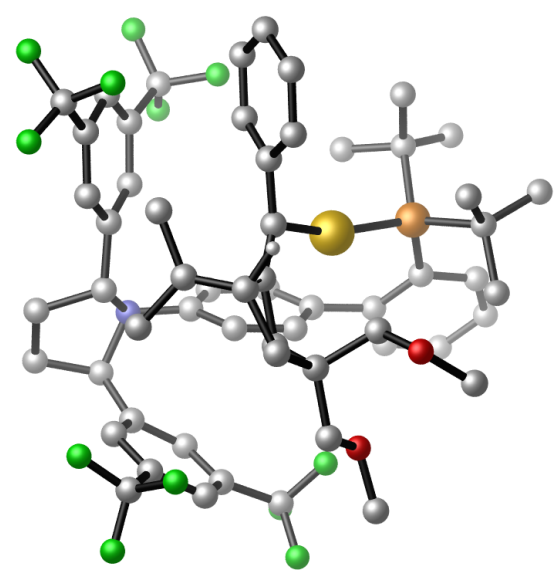

$E=-4167.35742293$ Hartrees

$G=-4166.380281$ Hartrees
B-TSInt1d-2d

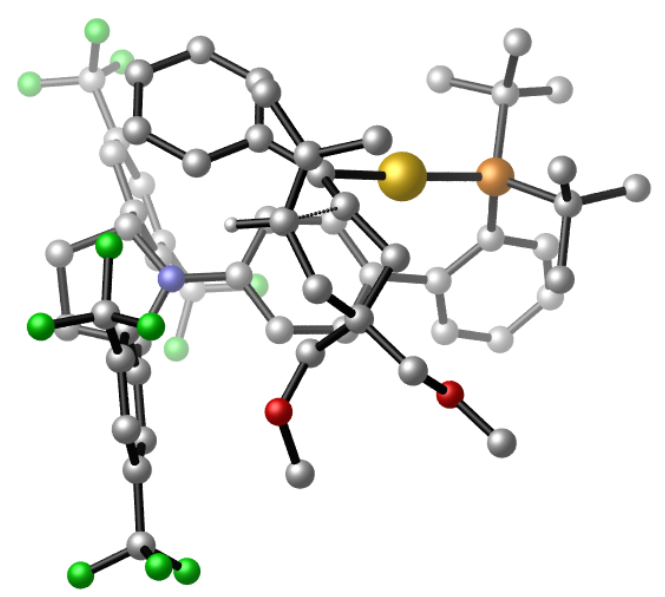

$E=-4167.36280837$ Hartrees

$G=-4166.391606$ Hartrees

$\begin{array}{lrrr}79 & -1.393523 & 7.182534 & 7.911018 \\ 6 & -4.446641 & 5.492664 & 7.741996 \\ 6 & -5.638686 & 4.937729 & 8.270595 \\ 6 & -6.751090 & 4.665795 & 7.465123 \\ 6 & -6.695748 & 4.960484 & 6.094524 \\ 6 & -5.518687 & 5.485233 & 5.549355 \\ 6 & -4.370766 & 5.747958 & 6.337689 \\ 6 & -3.171249 & 6.221216 & 5.590381 \\ 6 & -1.964364 & 5.479315 & 5.578428 \\ 6 & -0.913930 & 5.793948 & 4.711488 \\ 6 & -1.039618 & 6.857419 & 3.777277 \\ 6 & -2.207151 & 7.664118 & 3.851965 \\ 6 & -3.247006 & 7.340908 & 4.732109 \\ 6 & -0.409635 & 7.828622 & 1.561182 \\ 6 & 0.585873 & 7.265359 & 0.505577 \\ 6 & 1.755325 & 6.736423 & 1.352577 \\ 6 & 1.035686 & 6.127118 & 2.578277 \\ 6 & -0.262835 & 9.337843 & 1.723883 \\ 6 & -1.155583 & 10.205879 & 1.076986 \\ 6 & -0.970532 & 11.598057 & 1.145155 \\ 6 & 0.100620 & 12.140613 & 1.863235\end{array}$




\begin{tabular}{|c|c|c|c|c|c|c|c|}
\hline 6 & 3.007082 & 7.401673 & 3.995692 & 6 & 0.996010 & 11.270095 & 2.506851 \\
\hline 6 & 1.847166 & 7.003750 & 3.307353 & 6 & 0.815485 & 9.883216 & 2.443618 \\
\hline 6 & 2.708646 & 11.178765 & 3.939939 & 6 & -1.921959 & 12.501813 & 0.398940 \\
\hline 6 & 3.859896 & 6.359526 & 4.674087 & 6 & 2.178776 & 11.868985 & 3.224203 \\
\hline 6 & -2.862587 & 6.364773 & 0.996906 & 6 & 0.620106 & 4.678012 & 2.314632 \\
\hline 6 & -3.675068 & 5.381568 & 1.598168 & 6 & 1.551828 & 3.651363 & 2.552547 \\
\hline 6 & -4.915732 & 5.718160 & 2.164281 & 6 & 1.213278 & 2.312048 & 2.305624 \\
\hline 6 & -5.391097 & 7.035572 & 2.110001 & 6 & -0.064067 & 1.972526 & 1.831493 \\
\hline 6 & -4.608791 & 8.009659 & 1.470043 & 6 & -0.992613 & 2.996452 & 1.600266 \\
\hline 6 & -3.355816 & 7.683238 & 0.927515 & 6 & -0.655026 & 4.339435 & 1.834685 \\
\hline 6 & -5.698593 & 4.681002 & 2.932922 & 6 & 2.249825 & 1.230370 & 2.500190 \\
\hline 6 & -5.164973 & 9.404185 & 1.321975 & 6 & -2.405154 & 2.666988 & 1.180195 \\
\hline 6 & 0.545235 & 4.111254 & 8.730153 & 6 & -2.492122 & 4.535428 & 9.871989 \\
\hline 6 & -2.300598 & 5.077471 & 9.896749 & 6 & -4.042043 & 7.360786 & 10.056601 \\
\hline 15 & -1.262864 & 4.582568 & 8.344856 & 15 & -3.157862 & 6.063069 & 8.937765 \\
\hline 7 & -0.520050 & 6.174863 & 1.839505 & 7 & -0.083245 & 7.062875 & 2.784375 \\
\hline 9 & 4.026026 & 11.469969 & 3.750545 & 9 & -1.928361 & 13.771736 & 0.901766 \\
\hline 9 & 2.411190 & 11.522613 & 5.231353 & 9 & -3.204447 & 12.035119 & 0.451526 \\
\hline 9 & 1.980423 & 12.001880 & 3.131180 & 9 & -1.590342 & 12.597258 & -0.921856 \\
\hline 9 & 3.440724 & 6.177650 & 5.975255 & 9 & 1.799115 & 12.845056 & 4.103823 \\
\hline 9 & 5.170505 & 6.718821 & 4.735681 & 9 & 3.054442 & 12.446273 & 2.356108 \\
\hline 9 & 3.784211 & 5.145023 & 4.066622 & 9 & 2.884001 & 10.936885 & 3.942830 \\
\hline 9 & -7.042766 & 4.896610 & 2.858186 & 9 & 3.135674 & 1.547159 & 3.488679 \\
\hline 9 & -5.373900 & 4.710055 & 4.266966 & 9 & 2.979537 & 1.032667 & 1.361506 \\
\hline 9 & -5.451954 & 3.417293 & 2.494536 & 9 & 1.683597 & 0.032866 & 2.822906 \\
\hline 9 & -4.237070 & 10.294566 & 0.874910 & 9 & -3.254430 & 2.685573 & 2.252404 \\
\hline 9 & -6.217762 & 9.439826 & 0.461749 & 9 & -2.887578 & 3.575826 & 0.282791 \\
\hline 9 & -5.633931 & 9.887775 & 2.525282 & 9 & -2.502284 & 1.433566 & 0.611307 \\
\hline 1 & -1.143151 & 8.148312 & 2.433589 & 1 & -1.452991 & 7.614760 & 1.253541 \\
\hline 1 & 0.967133 & 8.200740 & 0.261185 & 1 & 0.886651 & 8.029109 & -0.228410 \\
\hline 1 & -0.574326 & 9.072633 & 0.350481 & 1 & 0.106358 & 6.433948 & -0.040102 \\
\hline 1 & 0.040006 & 6.409333 & -0.909139 & 1 & 2.416208 & 7.558063 & 1.680649 \\
\hline 1 & -1.454167 & 7.347656 & -1.118994 & 1 & 2.372808 & 5.993605 & 0.823011 \\
\hline 1 & -1.369750 & 4.958734 & 0.324540 & 1 & 1.694313 & 6.144117 & 3.464324 \\
\hline 1 & -2.306792 & 2.177425 & 9.596844 & 1 & -5.713433 & 4.728328 & 9.338994 \\
\hline 1 & -3.204665 & 0.032539 & 8.809385 & 1 & -7.654256 & 4.236727 & 7.910933 \\
\hline
\end{tabular}




\begin{tabular}{|c|c|c|c|c|c|c|c|}
\hline 1 & -3.395414 & -0.413573 & 6.335233 & 1 & -7.558231 & 4.766210 & 5.448159 \\
\hline 1 & -2.675852 & 1.343300 & 4.719322 & 1 & -5.457015 & 5.675868 & 4.472474 \\
\hline 1 & -0.163317 & 2.358803 & 4.391502 & 1 & -1.871466 & 4.591958 & 6.210461 \\
\hline 1 & 0.495147 & 3.832595 & 2.473511 & 1 & -0.011738 & 5.180120 & 4.740874 \\
\hline & -3.207333 & 5.206400 & 5.519354 & 1 & -4.148307 & 7.964396 & 4.742198 \\
\hline & -2.422324 & 6.752939 & 3.721352 & 1 & -2.311060 & 8.542115 & 3.208889 \\
\hline & 0.560369 & 10.094827 & 2.638733 & 1 & -2.006234 & 9.799364 & 0.519241 \\
\hline & 1.650996 & 5.942135 & 3.153203 & 1 & 1.501327 & 9.210409 & 2.960623 \\
\hline 1 & 4.223603 & 9.060789 & 4.694676 & 1 & 0.228809 & 13.222887 & 1.935765 \\
\hline & -6.359733 & 7.295796 & 2.543834 & 1 & -0.332328 & 0.928323 & 1.655957 \\
\hline 1 & -2.773112 & 8.471929 & 0.446906 & 1 & -1.402790 & 5.119858 & 1.668480 \\
\hline 1 & -3.319185 & 4.348581 & 1.658378 & 1 & 2.543418 & 3.897260 & 2.947449 \\
\hline 6 & -1.121427 & 8.524162 & 6.422113 & 6 & -0.129927 & 9.536422 & 7.443124 \\
\hline 6 & 0.054004 & 9.113264 & 7.076427 & 6 & 0.236823 & 8.323607 & 7.144724 \\
\hline 6 & 1.337817 & 8.588330 & 6.802197 & 6 & 1.387149 & 7.663387 & 6.527140 \\
\hline & -0.080461 & 10.116308 & 8.063590 & 6 & 1.827098 & 6.383977 & 6.939776 \\
\hline 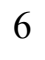 & 2.466691 & 9.086196 & 7.462007 & 1 & 1.295871 & 5.871327 & 7.749855 \\
\hline 1 & 1.434144 & 7.780817 & 6.072374 & 6 & 3.609902 & 6.432374 & 5.276632 \\
\hline 6 & 1.054190 & 10.609048 & 8.726022 & 1 & 4.470817 & 5.956687 & 4.795595 \\
\hline & -1.074917 & 10.489533 & 8.325590 & 6 & -2.967741 & 8.236997 & 10.747030 \\
\hline 6 & 2.330813 & 10.102808 & 8.423748 & 1 & -2.327653 & 7.664534 & 11.437051 \\
\hline 1 & 3.449669 & 8.661292 & 7.234987 & 1 & -2.313541 & 8.736894 & 10.011422 \\
\hline 1 & 0.937632 & 11.388079 & 9.487204 & 1 & -3.480656 & 9.019801 & 11.336555 \\
\hline 1 & 3.212339 & 10.488720 & 8.946374 & 6 & -4.967173 & 6.770283 & 11.140233 \\
\hline$r$ & -2.210097 & 8.871643 & 5.814135 & 1 & -5.774324 & 6.147993 & 10.722704 \\
\hline & -4.407202 & 10.690727 & 5.961782 & 1 & -4.409764 & 6.180168 & 11.886349 \\
\hline & -4.860770 & 10.982391 & 4.997910 & 1 & -5.451133 & 7.606814 & 11.678305 \\
\hline & -4.923680 & 11.256222 & 6.756619 & 6 & -4.860827 & 8.254629 & 9.092694 \\
\hline & -2.928312 & 11.010139 & 5.979199 & 1 & -4.235955 & 8.660408 & 8.275560 \\
\hline & -2.505162 & 11.265205 & 6.957563 & 1 & -5.708188 & 7.712884 & 8.640464 \\
\hline & -2.098657 & 11.182955 & 4.886144 & 1 & -5.264627 & 9.111399 & 9.663349 \\
\hline & -2.533318 & 10.997154 & 3.457021 & 6 & -1.543373 & 5.033329 & 10.984951 \\
\hline & -3.600365 & 10.771715 & 3.339609 & 1 & -0.784410 & 5.735075 & 10.593101 \\
\hline & -2.308822 & 11.910538 & 2.876089 & 1 & -2.091876 & 5.525581 & 11.805666 \\
\hline & -1.955023 & 10.178727 & 2.985032 & 1 & -1.012758 & 4.164326 & 11.416277 \\
\hline & -2.039523 & 6.579817 & 10.188474 & 6 & -3.574354 & 3.613143 & 10.472633 \\
\hline
\end{tabular}




\begin{tabular}{|c|c|c|c|c|c|c|c|}
\hline 1 & -0.972979 & 6.807854 & 10.346899 & 1 & -3.067589 & 2.795138 & 11.018089 \\
\hline 1 & -2.408879 & 7.222760 & 9.371504 & 1 & -4.231055 & 4.127919 & 11.191614 \\
\hline 1 & -2.586835 & 6.854767 & 11.109779 & 1 & -4.196010 & 3.148554 & 9.689761 \\
\hline 6 & -1.991608 & 4.292809 & 11.191423 & 6 & -1.683543 & 3.722474 & 8.833334 \\
\hline & -2.146629 & 3.206240 & 11.107870 & 1 & -1.264961 & 2.827198 & 9.329885 \\
\hline 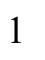 & -0.961475 & 4.470195 & 11.542224 & 1 & -2.324588 & 3.375081 & 8.003609 \\
\hline & -2.674746 & 4.653055 & 11.982995 & 1 & -0.842472 & 4.304493 & 8.415734 \\
\hline 6 & -3.785958 & 4.918107 & 9.494417 & 6 & -1.279883 & 10.432911 & 7.727152 \\
\hline 1 & -4.003888 & 5.445025 & 8.546752 & 1 & -1.233425 & 10.829667 & 8.758674 \\
\hline 1 & -4.084218 & 3.863868 & 9.376745 & 1 & -2.216066 & 9.855861 & 7.643141 \\
\hline & -4.418175 & 5.369346 & 10.281743 & 6 & -1.314967 & 11.603999 & 6.721144 \\
\hline 6 & 1.220144 & 5.279357 & 9.483080 & 6 & 0.055163 & 12.332275 & 6.841297 \\
\hline 1 & 1.126963 & 6.234146 & 8.933078 & 1 & 0.219836 & 12.936896 & 5.934937 \\
\hline 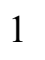 & 0.812868 & 5.415544 & 10.499071 & 1 & 0.029784 & 13.022416 & 7.703371 \\
\hline 1 & 2.298340 & 5.055710 & 9.586528 & 6 & 1.173768 & 11.327402 & 6.990662 \\
\hline 6 & 0.667763 & 2.799105 & 9.532132 & 1 & 1.664640 & 11.012606 & 6.065014 \\
\hline 1 & 1.740755 & 2.582110 & 9.689004 & 6 & 1.787910 & 10.956058 & 8.173167 \\
\hline 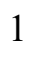 & 0.193127 & 2.859642 & 10.524183 & 6 & 1.343631 & 11.413164 & 9.538017 \\
\hline 1 & 0.230474 & 1.945734 & 8.986844 & 1 & 1.024321 & 10.541708 & 10.140480 \\
\hline 6 & 1.228398 & 3.929686 & 7.352729 & 1 & 2.198968 & 11.865988 & 10.072495 \\
\hline 1 & 2.284057 & 3.643499 & 7.515983 & 1 & 0.521407 & 12.143654 & 9.519876 \\
\hline 1 & 0.749621 & 3.133313 & 6.757086 & 6 & 3.000349 & 10.063073 & 8.167889 \\
\hline 1 & 1.219912 & 4.861345 & 6.760230 & 1 & 3.823874 & 10.555815 & 8.717956 \\
\hline 6 & -3.551837 & 8.453488 & 5.346217 & 1 & 2.783918 & 9.116647 & 8.697771 \\
\hline 1 & -3.661086 & 7.363938 & 5.459940 & 1 & 3.345390 & 9.817199 & 7.152584 \\
\hline 1 & -3.702046 & 8.682572 & 4.274262 & 6 & 2.931914 & 5.779321 & 6.322843 \\
\hline 6 & -4.634022 & 9.163753 & 6.187291 & 1 & 3.263791 & 4.790846 & 6.657632 \\
\hline & -0.663943 & 11.596373 & 5.073519 & 6 & 3.162259 & 7.693320 & 4.841001 \\
\hline & -0.381628 & 12.379227 & 4.348510 & 1 & 3.670253 & 8.211865 & 4.020303 \\
\hline 1 & -0.448291 & 11.949898 & 6.093165 & 6 & 2.054492 & 8.292862 & 5.452639 \\
\hline 1 & 0.000711 & 10.736286 & 4.884797 & 1 & 1.687939 & 9.256497 & 5.100938 \\
\hline 6 & -6.029048 & 8.743483 & 5.712154 & 6 & -1.505530 & 11.058032 & 5.282471 \\
\hline 1 & -6.795931 & 9.254464 & 6.332620 & 1 & -0.638783 & 10.431412 & 4.993508 \\
\hline 1 & -6.180855 & 9.049603 & 4.656054 & 1 & -2.401771 & 10.410350 & 5.243829 \\
\hline 6 & -4.437256 & 8.787420 & 7.681533 & 6 & -2.458437 & 12.558817 & 7.085012 \\
\hline & -4.406523 & 7.683952 & 7.774513 & 1 & -2.309055 & 12.950245 & 8.116950 \\
\hline
\end{tabular}




$\begin{array}{llllllll}1 & -3.463113 & 9.170030 & 8.039089 & 1 & -2.453152 & 13.424892 & 6.388783 \\ 8 & -6.133904 & 7.322402 & 5.832639 & 8 & -1.599407 & 12.138635 & 4.354358 \\ 8 & -5.460192 & 9.351177 & 8.503537 & 6 & -2.892816 & 12.268766 & 3.759213 \\ 6 & -7.438833 & 6.849741 & 5.501090 & 1 & -2.863847 & 13.180236 & 3.143110 \\ 1 & -7.737489 & 7.153095 & 4.475632 & 1 & -3.122332 & 11.407278 & 3.098763 \\ 1 & -7.402028 & 5.751752 & 5.546871 & 1 & -3.688057 & 12.355953 & 4.524946 \\ 1 & -8.201256 & 7.231097 & 6.212833 & 8 & -3.692602 & 11.844545 & 6.998281 \\ 6 & -6.348217 & 8.369137 & 9.048267 & 6 & -4.809584 & 12.669183 & 7.331121 \\ 1 & -6.816985 & 7.752365 & 8.256234 & 1 & -5.710246 & 12.041448 & 7.240911 \\ 1 & -5.819718 & 7.692812 & 9.753036 & 1 & -4.736156 & 13.055207 & 8.369912 \\ 1 & -7.130304 & 8.917917 & 9.597631 & 1 & -4.896775 & 13.533948 & 6.639635\end{array}$

B-TSInt3b-4b

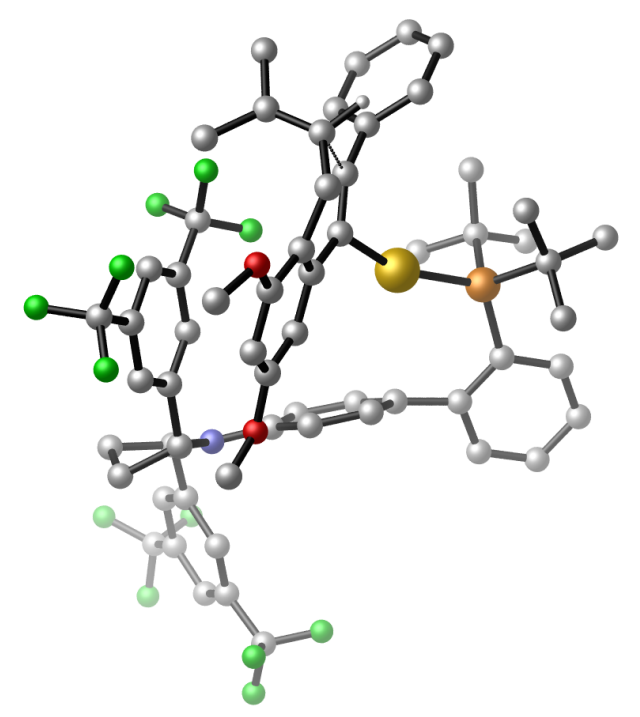

$E=-4241.18953982$ Hartrees

$G=-4240.246245$ Hartrees
B-TSInt3d-4d

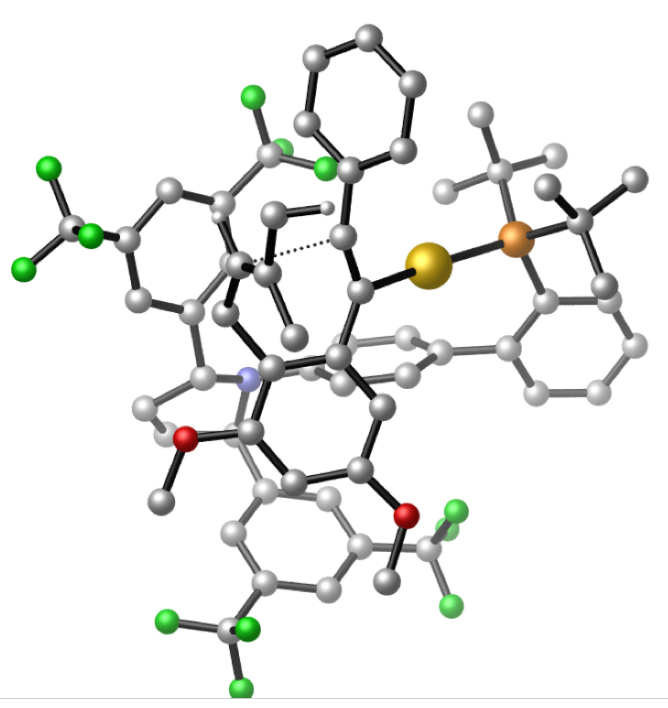

$E=-4241.18893406$ Hartrees

$G=-4240.243709$ Hartrees

$\begin{array}{llll}79 & -1.699784 & 6.896338 & 6.442532 \\ 6 & -4.163026 & 4.345760 & 6.445562 \\ 6 & -5.103157 & 3.598721 & 7.195843 \\ 6 & -6.305066 & 3.141411 & 6.641185 \\ 6 & -6.598555 & 3.426058 & 5.300186 \\ 6 & -5.664054 & 4.125696 & 4.527072\end{array}$

$\begin{array}{rrrr}79 & -1.340412 & 6.030325 & 5.920727 \\ 6 & -4.513609 & 4.400315 & 6.852156 \\ 6 & -5.320442 & 3.888021 & 7.898556 \\ 6 & -6.678906 & 3.600446 & 7.727665 \\ 6 & -7.271443 & 3.807889 & 6.474128 \\ 6 & -6.490373 & 4.303118 & 5.424872\end{array}$




\begin{tabular}{|c|c|c|c|c|c|c|c|}
\hline 6 & -5.114657 & 4.614434 & 5.578773 & 6 & -4.437265 & 4.581887 & 5.065021 \\
\hline 6 & -4.462417 & 5.122421 & 4.333943 & 6 & -3.498789 & 5.183112 & 4.067243 \\
\hline 6 & -4.438883 & 4.285926 & 3.197028 & 6 & -2.894251 & 4.325943 & 3.121189 \\
\hline 6 & -4.096285 & 4.766818 & 1.930551 & 6 & -2.085119 & 4.837643 & 2.103634 \\
\hline 6 & -3.788259 & 6.140413 & 1.741526 & 6 & -1.882999 & 6.230430 & 1.978324 \\
\hline 6 & -3.794519 & 6.985240 & 2.884054 & 6 & -2.487059 & 7.093976 & 2.912922 \\
\hline 6 & -4.114919 & 6.479083 & 4.150893 & 6 & -3.288711 & 6.570365 & 3.941986 \\
\hline 6 & -2.965386 & 7.982458 & 0.225748 & 6 & -0.382878 & 7.929413 & 0.862416 \\
\hline 6 & -3.136300 & 8.121696 & -1.305633 & 6 & -0.067042 & 8.211098 & -0.645160 \\
\hline 6 & -2.832936 & 6.698423 & -1.798234 & 6 & -0.899477 & 7.177346 & -1.449159 \\
\hline 6 & -3.522428 & 5.801049 & -0.732856 & 6 & -1.933042 & 6.636087 & -0.450506 \\
\hline 6 & -2.001825 & 2.825391 & 6.995493 & 6 & 0.862990 & 7.828830 & 1.736013 \\
\hline 6 & -2.508402 & 5.289827 & 8.985750 & 6 & 1.033196 & 6.825825 & 2.705071 \\
\hline & -2.700806 & 4.596524 & 7.184192 & 6 & 2.161296 & 6.822593 & 3.545000 \\
\hline 7 & -3.507371 & 6.640980 & 0.474269 & 6 & 3.158426 & 7.797095 & 3.417638 \\
\hline 1 & -3.560817 & 8.748342 & 0.756966 & 6 & 2.999298 & 8.790382 & 2.436612 \\
\hline 1 & -2.469170 & 8.884181 & -1.738093 & 6 & 1.865621 & 8.812789 & 1.611681 \\
\hline 1 & -4.180923 & 8.400269 & -1.531468 & 6 & 2.241351 & 5.754463 & 4.608127 \\
\hline 1 & -1.744166 & 6.513956 & -1.780823 & 6 & 3.983508 & 9.931813 & 2.388371 \\
\hline 1 & -3.204048 & 6.493663 & -2.814553 & 6 & -3.231497 & 7.436073 & -0.316001 \\
\hline 1 & -2.922009 & 4.882394 & -0.575982 & 6 & -4.305258 & 6.852222 & 0.386786 \\
\hline 1 & -4.877166 & 3.685751 & 8.872905 & 6 & -5.467773 & 7.583853 & 0.669352 \\
\hline 1 & -7.261128 & 3.209610 & 8.568463 & 6 & -5.610529 & 8.899474 & 0.209593 \\
\hline 1 & -8.332058 & 3.587880 & 6.312535 & 6 & -4.550945 & 9.483728 & -0.503733 \\
\hline 1 & -6.945713 & 4.476359 & 4.443906 & 6 & -3.368030 & 8.767625 & -0.751738 \\
\hline 1 & -4.726037 & 3.233449 & 3.302756 & 6 & -6.523563 & 6.964787 & 1.553203 \\
\hline 1 & -4.111901 & 4.076907 & 1.083238 & 6 & -4.711764 & 10.901151 & -0.995450 \\
\hline 1 & -4.146798 & 7.165189 & 5.005360 & 6 & -1.406412 & 3.496906 & 7.271917 \\
\hline 1 & -3.579751 & 8.051806 & 2.779949 & 6 & -3.136435 & 5.475320 & 9.108590 \\
\hline 6 & 0.237417 & 7.383998 & 5.477586 & 15 & -2.637764 & 4.949277 & 7.314808 \\
\hline 6 & -0.023731 & 8.729867 & 4.951855 & 7 & -1.155480 & 6.674071 & 0.832691 \\
\hline 6 & 0.802103 & 9.824740 & 5.310098 & 9 & 3.415060 & 5.783629 & 5.296102 \\
\hline 6 & -1.131800 & 8.906905 & 4.103710 & 9 & 2.085857 & 4.504925 & 4.090604 \\
\hline 6 & 0.523803 & 11.079043 & 4.712514 & 9 & 1.233296 & 5.910903 & 5.536442 \\
\hline 6 & -1.430662 & 10.175238 & 3.588251 & 9 & 3.638273 & 10.901180 & 3.299745 \\
\hline 1 & -1.748221 & 8.048686 & 3.833333 & 9 & 4.023180 & 10.533365 & 1.168690 \\
\hline
\end{tabular}




\begin{tabular}{|c|c|c|c|c|c|c|c|}
\hline 6 & -0.587758 & 11.265978 & 3.864610 & 9 & 5.247844 & 9.533531 & 2.700960 \\
\hline 1 & -0.789438 & 12.246431 & 3.435642 & 9 & -6.212868 & 7.132428 & 2.875489 \\
\hline 6 & 1.328004 & 6.846138 & 5.967792 & 9 & -6.634761 & 5.616593 & 1.348994 \\
\hline 6 & 1.924771 & 5.648106 & 6.515738 & 9 & -7.752695 & 7.515337 & 1.357940 \\
\hline 6 & 2.241674 & 4.557311 & 5.666977 & 9 & -3.664795 & 11.312421 & -1.762731 \\
\hline 6 & 2.190008 & 5.543137 & 7.904936 & 9 & -5.850854 & 11.061726 & -1.720438 \\
\hline 6 & 2.795041 & 3.386516 & 6.202653 & 9 & -4.798073 & 11.789308 & 0.056784 \\
\hline 1 & 2.031868 & 4.638699 & 4.600242 & 1 & -0.980026 & 8.783351 & 1.256917 \\
\hline 6 & 2.740068 & 4.369169 & 8.431291 & 1 & 1.008347 & 8.091102 & -0.853147 \\
\hline 6 & 3.047201 & 3.289266 & 7.581568 & 1 & -0.330647 & 9.251364 & -0.897018 \\
\hline & 3.480765 & 2.373505 & 7.996645 & 1 & -0.256648 & 6.341104 & -1.767965 \\
\hline & -1.078810 & 5.874760 & 9.134768 & 1 & -1.364312 & 7.601548 & -2.353890 \\
\hline & -0.291400 & 5.128860 & 8.946488 & 1 & -2.192962 & 5.583930 & -0.660044 \\
\hline & -0.906509 & 6.727731 & 8.455651 & 1 & -4.894889 & 3.360954 & 8.239850 \\
\hline & -0.958573 & 6.238966 & 10.172794 & 1 & -7.005436 & 2.571362 & 7.260134 \\
\hline 6 & -2.694745 & 4.277265 & 10.140037 & 1 & -7.541607 & 3.094768 & 4.852816 \\
\hline 1 & -3.684530 & 3.797991 & 10.172447 & 1 & -5.875809 & 4.343384 & 3.475415 \\
\hline 1 & -1.928171 & 3.485114 & 10.119711 & 1 & -3.067256 & 3.246451 & 3.186683 \\
\hline & -2.576390 & 4.823528 & 11.094489 & 1 & -1.614394 & 4.168314 & 1.375699 \\
\hline 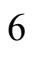 & -3.518713 & 6.455511 & 9.103005 & 1 & -3.832253 & 7.259232 & 4.594004 \\
\hline 1 & -3.405462 & 7.165584 & 8.262198 & 1 & -2.382199 & 8.177783 & 2.817371 \\
\hline 1 & -4.565679 & 6.111781 & 9.124974 & 1 & 0.286761 & 6.038803 & 2.826146 \\
\hline & -3.320023 & 7.007777 & 10.040473 & 1 & 1.747542 & 9.625824 & 0.889673 \\
\hline 6 & -0.544722 & 2.809470 & 7.499037 & 1 & 4.039950 & 7.788729 & 4.062876 \\
\hline 1 & 0.060835 & 3.601487 & 7.026145 & 1 & -6.537115 & 9.451354 & 0.387789 \\
\hline 1 & -0.476066 & 2.923734 & 8.593348 & 1 & -2.566649 & 9.253057 & -1.313312 \\
\hline 1 & -0.083769 & 1.839013 & 7.237045 & 1 & -4.233098 & 5.816719 & 0.731113 \\
\hline 6 & -2.841636 & 1.756800 & 7.723543 & 6 & -1.007241 & 8.856347 & 6.019907 \\
\hline 1 & -2.373092 & 0.769198 & 7.553847 & 6 & -1.713103 & 9.662108 & 5.024515 \\
\hline 1 & -2.885689 & 1.920086 & 8.811864 & 6 & -0.948826 & 10.328830 & 4.037567 \\
\hline 1 & -3.872740 & 1.711031 & 7.335345 & 6 & -3.118642 & 9.710924 & 5.023589 \\
\hline 6 & -2.019870 & 2.533763 & 5.475858 & 6 & -1.648972 & 10.914634 & 2.954782 \\
\hline 1 & -1.635721 & 1.510372 & 5.304870 & 6 & -3.789229 & 10.375245 & 3.984428 \\
\hline 1 & -3.040410 & 2.587763 & 5.059800 & 1 & -3.694016 & 9.225395 & 5.817145 \\
\hline 1 & -1.381106 & 3.240298 & 4.915562 & 6 & -3.059337 & 10.942188 & 2.916013 \\
\hline 1 & 3.028294 & 2.546838 & 5.539876 & 1 & -3.579574 & 11.382012 & 2.065660 \\
\hline
\end{tabular}




\begin{tabular}{|c|c|c|c|c|c|c|c|}
\hline 1 & 2.926851 & 4.292731 & 9.507331 & 6 & 0.107500 & 8.955278 & 6.665388 \\
\hline & -2.561607 & 10.252731 & 2.810570 & 6 & 1.048436 & 8.615889 & 7.684682 \\
\hline 8 & 1.389647 & 12.095010 & 5.028189 & 6 & 2.346159 & 8.144643 & 7.360796 \\
\hline 1 & 1.940879 & 6.380941 & 8.563693 & 6 & 0.668673 & 8.746706 & 9.047421 \\
\hline 6 & 1.872381 & 9.713683 & 6.384816 & 6 & 3.234548 & 7.789342 & 8.379192 \\
\hline & 2.523634 & 10.604587 & 6.329511 & 1 & 2.634469 & 8.055605 & 6.311562 \\
\hline 1 & 1.374226 & 9.762222 & 7.372424 & 6 & 1.564578 & 8.383229 & 10.059673 \\
\hline 6 & 2.746079 & 8.475780 & 6.366616 & 6 & 2.845319 & 7.904604 & 9.728300 \\
\hline 1 & 2.974406 & 8.046386 & 7.348756 & 1 & 3.544684 & 7.623768 & 10.522637 \\
\hline 6 & 3.579643 & 8.109644 & 5.320197 & 6 & -2.036501 & 6.430764 & 9.645495 \\
\hline 0 & 4.566393 & 6.988939 & 5.459857 & 1 & -1.028171 & 5.987343 & 9.639814 \\
\hline & 5.578840 & 7.349639 & 5.194991 & 1 & -1.996307 & 7.363793 & 9.057325 \\
\hline 1 & 4.592143 & 6.556578 & 6.471903 & 1 & -2.285331 & 6.695644 & 10.690446 \\
\hline & 4.323313 & 6.183082 & 4.739572 & 6 & -3.296965 & 4.329922 & 10.133322 \\
\hline 0 & 3.613721 & 8.871935 & 4.028915 & 1 & -4.035697 & 3.566531 & 9.845299 \\
\hline & 4.358504 & 9.687408 & 4.133649 & 1 & -2.338352 & 3.823596 & 10.334631 \\
\hline 1 & 3.944668 & 8.237486 & 3.191056 & 1 & -3.642618 & 4.769370 & 11.087637 \\
\hline 1 & 2.651389 & 9.348468 & 3.789415 & 6 & -4.443812 & 6.294348 & 8.979600 \\
\hline 6 & 1.271559 & 13.328110 & 4.301107 & 1 & -4.345448 & 7.083103 & 8.209422 \\
\hline 1 & 1.327183 & 13.150021 & 3.211799 & 1 & -5.317110 & 5.672612 & 8.726263 \\
\hline 1 & 0.330385 & 13.855930 & 4.546988 & 1 & -4.646637 & 6.790997 & 9.946675 \\
\hline & 2.125820 & 13.941553 & 4.622695 & 6 & -0.158467 & 3.874362 & 8.098909 \\
\hline & -2.935117 & 11.541742 & 2.298393 & 1 & 0.281971 & 4.830373 & 7.763888 \\
\hline & -3.140177 & 12.254908 & 3.118752 & 1 & -0.377625 & 3.942321 & 9.177447 \\
\hline & -2.149923 & 11.953584 & 1.638316 & 1 & 0.605086 & 3.085281 & 7.966176 \\
\hline 1 & -3.853274 & 11.373249 & 1.717376 & 6 & -2.025380 & 2.176750 & 7.777768 \\
\hline 6 & -4.937090 & 5.387868 & -1.142440 & 1 & -1.286570 & 1.366294 & 7.633453 \\
\hline 6 & -5.115987 & 4.313877 & -2.032651 & 1 & -2.279318 & 2.204819 & 8.848639 \\
\hline 6 & -6.065814 & 6.079215 & -0.674990 & 1 & -2.932605 & 1.906931 & 7.210072 \\
\hline 6 & -6.403862 & 3.946114 & -2.452565 & 6 & -1.007607 & 3.323417 & 5.788220 \\
\hline 1 & -4.245964 & 3.764056 & -2.407572 & 1 & -0.227361 & 2.542167 & 5.721633 \\
\hline 6 & -7.352446 & 5.702944 & -1.094214 & 1 & -1.865149 & 2.995291 & 5.179743 \\
\hline 1 & -5.936883 & 6.909997 & 0.024662 & 1 & -0.601578 & 4.250031 & 5.349309 \\
\hline 6 & -7.533573 & 4.635461 & -1.984580 & 1 & 4.231038 & 7.415520 & 8.123582 \\
\hline & -8.534787 & 4.348812 & -2.313619 & 1 & 1.265713 & 8.476267 & 11.108634 \\
\hline & -1.504772 & 8.119039 & 0.662569 & 8 & -5.154399 & 10.382495 & 4.076713 \\
\hline
\end{tabular}




$\begin{array}{rrrrrrrr}6 & -0.858250 & 9.364254 & 0.559619 & 8 & -0.857907 & 11.428575 & 1.956048 \\ 6 & -0.794702 & 7.026610 & 1.177447 & 1 & -0.331980 & 9.118267 & 9.286782 \\ 6 & 0.471979 & 9.508547 & 0.975411 & 6 & 0.557029 & 10.368210 & 4.128771 \\ 1 & -1.404869 & 10.230862 & 0.176880 & 1 & 0.941327 & 9.359120 & 3.905708 \\ 6 & 0.541741 & 7.173562 & 1.581602 & 1 & 0.969009 & 11.022469 & 3.341469 \\ 1 & -1.289045 & 6.058999 & 1.289252 & 6 & 1.164828 & 10.741493 & 5.465235 \\ 6 & 1.190169 & 8.406914 & 1.468644 & 1 & 2.171934 & 10.327173 & 5.602140 \\ 1 & 2.229932 & 8.519769 & 1.773024 & 6 & 0.726313 & 11.614090 & 6.429534 \\ 6 & -8.548361 & 6.420668 & -0.514427 & 6 & 1.558095 & 11.842513 & 7.666171 \\ 6 & -6.571470 & 2.751846 & -3.362729 & 1 & 0.964429 & 11.621386 & 8.575325 \\ 9 & -9.657621 & 6.290971 & -1.294976 & 1 & 2.464062 & 11.214673 & 7.689397 \\ 9 & -8.866635 & 5.929933 & 0.721500 & 1 & 1.860536 & 12.904989 & 7.738172 \\ 9 & -8.307799 & 7.755252 & -0.356884 & 6 & -0.576084 & 12.374695 & 6.375579 \\ 9 & -7.743392 & 2.795201 & -4.056587 & 1 & -0.438885 & 13.389856 & 6.790172 \\ 9 & -6.569011 & 1.583603 & -2.652189 & 1 & -0.982825 & 12.453814 & 5.356925 \\ 9 & -5.556575 & 2.662260 & -4.270760 & 1 & -1.339989 & 11.873045 & 7.000827 \\ 6 & 1.268827 & 5.964945 & 2.107555 & 6 & -1.502788 & 12.019479 & 0.818547 \\ 6 & 1.167575 & 10.842591 & 0.860593 & 1 & -2.122835 & 11.281339 & 0.280160 \\ 9 & 1.733735 & 5.165294 & 1.106392 & 1 & -2.132609 & 12.881492 & 1.107685 \\ 9 & 0.448313 & 5.183177 & 2.882217 & 1 & -0.688249 & 12.360372 & 0.163431 \\ 9 & 2.345995 & 6.302474 & 2.881564 & 6 & -5.883713 & 11.183324 & 3.134860 \\ 9 & 0.290229 & 11.891788 & 0.904525 & 1 & -5.575350 & 12.244878 & 3.189225 \\ 9 & 1.860306 & 10.956520 & -0.308836 & 1 & -5.748158 & 10.821706 & 2.101729 \\ 9 & 2.070456 & 11.032410 & 1.872387 & 1 & -6.941002 & 11.087171 & 3.421164\end{array}$




\section{Enyne 5b}

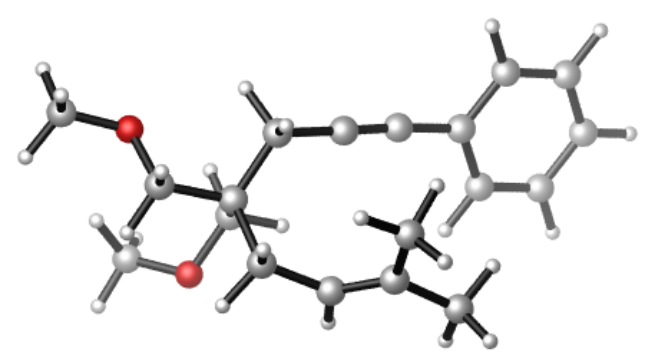

$\mathrm{E}=-890.034548813$ Hartrees

$G=-889.691653$ Hartrees
Enyne 9k

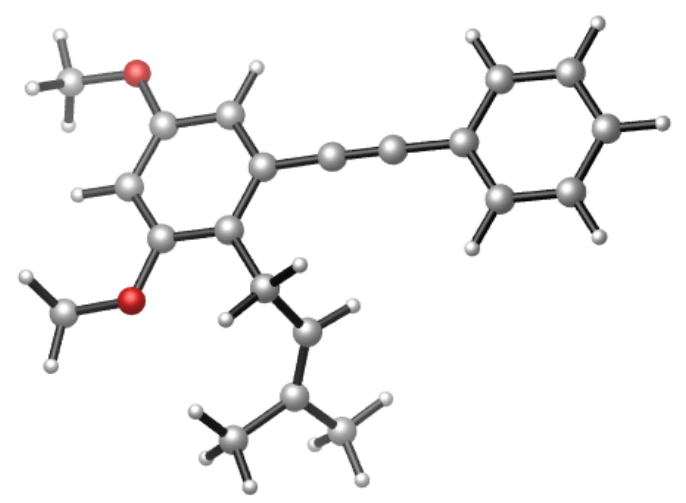

$$
\begin{gathered}
E=-963.859114793 \text { Hartrees } \\
G=-963.551049 \text { Hartrees }
\end{gathered}
$$

$\begin{array}{llll}6 & -5.187467 & -4.715371 & -4.531455 \\ 6 & -4.601578 & -4.078714 & -3.422135 \\ 6 & -4.168274 & -2.749952 & -3.515275 \\ 6 & -4.316619 & -2.032730 & -4.732263 \\ 6 & -4.909456 & -2.684618 & -5.847004 \\ 6 & -5.338899 & -4.013477 & -5.741429 \\ 1 & -5.524684 & -5.754571 & -4.453388 \\ 1 & -4.481628 & -4.621491 & -2.478098 \\ 1 & -3.711609 & -2.251466 & -2.654086 \\ 1 & -5.022626 & -2.134143 & -6.786801 \\ 1 & -5.793954 & -4.505235 & -6.608200 \\ 6 & -3.875493 & -0.683496 & -4.842354 \\ 6 & -3.491072 & 0.479958 & -4.953423 \\ 6 & -3.034741 & 1.819827 & -5.124310 \\ 6 & -3.290621 & 2.494069 & -6.351663 \\ 6 & -2.319972 & 2.446168 & -4.075599 \\ 6 & -2.777038 & 3.804221 & -6.494678 \\ 6 & -1.840962 & 3.754154 & -4.246433 \\ 1 & -2.130937 & 1.919840 & -3.136410 \\ 6 & -2.065665 & 4.442107 & -5.457358 \\ 1 & -1.692044 & 5.457250 & -5.592383 \\ 6 & -4.030308 & 1.818669 & -7.495472 \\ 1 & -4.805857 & 1.153937 & -7.068744\end{array}$




\begin{tabular}{rrrrrrrr}
1 & -0.778107 & 9.225626 & 9.813330 & 1 & -4.547366 & 2.579380 & -8.101071 \\
6 & 0.993826 & 10.319463 & 9.166043 & 6 & -3.086166 & 0.986322 & -8.341908 \\
6 & -2.423835 & 12.793842 & 6.787242 & 1 & -2.703240 & 0.089235 & -7.832490 \\
1 & -3.197863 & 13.550657 & 7.024298 & 6 & -2.651048 & 1.241173 & -9.596975 \\
1 & -1.669555 & 13.262084 & 6.130815 & 6 & -1.692149 & 0.295346 & -10.29034 \\
1 & -2.928565 & 11.995746 & 6.207797 & 1 & -0.749252 & 0.812845 & -10.55852 \\
6 & 1.826663 & 10.009646 & 10.425849 & 1 & -1.438516 & -0.577149 & -9.663298 \\
1 & 2.538088 & 10.844190 & 10.608733 & 1 & -2.120426 & -0.077872 & -11.24220 \\
1 & 1.156273 & 9.939626 & 11.313069 & 6 & -3.051607 & 2.445434 & -10.42089 \\
6 & 1.914352 & 10.384155 & 7.924899 & 1 & -3.658030 & 3.172305 & -9.859780 \\
1 & 2.391404 & 9.397576 & 7.767358 & 1 & -2.149109 & 2.975634 & -10.78401 \\
1 & 1.302469 & 10.610748 & 7.032184 & 1 & -3.612490 & 2.135154 & -11.32504 \\
8 & 2.539044 & 8.783512 & 10.248871 & 8 & -1.160555 & 4.282538 & -3.177671 \\
8 & 2.899346 & 11.418821 & 8.059670 & 8 & -3.028415 & 4.402930 & -7.704982 \\
6 & 3.296780 & 8.439179 & 11.405904 & 6 & -0.642570 & 5.613300 & -3.307668 \\
1 & 2.647178 & 8.307374 & 12.297866 & 1 & -0.138816 & 5.829595 & -2.354008 \\
1 & 3.806510 & 7.486966 & 11.186729 & 1 & 0.090628 & 5.685429 & -4.134247 \\
1 & 4.059037 & 9.213166 & 11.640649 & 1 & -1.451915 & 6.351389 & -3.470041 \\
6 & 4.224642 & 10.917851 & 8.243166 & 6 & -2.528758 & 5.726740 & -7.927764 \\
1 & 4.289186 & 10.216141 & 9.098661 & 1 & -2.972485 & 6.453848 & -7.220555 \\
1 & 4.593758 & 10.393225 & 7.335651 & 1 & -1.425192 & 5.763235 & -7.848661 \\
1 & 4.871651 & 11.790116 & 8.435840 & 1 & -2.829290 & 5.984407 & -8.953938 \\
6 & -0.742973 & 9.107595 & 7.707627 & & & & \\
6 & -1.330187 & 9.039208 & 6.633076 & & & & \\
& & & & & & \\
\hline
\end{tabular}




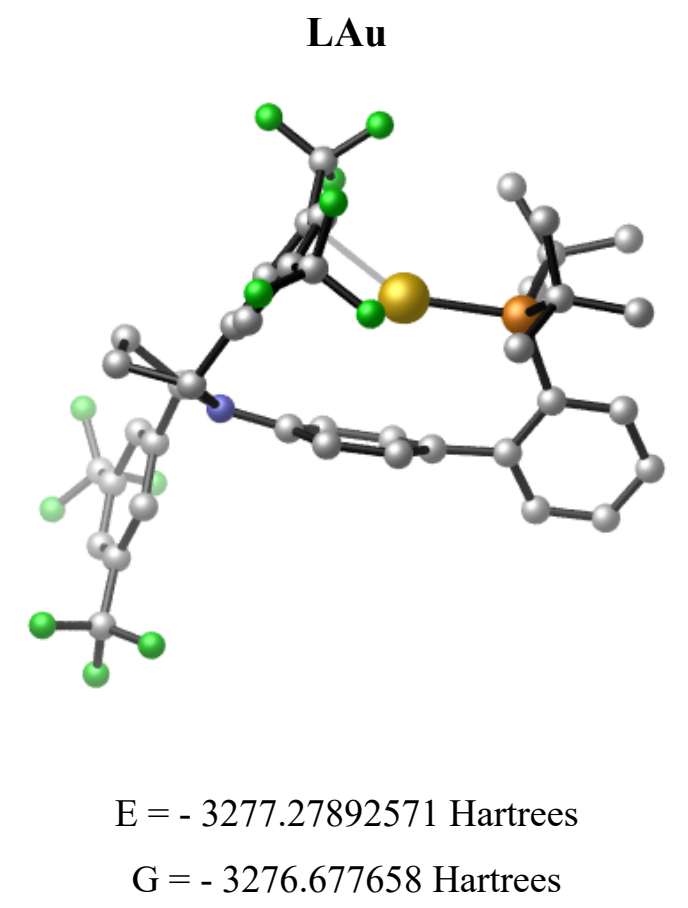

$\begin{array}{llll}79 & 0.220592 & 7.272394 & 7.101668 \\ 6 & 2.120281 & 4.225187 & 7.384029 \\ 6 & 2.243186 & 2.852457 & 7.714705 \\ 6 & 3.485645 & 2.205021 & 7.716901 \\ 6 & 4.646846 & 2.925816 & 7.402182 \\ 6 & 4.544330 & 4.281427 & 7.066817 \\ 6 & 3.299655 & 4.947891 & 7.037334 \\ 6 & 3.289756 & 6.381144 & 6.647867 \\ 6 & 3.854384 & 7.366958 & 7.481819 \\ 6 & 3.740130 & 8.730391 & 7.180655 \\ 6 & 3.037033 & 9.150855 & 6.021914 \\ 6 & 2.590267 & 8.152090 & 5.112657 \\ 6 & 2.701812 & 6.795006 & 5.434280 \\ 6 & 3.039414 & 11.547595 & 6.769550 \\ 6 & 1.995678 & 12.660138 & 6.457941 \\ 6 & 1.597422 & 12.400717 & 4.995537 \\ 6 & 1.644021 & 10.858448 & 4.869230 \\ 6 & 4.483658 & 12.029270 & 6.656667 \\ 6 & 5.091384 & 12.668790 & 7.752364 \\ 6 & 6.403209 & 13.155834 & 7.649929 \\ 6 & 7.129288 & 13.011327 & 6.456864 \\ 6 & 6.522488 & 12.368372 & 5.369292\end{array}$




\begin{tabular}{cccc}
6 & 5.209671 & 11.877259 & 5.465663 \\
6 & 7.065150 & 13.771608 & 8.860997 \\
6 & 7.253752 & 12.242706 & 4.053007 \\
6 & 0.337103 & 10.146838 & 5.236246 \\
6 & -0.517861 & 9.658747 & 4.239489 \\
6 & -1.681065 & 8.933639 & 4.570864 \\
6 & -2.045520 & 8.727616 & 5.902003 \\
6 & -1.188988 & 9.211054 & 6.932103 \\
6 & 0.005252 & 9.906067 & 6.590937 \\
6 & -2.475502 & 8.264252 & 3.470979 \\
6 & -1.744306 & 9.345810 & 8.341460 \\
6 & 0.185493 & 4.890857 & 9.563325 \\
6 & -0.822542 & 4.128194 & 6.569153 \\
15 & 0.468863 & 5.022070 & 7.666814 \\
7 & 2.727316 & 10.488781 & 5.793186 \\
9 & 7.668946 & 12.818581 & 9.632970 \\
9 & 6.163835 & 14.414485 & 9.658015 \\
9 & 8.027027 & 14.672329 & 8.514164 \\
9 & 8.602373 & 12.365150 & 4.197237 \\
9 & 6.853101 & 13.207396 & 3.171136 \\
9 & 7.008663 & 11.039299 & 3.457178 \\
9 & -1.923682 & 7.044853 & 3.176682 \\
9 & -2.472027 & 8.994561 & 2.325765 \\
9 & -3.766783 & 8.044940 & 3.831614 \\
9 & -0.752710 & 9.363481 & 9.281645 \\
9 & -2.428897 & 10.513818 & 8.456381 \\
9 & -2.601497 & 8.336166 & 8.648860 \\
1 & 2.878442 & 11.174740 & 7.801305 \\
1 & 2.410517 & 13.667674 & 6.617035 \\
1 & 1.123873 & 12.549374 & 7.125801 \\
1 & 2.346562 & 12.829368 & 4.307302 \\
1 & 0.610374 & 12.811856 & 4.731994 \\
1 & 1.908090 & 10.580466 & 3.832554 \\
\hline 1.364756 & 2.271389 & 7.994543 \\
\hline & 3.539489 & 1.143005 & 7.976532 \\
\hline 625625 & 2.435134 & 7.411615 \\
\hline 6 & & \\
\hline 6
\end{tabular}




\begin{tabular}{cccc}
1 & 5.440730 & 4.852511 & 6.802430 \\
1 & 4.357594 & 7.062593 & 8.406064 \\
1 & 4.180168 & 9.463788 & 7.861183 \\
1 & 2.307765 & 6.046247 & 4.739164 \\
1 & 2.115422 & 8.427948 & 4.168805 \\
1 & 4.539572 & 12.791030 & 8.690518 \\
1 & 4.752245 & 11.356442 & 4.619293 \\
1 & 8.151484 & 13.388463 & 6.380221 \\
1 & -2.964648 & 8.198307 & 6.161344 \\
1 & 0.642057 & 10.285225 & 7.394800 \\
1 & -0.268489 & 9.816428 & 3.185203 \\
6 & -0.918259 & 5.887585 & 9.983941 \\
1 & -1.093041 & 5.772799 & 11.069873 \\
1 & -0.614348 & 6.930885 & 9.802995 \\
1 & -1.876303 & 5.714135 & 9.469932 \\
6 & 1.522303 & 5.326098 & 10.209476 \\
1 & 1.858619 & 6.307436 & 9.825221 \\
1 & 1.369666 & 5.423398 & 11.300106 \\
1 & 2.325705 & 4.590933 & 10.038113 \\
6 & -0.207367 & 3.476097 & 10.035787 \\
1 & -1.179624 & 3.157046 & 9.625176 \\
1 & 0.552664 & 2.716073 & 9.797488 \\
1 & -0.306790 & 3.497843 & 11.136774 \\
6 & -0.789686 & 2.585635 & 6.642217 \\
1 & -0.931306 & 2.197653 & 7.663621 \\
1 & -1.623631 & 2.201432 & 6.026455 \\
1 & 0.142204 & 2.172696 & 6.224020 \\
6 & -0.497855 & 4.554420 & 5.115855 \\
1 & -1.190570 & 4.030494 & 4.431610 \\
1 & -0.627742 & 5.639462 & 4.957831 \\
1 & 0.532210 & 4.277283 & 4.829564 \\
6 & -2.230823 & 4.629724 & 6.961088 \\
1 & -2.550754 & 4.241316 & 7.942819 \\
1 & -2.293492 & 5.732035 & 6.981657 \\
\hline & -2.953685 & 4.265143 & 6.208257 \\
\hline
\end{tabular}




\section{X-ray crystallographic data}

The supplementary crystallographic data for this paper can be obtained free of charge from The Cambridge Crystallographic Data Centre via www.ccdc.cam.ac.uk/structures.

\section{Complex $(R, R)-\mathrm{C}$}

CCDC 1916876

The absolute configuration has been assigned.

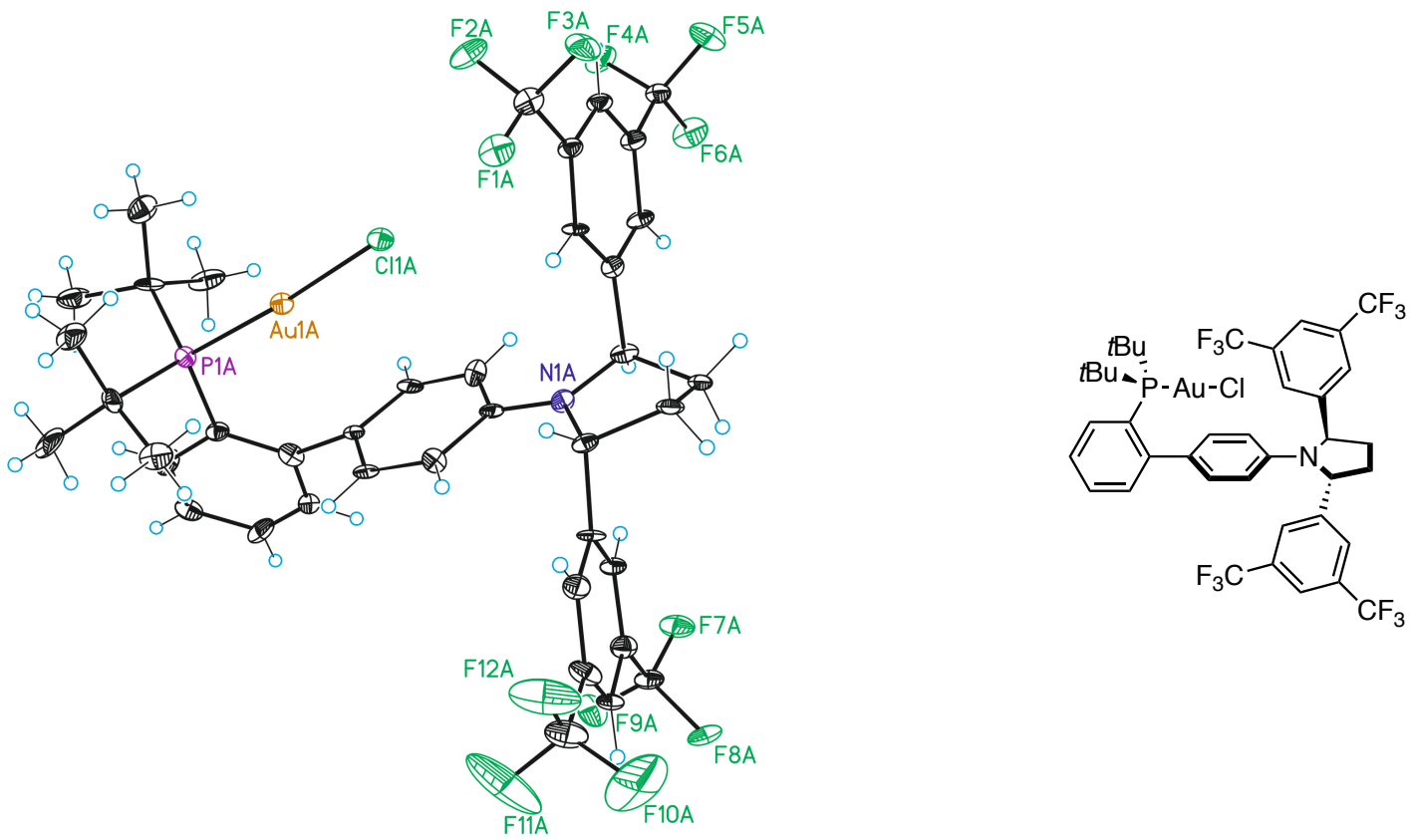




\section{Complex $(R, R)-\mathrm{D}$}

CCDC 1916871

The absolute configuration has been assigned.
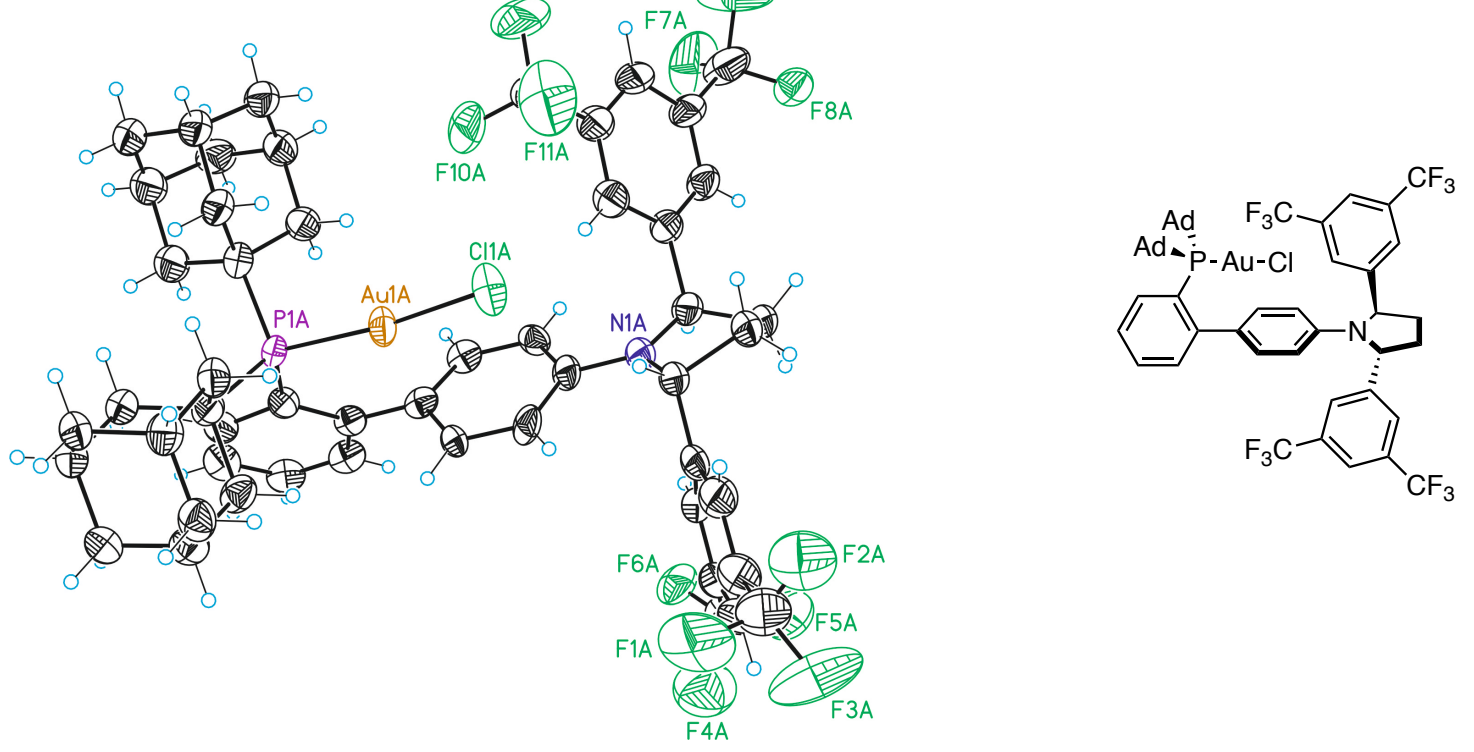

\section{Complex $(R, R)-\mathrm{E}$}

CCDC 1916870

The absolute configuration has been assigned.
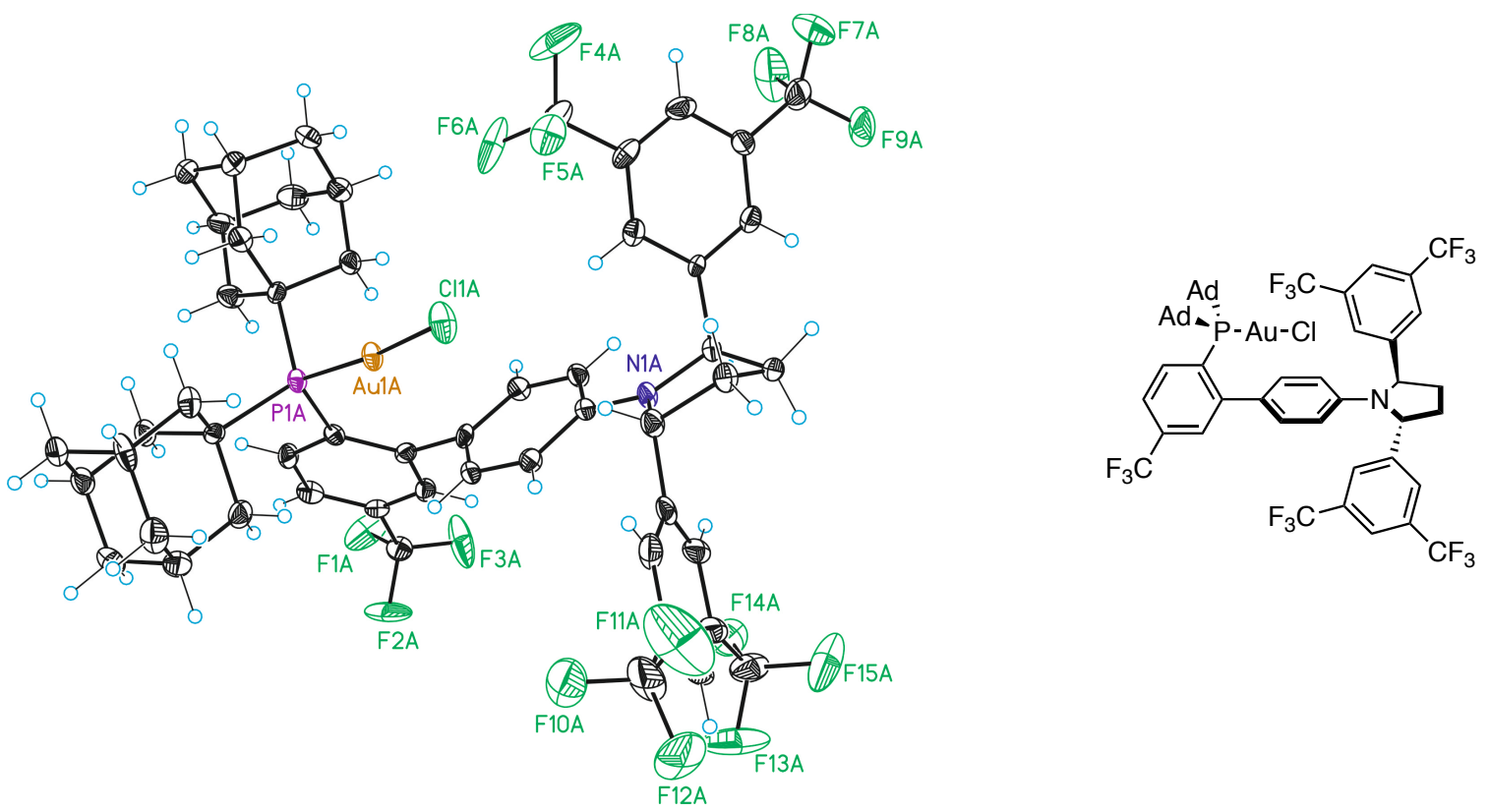


\section{Complex $(S, S)-\mathrm{F}$}

CCDC 1916877

The absolute configuration has been assigned.
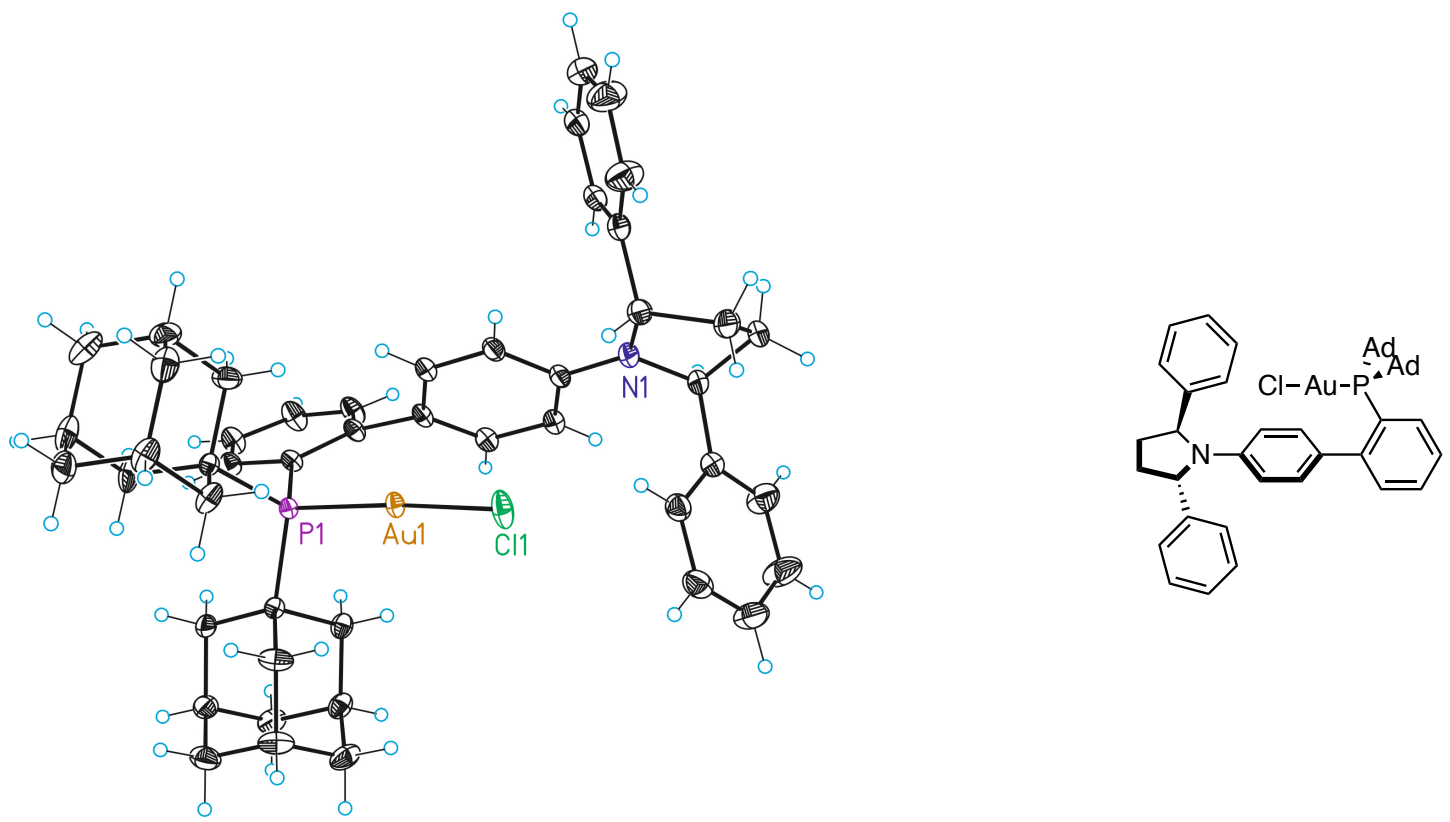

\section{Complex $(R, R)-G$}

CCDC 1916874

The absolute configuration has been assigned.
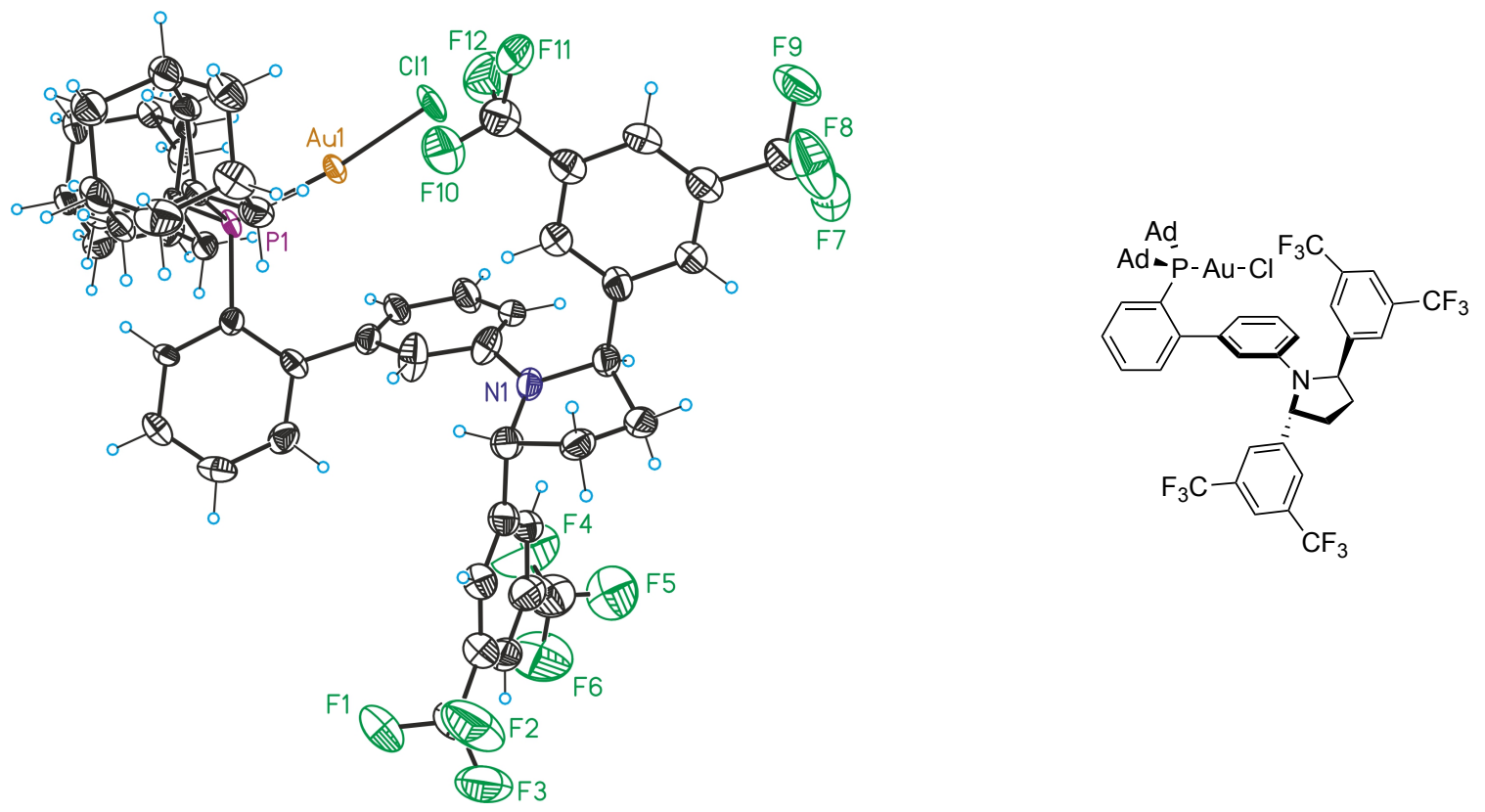
Chloride-bridged digold complex $(R, R)-C_{2}-C l$

CCDC 1916869

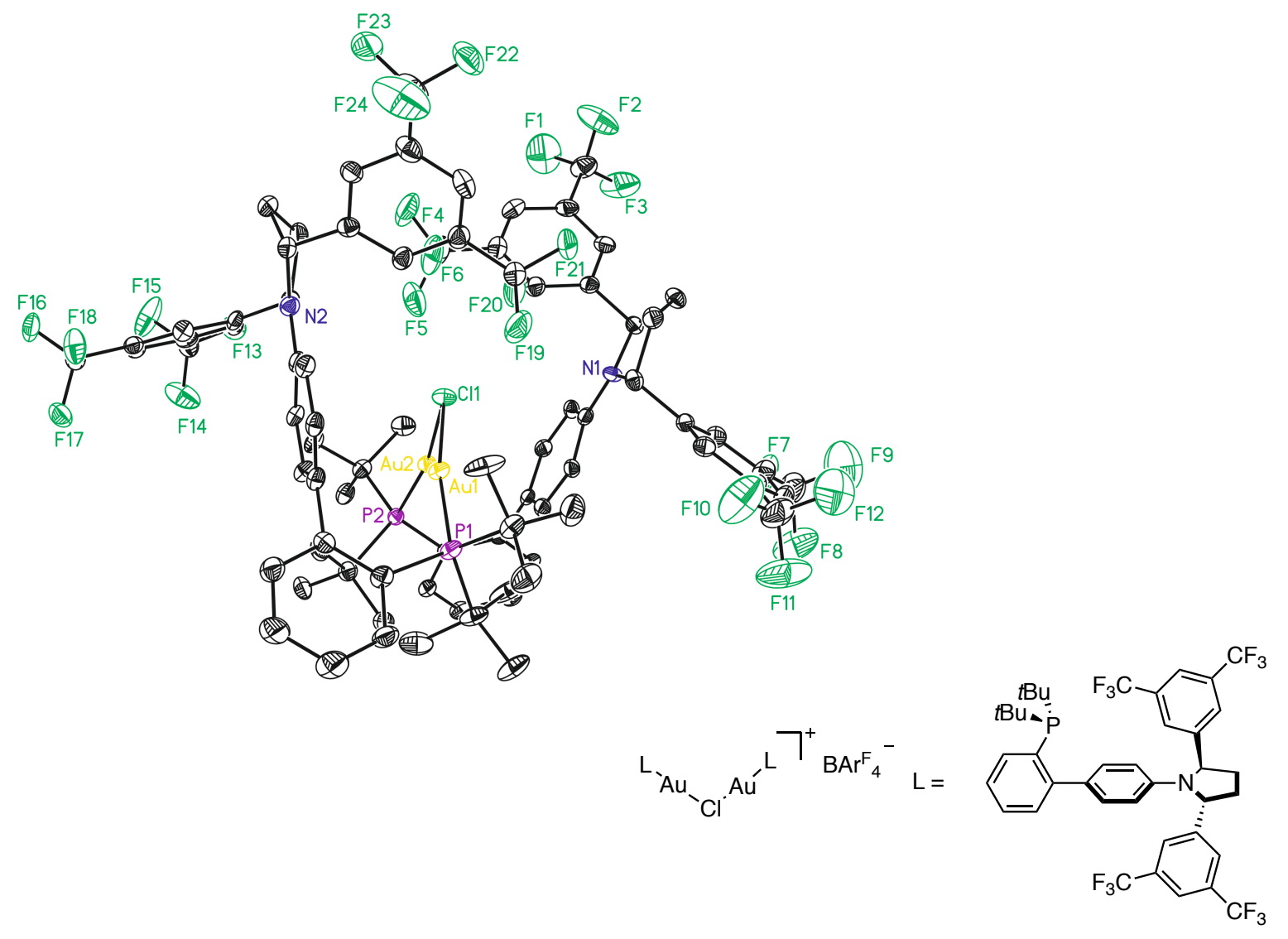




\section{Compound 6o}

CCDC 1916873

The absolute configuration has been assigned. ${ }^{37}$
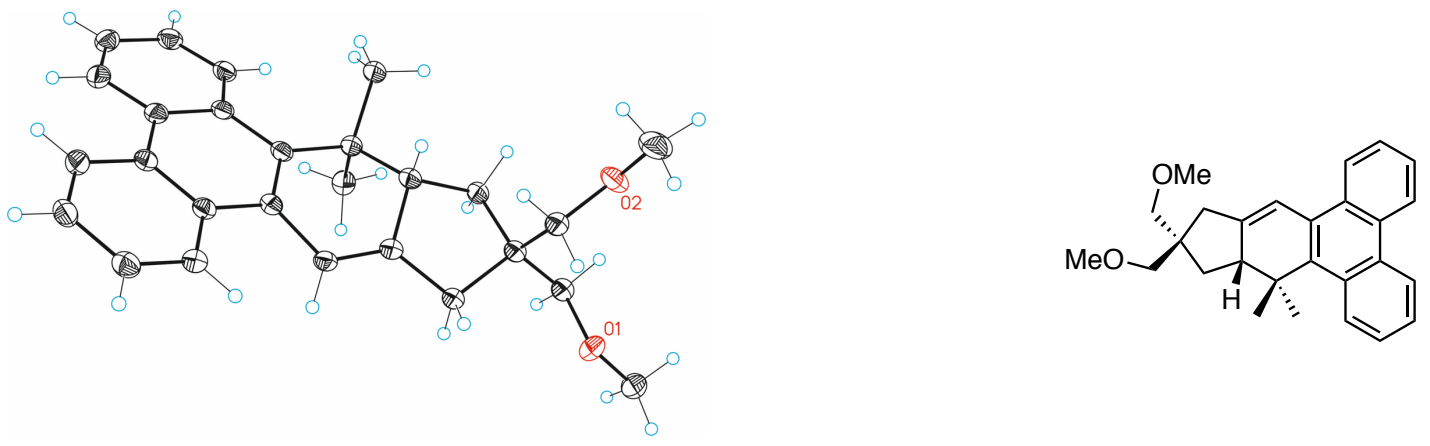

\section{Compound 10k}

CCDC 1916872

The absolute configuration assignment is based in the absolute structure parameter, ${ }^{37}$ which is in accordance with the absolute configuration assignments of compound $\mathbf{1 2}$.
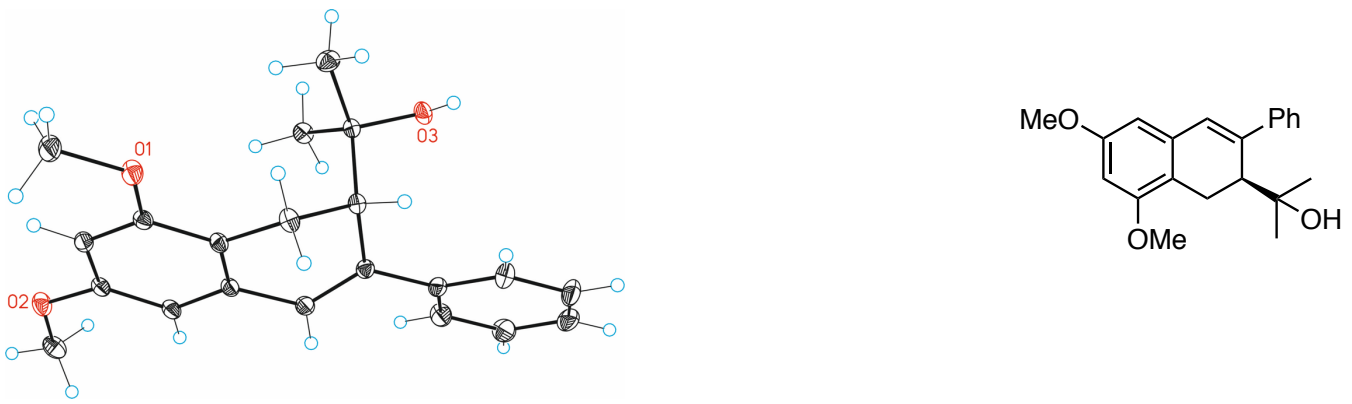

\section{Compound 12}

CCDC 1916875

The absolute configuration has been assigned.
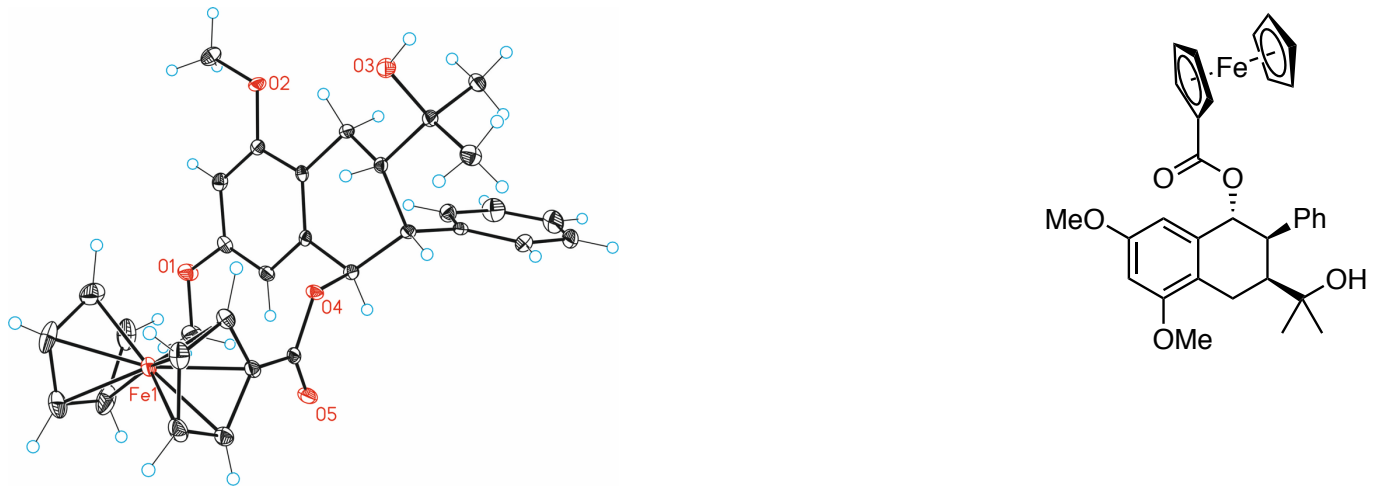


\section{Carexane I}

CCDC 1916866
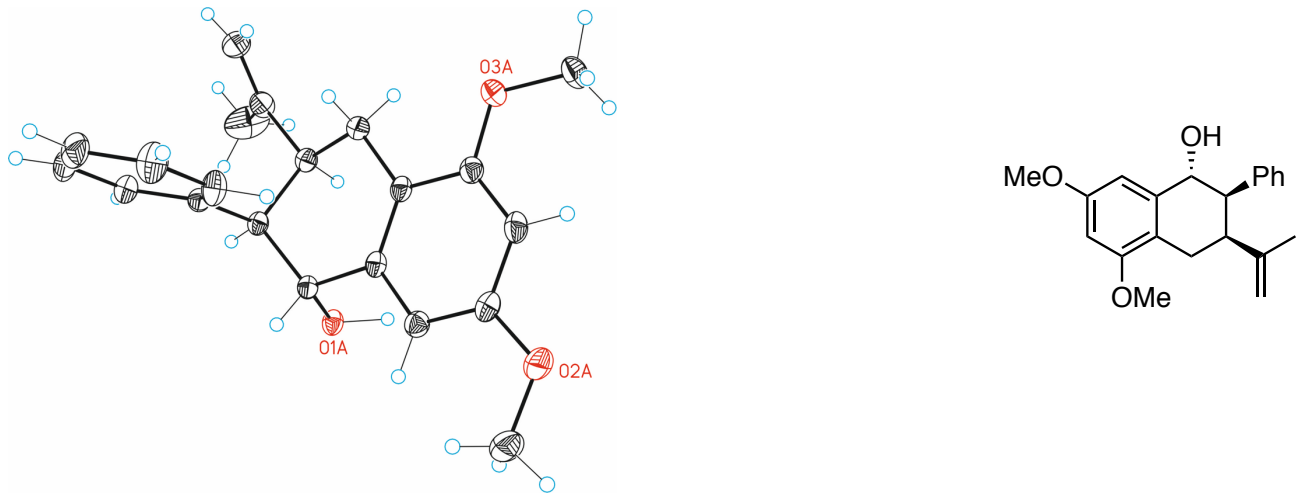

\section{Carexane $\mathbf{P}$}

CCDC 1916867
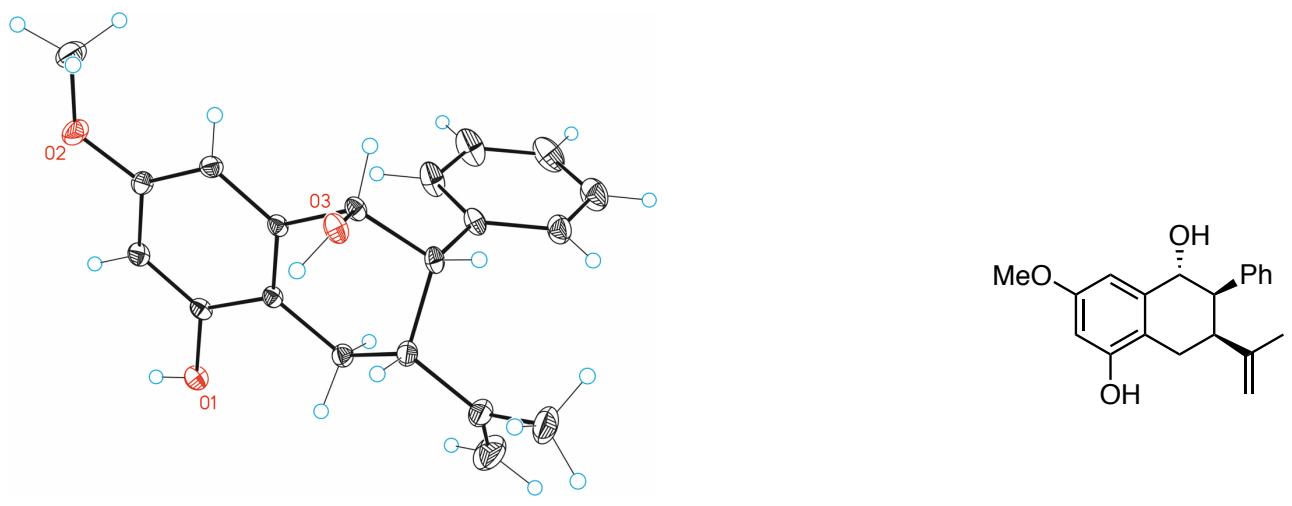

\section{Carexane O}

CCDC 1916868
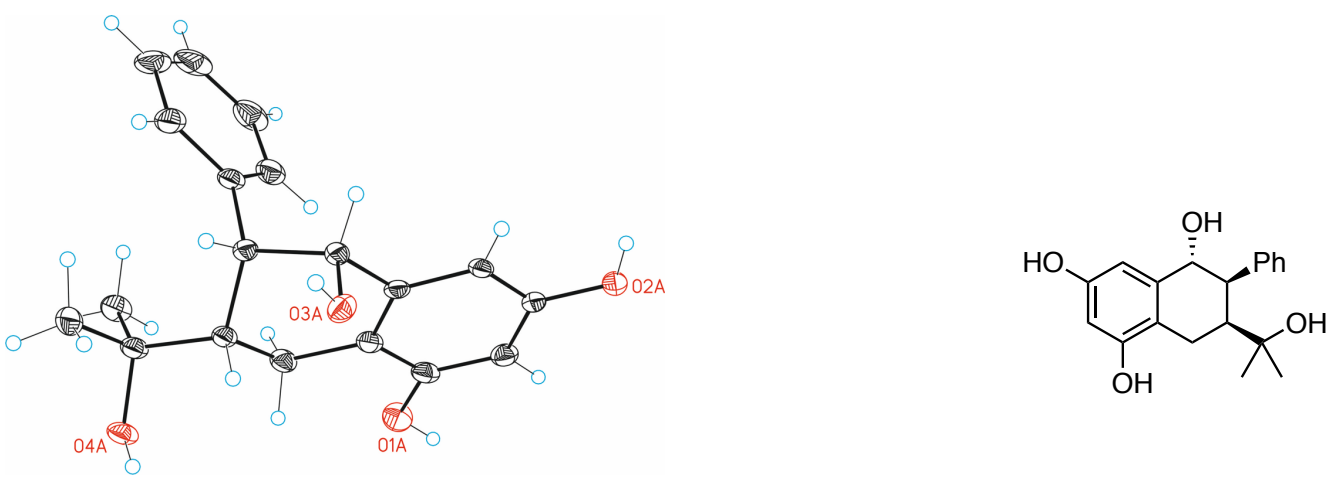


\section{NMR spectra}

${ }^{1} \mathrm{H}$ NMR: $500 \mathrm{MHz}, \mathrm{CDCl}_{3}$, compound 1a

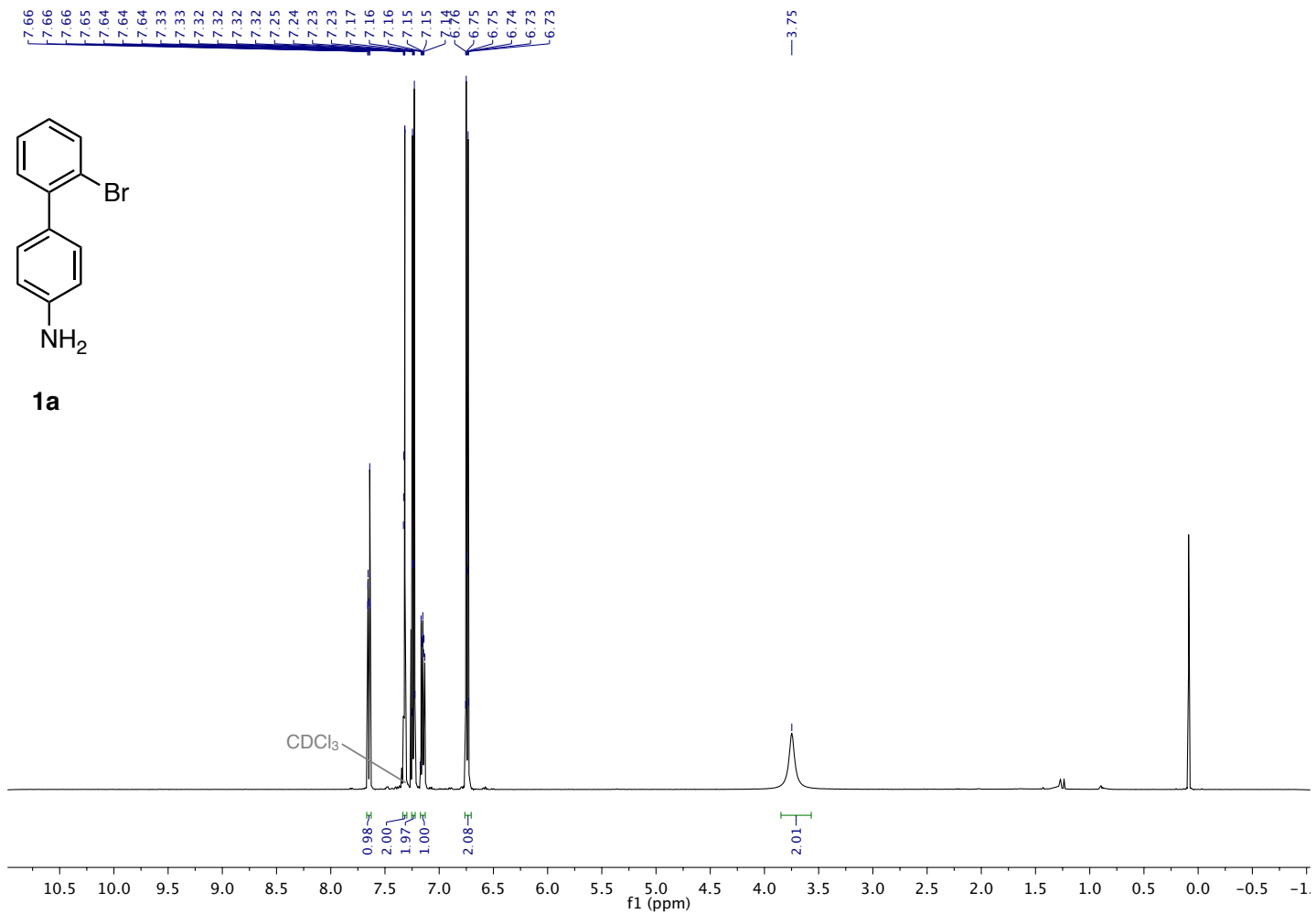

${ }^{13} \mathrm{C}$ NMR: $101 \mathrm{MHz}, \mathrm{CDCl}_{3}$, compound 1a<smiles>Nc1ccc(-c2ccccc2Br)cc1</smiles>

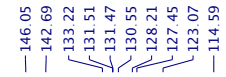

1a 
${ }^{1} \mathrm{H}$ NMR: $400 \mathrm{MHz}, \mathrm{CDCl}_{3}$, 2-bromo-4'-nitro-5-(trifluoromethyl)-1,1'-biphenyl

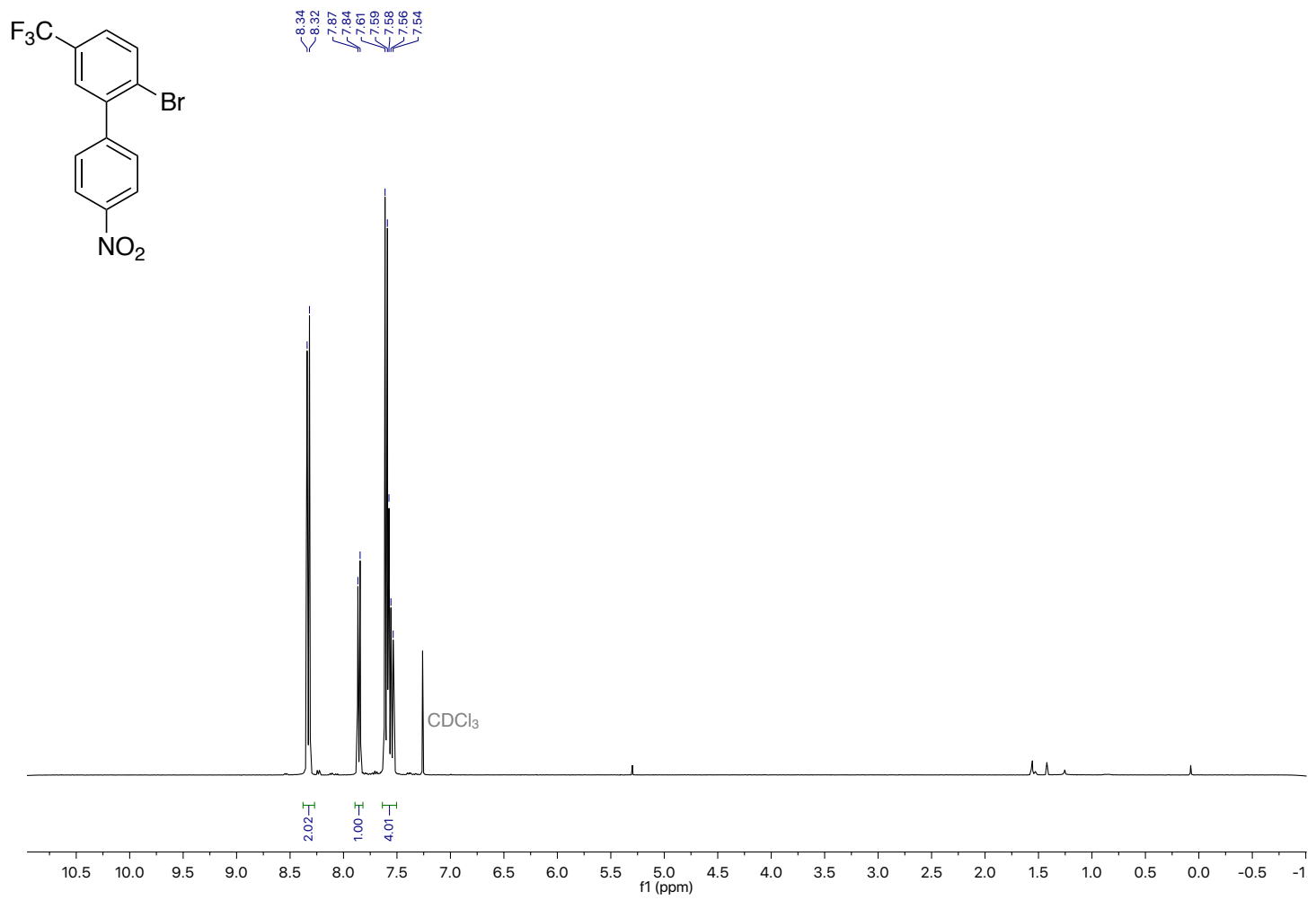

${ }^{13} \mathrm{C}$ NMR: $101 \mathrm{MHz}, \mathrm{CDCl}_{3}$, 2-bromo-4'-nitro-5-(trifluoromethyl)-1,1'-biphenyl

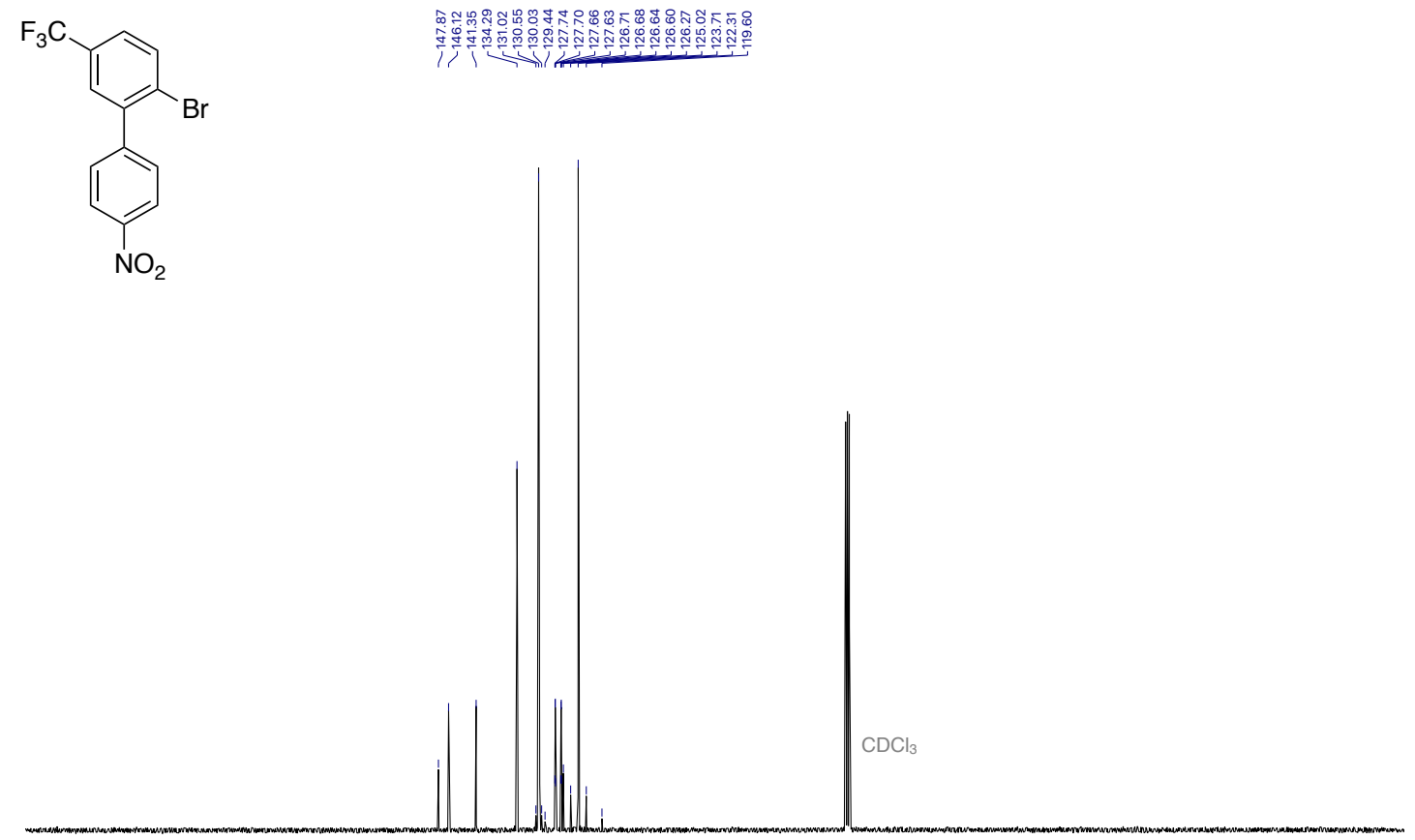

$\begin{array}{lllllllllllllllllllllllll}210 & 200 & 190 & 180 & 170 & 160 & 150 & 140 & 130 & 120 & 110 & \begin{array}{c}100 \\ \mathrm{f}(\mathrm{ppm})\end{array} & 90 & 80 & 70 & 60 & 50 & 40 & 30 & 20 & 10 & 0 & -10\end{array}$ 
${ }^{19} \mathrm{~F}\left\{{ }^{1} \mathrm{H}\right\}$ NMR: $376 \mathrm{MHz}, \mathrm{CDCl}_{3}$, 2-bromo-4'-nitro-5-(trifluoromethyl)-1,1'-biphenyl<smiles>[B]c1ccc(C(F)(F)F)cc1-c1ccc([N+](=O)[O-])cc1</smiles>
怘

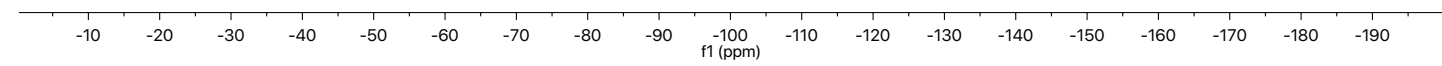

${ }^{1} \mathrm{H}$ NMR: $400 \mathrm{MHz}, \mathrm{CDCl}_{3}$, compound $\mathbf{1 b}$

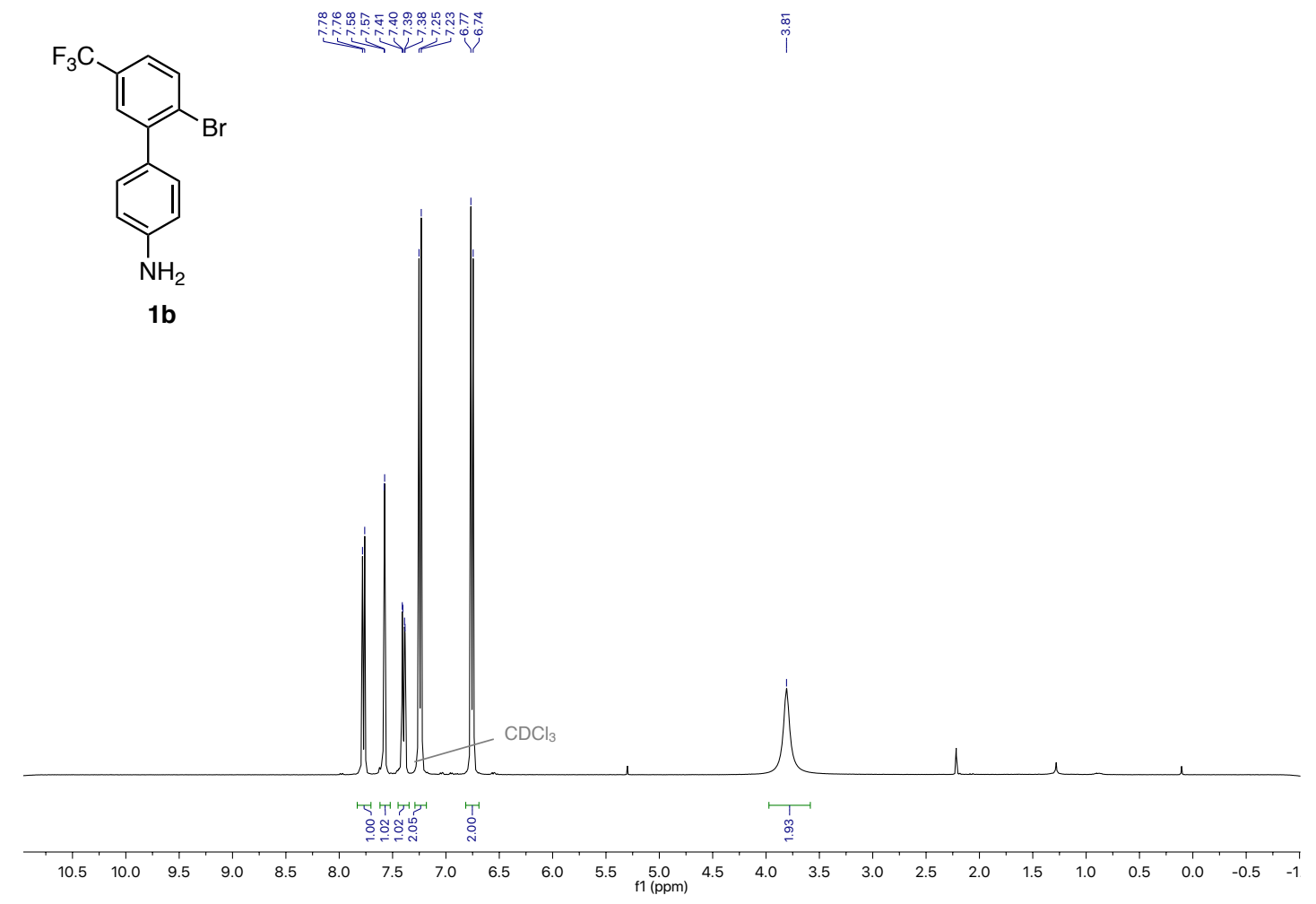


${ }^{13} \mathrm{C}$ NMR: $101 \mathrm{MHz}, \mathrm{CDCl}_{3}$, compound 1b

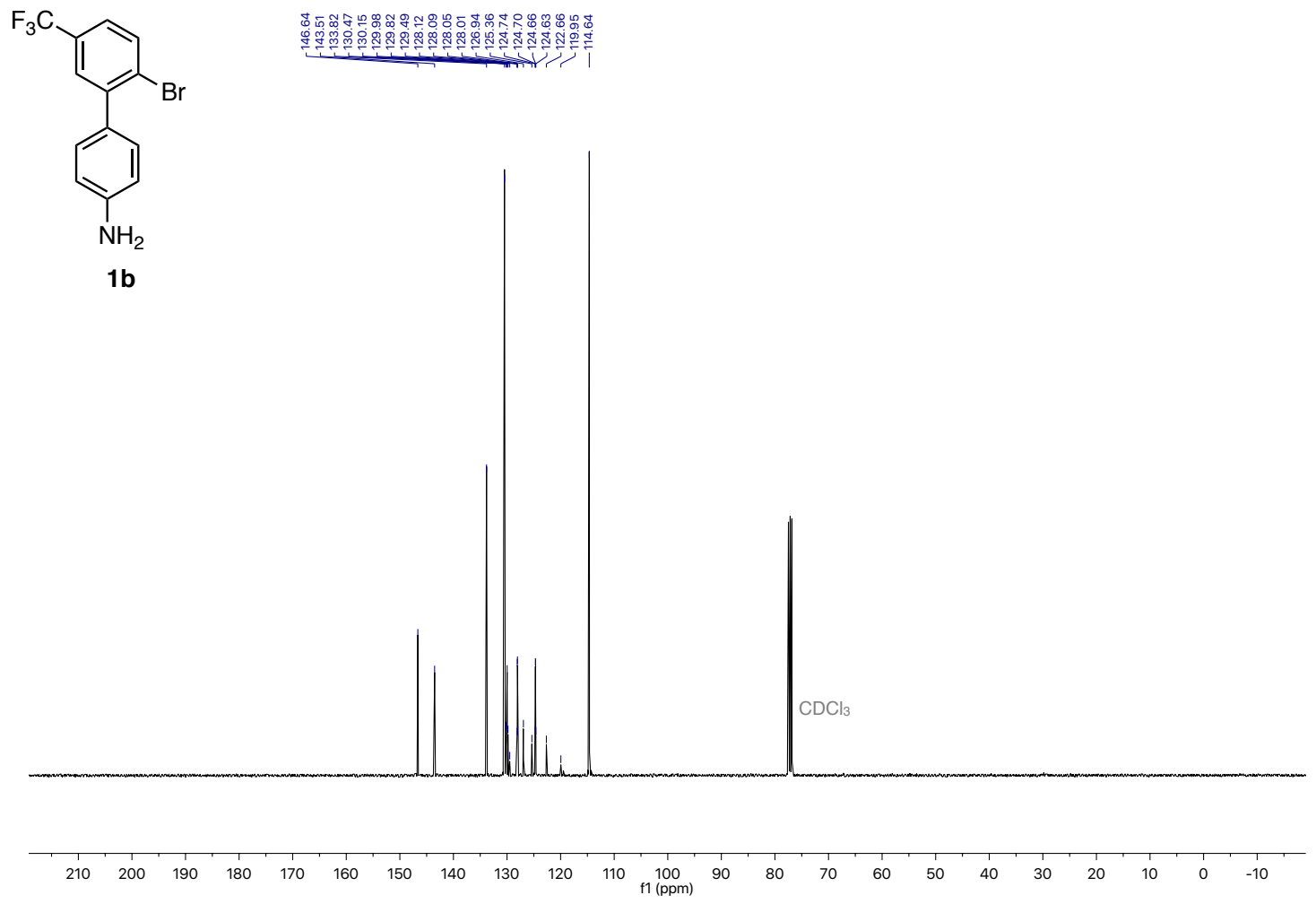

${ }^{19} \mathrm{~F}\left\{{ }^{1} \mathrm{H}\right\}$ NMR: $376 \mathrm{MHz}, \mathrm{CDCl}_{3}$, compound 1b

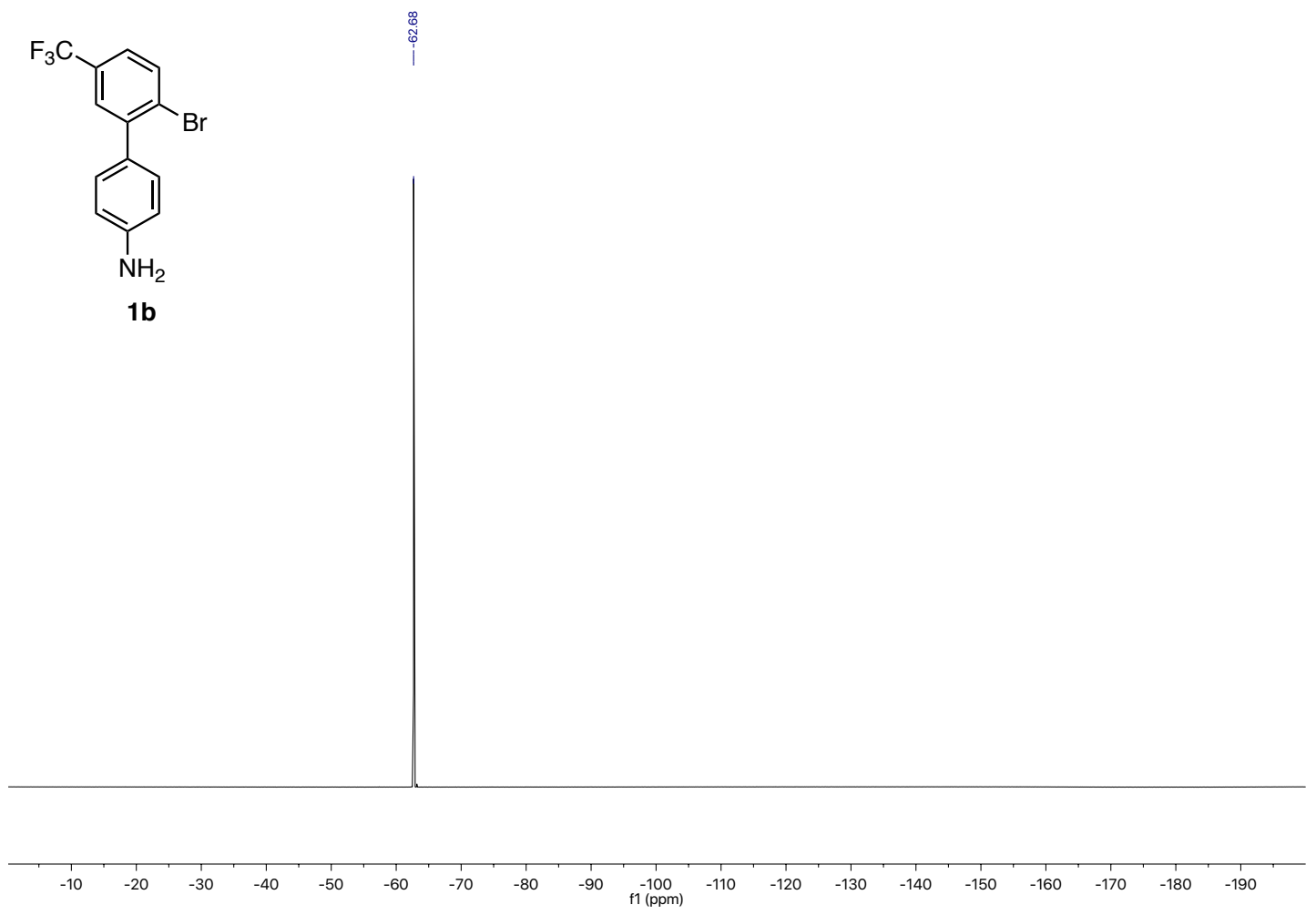


${ }^{1} \mathrm{H}$ NMR: $400 \mathrm{MHz}, \mathrm{CDCl}_{3}$, compound 1c

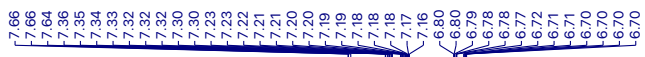<smiles>Nc1cccc(-c2ccccc2Br)c1</smiles>

$1 c$

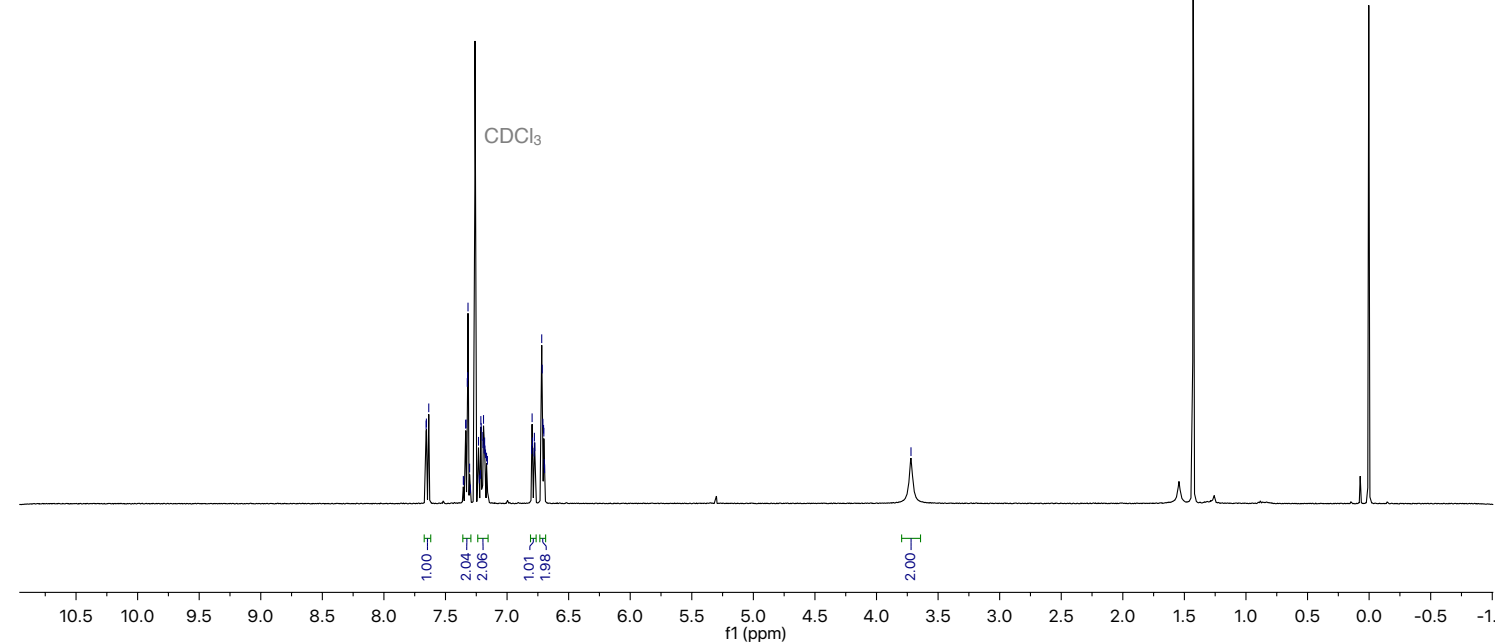

${ }^{13} \mathrm{C}$ NMR: $126 \mathrm{MHz}, \mathrm{CDCl}_{3}$, compound 1c

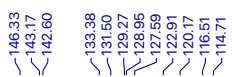<smiles>Nc1cccc(-c2ccccc2Br)c1</smiles> 
${ }^{1} \mathrm{H}$ NMR: $500 \mathrm{MHz}, \mathrm{CDCl}_{3}, 1$-([1,1':3',1"-terphenyl]-5'-yl)prop-2-en-1-ol

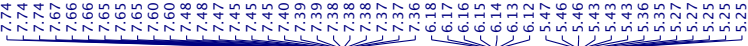

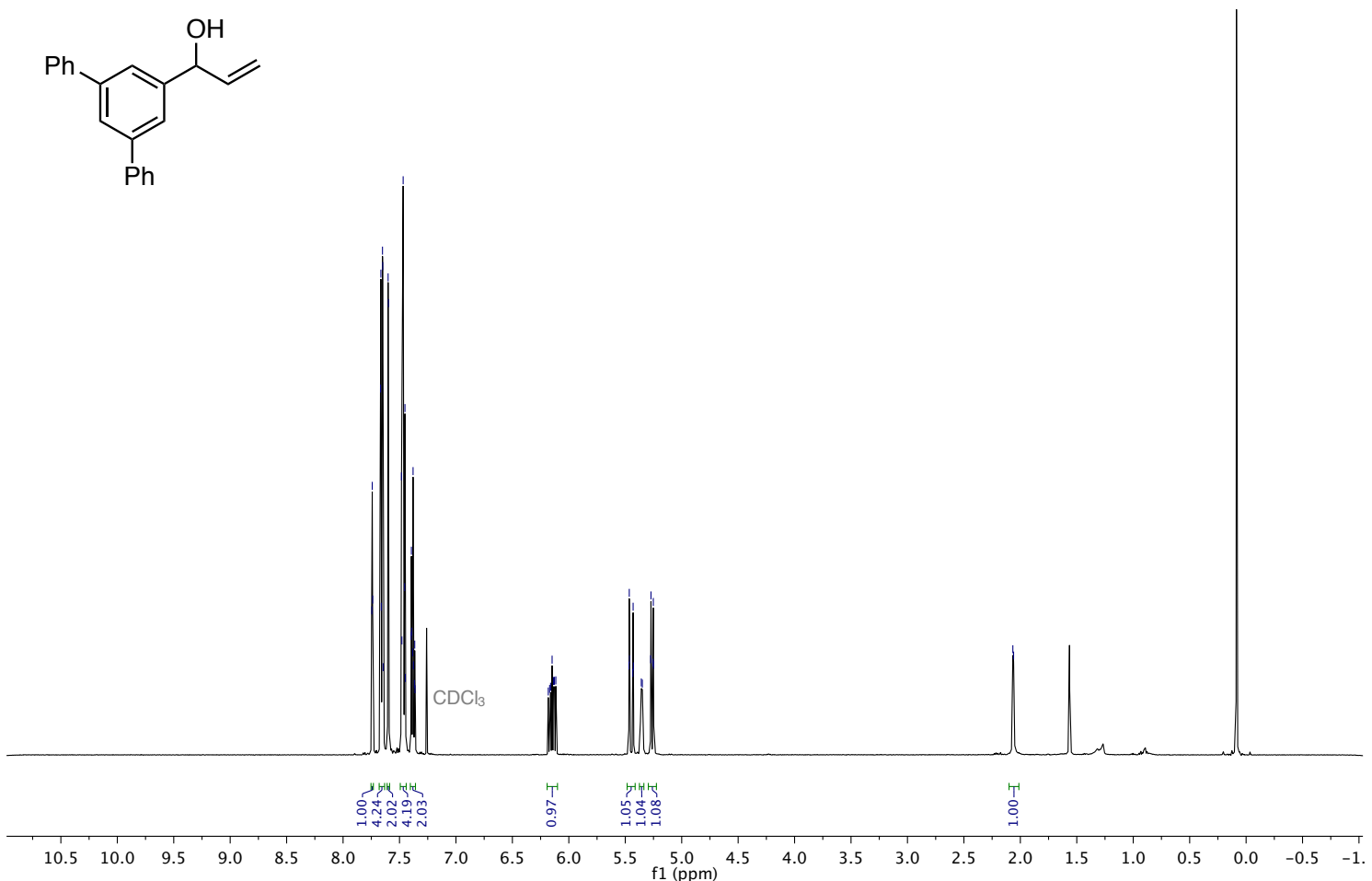

${ }^{13} \mathrm{C}$ NMR: $126 \mathrm{MHz}, \mathrm{CDCl}_{3}, 1-([1,1 ': 3$ ',1"-terphenyl]-5'-yl)prop-2-en-1-ol<smiles>C=CC(O)c1cc(-c2ccccc2)cc(-c2ccccc2)c1</smiles>

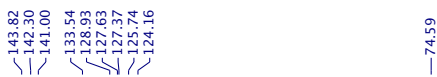


${ }^{1} \mathrm{H}$ NMR: $500 \mathrm{MHz}, \mathrm{CDCl}_{3},(1 S, 4 S, E)-1,4-\operatorname{di}\left(\left[1,1^{\prime}: 3\right.\right.$ ',1"-terphenyl]-5'-yl)but-2-ene-1,4-diol

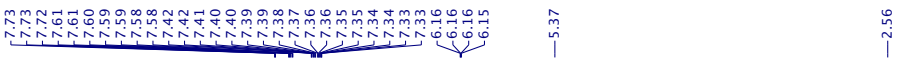

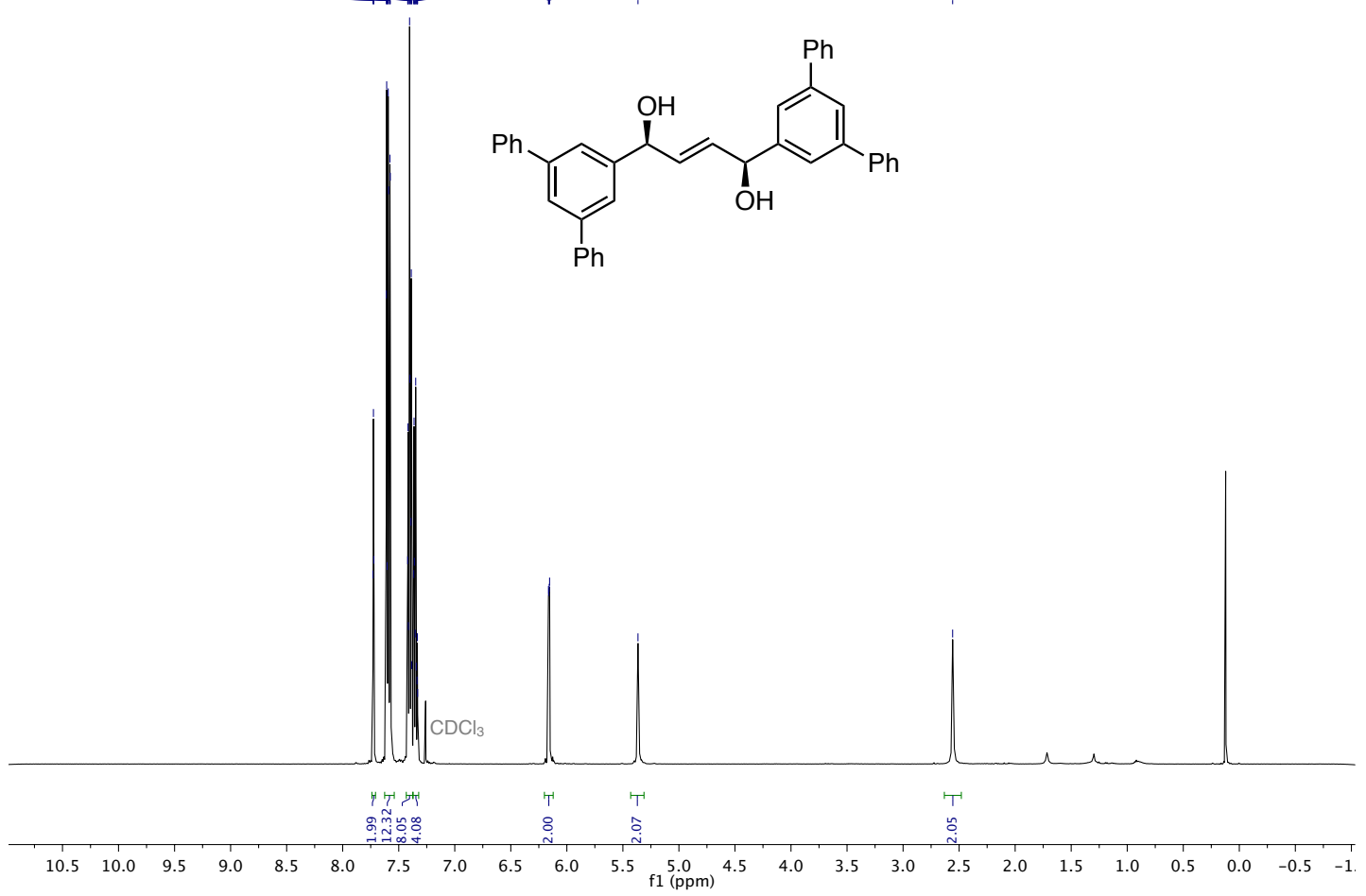

${ }^{13} \mathrm{C}$ NMR: $126 \mathrm{MHz}, \mathrm{CDCl}_{3},(1 S, 4 S, E)-1,4-\operatorname{di}\left(\left[1,1^{\prime}: 3\right.\right.$ ',1"-terphenyl]-5'-yl)but-2-ene-1,4-diol

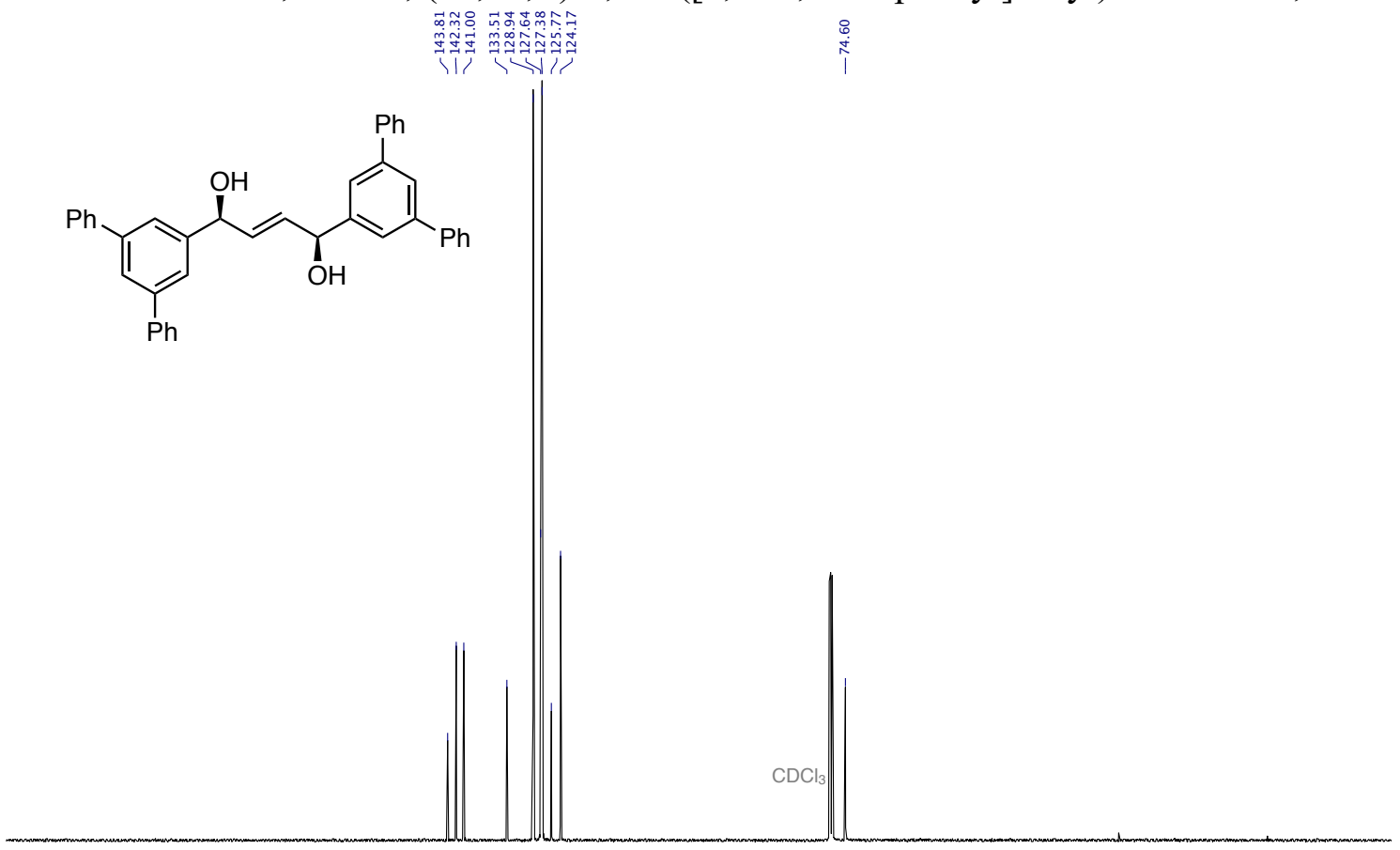

$\begin{array}{lllllllllllllllllllllllllllllll}20 & 210 & 200 & 190 & 180 & 170 & 160 & 150 & 140 & 130 & 120 & 110 & 100 & 90 & 80 & 70 & 60 & 50 & 40 & 30 & 20 & 10 & 0 & -10 & -2\end{array}$ 
${ }^{1} \mathrm{H}$ NMR: $400 \mathrm{MHz}, \mathrm{CDCl}_{3}$, compound 2a

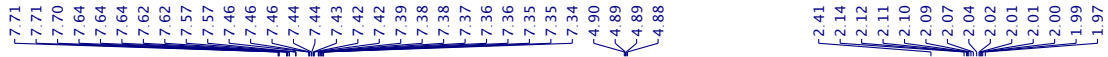

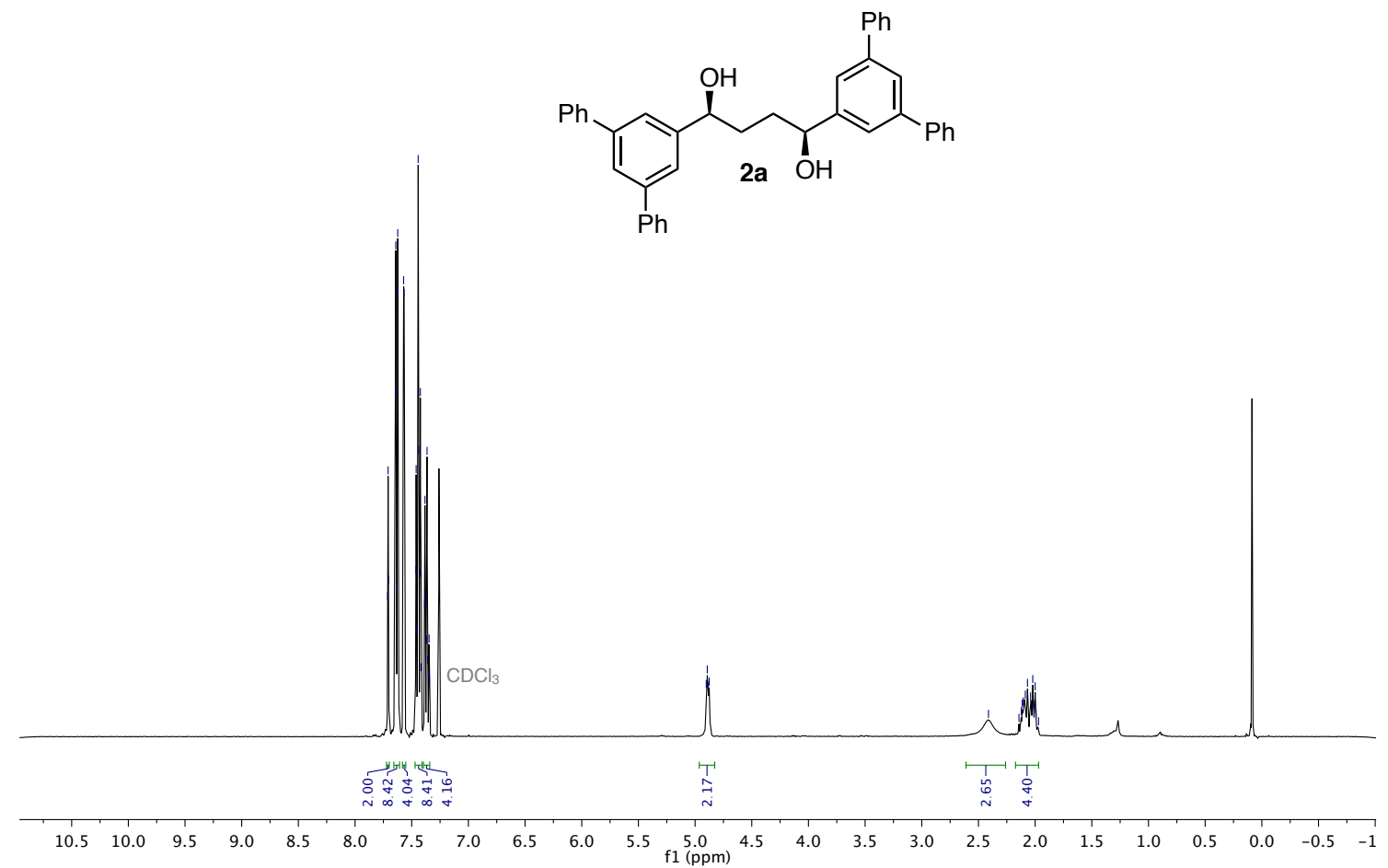

${ }^{13} \mathrm{C}$ NMR: $101 \mathrm{MHz}, \mathrm{CDCl}_{3}$, compound 2a

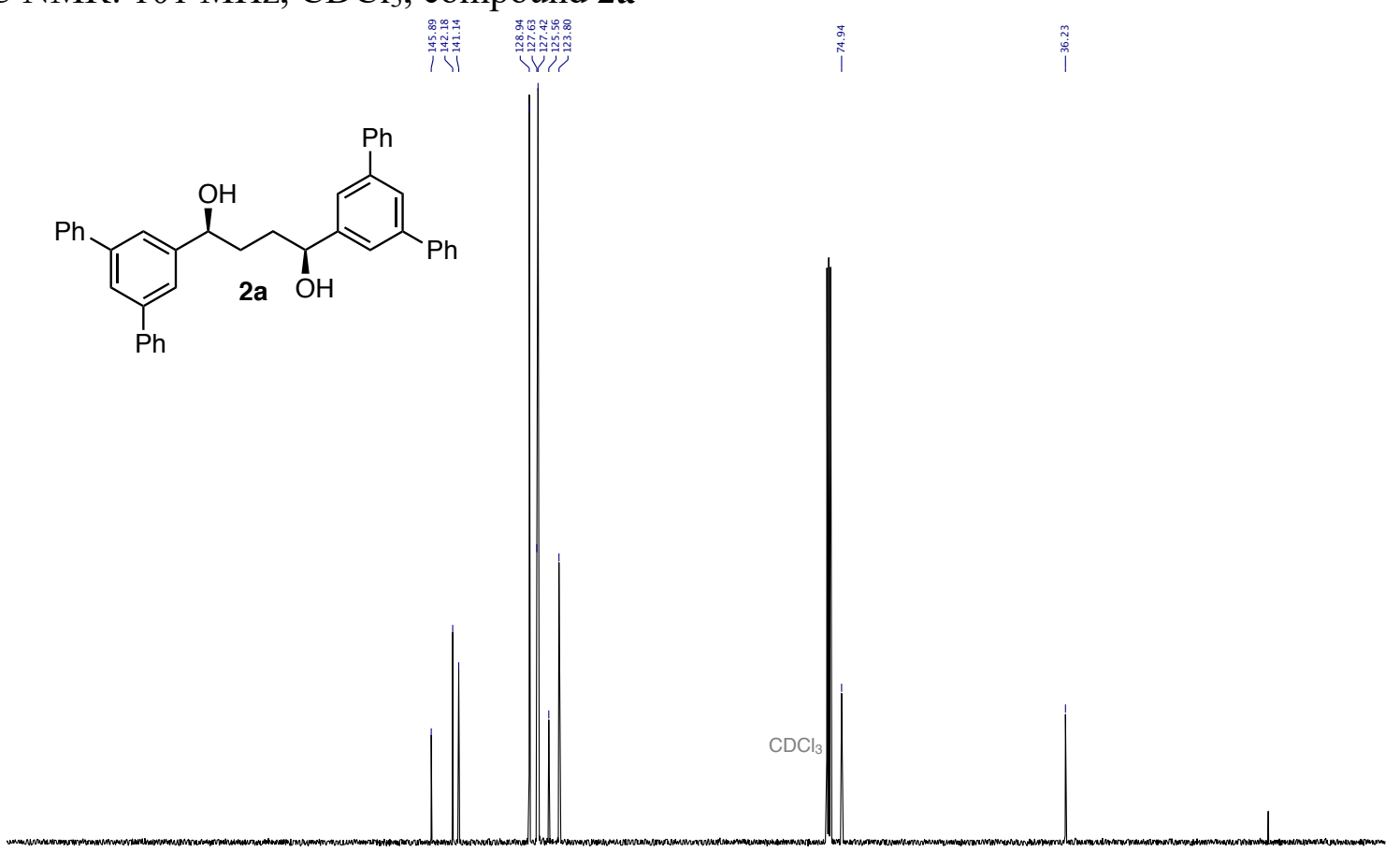

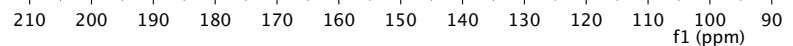


${ }^{1} \mathrm{H}$ NMR: $500 \mathrm{MHz}, \mathrm{CD}_{3} \mathrm{OD}, 1$-(perfluorophenyl)prop-2-en-1-ol 等<smiles>C=CC(O)c1c(F)c(F)c(F)c(F)c1F</smiles>
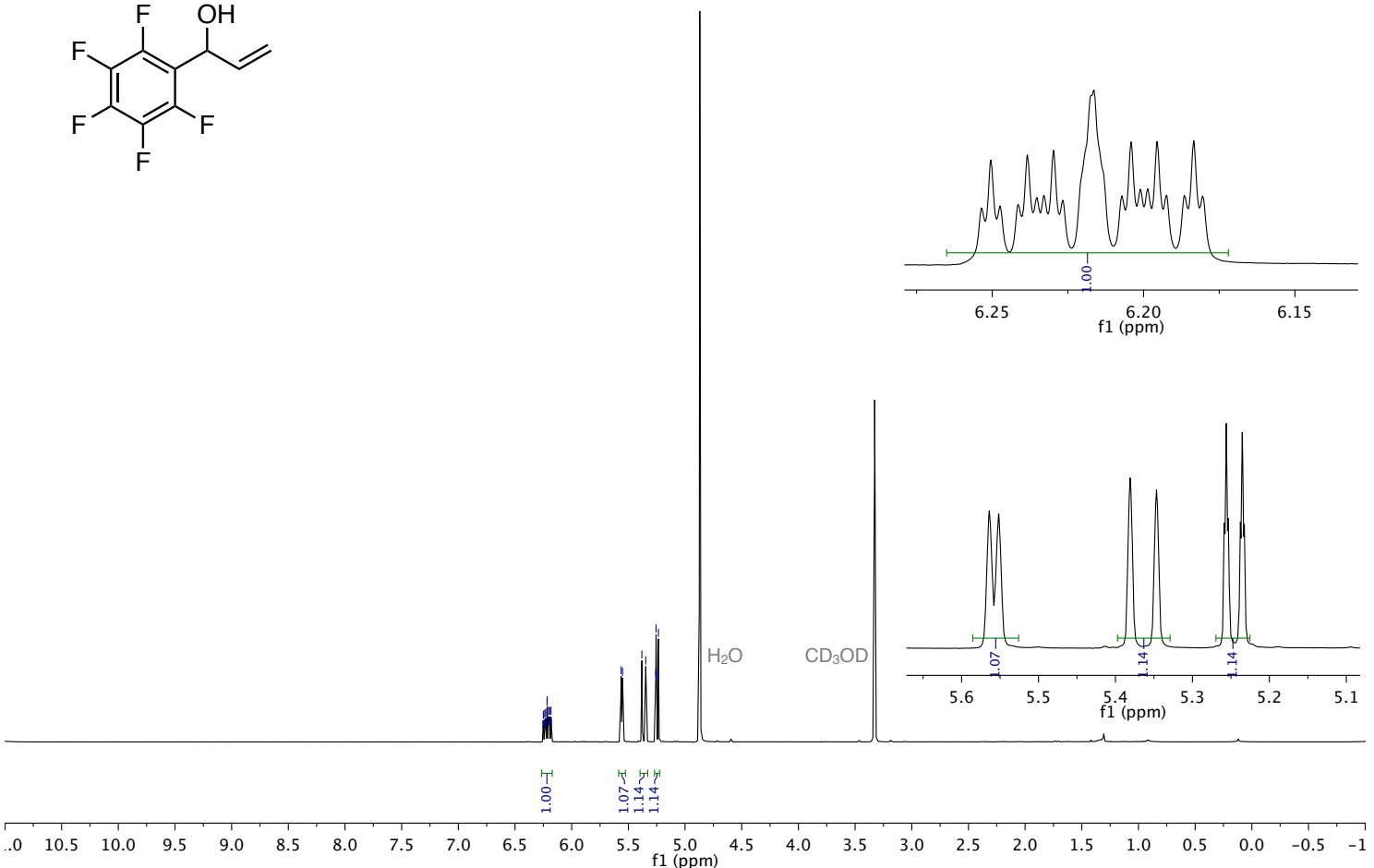

${ }^{13} \mathrm{C}$ NMR: $101 \mathrm{MHz}, \mathrm{CD}_{3} \mathrm{OD}, 1-($ perfluorophenyl)prop-2-en-1-ol

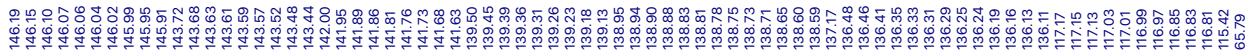<smiles>C=CC(O)c1c(F)c(F)c(F)c(F)c1F</smiles>
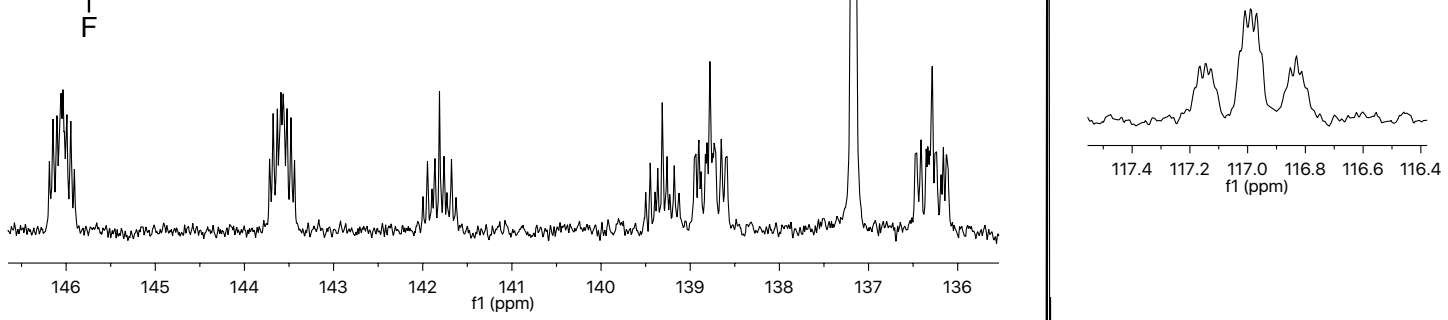

$210 \quad 200 \quad 190 \quad 180 \quad 170 \quad 160$ 
${ }^{19} \mathrm{~F}\left\{{ }^{1} \mathrm{H}\right\}$ NMR: $471 \mathrm{MHz}, \mathrm{CD}_{3} \mathrm{OD}$, compound 1-(perfluorophenyl)prop-2-en-1-ol<smiles>C=CC(O)c1c(F)c(F)c(F)c(F)c1F</smiles>

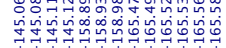
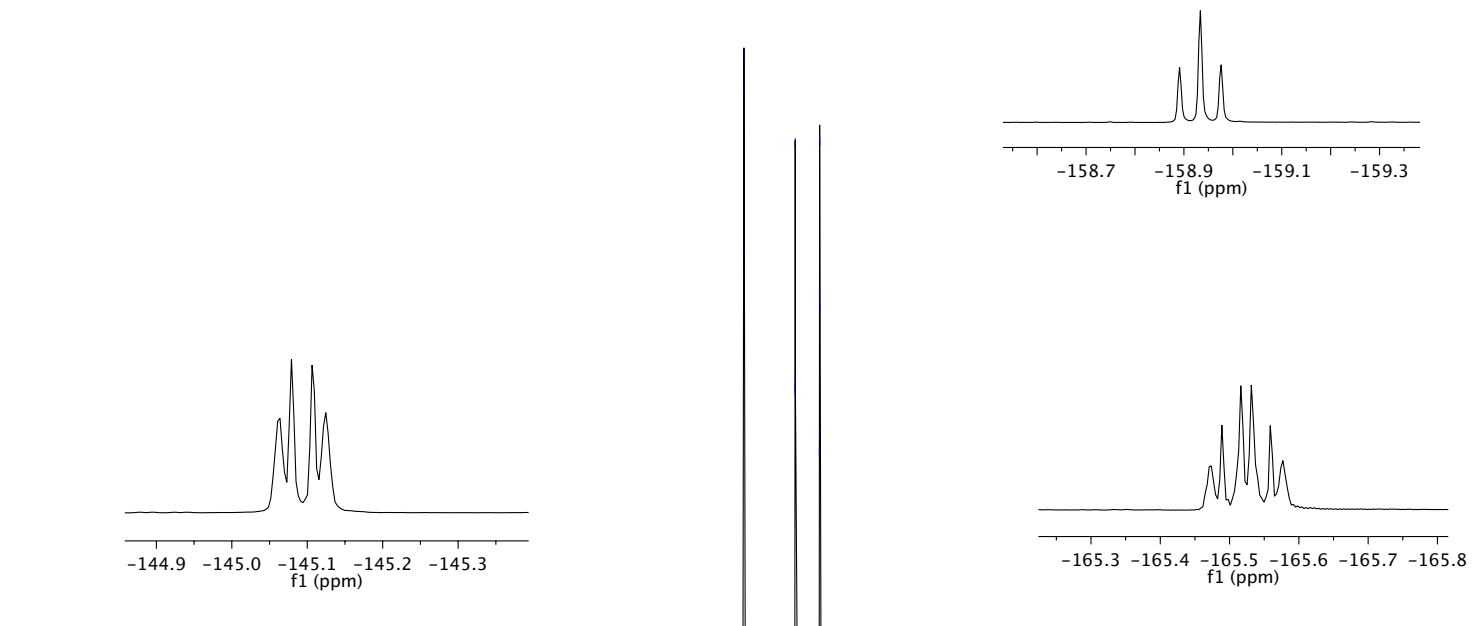

40

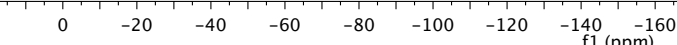

${ }^{1} \mathrm{H}$ NMR: $500 \mathrm{MHz}, \mathrm{CD}_{2} \mathrm{Cl}_{2}$, (1S,4S,E)-1,4-bis(perfluorophenyl)but-2-ene-1,4-diol

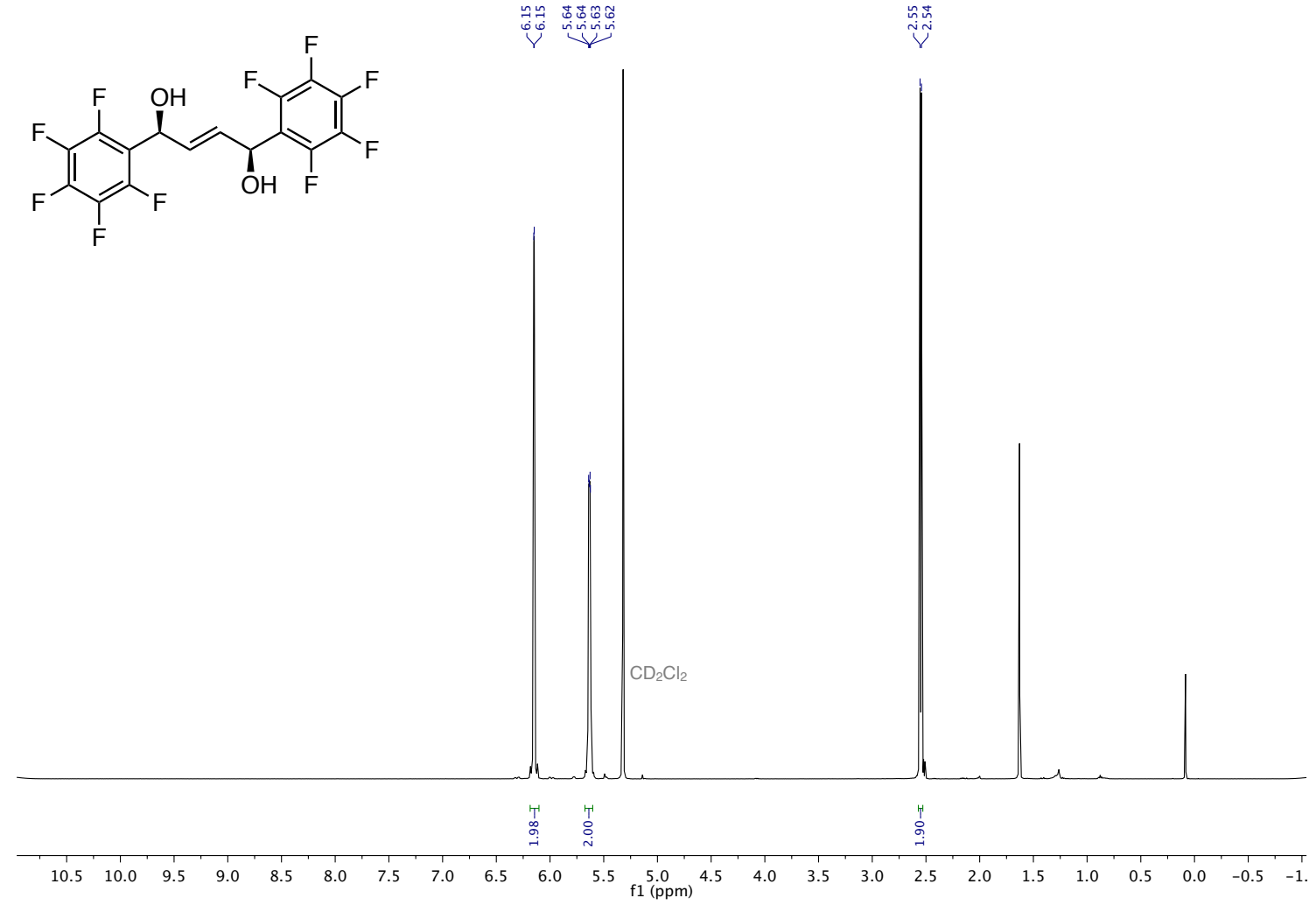


${ }^{13} \mathrm{C}$ NMR: $126 \mathrm{MHz}, \mathrm{CD}_{2} \mathrm{Cl}_{2},(1 S, 4 S, E)$-1,4-bis(perfluorophenyl)but-2-ene-1,4-diol

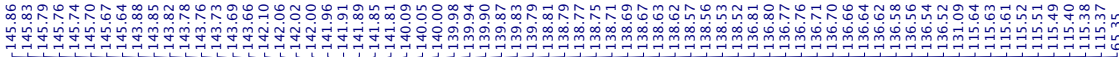

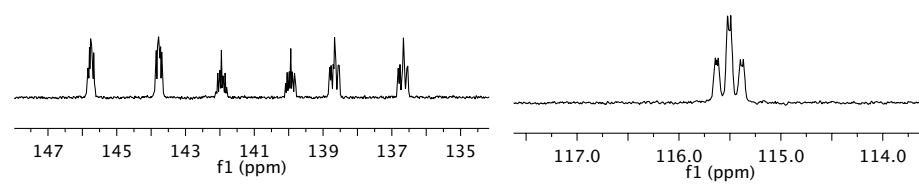<smiles>OC(/C=C/C(O)c1c(F)c(F)c(F)c(F)c1F)c1c(F)c(F)c(F)c(F)c1F</smiles>
Hull

${ }^{19} \mathrm{~F}\left\{{ }^{1} \mathrm{H}\right\}$ NMR: $471 \mathrm{MHz}, \mathrm{CD}_{2} \mathrm{Cl}_{2},(1 S, 4 S, E)$-1,4-bis(perfluorophenyl)but-2-ene-1,4-diol<smiles>OC(/C=C/C(O)c1c(F)c(F)c(F)c(F)c1F)c1c(F)c(F)c(F)c(F)c1F</smiles>
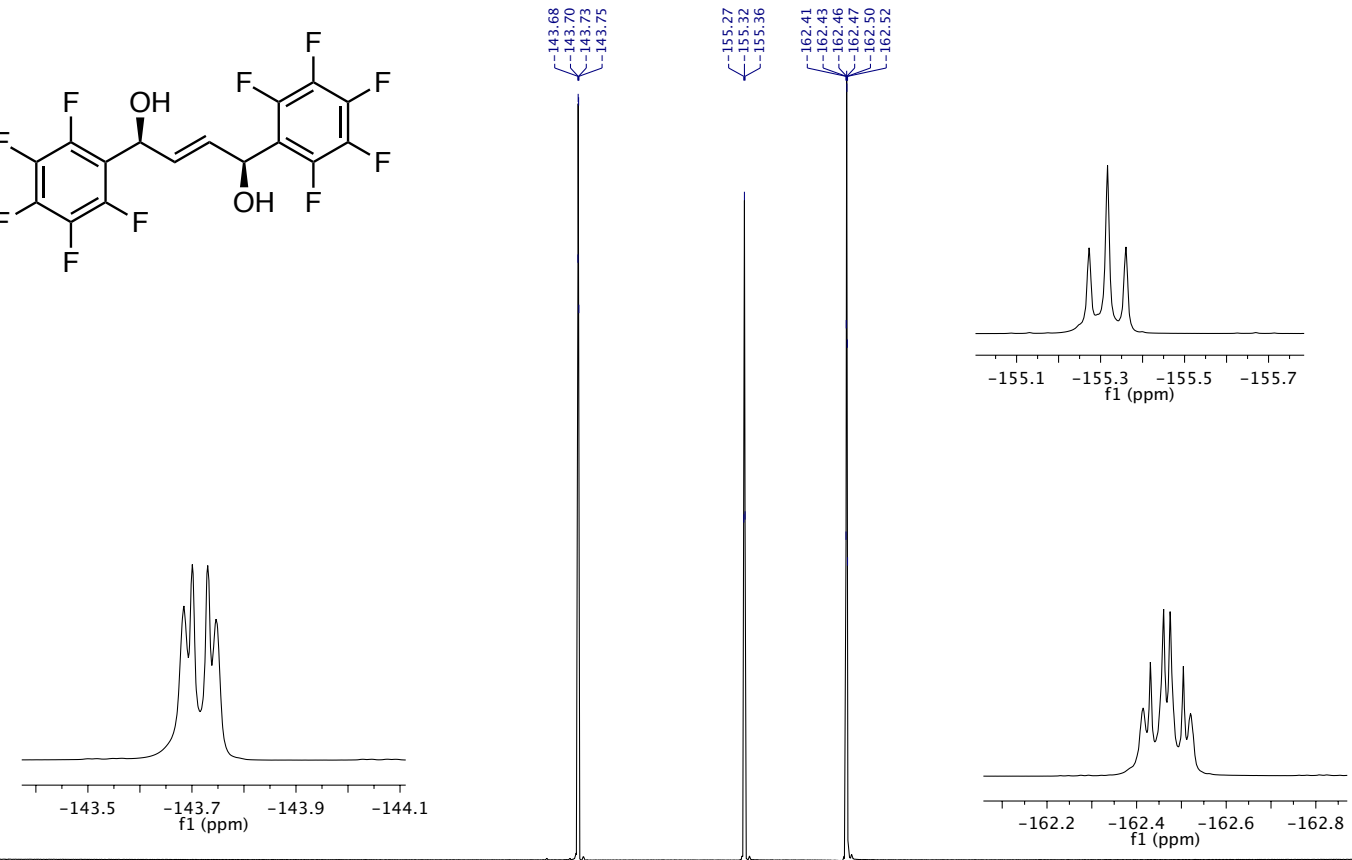

$\begin{array}{llllllllllllllllllll}-105 & -110 & -115 & -120 & -125 & -130 & -135 & -140 & -145 & \begin{array}{c}-150 \\ \mathrm{f} 1(\mathrm{ppm})\end{array} & -155 & -160 & -165 & -170 & -175 & -180 & -185 & -190 & -195\end{array}$ 
${ }^{1} \mathrm{H}$ NMR: $500 \mathrm{MHz}, \mathrm{CDCl}_{3}$, compound $\mathbf{2 b}$<smiles>OC(CCC(O)c1c(F)c(F)c(F)c(F)c1F)c1c(F)c(F)c(F)c(F)c1F</smiles>

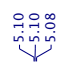

๕5ㅁำ

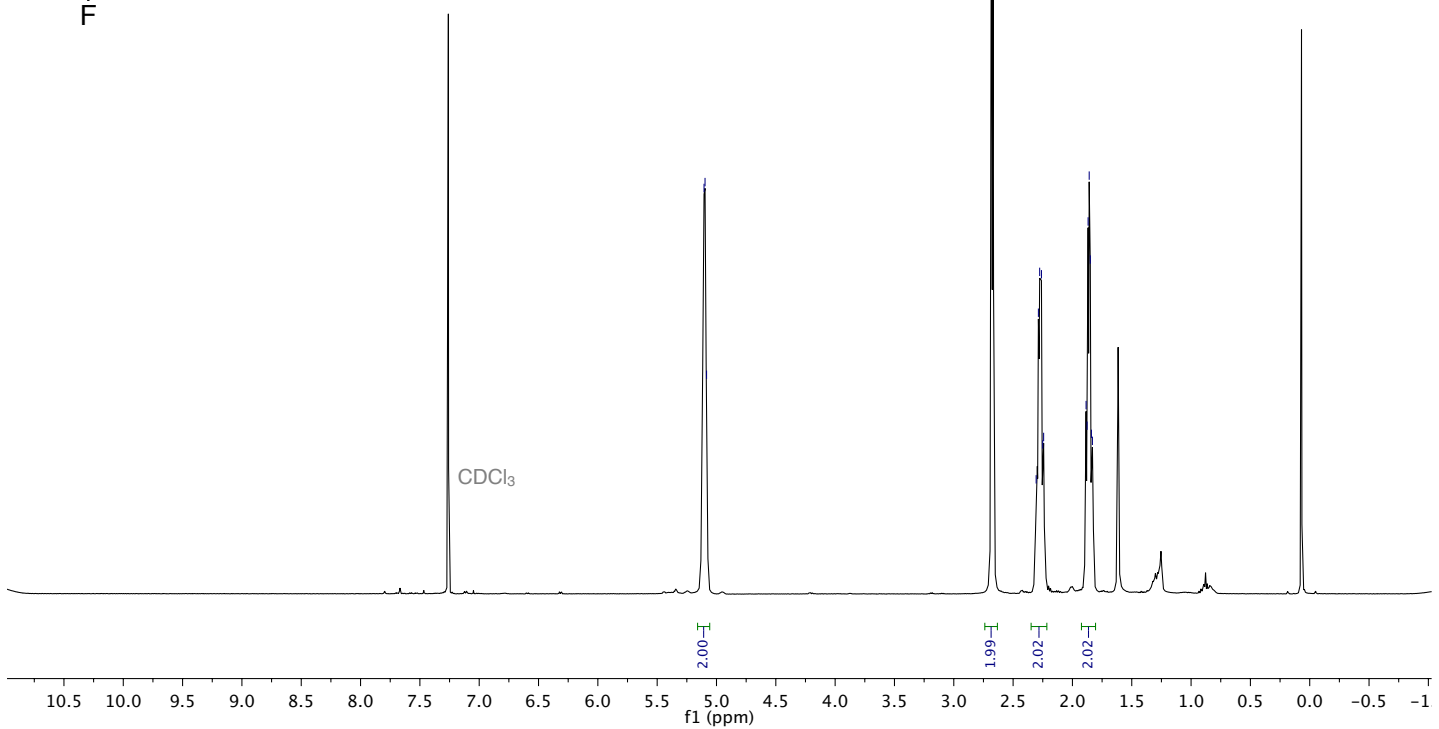

${ }^{13} \mathrm{C}$ NMR: $126 \mathrm{MHz}, \mathrm{CDCl}_{3}$, compound ab

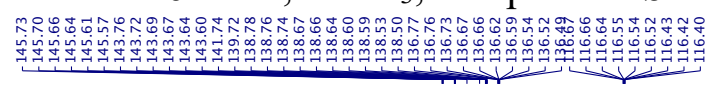

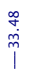
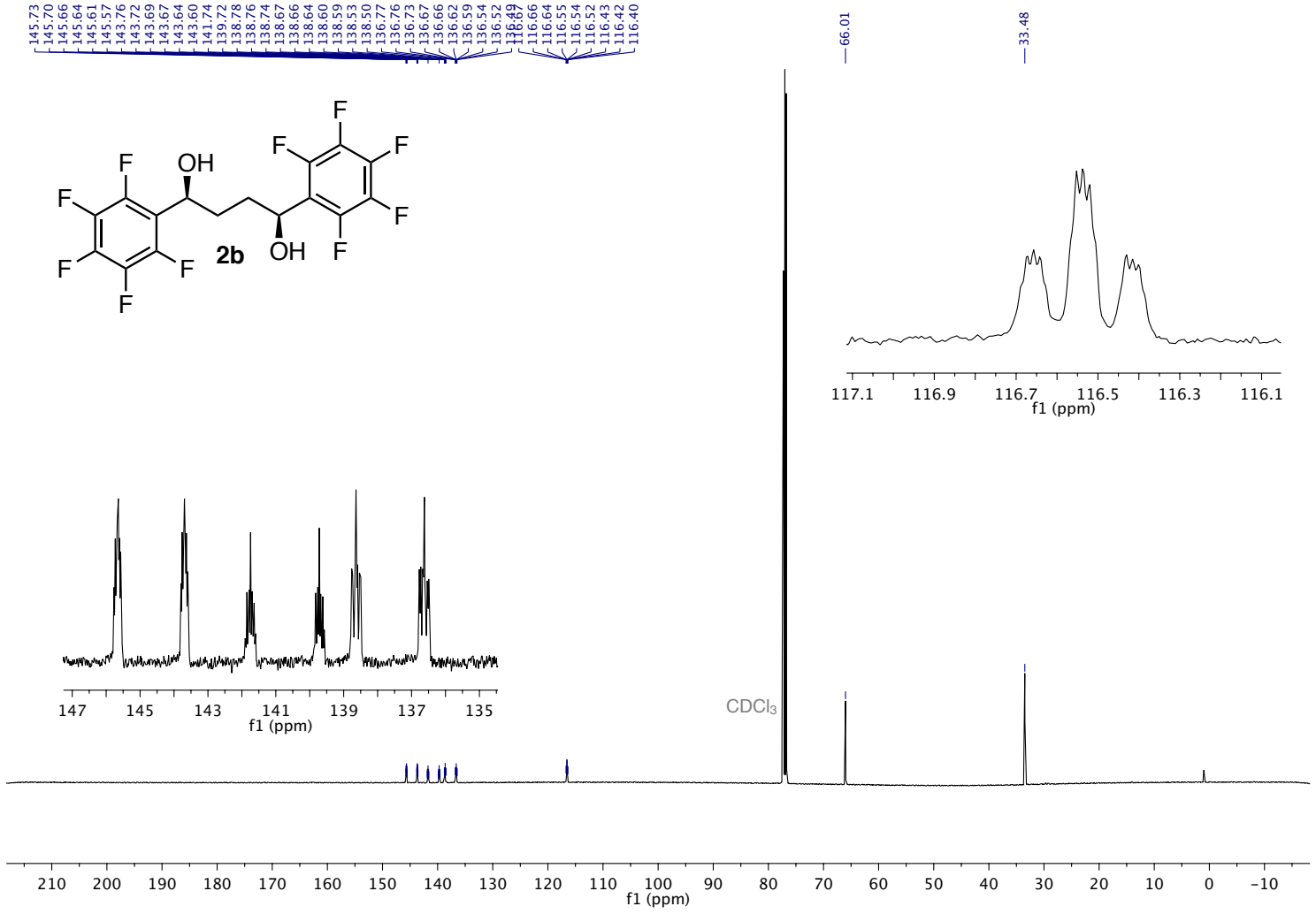

S196 
${ }^{19} \mathrm{~F}\left\{{ }^{1} \mathrm{H}\right\}$ NMR: $376 \mathrm{MHz}, \mathrm{CD}_{3} \mathrm{OD}$, compound 2b<smiles>OC(CCC(O)c1c(F)c(F)c(F)c(F)c1F)c1c(F)c(F)c(F)c(F)c1F</smiles>

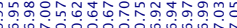
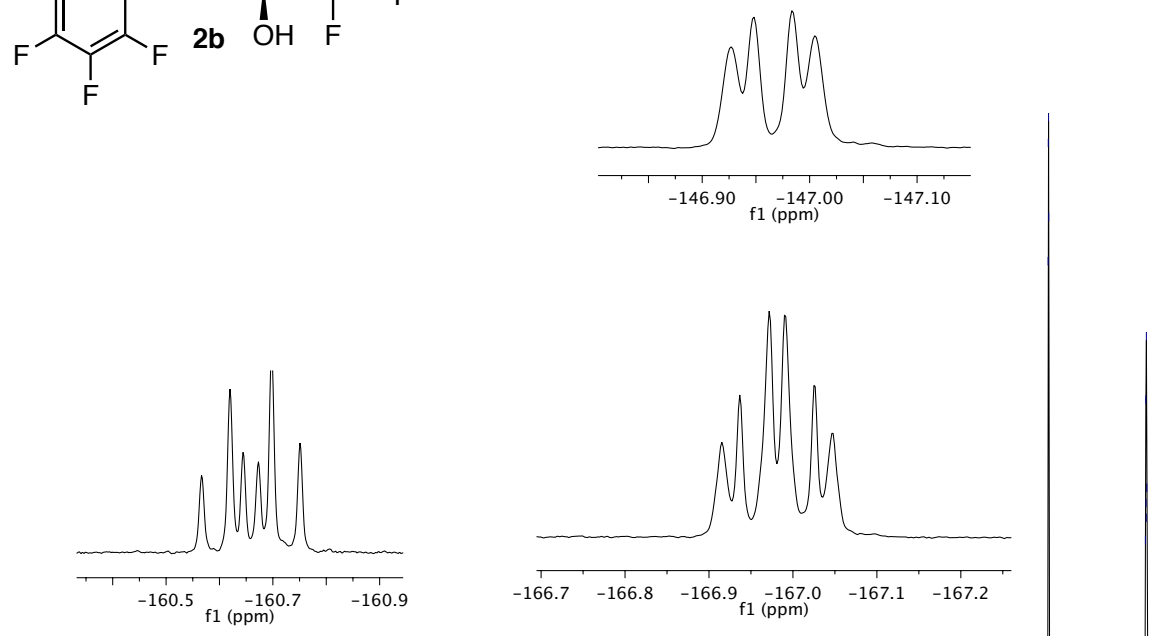

$\begin{array}{lllllll}-10 & -20 & -30 & -40 & -50 & -60 & -70\end{array}$

$\mathrm{f} 1(\mathrm{ppp}$

${ }^{1} \mathrm{H}$ NMR: $400 \mathrm{MHz}, \mathrm{CDCl}_{3}, 1$-(3,5-bis(trifluoromethyl)phenyl)prop-2-en-1-ol

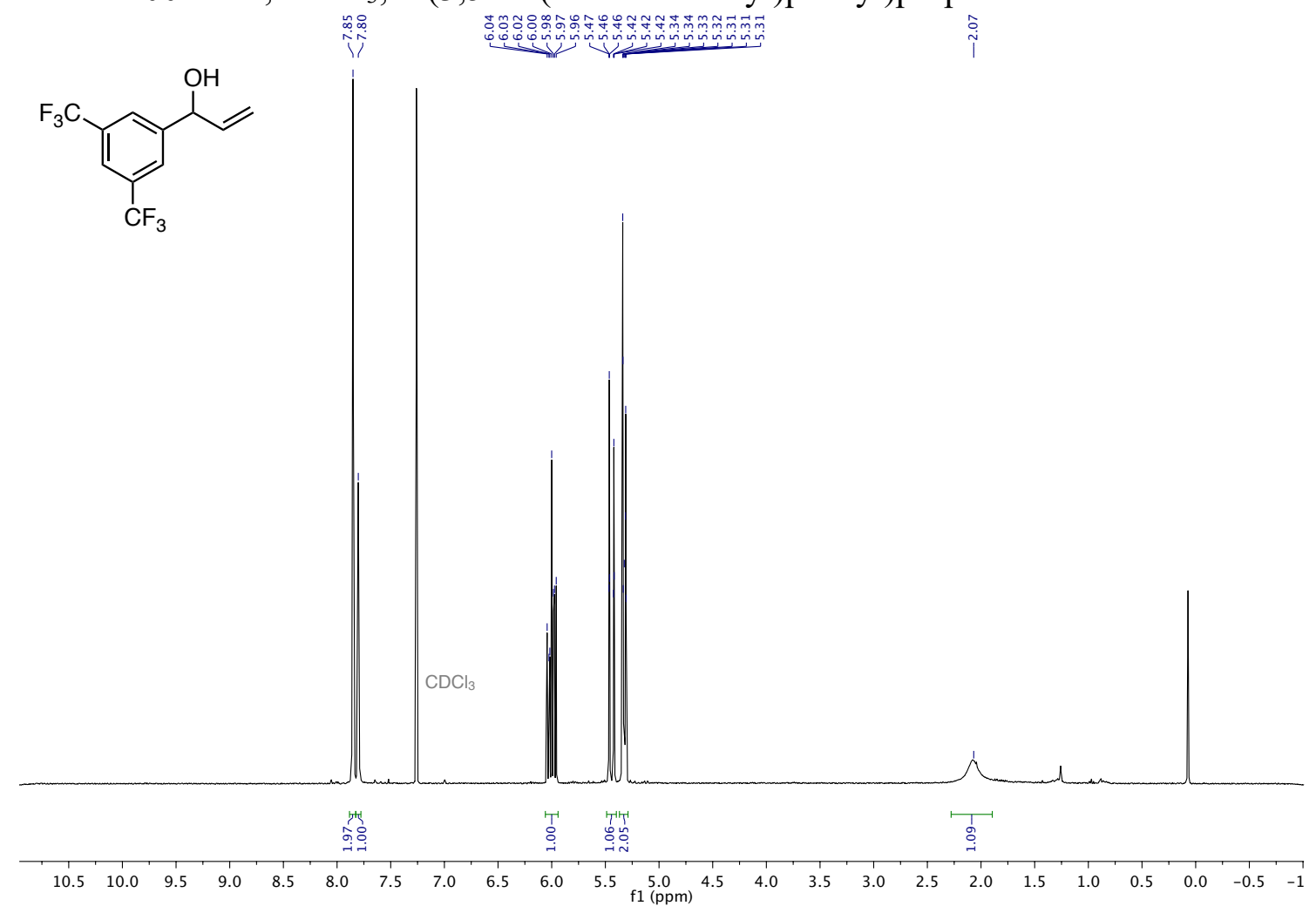


${ }^{13} \mathrm{C}$ NMR: $101 \mathrm{MHz}, \mathrm{CDCl}_{3}$, 1-(3,5-bis(trifluoromethyl)phenyl)prop-2-en-1-ol

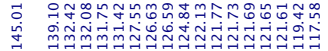<smiles>C=CC(O)c1cc(C(F)(F)F)cc(C(F)(F)F)c1</smiles>

1 人)

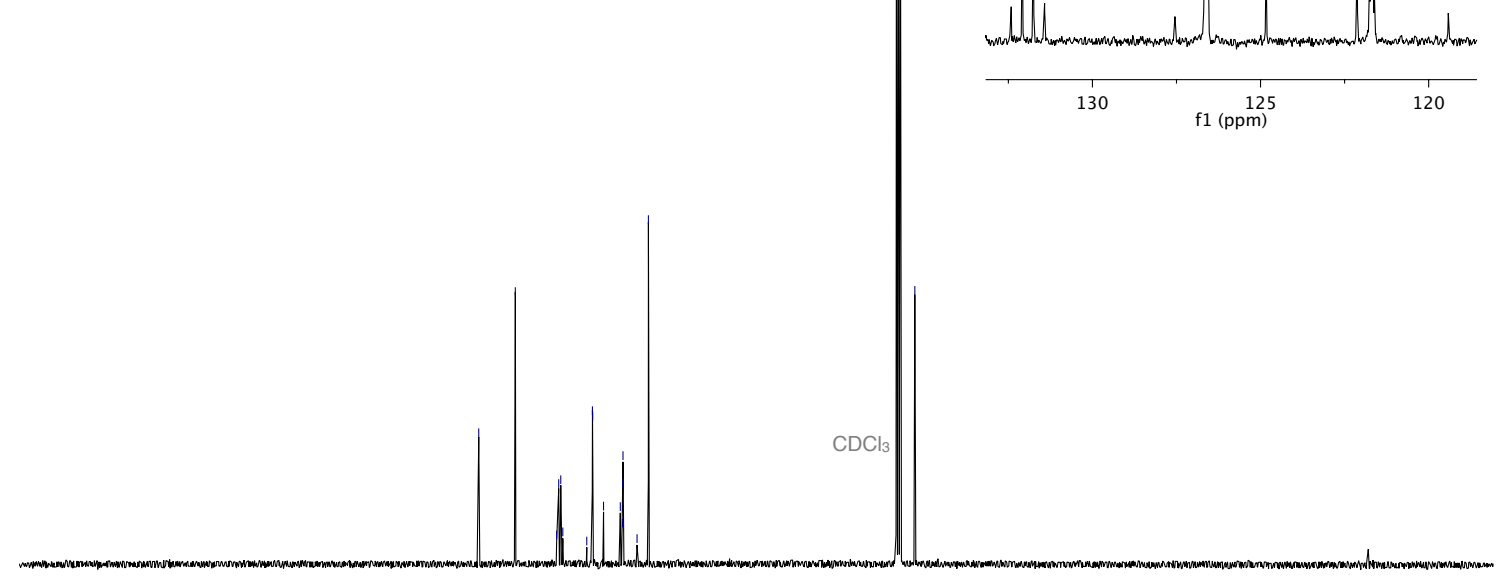

$\begin{array}{lllllllllllllllllllllll}210 & 200 & 190 & 180 & 170 & 160 & 150 & 140 & 130 & 120 & 110 & 100 & 90 & 80 & 70 & 60 & 50 & 40 & 30 & 20 & 10 & 0 & -10\end{array}$

${ }^{19} \mathrm{~F}\left\{{ }^{1} \mathrm{H}\right\}$ NMR: $376 \mathrm{MHz}, \mathrm{CDCl}_{3}, 1$-(3,5-bis(trifluoromethyl)phenyl)prop-2-en-1-ol

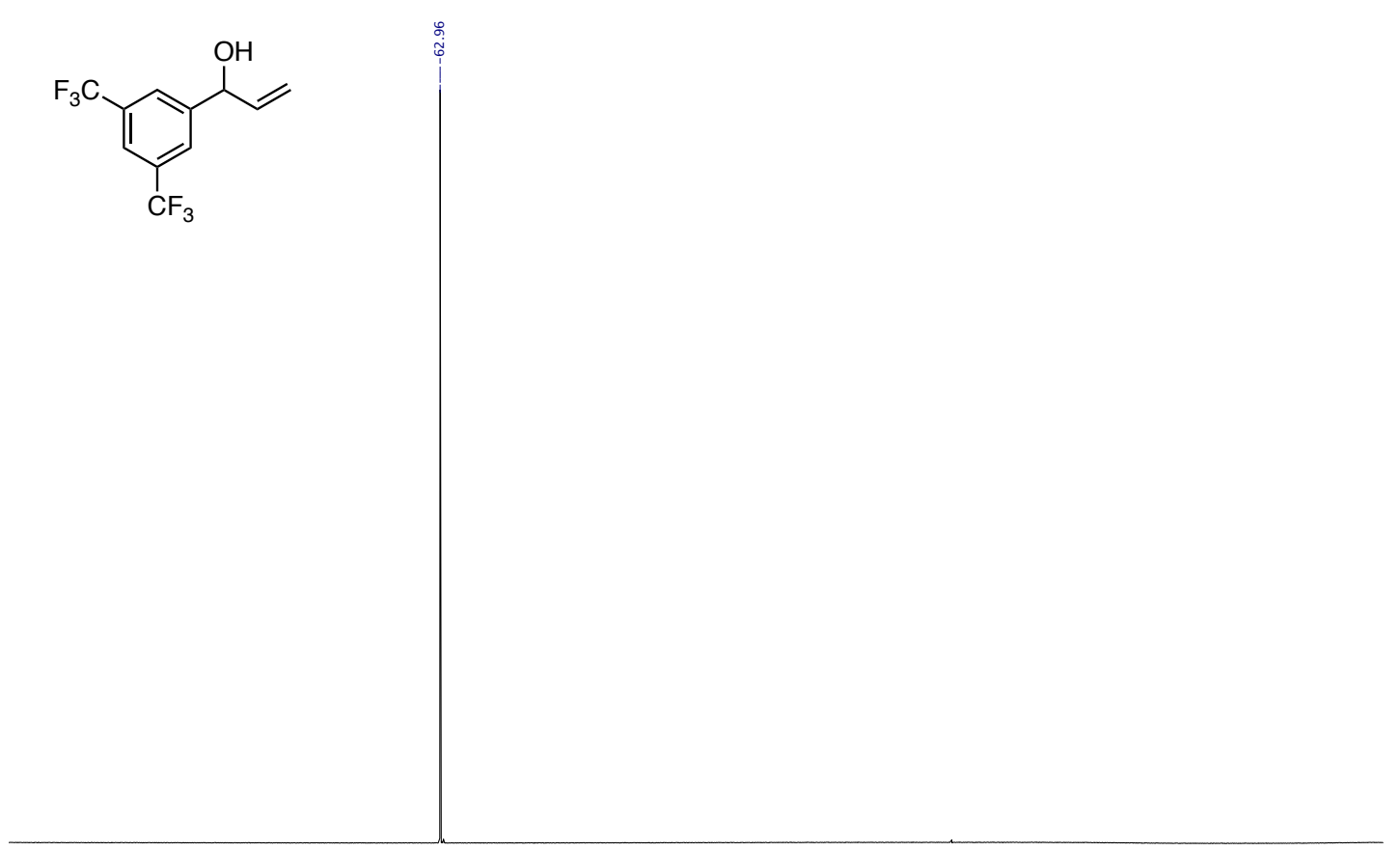

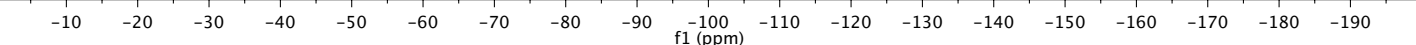


${ }^{1} \mathrm{H}$ NMR: $400 \mathrm{MHz}, \mathrm{CD}_{3} \mathrm{OD},(1 S, 4 S, E)$-1,4-Bis(3,5-bis(trifluoromethyl)phenyl)but-2-ene1,4-diol

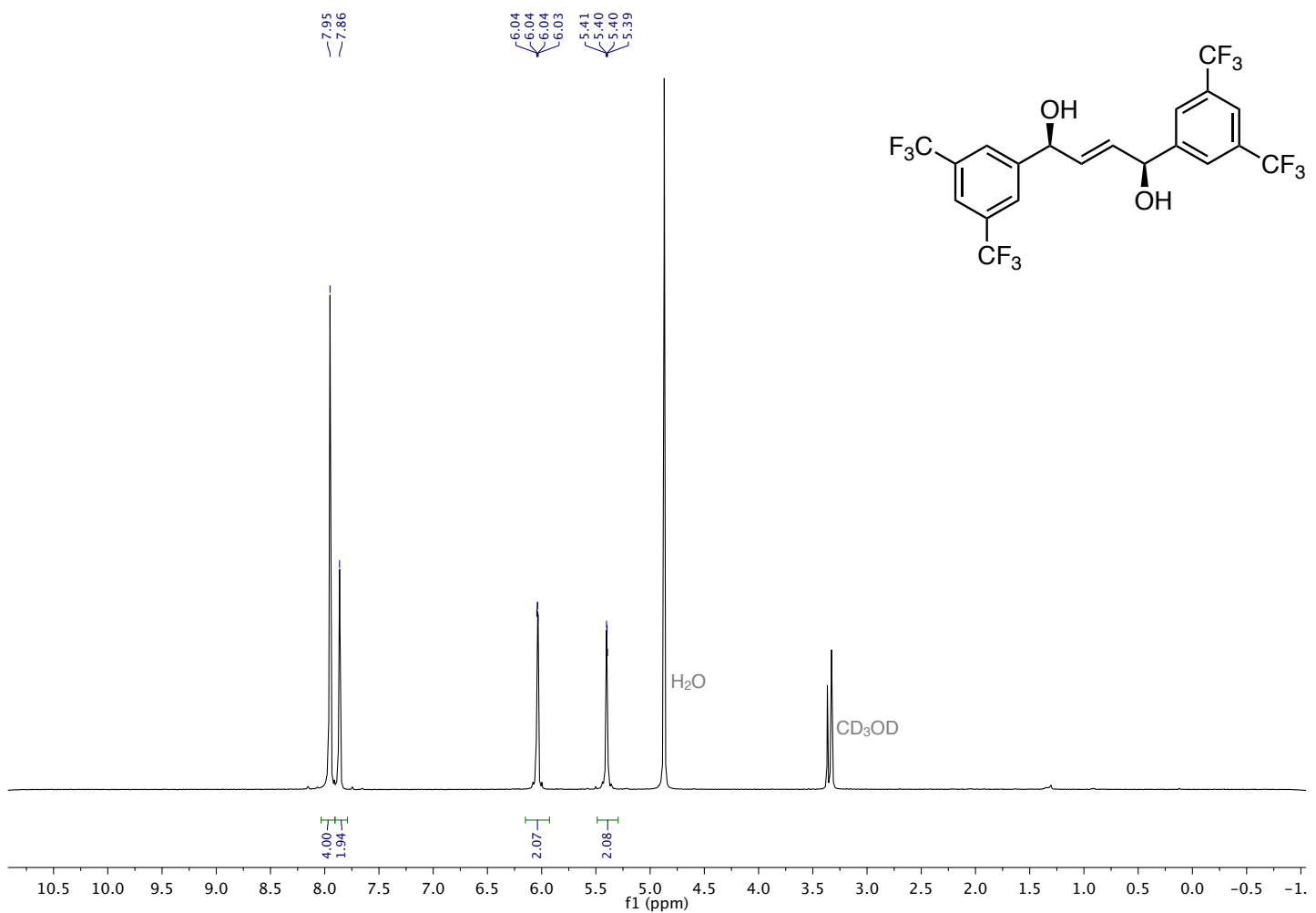

${ }^{13} \mathrm{C}$ NMR: $101 \mathrm{MHz}, \mathrm{CD}_{3} \mathrm{OD},(1 S, 4 S, E)$-1,4-Bis(3,5-bis(trifluoromethyl)phenyl)but-2-ene1,4-diol

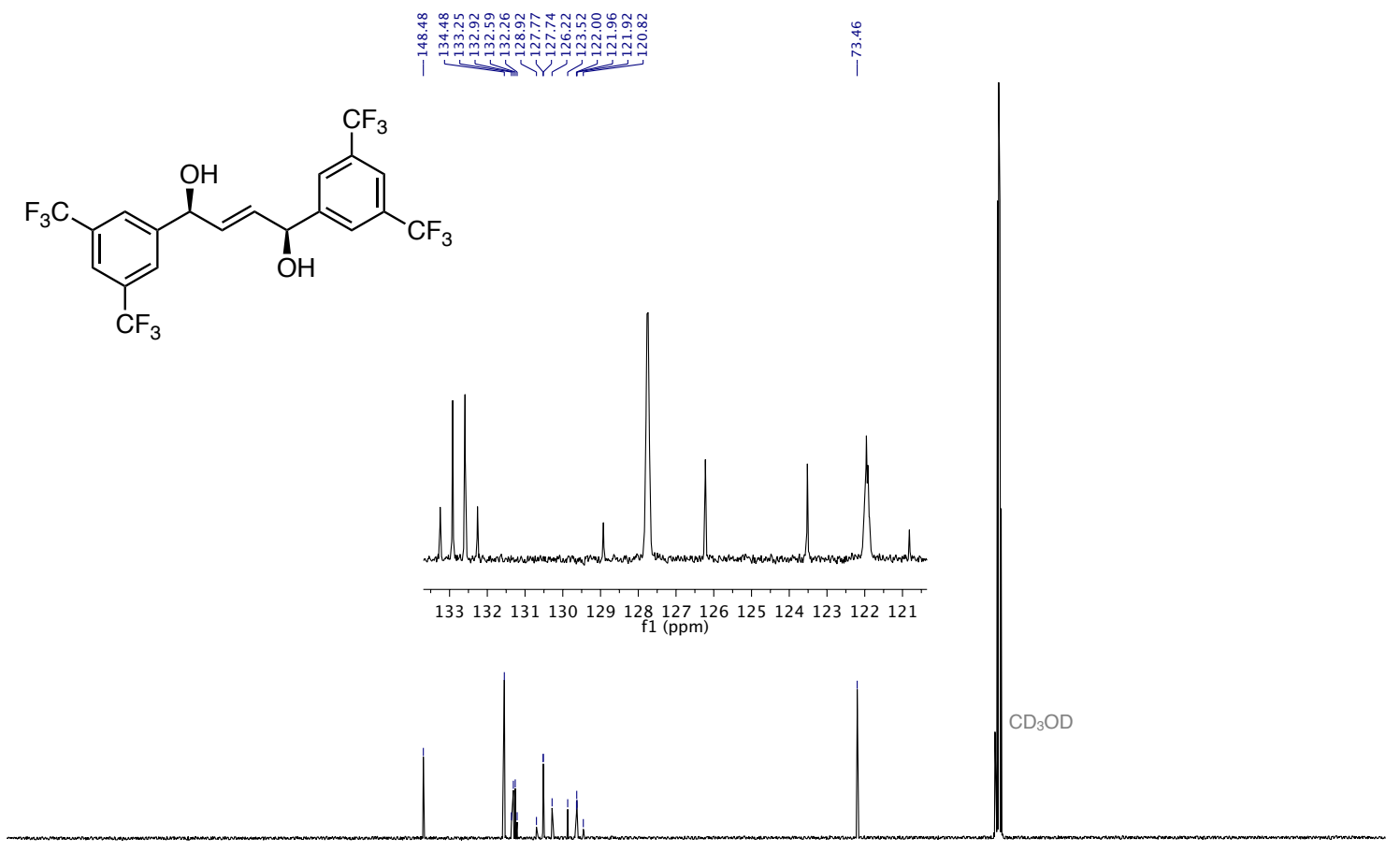

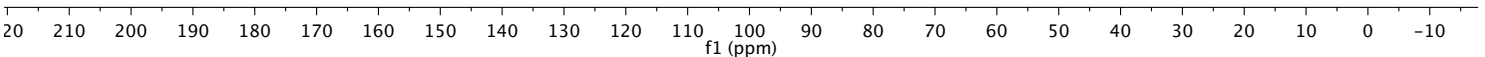


${ }^{19} \mathrm{~F}\left\{{ }^{1} \mathrm{H}\right\}$ NMR: $376 \mathrm{MHz}, \mathrm{CD}_{3} \mathrm{OD},(1 S, 4 S, E)$-1,4-Bis(3,5-bis(trifluoromethyl)phenyl)but-2ene-1,4-diol<smiles>OC(/C=C/c1cc(C(F)(F)F)cc(C(F)(F)F)c1)c1cc(C(F)(F)F)cc(C(F)(F)F)c1</smiles>

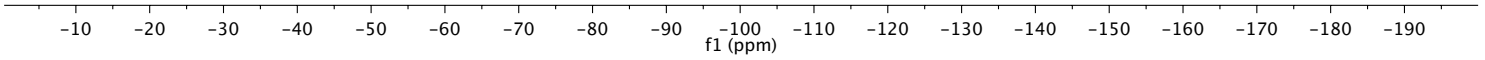

${ }^{1} \mathrm{H}$ NMR: $400 \mathrm{MHz}, \mathrm{CDCl}_{3}$, compound 2c

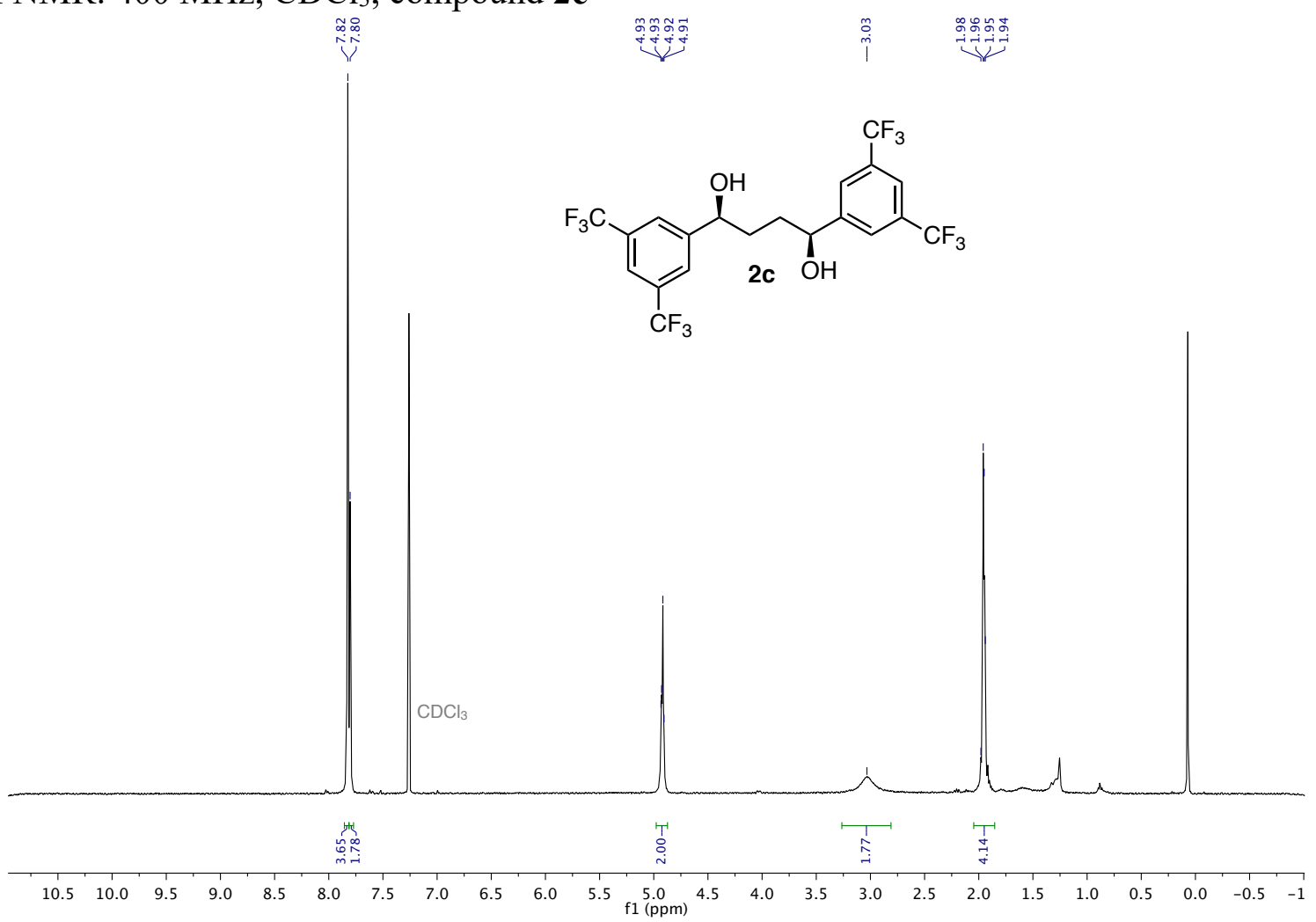


${ }^{13} \mathrm{C}$ NMR: $101 \mathrm{MHz}, \mathrm{CDCl}_{3}$, compound 2c

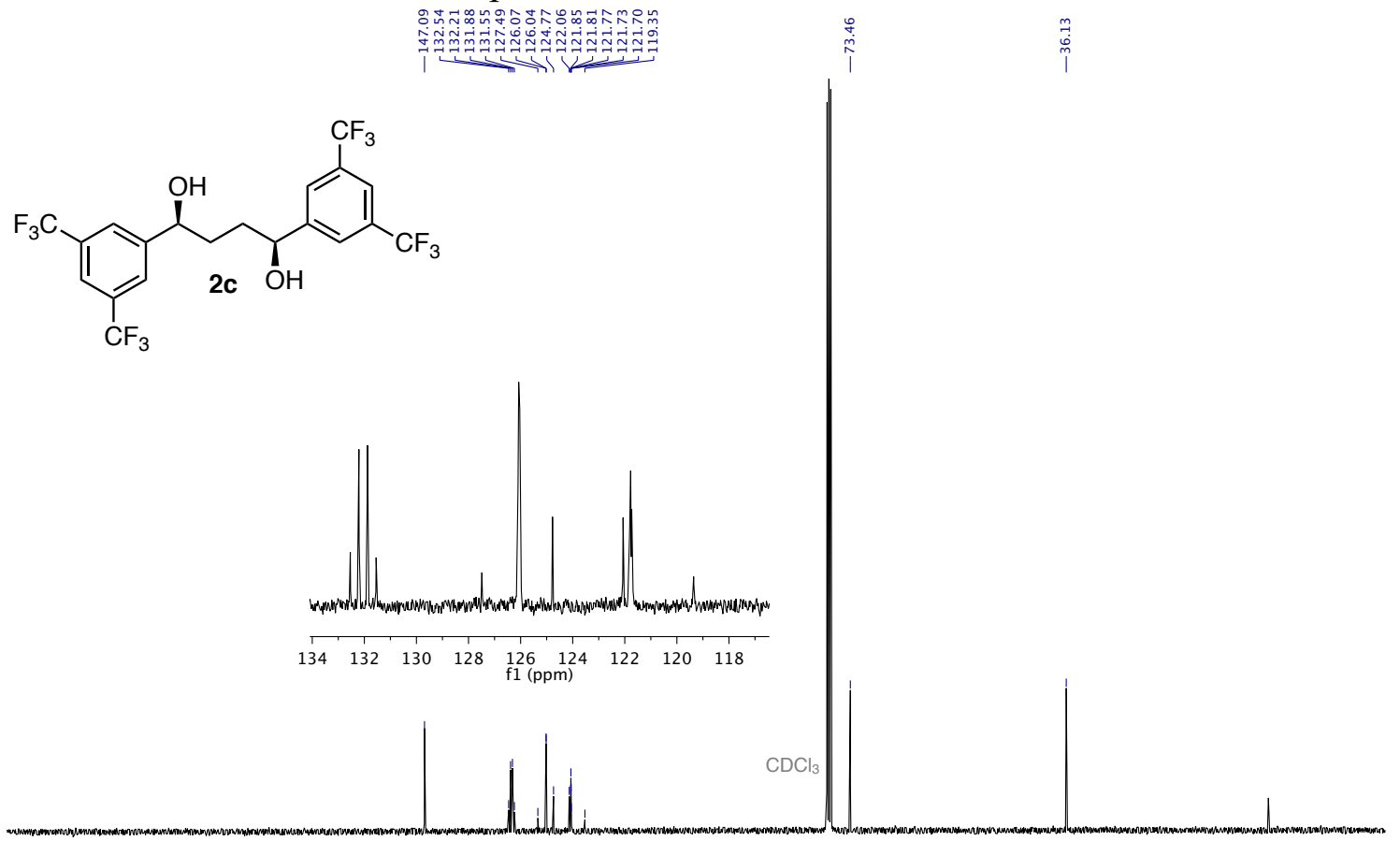

$\begin{array}{llllllllllllllllllllllllll}210 & 200 & 190 & 180 & 170 & 160 & 150 & 140 & 130 & 120 & 110 & 100 & 90 & 80 & 70 & 60 & 50 & 40 & 30 & 20 & 10 & 0 & -10\end{array}$

${ }^{19} \mathrm{~F}\left\{{ }^{1} \mathrm{H}\right\}$ NMR: $376 \mathrm{MHz}, \mathrm{CD}_{2} \mathrm{Cl}_{2}$, compound 2c

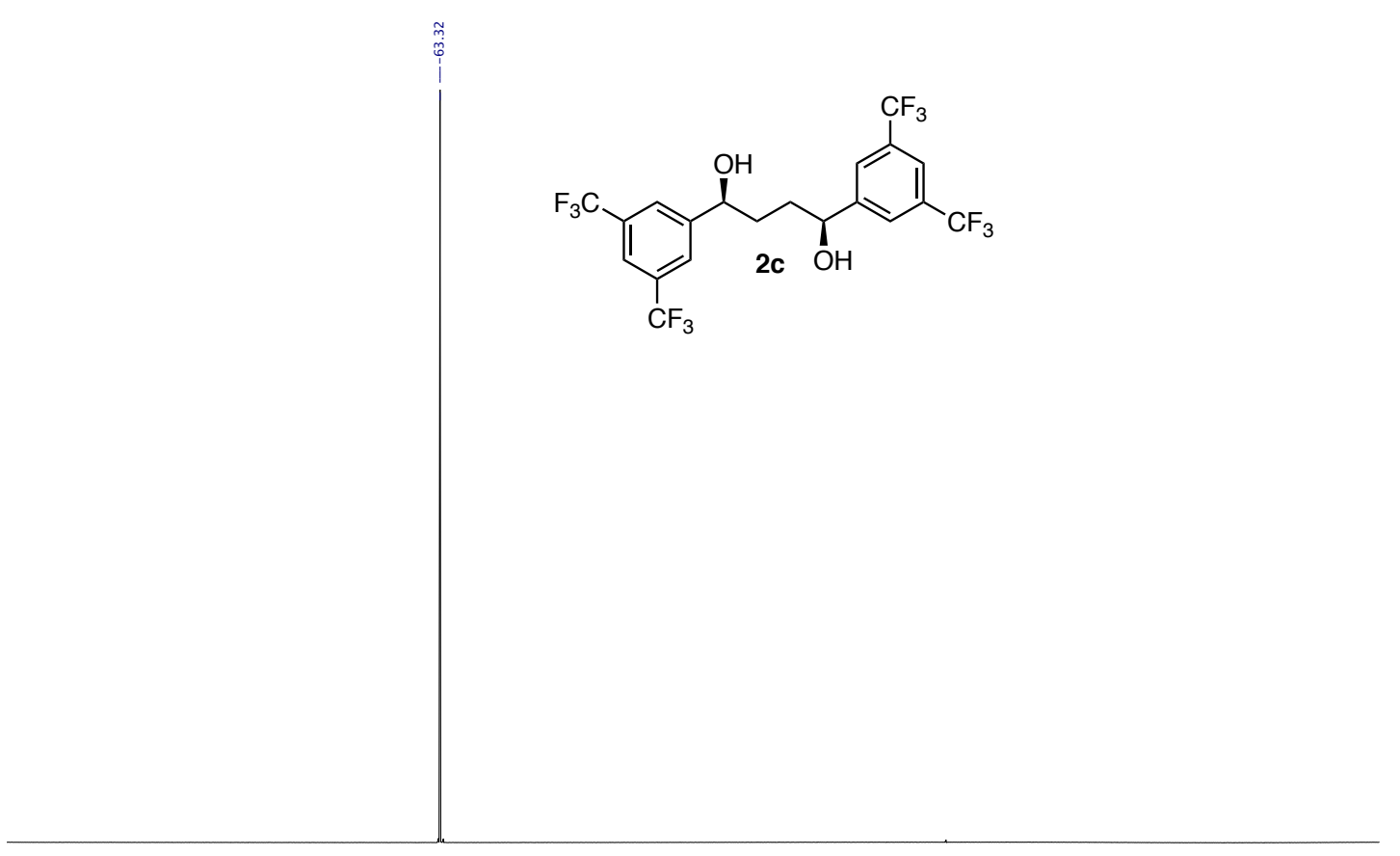

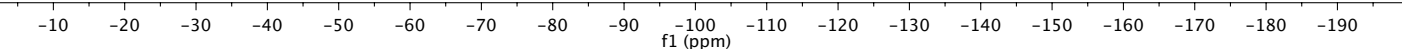


${ }^{1} \mathrm{H}$ NMR: $500 \mathrm{MHz}, \mathrm{CDCl}_{3}$, compound 3a

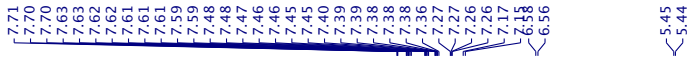

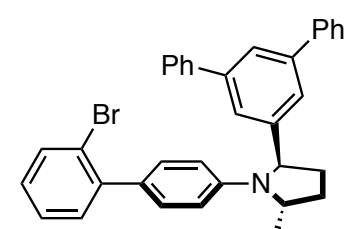

$3 a$

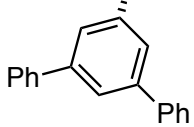

id

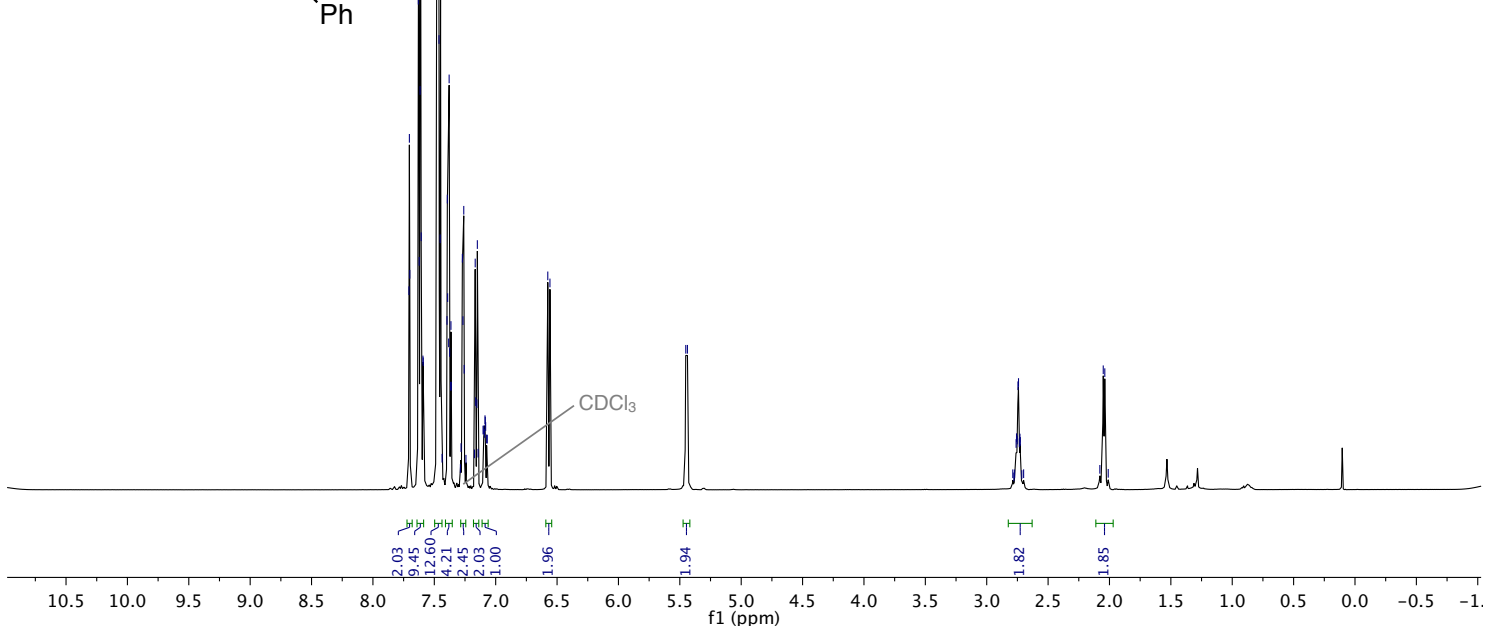

${ }^{13} \mathrm{C}$ NMR: $126 \mathrm{MHz}, \mathrm{CDCl}_{3}$, compound 3a<smiles>Brc1ccccc1-c1cc(-c2ccccc2)cc([C@@H]2CC[C@H]2c2cc(-c3ccccc3)cc(-c3ccccc3)c2)c1</smiles>

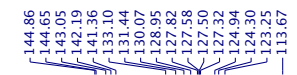


${ }^{1} \mathrm{H}$ NMR: $400 \mathrm{MHz}, \mathrm{CD}_{2} \mathrm{Cl}_{2}$, compound 4a

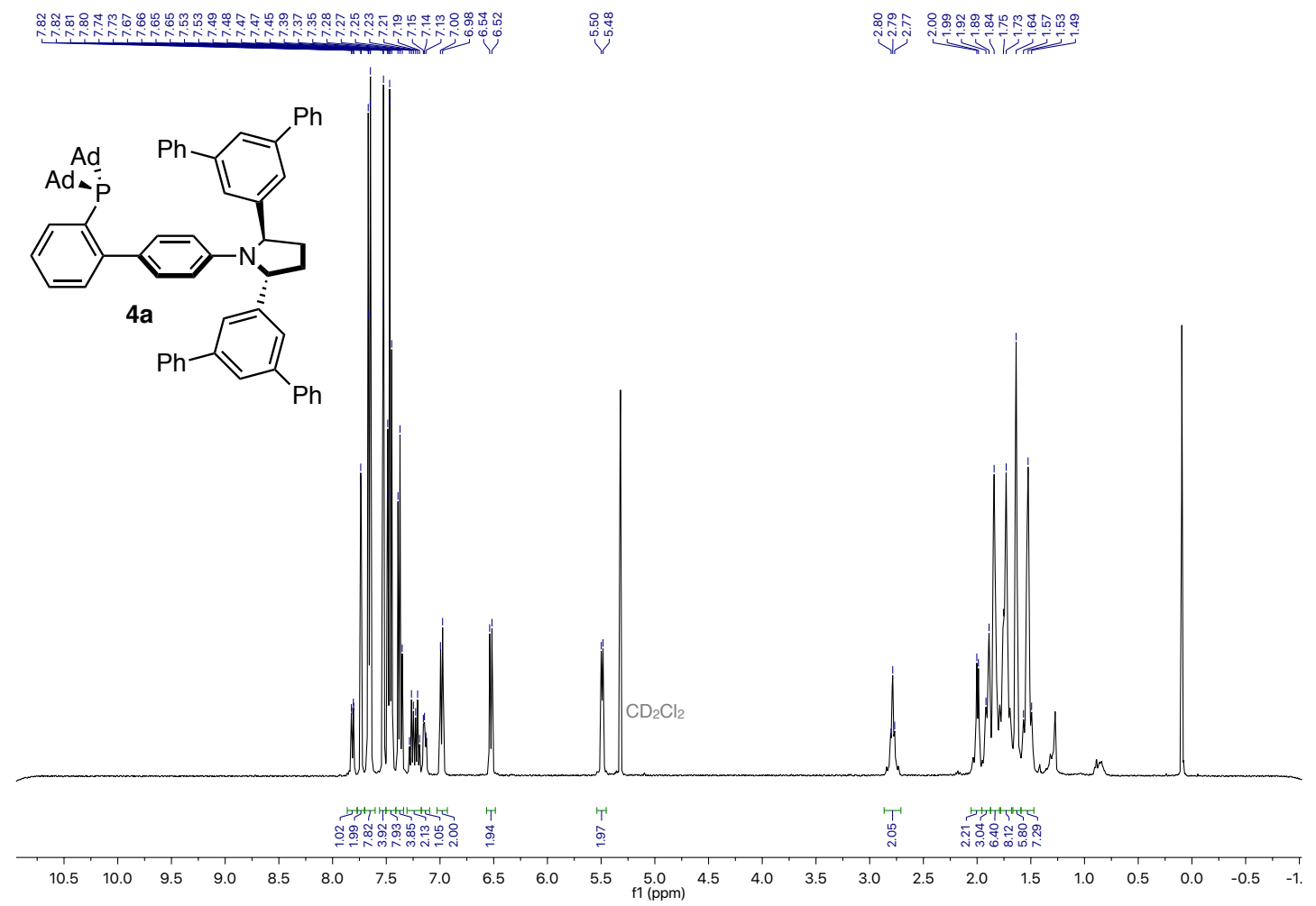

${ }^{13} \mathrm{C}$ NMR: $126 \mathrm{MHz}, \mathrm{CDCl}_{3}$, compound 4a

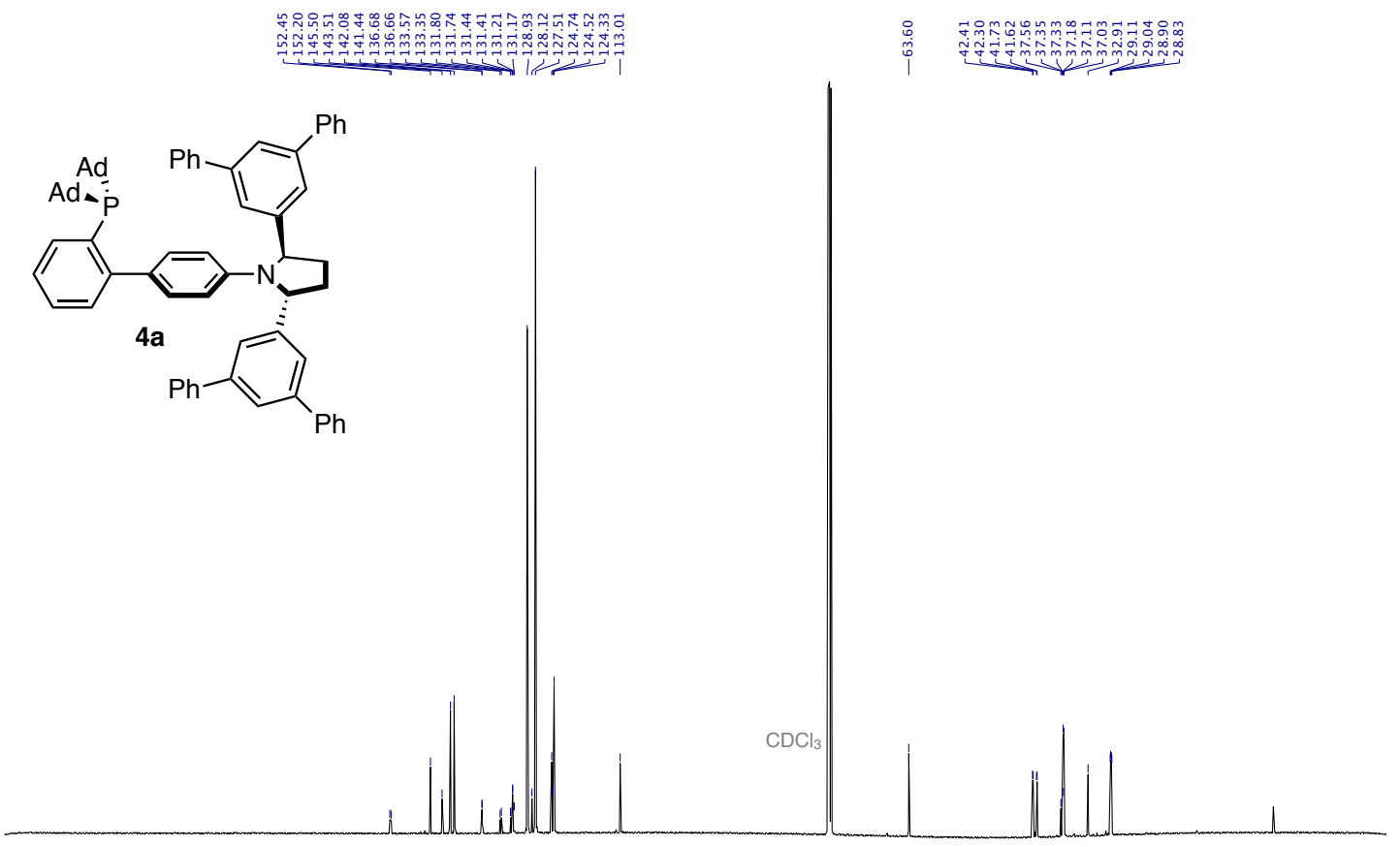

$\begin{array}{llllllllllllllllllllllllllll}1 & 1 & 200 & 190 & 180 & 170 & 160 & 150 & 140 & 130 & 120 & 110 & \begin{array}{c}100 \\ \mathrm{f} 1(\mathrm{ppm})\end{array} & 90 & 80 & 70 & 60 & 50 & 40 & 30 & 20 & 10 & 0 & -10\end{array}$ 
${ }^{31} \mathrm{P}\left\{{ }^{1} \mathrm{H}\right\} \mathrm{NMR}: 162 \mathrm{MHz}, \mathrm{CDCl}_{3}$, compound 4a

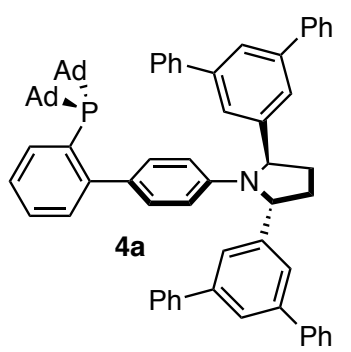

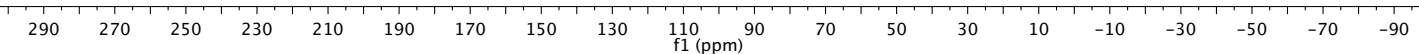

${ }^{1} \mathrm{H}$ NMR: $500 \mathrm{MHz}, \mathrm{CD}_{2} \mathrm{Cl}_{2}$, compound $(R, R)-\mathbf{A}$

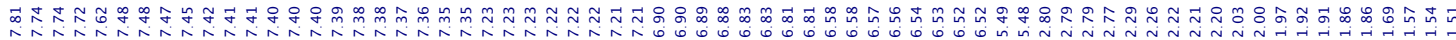<smiles></smiles>

$(R, R)-\mathbf{A}$<smiles></smiles> 
${ }^{13} \mathrm{C}$ NMR: $126 \mathrm{MHz}, \mathrm{CD}_{2} \mathrm{Cl}_{2}$, compound $(R, R)-\mathbf{A}$ 等

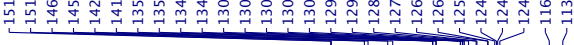

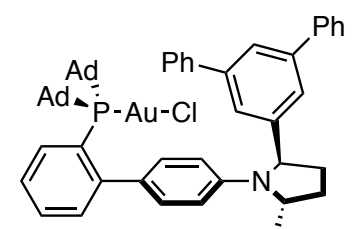

$(R, R)-\mathbf{A}$<smiles>c1ccc(-c2cccc(-c3ccccc3)c2)cc1</smiles>

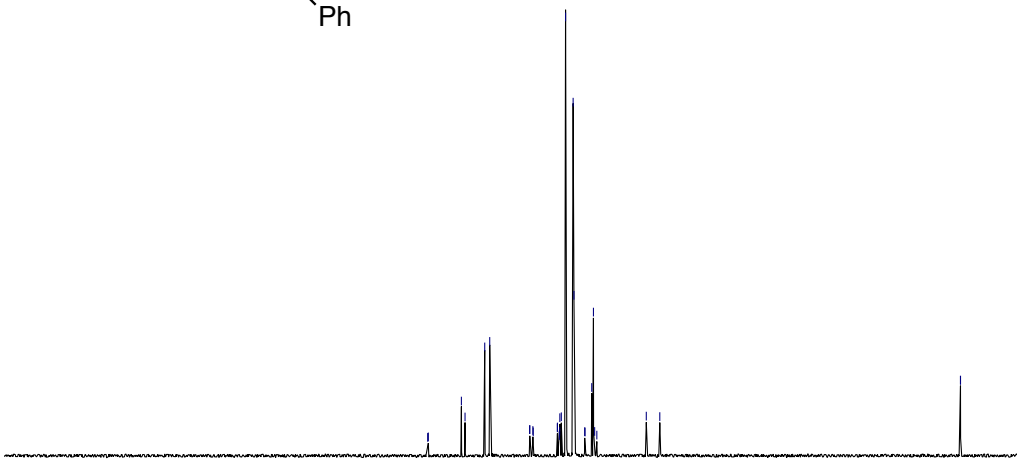

${ }^{31} \mathrm{P}\left\{{ }^{1} \mathrm{H}\right\} \mathrm{NMR}: 162 \mathrm{MHz}, \mathrm{CD}_{2} \mathrm{Cl}_{2}$, compound $(R, R)-\mathbf{A}$

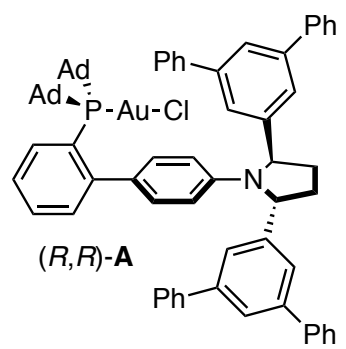

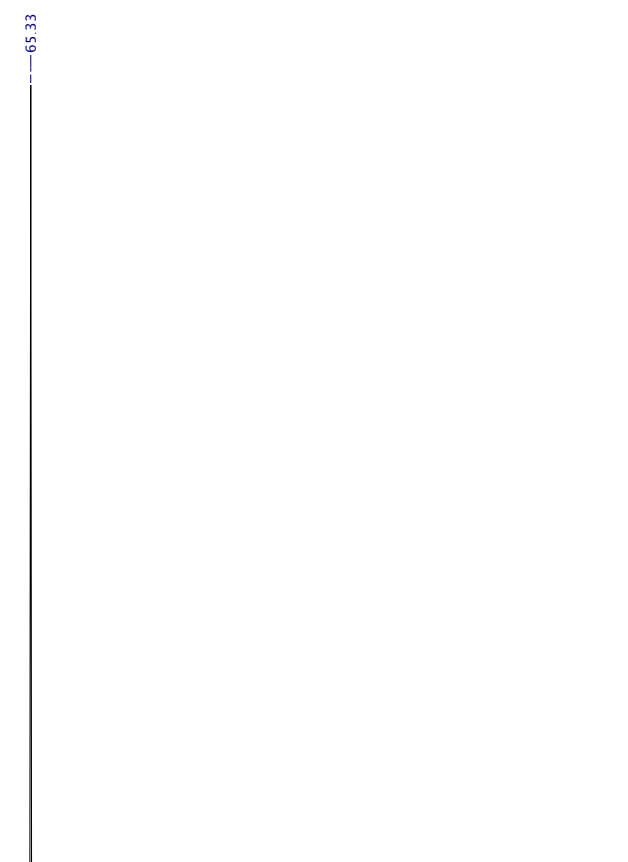


${ }^{1} \mathrm{H}$ NMR: $400 \mathrm{MHz}, \mathrm{CD}_{2} \mathrm{Cl}_{2}$, compound $\mathbf{3 b}$

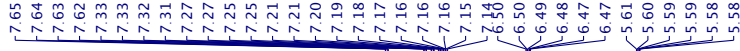<smiles>Fc1c(F)c(F)c([C@H]2CC[C@H]2c2c(F)c(F)c(F)c(F)c2F)c(F)c1F</smiles>

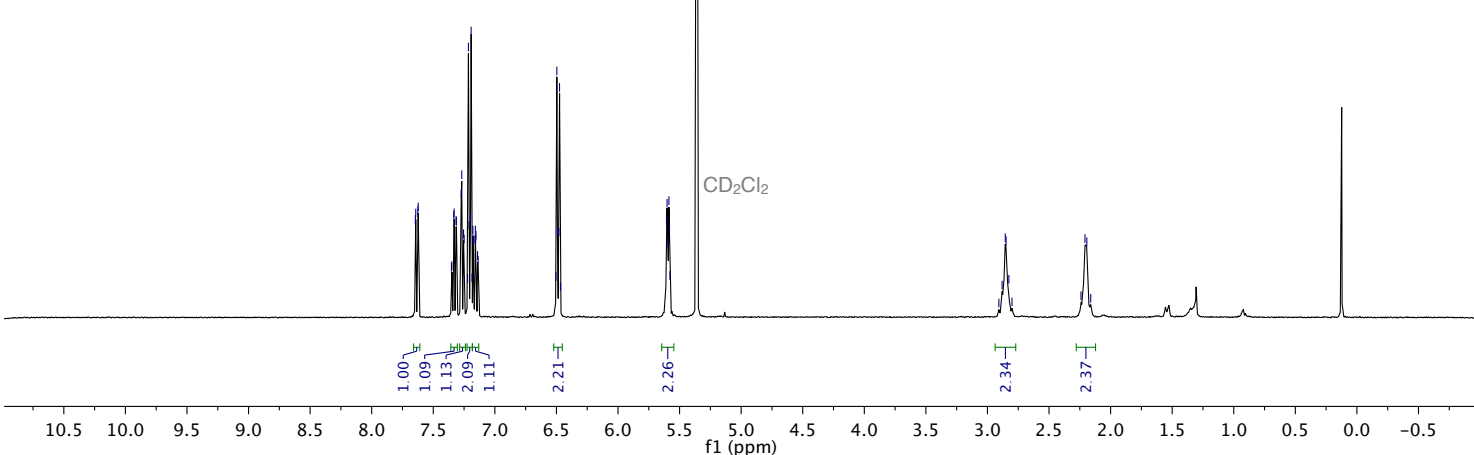

${ }^{13} \mathrm{C}$ NMR: $101 \mathrm{MHz}, \mathrm{CD}_{2} \mathrm{Cl}_{2}$, compound 3b

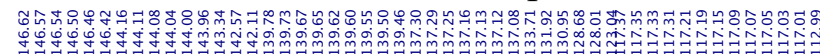
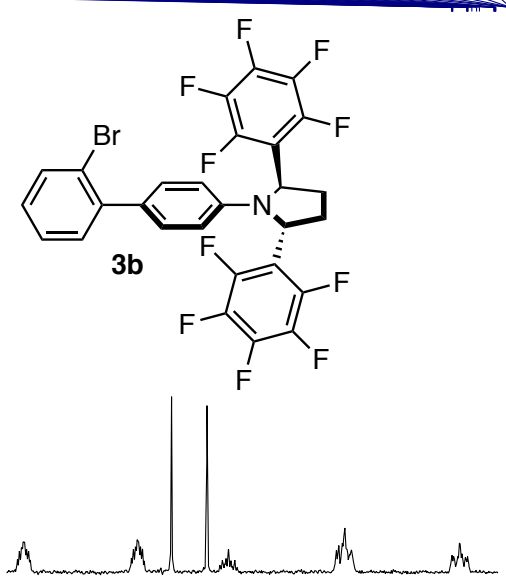

$\begin{array}{llllllllll}146 & 145 & 144 & 143 & 142 & 141 & 140 & 139 & 138 & 137\end{array}$
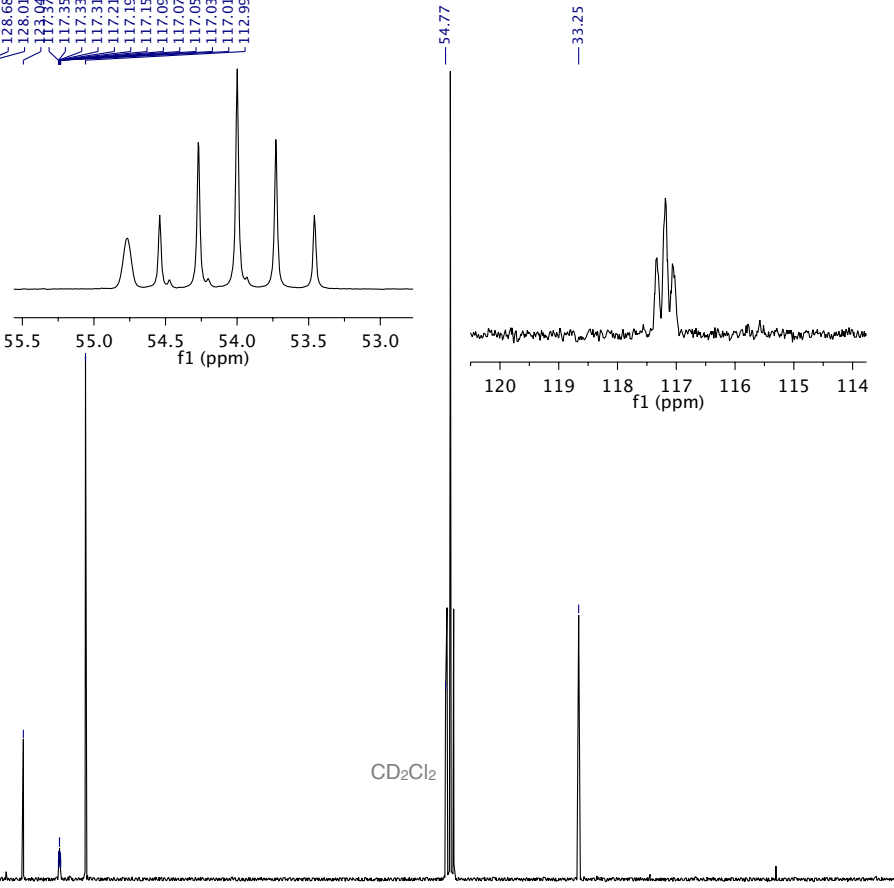

$\begin{array}{llllll}120 & 119 & 118_{\mathrm{f} 1(\mathrm{ppm})}^{117} & 116 & 115 & 114\end{array}$

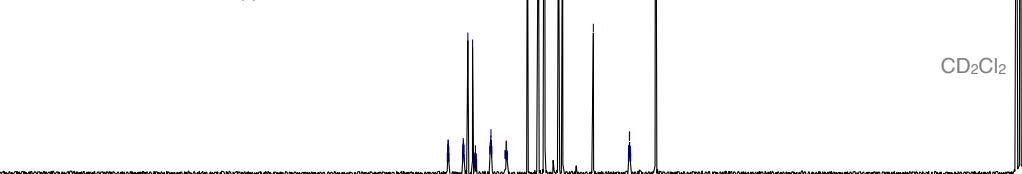

$\begin{array}{lllllllllllllllllllllllll}210 & 200 & 190 & 180 & 170 & 160 & 150 & 140 & 130 & 120 & 110 & 100 & 90 & 80 & 70 & 60 & 50 & 40 & 30 & 20 & 10 & 0 & -10\end{array}$ 
${ }^{19} \mathrm{~F}\left\{{ }^{1} \mathrm{H}\right\}$ NMR: $376 \mathrm{MHz}, \mathrm{CD}_{2} \mathrm{Cl}_{2}$, compound 3b
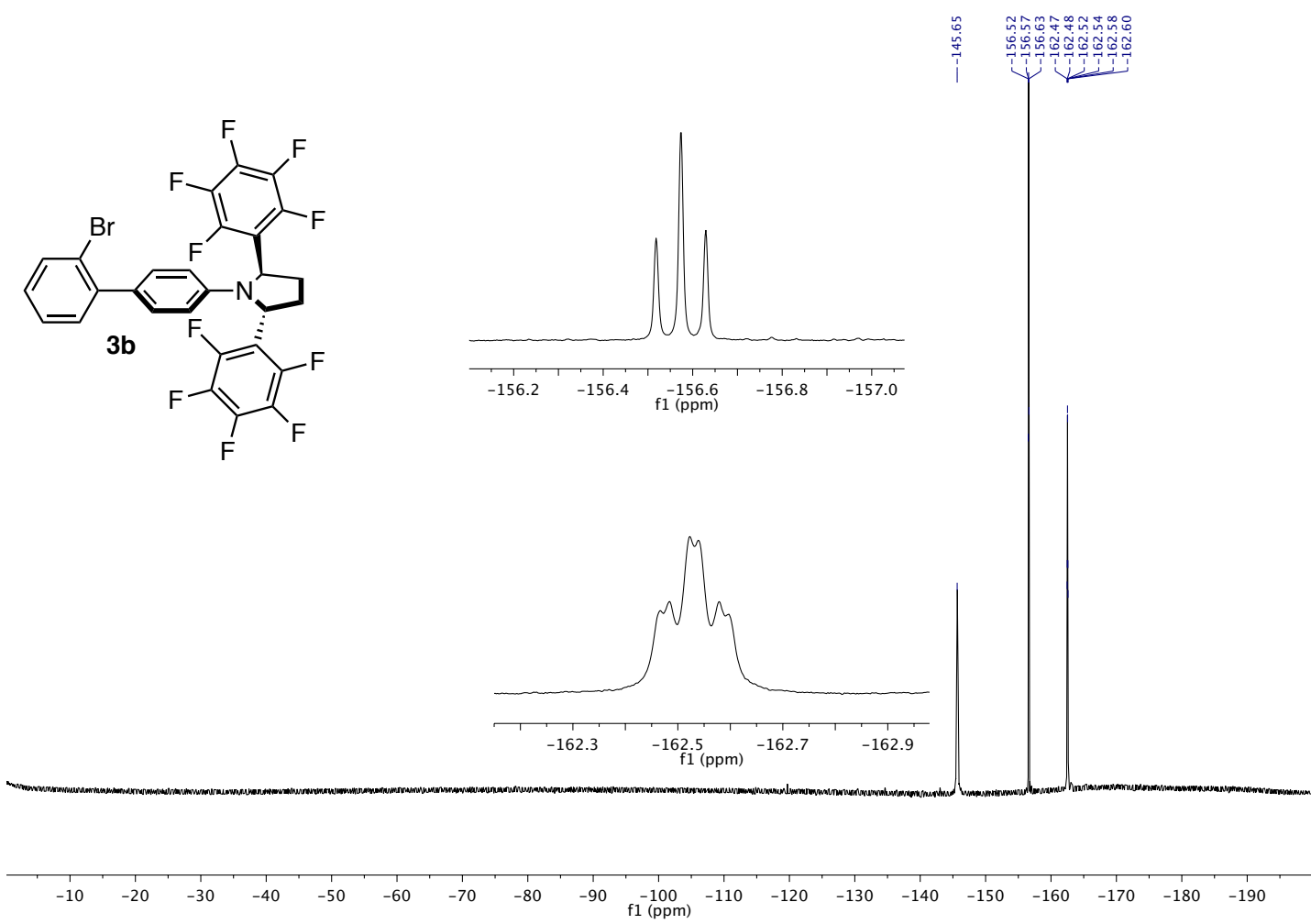

${ }^{1} \mathrm{H}$ NMR: $500 \mathrm{MHz}, \mathrm{CD}_{2} \mathrm{Cl}_{2}$, compound 4b

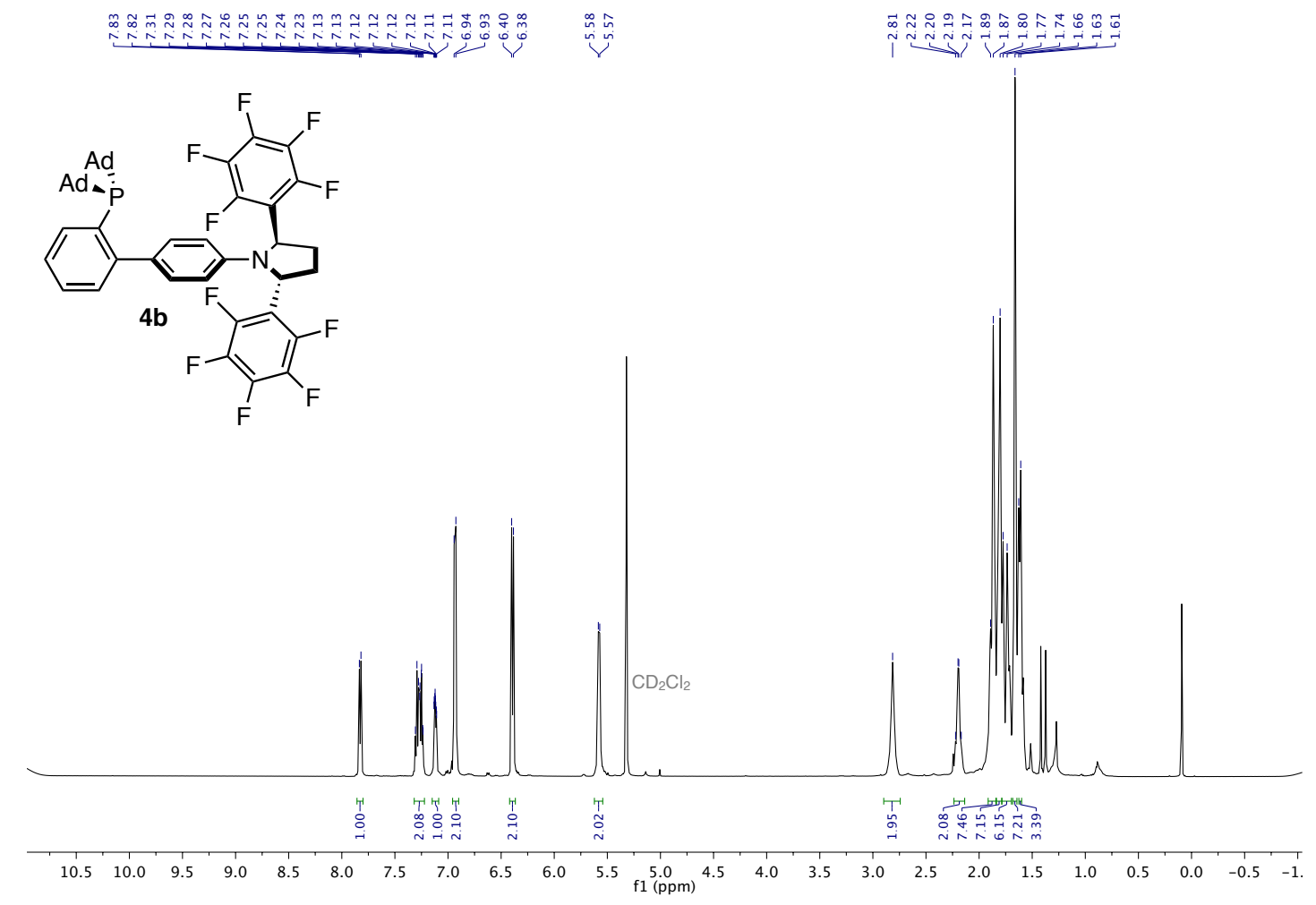


${ }^{13} \mathrm{C}$ NMR: $126 \mathrm{MHz}, \mathrm{CD}_{2} \mathrm{Cl}_{2}$, compound $\mathbf{4 b}$

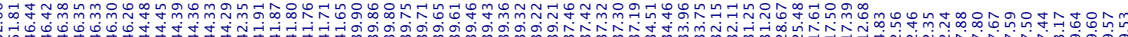

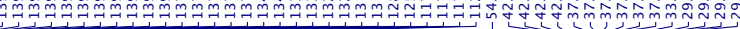<smiles>Fc1c(F)c(F)c(N2CCCN2c2c(F)c(F)c(F)c(-c3ccccc3P=[W])c2F)c(F)c1F</smiles>

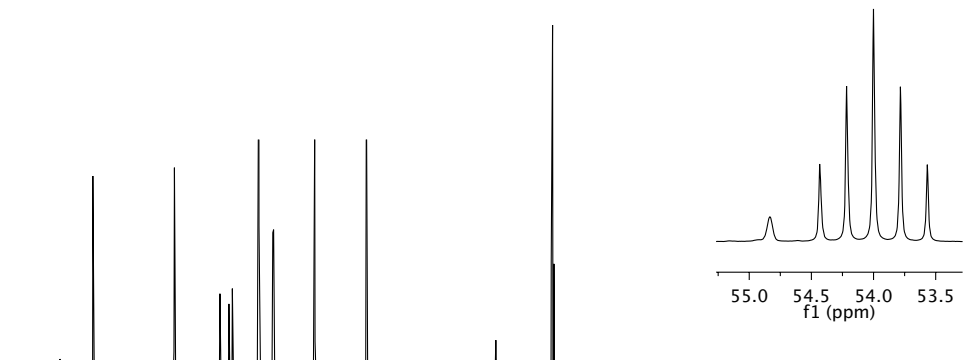

${ }^{31} \mathrm{P}\left\{{ }^{1} \mathrm{H}\right\}$ NMR: $162 \mathrm{MHz}, \mathrm{CD}_{2} \mathrm{Cl}_{2}$, compound $\mathbf{4 b}$<smiles>Fc1cc(-c2ccccc2P=[W])ccc1N1C(c2c(F)c(F)c(F)c(F)c2F)CCN1c1c(F)c(F)c(F)c(F)c1F</smiles>

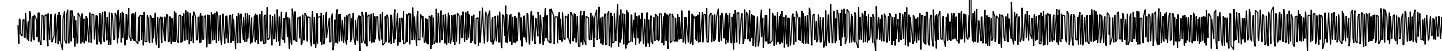

$\begin{array}{lllllllllllllllllllllll}320 & 300 & 280 & 260 & 240 & 220 & 200 & 180 & 160 & 140 & 120 & 100 & 80 & 60 & 40 & 20 & 0 & -20 & -40 & -60 & -80 & -100 & -120\end{array}$ 
${ }^{19} \mathrm{~F}\left\{{ }^{1} \mathrm{H}\right\}$ NMR: $376 \mathrm{MHz}, \mathrm{CD}_{2} \mathrm{Cl}_{2}$, compound 4b<smiles>N#[PH](F)(F)c1ccccc1-c1ccc(N2CN(c3c(F)c(F)c(F)c(F)c3F)CCC2c2c(F)c(F)c(F)c(F)c2F)c(F)c1F</smiles>
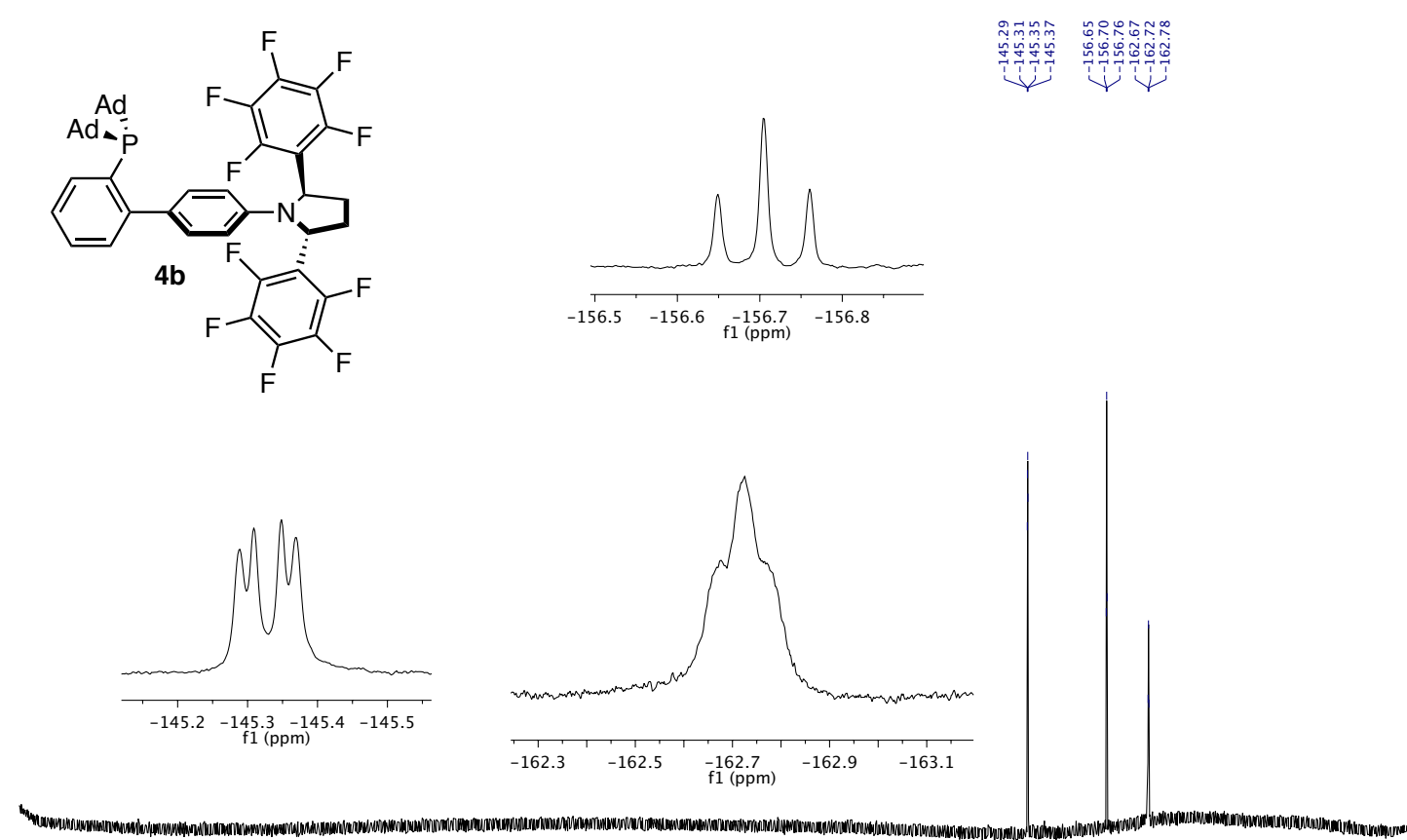

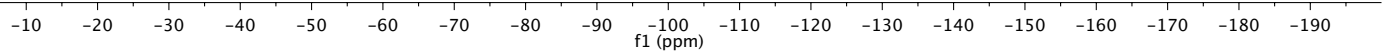

${ }^{1} \mathrm{H}$ NMR: $500 \mathrm{MHz}, \mathrm{CD}_{2} \mathrm{Cl}_{2}$, compound $(R, R)-\mathbf{B}$

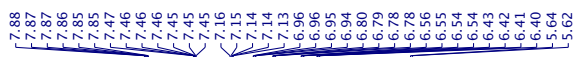
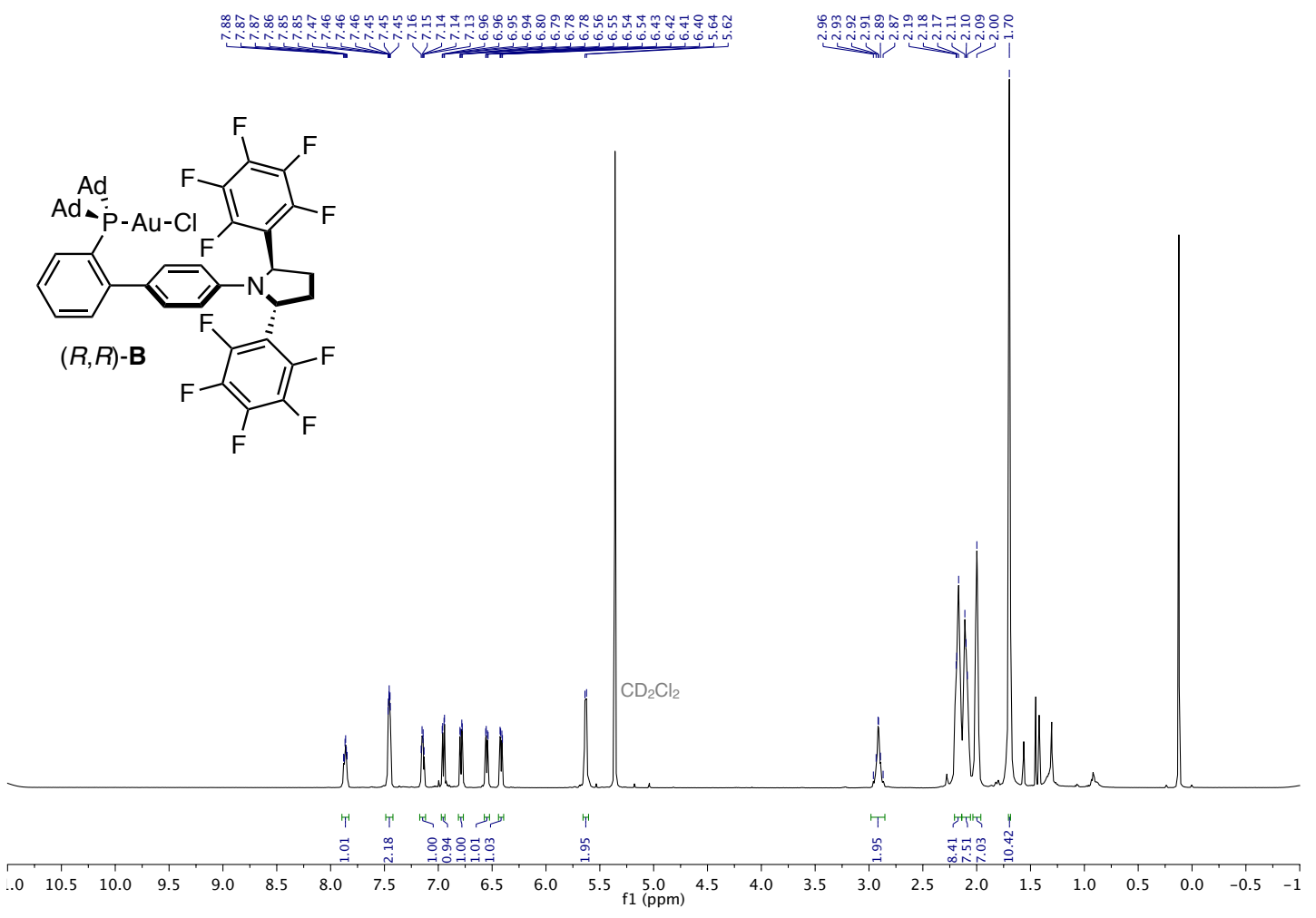
${ }^{13} \mathrm{C}$ NMR: $126 \mathrm{MHz}, \mathrm{CD}_{2} \mathrm{Cl}_{2}$, compound $(R, R)-\mathbf{B}$

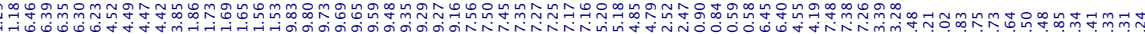

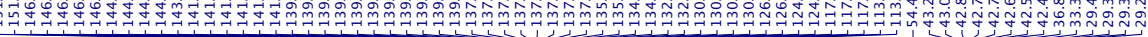
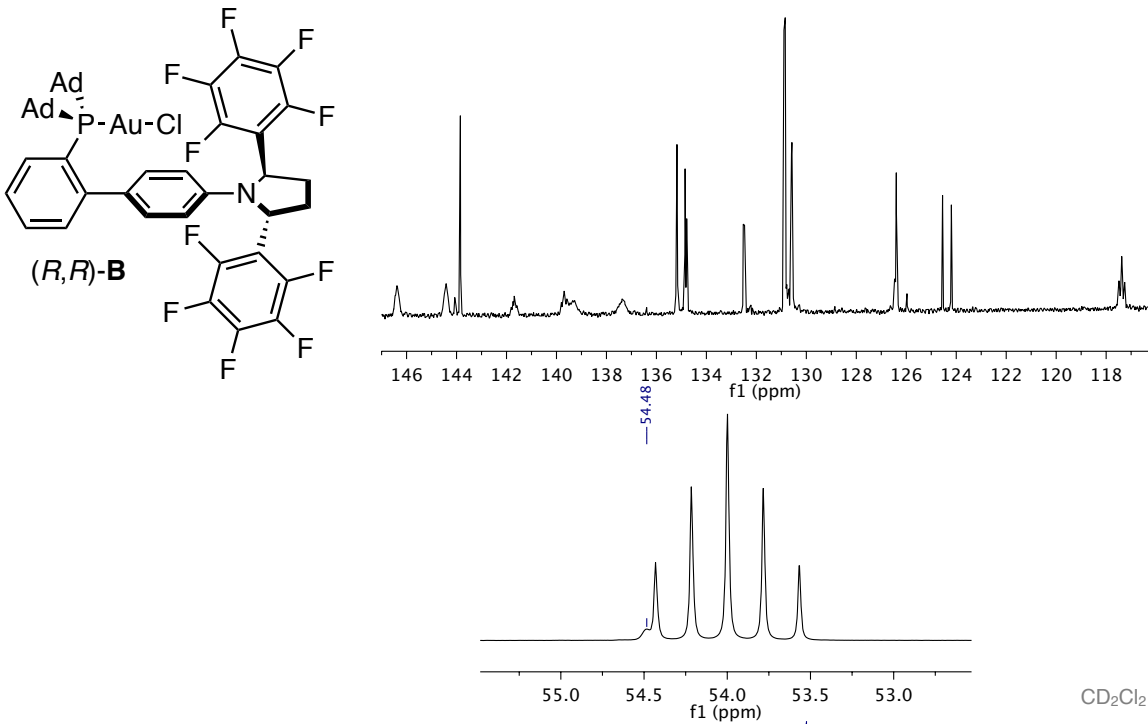

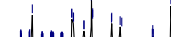

$\begin{array}{llllllllllllllllllllllllllllllllllllll}300 & 290 & 280 & 270 & 260 & 250 & 240 & 230 & 220 & 210 & 200 & 190 & 180 & 170 & 160 & 150 & 140 & 130 & 120 & 110 & 100 & 90 & 80 & 70 & 60 & 50 & 40 & 30 & 20 & 10 & 0 & -10 & -21\end{array}$

${ }^{31} \mathrm{P}\left\{{ }^{1} \mathrm{H}\right\}$ NMR: $203 \mathrm{MHz}, \mathrm{CD}_{2} \mathrm{Cl}_{2}$, compound $(R, R)$-B

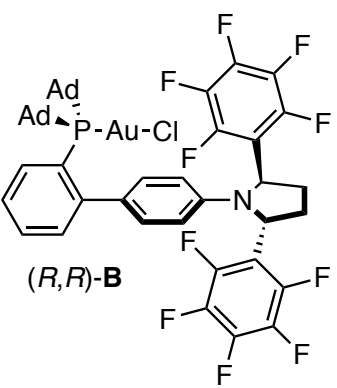

$\mathrm{CD}_{2} \mathrm{Cl}_{2}$

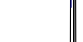


${ }^{19} \mathrm{~F}\left\{{ }^{1} \mathrm{H}\right\}$ NMR: $471 \mathrm{MHz}, \mathrm{CD}_{2} \mathrm{Cl}_{2}$, compound $(R, R)-\mathbf{B}$
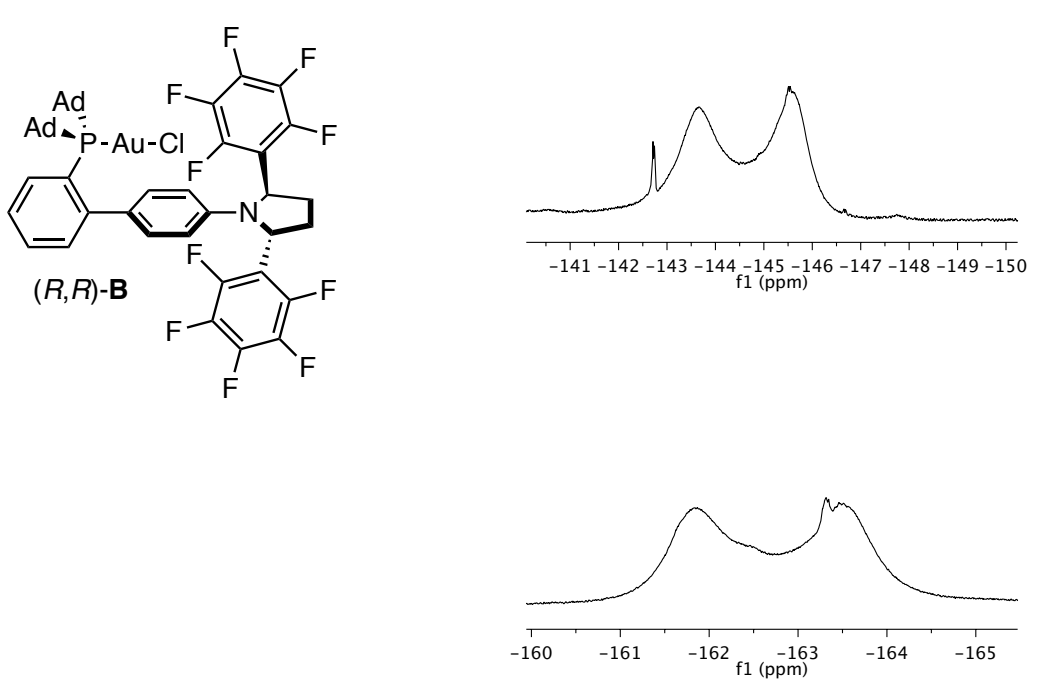

0

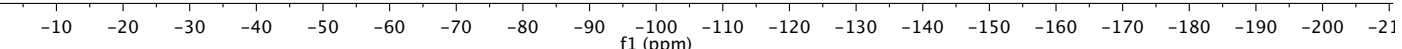

${ }^{1} \mathrm{H}$ NMR: $500 \mathrm{MHz}, \mathrm{CDCl}_{3}$, compound 3c

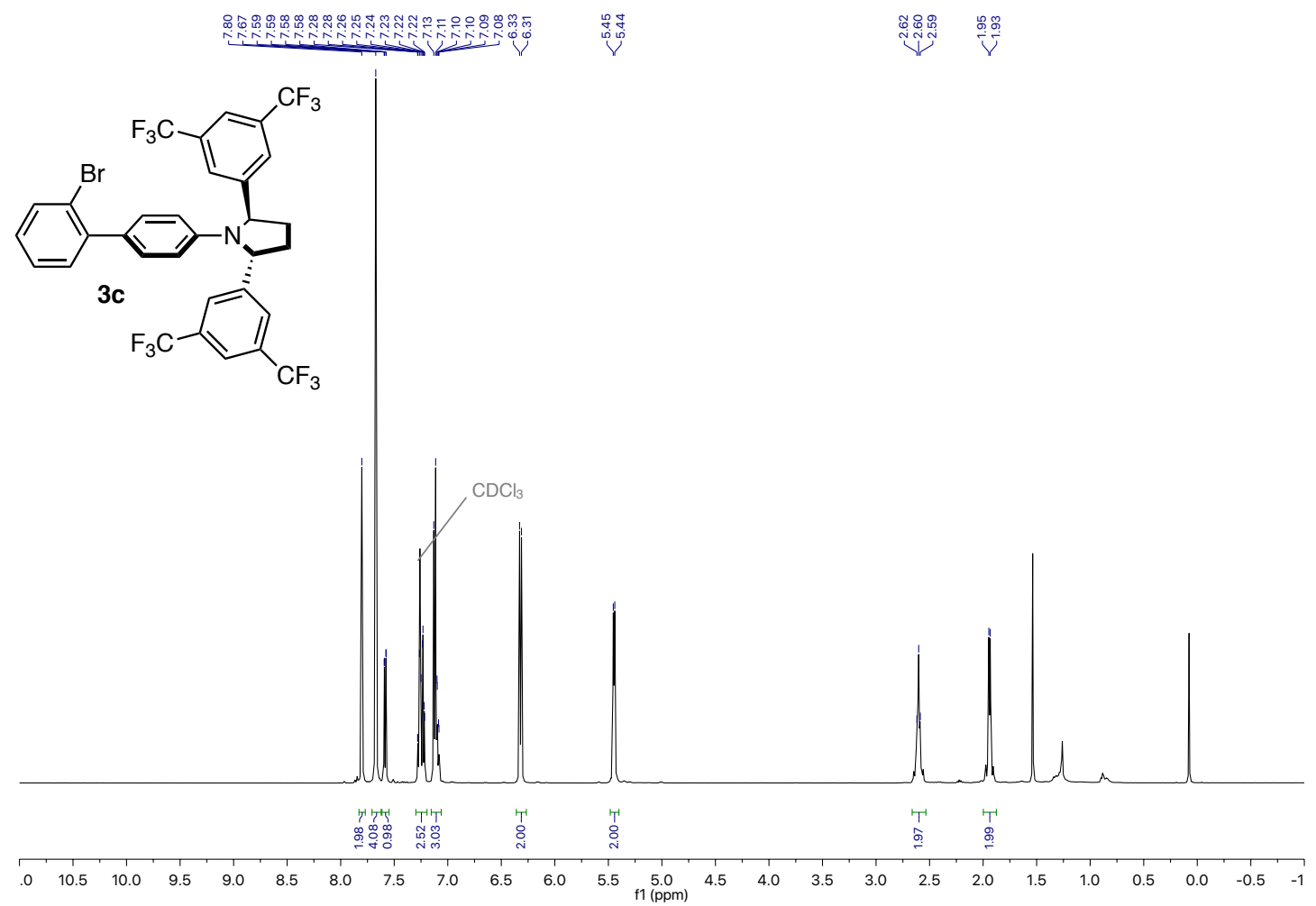


${ }^{13} \mathrm{C} \mathrm{NMR}: 126 \mathrm{MHz}, \mathrm{CDCl}_{3}$, compound 3c

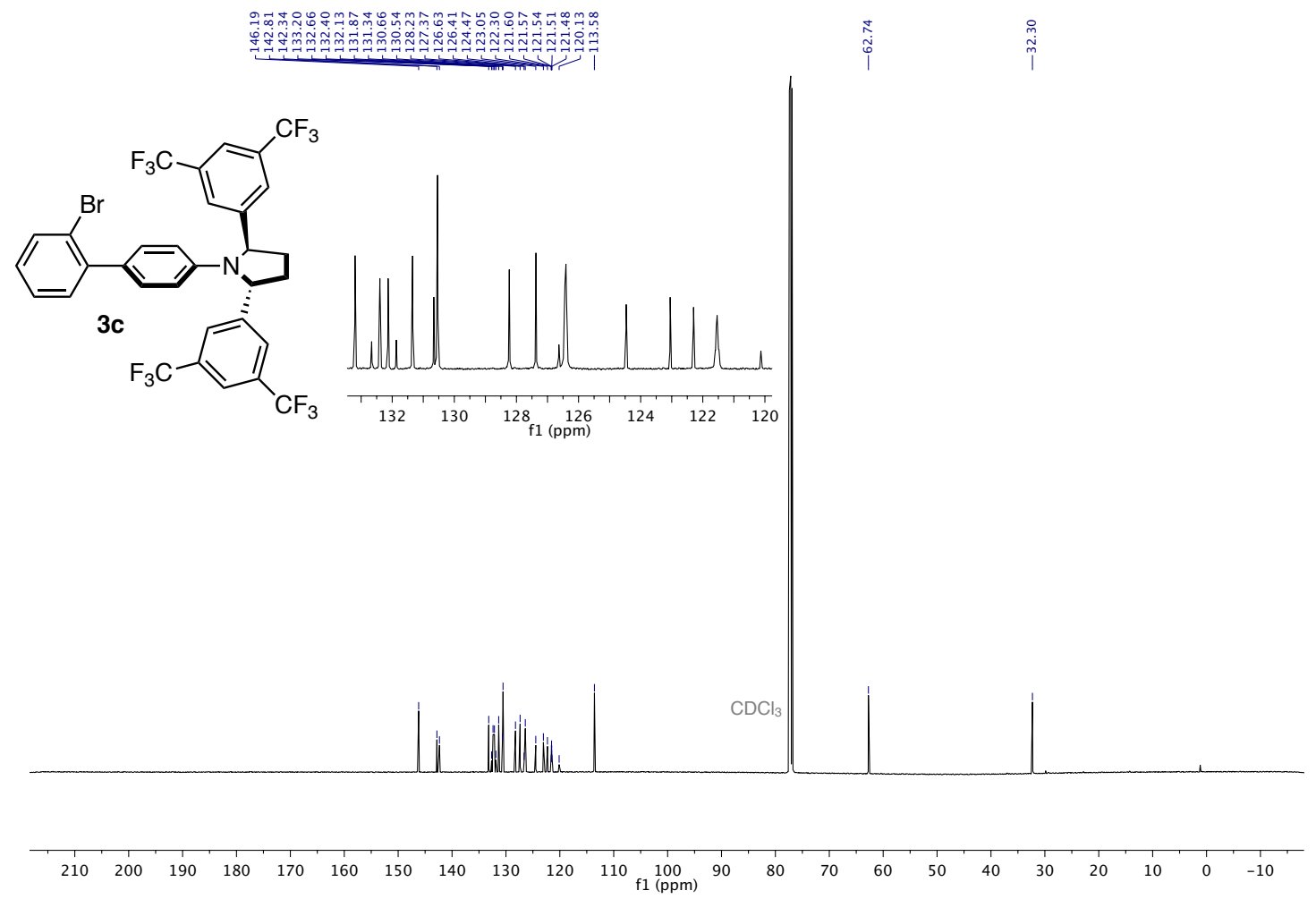

${ }^{19} \mathrm{~F}\left\{{ }^{1} \mathrm{H}\right\}$ NMR: $376 \mathrm{MHz}, \mathrm{CDCl}_{3}$, compound 3c

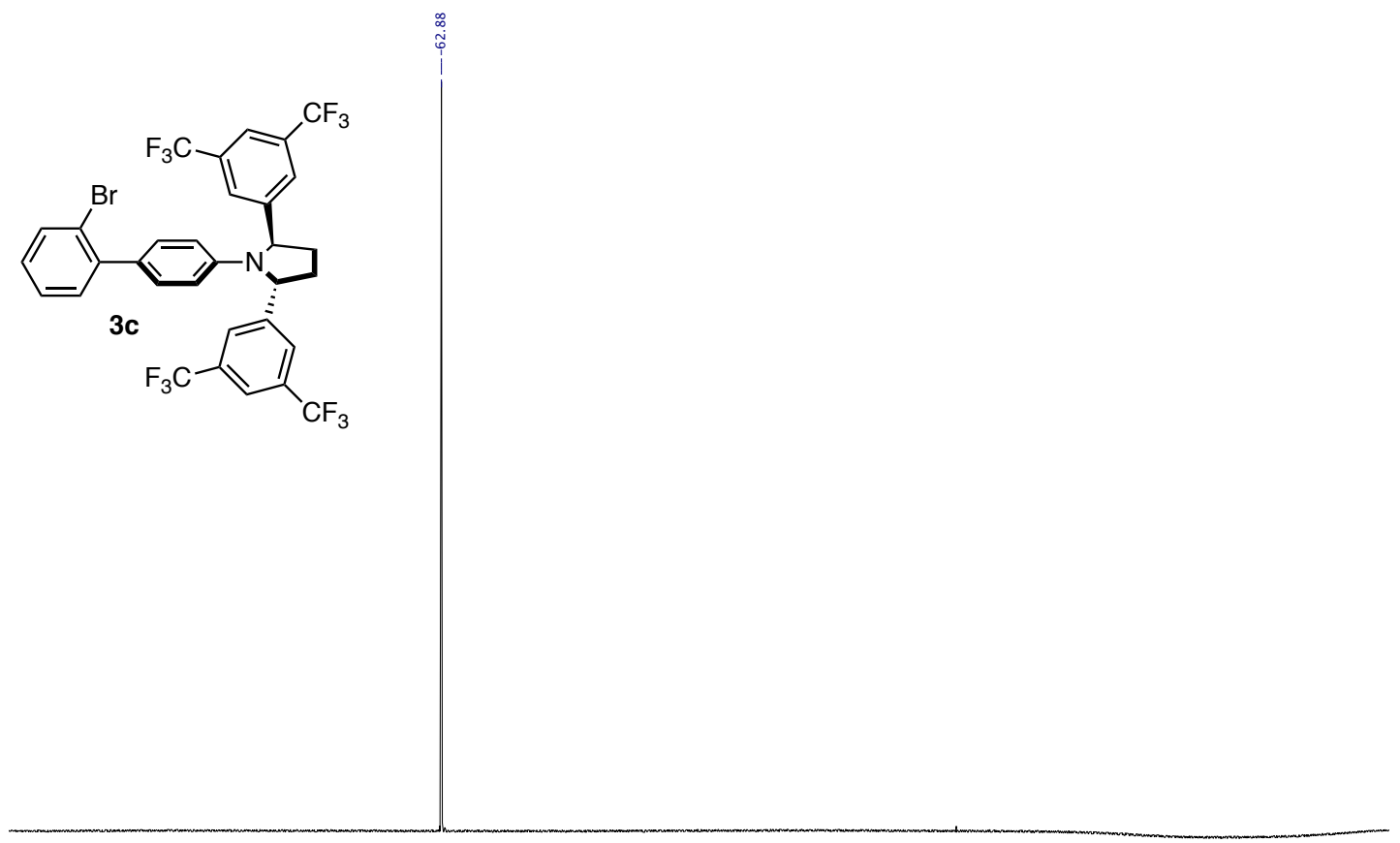

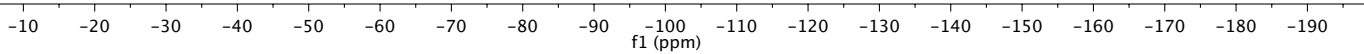


${ }^{1} \mathrm{H}$ NMR: $400 \mathrm{MHz}, \mathrm{CD}_{2} \mathrm{Cl}_{2}$, compound 4c

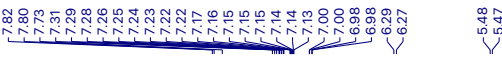

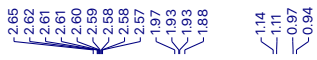<smiles>CCN(c1cc(C(F)(F)F)cc(C(F)(F)F)c1)C(C)c1cc(C(F)(F)F)cc(C(F)(F)F)c1</smiles>

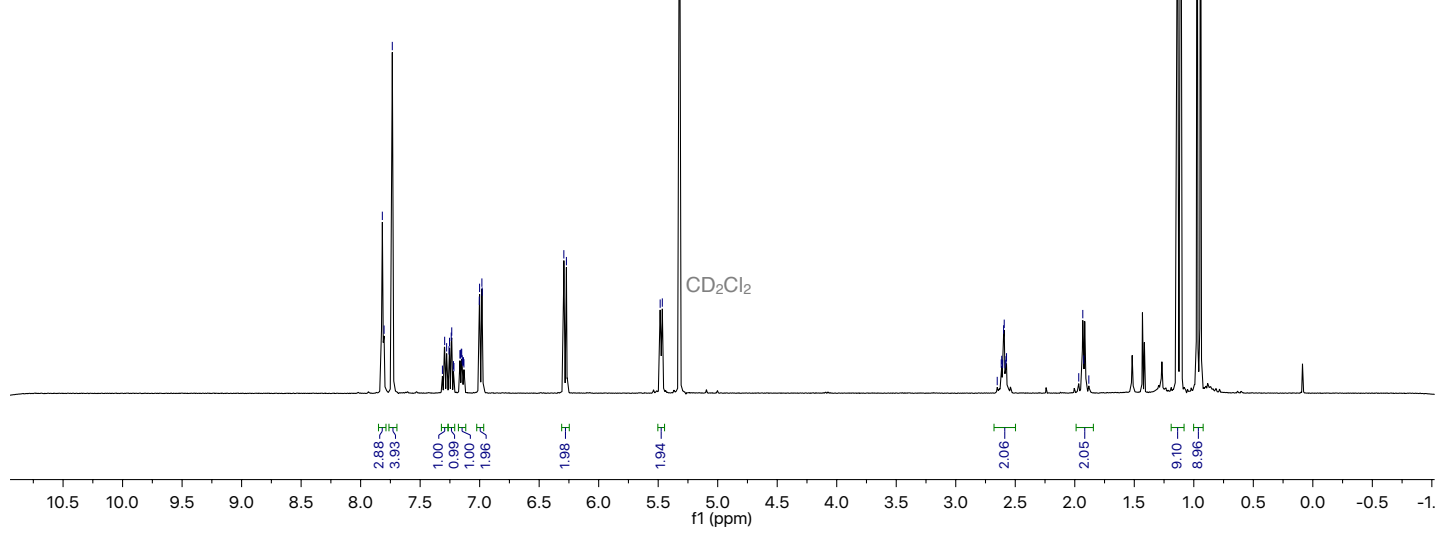

${ }^{13} \mathrm{C}$ NMR: $126 \mathrm{MHz}, \mathrm{CD}_{2} \mathrm{Cl}_{2}$, compound 4c
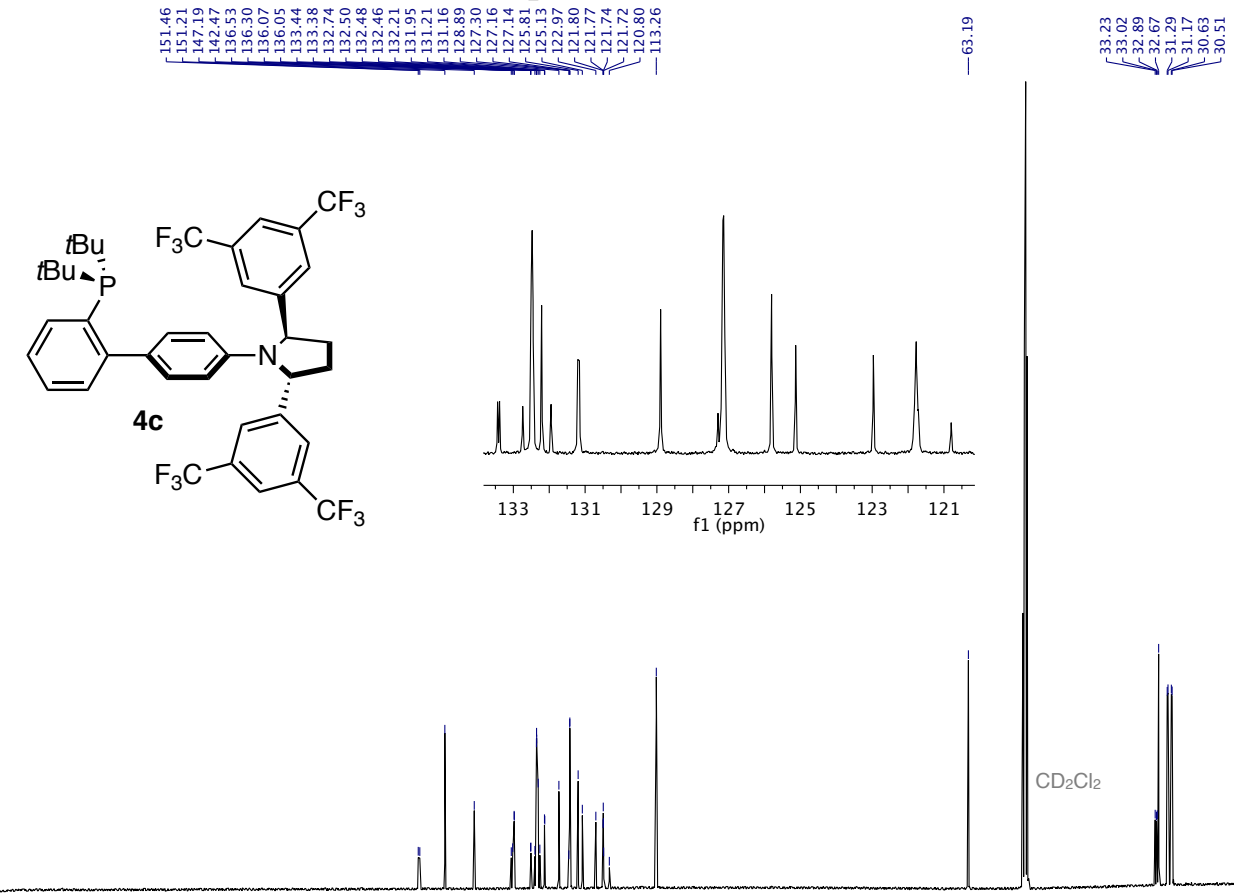

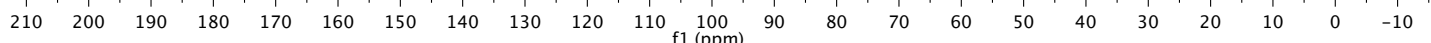


${ }^{31} \mathrm{P}\left\{{ }^{1} \mathrm{H}\right\}$ NMR: $203 \mathrm{MHz}, \mathrm{CD}_{2} \mathrm{Cl}_{2}$, compound 4c

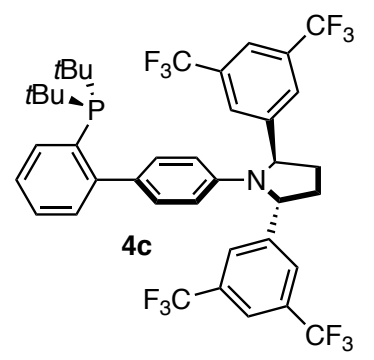

$\begin{array}{lllllllllllllllllllllllllllll}380 & 360 & 340 & 320 & 300 & 280 & 260 & 240 & 220 & 200 & 180 & 160 & 140 & 120 & 100 & 80 & 60 & 40 & 20 & 0 & -20 & -40 & -60 & -80 & -100 & -120 & -140 & -160 & -180\end{array}$

${ }^{19} \mathrm{~F}\left\{{ }^{1} \mathrm{H}\right\}$ NMR: $376 \mathrm{MHz}, \mathrm{CD}_{2} \mathrm{Cl}_{2}$, compound 4c

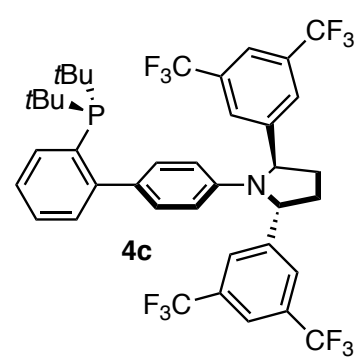

$\begin{array}{lllllllllllllllllll}-10 & -20 & -30 & -40 & -50 & -60 & -70 & -80 & -90 & -100 & -110 & -120 & -130 & -140 & -150 & -160 & -170 & -180 & -190\end{array}$ 
${ }^{1} \mathrm{H}$ NMR: $400 \mathrm{MHz}, \mathrm{CD}_{2} \mathrm{Cl}_{2}$, compound $(R, R)-\mathbf{C}$

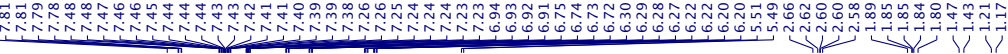

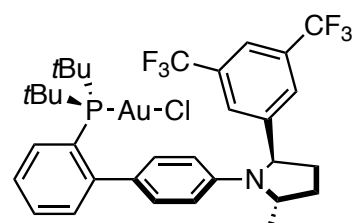

$(R, R)-\mathrm{C}$<smiles>FC(F)(F)c1cccc(C(F)(F)F)c1</smiles>
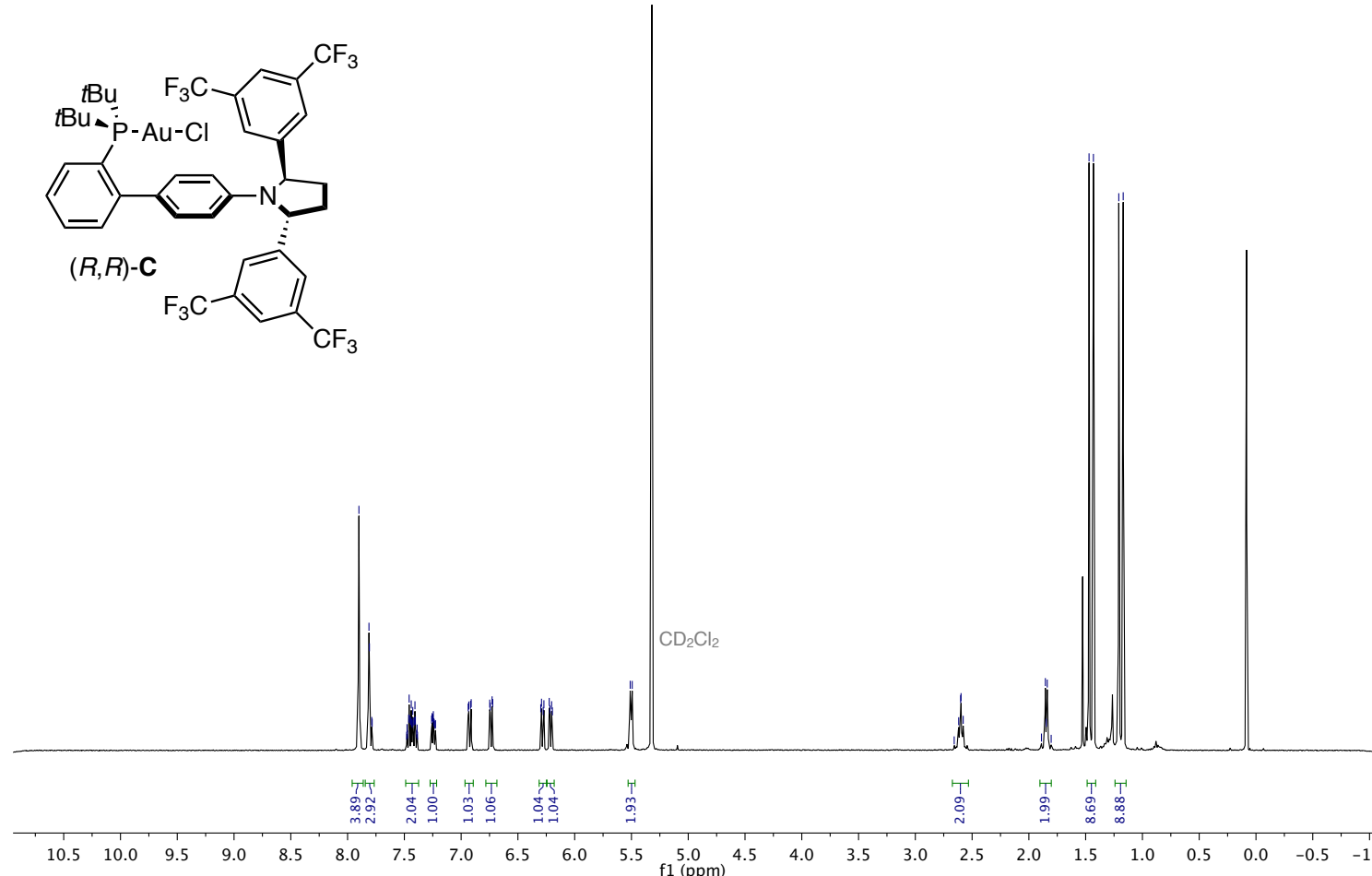

${ }^{13} \mathrm{C}$ NMR: $126 \mathrm{MHz}, \mathrm{CD}_{2} \mathrm{Cl}_{2}$, compound $(R, R)-\mathrm{C}$

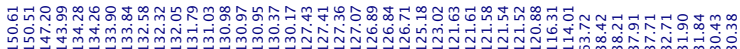<smiles>[18OH]P([18F])c1ccccc1-c1cccc(C2CCN2c2cc(C(F)(F)F)cc(C(F)(F)F)c2)c1</smiles>

$(R, R)-\mathrm{C}$

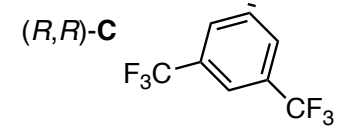

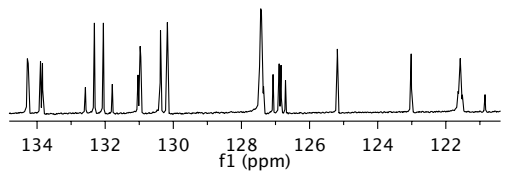

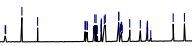

逍

$\begin{array}{llllllllllllllllllllllllllllllllll}50 & 240 & 230 & 220 & 210 & 200 & 190 & 180 & 170 & 160 & 150 & 140 & 130 & 120 & 110 & 100 & 90 & 80 & 70 & 60 & 50 & 40 & 30 & 20 & 10 & 0 & -10 & -20 & -30 & -40\end{array}$ 
${ }^{31} \mathrm{P}\left\{{ }^{1} \mathrm{H}\right\}$ NMR: $162 \mathrm{MHz}, \mathrm{CD}_{2} \mathrm{Cl}_{2}$, compound $(R, R)-\mathbf{C}$

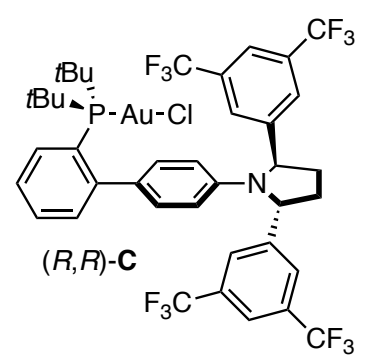

$\begin{array}{lllllllllllllllllllllllllll}320 & 300 & 280 & 260 & 240 & 220 & 200 & 180 & 160 & 140 & 120 & 100 & 80 & 60 & 40 & 20 & 0 & -20 & -40 & -60 & -80 & -100 & -120\end{array}$

${ }^{19} \mathrm{~F}\left\{{ }^{1} \mathrm{H}\right\}$ NMR: $376 \mathrm{MHz}, \mathrm{CD}_{2} \mathrm{Cl}_{2}$, compound $(R, R)-\mathrm{C}$

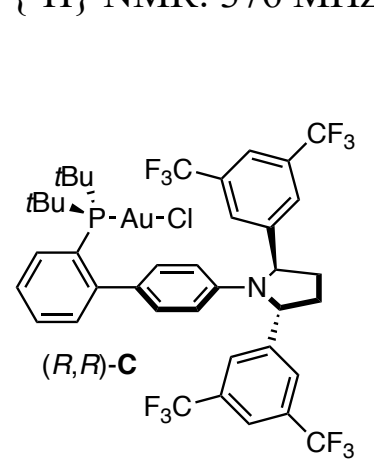

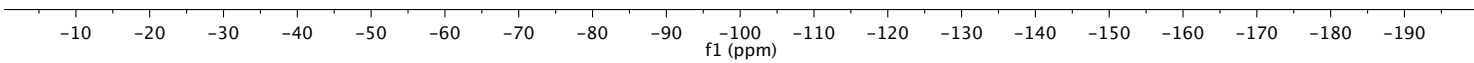


${ }^{1} \mathrm{H}$ NMR: $500 \mathrm{MHz}, \mathrm{CD}_{2} \mathrm{Cl}_{2}$, compound $\mathbf{4 d}$

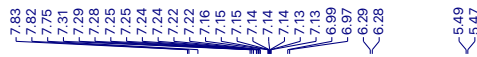

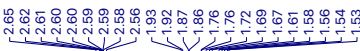<smiles>Cc1cc(C(=O)N(C)c2cc(C(F)(F)F)cc(C(F)(F)F)c2)cc(C(F)(F)F)c1</smiles>

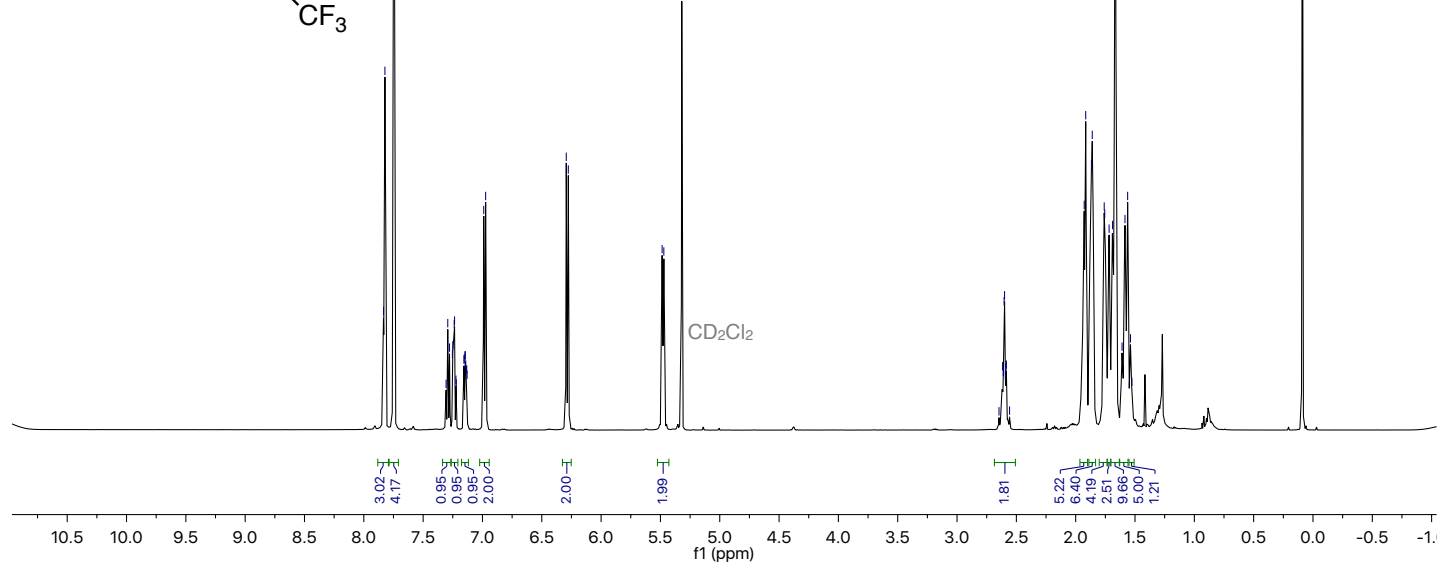

${ }^{13} \mathrm{C}$ NMR: $126 \mathrm{MHz}, \mathrm{CD}_{2} \mathrm{Cl}_{2}$, compound $\mathbf{4 d}$

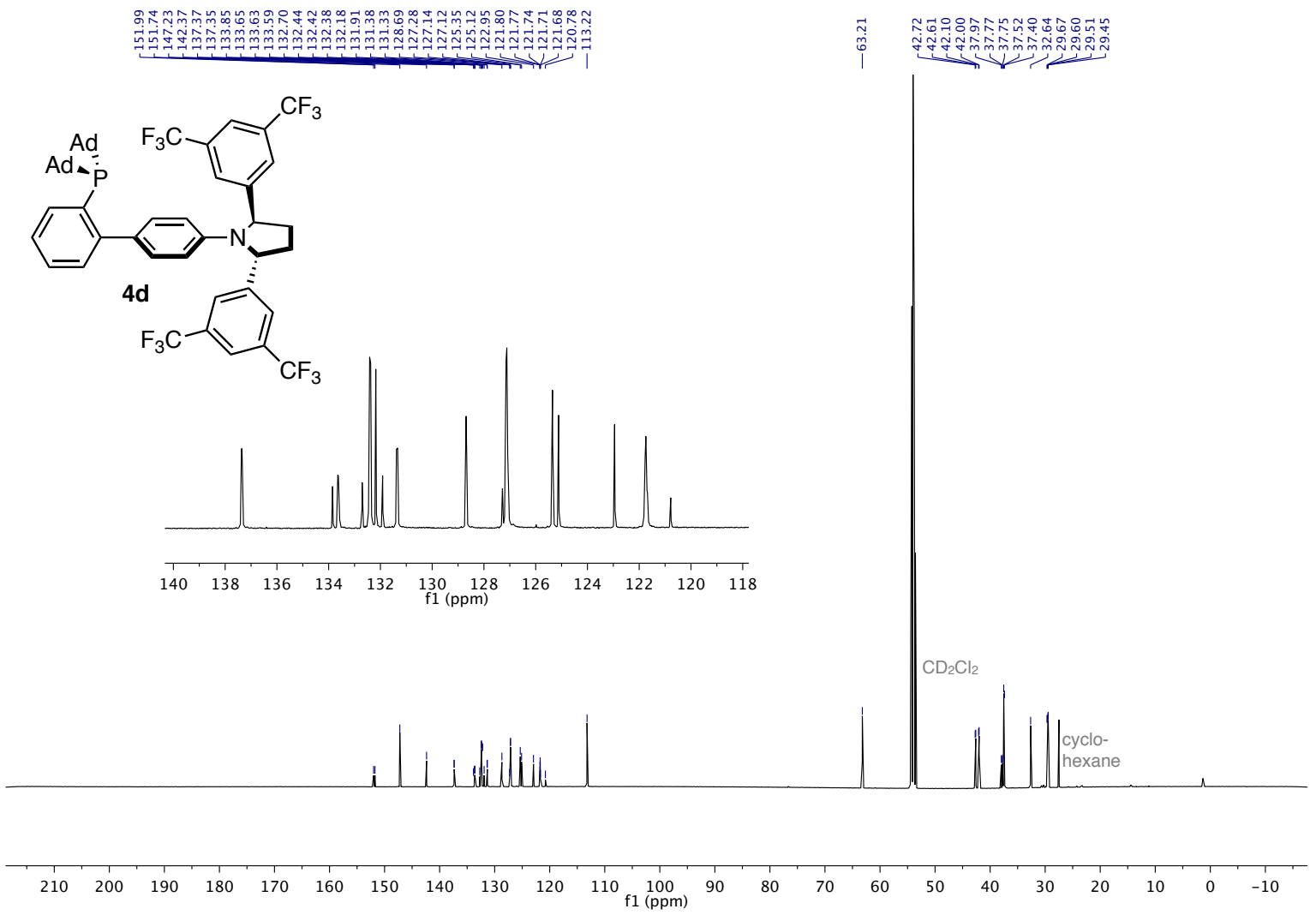


${ }^{31} \mathrm{P}\left\{{ }^{1} \mathrm{H}\right\}$ NMR: $203 \mathrm{MHz}, \mathrm{CD}_{2} \mathrm{Cl}_{2}$, compound 4d

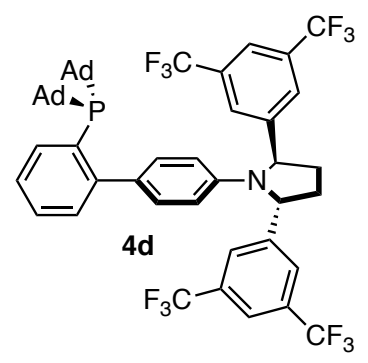

$\begin{array}{lllllllllllllllllllllllllllllllll}380 & 360 & 340 & 320 & 300 & 280 & 260 & 240 & 220 & 200 & 180 & 160 & 140 & 120 & 100 & 80 & 60 & 40 & 20 & 0 & -20 & -40 & -60 & -80 & -100 & -120 & -140 & -160 & -180\end{array}$

${ }^{19} \mathrm{~F}\left\{{ }^{1} \mathrm{H}\right\}$ NMR: $471 \mathrm{MHz}, \mathrm{CD}_{2} \mathrm{Cl}_{2}$, compound 4d

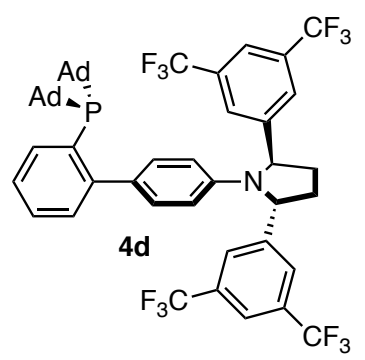

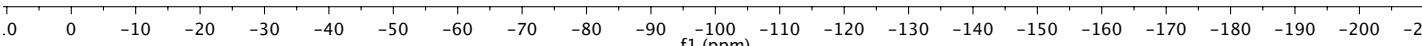


${ }^{1} \mathrm{H}$ NMR: $500 \mathrm{MHz}, \mathrm{CDCl}_{3}$, compound $(R, R)$-D

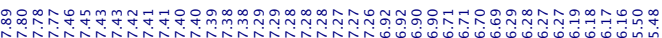

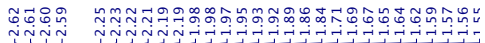<smiles>COP([Ge])(=[W])c1ccccc1-c1cccc(C2CCN2c2cc(C(F)(F)F)cc(C(F)(F)F)c2)c1</smiles>

$(R, R)$-D<smiles>FC(F)(F)c1cccc(C(F)(F)F)c1</smiles>
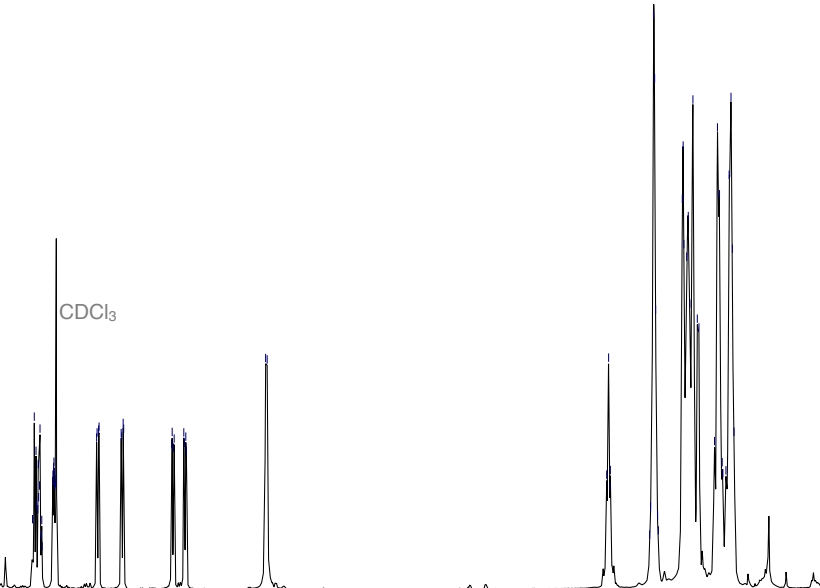

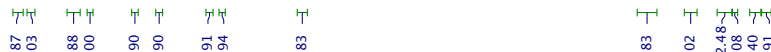

$\begin{array}{lllllllllllllllllllllllll}10.5 & 10.0 & 9.5 & 9.0 & 8.5 & 8.0 & 7.5 & 7.0 & 6.5 & 6.0 & 5.5 & \begin{array}{l}5.0 \\ \mathrm{f} 1\end{array}(\mathrm{ppm}) & 4.5 & 4.0 & 3.5 & 3.0 & 2.5 & 2.0 & 1.5 & 1.0 & 0.5 & 0.0 & -0.5 & -1\end{array}$

${ }^{13} \mathrm{C}$ NMR: $126 \mathrm{MHz}, \mathrm{CDCl}_{3}$, compound $(R, R)$-D

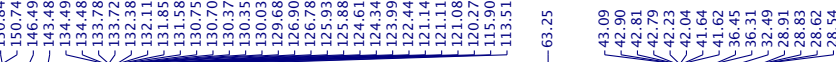

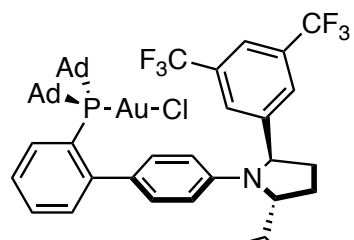

$(R, R)$-D<smiles>FC(F)(F)c1cccc(C(F)(F)F)c1</smiles>

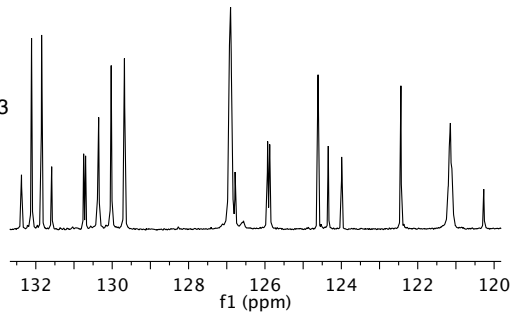

illiditili

$\begin{array}{llllllllllll}210 & 200 & 190 & 180 & 170 & 160 & 150 & 140 & 130 & 120 & 110 & 100\end{array}$ 
${ }^{31} \mathrm{P}\left\{{ }^{1} \mathrm{H}\right\}$ NMR: $162 \mathrm{MHz}, \mathrm{CDCl}_{3}$, compound $(R, R)-\mathbf{D}$

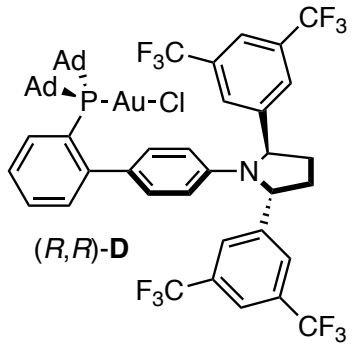

$\begin{array}{lllllllllllllllllllllllll}320 & 300 & 280 & 260 & 240 & 220 & 200 & 180 & 160 & 140 & 120 & 100 & 80 & 60 & 40 & 20 & 0 & -20 & -40 & -60 & -80 & -100 & -120\end{array}$

${ }^{19} \mathrm{~F}\left\{{ }^{1} \mathrm{H}\right\}$ NMR: $471 \mathrm{MHz}, \mathrm{CDCl}_{3}$, compound $(R, R)-\mathbf{D}$

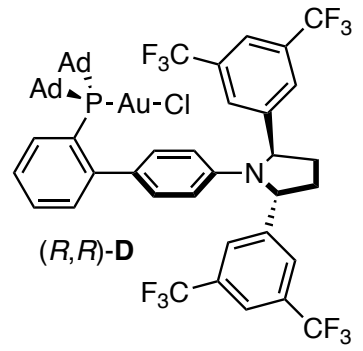

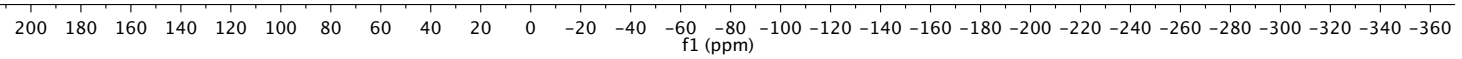


${ }^{1} \mathrm{H}$ NMR: $500 \mathrm{MHz}, \mathrm{CDCl}_{3}$, compound 3d

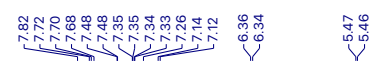<smiles>FC(F)(F)c1cc(C2CCC2c2cc(C(F)(F)F)cc(C(F)(F)F)c2)cc(C(F)(F)F)c1</smiles>

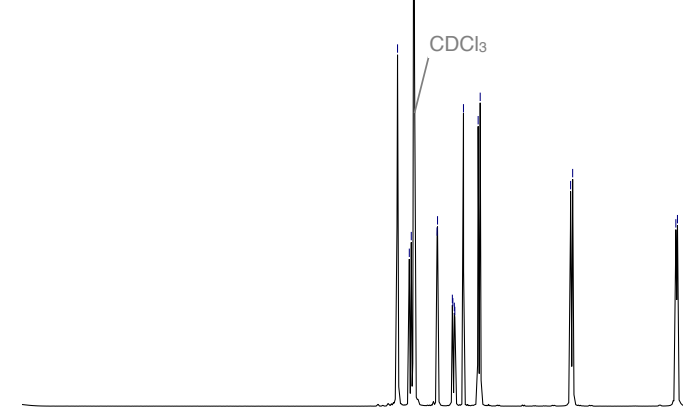

\section{Iึ}

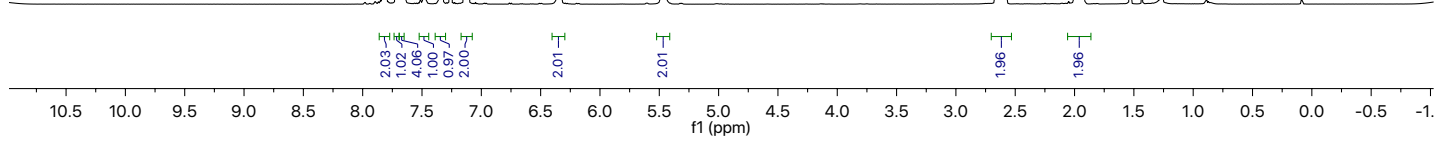

${ }^{13} \mathrm{C}$ NMR: $126 \mathrm{MHz}, \mathrm{CDCl}_{3}$, compound 3d

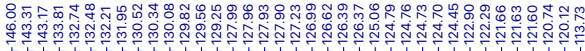

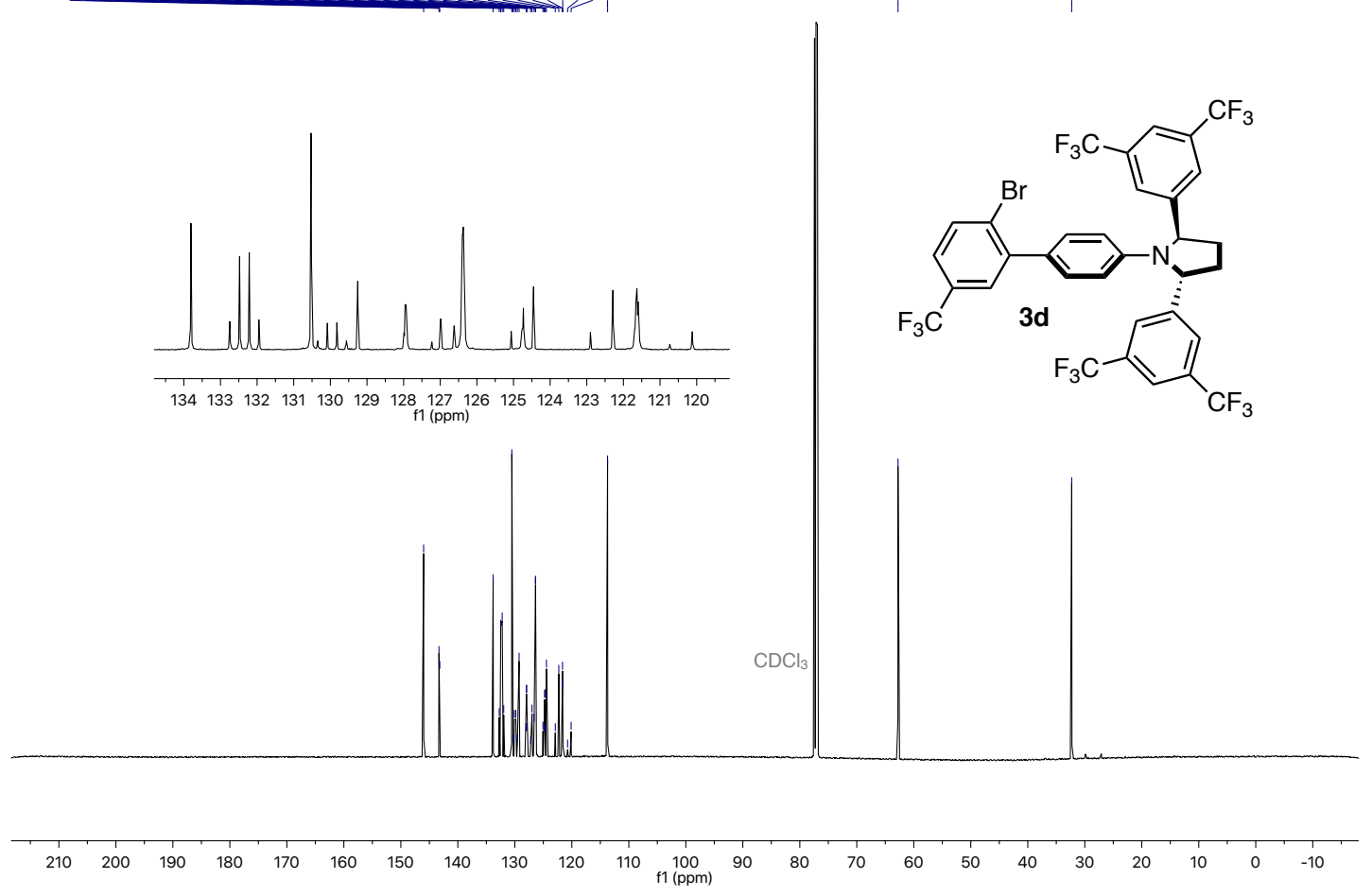


${ }^{19} \mathrm{~F}\left\{{ }^{1} \mathrm{H}\right\}$ NMR: $471 \mathrm{MHz}, \mathrm{CDCl}_{3}$, compound 3d

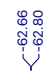
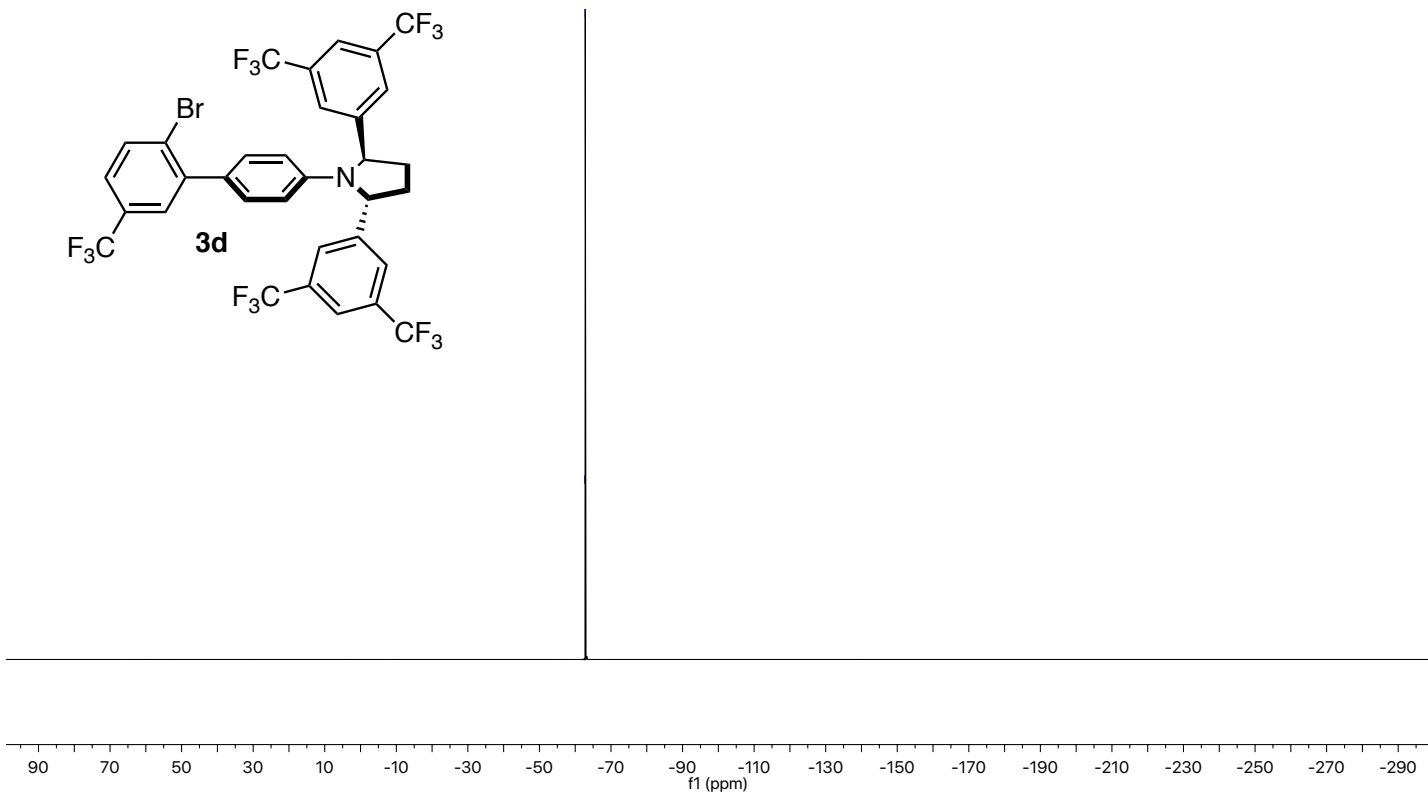

${ }^{1} \mathrm{H}$ NMR: $500 \mathrm{MHz}, \mathrm{CDCl}_{3}$, compound 4e

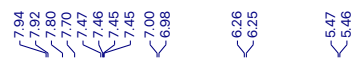

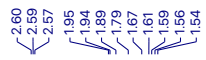<smiles>FC(F)(F)c1cc(-c2ccc(N(c3cc(C(F)(F)F)cc(C(F)(F)F)c3)C3CCC3c3cc(C(F)(F)F)cc(C(F)(F)F)c3)cc2)cc(C(F)(F)F)c1</smiles>

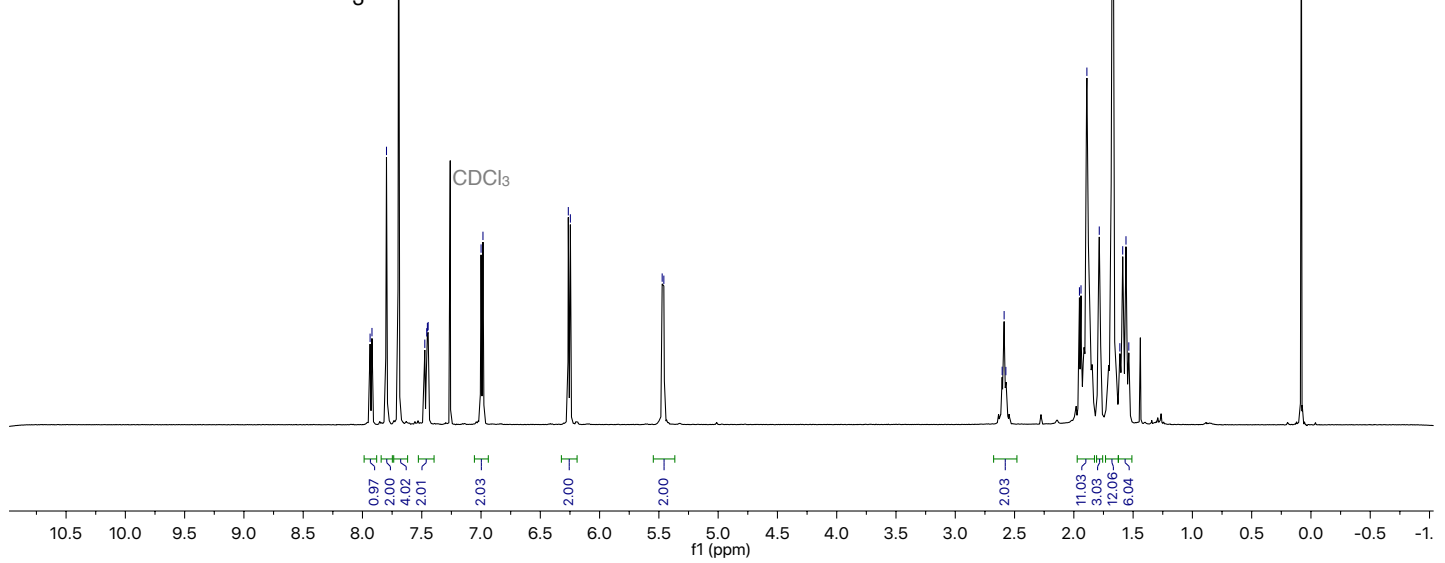


${ }^{13} \mathrm{C} \mathrm{NMR}: 126 \mathrm{MHz}, \mathrm{CDCl}_{3}$, compound 4e

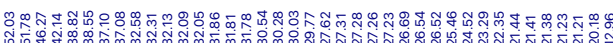
ن

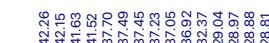

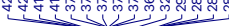
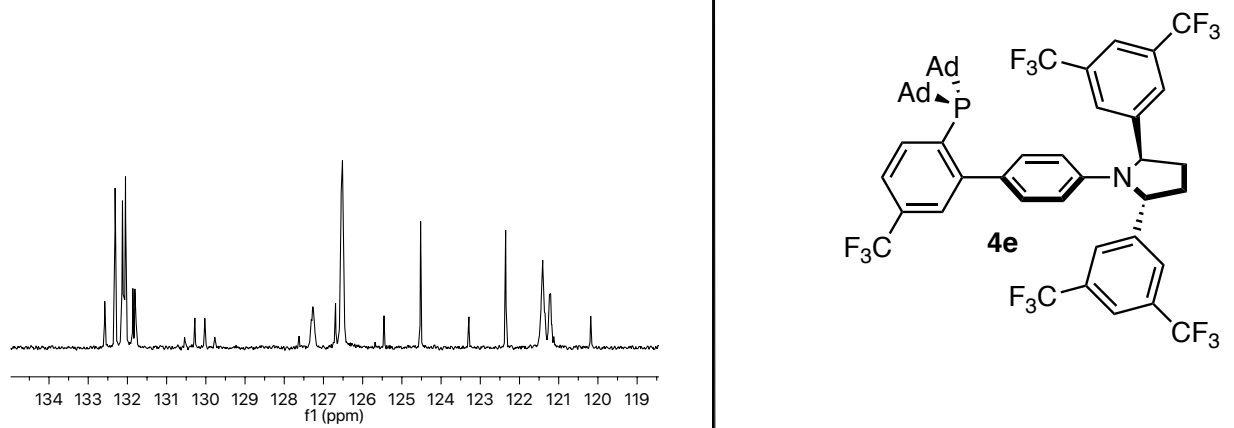

$\begin{array}{llllllllllllllll}134 & 133 & 132 & 131 & 130 & 129 & 128 & 127 & 126 & 125 & 124 & 123 & 122 & 121 & 120 & 119\end{array}$
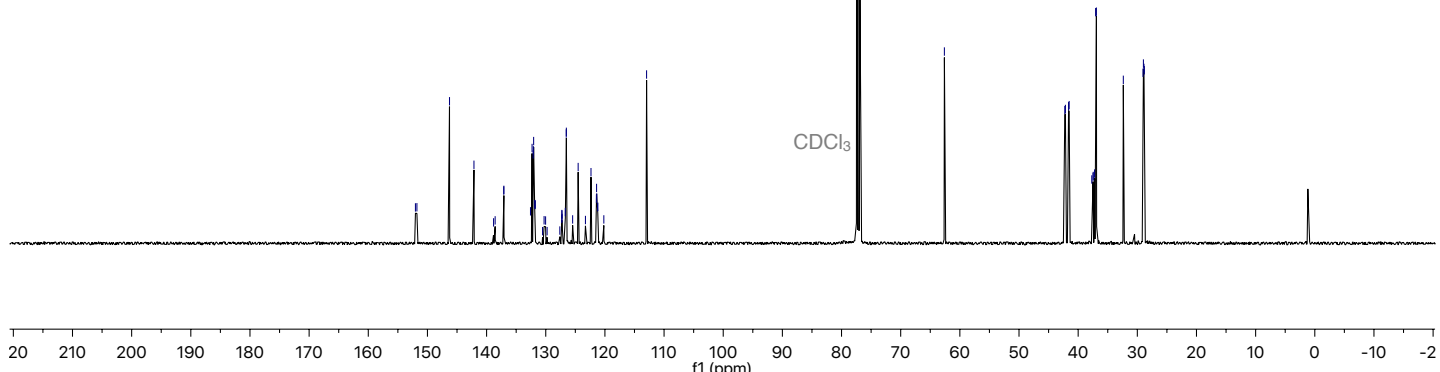

${ }^{31} \mathrm{P}\left\{{ }^{1} \mathrm{H}\right\}$ NMR: $202 \mathrm{MHz}, \mathrm{CDCl}_{3}$, compound 4e<smiles>FC(F)(F)c1cc(C2CCC2c2cc(C(F)(F)F)cc(C(F)(F)F)c2)cc(C(F)(F)F)c1</smiles>

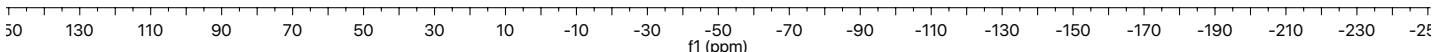


${ }^{19} \mathrm{~F}\left\{{ }^{1} \mathrm{H}\right\}$ NMR: $376 \mathrm{MHz}, \mathrm{CDCl}_{3}$, compound $4 \mathbf{e}$

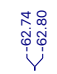
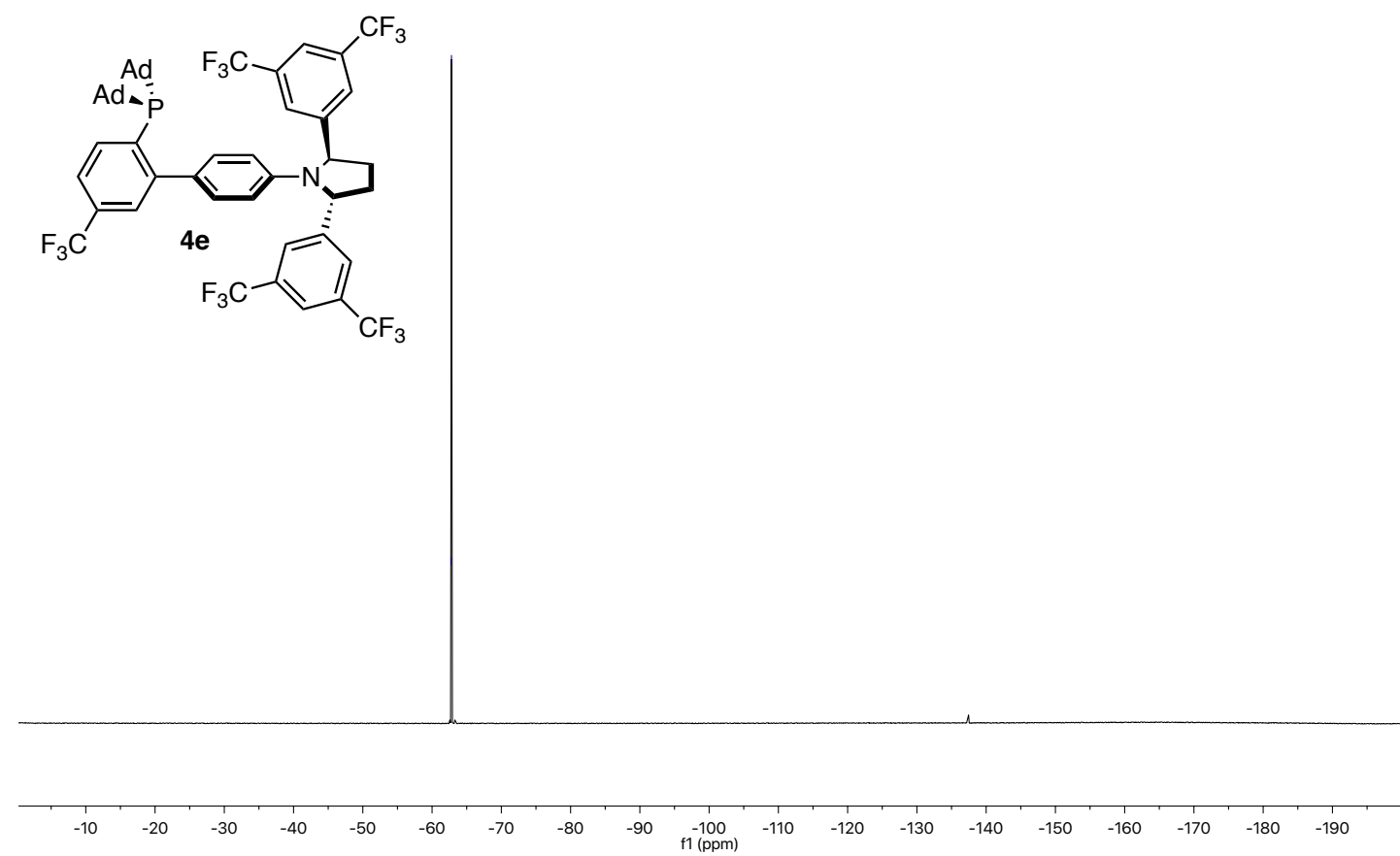

${ }^{1} \mathrm{H}$ NMR: $500 \mathrm{MHz}, \mathrm{CDCl}_{3}$, compound $(R, R)-\mathbf{E}$

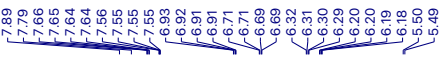

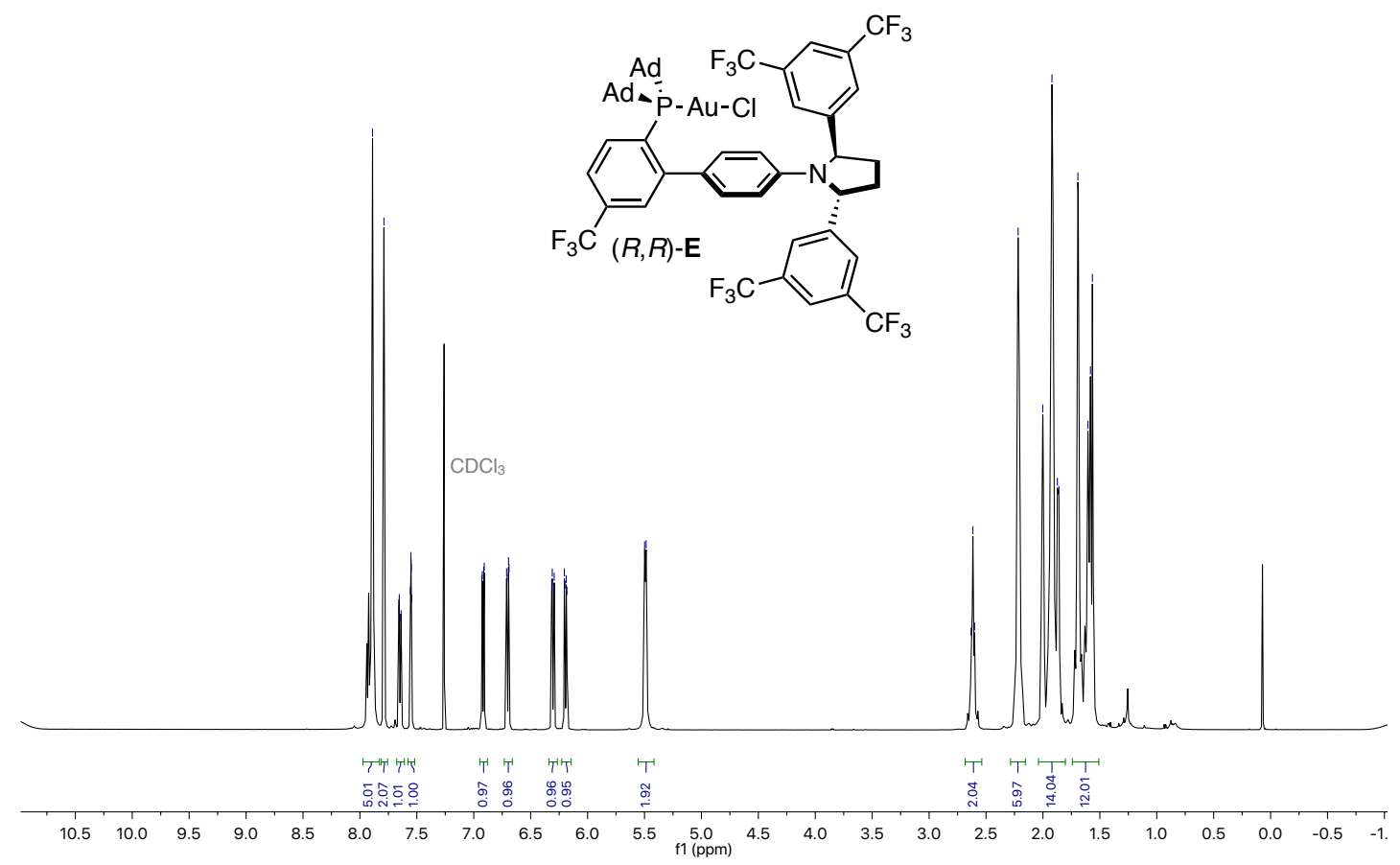


${ }^{13} \mathrm{C}$ NMR: $126 \mathrm{MHz}, \mathrm{CDCl}_{3}$, compound $(R, R)-\mathbf{E}$
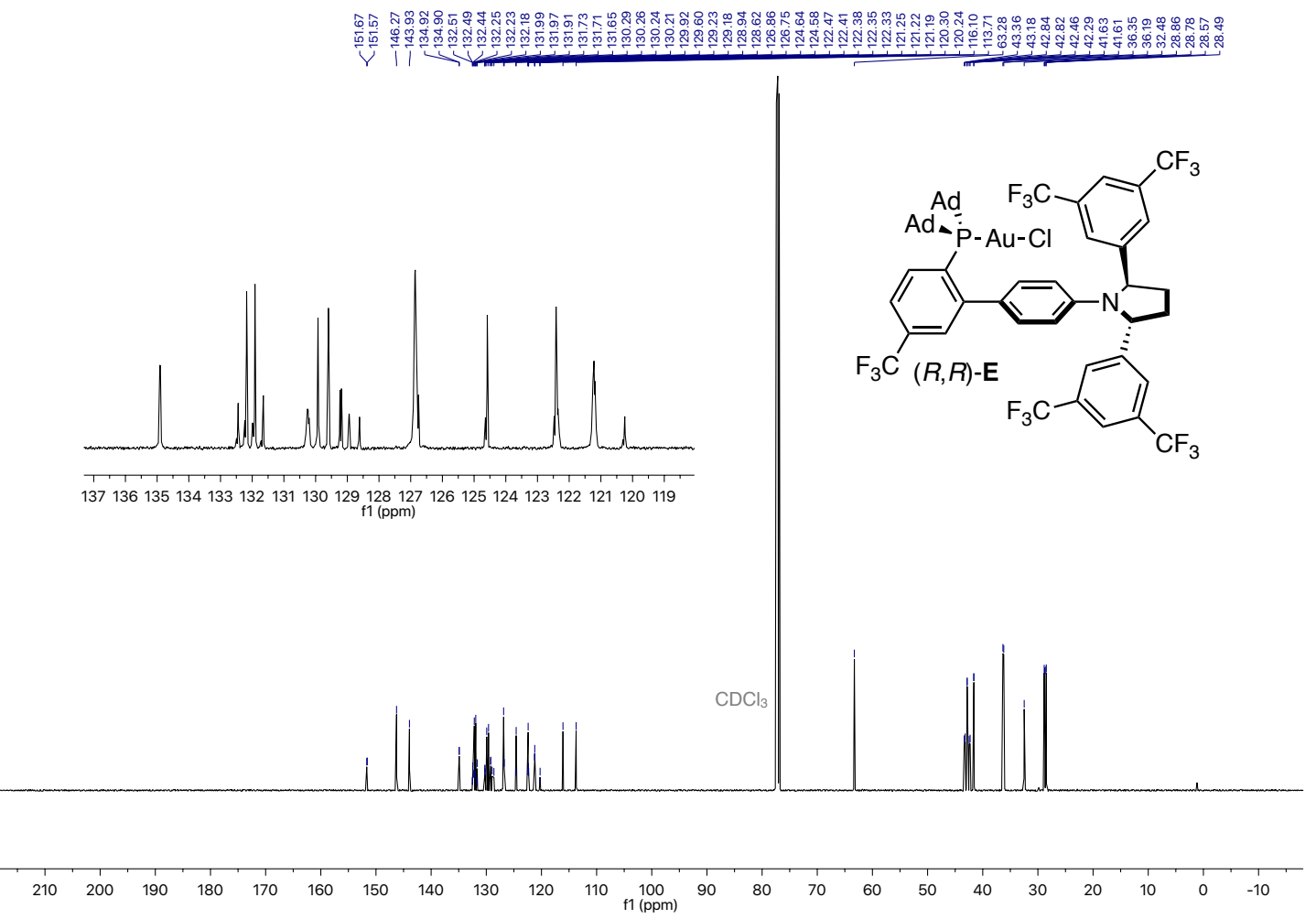

${ }^{31} \mathrm{P}\left\{{ }^{1} \mathrm{H}\right\}$ NMR: $203 \mathrm{MHz}, \mathrm{CDCl}_{3}$, compound $(R, R)-\mathbf{E}$
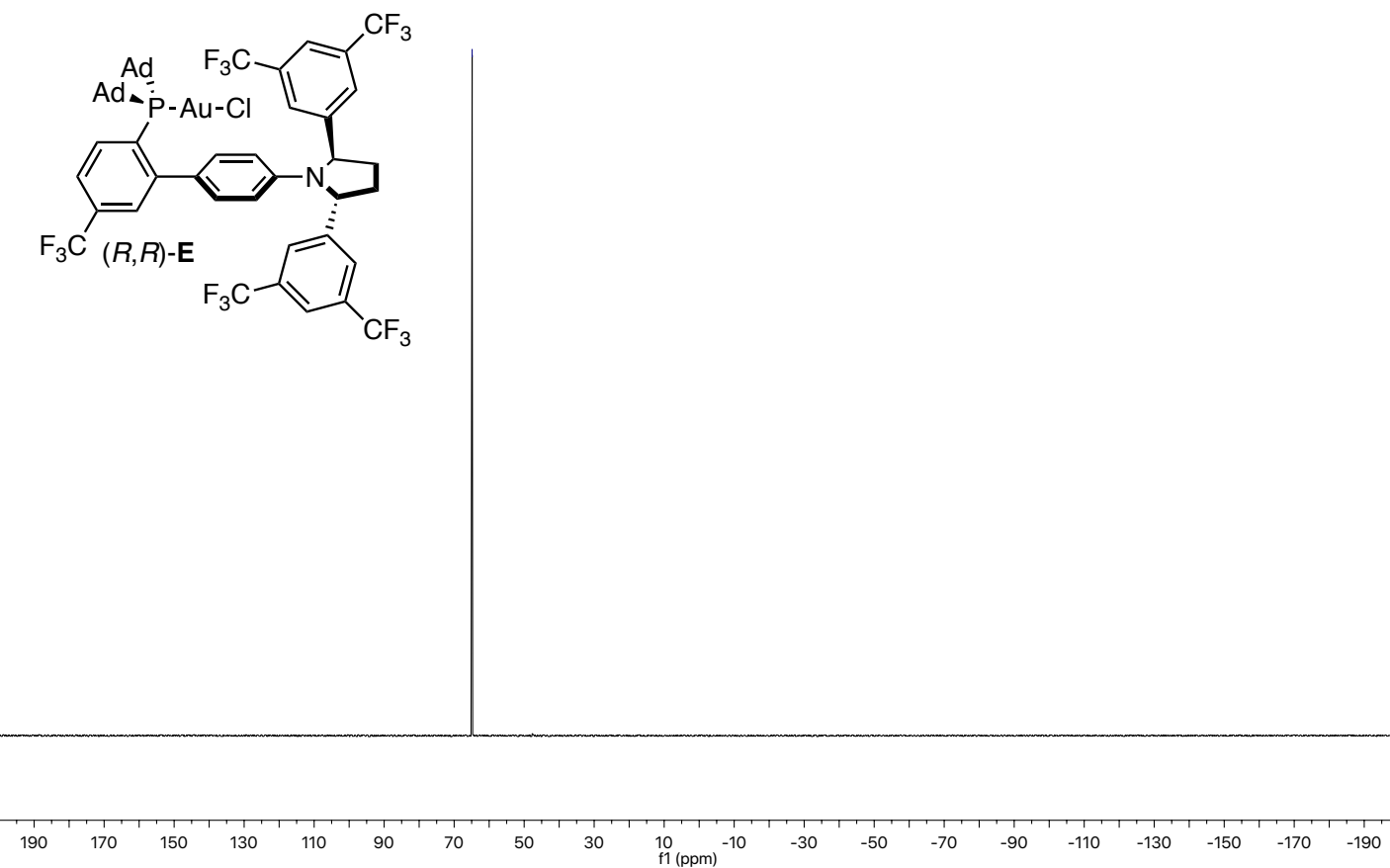
${ }^{19} \mathrm{~F}\left\{{ }^{1} \mathrm{H}\right\}$ NMR: $471 \mathrm{MHz}, \mathrm{CDCl}_{3}$, compound $(R, R)-\mathbf{E}$

䠉

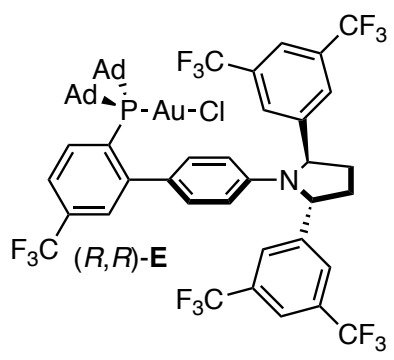

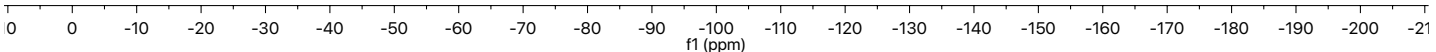

${ }^{1} \mathrm{H}$ NMR: $400 \mathrm{MHz}, \mathrm{CD}_{2} \mathrm{Cl}_{2}$, compound 3e

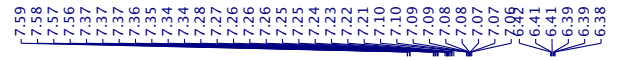

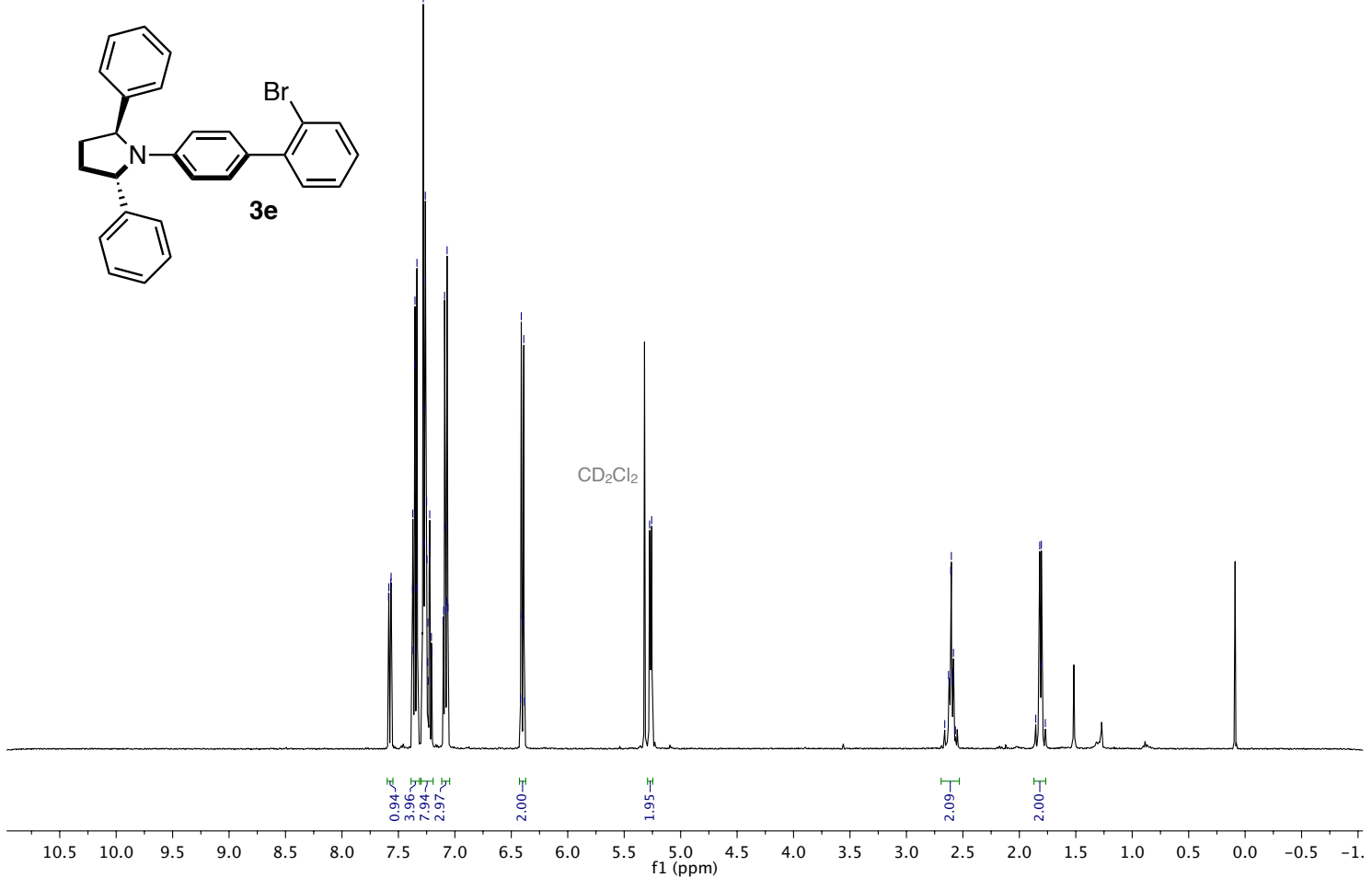


${ }^{13} \mathrm{C}$ NMR: $101 \mathrm{MHz}, \mathrm{CD}_{2} \mathrm{Cl}_{2}$, compound 3e

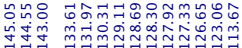

()<smiles>Brc1ccccc1-c1ccc(N2C(c3ccccc3)CCC2c2ccccc2)cc1</smiles>

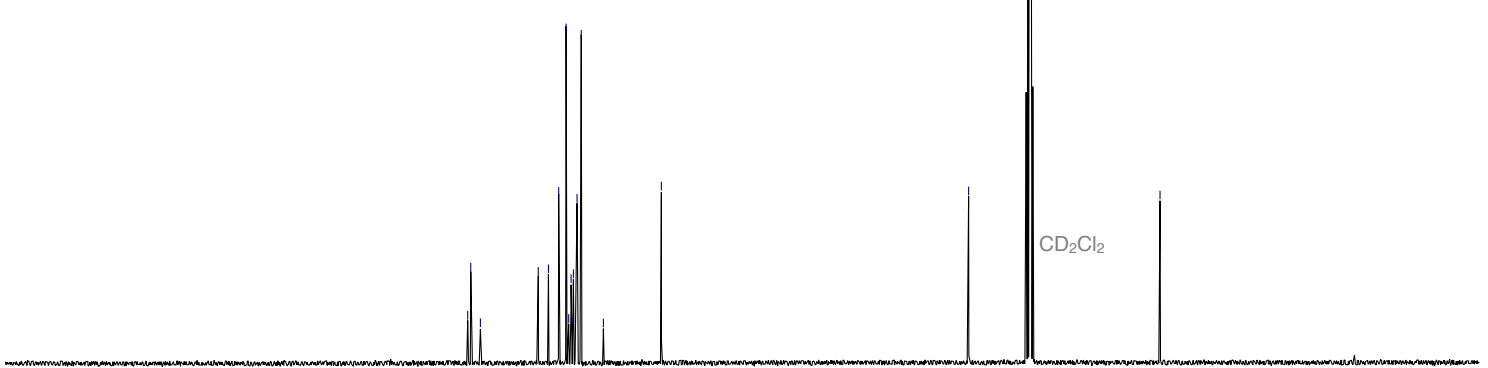

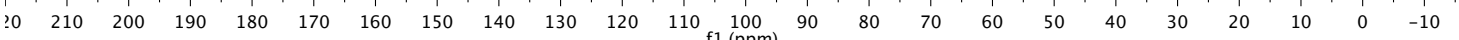

${ }^{1} \mathrm{H}$ NMR: $400 \mathrm{MHz}, \mathrm{CDCl}_{3}$, compound $\mathbf{4 f}$

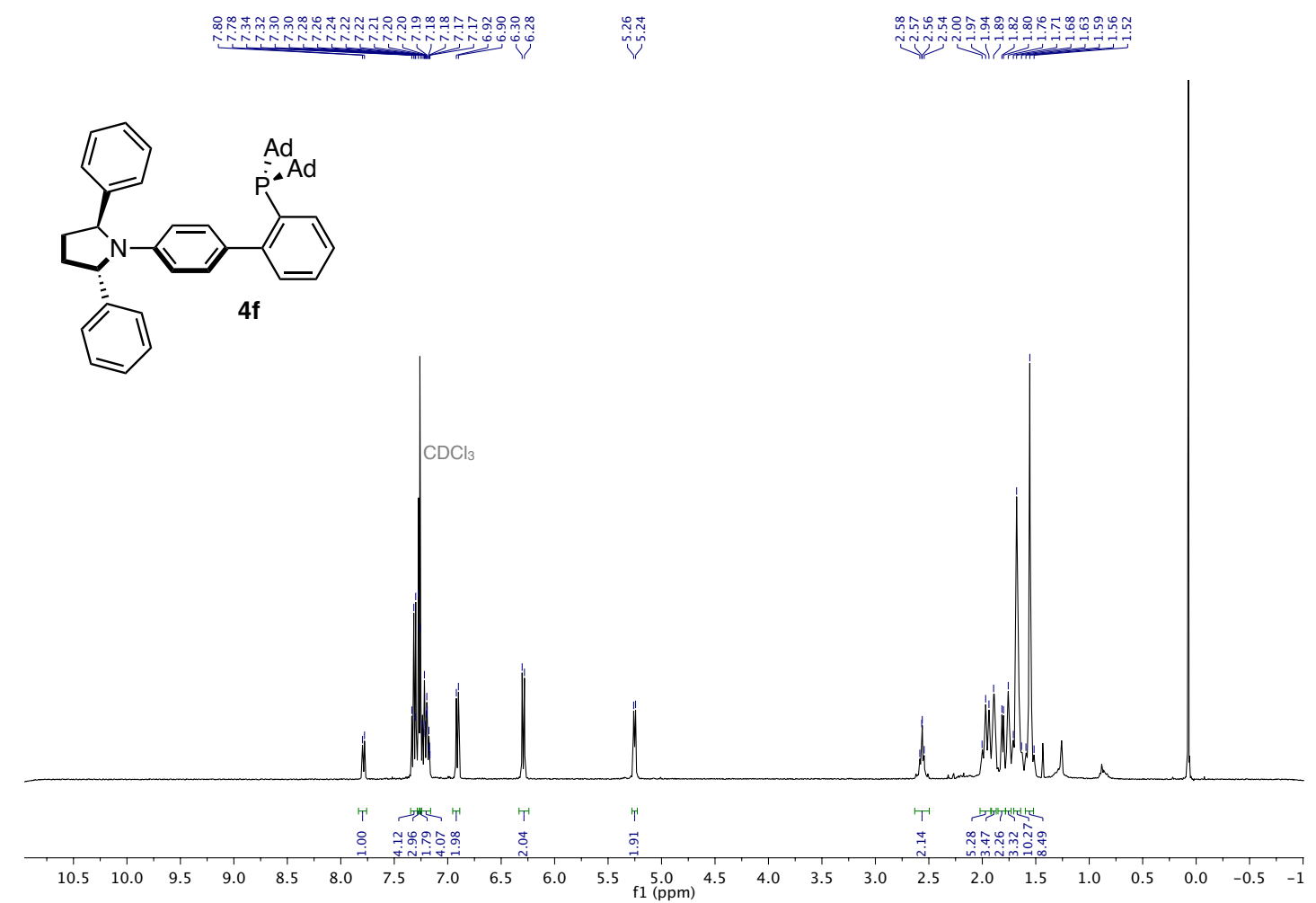


${ }^{13} \mathrm{C}$ NMR: $101 \mathrm{MHz}, \mathrm{CDCl}_{3}$, compound $4 \mathbf{f}$

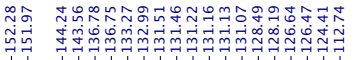

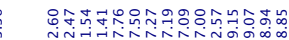

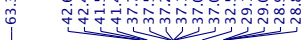<smiles>[Te]c1ccccc1-c1ccc(N2C(c3ccccc3)CCC2c2ccccc2)cc1</smiles>

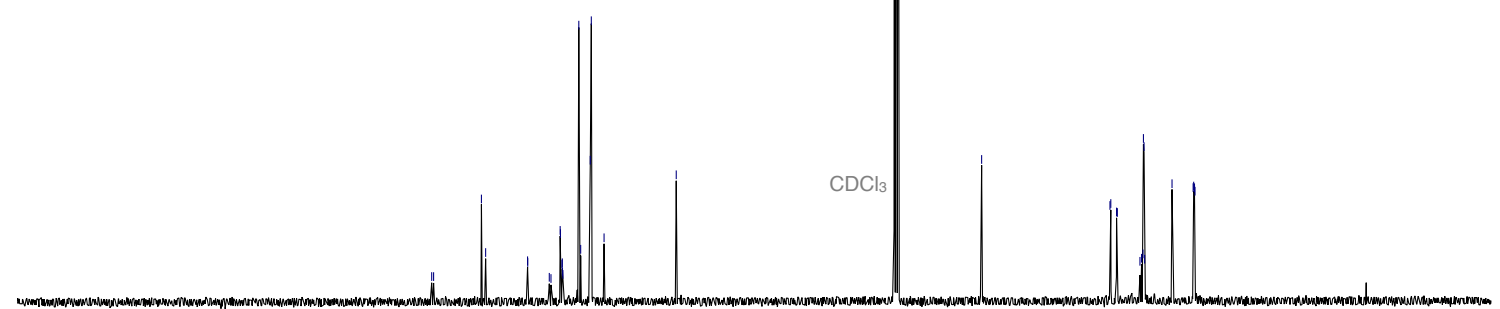

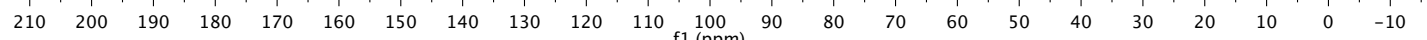

${ }^{31} \mathrm{P}\left\{{ }^{1} \mathrm{H}\right\}$ NMR: $162 \mathrm{MHz}, \mathrm{CDCl}_{3}$, compound $\mathbf{4 f}$<smiles>[Te]c1ccccc1-c1ccc(N2C(c3ccccc3)CCC2c2ccccc2)cc1</smiles>

$250 \quad 150 \quad \begin{array}{r}100 \\ 2100\end{array}$

50

$-150$ 
${ }^{1} \mathrm{H}$ NMR: $400 \mathrm{MHz}, \mathrm{CD}_{2} \mathrm{Cl}_{2}$, compound $(S, S)$-F

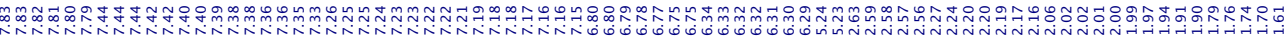<smiles>Cl[As](Cl)(Cl)c1ccccc1-c1ccc(N2[C@H](c3ccccc3)CC[C@H]2c2ccccc2)cc1</smiles>
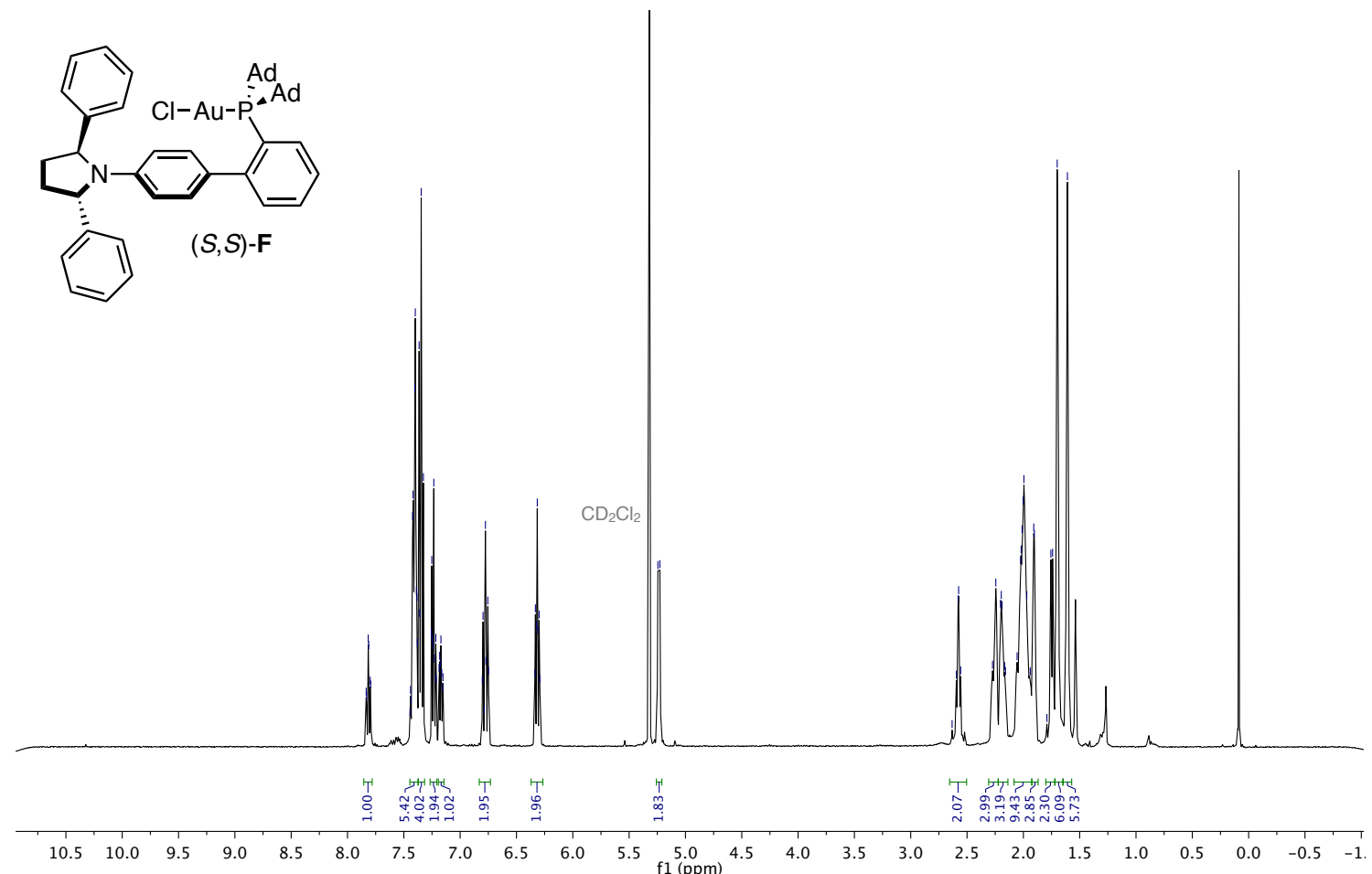

${ }^{13} \mathrm{C}$ NMR: $101 \mathrm{MHz}, \mathrm{CD}_{2} \mathrm{Cl}_{2}$, compound $(S, S)-\mathbf{F}$
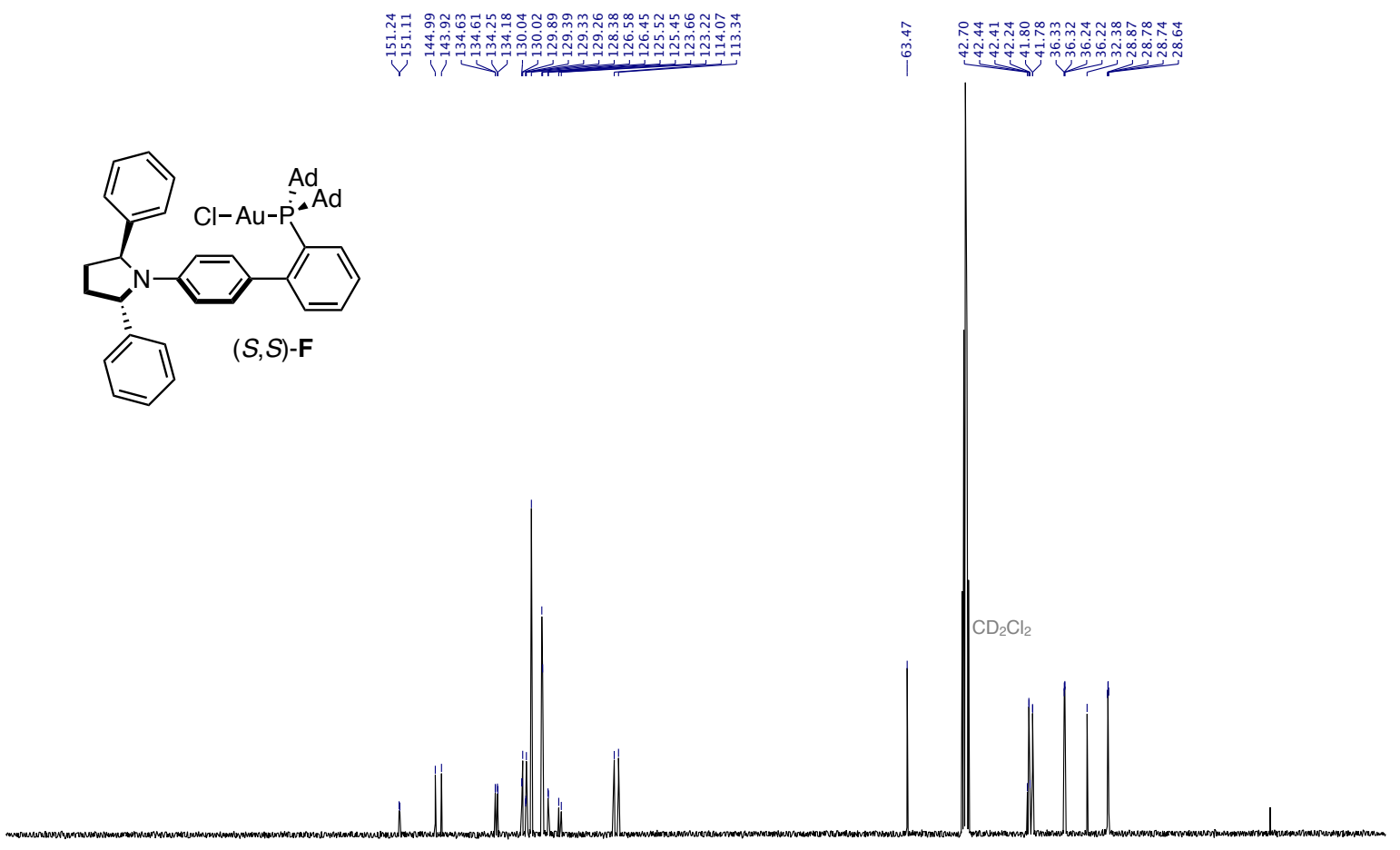

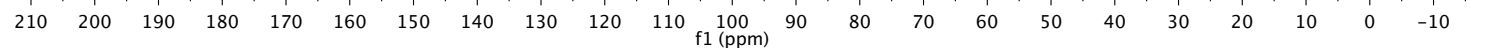


${ }^{31} \mathrm{P}\left\{{ }^{1} \mathrm{H}\right\} \mathrm{NMR}: 202 \mathrm{MHz}, \mathrm{CDCl}_{3}$, compound $(S, S)-\mathbf{F}$
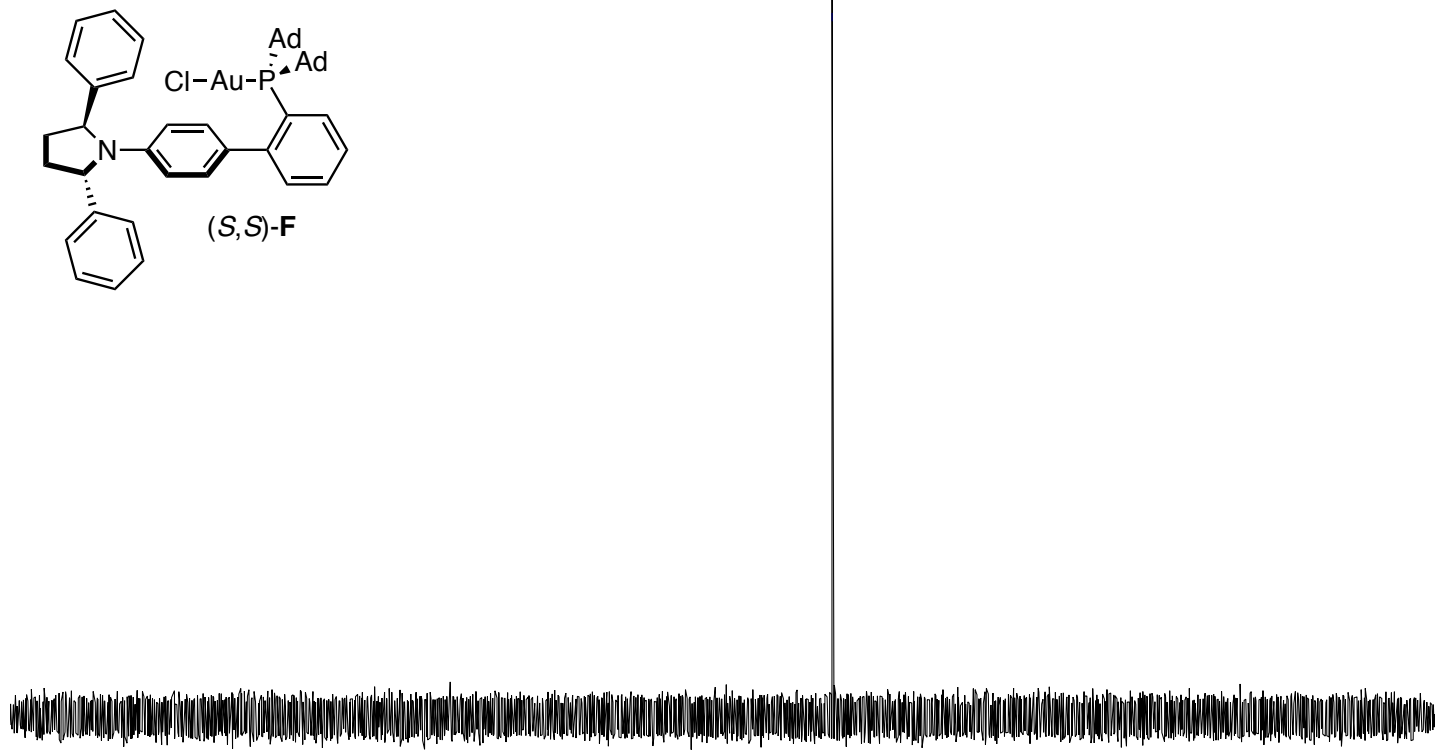

\begin{tabular}{|lllllllllllllllllllllllllll}
\hline 340 & 320 & 300 & 280 & 260 & 240 & 220 & 200 & 180 & 160 & 140 & 120 & 100 & 80 & 60 & 40 & 20 & 0 & -20 & -40 & -60 & -80 & -100 & -120 & -14 \\
\hline
\end{tabular}

${ }^{1} \mathrm{H}$ NMR: $500 \mathrm{MHz}, \mathrm{CDCl}_{3}$, compound $\mathbf{3 f}$

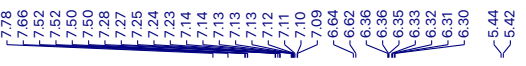

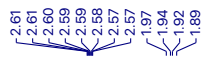<smiles>FC(F)(F)c1ccc(N(CCC(c2cccc(C(F)(F)F)c2)c2ccccc2Br)Cc2cc(C(F)(F)F)cc(C(F)(F)F)c2)cc1</smiles>

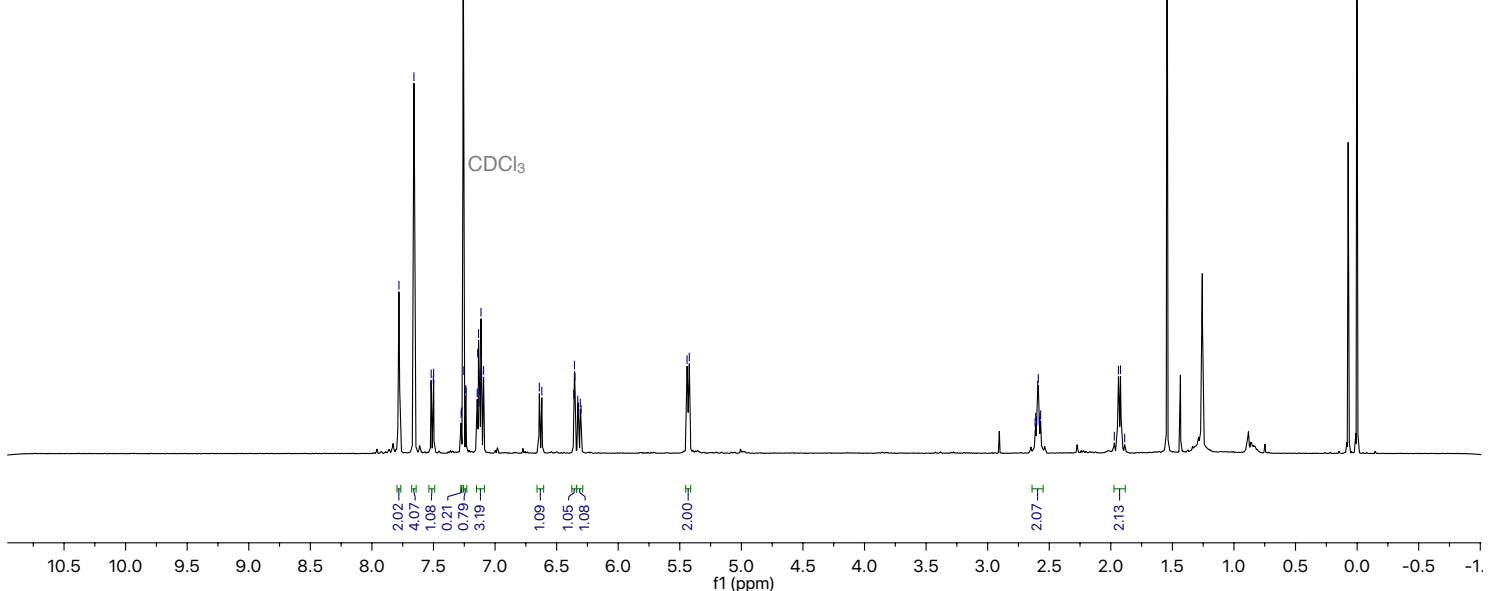


${ }^{13} \mathrm{C}$ NMR: $126 \mathrm{MHz}, \mathrm{CDCl}_{3}$, compound $3 f$

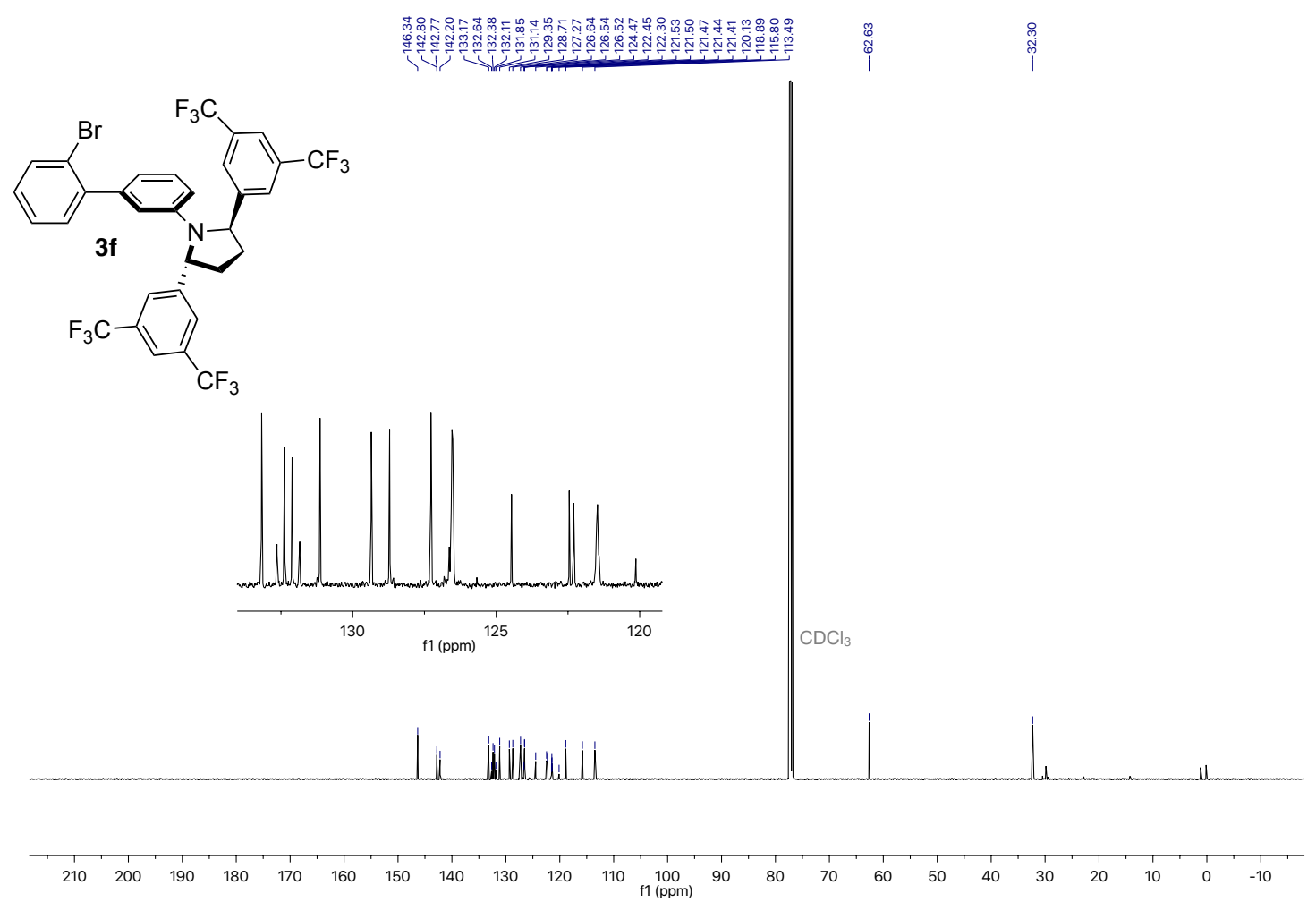

${ }^{19} \mathrm{~F}\{\mathrm{H}\}$ NMR: $376 \mathrm{MHz}, \mathrm{CDCl}_{3}$, compound $\mathbf{3 f}$

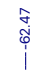

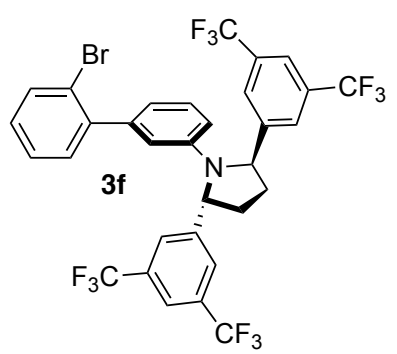

\begin{tabular}{llllllllllllllllllllllllllllllllll}
\hline 30 & 20 & 10 & 0 & -10 & -20 & -30 & -40 & -50 & -60 & -70 & -80 & -90 & -100 & -110 & -120 & -130 & -140 & -150 & -160 & -170 & -180 & -190 & -200 & -210 & -220 & $-23 c$
\end{tabular} 
${ }^{1} \mathrm{H}$ NMR: $500 \mathrm{MHz}, \mathrm{CDCl}_{3}$, compound $\mathbf{4 g}$

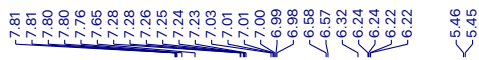<smiles></smiles><smiles>FC(F)(F)c1cccc(C(F)(F)F)c1</smiles>

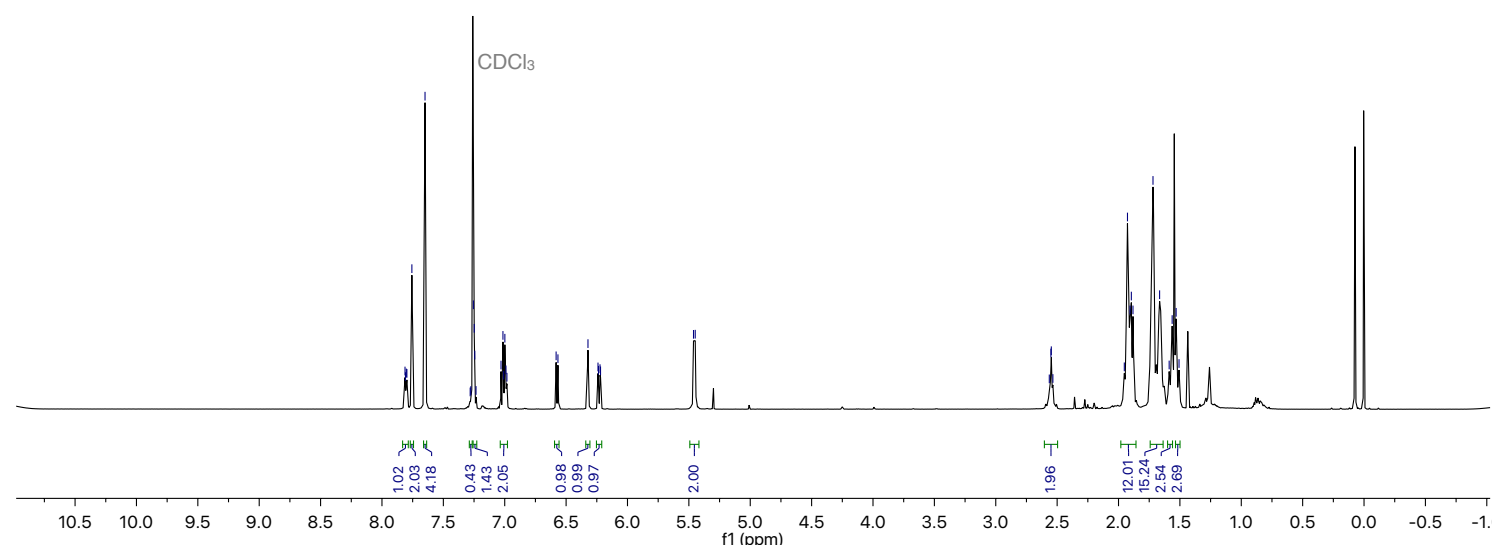

${ }^{13} \mathrm{C}$ NMR: $126 \mathrm{MHz}, \mathrm{CDCl}_{3}$, compound 4g

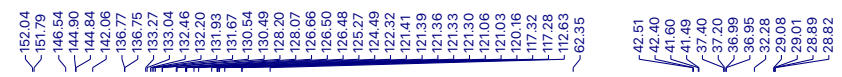<smiles></smiles><smiles>FC(F)(F)c1cccc(C(F)(F)F)c1</smiles>

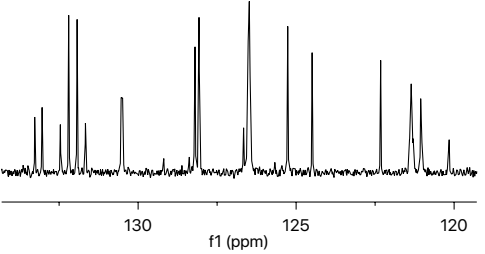

- 1 i dillin:

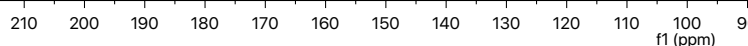


${ }^{31} \mathrm{P}\{\mathrm{H}\} \mathrm{NMR}: 162 \mathrm{MHz}, \mathrm{CDCl}_{3}$, compound 4g

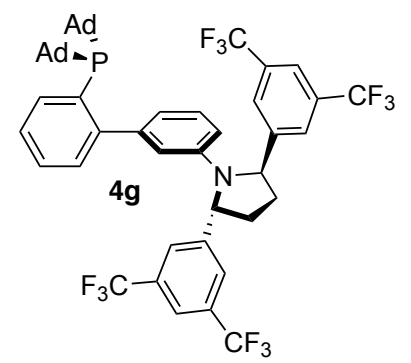

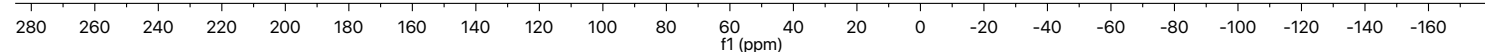

${ }^{19} \mathrm{~F}\{\mathrm{H}\}$ NMR: $376 \mathrm{MHz}, \mathrm{CDCl}_{3}$, compound 4g

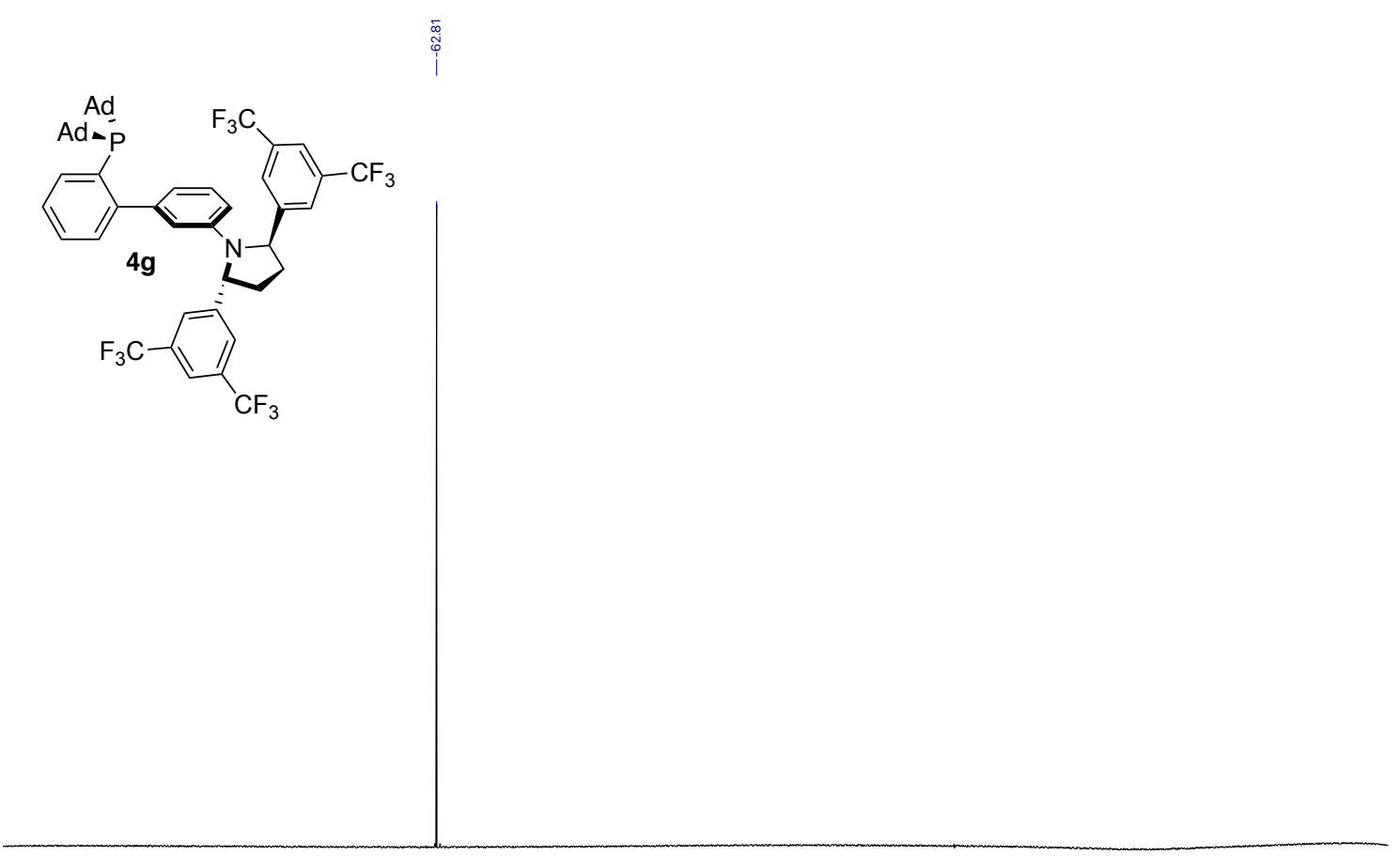

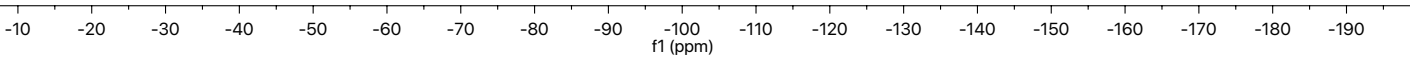


${ }^{1} \mathrm{H}$ NMR: $400 \mathrm{MHz}, \mathrm{CDCl}_{3}$, compound $(R, R)-\mathbf{G}$

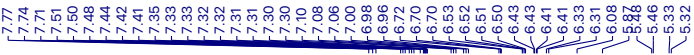

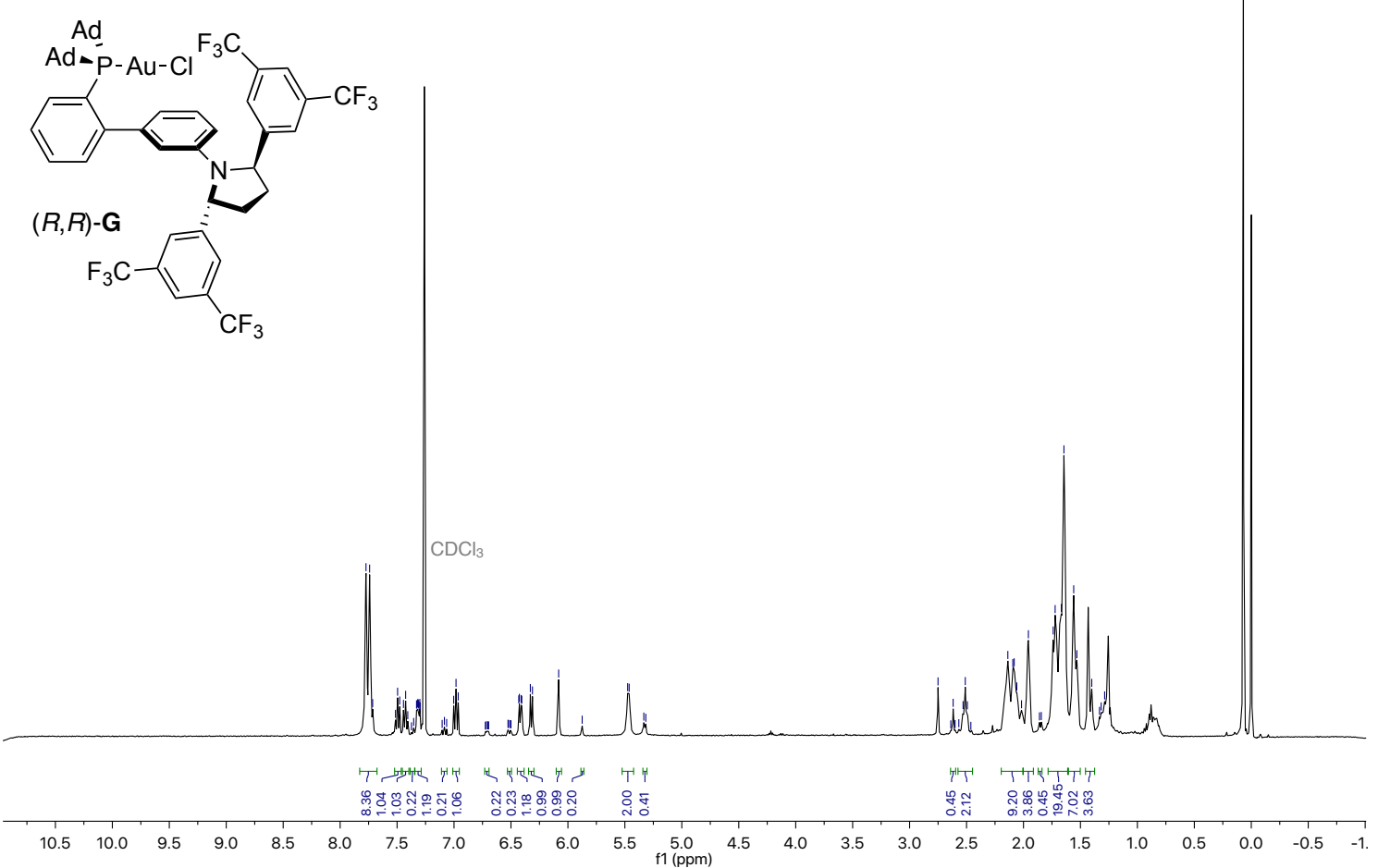

${ }^{13} \mathrm{C}$ NMR: $126 \mathrm{MHz}, \mathrm{CDCl}_{3}$, compound $(R, R)-\mathbf{G}$

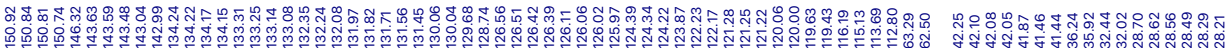
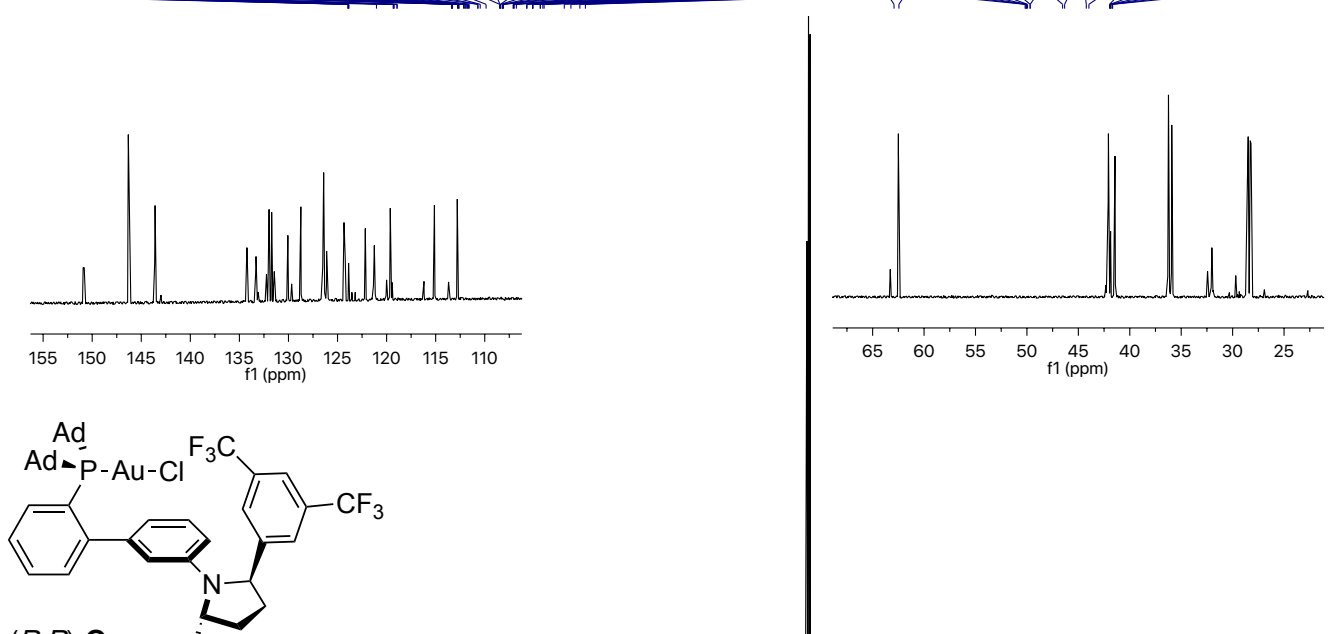

$(R, R)-\mathbf{G}$

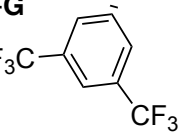

Li midulinid

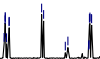

$\begin{array}{llllllllllllllllllllllllllll}210 & 200 & 190 & 180 & 170 & 160 & 150 & 140 & 130 & 120 & 110 & 100 & 90 & 80 & 70 & 60 & 50 & 40 & 30 & 20 & 10 & 0 & -10\end{array}$ 
${ }^{31} \mathrm{P}\{\mathrm{H}\} \mathrm{NMR}: 162 \mathrm{MHz}, \mathrm{CDCl}_{3}, \operatorname{compound}(R, R)-\mathbf{G}$

量器
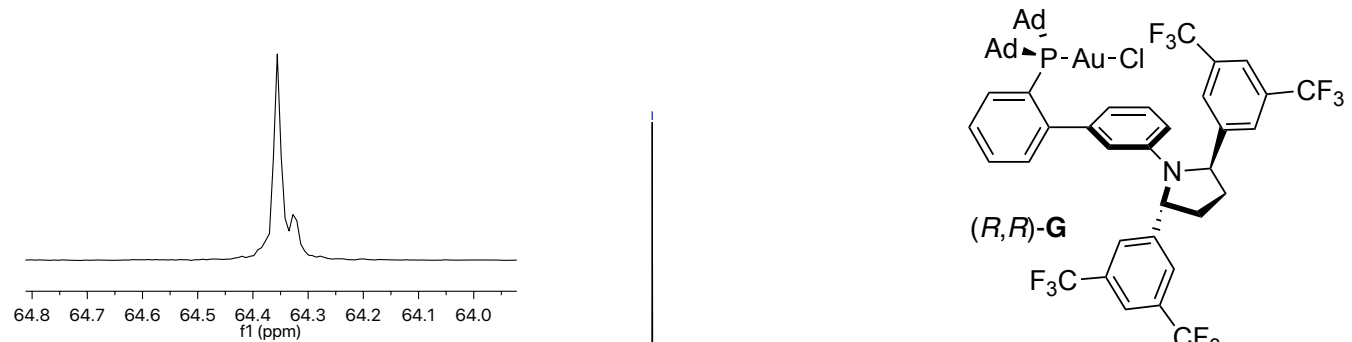

$(R, R)-\mathrm{G}$
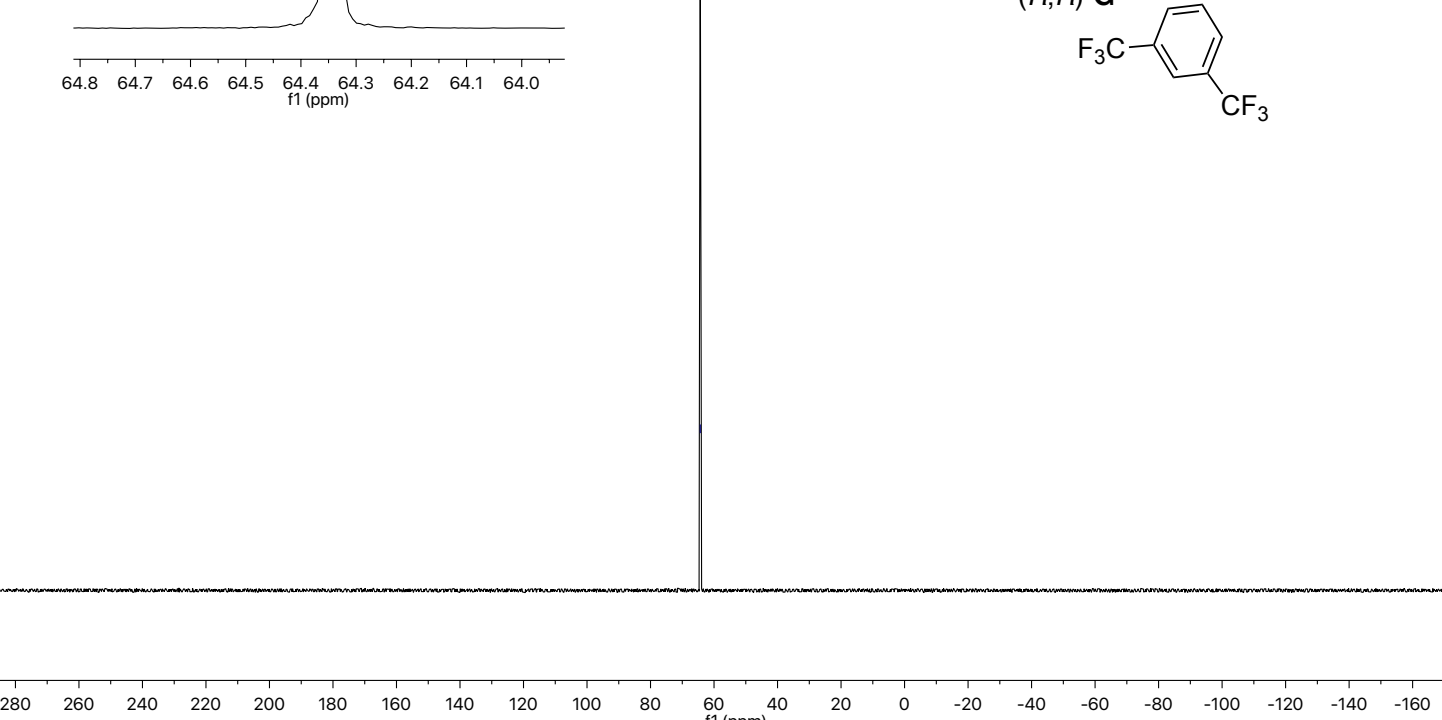

${ }^{19} \mathrm{~F}\{\mathrm{H}\}$ NMR: $471 \mathrm{MHz}, \mathrm{CDCl}_{3}, \operatorname{compound}(R, R)-\mathbf{G}$

บั

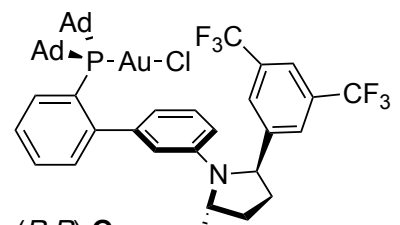

$(R, R)$-G

$\mathrm{CF}_{3}$
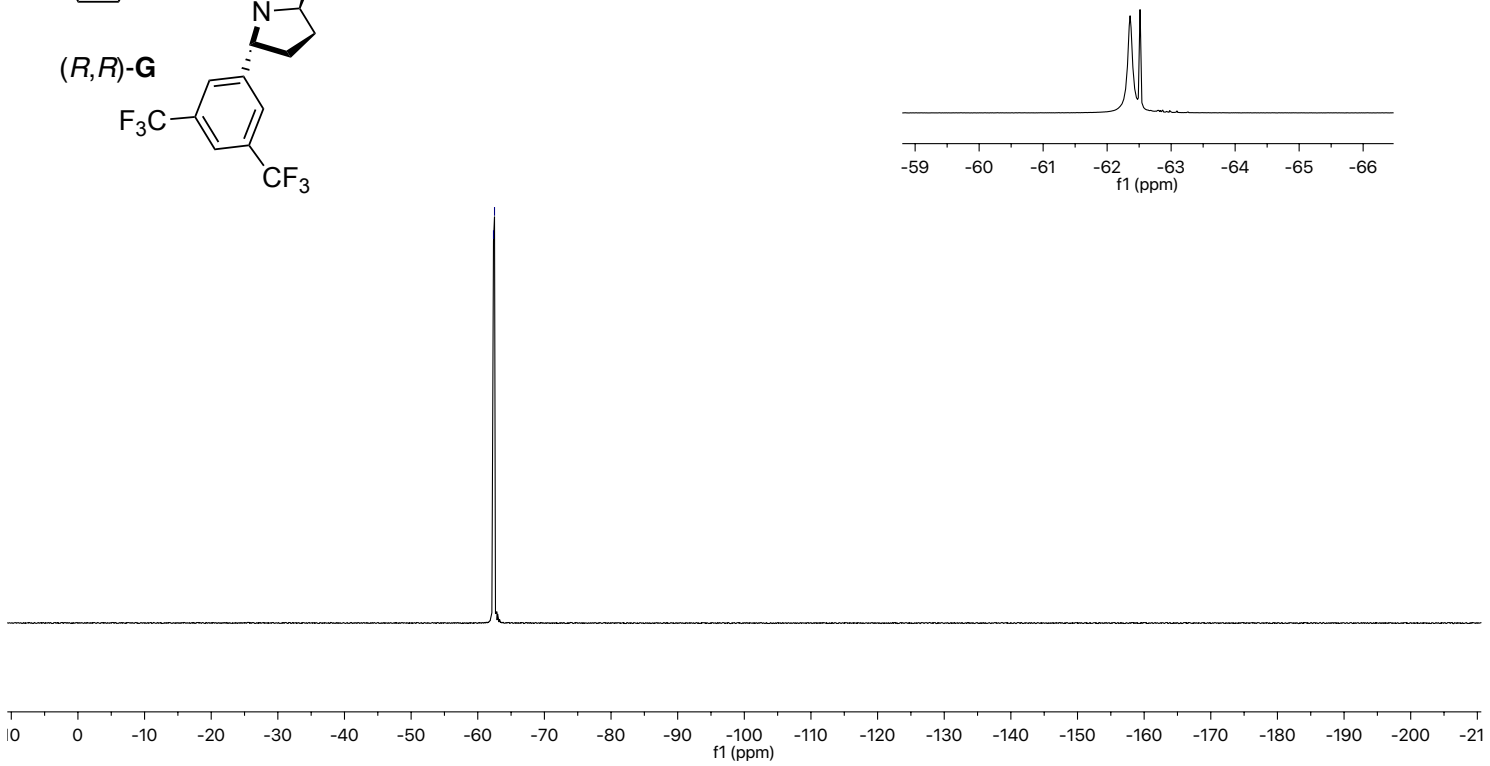
2D NOESY: $400 \mathrm{MHz}, \mathrm{CDCl}_{3}, 213 \mathrm{~K}$, compound $(R, R)-\mathbf{G}$

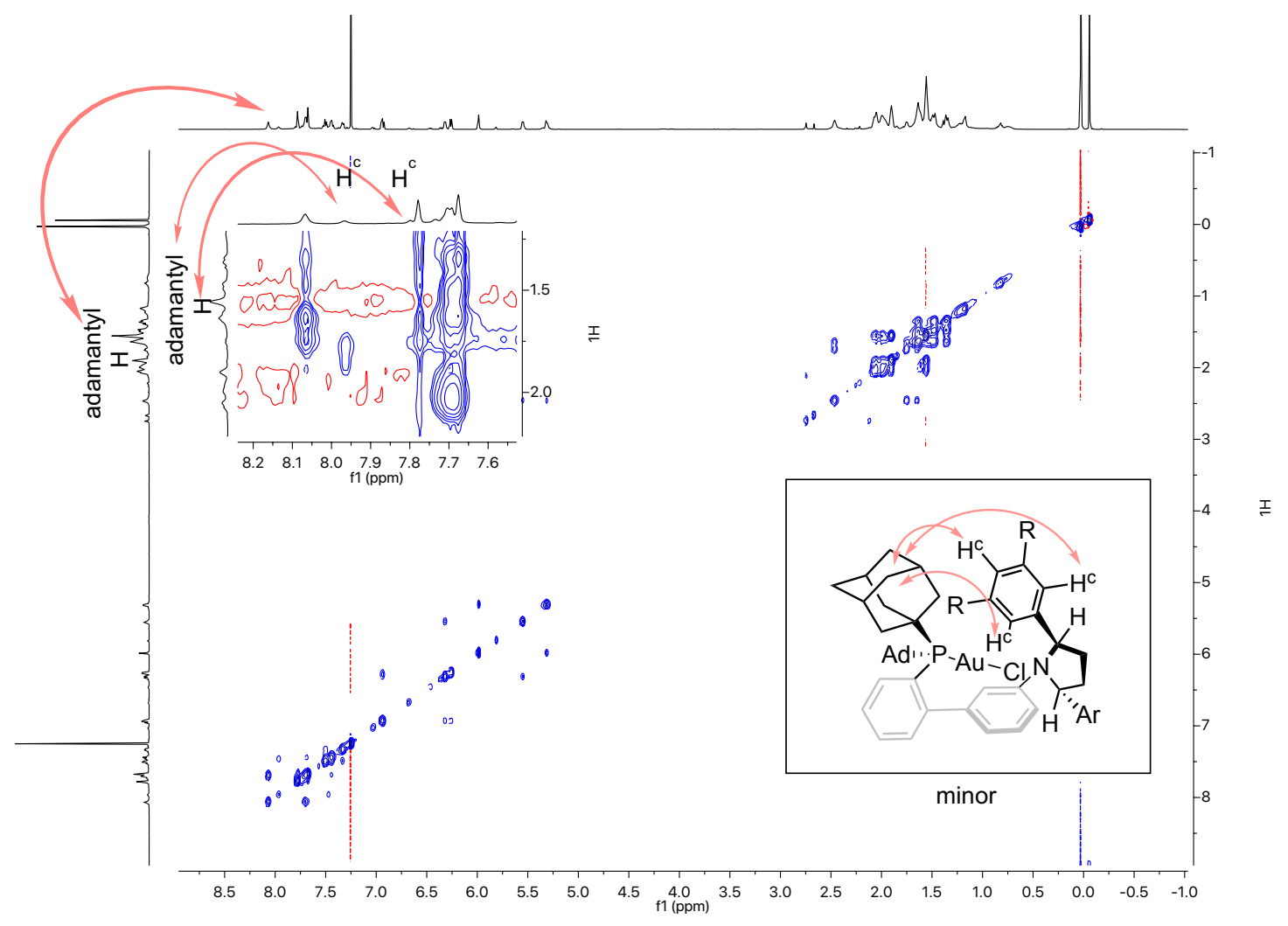


${ }^{1} \mathrm{H}$ NMR: $500 \mathrm{MHz}, \mathrm{CD}_{2} \mathrm{Cl}_{2}$, compound $(R, R)-\mathrm{C}_{2}-\mathrm{Cl}$

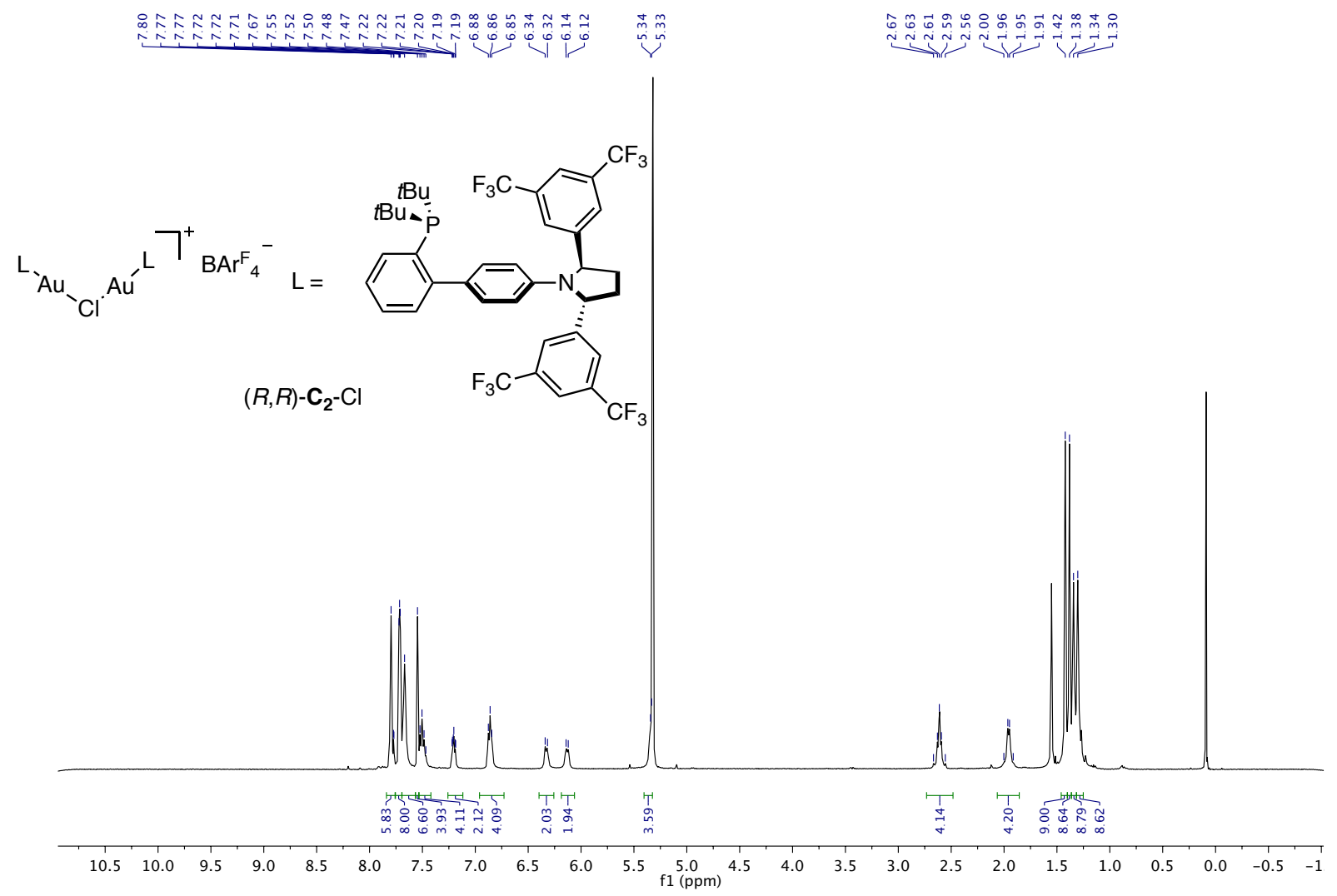

${ }^{13} \mathrm{C}$ NMR: $126 \mathrm{MHz}, \mathrm{CD}_{2} \mathrm{Cl}_{2}$, compound $(R, R)-\mathbf{C}_{2}-\mathrm{Cl}$

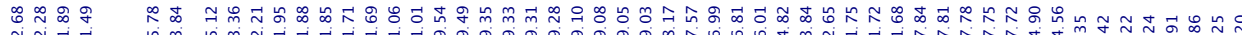

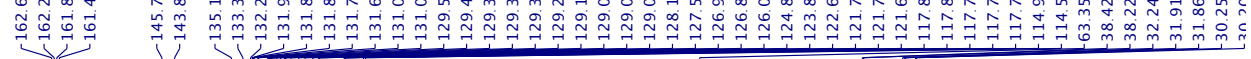

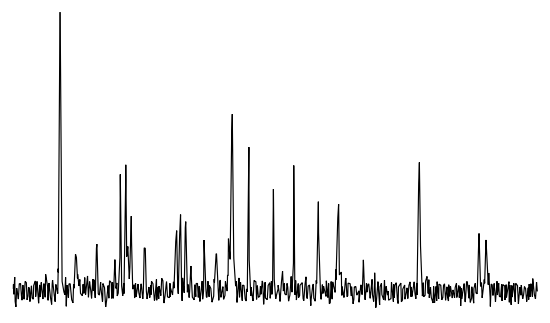

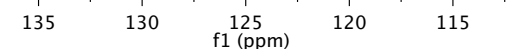
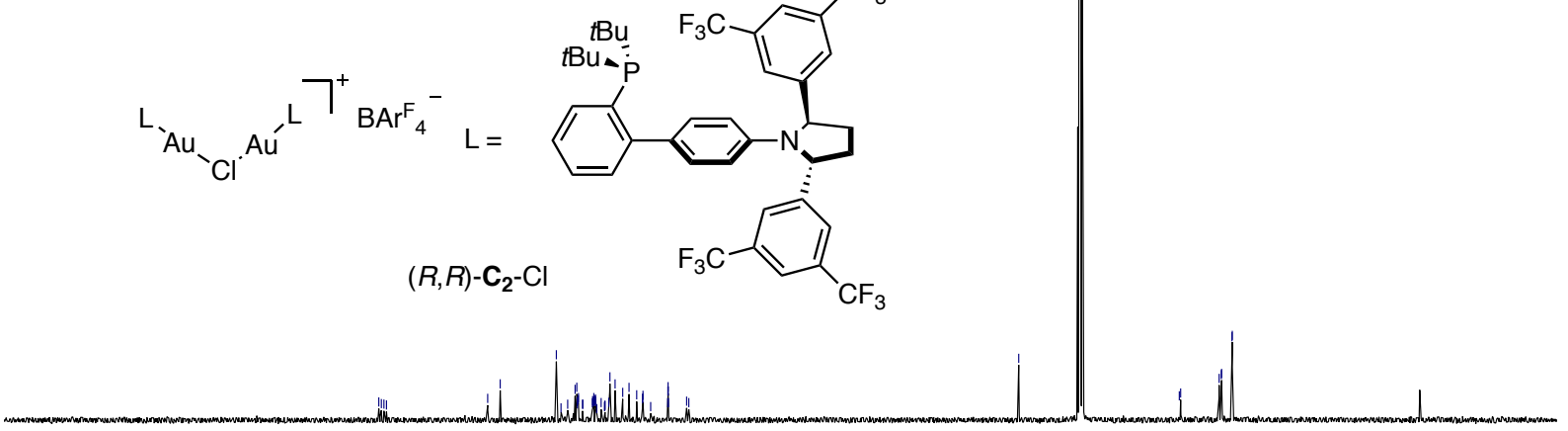

$\begin{array}{llllllllllllllllllllllllll}20 & 210 & 200 & 190 & 180 & 170 & 160 & 150 & 140 & 130 & 120 & 110 & 100 & 90 & 80 & 70 & 60 & 50 & 40 & 30 & 20 & 10 & 0 & -10 & -2\end{array}$ 
${ }^{31} \mathrm{P}\left\{{ }^{1} \mathrm{H}\right\}$ NMR: $162 \mathrm{MHz}, \mathrm{CD}_{2} \mathrm{Cl}_{2}$, compound $(R, R)-\mathbf{C}_{2}-\mathrm{Cl}$

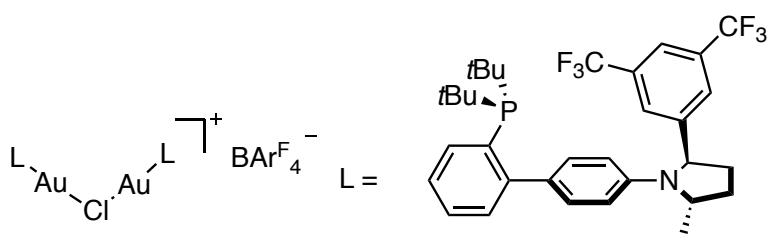

$(R, R)-\mathbf{C}_{2}-\mathrm{Cl}$

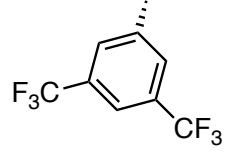

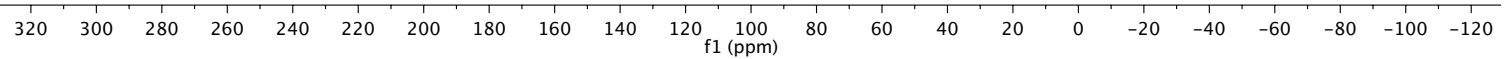

${ }^{19} \mathrm{~F}\left\{{ }^{1} \mathrm{H}\right\}$ NMR: $376 \mathrm{MHz}, \mathrm{CD}_{2} \mathrm{Cl}_{2}$, compound $(R, R)-\mathrm{C}_{2}-\mathrm{Cl}$

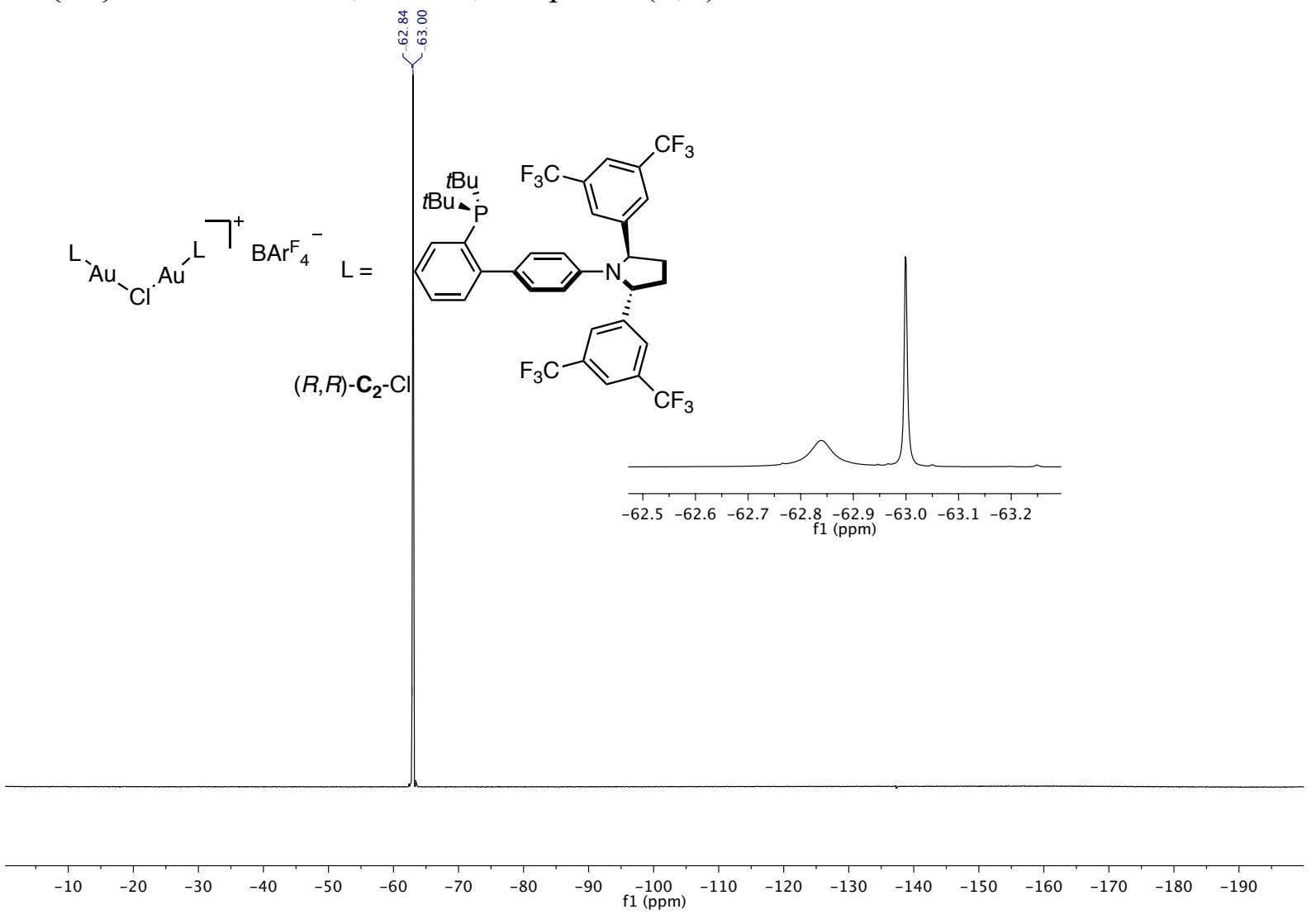


${ }^{1} \mathrm{H}$ NMR: $400 \mathrm{MHz}, \mathrm{CD}_{2} \mathrm{Cl}_{2}$, compound $\mathbf{5 b}$
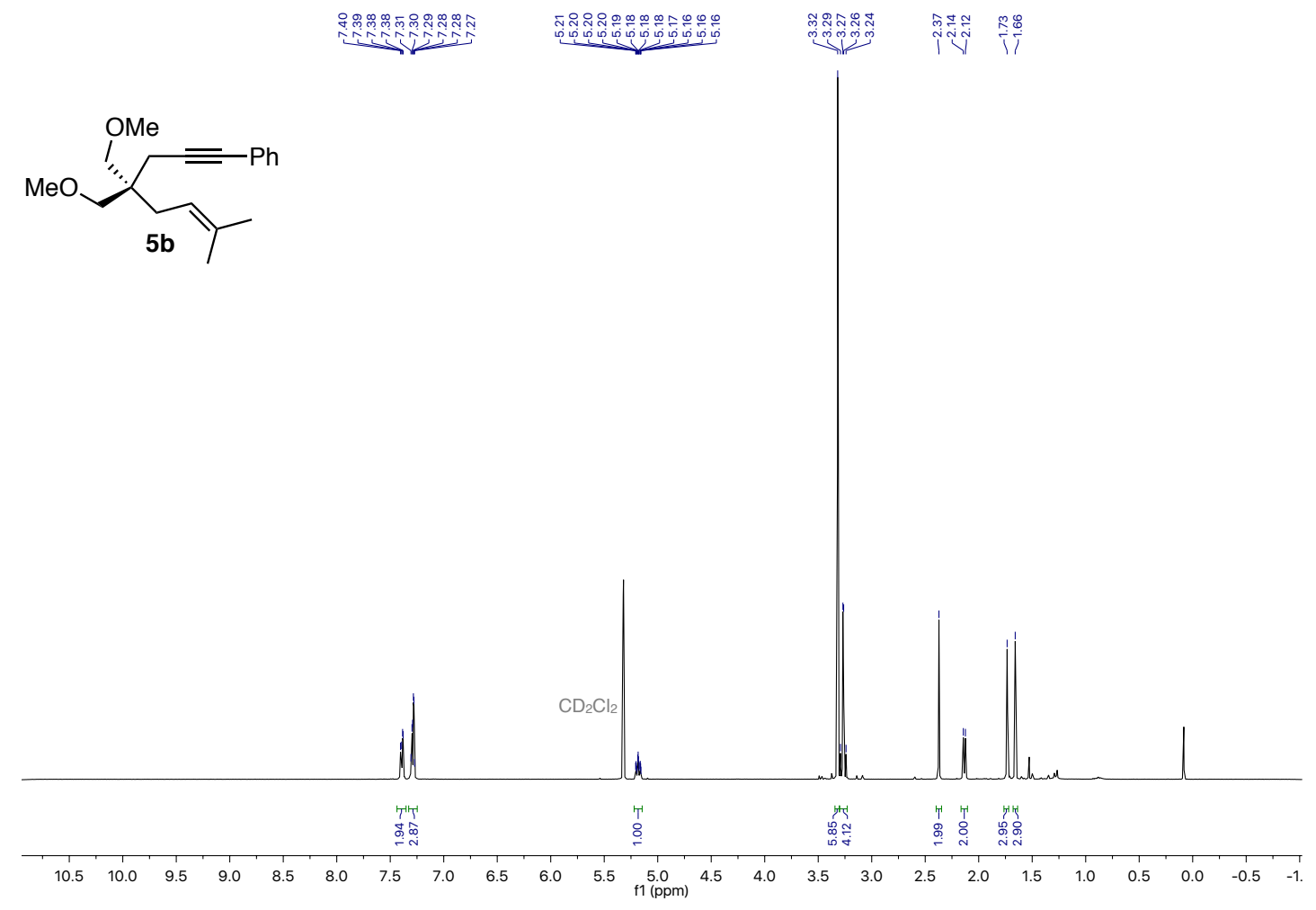

${ }^{13} \mathrm{C}$ NMR: $101 \mathrm{MHz}, \mathrm{CD}_{2} \mathrm{Cl}_{2}$, compound $\mathbf{5 b}$

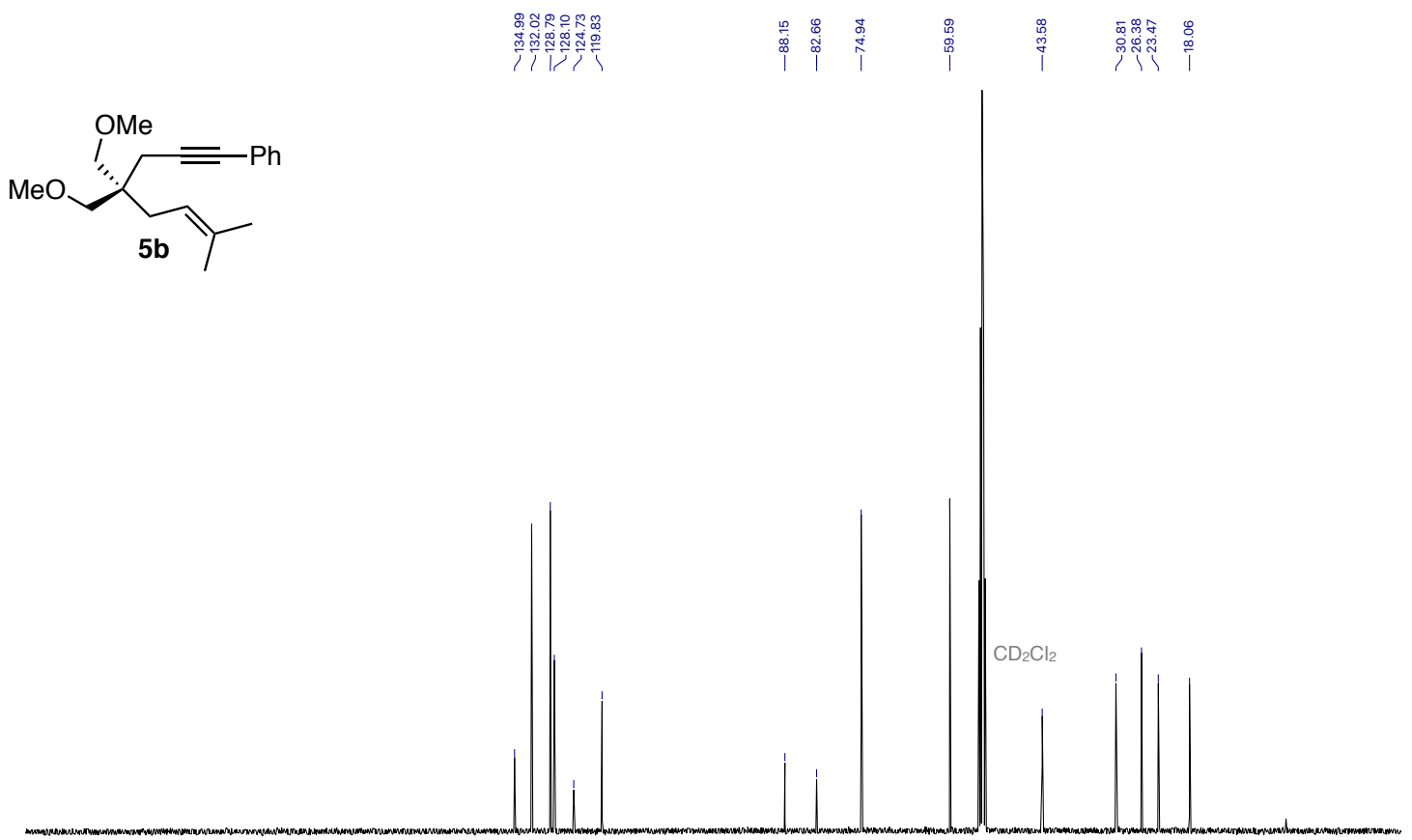

$\begin{array}{llllllllllllllllllllllllllllllllllllllll}210 & 200 & 190 & 180 & 170 & 160 & 150 & 140 & 130 & 120 & 110 & 100 & 90 & 80 & 70 & 60 & 50 & 40 & 30 & 20 & 10 & 0 & -10\end{array}$ 
${ }^{1} \mathrm{H}$ NMR: $500 \mathrm{MHz}, \mathrm{CD}_{2} \mathrm{Cl}_{2}$, compound $\mathbf{5 c}$

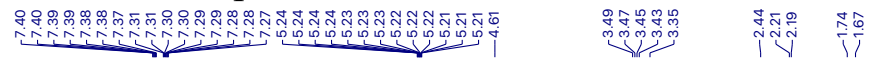
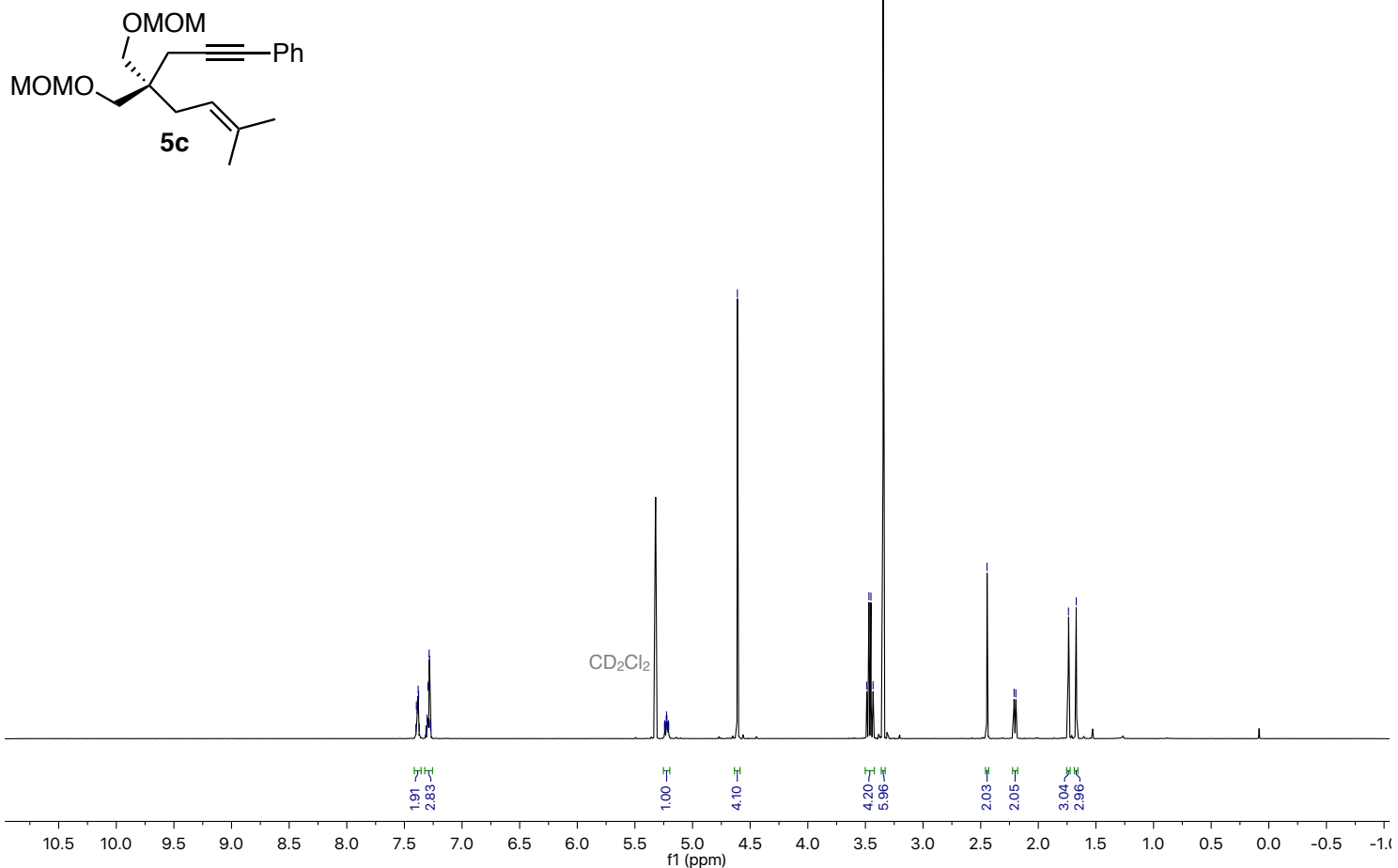

${ }^{13} \mathrm{C} \mathrm{NMR}: 126 \mathrm{MHz}, \mathrm{CD}_{2} \mathrm{Cl}_{2}$, compound 5c

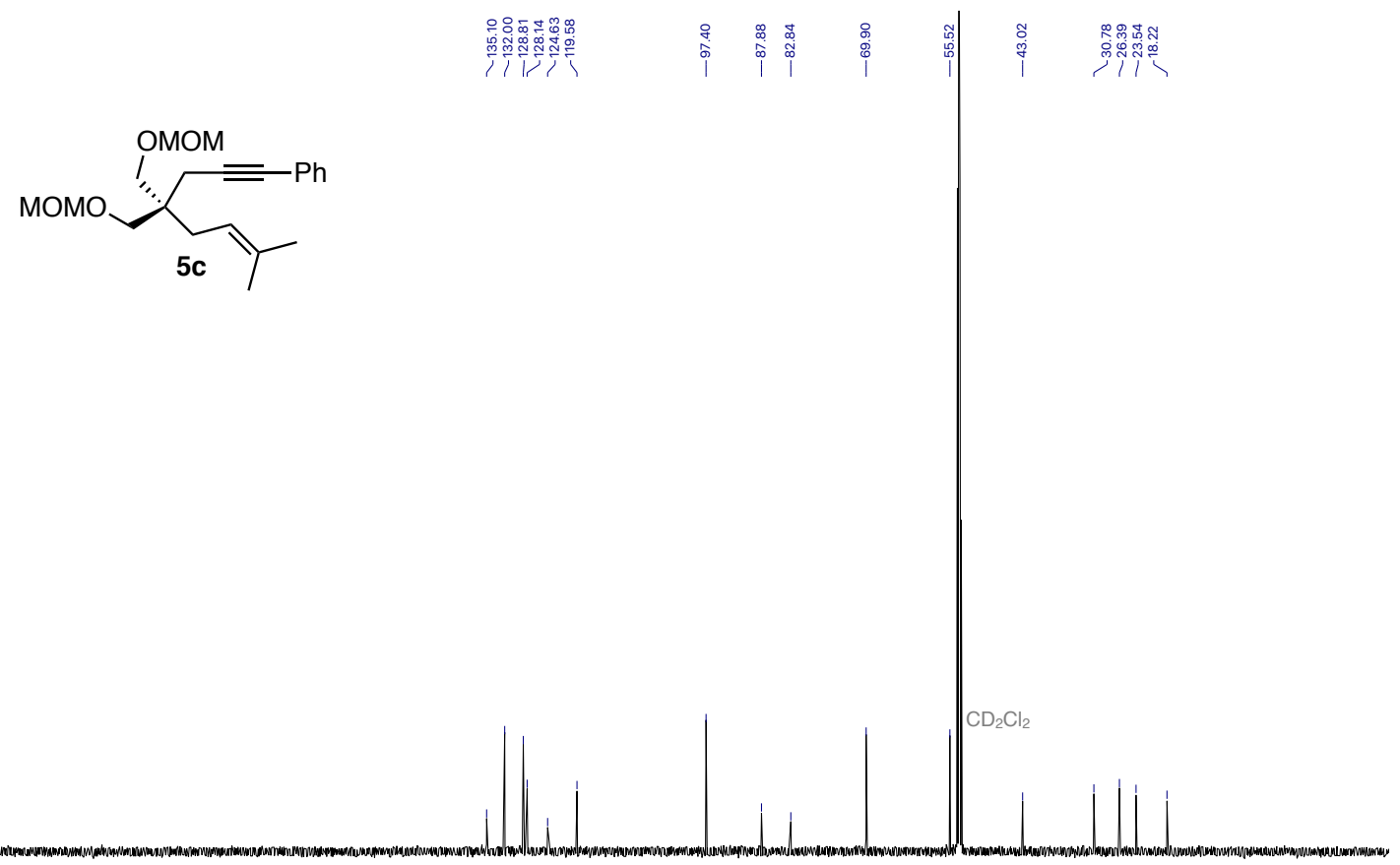

$\begin{array}{lllllllllllllllllllllllllllllllllllll} & 20 & 210 & 200 & 190 & 180 & 170 & 160 & 150 & 140 & 130 & 120 & 110 & 100 & 90 & 80 & 70 & 60 & 50 & 40 & 30 & 20 & 10 & 0 & -10 & \end{array}$ 
${ }^{1} \mathrm{H}$ NMR: $300 \mathrm{MHz}, \mathrm{CDCl}_{3}$, compound 5d

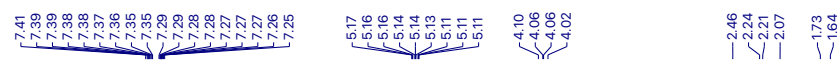<smiles>CC(=O)OCC(CC#Cc1ccccc1)(CC=C(C)C)COC(C)=O</smiles>

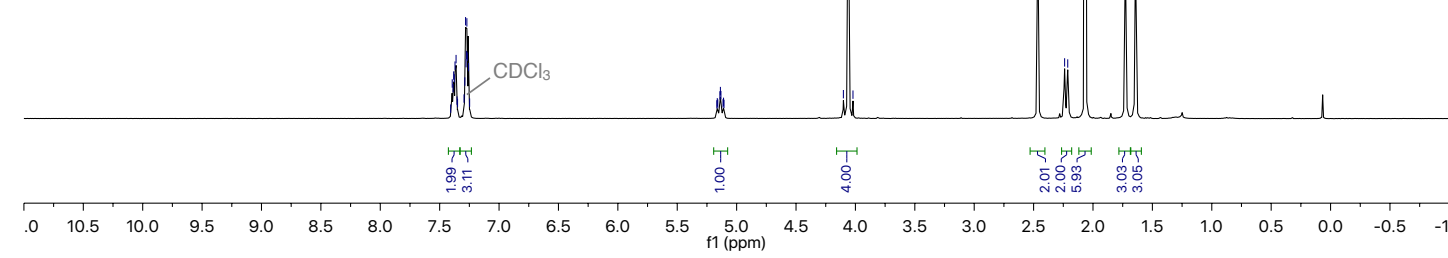

${ }^{13} \mathrm{C}$ NMR: $101 \mathrm{MHz}, \mathrm{CDCl}_{3}$, compound 5d

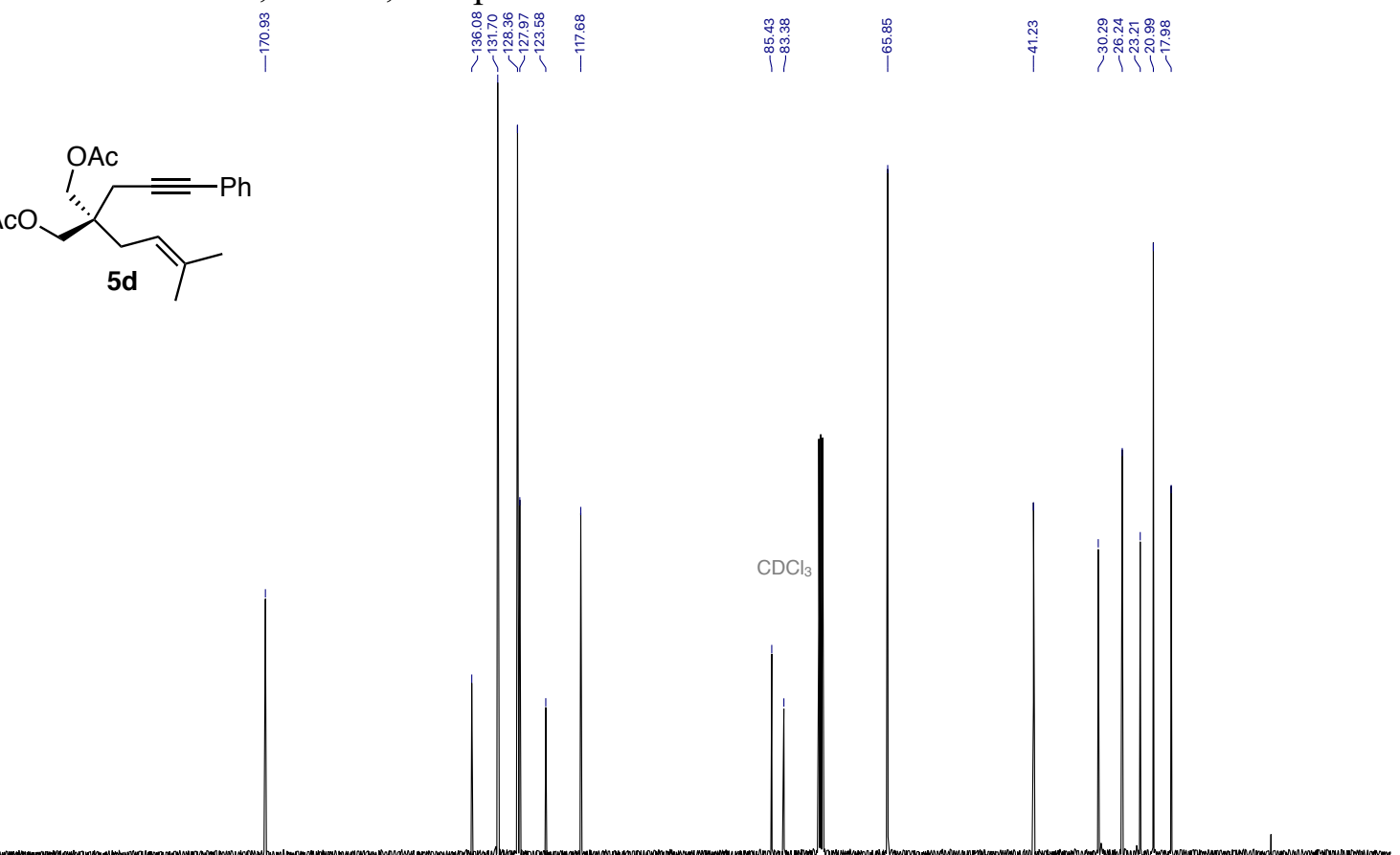

$\begin{array}{lllllllllllllllllllllll}210 & 200 & 190 & 180 & 170 & 160 & 150 & 140 & 130 & 120 & 110 & 100 & 90 & 80 & 70 & 60 & 50 & 40 & 30 & 20 & 10 & 0 & -10\end{array}$ 
${ }^{1} \mathrm{H}$ NMR: $500 \mathrm{MHz}, \mathrm{CDCl}_{3}, 4,4-\mathrm{Bis}$ (methoxymethyl)-7-methyloct-6-en-1-yne $\mathrm{CD}_{2} \mathrm{Cl}_{2}$

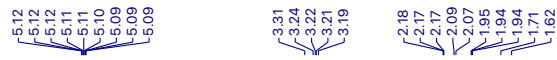
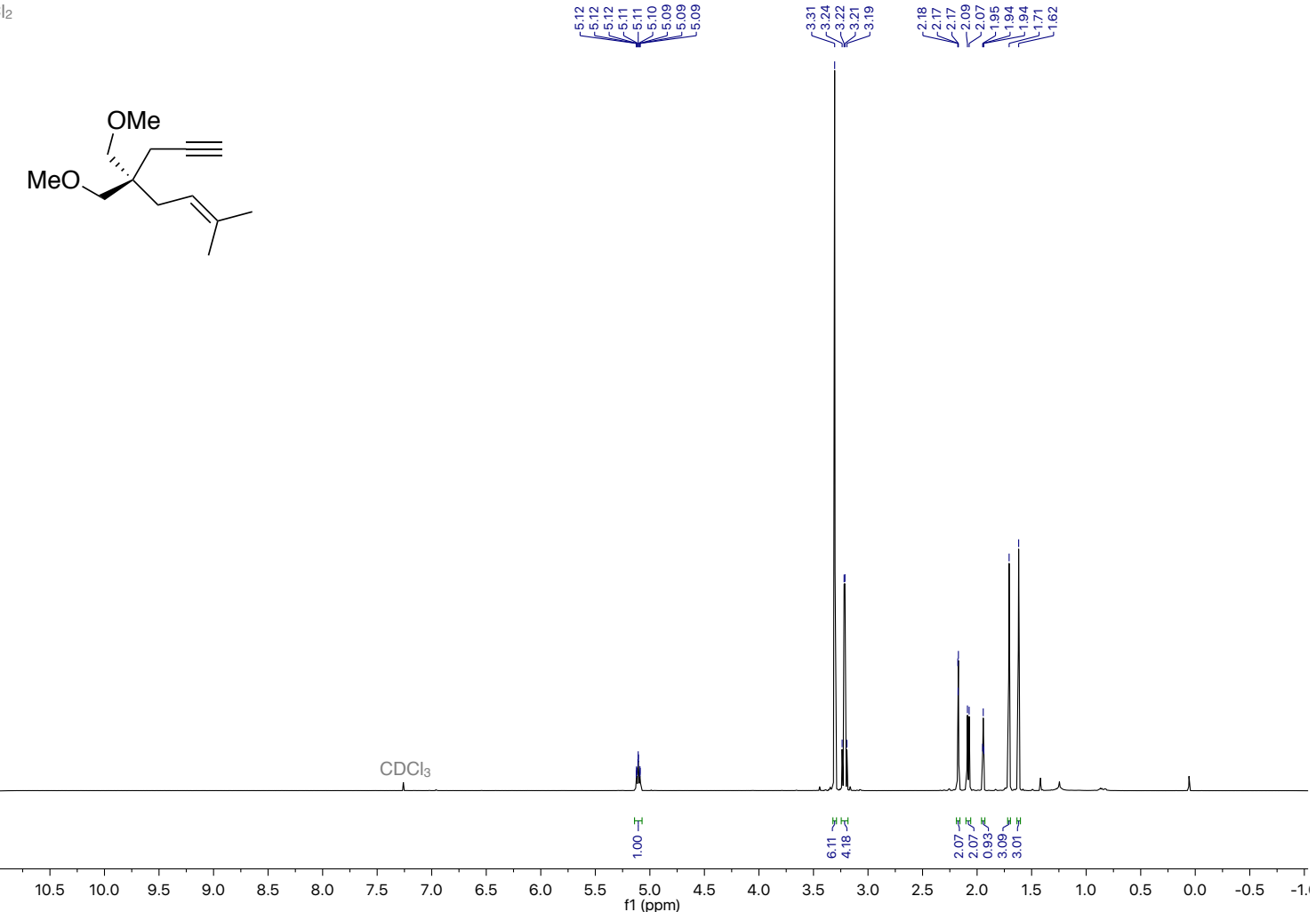

${ }^{13} \mathrm{C}$ NMR: $126 \mathrm{MHz}, \mathrm{CDCl}_{3}, 4,4-\mathrm{Bis}$ (methoxymethyl)-7-methyloct-6-en-1-yne
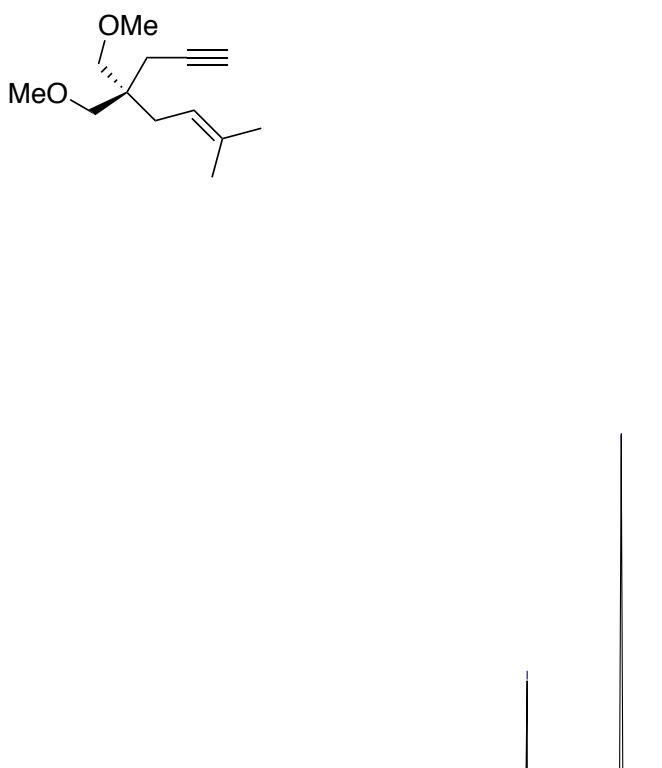

$\begin{array}{lllllllllllll}20 & 210 & 200 & 190 & 180 & 170 & 160 & 150 & 140 & 130 & 120 & 110 & \begin{array}{c}100 \\ \mathrm{f} 1(\mathrm{ppm})\end{array}\end{array}$

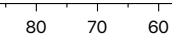

$\begin{array}{llllllll}50 & 40 & 30 & 20 & 10 & 0 & -10 & -2\end{array}$ 
${ }^{1} \mathrm{H}$ NMR: $500 \mathrm{MHz}, \mathrm{CDCl}_{3}$, compound 5e

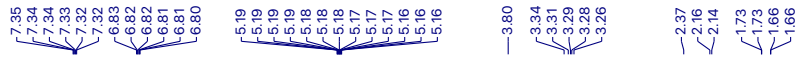
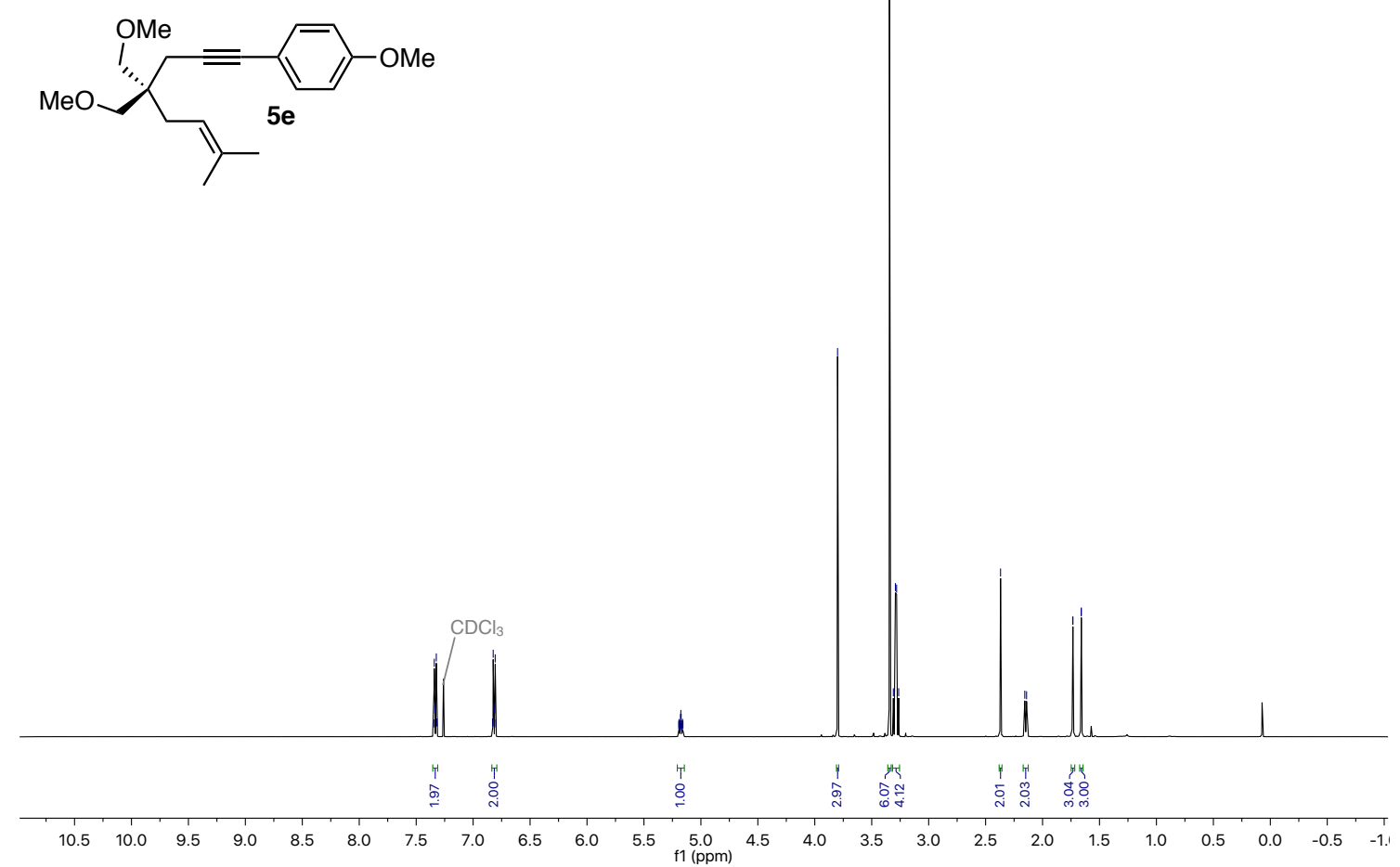

${ }^{13} \mathrm{C} \mathrm{NMR}: 126 \mathrm{MHz}, \mathrm{CDCl}_{3}$, compound 5e

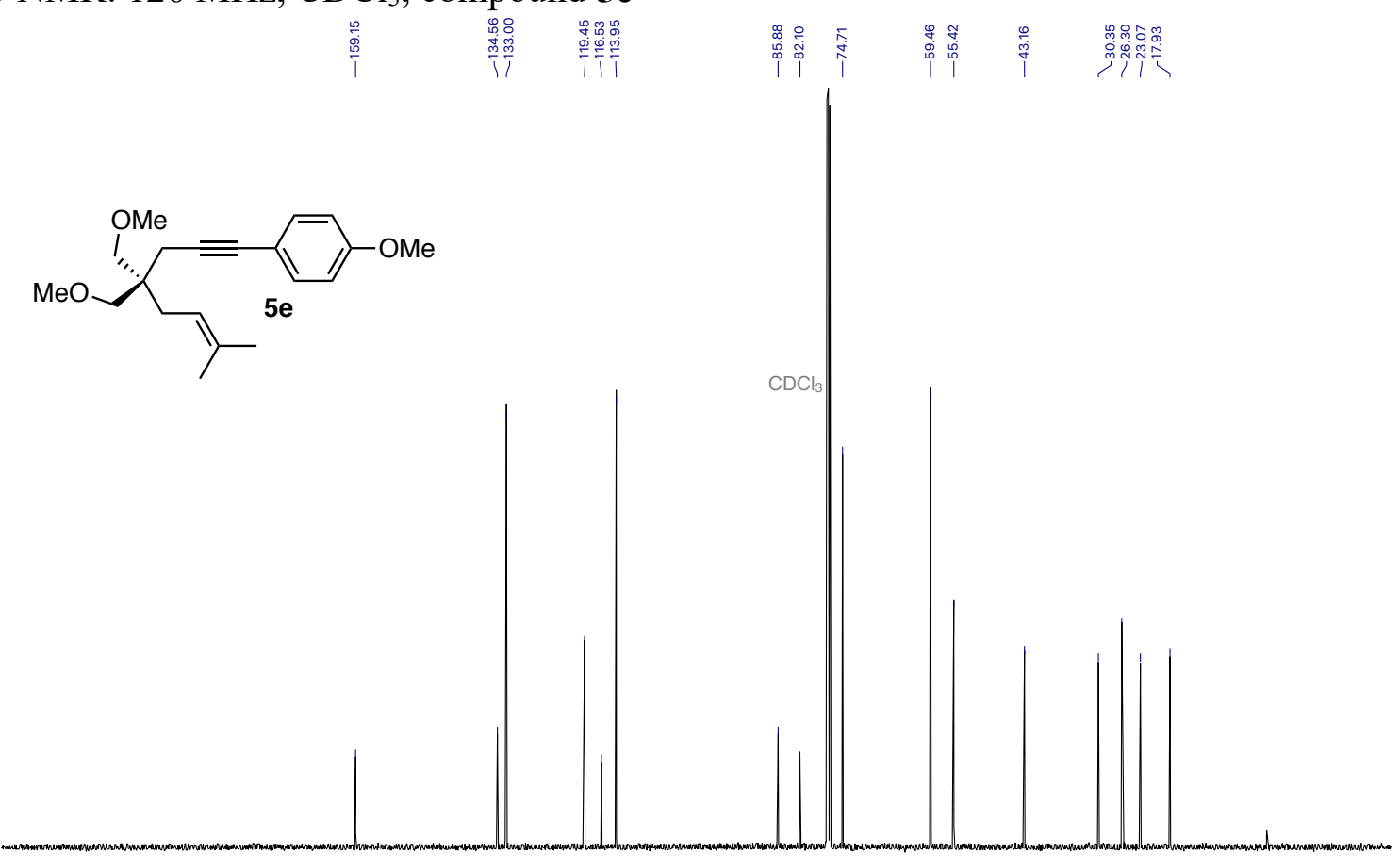

\begin{tabular}{lllllllllllllllllllllllllllllll}
\hline 20 & 210 & 200 & 190 & 180 & 170 & 160 & 150 & 140 & 130 & 120 & 110 & $\begin{array}{c}100 \\
\mathrm{f}(\mathrm{ppm})\end{array}$ & 90 & 80 & 70 & 60 & 50 & 40 & 30 & 20 & 10 & 0 & -10 & -2
\end{tabular} 
${ }^{1} \mathrm{H}$ NMR: $400 \mathrm{MHz}, \mathrm{CDCl}_{3}$, compound $\mathbf{5 f}$

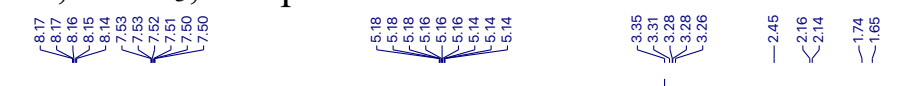<smiles>COCC(CC#Cc1ccc([N+](=O)[O-])cc1)(CC=C(C)C)COC</smiles>

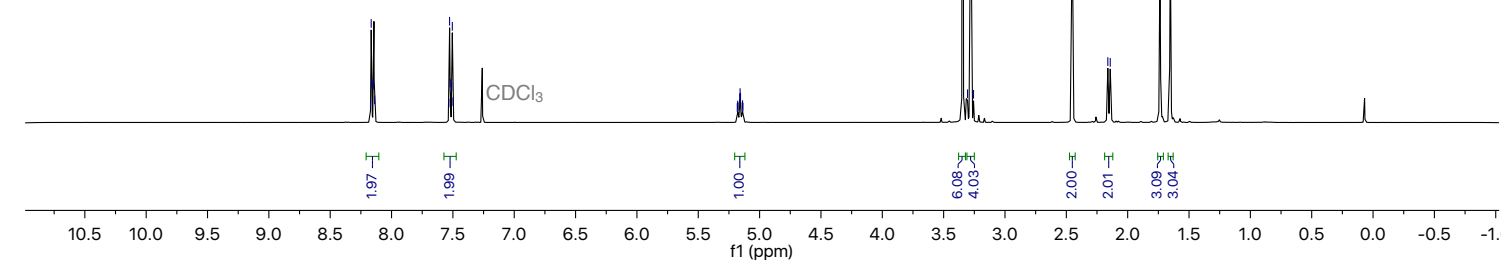

${ }^{13} \mathrm{C}$ NMR: $101 \mathrm{MHz}, \mathrm{CDCl}_{3}$, compound $\mathbf{5 f}$

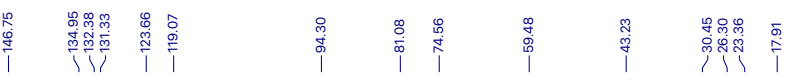<smiles>COCC(CC#Cc1ccc([N+](=O)[O-])cc1Br)(CC=C(C)C)COC</smiles>

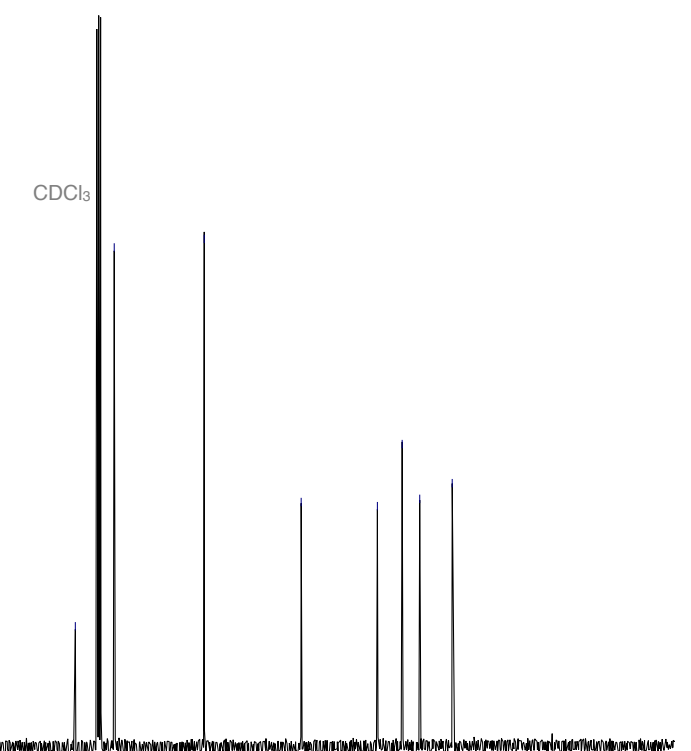

$\begin{array}{lllllllllllllllllllllllllllll}210 & 200 & 190 & 180 & 170 & 160 & 150 & 140 & 130 & 120 & 110 & \begin{array}{c}100 \\ \mathrm{f} 1(\mathrm{ppm})\end{array} & 90 & 80 & 70 & 60 & 50 & 40 & 30 & 20 & 10 & 0 & -10\end{array}$ 
${ }^{1} \mathrm{H}$ NMR: $400 \mathrm{MHz}, \mathrm{CDCl}_{3}$, compound 5g

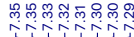

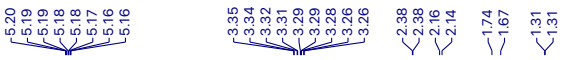
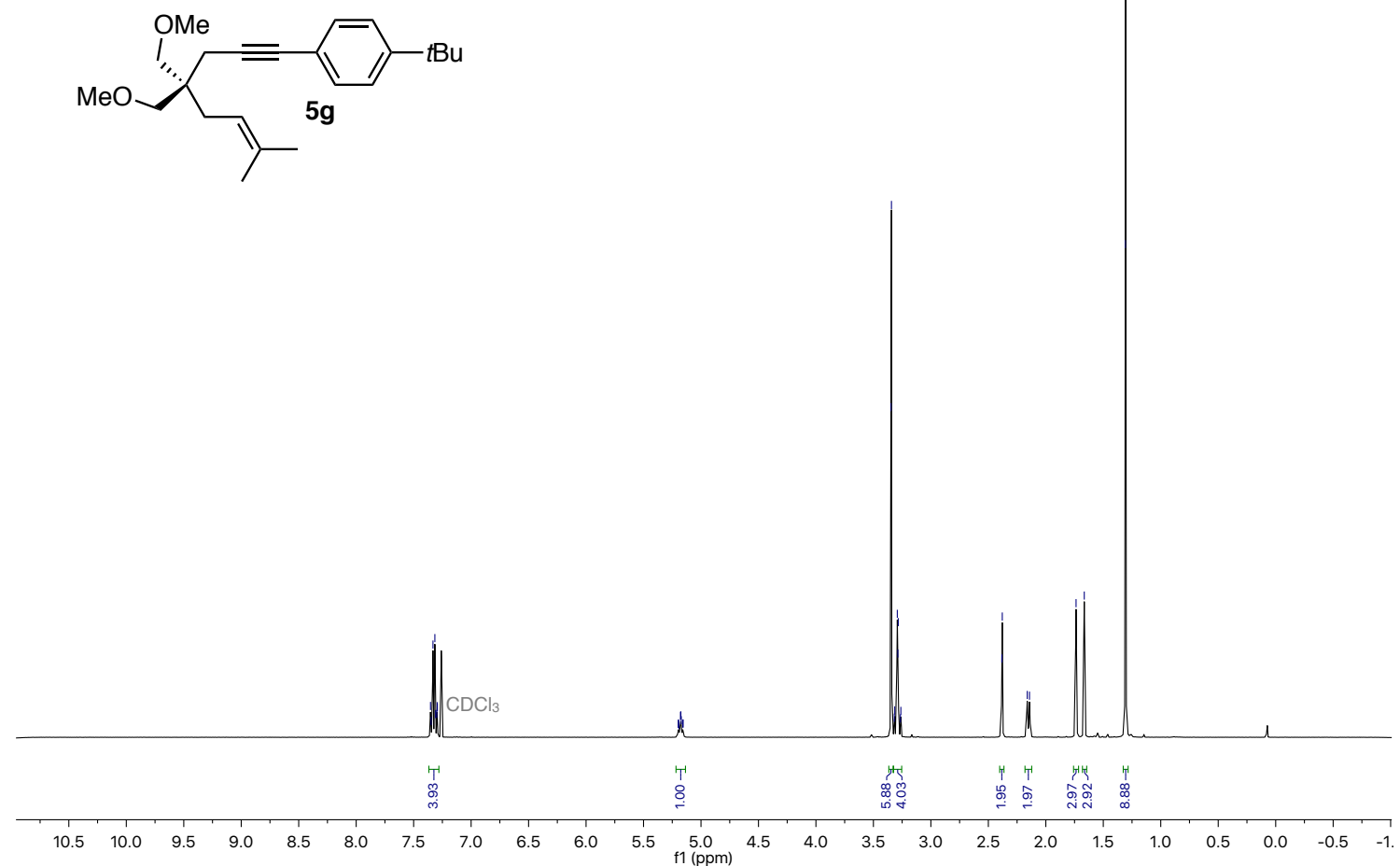

${ }^{13} \mathrm{C}$ NMR: $101 \mathrm{MHz}, \mathrm{CDCl}_{3}$, compound 5g

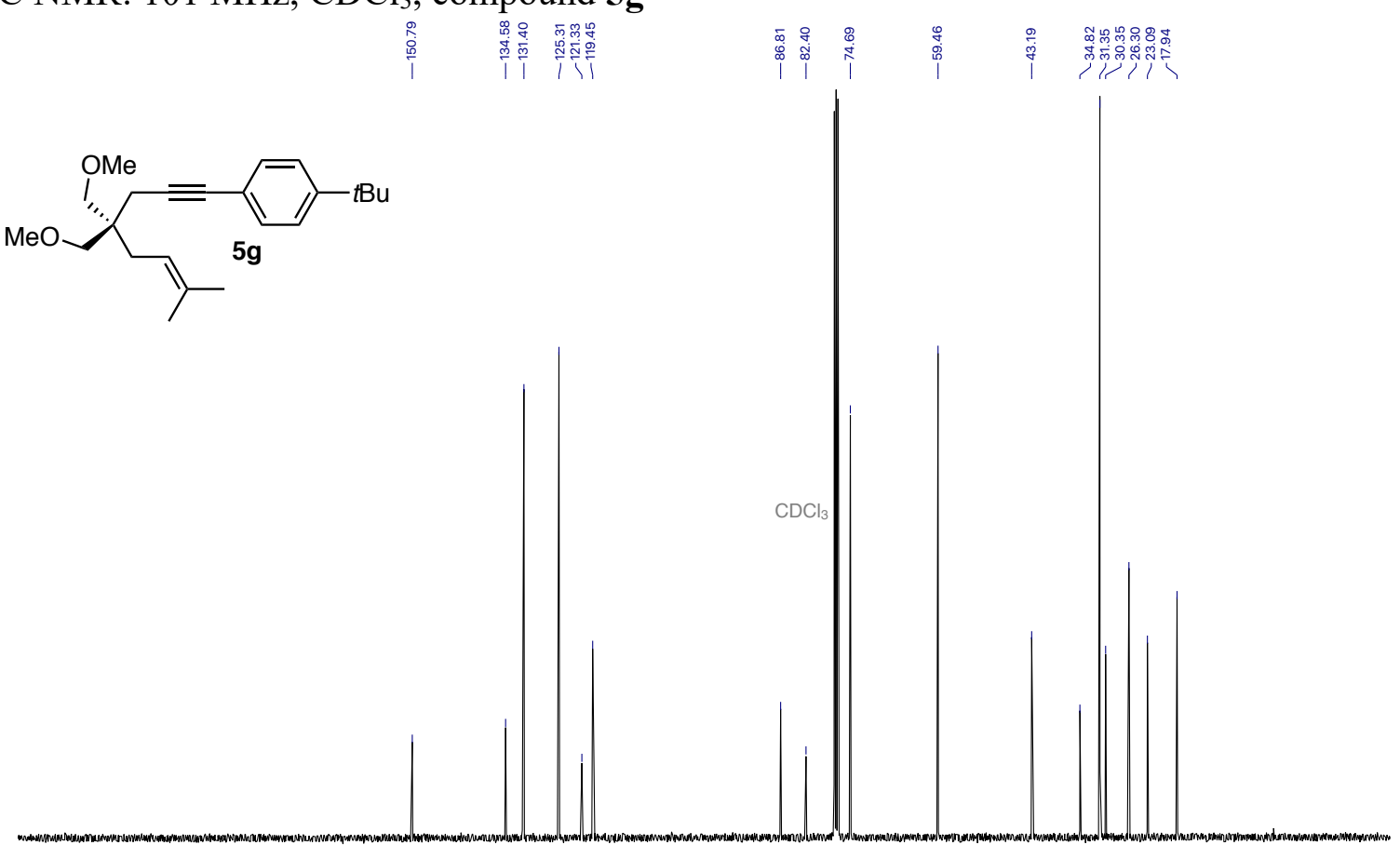

$\begin{array}{lllllllllllllllllllllllll}210 & 200 & 190 & 180 & 170 & 160 & 150 & 140 & 130 & 120 & 110 & \begin{array}{c}100 \\ \mathrm{f}(\mathrm{ppm})\end{array} & 90 & 80 & 70 & 60 & 50 & 40 & 30 & 20 & 10 & 0 & -10\end{array}$ 
${ }^{1} \mathrm{H}$ NMR: $400 \mathrm{MHz}, \mathrm{CDCl}_{3}$, compound $\mathbf{5 h}$

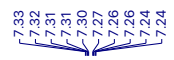

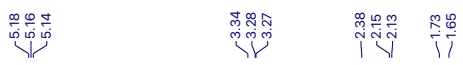
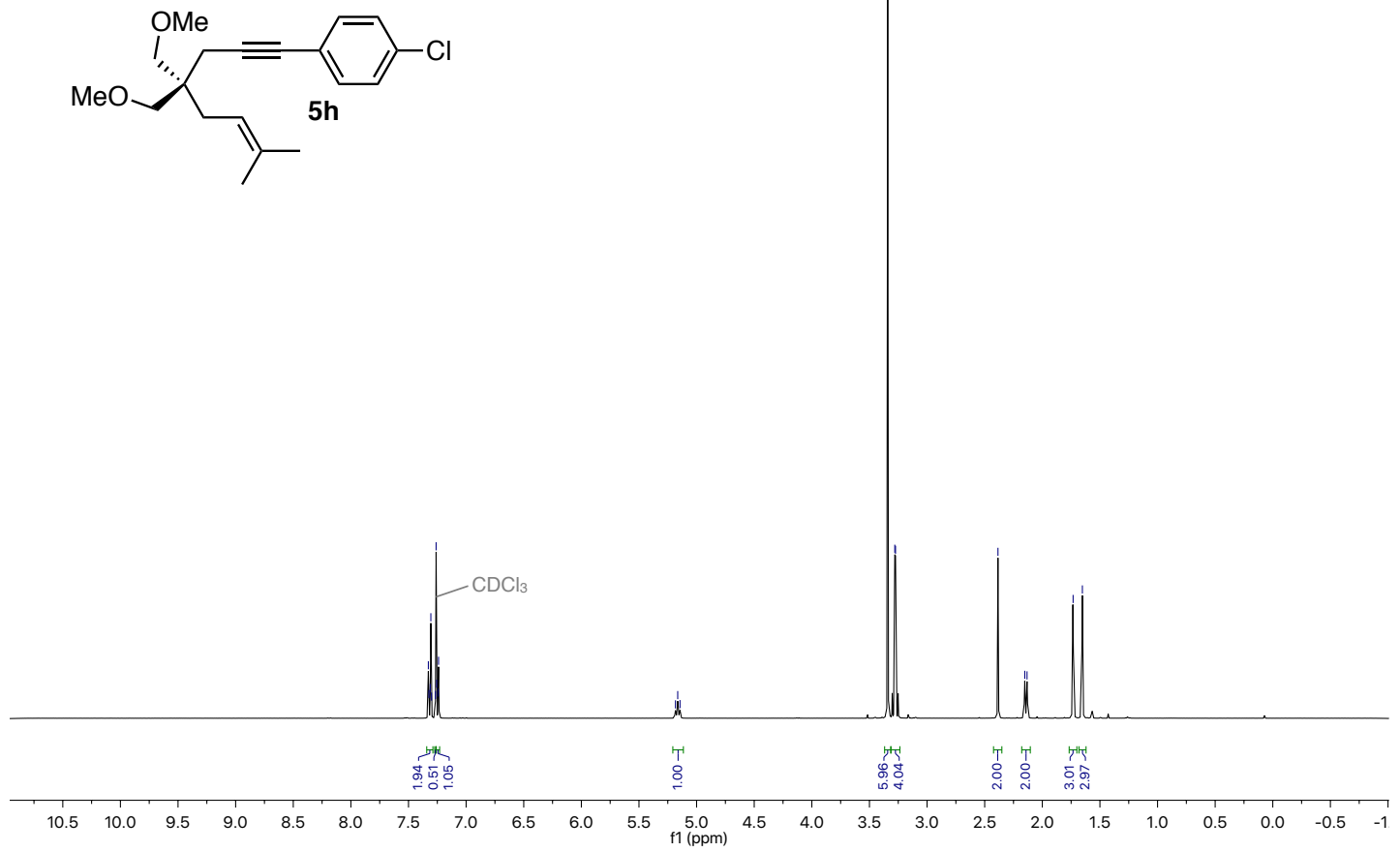

${ }^{13} \mathrm{C}$ NMR: $101 \mathrm{MHz}, \mathrm{CDCl}_{3}$, compound $\mathbf{5 h}$

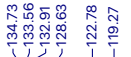

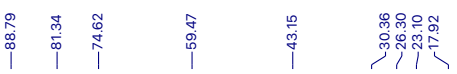<smiles>COCC(CC#Cc1ccc(Cl)cc1)(CC=C(C)C)COC</smiles>

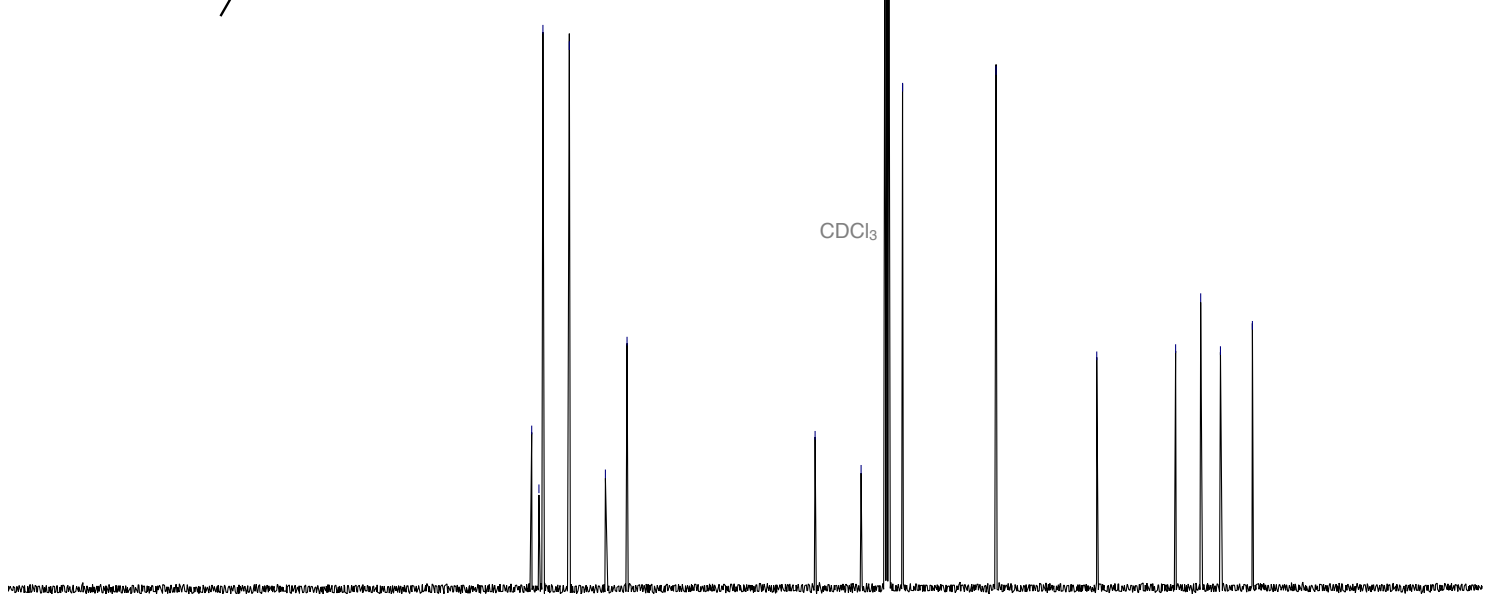

$\begin{array}{lllllllllllllllllllllllllll}210 & 200 & 190 & 180 & 170 & 160 & 150 & 140 & 130 & 120 & 110 & 100 & 90 & 80 & 70 & 60 & 50 & 40 & 30 & 20 & 10 & 0 & -10\end{array}$ 
${ }^{1} \mathrm{H}$ NMR: $400 \mathrm{MHz}, \mathrm{CDCl}_{3}$, compound 5i

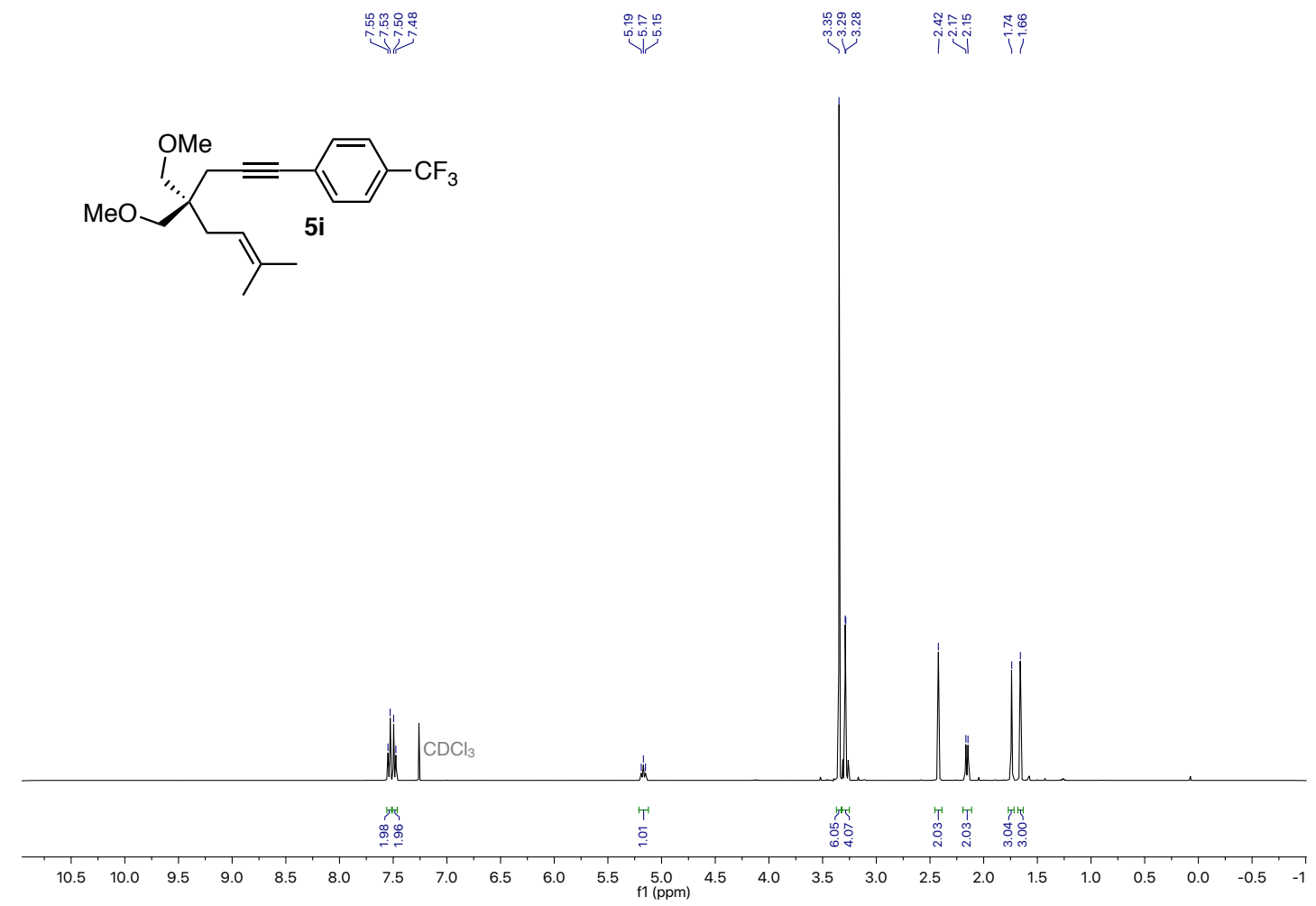

${ }^{13} \mathrm{C}$ NMR: $101 \mathrm{MHz}, \mathrm{CDCl}_{3}$, compound 5i

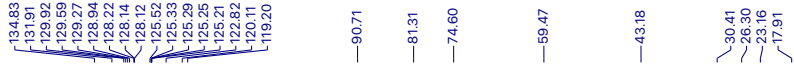

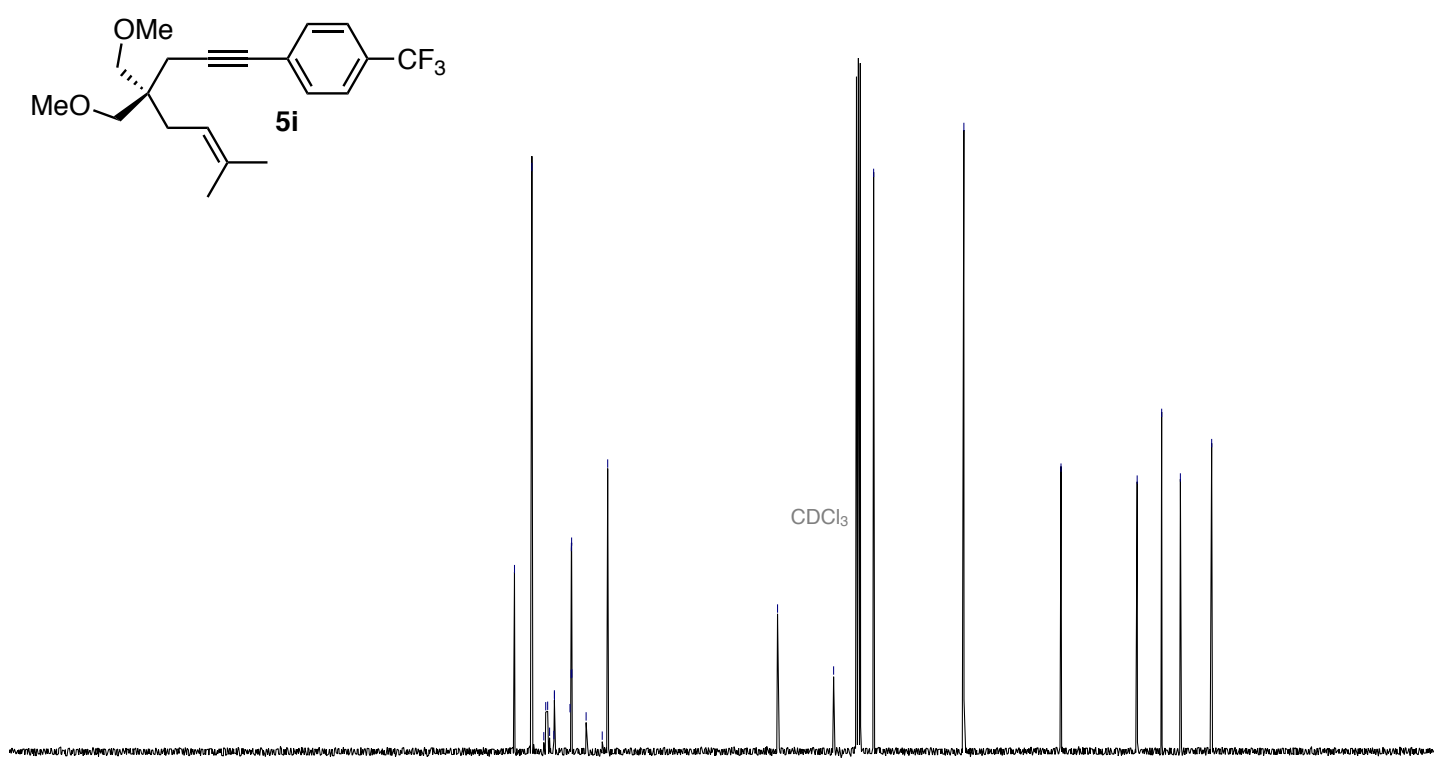

$\begin{array}{lllllllllllllllllllllllllll}210 & 200 & 190 & 180 & 170 & 160 & 150 & 140 & 130 & 120 & 110 & \begin{array}{c}100 \\ \mathrm{f} 1(\mathrm{ppm})\end{array} & 90 & 80 & 70 & 60 & 50 & 40 & 30 & 20 & 10 & 0 & -10\end{array}$ 
${ }^{19} \mathrm{~F}\left\{{ }^{1} \mathrm{H}\right\}$ NMR: $376 \mathrm{MHz}, \mathrm{CDCl}_{3}$, compound 5i

喿<smiles>COCC(CC#Cc1ccc(C(F)(F)F)cc1)(CC=C(C)C)COC</smiles>

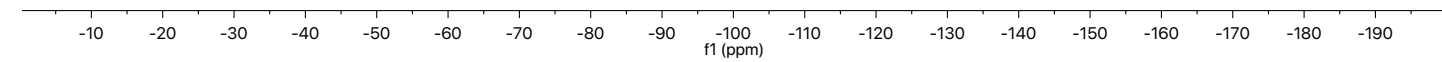

${ }^{1} \mathrm{H}$ NMR: $400 \mathrm{MHz}, \mathrm{C}_{6} \mathrm{D}_{6}$, compound 5j

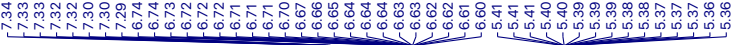

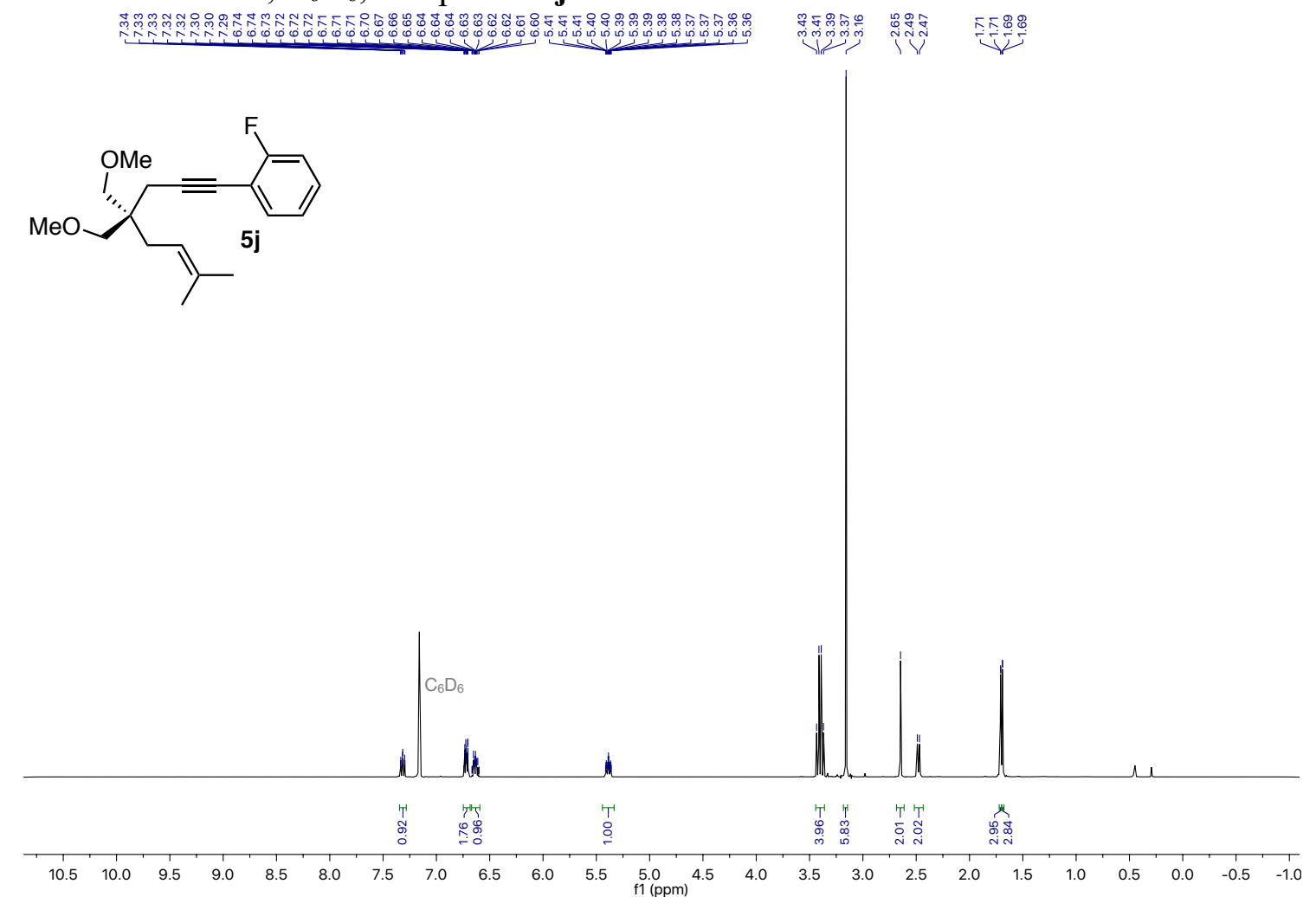


${ }^{13} \mathrm{C}$ NMR: $101 \mathrm{MHz}, \mathrm{C}_{6} \mathrm{D}_{6}$, compound $\mathbf{5 j}$

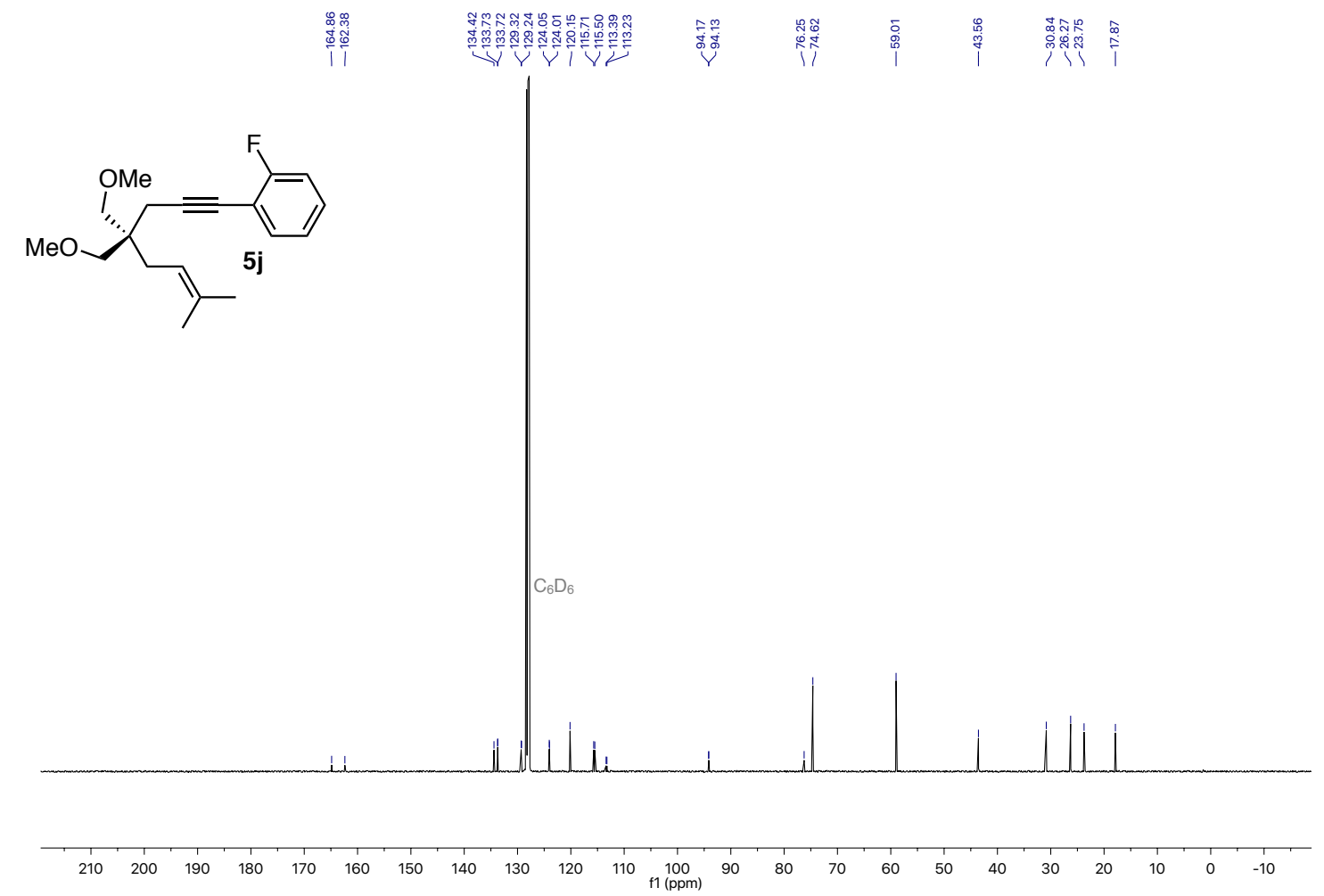

${ }^{19} \mathrm{~F}\left\{{ }^{1} \mathrm{H}\right\}$ NMR: $376 \mathrm{MHz}, \mathrm{C}_{6} \mathrm{D}_{6}$, compound $\mathbf{5 j}$
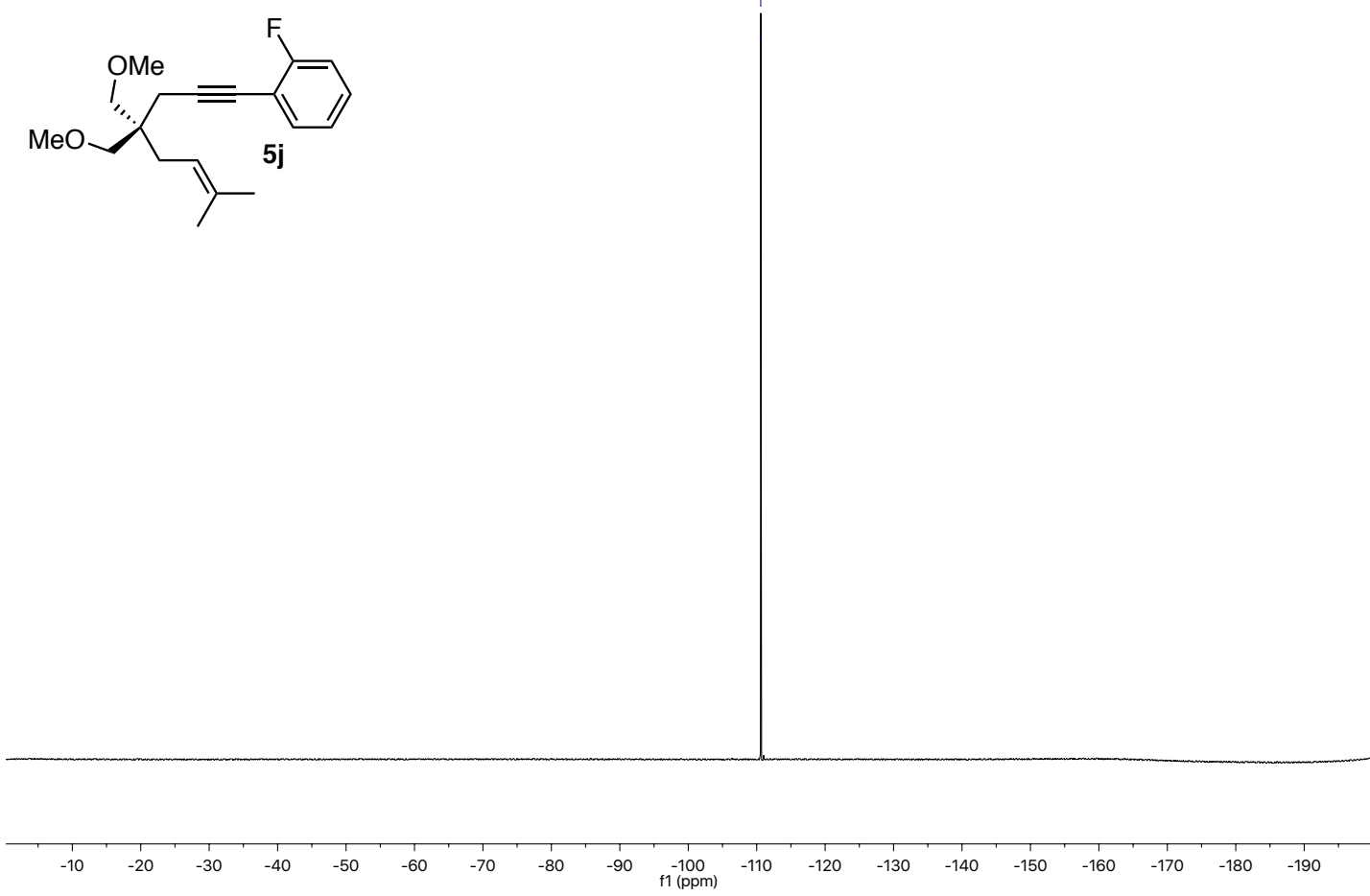
${ }^{1} \mathrm{H}$ NMR: $400 \mathrm{MHz}, \mathrm{CDCl}_{3}$, compound 5k

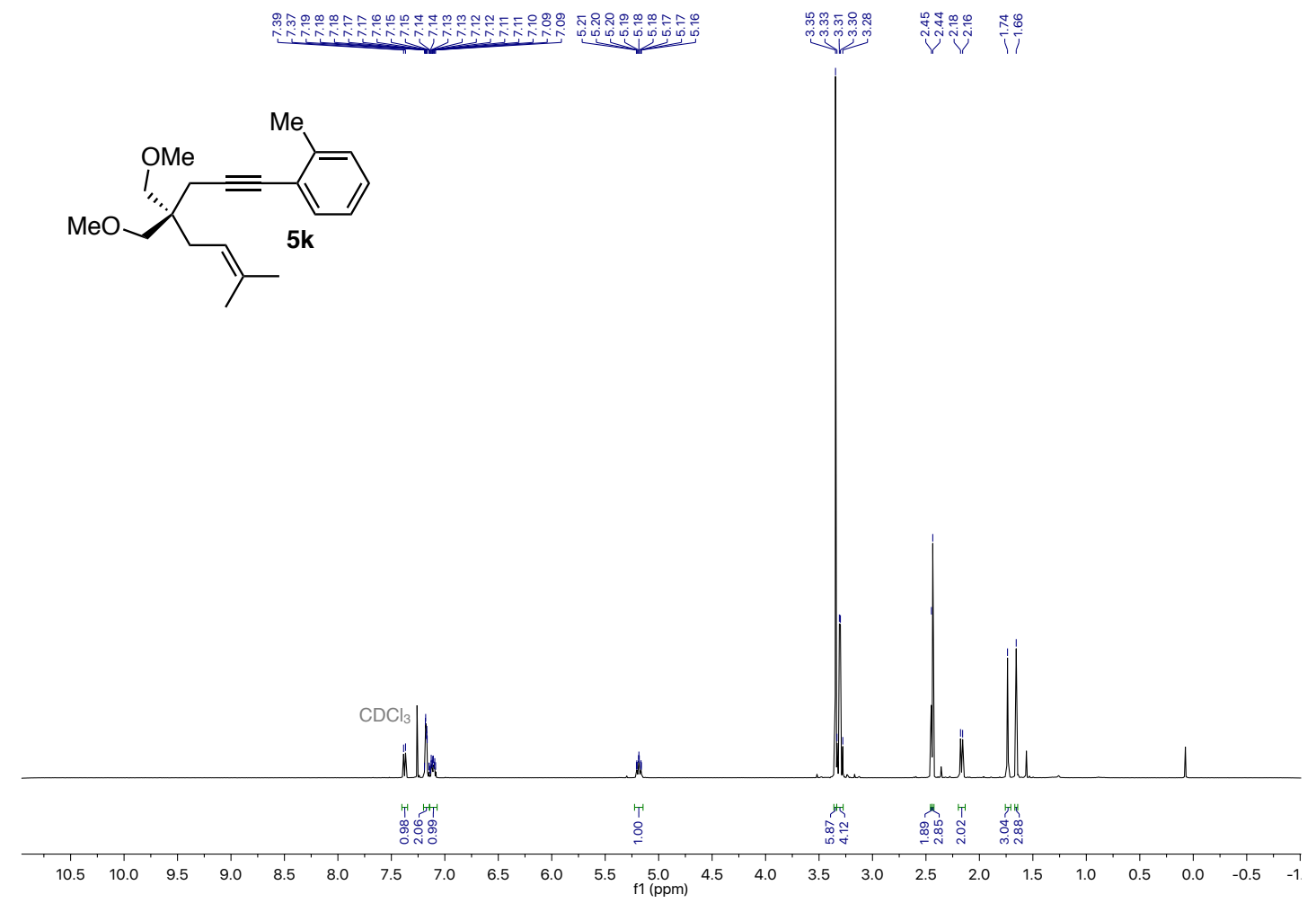

${ }^{13} \mathrm{C}$ NMR: $101 \mathrm{MHz}, \mathrm{CDCl}_{3}$, compound 5k

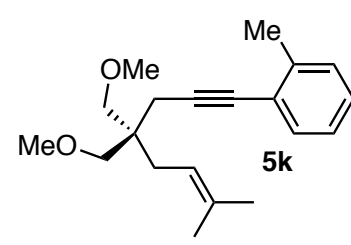

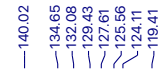

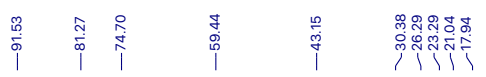

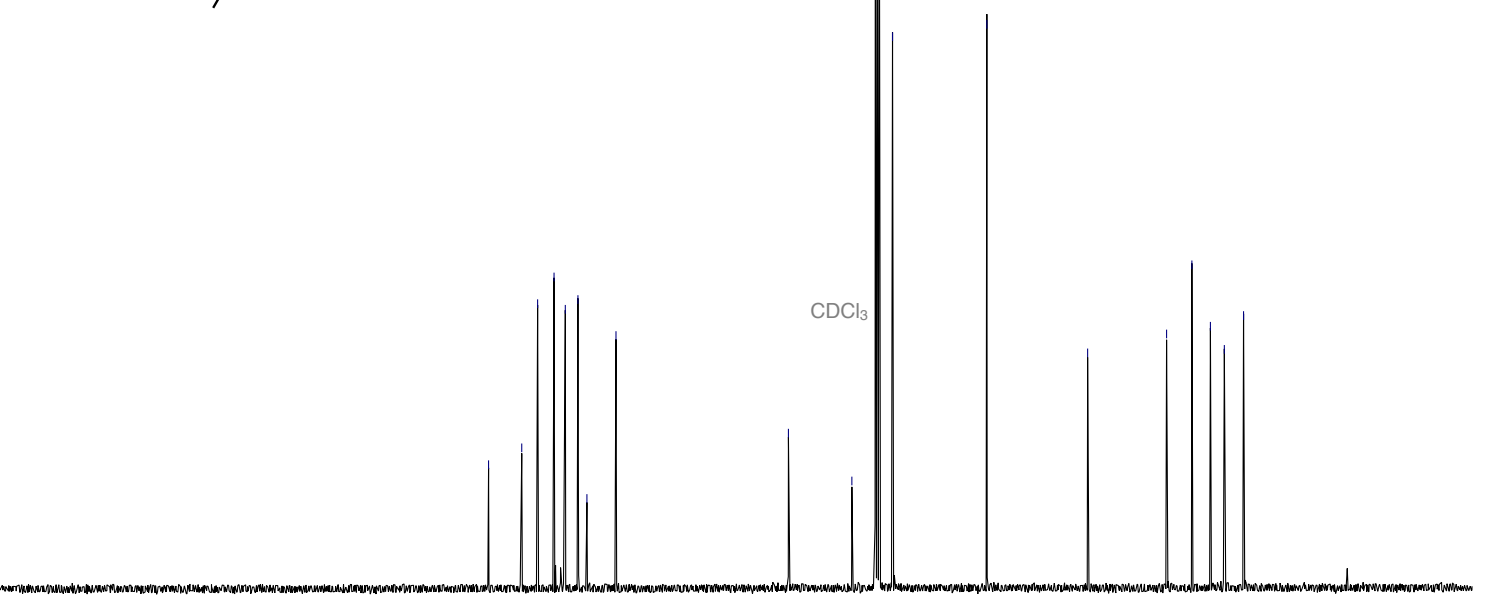

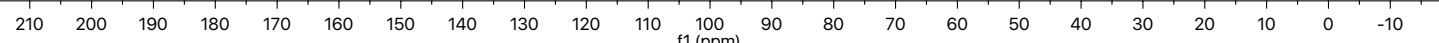


${ }^{1} \mathrm{H}$ NMR: $400 \mathrm{MHz}, \mathrm{CD}_{2} \mathrm{Cl}_{2}$, compound $5 \mathbf{l}$

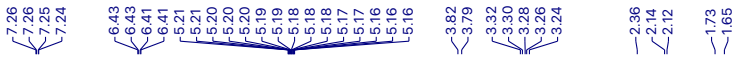
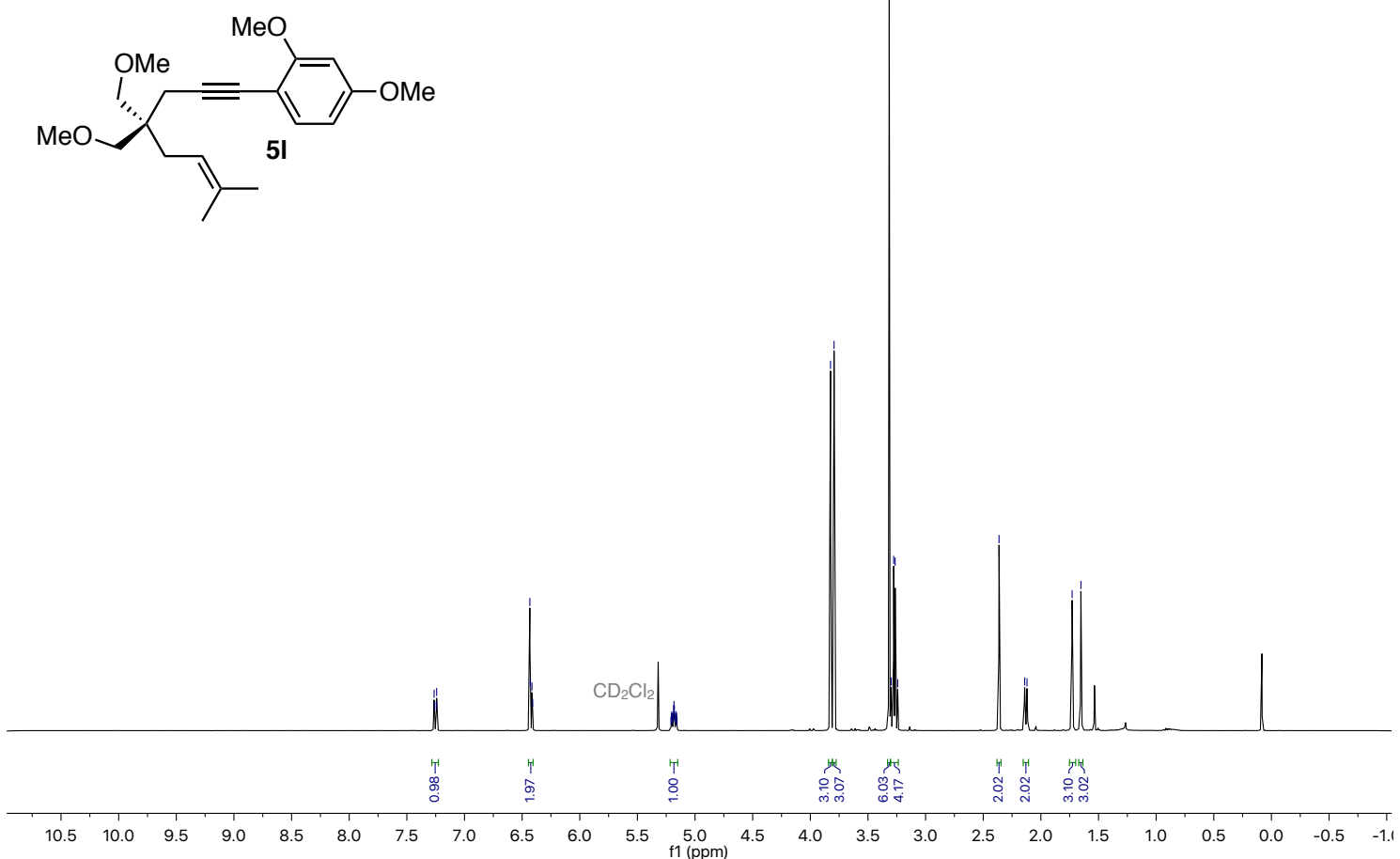

${ }^{13} \mathrm{C}$ NMR: $101 \mathrm{MHz}, \mathrm{CD}_{2} \mathrm{Cl}_{2}$, compound 5l
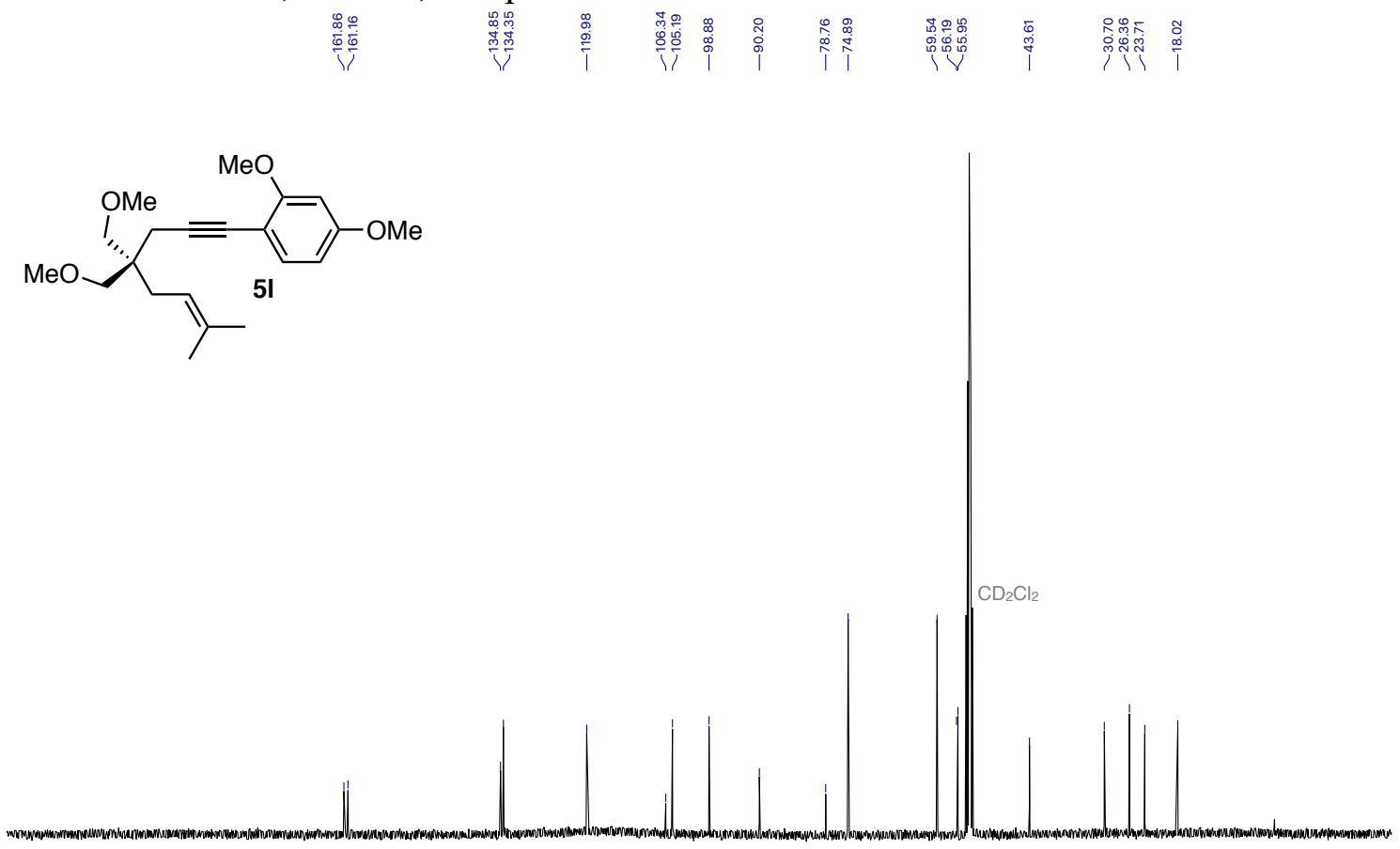

\begin{tabular}{llllllllllllllllllllllllllllll}
\hline 0 & 210 & 200 & 190 & 180 & 170 & 160 & 150 & 140 & 130 & 120 & 110 & $\begin{array}{c}100 \\
\mathrm{f} 1(\mathrm{ppm})\end{array}$ & 90 & 80 & 70 & 60 & 50 & 40 & 30 & 20 & 10 & 0 & -10
\end{tabular} 
${ }^{1} \mathrm{H}$ NMR: $500 \mathrm{MHz}, \mathrm{C}_{6} \mathrm{D}_{6}$, compound 5m

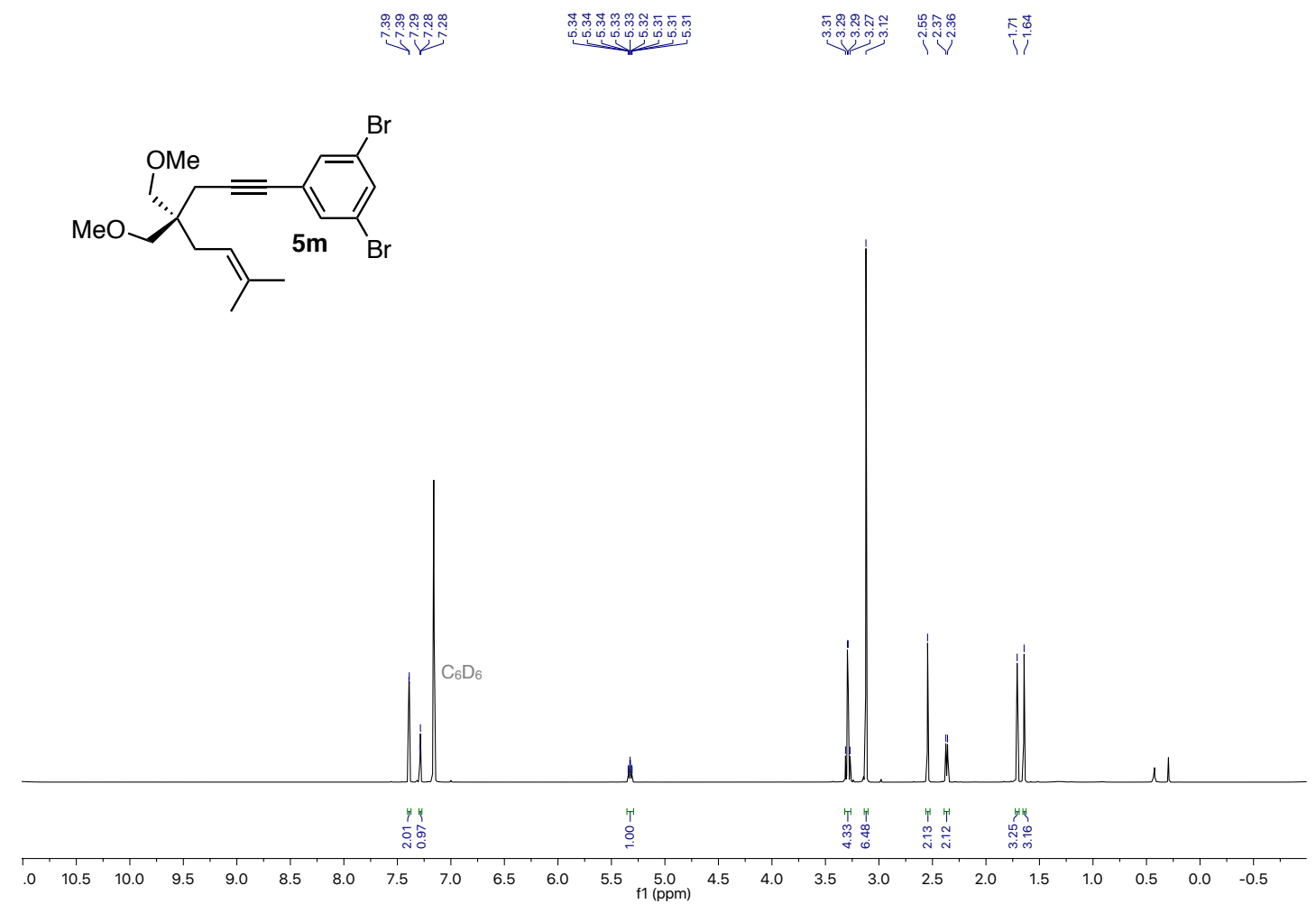

${ }^{13} \mathrm{C}$ NMR: $126 \mathrm{MHz}, \mathrm{C}_{6} \mathrm{D}_{6}$, compound 5 m

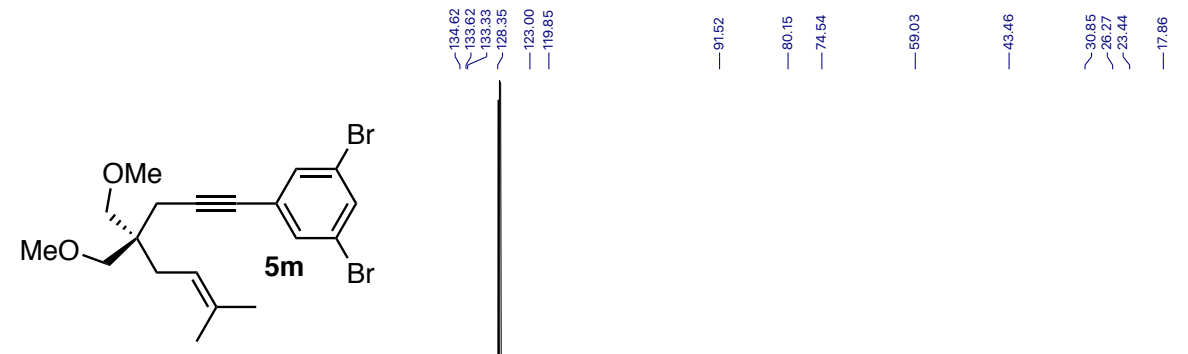

$\begin{array}{lllllllllllllllllllllllllll}1 & 110 & 200 & 190 & 180 & 170 & 160 & 150 & 140 & 130 & 120 & 110 & \underset{f 1}{1(\mathrm{ppm})} & 90 & 80 & 70 & 60 & 50 & 40 & 30 & 20 & 10 & 0 & -10\end{array}$ 
${ }^{1} \mathrm{H}$ NMR: $400 \mathrm{MHz}, \mathrm{CDCl}_{3}$, compound 5n
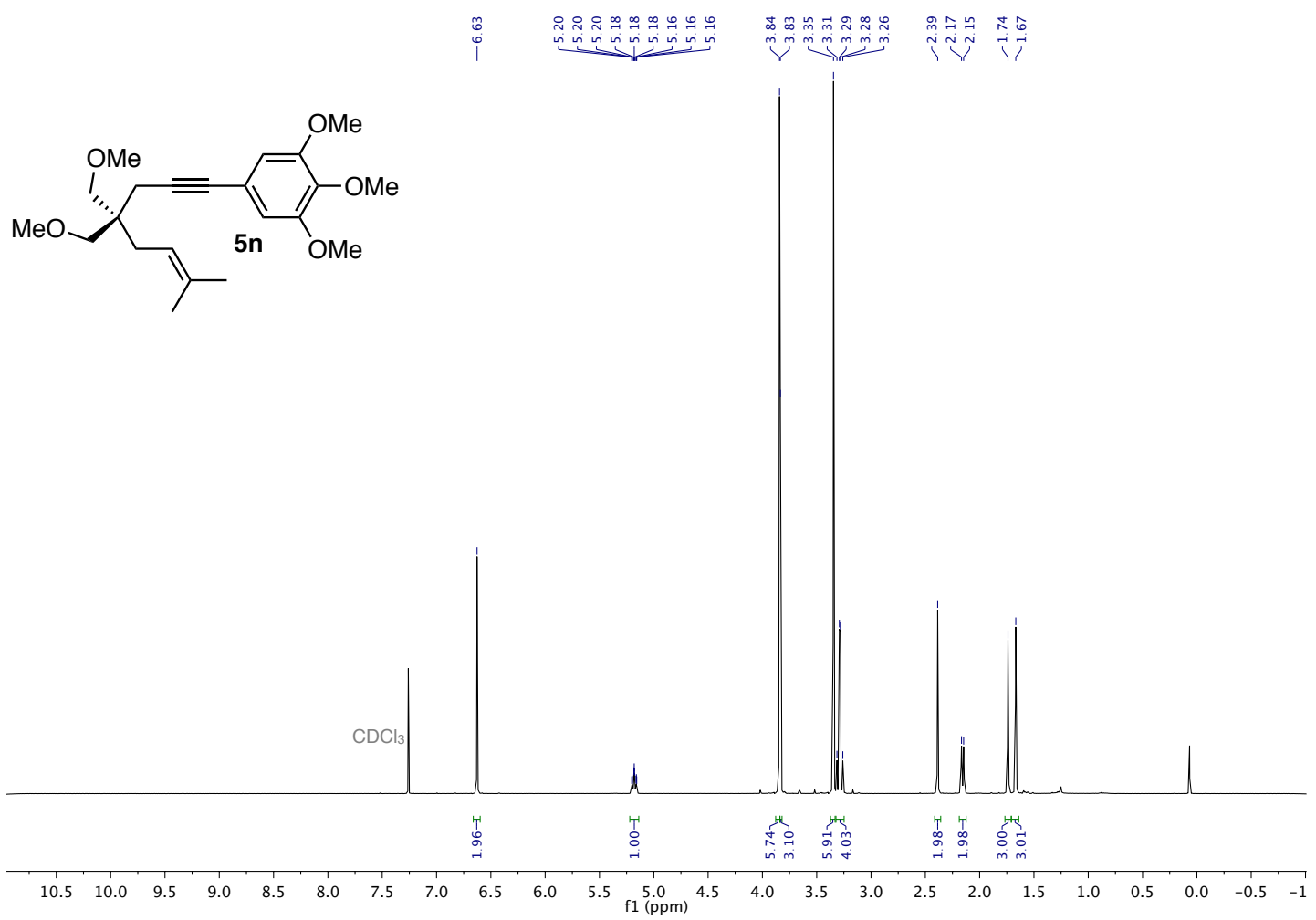

${ }^{13} \mathrm{C}$ NMR: $101 \mathrm{MHz}, \mathrm{CDCl}_{3}$ compound 5n

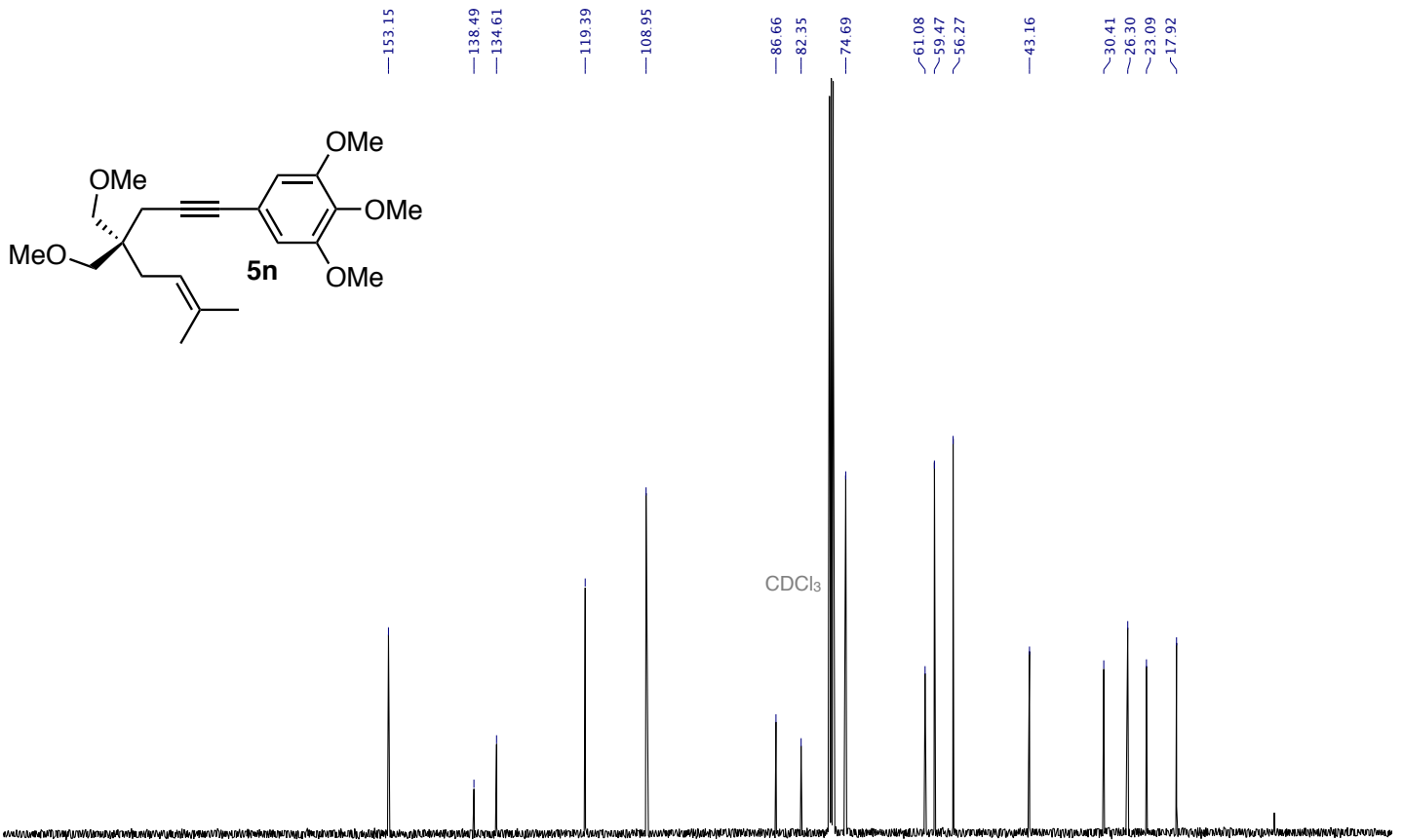

$\begin{array}{lllllllllllllllllllllll}210 & 200 & 190 & 180 & 170 & 160 & 150 & 140 & 130 & 120 & 110 & \begin{array}{l}100 \\ \mathrm{f} 1(\mathrm{ppm})\end{array} & 90 & 80 & 70 & 60 & 50 & 40 & 30 & 20 & 10 & 0 & -10\end{array}$ 
${ }^{1} \mathrm{H}$ NMR: $400 \mathrm{MHz}, \mathrm{C}_{6} \mathrm{D}_{6}$, compound 5 o

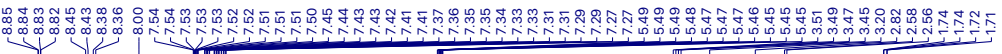
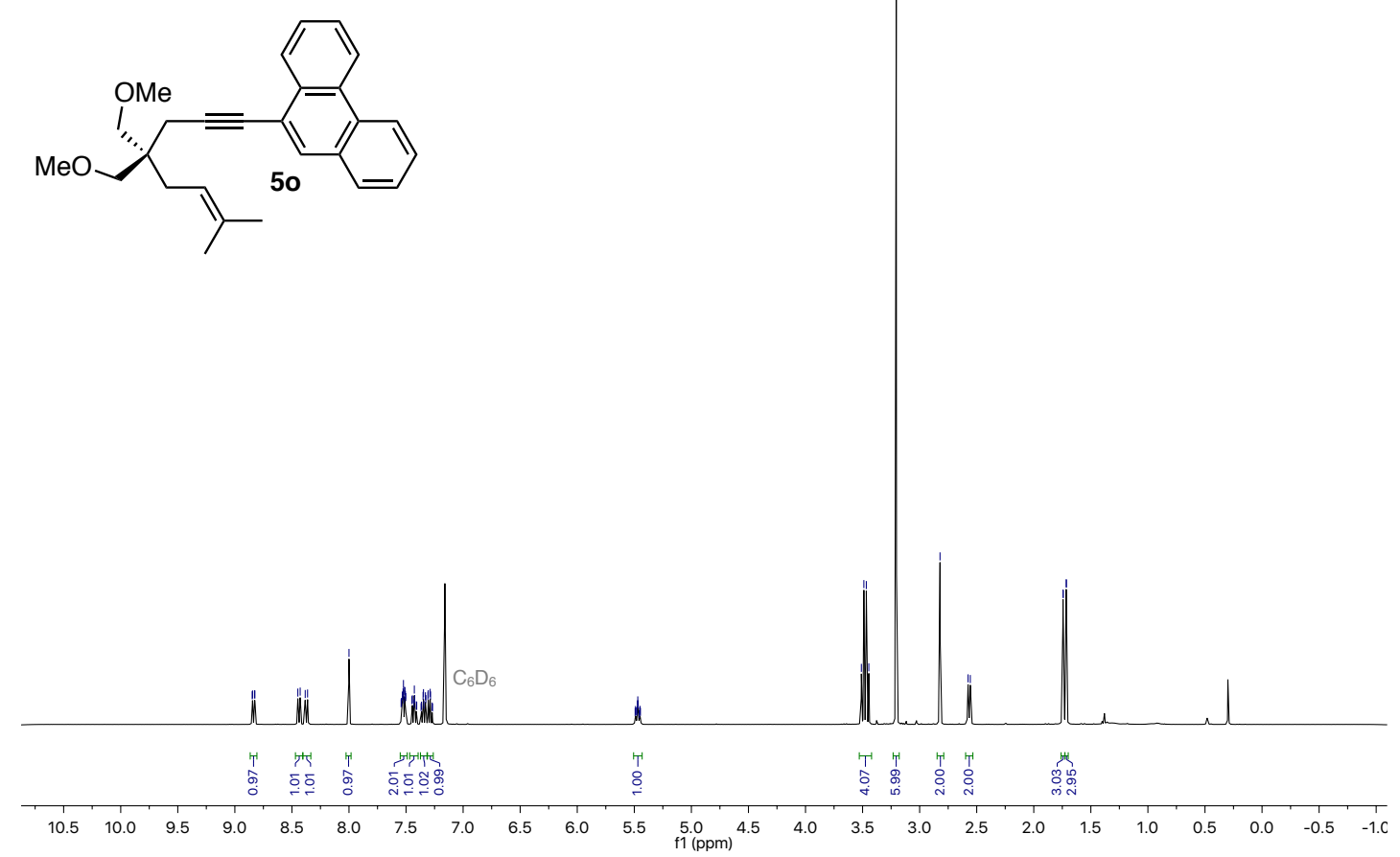

${ }^{13} \mathrm{C}$ NMR: $101 \mathrm{MHz}, \mathrm{C}_{6} \mathrm{D}_{6}$, compound 50

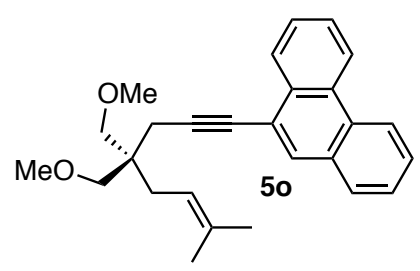

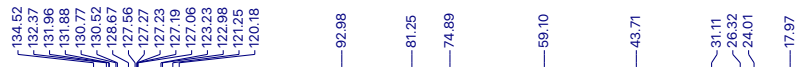

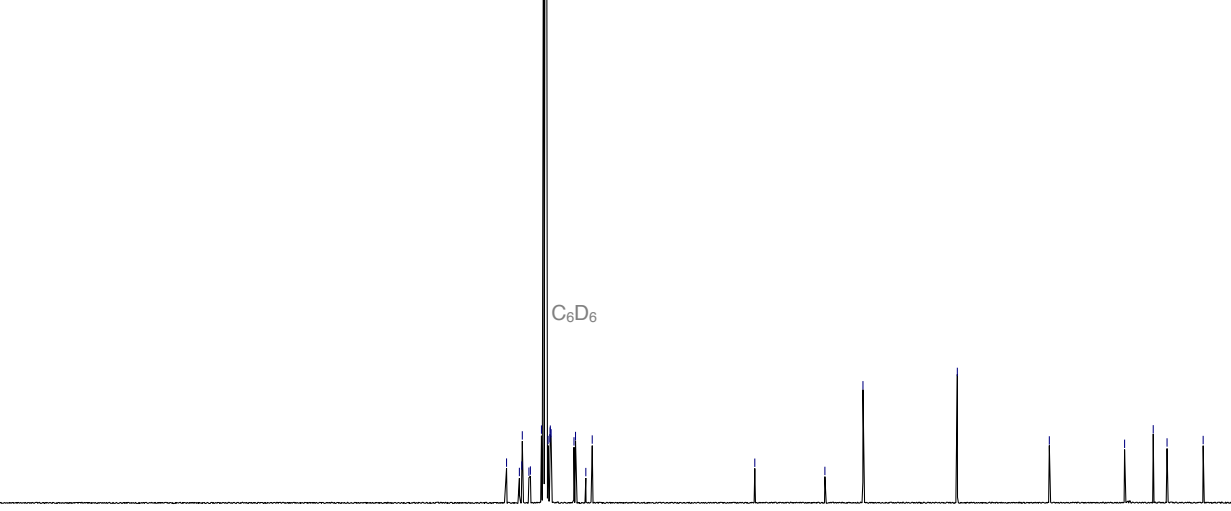

$\begin{array}{llllllllllllllllllllllllll}1 & 10 & 200 & 190 & 180 & 170 & 160 & 150 & 140 & 130 & 120 & 110 & \underset{f 1}{1(\mathrm{ppm})} & 90 & 80 & 70 & 60 & 50 & 40 & 30 & 20 & 10 & 0 & -10\end{array}$ 
${ }^{1} \mathrm{H}$ NMR: $400 \mathrm{MHz}, \mathrm{CDCl}_{3},(E)-4,4-B i s(m e t h o x y m e t h y l)-7,11$-dimethyldodeca-6,10-dien-1yne

$\underbrace{ \pm}$
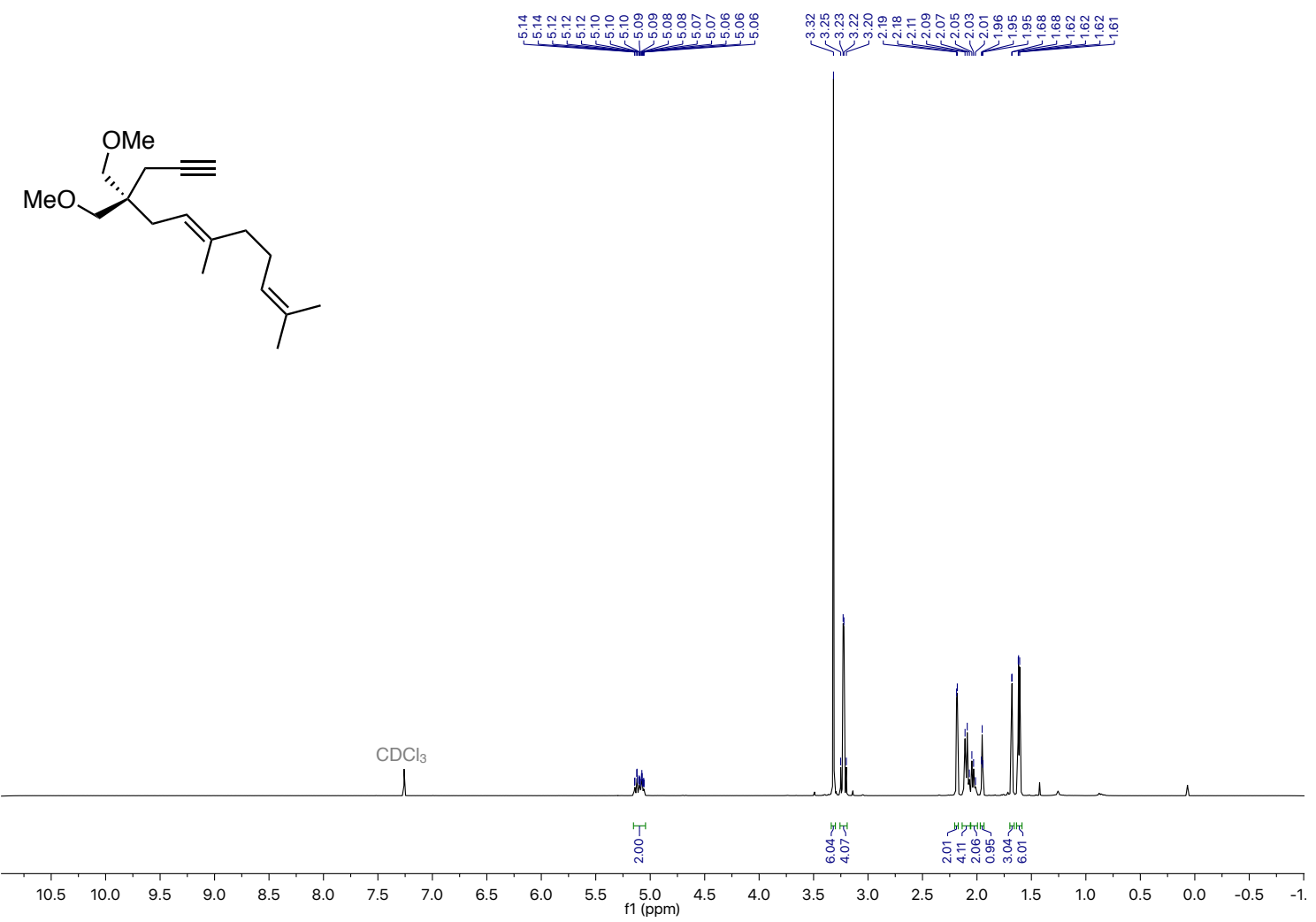

${ }^{13} \mathrm{C}$ NMR: $101 \mathrm{MHz}, \mathrm{CDCl}_{3},(E)-4,4-B i s(m e t h o x y m e t h y l)-7,11$-dimethyldodeca-6,10-dien-1yne

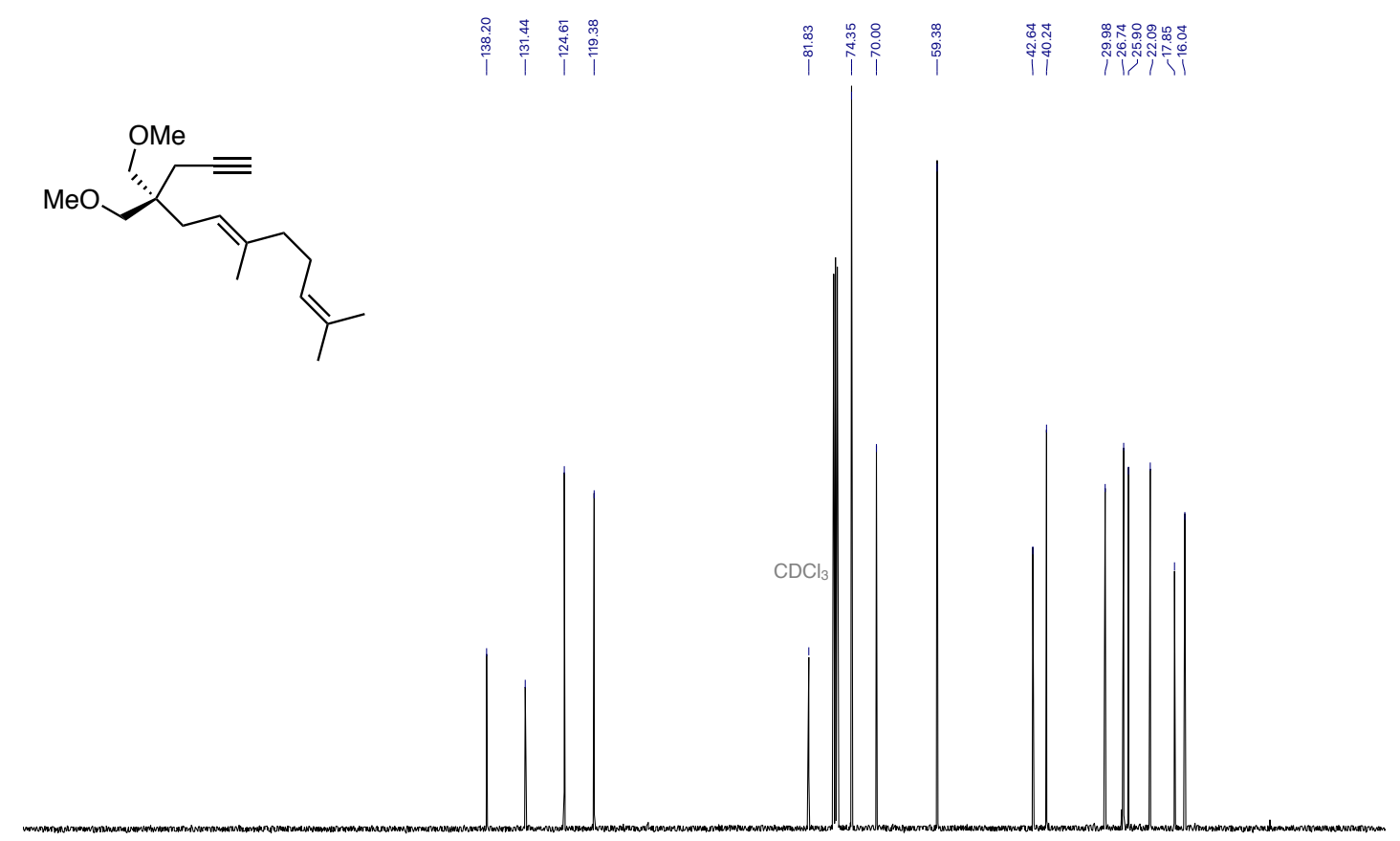

$\begin{array}{lllllllllllllllllllllllllll}210 & 200 & 190 & 180 & 170 & 160 & 150 & 140 & 130 & 120 & 110 & \begin{array}{c}100 \\ \mathrm{f} 1(\mathrm{ppm})\end{array} & 90 & 80 & 70 & 60 & 50 & 40 & 30 & 20 & 10 & 0 & -10\end{array}$ 
${ }^{1} \mathrm{H}$ NMR: $400 \mathrm{MHz}, \mathrm{CD}_{2} \mathrm{Cl}_{2}$, compound $\mathbf{5 p}$

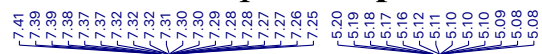
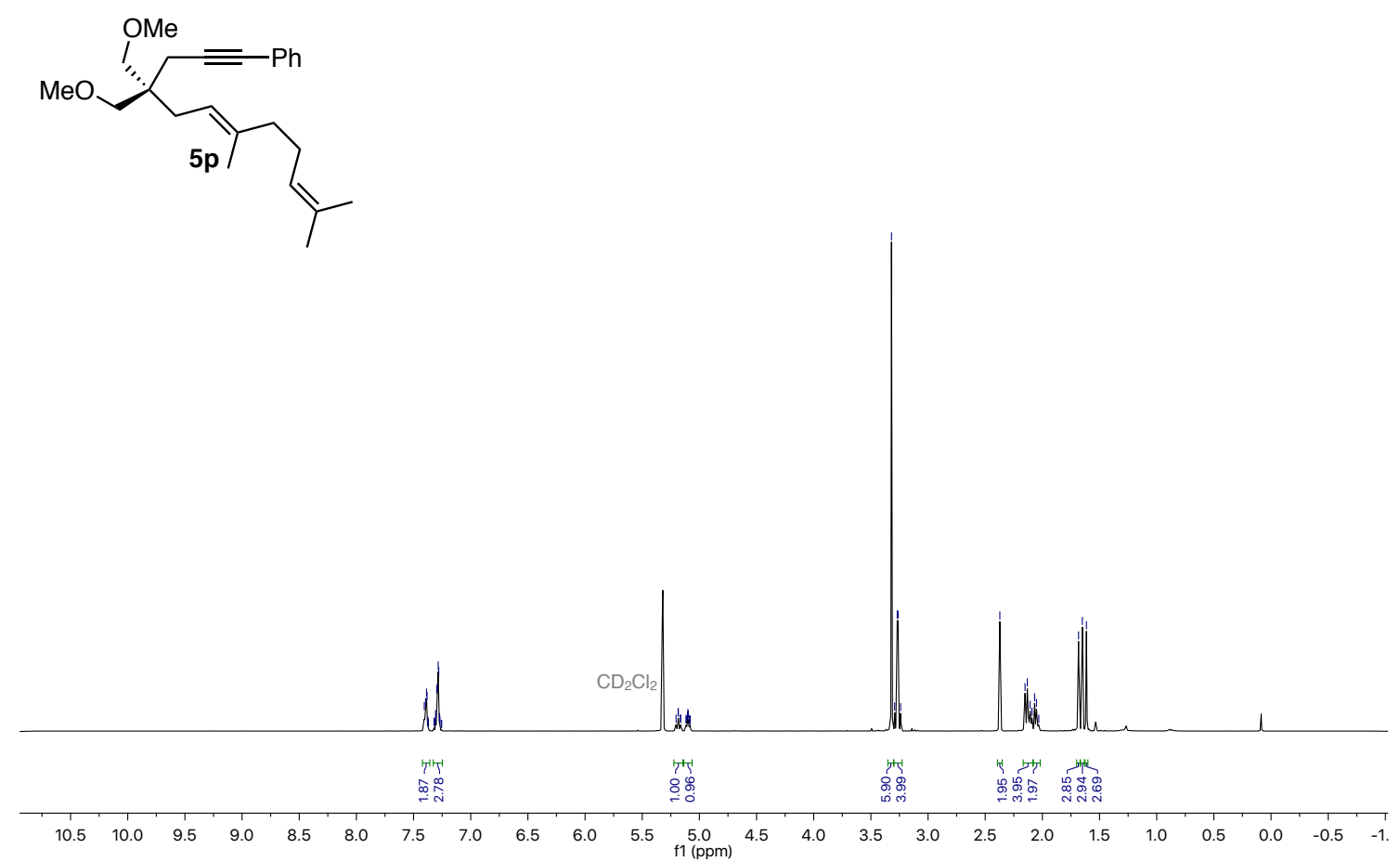

${ }^{13} \mathrm{C}$ NMR: $101 \mathrm{MHz}, \mathrm{CD}_{2} \mathrm{Cl}_{2}$, compound 5p

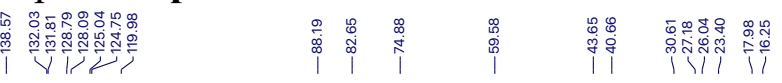
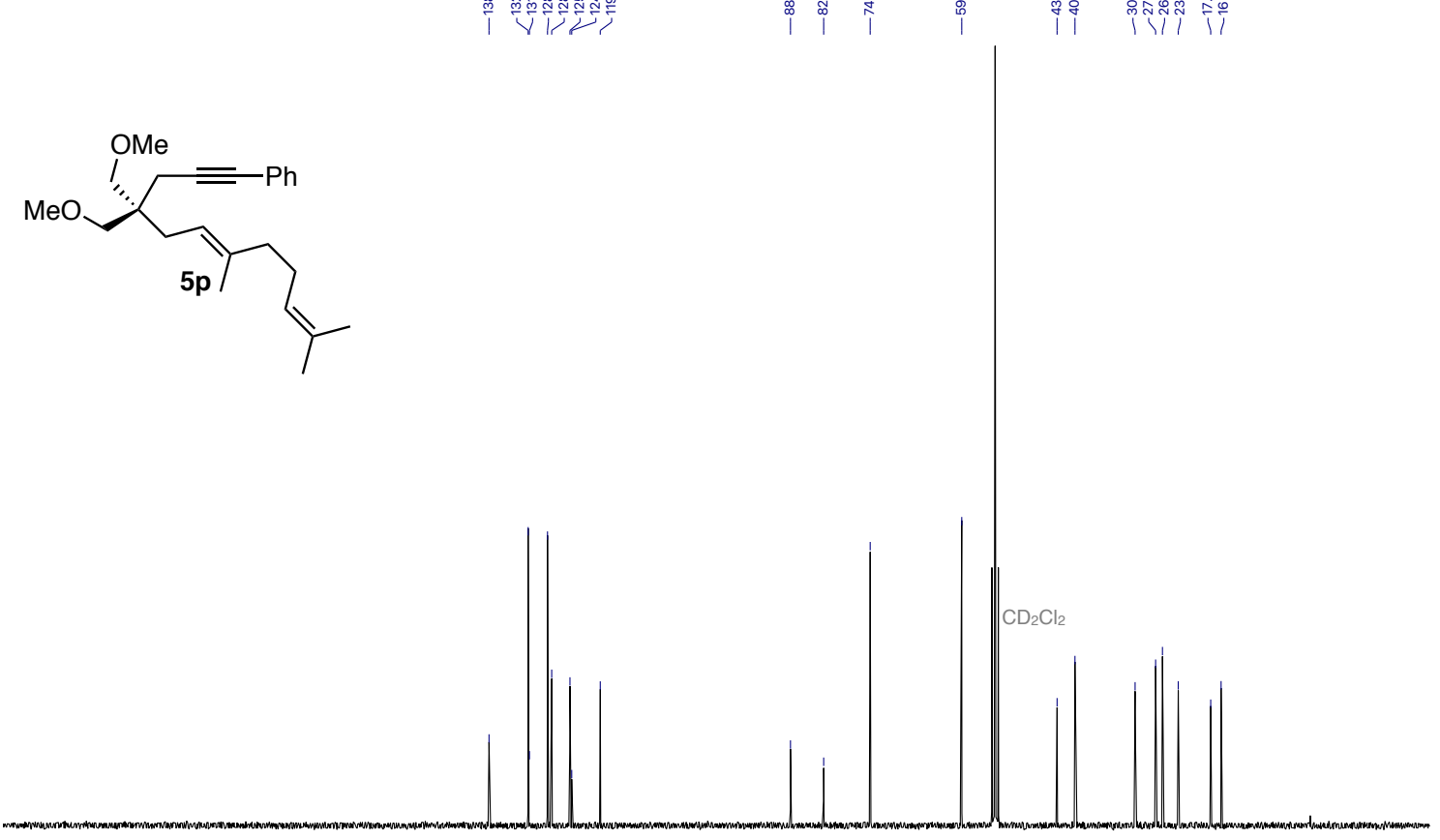

$\begin{array}{lllllllllllllllllllllllllll} & 210 & 200 & 190 & 180 & 170 & 160 & 150 & 140 & 130 & 120 & 110 & 100 & 90 & 80 & 70 & 60 & 50 & 40 & 30 & 20 & 10 & 0 & -10\end{array}$ 
${ }^{1} \mathrm{H}$ NMR: $400 \mathrm{MHz}, \mathrm{CDCl}_{3}$, 2-Cinnamyl-2-(3-phenylprop-2-yn-1-yl)propane-1,3-diol

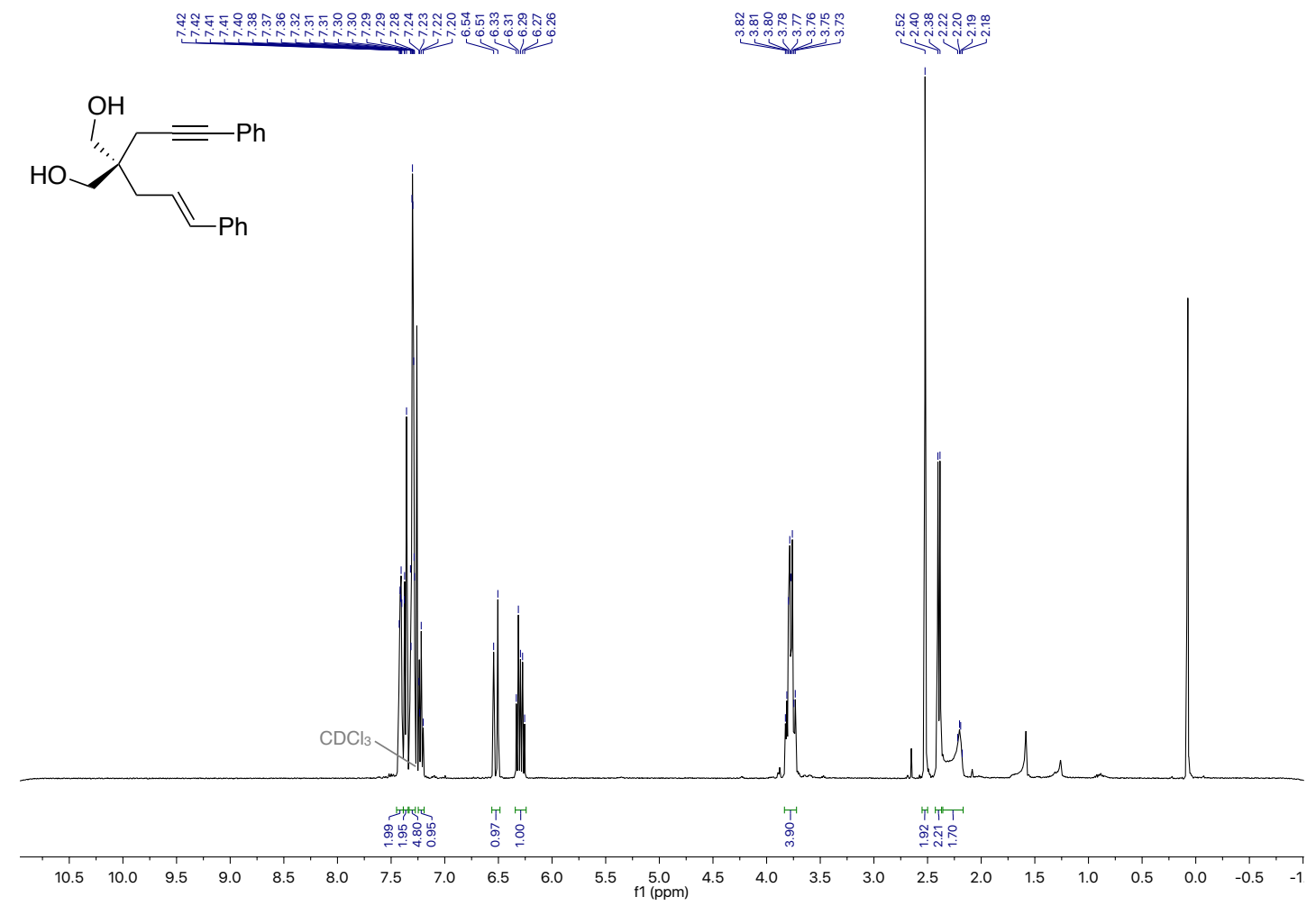

${ }^{13} \mathrm{C}$ NMR: $101 \mathrm{MHz}, \mathrm{CDCl}_{3}$, 2-Cinnamyl-2-(3-phenylprop-2-yn-1-yl)propane-1,3-diol

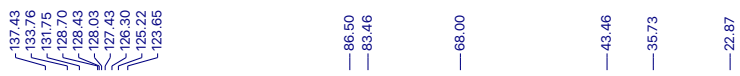
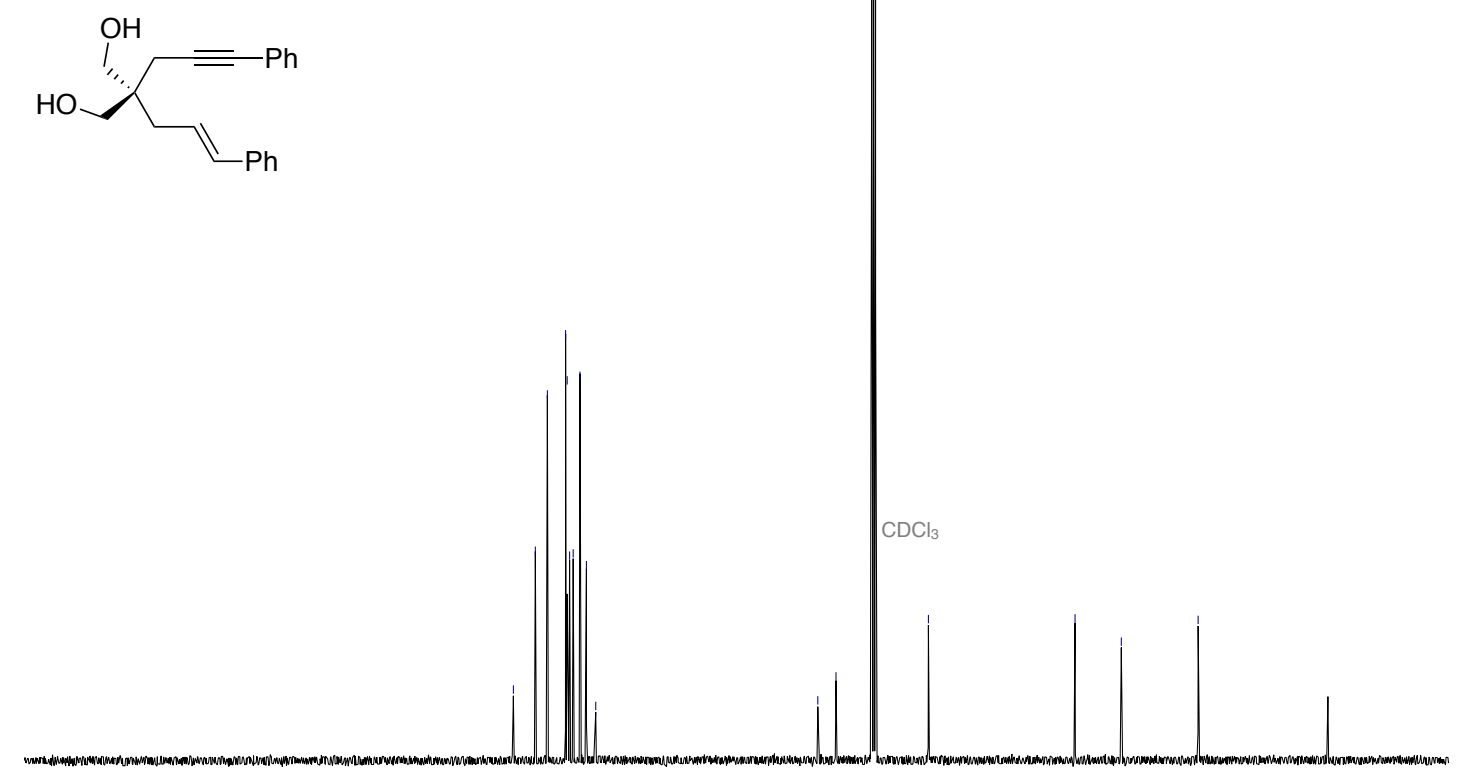

$\begin{array}{llllllllllllllllllllllllll}1 & 1 & 200 & 190 & 180 & 170 & 160 & 150 & 140 & 130 & 120 & 110 & \begin{array}{c}100 \\ \mathrm{f} 1(\mathrm{ppm})\end{array} & 90 & 80 & 70 & 60 & 50 & 40 & 30 & 20 & 10 & 0 & -10\end{array}$ 
${ }^{1} \mathrm{H}$ NMR: $400 \mathrm{MHz}, \mathrm{CD}_{2} \mathrm{Cl}_{2}$, compound 5q

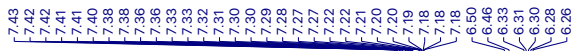
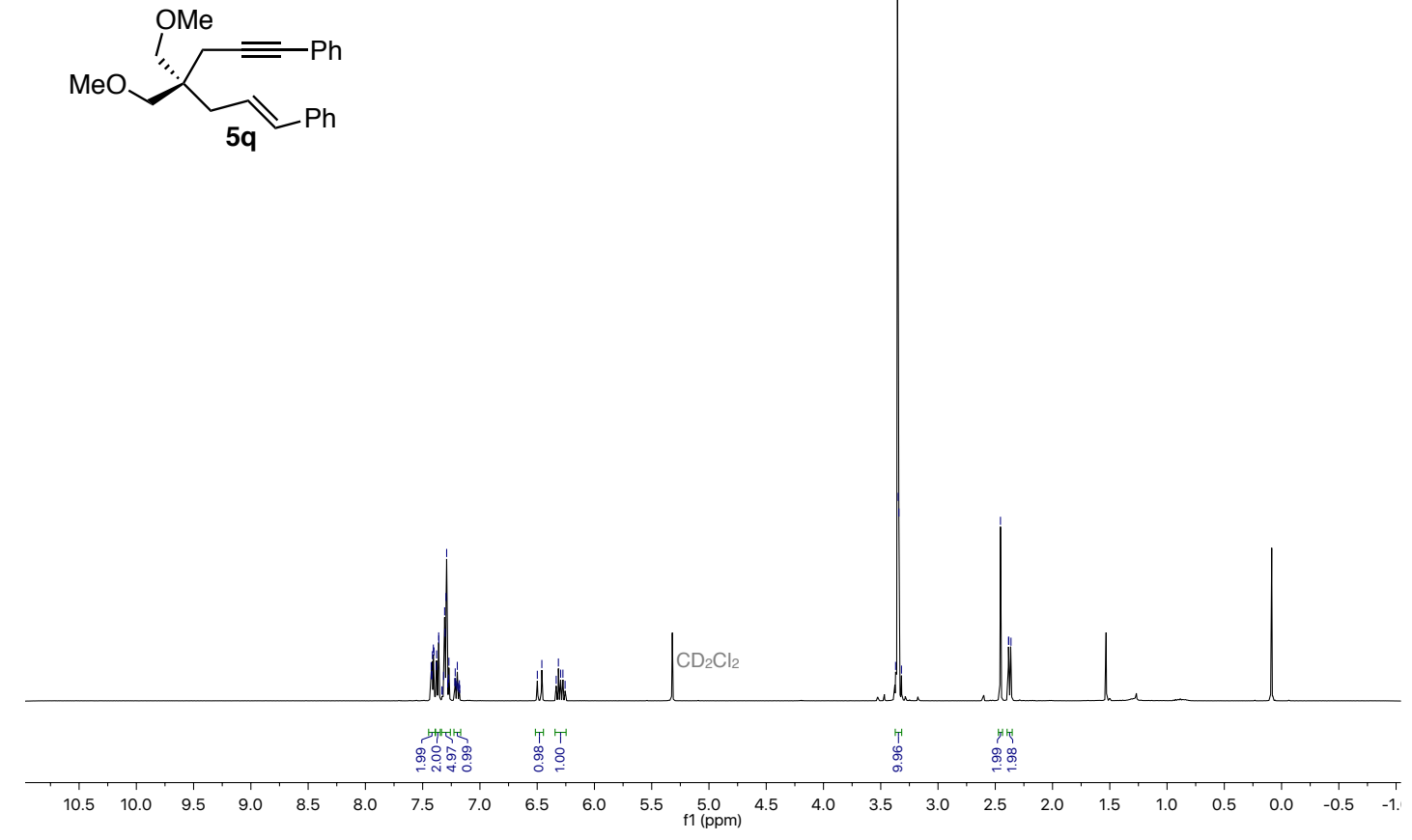

${ }^{13} \mathrm{C}$ NMR: $101 \mathrm{MHz}, \mathrm{CD}_{2} \mathrm{Cl}_{2}$, compound 5q

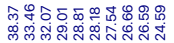

$\underline{\underline{m}}$
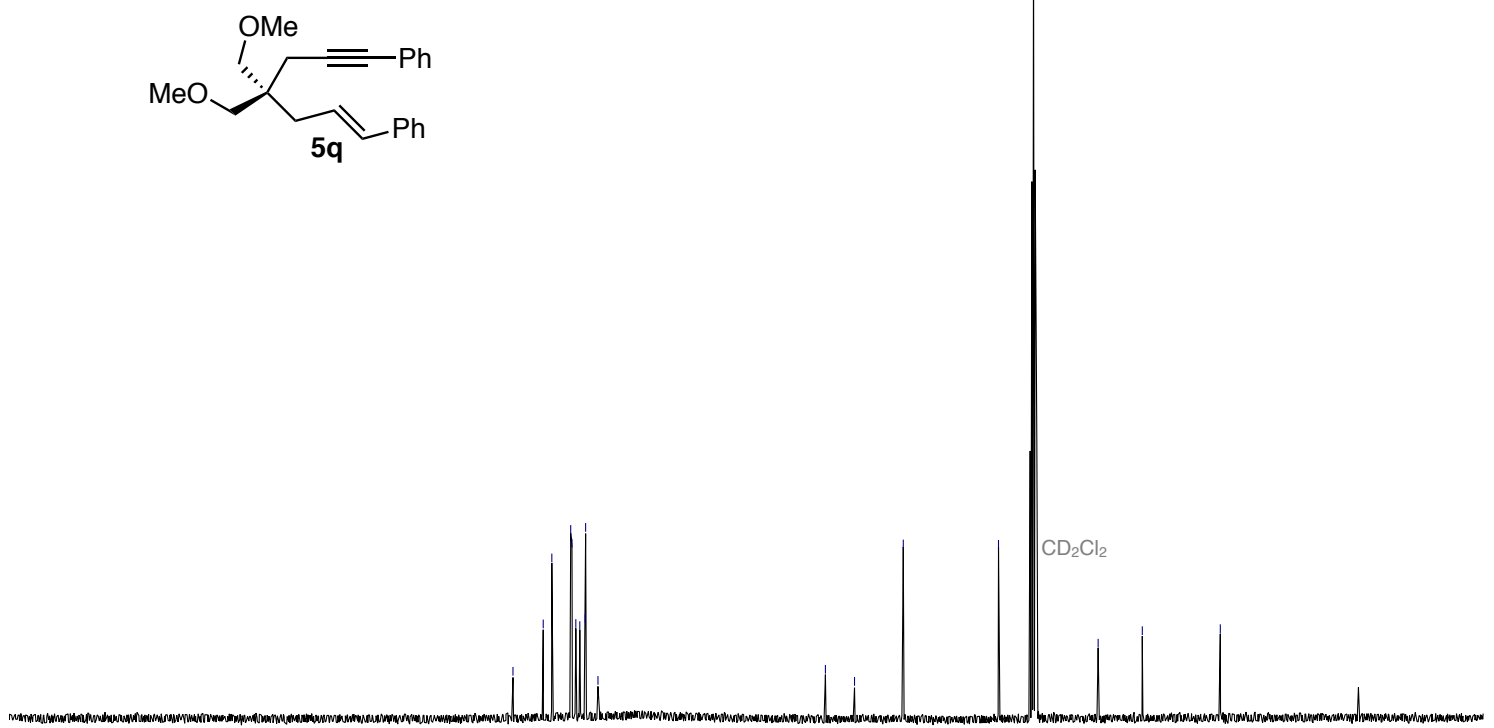

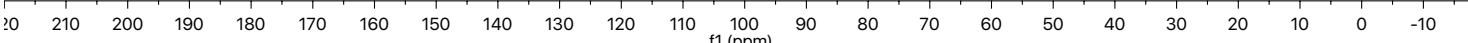


${ }^{1} \mathrm{H}$ NMR: $400 \mathrm{MHz}, \mathrm{CD}_{2} \mathrm{Cl}_{2}$, compound $\mathbf{6 b}$

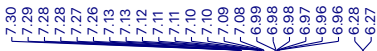<smiles>COCC1(COC)CC2=Cc3ccccc3C(C)(C)[C@H]2C1</smiles>

$6 b$

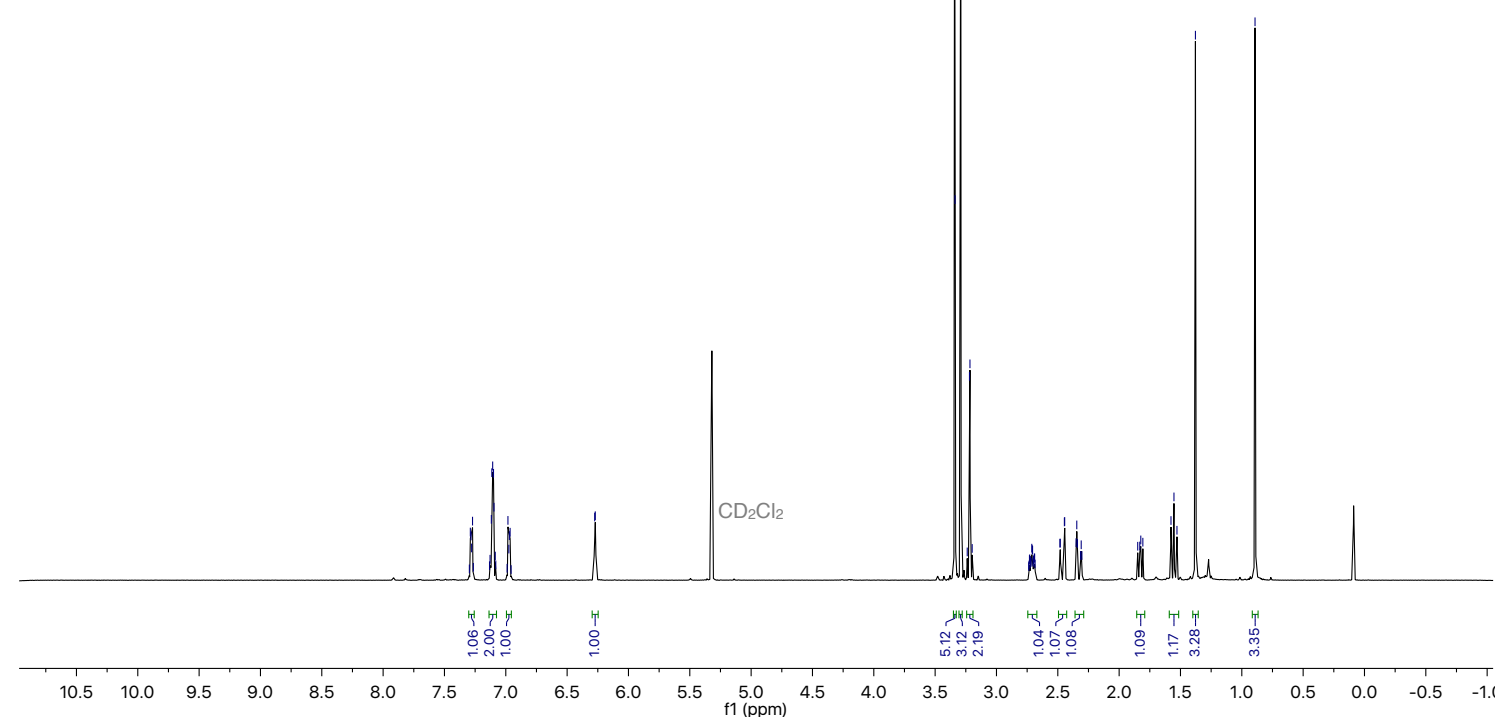

${ }^{13} \mathrm{C}$ NMR: $126 \mathrm{MHz}, \mathrm{CD}_{2} \mathrm{Cl}_{2}$, compound $\mathbf{6 b}$ 1)

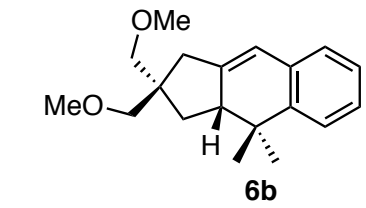

$6 b$

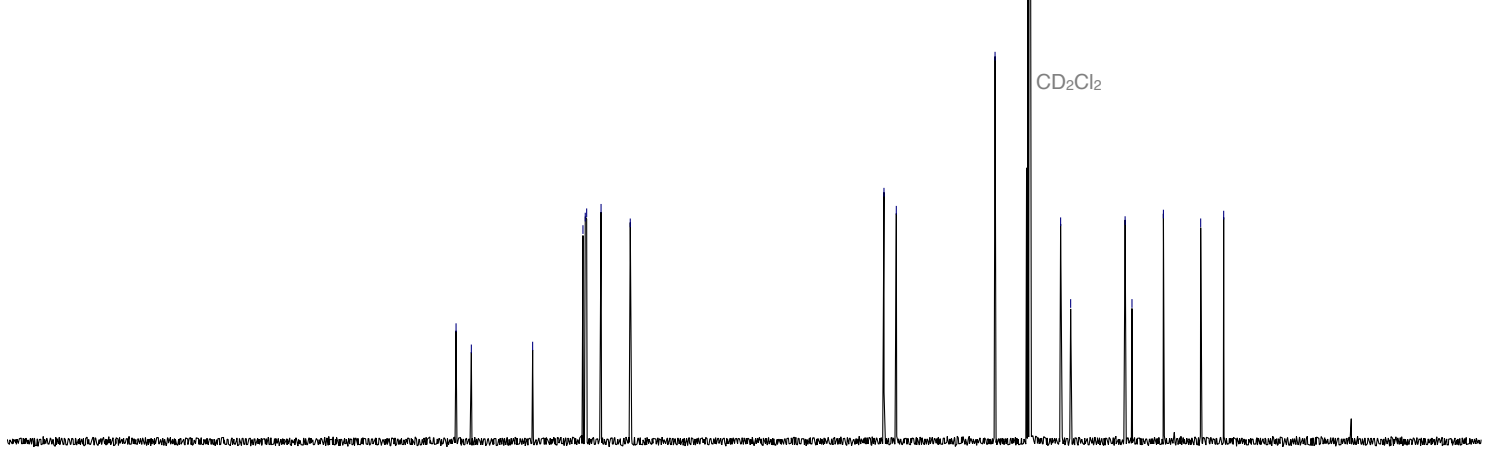

\begin{tabular}{lllllllllllllllllllllllllllllll}
\hline & 20 & 210 & 200 & 190 & 180 & 170 & 160 & 150 & 140 & 130 & 120 & 110 & 100 & 90 & 80 & 70 & 60 & 50 & 40 & 30 & 20 & 10 & 0 & -10
\end{tabular} 
${ }^{1} \mathrm{H}$ NMR: $500 \mathrm{MHz}, \mathrm{CD}_{2} \mathrm{Cl}_{2}$, compound 6c

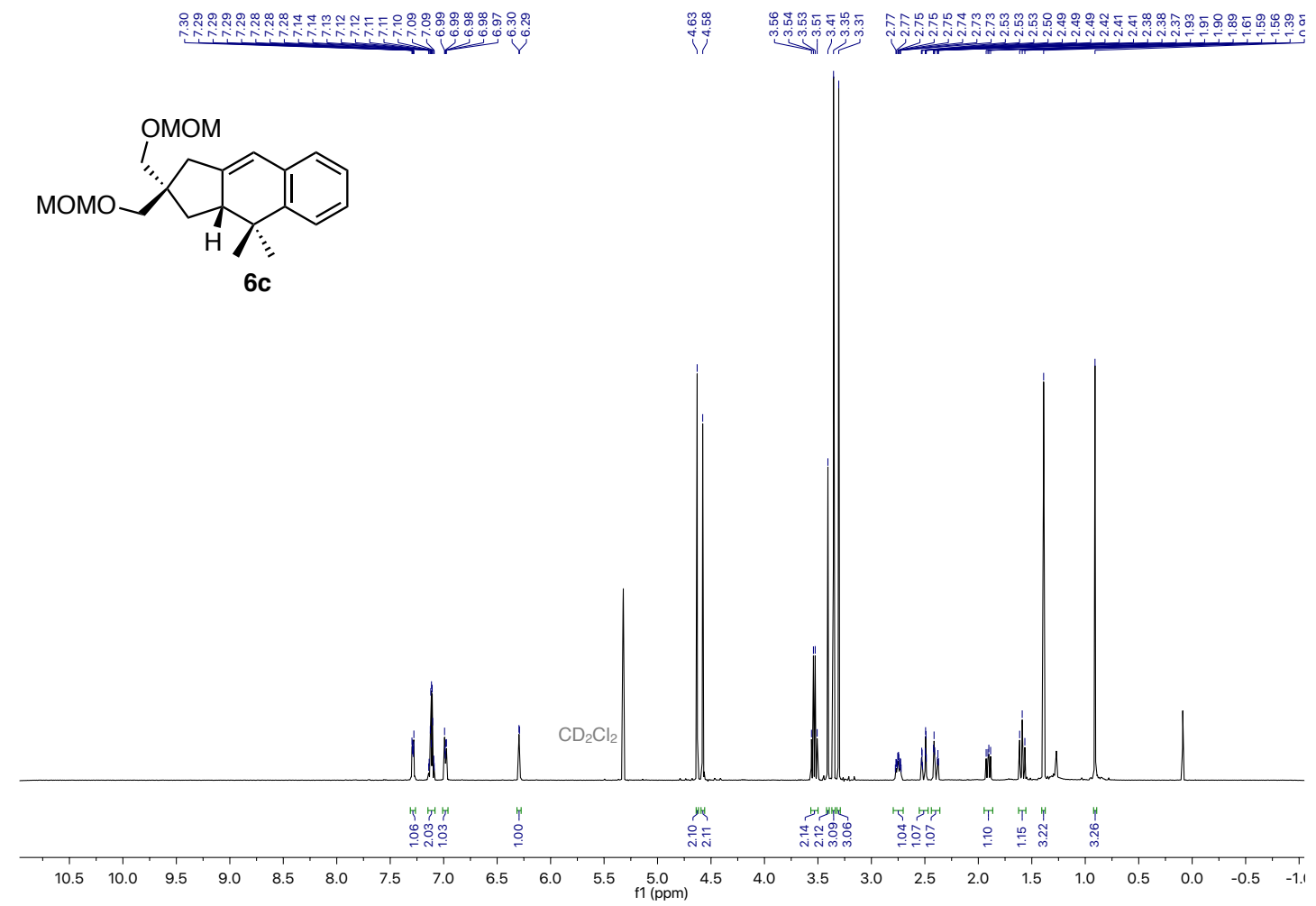

${ }^{13} \mathrm{C}$ NMR: $126 \mathrm{MHz}, \mathrm{CD}_{2} \mathrm{Cl}_{2}$, compound 6c
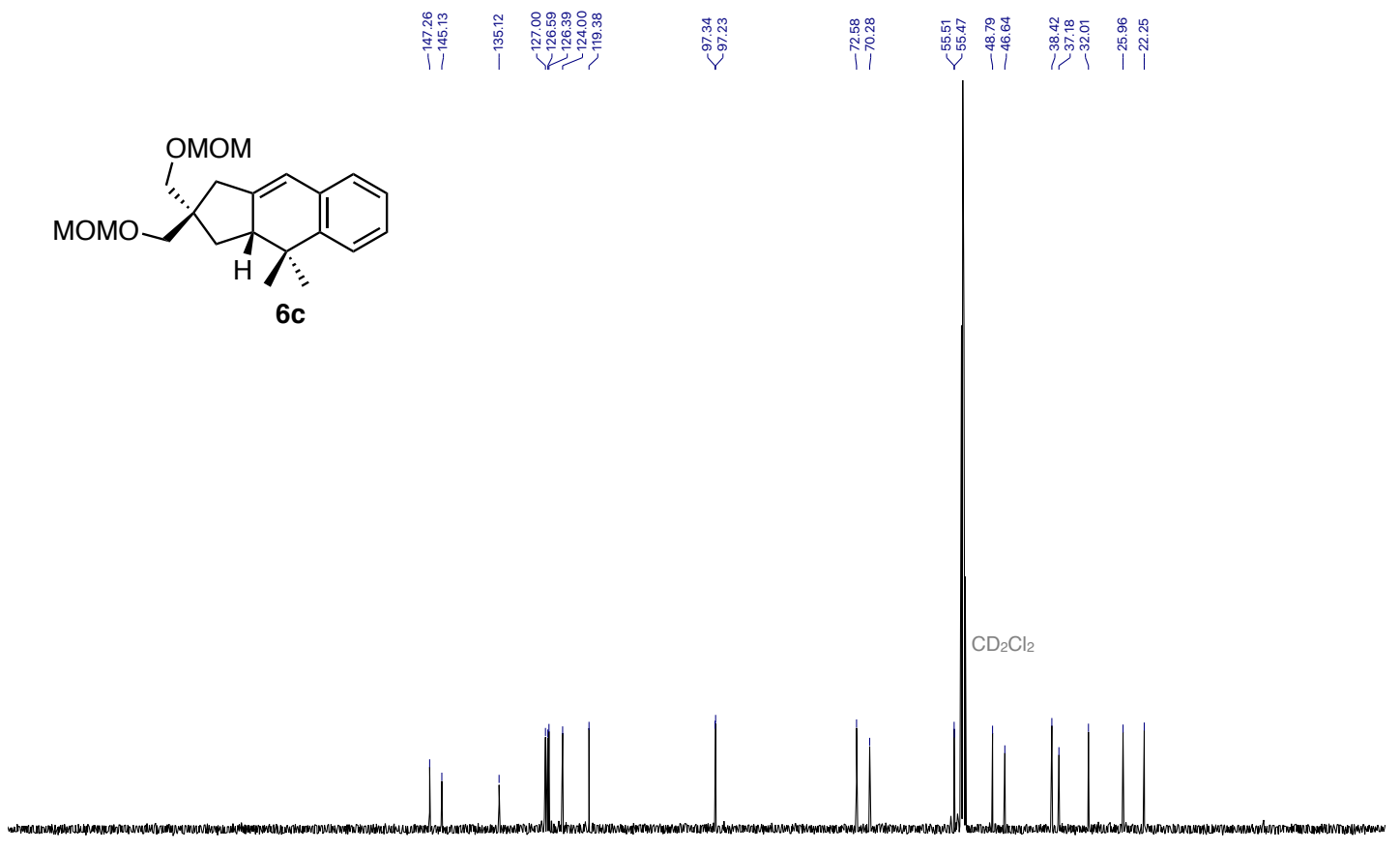

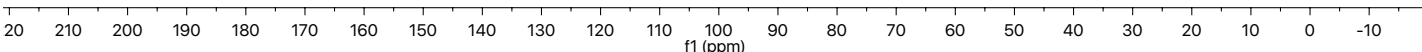


${ }^{1} \mathrm{H}$ NMR: $500 \mathrm{MHz}, \mathrm{CDCl}_{3}$, compound $\mathbf{6 d}$<smiles>CC(=O)OCC1(COC(C)=O)CC2=Cc3ccccc3[C@@H](C)[C@H]2C1</smiles>

$6 d$

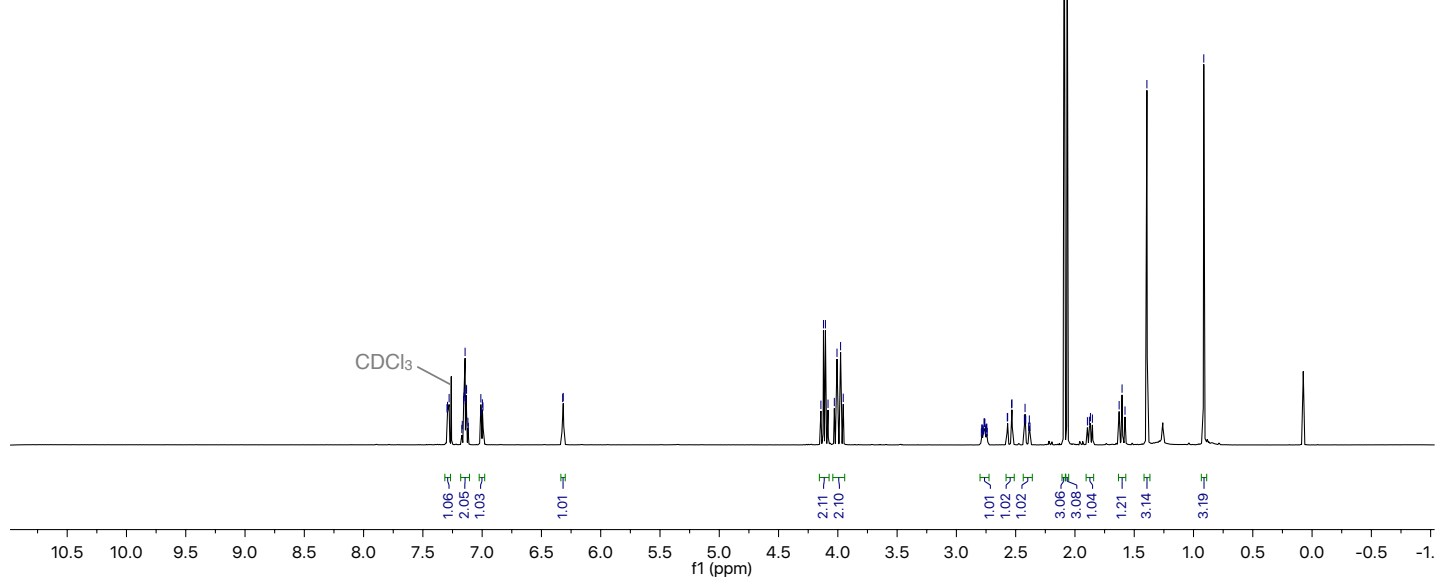

${ }^{13} \mathrm{C}$ NMR: $126 \mathrm{MHz}, \mathrm{CDCl}_{3}$, compound 6d

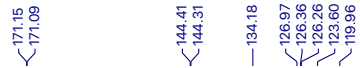

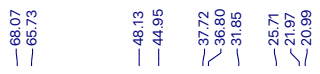<smiles>CC(=O)OCC1(CO)CC2=Cc3ccccc3[C@@H](C)[C@H]2C1</smiles>

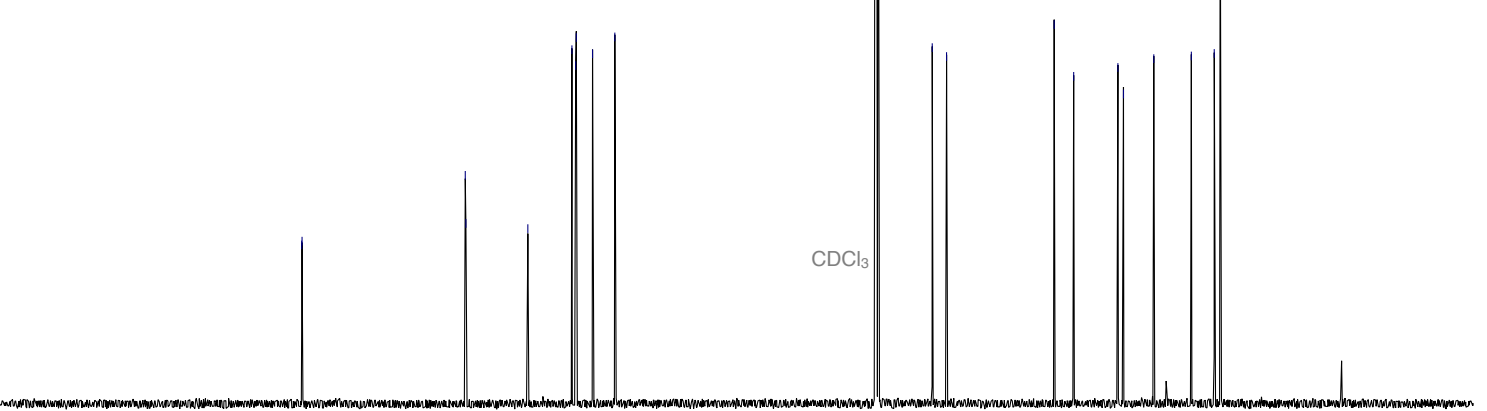

$\begin{array}{llllllllllllllllllllllllllllllllll} & 20 & 210 & 200 & 190 & 180 & 170 & 160 & 150 & 140 & 130 & 120 & 110 & 100 & 90 & 80 & 70 & 60 & 50 & 40 & 30 & 20 & 10 & 0 & -10 & -2\end{array}$ 
${ }^{1} \mathrm{H}$ NMR: $400 \mathrm{MHz}, \mathrm{CDCl}_{3}$, compound $6 \mathbf{e}$

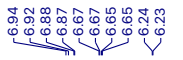<smiles>COCC1(COC)CC2=Cc3ccc(OC)cc3[C@@H](C)[C@H](C)C2C1</smiles>

$6 e$

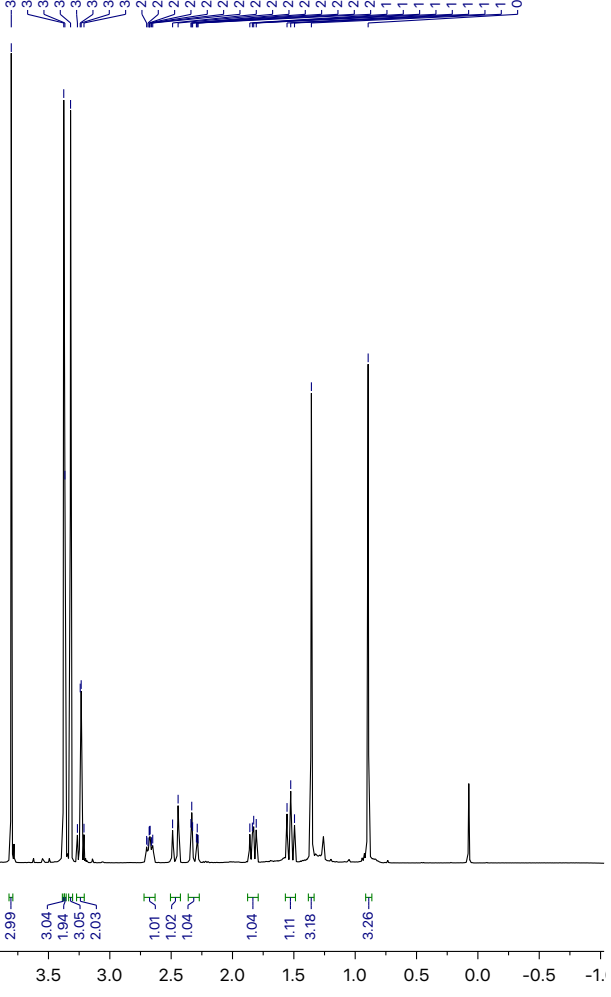

${ }^{13} \mathrm{C}$ NMR: $101 \mathrm{MHz}, \mathrm{CDCl}_{3}$, compound 6e

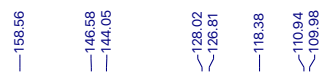
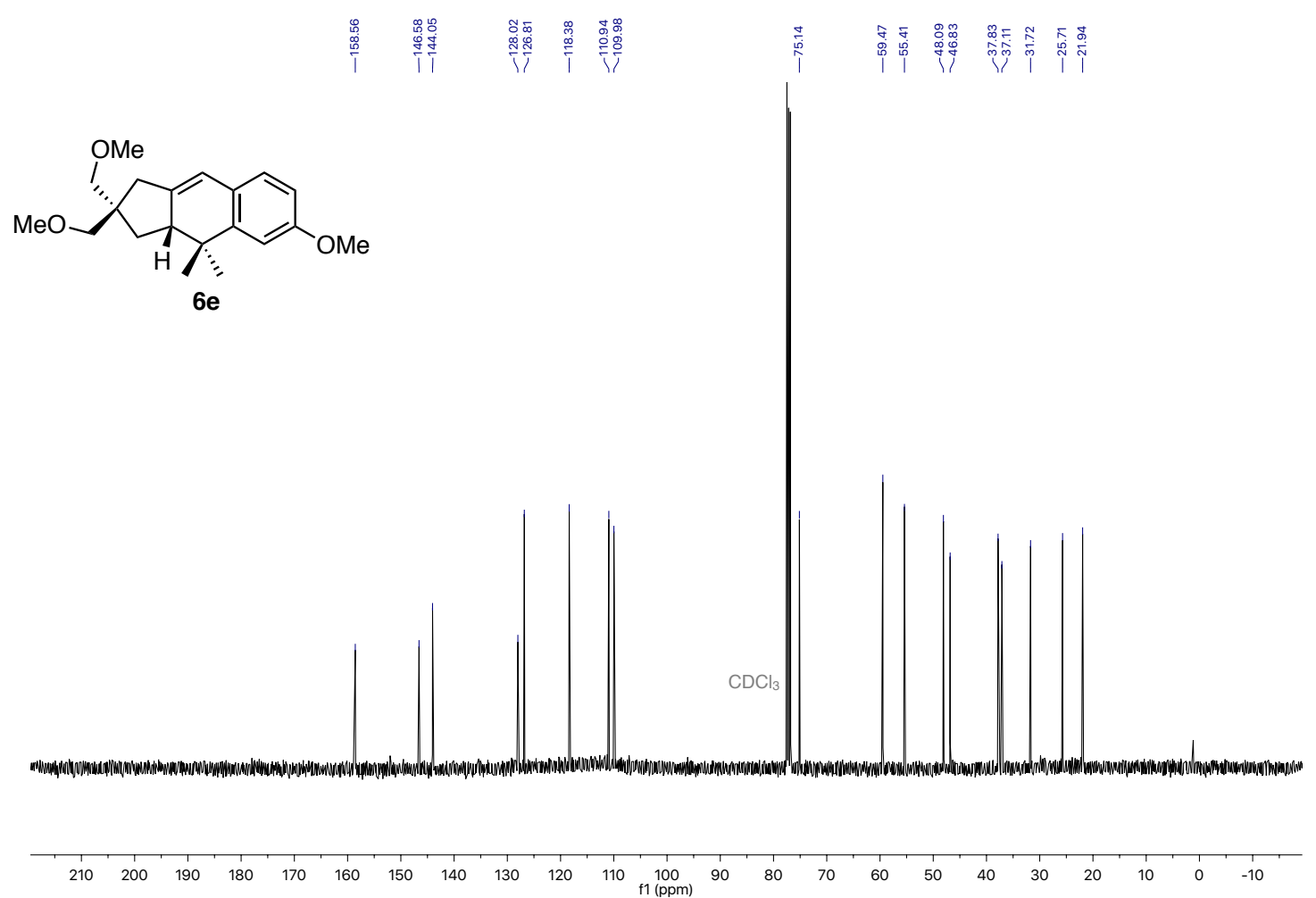

$80 \quad 70 \quad 60$

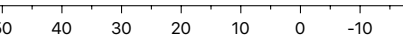


${ }^{1} \mathrm{H}$ NMR: $400 \mathrm{MHz}, \mathrm{CDCl}_{3}$, compound $6 \mathbf{f}$

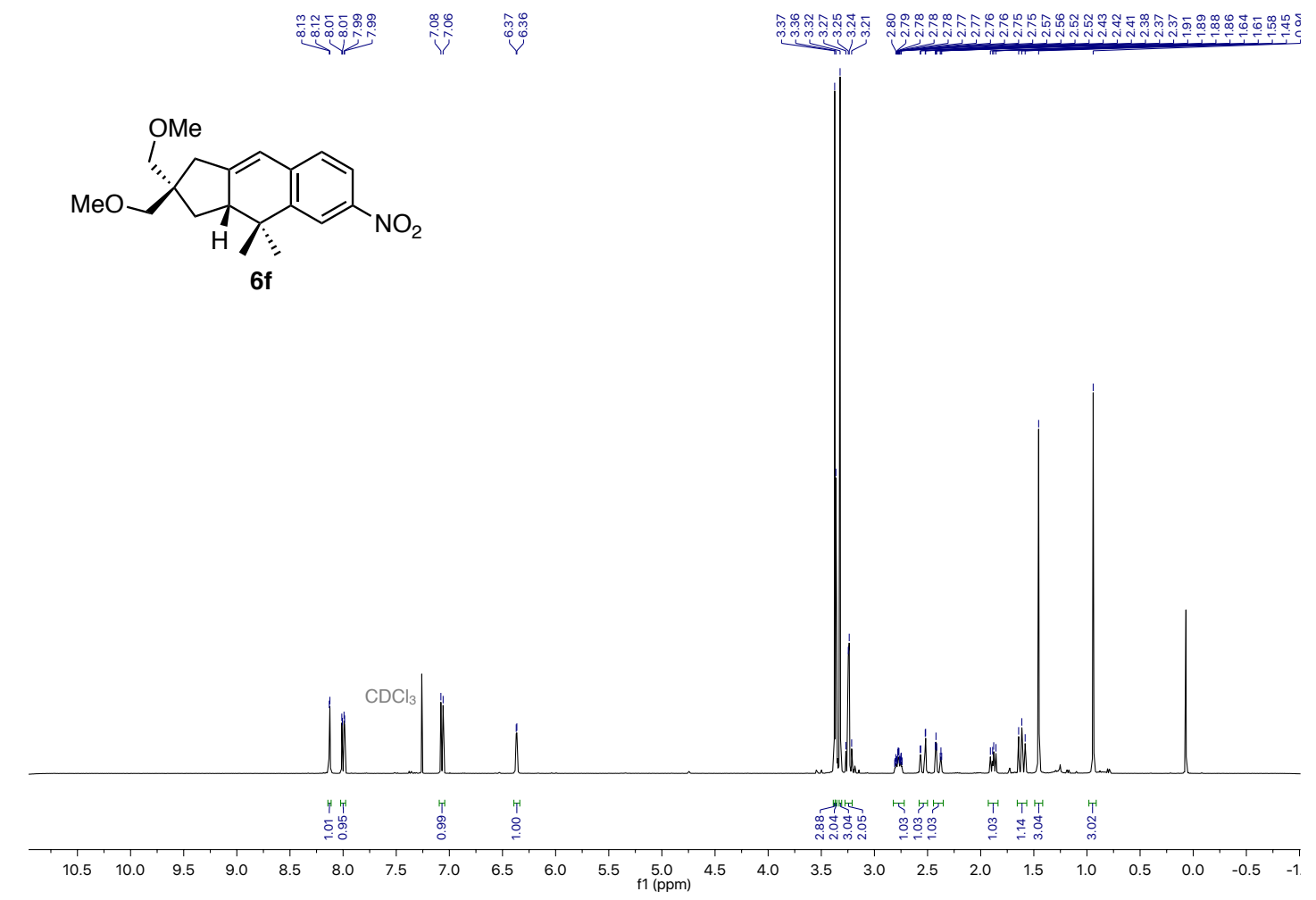

${ }^{13} \mathrm{C}$ NMR: $101 \mathrm{MHz}, \mathrm{CDCl}_{3}$, compound $\mathbf{6 f}$

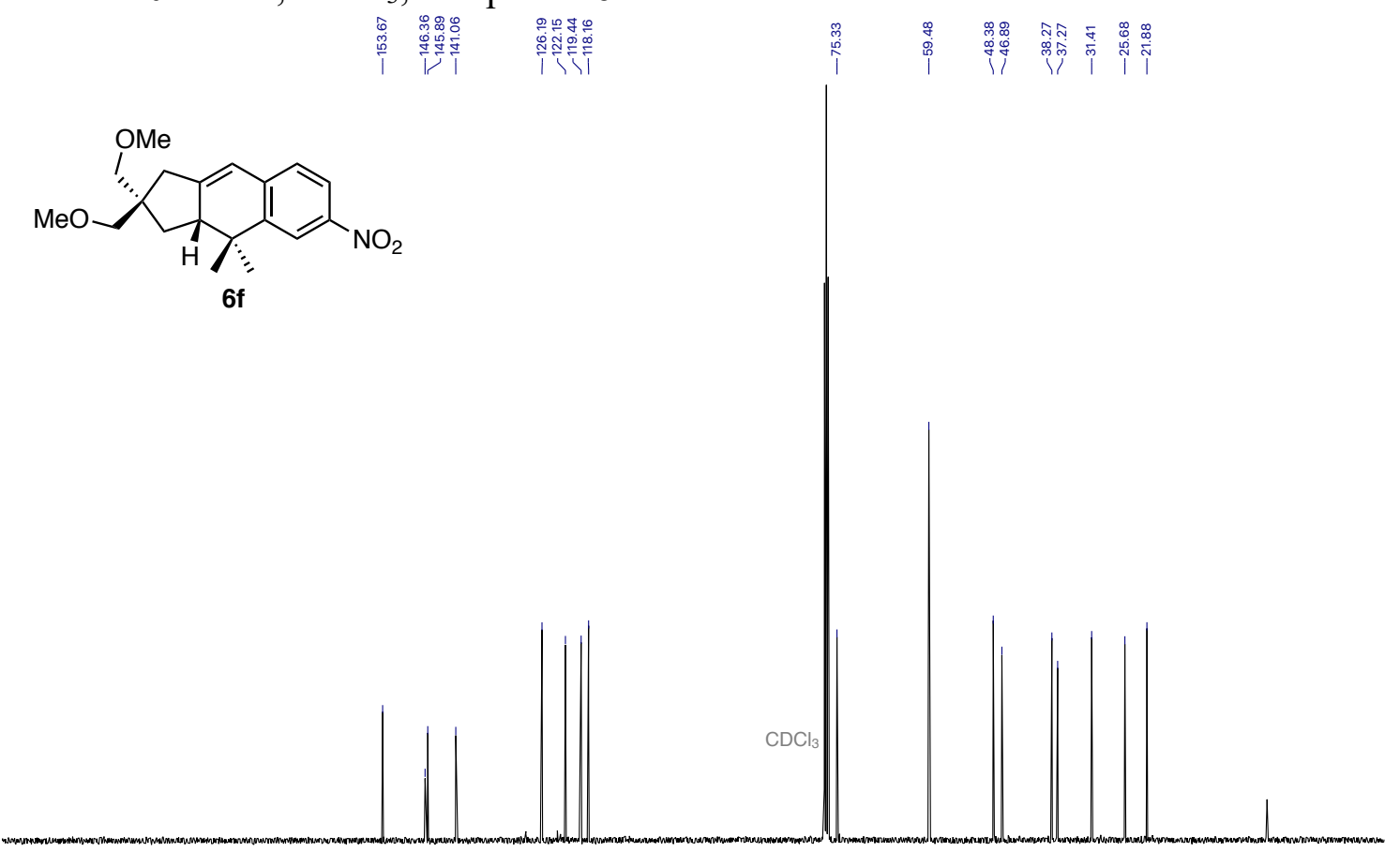

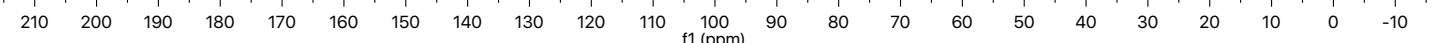


${ }^{1} \mathrm{H}$ NMR: $500 \mathrm{MHz}, \mathrm{CD}_{2} \mathrm{Cl}_{2}$, compound $\mathbf{6 g}$

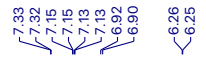
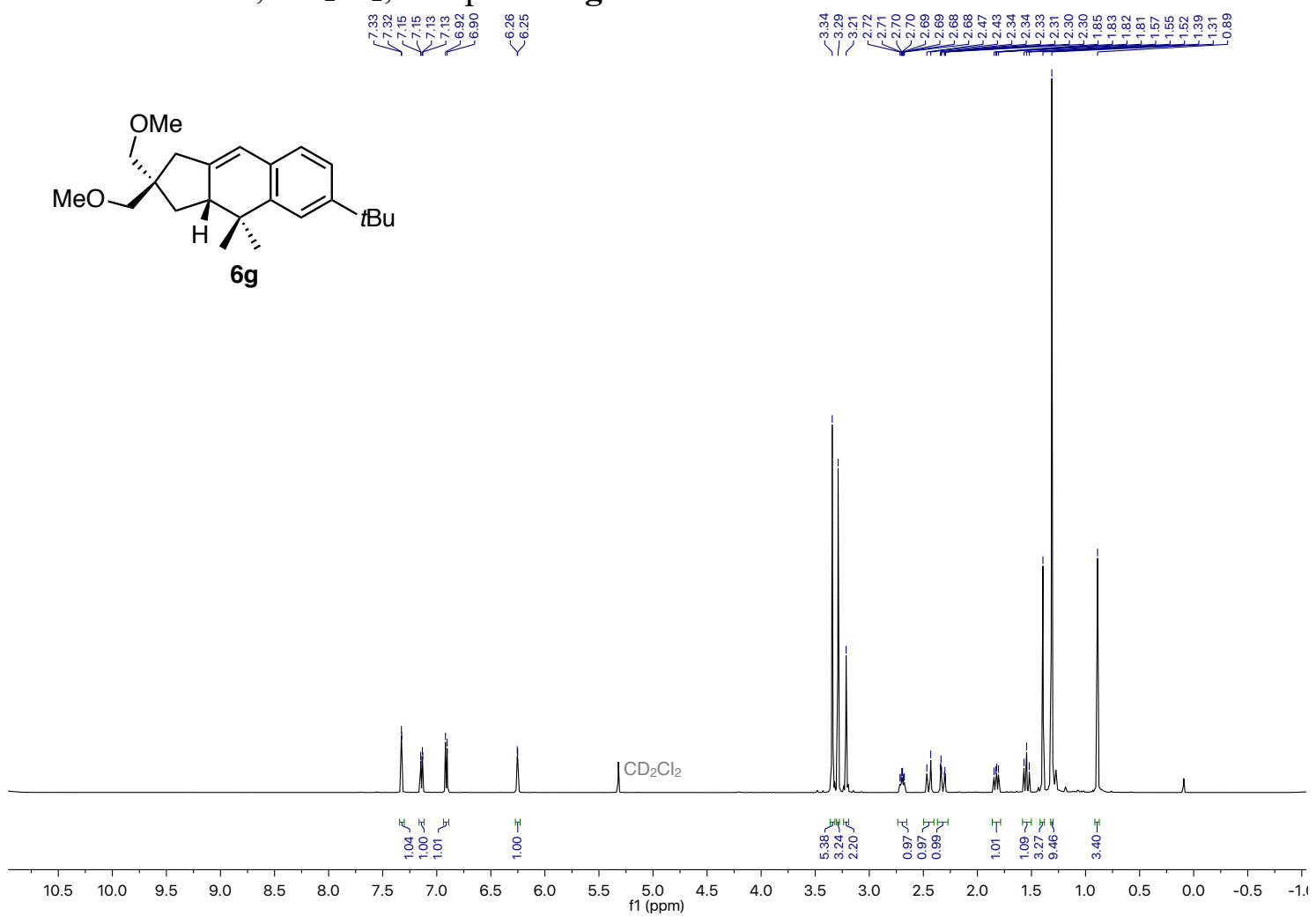

${ }^{13} \mathrm{C}$ NMR: $126 \mathrm{MHz}, \mathrm{CD}_{2} \mathrm{Cl}_{2}$, compound $6 \mathrm{~g}$

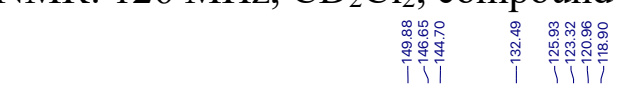

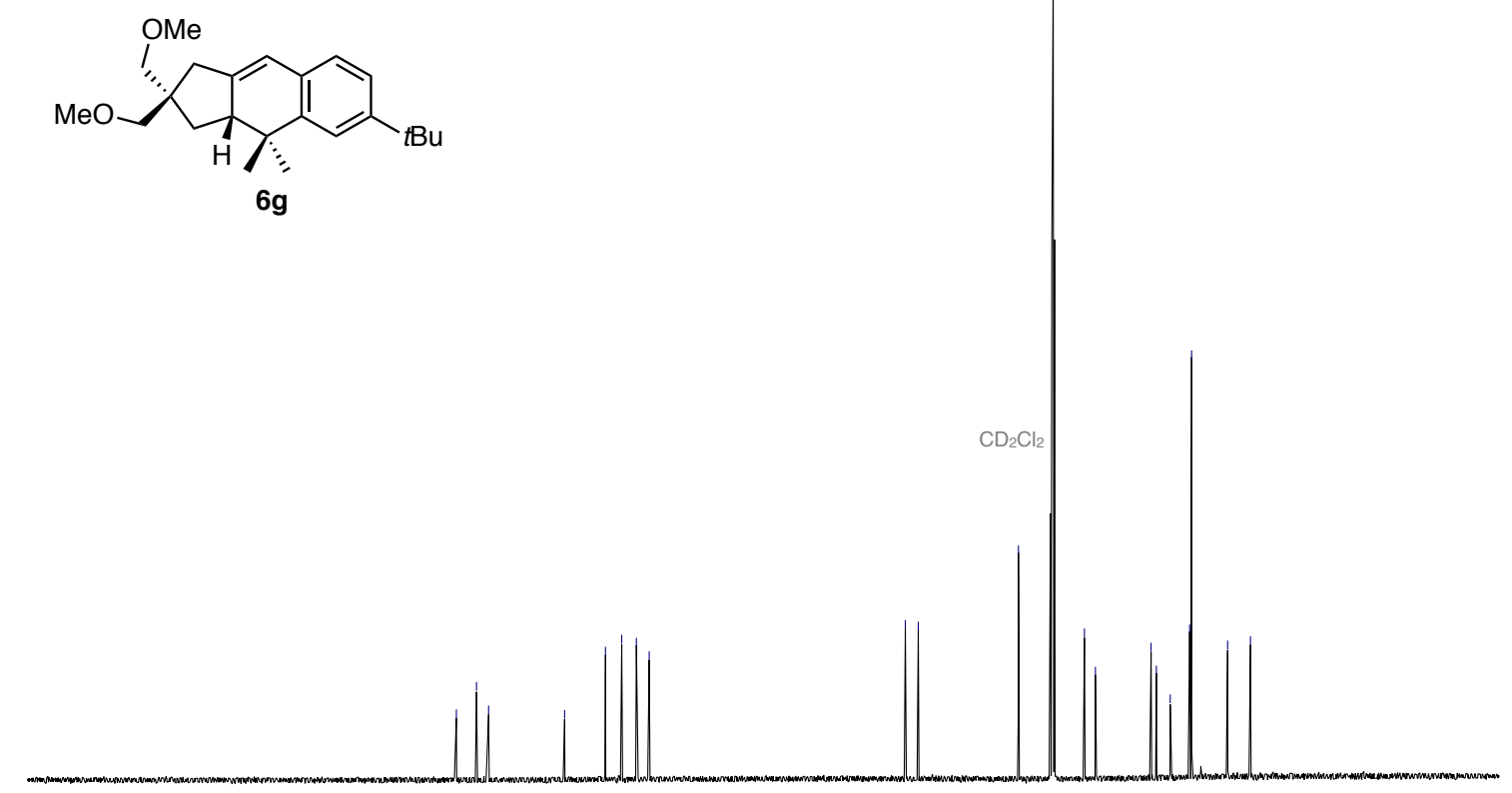

$6 \mathrm{~g}$

$\begin{array}{lllllllllllllllllllllllllll}210 & 200 & 190 & 180 & 170 & 160 & 150 & 140 & 130 & 120 & 110 & \begin{array}{c}100 \\ \mathrm{f} 1(\mathrm{ppm})\end{array} & 90 & 80 & 70 & 60 & 50 & 40 & 30 & 20 & 10 & 0 & -10\end{array}$ 
${ }^{1} \mathrm{H}$ NMR: $500 \mathrm{MHz}, \mathrm{CDCl}_{3}$, compound $\mathbf{6 h}$

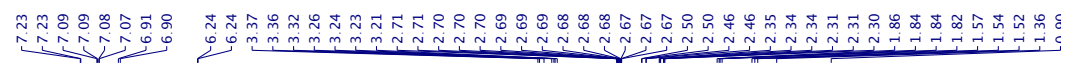<smiles>COC[C@@H]1c2cc(Cl)ccc2C=C2C[C@H](COC)C(C)(C)C[C@@H]21</smiles>

$6 \mathrm{~h}$

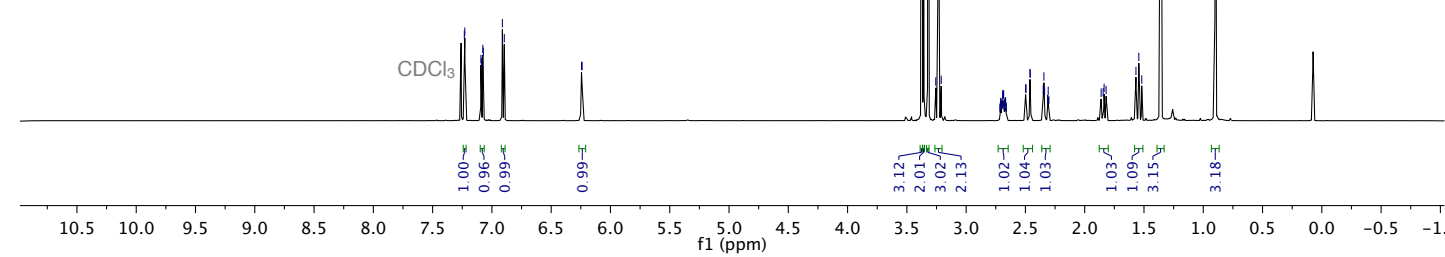

${ }^{13} \mathrm{C}$ NMR: $126 \mathrm{MHz}, \mathrm{CDCl}_{3}$, compound $\mathbf{6 h}$
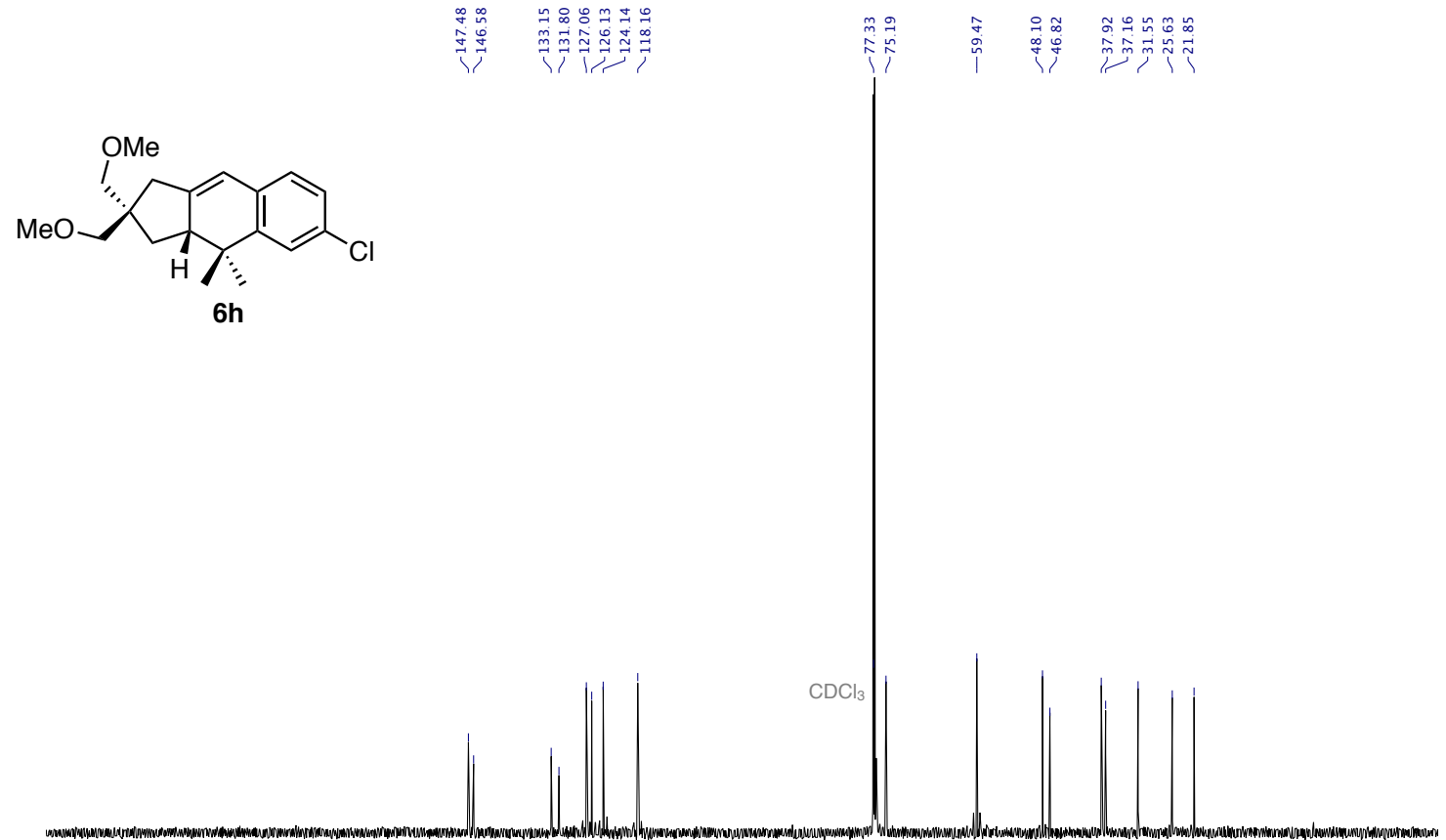

\begin{tabular}{lllllllllllllllllllllllll}
\hline & 210 & 200 & 190 & 180 & 170 & 160 & 150 & 140 & 130 & 120 & 110 & $\underset{\mathrm{f} 1(\mathrm{ppm})}{100}$ & 90 & 80 & 70 & 60 & 50 & 40 & 30 & 20 & 10 & 0 & -10 & -2
\end{tabular} 
${ }^{1} \mathrm{H}$ NMR: $400 \mathrm{MHz}, \mathrm{CDCl}_{3}$, compound 6i

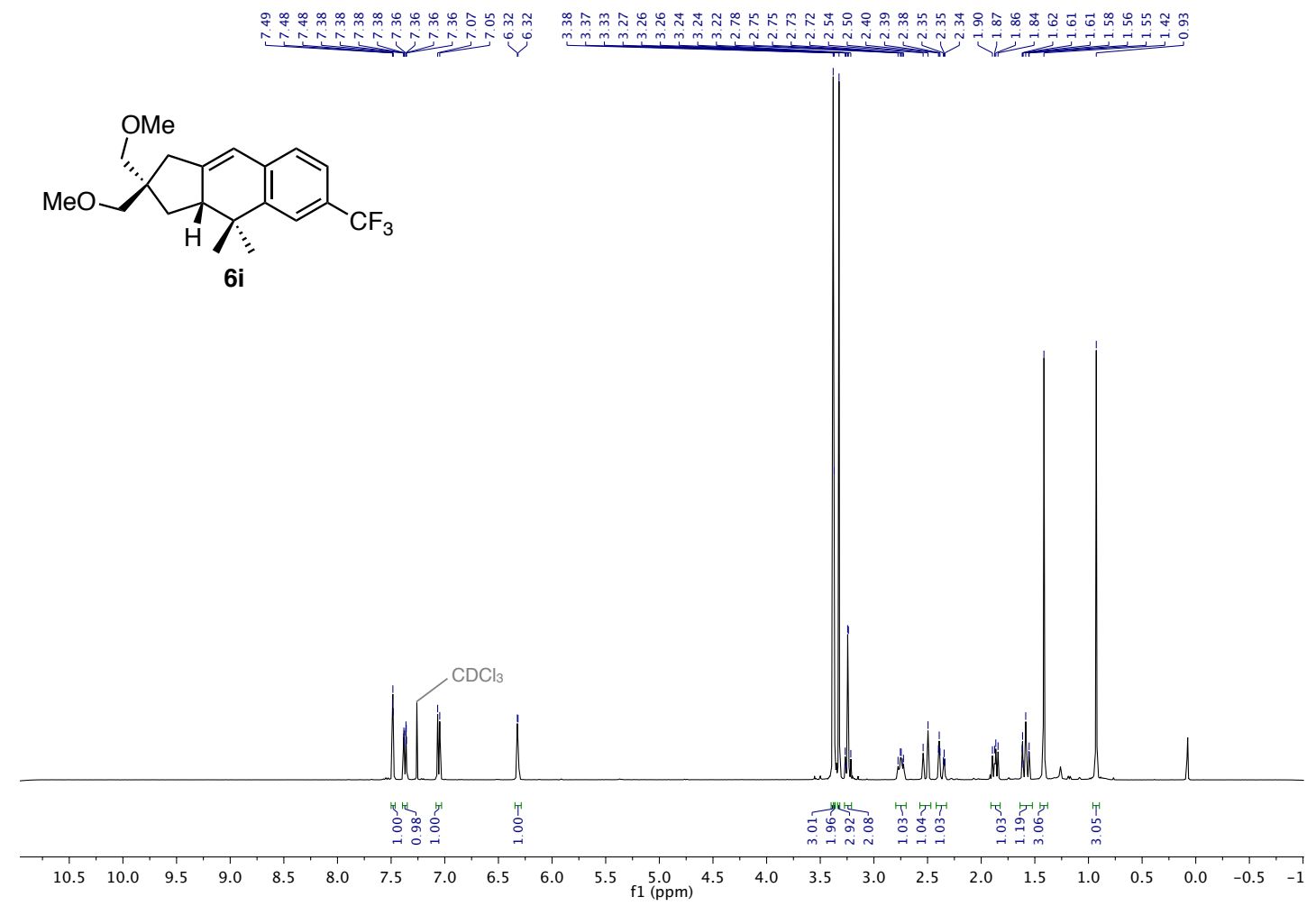

${ }^{13} \mathrm{C}$ NMR: $101 \mathrm{MHz}, \mathrm{CDCl}_{3}$, compound 6i

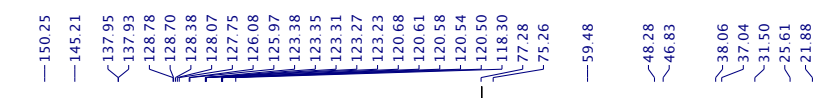<smiles>COCC1(COC)CC2=Cc3ccc(C(F)(F)F)cc3[C@@H](C)[C@@H](C1)[C@@H]2C</smiles>

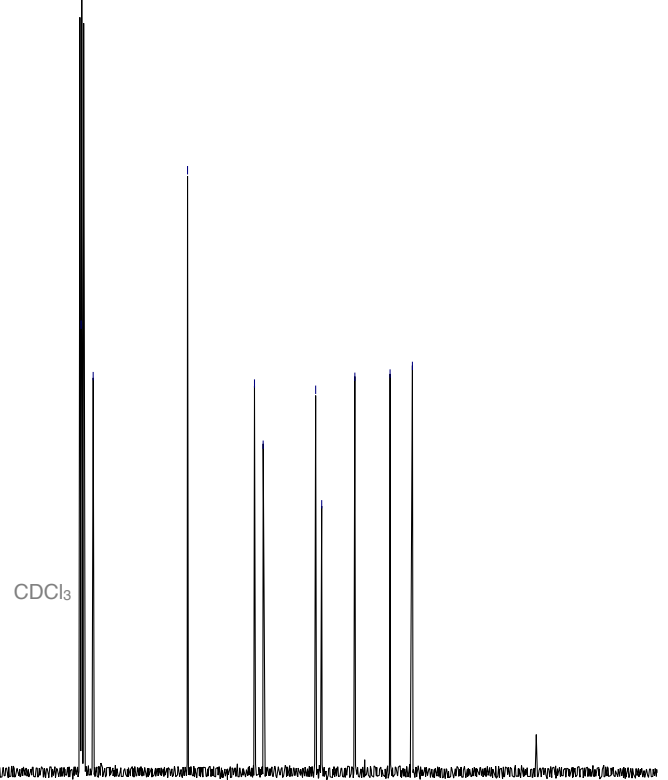

$\begin{array}{lllllllllllllllllllllllll}210 & 200 & 190 & 180 & 170 & 160 & 150 & 140 & 130 & 120 & 110 & 100 & 90 & 80 & 70 & 60 & 50 & 40 & 30 & 20 & 10 & 0 & -10\end{array}$ 
${ }^{19} \mathrm{~F}\left\{{ }^{1} \mathrm{H}\right\}$ NMR: $376 \mathrm{MHz}, \mathrm{CDCl}_{3}$, compound $6 \mathbf{i}$

ก
บิ
$\mid$<smiles>COCC1(COC)CC2=Cc3ccc(C(F)(F)F)cc3[C@@H](C)[C@@H](C1)[C@@H]2C</smiles>

$6 i$

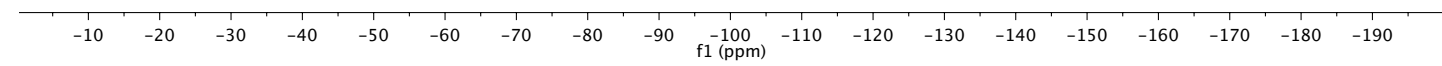

${ }^{1} \mathrm{H}$ NMR: $400 \mathrm{MHz}, \mathrm{CDCl}_{3}$, compound $\mathbf{6 j}$

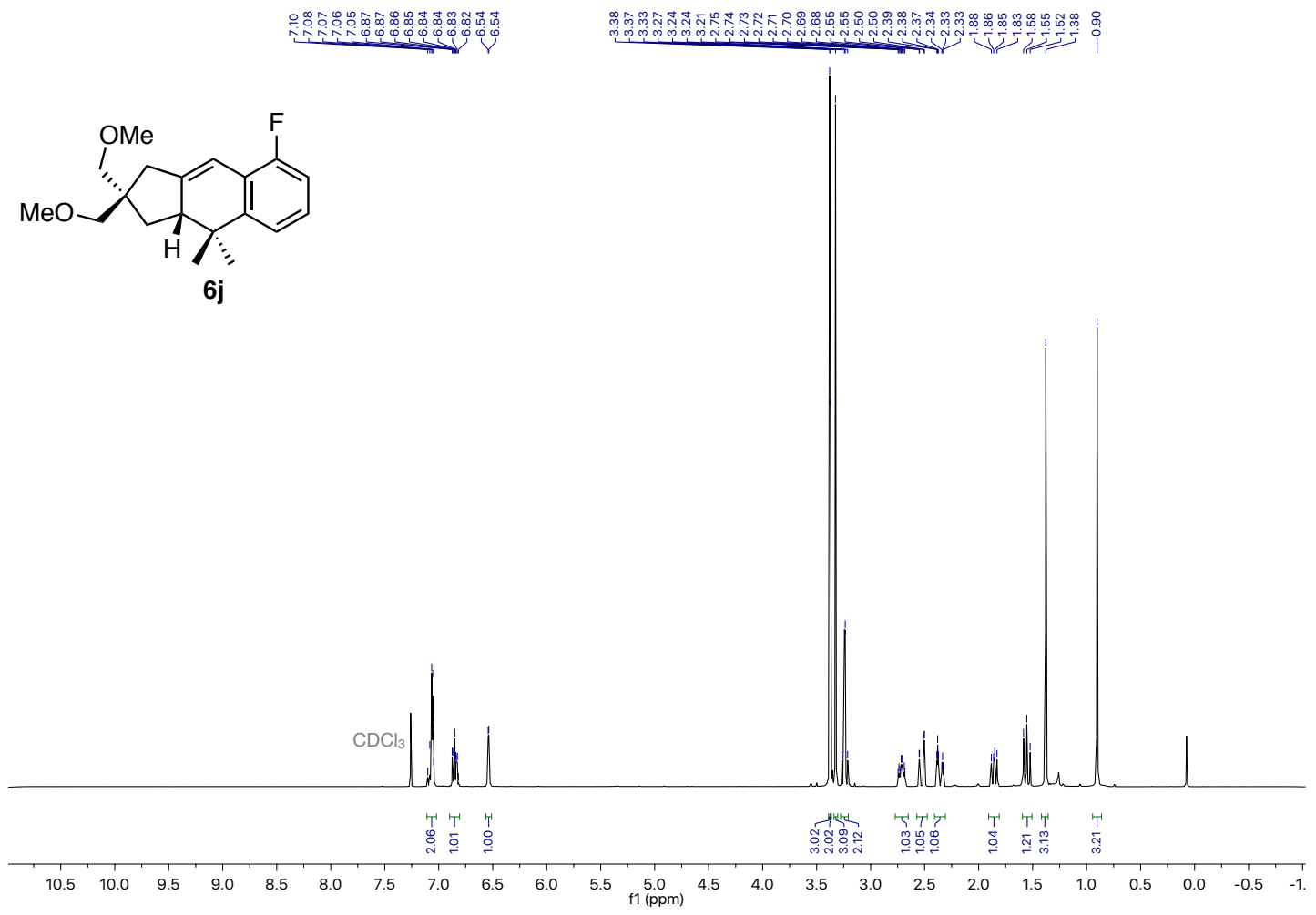


${ }^{13} \mathrm{C}$ NMR: $101 \mathrm{MHz}, \mathrm{CDCl}_{3}$, compound $\mathbf{6 j}$

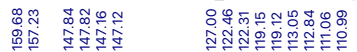

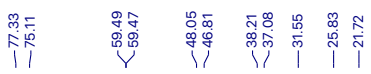<smiles>COCC1(COC)CC2=Cc3c(F)cccc3C(C)(C)[C@H]2C1</smiles>

$6 \mathbf{j}$

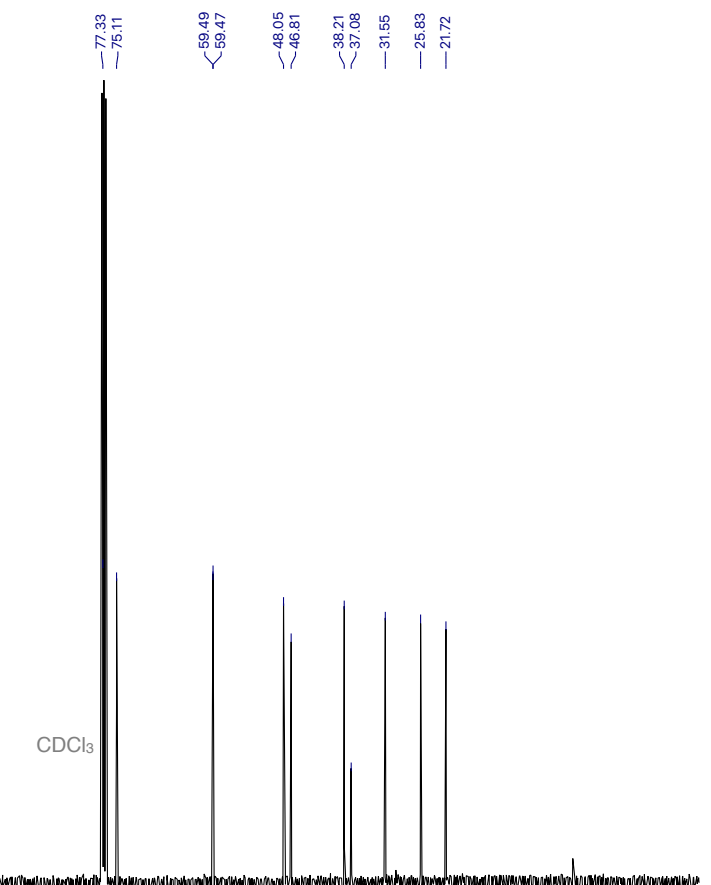

$\begin{array}{llllllllllll}210 & 200 & 190 & 180 & 170 & 160 & 150 & 140 & 130 & 120 & 110 & \begin{array}{c}100 \\ \mathrm{f} 1(\mathrm{ppm})\end{array}\end{array}$

${ }^{19} \mathrm{~F}\left\{{ }^{1} \mathrm{H}\right\}$ NMR: $376 \mathrm{MHz}, \mathrm{CDCl}_{3}$, compound $\mathbf{6 j}$<smiles>COCC1(COC)CC2=Cc3c(F)cccc3C(C)(C)C2C1</smiles>

6j

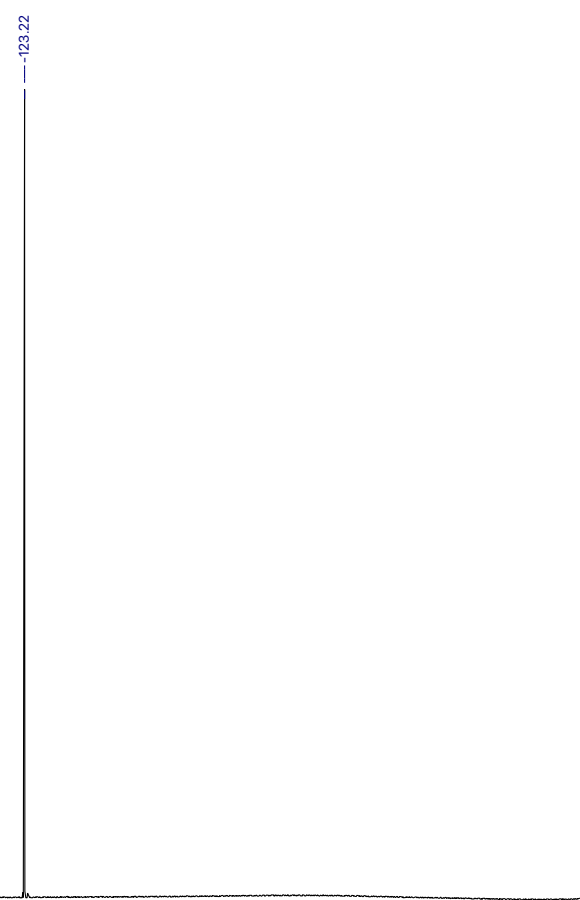

$\begin{array}{lllllllllllllllllllllll}-10 & -20 & -30 & -40 & -50 & -60 & -70 & -80 & -90 & -100 & -110 & -120 & -130 & -140 & -150 & -160 & -170 & -180 & -190\end{array}$ 
${ }^{1} \mathrm{H}$ NMR: $500 \mathrm{MHz}, \mathrm{CD}_{2} \mathrm{Cl}_{2}$, compound 6k

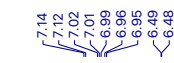

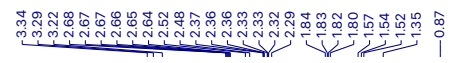
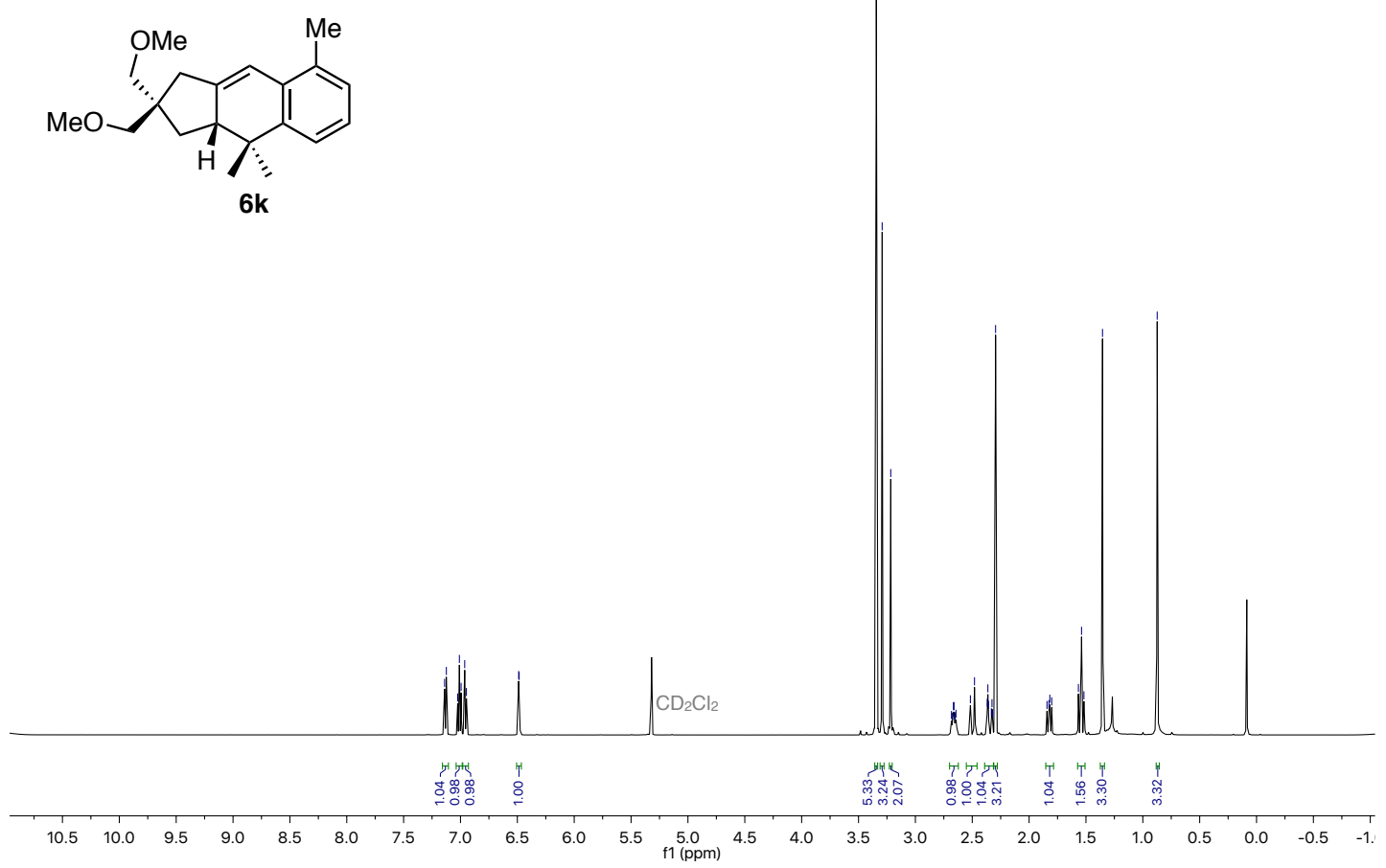

${ }^{13} \mathrm{C}$ NMR: $126 \mathrm{MHz}, \mathrm{CD}_{2} \mathrm{Cl}_{2}$, compound $\mathbf{6 k}$
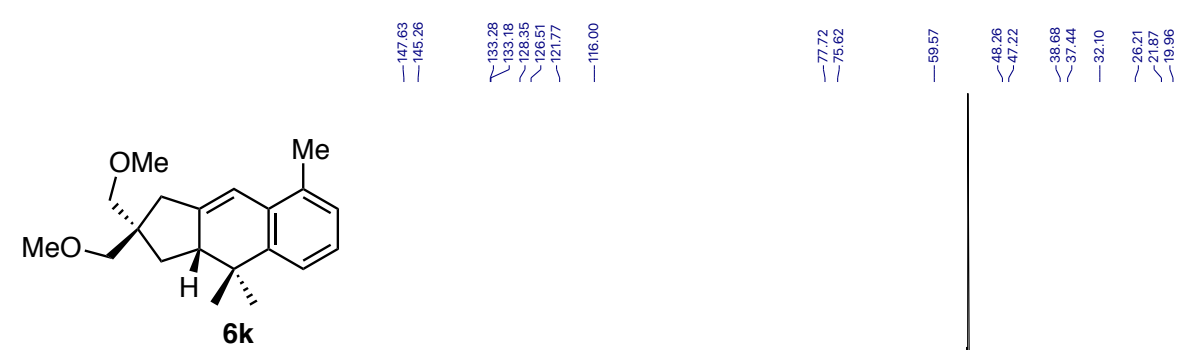

6k

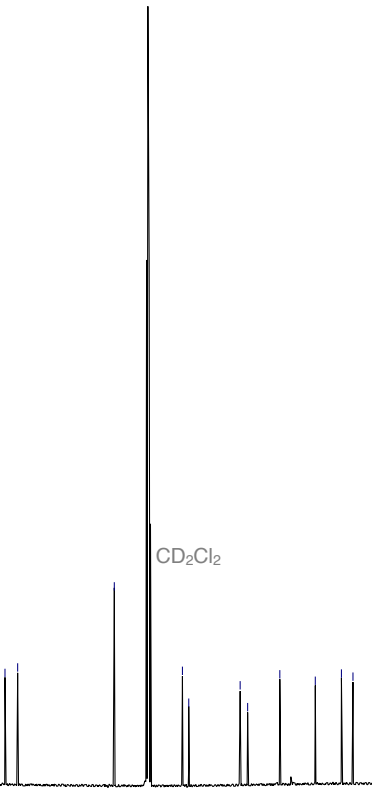

$\begin{array}{lllllllllllllllllllllllllllll}210 & 200 & 190 & 180 & 170 & 160 & 150 & 140 & 130 & 120 & 110 & 100 & 90 & 80 & 70 & 60 & 50 & 40 & 30 & 20 & 10 & 0 & -10\end{array}$ 
${ }^{1} \mathrm{H}$ NMR: $400 \mathrm{MHz}, \mathrm{CDCl}_{3}$, compound $6 \mathbf{1}$

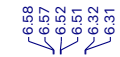

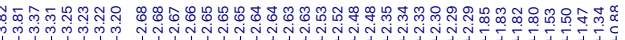

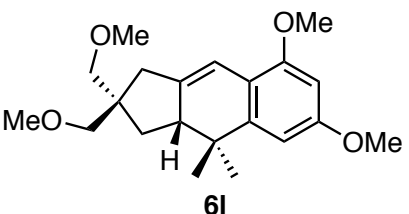

61
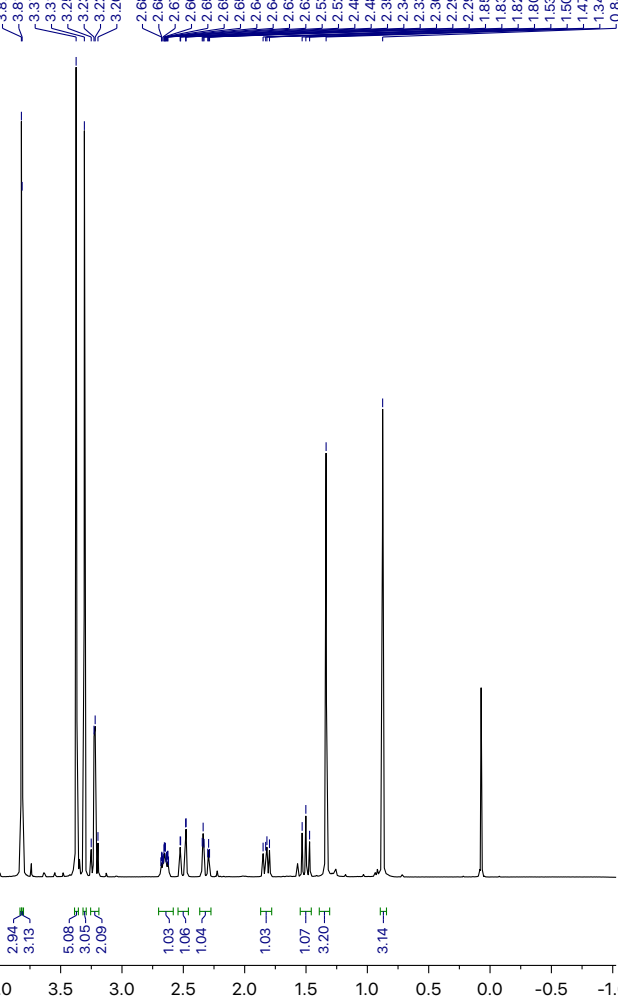

${ }^{13} \mathrm{C}$ NMR: $101 \mathrm{MHz}, \mathrm{CDCl}_{3}$, compound $6 \mathbf{I}$<smiles>COCC1(COC)CC2=Cc3c(OC)cc(OC)cc3C(C)(C)[C@H]2C1</smiles>

61 
${ }^{1} \mathrm{H}$ NMR: $400 \mathrm{MHz}, \mathrm{CDCl}_{3}$, compound 6m

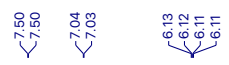

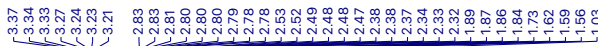

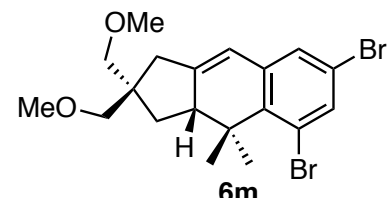

$6 \mathrm{~m}$

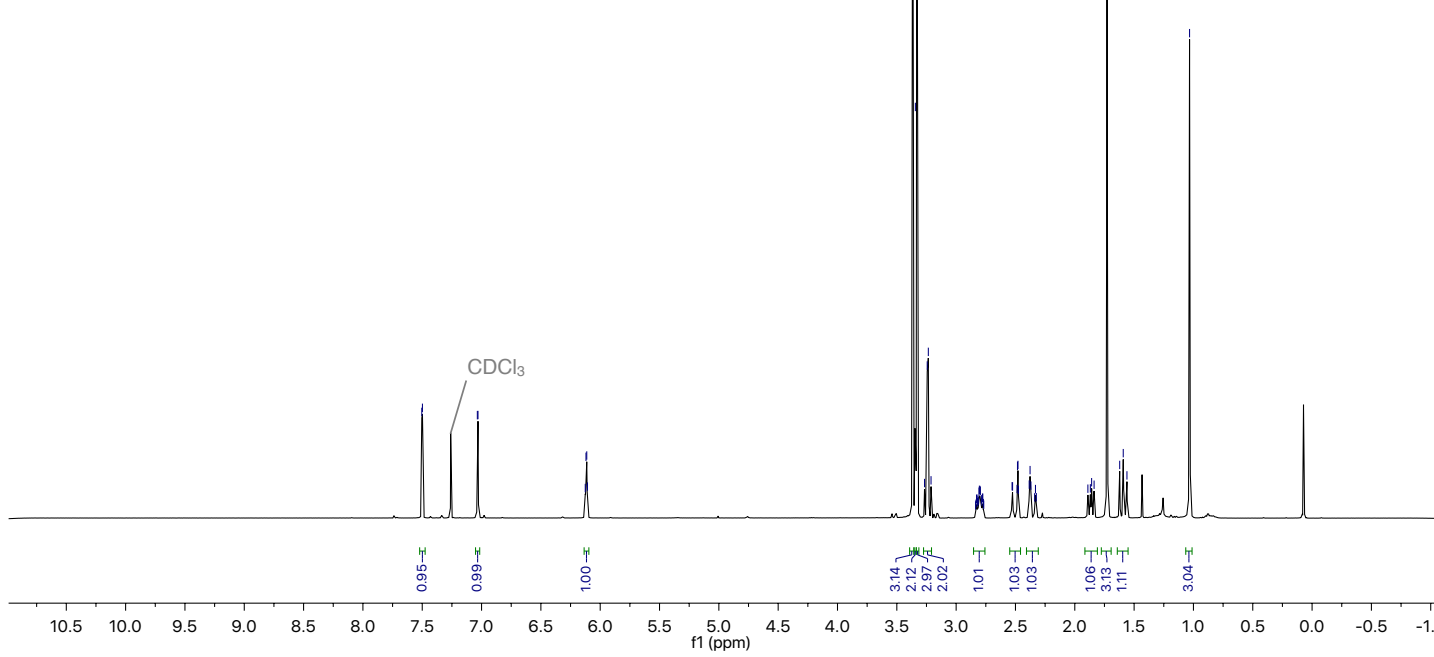

${ }^{13} \mathrm{C}$ NMR: $101 \mathrm{MHz}, \mathrm{CDCl}_{3}$, compound 6m

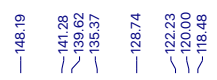<smiles>[2H][C@H]1[C@H]([18OH])c2c(Br)cc(Br)cc2C=C2CC(COC)(COC)C[C@@H]21</smiles>

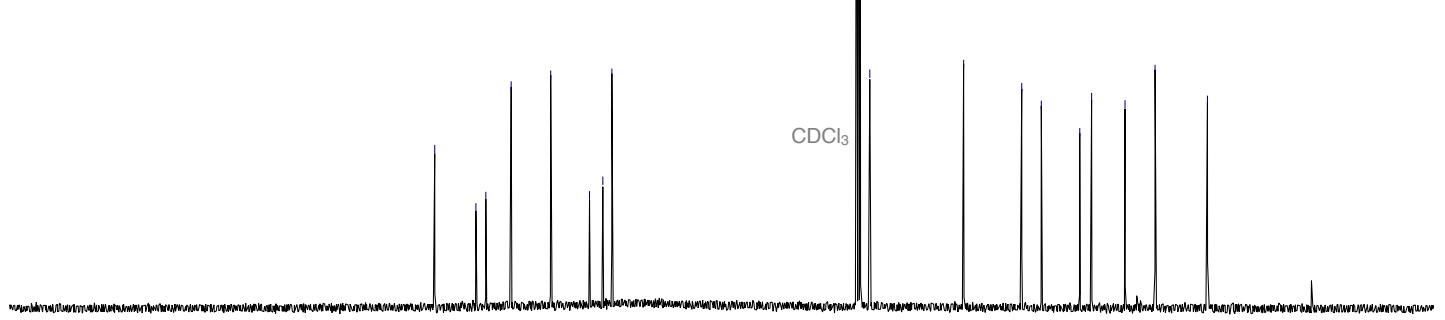

$\begin{array}{llllllllllllllllllllllllll}10 & 200 & 190 & 180 & 170 & 160 & 150 & 140 & 130 & 120 & 110 & 100 & 10 & 100 & 10 & 70 & 60 & 50 & 40 & 30 & 20 & 10 & 0 & -10\end{array}$ 
${ }^{1} \mathrm{H}$ NMR: $500 \mathrm{MHz}, \mathrm{CDCl}_{3}$, compound 6n

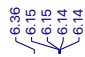

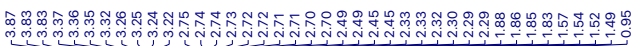<smiles>COCC1(COC)CC2=Cc3cc(OC)c(OC)c(OC)c3[C@@H](C(C)C)[C@H](C2)C1</smiles>
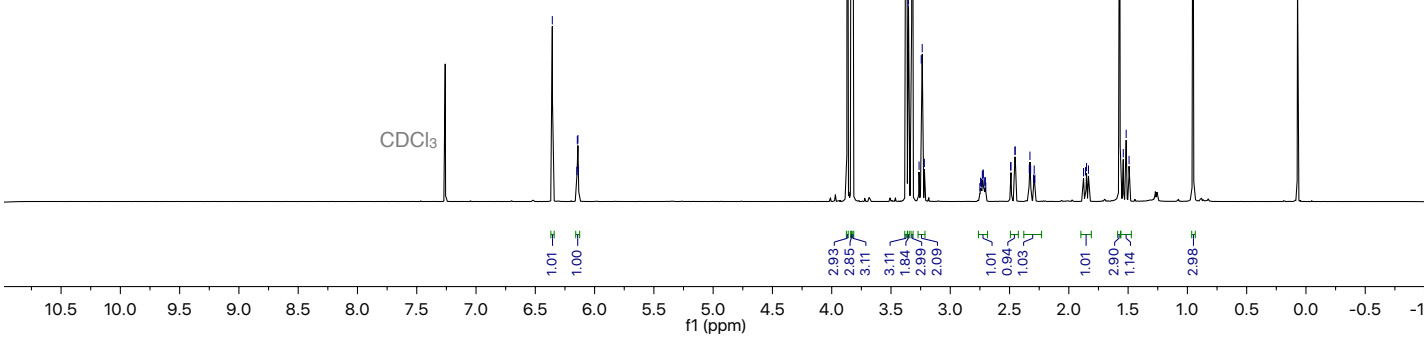

${ }^{13} \mathrm{C}$ NMR: $126 \mathrm{MHz}, \mathrm{CDCl}_{3}$, compound 6n

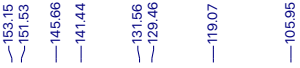<smiles>COCC1(COC)CC2=Cc3cc(OC)c(OC)c(OC)c3[C@@H]([18OH])[C@H](C2)[C@H]1c1ccccc1</smiles>

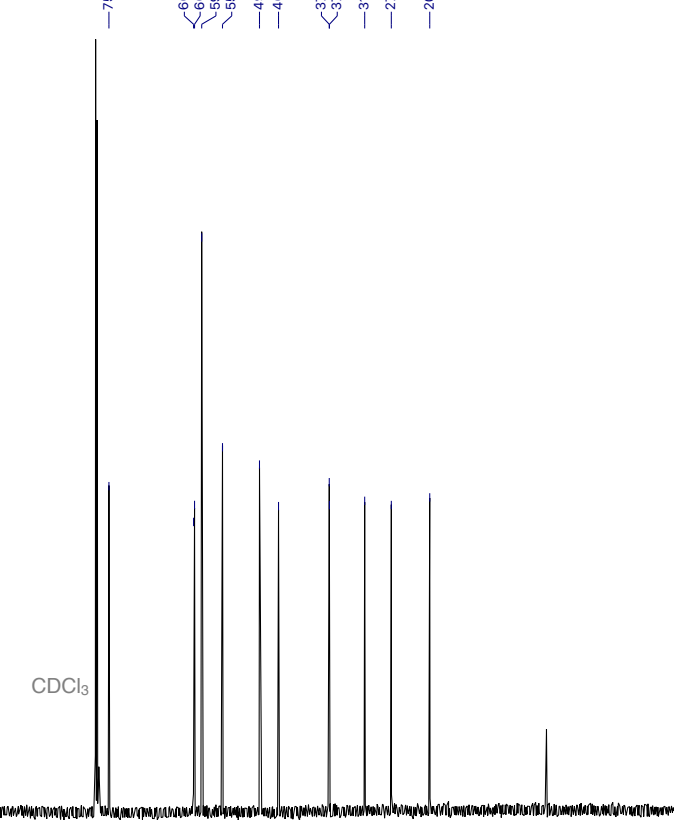

$\begin{array}{lllllllllllllllllllllllllllllllllllll}1 & 20 & 210 & 200 & 190 & 180 & 170 & 160 & 150 & 140 & 130 & 120 & 110 & 100 & 90 & 80 & 70 & 60 & 50 & 40 & 30 & 20 & 10 & 0 & -10 & -2\end{array}$ 
${ }^{1} \mathrm{H}$ NMR: $300 \mathrm{MHz}, \mathrm{C}_{6} \mathrm{D}_{6}$, compound $6 \mathbf{0}$

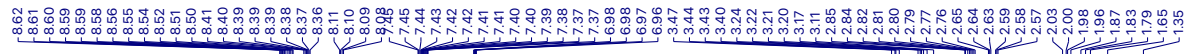<smiles>COCC1(COC)CC2=Cc3c(c4ccccc4c4ccccc34)[C@@](C)(COC)[C@H]2C1</smiles>

60

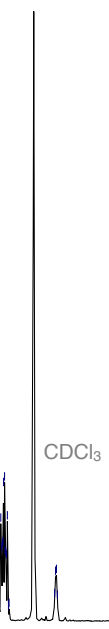

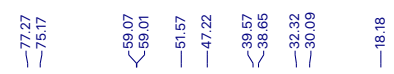<smiles>[2H][C@@]1([18OH])c2c(c3ccccc3c3ccccc23)C=C2CC(COC)(COC)C[C@@H]21</smiles>

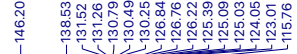
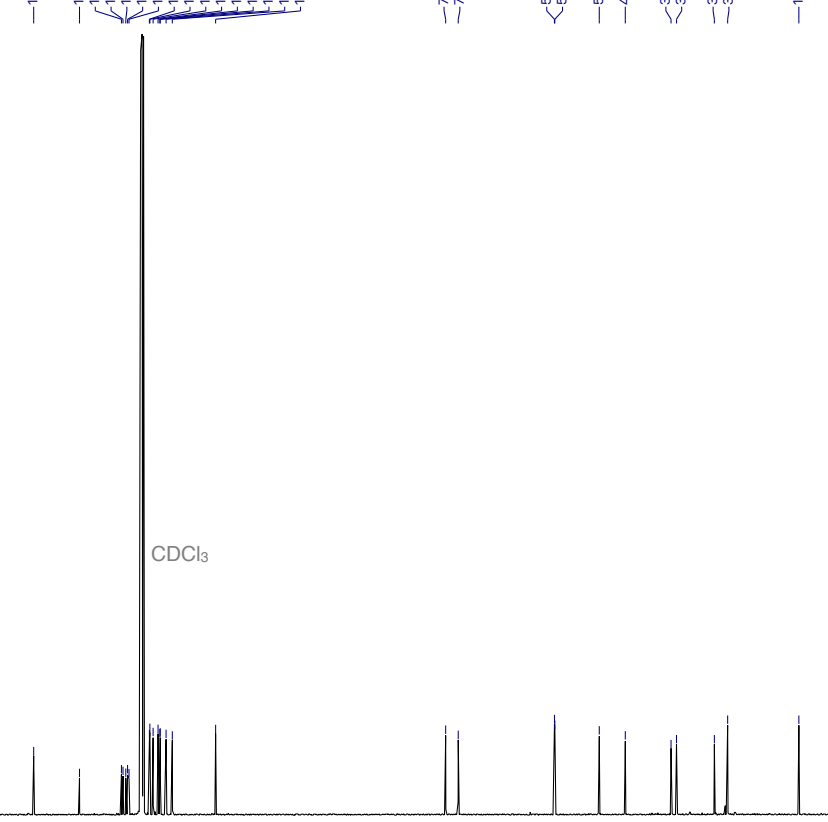

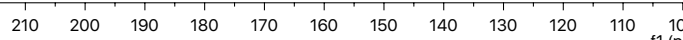


${ }^{1} \mathrm{H}$ NMR: $500 \mathrm{MHz}, \mathrm{CD}_{2} \mathrm{Cl}_{2}$, compound $\mathbf{6 p}$

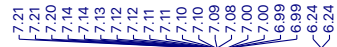
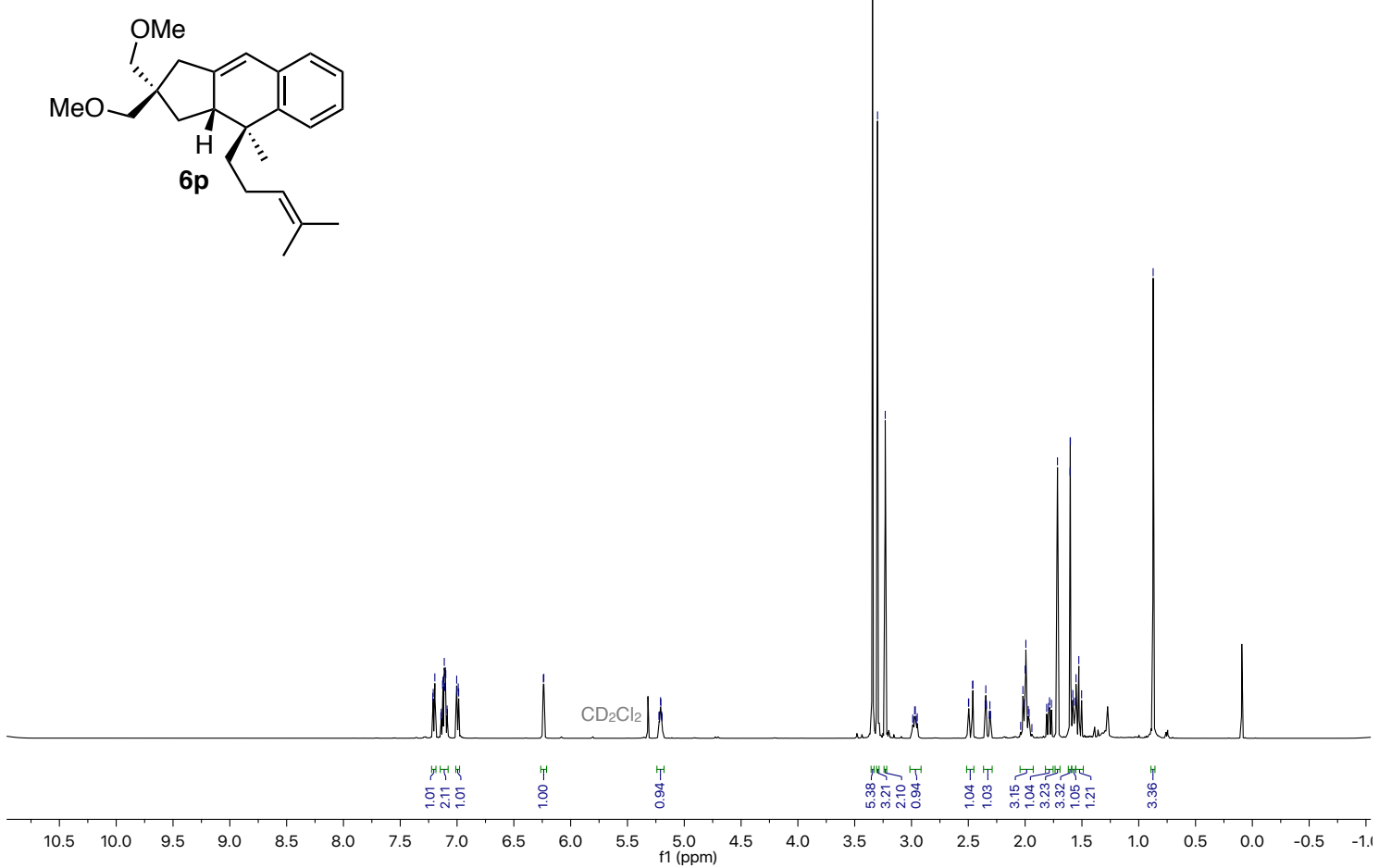

${ }^{13} \mathrm{C}$ NMR: $126 \mathrm{MHz}, \mathrm{CD}_{2} \mathrm{Cl}_{2}$, compound 6p
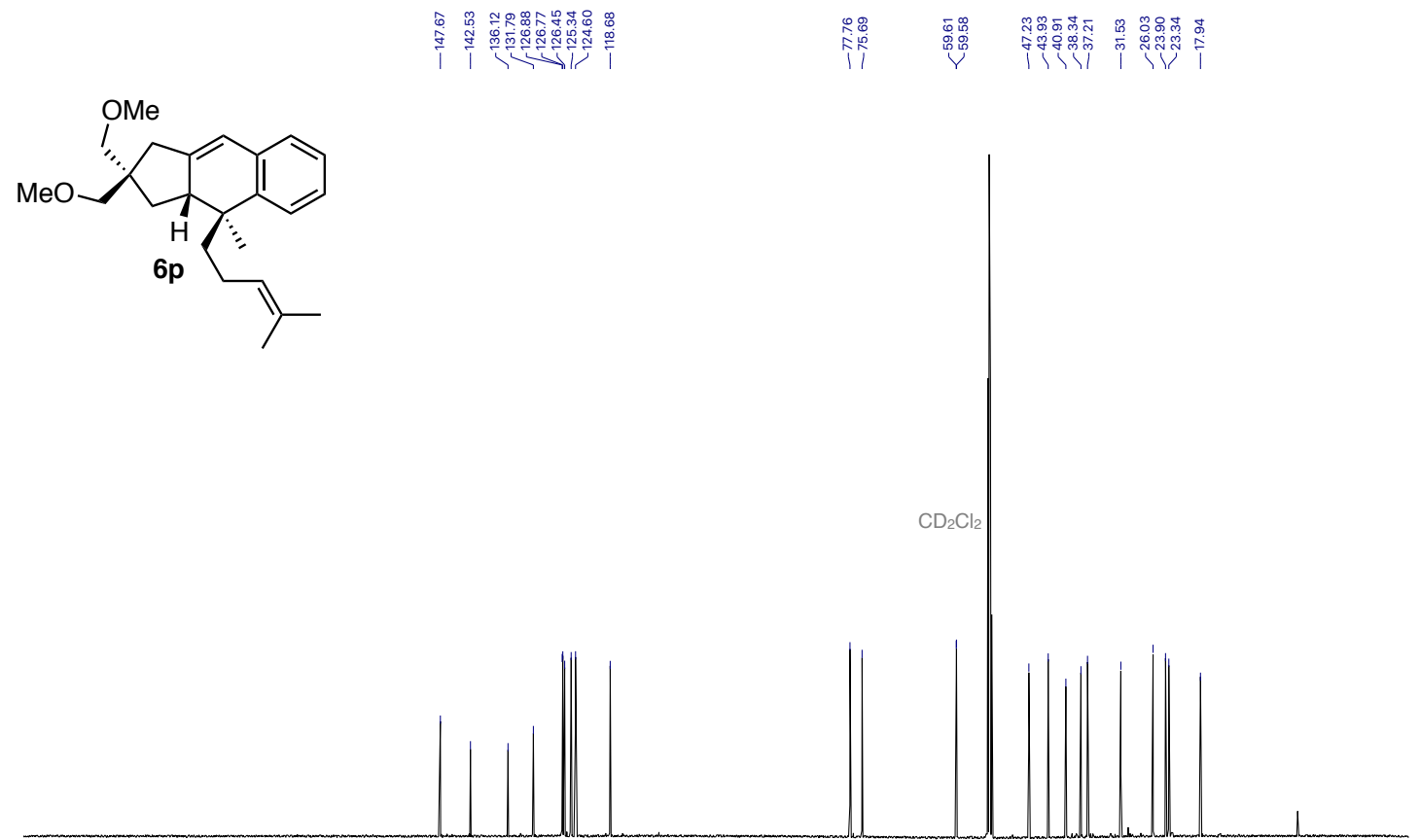

$210 \quad 200 \quad 190 \quad 180 \quad 170 \quad 160$
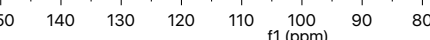
${ }^{1} \mathrm{H}$ NMR: $400 \mathrm{MHz}, \mathrm{CD}_{2} \mathrm{Cl}_{2}$, compound $\mathbf{6 q}$

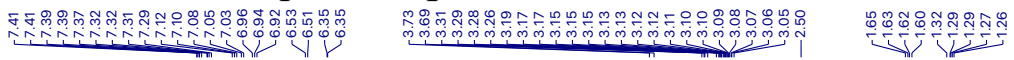
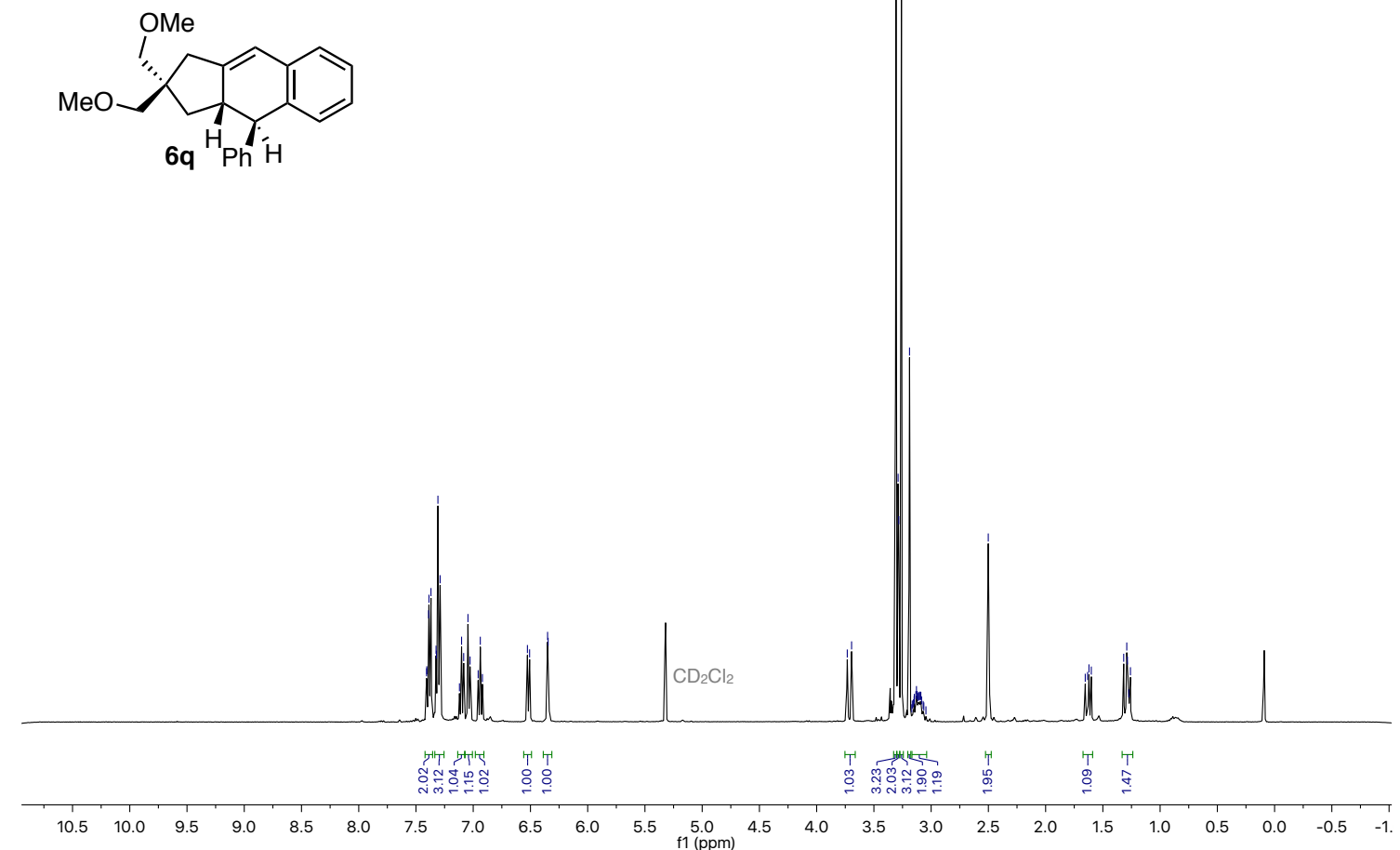

${ }^{13} \mathrm{C}$ NMR: $101 \mathrm{MHz}, \mathrm{CD}_{2} \mathrm{Cl}_{2}$, compound 6q

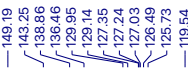<smiles>COCC1(COC)CC2=Cc3ccccc3C(c3ccccc3)C2(Cc2ccccc2)C1</smiles>

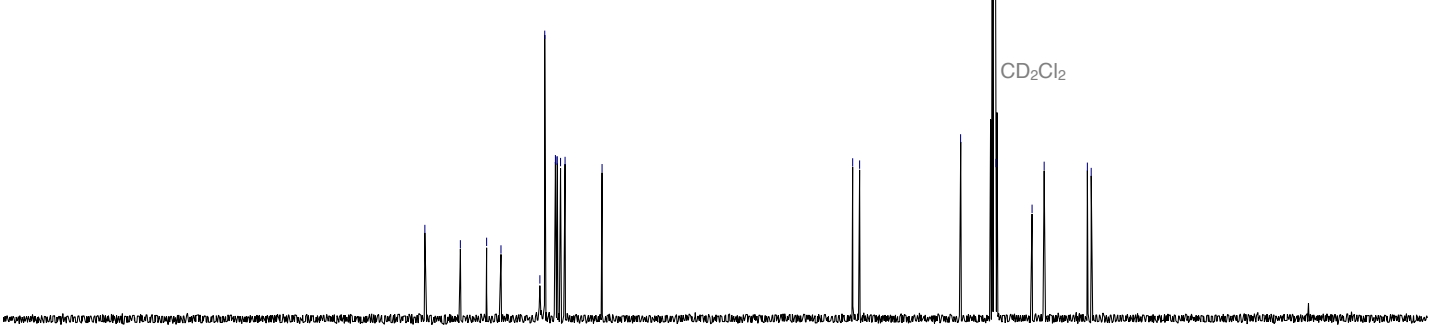

$\begin{array}{lllllllllllllllllllllll}210 & 200 & 190 & 180 & 170 & 160 & 150 & 140 & 130 & 120 & 110 & 100 & 90 & 80 & 70 & 60 & 50 & 40 & 30 & 20 & 10 & 0 & -10\end{array}$ 
${ }^{1} \mathrm{H}$ NMR: $500 \mathrm{MHz}, \mathrm{CDCl}_{3}$, 1-Bromo-4-chloro-2-(phenylethynyl)benzene

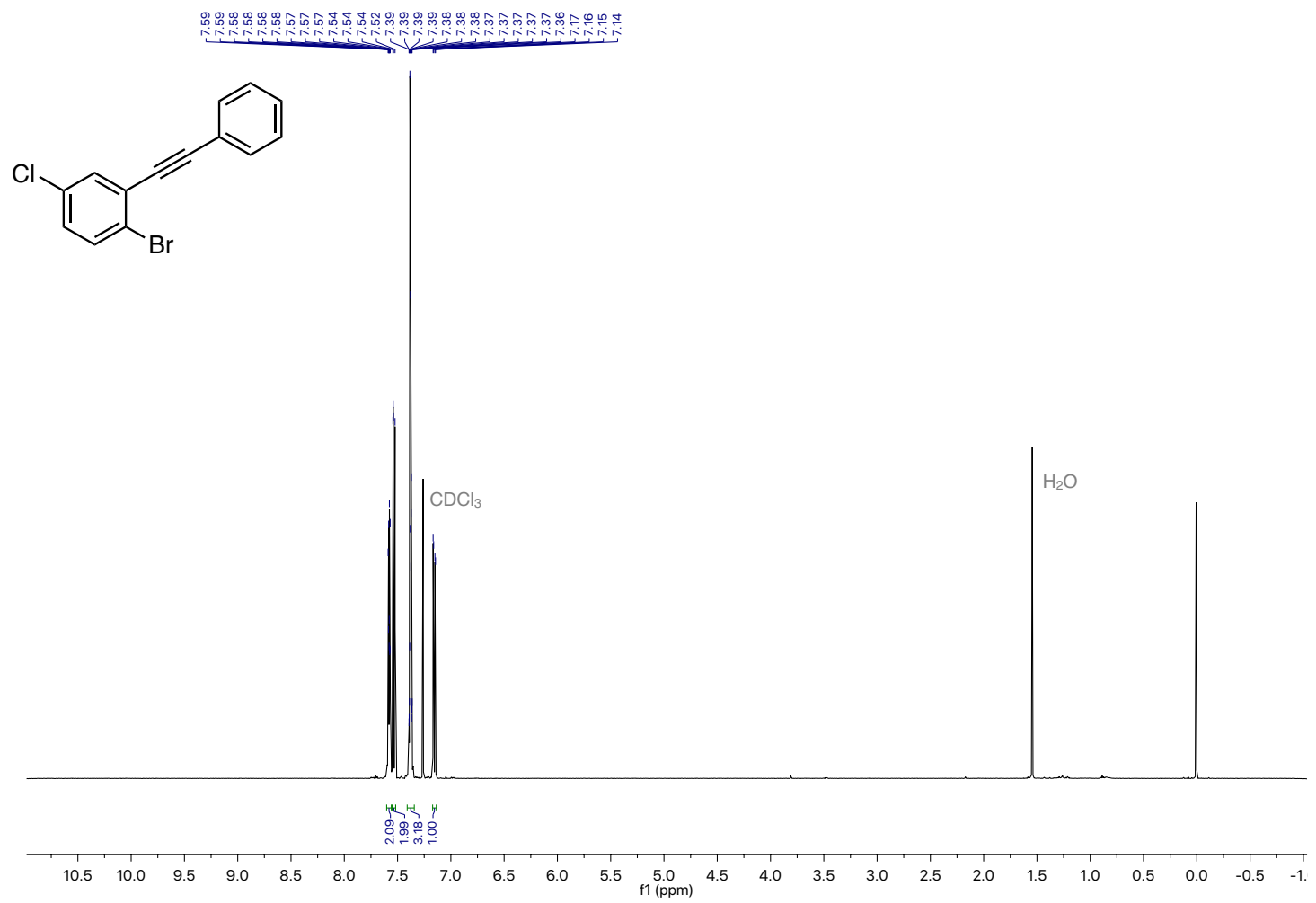

${ }^{13} \mathrm{C}$ NMR: $126 \mathrm{MHz}, \mathrm{CDCl}_{3}$, 1-Bromo-4-chloro-2-(phenylethynyl)benzene

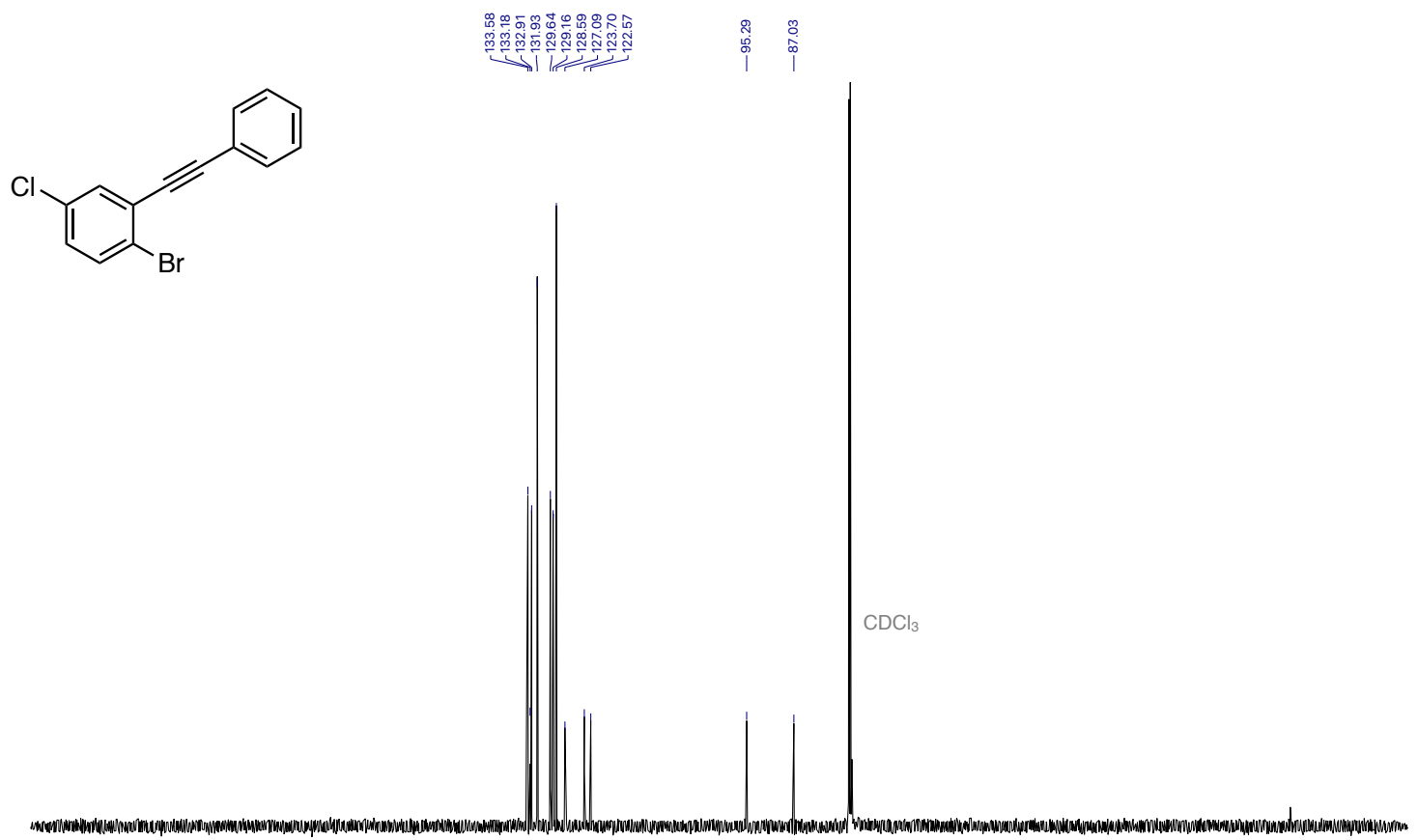

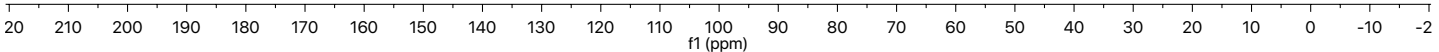


${ }^{1} \mathrm{H}$ NMR: $400 \mathrm{MHz}, \mathrm{CDCl}_{3}$, compound 9b

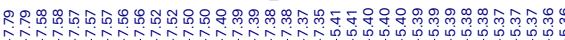

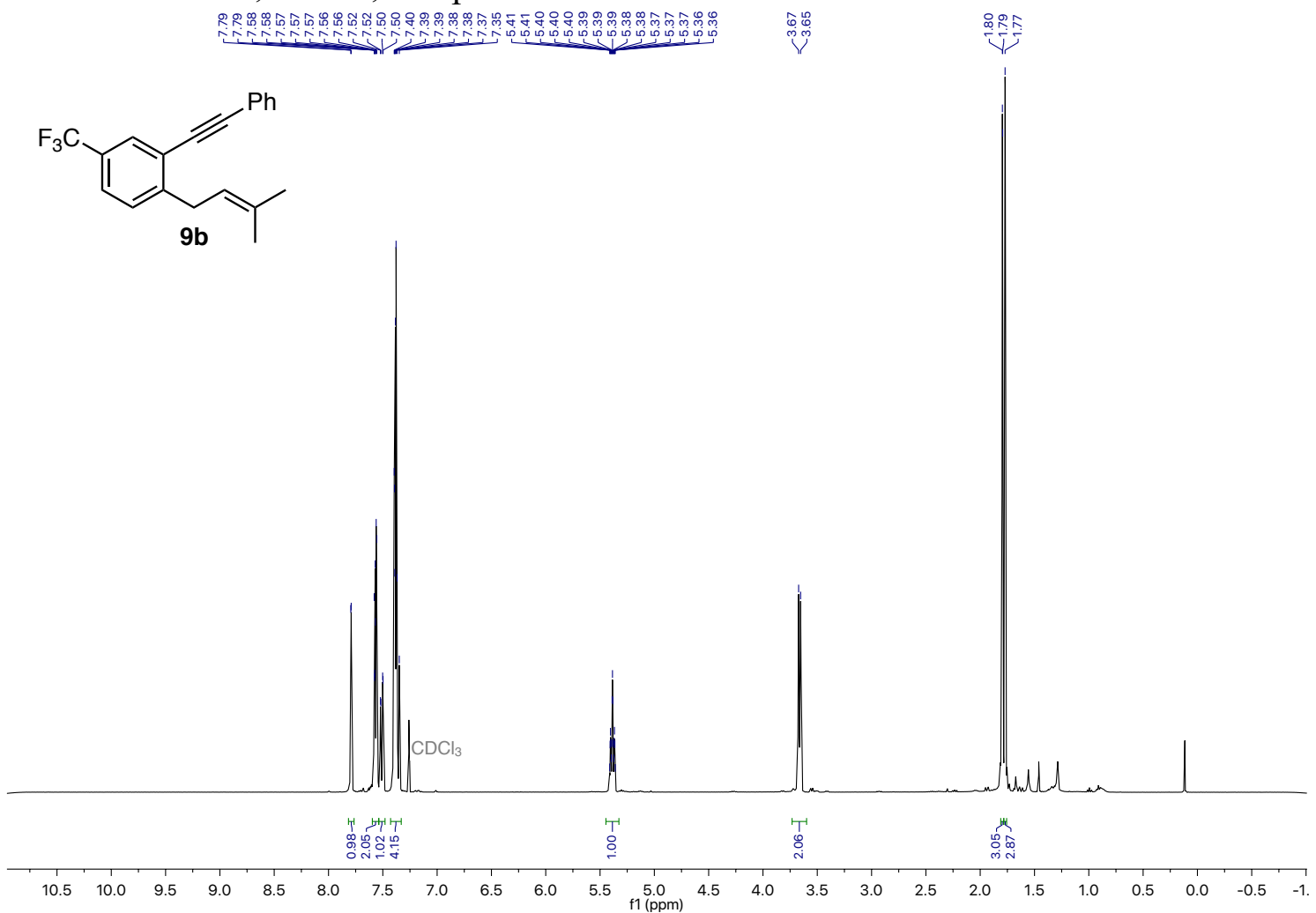

${ }^{13} \mathrm{C}$ NMR: $101 \mathrm{MHz}, \mathrm{CDCl}_{3}$, compound 9b<smiles>CC(C)=CCc1ccc(C(F)(F)F)cc1C#Cc1ccccc1</smiles>

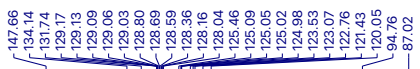

9b

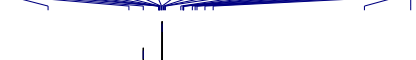


${ }^{19} \mathrm{~F}\left\{{ }^{1} \mathrm{H}\right\}$ NMR: $376 \mathrm{MHz}, \mathrm{CDCl}_{3}$, compound 9b

i<smiles>CC(C)=CCc1ccc(C(F)(F)F)cc1C#Cc1ccccc1</smiles>

9b

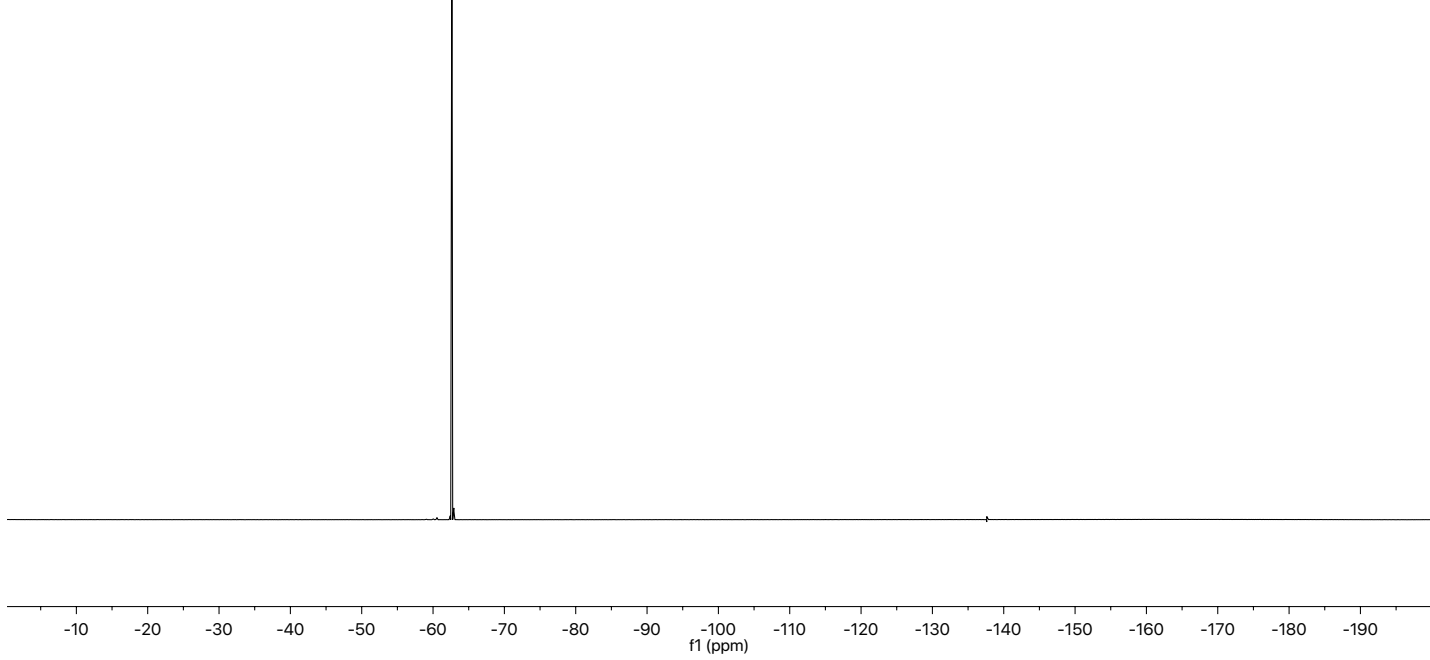

${ }^{1} \mathrm{H}$ NMR: $500 \mathrm{MHz}, \mathrm{CDCl}_{3}$, compound 9c

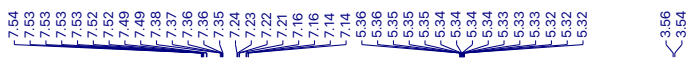<smiles>CC(C)=CCc1ccc(Cl)cc1C#Cc1ccccc1</smiles>

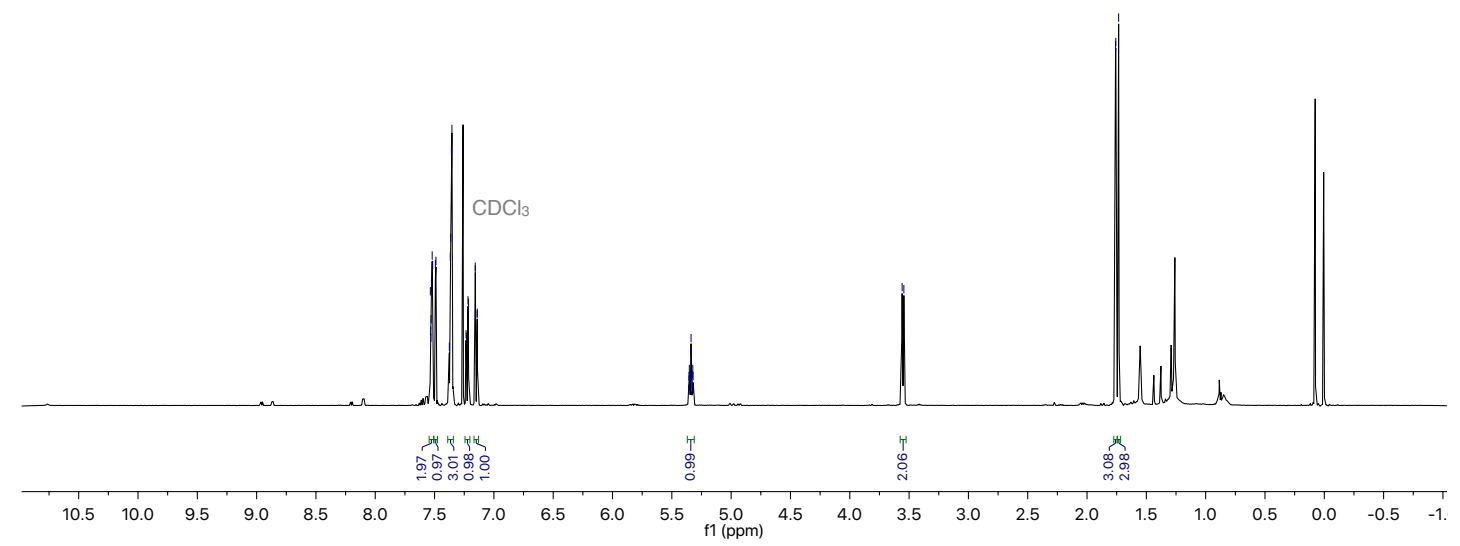


${ }^{13} \mathrm{C} \mathrm{NMR}: 126 \mathrm{MHz}, \mathrm{CDCl}_{3}$, compound 9c
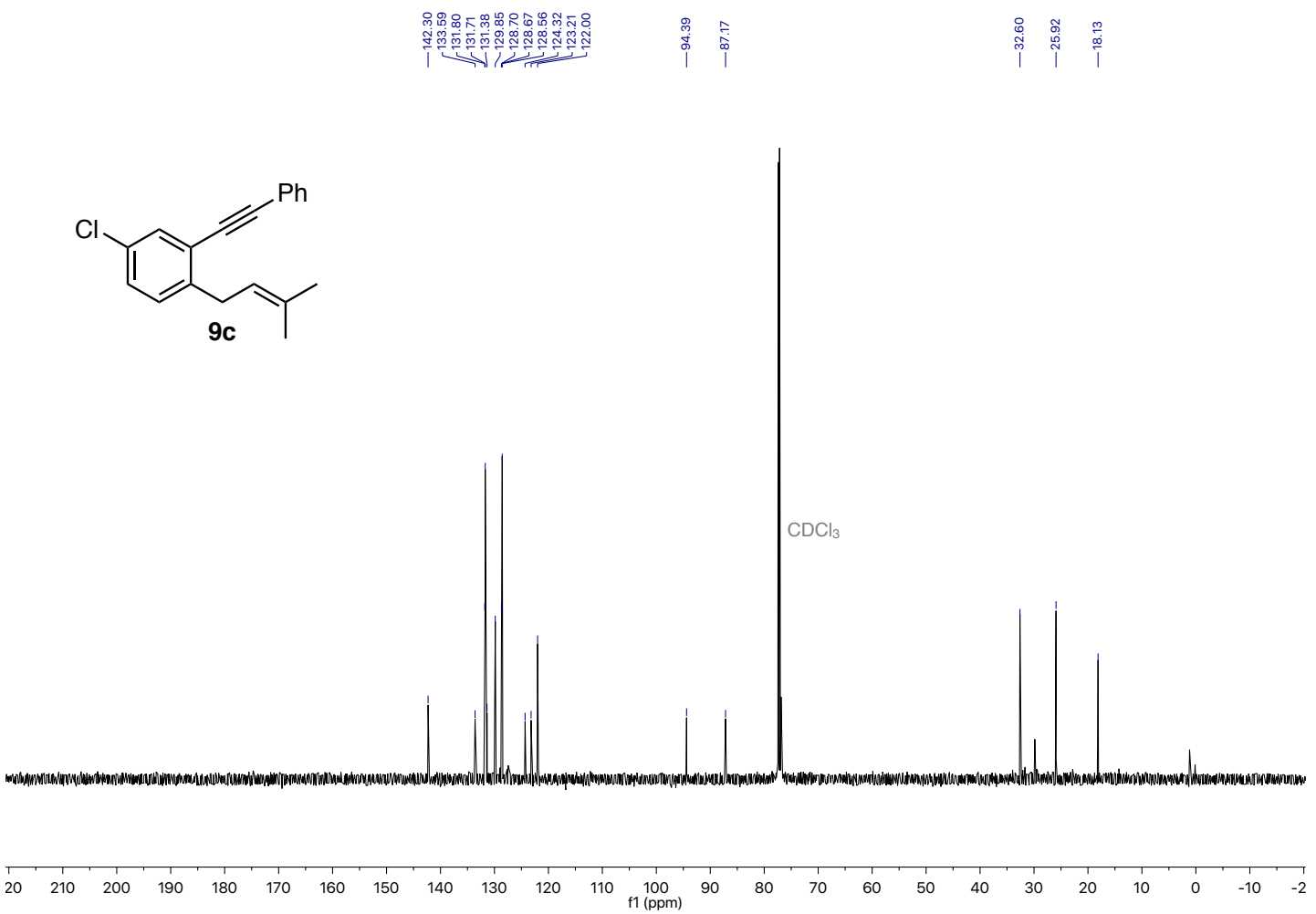

${ }^{1} \mathrm{H}$ NMR: $500 \mathrm{MHz}, \mathrm{CDCl}_{3}$, compound 9d

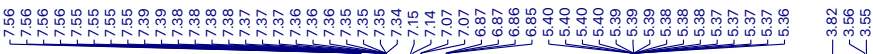

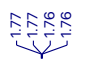<smiles>COc1ccc(CC#Cc2ccccc2)c(CC=C(C)C)c1</smiles>

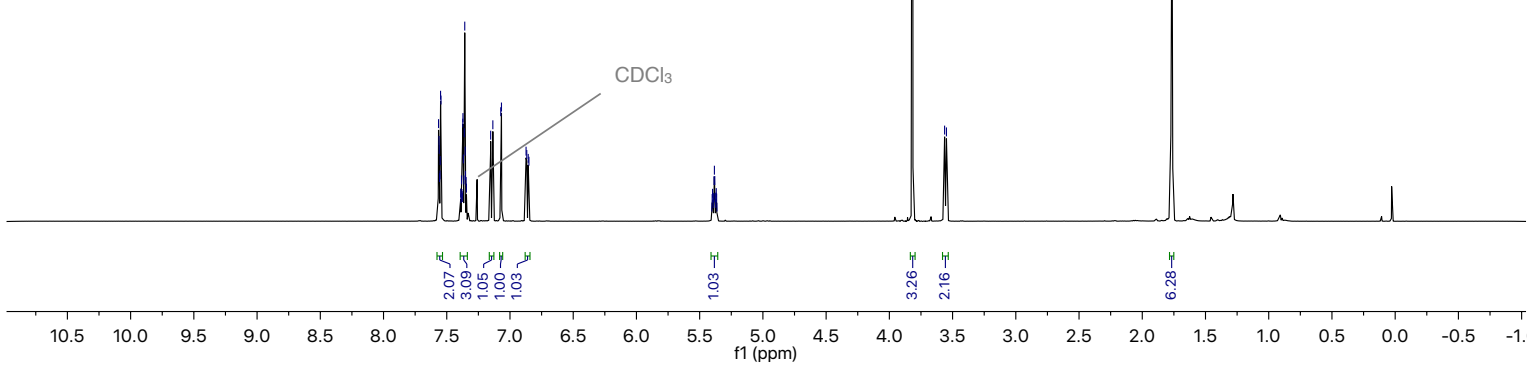


${ }^{13} \mathrm{C} \mathrm{NMR}: 126 \mathrm{MHz}, \mathrm{CDCl}_{3}$, compound 9d

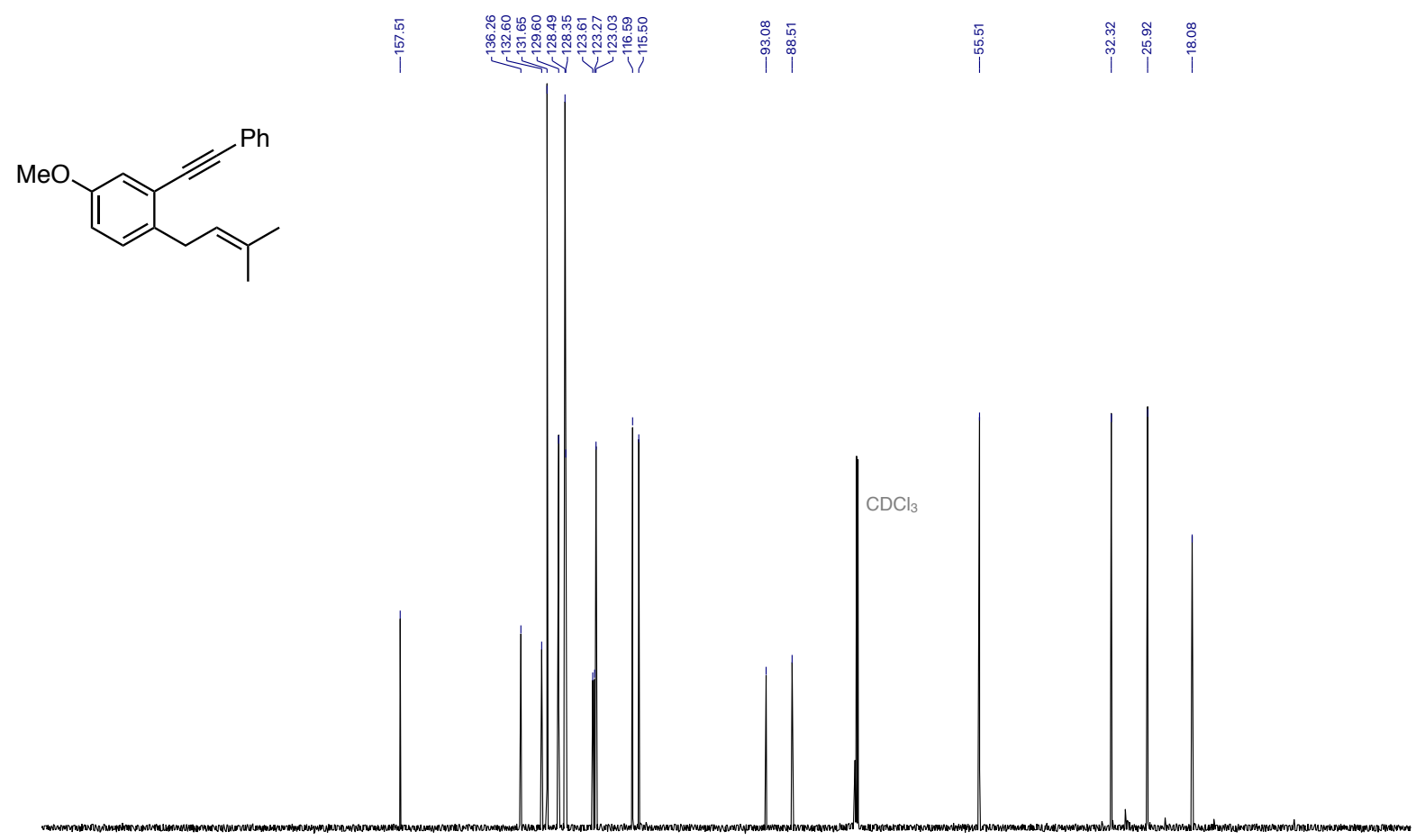

\begin{tabular}{|lllllllllllllllllllllllllll}
\hline 20 & 210 & 200 & 190 & 180 & 170 & 160 & 150 & 140 & 130 & 120 & 110 & $\begin{array}{c}100 \\
\mathrm{f} 1(\mathrm{ppm})\end{array}$ & 90 & 80 & 70 & 60 & 50 & 40 & 30 & 20 & 10 & 0 & -10 & -2
\end{tabular}

${ }^{1} \mathrm{H}$ NMR: $400 \mathrm{MHz}, \mathrm{CD}_{2} \mathrm{Cl}_{2}$, compound 9i<smiles>CC(C)=CC=C(C)C#Cc1ccc(Cl)cc1</smiles>

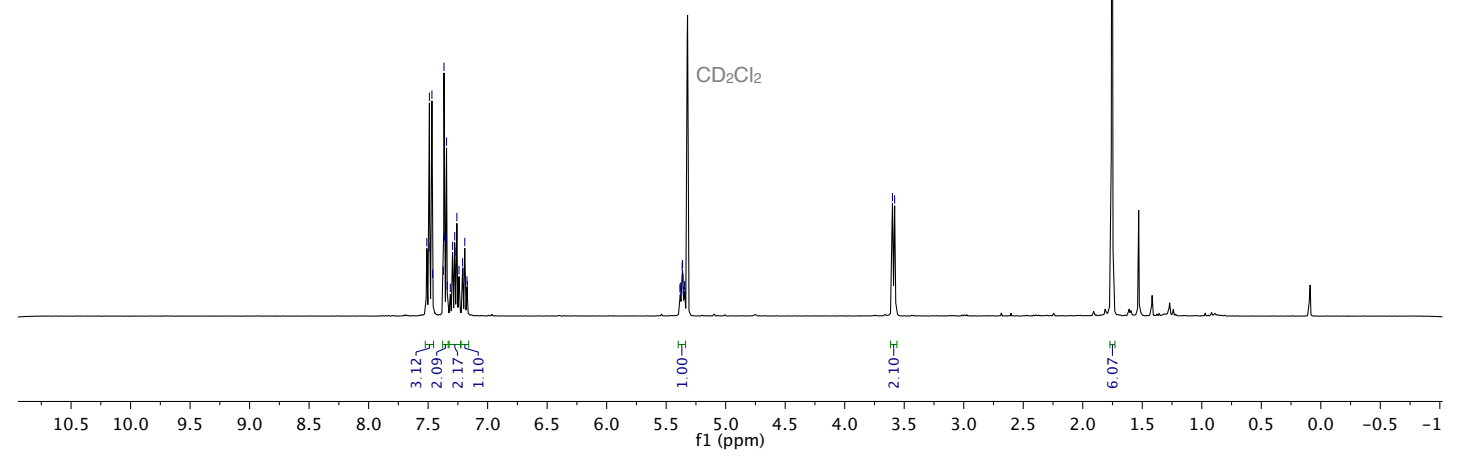


${ }^{13} \mathrm{C}$ NMR: $101 \mathrm{MHz}, \mathrm{CD}_{2} \mathrm{Cl}_{2}$, compound 9i

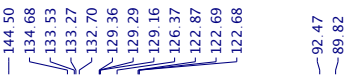

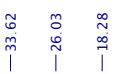<smiles>CC(C)=CCc1ccccc1C#Cc1ccc(Cl)cc1</smiles>

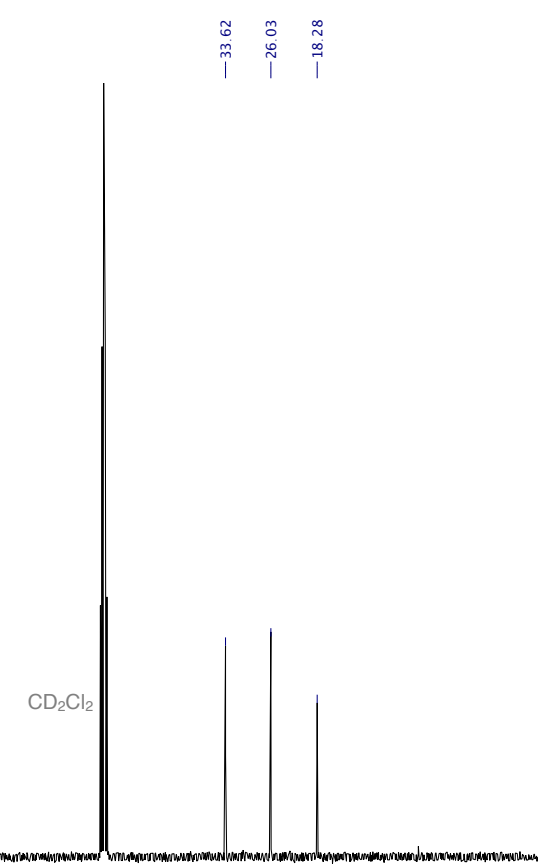

$\begin{array}{lllllllllllllllllllllll}210 & 200 & 190 & 180 & 170 & 160 & 150 & 140 & 130 & 120 & 110 & 100 & 90 & 80 & 70 & 60 & 50 & 40 & 30 & 20 & 10 & 0 & -10\end{array}$

${ }^{1} \mathrm{H}$ NMR: $500 \mathrm{MHz}, \mathrm{CDCl}_{3}$, 1,3-Dibenzyloxy-5-(phenylethynyl)benzene

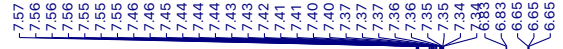<smiles>C(#Cc1cc(OCc2ccccc2)cc(OCc2ccccc2)c1)c1ccccc1</smiles>

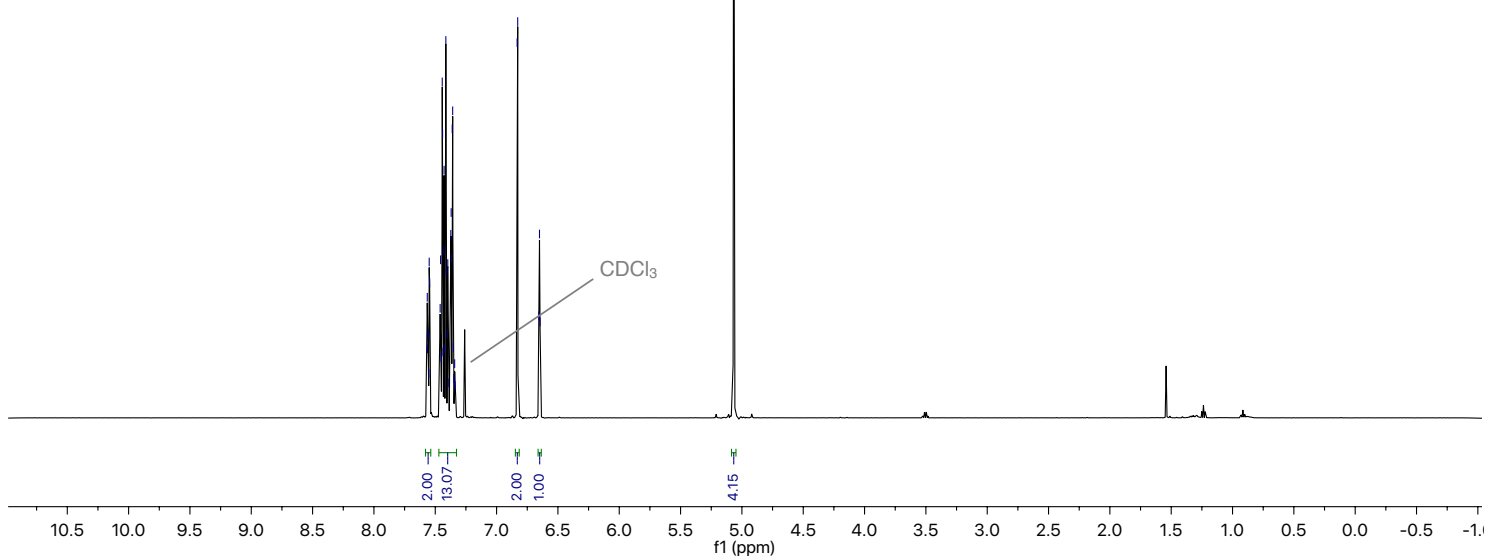


${ }^{13} \mathrm{C}$ NMR: $126 \mathrm{MHz}, \mathrm{CDCl}_{3}, 1,3$-dibenzyloxy-5-(phenylethynyl)benzene<smiles>Oc1cc(C#Cc2ccccc2)cc(OCc2ccccc2)c1</smiles>

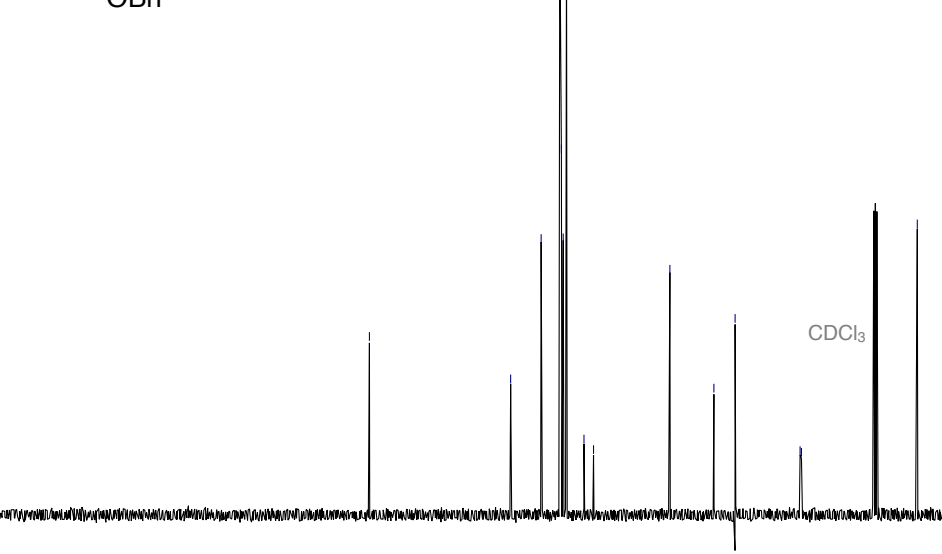

\begin{tabular}{llllllllllllllllllllllllllllllllllll}
\hline 20 & 210 & 200 & 190 & 180 & 170 & 160 & 150 & 140 & 130 & 120 & 110 & 100 & 90 & 80 & 70 & 60 & 50 & 40 & 30 & 20 & 10 & 0 & -10 & -2
\end{tabular}

${ }^{1} \mathrm{H}$ NMR: $300 \mathrm{MHz}, \mathrm{CDCl}_{3}$, 2-Bromo-1,5-dimethoxy-3-(phenylethynyl)benzene<smiles>COc1cc(C#Cc2ccccc2)c(Br)c(OC)c1</smiles>

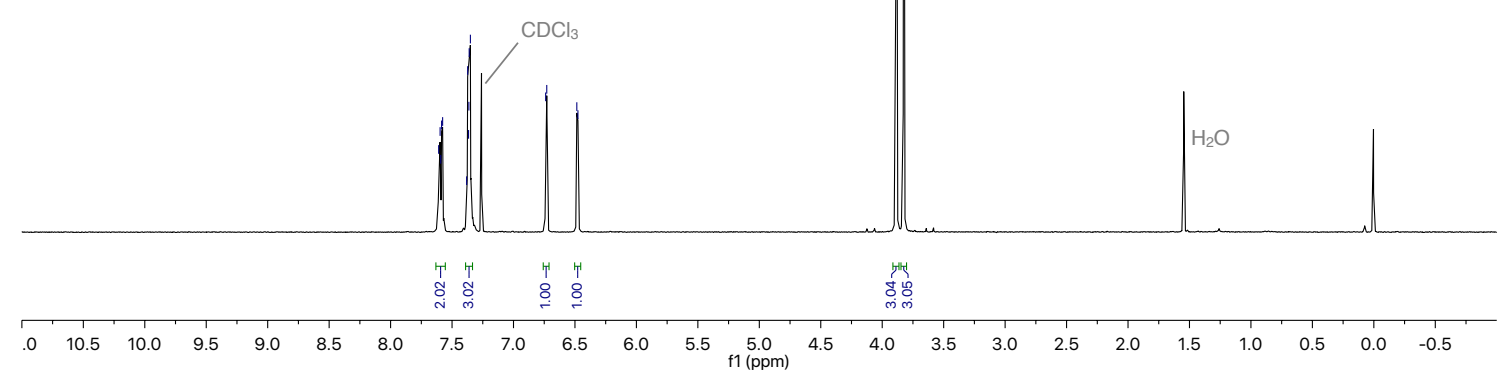


${ }^{13} \mathrm{C}$ NMR: $75 \mathrm{MHz}, \mathrm{CDCl}_{3}$, 2-Bromo-1,5-dimethoxy-3-(phenylethynyl)benzene

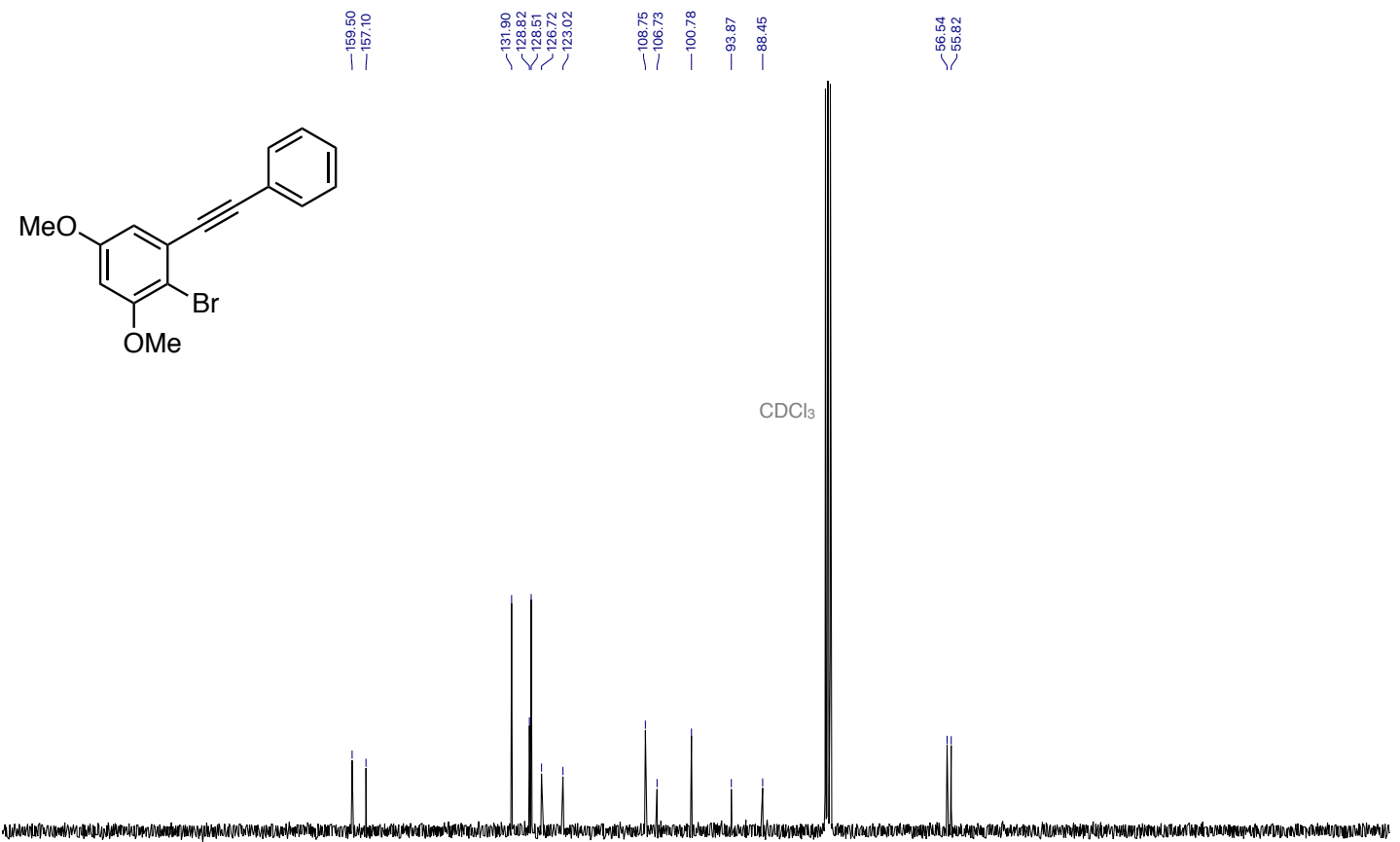

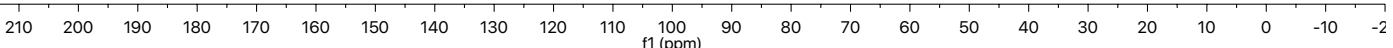

${ }^{1} \mathrm{H}$ NMR: $500 \mathrm{MHz}, \mathrm{CDCl}_{3}$, 2-Bromo-1,5-dibenzyloxy-3-(phenylethynyl)benzene

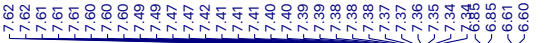
兹㩊<smiles>Oc1cc(C#Cc2ccccc2)c(Br)c(OC(Br)(Br)c2ccccc2)c1</smiles>

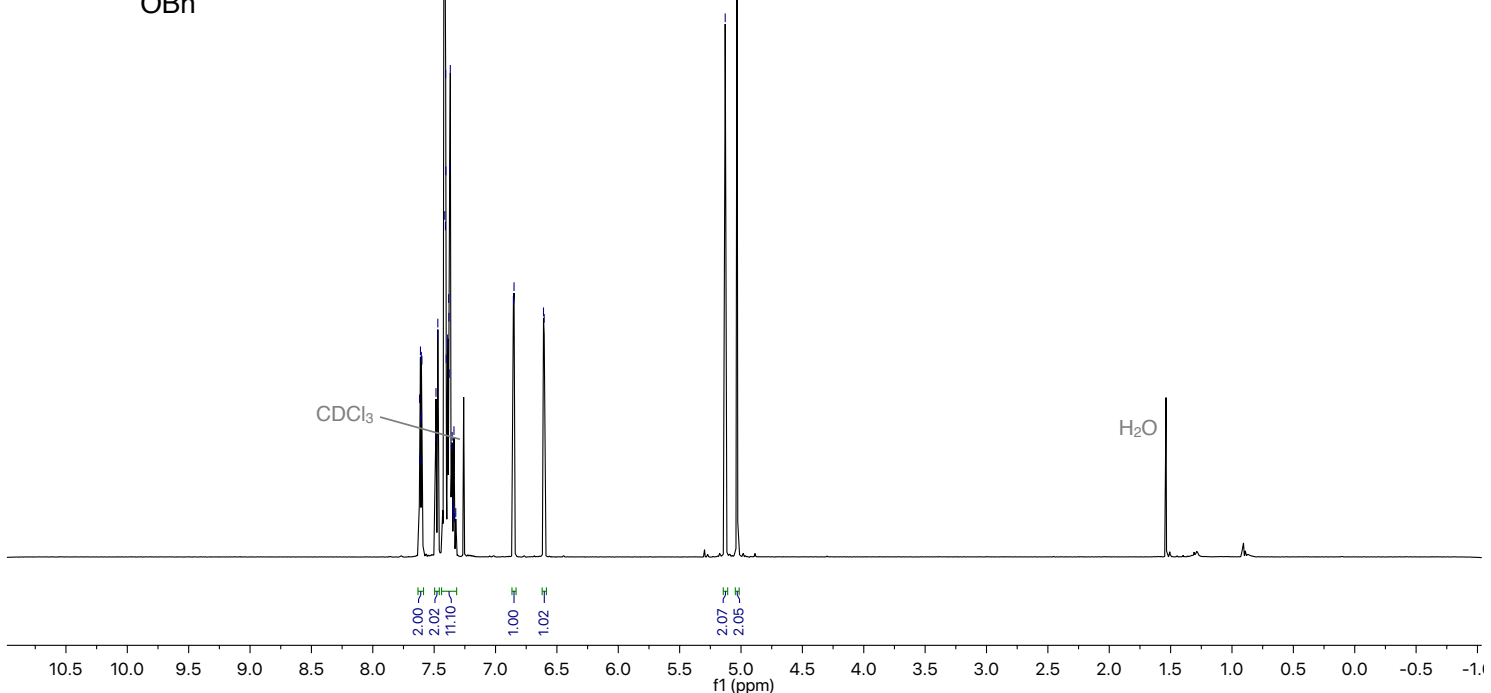


${ }^{13} \mathrm{C}$ NMR: $126 \mathrm{MHz}, \mathrm{CDCl}_{3}$, 2-bromo-1,5-dibenzyloxy-3-(phenylethynyl)benzene

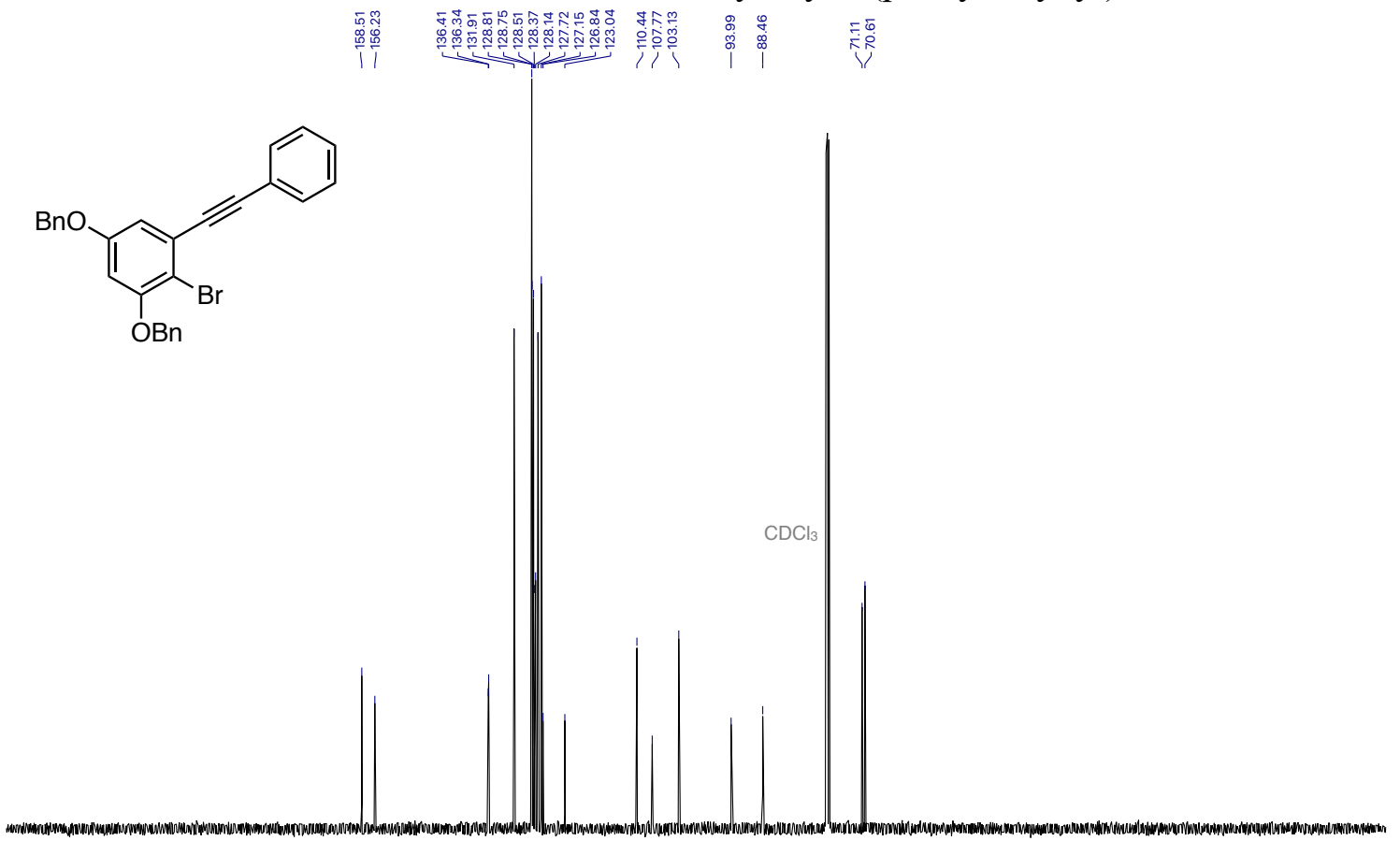

\begin{tabular}{lllllllllllllllllllllllllllllllll}
\hline 20 & 210 & 200 & 190 & 180 & 170 & 160 & 150 & 140 & 130 & 120 & 110 & 100 & 90 & 80 & 70 & 60 & 50 & 40 & 30 & 20 & 10 & 0 & -10 & -2
\end{tabular}

${ }^{1} \mathrm{H}$ NMR: $400 \mathrm{MHz}, \mathrm{CDCl}_{3}$, compound 9k

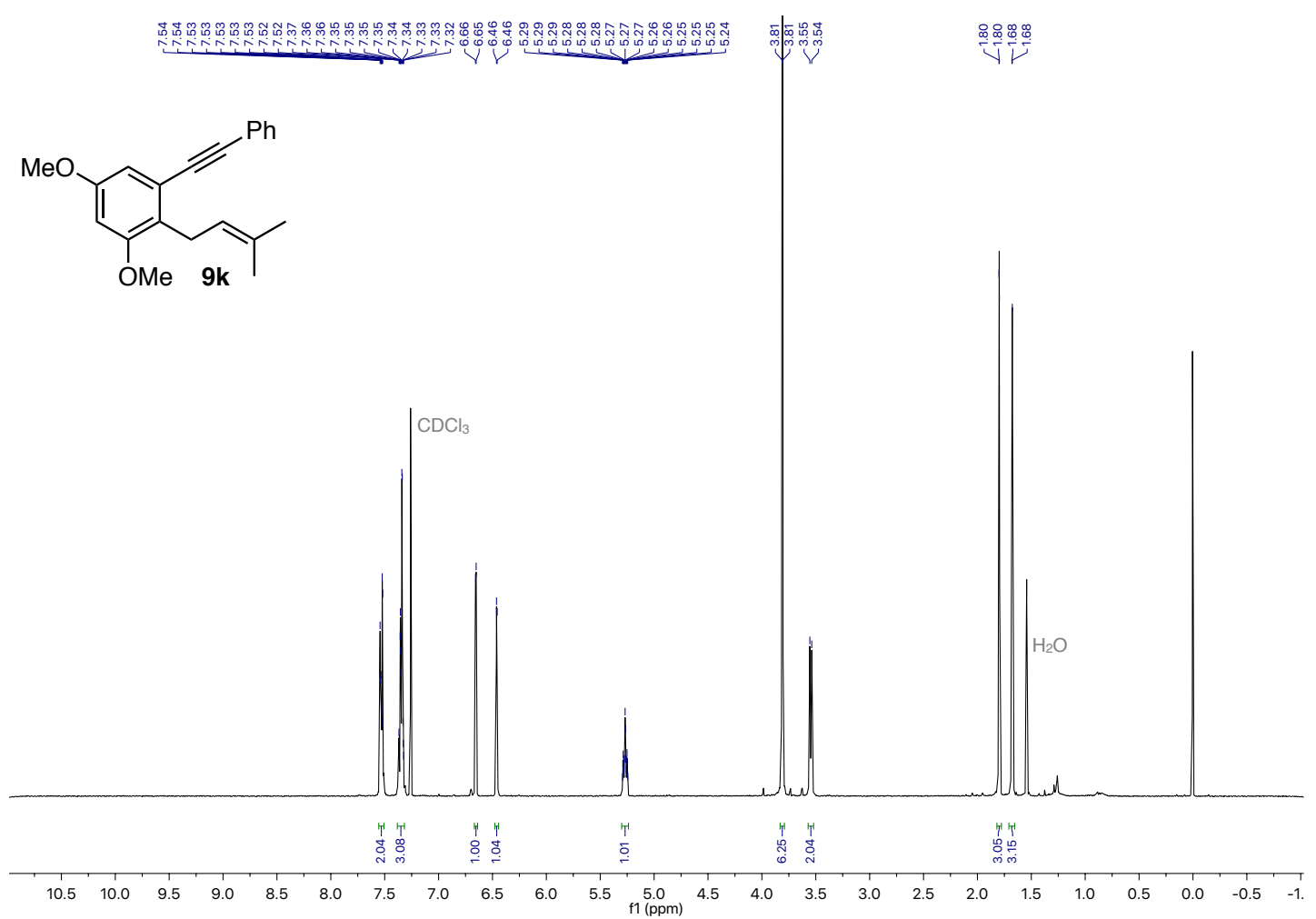


${ }^{13} \mathrm{C}$ NMR: $101 \mathrm{MHz}, \mathrm{CDCl}_{3}$, compound 9k
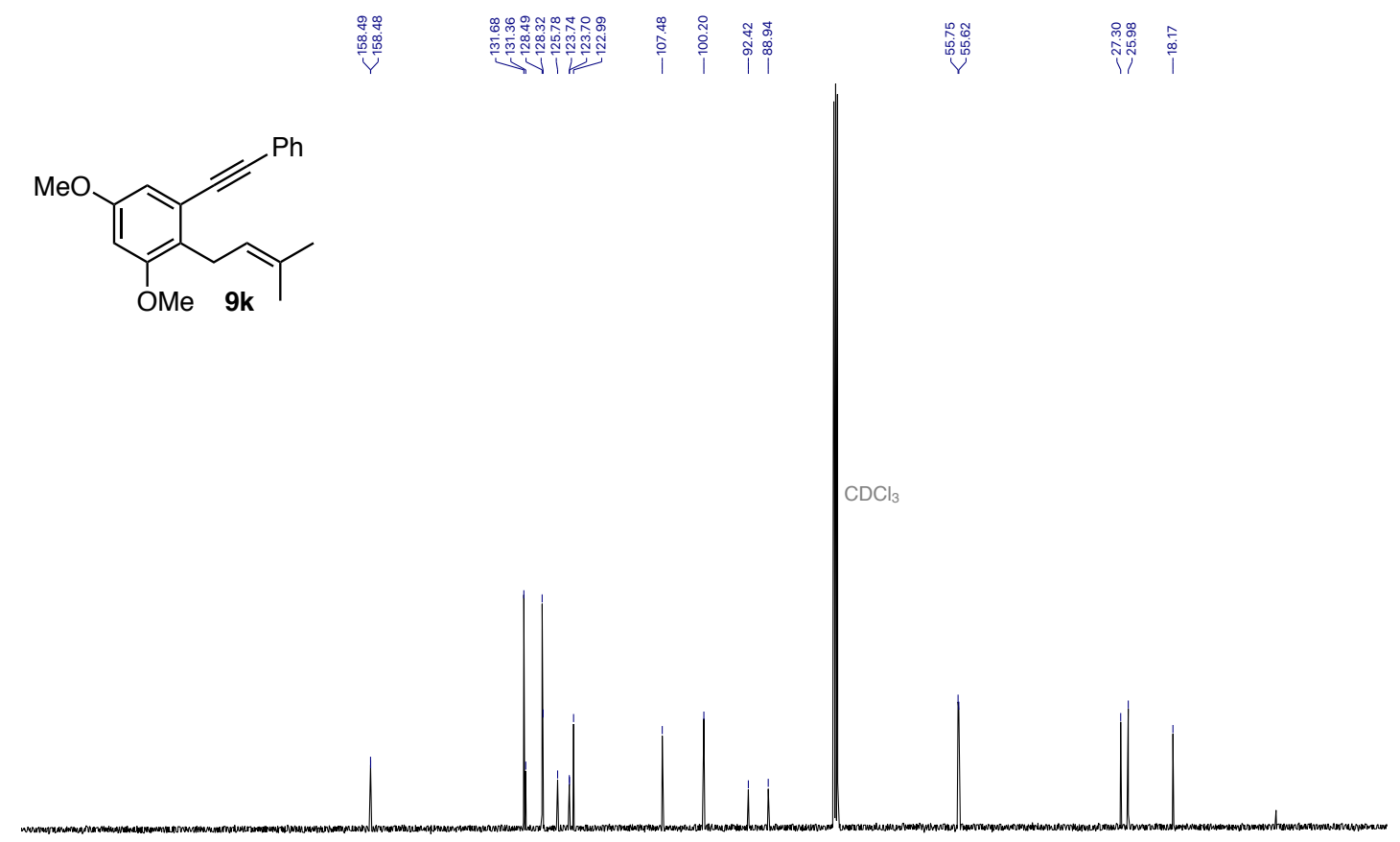

$\begin{array}{lllllllllllllllllllllllll}210 & 200 & 190 & 180 & 170 & 160 & 150 & 140 & 130 & 120 & 110 & \begin{array}{c}100 \\ \mathrm{f}(\mathrm{ppm})\end{array} & 90 & 80 & 70 & 60 & 50 & 40 & 30 & 20 & 10 & 0 & -10\end{array}$

${ }^{1} \mathrm{H}$ NMR: $400 \mathrm{MHz}, \mathrm{CDCl}_{3}$, compound 91

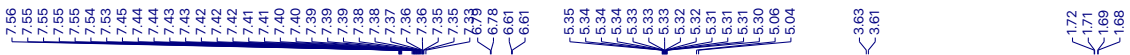<smiles>CC(C)=CCc1c(C#Cc2ccccc2)cc(OCc2ccccc2)cc1OCc1ccccc1</smiles>
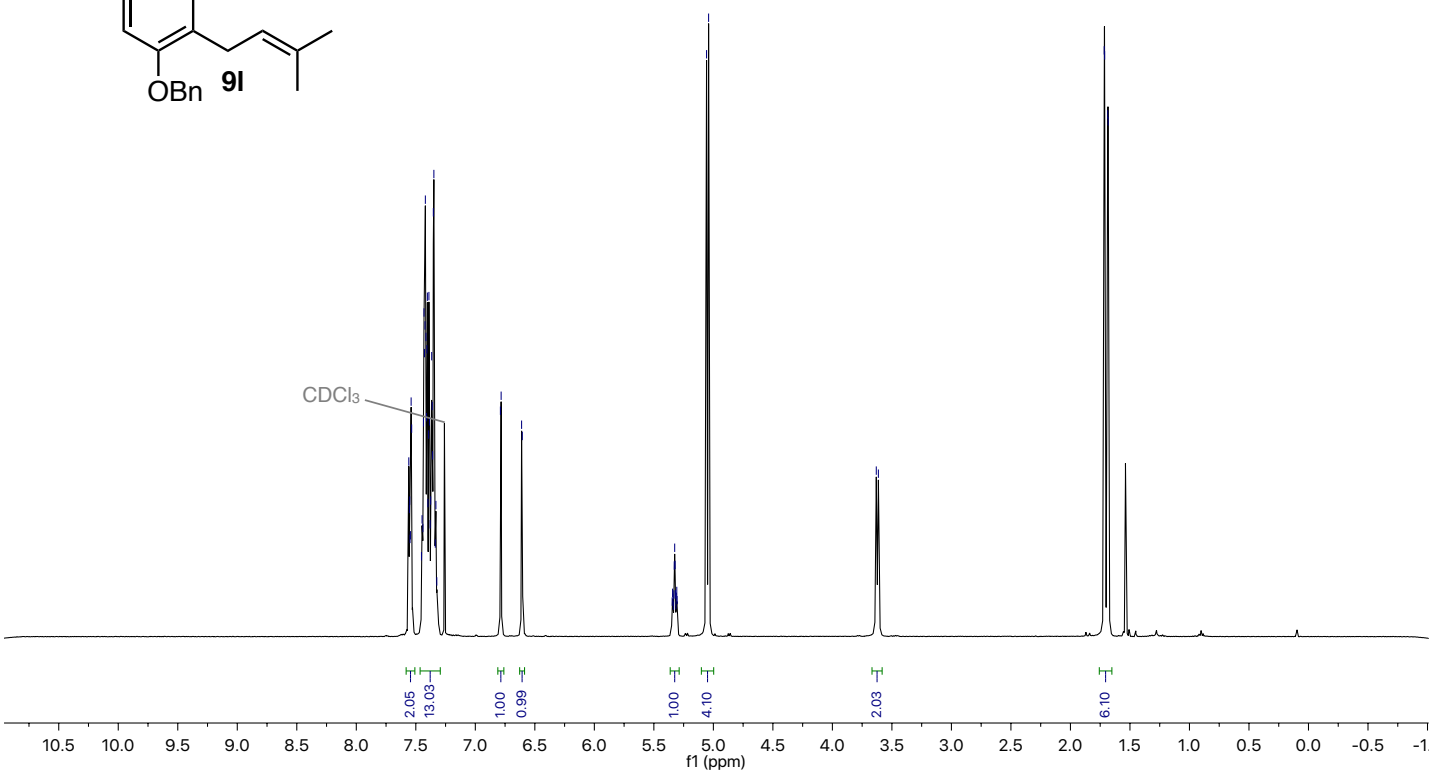
${ }^{13} \mathrm{C}$ NMR: $101 \mathrm{MHz}, \mathrm{CDCl}_{3}$, compound 91
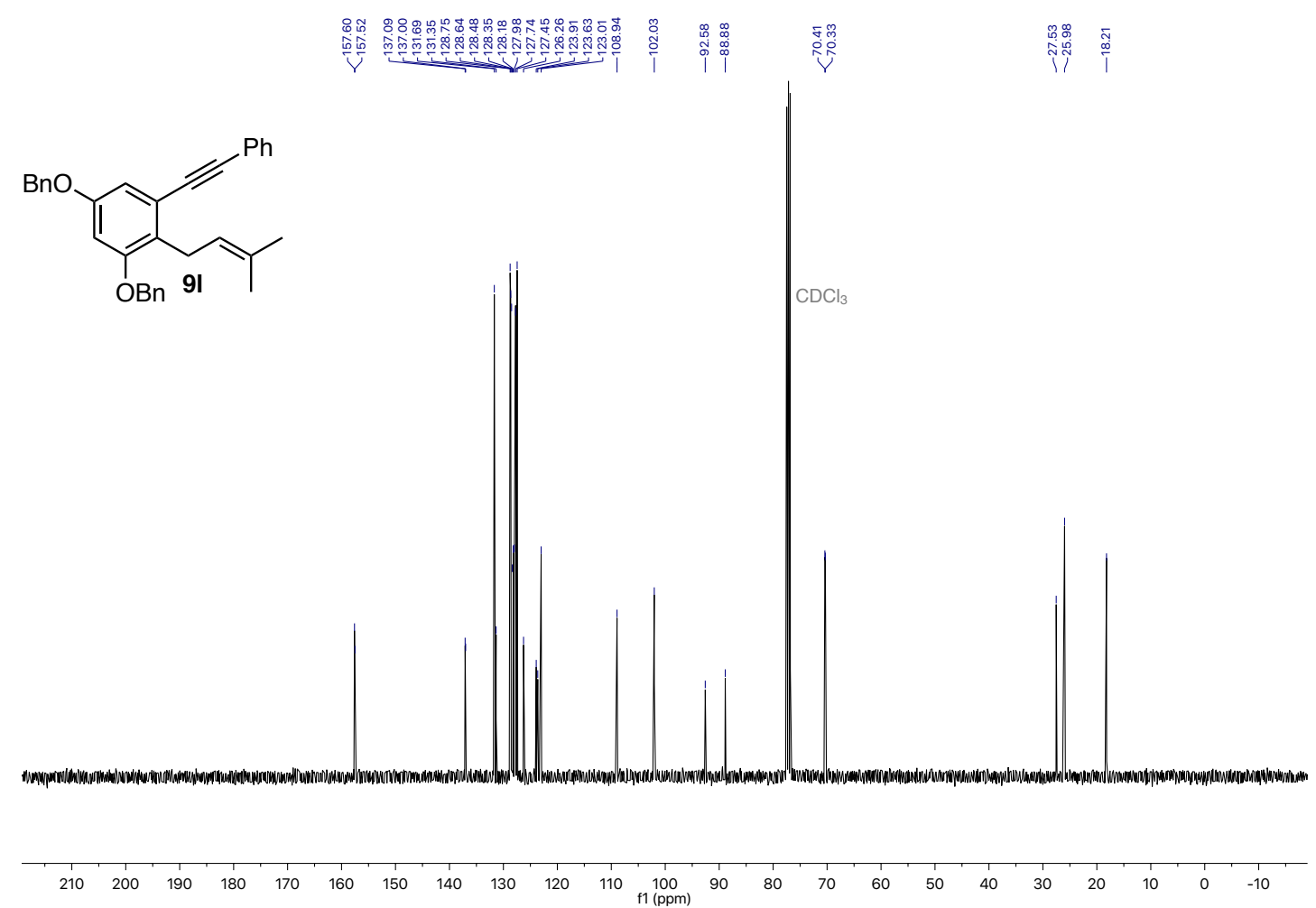

${ }^{1} \mathrm{H}$ NMR: $400 \mathrm{MHz}, \mathrm{CD}_{2} \mathrm{Cl}_{2}$, compound 10a

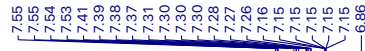

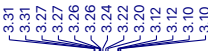<smiles>CC(C)(O)C1Cc2ccccc2C=C1c1ccccc1</smiles>

$10 a$

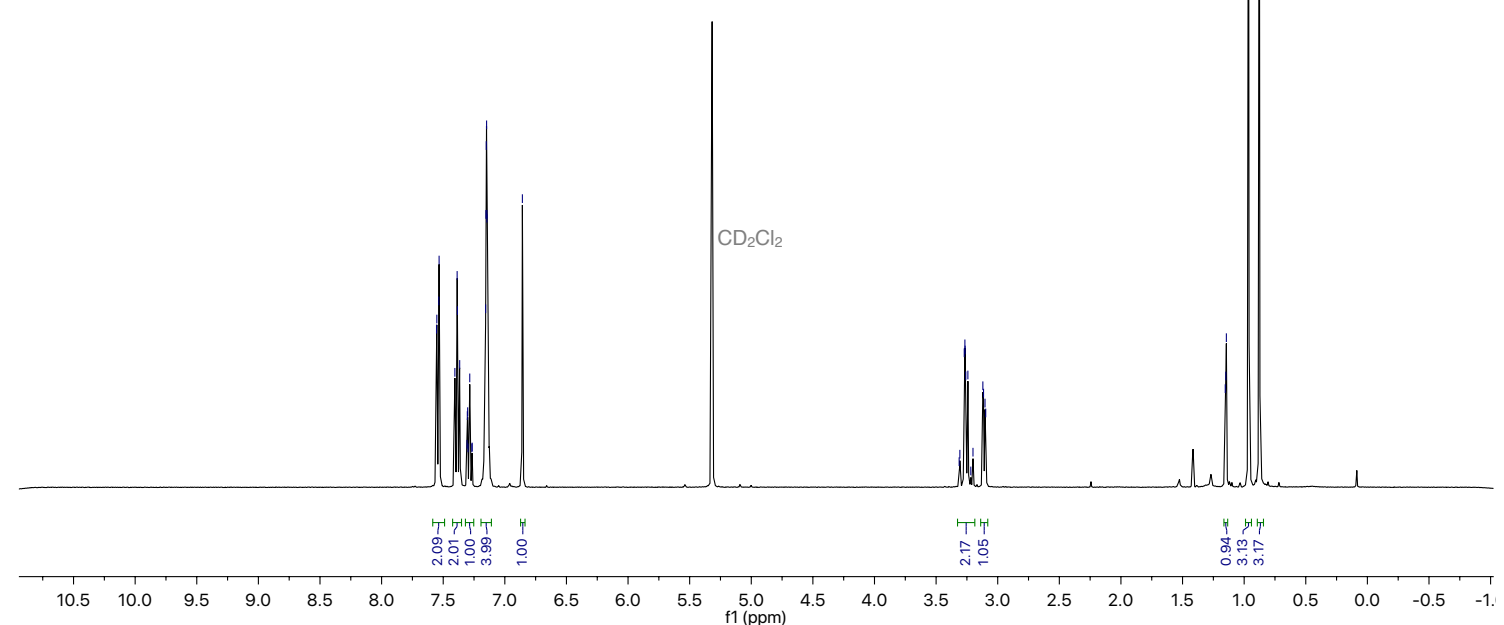


${ }^{13} \mathrm{C}$ NMR: $126 \mathrm{MHz}, \mathrm{CD}_{2} \mathrm{Cl}_{2}$, compound 10a

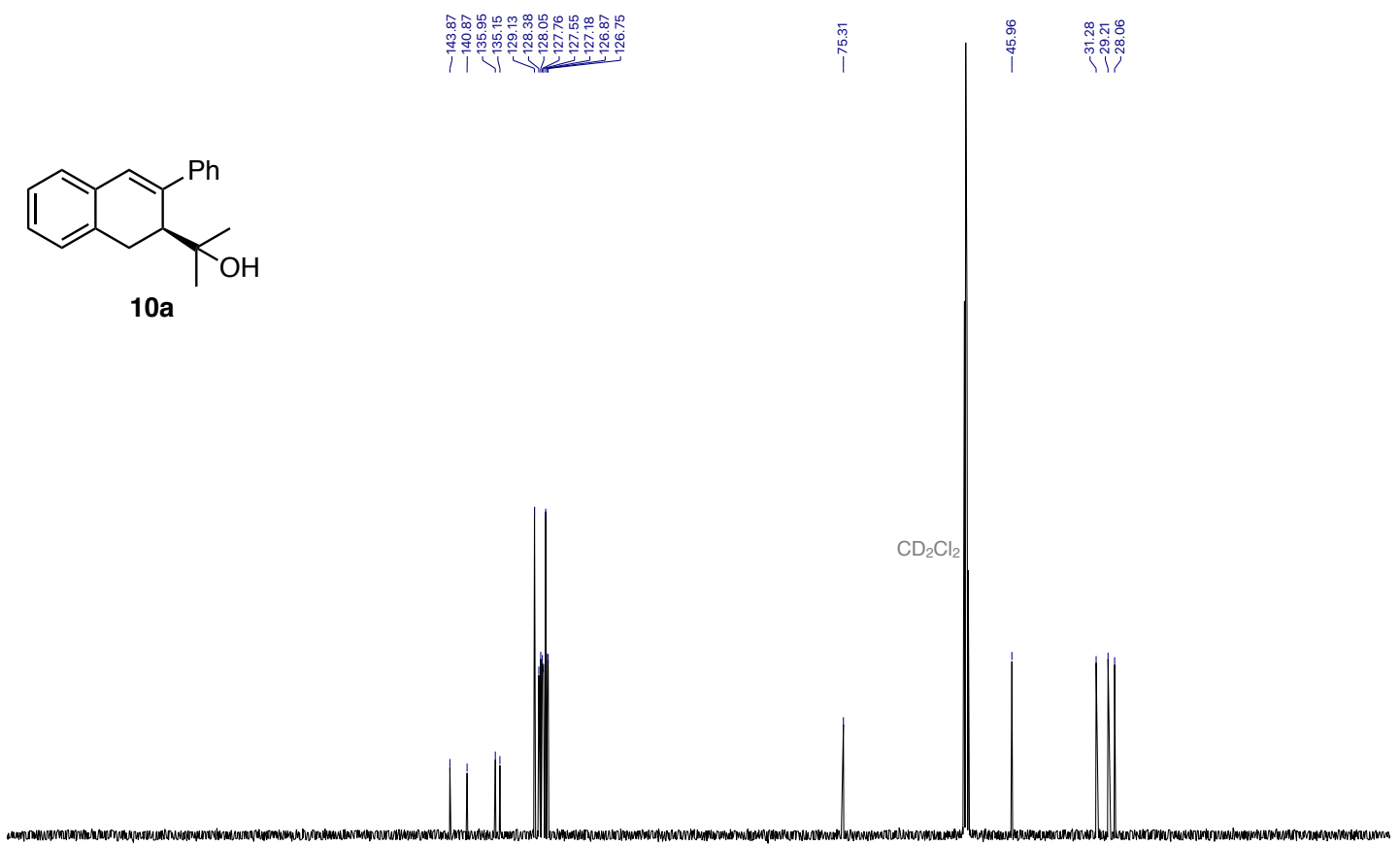

\begin{tabular}{lllllllllllllllllllllllllllll}
\hline 20 & 210 & 200 & 190 & 180 & 170 & 160 & 150 & 140 & 130 & 120 & 110 & $\begin{array}{c}100 \\
100\end{array}$ & 90 & 80 & 70 & 60 & 50 & 40 & 30 & 20 & 10 & 0 & -10
\end{tabular}

${ }^{1} \mathrm{H}$ NMR: $400 \mathrm{MHz}, \mathrm{CD}_{2} \mathrm{Cl}_{2}$, compound 10b

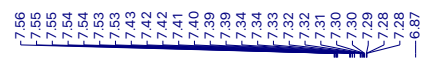<smiles>CC(C)(O)C1Cc2ccc(C(F)(F)F)cc2C=C1c1ccccc1</smiles>

10b

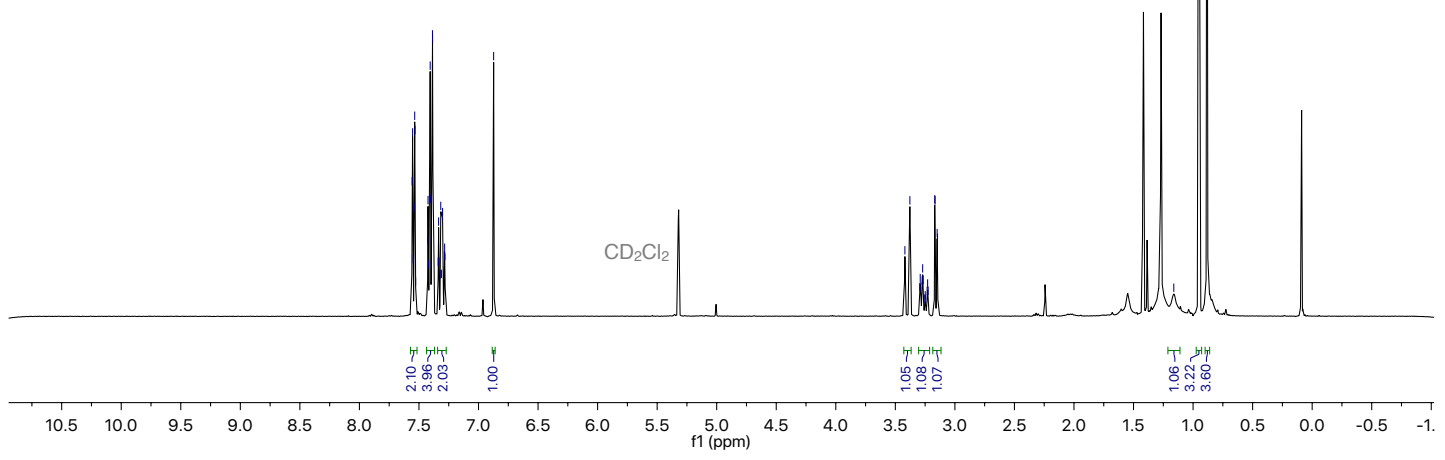


${ }^{13} \mathrm{C}$ NMR: $101 \mathrm{MHz}, \mathrm{CD}_{2} \mathrm{Cl}_{2}$, compound 10b

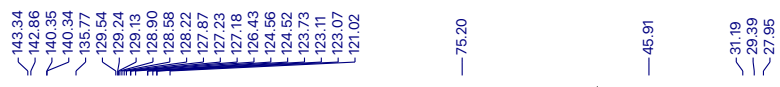<smiles>CC(C)(O)C1Cc2ccc(C(F)(F)F)cc2C=C1c1ccccc1</smiles>

$10 \mathrm{~b}$

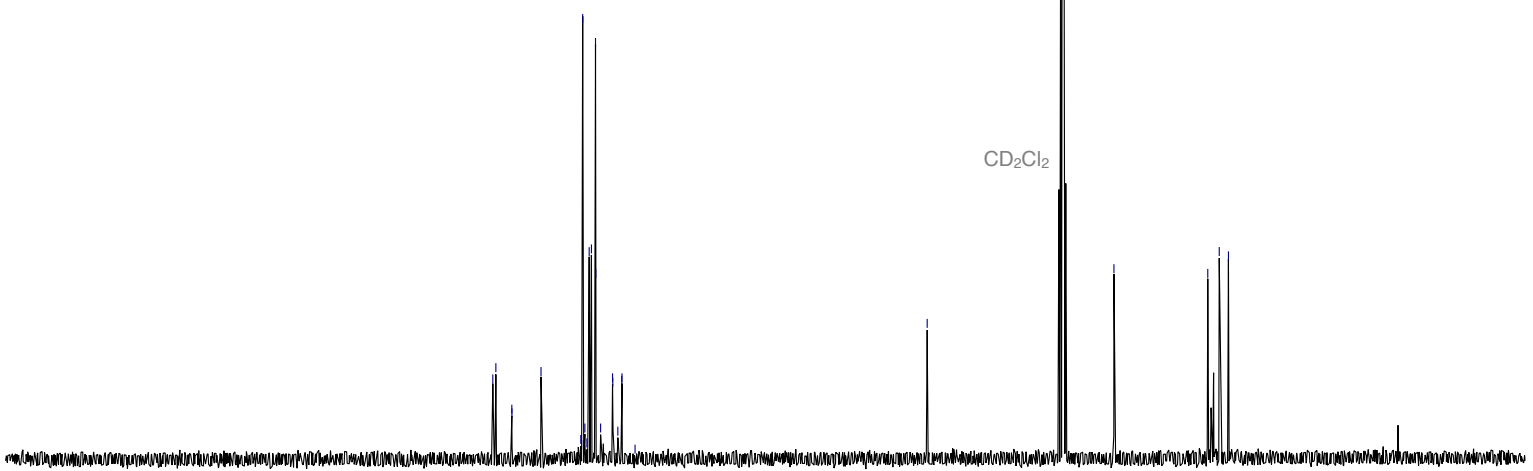

$\begin{array}{lllllllllllllllllllllll}210 & 200 & 190 & 180 & 170 & 160 & 150 & 140 & 130 & 120 & 110 & \begin{array}{l}100 \\ \mathrm{f} 1(\mathrm{ppm})\end{array} & 90 & 80 & 70 & 60 & 50 & 40 & 30 & 20 & 10 & 0 & -10\end{array}$

${ }^{19} \mathrm{~F}\left\{{ }^{1} \mathrm{H}\right\}$ NMR: $376 \mathrm{MHz}, \mathrm{CD}_{2} \mathrm{Cl}_{2}$, compound 10b

$\underset{\substack{0 \\ \vdots}}{i}$<smiles>CC(C)(O)C1Cc2ccc(C(F)(F)F)cc2C=C1c1ccccc1</smiles>

$10 b$

$\begin{array}{lllllllllllllllllll}-10 & -20 & -30 & -40 & -50 & -60 & -70 & -80 & -90 & -100 & -110 & -120 & -130 & -140 & -150 & -160 & -170 & -180 & -190\end{array}$ 
${ }^{1} \mathrm{H}$ NMR: $400 \mathrm{MHz}, \mathrm{CDCl}_{3}$, compound 10c

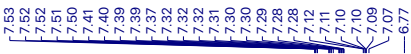<smiles>CC(C)(O)C1Cc2ccc(Cl)cc2C=C1c1ccccc1</smiles>

$10 \mathrm{c}$

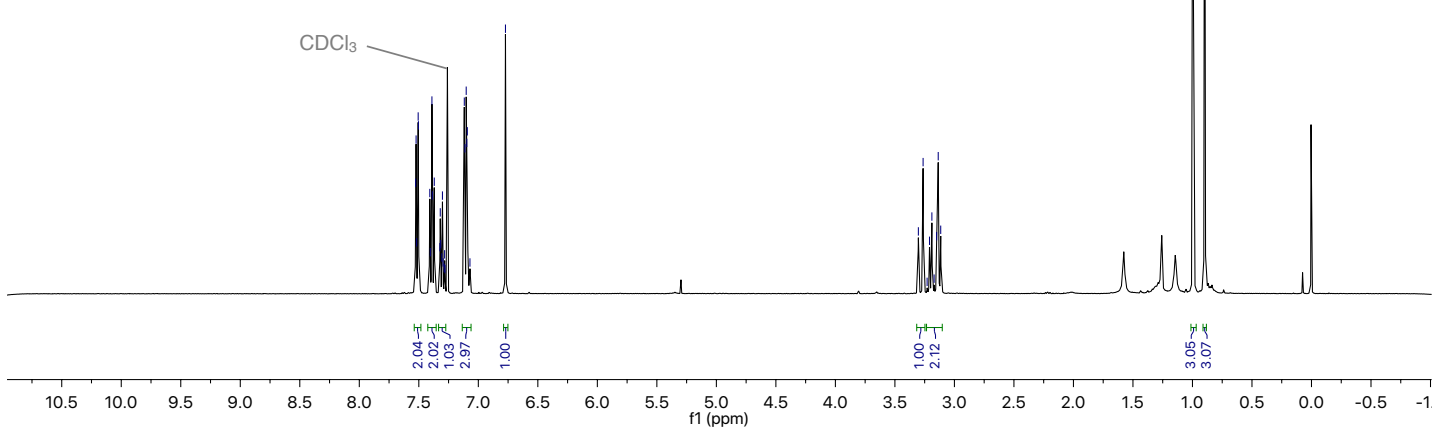

${ }^{13} \mathrm{C}$ NMR: $101 \mathrm{MHz}, \mathrm{CDCl}_{3}$, compound 10c
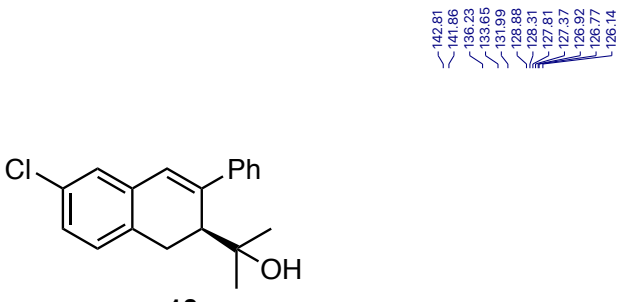

10c

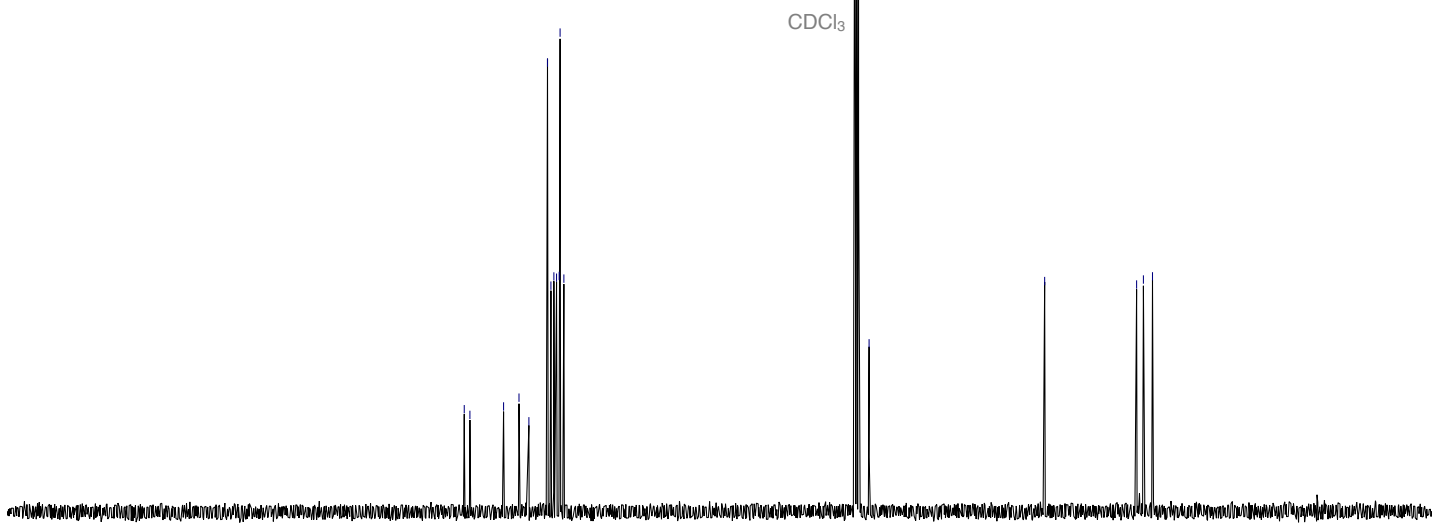

$\begin{array}{llllllllllllllllllllllll}210 & 200 & 190 & 180 & 170 & 160 & 150 & 140 & 130 & 120 & 110 & \begin{array}{c}100 \\ \mathrm{f} 1(\mathrm{ppm})\end{array} & 90 & 80 & 70 & 60 & 50 & 40 & 30 & 20 & 10 & 0 & -10\end{array}$ 
${ }^{1} \mathrm{H}$ NMR: $500 \mathrm{MHz}, \mathrm{CDCl}_{3}$, compound 10d

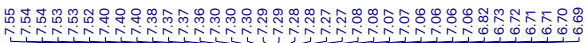

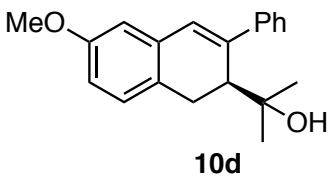

$10 d$

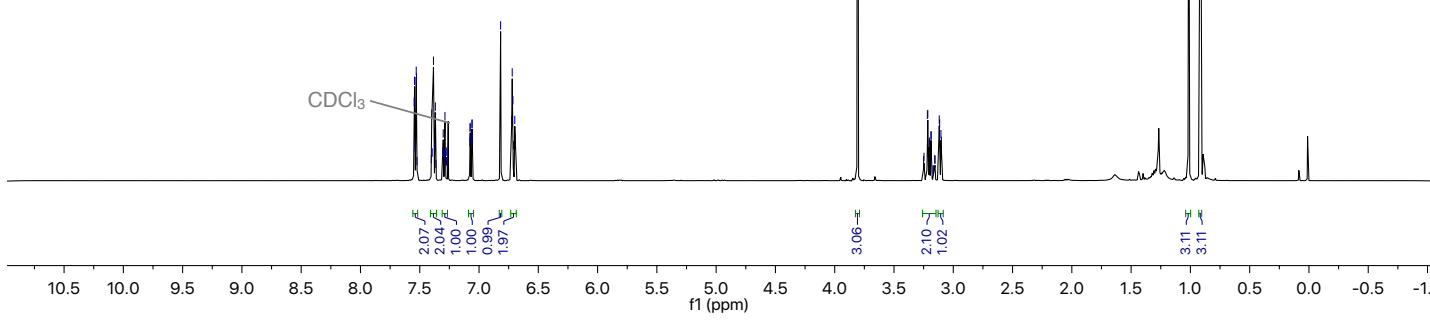

${ }^{13} \mathrm{C}$ NMR: $126 \mathrm{MHz}, \mathrm{CDCl}_{3}$, compound 10d

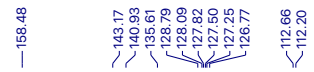

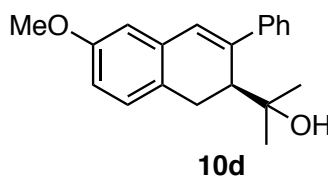

$\begin{array}{lllllllllllllllllllllllllllllllllllllllll}20 & 210 & 200 & 190 & 180 & 170 & 160 & 150 & 140 & 130 & 120 & 110 & 100 & 90 & 80 & 70 & 60 & 50 & 40 & 30 & 20 & 10 & 0 & -10 & -2\end{array}$ 
${ }^{1} \mathrm{H}$ NMR: $400 \mathrm{MHz}, \mathrm{CD}_{2} \mathrm{Cl}_{2}$, compound 10e

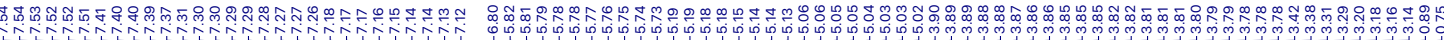<smiles>C=CCOC(C)(C)C1Cc2ccccc2C=C1c1ccccc1</smiles>

$10 \mathrm{e}$

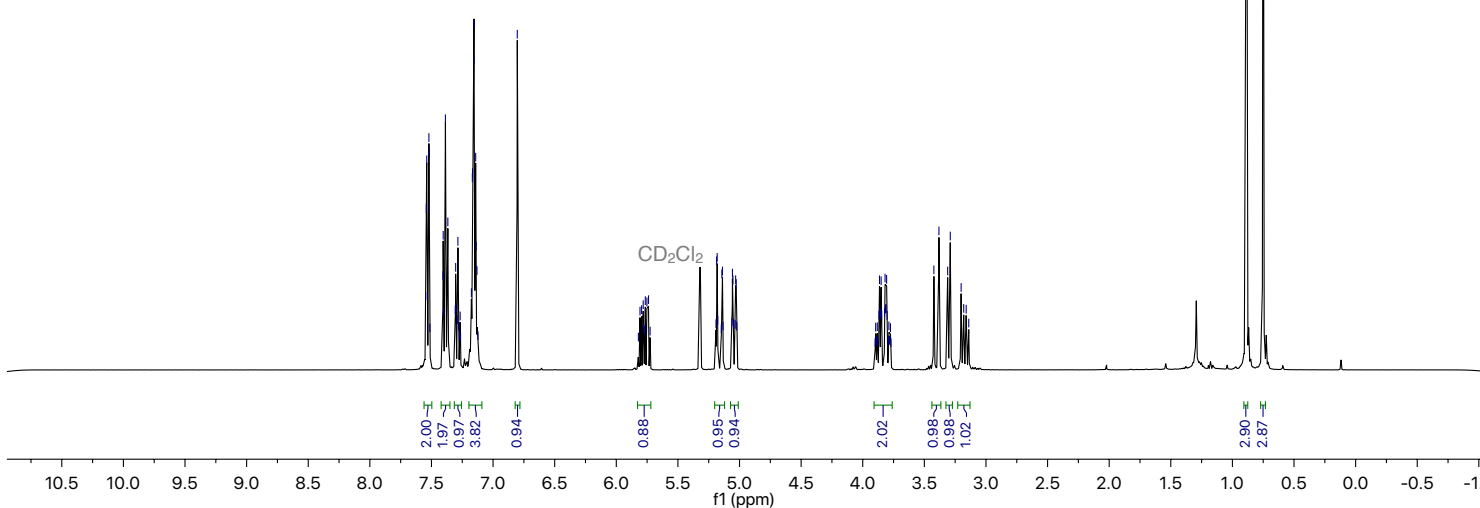

${ }^{13} \mathrm{C}$ NMR: $126 \mathrm{MHz}, \mathrm{CD}_{2} \mathrm{Cl}_{2}$, compound $\mathbf{1 0 e}$

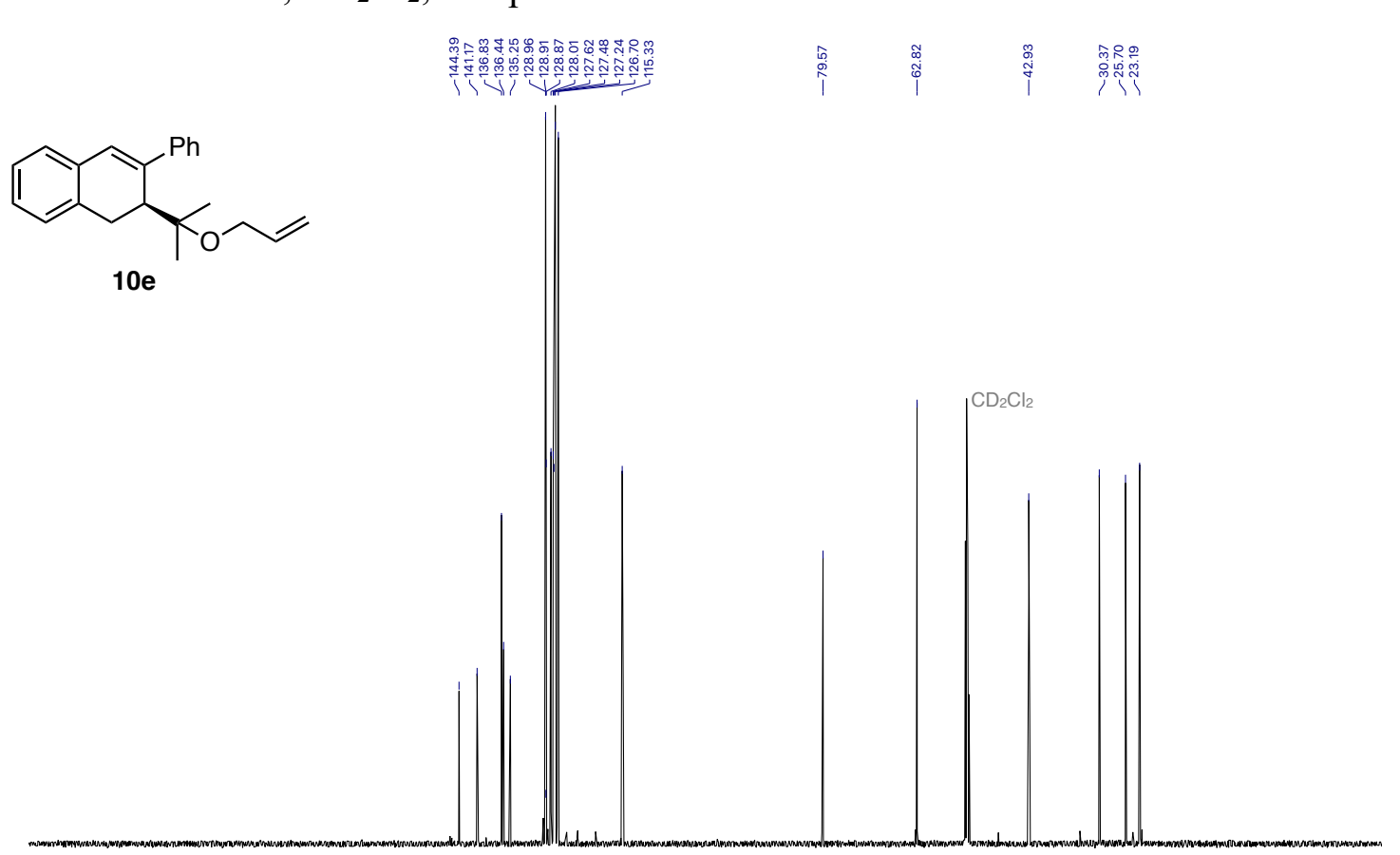

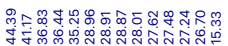

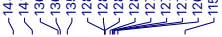

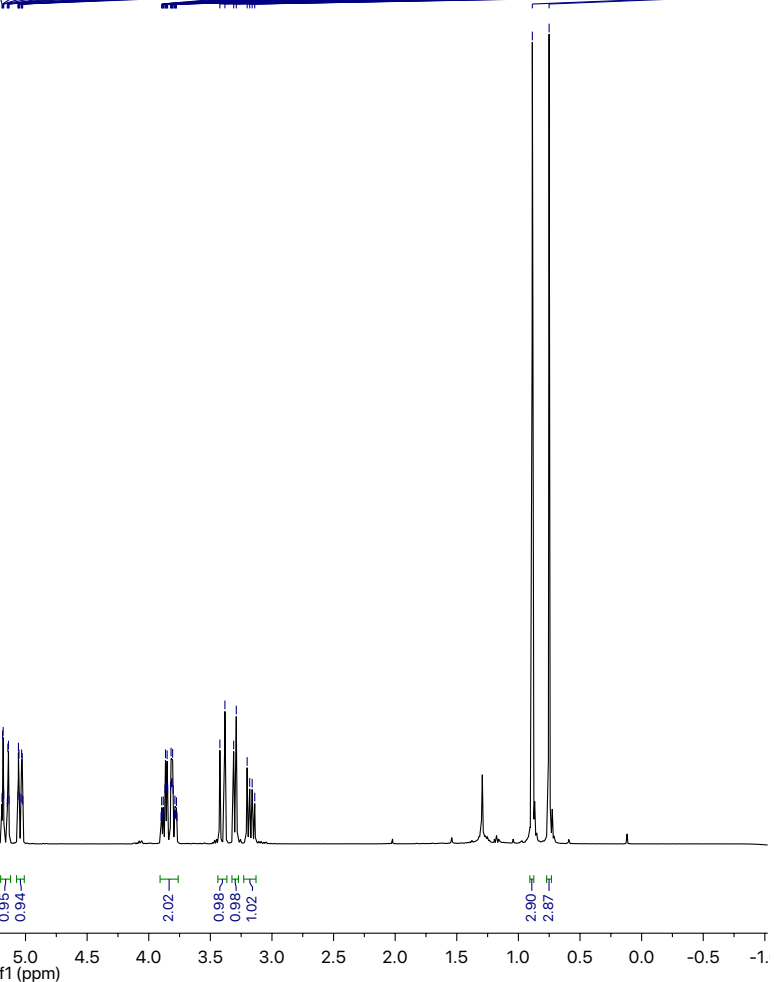

\begin{tabular}{llllllllllllllllllllllll} 
& 20 & 210 & 200 & 190 & 180 & 170 & 160 & 150 & 140 & 130 & 120 & 110 & 100 & 1 & 1 & 1 & 1 & 1 & 1 & 1 & 1 & 1 & 1 \\
\hline
\end{tabular} 
${ }^{1} \mathrm{H}$ NMR: $400 \mathrm{MHz}, \mathrm{CD}_{2} \mathrm{Cl}_{2}$, compound $\mathbf{1 0 f}$

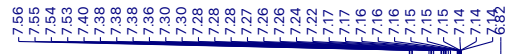

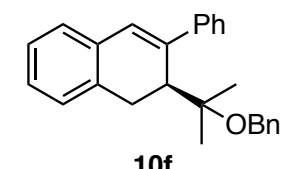

$10 \mathrm{f}$

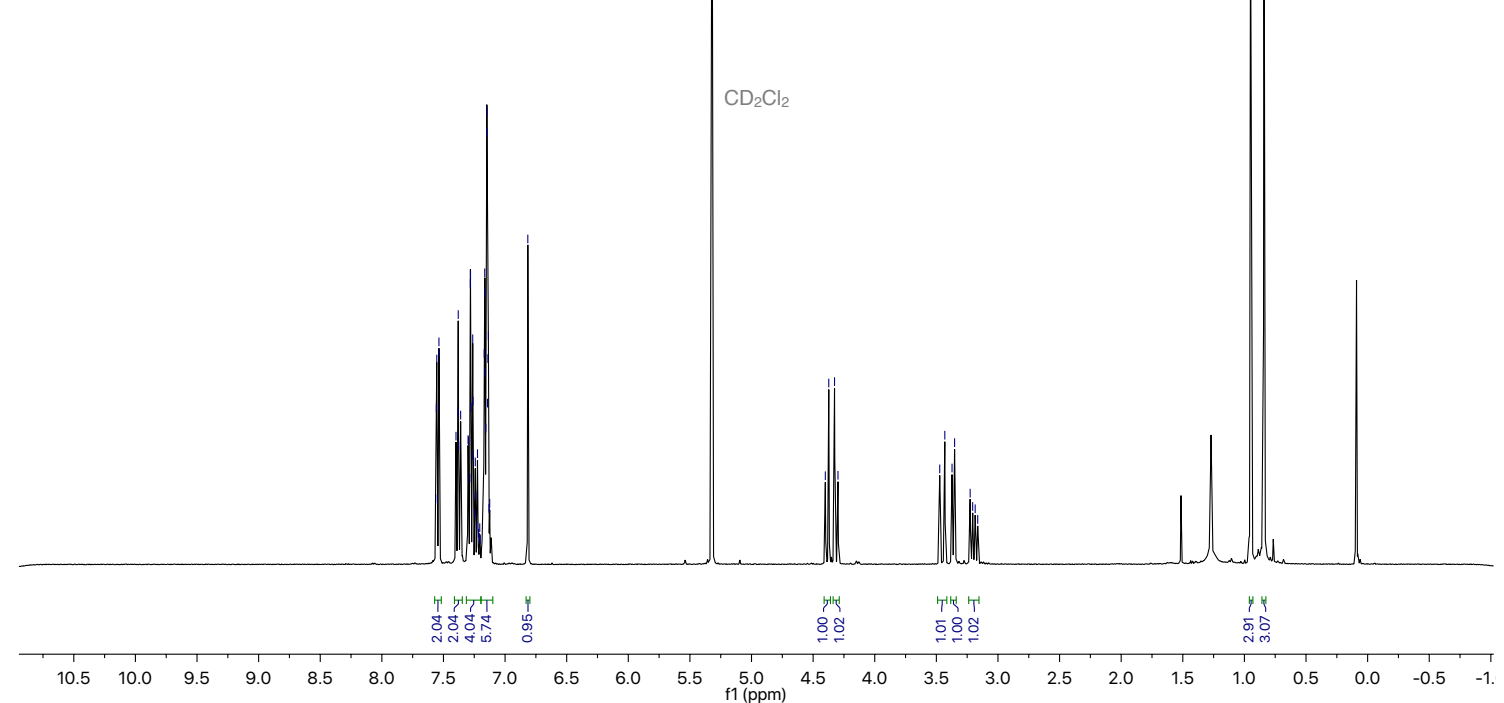

${ }^{13} \mathrm{C}$ NMR: $126 \mathrm{MHz}, \mathrm{CD}_{2} \mathrm{Cl}_{2}$, compound $\mathbf{1 0 f}$

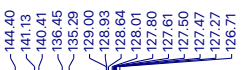<smiles>CC(C)(Br)C1Cc2ccccc2C=C1c1ccccc1</smiles>
$10 \mathrm{f}$

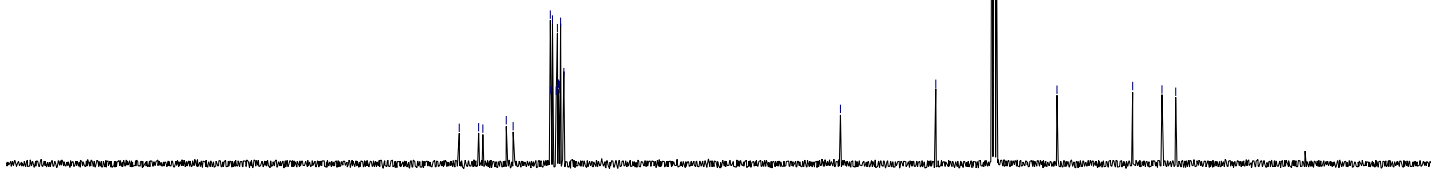

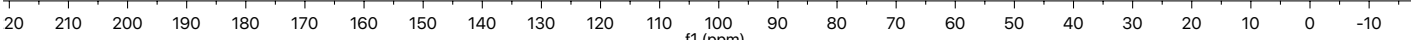


${ }^{1} \mathrm{H}$ NMR: $400 \mathrm{MHz}, \mathrm{CD}_{2} \mathrm{Cl}_{2}$, compound $10 \mathrm{~g}$

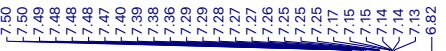<smiles>CC(=O)OC(C)(C)C1Cc2ccccc2C=C1c1ccccc1</smiles>

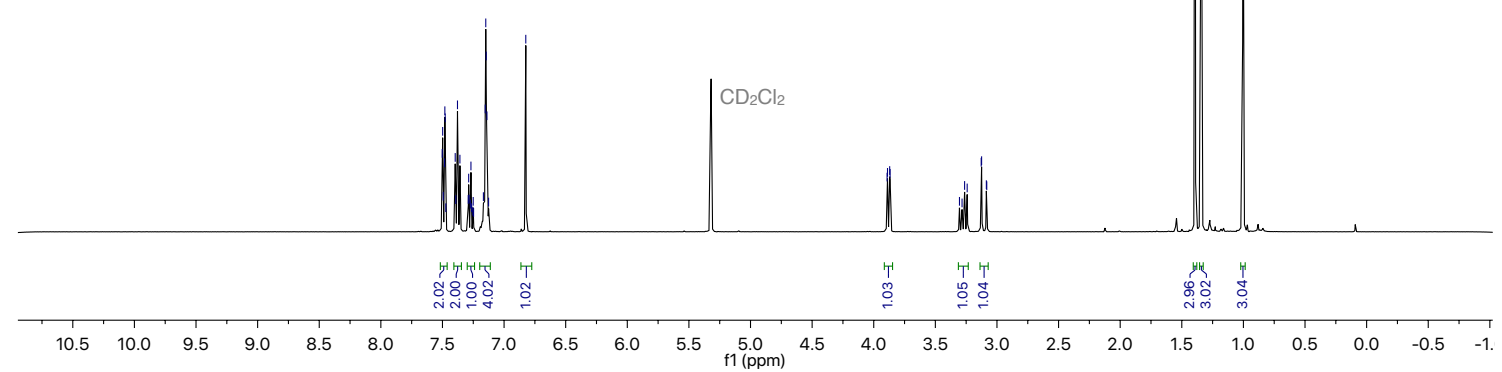

${ }^{13} \mathrm{C}$ NMR: $126 \mathrm{MHz}, \mathrm{CD}_{2} \mathrm{Cl}_{2}$, compound $\mathbf{1 0 g}$<smiles>CC(=O)OC(C)(C)C1Cc2ccccc2C=C1c1ccccc1</smiles>

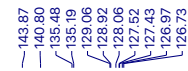
11 Ac

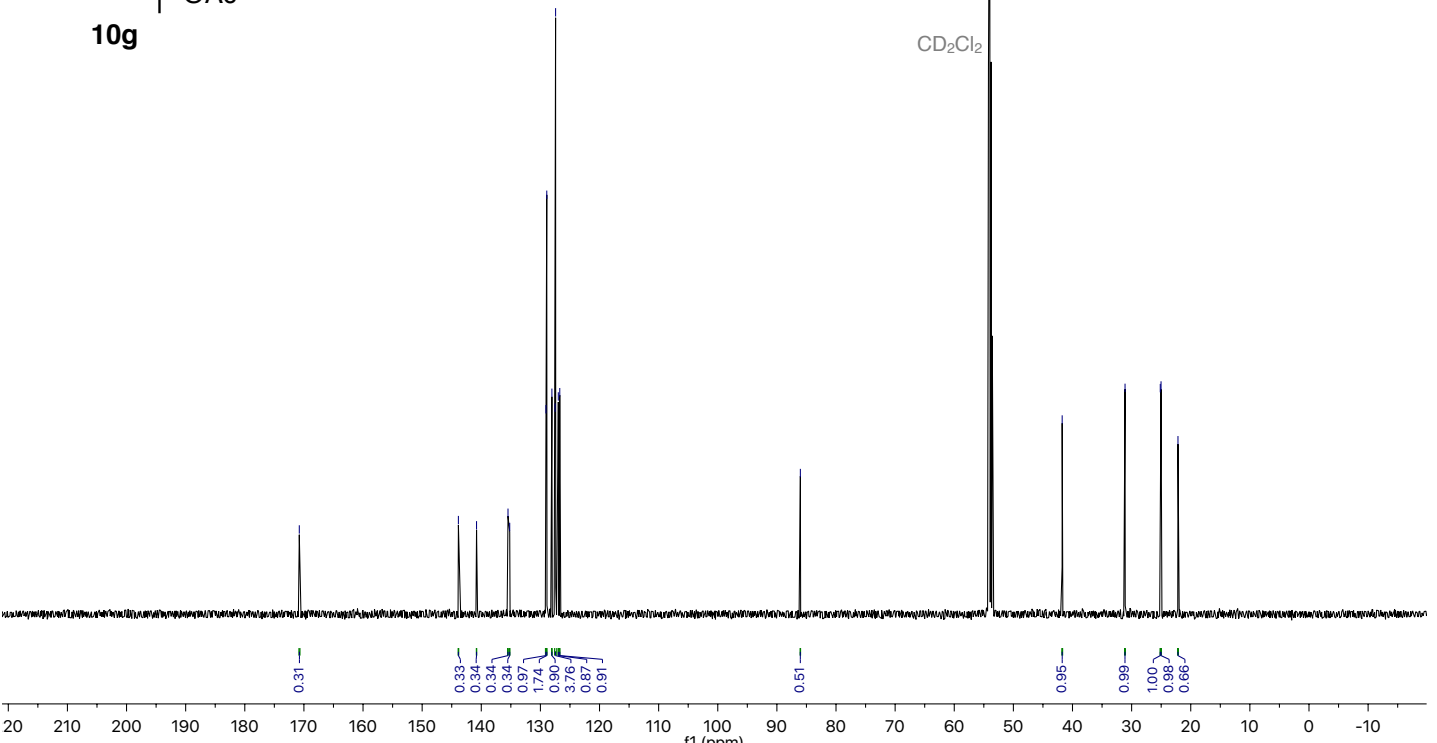


${ }^{1} \mathrm{H}$ NMR: $400 \mathrm{MHz}, \mathrm{CD}_{2} \mathrm{Cl}_{2}$, compound 10h

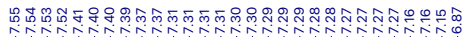

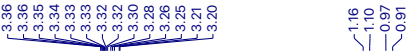<smiles>CC(F)(F)C1Cc2ccccc2C=C1c1ccccc1</smiles>

$10 \mathrm{~h}$

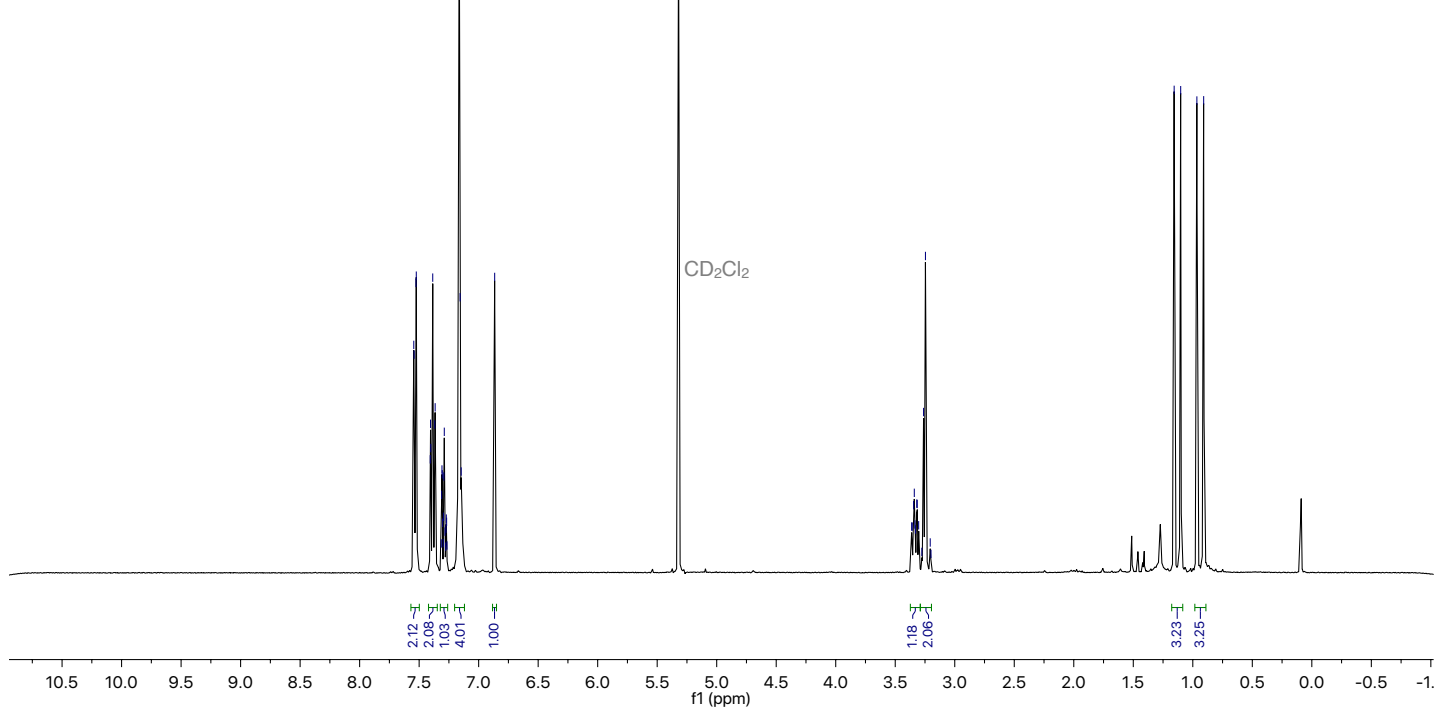

${ }^{13} \mathrm{C}$ NMR: $126 \mathrm{MHz}, \mathrm{CD}_{2} \mathrm{Cl}_{2}$, compound $\mathbf{1 0 h}$

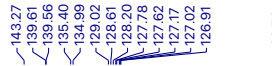<smiles>CC(F)(F)C1Cc2ccccc2C=C1c1ccccc1</smiles>

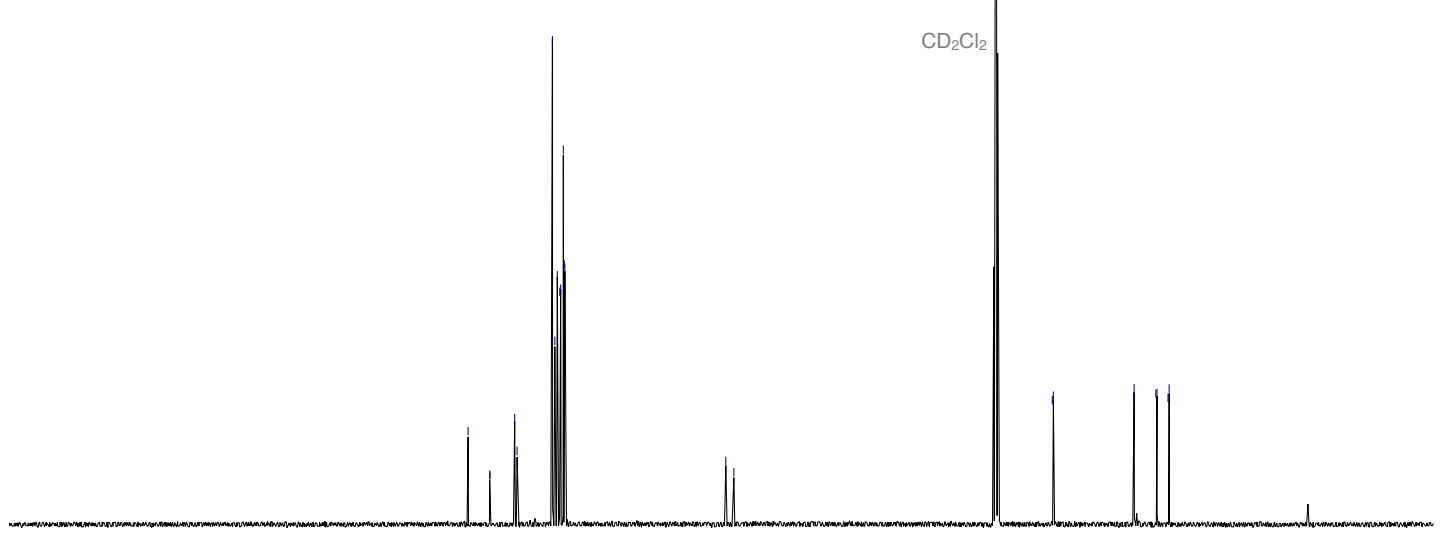

$\begin{array}{llllllllllllllllllllllllllllllllll} & 20 & 210 & 200 & 190 & 180 & 170 & 160 & 150 & 140 & 130 & 120 & 110 & 100 & 90 & 80 & 70 & 60 & 50 & 40 & 30 & 20 & 10 & 0 & -10\end{array}$ 
${ }^{19} \mathrm{~F}\left\{{ }^{1} \mathrm{H}\right\}$ NMR: $376 \mathrm{MHz}, \mathrm{CD}_{2} \mathrm{Cl}_{2}$, compound 10h
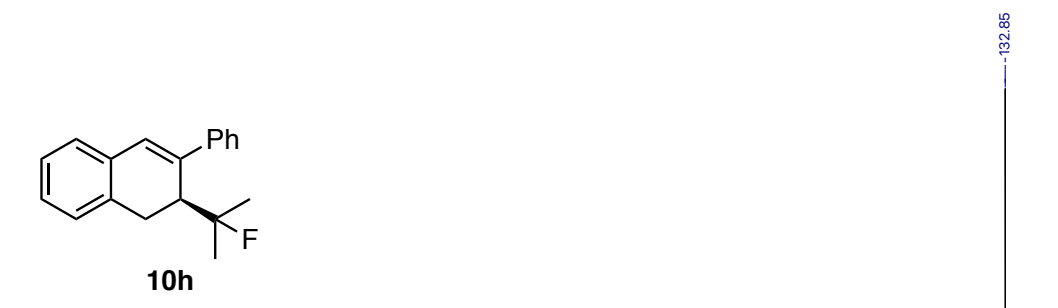

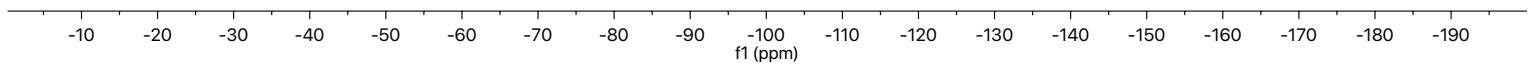

${ }^{1} \mathrm{H}$ NMR: $500 \mathrm{MHz}, \mathrm{CD}_{2} \mathrm{Cl}_{2}$, compound 10i

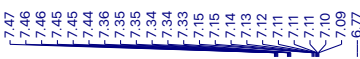

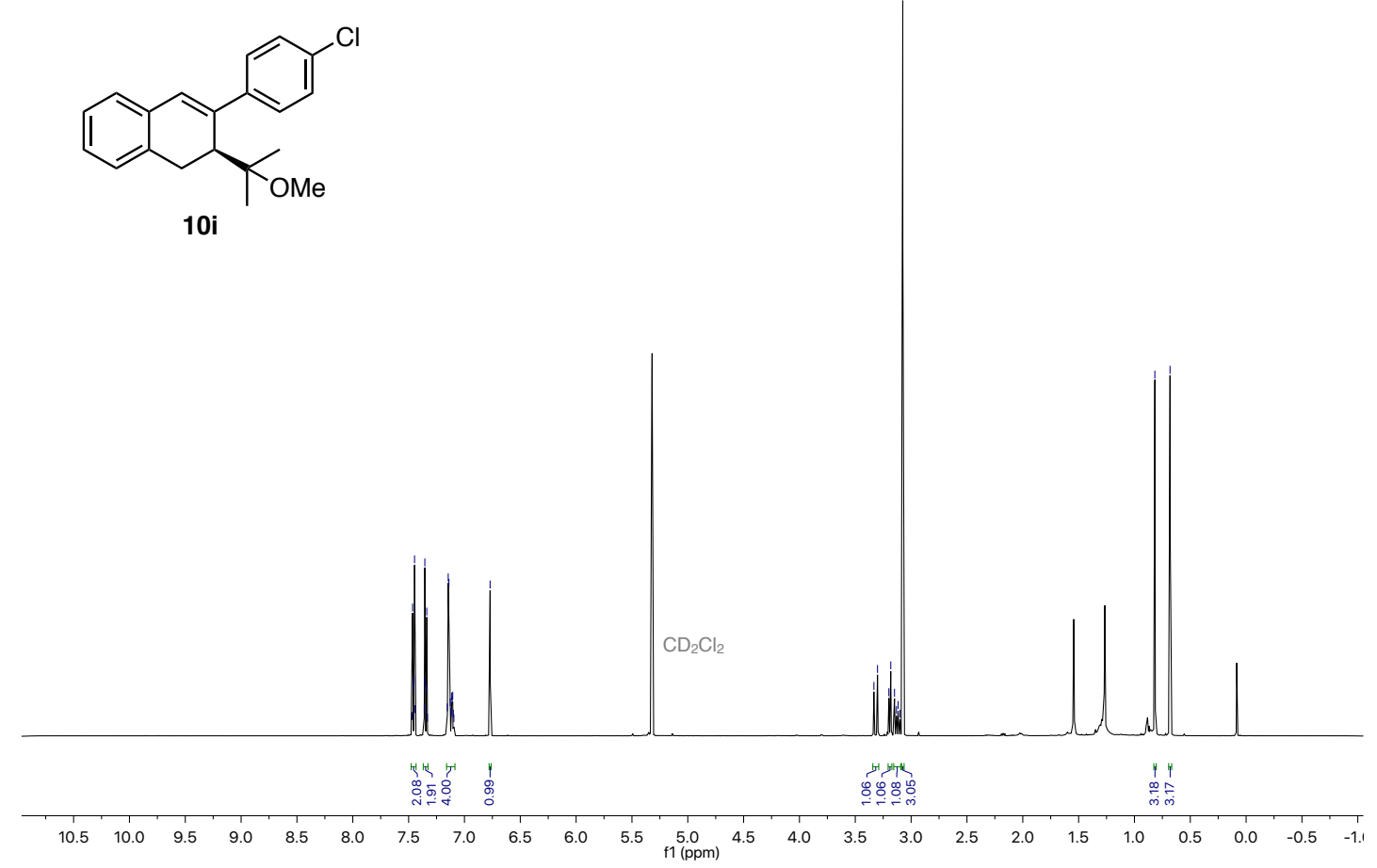

$10 \mathrm{i}$

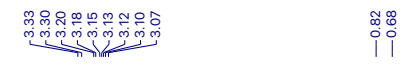

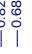


${ }^{13} \mathrm{C}$ NMR: $126 \mathrm{MHz}, \mathrm{CD}_{2} \mathrm{Cl}_{2}$, compound 10i
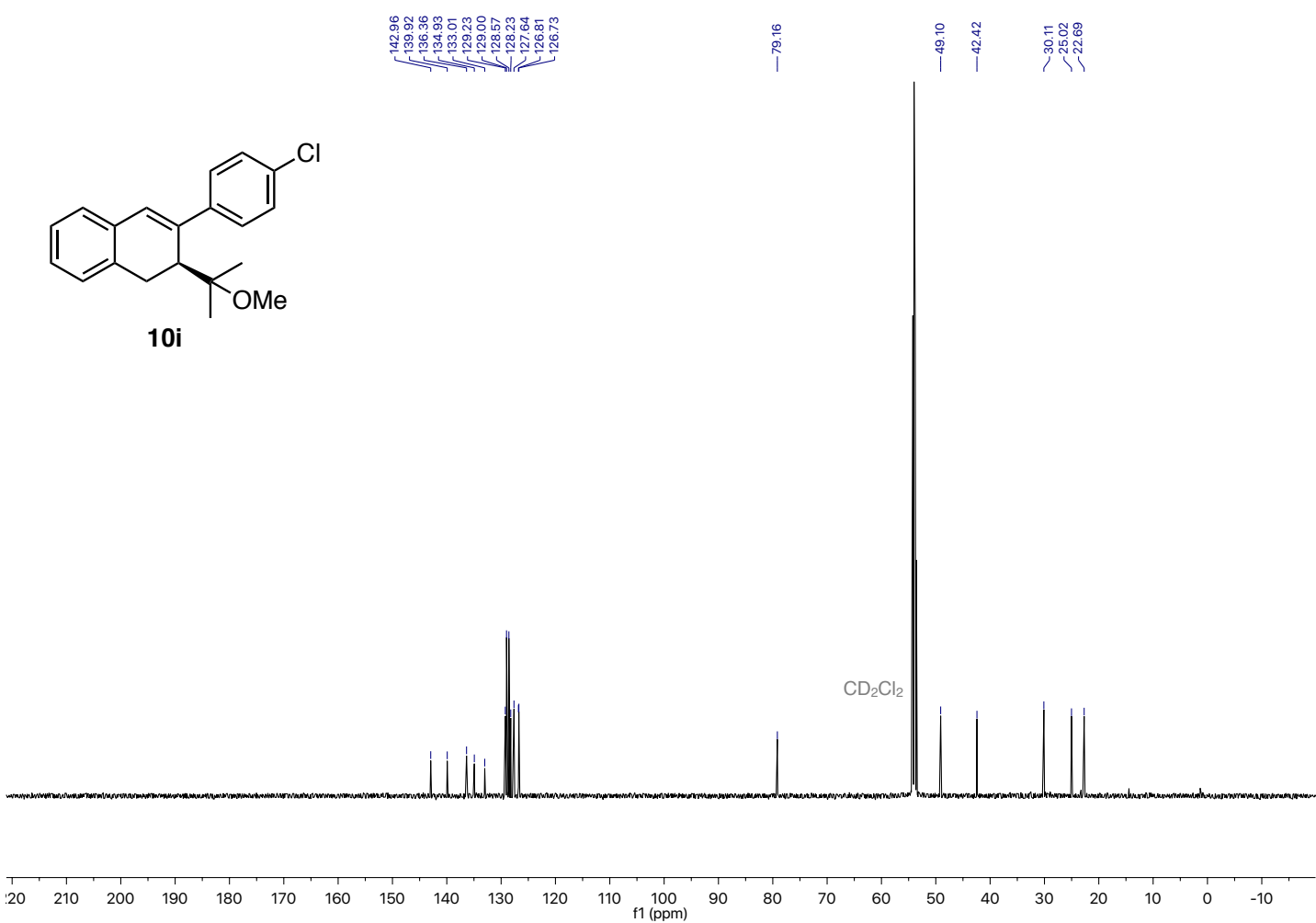

${ }^{1} \mathrm{H}$ NMR: $400 \mathrm{MHz}, \mathrm{CDCl}_{3}$, compound 10k

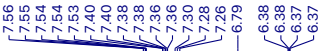<smiles>COc1cc2c(c(OC)c1)CC(C(C)(C)O)C(c1ccccc1)=C2</smiles>

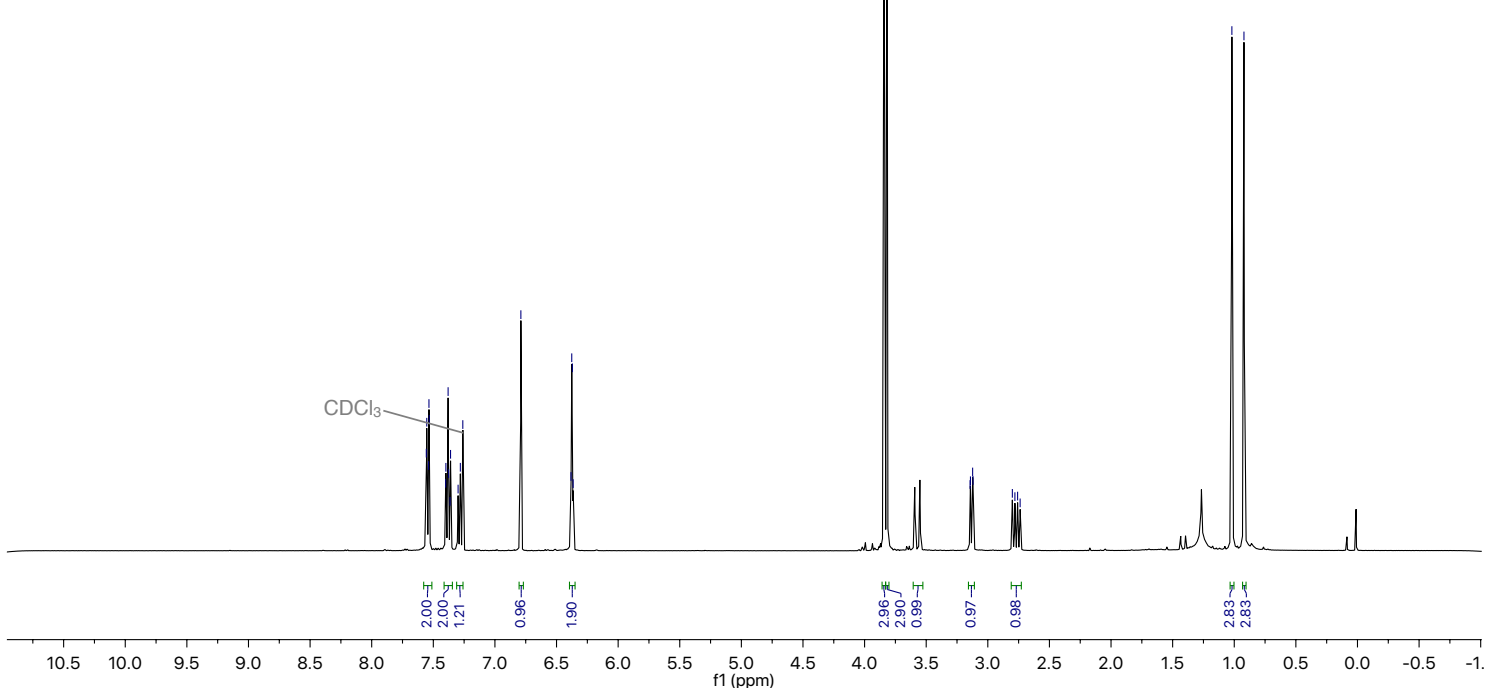


${ }^{13} \mathrm{C}$ NMR: $101 \mathrm{MHz}, \mathrm{CDCl}_{3}$, compound 10k
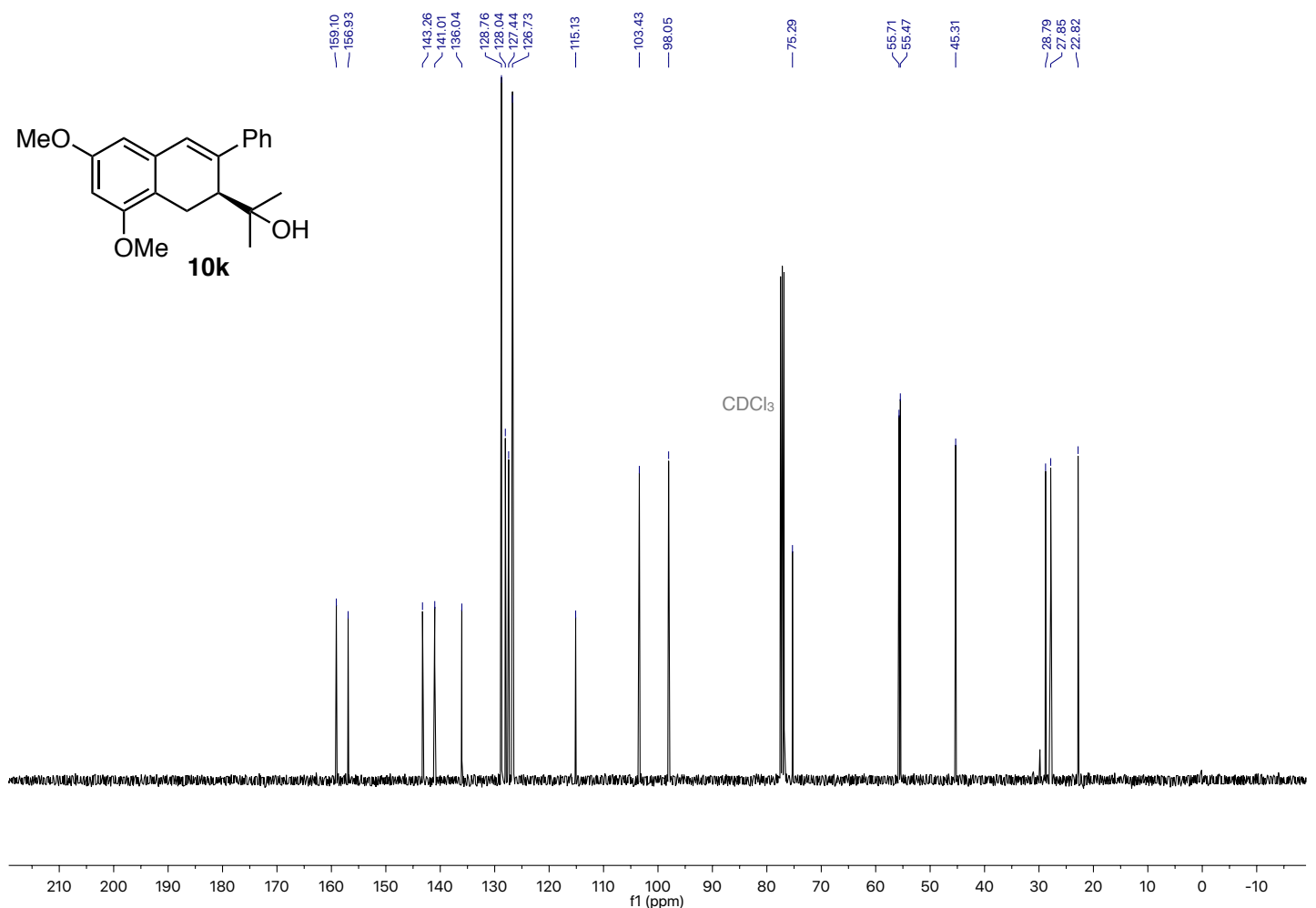

${ }^{1} \mathrm{H}$ NMR: $500 \mathrm{MHz}, \mathrm{CDCl}_{3}$, compound 101

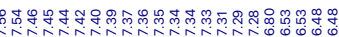

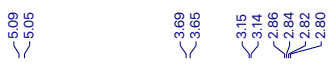<smiles>CC(C)(C)Oc1cc(O)c2c(c1)C=C(c1ccccc1)C(C(C)(C)O)C2</smiles>
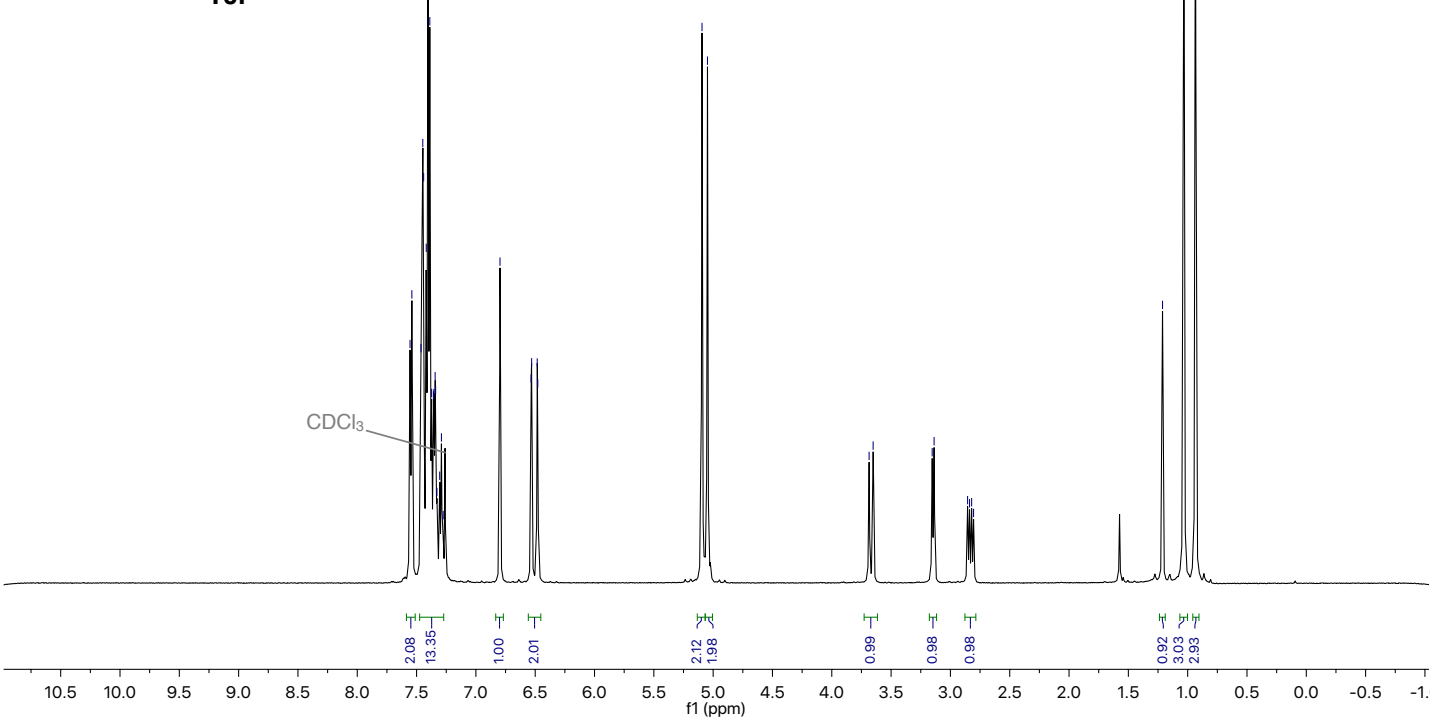
${ }^{13} \mathrm{C}$ NMR: $126 \mathrm{MHz}, \mathrm{CDCl}_{3}$, compound 101
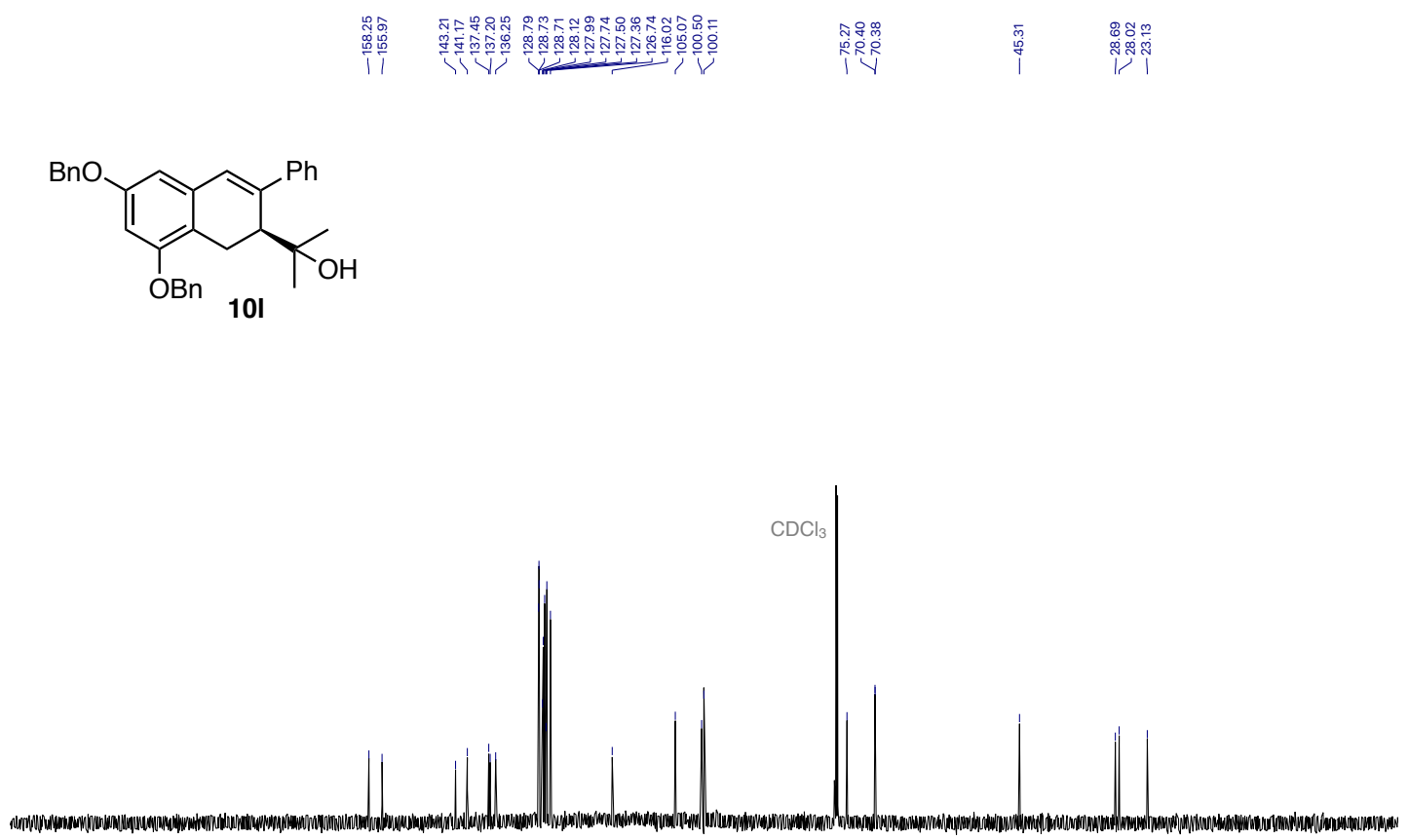

$\begin{array}{lllllllllllllllllllllllllllll}1 & 20 & 210 & 200 & 190 & 180 & 170 & 160 & 150 & 140 & 130 & 120 & 110 & \begin{array}{c}100 \\ \mathrm{f}(\mathrm{ppm})\end{array} & 90 & 80 & 70 & 60 & 50 & 40 & 30 & 20 & 10 & 0 & -10 & -2\end{array}$

${ }^{1} \mathrm{H}$ NMR: $500 \mathrm{MHz}, \mathrm{CDCl}_{3}$, compound 11a

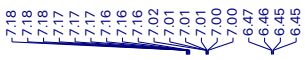
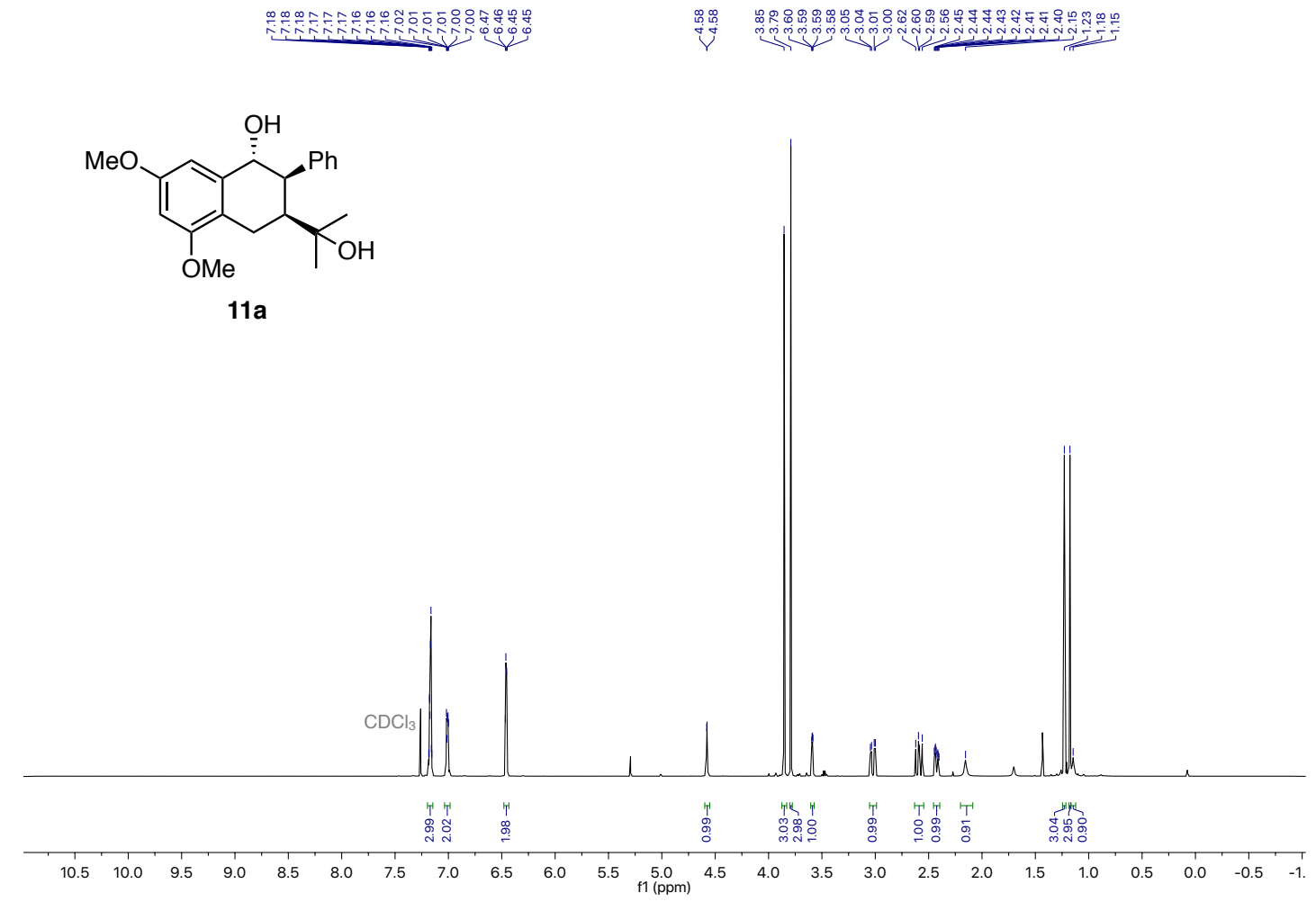

$11 \mathrm{a}$ 
${ }^{13} \mathrm{C}$ NMR: $126 \mathrm{MHz}, \mathrm{CDCl}_{3}$, compound 11a
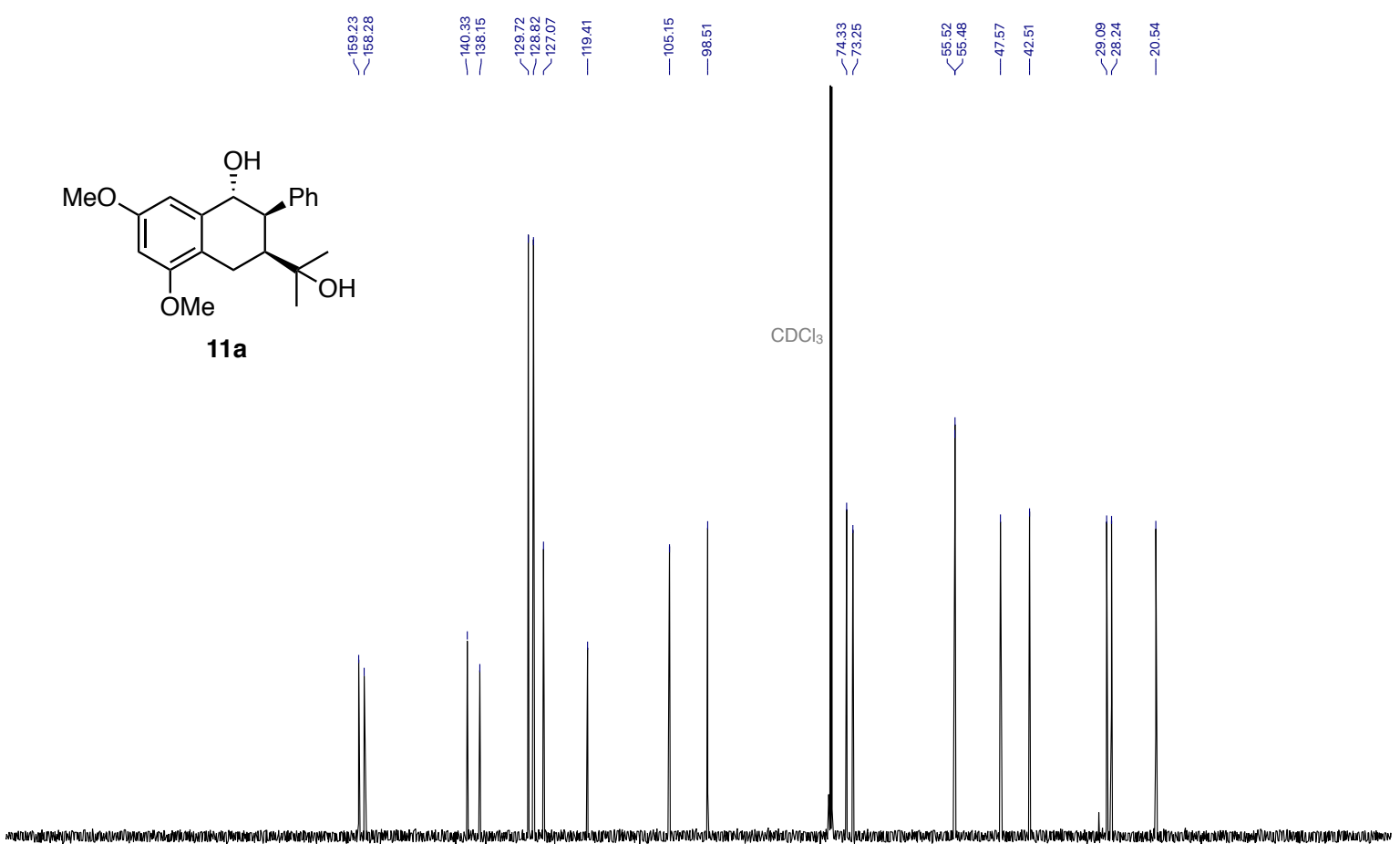

\begin{tabular}{|lllllllllllllllllllllllllll}
\hline 20 & 210 & 200 & 190 & 180 & 170 & 160 & 150 & 140 & 130 & 120 & 110 & $\begin{array}{c}100 \\
\mathrm{f} 1(\mathrm{ppm})\end{array}$ & 90 & 80 & 70 & 60 & 50 & 40 & 30 & 20 & 10 & 0 & -10 & -2
\end{tabular}

${ }^{1} \mathrm{H}$ NMR: $500 \mathrm{MHz}, \mathrm{CDCl}_{3}$, compound 12

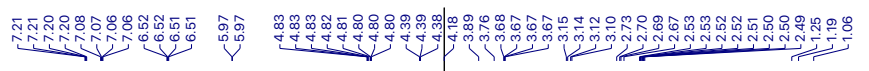
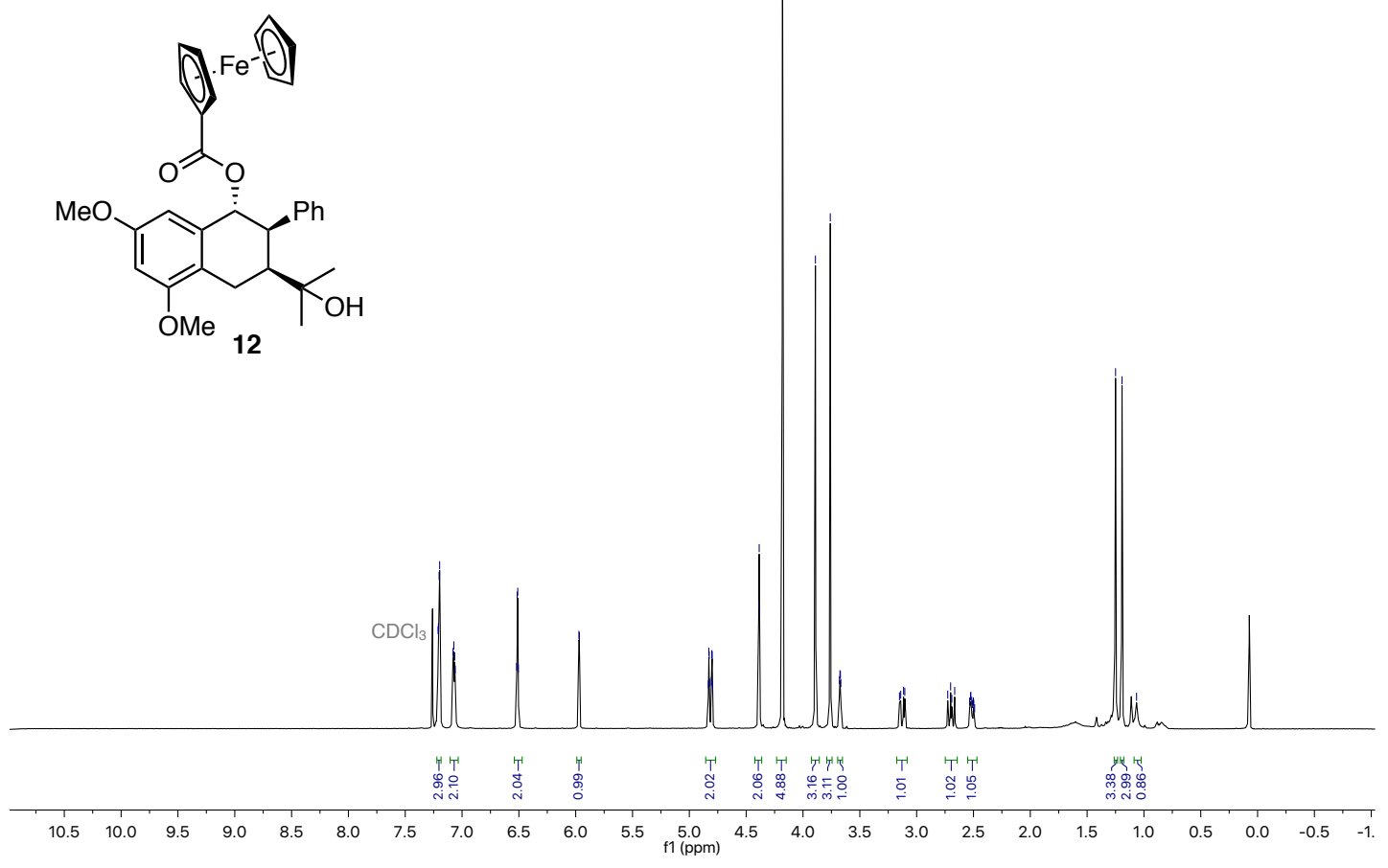
${ }^{13} \mathrm{C}$ NMR: $126 \mathrm{MHz}, \mathrm{CDCl}_{3}$, compound 12

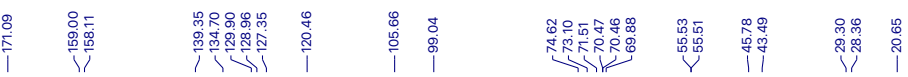
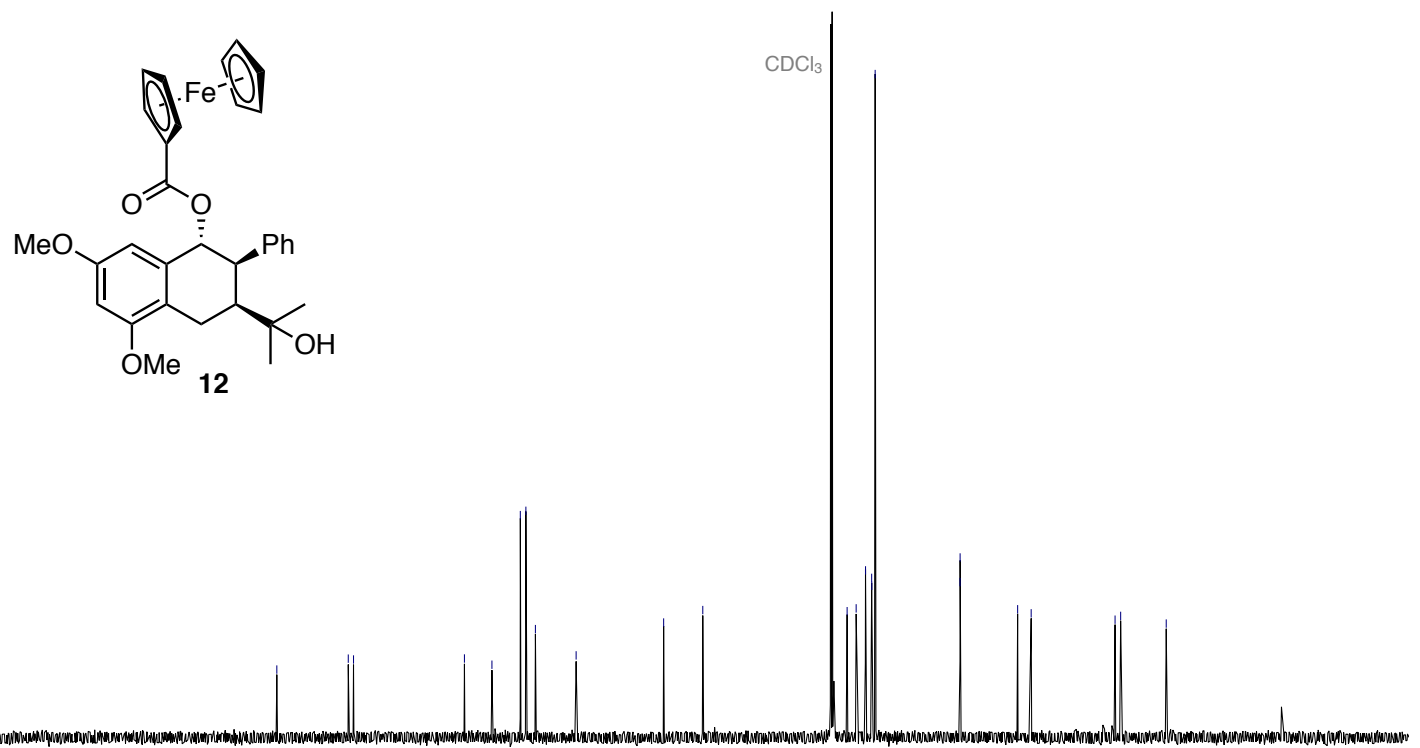

$\begin{array}{llllllllllllllllllllllllllll}1 & 20 & 210 & 200 & 190 & 180 & 170 & 160 & 150 & 140 & 130 & 120 & 110 & \begin{array}{c}100 \\ 100\end{array} & 90 & 80 & 70 & 60 & 50 & 40 & 30 & 20 & 10 & 0 & -10 & -2\end{array}$

${ }^{1} \mathrm{H}$ NMR: $500 \mathrm{MHz}, \mathrm{CDCl}_{3}$, compound 13

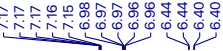

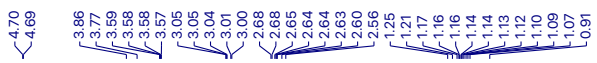<smiles>COc1cc(OC)c2c(c1)[C@H](O[18OH])[C@H](c1ccccc1)C(C(C)(C)O)C2</smiles>

13

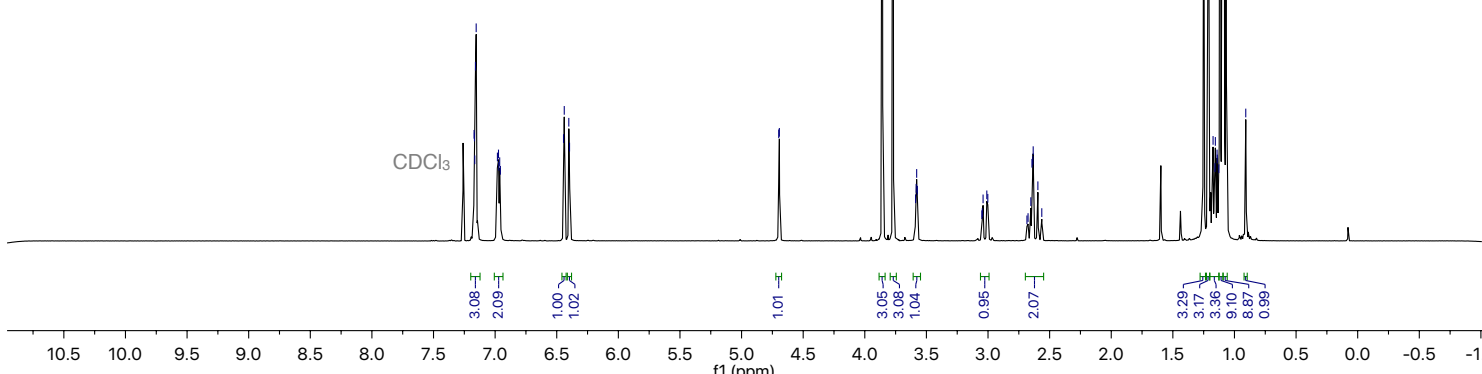


${ }^{13} \mathrm{C}$ NMR: $126 \mathrm{MHz}, \mathrm{CDCl}_{3}$, compound 13
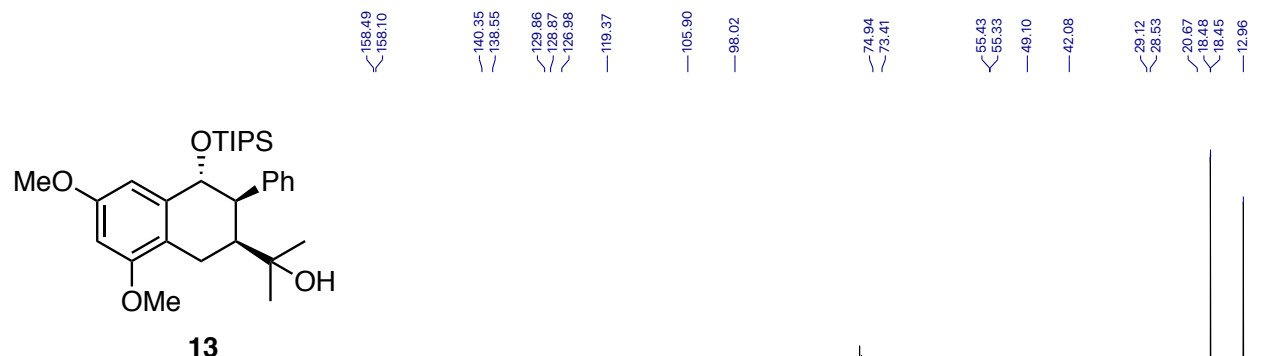

13

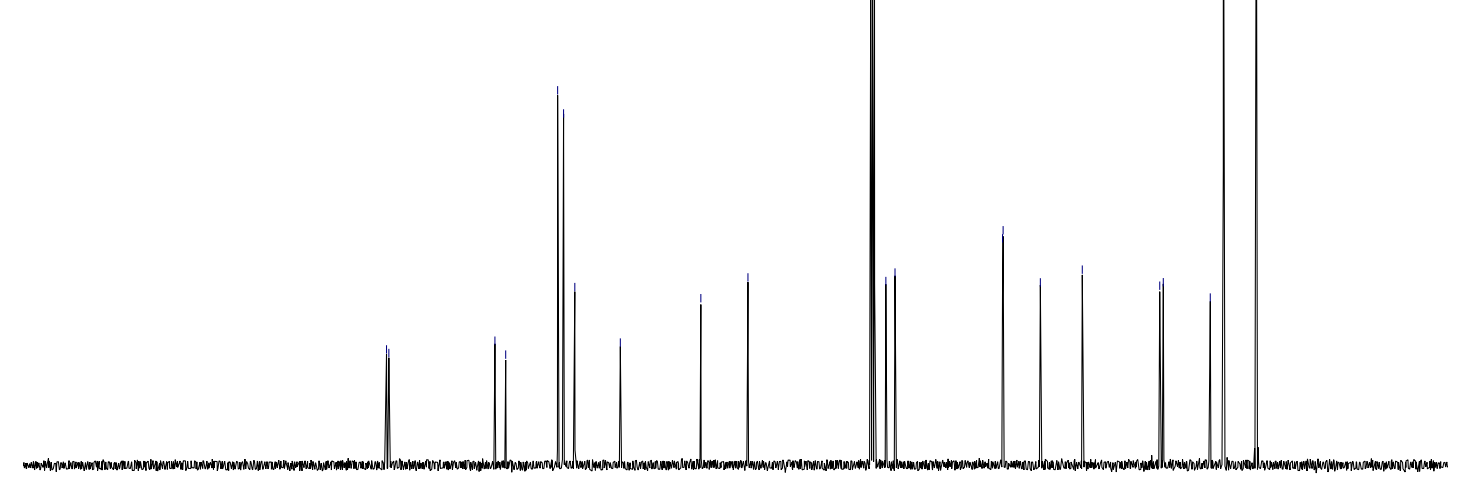

$\begin{array}{llllllllllllllllllllllll}1 & 210 & 200 & 190 & 180 & 170 & 160 & 150 & 140 & 130 & 120 & 110 & \begin{array}{c}1 \\ \mathrm{f}(\mathrm{ppm})\end{array} & 90 & 80 & 70 & 60 & 50 & 40 & 30 & 20 & 10 & 0 & -10\end{array}$

${ }^{1} \mathrm{H}$ NMR: $400 \mathrm{MHz}, \mathrm{CDCl}_{3}$, compound 14

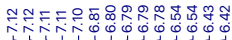
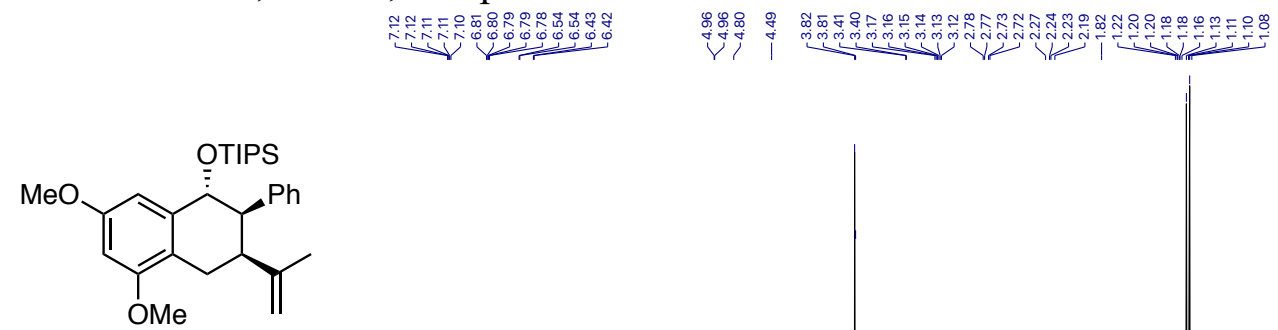

14

Y)

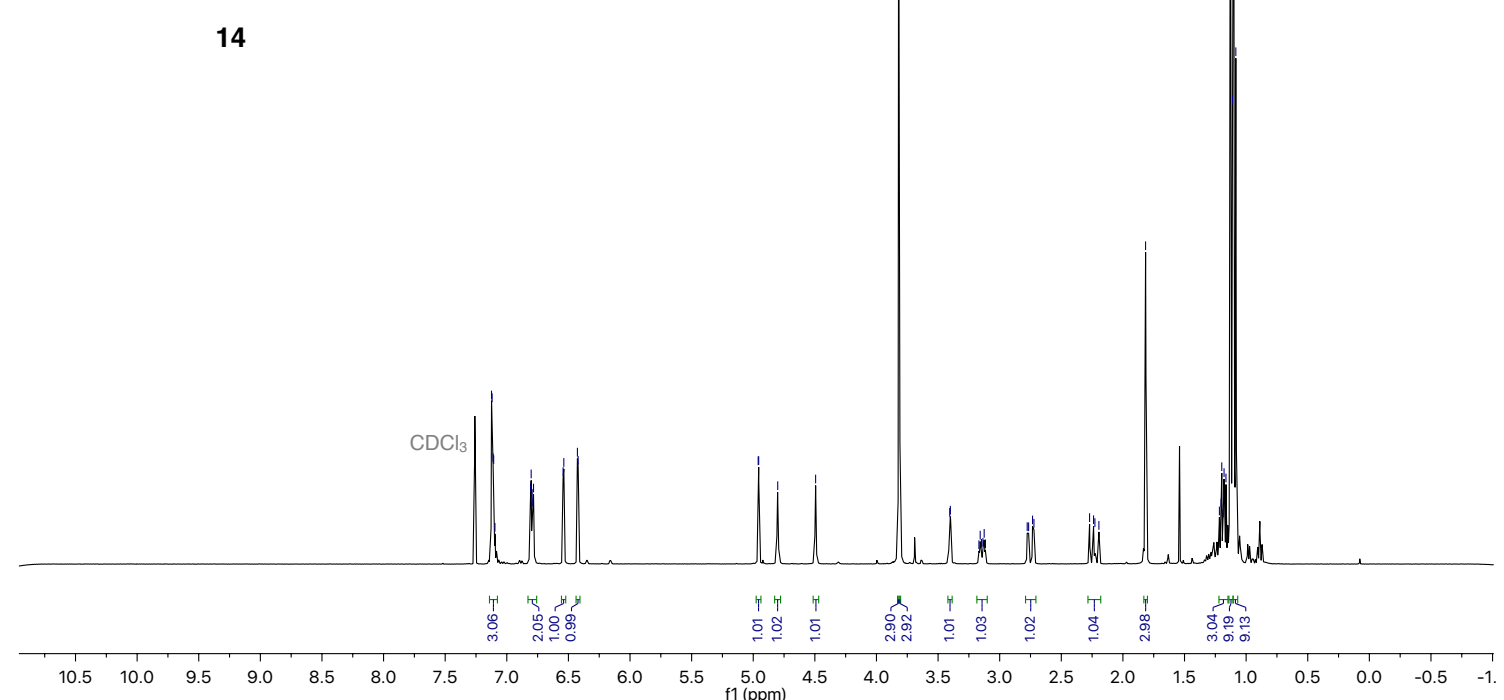


${ }^{13} \mathrm{C}$ NMR: $101 \mathrm{MHz}, \mathrm{CDCl}_{3}, 14$

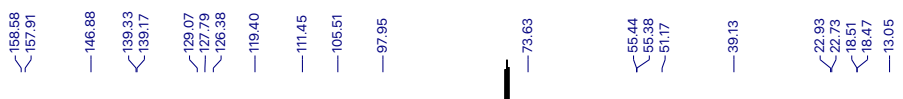<smiles>C=C(C)C1Cc2c(OC)cc(OC)cc2C(O)C1c1ccccc1</smiles>

14

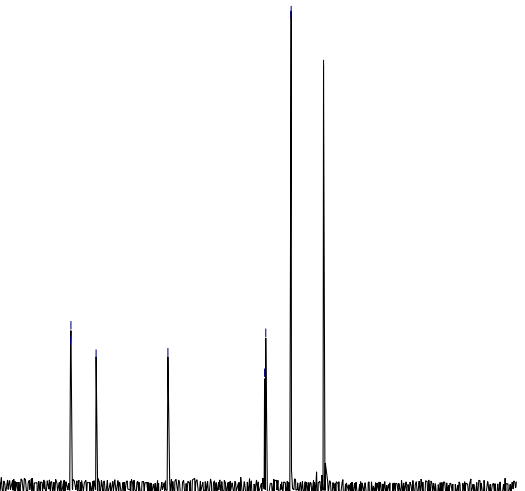

${ }^{1} \mathrm{H}$ NMR: $400 \mathrm{MHz}, \mathrm{CD}_{3} \mathrm{OD}$, carexane I

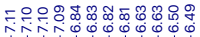<smiles>C=C(C)C1Cc2c(OC)cc(OC)cc2[C@@H](O)[C@@H]1c1ccccc1</smiles>

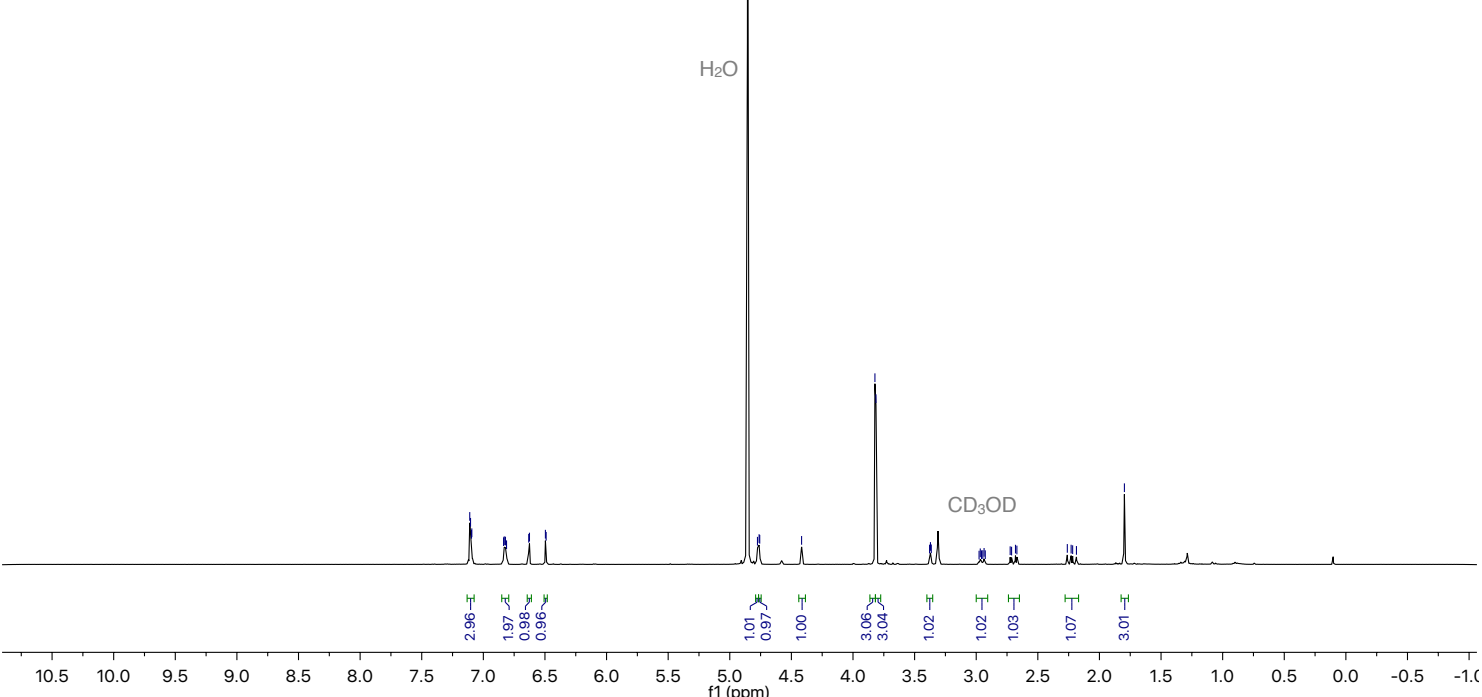


${ }^{13} \mathrm{C}$ NMR: $101 \mathrm{MHz}, \mathrm{CD}_{3} \mathrm{OD}$, carexane I<smiles>C=C(C)C1Cc2c(OC)cc(OC)cc2C(O)C1c1ccccc1</smiles>

${ }^{1} \mathrm{H}$ NMR: $500 \mathrm{MHz}, \mathrm{CD}_{2} \mathrm{Cl}_{2},(5 R, 6 R, 7 S)-3-M e t h o x y-6-p h e n y l-7-($ prop-1-en-2-yl)-5-((triisopropylsilyl)oxy)-5,6,7,8-tetrahydronaphthalen-1-ol and $(6 S, 7 R, 8 R)-4-M e t h o x y-7-p h e n y l-6-$ (prop-1-en-2-yl)-8-((triisopropylsilyl)oxy)-5,6,7,8-tetrahydronaphthalen-2-ol

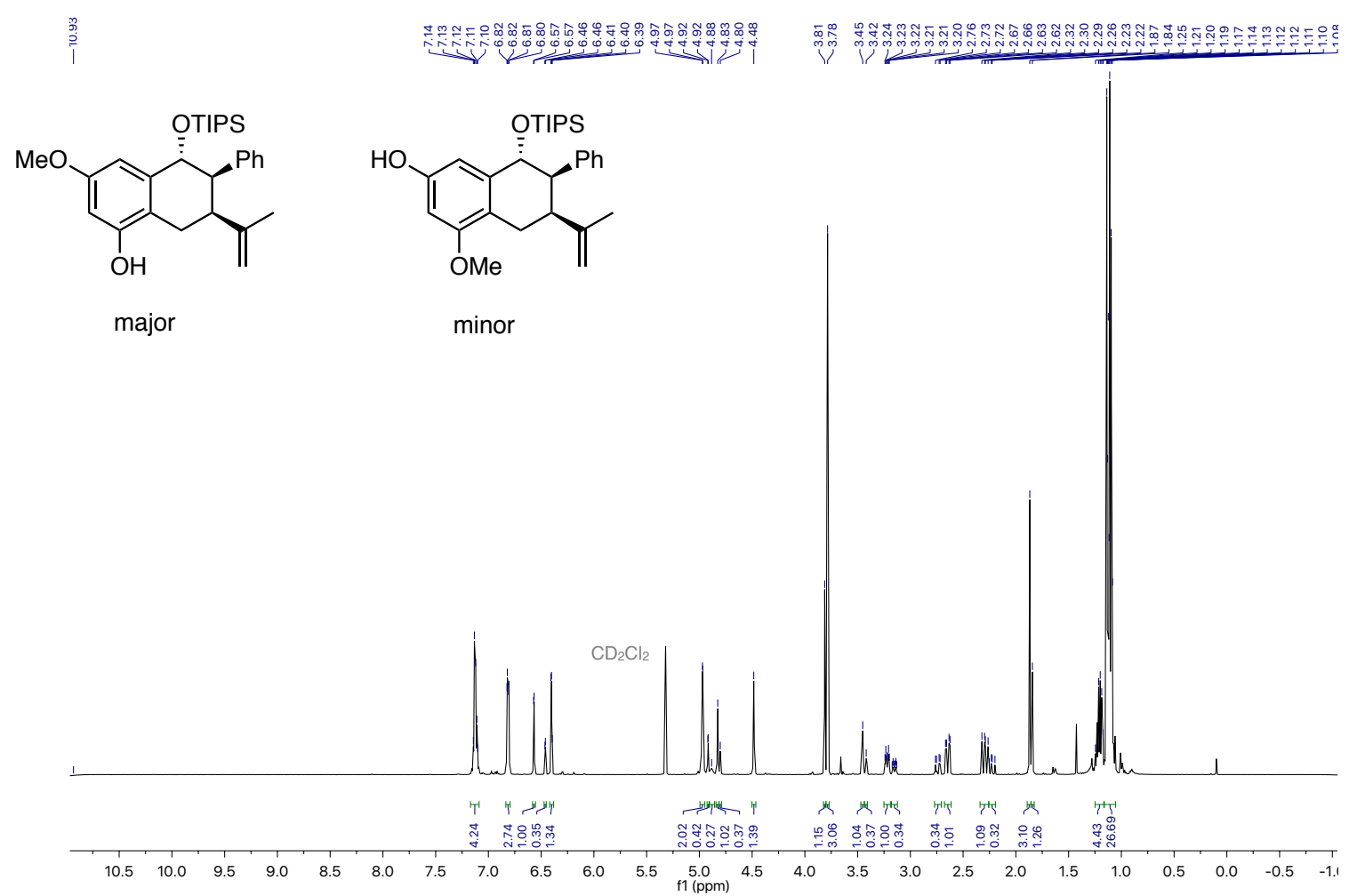


${ }^{13} \mathrm{C}$ NMR: $126 \mathrm{MHz}, \mathrm{CD}_{2} \mathrm{Cl}_{2},(5 R, 6 R, 7 S)$-3-Methoxy-6-phenyl-7-(prop-1-en-2-yl)-5-((triisopropylsilyl)oxy)-5,6,7,8-tetrahydronaphthalen-1-ol and (6S,7R,8R)-4-Methoxy-7-phenyl-6(prop-1-en-2-yl)-8-((triisopropylsilyl)oxy)-5,6,7,8-tetrahydronaphthalen-2-ol<smiles>C=C(C)[C@H]1Cc2c(O)cc(OC)cc2[C@H]([O-])[C@@H]1c1ccccc1</smiles>

major

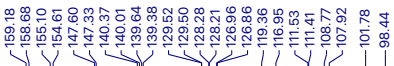<smiles>C=C(C)[C@H]1Cc2c(OC)cc(O)cc2[C@H](OC)[C@@H]1c1ccccc1</smiles>

minor

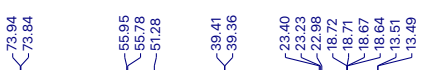

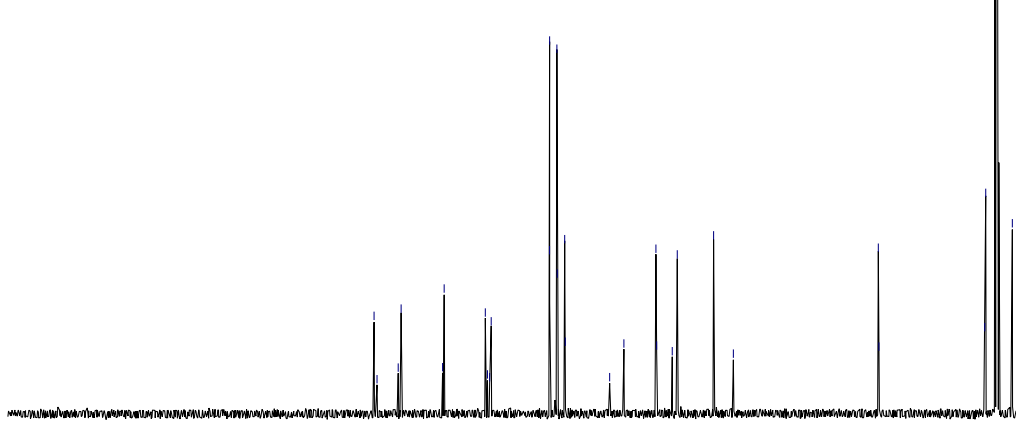

$20 \quad 210 \quad 200 \quad 190 \quad 180 \quad 170 \quad 160$
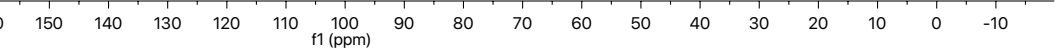

${ }^{1} \mathrm{H}$ NMR: $500 \mathrm{MHz}, \mathrm{CD}_{3} \mathrm{OD}$, carexane P
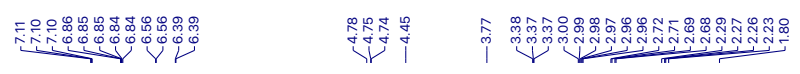<smiles>C=C(C)[C@H]1Cc2c(O)cc(OC)cc2[C@@H](O)[C@@H]1c1ccccc1</smiles>

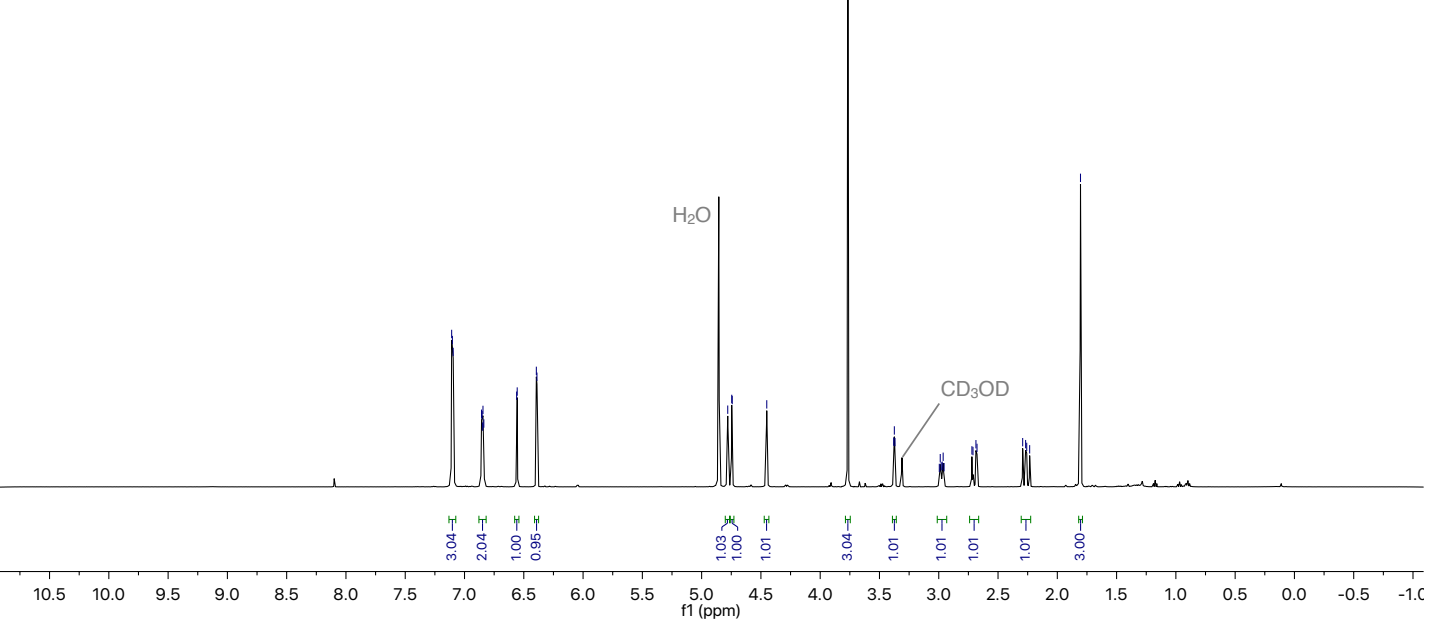


${ }^{13} \mathrm{C}$ NMR: $126 \mathrm{MHz}, \mathrm{CD}_{3} \mathrm{OD}$, carexane $\mathrm{P}$<smiles>C=C(C)C1Cc2c(O)cc(OC)cc2C(O)C1c1ccccc1</smiles>

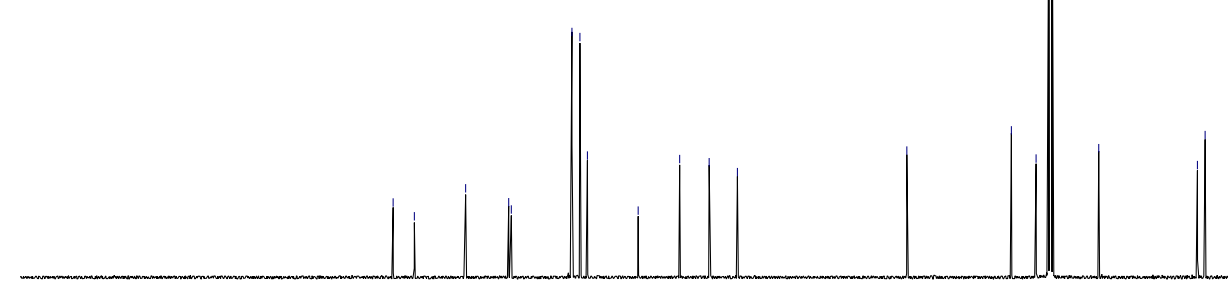

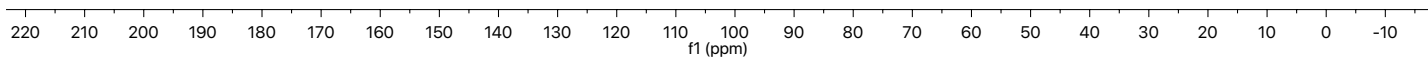

${ }^{1} \mathrm{H}$ NMR: $500 \mathrm{MHz}, \mathrm{CDCl}_{3}$, compound 11b

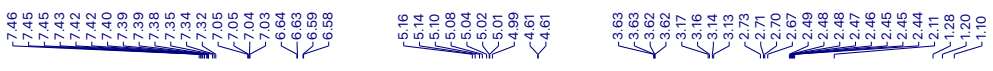<smiles>CC(C)(O)C1Cc2c(OCc3ccccc3)cc(Oc3ccccc3)cc2[C@@H](O)[C@@H]1c1ccccc1</smiles>

11b

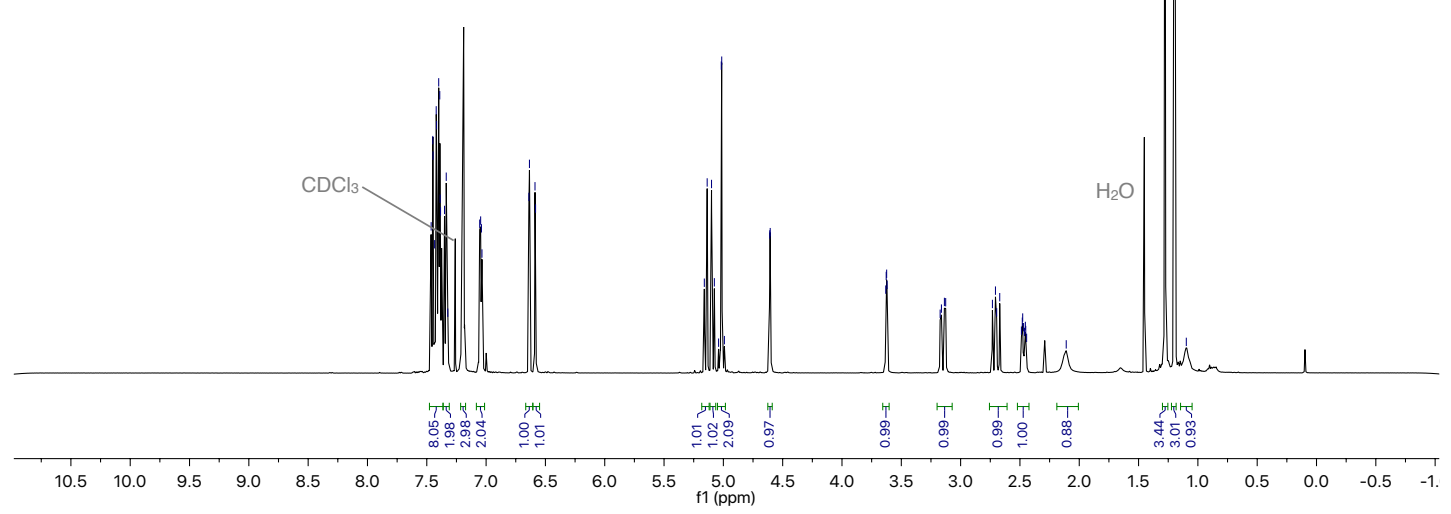


${ }^{13} \mathrm{C}$ NMR: $126 \mathrm{MHz}, \mathrm{CDCl}_{3}$, compound 11b

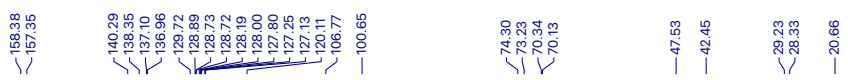<smiles>CC(C)(C)Oc1cc(OCc2ccccc2)c2c(c1)[C@@H](O)[C@H](c1ccccc1)C(C(C)(C)O)C2</smiles>

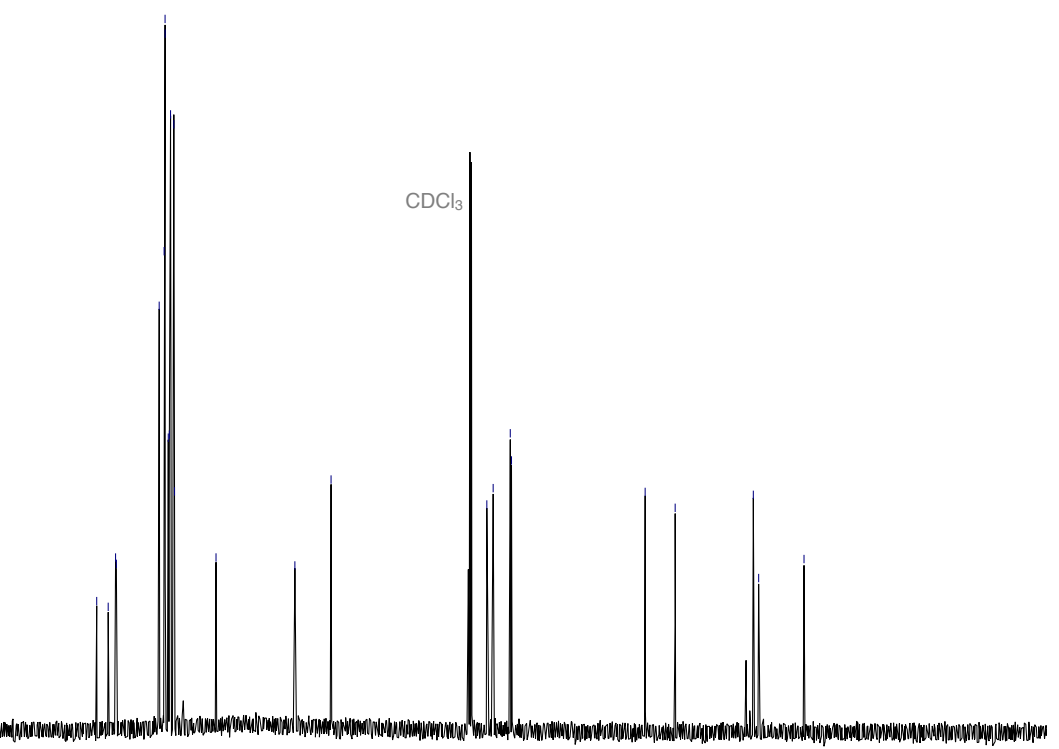

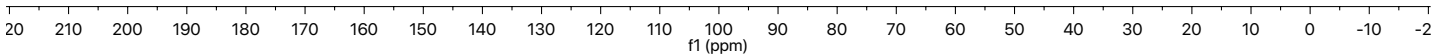

${ }^{1} \mathrm{H}$ NMR: $500 \mathrm{MHz}, \mathrm{CD}_{3} \mathrm{OD}$, Carexane O

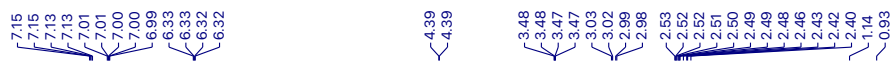<smiles>CC(C)(O)[C@H]1Cc2c(O)cc(O)cc2[C@@H](O)[C@@H]1c1ccccc1</smiles>
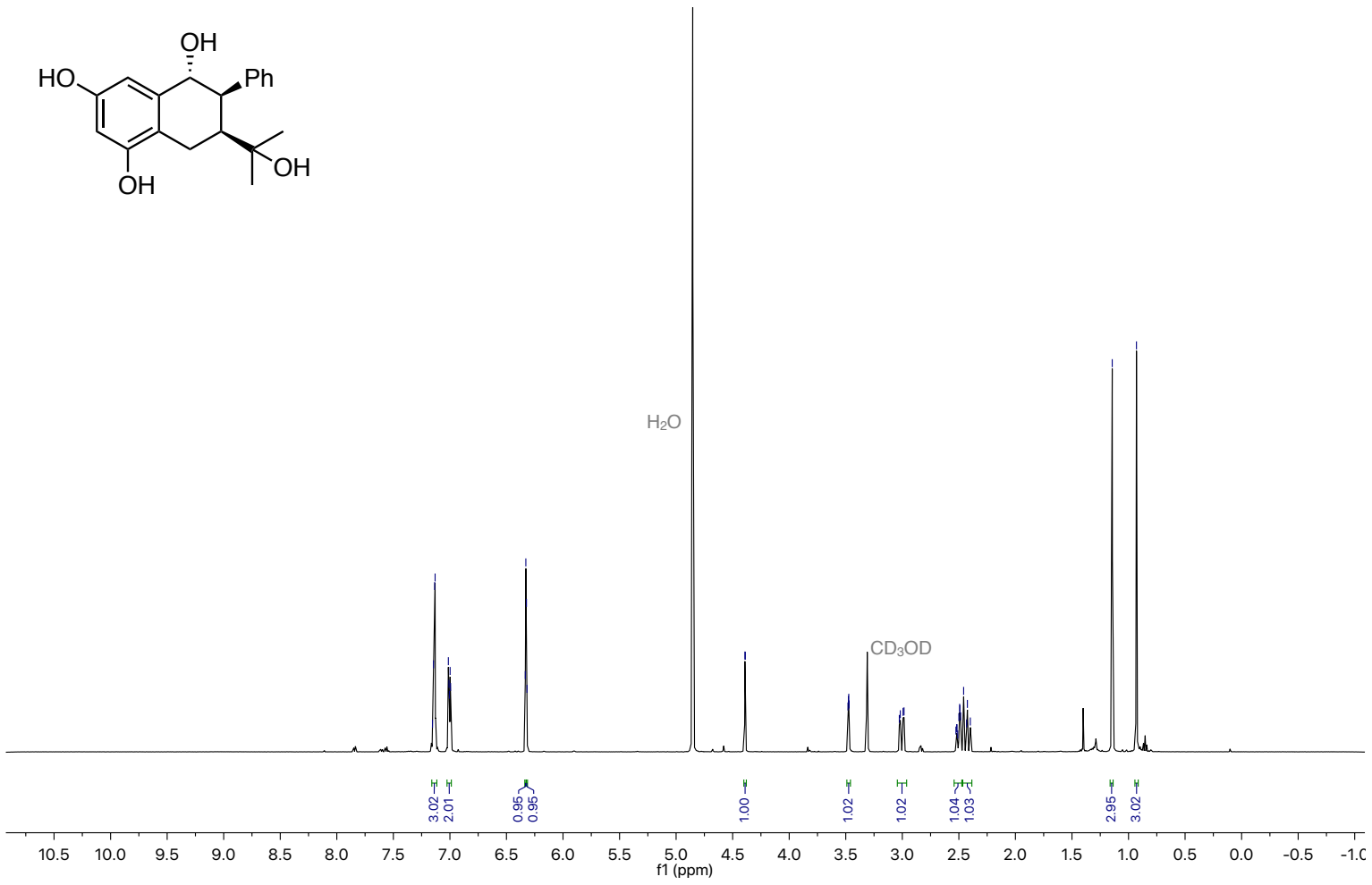
${ }^{13} \mathrm{C}$ NMR: $126 \mathrm{MHz}, \mathrm{CD}_{3} \mathrm{OD}$, Carexane $\mathrm{O}$

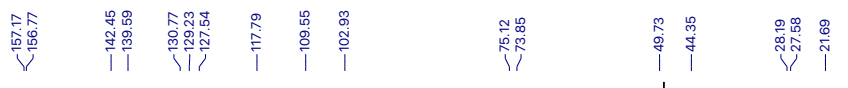<smiles>CC(C)(O)C1Cc2c(O)cc(O)cc2[C@@H](O)C1c1ccccc1</smiles>

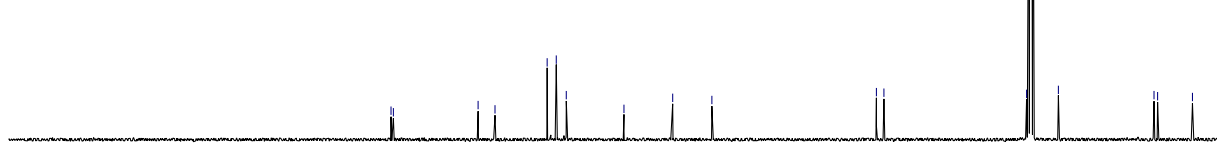

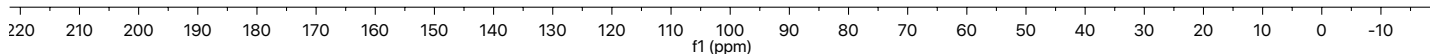




\section{UPC2 and HPLC traces}

Chromatogram of racemic 1-([1,1':3',1"-Terphenyl]-5'-yl)prop-2-en-1-ol
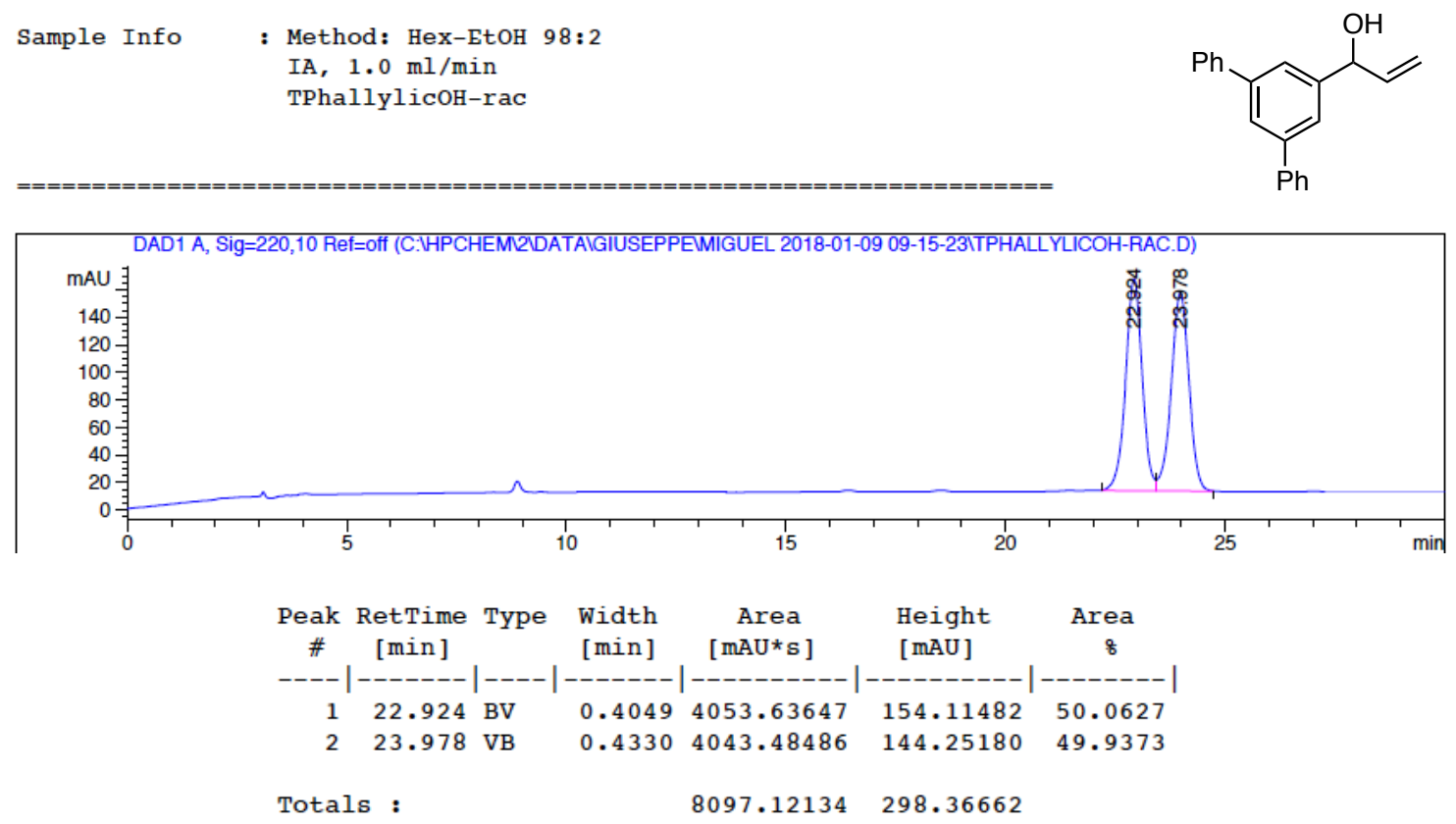

Chromatogram of enantioenriched 1-([1,1':3',1"-Terphenyl]-5'-yl)prop-2-en-1-ol
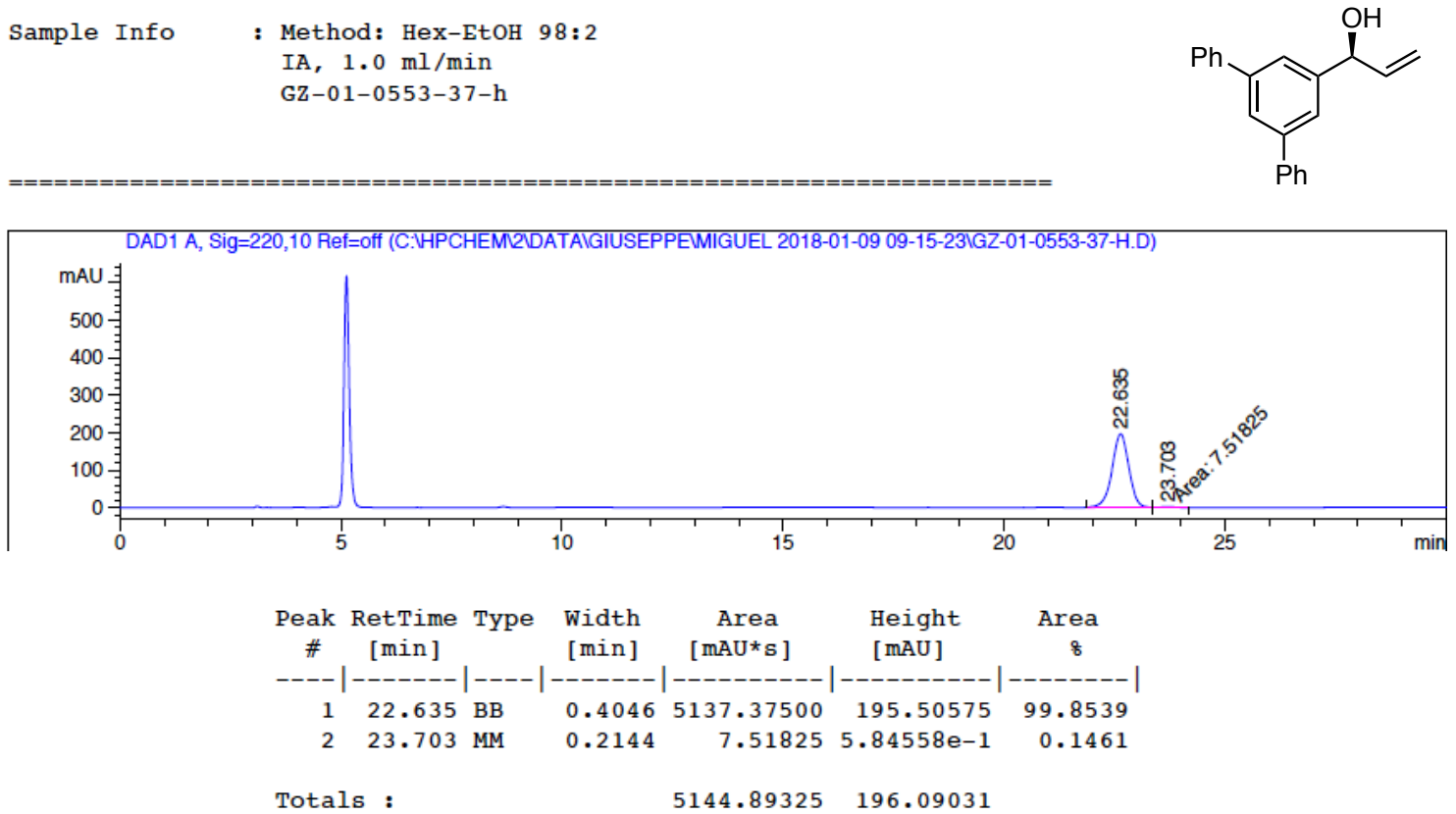
Chromatogram of racemic 1-(Perfluorophenyl)prop-2-en-1-ol
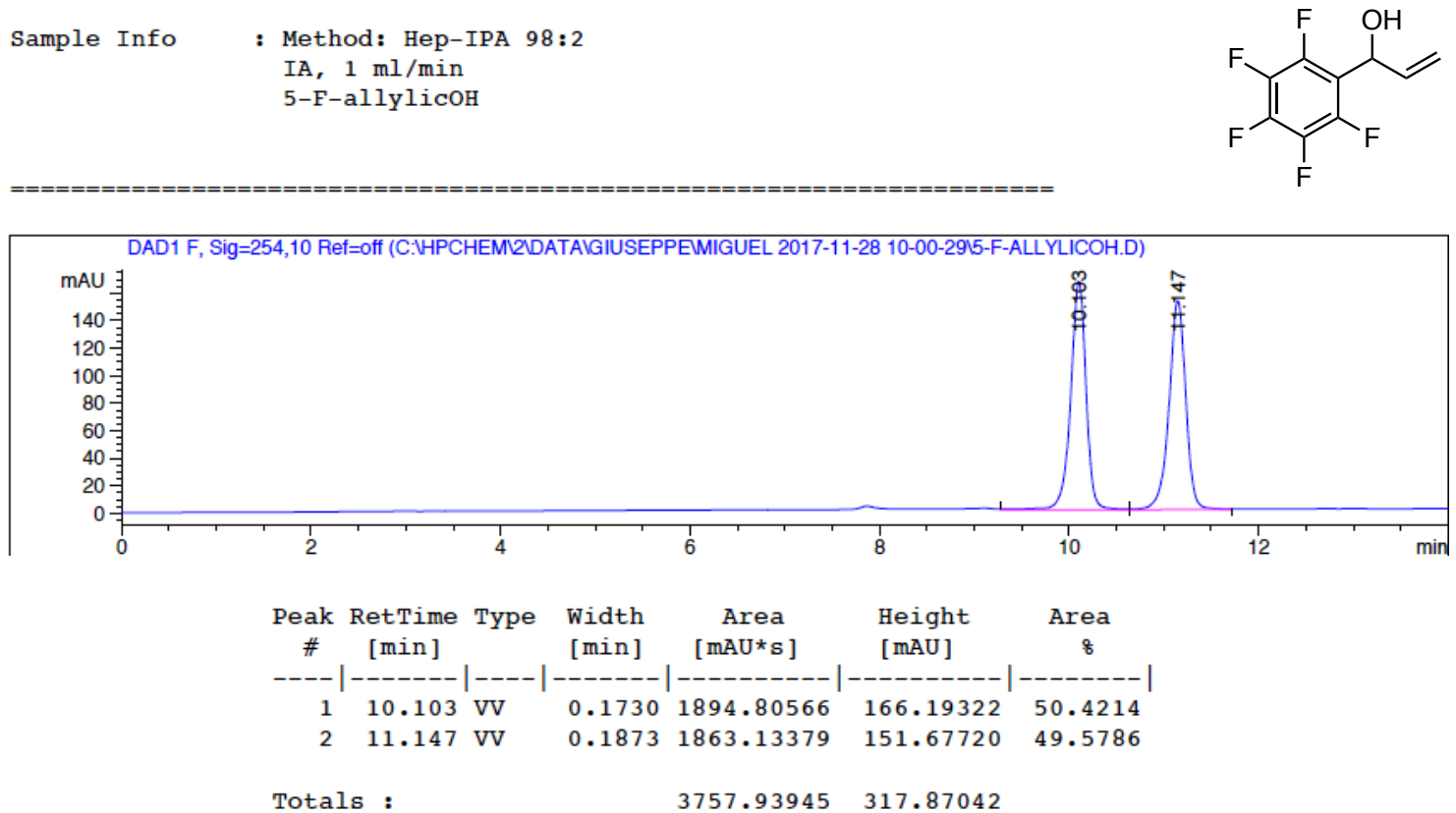

Chromatogram of enantioenriched 1-(Perfluorophenyl)prop-2-en-1-ol
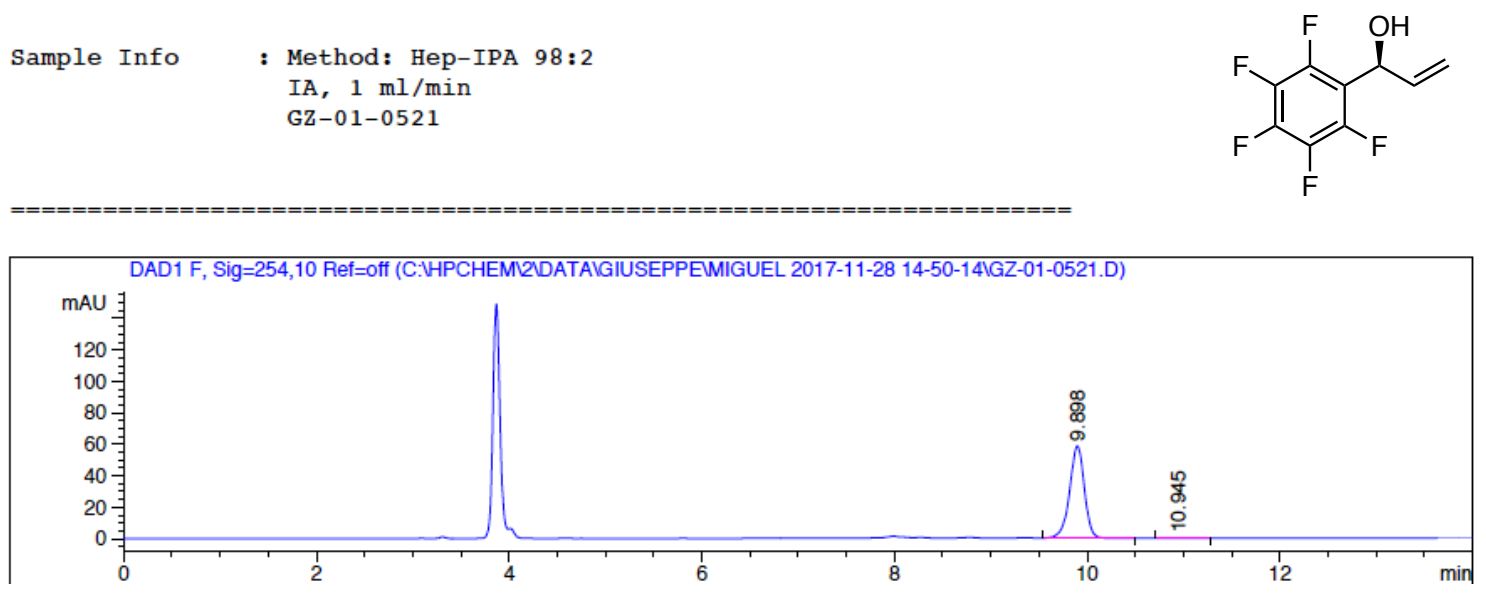

Signal 1: DAD1 F, Sig=254,10 Ref=off

\begin{tabular}{|c|c|c|c|c|c|c|}
\hline $\begin{array}{c}\text { Peak } \\
\#\end{array}$ & $\begin{array}{l}\text { RetTime } \\
\text { [min] }\end{array}$ & Type & $\begin{array}{l}\text { Width } \\
\text { [min] }\end{array}$ & $\begin{array}{c}\text { Area } \\
{\left[\mathrm{mAU} \mathbf{U}^{*} \mathrm{~s}\right]}\end{array}$ & $\begin{array}{l}\text { Height } \\
\text { [mAU] }\end{array}$ & $\begin{array}{c}\text { Area } \\
8\end{array}$ \\
\hline 1 & 9.898 & VB & 0.1656 & 632.39441 & 58.75846 & 99.2434 \\
\hline 2 & 10.945 & BB & 0.2225 & 4.82093 & $3.18753 \mathrm{e}-1$ & 0.7566 \\
\hline Tota & $5:$ & & & 637.21534 & 59.07721 & \\
\hline
\end{tabular}


Chromatogram of racemic 1-(3,5-Bis(trifluoromethyl)phenyl)prop-2-en-1-ol

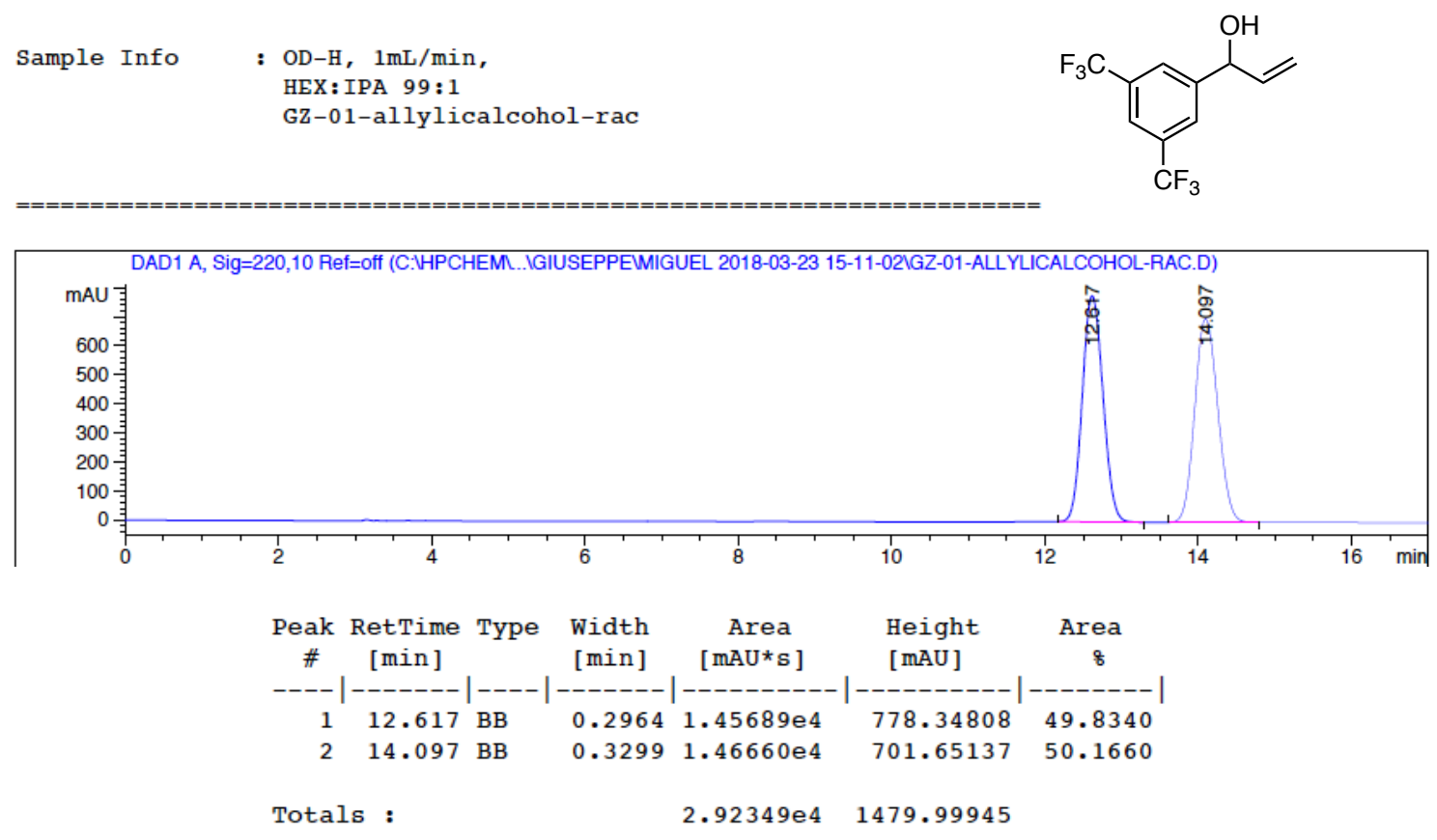

Chromatogram of enantioenriched 1-(3,5-Bis(trifluoromethyl)phenyl)prop-2-en-1-ol

$$
\begin{aligned}
\text { Sample Info } \quad \text { : } & \text { OD-H, } 1 \mathrm{~mL} / \mathrm{min}, \\
& \text { HEX:IPA } 99: 1 \\
& \text { resolution-CF3 }
\end{aligned}
$$
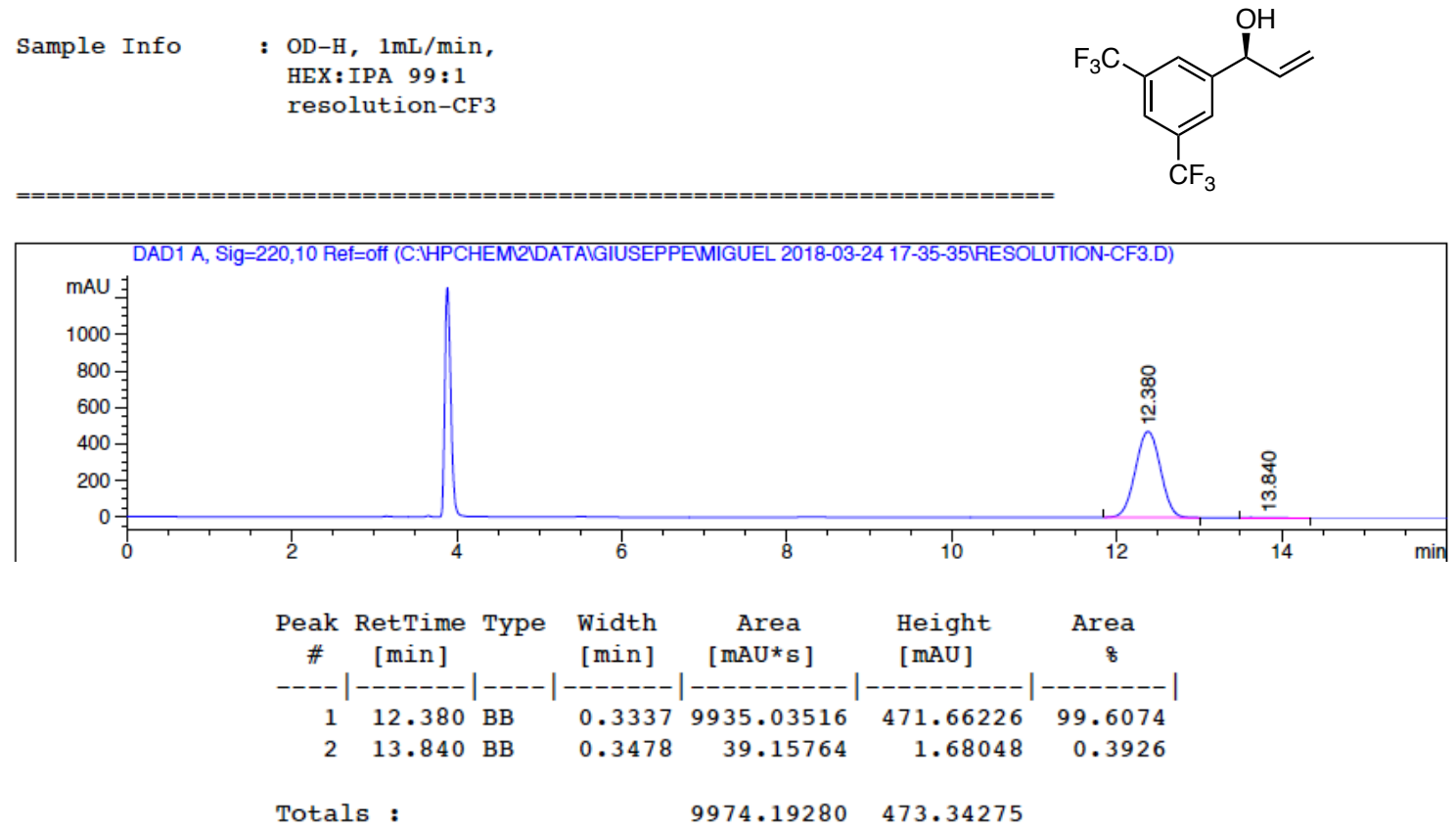
Chromatogram of racemic $\mathbf{6 a}$

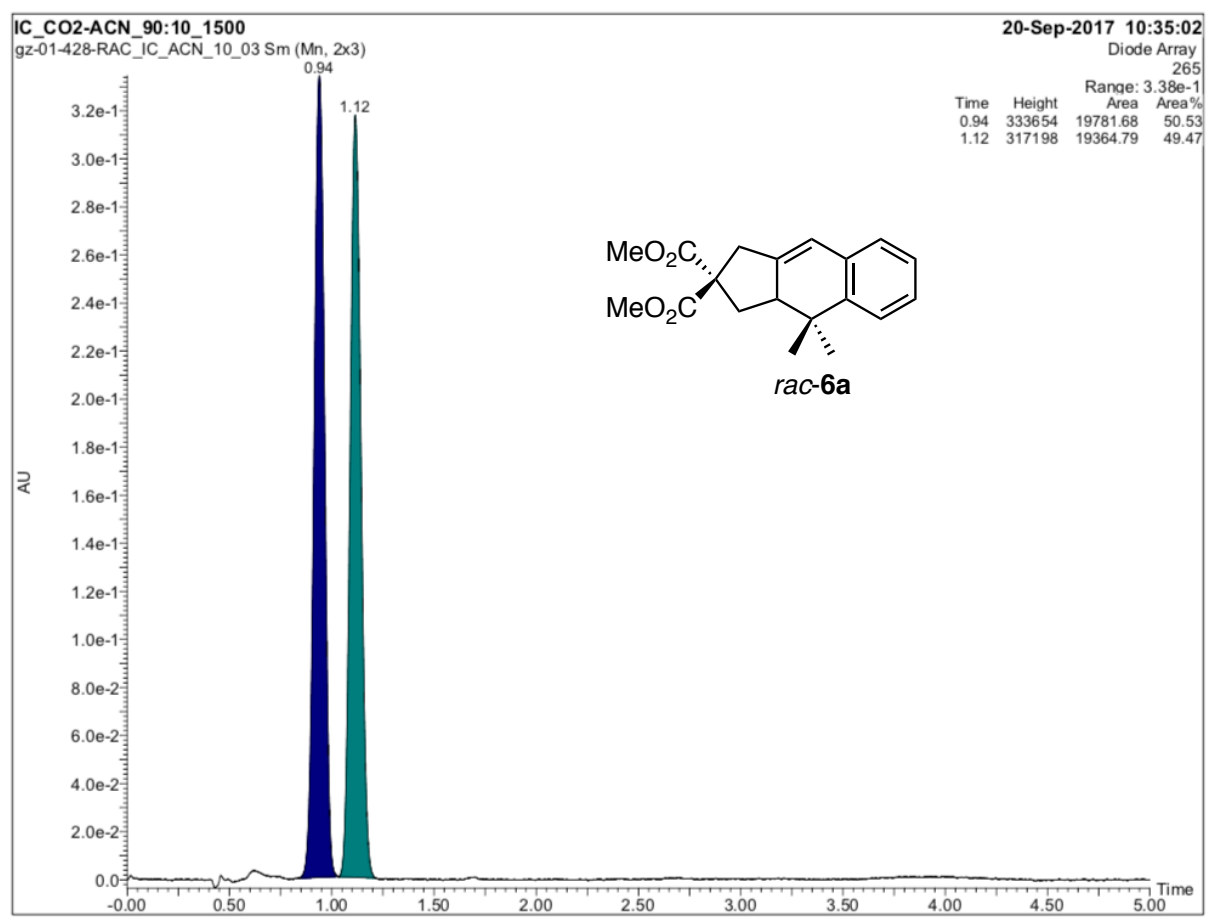

Chromatogram of enantioenriched $\mathbf{6 a}$

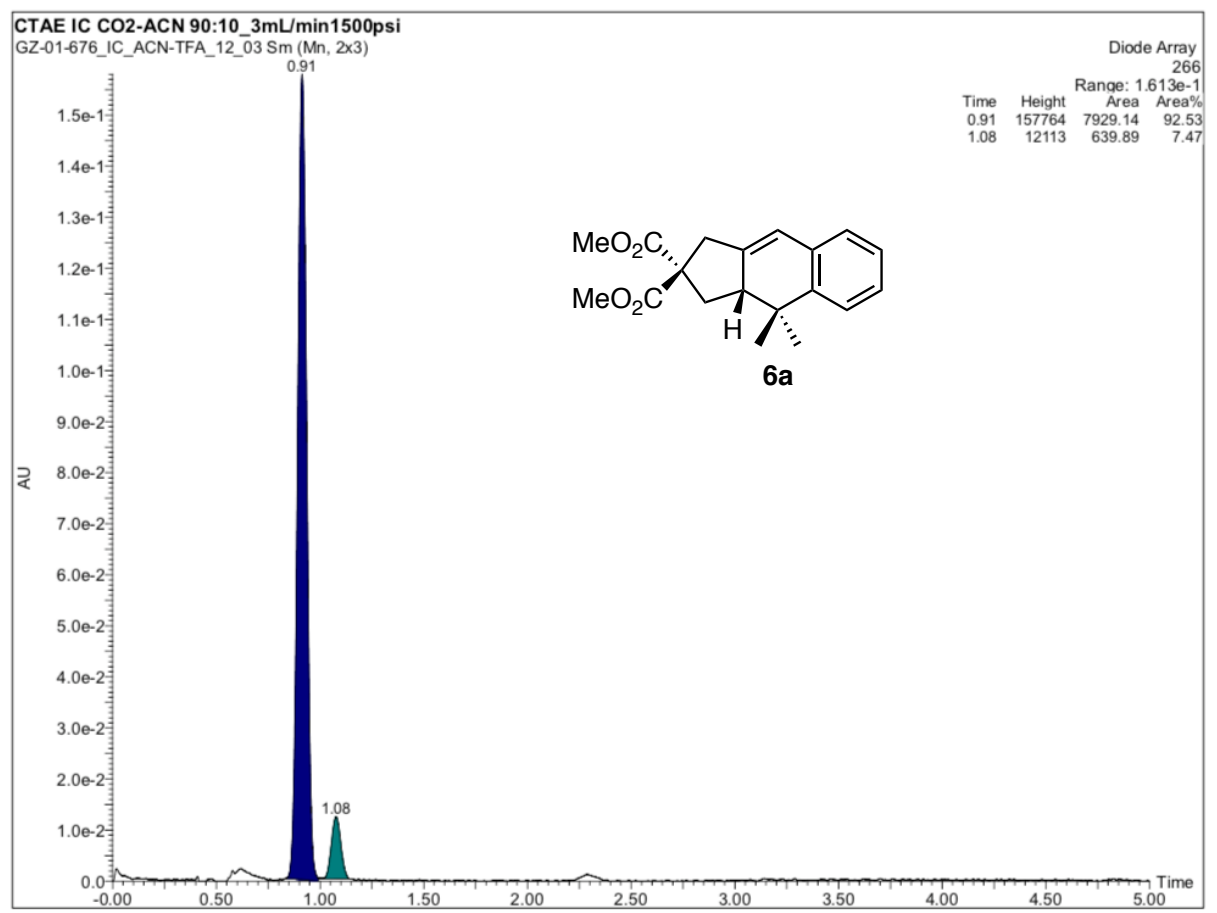


Chromatogram of racemic $\mathbf{6 b}$

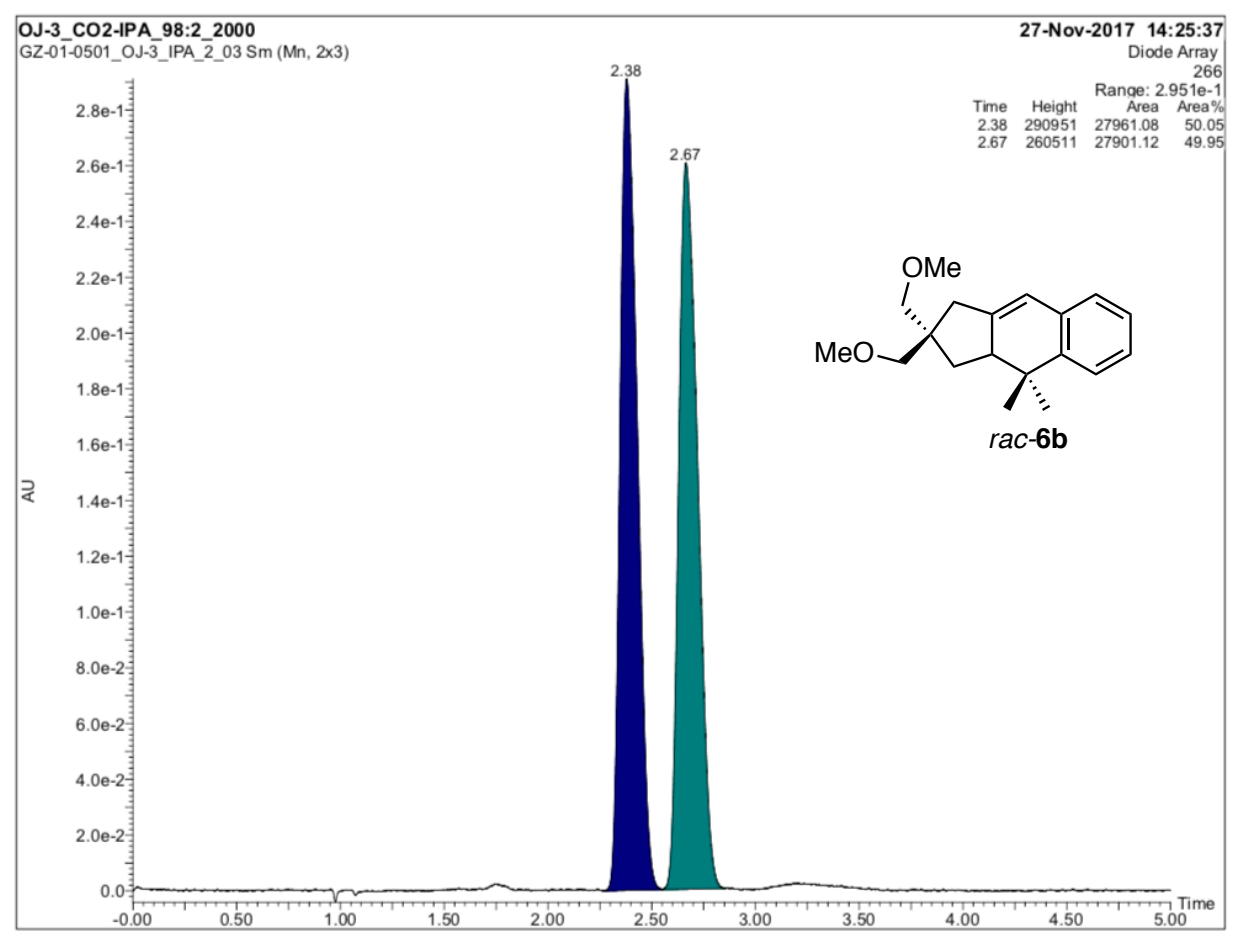

Chromatogram of enantioenriched $\mathbf{6 b}$

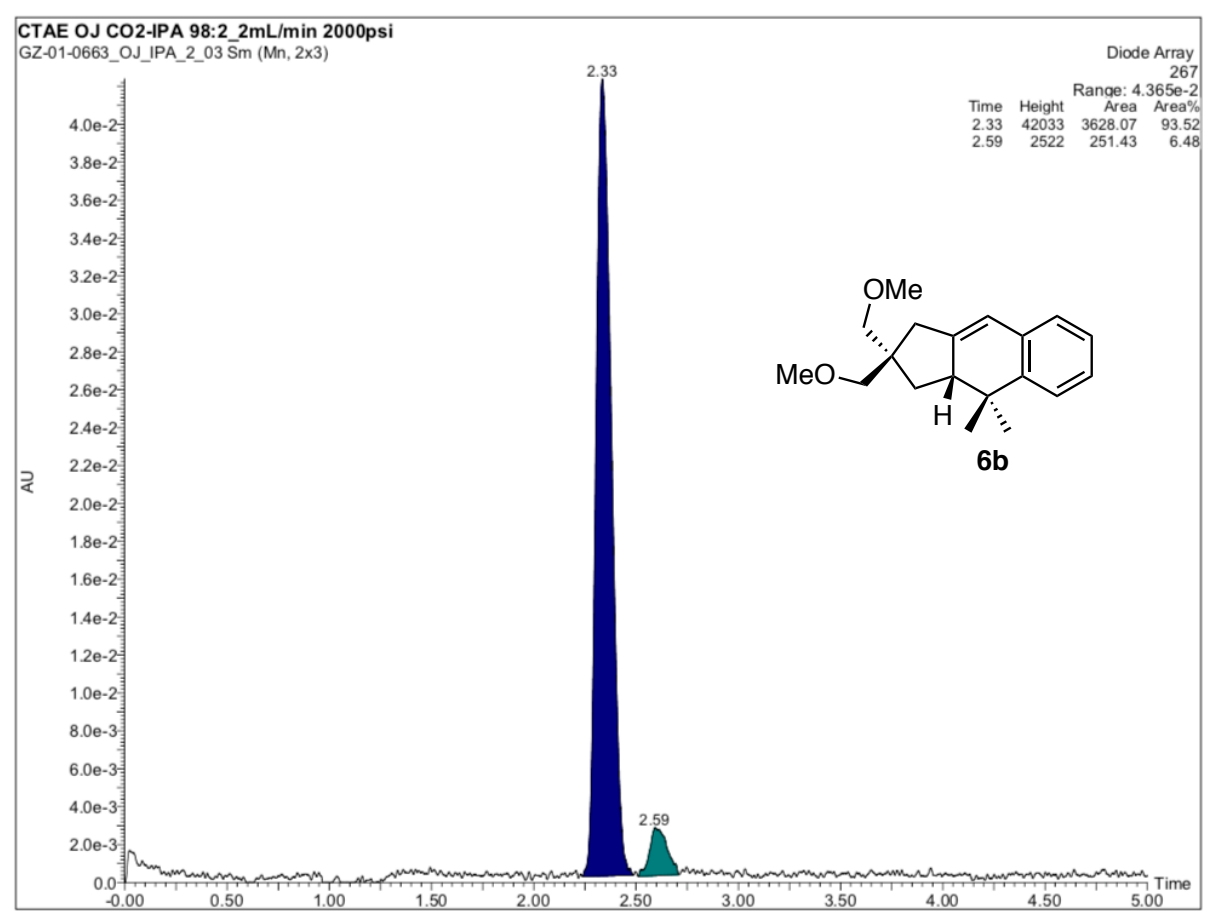


Chromatogram of racemic $\mathbf{6 c}$

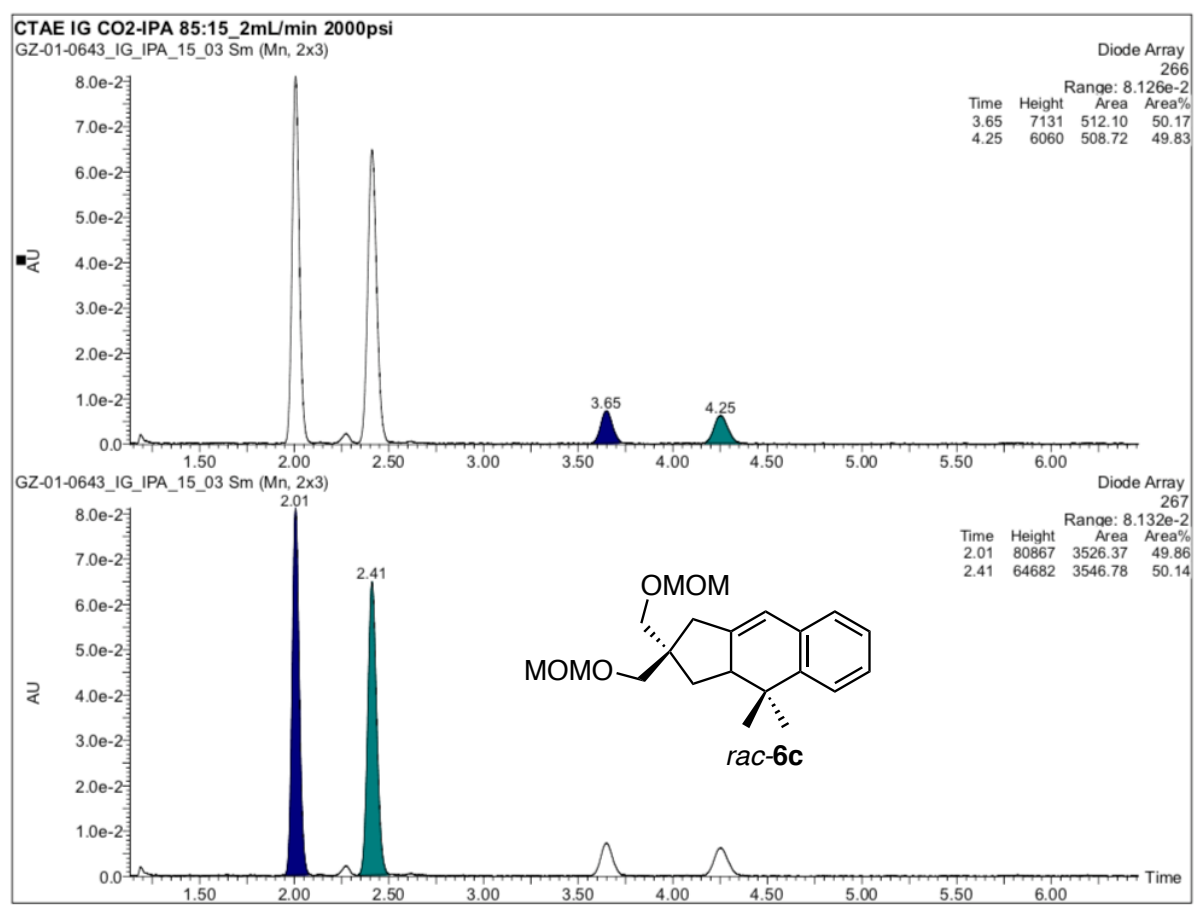

Chromatogram of enantioenriched $\mathbf{6 c}$

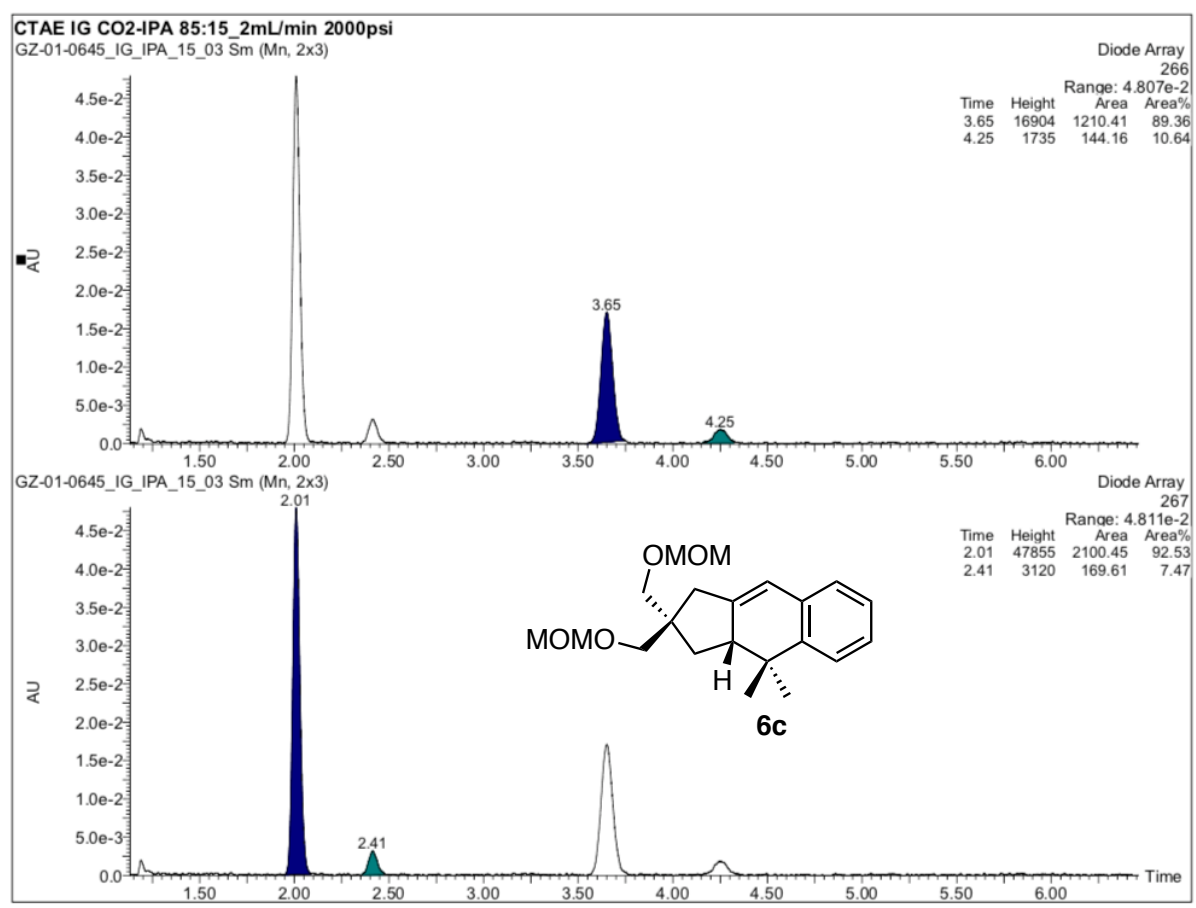


Chromatogram of racemic $\mathbf{6 d}$

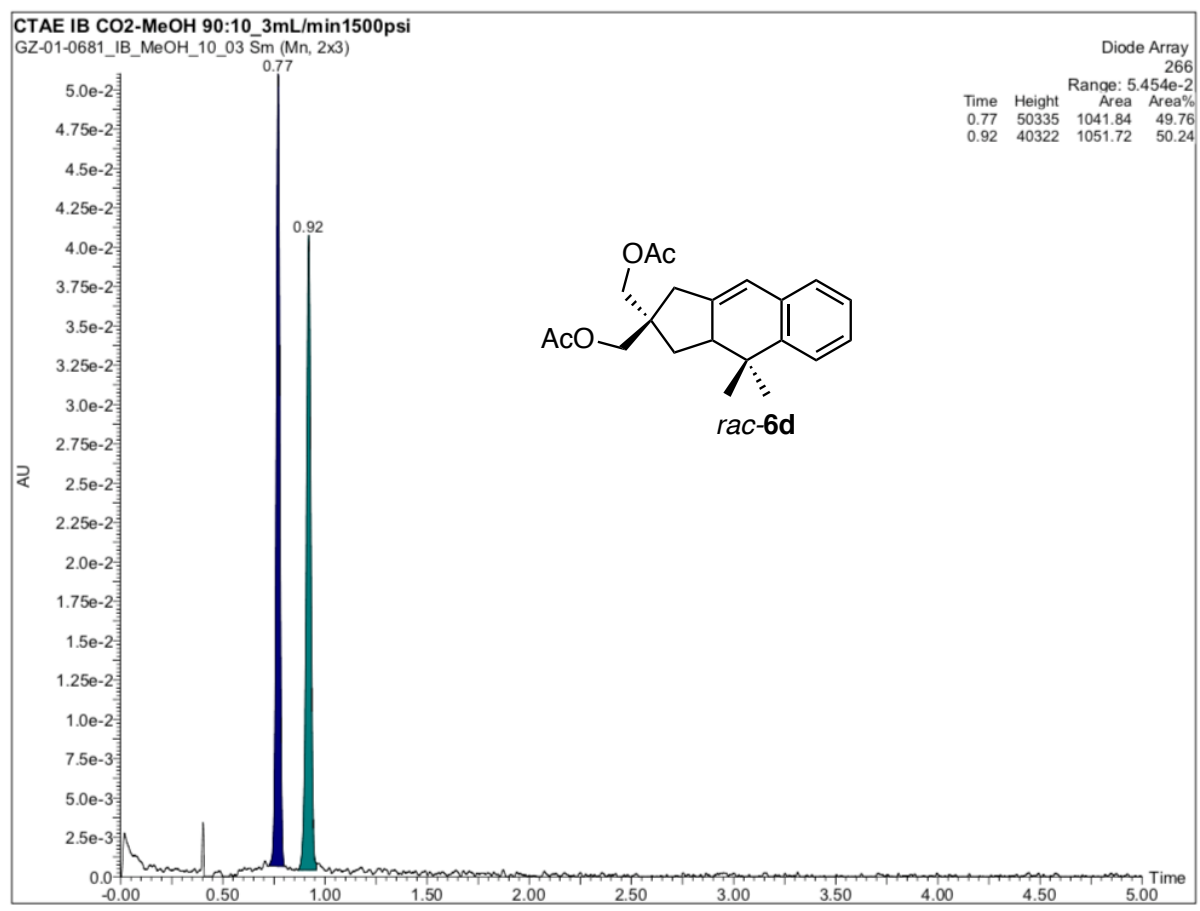

Chromatogram of enantioenriched $\mathbf{6 d}$

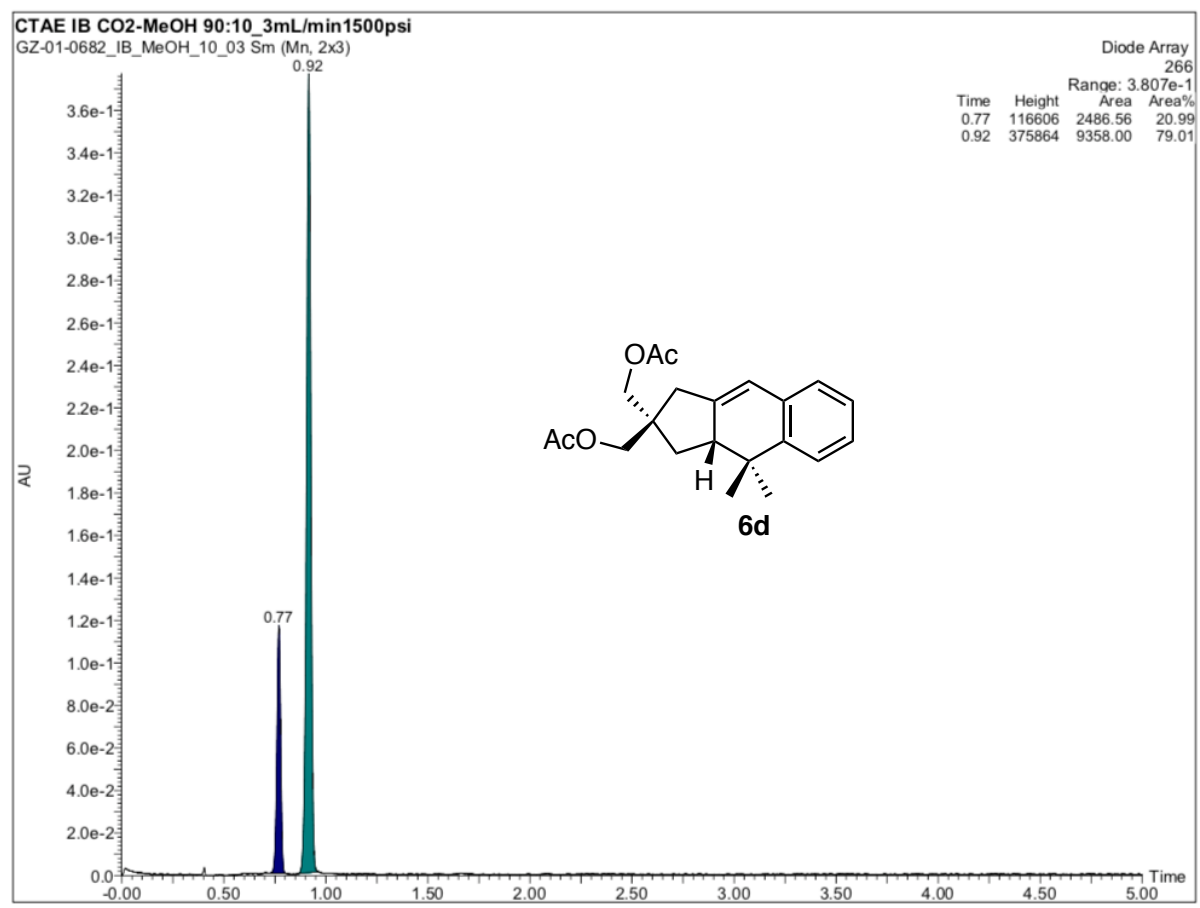


Chromatogram of racemic $\mathbf{6 e}$

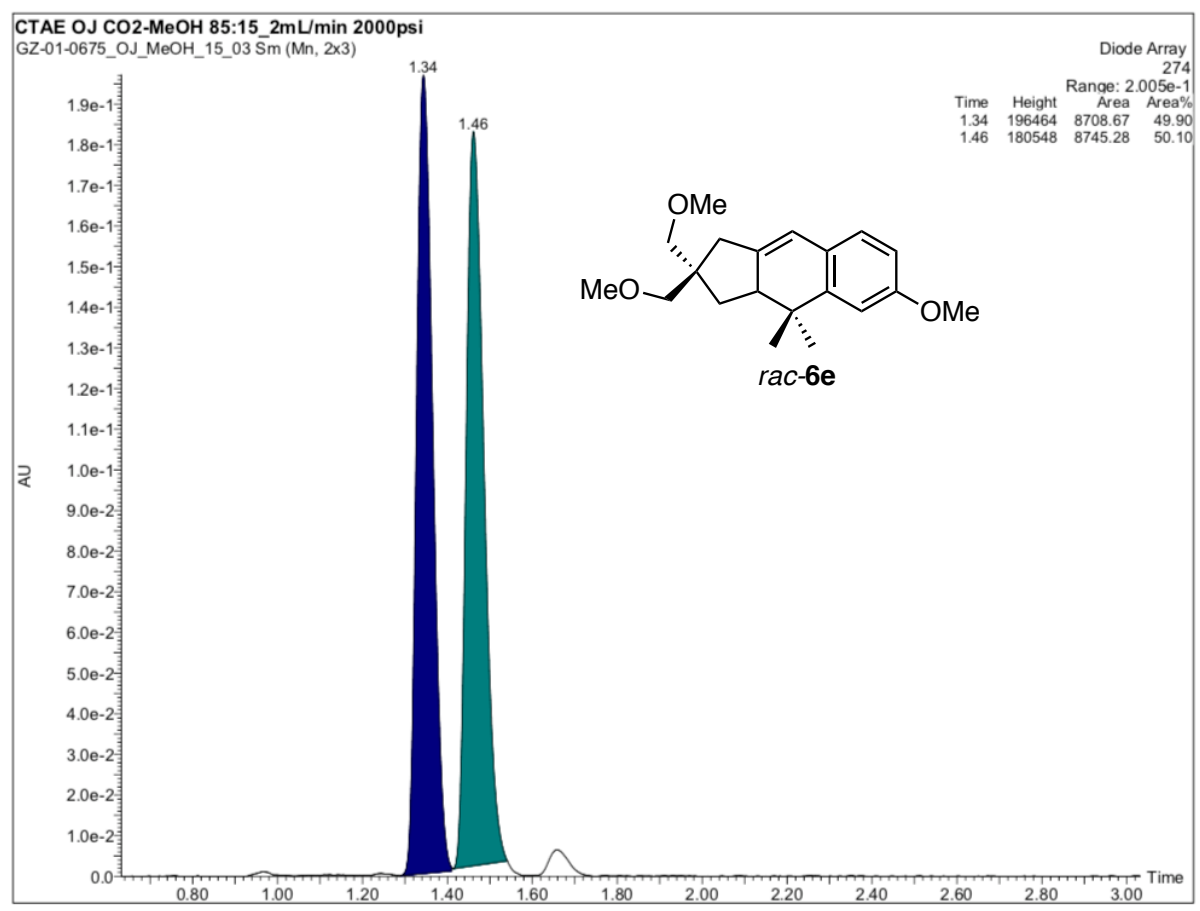

Chromatogram of enantioenriched $\mathbf{6 e}$

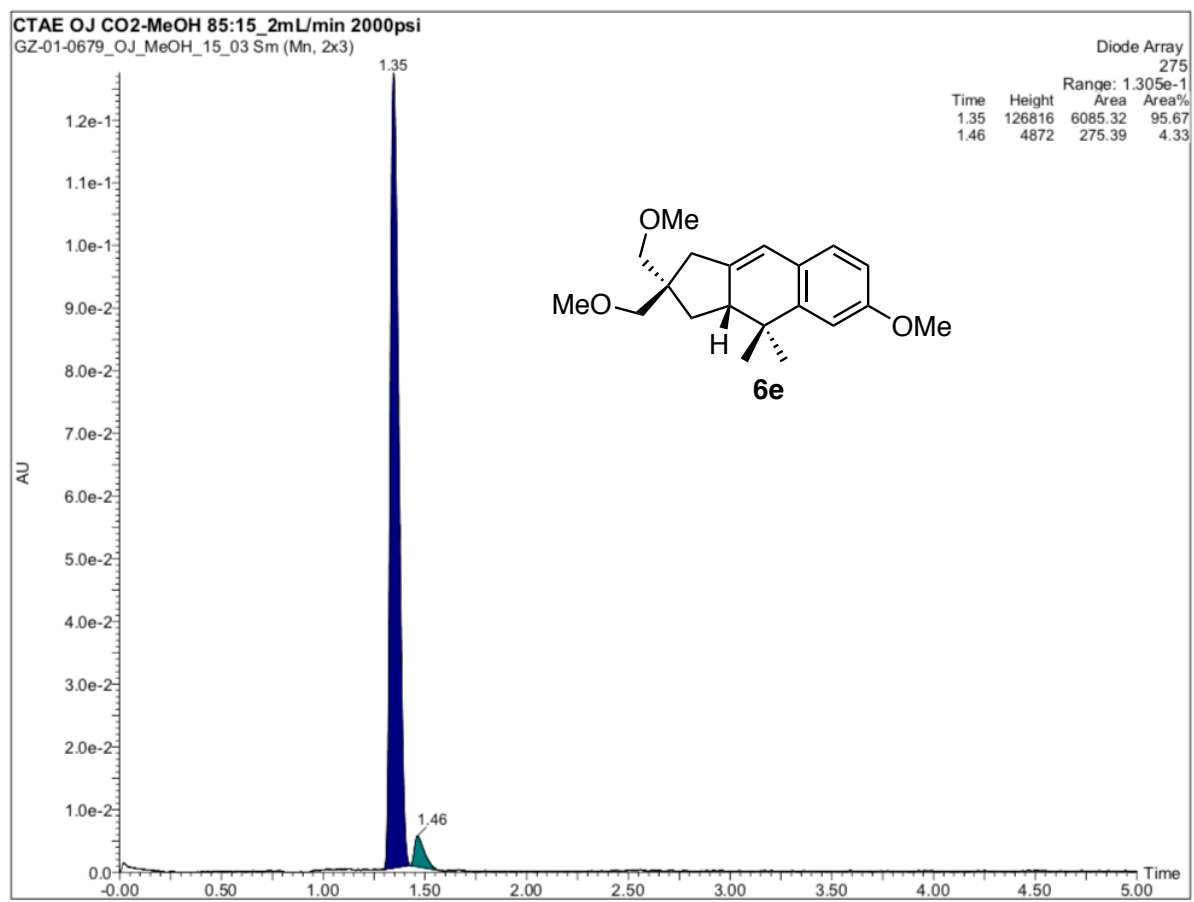


Chromatogram of racemic $\mathbf{6} \mathbf{f}$

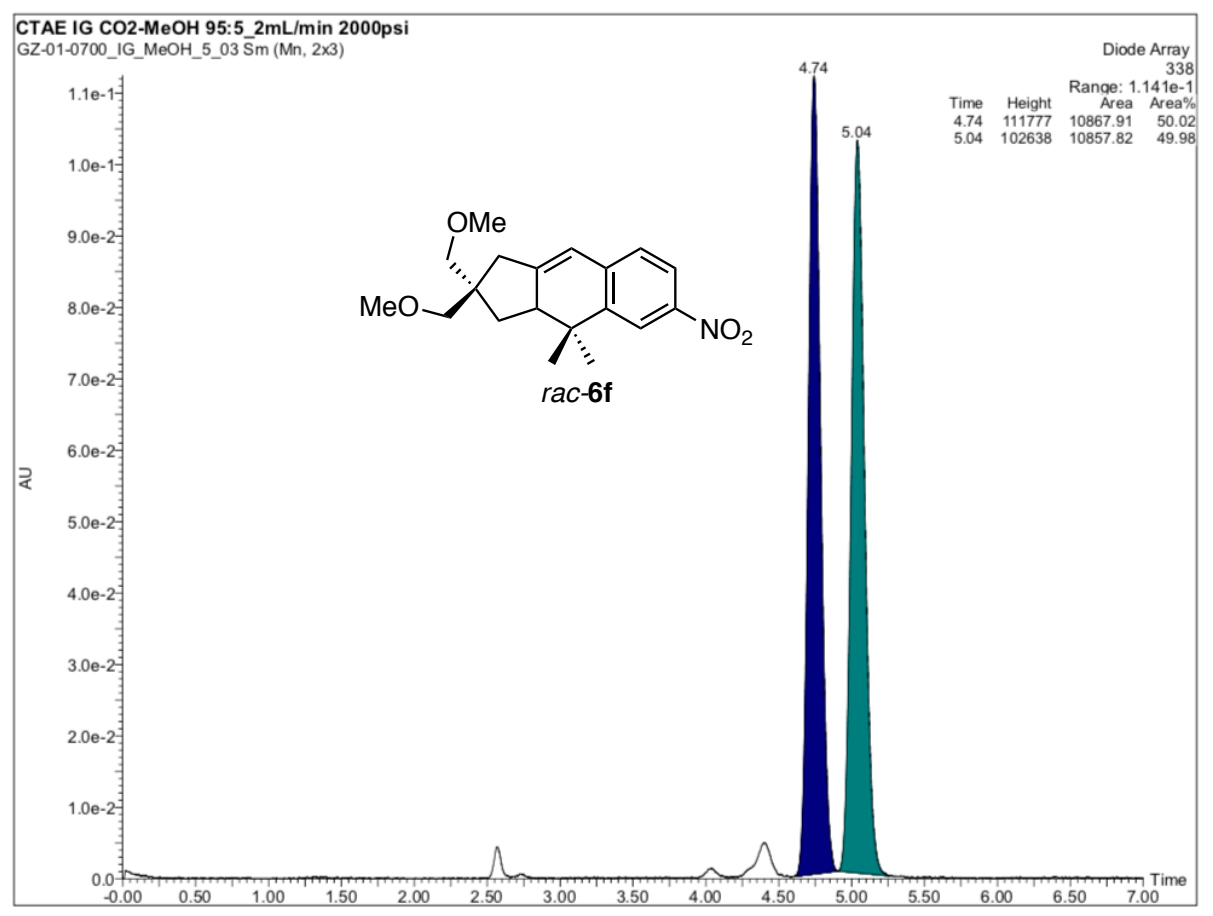

Chromatogram of enantioenriched $\mathbf{6 f}$

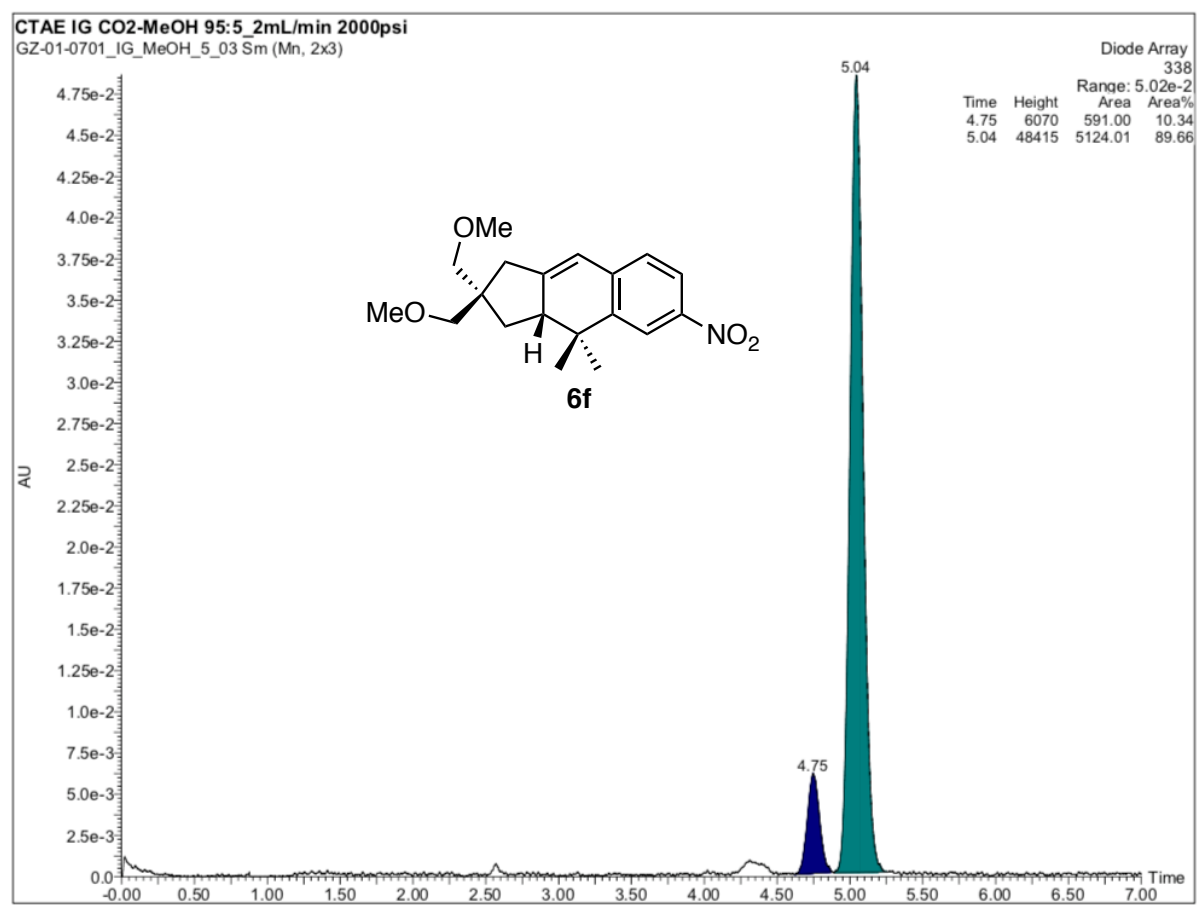


Chromatogram of racemic $\mathbf{6 g}$

$$
\begin{aligned}
\text { Sample Info : } & \text { OD-H, } 1 \mathrm{~mL} / \mathrm{min}, \\
& \text { HEX } 100 \text { \% } \\
& \text { GZ-01-0719-rac }
\end{aligned}
$$
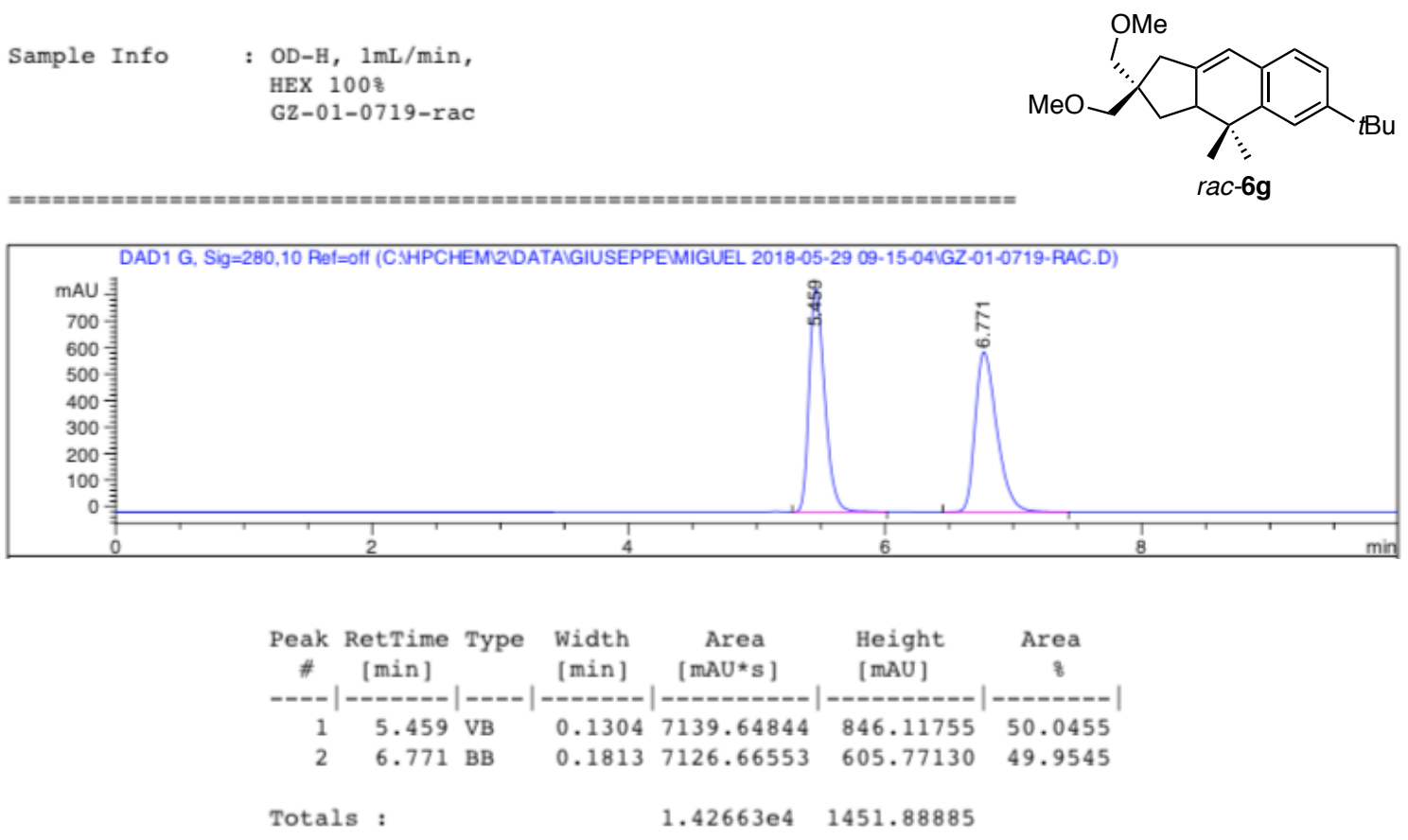

\section{Chromatogram of enantioenriched $\mathbf{6 g}$}

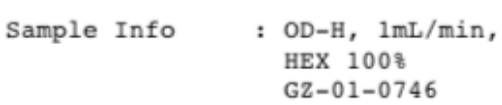

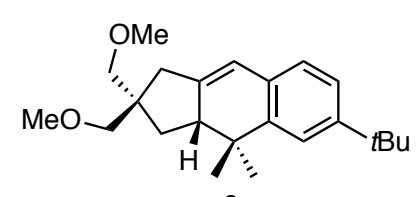

$6 \mathrm{~g}$

\begin{tabular}{|c|c|c|c|c|c|c|}
\hline $\begin{array}{c}\text { Peak } \\
\#\end{array}$ & $\begin{array}{c}\text { RetTime } \\
\text { [min] }\end{array}$ & Type & $\begin{array}{l}\text { Width } \\
\text { [min] }\end{array}$ & $\begin{array}{c}\text { Area } \\
{\left[\mathrm{mAU}{ }^{*} \mathrm{~s}\right]}\end{array}$ & $\begin{array}{l}\text { Height } \\
\text { [mAU] }\end{array}$ & $\begin{array}{c}\text { Area } \\
\text { ? }\end{array}$ \\
\hline$-\infty$ & | - - & & ------ & --n--n- & | --n--no- & ------n \\
\hline 1 & 5.589 & vV & 0.1307 & 1196.21521 & 141.46545 & 8.8353 \\
\hline 2 & 6.949 & BB & 0.1854 & $1.23429 \mathrm{e} 4$ & 1018.52521 & 91.1647 \\
\hline Tota. & ls : & & & $1.35391 \mathrm{e} 4$ & 1159.99066 & \\
\hline
\end{tabular}

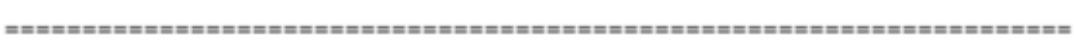

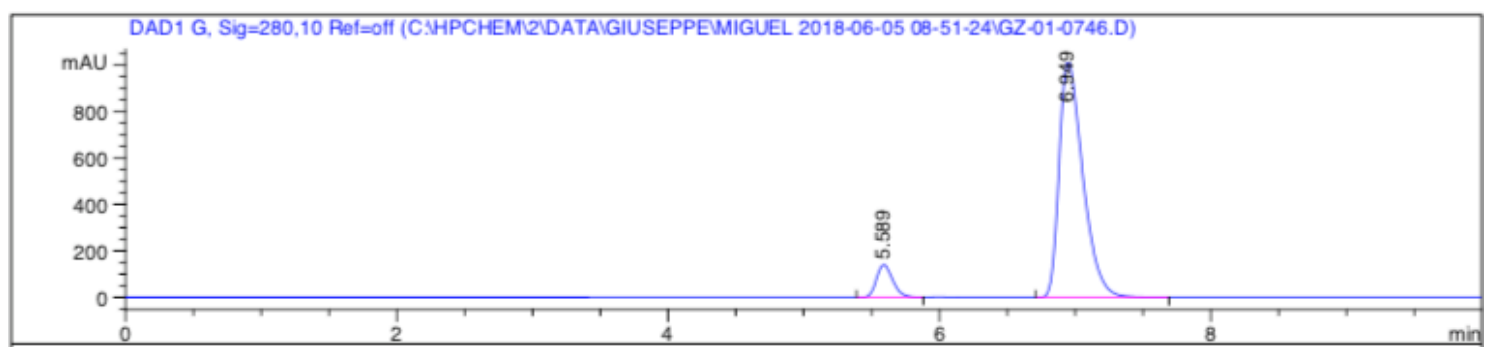


Chromatogram of racemic $6 \mathrm{~h}$

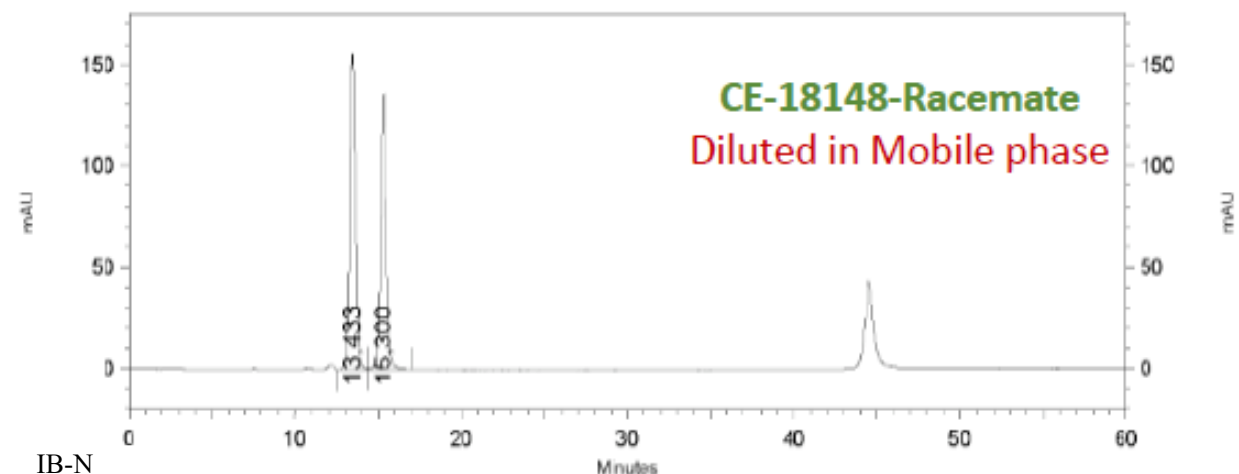

$0.5 \mathrm{~mL} / \mathrm{min}$

$n$ heptane/MtBE 98:2

1: 280

nm, 4

$\mathrm{nm}$

Mnubes

$\mathrm{OMe}$

Results

\begin{tabular}{rrrrrrr} 
Pk \# & $\begin{array}{c}\text { RT } \\
\text { (min) }\end{array}$ & Area Area of & \multicolumn{2}{c}{$\begin{array}{r}\text { Theoretical } \\
\text { plates }\end{array}$ (USP) } & $\begin{array}{r}\text { Resolution } \\
\text { (USP) }\end{array}$ & Asymmetry \\
\hline 1 & 13,43 & 13931269 & 49,82 & 8501 & 0,00 & 1,08 \\
2 & 15,30 & 14032579 & 50,18 & 8459 & 2,99 & 1,09
\end{tabular}

\begin{tabular}{|l|l|l|l|l|l|}
\hline Totals & 27963848 & 100,00 & & \\
\hline
\end{tabular}

Chromatogram of enantioenriched $\mathbf{6 h}$

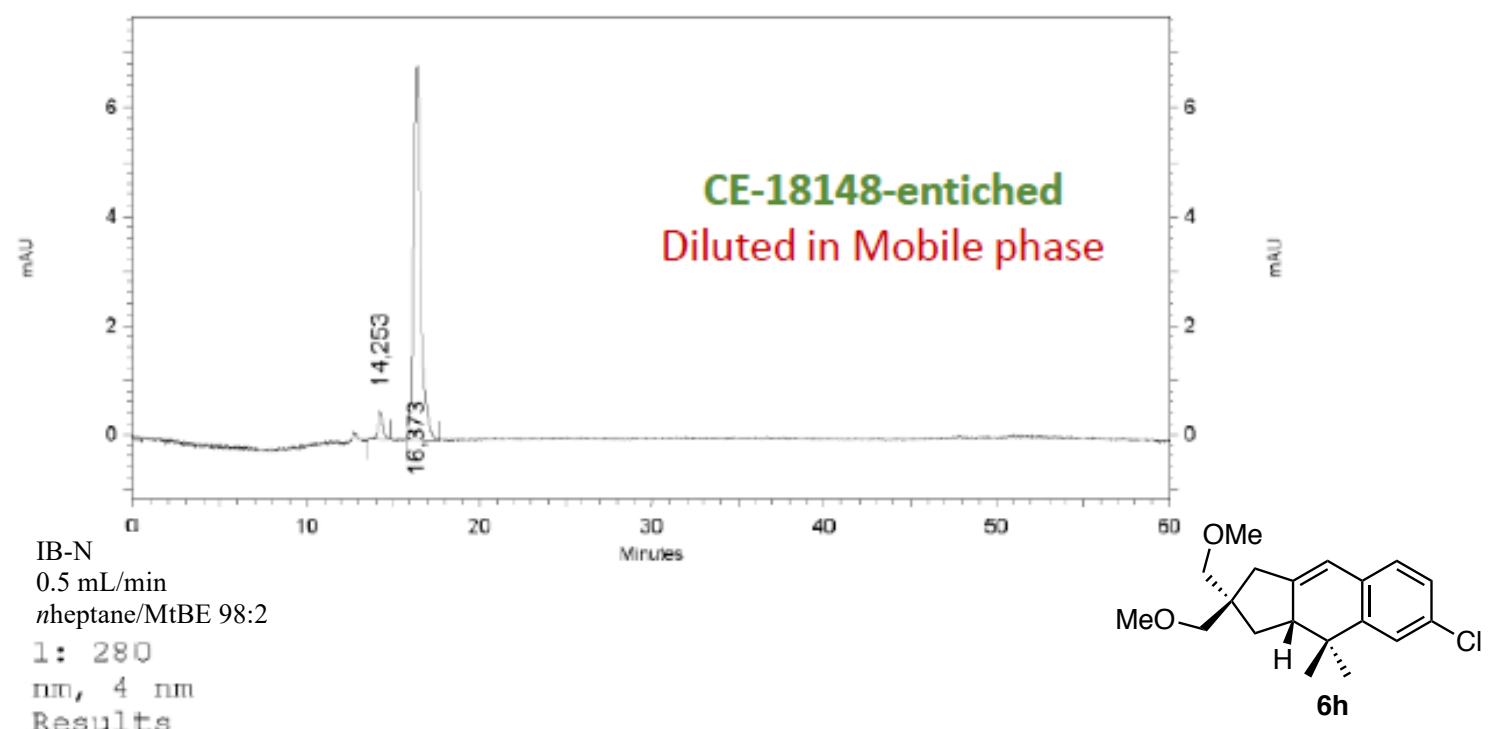

\begin{tabular}{rrrrrr} 
Pk \# & RT (min) & Area & Area * & $\begin{array}{c}\text { Theoretical } \\
\text { plates }\end{array}$ & $\begin{array}{r}\text { Resolution } \\
\text { (USP) }\end{array}$ \\
\hline 1 & 14,25 & 46472 & 5,70 & 8614 & 0,00 \\
2 & 16,37 & 769321 & 94,30 & 8616 & 3,21
\end{tabular}

\begin{tabular}{|l|l|l|l|}
\hline Totals & 815793 & 100,00 & \\
\hline
\end{tabular}


Chromatogram of racemic $\mathbf{6 i}$

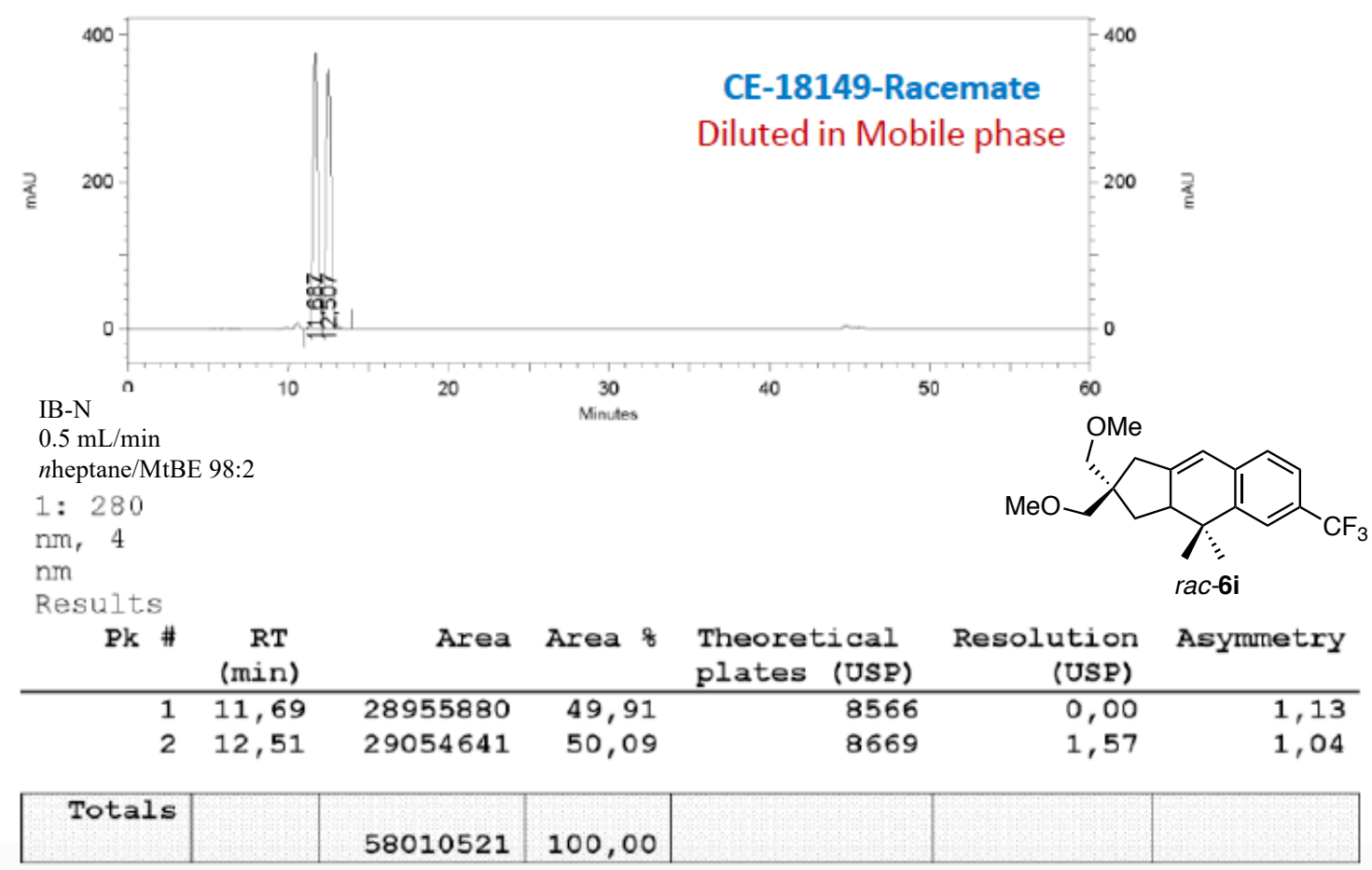

Chromatogram of enantioenriched $\mathbf{6 i}$

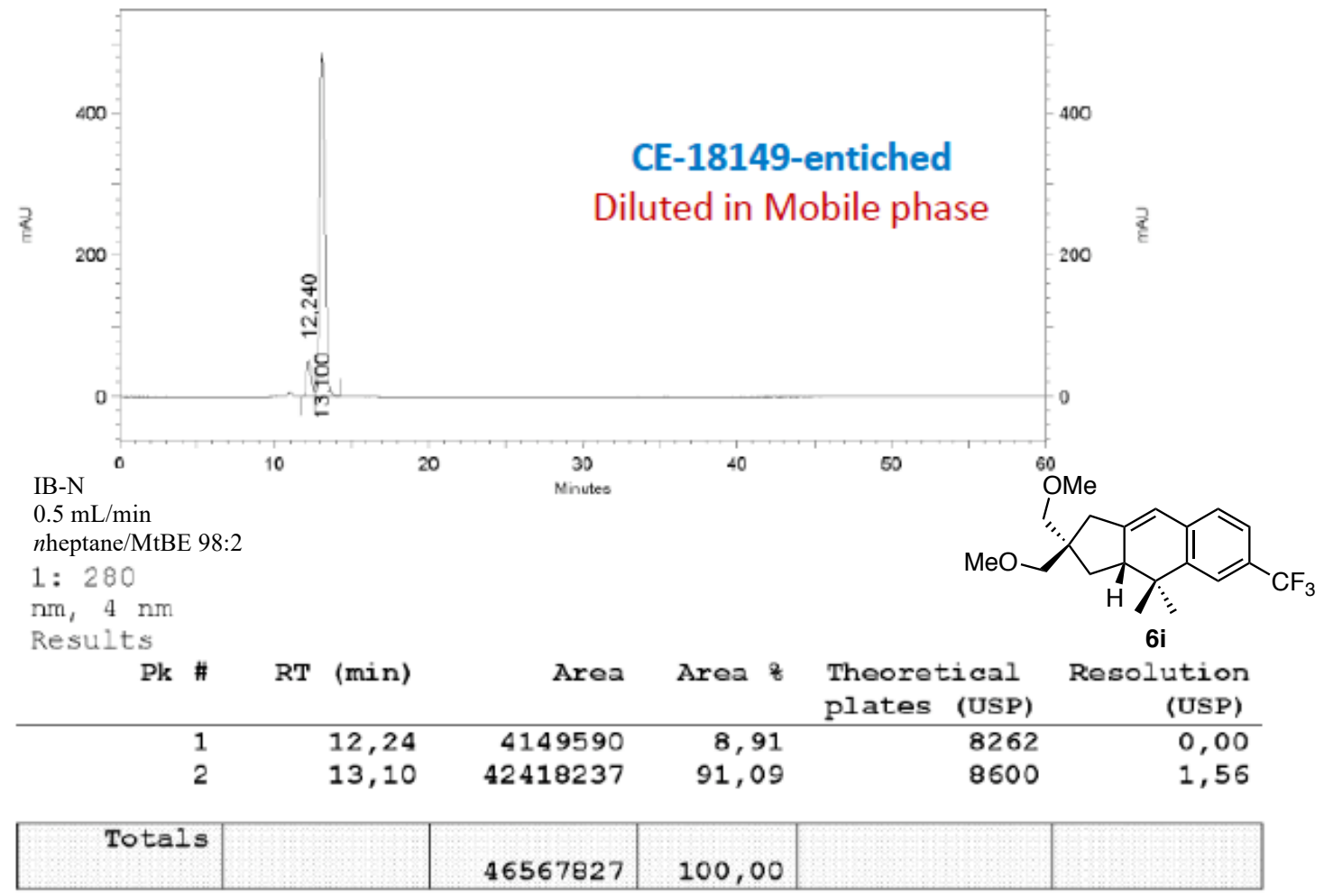


Chromatogram of racemic $\mathbf{6 j}$

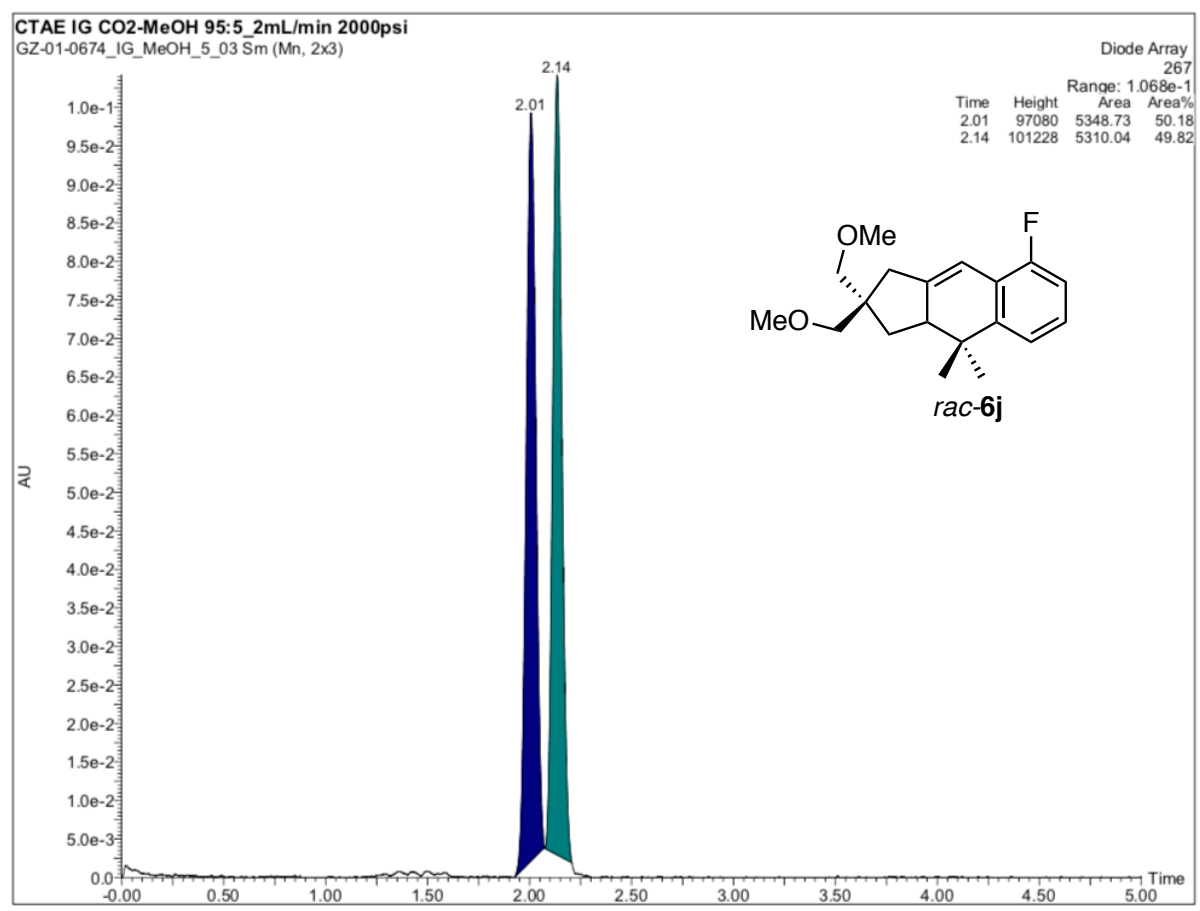

Chromatogram of enantioenriched $\mathbf{6 j}$

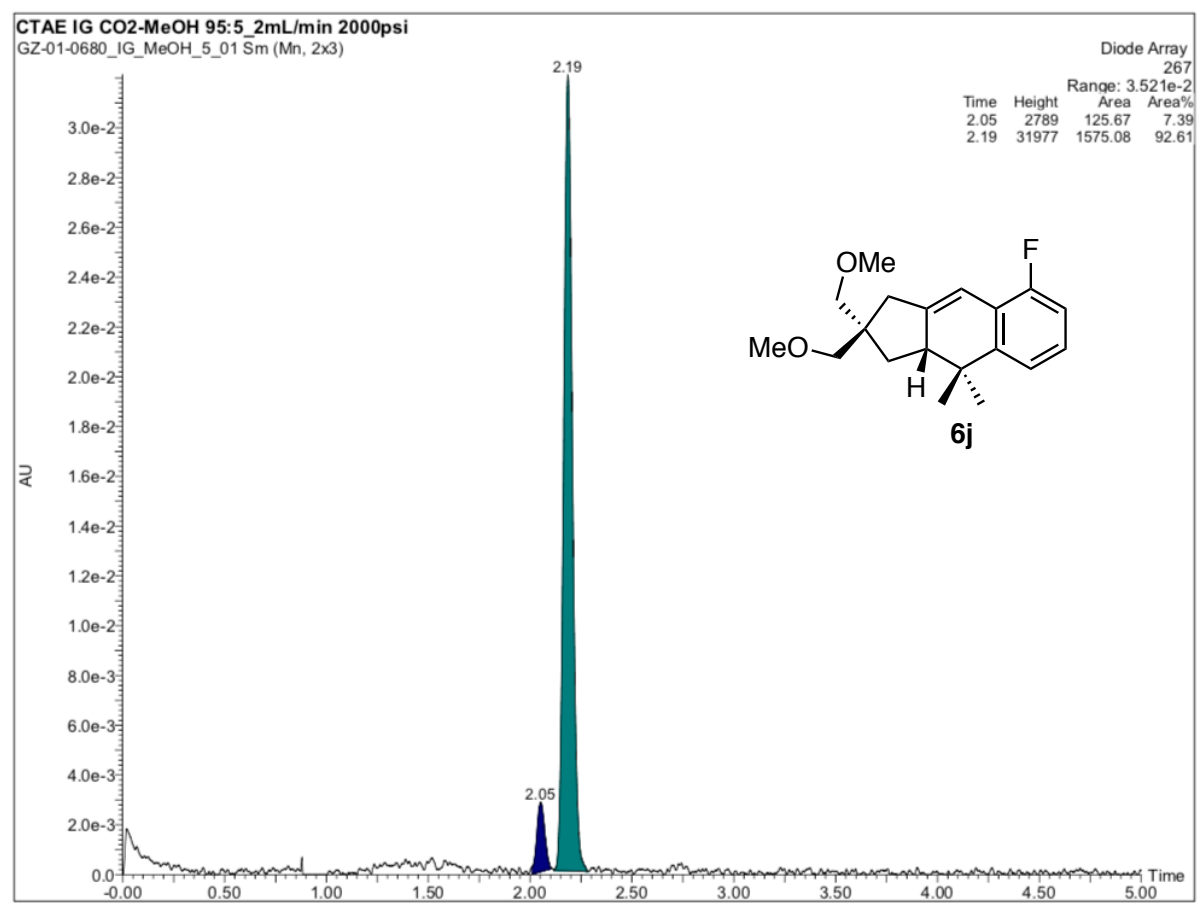


Chromatogram of racemic $\mathbf{6 k}$

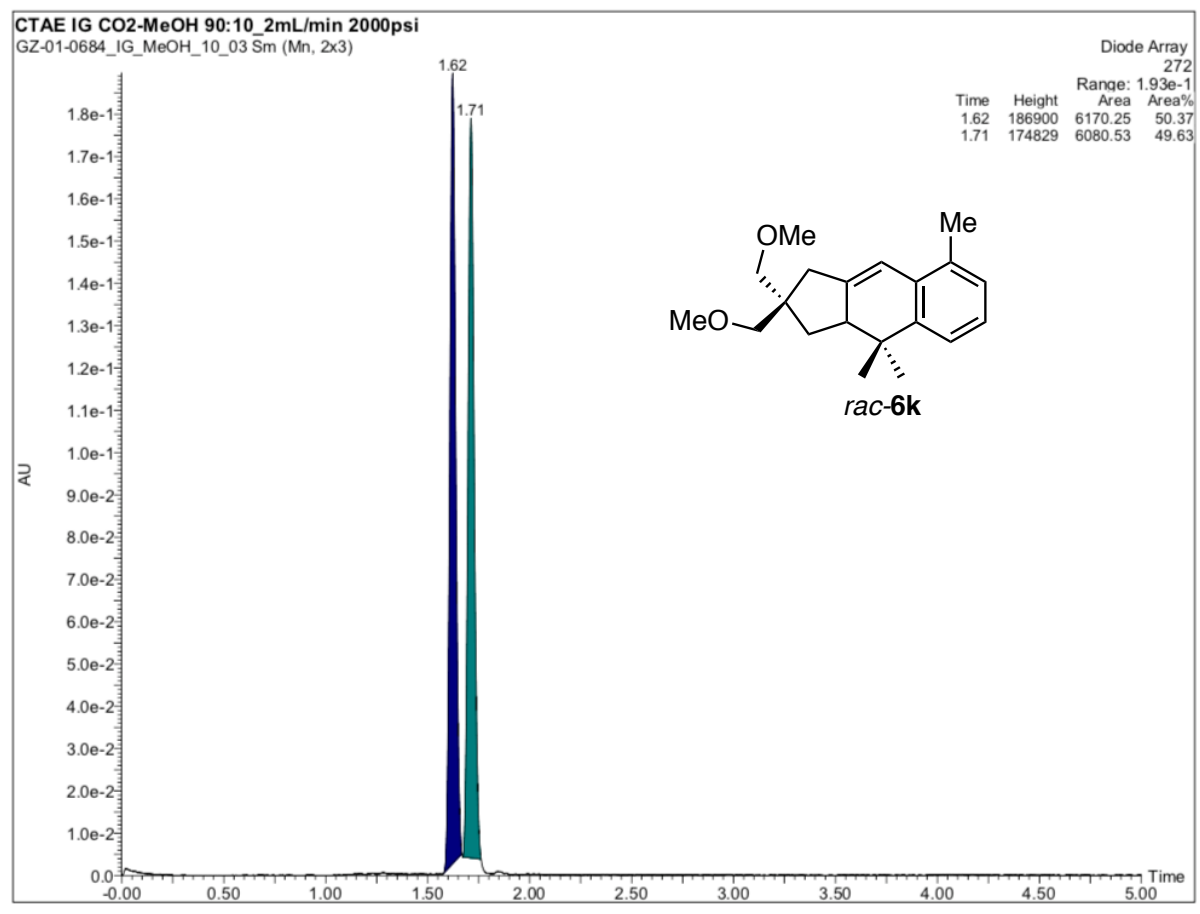

Chromatogram of enantioenriched $\mathbf{6 k}$

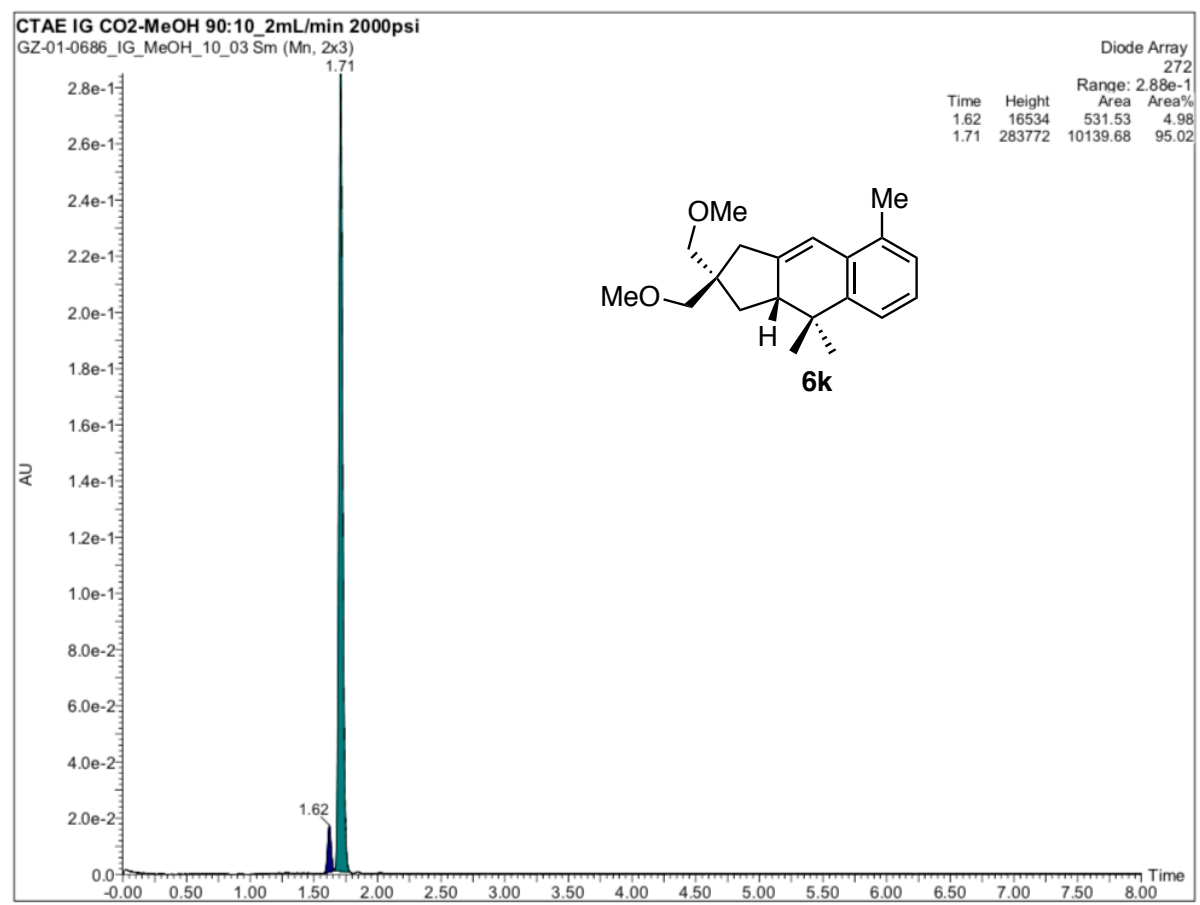


Chromatogram of racemic $\mathbf{6 l}$

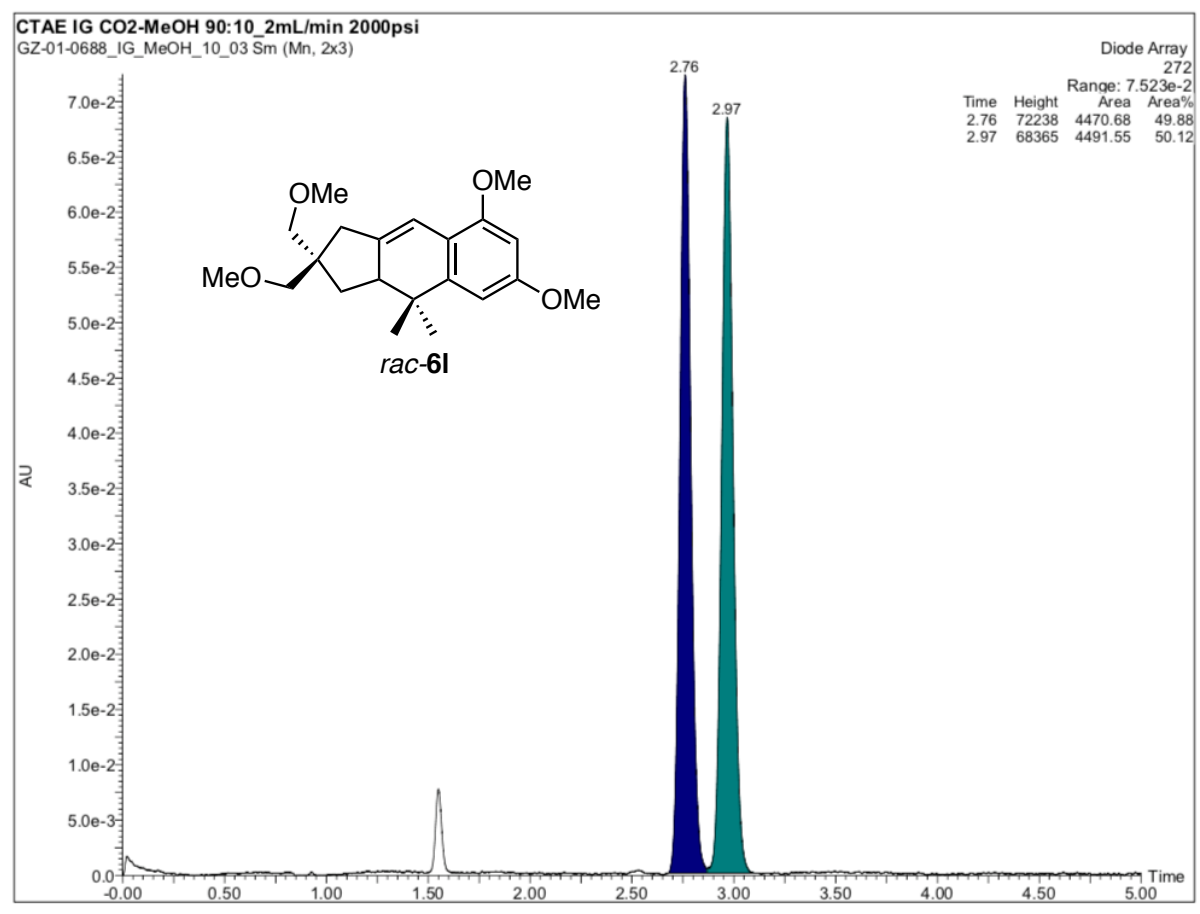

Chromatogram of enantioenriched $\mathbf{6 l}$

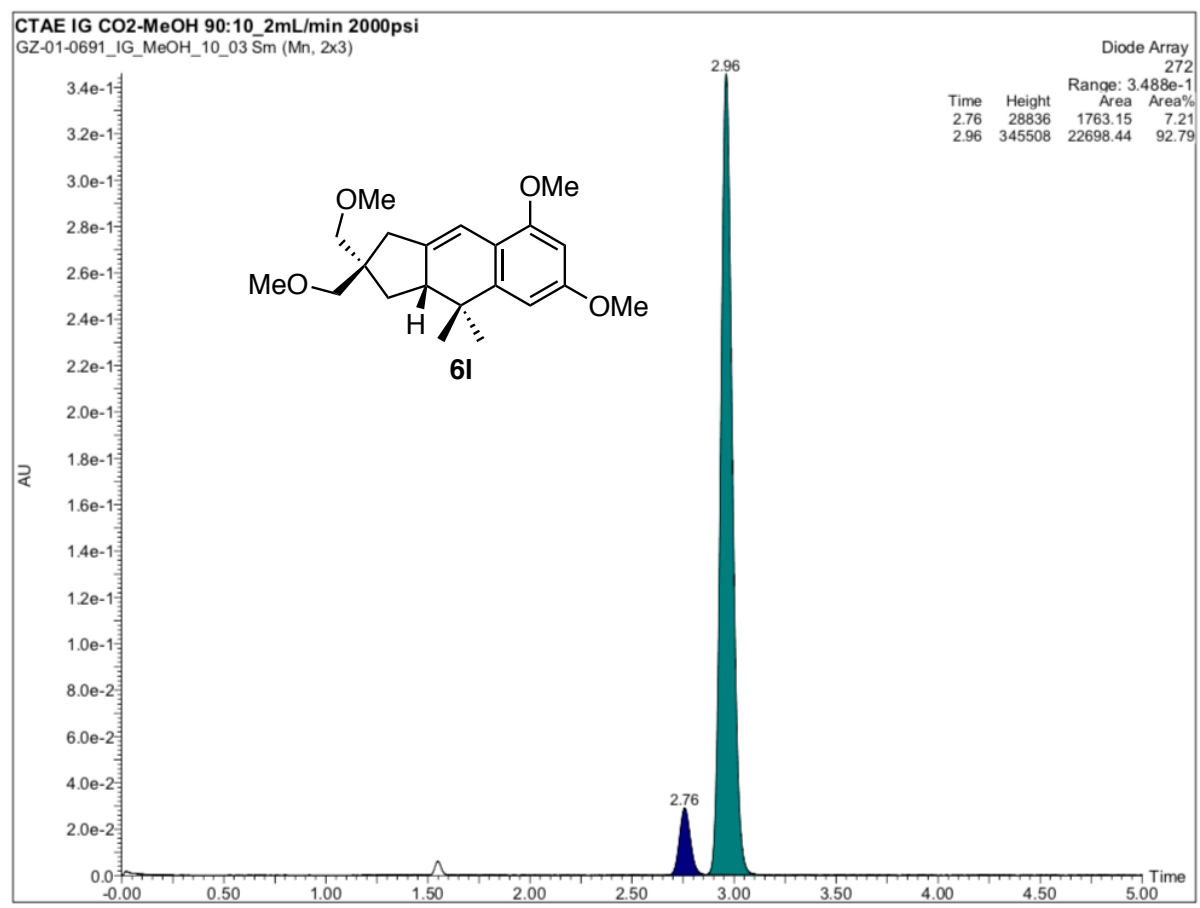




\section{Chromatogram of racemic $\mathbf{6 m}$}

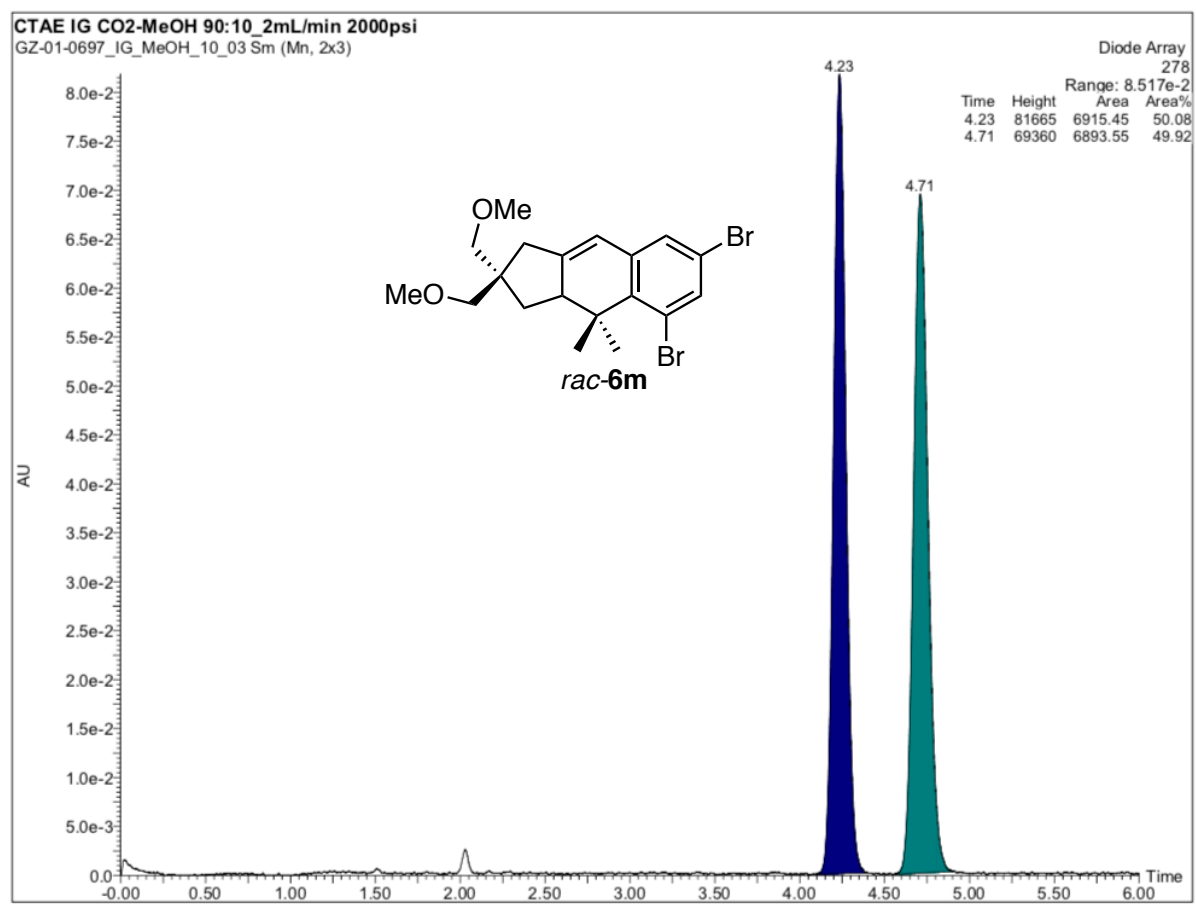

Chromatogram of enantioenriched $\mathbf{6 m}$

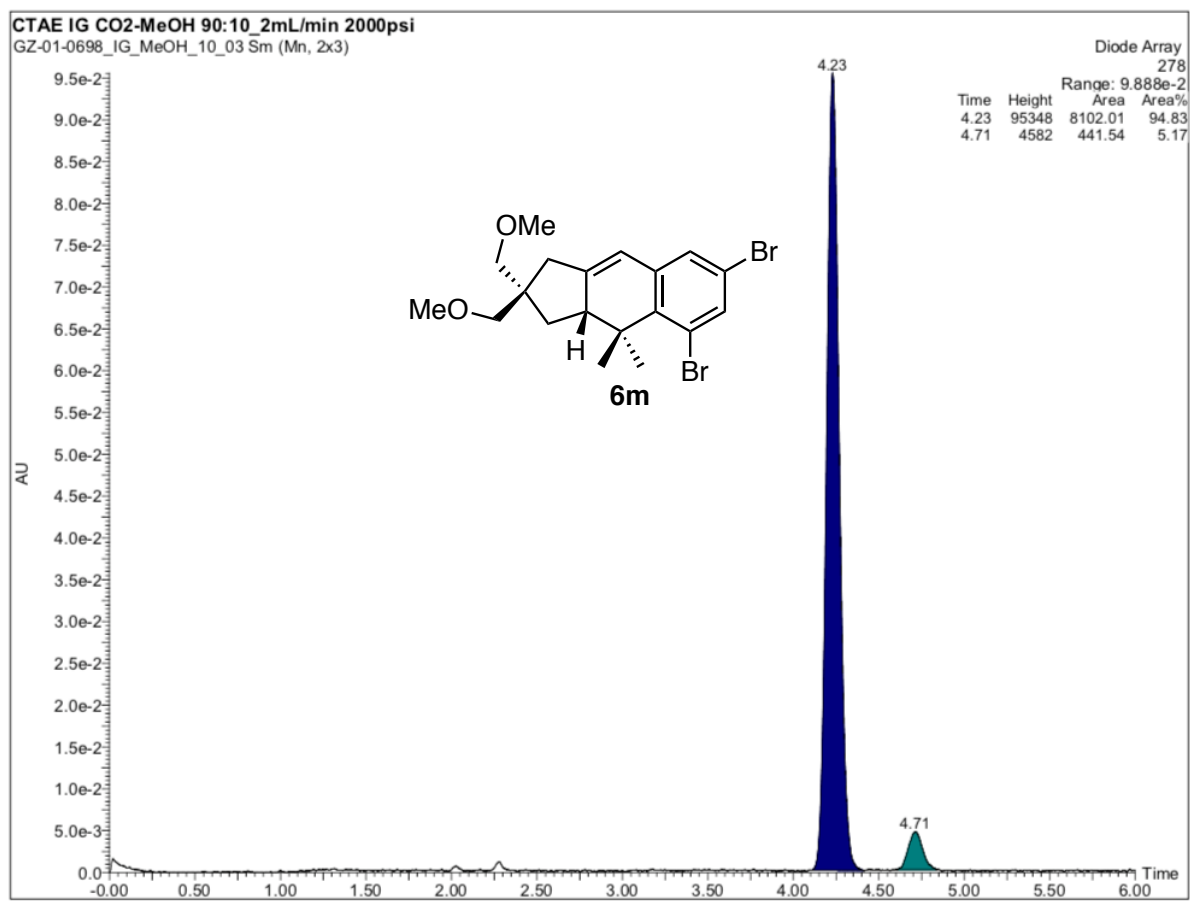


Chromatogram of racemic 6n

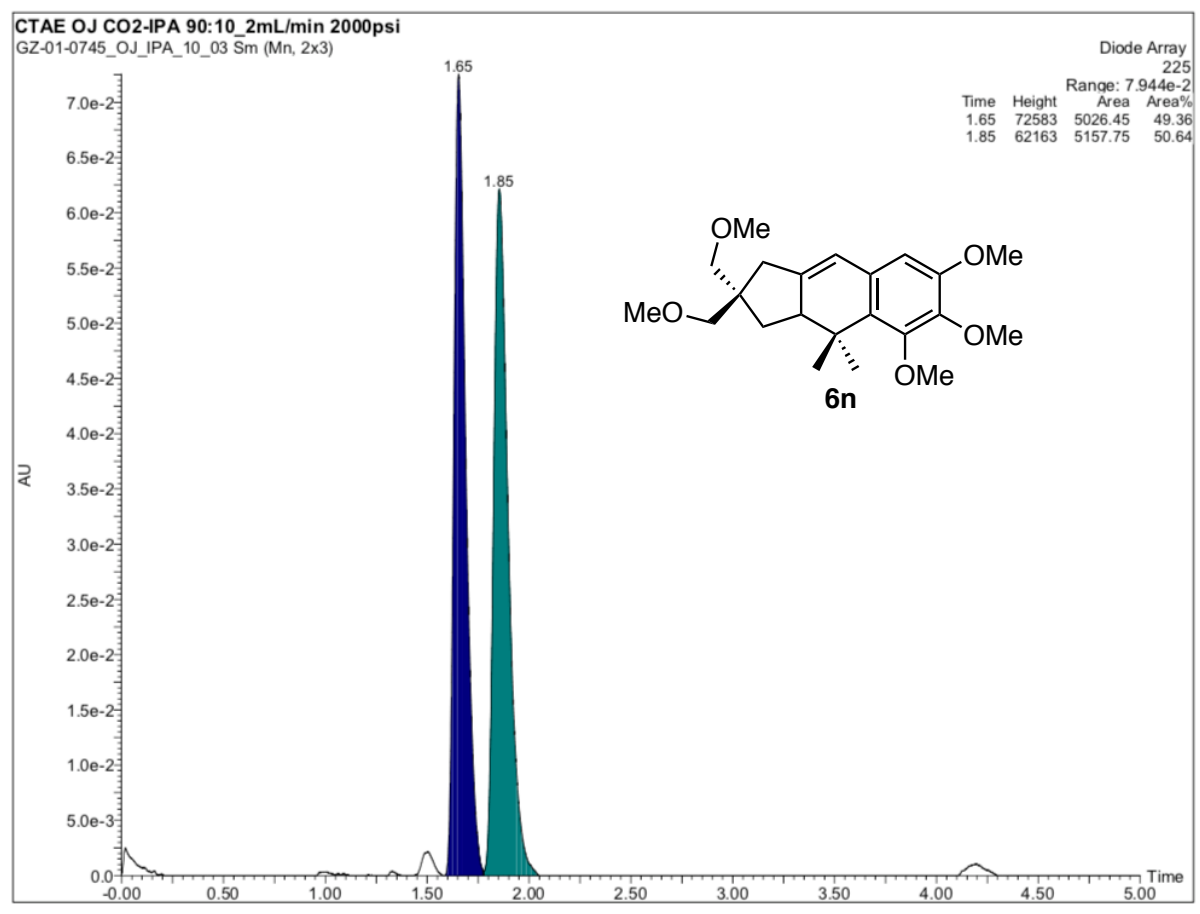

Chromatogram of enantioenriched $\mathbf{6 n}$

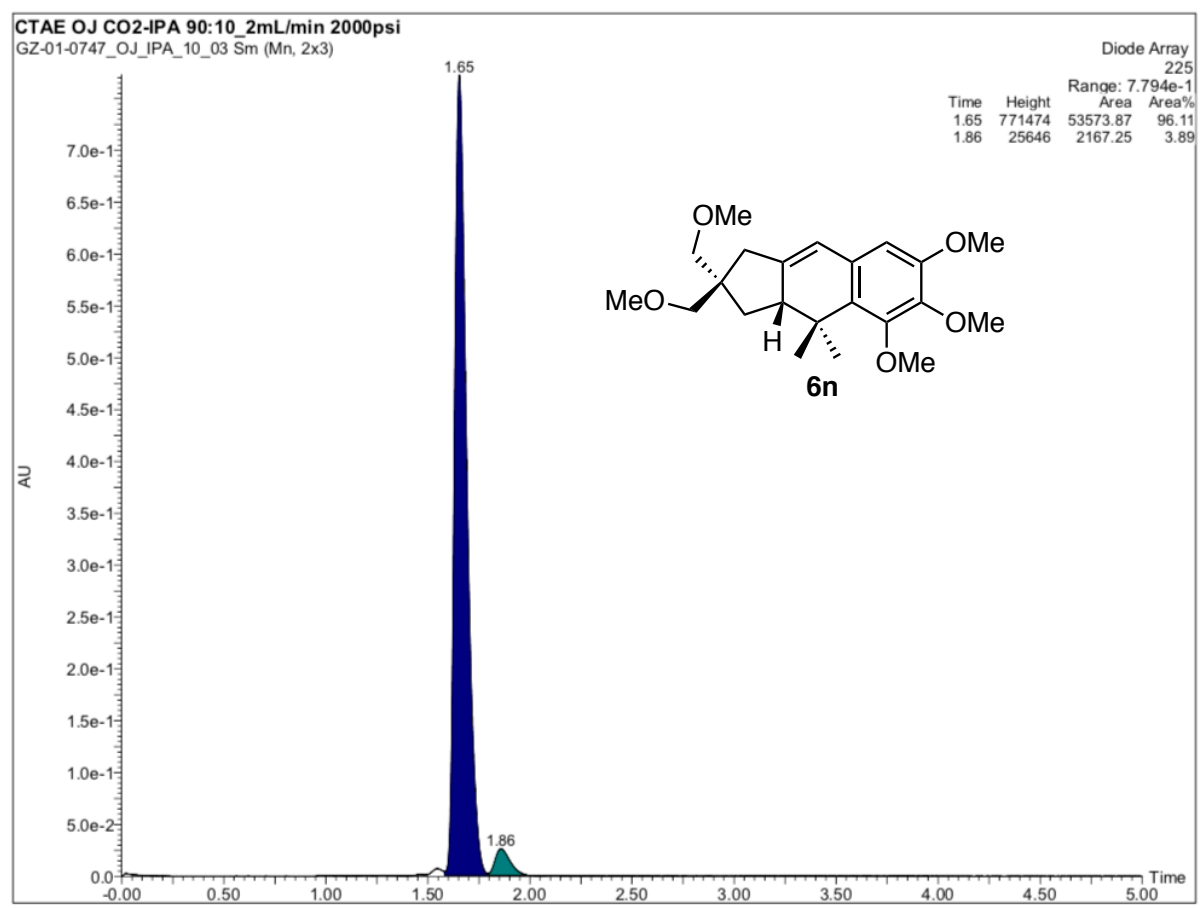


Chromatogram of racemic 60

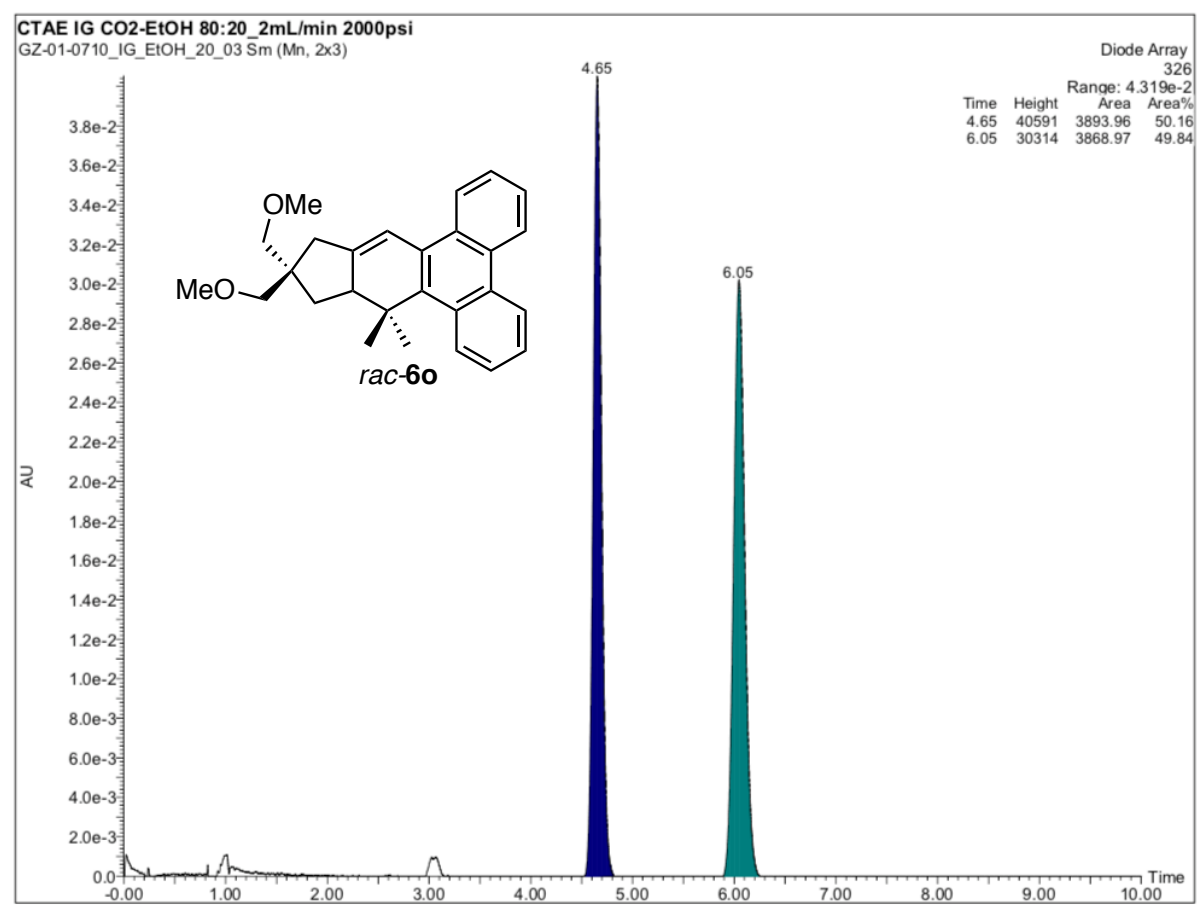

Chromatogram of enantioenriched 60

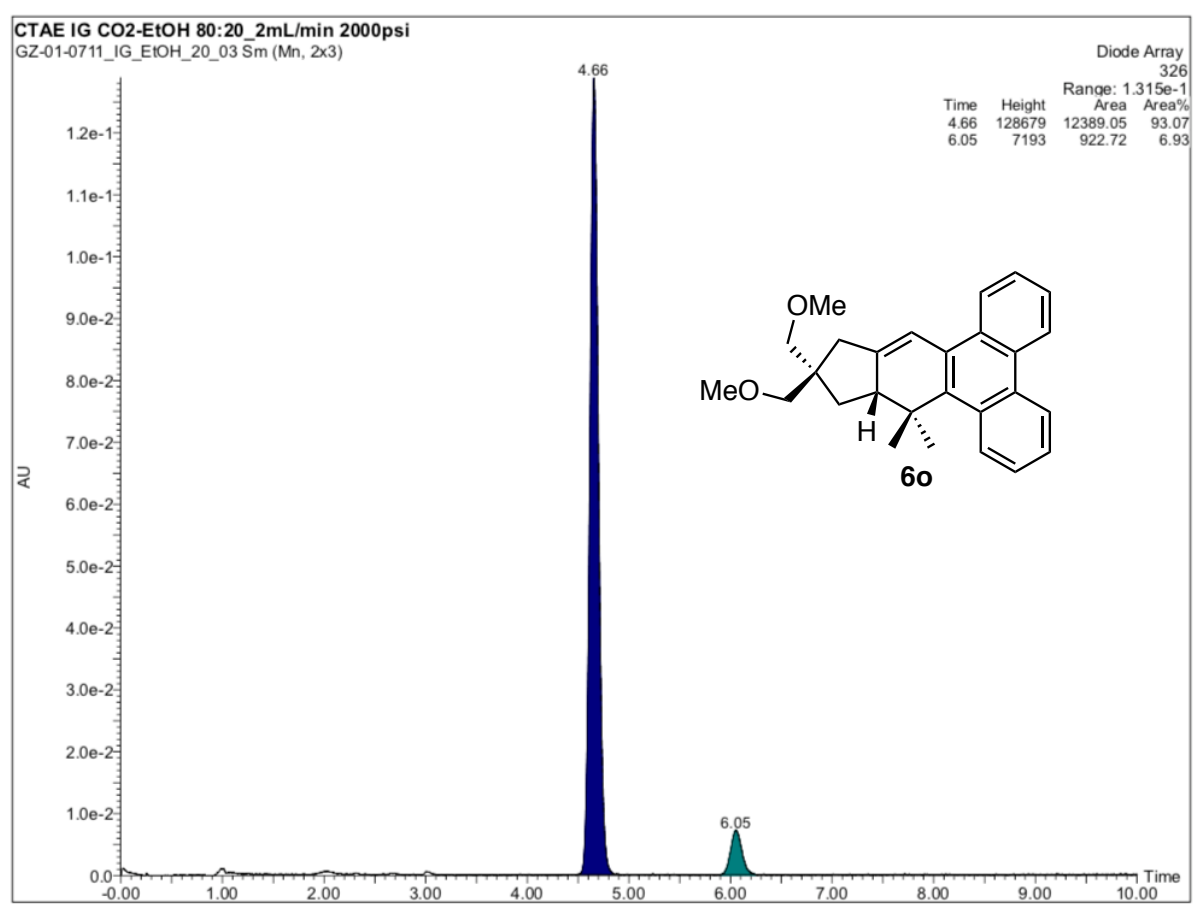


Chromatogram of racemic $\mathbf{6 p}$

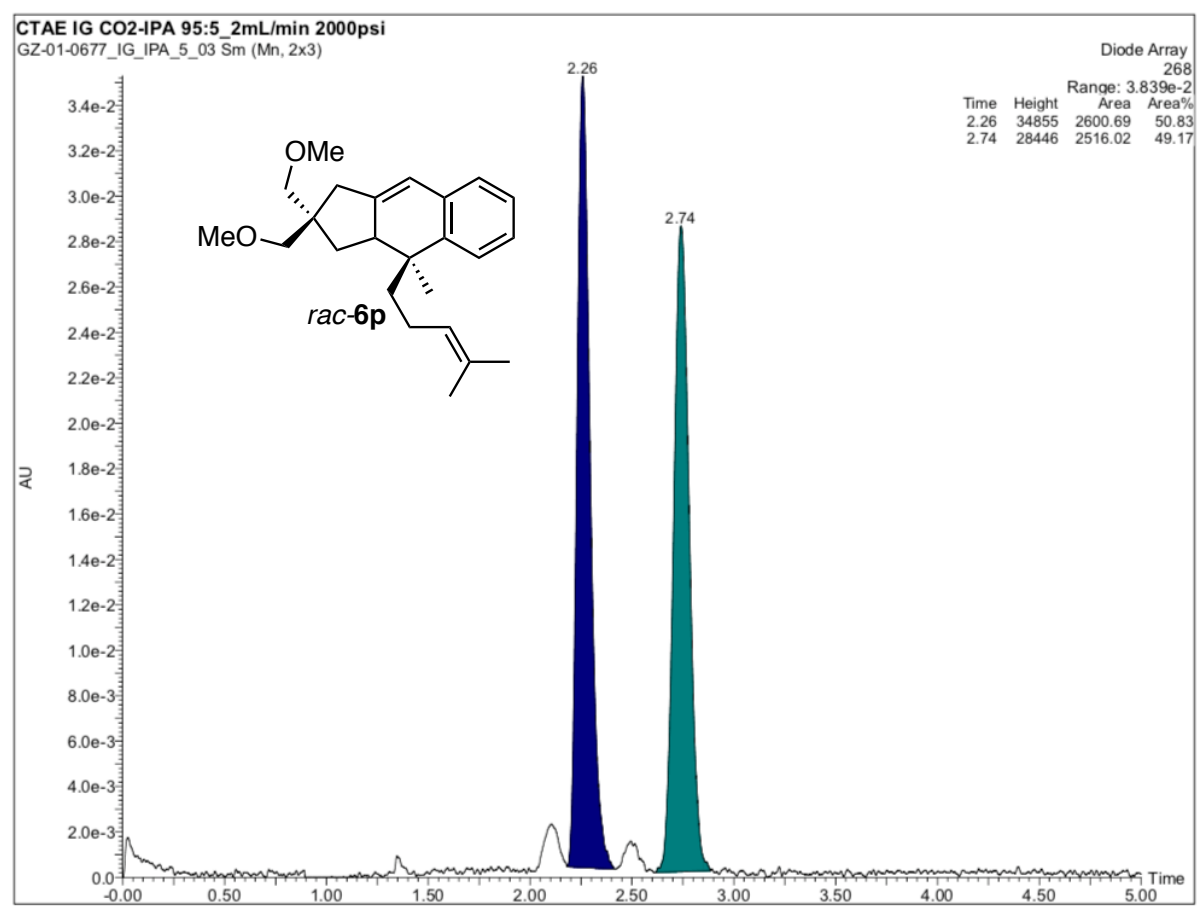

Chromatogram of enantioenriched $\mathbf{6 p}$

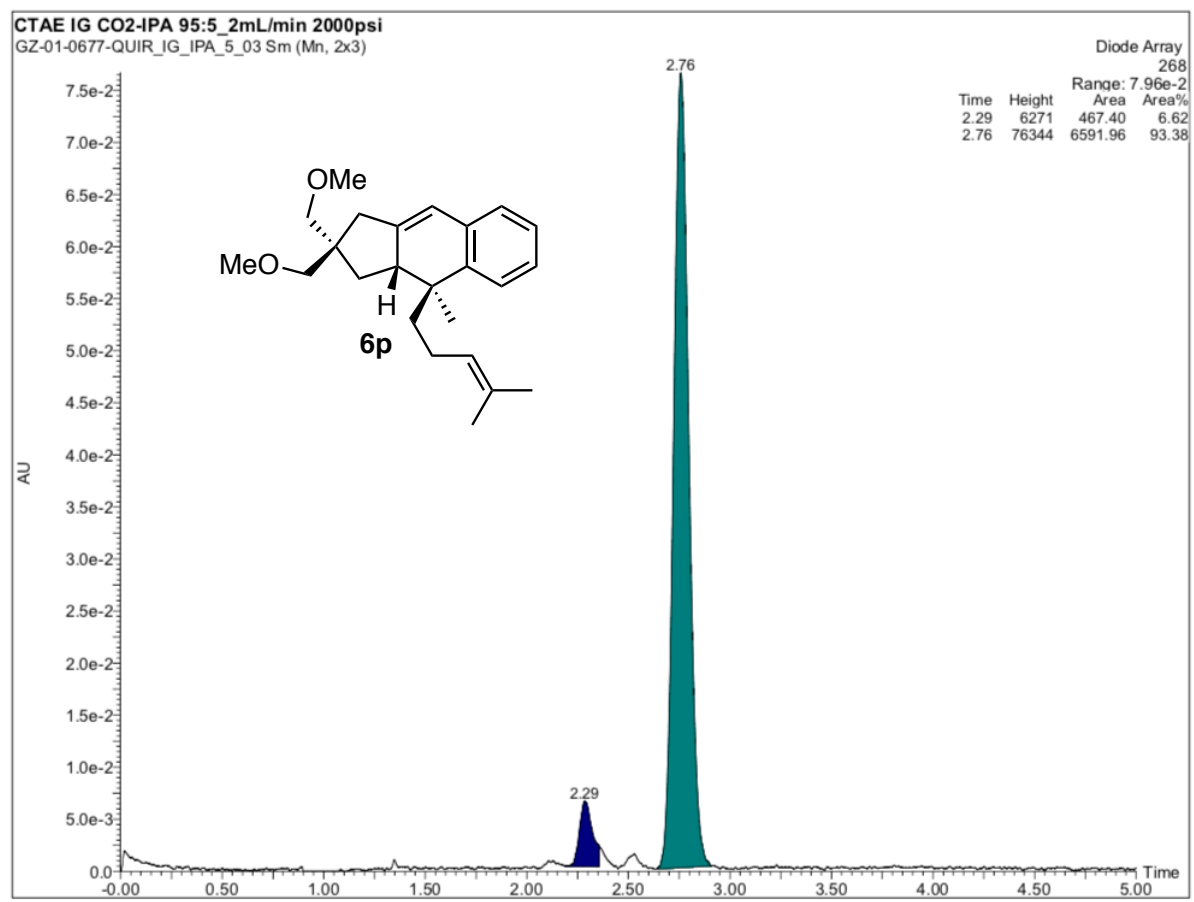


Chromatogram of racemic $\mathbf{6 q}$

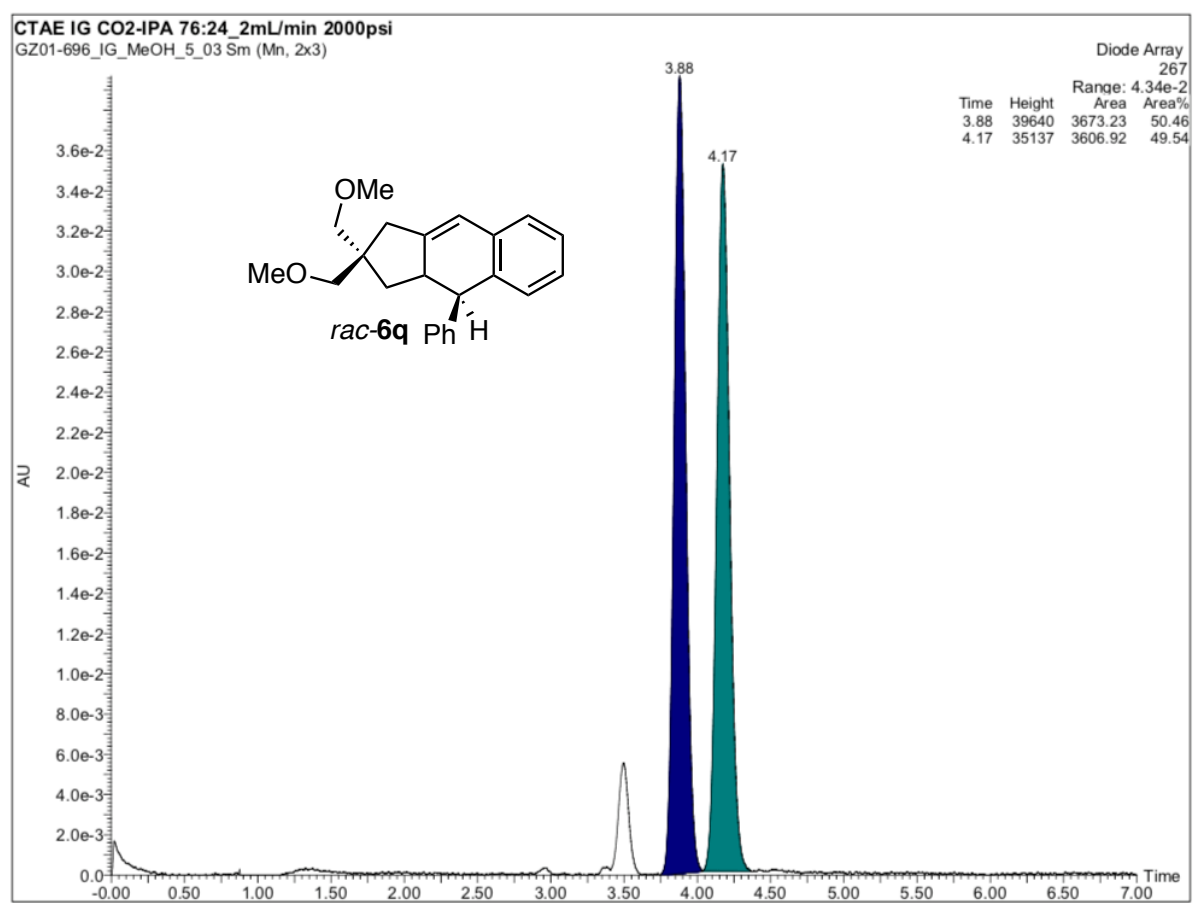

Chromatogram of enantioenriched $\mathbf{6 q}$

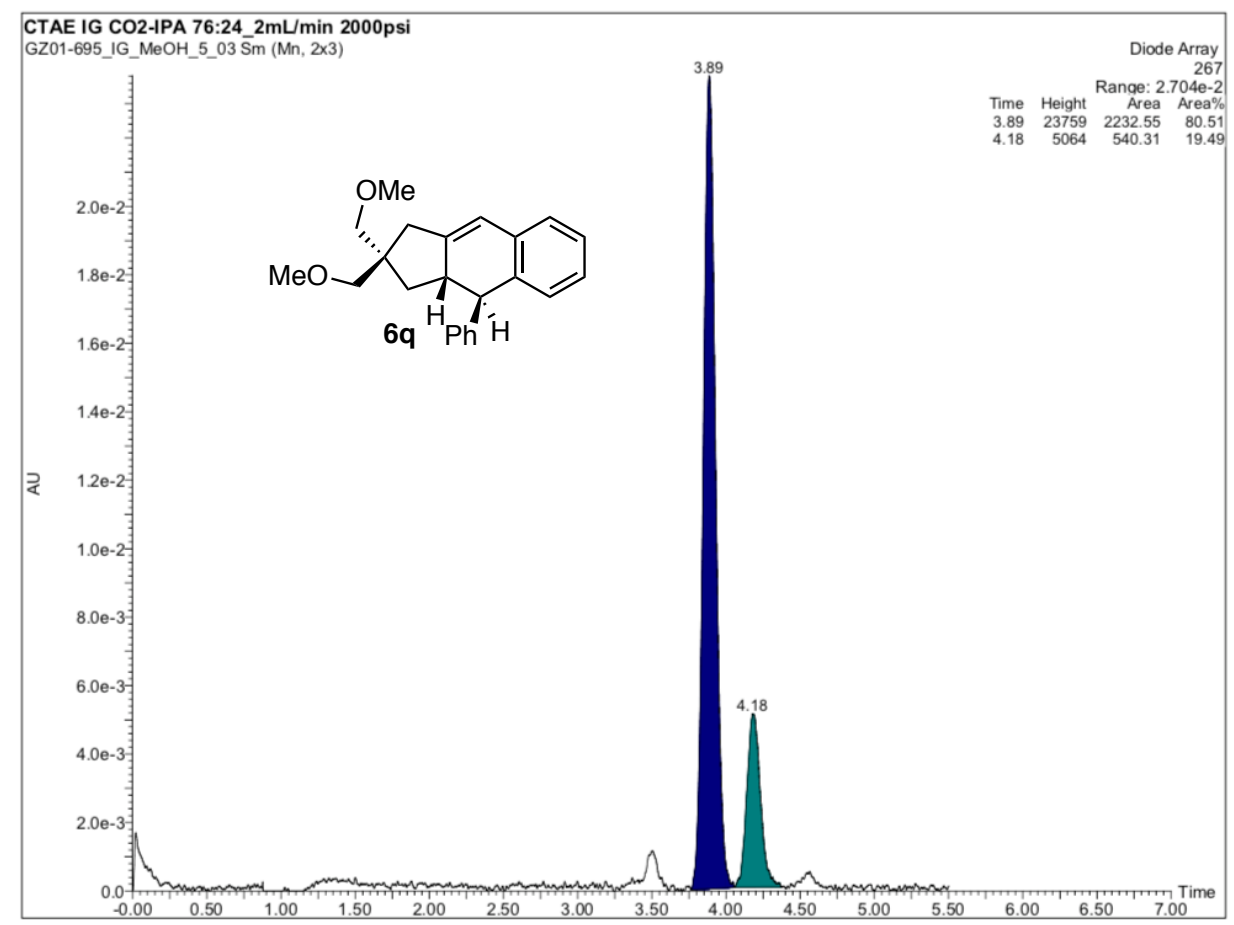


Chromatogram of racemic $\mathbf{1 6}$

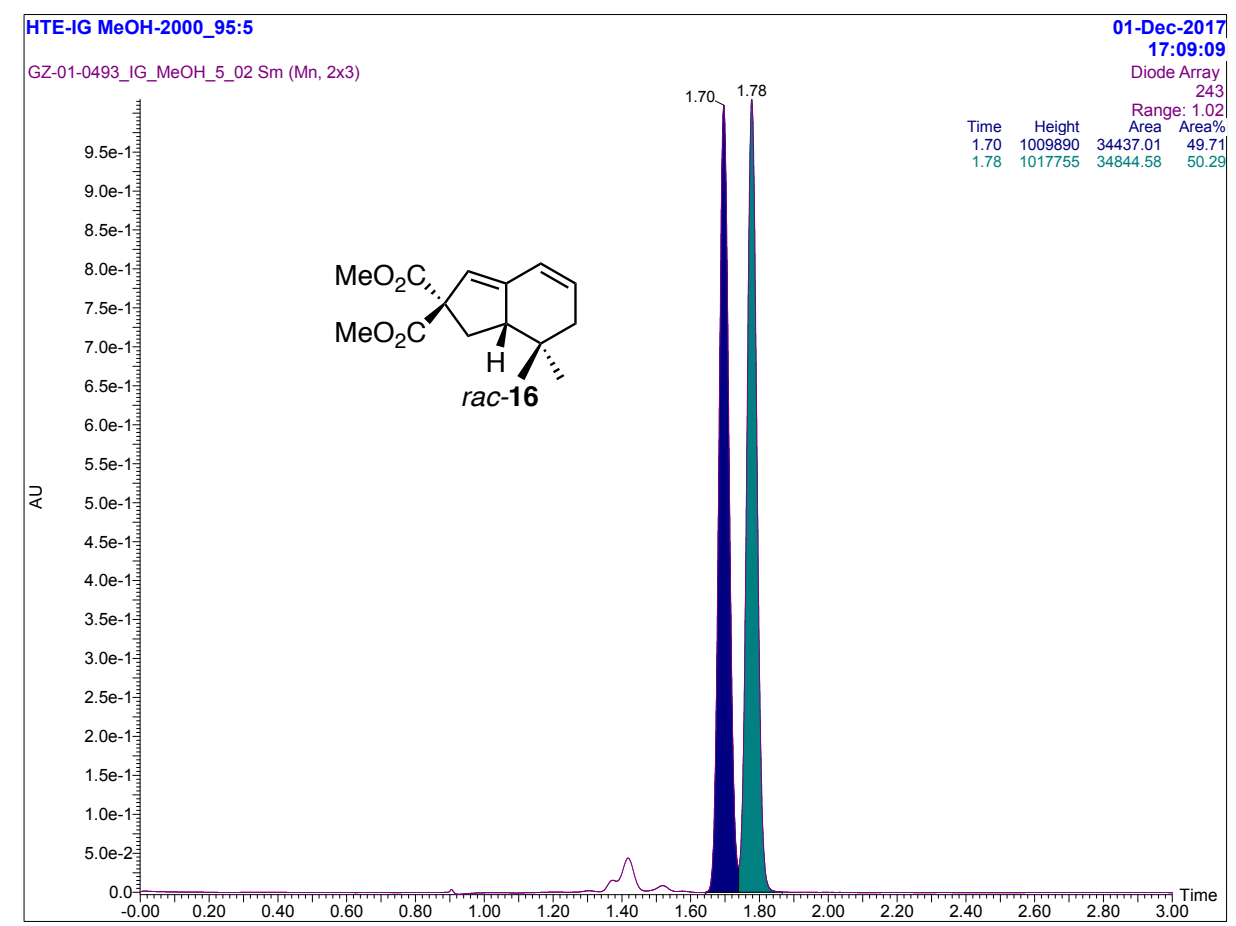

Chromatogram of enantioenriched $\mathbf{1 6}$

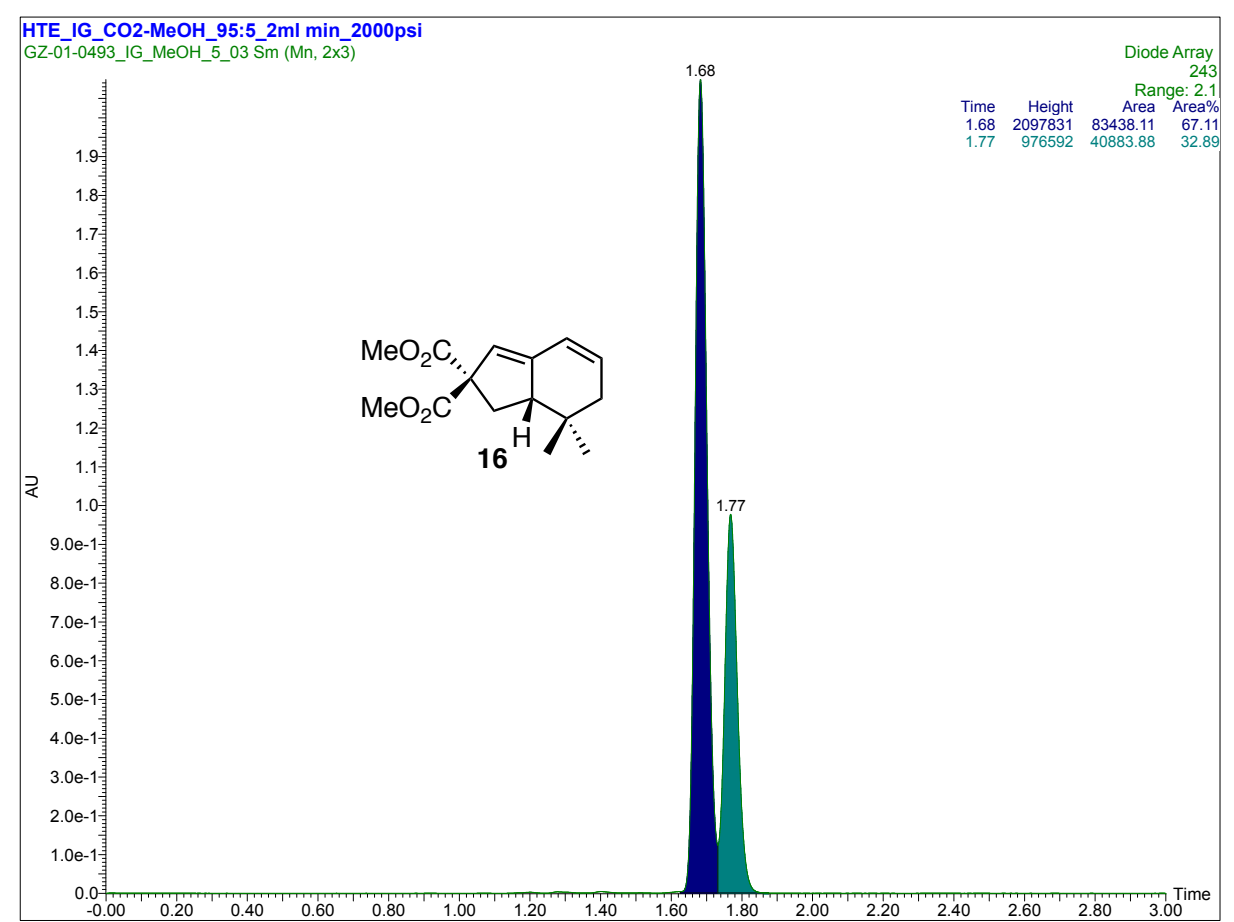




\section{Chromatogram of racemic 8a}
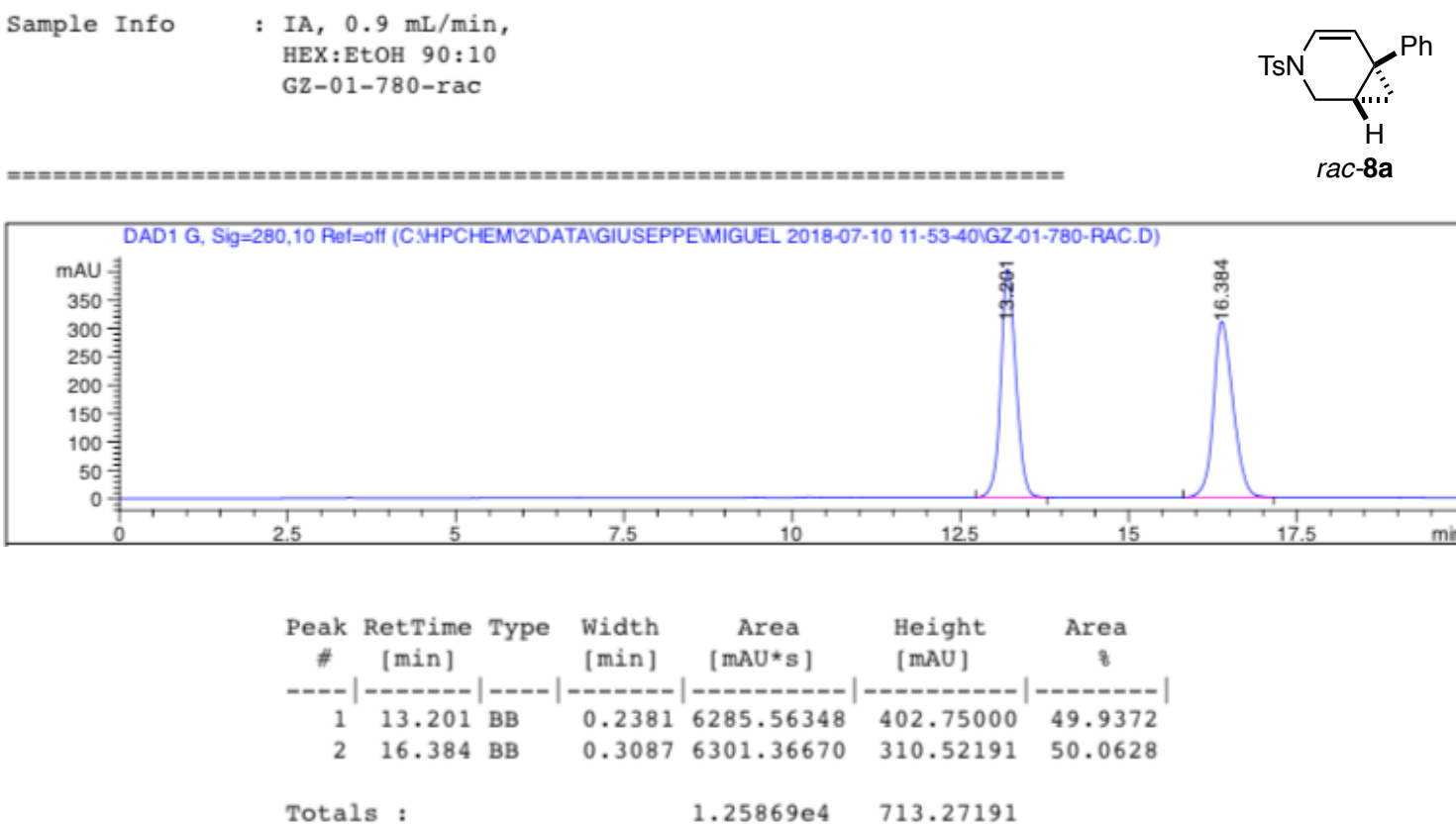

\section{Chromatogram of enantioenriched 8a}
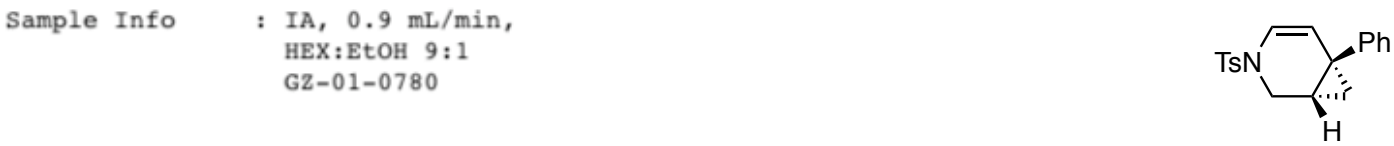

$8 \mathbf{a}$

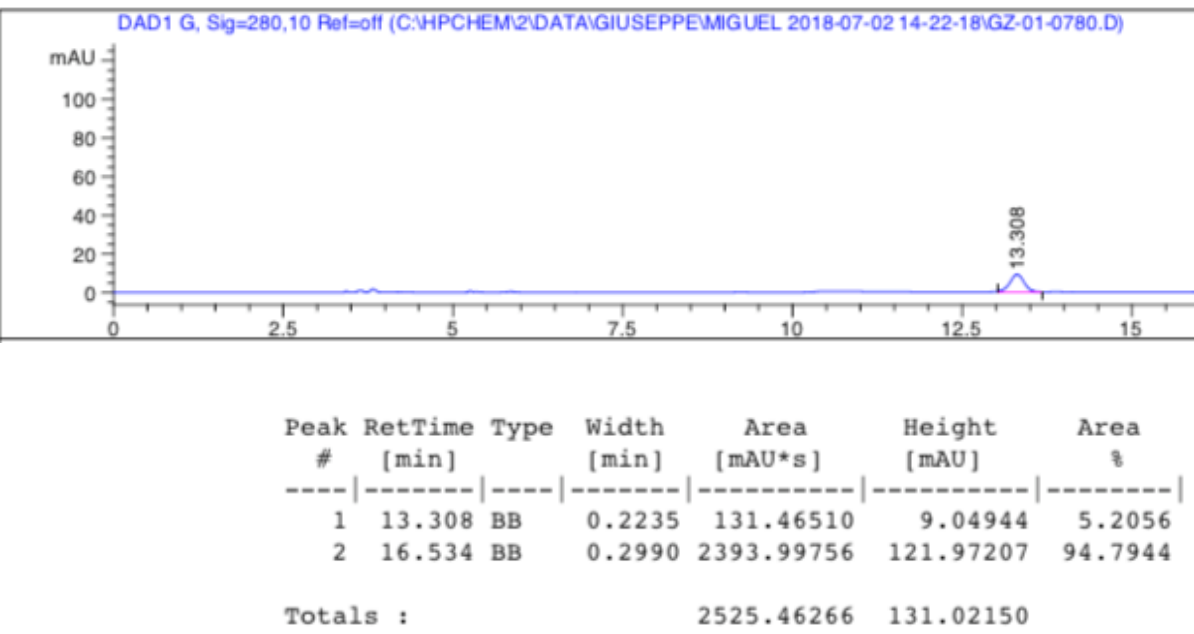


Chromatogram of racemic $\mathbf{8 b}$

$$
\begin{aligned}
& \text { Sample Info : IA, } 1 \mathrm{~mL} / \mathrm{min} \text {, } \\
& \text { HEX : IPA } 95: 5 \\
& \text { GZ-01-0785-rac }
\end{aligned}
$$
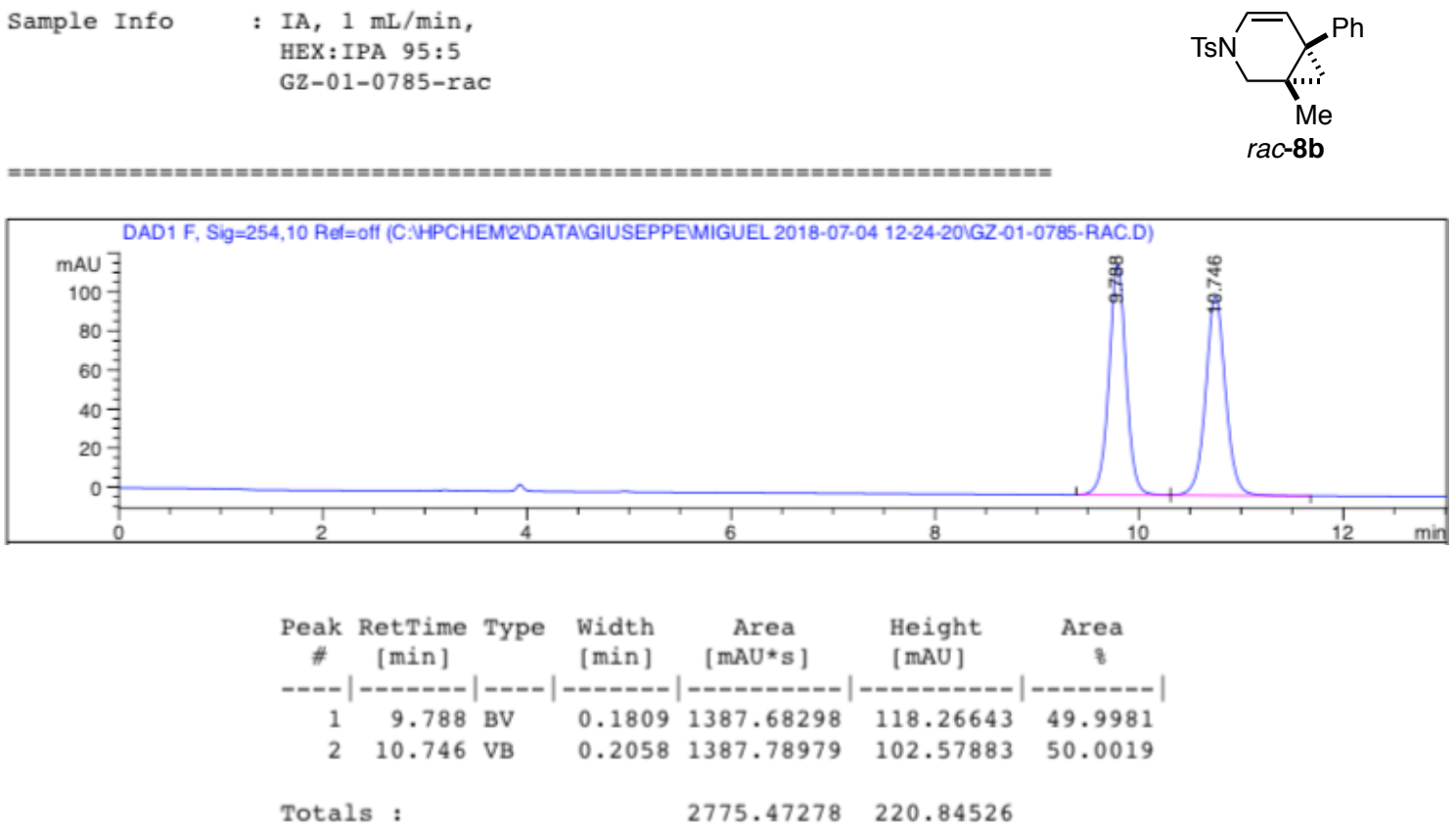

\section{Chromatogram of enantioenriched $\mathbf{8 b}$}
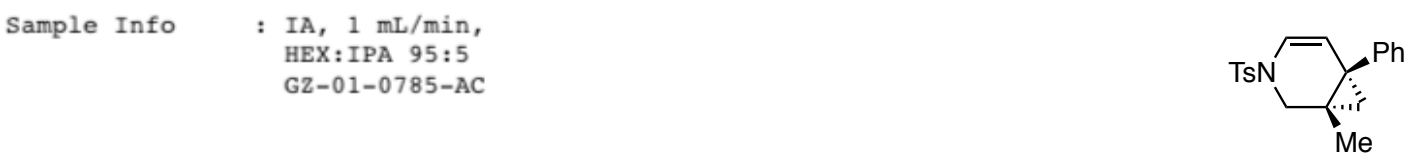

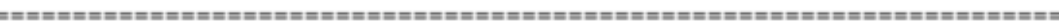

$8 \mathbf{b}$

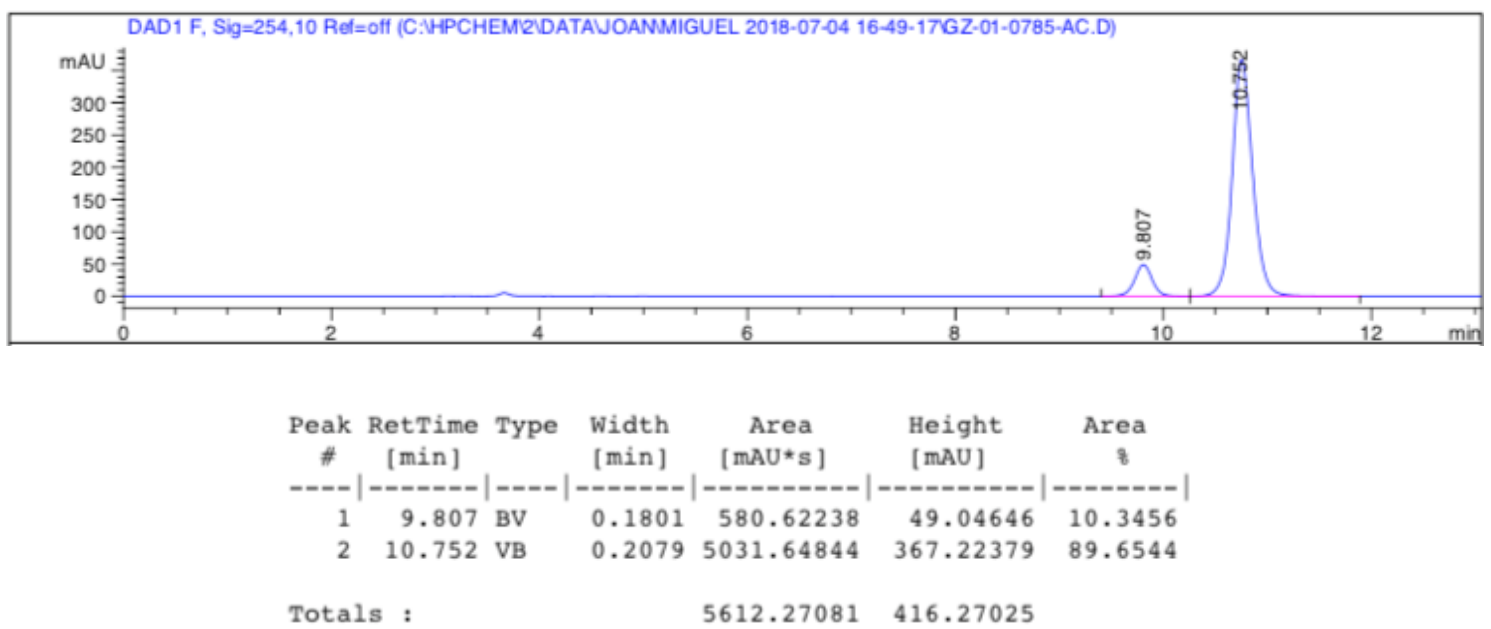


Chromatogram of racemic 8c
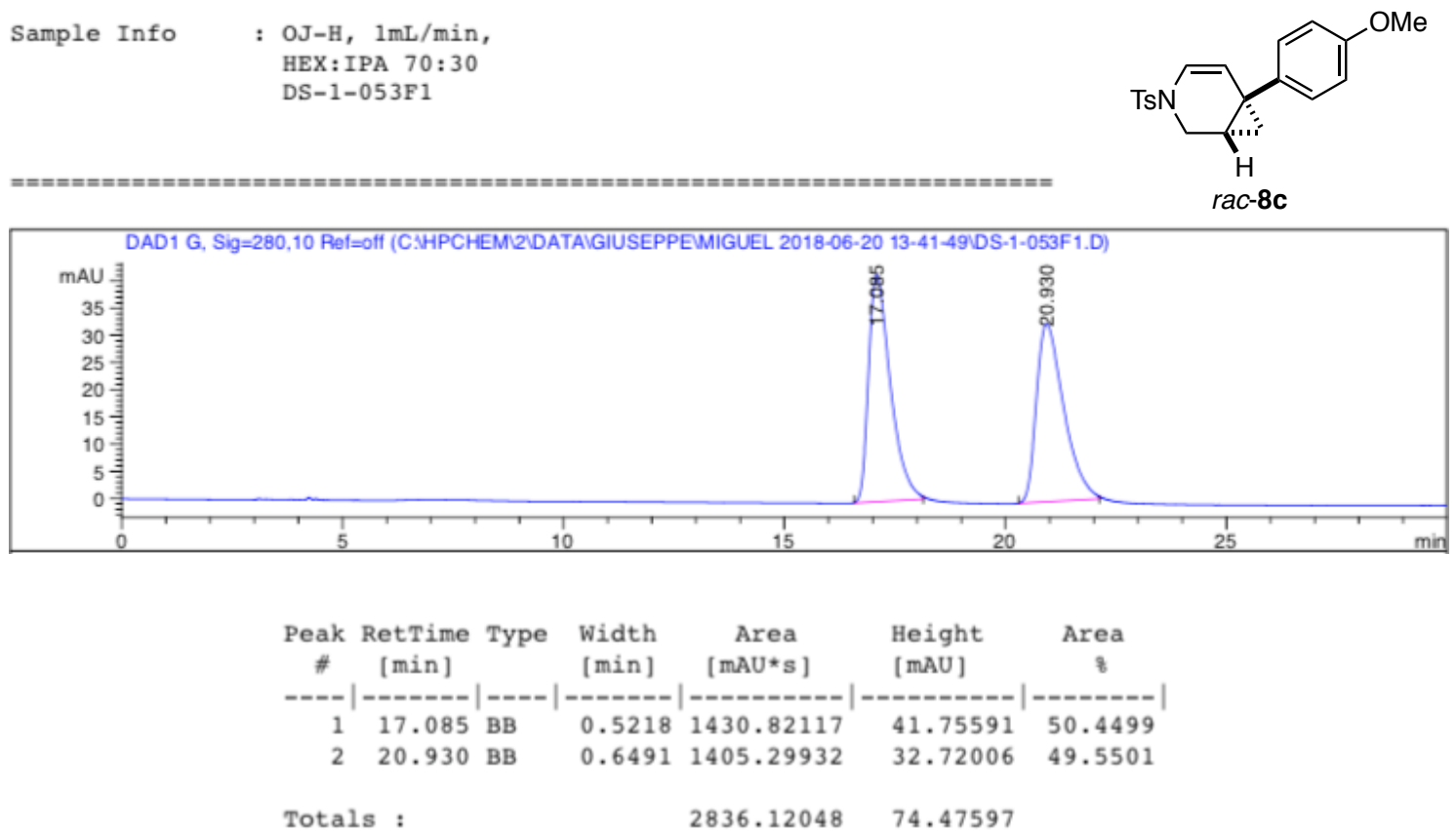

Chromatogram of enantioenriched 8c
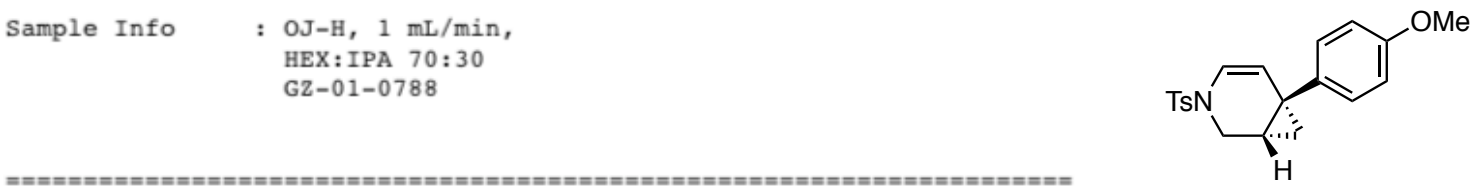

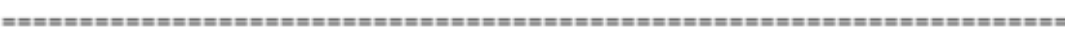

8c

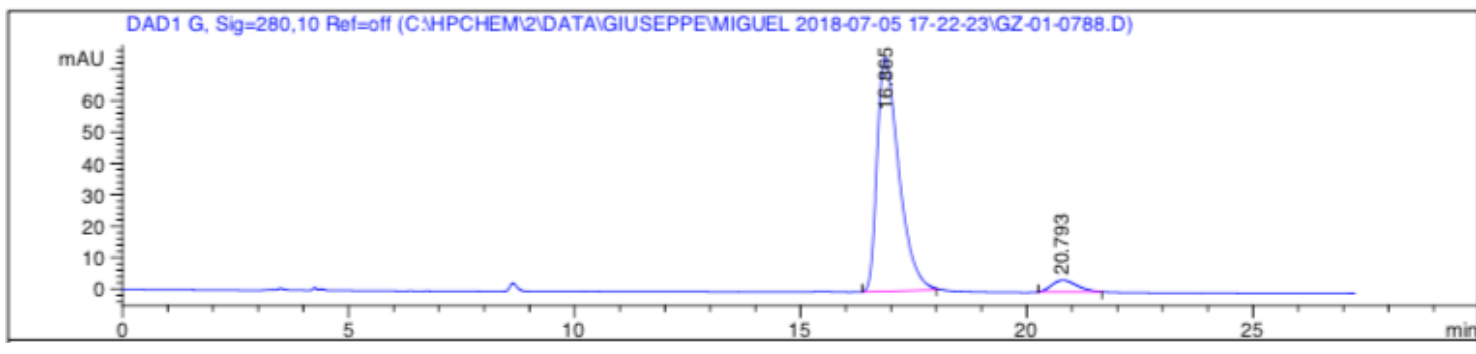

\begin{tabular}{|c|c|c|c|c|c|c|}
\hline $\begin{array}{c}\text { Peak } \\
\#\end{array}$ & $\begin{array}{c}\text { RetTime } \\
\text { [min] }\end{array}$ & Type & $\begin{array}{l}\text { Width } \\
\text { [min] }\end{array}$ & $\begin{array}{c}\text { Area } \\
{\left[\mathrm{mAU}{ }^{*} \mathrm{~S}\right]}\end{array}$ & $\begin{array}{l}\text { Height } \\
\text { [mAU] }\end{array}$ & $\begin{array}{c}\text { Area } \\
\text { के }\end{array}$ \\
\hline--- & 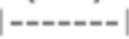 & & - & ----n--0- & -- & ------- \\
\hline 1 & 16.865 & BB & 0.5098 & 2490.90942 & 74.55279 & 94.3436 \\
\hline 2 & 20.793 & BB & 0.5603 & 149.34204 & 3.80205 & 5.6564 \\
\hline Tota & Is : & & & 2640.25146 & 78.35484 & \\
\hline
\end{tabular}


Chromatogram of racemic 8d

$$
\begin{aligned}
\text { Sample Info : } & \text { IC, } 1 \mathrm{~mL} / \mathrm{min}, \\
& \text { HEX }: \text { IPA } 8: 2 \\
& \text { MsN-cyclization }
\end{aligned}
$$
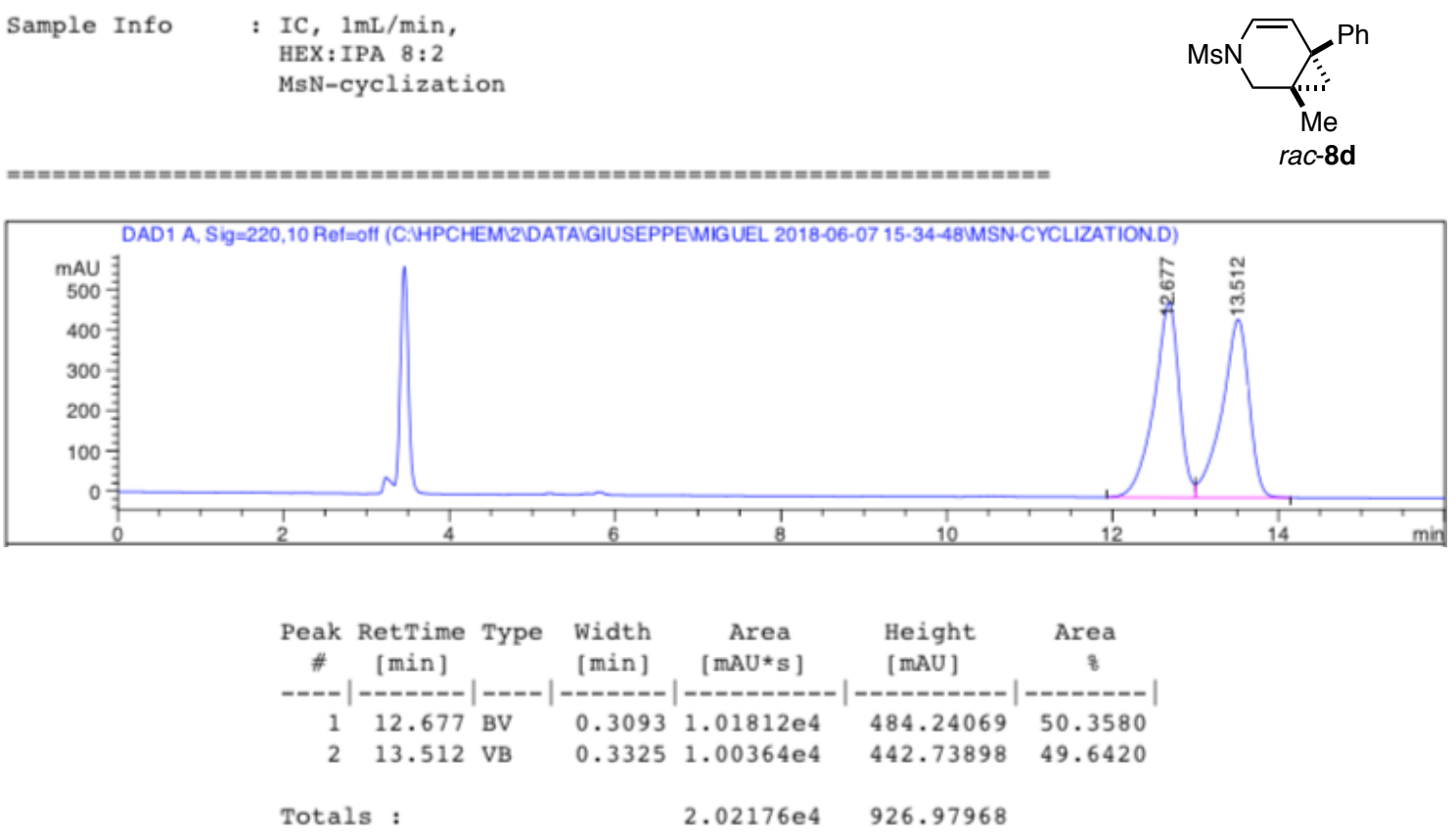

Chromatogram of enantioenriched $\mathbf{8 d}$
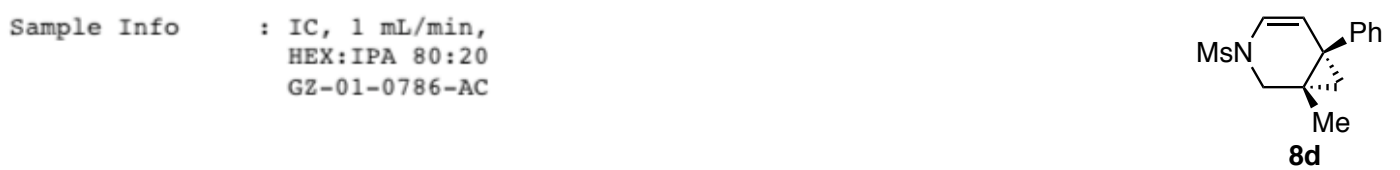

\begin{tabular}{|c|c|c|c|c|c|c|}
\hline $\begin{array}{c}\text { Peak } \\
\#\end{array}$ & $\begin{array}{l}\text { RetTime } \\
\text { [min] }\end{array}$ & Type & $\begin{array}{l}\text { Width } \\
\text { [min] }\end{array}$ & $\begin{array}{c}\text { Area } \\
{[\mathrm{mAU} * \mathrm{~s}]}\end{array}$ & $\begin{array}{l}\text { Height } \\
\text { [mAU] }\end{array}$ & $\begin{array}{c}\text { Area } \\
\text { ? }\end{array}$ \\
\hline 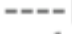 & 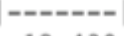 & & --- & $-1-0-1$ & 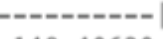 & $2-2-2-2$ \\
\hline 1 & 12.430 & BV & 0.3014 & 3045.37598 & 148.40620 & 26.1755 \\
\hline 2 & 13.220 & VB & 0.3256 & 8589.08398 & 389.06757 & 73.8245 \\
\hline Tota. & is : & & & $1.16345 \mathrm{e} 4$ & 537.47377 & \\
\hline
\end{tabular}

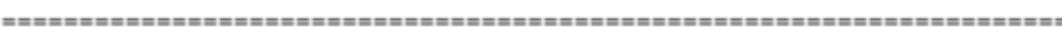

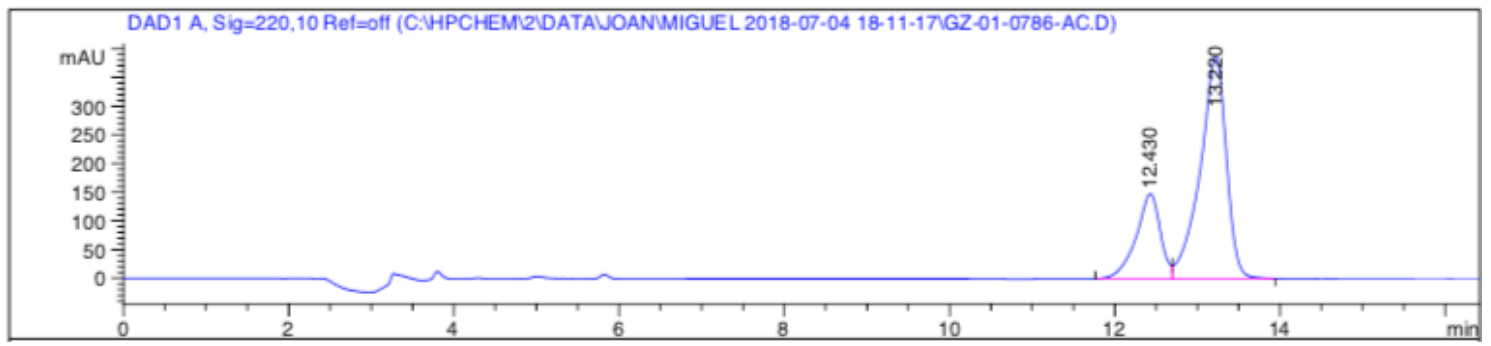


Chromatogram of racemic 8e
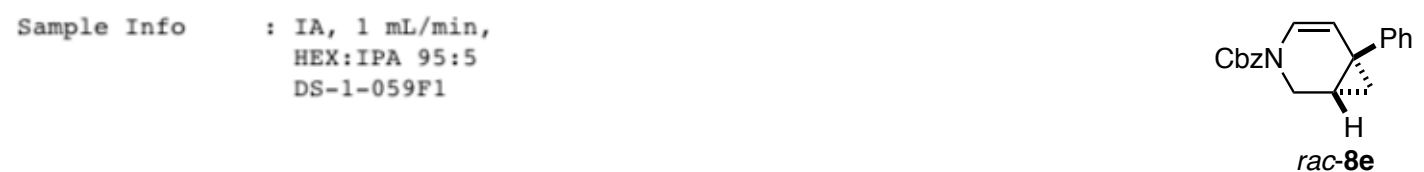

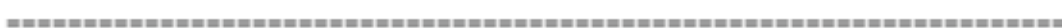

rac-8e

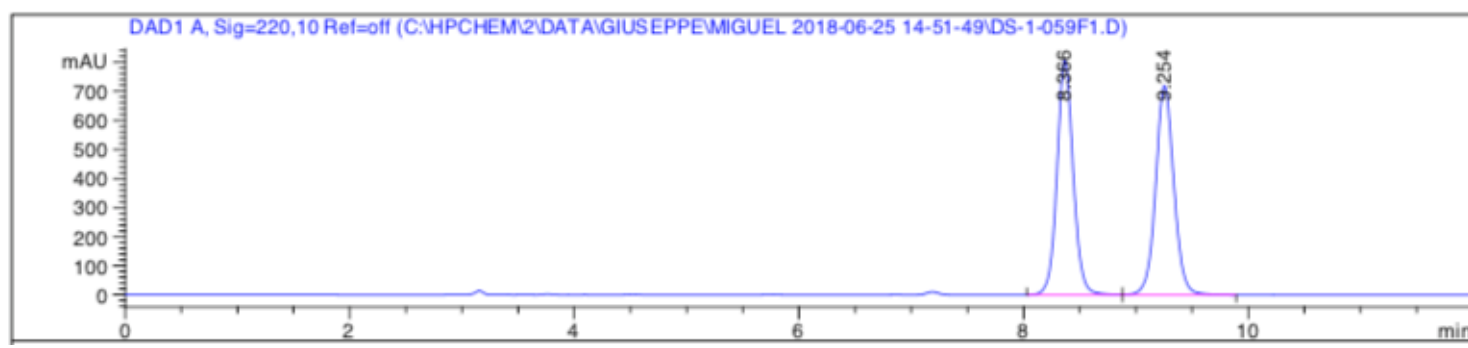

\begin{tabular}{|c|c|c|c|c|c|c|}
\hline $\begin{array}{c}\text { Peak } \\
\#\end{array}$ & $\begin{array}{c}\text { RetTime } \\
\text { [min] }\end{array}$ & Type & $\begin{array}{l}\text { Width } \\
\text { [min] }\end{array}$ & $\begin{array}{c}\text { Area } \\
{[\mathrm{mAU} * \mathrm{~S}]}\end{array}$ & $\begin{array}{l}\text { Height } \\
\text { [mAU] }\end{array}$ & $\begin{array}{c}\text { Area } \\
\text { เิ }\end{array}$ \\
\hline & & & & $-----0---$ & | - - - - - - & -------- \\
\hline 1 & 8.366 & BV & 0.1534 & 8125.12793 & 807.58972 & 49.8914 \\
\hline 2 & 9.254 & vB & 0.1741 & 8160.50977 & 720.53467 & 50.1086 \\
\hline Total & & & & $1.62856 \mathrm{e} 4$ & 1528.12439 & \\
\hline
\end{tabular}

Chromatogram of enantioenriched $\mathbf{8 e}$

$$
\begin{aligned}
\text { Sample Info : IA, } 1 \mathrm{~mL} / \mathrm{min}, \\
\\
\text { HEX:IPA } 95: 5 \\
\text { GZ-01-0787-AC }
\end{aligned}
$$

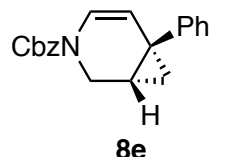

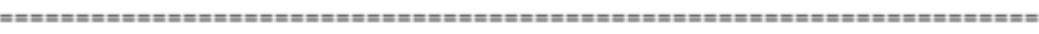
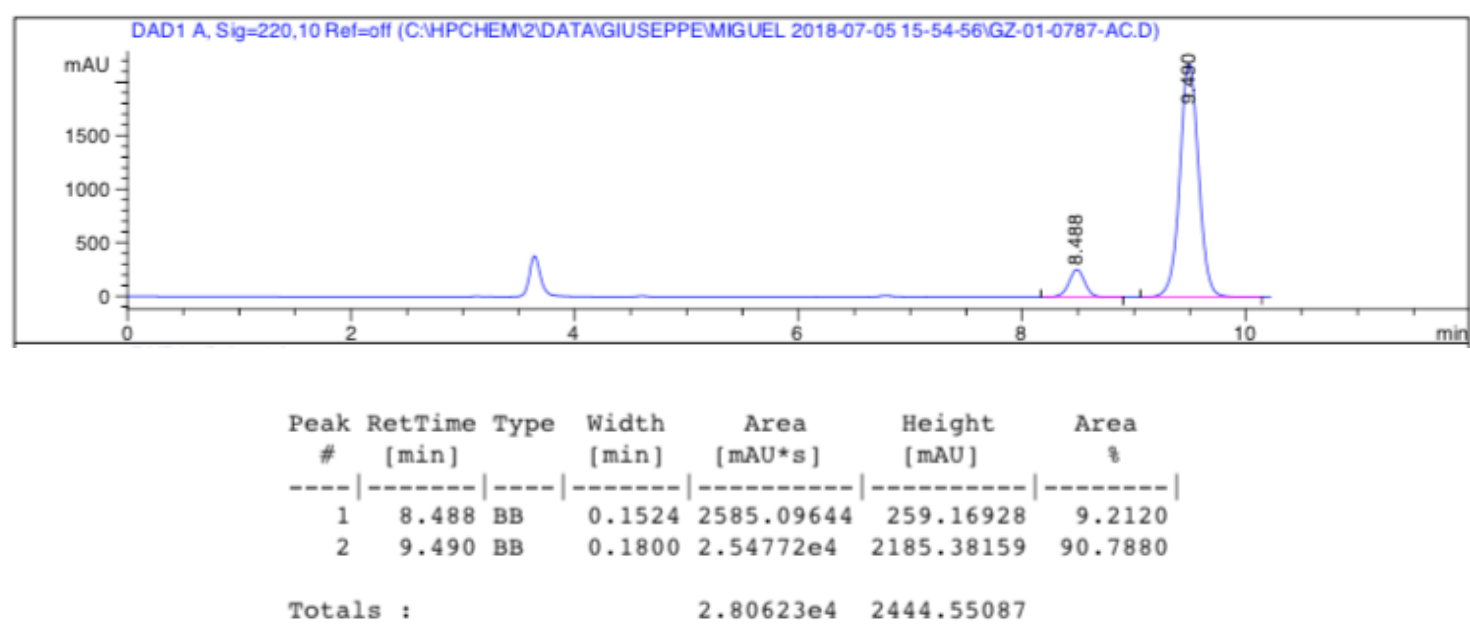
Chromatogram of racemic $\mathbf{8 f}$

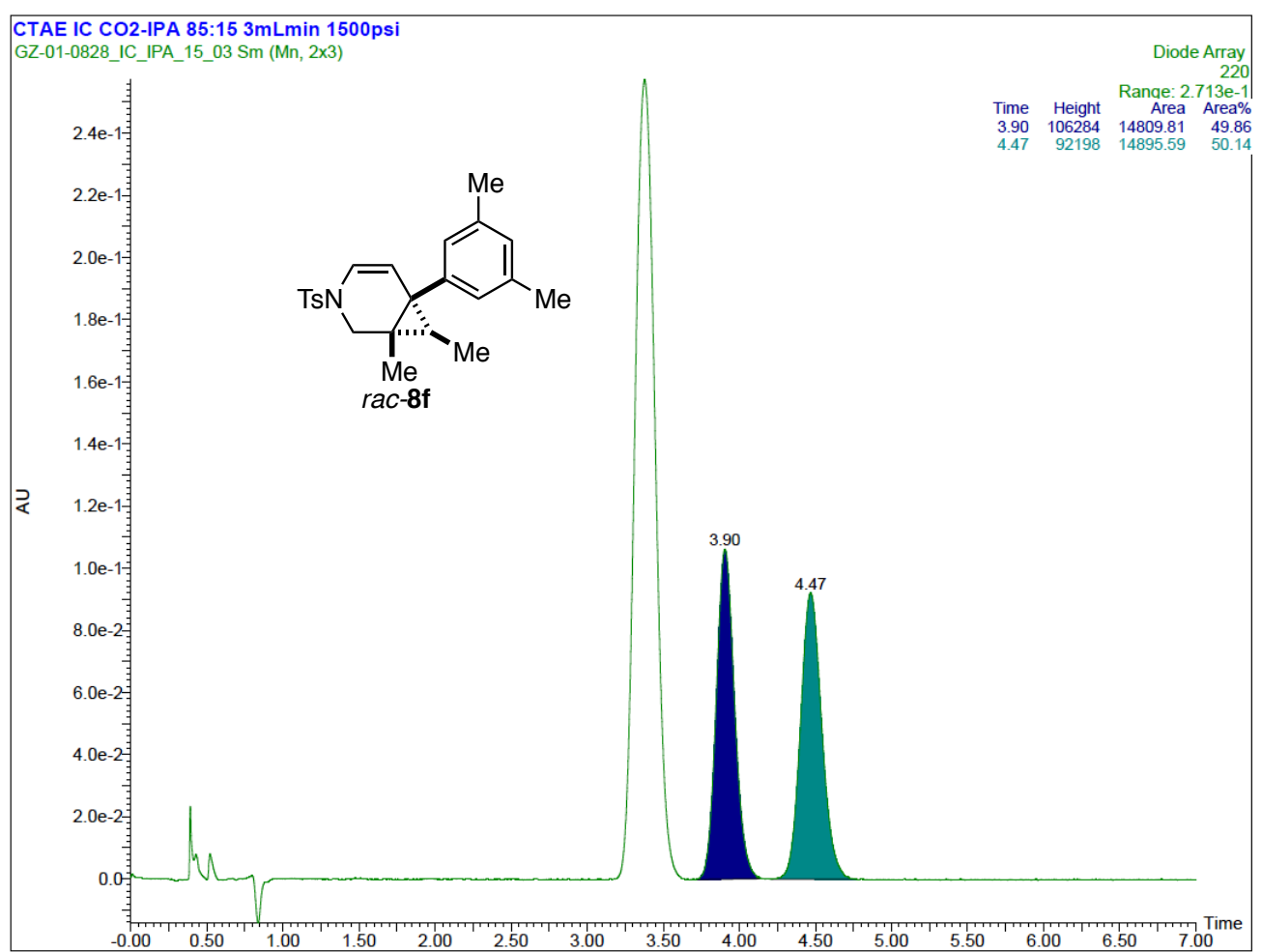

Chromatogram of enantioenriched $\mathbf{8 f}$

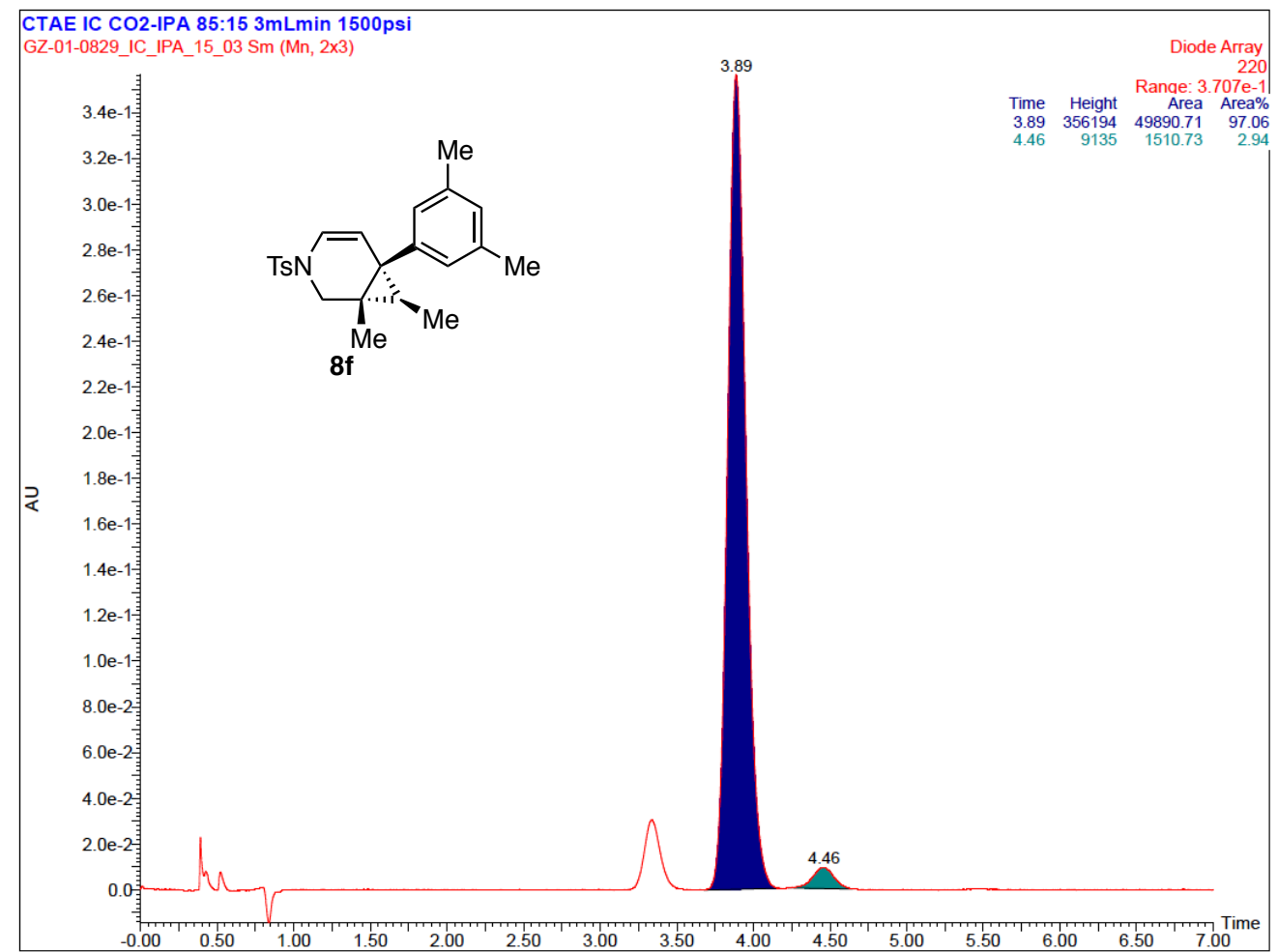


Chromatogram of racemic $\mathbf{8 g}$
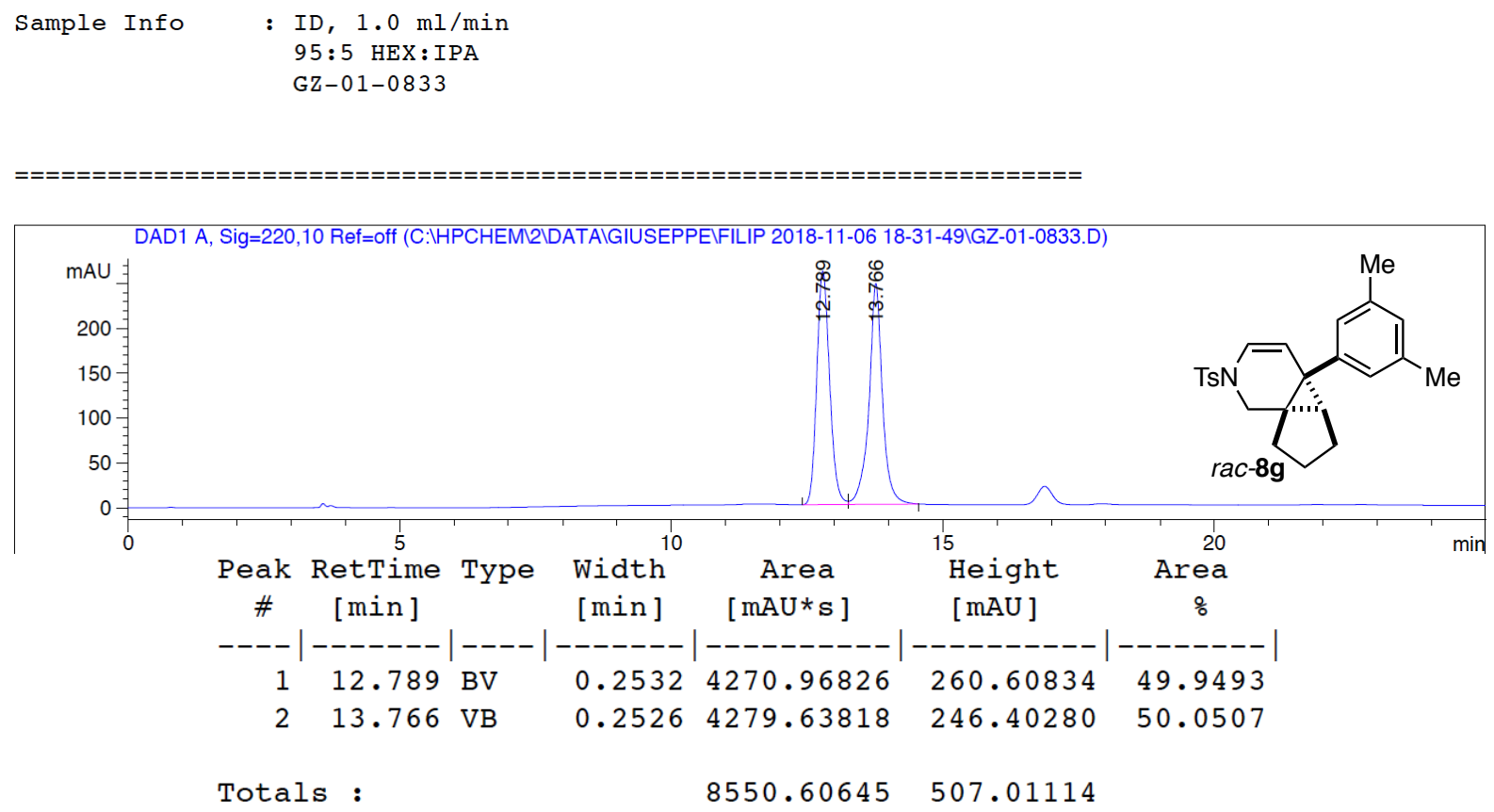

Chromatogram of enantioenriched $\mathbf{8 g}$
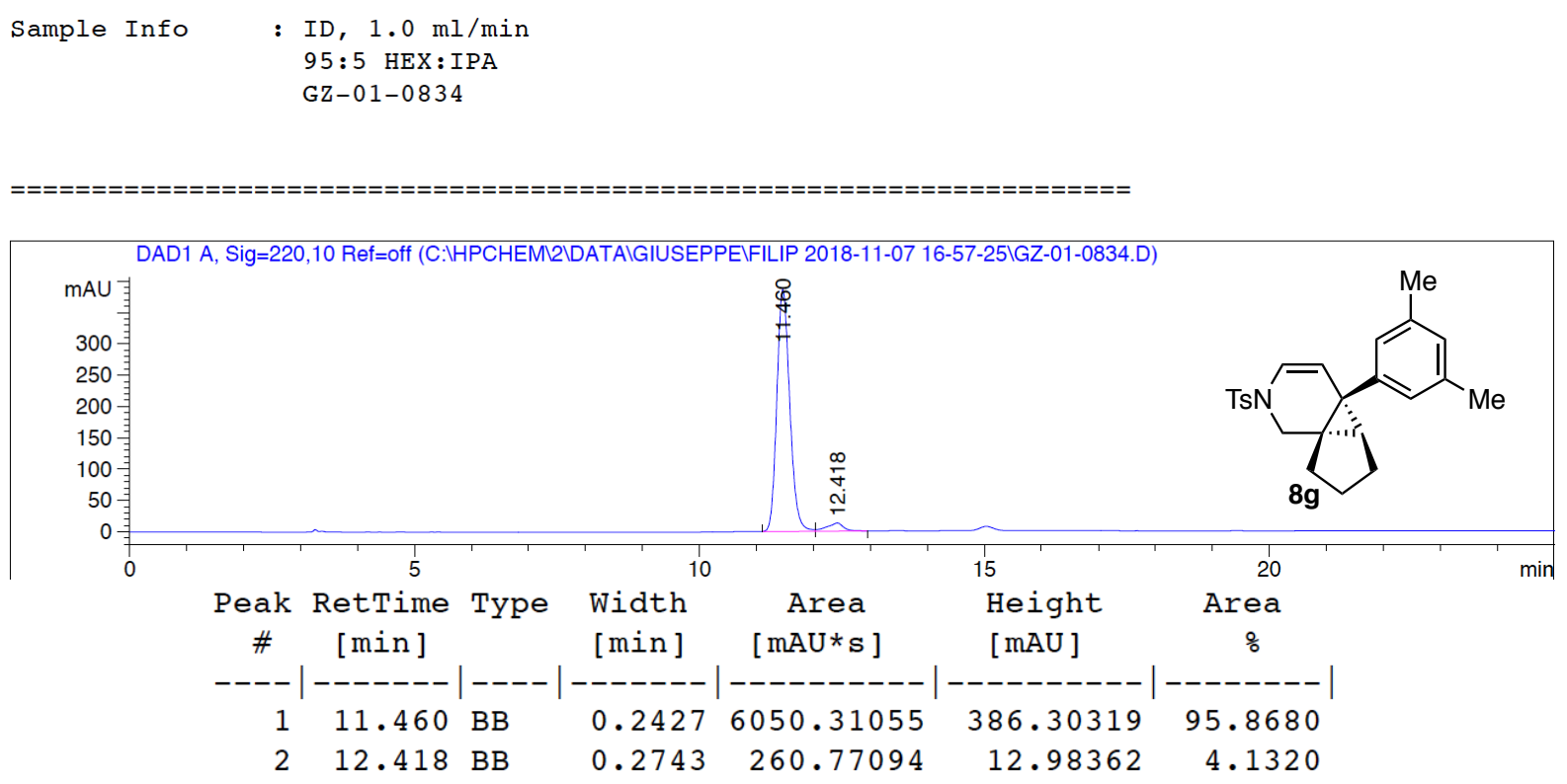
Chromatogram of racemic 10a

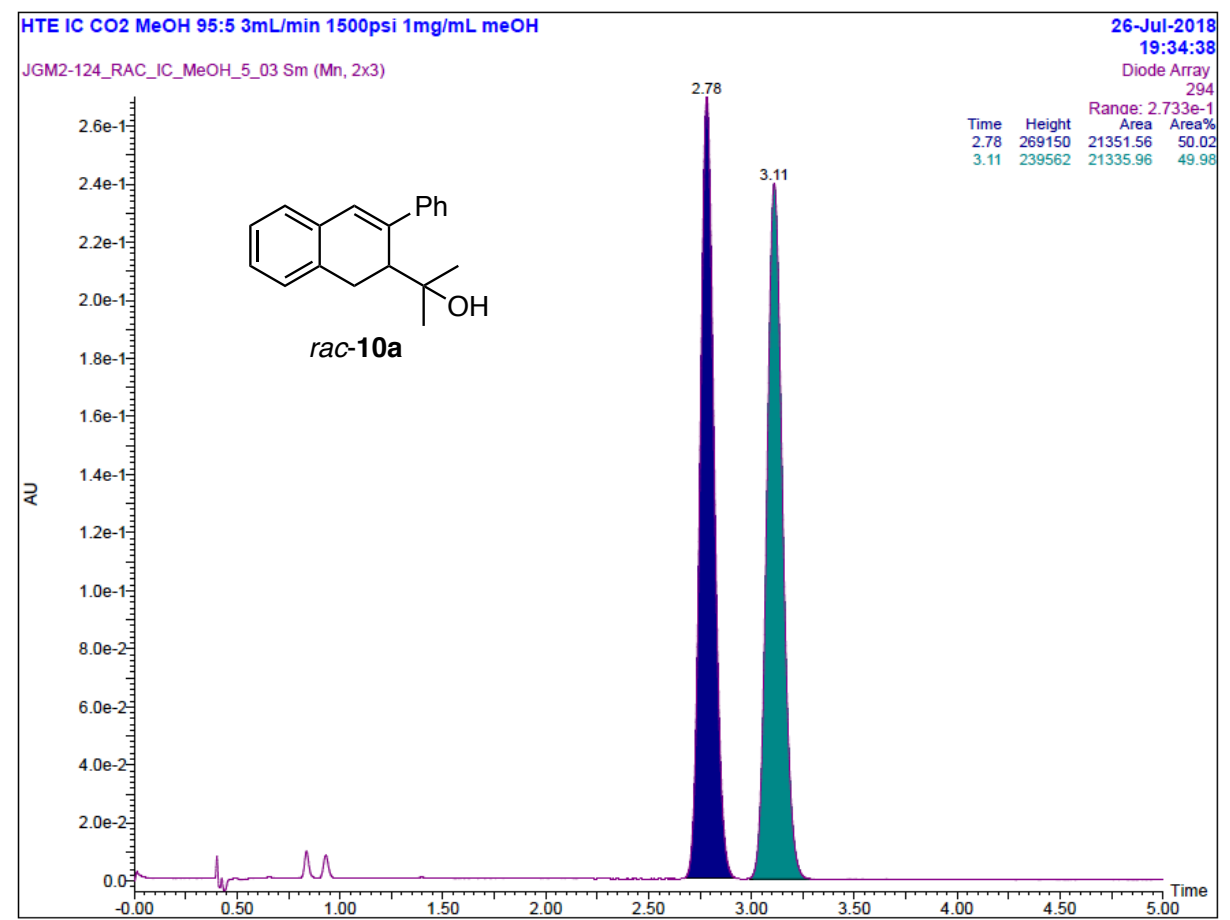

Chromatogram of enantioenriched 10a

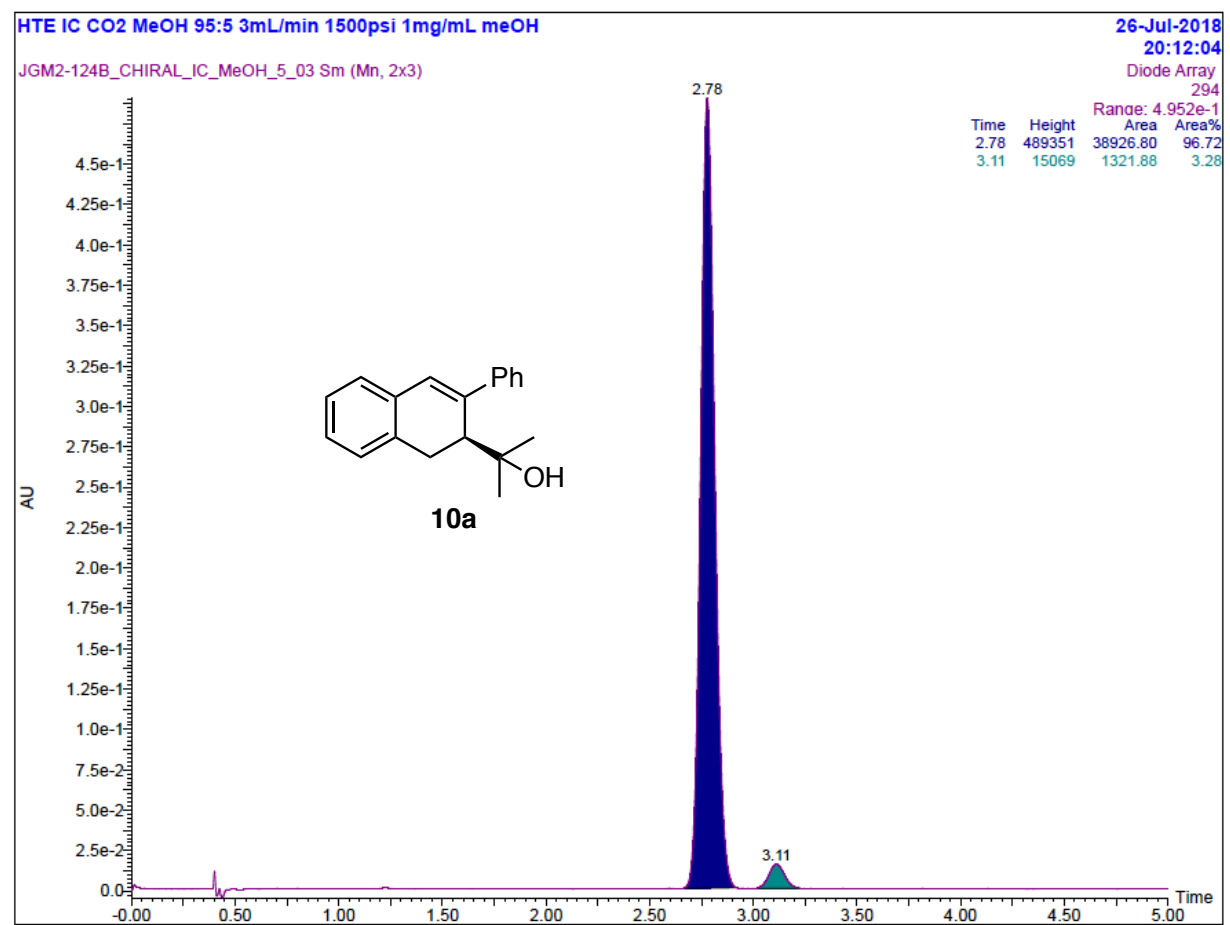


Chromatogram of racemic $\mathbf{1 0 b}$

$$
\begin{array}{ll}
\text { Sample Info } \quad & \text { OJ-H, } 1 \mathrm{~mL} / \mathrm{min}, \\
& \text { HEX:IPA } 9: 1 \\
& \text { JGM2-273 }
\end{array}
$$

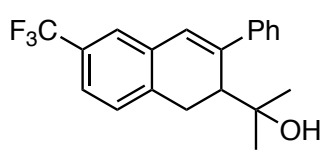

rac-10b
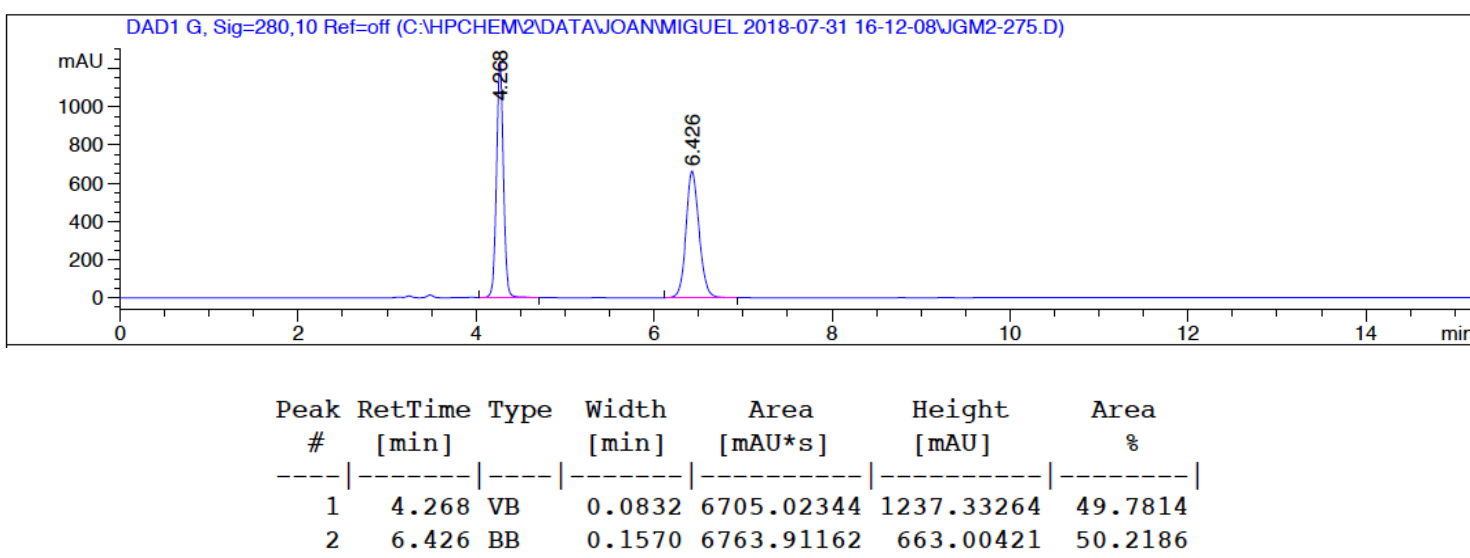

Totals :

$1.34689 \mathrm{e} 4 \quad 1900.33685$

\section{Chromatogram of enantioenriched 10b}

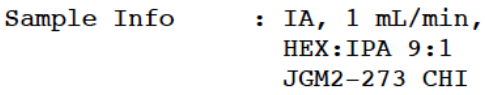

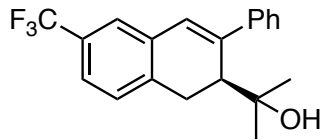

$10 b$

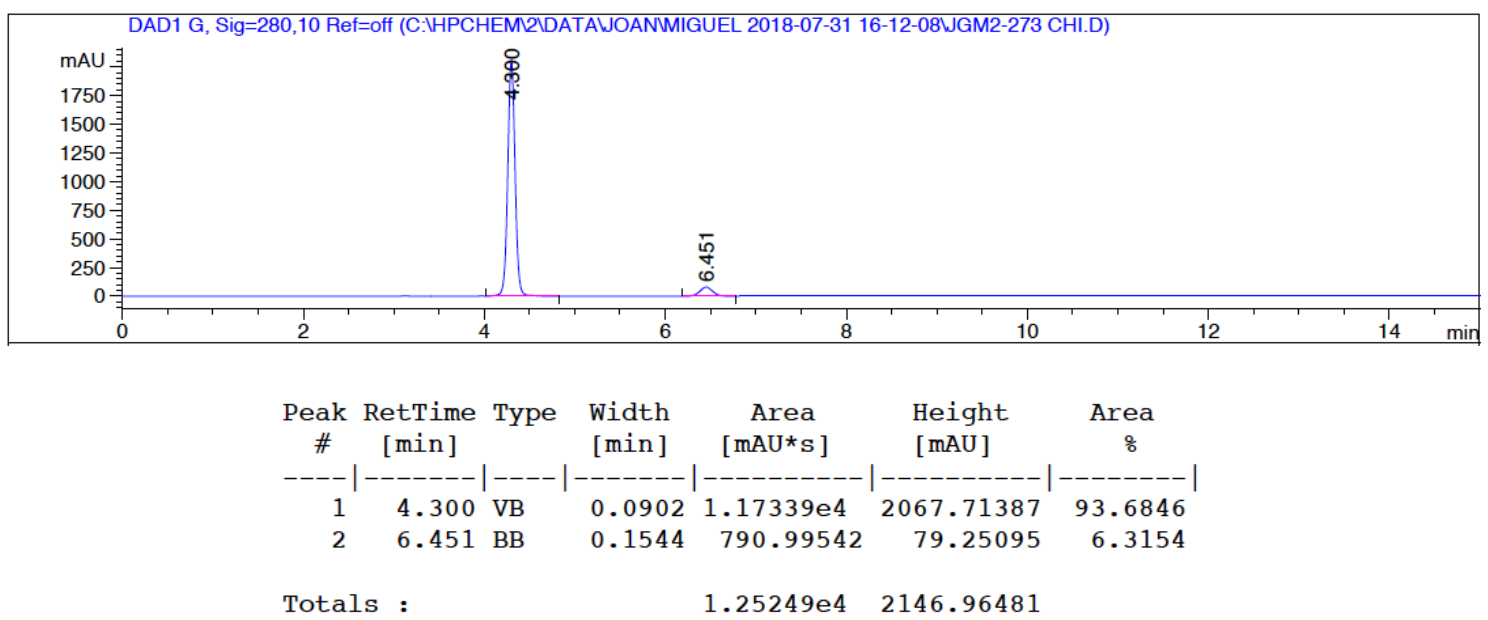


Chromatogram of racemic 10c
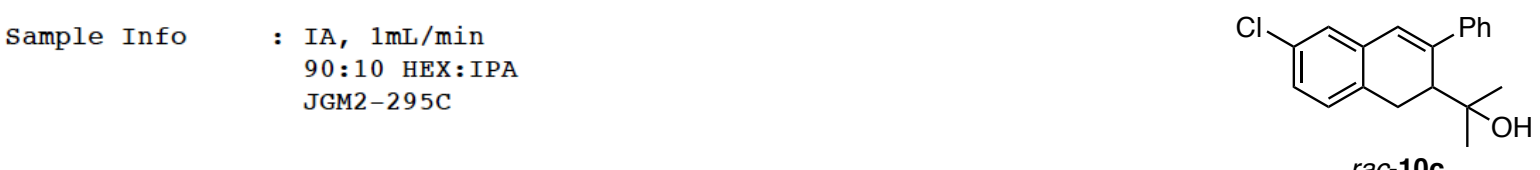

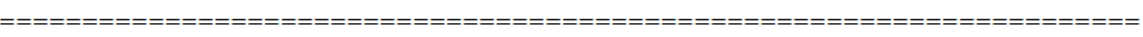

rac-10c

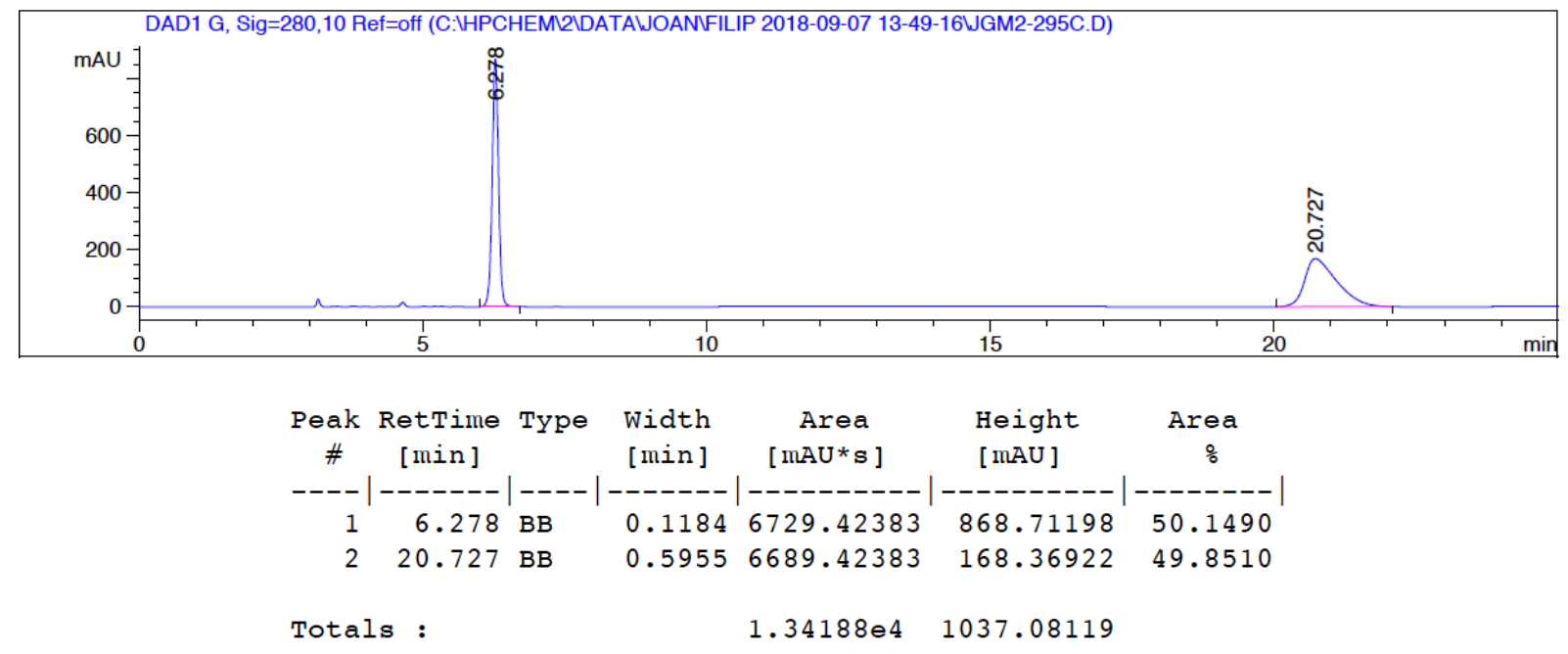

\section{Chromatogram of enantioenriched 10c}
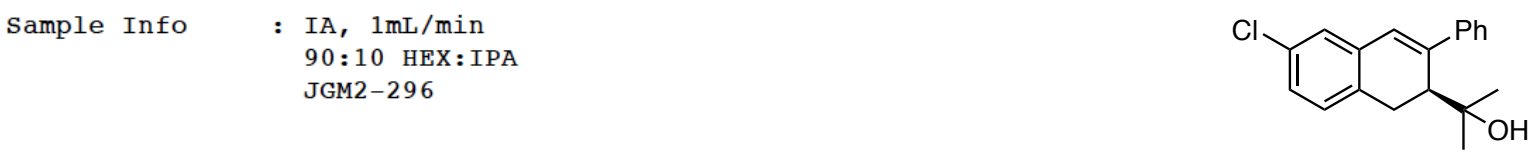

10c

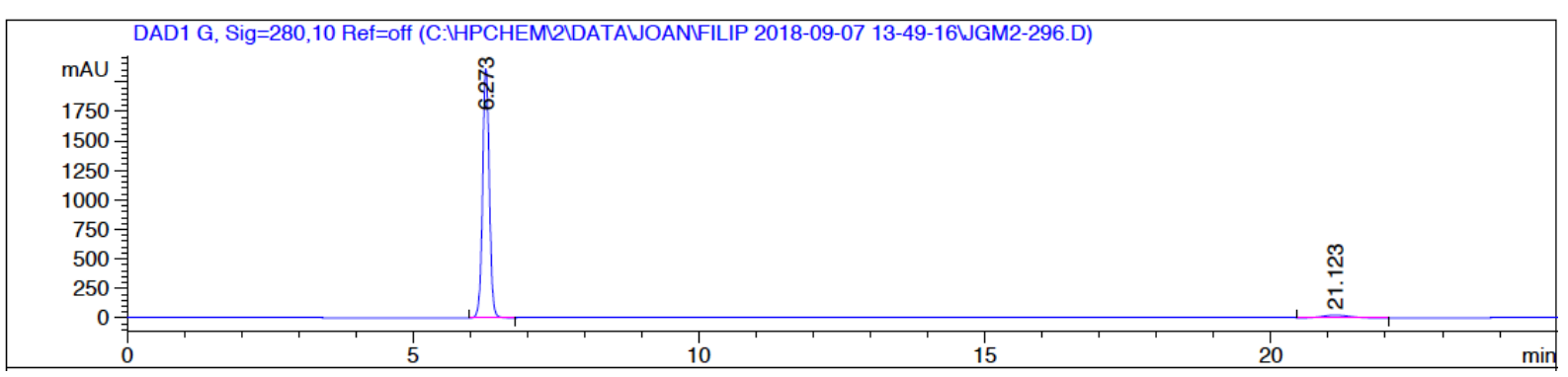


Chromatogram of racemic 10d

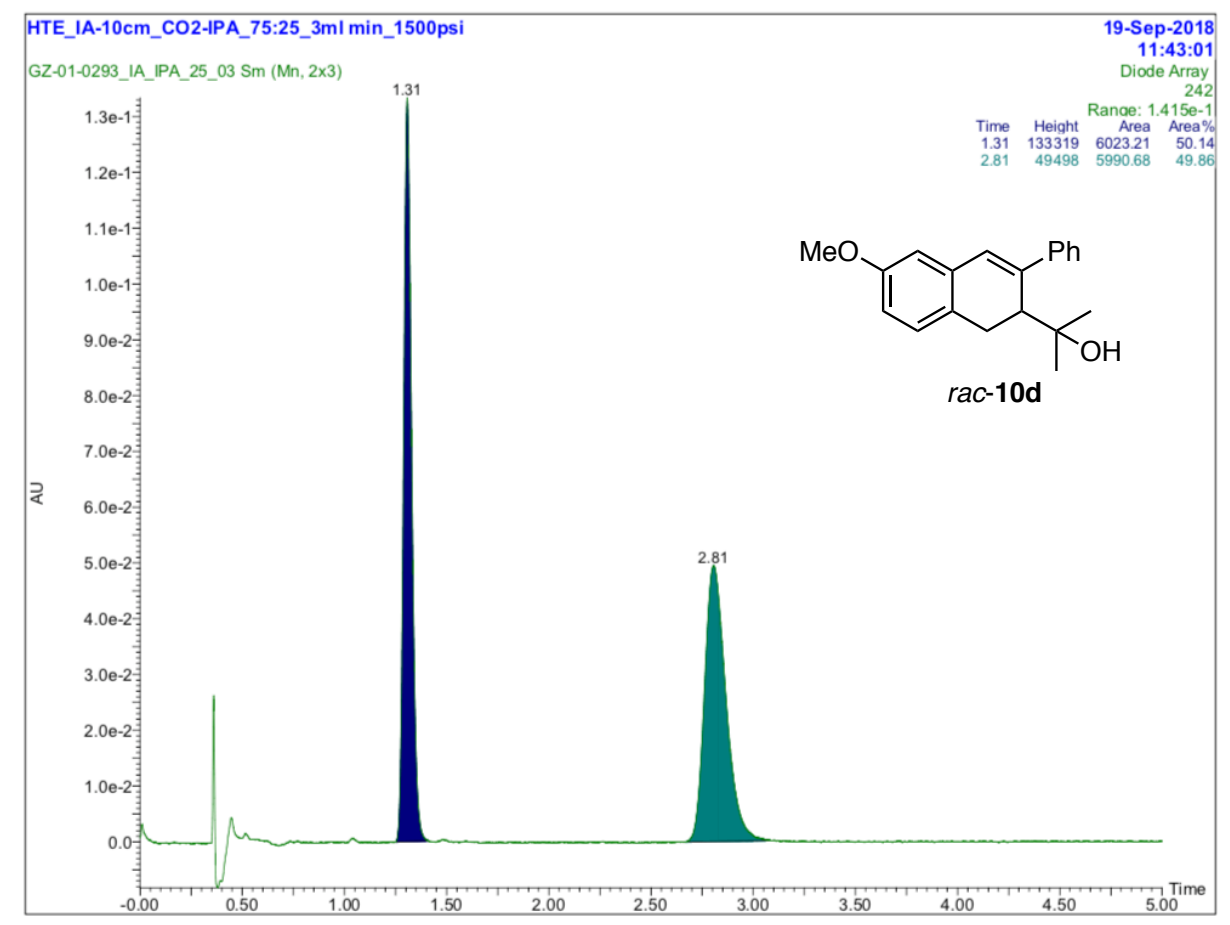

Chromatogram of enantioenriched 10d

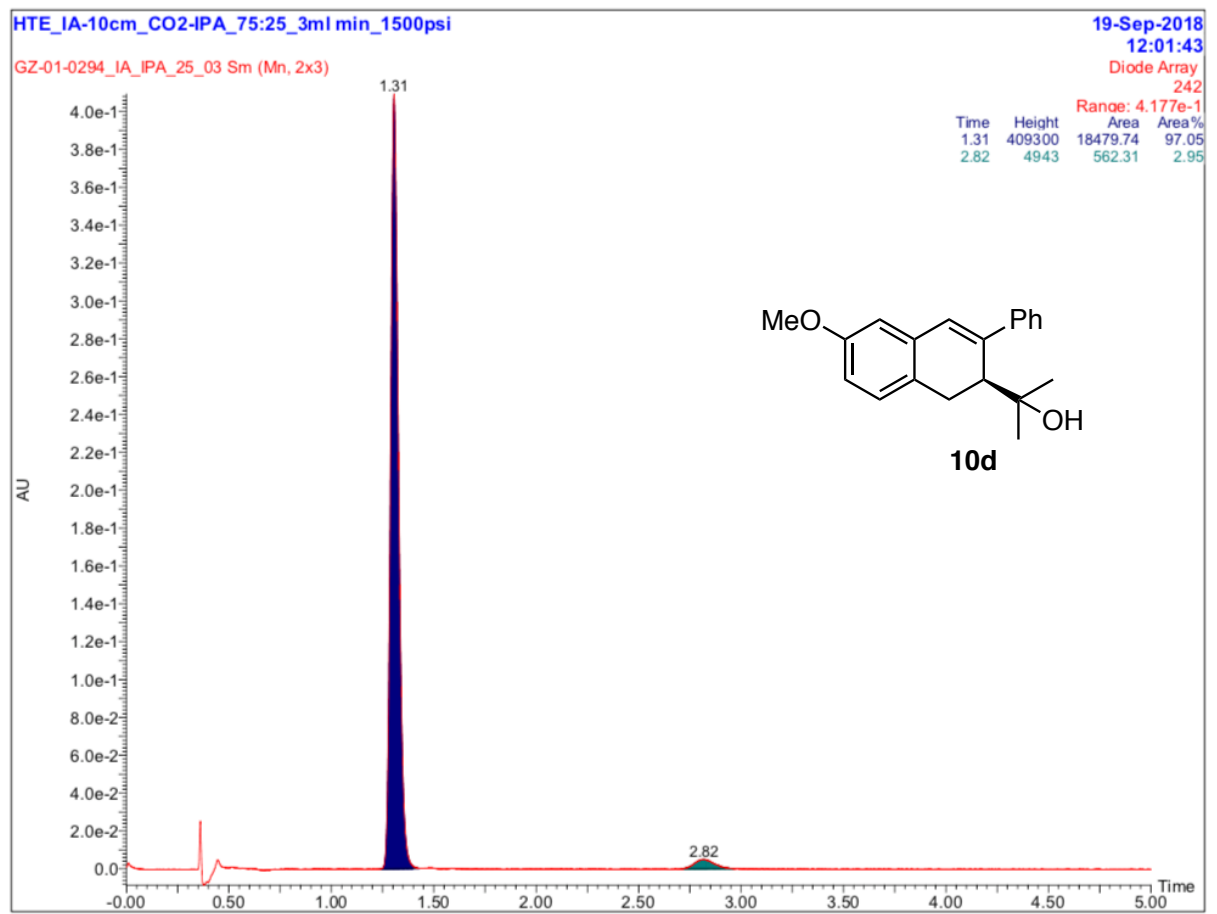


Chromatogram of racemic 10e

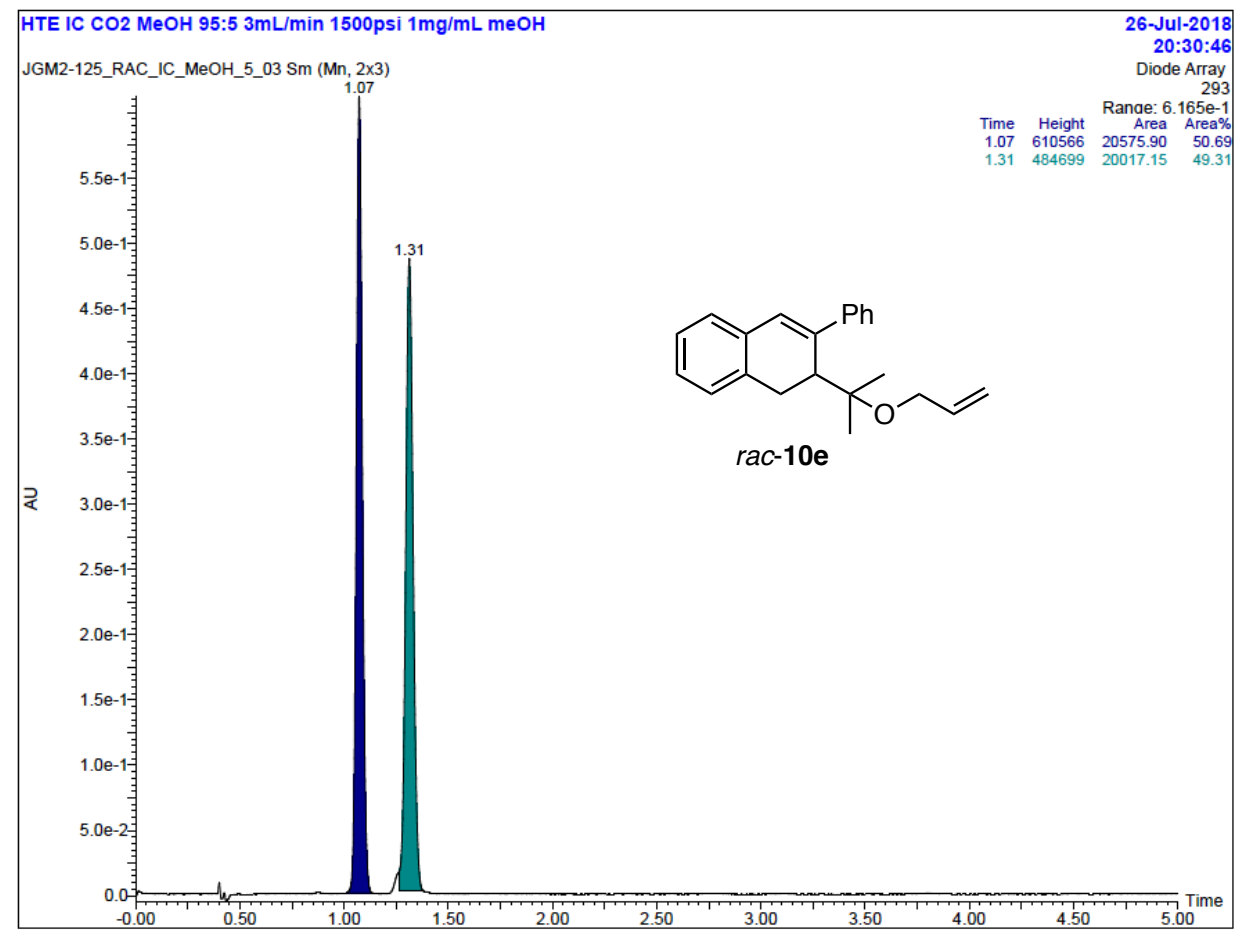

Chromatogram of enantioenriched 10e

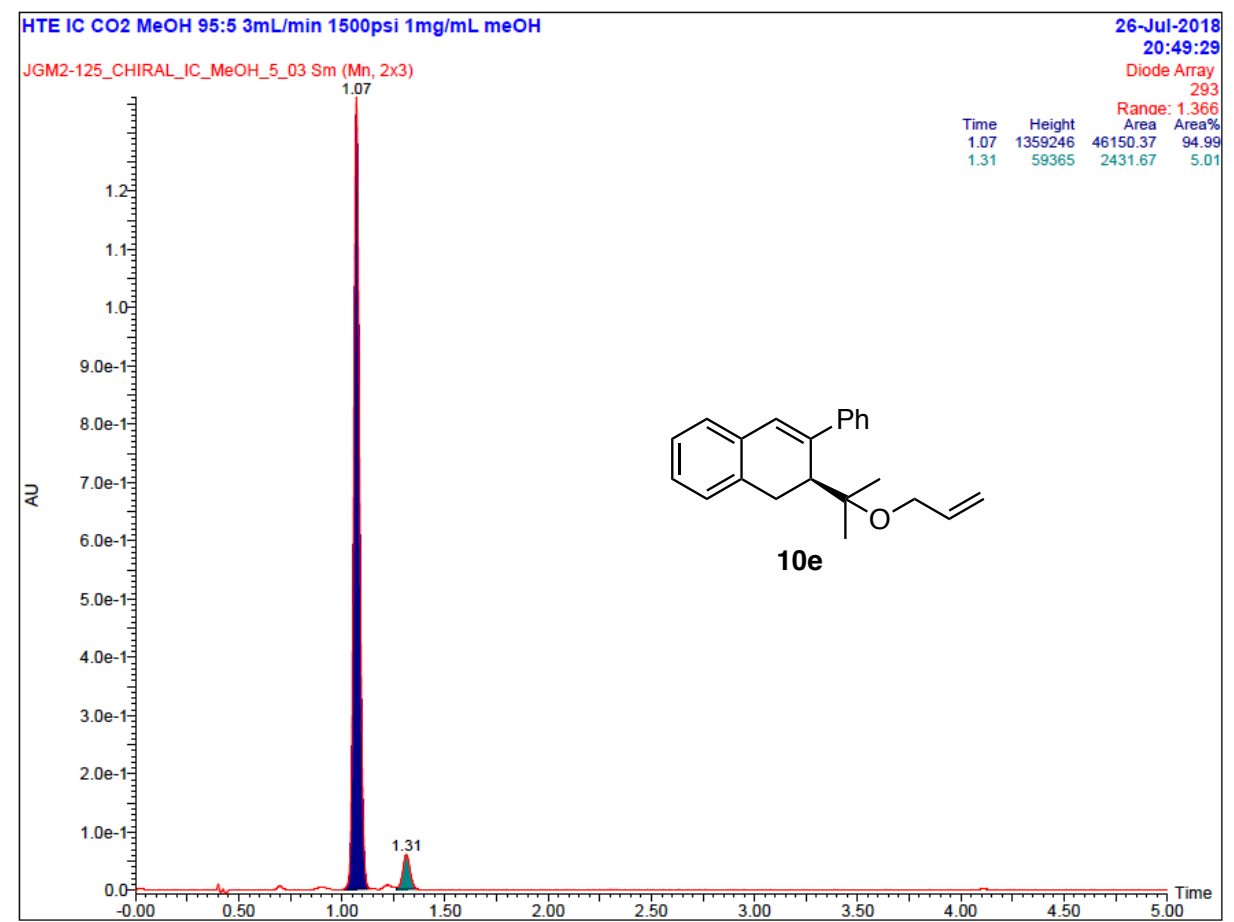


Chromatogram of racemic $\mathbf{1 0 f}$

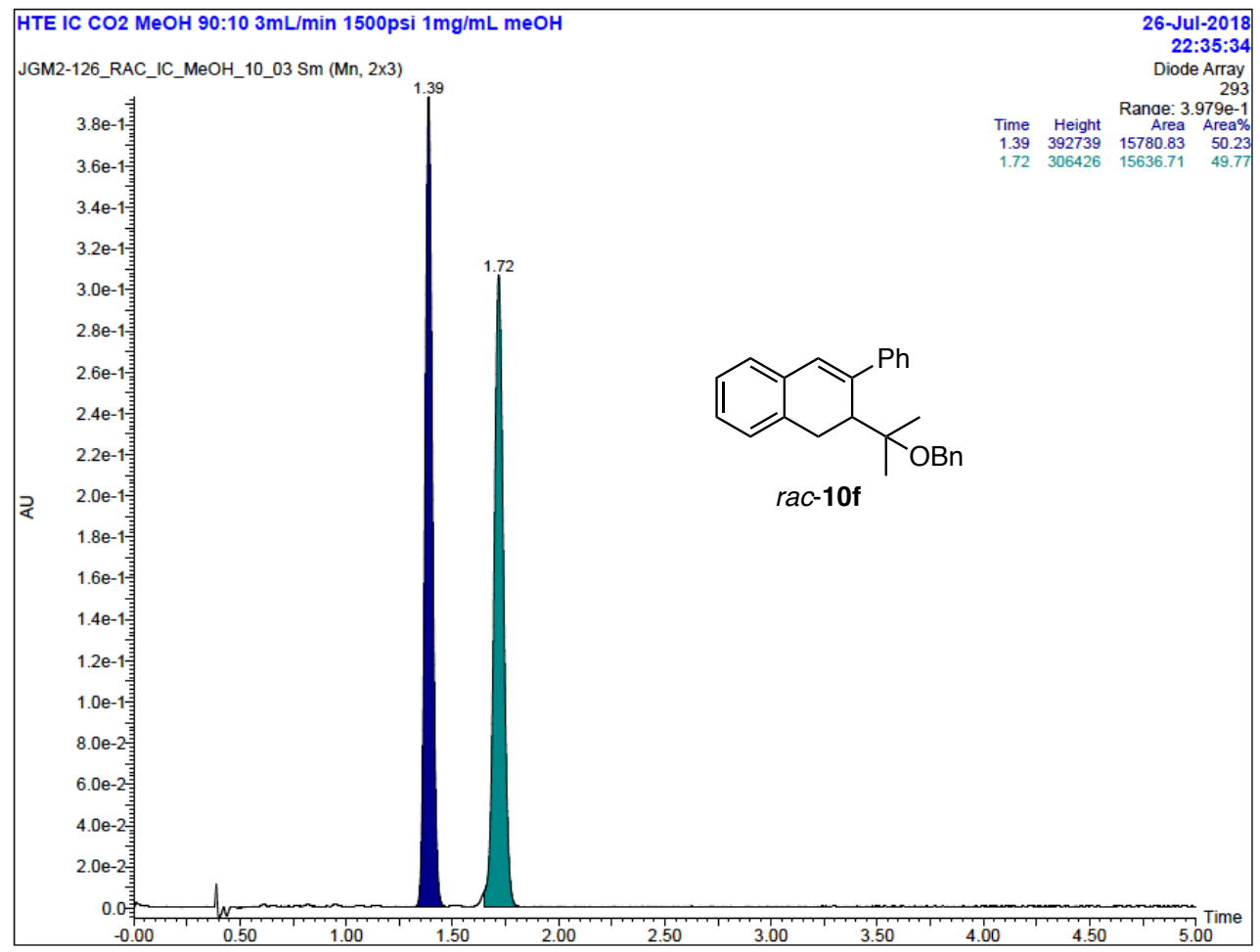

Chromatogram of enantioenriched $\mathbf{1 0 f}$

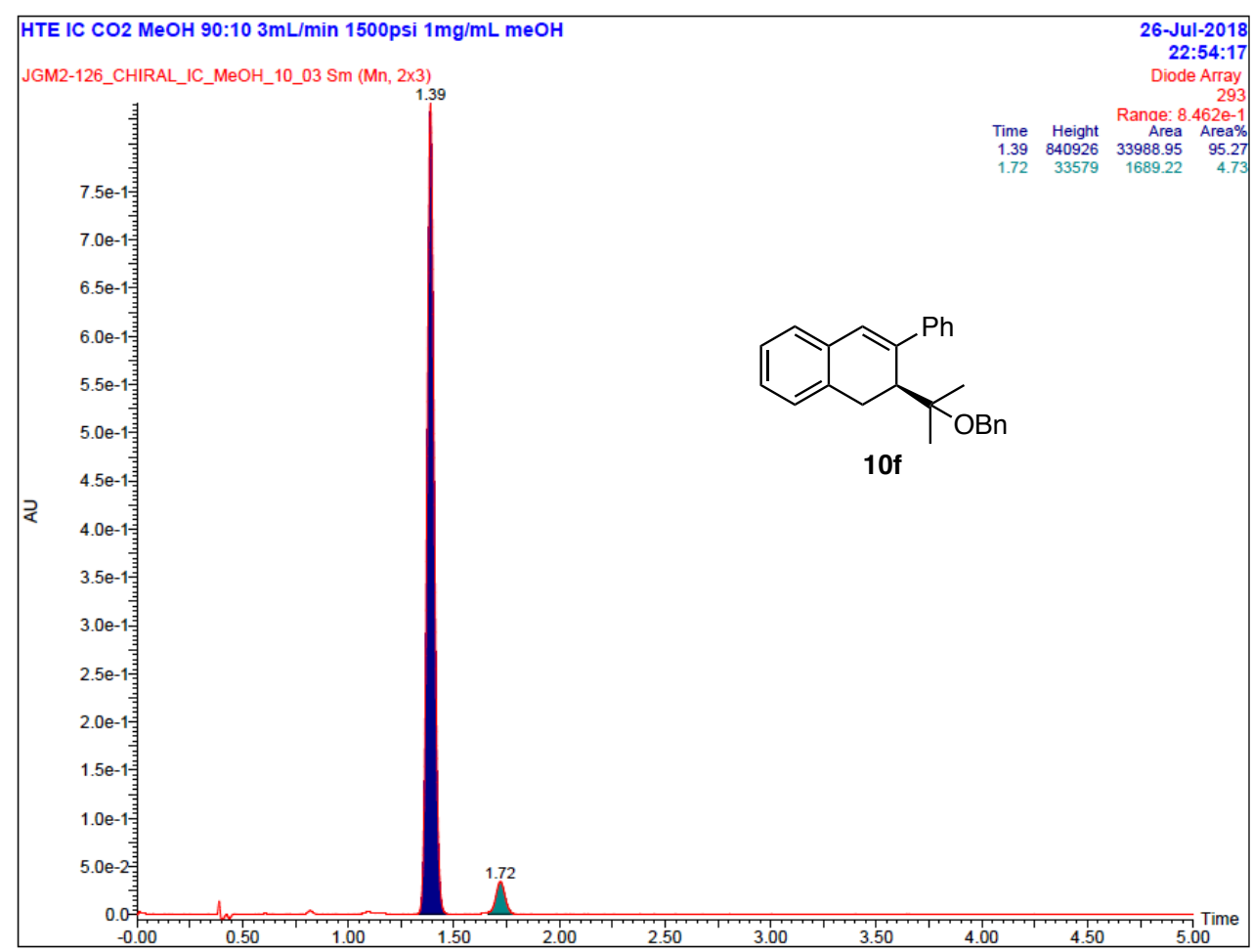


Chromatogram of racemic $\mathbf{1 0 g}$

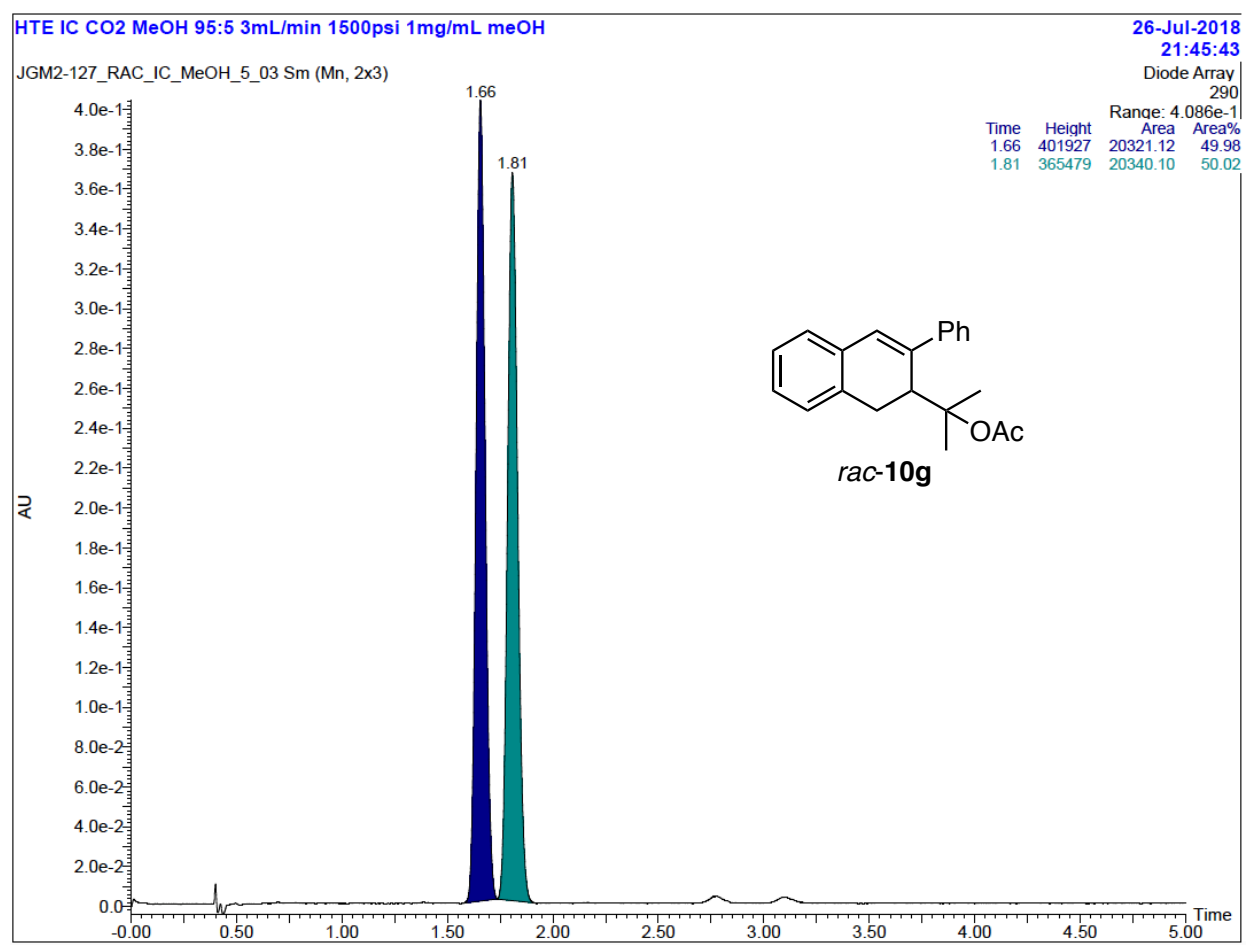

Chromatogram of enantioenriched $\mathbf{1 0 g}$

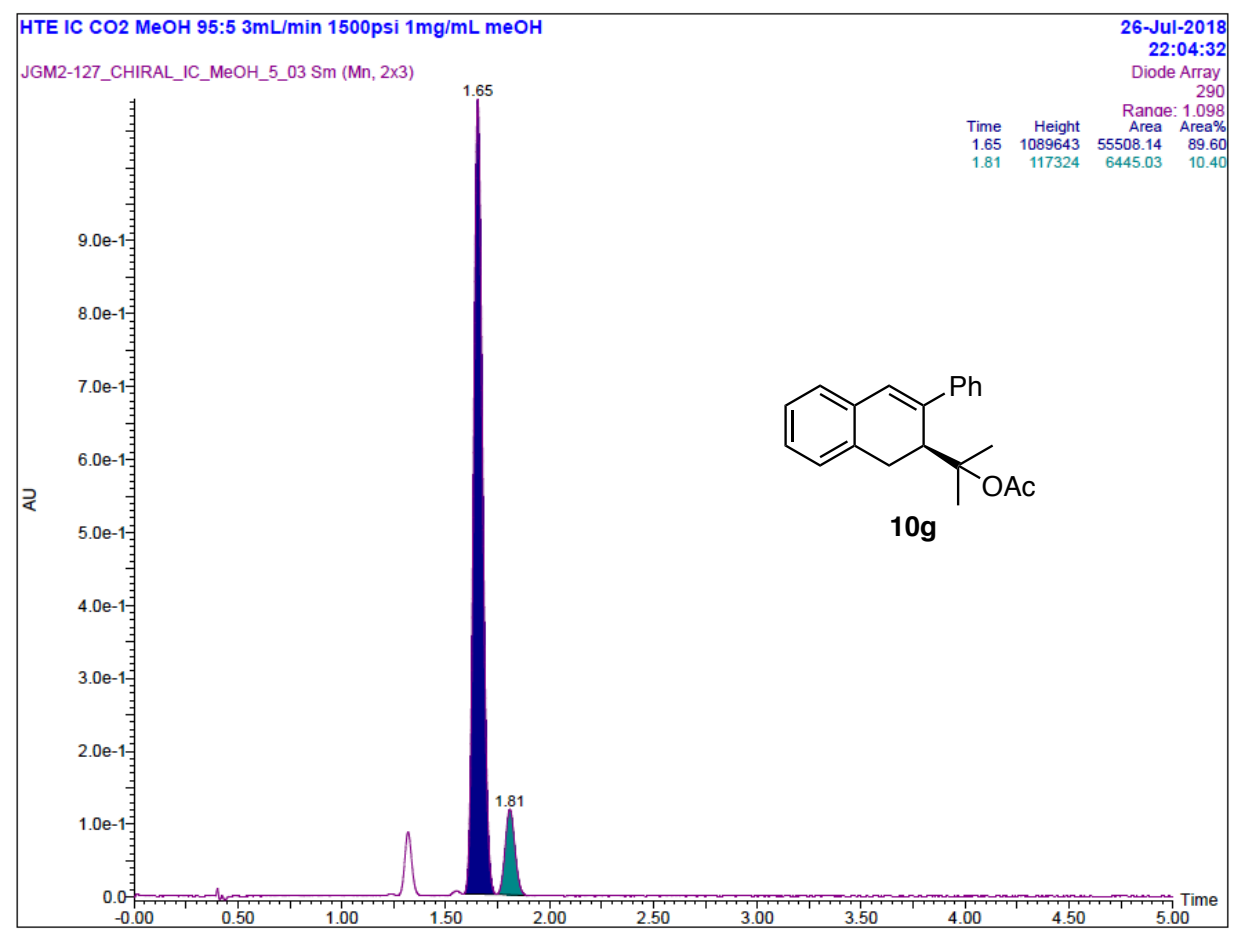


Chromatogram of racemic $\mathbf{1 0 h}$

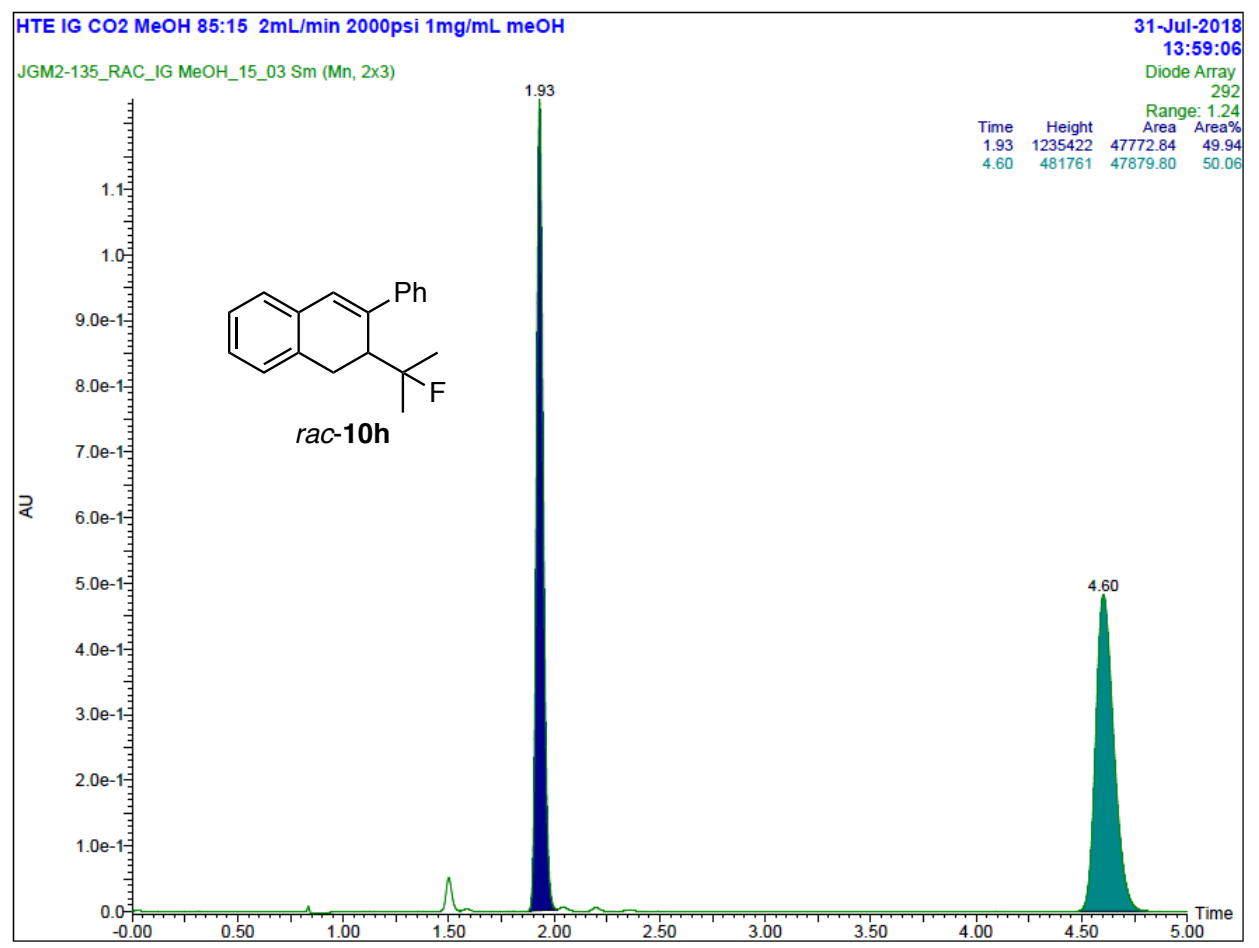

Chromatogram of enantioenriched $\mathbf{1 0 h}$

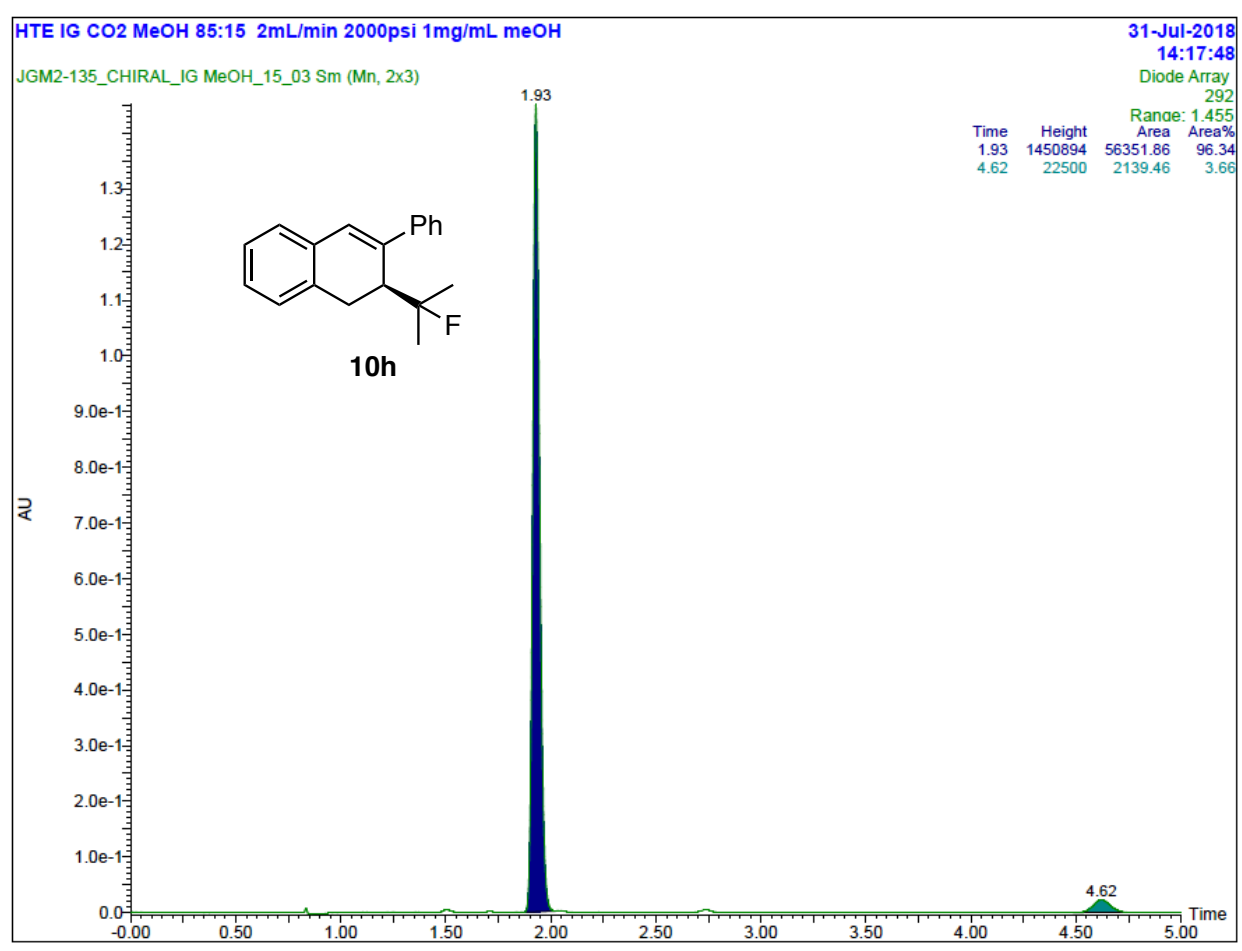


Chromatogram of racemic 10i
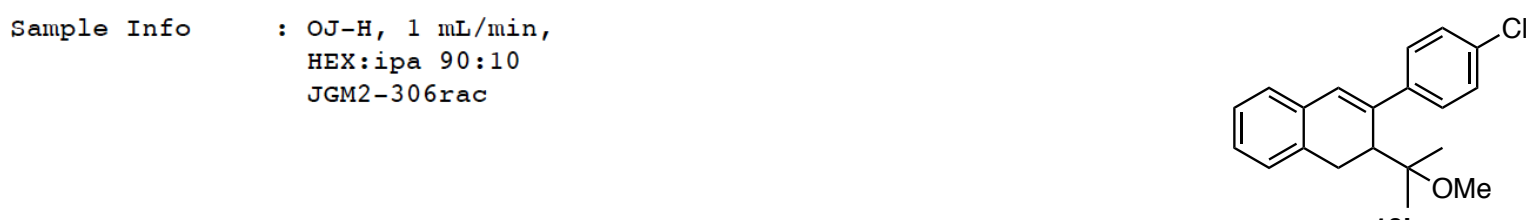

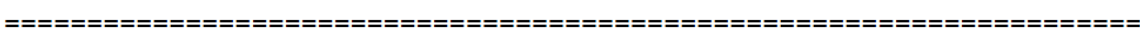

rac-10i

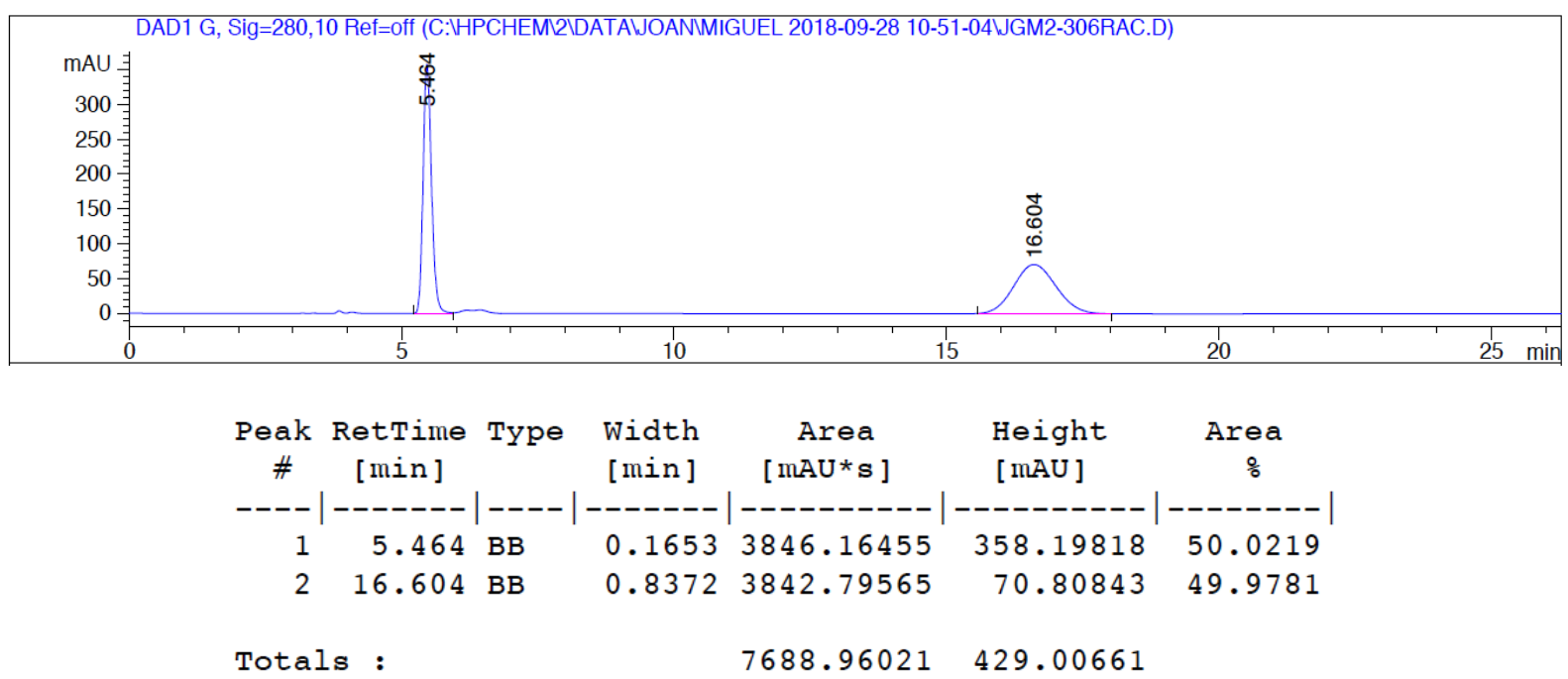

Chromatogram of enantioenriched 10i
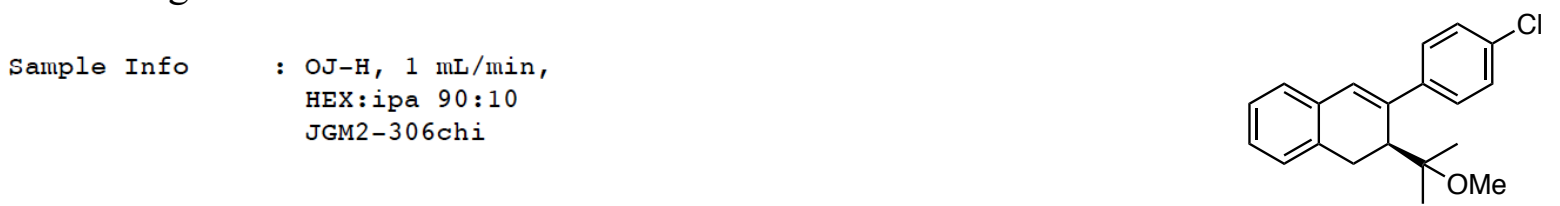

$10 \mathrm{i}$

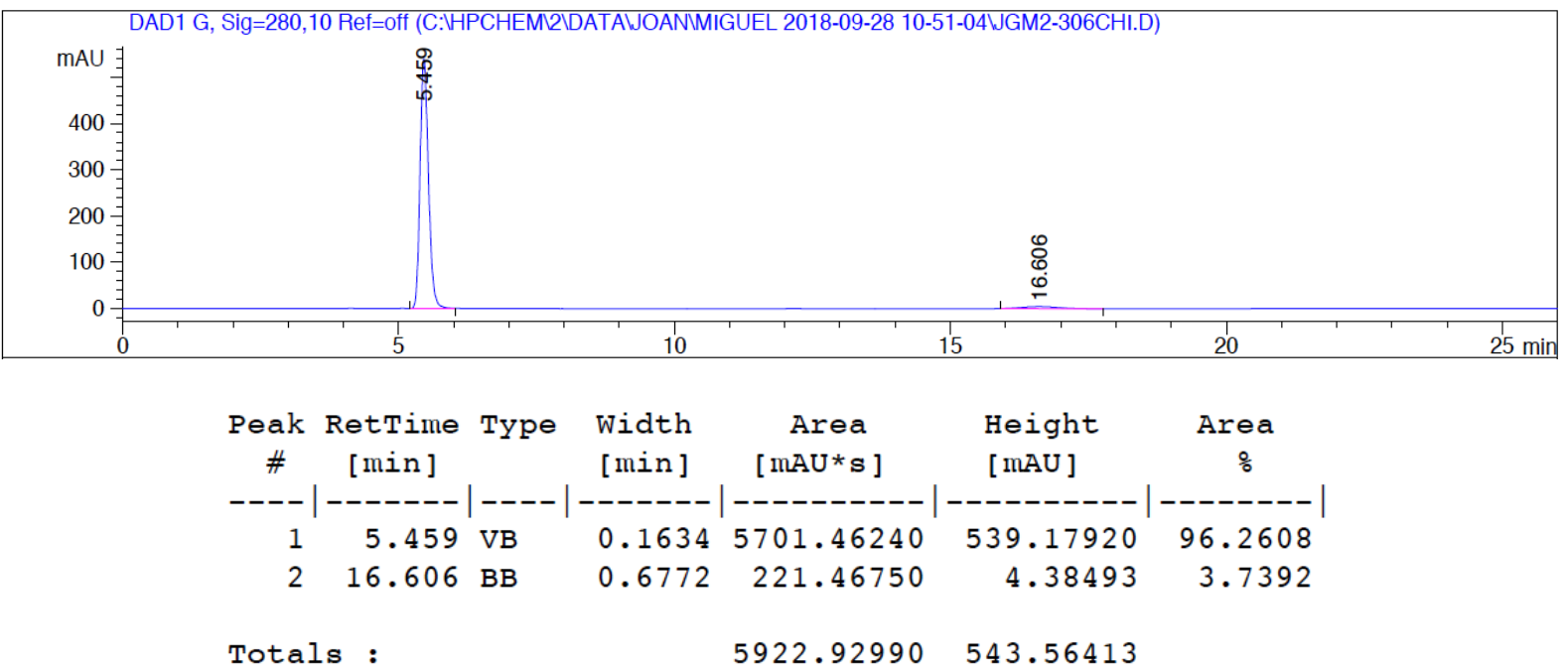


Chromatogram of racemic $\mathbf{1 0 j}$

$$
\begin{aligned}
\text { Sample Info : } & \text { IA, } 1 \mathrm{ml} / \mathrm{min} \\
& 93: 7 \mathrm{HEX}: \mathrm{IPA} \\
& \mathrm{GZ}-01-0813
\end{aligned}
$$

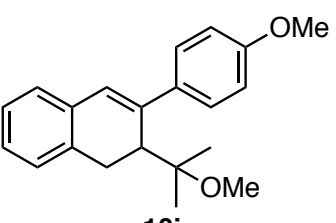

rac-10j

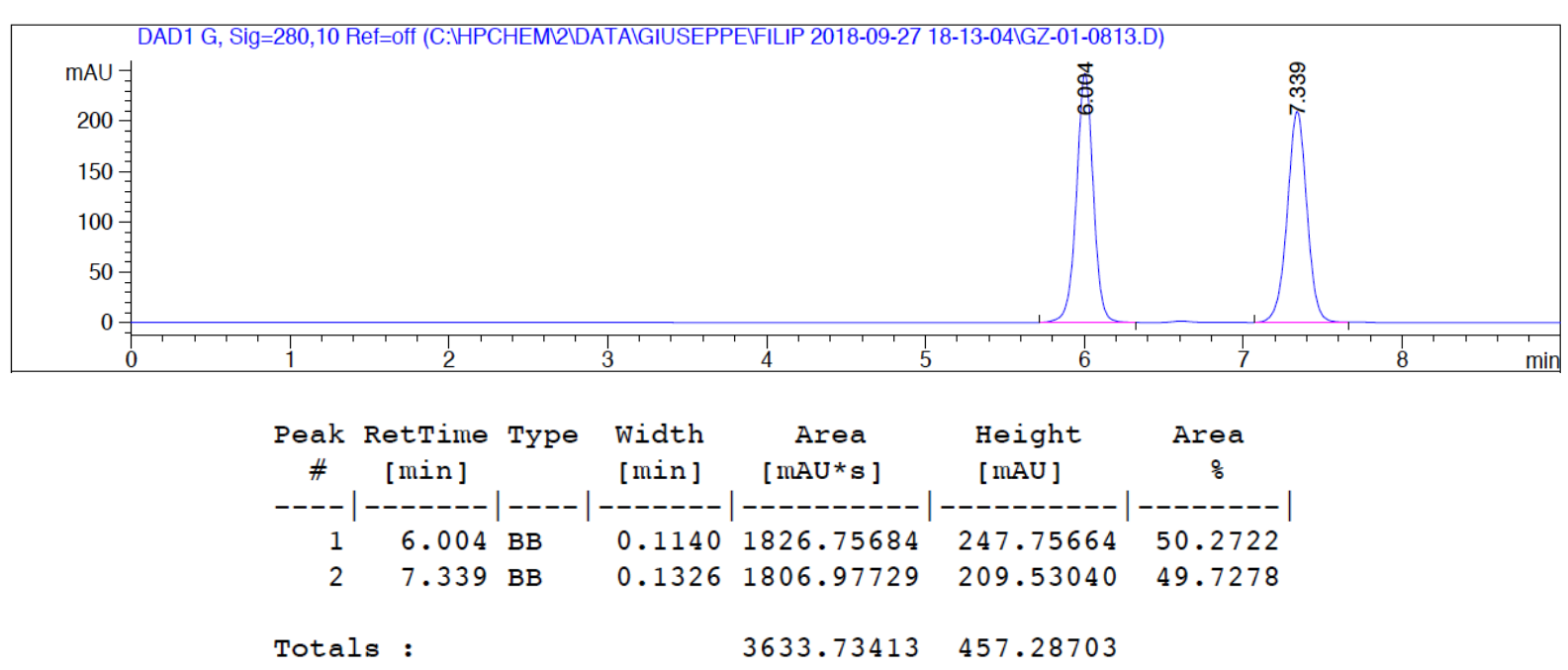

Chromatogram of enantioenriched $\mathbf{1 0 j}$

$$
\begin{aligned}
\text { Sample Info : } & \text { IA, } 1 \mathrm{ml} / \mathrm{min} \\
& 98: 2 \mathrm{HEX}: \mathrm{IPA} \\
& \text { JGM } 2-305
\end{aligned}
$$

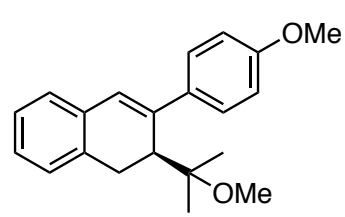

10j

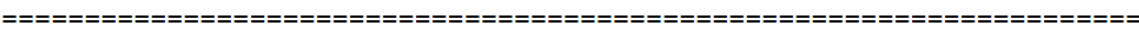

DAD1 A, Sig=220,10 Ref=off (C:VHPCHEM2LATAIGIUSEPPEIFILIP 2018-09-27 18-36-20WGM2-305.D)

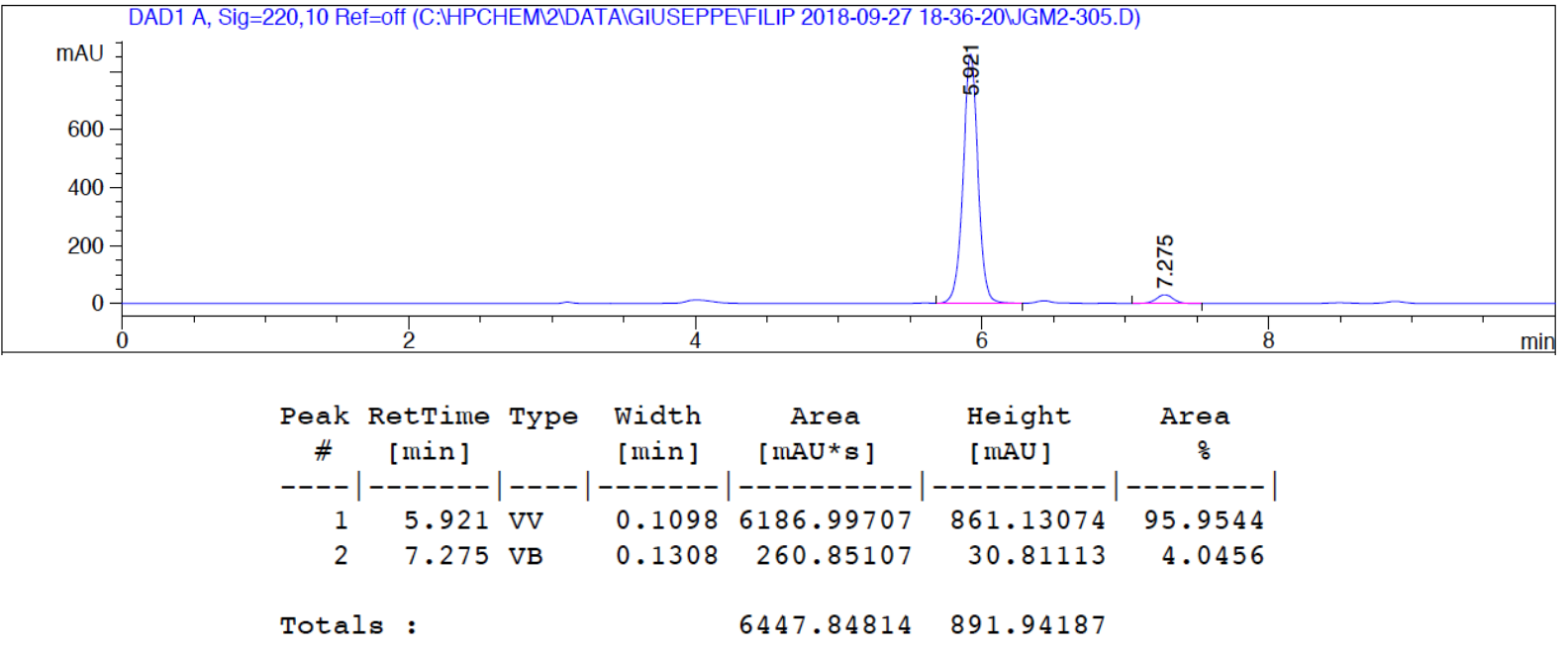


Chromatogram of enantioenriched $\mathbf{1 0} \mathbf{j}$ interconverted from $\mathbf{1 0} \mathbf{i}$
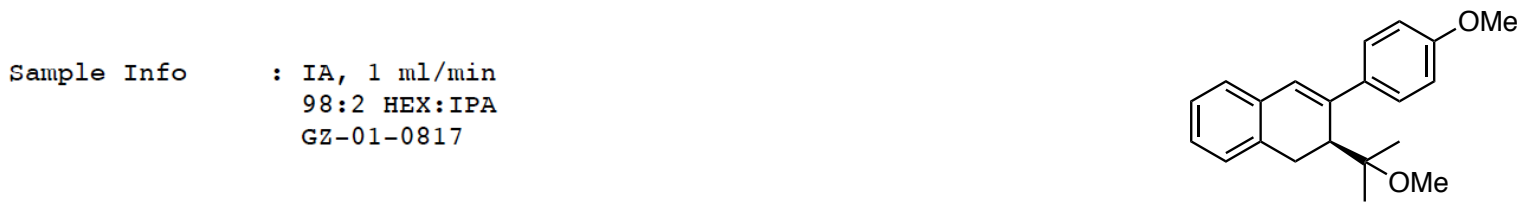

10j

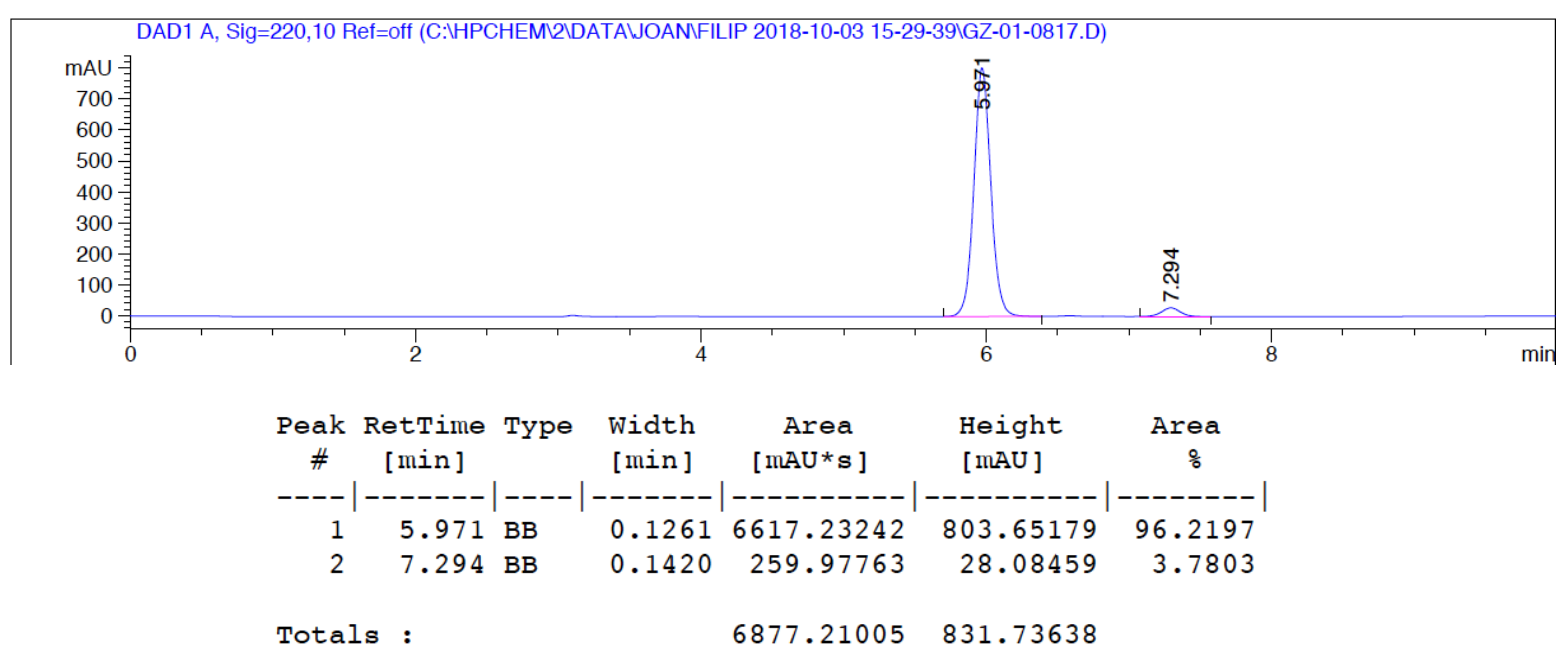




\section{Chromatogram of racemic 10k}

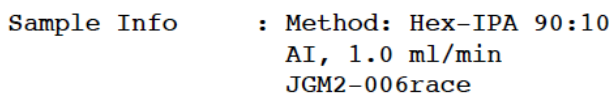

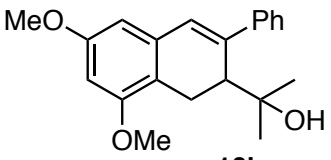

rac-10k
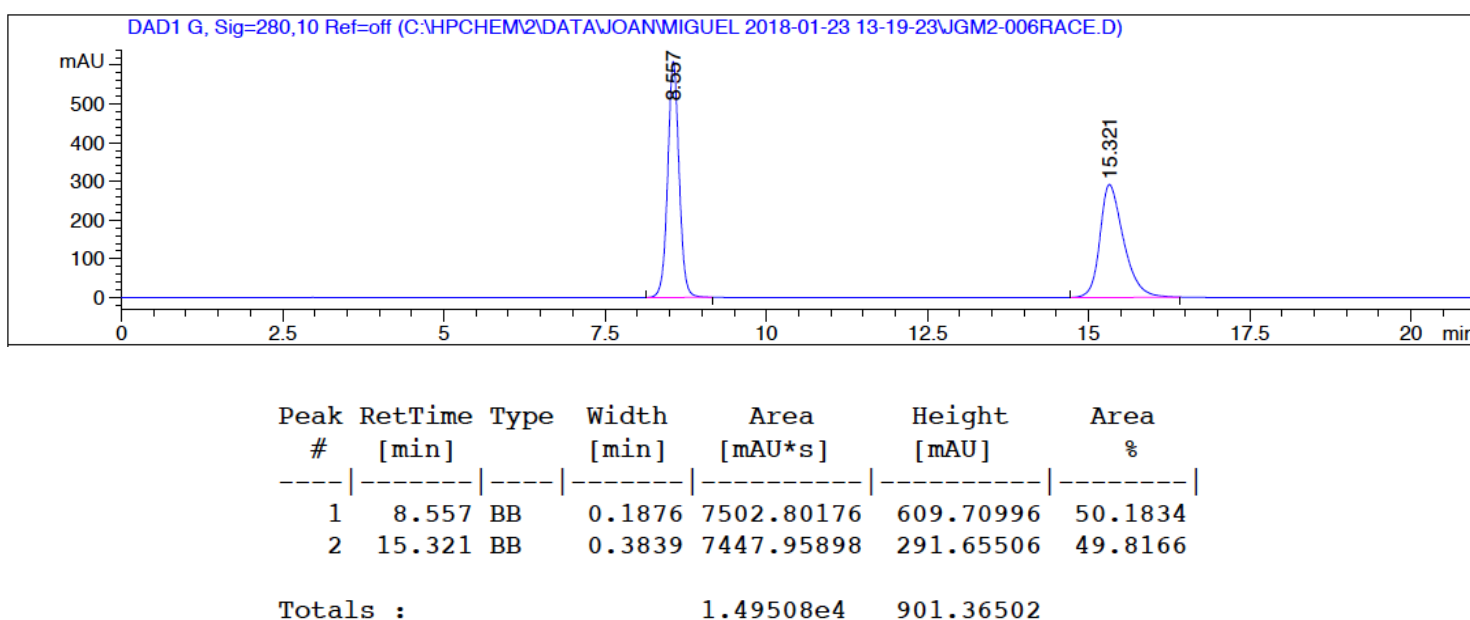

\section{Chromatogram of enantioenriched $\mathbf{1 0 k}$}
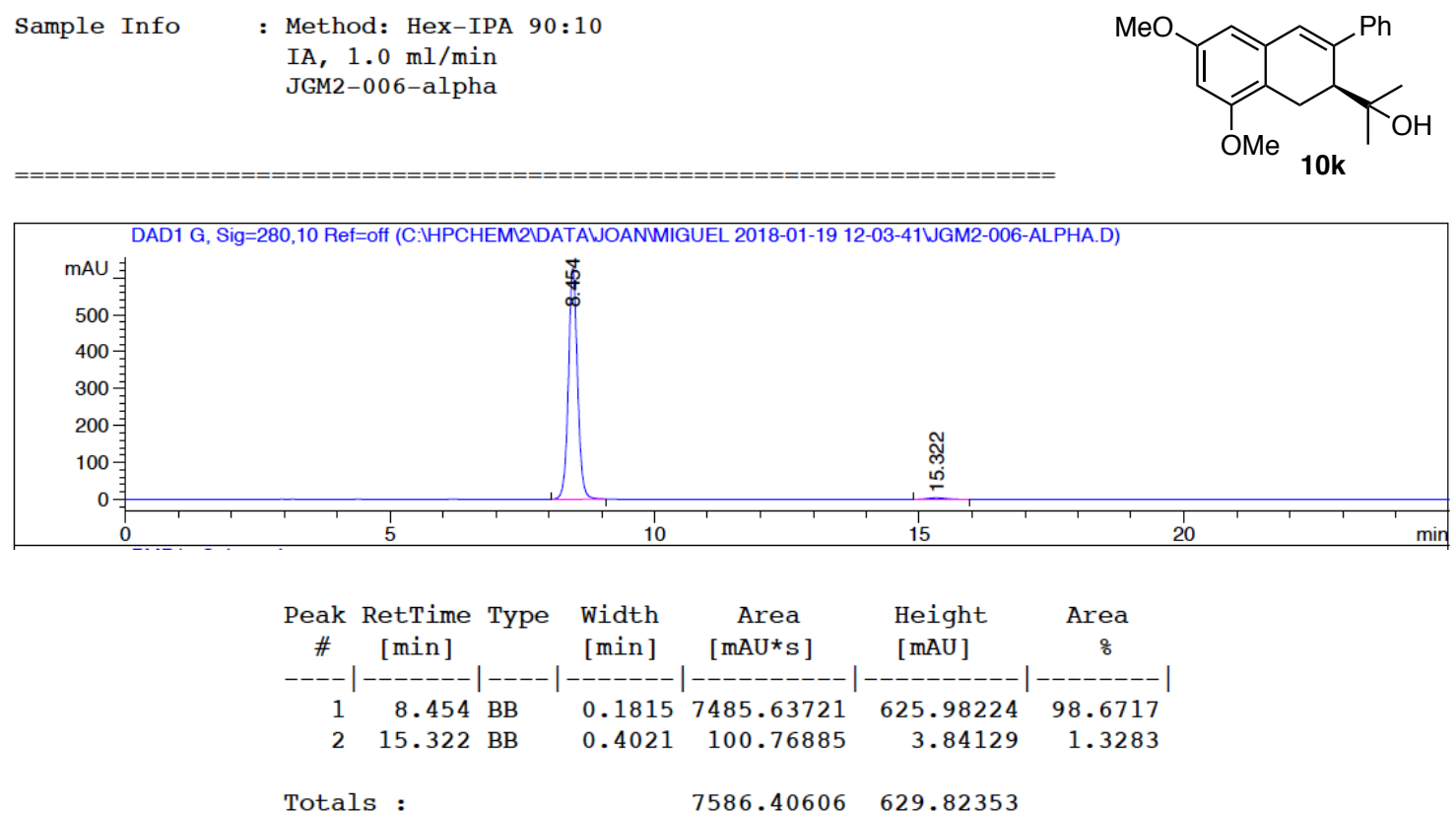


\section{Chromatogram of racemic $\mathbf{1 0 l}$}
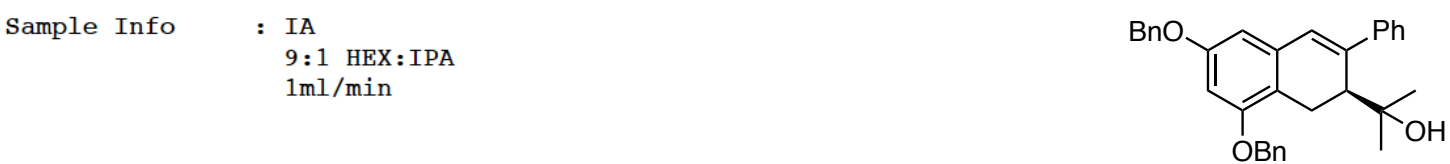

rac-10l

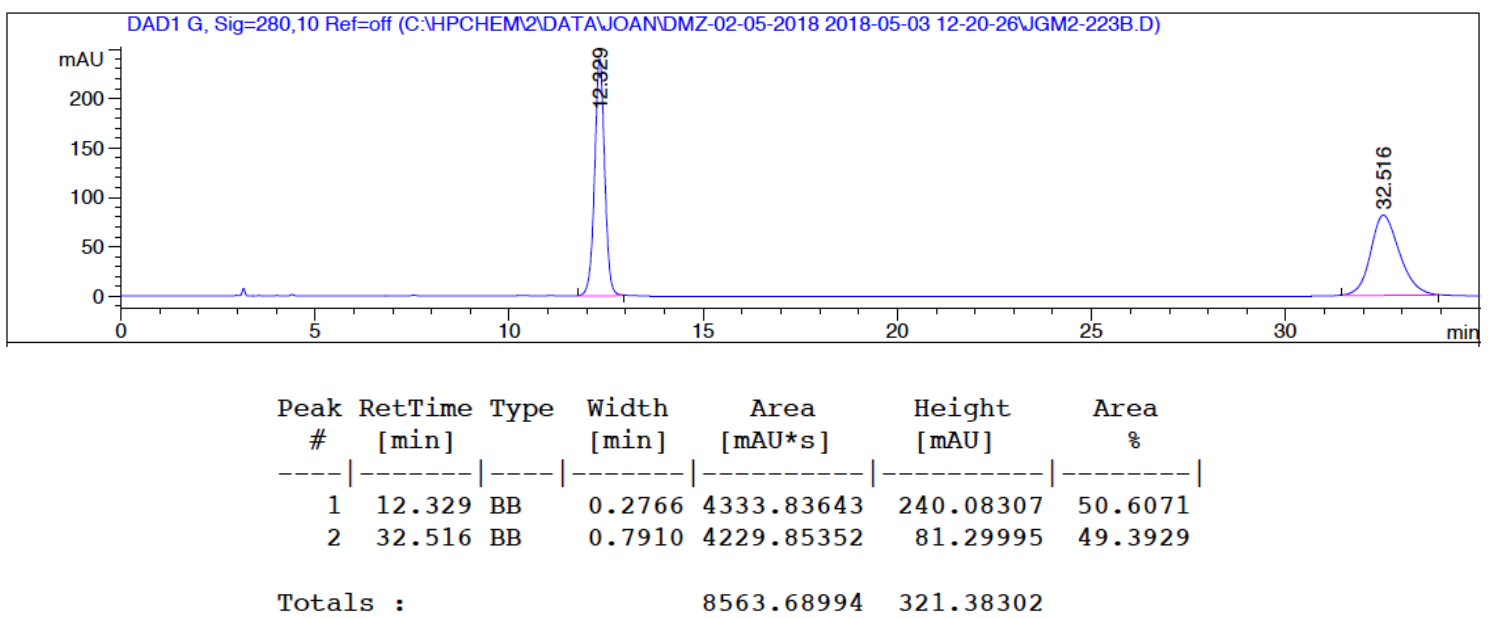

\section{Chromatogram of enantioenriched $\mathbf{1 0 1}$}
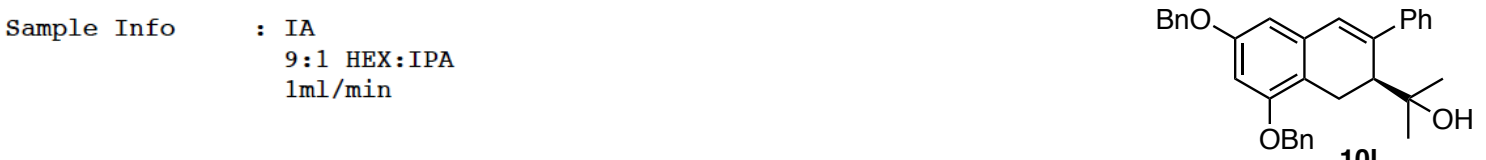

10

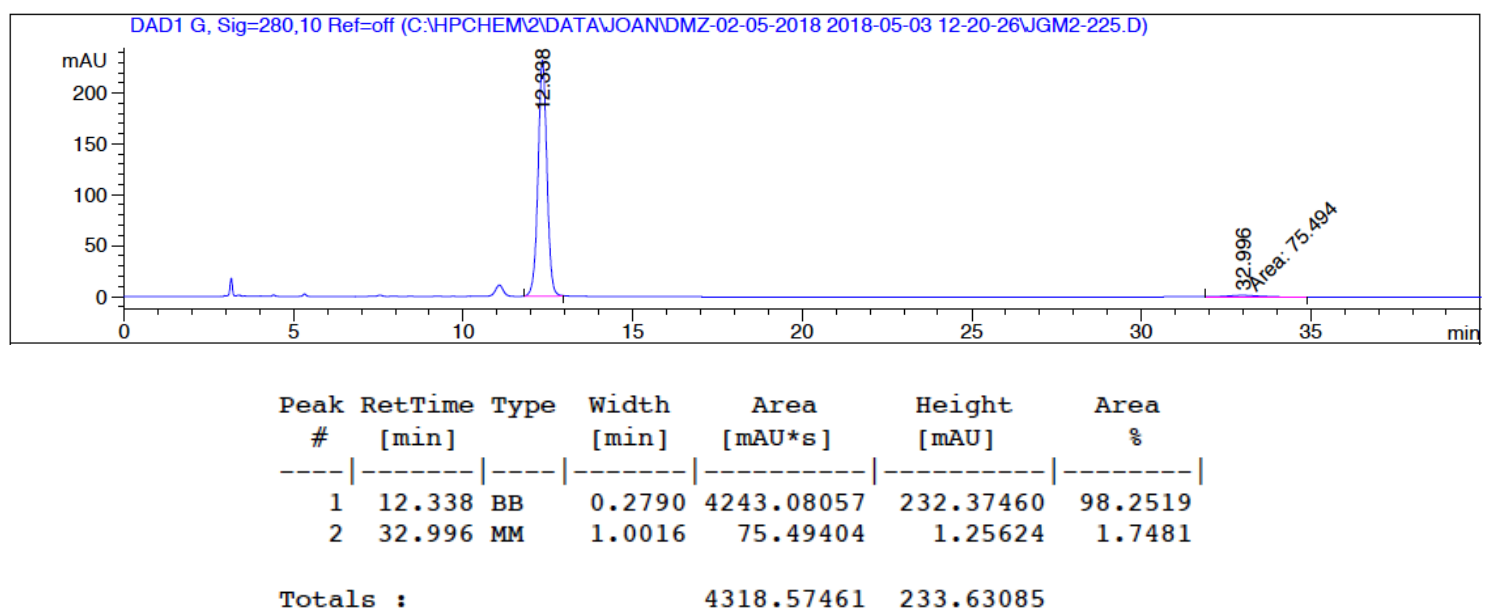




\section{Chromatogram of racemic $\mathbf{1 8}$}
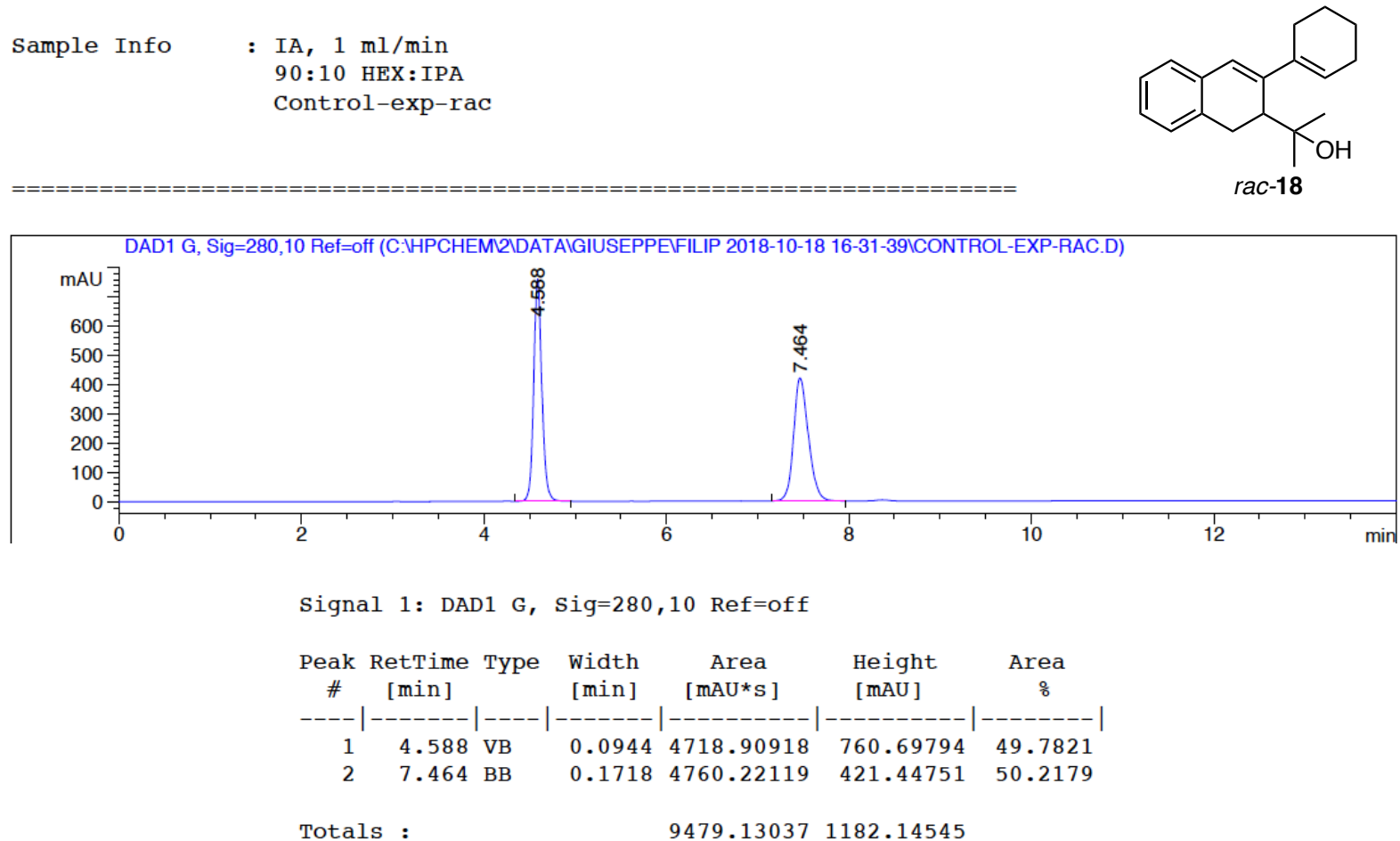

Chromatogram of enantioenriched $\mathbf{1 8}$
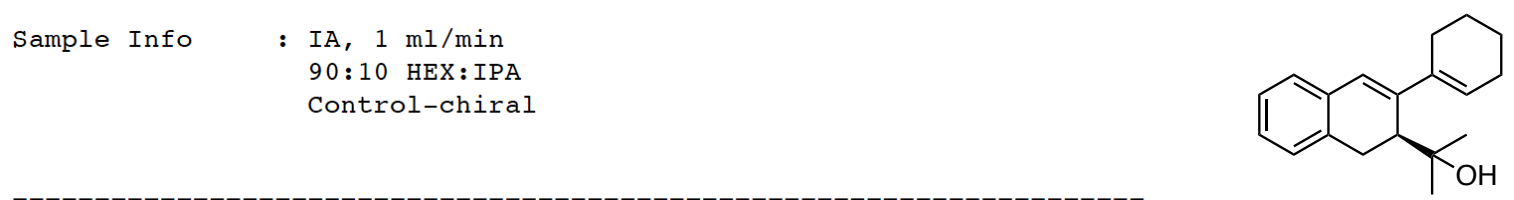

18

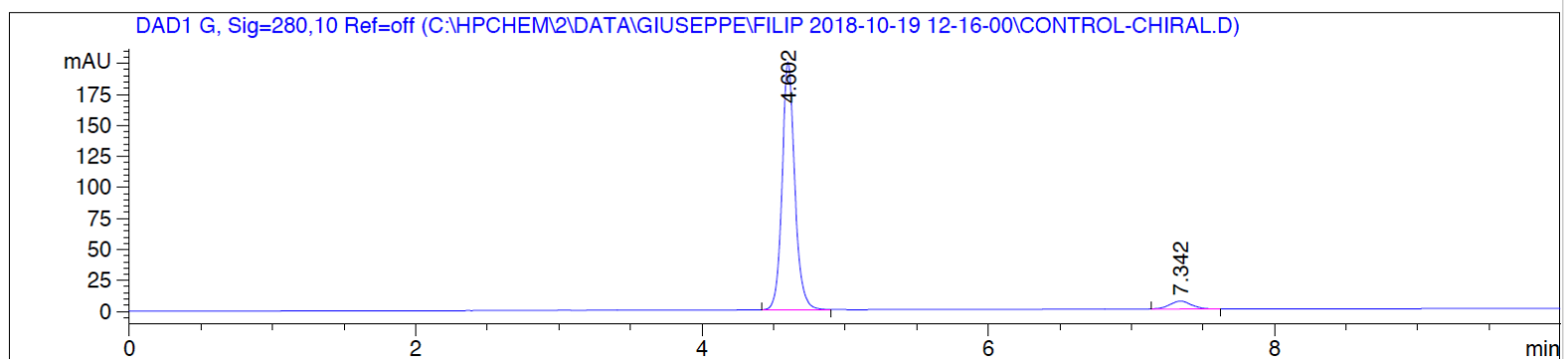

Signal 1: DAD1 G, Sig=280,10 Ref=off

\begin{tabular}{|c|c|c|c|c|c|c|}
\hline $\begin{array}{c}\text { Peak } \\
\quad \#\end{array}$ & $\begin{array}{c}\text { RetTime } \\
\text { [min] }\end{array}$ & Type & $\begin{array}{l}\text { Width } \\
\text { [min] }\end{array}$ & $\begin{array}{c}\text { Area } \\
{[\mathrm{mAU} * \mathrm{~s}]}\end{array}$ & $\begin{array}{l}\text { Height } \\
\text { [mAU] }\end{array}$ & $\begin{array}{c}\text { Area } \\
\%\end{array}$ \\
\hline 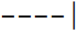 & & & ---1 & $----------\mid$ & $----------\mid$ & --ー--ー-ー \\
\hline 1 & 4.602 & BB & 0.0938 & 1231.65991 & 200.12206 & 94.7694 \\
\hline 2 & 7.342 & BB & 0.1625 & 67.97930 & 6.37065 & 5.2306 \\
\hline
\end{tabular}


Chromatogram of racemic $\mathbf{1 2}$
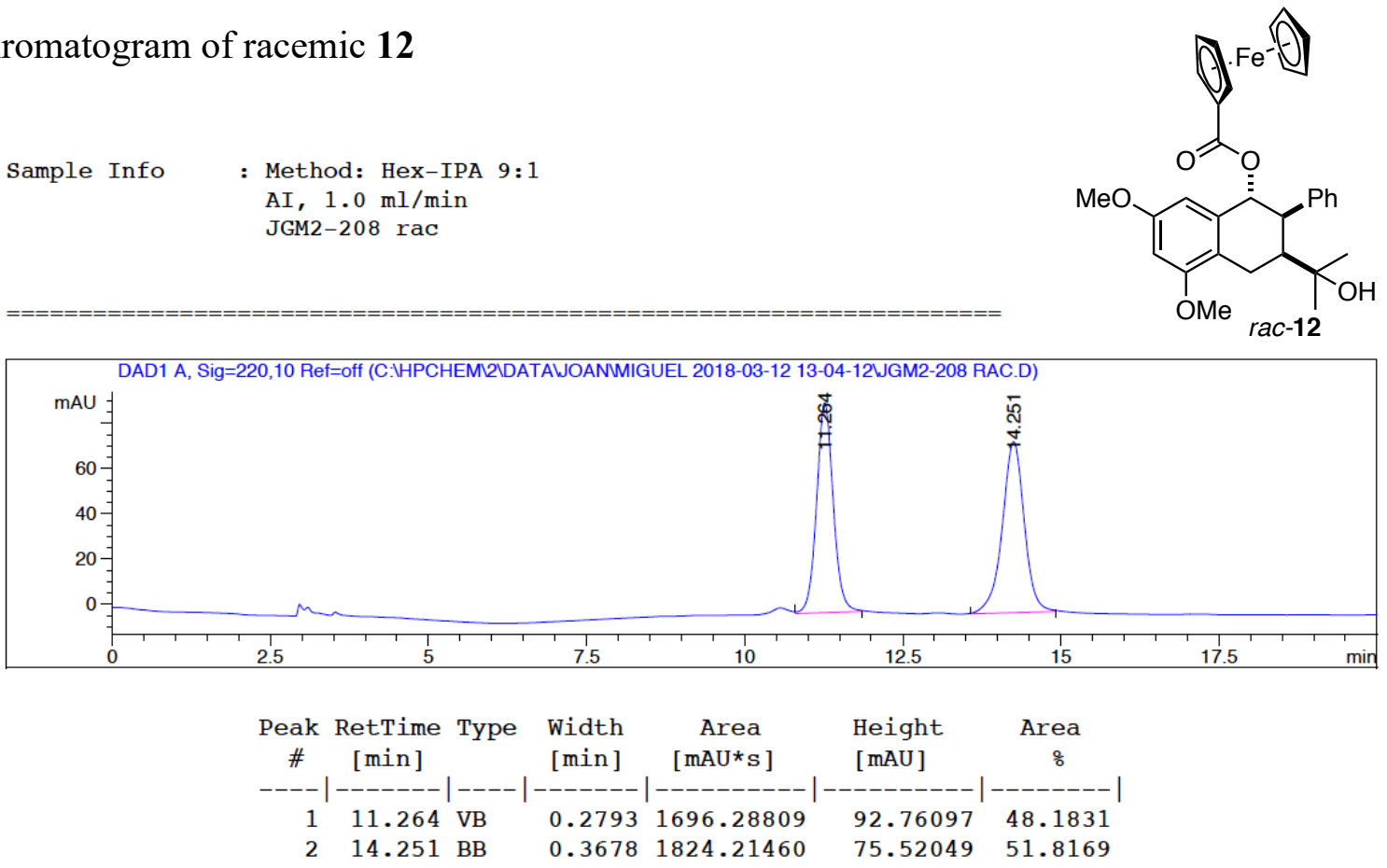

Totals :

$3520.50269 \quad 168.28146$

\section{Chromatogram of enantioenriched $\mathbf{1 2}$}
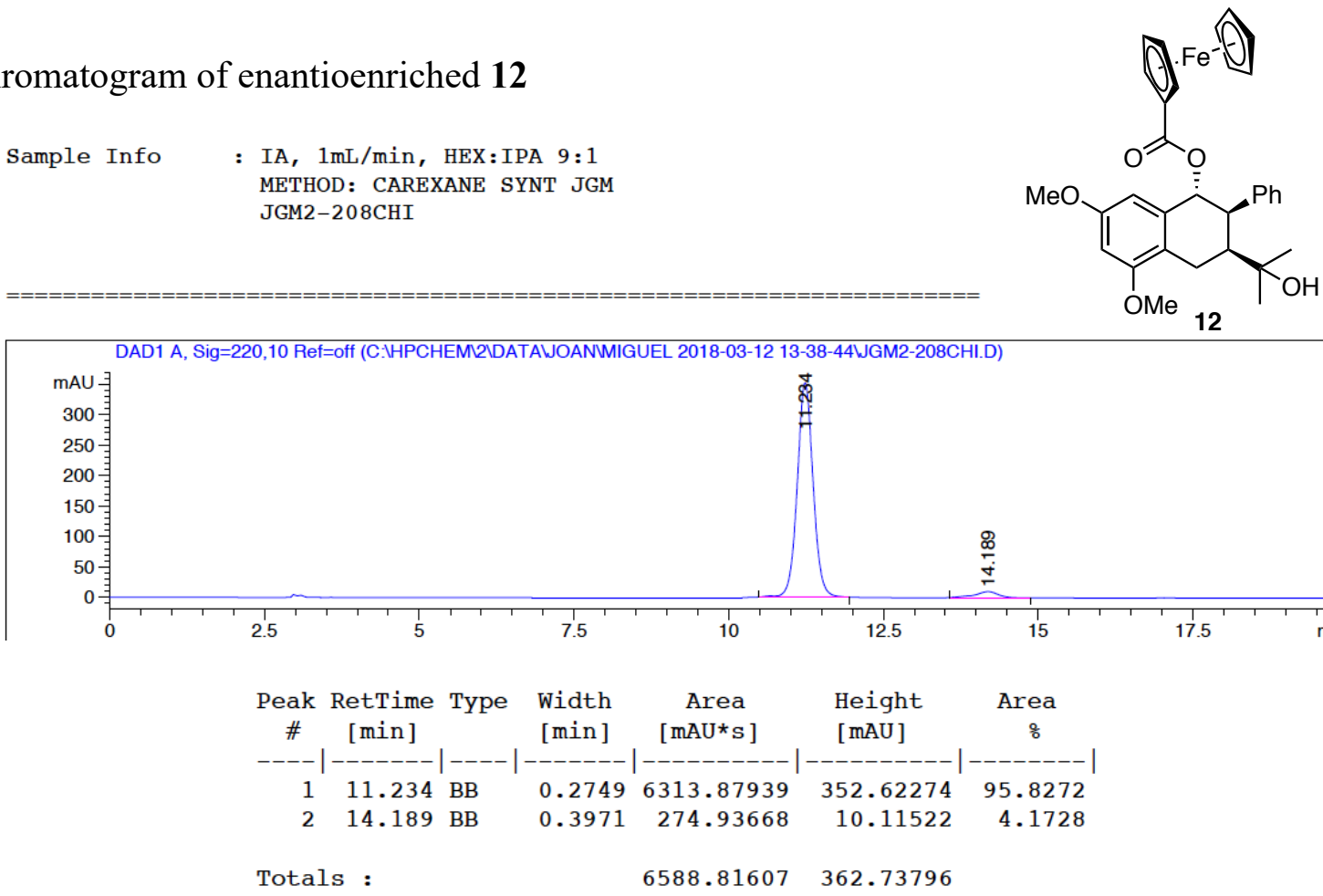
Chromatogram of racemic Carexane I
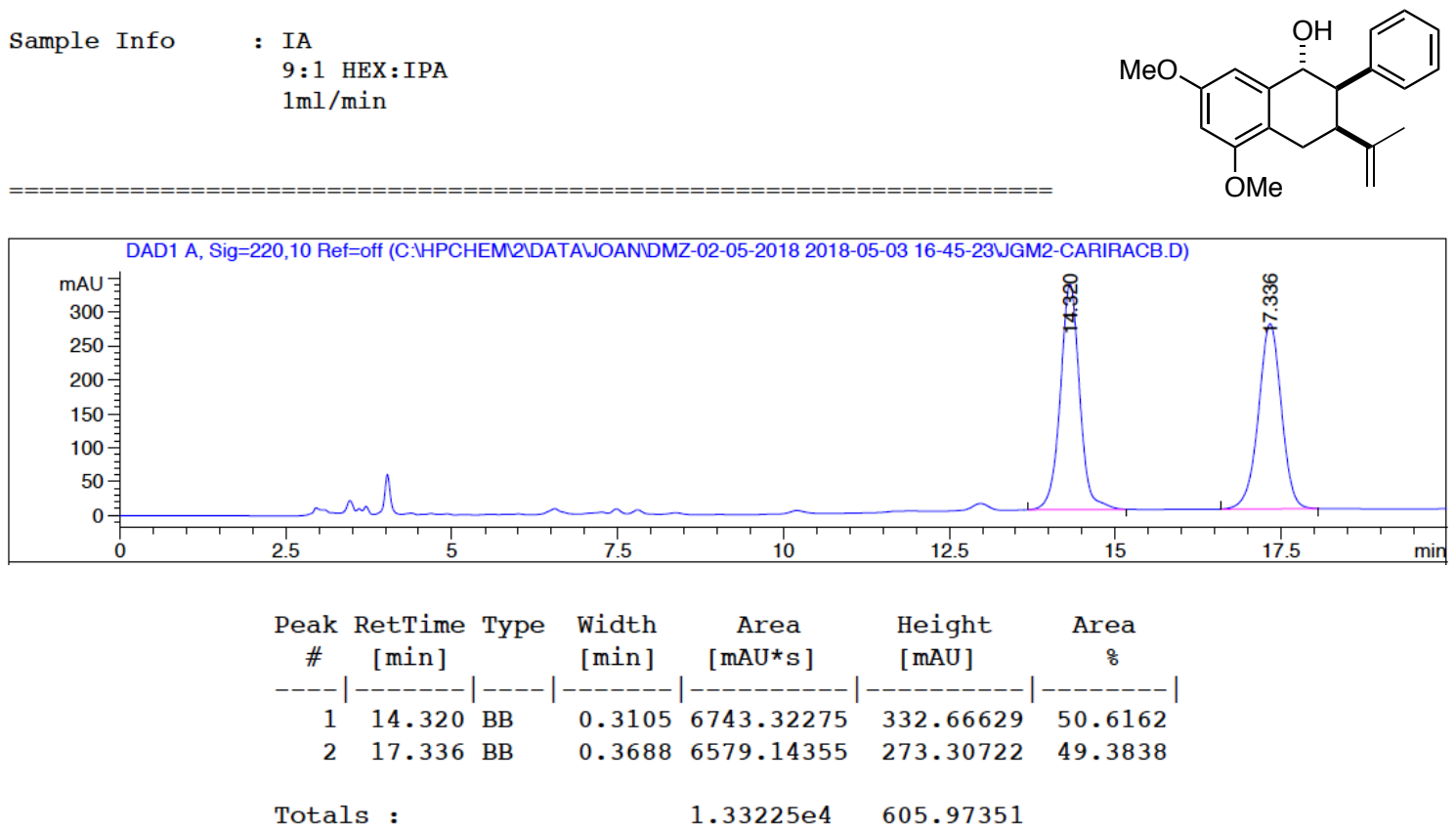

Chromatogram of enantioenriched Carexane I
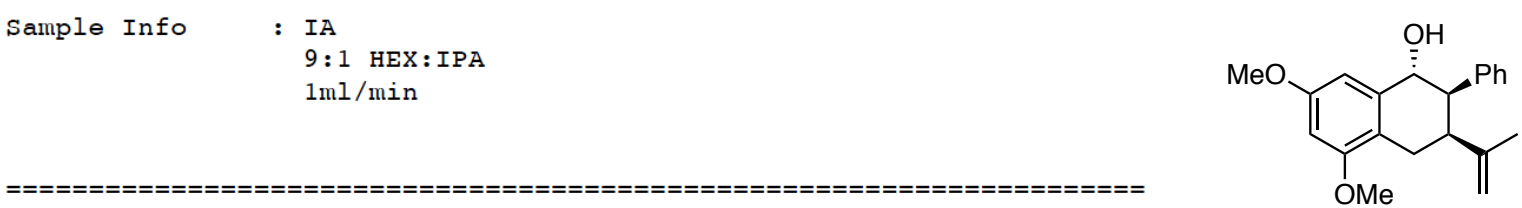

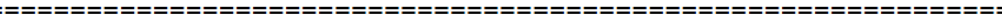

DAD1 A, Sig=220,10 Ref=off (C:UHPCHEM2IDATAWOANDMZ-02-05-2018 2018-05-03 16-45-23WGM2-226.D)

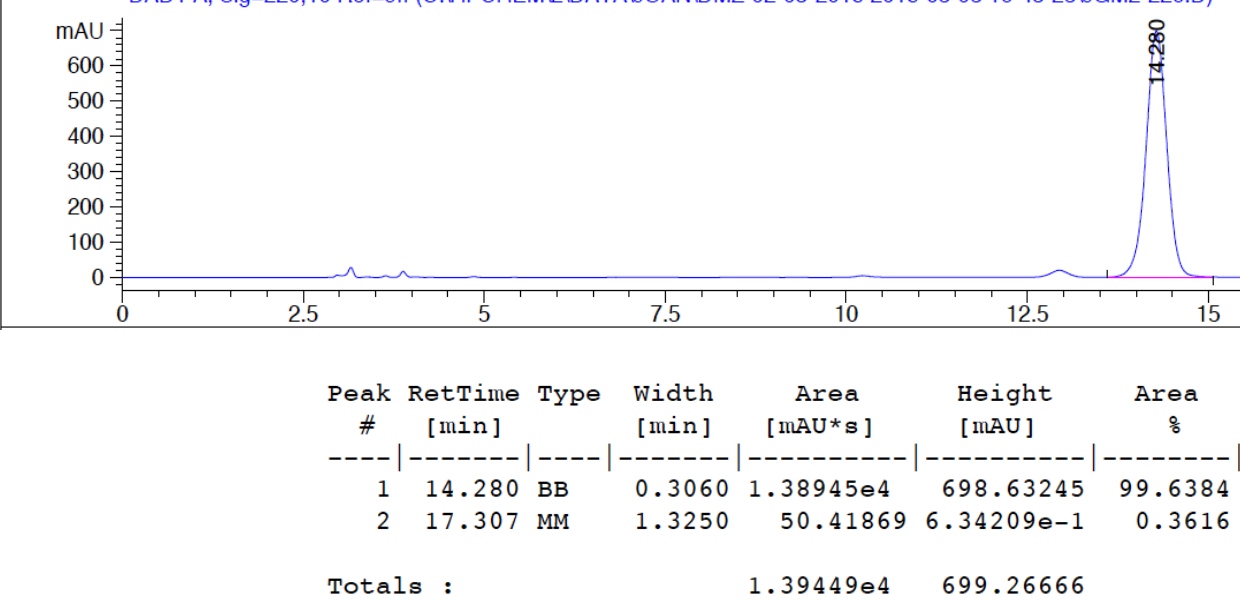


Chromatogram of racemic Carexane $\mathrm{P}$
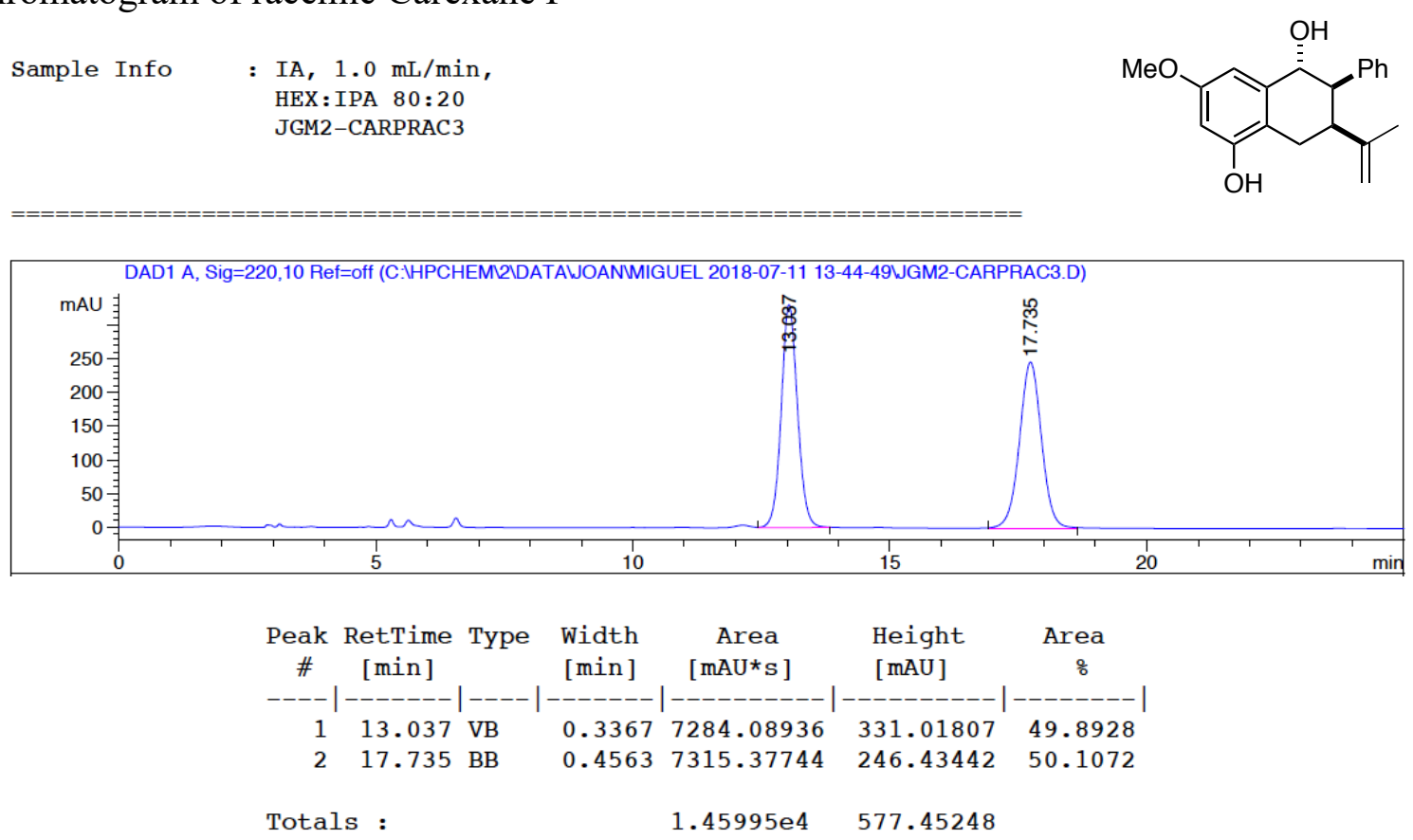

Chromatogram of enantioenriched Carexane $\mathrm{P}$

Sample Info: IA, $1.0 \mathrm{~mL} / \mathrm{min}$,
HEX:IPA $80: 20$

JGM $2-C A R P 3$
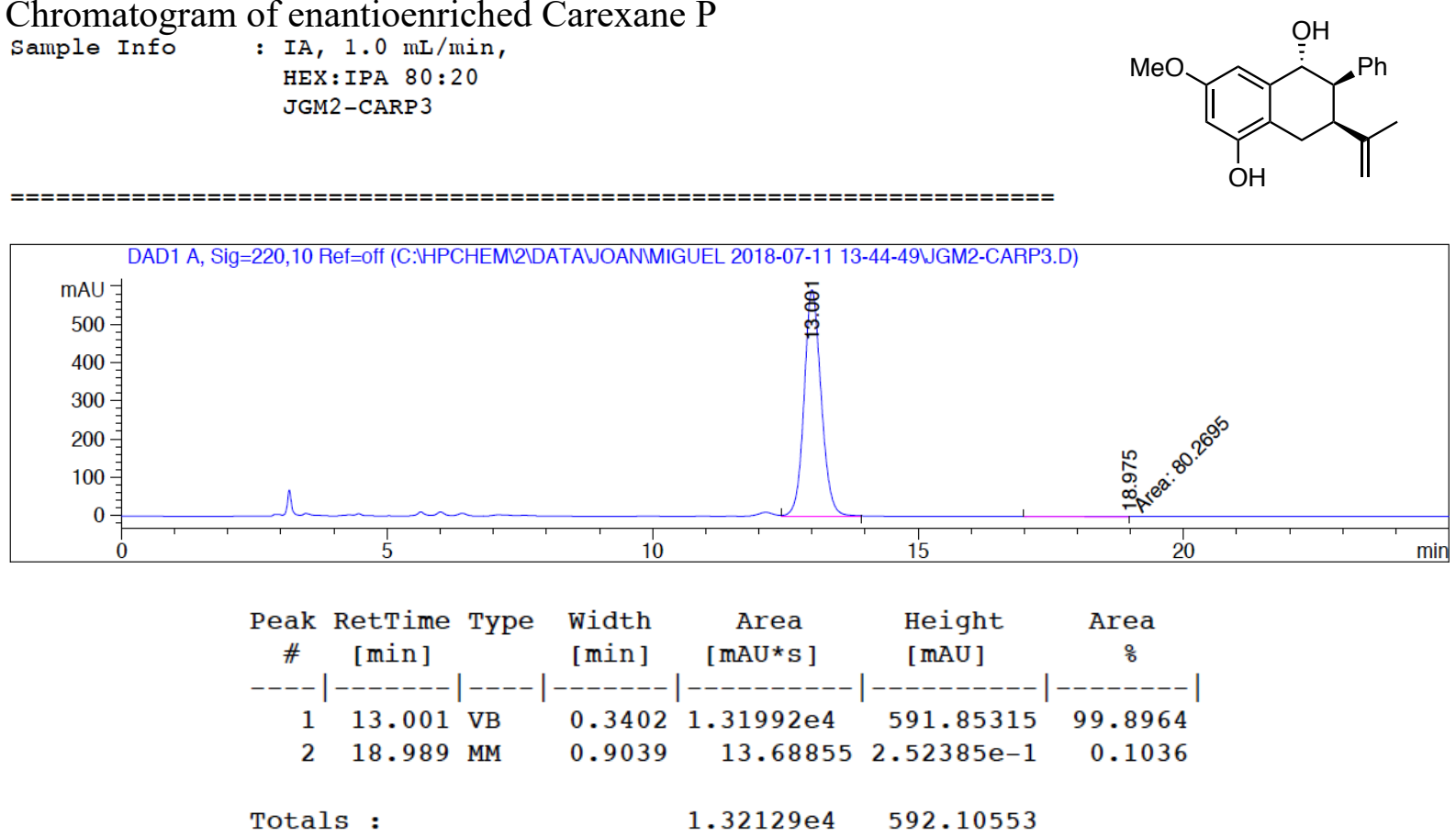
Chromatogram of racemic Carexane $\mathrm{O}$
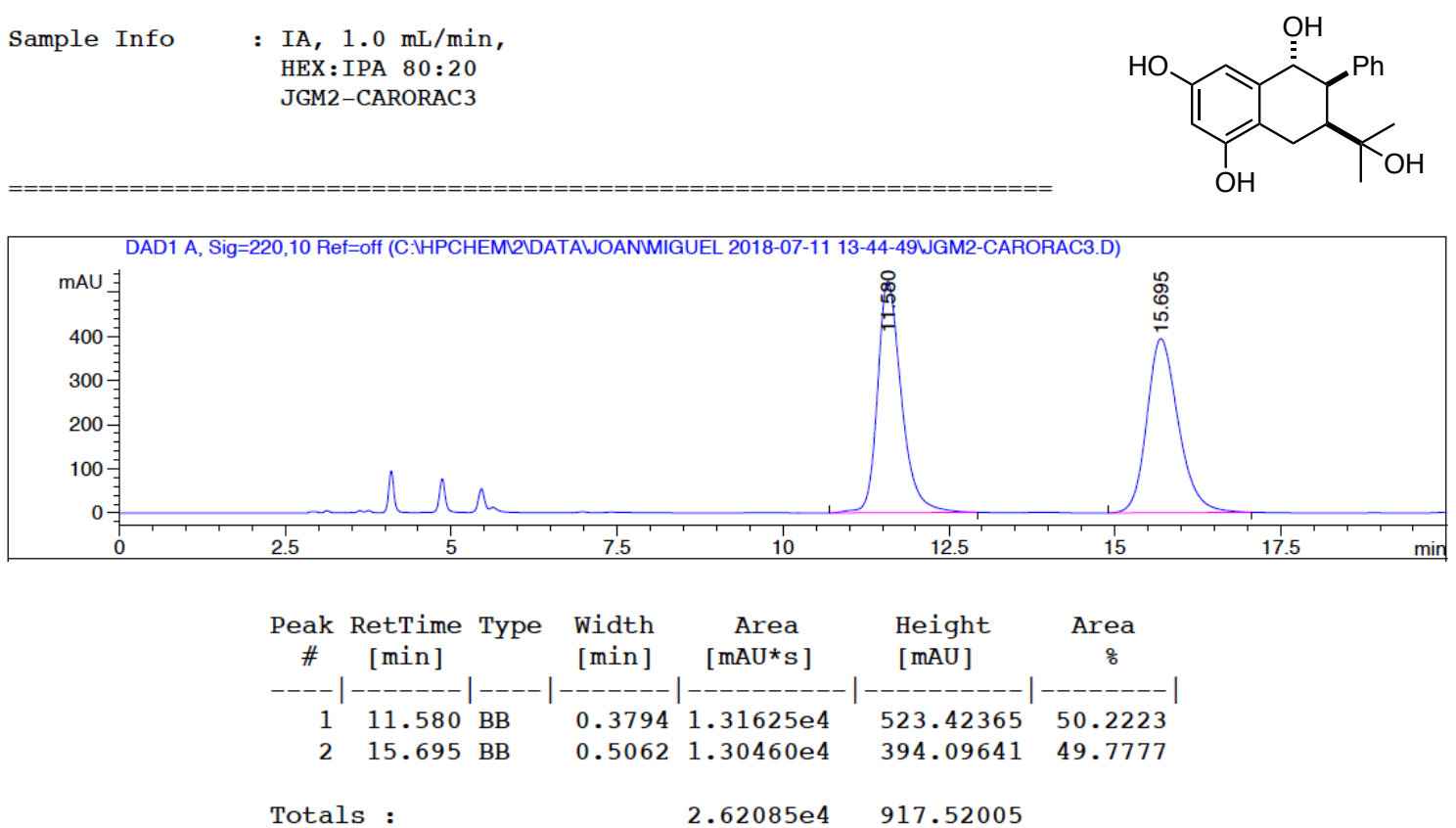

Chromatogram of enantioenriched Carexane O
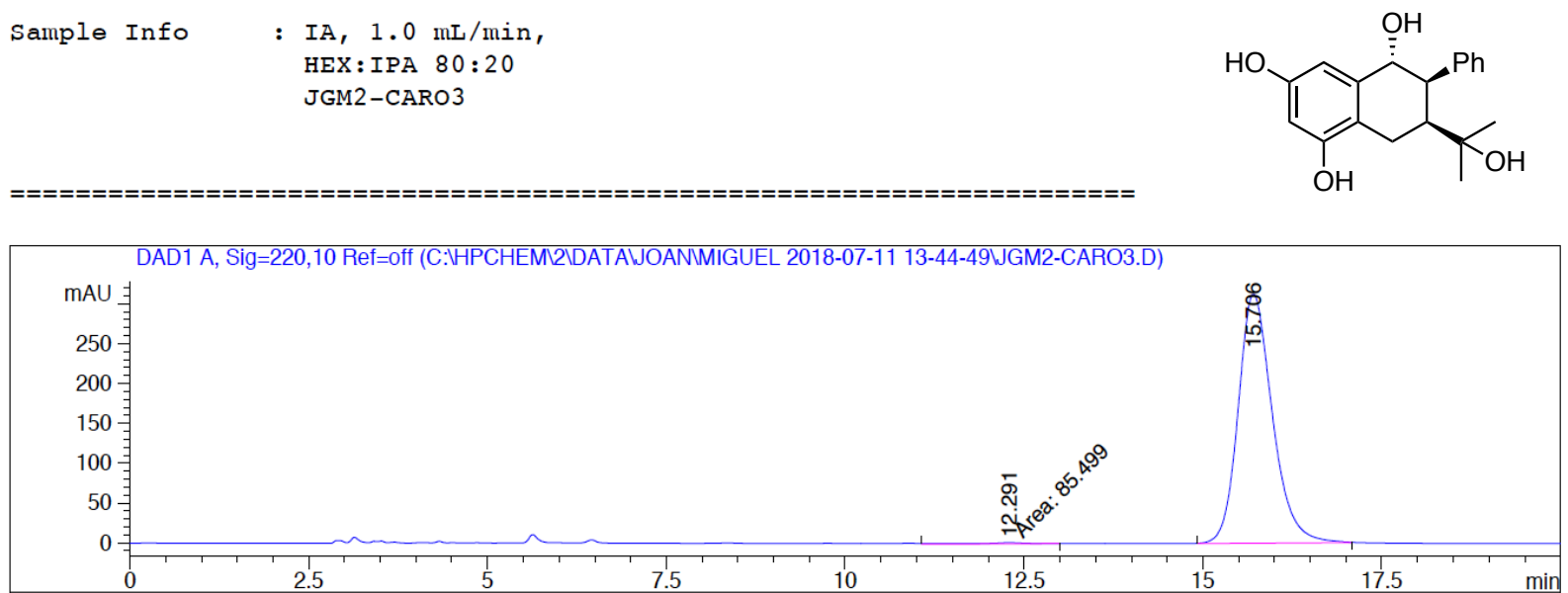

\begin{tabular}{|c|c|c|c|c|c|c|}
\hline $\begin{array}{c}\text { Peak } \\
\#\end{array}$ & $\begin{array}{c}\text { RetTime } \\
\text { [min] }\end{array}$ & Type & $\begin{array}{l}\text { Width } \\
\text { [min] }\end{array}$ & $\begin{array}{c}\text { Area } \\
{[\mathrm{mAU} * \mathbf{s}]}\end{array}$ & $\begin{array}{l}\text { Height } \\
\text { [mAU ] }\end{array}$ & $\begin{array}{c}\text { Area } \\
\%\end{array}$ \\
\hline-- & -- & & ------- & $|----------|$ & ---------- & -----ー-- \\
\hline 1 & 12.291 & MM & 0.9085 & 85.49901 & 1.56851 & 0.8147 \\
\hline 2 & 15.706 & BB & 0.5083 & $1.04085 e 4$ & 312.69321 & 99.1853 \\
\hline ota & Is: & & & $1.04940 e 4$ & 314.26171 & \\
\hline
\end{tabular}




\section{CD spectra}

The CD-spectra have been measured in $\mathrm{MeCN}$ at the given concentrations. The intensities are given as $\Delta \varepsilon$.
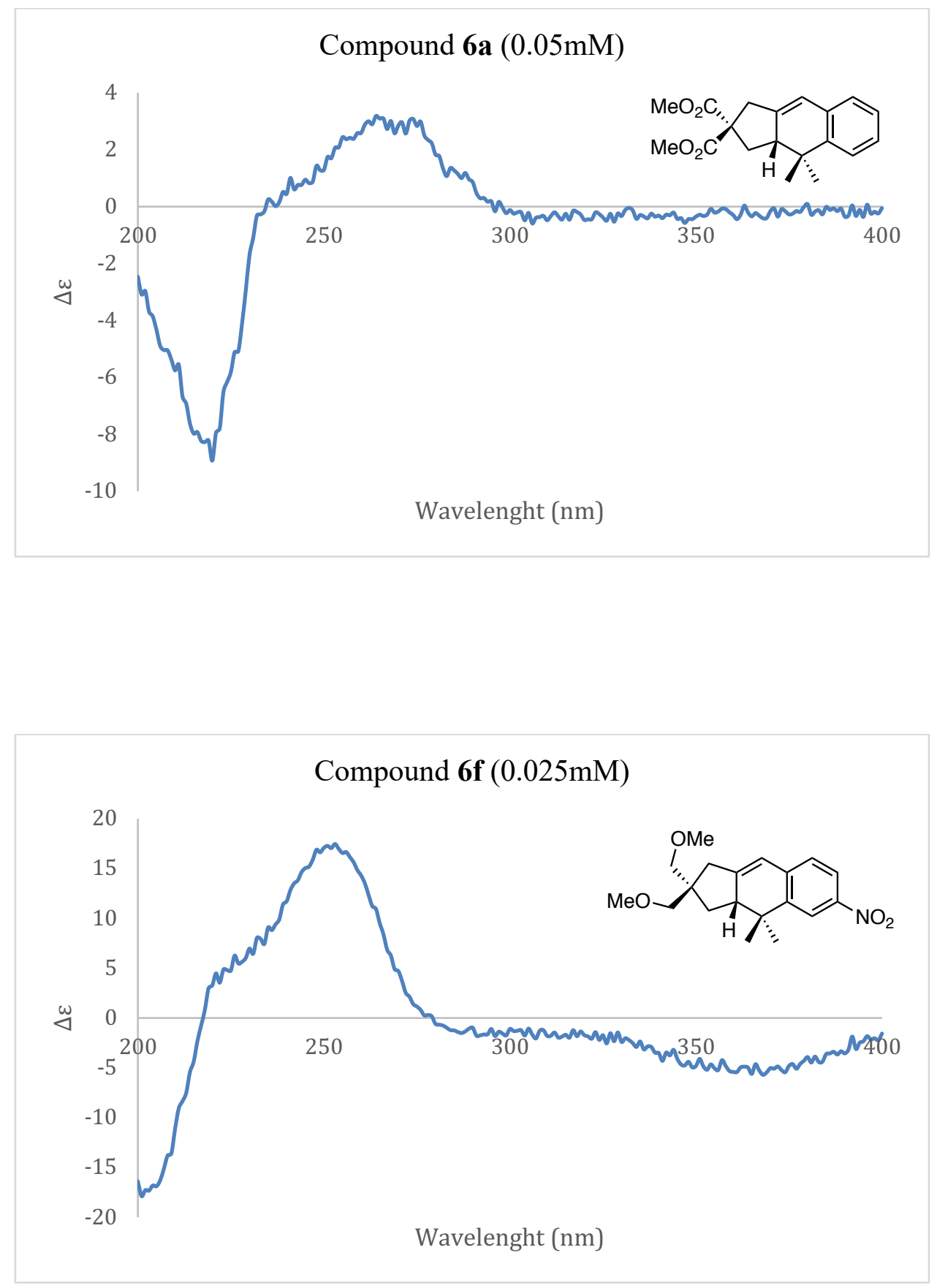

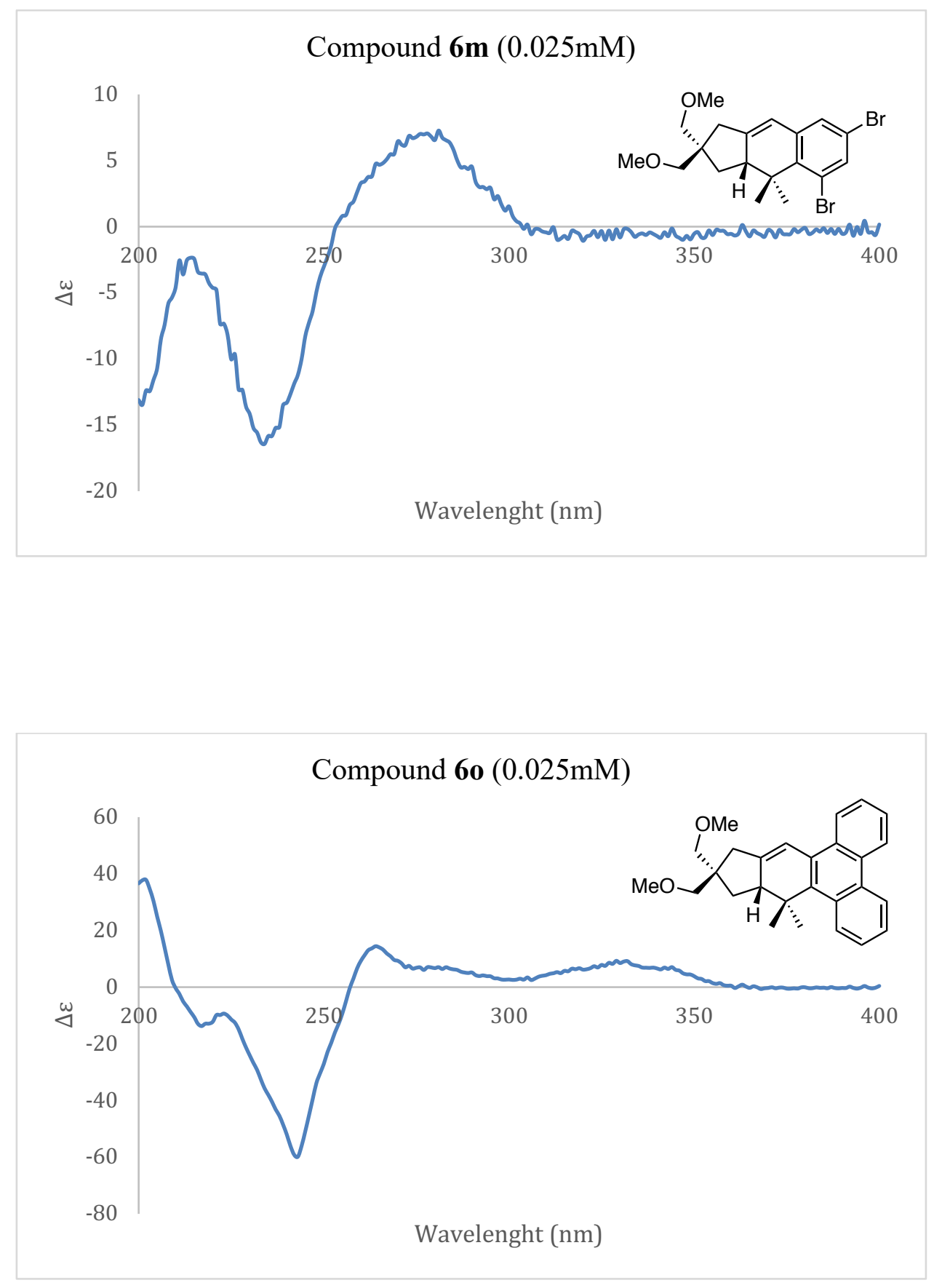

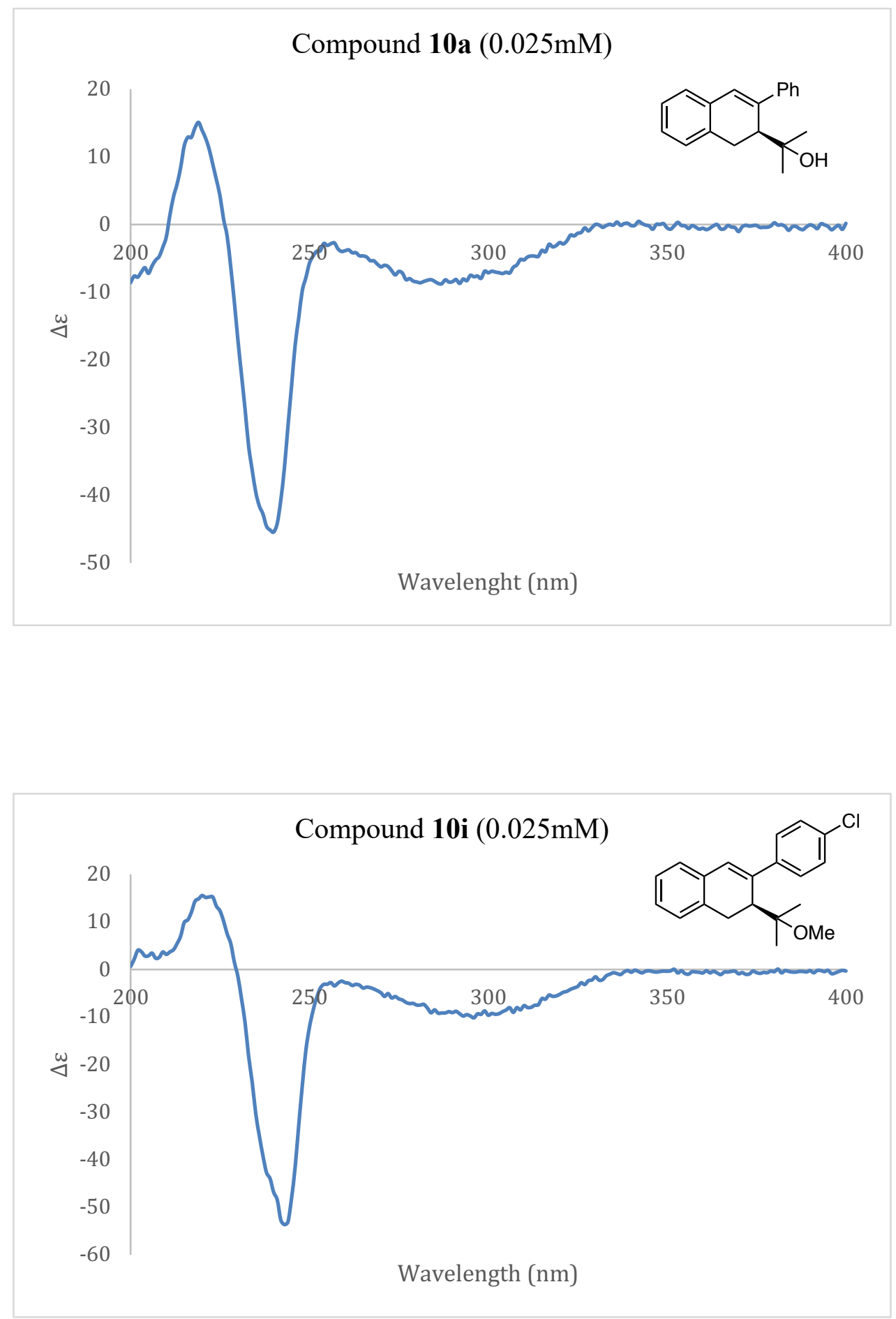

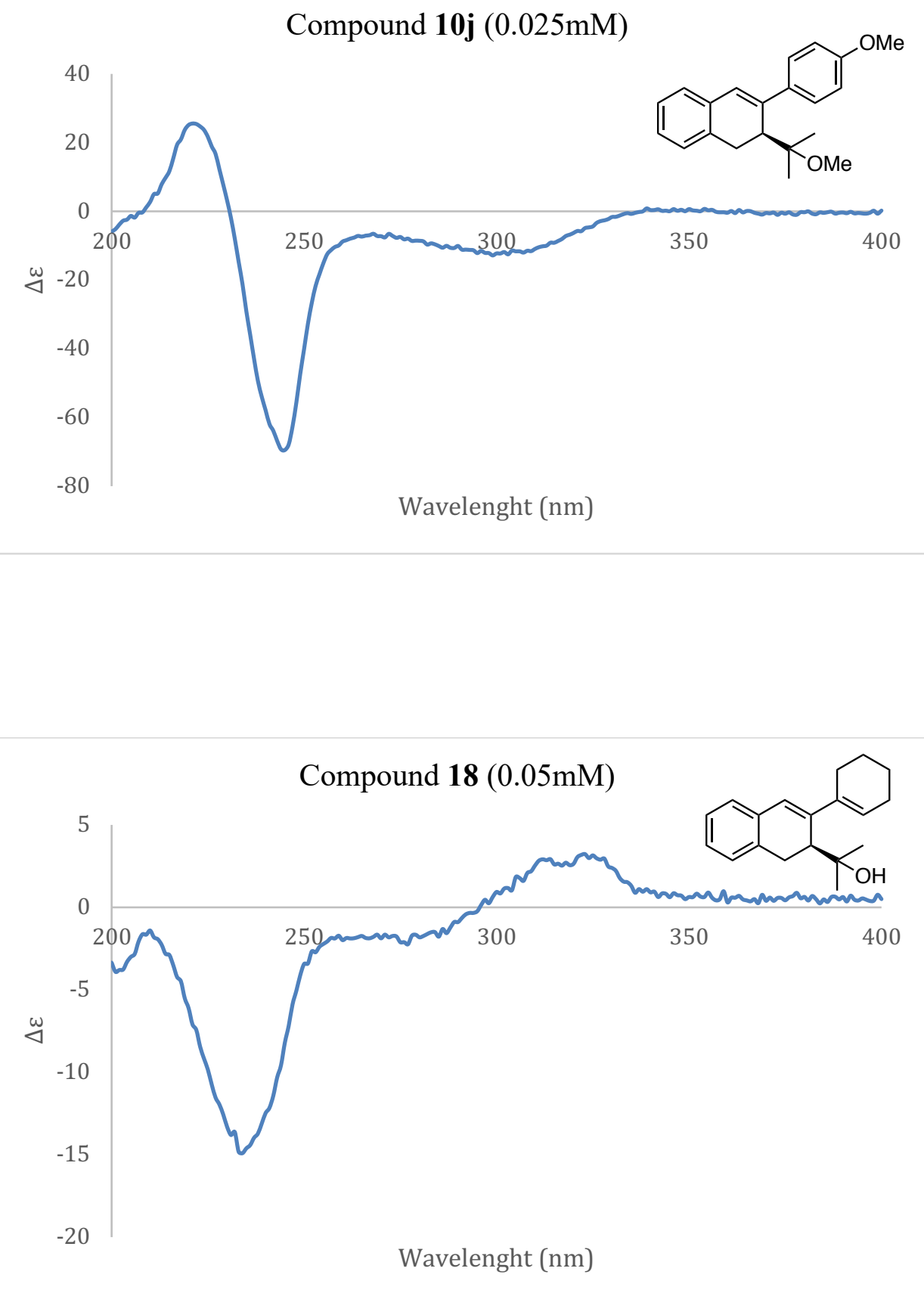
${ }^{1}$ Barker, C. A.; Zeng, X.; Bettington, S.; Batsanov, A. S.; Bryce, M. R.; Beeby. A. Porphyrin, Phtalocyanine and Porphyrazine Derivatives with Multifluorenyl Substituents as Efficient Deep-Red Emitters. Chem. Eur. J. 2007, 13, 6710-6717.

${ }^{2}$ The procedure was adapted from reported procedures: a) Kirillova, M. S.; Muratore, M. E.; Dorel, R.; Echavarren, A. M. Concise Total Synthesis of Lundurines A-C Enabled by Gold Catalysis and a Homodienyl Retro-Ene/Ene Isomerization. J. Am. Chem. Soc. 2016, 138, 3671-3674; b) Štambaský, J.; Malkov, A. V.; Kočovský, P. Synthesis of Enantiopure 1-Arylprop-2-en-1-ols and Their tert-Butyl Carbonates. J. Org. Chem. 2008, 73, 9148-9150.

${ }^{3}$ Kemppainen, E. K.; Sahoo, G.; Valkonen, A.; Pihko, P. M. Mukaiyama-Michael Reactions with Acrolein and Methacrolein: A Catalytic Enantioselective Synthesis of the C17-C28 Fragment of Pectenotoxins. Org. Lett. 2012, 14, 1086-1089.

${ }^{4}$ Surendra, K.; Corey, E. J. Diiodoindium(III) Cation, $\operatorname{InI}_{2}{ }^{+}$, a Potent Yneophile. Generation and Application to Cationic Cyclization by Selective $\pi$-Activation of C $\equiv$ C. J. Am. Chem. Soc. 2014, 136, 10918-10920.

${ }^{5}$ Benedetti, E.; Simonneau, A.; Hours, A.; Amouri, H.; Penoni, A.; Palmisano, G.; Malacria, M.; Goddard, J.; Fensterbank, L. (Pentamethylcyclopentadienyl)Iridium Dichloride Dimer $\left\{\left[\operatorname{IrCp} * \mathrm{Cl}_{2}\right]_{2}\right\}$ : A Novel Efficient Catalyst for the Cycloisomerizations of Homopropargylic Diols and N-Tethered Enynes. Adv. Synth. Catal. 2011, 353, 1908-1912.

${ }^{6}$ Nieto-Oberhuber, C.; López, S.; Muñoz, M. P.; Jiménez-Núñez, E.; Buñuel, E.; Cárdenas, D. J.; Echavarren, A. M. Gold(I)-Catalyzed Intramolecular Cyclopropanation of Dienynes. Chem. Eur. J. 2006, 12, 1694-1702.

${ }^{7}$ Nieto-Oberhuber, C.; López, S.; Echavarren, A. M. Intramolecular [4+2] Cycloadditions of 1,3-Enynes or Arylalkynes with Alkenes with Highly Reactive Cationic Phosphine Au(I) Complexes. J. Am. Chem. Soc. 2005, 127, 6178-6179.

${ }^{8}$ Nieto-Oberhuber, C.; Pérez-Galán, P.; Herrero-Gómez, E.; Lauterbach, T.; Rodríguez, C.; López, S.; Bour, C.; Rosellón, A.; Cárdenas, D. J.; Echavarren, A. M. Gold(I)-Catalyzed Intramolecular [4+2] Cycloadditions of Arylalkynes or 1,3-Enynes with Alkenes: Scope an Mechanism. J. Am. Chem. Soc. 2008, 130, 269-279.

${ }^{9}$ Cabrera-Lobera, N.; Rodríguez-Salamanca, P.; Nieto-Carmona, J. C.; Buñuel, E.; Cárdenas, D. J. Iron-Catalyzed Hydroborylative Cyclization of 1,6-Enynes. Chem. Eur. J. 2018, 24, 784-788.

${ }^{10}$ Brissy, D.; Skander, M.; Jullien, H.; Retailleau, P.; Marinetti, A. Platinum(II) Catalysts for Highly Enantioselective 1,6-Enyne Cycloisomerizations. Synthetic, Structural, and Catalytic Studies. Org. Lett. 2009, $11,2137-2139$.

${ }^{11}$ Teller, H.; Corbet, M.; Mantilli, L.; Gopakumar, G.; Goddard, R.; Thiel, W.; Fürstner A. One-Point Binding Ligands for Asymmetric Gold Catalysis: Phosphoramidites with a TADDOL-Related but Acyclic Backbone. J. Am. Chem. Soc. 2012, 134, 15331-15342.

${ }^{12}$ Lin, A.; Zhang, Z.-W.; Yang, J. Iron-Catalyzed Reductive Cyclization of 1,6-Enynes. Org. Lett. 2014, 16, 386389.

${ }^{13}$ Zhang, P.-C.; Wang, Y.; Zhang, Z.-M.; Zhang, J. Gold(I)/Xiang-Phos-Catalyzed Asymmetric Intramolecular Cyclopropanation of Indanes and Trisubstituted Alkenes. Org. Lett. 2018, 20, 7049-7052.

${ }^{14}$ Liang, B.; Dai, M.; Chen, J.; Yang, Z. Copper-Free Sonogashira Coupling Reaction with $\mathrm{PdCl}_{2}$ in Water under Aerobic Conditions. J. Org. Chem. 2005, 70, 391-393.

${ }^{15}$ Watanabe, T.; Abe, H.; Mutoh, Y.; Saito, S. Ruthenium-Catalyzed Cycloisomerization of 2-Alkynylstyrenes via 1,2-Carbon Migration That Leads to Substituted Naphtalenes. Chem. Eur. J. 2018, 24, 11545-11549.

${ }^{16}$ Shaikh, A. C.; Ranade, D. S.; Rajamohanan, P. R.; Kulkarni, P. P.; Patil, N. T. Oxidative Intramolecular 1,2Amino-Oxygenation of Alkynes under $\mathrm{Au}^{\mathrm{I}} / \mathrm{Au}^{\mathrm{III}}$ Catalysis: Discovery of a Pyridinium-Oxazole Dyad as an Ionic Fluorophore. Angew. Chem. Int. Ed. 2017, 56, 757-761.

${ }^{17}$ Sanjuán, A. M.; Martínez, A.; García-García, P.; Fernández-Rodríguez, M. A.; Sanz, R. Gold(I)-catalyzed 6endo hydroxycyclization of 7-substituted-1,6-enynes. Beilstein J. Org. Chem. 2013, 9, 2242-2249.

${ }^{18}$ Xu, L.; Liu, Z.; Dong, W.; Song, J.; Miao, M.; Xu, J.; Ren, H. Copper-free arylation of 3,3-substituted allylic halides with triazene-softened aryl Grignard reagents. Org. Biomol. Chem. 2015, 13, 6333-6337.

${ }^{19}$ The procedure was adapted from a reported procedure: see ref. 17. 
${ }^{20}$ Truong, T.; Daugulis, O. Transition-Metal-Free Alkynylation of Aryl Chlorides. Org. Lett. 2011, 13, 41724175.

${ }^{21}$ Wagh, S. B.; Hsu, Y.-C.; Liu, R.-S. Two Distinct Cyclizations of 2-Propenyl-1-ethynyl Benzenes with Aryldiazo Esters Using Au and Rh/Au Catalysts Respectively. ACS Catal. 2016, 6, 7160-7166.

${ }^{22}$ The procedure was adapted from a reported procedure: Yao, T.; Larock, R. C. Synthesis of Isocoumarins and $\alpha$-Pyrones via Electrophilic Cyclization. J. Org. Chem. 2003, 68, 5936-5942.

${ }^{23}$ The procedure was adapted from a reported procedure: Tolonai, G. L.; Petho B.; Králl, P.; Novák, Z. PalladiumCatalyzed Methoxylation of Aromatic Chlorides with Borate Salts. Adv. Synth. Catal. 2014, 356, 125-129.

${ }^{24}$ Holstein, P. M.; Holstein, J. J.; Escudero-Adán, E. C.; Baudoin, O.; Echavarren, A. M. Ferrocene derivatives of liquid chiral molecules allow assignment of absolute configuration by X-ray crystallography. Tetrahedron: Asymmetry 2017, 28, 1321-1329.

${ }^{25}$ Fiorentino, A.; D’Abrosca, B.; Pacifico, S.; Natale, A.; Monaco, P. Structures of bioactive carexanes from the roots of Carex distachya Desf. Phytochemistry 2006, 67, 971-977.

${ }^{26}$ Fiorentino, A.; D'Abrosca, B.; Pacifico, S.; Iacovino, R.; Izzo, A.; Uzzo, P.; Russo, A.; Di Blasio, B.; Monaco, P. Carexanes from Carex distachya Desf.: revised stereochemistry and characterization of four novel polyhydroxylated prenylstilbenes. Tetrahedron 2008, 64, 7782-7786.

${ }^{27}$ a) Milo, A.; Elizabeth, N. B.; Sigman, M. S. Interrogating selectivity in catalysis using molecula vibrations. Nature 2014, 507, 210-215; b) Santiago, C. B.; Milo, A.; Sigman, M. S. Developing a Modern Approach To Account for Steric Effects in Hammett-Type Correlations. J. Am. Chem. Soc. 2016, 138, 13424-13430.

${ }^{28}$ a) Herrero-Gómez, E.; Nieto-Oberhuber, C.; López, S.; Benet-Buchholz, J.; Echavarren, A. M. Cationic $\eta^{1} / \eta^{2}-$ Gold(I) Complexes of Simple Arenes. Angew. Chem. Int. Ed. 2006, 45, 5455-5459; b) Pérez-Galán, P.; Delpont, N.; Herrero-Gómez, E.; Maseras, F.; Echavarren, A. M. Metal-Arene Interactions in Dialkylbiarylphosphane Complexes of Copper, Silver, and Gold. Chem. Eur. J. 2010, 16, 5324-5332.

${ }^{29}$ Gaussian 09, Revision B.1, Frisch, M. J., Trucks, G. W., Schlegel, H. B., Scuseria, G. E., Robb, M. A., Cheeseman, J. R., Scalmani, G., Barone, V., Mennucci, B., Petersson, G. A., Nakatsuji, H., Caricato, M., Li, X., Hratchian, H. P., Izmaylov, A. F., Bloino, J., Zheng, G., Sonnenberg, J. L., Hada, M., Ehara, M., Toyota, K., Fukuda, R., Hasegawa, J., Ishida, M., Nakajima, T., Honda, Y., Kitao, O., Nakai, H., Vreven, T., Montgomery, J. A., Peralta, Jr. J. E., Ogliaro, F., Bearpark, M., Heyd, J. J., Brothers, E., Kudin, K. N., Staroverov, V. N., Kobayashi, R., Normand, J., Raghavachari, K., Rendell, A., Burant, J. C., Iyengar, S. S., Tomasi, J., Cossi, M., Rega, N., Millam, J. M., Klene, M., Knox, J. E., Cross, J. B., Bakken, V., Adamo, C., Jaramillo, J., Gomperts, R., Stratmann, R. E., Yazyev, O., Austin, A. J., Cammi, R., Pomelli, C., Ochterski, J. W., Martin, R.L., Morokuma, K., Zakrzewski, V. G., Voth, G. A., Salvador, P., Dannenberg, J. J., Dapprich, S., Daniels, A. D., Farkas, Ö., Foresman, J. B., Ortiz, J. V., Cioslowski, J., Fox, D. J. Gaussian, Inc., Wallingford CT 2009.

${ }^{30}$ a) Kang, R.; Chen, H.; Shaik, S.; Yao, J. Assessment of Theoretical Methods for Complexes of Gold(I) and Gold(III) with Unsaturated Aliphatic Hydrocarbon: Which Density Functional Should We Choose? J. Chem. Theory Comput. 2011, 7, 4002-4011; b) Ciancaleoni, G.; Rampino, S.; Zuccaccia, D.; Tarantelli, F.; Belanzoni, P.; Belpassi, L. An ab Initio Benchmark and DFT Validation Study on Gold(I)-Catalyzed Hydroamination of Alkynes. J. Chem. Theory Comput. 2014, 10, 1021-1034; c) García-Morales, C.; Ranieri, B.; Escofet, I.; López-Suarez, L.; Obradors, C.; Konovalov, A. I.; Echavarren, A. M. Enantioselective Synthesis of Cyclobutenes by Intermolecular [2+2] Cycloaddition with Non- $C_{2}$ Symmetric Digold Catalysts. J. Am. Chem. Soc. 2017, 139, 13628-13631.

${ }^{31}$ Andrae, D.; Häußermann, U.; Dolg, M.; Stoll, H.; Preuß, H. Energy-adjusted ab initio pseudopotentials for the second and third row transition elements. Theor. Chim. Acta. 1990, 77, 123-141.

${ }^{32}$ Hehre, W. J.; Ditchfield, R.; Pople, J. A. Self-Consistent Molecular Orbital Methods. XII. Further Extensions of Gaussian-Type Basis Sets for Use in Molecular Orbital Studies of Organic Molecules. J. Chem. Phys. 1972, $56,2257-2261$.

${ }^{33}$ Cancès, E.; Mennucci, B.; Tomasi, J. A new integral equation formalism for the polarizable continuum model: Theoretical background and applications to isotropic and anisotropic dielectrics. J. Chem. Phys. 1997, 107, 3032-3041.

${ }^{34}$ Gonzalez, C.; Schlegel, H. B. Reaction path following in mass-weighted internal coordinates. J. Phys. Chem. 1990, 94, 5523-5527. 
35 a) Johnson, E. R.; Keinan, S.; Mori-Sánchez, P.; Contreras-García, J.; Cohen, A. J.; Yang, W. Revealing Noncovalent Interactions. J. Am. Chem. Soc. 2010, 132, 6498-6506; b) Contreras-García, J.; Johnson, E. R.; Keinan, S.; Chaudret, R.; Piquemal, J.-P.; Beratan, D. N.; Yang, W. NCIPLOT: A Program for Plotting Noncovalent Interaction Regions. J. Chem. Theory Comput. 2011, 7, 625-632.

${ }^{36}$ Humphrey, W.; Dalke, A.; Schulten, K. VMD: Visual molecular dynamics. J. Mol. Graphics 1996, 14, 33-38.

${ }^{37}$ Escudero-Adán, E. C.; Benet-Buchholz, J.; Ballester, P. The use of Mo K $\alpha$ radiation in the assignment of the absolute configuration of light-atom molecules; the importance of high-resolution data. Acta Cryst. B 2014, $70,660-668$. 